\title{
EXPLORING DISCRETE DYNAMICS
}

The DDLab Manual 
Dr. Andy Wuensche is a former architect, the inventor of reverse algorithms for cellular automata and discrete dynamical networks, the author of many academic publications including the book The Global Dynamics of Cellular Automata (with Mike Lesser) in the Santa Fe Institute's Studies in the Sciences of Complexity. He created and continues to develop the classic software Discrete Dynamics Laboratory (DDLab), widely used in research and education. He has lectured at universities and conferences throughout the world. He is an independent academic, a visiting Professor at the International Center of Unconventional Computing, UWE, Bristol, and a visiting research fellow at the Dept. of Informatics, University of Sussex.

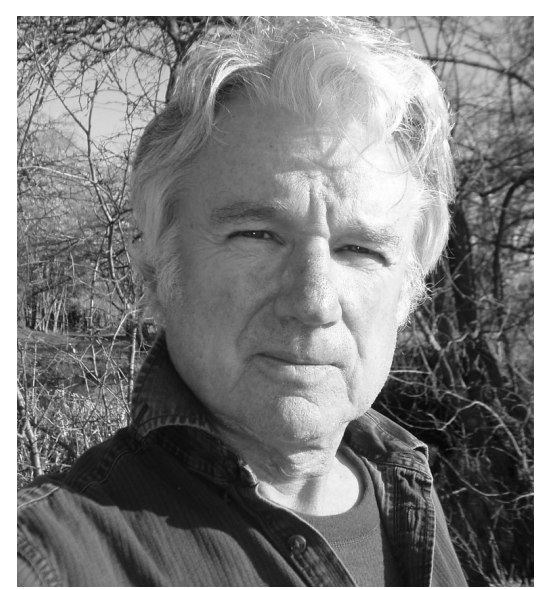




\section{Andrew Wuensche}

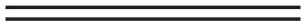

\section{EXPLORING DISCRETE DYNAMICS}

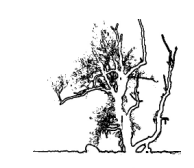

Luniver Press

2011 
Published by Luniver Press

Frome BA11 3EY United Kingdom

British Library Cataloguing-in-Publication Data

A catalogue record for this book is available from the British Library

\section{EXPLORING DISCRETE DYNAMICS}

Copyright (c) Luniver Press 2011

Cover images created with DDLab: The background is the space-time pattern of a one-dimensional cellular automaton, $v 8 k 3, n=800,490$ time-steps, from the kcode sample (index 17) described in figure 33.5. The single basin of attraction on the front cover is from a one-dimensional cellular automaton, $v 5 k 2, n=6$, seed(dec)00444, rcode(hex)0380232281c884313508.

All rights reserved. This book, or parts thereof, may not be reproduced in any form or by any means, electronic or mechanical, including photocopying, recording or by any information storage and retrieval system, without permission in writing from the copyright holder.

ISBN-10: 1-905986-31-9

ISBN-13: 978-1-905986-31-6

While every attempt is made to ensure that the information in this publication is correct, no liability can be accepted by the authors or publishers for loss, damage or injury caused by any errors in, or omission from, the information given. 
To Stephanie 



\section{Chapters}

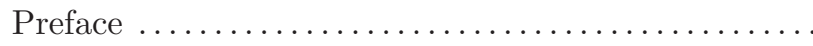

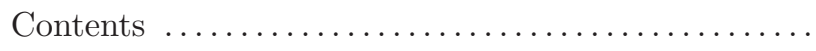

ix

List of Figures $\ldots \ldots \ldots \ldots \ldots \ldots \ldots \ldots \ldots \ldots \ldots . \ldots \ldots$

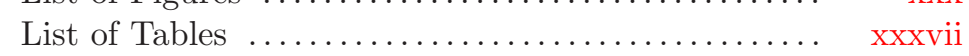

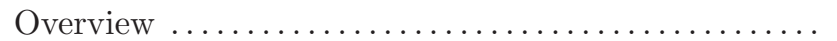

2 Summary of DDLab functions $\ldots \ldots \ldots \ldots \ldots \ldots \ldots$

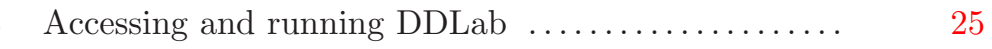

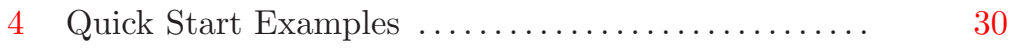

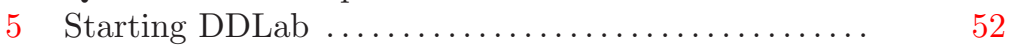

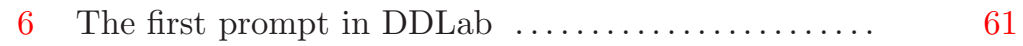

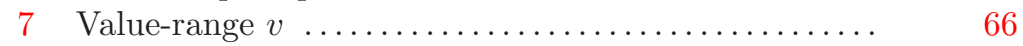

8 1d network size $n$, or range- $n \ldots \ldots \ldots \ldots \ldots \ldots \ldots \ldots$

9 Neighborhood $k$, or mixed $k \ldots \ldots \ldots \ldots \ldots \ldots \ldots \ldots$

10 The local neighborhood, and network geometry .... 82

11 Setting the wiring, quick settings ........... 88

12 Setting special wiring $\ldots \ldots \ldots \ldots \ldots \ldots \ldots \ldots \ldots \ldots \ldots \ldots \ldots$

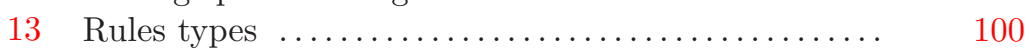

14 Rulemix options ...................... 110

15 Setting Canalyzation in a random rulemix ........ 124

16 Setting a singe rule .................... 131

17 Reviewing network architecture .............. 156

18 Transforming rules $\ldots \ldots \ldots \ldots \ldots \ldots \ldots \ldots \ldots \ldots$

19 File/print network architecture ............... 194

20 The network-graph, and attractor jump-graph ..... 204

21 The Seed or initial state .................. 227

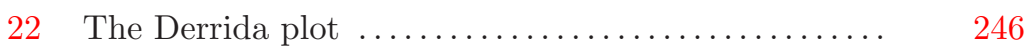

23 Graphic conventions for attractor basins ......... 252

24 Output parameters for attractor basins ......... 258

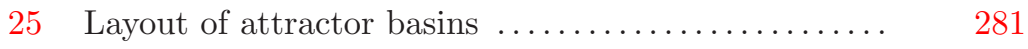

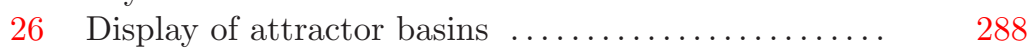

27 Pausing attractor basins, and data ........... 299

28 Mutation of attractor basins ............... 314

29 Final options for attractor basins ............ 322

30 Drawing attractor basins, and changes on-the-fly ... 343

31 Output parameters for space-time patterns ....... 351

32 Drawing space-time patterns, and changes on-the-fly 386

33 Classifying rule space .................. 437

34 Learning, forgetting, and highlighting .......... 459

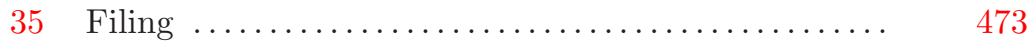

36 Vector PostScript capture of DDLab output ....... 479

37 Glossary ............................. 481

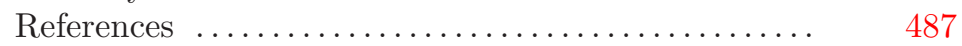

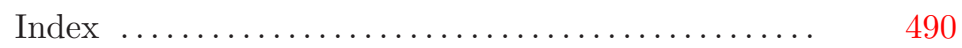





\section{Preface}

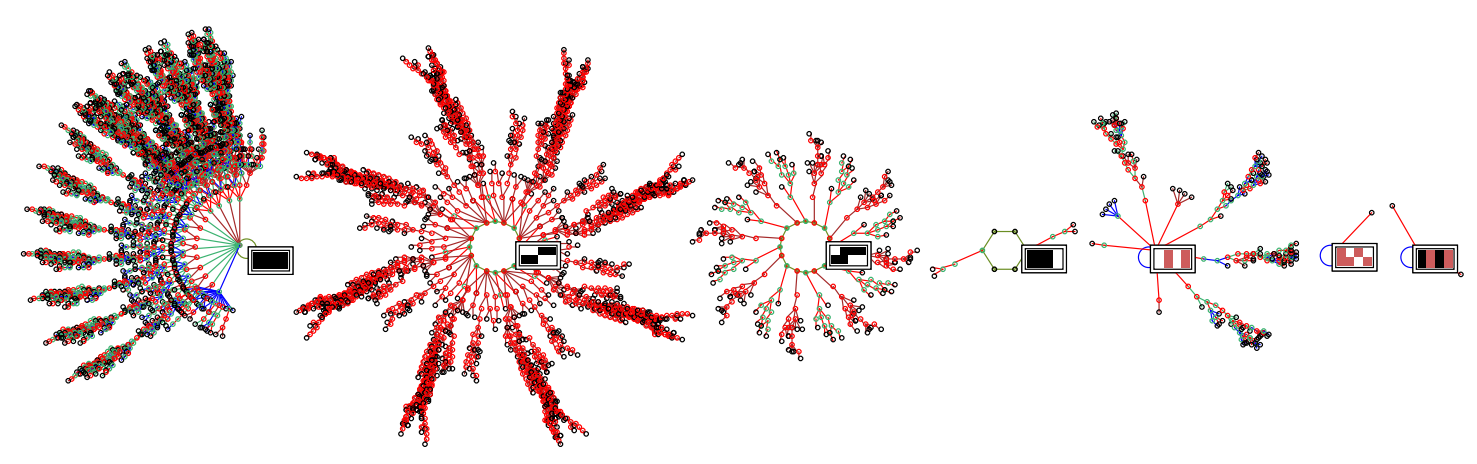

Figure 1: The basin of attraction field of a 3-value Cellular Automaton, $v=3, k=3, n=8$. There are in fact 17 basins, but equivalent basins have been suppressed leaving just the 7 prototypes. One attractor state is shown for each basin. The rcode rule-table is 020120122021201201011011022.

\section{Dynamics on networks, and of networks}

Networks of sparsely inter-connected elements with discrete values and updating in parallel are central to a wide range of natural and artificial phenomena drawn from many areas of science: from physics to biology to cognition, to social science and economics; to parallel computation, emergence, self-organization and artificial life; to complex systems of all kinds.

Dynamics upon or on a network are its changing patterns of cell-states, while the network "architecture", its connections and logic, remain fixed. DDLab studies these changing patterns and how they "fall" along trajectories into various attractors, revealing the fine detail of basins of attraction. DDLab also deploys flexible methods for constructing and changing the network's architecture - the dynamics of the network - which of course has consequences for the dynamics on the network.

To give an oversimplified example, take a network of neurons in a brain; the pattern of firing and synaptic activity is the dynamics on the neural network sustaining automatic behavior, whereas network plasticity, changes in synaptic micro-circuitry and connections between neurons, is the dynamics of the network supporting learning and lifetime adaptation.

Networks like this are sometimes called "decision-making" because each element "decides" how to change based on the signals arriving from other elements along its input connections, mediated by its own internal logic, and each element provides outputs to other elements making 
their "decisions". The many decisions make a collective by simultaneous iteration and massive feedback creating the possibility of all sorts of "emergent" dynamic patterns.

We can unravel the dynamics and gain some insight into the basic principles by studying idealized networks were all aspects are discretized: value, space, and time, where dynamics on the network are simplified into discrete time-steps updating synchronously, in parallel, or in some partial order.

Cellular automata and random Boolean networks are the most common idealized dynamical networks that have been studied, and together with their applications support a very large body of literature. Applications include complex systems and emergent patterns[25, 23, 24], collision-based computing[1], neural networks, memory and learning[28], genetic regulatory networks[15, 20, 11, 27, 30], and theories of networks in general. The idealized networks are interesting in themselves as mathematical/physical/dynamical systems. Because the dynamics resist analysis by classical mathematics, computer simulation, a kind of experimental mathematics, is unavoidable.

\section{DDLab software}

DDLab is able to construct these networks and investigate many aspects of their dynamical behavior. The software utilizes interactive graphics and other methods to study cellular automata (CA), random Boolean networks (RBN)[15], and the general case of discrete dynamical networks (DDN), where the "Boolean" attribute is extended to multi-value. Together with hybrid networks, and networks of interacting sub-networks, there is a huge diversity of behavior to be explored mostly terra incognita.

There are currently compiled versions of DDLab for Linux, Unix, Irix, Mac, Cygwin and DOS. The code is written in C, and is open source "free software" under the GNU General Public License. As well as generating space-time patterns in one, two or three dimensions, DDLab generates attractor basins, graphs that link network states according to their transitions, representing "global" dynamics[25], analogous to Poincaré's "phase portrait" which provided powerful insights in continuous dynamical systems. A key insight is that the dynamics on networks converge, thus fall into a number of basins of attraction. The state transition graphs, consisting of trees rooted on attractor cycles, represent a network's memory, its ability to hierarchically categorize its patterns of activation (state-space) as a function of the precise network architecture[27].

Relating this to space-time patterns in CA, high convergence implies order, low convergence implies disorder or chaos[25]. The most interesting emergent structures occur at the transition, sometimes called the "edge of chaos" [17, 32].

DDLab is an applications program, it does not require writing code. Network parameters and the graphics presentation can be flexibly set, reviewed and altered, including changes "on-the-fly". A wide variety of data, measures, analysis, and statistics are available. DDLab produces graphic images of all aspects of the data, the networks, attractor basins, and moving images of space-time patterns. Many of the images can be captured as PostScript files for publication. This book provides a comprehensive operating manual for the current multi-value version of DDLab at the time of publication.

\section{Update: multi-value, totalistic rules, vector graphics}

Together with many other new features, improvements and bug fixes, the significant updates from the old binary version of DDLab and its online manual[34] are as follows: 
- DDLab is generalized for multi-value logic. Up to 8 values (or cell-states, or colors) are now possible, instead of just Boolean logic (two values: 0,1 ). Of course, with just 2 values selected, DDLab behaves as before[34, 37]. The multi-value version opens up new possibilities for dynamical behavior and modelling.

- DDLab can be constrained to run "forward-only" for various types of totalistic rules which depend on just the totals of each value in a neighborhood, including outer-totalistic rules and reaction-diffusion rules. This reduces the relative sizes of rule-tables allowing larger neighborhoods, with an upper limit of 25 instead of 13, at the cost of disabling basin of attraction functions. In $2 \mathrm{~d}$ the neighborhoods are predefined to make hexagonal as well square lattices. Many interesting cellular automaton rules with "Life-like" and other complex dynamics can be found in totalistic multi-value rule-space, in $3 \mathrm{~d}$ as well as $2 \mathrm{~d}[39,40]$.

- Most of DDLab's graphic output, including space-time patterns and attractor basins, the network-graph and the attractor jump-graph, can be captured as vector PostScript files, which is a preferred format for publication than the previously available bitmap images.

\section{Acknowledgements}

The precursor of DDLab was the Atlas software, included on diskette inside the back cover of The Global Dynamics of Cellular Automata, An Atlas of Basin of Attraction Fields of One-Dimensional Cellular Automata[25], by Andrew Wuensche and Mike Lesser, published in 1992 in the Santa Fe Institute's Studies in the Sciences of Complexity. The Atlas software was compiled in DOS with the operating instructions in one chapter of the book. The book and original software can be found at www.ddlab.org.

Many people have influenced DDLab by contributing ideas, suggesting new features, providing encouragement, criticism, and helping with programming. I reserve all the blame for its shortcomings. I would like to thank Mike Lesser, Grant Warrell, Crayton Walker, Chris Langton, Stuart Kauffman, Wentian Li, Pedro P.B. de Oliveira, Inman Harvey, Phil Husbands, Guillaume Barreau, Josh Smith, Raja Das, Christian Reidys, Brosl Hasslacher, Steve Harris, Simon Frazer, Burt Voorhees, John Myers, Roland Somogyi, Lee Altenberg, Andy Adamatzky, Mark Tilden, Rodney Douglas, Terry Bossomaier, Ed Coxon, Oskar Itzinger, Pietro di Fenizio, Pau Fernandez, Ricard Sole, Paolo Patelli, Jose Manuel Gomez Soto, Dave Burraston, Genaro Martnez, and many other friends and colleagues (to whom I apologize for not listing). Also DDLab users who have provided feedback, and researchers and staff at the Santa Fe Institute in the 1990s. 



\section{Contents}

Chapters vii

Preface $\quad$ ix

Contents $\quad$ xiii

List of figures $\quad$ xxx

List of tables $\quad$ xxxvii

1 Overview 1

1.1 Introduction . . . . . . . . . . . . . . . . . . . . . . . . 1

1.2 Source code and platforms . . . . . . . . . . . . . . . . . . . . . . . 2

1.3 Discrete dynamical networks . . . . . . . . . . . . . . . . . . . . . . . 3

1.4 Space-time patterns and attractor basins . . . . . . . . . . . . . . . . . . . . . . . . . . . . . . . . . . . . .

1.4.1 Totalistic rules - forwards only . . . . . . . . . . . . . . . . 7

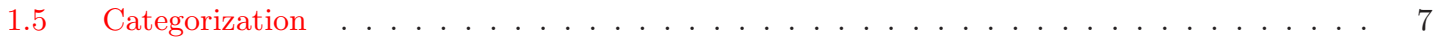

1.6 Network size limits . . . . . . . . . . . . . . . . . . . . . . . . . . 7

1.7 Parameters and options . . . . . . . . . . . . . . . . . . . . . . 7

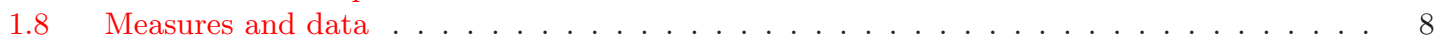

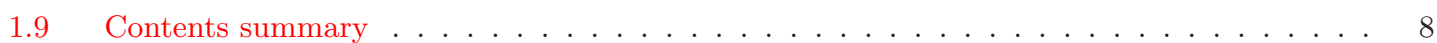

2 Summary of DDLab functions 9

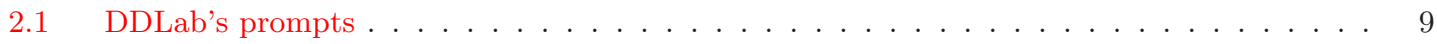

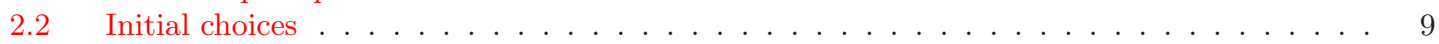

2.2.1 Totalistic rules, forwards-only, TFO-mode . . . . . . . . . . . . . . . . 9

2.2.2 Basin field or initial state . . . . . . . . . . . . . . . . . . . . . . . 9 9

2.3 Setting the value-range . . . . . . . . . . . . . . . . . . . . . . . . 10

2.4 Setting the network size . . . . . . . . . . . . . . . . . . . . . 10

2.5 The neighborhood $k$ or $k$-mix . . . . . . . . . . . . . . . . . . . . 10

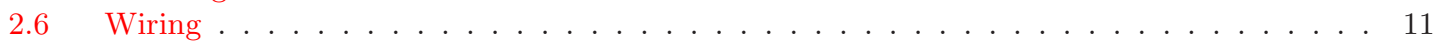

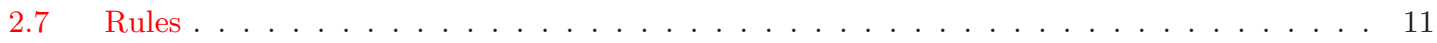

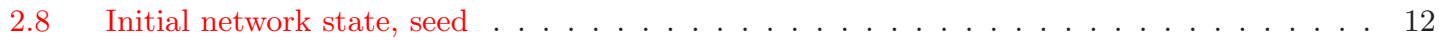

2.9 Networks of sub-networks . . . . . . . . . . . . . . . . . . . . . 12

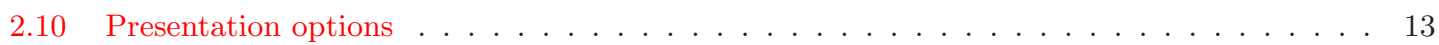

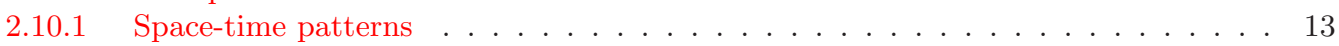

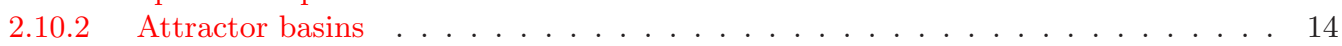

2.10 .3 Interrupting a run . . . . . . . . . . . . . . . . . . . . . 15

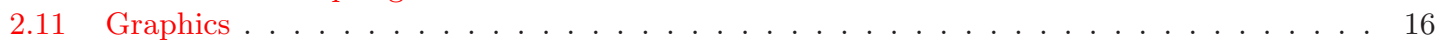

2.12 Filing and Printing . . . . . . . . . . . . . . . . . . . . . 16 
2.12.1 Filing network parameters . . . . . . . . . . . . . . . . . . . 17

2.12 .2 Filing data . . . . . . . . . . . . . . . . . . . . . . 17

2.13 Vector PostScript images . . . . . . . . . . . . . . . . . . . . . . . . 17

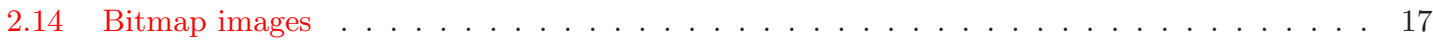

2.15 Printing the screen image . . . . . . . . . . . . . . . . . . . . 17

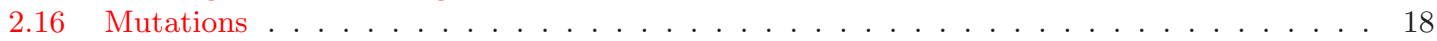

2.16.1 Running Forward . . . . . . . . . . . . . . . . . . . . . 18

2.16.2 Running Backward . . . . . . . . . . . . . . . . . . . . 18

2.17 Quantitative, statistical and analytical measures . . . . . . . . . . . . . . . 18

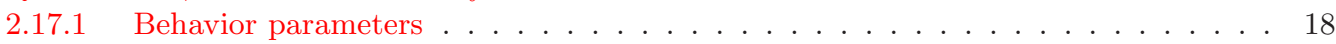

2.17 .2 Network connectivity . . . . . . . . . . . . . . . . . . . . . . . . 19

2.17.3 Measures on local dynamics . . . . . . . . . . . . . . . . . . . . . . 19

2.17.4 Measures on global dynamics . . . . . . . . . . . . . . . . . . . . 21

2.18 Reverse algorithms . . . . . . . . . . . . . . . . . . . . . . . . . 21

2.18.1 1d CA wiring reverse algorithm . . . . . . . . . . . . . . . . . . . . . . . . . . . . . . . . . . . . . . . . . . . . .

2.18.2 nonlocal wiring algorithm . . . . . . . . . . . . . . . . . . 23

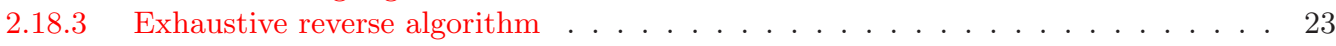

2.19 Random map . . . . . . . . . . . . . . . . . . . . . . . . . . . 23

2.20 Sequential updating . . . . . . . . . . . . . . . . . . . . . . . . 23

2.20 .1 Neutral order components . . . . . . . . . . . . . . . . . . . . . . . 24

2.21 Sculpting attractor basins . . . . . . . . . . . . . . . . . . . . . . . 24

3 Accessing and running DDLab $\mathbf{2 5}$

3.1 The DDLab web site . . . . . . . . . . . . . . . . . . . . . . . . . 25

3.2 DDLab at SourceForge . . . . . . . . . . . . . . . . . . . . . . . . . . . . . . . . . . . . . . . . . . . . . . . . . . . . . . .

3.3 Latest version of DDLab . . . . . . . . . . . . . . . . . . . . . . . . . . 25

3.4 Unzipping and running - Linux-like versions . . . . . . . . . . . . . . . . . . . . 26

3.4.1 Unix library files . . . . . . . . . . . . . . . . . . . . . . . 26

3.5 Unzipping and running - DOS . . . . . . . . . . . . . . . . . . . . . . 27

3.6 The Quick Start Examples . . . . . . . . . . . . . . . . . . . . . . . . 27

3.7 Extra data files . . . . . . . . . . . . . . . . . . . . . . . . . 27

3.7.1 Complex rule collections . . . . . . . . . . . . . . . . . . . . . 27

3.7.2 Selected $2 \mathrm{~d}$ complex rules, $v=3 \ldots \ldots \ldots \ldots \ldots \ldots$

3.7.3 Selected seeds . . . . . . . . . . . . . . . . . . . . . . . . 28

3.7.4 Sorted rule samples . . . . . . . . . . . . . . . . . . . . . . . . . 28

3.7.5 Byl's self reproducing loop . . . . . . . . . . . . . . . . . . . . . . 29

3.8 Copyright, License and Registration . . . . . . . . . . . . . . . . . . . . . . . . . . 29

3.9 Previous versions of DDLab . . . . . . . . . . . . . . . . . . . . . . . . . . . . . . 29

4 Quick Start Examples 30

4.1 The DDLab screen . . . . . . . . . . . . . . . . . . . . . . . . 30

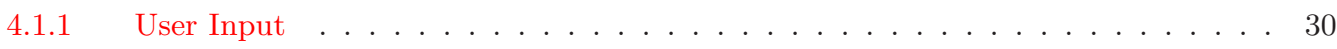

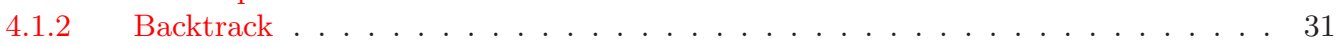

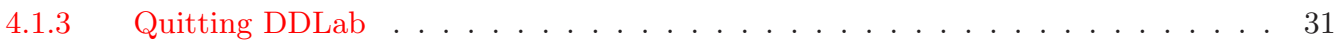

4.1 .4 Skipping Forward . . . . . . . . . . . . . . . . . . . . . . . 31

4.1.5 The graphics setup - Linux-like systems . . . . . . . . . . . . . . . . 31

4.1.6 The graphics setup - DOS, or Windows command line . . . . . . . . . . . . 31

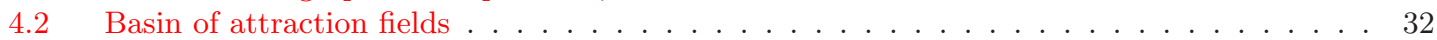

4.2.1 Changing basin parameters . . . . . . . . . . . . . . . . . . . 32

4.3 Backwards space-time patterns, and state-space matrix . . . . . . . . . . . . . . . . 33

4.4 Basin of attraction fields for a range of network sizes . . . . . . . . . . . . . . . . . . 34 
4.5 A single basin of attraction . . . . . . . . . . . . . . . . . . . . 35

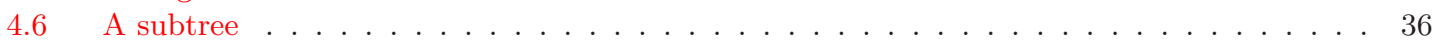

4.7 Space-time patterns — SEED-mode or TFO-mode . . . . . . . . . . . . . . . 37

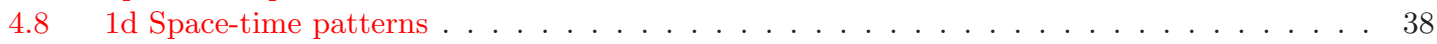

4.8.1 1d ring of cells, and scrolling the ring . . . . . . . . . . . . . . . . . . 39

4.8.2 Scrolling the ring . . . . . . . . . . . . . . . . . . . . . 39

4.8.3 Multi-value 1d space-time patterns in TFO-mode . . . . . . . . . . . . . . 41

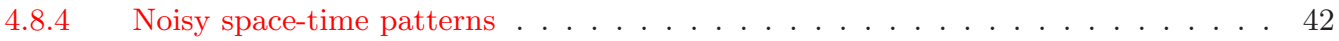

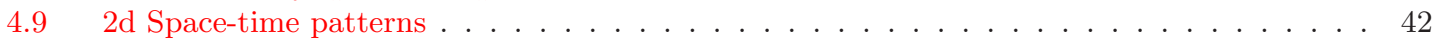

4.9 .12 d space-time patterns - game-of-Life . . . . . . . . . . . . . . . . . 42

4.9.2 2d Space-time patterns — binary totalistic rules . . . . . . . . . . . . . . 45

4.9.3 Multi-value 2d space-time patterns in TFO-mode . . . . . . . . . . . . . 46

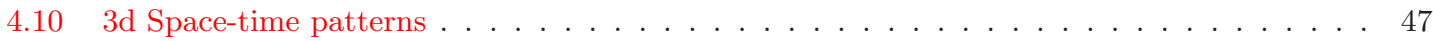

4.10.1 3d Space-time patterns - examples . . . . . . . . . . . . . . . . . 47

4.11 "Screen-saver" demo . . . . . . . . . . . . . . . . . . . . . . . . . . . . 48

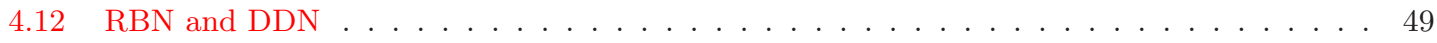

5 Starting DDLab $\mathbf{5 2}$

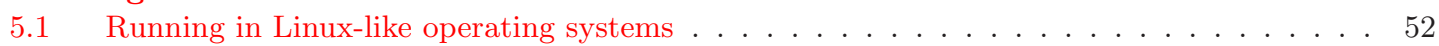

5.2 Running in DOS or Windows . . . . . . . . . . . . . . . . . . . . . . . 53

5.2.1 Running in a pure DOS environment . . . . . . . . . . . . . . . . . 54

5.2.2 Running in Windows95, 98, and later editions . . . . . . . . . . . . . . 54

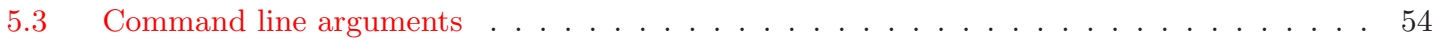

5.4 The UNREGISTERED banner . . . . . . . . . . . . . . . . . . . . . . . . 55

5.5 Title bar . . . . . . . . . . . . . . . . . . . . . . . . . . . . . 55

5.6 Saving, Loading and Printing the DDLab screen . . . . . . . . . . . . . . . . . . 55

5.6 .1 Saving and Loading the screen in DDLab's own format . . . . . . . . . . . . . 56

5.6.2 Saving and Printing the screen in Linux-like systems . . . . . . . . . . . . . . 56

$5.6 .3 \quad$ Printing the screen in DOS . . . . . . . . . . . . . . . . . 57

5.7 DDLab version and graphics info . . . . . . . . . . . . . . . . . . . . 58

5.8 The mouse cursor in DOS and Windows . . . . . . . . . . . . . . . . . . . . 58

5.9 Memory (DOS only) . . . . . . . . . . . . . . . . . . . . . . 58

$5.9 .1 \quad$ Virtual Memory . . . . . . . . . . . . . . . . . . . . 59

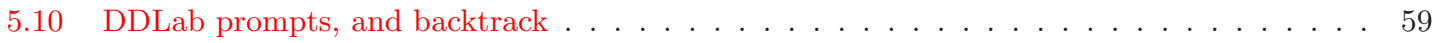

5.10.1 Prompts: main sequence and pop-up windows . . . . . . . . . . . . . . . 59

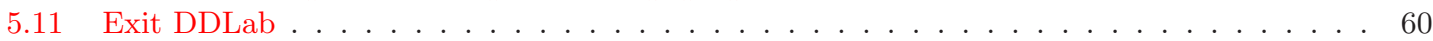

6 The first prompt in DDLab 61

6.1 TFO-mode, SEED-mode, FIELD-mode . . . . . . . . . . . . . . . . . . 61

6.2 The first prompt . . . . . . . . . . . . . . . . . . . . . . . . 62 62

6.2.1 TFO-mode: totalistic forwards only . . . . . . . . . . . . . . . . 62

6.2.2 FIELD, or a run requiring a SEED . . . . . . . . . . . . . . . . . 62

6.2.3 Notice of the current mode . . . . . . . . . . . . . . . . . . . . 62

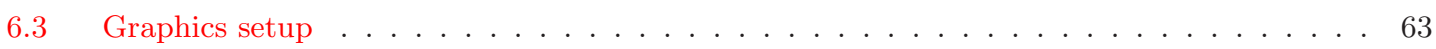

6.3.1 The DDLab window resolution, Linux-like systems . . . . . . . . . . . . . . 63

6.3.2 The DDLab window resolution in DOS . . . . . . . . . . . . . . . . 63

6.3.3 White or black background . . . . . . . . . . . . . . . . . . . . . 64

6.3.4 The color palette . . . . . . . . . . . . . . . . . . . . 6 64

6.4 Changing the font size and line spacing . . . . . . . . . . . . . . . . . . . . . 64

6.4.1 Font size and line spacing, Linux-like systems . . . . . . . . . . . . . . . . . . 64 . . . . . . . . . . . . . . . .

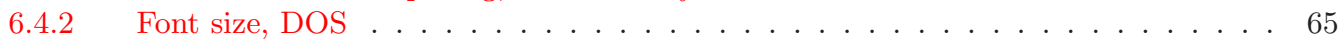




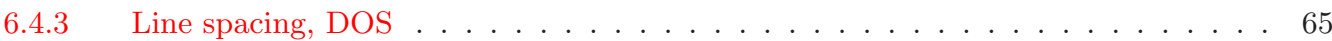

6.5 Changing the flashing cursor speed . . . . . . . . . . . . . . . . . . . . . . 65

6.6 Random number seed . . . . . . . . . . . . . . . . . . . . . . 65

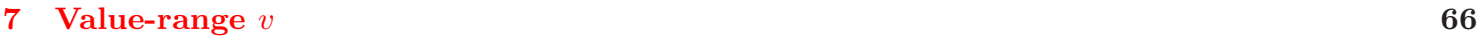

7.1 The value-range prompt . . . . . . . . . . . . . . . . . . . . . . . . . 66

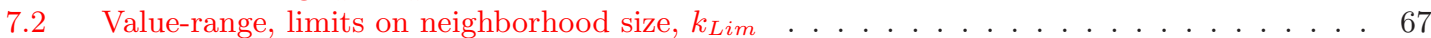

7.3 Value-range, limits on network size, $n_{L i m} \ldots \ldots \ldots$. . . . . . . . . . . . . . . 67

8 1d network size $n$, or range- $n \quad 68$

8.1 Setting range of sizes, $1 \mathrm{~d} \ldots \ldots \ldots \ldots$

8.2 Setting the size of one network, $1 \mathrm{~d} \ldots \ldots . \ldots . \ldots$

8.3 Network size limits . . . . . . . . . . . . . . . . . . . . . . . . . . 70

8.3.1 Computational and speed limitations for attractor basins . . . . . . . . . . 70

8.4 Times for basin of attraction fields . . . . . . . . . . . . . . . . . . . . . 71

8.4.1 Examples for $1 \mathrm{~d}$ CA . . . . . . . . . . . . . . . . . . . . 72

8.4.2 Examples for RBN and DDN . . . . . . . . . . . . . . . . . . 74

9 Neighborhood $k$, or mixed- $k \quad \mathbf{7 5}$

9.1 Selecting $k$, or a $k$-mix, for $1 \mathrm{~d}$ networks . . . . . . . . . . . . . . . . . . 75

$9.1 .1 \quad k_{\text {Lim }}$ and minimum $k \ldots \ldots \ldots \ldots \ldots \ldots \ldots$

9.2 Effective $k=0 \ldots \ldots \ldots \ldots \ldots \ldots$

9.3 Specifying the $k$-mix . . . . . . . . . . . . . . . . . . . . . 76

9.4 Loading a $k$-mix file . . . . . . . . . . . . . . . . . . . . . . . . . 76

9.5 Setting the $k$-mix by hand . . . . . . . . . . . . . . . . . . . . . 77

9.6 Setting a normal distribution . . . . . . . . . . . . . . . . . . . . . . . . . 77

9.7 Specify each $k$, or power-law distribution . . . . . . . . . . . . . . . . . . . . . . . 77

9.7.1 Specify the percentage of different $k \ldots \ldots \ldots \ldots \ldots$

9.7 .2 Setting a power-law distribution of $k \ldots \ldots \ldots \ldots$

9.7.3 Showing the distribution . . . . . . . . . . . . . . . . . . 78

9.8 Setting the $k$-mix at random . . . . . . . . . . . . . . . . . . . . . . . 79

9.9 Setting a $k$-mix with uniform $k \ldots \ldots \ldots \ldots$

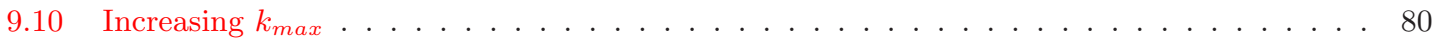

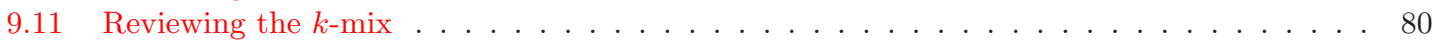

9.11.1 Reviewing the $k$-mix in large networks . . . . . . . . . . . . . . . . . . 81

9.11 .2 Jumping to a new cell index . . . . . . . . . . . . . . . . . . . . . . . . 81

9.11.3 Saving the $k$-mix file . . . . . . . . . . . . . . . . . . . . 81

9.12 Reviewing the $k$-mix within "network architecture" . . . . . . . . . . . . . . . . . 81

10 The local neighborhood, and network geometry 82

10.1 The CA neighborhood . . . . . . . . . . . . . . . . . . . . 82

10.1.1 The pseudo-neighborhood, RBN and DDN . . . . . . . . . . . . . . . . . . 82

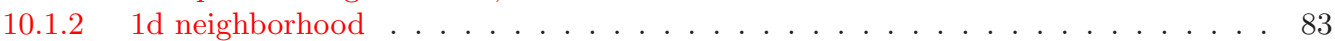

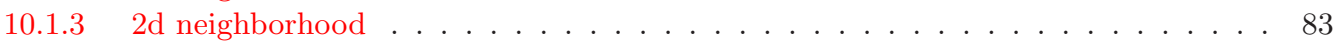

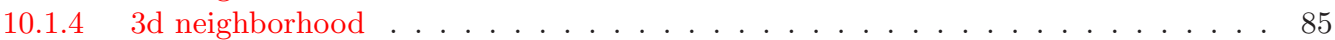

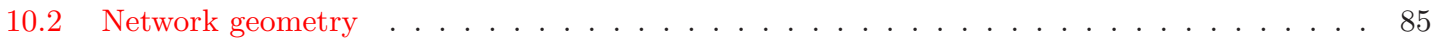

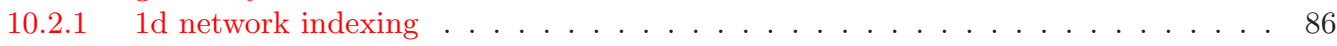

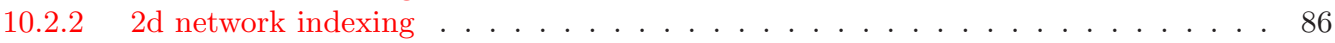

10.2.3 3d network indexing . . . . . . . . . . . . . . . . . . . 87

11 Setting the wiring, quick settings 88

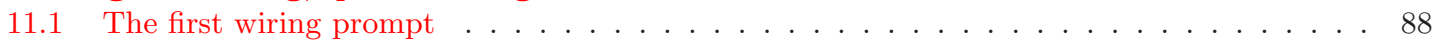


11.2 Special wiring . . . . . . . . . . . . . . . . . . . . . . . . 88

11.3 Loading the wiring scheme . . . . . . . . . . . . . . . . . . . . . . . . . . . . . 88

11.4 Random 1d wiring . . . . . . . . . . . . . . . . . . . . . . . . . 88

11.5 Local $1 \mathrm{~d}$ wiring . . . . . . . . . . . . . . . . . . . . . . . . . . . . 89

11.6 Local $2 \mathrm{~d}$ or $3 \mathrm{~d}$ wiring . . . . . . . . . . . . . . . . . . . . . . . . . . . 89

11.6.1 Setting $2 \mathrm{~d}$ and $3 \mathrm{~d}$ network size . . . . . . . . . . . . . . . . . 89

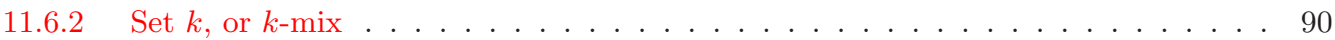

11.7 Networks too big for a basin field . . . . . . . . . . . . . . . . . . . . . . 90

12 Setting special wiring $\quad 91$

12.1 Setting up the network geometry . . . . . . . . . . . . . . . . . . . . 91

12.2 Hypercube wiring . . . . . . . . . . . . . . . . . . . . . . . . . . 92

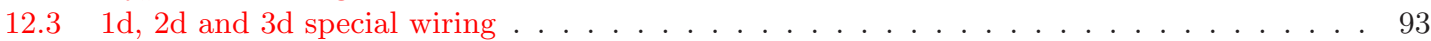

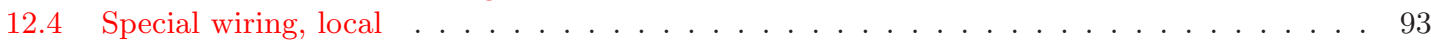

12.4.1 Local $1 \mathrm{~d}$ treated as random . . . . . . . . . . . . . . . . . . . . . . . . . . . . . . . . . . . . . . . . . . . . .

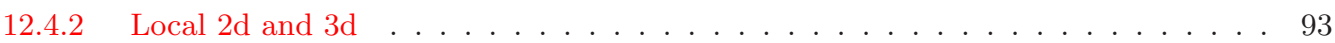

12.5 Special wiring, random . . . . . . . . . . . . . . . . . . . . . . . 94

12.5.1 Applying wiring biases to parts of the network . . . . . . . . . . . . . . . . 94

12.5.2 Confining random wiring to a set zone . . . . . . . . . . . . . . . . . . . 94

12.5.3 Setting local wiring as random . . . . . . . . . . . . . . . . . . . . . . . . . . . . . . . . . . . . . .

12.5.4 Release wires from zone . . . . . . . . . . . . . . . . . . . . . . . 95

12.5.5 Suppress periodic boundary conditions . . . . . . . . . . . . . . . . . 96

12.5.6 Self-wiring. . . . . . . . . . . . . . . . . . . . 96

12.5.7 Distinct wiring . . . . . . . . . . . . . . . . . . . . . . 96

12.5.8 Suppress links to $3 \mathrm{~d}$ layers . . . . . . . . . . . . . . . . . . . . . . . . . . . . . . . . . . . . . . 96

12.5.9 Force a direct link to a 3 d layer . . . . . . . . . . . . . . . . . . . . . 96

12.5.10 Force random links to specific 3d layers . . . . . . . . . . . . . . . . . . . 97

12.5.11 Same random wiring everywhere . . . . . . . . . . . . . . . . . 97

12.6 Wiring by hand . . . . . . . . . . . . . . . . . . . . . . . . 97

12.7 Reviewing wiring . . . . . . . . . . . . . . . . . . . . . . 98

13 Rule types $\quad 100$

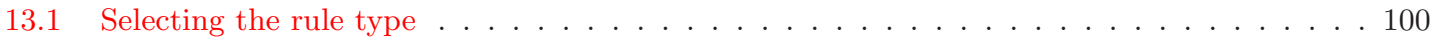

13.1.1 Select full rule-tables, rcode . . . . . . . . . . . . . . . . . . . . . 100

13.1.2 Select kcode or tcode in TFO-mode. . . . . . . . . . . . . . . . . . . . . 101

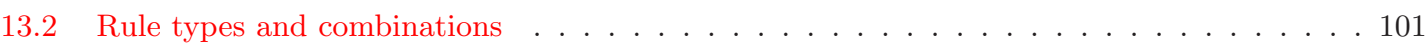

13.2.1 Rule-table size implications . . . . . . . . . . . . . . . . . . . . . . . 102

13.3 Binary full rule-table - rcode . . . . . . . . . . . . . . . . . . . . . . . . 102

13.3.1 The binary neighborhood matrix . . . . . . . . . . . . . . . . 103

13.4 Binary totalistic rules . . . . . . . . . . . . . . . . . . . . . . . . . . 104

13.5 Multi-value full rule-table - rcode . . . . . . . . . . . . . . . . . . . . . . . . . . 104

13.5.1 The multi-value neighborhood matrix . . . . . . . . . . . . . . 105

13.6 Multi-value totalistic rules, tcode and kcode . . . . . . . . . . . . . . . . . . . 105

13.6.1 k-totalistic rules - kcode . . . . . . . . . . . . . . . . . . . 106

13.6 .2 kcode $v=3$ matrix . . . . . . . . . . . . . . . . . . . . . . . . . . 107

13.6.3 t-totalistic rules - tcode . . . . . . . . . . . . . . . . . . . . . . 107

13.7 Outer-totalistic rules . . . . . . . . . . . . . . . . . . . . . . . . . 108

13.8 Reaction-Diffusion dynamics . . . . . . . . . . . . . . . . . . . . . . 108

13.8.1 Reaction-Diffusion from outer-kcode . . . . . . . . . . . . . . . . 109

13.8.2 Reaction-Diffusion from a full rule-table - rcode . . . . . . . . . . . . . . . . . 109

13.8.3 Selecting the threshold interval . . . . . . . . . . . . . . . . . . 109 
14 Rulemix options

14.1 Single rule or rulemix, and other options . . . . . . . . . . . . . . . . . . . . . . . . . . 110

14.1.1 Summary of rulemix and other options . . . . . . . . . . . . . . . . . . 111

14.1.2 Density-bias $(\lambda$-parameter $) \ldots \ldots \ldots \ldots \ldots \ldots$

14.2 Outer-totalistic kcode or tcode . . . . . . . . . . . . . . . . . . . . . 112

14.2.1 Setting reaction-diffusion by outer-kcode . . . . . . . . . . . . . . . . . 112

14.2.2 The game-of-Life and other Life-like rules by outer-kcode . . . . . . . . . . . . . 112

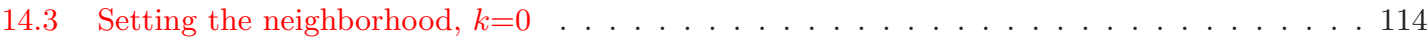

14.4 Methods for setting the rulemix or rule-subset . . . . . . . . . . . . . . . . . . . . . 114

14.4.1 Setting a rulemix directly . . . . . . . . . . . . . . . . . . 115

14.4.2 Setting a rulemix indirectly - specify a rule-subset . . . . . . . . . . . . 115

14.4.3 A rulemix with just one rule . . . . . . . . . . . . . . . . . . 116

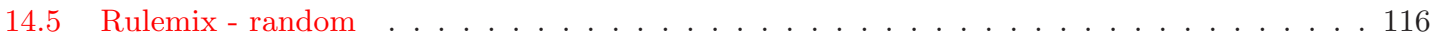

14.6 Rulemix by hand . . . . . . . . . . . . . . . . . . . . . . . . . . 117

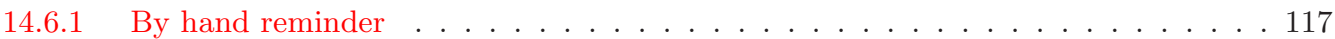

14.6.2 By hand single rule prompt . . . . . . . . . . . . . . . . . . . 117

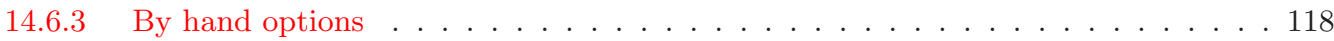

14.6.4 Change the selection method or rule index . . . . . . . . . . . . . . . . 118

14.6.5 Complete the rulemix automatically . . . . . . . . . . . . . . . . . 119

14.6.6 Copy rules automatically for a $k$-mix . . . . . . . . . . . . . . . . . . . . . 119

14.6.7 Mixed $k$ where all $k$ 's (and rules) are the same . . . . . . . . . . . . . . . . 119

14.7 Rulemix - majority . . . . . . . . . . . . . . . . . . . . . . . 120

14.8 Rulemix - majority with shifted uniform outputs . . . . . . . . . . . . . . . . . 120

14.9 Rulemix for large networks, or large $k$. . . . . . . . . . . . . . . . . . . . 120

14.10 The all 0 s output . . . . . . . . . . . . . . . . . . . . . . . . . 121

14.11 Amending the neighborhood matrix . . . . . . . . . . . . . . . . . . 121

14.12 List Post functions . . . . . . . . . . . . . . . . . . . . . . . . . . . . . . 122

14.12.1 Initial Post-function prompt . . . . . . . . . . . . . . . . . . . . . 122

14.12.2 Restrict Post-functions . . . . . . . . . . . . . . . . . . . . . . . . . . . 123

14.12.3 Set Post-function sample size . . . . . . . . . . . . . . . . . . . . . . 123

14.12.4 Final Post-function prompt . . . . . . . . . . . . . . . . . . 123

15 Setting Canalyzation in a random rule-mix 124

15.1 Selecting Canalyzing . . . . . . . . . . . . . . . . . . . . . . . . . . 124

15.1.1 Selecting canalyzing from the rcode-mix . . . . . . . . . . . . . . . . 124

15.1.2 Selecting canalyzing from wiring graphic - transform rule . . . . . . . . . . . 125

15.1.3 Selecting canalyzing from the Derrida plot . . . . . . . . . . . . . . . . 125

15.2 The first canalyzing prompt . . . . . . . . . . . . . . . . . . . . . . . . 125

15.3 Canalyzing percentage or number . . . . . . . . . . . . . . . . . . . . 125

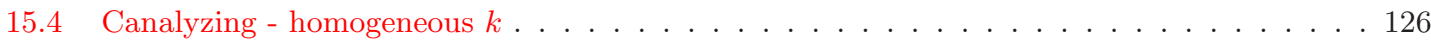

15.5 Canalyzing - mixed $k \ldots \ldots \ldots \ldots \ldots \ldots \ldots$

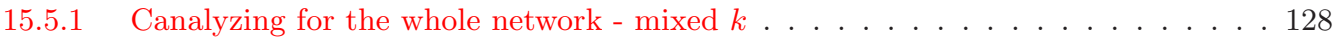

15.5.2 Canalyzing for a particular $k$ in a mixed $k$ network . . . . . . . . . . . . . . . . 129

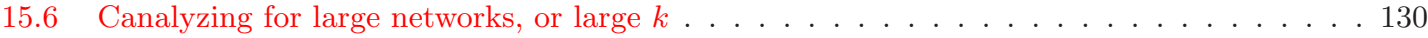

16 Setting a singe rule 131

16.1 The first single rule prompt . . . . . . . . . . . . . . . . . . . . . . . . . 131

16.1.1 Single rcode prompt examples . . . . . . . . . . . . . . . . . . . 131

16.1.2 TFO-mode single rule prompt examples . . . . . . . . . . . . . . . . . . 132

16.2 Methods for setting a rule . . . . . . . . . . . . . . . . . . . . . . 132

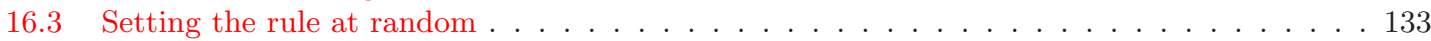




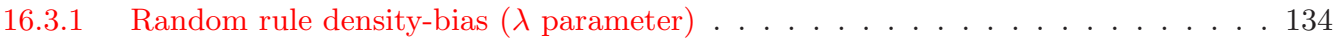

16.3.2 Random rule value-bias . . . . . . . . . . . . . . . . . . . . . . 135

16.3.3 Random rule parameters . . . . . . . . . . . . . . . . . . . . 136

16.4 Setting the rule as bits or values . . . . . . . . . . . . . . . . . . . . . . 136

16.4.1 Rules: bits/values reminder window . . . . . . . . . . . . . . . . . . 137

16.4.2 Rules: setting bits/values with the keyboard and mouse . . . . . . . . . . . . . 138

16.4.3 Rules: save as 1d seed or PostScript . . . . . . . . . . . . . . . . . . . 139

16.4.4 Rules: PostScript prompt . . . . . . . . . . . . . . . . . . . . . . . 140

16.5 Setting the rule in hex . . . . . . . . . . . . . . . . . . . . . . . . 141

16.6 Setting the rule in decimal . . . . . . . . . . . . . . . . . . . . . . . . . 141

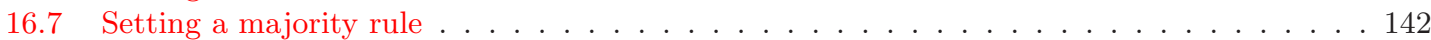

16.8 Majority with shifted uniform outputs . . . . . . . . . . . . . . . . . . . . . . . 144

16.9 Setting Altenberg rules . . . . . . . . . . . . . . . . . . . . . . . 145

16.10 The game-of-Life and other Life-like rules - rcode . . . . . . . . . . . . . . . . . . . . 145

16.10.1 Setting Life-like rules - rcode . . . . . . . . . . . . . . . . . . . . . . . . 146

16.11 Setting a chain-rule . . . . . . . . . . . . . . . . . . . . . . 147

16.12 Setting reaction-diffusion - rcode . . . . . . . . . . . . . . . . . . . . . . . 148

16.13 Repeating the last rule . . . . . . . . . . . . . . . . . . . . . . . . . . . . 148

16.14 Loading a single rule . . . . . . . . . . . . . . . . . . . . . . . . . . . . . . . 148

16.15 Automatic saving of last rule . . . . . . . . . . . . . . . . . . . . . . . . . 148

16.16 Single rule file encoding . . . . . . . . . . . . . . . . . . . . . . . . . . . 149

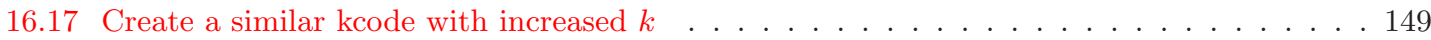

16.18 Show the rule in the terminal . . . . . . . . . . . . . . . . . . . . . . . . 150

16.18.1 Immediate rule data . . . . . . . . . . . . . . . . . . . . . 150

16.18.2 Immediate rule data for a rulemix by hand . . . . . . . . . . . . . . . . . . 151

16.18.3 Rule data in more detail — vertical layout . . . . . . . . . . . . . . . . . . . . 151

16.18.4 Additional rule data options for kcode . . . . . . . . . . . . . . . . . . . . . . . 152

16.18.5 Swapping kcode values . . . . . . . . . . . . . . . . . . . . 152

16.19 The rule window . . . . . . . . . . . . . . . . . . . . . . . . . . 152

16.19.1 Decoding the rule window . . . . . . . . . . . . . . . . . . . 153

16.20 Complementary values . . . . . . . . . . . . . . . . . . . . . . . . 154

16.21 Transforming the single rule . . . . . . . . . . . . . . . . . . . . . . . 155

17 Reviewing network architecture 15

17.1 The network architecture prompt . . . . . . . . . . . . . . . . . . . . 156

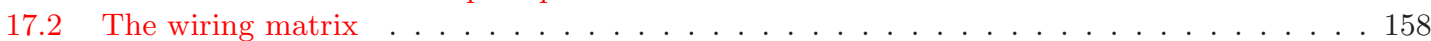

17.2.1 Viewing the wiring matrix and creating vector PostScript . . . . . . . . . . . . . 159

17.2.2 Amending the matrix . . . . . . . . . . . . . . . . . . . . 159

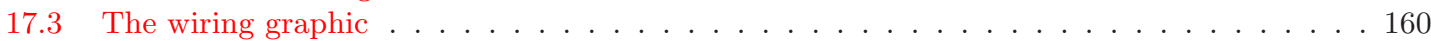

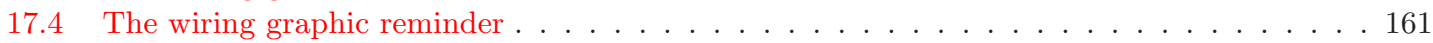

17.4.1 Wiring graphic options summary . . . . . . . . . . . . . . . . . . 161

17.5 Wiring graphic, $1 \mathrm{~d} \ldots \ldots \ldots \ldots \ldots \ldots$

17.5.1 Data - 1d wiring graphic . . . . . . . . . . . . . . . . . 165

17.5.2 Moving or jumping between cells, 1d . . . . . . . . . . . . . . 166

17.5.3 Defining a block, $1 \mathrm{~d} \ldots \ldots \ldots \ldots \ldots$

17.5.4 Toggling the block, $1 \mathrm{~d}$. . . . . . . . . . . . . . . . . . . 168

17.5.5 Include the pseudo-neighborhood, or direct wiring only, 1d . . . . . . . . . . 168

17.5.6 Recursive inputs to a cell, $1 \mathrm{~d} \ldots \ldots \ldots$. . . . . . . . . . . . . 168

17.5.7 Recursive outputs from a cell, 1d . . . . . . . . . . . . . . . . 168

17.5.8 Untangling the wiring . . . . . . . . . . . . . . . . . . . 171

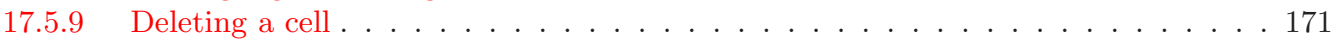


17.6 Wiring graphic, $2 \mathrm{~d} \ldots \ldots \ldots \ldots \ldots \ldots \ldots \ldots$

17.6.1 Data - 2d wiring graphic . . . . . . . . . . . . . . . . . . . . . 172

17.6.2 Moving or jumping between cells, 2d . . . . . . . . . . . . . . . . . 173

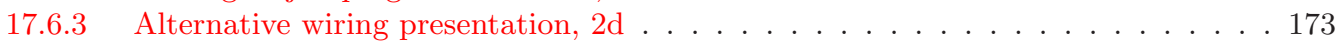

17.6.4 Include the pseudo-neighborhood, or direct wiring only, 2d . . . . . . . . . . . 173

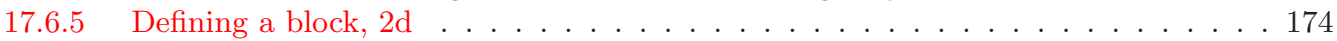

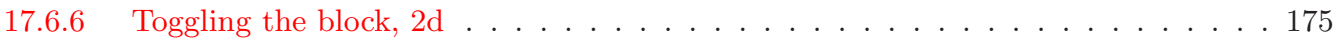

17.6.7 Expand/Contract the scale, 2d . . . . . . . . . . . . . . . . . 175

17.6.8 Shifting the $2 \mathrm{~d}$ graphic up and down . . . . . . . . . . . . . . . . . . . 175

17.7 Wiring graphic, $3 \mathrm{~d} \ldots \ldots \ldots \ldots \ldots \ldots \ldots$

17.7.1 Data - 3d wiring graphic . . . . . . . . . . . . . . . . 176

17.7.2 Moving or jumping between cells, 3d . . . . . . . . . . . . . . . . . . 176

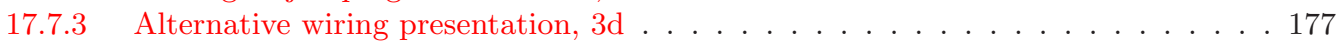

17.7.4 Include the pseudo-neighborhood, or direct wiring only, 3d . . . . . . . . . . 177

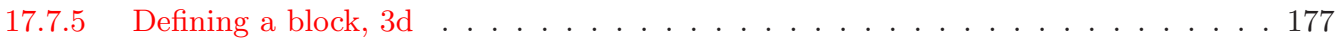

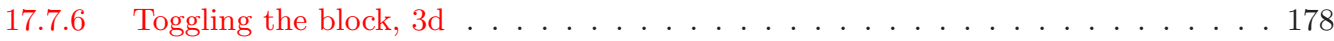

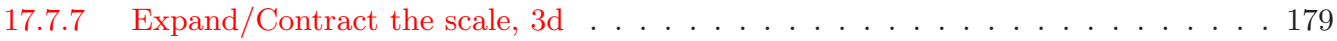

17.7.8 Shifting the $3 \mathrm{~d}$ graphic up and down . . . . . . . . . . . . . . . . . . . 179

17.8 Further options for the $1 \mathrm{~d}, 2 \mathrm{~d}$ and $3 \mathrm{~d}$ wiring graphics . . . . . . . . . . . . . . . . . 180

17.8.1 Decoding wiring graphic data - 1d, 2d and 3d . . . . . . . . . . . . . . 180

17.8.2 Computing the (weighted) average $\lambda$ and $Z$ parameters . . . . . . . . . . . . . 180

17.8.3 Options for learning pre-images . . . . . . . . . . . . . . . . . . 181

17.8.4 Hand rewiring. . . . . . . . . . . . . . . . . . . . . . . . 181

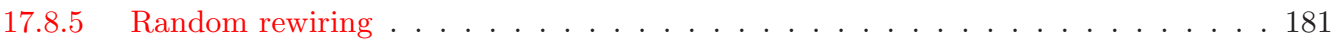

17.8.6 Biased random rewiring . . . . . . . . . . . . . . . . . . . . 182

17.8.7 Local 1d, $2 \mathrm{~d}$ or $3 \mathrm{~d}$ wiring . . . . . . . . . . . . . . . . . . . . . 182

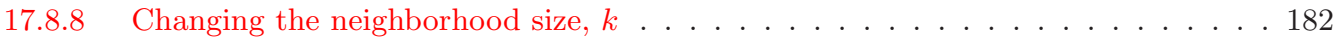

17.8 Kill a cell . . . . . . . . . . . . . . . . . . . . . . . . 183

17.8.10 Revising and copying the rule . . . . . . . . . . . . . . . . . . . . . . 183

17.8.11 Transforming the rule . . . . . . . . . . . . . . . . . . . . 183

17.8.12 Filing, from the wiring graphic . . . . . . . . . . . . . . . . . . . . . . 184

17.8.13 The histogram of the network's $k$ and output distribution . . . . . . . . . . . . . 184

17.8.14 Creating a vector PostScript file of the wiring graphic . . . . . . . . . . . . . 185

18 Transforming rules $\quad 186$

18.1 Options for transforming rules . . . . . . . . . . . . . . . . . . . . . . . . 187

18.1.1 Transform options, single rule . . . . . . . . . . . . . . . . . . . . . . 187

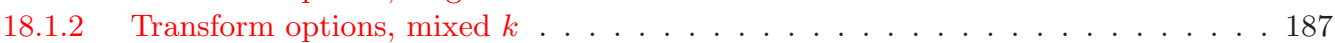

18.1.3 Transform options, mixed rule, homogeneous $k$. . . . . . . . . . . . . . . 187

18.1.4 Transform all cells in a mixed rule network . . . . . . . . . . . . . . . . . . . . 188

18.2 Saving or printing the transformed rule . . . . . . . . . . . . . . . . . . . . 188

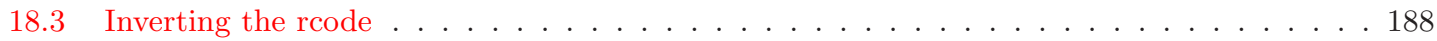

18.4 Solidifying the rule . . . . . . . . . . . . . . . . . . . . . . . . . 189

18.5 Equivalence classes and rule clusters . . . . . . . . . . . . . . . . . . . . 189

18.5.1 Complementary transformation . . . . . . . . . . . . . . . . . . . 189

18.5.2 Equivalent rcode by the Negative transformation . . . . . . . . . . . . . . . . . 189

18.5.3 Equivalent rcode by the Reflection transformation . . . . . . . . . . . . . . . . . 189

18.6 Setting canalyzing inputs, single rcode . . . . . . . . . . . . . . . . . . . 191

18.6.1 Canalyzing inputs at random, single rcode . . . . . . . . . . . . . . . . . . 191

18.6.2 Canalyzing inputs explicitly, single rcode . . . . . . . . . . . . . . . . . . 191

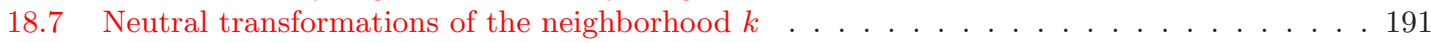


18.7.1 Equivalent rules with greater $k \ldots \ldots . . . . . . . . . . . . . . .191$

18.7.2 Reducing $k_{\max }$ to the maximum $k$ in the network . . . . . . . . . . . . . . . . 192

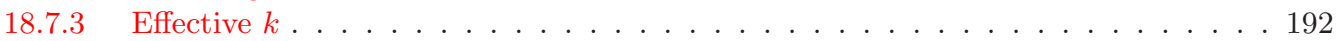

18.7.4 Reverse engineering - loading an exhaustive map . . . . . . . . . . . . . . 192

19 File/print network architecture 19

19.1 Network filing options . . . . . . . . . . . . . . . . . . . . . . . . . . . . . 194

19.2 Wiring/rulemix filenames . . . . . . . . . . . . . . . . . . . . . . . 195

19.3 Wiring/rulemix encoding . . . . . . . . . . . . . . . . . . . . . 195

19.3.1 Mixed- $k$ encoding . . . . . . . . . . . . . . . . . . 196

19.4 Loading networks and sub-networks . . . . . . . . . . . . . . . . . . . . . . . . 196

19.4.1 loading networks - compatibility . . . . . . . . . . . . . . . . . . 197

19.4.1.1 Compatibility with $v \ldots \ldots \ldots$. . . . . . . . . . . 197

19.4.1.2 Compatibility with $k \ldots \ldots \ldots$. . . . . . . . . . . . . . . . . . . . . . . . . . . . . 198

19.4.1.3 Compatibility with $n \ldots \ldots$. . . . . . . . . . . 198

19.4.1.4 Compatibility with edge sizes and dimensions . . . . . . . . . . 198

19.4.2 Loading a complicated network into a CA and vice versa . . . . . . . . . . . . 199

19.4.3 Loading sub-networks in a set position . . . . . . . . . . . . . . . . . . . 199

19.4.4 Loading $k$-mix networks . . . . . . . . . . . . . . . . . . . . . . . . . . 201

19.5 Saving just the $k$-mix . . . . . . . . . . . . . . . . . . . . . . . . . . . . . 202

19.6 Printing network data to the terminal or file . . . . . . . . . . . . . . . . . . . . 202

20 The network-graph, and attractor jump-graph 204

20.1 Unravelling the jump-graph and the network-graph . . . . . . . . . . . . . . . . . . . 204

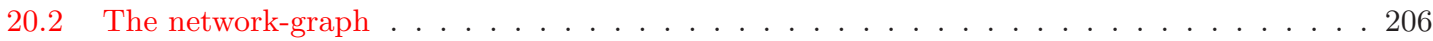

20.2.1 The network-graph reminder . . . . . . . . . . . . . . . . 206

20.3 The jump-graph of the basin of attraction field . . . . . . . . . . . . . . . . . . 207

20.3.1 Selecting the jump-graph . . . . . . . . . . . . . . . . . . . . 207

20.3 .2 The jump-graph reminder . . . . . . . . . . . . . . . . . . . . . . 208

20.4 Initial graph options . . . . . . . . . . . . . . . . . . . . . . . . . . . . 210

20.5 Dragging nodes or fragments . . . . . . . . . . . . . . . . . . . . . 212

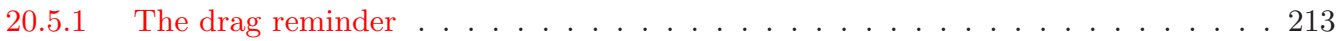

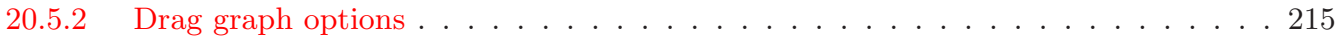

20.5.3 Defining a block . . . . . . . . . . . . . . . . . . . 217

20.6 Probabilistic "ant" . . . . . . . . . . . . . . . . . . . . . . . 217

20.6.1 Show ant hits . . . . . . . . . . . . . . . . . . . . . 218

20.7 Redraw basins at jump-graph nodes . . . . . . . . . . . . . . . . . . . . . . 219

20.7.1 PostScript of jump-graph basins . . . . . . . . . . . . . . . . . . . 221

20.8 PostScript of the network-graph or jump-graph . . . . . . . . . . . . . . . . . . . . . . 222

20.9 Graph layout file . . . . . . . . . . . . . . . . . . . . . . . . 222

20.10 Revise settings . . . . . . . . . . . . . . . . . . . . . . . . . . 222

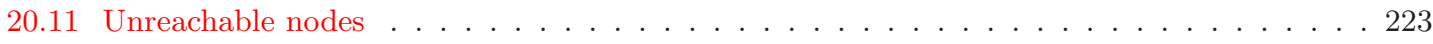

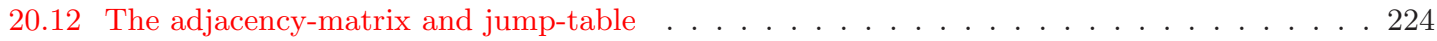

20.12.1 Printing and scanning tables . . . . . . . . . . . . . . . . . . . . . 225

20.13 Space-time patterns within the network-graph . . . . . . . . . . . . . . . . 225

21 The Seed or initial state $\quad 227$

21.1 The seed prompt . . . . . . . . . . . . . . . . . . . . . . . . . . . . . . . . . . . . . . . . . . . . . . . .

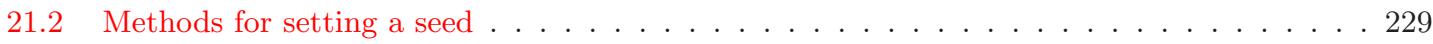

21.3 Setting the seed at random . . . . . . . . . . . . . . . . . . . . . 230

21.3.1 Non-zero seed density bias . . . . . . . . . . . . . . . . . . 231 
21.4 Setting the seed as bits or values . . . . . . . . . . . . . . . . . . . . . . 233

21.4 Seed: bits/values reminder window . . . . . . . . . . . . . . . . . . . . 233

21.4 .2 Seed: bits/values options summary . . . . . . . . . . . . . . . . . . . . . 234

21.4.3 Seed: bits/values current settings inset . . . . . . . . . . . . . . . . . . . 235

21.4.4 Seed: setting bits/values with the keyboard and mouse . . . . . . . . . . . . . 236

21.4.5 setting bits/values: $1 \mathrm{~d}$ segments . . . . . . . . . . . . . . . . . 236

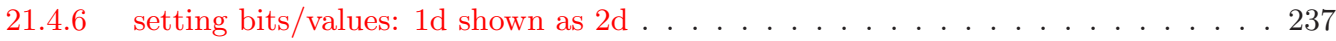

21.4.7 Setting bits/values: filing and PostScript . . . . . . . . . . . . . . . . . 237

21.4.8 Setting bits/values: saving a patch . . . . . . . . . . . . . . . . . . 238

21.4.9 Setting bits/values: PostScript prompt . . . . . . . . . . . . . . . . 239

21.4.10 Setting bits/values: PostScript 3d image . . . . . . . . . . . . . . . . . . . 241

21.5 Setting the seed in hex . . . . . . . . . . . . . . . . . . . . . . . . 242

21.6 Setting the seed in decimal . . . . . . . . . . . . . . . . . . . . . . . 243

21.7 Loading a seed . . . . . . . . . . . . . . . . . . . . . . . . . . . . 243

21.7.1 Constraints for loading a seed . . . . . . . . . . . . . . . . . . . . . . . 244

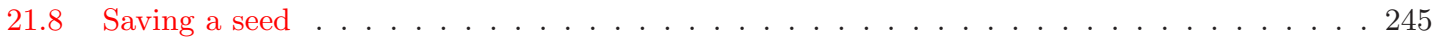

21.9 Seed file encoding . . . . . . . . . . . . . . . . . . . . . . . . 245

22 The Derrida plot 246

22.1 Selecting the Derrida plot . . . . . . . . . . . . . . . . . . . . . . . . . . 246

22.2 Derrida plot options . . . . . . . . . . . . . . . . . . . . . . . . . . 247

22.2.1 Derrida plot parameters . . . . . . . . . . . . . . . . . . 248

22.3 Data within the Derrida plot . . . . . . . . . . . . . . . . . . . . . . 249

22.3.1 Network data . . . . . . . . . . . . . . . . . . . . . . . . . 249

22.3 .2 Derrida data . . . . . . . . . . . . . . . . . . . . . . . . 249

22.4 Interrupting the Derrida plot . . . . . . . . . . . . . . . . . . . . 250

22.5 Completing the Derrida plot . . . . . . . . . . . . . . . . . . . . . . 250

22.6 The Derrida coefficient . . . . . . . . . . . . . . . . . . . . 250

23 Graphic conventions for attractor basins 252

23.1 Basins of Attraction - the idea . . . . . . . . . . . . . . . . . . . . . . . . 252

23.2 Network states, nodes . . . . . . . . . . . . . . . . . . . . . . . 253

23.3 Attractor cycles . . . . . . . . . . . . . . . . . . . . . . . 255

23.4 Transient trees . . . . . . . . . . . . . . . . . . . . . . . . 255

23.4.1 Transient tree colors . . . . . . . . . . . . . . . . . . . . . 255

23.4 Transient trees for uniform states . . . . . . . . . . . . . . . . . . . . . 256

23.4 .3 Subtree only . . . . . . . . . . . . . . . . . . . . . . . . 257

24 Output parameters for attractor basins $\mathbf{2 5 8}$

24.1 The first output parameter prompt for attractor basins . . . . . . . . . . . . . . . 258

24.1.1 Output parameter prompt for attractor basins — options summary . . . . . . . . 259

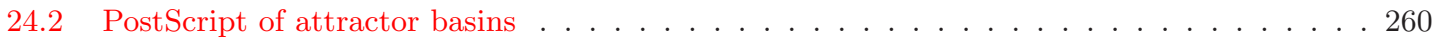

24.3 Activate the jump-graph . . . . . . . . . . . . . . . . . . . . . 260

24.4 Miscellaneous (hard to categorize) options . . . . . . . . . . . . . . . . . . . . 261

24.5 State-space matrix . . . . . . . . . . . . . . . . . . . . . . . . 261

24.5.1 State-space matrix - options summery . . . . . . . . . . . . . . . . . . . . . . 262

24.5.2 Toggle the matrix on-the-fly . . . . . . . . . . . . . . . . . . . . . . . . 264

24.6 In-degree frequency histogram . . . . . . . . . . . . . . . . . . . . . . . . . 264

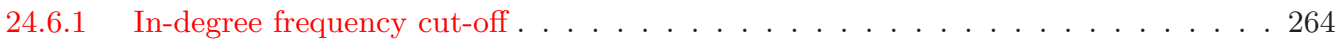

24.6.2 Drawing the in-degree histogram . . . . . . . . . . . . . . . . . . . 265

24.6.3 In-degree data and prompts . . . . . . . . . . . . . . . . . . . . 267 
24.6.4 in-degree window - data decode . . . . . . . . . . . . . . . . . 267

24.6.5 in-degree window - options summery . . . . . . . . . . . . . . . . . 267

24.6 .6 In-degree $\log$ plot . . . . . . . . . . . . . . . . . . . . . . . 268

24.6.7 Rescaling the $\mathrm{x} / \mathrm{y}$-axis . . . . . . . . . . . . . . . . . 268

24.7 Density classification problem — attractor basins . . . . . . . . . . . . . . . . 269

24.8 Screen-saver demo . . . . . . . . . . . . . . . . . . . . . . . . 270

$24.9 G$-density, $Z$ and $\lambda \ldots \ldots \ldots \ldots \ldots \ldots \ldots \ldots \ldots$

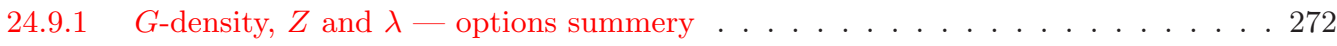

24.9.2 G-density plotted against network size . . . . . . . . . . . . . . . . . 272

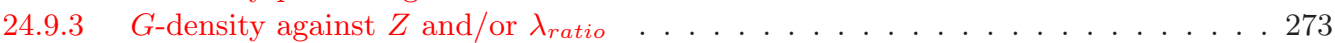

24.9.3.1 G-density for tcode, or rcode $k \leq 3 \ldots \ldots . . . . . . . . .274$

24.9.3.2 G-density for $k>3$ rcode . . . . . . . . . . . . . 274

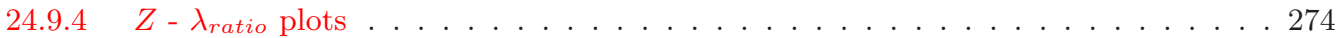

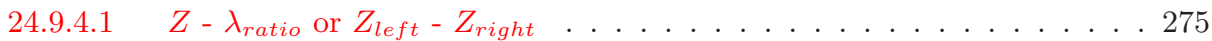

24.9.4.2 $Z-\lambda_{\text {ratio or }} Z_{\text {left }}-Z_{\text {right }}$ for $k \geq 4$ rcode . . . . . . . . . . . . 275

24.9.4.3 $Z-\lambda_{\text {ratio }}$ or $Z_{\text {left }}-Z_{\text {right }}$ for tcode . . . . . . . . . . . . . . . 276

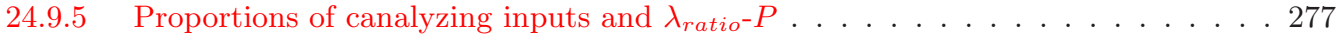

24.10 "Backwards" space-time patterns . . . . . . . . . . . . . . . . . . . . . 278

24.10.1 Scroll space-time patterns . . . . . . . . . . . . . . . . . . . . 278

24.11 Basin speed . . . . . . . . . . . . . . . . . . . . . . . . 280

24.12 Basin on-the-fly options . . . . . . . . . . . . . . . . . . . . . . . 280

25 Layout of attractor basins 281

25.1 The layout preview . . . . . . . . . . . . . . . . . . . . . . . . . . . 281

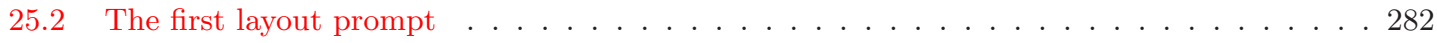

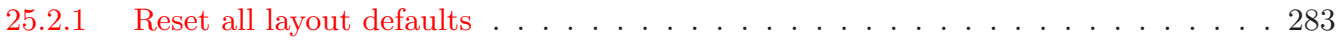

25.2.2 Mutants for single basins on one screen . . . . . . . . . . . . . . . . . . 283

25.2.3 Basin scale, attractor radius . . . . . . . . . . . . . . . . . . . . . . . . . 283

25.2.4 Basin start position . . . . . . . . . . . . . . . . . . . . . . . . 284

25.2.5 Show the field as successive basins . . . . . . . . . . . . . . . . . . . . . . . . . . 284

25.2.6 Basin spacing and stagger rows . . . . . . . . . . . . . . . . . . . . . . . . 284

25.2.7 Select minimum right border width . . . . . . . . . . . . . . . . . . . . . . 284

25.2.8 Amend the spacing increase for a range of $n$. . . . . . . . . . . . . . . . 285

25.2.9 Accept or revise layout parameters . . . . . . . . . . . . . . . . . . 285

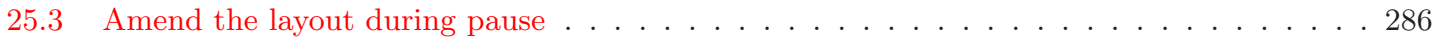

25.3.1 Amend the orientation and fan angle during pause . . . . . . . . . . . . . . 287

25.3.2 Amend the spacing and right border during pause . . . . . . . . . . . . . . . 287

25.3.3 Amend the next position during pause . . . . . . . . . . . . . . . . . . . . 287

25.3.4 Amend the spacing increase during pause . . . . . . . . . . . . . . . . 287

26 Display of attractor basins $\quad 288$

26.1 Compression of equivalent CA dynamics . . . . . . . . . . . . . . . . . . . . . 288

26.1.1 Suppress compression . . . . . . . . . . . . . . . . . . . . . 289

26.1.2 Pre-images of uniform states . . . . . . . . . . . . . . . . . . . 290

26.1.3 Suppress copies of trees (and subtrees) . . . . . . . . . . . . . . . . . . . 291

26.2 Node display . . . . . . . . . . . . . . . . . . . . . . . . . . . . . 292

26.2.1 Node colors . . . . . . . . . . . . . . . . . . . . . . . . . . . 294

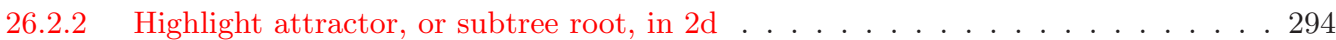

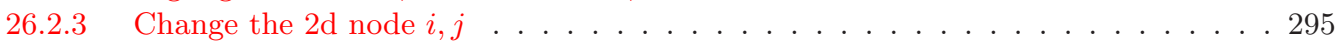

26.2.4 Alter scale, divisions and dots — node as bits/values . . . . . . . . . . . . . 296

26.2.5 Alter decimal or hex node scale . . . . . . . . . . . . . . . . . . . 296 
26.3 Change orientation, fan angle, edge color . . . . . . . . . . . . . . . . . . 296

26.3 .1 Orientation . . . . . . . . . . . . . . . . . . . . . 297

26.3.2 Pre-image fan angle . . . . . . . . . . . . . . . . . . . . 297

26.3.3 Edge color . . . . . . . . . . . . . . . . . . . . . . . 298

27 Pausing attractor basins, and data 292

27.1 Pause stages hierarchy . . . . . . . . . . . . . . . . . . . . . . . 299

27.1.1 Pause after each field for a range of fields . . . . . . . . . . . . . . . . . . 300

27.1.2 Pause after each basin, tree, or pre-image fan . . . . . . . . . . . . . . . 300

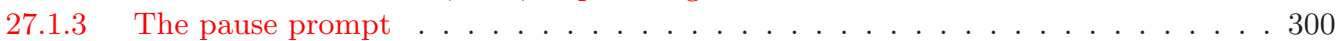

27.2 Attractor basin complete - data window . . . . . . . . . . . . . . . . . . 300

27.2.1 Data on basin of attraction fields . . . . . . . . . . . . . . . . . . 301

27.2.2 Errors in basin of attraction fields . . . . . . . . . . . . . . . . . . . . . 302

27.2.3 Data on basins . . . . . . . . . . . . . . . . . . . . . 302

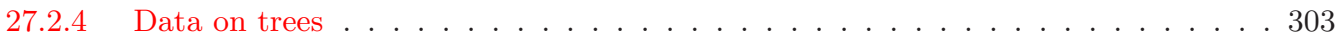

27.2.5 Data on pre-image fans . . . . . . . . . . . . . . . . . . . . . . . . . . . . . . . . . . . . . . . . . . . . . . . . . . . . . .

27.2.6 Data on subtrees . . . . . . . . . . . . . . . . . . . . . 303

27.2.7 Data on subtrees from a uniform state . . . . . . . . . . . . . . . 305

27.3 Print or save data . . . . . . . . . . . . . . . . . . . . . . . . . . . . . . . . . . . . . . . . . . . .

27.4 Data format . . . . . . . . . . . . . . . . . . . . . . . . . . . 307

27.4.1 Network parameters data . . . . . . . . . . . . . . . . . . . . 307

27.4 Basin field data . . . . . . . . . . . . . . . . . . . . . . . . . . . . . . . . . . . . . . . . . . . . . . . . .

27.4 Key to basin data order . . . . . . . . . . . . . . . . . . . . . . 308

27.4 Tree data . . . . . . . . . . . . . . . . . . . . . . . . . . . . 308

27.4.5 Key to tree data order . . . . . . . . . . . . . . . . . . . . . . . . . . . . . . . . . . . . . . . . . . . . . . . . . . . . . .

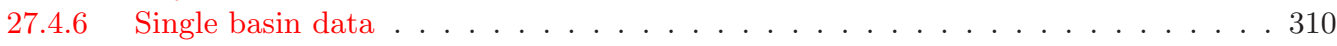

27.4 Subtree data . . . . . . . . . . . . . . . . . . . . . . . 310

27.5 List of states . . . . . . . . . . . . . . . . . . . . . . . . 311

27.5.1 Just the list of states . . . . . . . . . . . . . . . . . . . . . . 311

27.5.2 The list of states with basin data . . . . . . . . . . . . . . . . . . . 312

27.5.3 The list of states with basin and tree data . . . . . . . . . . . . . . . 313

28 Mutation of attractor basins 314

28.1 The first mutation prompt . . . . . . . . . . . . . . . . . . . . . . . 314

28.1.1 The first mutation prompt - options summary . . . . . . . . . . . . . . . . 314

28.2 Mutate wiring . . . . . . . . . . . . . . . . . . . . . . . . 315

28.3 Special mutation options . . . . . . . . . . . . . . . . . . . . . . . 317

28.3.1 Bit-flip or value-flip in sequence . . . . . . . . . . . . . . . . . . . . . . 319

28.4 Flip bits or values . . . . . . . . . . . . . . . . . . . . . . . . . . . . 320

28.5 No pause before next mutant . . . . . . . . . . . . . . . . . . . . . 321

29 Final options for attractor basins $\quad 322$

29.1 Subtree or single basin . . . . . . . . . . . . . . . . . . . . . . . . . . . . 322

29.2 Subtree: Run forward before running backwards . . . . . . . . . . . . . . . . . . . . 323

29.3 Single basin of attraction . . . . . . . . . . . . . . . . . . . . . . . . . . . . . . . . . . . . . . . . . . . . . . . . . . . . .

29.3.1 Single basin history limit . . . . . . . . . . . . . . . . . . . . . . 323

29.3.2 Interrupting while looking for attractor . . . . . . . . . . . . . . . . . . . 323

29.4 Final attractor basin prompt . . . . . . . . . . . . . . . . . . . . . . 324

29.4.1 Final attractor basin prompt - conditions and reminders . . . . . . . . . . . . . 324

29.4.2 Final attractor basin prompt — options summary . . . . . . . . . . . . . . 324

29.5 Limit backward steps . . . . . . . . . . . . . . . . . . . . . . . . . . 325 
29.6 Viewing the partial pre-image stack . . . . . . . . . . . . . . . . . . . . . 327

29.6.1 Partial pre-image stack, local wiring . . . . . . . . . . . . . . . . . . . 327

29.6.2 Partial pre-image stack, nonlocal wiring . . . . . . . . . . . . . . . . . . 327

29.6.3 Reorder to optimize the nonlocal reverse algorithm . . . . . . . . . . . . . . . 329

29.7 Exhaustive algorithm . . . . . . . . . . . . . . . . . . . . . . . . . . 329

29.7.1 Generating the exhaustive list . . . . . . . . . . . . . . . . . . . . 331

29.7.2 Saving the exhaustive pairs . . . . . . . . . . . . . . . . . . 331

29.7.3 Printing the exhaustive pairs in the terminal . . . . . . . . . . . . . . 331

29.8 Random map . . . . . . . . . . . . . . . . . . . . . . . . . . 332

29.8.1 Loading the random map or exhaustive pairs . . . . . . . . . . . . . . . . . . 334

29.8.2 Biasing the random map by Hamming distance . . . . . . . . . . . . . . . . . . 334

29.8.3 Random map data . . . . . . . . . . . . . . . . . . . . . . . . . . . . . . . . . . . . . . . . . . . . . . . . .

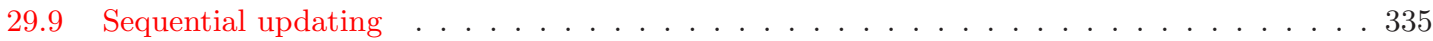

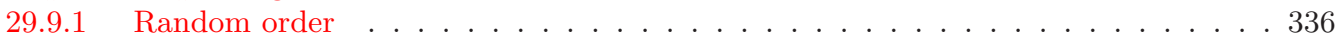

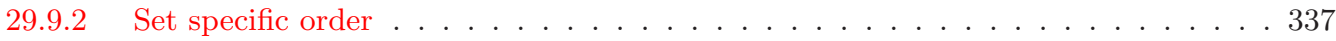

29.9 .3 List all orders . . . . . . . . . . . . . . . . . . . . . . . . 337

29.9.4 List all orders — set order seed . . . . . . . . . . . . . . . . . . . 338

29.9.5 Drawing the attractor basin with sequential updating . . . . . . . . . . . . 338

29.10 Neutral order components . . . . . . . . . . . . . . . . . . . . . . . . . . . . 339

29.10 .1 Neutral subtree . . . . . . . . . . . . . . . . . . . . . . . . 340

29.10 .2 Neutral field . . . . . . . . . . . . . . . . . . . . . 341

30 Drawing attractor basins, and changes on-the-fly 343

30.1 The progress bar for basin of attraction fields . . . . . . . . . . . . . . . . . . . . . 343

30.2 Attractor basins, interrupting and changing . . . . . . . . . . . . . . . . . . . . . . . . . . . . . . . . . . . . . . . . . . . . . .

30.2.1 Abandoning a tree and continuing with the next tree . . . . . . . . . . . . 345

30.2.2 Errors in attractor basins . . . . . . . . . . . . . . . . . . . . 346

30.2.3 Abandon the attractor basin . . . . . . . . . . . . . . . . . . . . . 346

30.3 Attractor basin options, on-the-fly . . . . . . . . . . . . . . . . . . . . . . . 346

30.4 Attractor basin complete prompt . . . . . . . . . . . . . . . . . . . . . . . 347

30.5 Further attractor basin complete options . . . . . . . . . . . . . . . . . . . . 348

30.5.1 Attractor basin - revising rule/s . . . . . . . . . . . . . . . . . . . . . 349

30.5.2 Attractor basin - revising the seed . . . . . . . . . . . . . . . 349

31 Output parameters for space-time patterns 351

31.1 The first output parameter prompt for space-time patterns . . . . . . . . . . . . . 351

31.1.1 Output parameter prompt for space-time patterns — options summary . . . . . 352

31.2 Miscellaneous options - space-time patterns . . . . . . . . . . . . . . . . 353

31.2.1 Color cells by value or neighborhood . . . . . . . . . . . . . . . . . . . 353

31.2.2 State-space matrix and return map . . . . . . . . . . . . . . . . . . . . . 354

31.2.2.1 State-space matrix . . . . . . . . . . . . . . . . . . . . . . . . . . . . . . . . . . . . . . . . . . . . . . . . . . .

31.2.2.2 Return map . . . . . . . . . . . . . . . . . . . . . . . . . . . . . . . . . . . . . . . . . . . . . . . . .

31.2.3 Frozen generation size . . . . . . . . . . . . . . . . . . . . 355

31.2.4 Cell scale . . . . . . . . . . . . . . . . . . . . . . . 356

31.2.5 Space-time patters in other than the native dimension . . . . . . . . . . . . . 356

31.2.6 Scrolling 1d space-time patterns . . . . . . . . . . . . . . . . . 356

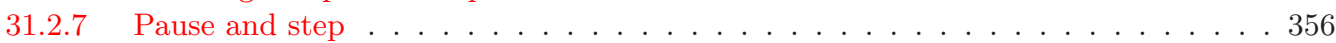

31.2 .8 Glider rule order . . . . . . . . . . . . . . . . . . . . . . . 357

31.2.9 Inverting the kcode in TFO-mode . . . . . . . . . . . . . . . . . . . . 357

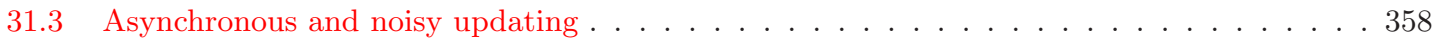

31.3.1 Probabilistic updating . . . . . . . . . . . . . . . . . . . 358 
31.3.2 sequential updating - space-time patterns . . . . . . . . . . . . . . . 359

31.3.3 partial order updating - space-time patterns . . . . . . . . . . . . . . . 361

31.4 Input-entropy and pattern density . . . . . . . . . . . . . . . . . . . . . . . . . 361

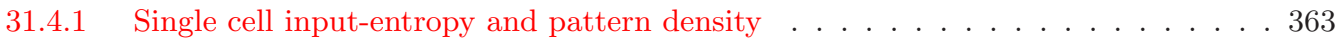

31.4.2 Generation size - moving window of time-steps . . . . . . . . . . . . . . 363

31.4.3 On-the-fly changes to input-entropy and pattern density . . . . . . . . . . . . . 363

31.5 Damage, the difference between two networks . . . . . . . . . . . . . . . . . . . 364

31.5.1 Duplicate the network and seed . . . . . . . . . . . . . . . . . . 365

31.5.2 The Damage Histogram . . . . . . . . . . . . . . . . . . . . . . . 366

31.5.3 Drawing the damage histogram . . . . . . . . . . . . . . . . . . . . 367

31.5.4 Pausing the damage histogram . . . . . . . . . . . . . . . . . . . . 369

31.6 Attractor histogram . . . . . . . . . . . . . . . . . . . . . . 371

31.6.1 Density classification problem — attractor histogram . . . . . . . . . . . . 371

31.6.2 Drawing the attractor histogram . . . . . . . . . . . . . . . . . . . 372

31.6.2.1 Histogram window information . . . . . . . . . . . . . . . . 374

31.6.3 Pausing the attractor histogram . . . . . . . . . . . . . . . . . . . 375

31.6.4 Rescaling the attractor histogram . . . . . . . . . . . . . . . . . 375

31.6.5 Attractor histogram data . . . . . . . . . . . . . . . . . 376

31.6.5.1 Attractor histogram screen data . . . . . . . . . . . . . . 376

31.6.5.2 Attractor histogram data decode . . . . . . . . . . . . . . . 377

31.6.5.3 Histogram data for density rules . . . . . . . . . . . . . . . . . . . . . . . . . . . . . . . . . . . . . .

31.6.6 Print/Save attractor state data . . . . . . . . . . . . . . . . . . . . . . . . . . . . . . . . . . . . . . . . . . . . . . . . . . . .

31.6.7 Sorting the attractor histogram . . . . . . . . . . . . . . . . . . . . . 379

31.6.8 Attractor histogram jump-graph . . . . . . . . . . . . . . . . . . . 379

31.7 Skeleton (fuzzy attractor) histogram . . . . . . . . . . . . . . . . . . . . . . 380

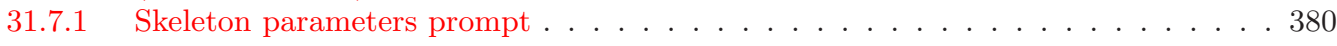

31.7.2 Drawing the Skeleton histogram . . . . . . . . . . . . . . . . . . 382

31.7.3 Pausing the skeleton histogram . . . . . . . . . . . . . . . . . . . 382

31.7.4 Skeleton histogram data . . . . . . . . . . . . . . . . . . . . . 383

31.7.4.1 Skeleton histogram data decode . . . . . . . . . . . . . . . . . . . . . . . . . . . . . . . . . . . .

31.7.5 Sorting the skeleton histogram . . . . . . . . . . . . . . . . . . 385

32 Drawing space-time patterns, and changes on-the-fly 386

32.1 On-the-fly key index . . . . . . . . . . . . . . . . . . . . . . . . . . . . . . . . . . . . . . . . . . . . . . . . . .

32.2 On-the-fly prompts - bottom title bar . . . . . . . . . . . . . . . . . . . . . 388

32.3 Summary of on-the-fly options for space-time patterns . . . . . . . . . . . . . . . . . 388

32.4 Updating (tog) . . . . . . . . . . . . . . . . . . . . . . . . . . . . 394

32.4 U/Y..sync-seq/sync-porder . . . . . . . . . . . . . . . . . . . . . . . 394

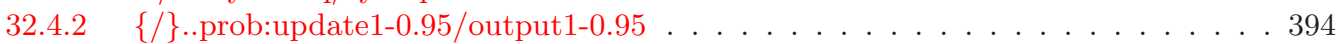

32.5 Change rule . . . . . . . . . . . . . . . . . . . . . . . . . 394

32.5.1 r,R/K/k..rnd rule/tcode/kcode . . . . . . . . . . . . . . . . . . 395

32.5.2 [/]..iso/togflip(on) . . . . . . . . . . . . . . . . . . . . 396

32.5.3 O/A/M/C..Orig/Alt/Maj/Chain . . . . . . . . . . . . . . . . 396

32.5.4 1/2..rnd bitflip/restore . . . . . . . . . . . . . . . . . . 396

32.5.5 Z/z..force Z higher/lower . . . . . . . . . . . . . . . . . . . . . . . . . 397

32.5.6 b/B..flip all0s- $>0 /$ allVs- $>$ V . . . . . . . . . . . . . . . . . . . . 397

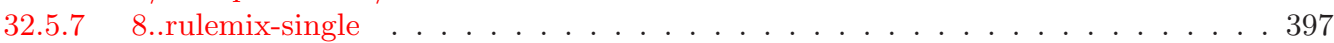

32.6 Rule samples . . . . . . . . . . . . . . . . . . . . . . . . . . 398

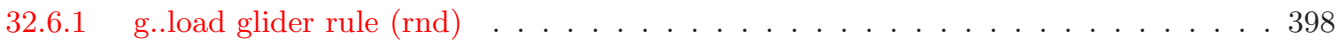

32.6.1.1 creating a glider rule collection . . . . . . . . . . . . . . . . . 398

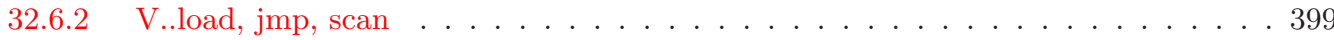




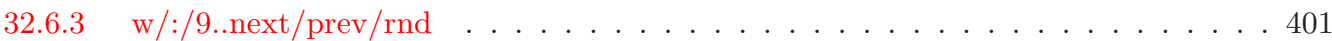

32.6 .4 uE..create sample . . . . . . . . . . . . . . . . . . . . . . . 401

32.7 Change wiring . . . . . . . . . . . . . . . . . . . . . . . . . 402

32.7.1 m/W..move 1 wires . . . . . . . . . . . . . . . . . . . . . . 402

32.7.2 7..nonlocal-local . . . . . . . . . . . . . . . . . . . . 402

32.8 Change seed/size . . . . . . . . . . . . . . . . . . . . . . . . . . 403

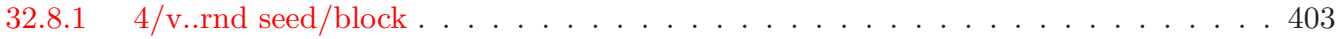

32.8.2 l/L..rnd value/block . . . . . . . . . . . . . . . . . . . . . . . . 403

32.8.3 o/ ..original/last . . . . . . . . . . . . . . . . . . . . . . . . . . . 404

32.8.4 5/6..singleton pos $/$ neg . . . . . . . . . . . . . . . . . . . . . . . 404

32.8.5 5/6..singleton zero/rnd . . . . . . . . . . . . . . . . . . . . . . 404

32.8.6 N/n..inc/decrease 1 cell . . . . . . . . . . . . . . . . . . . . . . . . . . . 404

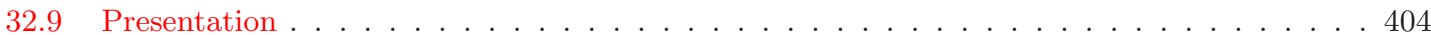

32.9.1 x..tog slant(off) . . . . . . . . . . . . . . . . . . . . . . . 404

32.9.2 x..tog hex(off) . . . . . . . . . . . . . . . . . . . . 405

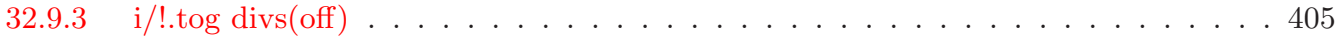

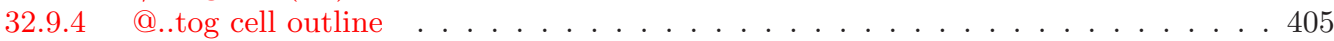

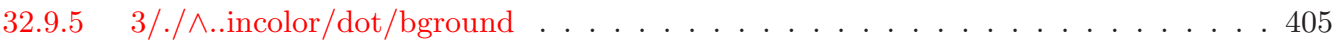

32.9.6 d/-..colors:swap/black-blue ........................ . 406

32.9.7 d/-..tog shuffle colors/restore . . . . . . . . . . . . . . . . . . . . . 407

32.9.8 S..tog space-time display . . . . . . . . . . . . . . . . . . . . . . 407

32.9.9 P..tog skip steps $=1$ (off) . . . . . . . . . . . . . . . . . . . . . 407

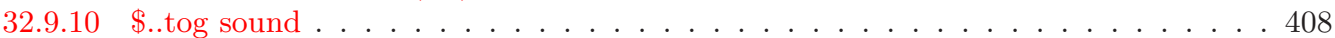

32.9.11 e/c..expand/contr scale . . . . . . . . . . . . . . . . . . . 408

$32.101 \mathrm{~d} 2 \mathrm{~d} 3 \mathrm{~d} \ldots \ldots \ldots \ldots \ldots \ldots \ldots$

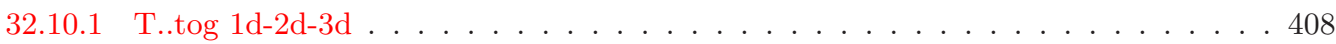

32.10 .2 t..tog $2 \mathrm{~d}-2 \mathrm{~d}+$ time . . . . . . . . . . . . . . . . . . . . 409

32.10 .3 I..tog balls . . . . . . . . . . . . . . . . . . . . . . . . 410

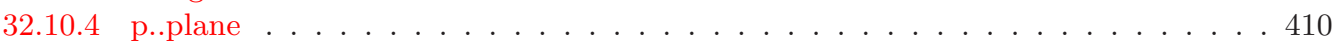

32.10 .5 J..invisible . . . . . . . . . . . . . . . . . . . . . . . 410

32.11 Frozen/Filter . . . . . . . . . . . . . . . . . . . . . . . . . 410

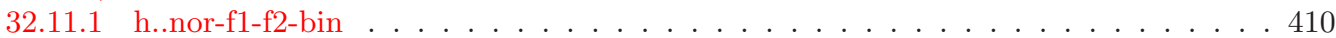

32.11 .2 H..f-gens $($ now 20)/bins(10) . . . . . . . . . . . . . . . . . . 413

32.11 .3 frozen generations . . . . . . . . . . . . . . . . . . . . . . . . . . . . . . . . . . . . . . . . . . . . . . . . .

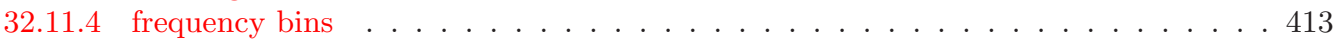

$32.11 .5 \mathrm{f} / \mathrm{F} / \mathrm{a}$. filter$/$ undo/all . . . . . . . . . . . . . . . . . . . . . . . . . . 414

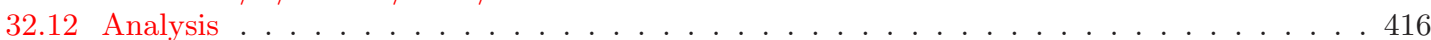

32.12.1 0/\%..tog lookuphist:1-2/1-time . . . . . . . . . . . . . . . . 4 418

32.12 .2 )/(.lookhist: amplify/restore . . . . . . . . . . . . . . . . . . . 418

32.12 .3 s..tog entropy-density . . . . . . . . . . . . . . . . . . . . . . . 419

32.12 .4 j..tog ent-in-both . . . . . . . . . . . . . . . . . . . . 419

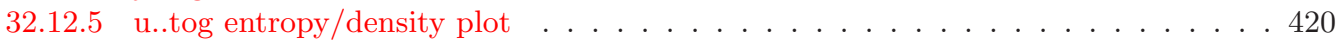

32.12 .6 G..a-gens (now 10) . . . . . . . . . . . . . . . . . . . . 420

32.12.7 D..return map . . . . . . . . . . . . . . . . . . . . . . . 420

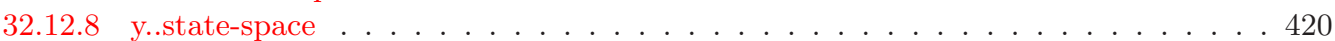

$32.12 .9=$..tog diff (keep damage) . . . . . . . . . . . . . . . . . . . . . . . . 420

32.13 Miscellaneous ... . . . . . . . . . . . . . . . . . . . . . . . 421

32.13 .1 X..index display . . . . . . . . . . . . . . . . . . . . . . . 421

32.13.2 *..tog end pause $($ on) . . . . . . . . . . . . . . . . . . . . . . . . 421

32.13.3 \#/\&.tog scrolling . . . . . . . . . . . . . . . . . . . . . . 421

32.13.3.1 1d scrolling . . . . . . . . . . . . . . . . . . 421 
32.13.3.2 2d diagonal scrolling . . . . . . . . . . . . . . . . . . 421

32.13.3.3 network-graph scrolling .................... . 421

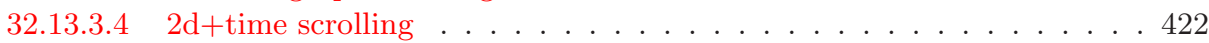

$32.13 .4+$ +.tog time-step pause . . . . . . . . . . . . . . . . . . . . . 422

$32.13 .5<$..slow $/$ max speed . . . . . . . . . . . . . . . . . . . . . 422

32.13 .6 q/Q..pause/no-options . . . . . . . . . . . . . . . . . . . . . . 422

32.14 Interrupting space-time patterns . . . . . . . . . . . . . . . . . . . . . . 423

32.15 Rule details for space-time patterns . . . . . . . . . . . . . . . . . . . . . . . . . . 423

32.16 Space-time pattern interrupt/pause prompt . . . . . . . . . . . . . . . . . . . . . . 424

32.16.1 Revising rule/net . . . . . . . . . . . . . . . . . . . . . . 425

32.16.2 Classified samples of rule-space $-\mathrm{load} /$ keep . . . . . . . . . . . . . . . . . . 426

32.16.3 Directly scanning for PostScript . . . . . . . . . . . . . . . . . . . . . . . . . 426

32.16.4 Revising the seed and native PostScript . . . . . . . . . . . . . . . . . . . . . . 426

32.16.5 Space-time patterns on the network-graph . . . . . . . . . . . . . . . . . . . 4427

32.16.6 Network border . . . . . . . . . . . . . . . . . . . . . . . . . . 428

32.16.7 Finer control of filtering . . . . . . . . . . . . . . . . . . . . . . . . . 428

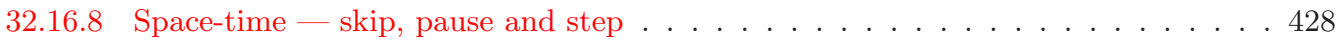

32.16 .9 Miscellaneous options . . . . . . . . . . . . . . . . . . . . . . . . 429

32.17 Quit and further options . . . . . . . . . . . . . . . . . . . . . . . . . 430

32.18 Directly scanning space-time patterns for vector PostScript . . . . . . . . . . . . . . . 431

32.19 Network-graph layout of space-time patterns . . . . . . . . . . . . . . . . . . . 433

32.19.1 On-the-fly options within the network-graph . . . . . . . . . . . . . . . . . . 434

32.19.2 Network-graph space-time pattern as vector PostScript . . . . . . . . . . . . 436

33 Classifying rule space $\quad 437$

33.1 Input entropy and variability (min-max or sdev) . . . . . . . . . . . . . . . . . . . 440

33.2 Creating a rule sample — initial prompts . . . . . . . . . . . . . . . . . . . . . 440

33.2.1 Biasing random rules . . . . . . . . . . . . . . . . . . . . . . . . 442

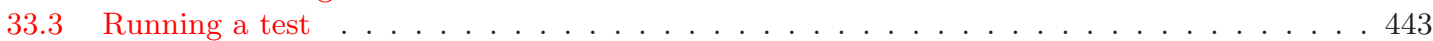

33.4 Creating an automatic rule sample . . . . . . . . . . . . . . . . . . . . . . . . . . . 444

33.5 Loading, sorting and displaying a sample . . . . . . . . . . . . . . . . . . . . 447

33.6 The rule sample scatter plot . . . . . . . . . . . . . . . . . . . . . . . . . . . . 448

33.6.1 Probing the scatter plot with the mouse, and selecting rules . . . . . . . . . . . . 448

33.6.2 The scatter plot as a $2 \mathrm{~d}$ frequency histogram . . . . . . . . . . . . . . . . . 451

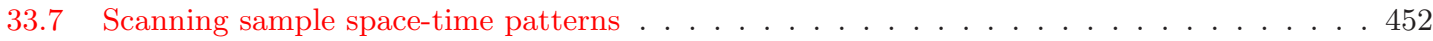

33.7.1 Scanning on-the fly . . . . . . . . . . . . . . . . . . . . . . . . . . . . . . . . . . . . . . . . . . . . . . . . . . .

33.7.2 Scanning automatically in blocks of time-steps . . . . . . . . . . . . . . . . 4 . . . . . . . . . . . . . . . . . . . .

33.7.3 Scanning $2 \mathrm{~d}$ or $3 \mathrm{~d}$ samples . . . . . . . . . . . . . . . . . . . . . . . . 454

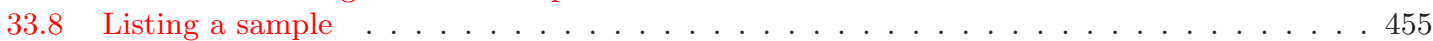

33.8.1 Selecting a rule from the list . . . . . . . . . . . . . . . . . . . . 457

33.9 Listing by plot coordinates or probing with the mouse . . . . . . . . . . . . . . . . . 457

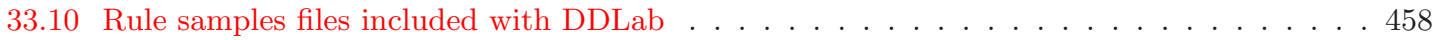

33.11 Rule sample encoding . . . . . . . . . . . . . . . . . . . . . . . . . . . . . . . . . . . . . . . . . . . . . . . . . . .

34 Learning, forgetting, and highlighting 459

34.1 Learning/forgetting methods . . . . . . . . . . . . . . . . . . . . . . . 460

34.1.1 Highlighting states in attractor basins . . . . . . . . . . . . . . . . . . . . . 461

34.1 Basin layout for learning . . . . . . . . . . . . . . . . . . . . . 461 . . . . . . . . . . . . . . . . . . . . . . . .

34.2 Selecting the learn/forget/highlight window . . . . . . . . . . . . . . . . . . . 462

34.2.1 Selecting the wiring graphic . . . . . . . . . . . . . . . . . . . . . . . . . . . . . . . . . . . . . . . . . . . . . .

34.2.2 Selecting the network architecture prompt . . . . . . . . . . . . . . 463 


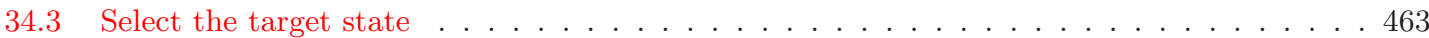

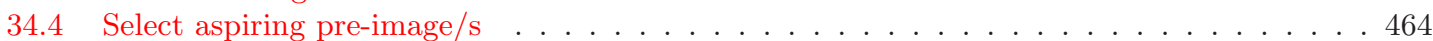

34.4.1 Odd or even parity . . . . . . . . . . . . . . . . . . . . . . . . . . . . . . . . . . . . . . . . . . . . . . . . . . . . . . . .

34.4.2 Range of decimal equivalents . . . . . . . . . . . . . . . . . . . . . . . . . . . . . . . . . . . . . . . . . . . . . . .

34.4.3 Pre-images according to Hamming distance . . . . . . . . . . . . . . . . . 465

34.4.4 List of aspiring pre-images . . . . . . . . . . . . . . . . . . . . . . . . . . . . . . . . . . . . . . . . . . . . . . . . .

34.4.5 Review target state and aspiring pre-images . . . . . . . . . . . . . . . . . . . . . . . . . . . . . . . . . . . . . . . . . .

34.5 Learn, forget, or highlight only . . . . . . . . . . . . . . . . . . . . . 466

34.5.1 1d CA - local wiring and highlighting . . . . . . . . . . . . . . . . 467

34.5.2 Nonlocal wiring — learn, forget, or highlight only . . . . . . . . . . . . . . . . 467

34.5.3 Learning/forgetting by wire moves or bit/value-flips . . . . . . . . . . . . . . . . 468

34.6 Learning/forgetting by bit/value-flips . . . . . . . . . . . . . . . . . . . . . . 468

34.7 Learning/forgetting by wire-moves . . . . . . . . . . . . . . . . . . . . . . 469

34.8 Highlighting options . . . . . . . . . . . . . . . . . . . . . . . . . 472

34.9 Learning/forgetting/highlighting complete . . . . . . . . . . . . . . . . . . . . . . . 472

35 Filing

35.1 File types, default filenames, and extensions . . . . . . . . . . . . . . . . . . . . 474

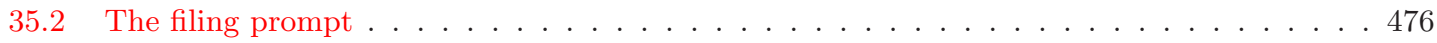

35.3 Loading constraints on the value-range $[v, k]$ - rules . . . . . . . . . . . . . . . . . 477

35.4 Loading constraints on the value-range $[v, n]$ - seeds . . . . . . . . . . . . . . . . 477

35.5 Changing the directory . . . . . . . . . . . . . . . . . . . . . . 477

35.6 List files . . . . . . . . . . . . . . . . . . . . . . . . . . . . . . . . . . . . . .

36 Vector PostScript capture of DDLab output 479

36.1 Cropping and editing vector PostScript . . . . . . . . . . . . . . . . . . . . . . 480

36.1.1 Cropping the PostScript image . . . . . . . . . . . . . . . . . . . . . . . 480

36.1.2 Amending the width of lines . . . . . . . . . . . . . . . . . . . . . 440

37 Glossary

\begin{tabular}{ll} 
References & 487 \\
\hline
\end{tabular}

Index 


\section{List of Figures}

1.1 The basin of attraction field of a Cellular Automaton . . . . . . . . . . . . . . . . 1

1.2 Themes in DDLab . . . . . . . . . . . . . . . . . . . . . . . . 2

1.3 The space-time pattern of a $1 \mathrm{~d}$ complex $\mathrm{CA}$ with interacting gliders . . . . . . . . . 5

1.4 The basin of attraction field of a complex CA . . . . . . . . . . . . . . . . . 6

1.5 A detail of a basin of attraction . . . . . . . . . . . . . . . . . . 6

2.1 Cell value color scheme . . . . . . . . . . . . . . . . . . . . . . . . . . 10

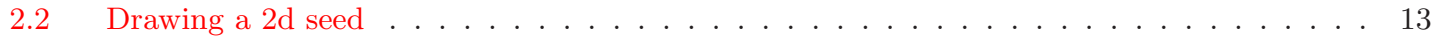

2.3 Space-time patterns of a $1 \mathrm{~d}$ CA . . . . . . . . . . . . . . . . . . . . 14

2.4 The basin of attraction field of a random Boolean network . . . . . . . . . . . . . . . 15

2.5 A basin of attraction of a random Boolean network . . . . . . . . . . . . . . . . 16

2.6 Order-chaos measures for RBN . . . . . . . . . . . . . . . . . . . . . . . 19

2.7 1d CA space-time patterns showing ordered, complex and chaotic dynamics . . . . . . . 20

2.8 Subtrees of ordered-complex-chaotic CA . . . . . . . . . . . . . . . . . . . . . 22

4.1 A basin of attraction field . . . . . . . . . . . . . . . . . . . . . 32

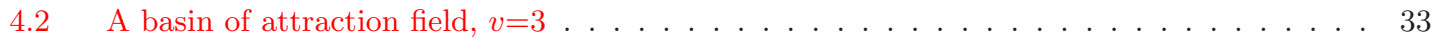

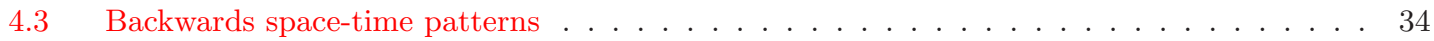

4.4 The state-apace matrix . . . . . . . . . . . . . . . . . . . . . 34

4.5 Basin of attraction fields for a range of network size . . . . . . . . . . . . . . . . . 35

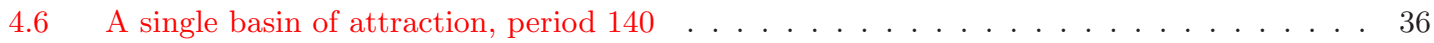

4.7 A single basin with a point attractor . . . . . . . . . . . . . . . . . . . 36

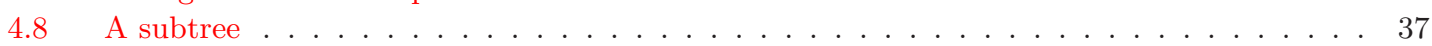

4.9 A 1d space-time pattern . . . . . . . . . . . . . . . . . . . . 39

4.10 A 1d CA shown as a scrolling ring of cells. . . . . . . . . . . . . . . . . . . . . . 40

4.11 A 1d CA and filtered Altenberg rule . . . . . . . . . . . . . . . . . . . . . 41

4.12 Space-time patterns of the 2 d game-of-Life . . . . . . . . . . . . . . . . . . . . 43

4.13 Space-time snapshots of the $2 \mathrm{~d}$ game-of-Life - frozen options . . . . . . . . . . . . . . 43

4.14 Space-time patterns of the 2 d game-of-Life scrolling diagonally . . . . . . . . . . . . . . . 44

$4.152 \mathrm{~d}$ space-time patterns, $v 2 k 7 \mathrm{CA}$ on a hexagonal grid . . . . . . . . . . . . . . . . 45

4.162 2d space-time patterns, v3k6 k-totalistic $2 \mathrm{~d}$ CA . . . . . . . . . . . . . . . . . 46

4.17 Examples of $3 \mathrm{~d} v 2 k 7 \mathrm{CA} \ldots \ldots \ldots \ldots$

4.18 The screen-saver . . . . . . . . . . . . . . . . . . . . . . . . . . . . . 49

5.1 Typical graphical user interface when starting DDLab . . . . . . . . . . . . . . . . . . 53

5.2 The main sequence of prompts . . . . . . . . . . . . . . . . . . . . . . . 57

6.1 A very small DDLab window in Linux . . . . . . . . . . . . . . . . . . . . . . . 64 
$7.1 \quad$ Value color key, $\mathrm{v}=2$ to $8 \ldots \ldots \ldots$

8.1 Basin of attraction fields for a range of network size . . . . . . . . . . . . . . . . . 69

8.2 Single basins for a range of network size . . . . . . . . . . . . . . . . . . . . . . 70

8.3 Subtrees for a range of network size . . . . . . . . . . . . . . . . . . . . . . . 71

8.4 A subtree for a $1 \mathrm{~d}$ CA chain-rule . . . . . . . . . . . . . . . . . . . . . . . 72

8.5 Basin of attraction fields for a range of network sizes, $k=5$. . . . . . . . . . . . . . 72

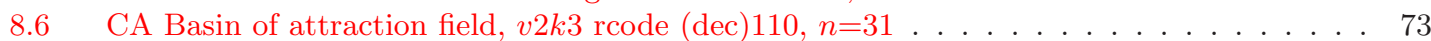

8.7 Basin of attraction fields for a range of network sizes, RBN, v2k3 . . . . . . . . . . . . 74

9.1 A normal $k$ distribution . . . . . . . . . . . . . . . . . . . . . . 77

9.2 A power-law $k$ distribution . . . . . . . . . . . . . . . . . . . . . 79

9.3 An example $k$-mix . . . . . . . . . . . . . . . . . . . . . . . . . 80

10.1 Neighborhood indexing 1d . . . . . . . . . . . . . . . . . . . . 83

$10.21 \mathrm{~d}$ neighborhood templates . . . . . . . . . . . . . . . . . . . . 83

10.32 d neighborhood templates . . . . . . . . . . . . . . . . . . . . . 84

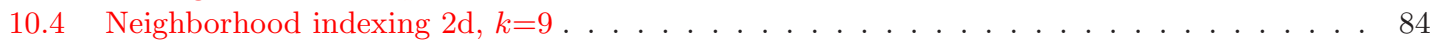

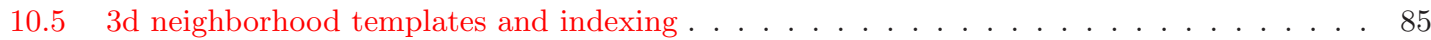

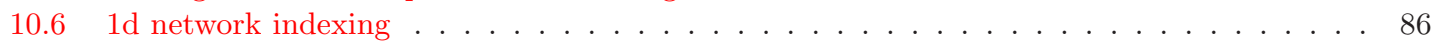

10.72 d network indexing . . . . . . . . . . . . . . . . . . . . . . . 86

10.83 d network indexing . . . . . . . . . . . . . . . . . . . . . . . . . . . 87

10.93 d network indexing, $i, j, h=1 \ldots \ldots \ldots$

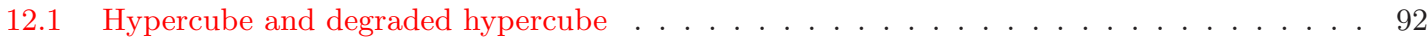

12.2 Confining $1 \mathrm{~d}$ random wiring within a local zone . . . . . . . . . . . . . . . . . . . 95

12.3 Confining $2 \mathrm{~d} k=9$ random wiring within a local zone . . . . . . . . . . . . . . . . . 95

12.4 Confining 3d random wiring within a local zone . . . . . . . . . . . . . . . . . . . 95

12.5 Setting wiring by hand on the blank wiring matrix . . . . . . . . . . . . . . . . . . . 98

13.1 The binary neighborhood neighborhood matrix $k=1$ to 9 . . . . . . . . . . . . . . 103

13.2 Multi-value neighborhood matrix examples . . . . . . . . . . . . . . . . . . 105

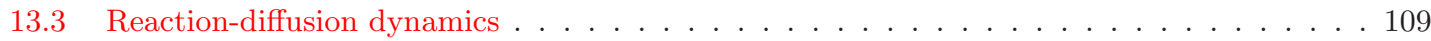

14.1 Glider guns in $v=8$ Life . . . . . . . . . . . . . . . . . . . . . . . . . . . . . 113

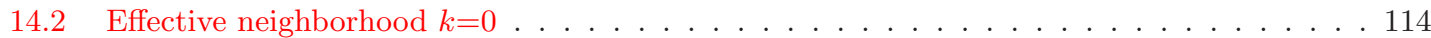

14.3 2d CA perturbed by a chaotic block of rules . . . . . . . . . . . . . . . . . . . 116

14.4 Shifted majority kcode-mix with random wiring . . . . . . . . . . . . . . . . . . 120

14.5 Multi-value neighborhood matrix, part only . . . . . . . . . . . . . . . . . 121

15.1 Canalyzing frequency/saturation - homogeneous $k \ldots \ldots . . . . . . . . . . .126$

15.2 Canalyzing saturation $-2 \mathrm{~d}$, mixed $k \ldots \ldots \ldots \ldots \ldots \ldots$

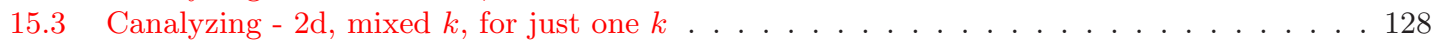

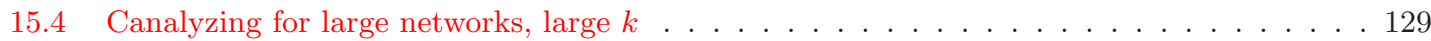

16.1 Setting bits starting with all $0 \mathrm{~s} \ldots \ldots \ldots \ldots \ldots$

16.2 Setting values — alternative presentations . . . . . . . . . . . . . . . . . . . 137

16.3 Drawing bits or values on the rcode pattern . . . . . . . . . . . . . . . . . . . . . . . 139

16.4 Setting rcode in hex . . . . . . . . . . . . . . . . . . . . . . . . . . 141

16.5 Majority rcode $v 2 k 9$. . . . . . . . . . . . . . . . . . . . . . . . . . . . . . . . . . . . . . 142

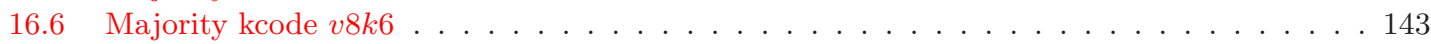

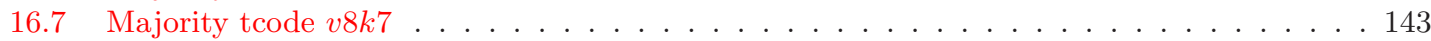


16.8 Flipped $v=2$ majority rcode with random wiring . . . . . . . . . . . . . . . . . . . . . . 144

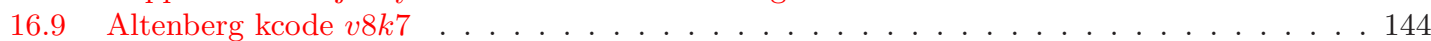

16.10 The game-of-Life rule . . . . . . . . . . . . . . . . . . . . . . . . . . . 145

16.11 Fredkin's replicator . . . . . . . . . . . . . . . . . . . . . . . . 145

16.12 Glider guns in $v=3$ Life . . . . . . . . . . . . . . . . . . . . . . . . . . . . . . . 146

16.13 Encryption with chain-rules . . . . . . . . . . . . . . . . . . . . 147

16.14 Analogous kcode with increased $k$. . . . . . . . . . . . . . . . . . . . . . . 150

16.15 Equivalent kcode - two values swapped, v3k5 . . . . . . . . . . . . . . . . . 153

16.16 Rule window examples . . . . . . . . . . . . . . . . . . . . . . . . . . . . . . . . . . . .

17.1 Wiring matrix examples . . . . . . . . . . . . . . . . . . . . . . 158

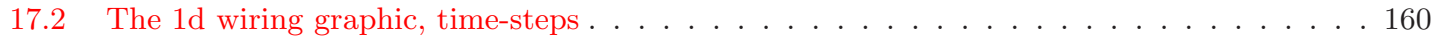

17.3 The $1 \mathrm{~d}$ circle wiring graphic . . . . . . . . . . . . . . . . . . . . . . . . . . 164

17.4 The 1d graphic, showing wiring to a block . . . . . . . . . . . . . . . . . . 165

17.5 The 1d graphic, showing the wiring of the whole network . . . . . . . . . . . . . 166

17.6 Include the pseudo-neighborhood or direct wiring, 1d . . . . . . . . . . . . . . . . 167

17.7 Recursive inputs (direct and indirect) to a cell . . . . . . . . . . . . . . . . . . . 169

17.8 Recursive outputs (direct and indirect) from a cell . . . . . . . . . . . . . . . . . . 170

17.9 Untangling the wiring . . . . . . . . . . . . . . . . . . . . . . 171

17.10 The $2 \mathrm{~d}$ wiring graphic, direct wiring or pseudo-neighborhood . . . . . . . . . . . . . 172

17.11 Alternative ways of showing cell connections, 2d . . . . . . . . . . . . . . . 173

17.12 2d block, alternative presentations . . . . . . . . . . . . . . . . . . . . . 174

17.13 3d wiring graphic . . . . . . . . . . . . . . . . . . . . . . . . 176

17.14 3d wiring graphic showing pseudo-neighborhood . . . . . . . . . . . . . . . . . 177

17.15 Alternative ways of showing cell connections, $3 \mathrm{~d}$. . . . . . . . . . . . . . . . . 178

17.16 3d block, alternative presentations . . . . . . . . . . . . . . . . . . . . 179

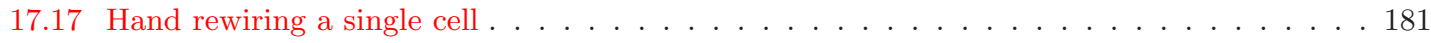

17.18 Histograms of a power-law distribution of network links . . . . . . . . . . . . . . . 185

18.1 Transforming rcode . . . . . . . . . . . . . . . . . . . . . . . . 188

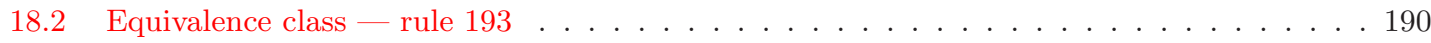

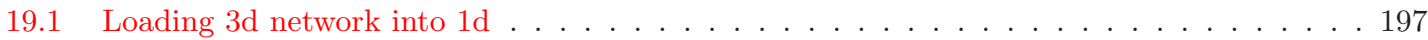

19.2 Loading a $3 \mathrm{~d}$ CA into a $2 \mathrm{~d}$ CA . . . . . . . . . . . . . . . . . . . . . . . . . . . . . . . . . . . . . . . . . . . . . . . . . . . . .

19.3 Loading a DDN into a CA . . . . . . . . . . . . . . . . . . . . . . . . . . 199

19.4 Loading a $2 \mathrm{~d}$ CA into a larger $2 \mathrm{~d}$ CA . . . . . . . . . . . . . . . . . . . 200

19.5 Loading a small 3d DDN into a 3d DDN . . . . . . . . . . . . . . . . . . . 200

19.6 Space-time snapshot of a 2d CA inside another $2 \mathrm{~d}$ CA with different $k$. . . . . . . . 201

20.1 Simple network-graphs . . . . . . . . . . . . . . . . . . . . . . 205

20.2 Power-law network-graphs) . . . . . . . . . . . . . . . . . . . . . 206

20.3 The jump-graph + basins . . . . . . . . . . . . . . . . . . . . . . . . 208

20.4 Screen shot of the basin of attraction field and jump-graph . . . . . . . . . . . . . . 209

20.5 Dragging the jump-graph, further examples . . . . . . . . . . . . . . . . . . . . 210

20.6 Unscrambling the network-graph of a scale-free RBN . . . . . . . . . . . . . . . . . . 211

20.7 Dragging network-graph nodes and fragments $-1 \mathrm{~d} \ldots \ldots . . . . . . . . . .213$

20.8 Dragging network-graph nodes and fragments $-2 \mathrm{~d}$. . . . . . . . . . . . . . . 213

20.9 Dragging network-graph nodes and fragments $-3 \mathrm{~d}$. . . . . . . . . . . . . . . . . 214

20.10 Probabilistic ant hits . . . . . . . . . . . . . . . . . . . . . . . . . . . 219

20.11 Inserting basins in the jump-graph . . . . . . . . . . . . . . . . . . . . . . . 220

20.12 Basins of attraction at the jump-graph nodes . . . . . . . . . . . . . . . . . . 221

20.13 Disconnecting and isolating unreachable nodes . . . . . . . . . . . . . . . . . 223 
20.14 A 2d CA shown as a scrolling ring of cells. . . . . . . . . . . . . . . . . . . . . 226

21.1 Seed array indexing, $1 \mathrm{~d} \ldots \ldots \ldots \ldots \ldots \ldots \ldots \ldots$

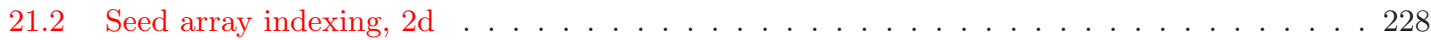

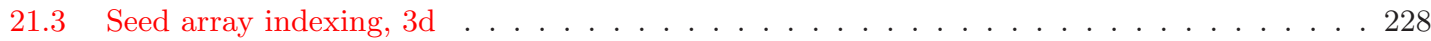

21.4 Setting a seed at random . . . . . . . . . . . . . . . . . . . . . . 231

21.5 Setting the density bias . . . . . . . . . . . . . . . . . . . . . . 232

21.6 Drawing bits or values on a $2 \mathrm{~d}$ seed . . . . . . . . . . . . . . . . . . . . . . . . . . 233

21.7 Examples of PostScript seed output . . . . . . . . . . . . . . . . . . . . . 237

21.8 Symbols for PostScript seed output . . . . . . . . . . . . . . . . . . . . . . 240

$21.93 \mathrm{~d}$ bit/value seed . . . . . . . . . . . . . . . . . . . . . . . . 241

21.10 Loading a $2 \mathrm{~d}$ seed into a $3 \mathrm{~d}$ network . . . . . . . . . . . . . . . . . . . . . . . 242

21.11 Setting the seed in hex . . . . . . . . . . . . . . . . . . . . . . 242

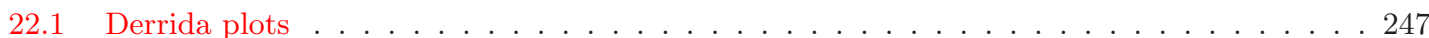

22.2 The Derrida coefficient . . . . . . . . . . . . . . . . . . . . . 251

23.1 Basins of attraction - the idea . . . . . . . . . . . . . . . . . . 253

23.2 Point attractors $($ period $=1) \ldots \ldots \ldots \ldots \ldots \ldots$. . . . . . . . . . . . . . . 254

23.3 The pre-image fan of a single pre-image of a point attractor . . . . . . . . . . . . . . . . 254

23.4 Attractors with period 2 . . . . . . . . . . . . . . . . . . . . . . . 254

23.5 Attractors with periods $\geq 3 \ldots \ldots \ldots \ldots \ldots \ldots \ldots$

23.6 Subtrees from a root state . . . . . . . . . . . . . . . . . . . . 256

24.1 The state-space matrix, $n=6$. . . . . . . . . . . . . . . . . . . . . . 262

24.2 The state-space matrix, $n=12,13 \ldots \ldots \ldots \ldots$

24.3 State-space matrix, further examples . . . . . . . . . . . . . . . . . . . . . . . . . . . . 264

24.4 In-degree histogram of a basin of attraction field, $n=18 \ldots \ldots$. . . . . . . . . . . . 265

24.5 Rescaling the in-degree histogram of a subtree . . . . . . . . . . . . . . . . . 266

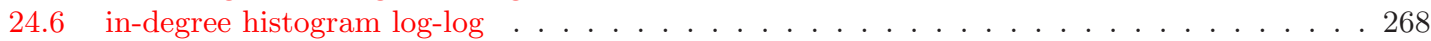

24.7 Density classification in emergent computation . . . . . . . . . . . . . . . . . 269

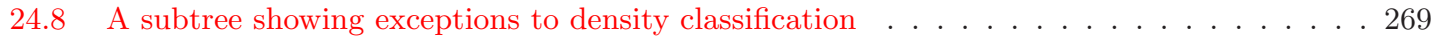

24.9 Basin of attraction field of a density classification rule . . . . . . . . . . . . . . . . . . 270

24.10 A view of rule-space . . . . . . . . . . . . . . . . . . . . . . . 271

24.11 The attractor basin window while drawing graphs . . . . . . . . . . . . . . . . . . . . . 273

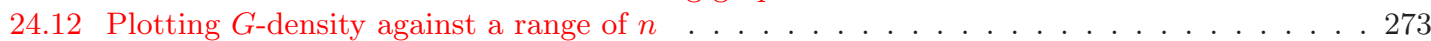

24.13 G-density against both $\lambda_{\text {ratio }}$ and $Z \ldots \ldots \ldots \ldots \ldots$. . . . . . . . . . . . . 274

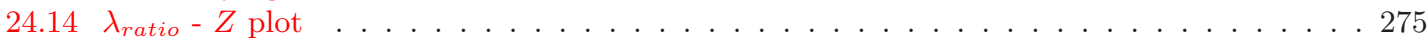

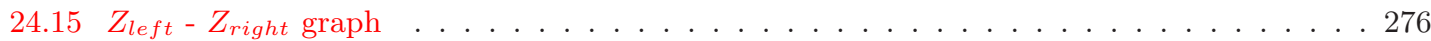

24.16 "Backwards" space-time patterns . . . . . . . . . . . . . . . . . . . . 279

25.1 Layout preview, single basin . . . . . . . . . . . . . . . . . . . . . . . . . . . . . . . . 282

25.2 Layout preview, basin field . . . . . . . . . . . . . . . . . . . . . . . . . . 282

25.3 The layout preview . . . . . . . . . . . . . . . . . . . . . . . 283

25.4 The basin of attracion field for a range of network size . . . . . . . . . . . . . . . . . 285

25.5 Amending the layout of a basin of attraction field on-the-fly . . . . . . . . . . . . . 286

26.1 Compression of equivalent dynamics . . . . . . . . . . . . . . . . . . . . . . . 289

26.2 Compression suppressed . . . . . . . . . . . . . . . . . . . . . . . . . 289

26.3 CA uniform state attractors . . . . . . . . . . . . . . . . . . . . . . 290

26.4 Suppressing equivalent subtrees . . . . . . . . . . . . . . . . . . . . . . . 291

26.5 Alternative node display . . . . . . . . . . . . . . . . . . . . . . . 292 
26.6 Symmetrical states only . . . . . . . . . . . . . . . . . . . . . . 293

26.7 Just leaf-states or exclude leaf-states . . . . . . . . . . . . . . . . . . . . . . . . . 293

26.8 Bit pattern colors of a uniform state's pre-images . . . . . . . . . . . . . . . . . . . . . 294

26.9 Highlighting all non-equivalent attractor states . . . . . . . . . . . . . . . . . . 295

26.10 Highlighting all attractor states in a RBN . . . . . . . . . . . . . . . . . . . . . . 295

26.11 Changing the orientation of attractor basins . . . . . . . . . . . . . . . . . . . 297

26.12 Decreasing the pre-image fan-angle of a subtree . . . . . . . . . . . . . . . . . . . . . 297

26.13 Increasing the pre-image fan-angle of a basin of attraction . . . . . . . . . . . . . . . . 298

26.14 Alternative edge start colors . . . . . . . . . . . . . . . . . . . . . . . . . . 298

27.1 A basin of attraction field of a CA illustrating data . . . . . . . . . . . . . . . . 301

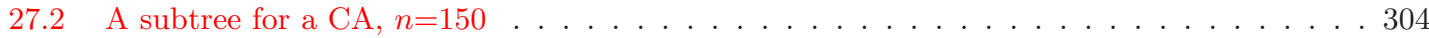

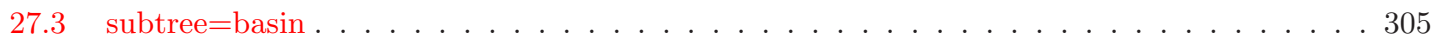

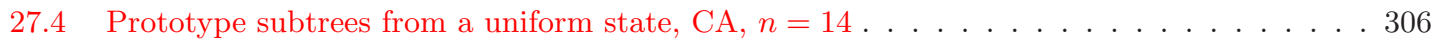

27.5 Detail of basin rule 110 . . . . . . . . . . . . . . . . . . . . . . . 310

27.6 Listing states of a basin of attraction field . . . . . . . . . . . . . . . . . . . . 311

28.1 Mutation by one wire move . . . . . . . . . . . . . . . . . . . . . . 316

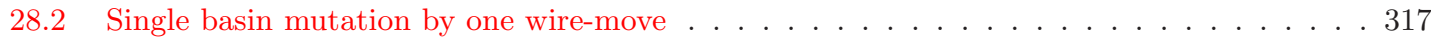

28.3 Single basin mutation by one bit-flip . . . . . . . . . . . . . . . . . . . . . . . . . . . 318

28.4 Mutation of field by one bit-flip . . . . . . . . . . . . . . . . . . . . . . . . . 319

28.5 Single basin mutation by one value-flip . . . . . . . . . . . . . . . . . . . . . . . 320

29.1 Basin of attraction — limiting backward steps . . . . . . . . . . . . . . . . . . 326

29.2 Partial pre-image stack of a $1 \mathrm{~d}$ local CA . . . . . . . . . . . . . . . . . . . . . . . . . . . . . . . . . . . . . . . . . . . . . . . . .

29.3 Partial pre-image stack of a $1 \mathrm{~d}$ nonlocal CA . . . . . . . . . . . . . . . . . . . . 328

29.4 The final partial pre-image histogram, not reordered . . . . . . . . . . . . . . . . . . . . 329

$29.5 \mathrm{CA}, \mathrm{DDN} / \mathrm{RBN}$, and random maps . . . . . . . . . . . . . . . . . . . . . . . . . . . 330

29.6 Random map graphs with different Hamming bias . . . . . . . . . . . . . . . . . . . 333

29.7 Basin of attraction field with sequential updating . . . . . . . . . . . . . . . . . . . . . . . . . . . . . . . . . . . . . . . . .

29.8 The rule window for sequential updating . . . . . . . . . . . . . . . . . . . . . . 338

29.9 Neutral subtree . . . . . . . . . . . . . . . . . . . . . . . . . . . 339

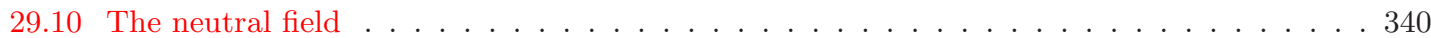

30.1 The basin of attraction field progress bar. . . . . . . . . . . . . . . . . . . . . . 343

30.2 Drawing a basin of attraction field . . . . . . . . . . . . . . . . . . . . . . . 344

31.1 1d space-time pattern, colors by value and neighborhood . . . . . . . . . . . . . 353

31.2 2d space-time pattern, colors by value and neighborhood . . . . . . . . . . . . . . 353

31.3 3d space-time pattern, colors by value and neighborhood . . . . . . . . . . . . . . . 354

31.4 State space matrix . . . . . . . . . . . . . . . . . . . . . . . . . . . 354

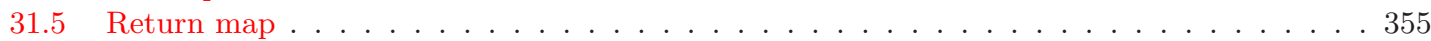

31.6 Probabilistic updating . . . . . . . . . . . . . . . . . . . . . . 359

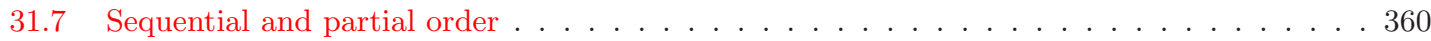

31.8 Pattern Density and Input-Entropy plots . . . . . . . . . . . . . . . . . . . . . . . 362

31.9 The difference in the dynamics between two $1 \mathrm{~d}$ CA . . . . . . . . . . . . . . . . 365

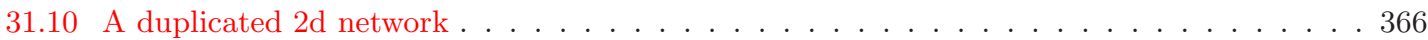

31.11 A duplicated $2 \mathrm{~d}$ seed . . . . . . . . . . . . . . . . . . . . . . . . . . . . . 366

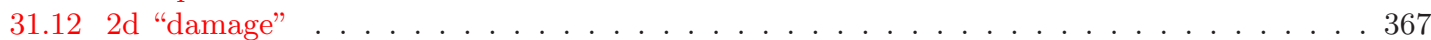

31.132 d automatic statistics on damage . . . . . . . . . . . . . . . . . . . . . . . 368

31.14 The damage histogram showing actual damage . . . . . . . . . . . . . . . . . . . . . 369

31.15 The damage histogram showing damage bins . . . . . . . . . . . . . . . . . . 370 
31.16 The attractor histogram for density classification . . . . . . . . . . . . . . . . . . 372

31.17 The attractor frequency histogram . . . . . . . . . . . . . . . . . . . . . 373

31.18 The attractor histogram sorted by different measures . . . . . . . . . . . . . . . . . . . . . 374

31.19 The attractor histogram jump-graph . . . . . . . . . . . . . . . . . . . . . 378

31.20 The skeleton histogram . . . . . . . . . . . . . . . . . . . . . . . . . . 381

31.21 Examples of frozen skeletons . . . . . . . . . . . . . . . . . . . . . . . 381

31.22 The skeleton frequency histogram . . . . . . . . . . . . . . . . . . . . . . . . . 384

32.1 On-the-fly key index for space-time patterns . . . . . . . . . . . . . . . . . . . 387

32.2 Setting a random k-rcode on-the-fly . . . . . . . . . . . . . . . . . . . . . . . . . . . . 394

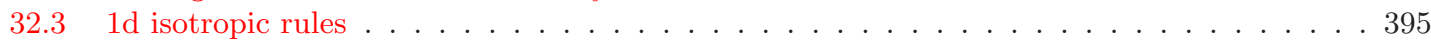

32.4 Mutate/restore on-the-fly by a random bit/value flip . . . . . . . . . . . . . . . . . . 396

32.5 Info when changing $Z$ on-the-fly . . . . . . . . . . . . . . . . . . . . . . 397

32.6 Examples of $v 2 k 5$ complex space-time patterns with interacting gliders . . . . . . . . . . 398

32.7 Space-time 2 d snapshots of complex kcode . . . . . . . . . . . . . . . . . . . . 399

32.8 Loading complex rules on-the-fly . . . . . . . . . . . . . . . . . . . . . . . 4400

32.9 Info when loading a complex rule on-the-fly . . . . . . . . . . . . . . . . . . . . 400

32.10 Randomizing wiring by stages . . . . . . . . . . . . . . . . . . . . . . . . . 401

32.11 A positive and negative singleton seed . . . . . . . . . . . . . . . . . . . . . . . . . . 403

32.12 Slanting $1 \mathrm{~d}$ space-time patterns . . . . . . . . . . . . . . . . . . . . 405

32.13 Hex or square 2 d lattice . . . . . . . . . . . . . . . . . . . . . . . . . . . 405

32.14 The cell outline in network-graph space-time-patterns . . . . . . . . . . . . . . . . . . 406

32.15 Skipping time-steps . . . . . . . . . . . . . . . . . . . . . . . . 407

32.16 Time-step spacing in scrolling network-graph STP . . . . . . . . . . . . . . . . . . . . 408

32.17 A $3 \mathrm{~d}$ network shown in $2 \mathrm{~d}$. . . . . . . . . . . . . . . . . . . . . . . . . . 4409

32.18 3d cells shown as balls or parallelograms . . . . . . . . . . . . . . . . . . . . 410

32.19 Normal, frozen, and binned, 2 d space-time patterns . . . . . . . . . . . . . . . . . . . . 411

32.20 Color key — normal space-time patterns . . . . . . . . . . . . . . . . . . . . . . 412

32.21 Color key — $\mathrm{f} 1$ frozen space-time patterns . . . . . . . . . . . . . . . . . . . . . . . . . 412

32.22 Color key $-\mathrm{f} 2$ frozen space-time patterns . . . . . . . . . . . . . . . . . . . . . 412

32.23 Color key — bins frozen space-time patterns . . . . . . . . . . . . . . . . . . . . . 412

32.24 frequency bins set by hand . . . . . . . . . . . . . . . . . . . . . . . . . 413

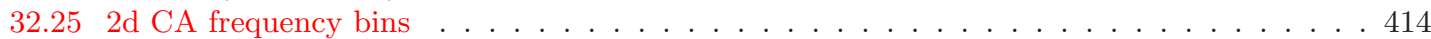

32.26 Examples of filtering space-time patterns . . . . . . . . . . . . . . . . . . . . 415

32.27 Filtered lookup-frequency histogram . . . . . . . . . . . . . . . . . . . . . . 415

32.28 Filtering a complicated background domain . . . . . . . . . . . . . . . . . . . . 416

32.29 Filtering showing discontinuities within a chaotic domain . . . . . . . . . . . . . . . 416

32.30 Input frequency histogram presentation . . . . . . . . . . . . . . . . . . . . . . . . 417

32.31 The input frequency histogram in $3 \mathrm{~d}$. . . . . . . . . . . . . . . . . . . . . . . . . 418

32.32 Entropy/density scatter plot . . . . . . . . . . . . . . . . . . . . 419

32.33 Directly scanned 1d space-time patterns for vector PostScript . . . . . . . . . . . . . . . 441

32.34 PostScript 1d space-time patterns, dots and divisions . . . . . . . . . . . . . . . . . . 432

32.35 1d CA space-time patterns in network-graph layout . . . . . . . . . . . . . . . . . . 444

32.36 2d CA space-time patterns within the network-graph . . . . . . . . . . . . . . . 435

32.37 Network-graph vector PostScript . . . . . . . . . . . . . . . . . . . . 436

33.1 A 2d histogram of an entropy-variability scatter plot . . . . . . . . . . . . . . . . 447

33.2 Input-entropy variability . . . . . . . . . . . . . . . . . . . . . . . 448

$33.31 \mathrm{~d} v 8 k 5$ tcode sample . . . . . . . . . . . . . . . . . . . . . . . . . . 439

33.4 2d histogram - other presentations . . . . . . . . . . . . . . . . . . . . . 439

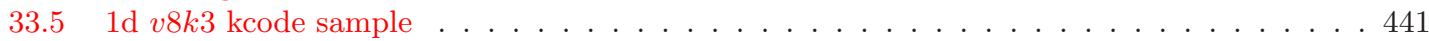


33.6 Running a test . . . . . . . . . . . . . . . . . . . . . . . . . . . . . . . . . . . . . . . . . . . . . . . . . . . . . .

33.7 Creating an automatic sample. . . . . . . . . . . . . . . . . . . . . . . . 445

33.8 Loading, sorting and displaying a sample . . . . . . . . . . . . . . . . . . . . . 446

33.9 The scatter plot color scheme . . . . . . . . . . . . . . . . . . . . . . . . . . 448

33.10 rule sample scatter plot . . . . . . . . . . . . . . . . . . . . . . . . . . . 449

$33.112 \mathrm{~d}$ frequency histogram of the rule sample scatter plot . . . . . . . . . . . . . . . . 449

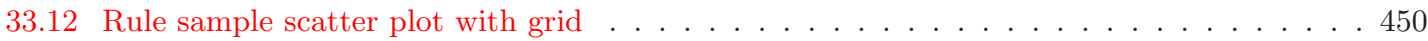

33.13 2d frequency histogram at a lower resolution . . . . . . . . . . . . . . . . . . . 450

33.14 rule sample scatter plot, $Z$-parameter and $\lambda$-ratio . . . . . . . . . . . . . . . . . . . . . 451

33.15 On-the-fly info when scanning sample space-time patterns . . . . . . . . . . . . . . . 453

$33.162 \mathrm{~d} v 4 k 7$ kcode sample . . . . . . . . . . . . . . . . . . . . . . . . . . . . 4454

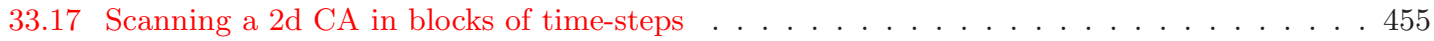

33.18 Listing the rule sample . . . . . . . . . . . . . . . . . . . . . . . 456

33.19 Listing by plot coordinates or probing with the mouse . . . . . . . . . . . . . . . . . 457

34.1 The basin of attraction field of a RBN, before learning . . . . . . . . . . . . . . . . . . . 459

34.2 The basin of attraction field of a RBN after learning . . . . . . . . . . . . . . . . . . . 460

34.3 The RBN networks before and after learning . . . . . . . . . . . . . . . . . . . . 461

34.4 Nodes other than target and pre-image suppressed . . . . . . . . . . . . . . . . . . 462

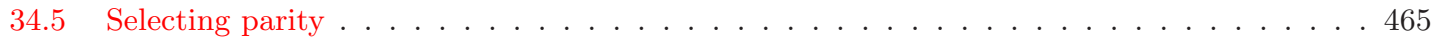

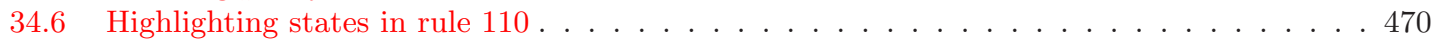

34.7 Highlighting states in a $v=4 \mathrm{CA}$. . . . . . . . . . . . . . . . . . . . . . . . . . . . . 471

35.1 Listing files . . . . . . . . . . . . . . . . . . . . . . . . . . . . . . . . . . . . . . . . . 


\section{List of Tables}

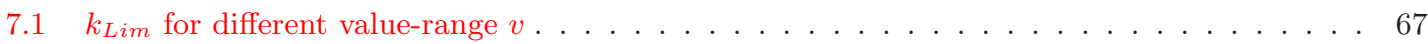

7.2 Basin of attraction field: $n_{\text {Lim }}$ for different value-range $v \ldots \ldots$. . . . . . . . . . . . . 67

8.1 Times for increasing $k$ and $n$ for $v=2$, basin of attraction fields, 1 d CA. . . . . . . . . . 73

8.2 Times for increasing $v$ and $n$ for $k=3$, basin of attraction fields, 1 d CA. . . . . . . . . . . 73

8.3 Times for increasing $k$ and $n$ for $v=2$, basin of attraction fields, RBN. . . . . . . . . . . . . 74

8.4 Times for increasing $v$ and $n$ for $k=3$, basin of attraction fields, DDN. . . . . . . . . . . . . 74

9.1 Effective $k=0$ neutral rules . . . . . . . . . . . . . . . . . . . . . . . . 76

13.1 The size of rule-tables $k=1$ to 13 . . . . . . . . . . . . . . . . . . . . 102

13.2 The size of rule-tables . . . . . . . . . . . . . . . . . . . . . . . . . . . . . 104

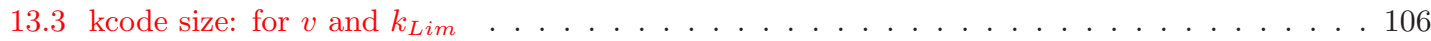

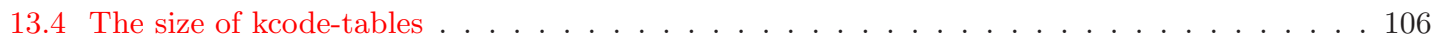

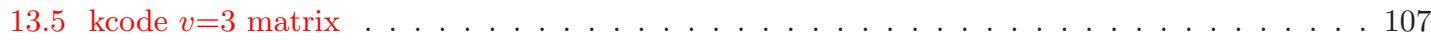

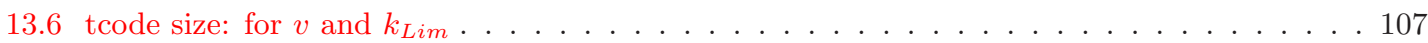

14.1 Post function list $v 2 k 3 \ldots \ldots \ldots \ldots \ldots \ldots$. . . . . . . . . . . . . . . . . . . 122

14.2 Post function list $v 2 k 5$. . . . . . . . . . . . . . . . . . . . . . . . . . . . . . . . . 122

14.3 Post function list (multi-value) $v 3 k 5 \ldots$. . . . . . . . . . . . . . . . . . . . . . . . . . . . . . . . . . . . . . . .

16.1 Rules in decimal - rule-table size limilations . . . . . . . . . . . . . . . . . . . . . . 142

16.2 Immediate rule data in the terminal . . . . . . . . . . . . . . . . . . . . . . . 150

16.3 Rule details in the terminal . . . . . . . . . . . . . . . . . . . . . . . . . . 151

16.4 Horizontal and matrix layout options for kcode in the terminal . . . . . . . . . . . . . . 152

16.5 Swapped kcode - some terminal layouts . . . . . . . . . . . . . . . . . . . . . . . . 152

20.1 The RBN used in the network-graph and jump-graph examples . . . . . . . . . . . . . . 205

20.2 The adjacency-matrix of the network-graph . . . . . . . . . . . . . . . . . . . . . 224

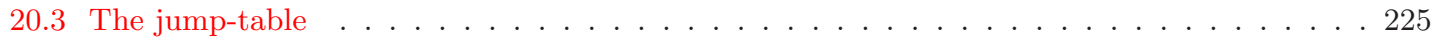

21.1 Seeds in decimal - network size limitations . . . . . . . . . . . . . . . . . . . . . . . 243

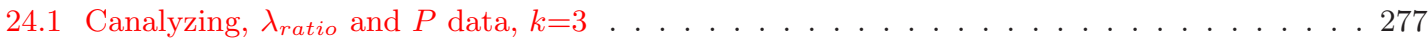

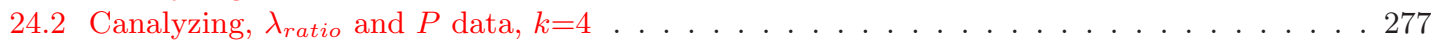

26.1 Alternative presentations bit/value nodes . . . . . . . . . . . . . . . . . . 296

29.1 Maximum network size for the exhaustive testing algorithm . . . . . . . . . . . . . 331

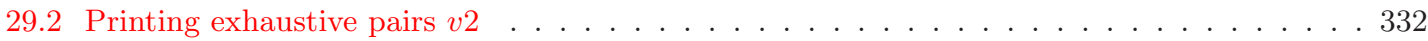

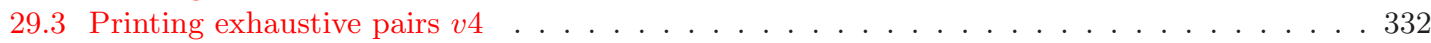





\section{Chapter 1}

\section{Overview}

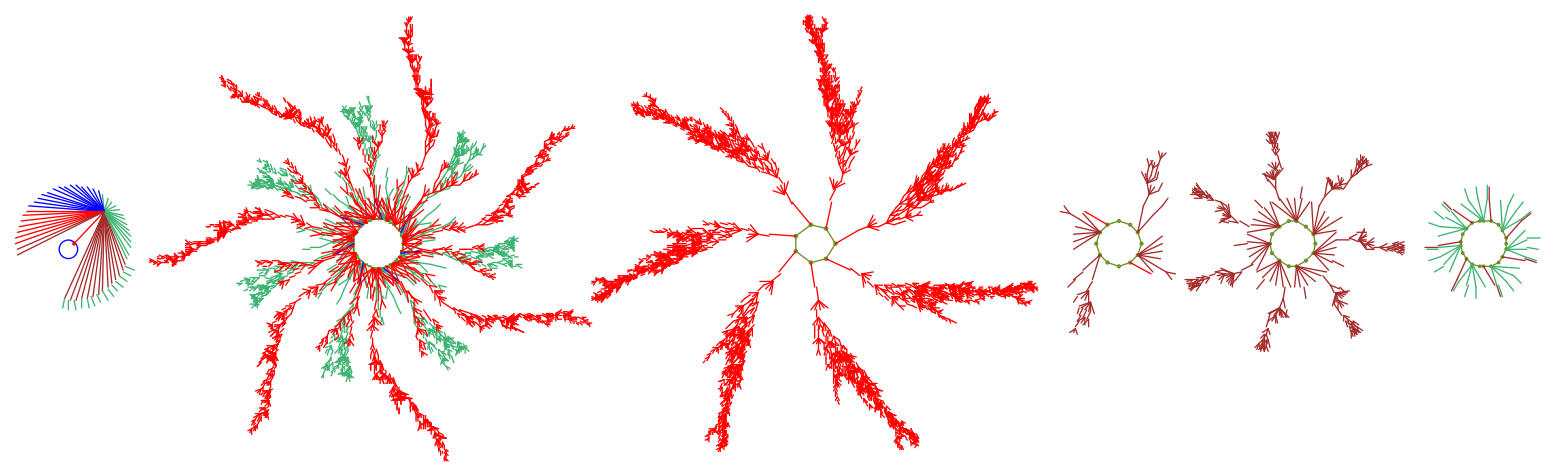

Figure 1.1: The basin of attraction field of a Cellular Automaton, $v=2, k=3, n=14$. There are in fact 15 basins, but equivalent basins have been suppressed leaving just the 6 prototypes. State nodes have been omitted. This is one of the computationally universal "elementary" rules, 193 (equivalent to the famous rule 110[22])

\subsection{Introduction}

DDLab is interactive graphics software, able to construct, visualize and manipulate a hierarchy of discrete systems: Cellular Automata, Random Boolean Networks, Discrete Dynamical Networks in general, intermediate or hybrid networks, and random maps, and investigate and visualize many aspects of the dynamics on the networks, both from the time-series perspective - space-time patterns, and from the state-space perspective - attractor basins, with interactive graphical methods, as well as data gathering, analysis, and statistics. The term "attractor basin" refers to any type of state transition graph: a basin of attraction fields, single basins, or subtree.

Figure 1.2 gives a glimpse of the main themes in DDLab, and also the broad and slippery categories of the systems available, listed below (see also figure 29.5),

- CA: Cellular Automata: a local neighborhood of $k$ inputs (1d, 2d, 3d) and one rule (but possibly a mix of rules to extend the definition).

- RBN: Random Boolean Networks: random wiring of $k$ inputs (but possibly with mixed $k$ ) and a mix of rules (but possibly just one rule). 


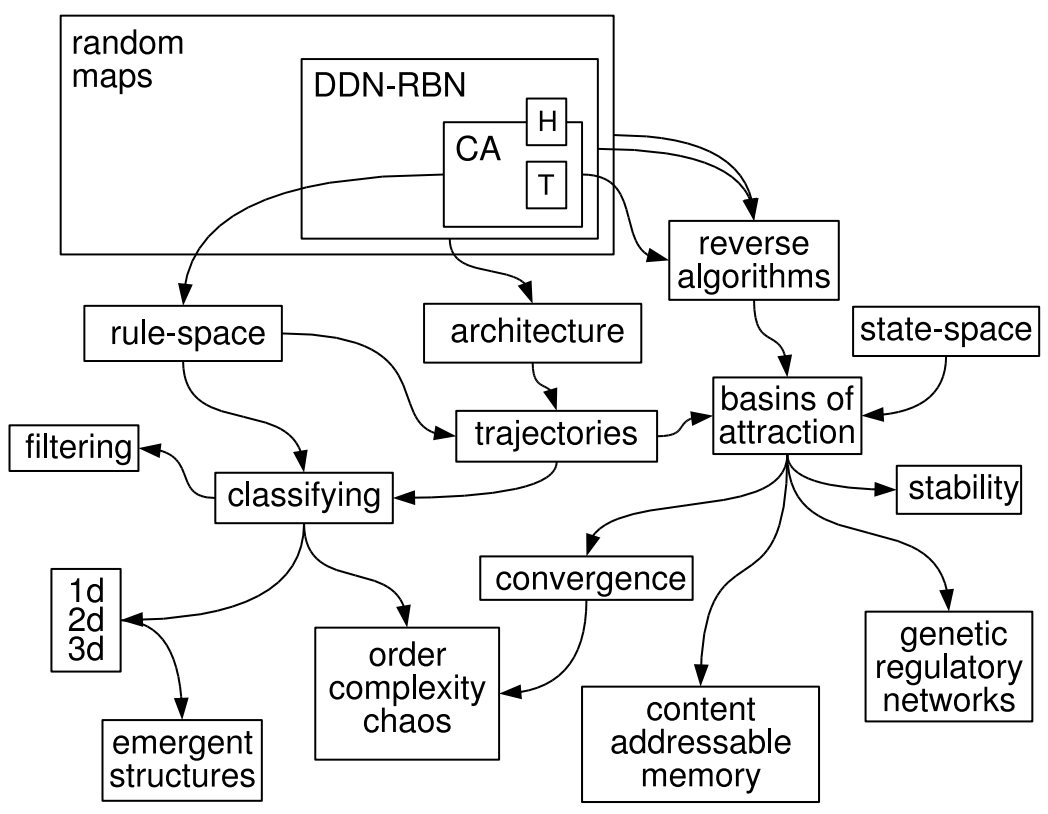

Figure 1.2: The various themes, methods, functions and applications in DDLab, loosely connected. Top Left: The expanding hierarchy of networks: CA $\rightarrow$ RBN/DDN $\rightarrow$ within the super-set of random maps (directed graphs with out-degree one), imposing decreasing constraints on the dynamics. There are also multiple sub-categories, for example totalistic rules $(T)$, hybrids $(H)$ and networks of networks.

- DDN: Discrete Dynamical Networks: as RBN, but allowing a value-range $v \geq 2$. Binary CA are a special case of RBN, and RBN and multi-value CA are special cases of DDN.

- Random maps: directed graphs with out-degree one, where each state in state-space is assigned a successor. CA, RBN and DDN, which are usually sparsely connected $(k \ll n)$ are all special cases of random maps, but if fully connected $(k=n)$, rulemix CA and the rest are equivalent to random maps.

DDLab is used widely in research and education, and has been applied to study complexity, emergence and self-organization[25, 32, 40], in the behavior of bio-molecular networks such as neural $[27,28]$ and genetic $[15,20,11,30]$ networks, and in many other disparate areas. The results in publications [25] to [40] may be implemented with DDLab.

As well as scientific applications, DDLab's imagery has featured in art exhibitions[33], as the light show at events, and has been applied for generative music[4].

\section{$1.2 \quad$ Source code and platforms}

The DDLab code is free software under the GNU General Public License (GPL) as published by the Free Software Foundation. Registration is subject to a modest fee, whereby the annoying UNREGISTERED banner can be easily removed (section 5.4). 
Compiled versions of DDLab are maintained for UNIX based (Linux-like) operating systems, and also for DOS, as follows:

- Linux/PC: compiled in Ubuntu 6.06.

- UNIX/XWindows/Sun: compiled in SunOS 5.8. The libraries libX11.so.6.1 and libsunmath.so.1 need to be accessible in your system.

- MAC OSX with X11: compiled in Mac OSX 10.4.10.

- Irix/SGI: compiled for IRIX 6.5.27 with MIPSpro C 7.4.3m and -n32.

- CygwinX/Windows/PC: this is a Linux environment running in MSWindows98 and above. The default packages plus xorg-x11-devel, xorg-x11-fnts, must be installed. The CygwinX version is preferable to the DOS version below.

- DOS/PC: compiled with WatcomC version 11. There is no native MSWindows version of DDLab at present.

The manual covers all versions, which function in essentially the same way, though aspects of the graphics presentation might be slightly different. The manual will refer to the Linux/UNIX/MAC/Irix/CygwinX versions as Linux-like, as opposed to the DOS version.

DDLab is in the process of continual development. New features are being added in response to various research needs. Some aspects of this manual may be out of date or not applicable to particular platforms.

For information on the latest versions, downloading, installing, documentation, registration, platforms, source code, updates, examples of output, applications, and related publications, consult one of the DDLab web sites below:

www. ddlab.org

www.cogs.susx.ac.uk/users/andywu/ddlab.html (Univ. of Sussex)

uncomp.uwe.ac.uk/wuensche/ddlab.html (Univ. of the West of England)

\subsection{Discrete dynamical networks}

A discrete dynamical network in DDLab can be imagined as a software simulation of a collection light bulbs which transmit information to each other about their color state (on/off for binary), and change color according to arriving signals. More abstractly, the network is made up of automatons, elements ${ }^{1}$ or "cells", connected to each other by directed links or "wires", where a wire has an input and output terminal. A cell takes on a value (or color, or cell-state), and transmits this value down its output wires. Its value is updated as a function of the values on its input wires.

Updating is usually done in parallel, synchronously, in discrete time-steps, but may also be sequential in a predetermined or random partial order. These dynamics are deterministic, but noise can be added.

\footnotetext{
${ }^{1}$ The term "cell" (as in cellular automata) denotes a network "element", and should not be confused with a biological cell. In random Boolean network models of genetic regulatory networks, a network element represents a gene, whereas in neural network models, a network element represents a neuron.
} 
This is the system in a nutshell. It remains to set up the network according to its various parameters,

- The "value-range", $v$ : The range of values (cell-states) available to a cell. In other words, the number of possible internal states of the cell, or colors, or letters in its "alphabet". In early versions of DDLab this was limited to just 2 values $(0,1)$, but can now be selected from 2 to 8 .

- The system size, $n$ : The number of network elements. $n \leq 31$ for basin of attraction fields, or $n \leq 65025$ otherwise.

- The network "geometry": How the elements are arranged in space: in a 1d, $2 \mathrm{~d}$ or $3 \mathrm{~d}$ lattice $\overline{\text { with axial dimensions } i, j}, h$, or some other arrangement. The network geometry may have real meaning (depending on the "wiring scheme" below), or it may simply allow convenient indexing and representation.

- The connectivity: The number of input wires, $k$, to each cell, or the " $k$-mix" if $k$ is not homogeneous. $k$, may vary from 0 to 13 , or to 25 for totalistic rules. Maximum $k$ is reduced for greater value-range $v$. The $k$-mix and "wiring scheme" (below) can be biased, for instance to make scale-free networks.

- The "wiring scheme": defining the location of the output terminals of each cell's input wires, the element's "neighborhood". Cellular automata have a homogeneous nearest neighbor (or next nearest etc.) local neighborhood throughout the network, in $1 \mathrm{~d}, 2 \mathrm{~d}$ or $3 \mathrm{~d}$. RBN and DDN may have a completely arbitrary wiring scheme (a "pseudo-neighborhood"). The wiring can be assigned at random, or may be biased - for example, by confining an element's pseudo-neighborhood close to itself. The wiring can be assigned to create a hybrid of $\mathrm{CA} / \mathrm{DDN}$, or a network of sub-networks in any combination.

The wiring scheme defines the lattice and its boundary conditions. CA wiring usually requires "periodic boundary conditions" where lattice edges wrap around to their opposite edges.

- The "rule scheme": the rules or logical functions in the network. Each element applies a rule to its inputs to compute its output. Usually this is made into a lookup-table, the "rule-table", listing the outputs of all possible input patterns (rcode), or some subset of input patterns (e.g. kcode or tcode). Cellular automata have a homogeneous rule scheme, the same rule throughout the network. DDN may have a completely arbitrary rule scheme. The rule scheme can be a CA/DDN hybrid. Rules and rule schemes can be biased in various ways.

A special case of a rule scheme are outer-totalistic rules where the rule to be applied depends on a cell's current value, thus requiring $v$ separate rules. Outer totalistic rules are used to implement reaction-diffusion, the game-of-Life, and other Life-like rules.

DDLab is able to create and modify these networks, and graphically represent and analyze both the networks themselves, and the local and global dynamics on the networks - the changing patterns made by the complex web of feedback. 
Acronym glossary: (chapter 37 provides a detailed glossary)

- CA: cellular automata: homogeneous wiring/rules; a local universal wiring template (for example: nearest neighbor) and a universal rule, the same rule everywhere.

- RBN: random Boolean networks: $v=2$, arbitrary wiring and heterogeneous rules, Kauffman's famous model[14], but possibly with heterogeneous connectivity $k$.

- DDN: discrete dynamical networks: as RBN, but allowing for a value-range $v>2$ (CA and RBN are special cases of DDN).

\subsection{Space-time patterns and attractor basins}

DDLab has two alternative ways of looking at network dynamics. Local dynamics, running the network forwards for space-time patterns, and global dynamics, running the network backwards for attractor basins - the idea is explained in figure 23.1.

Running "backwards" generates multiple predecessors rather than a trajectory of unique successors. This procedure reconstructs the branching subtree of ancestor patterns rooted on a particular state. States without predecessors will be disclosed, the so called "garden-of-Eden" states, the "leaves" of the subtree. subtrees, basins of attraction (with a topology of trees rooted on attractor cycles), or the entire basin of attraction field (referred to collectively as "attractor basins") can be displayed in real time as directed graphs (state transition graphs), with many alternative presentation options, and methods for gathering/analyzing data. The attractor basins of "random maps" may be generated, with or without some bias in the mapping.

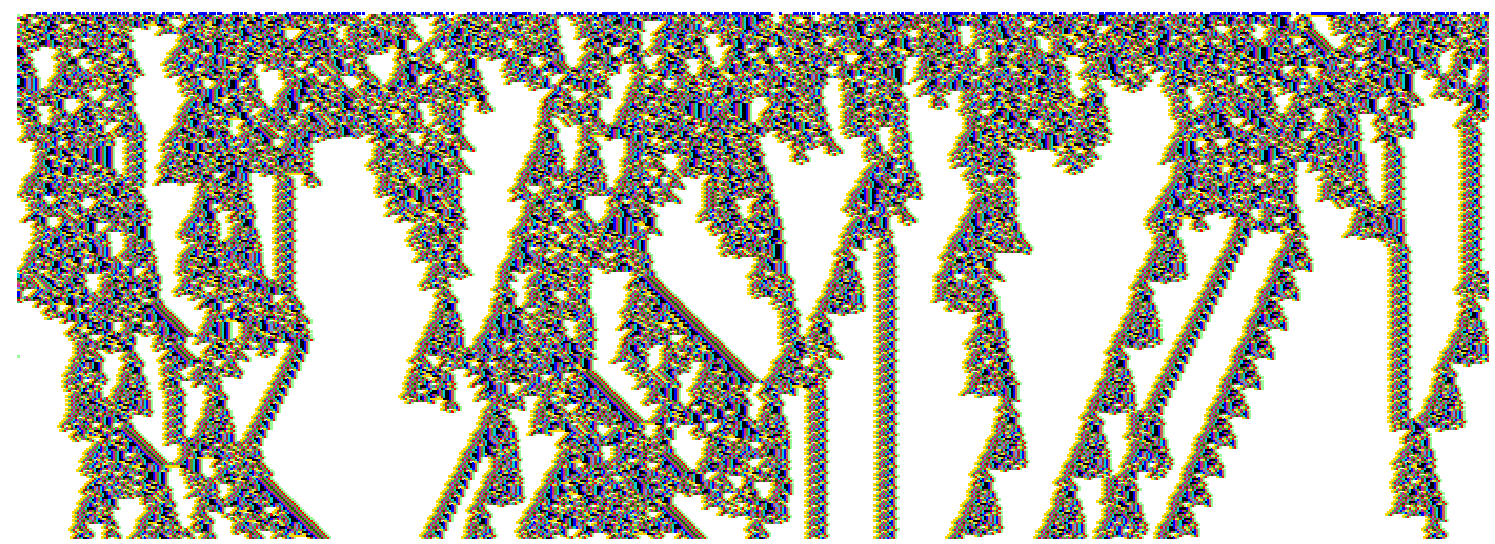

Figure 1.3: The space-time pattern of a $1 \mathrm{~d}$ complex CA with interacting gliders. 308 timesteps from a random initial state. Value-range $v=2$, neighborhood size $k=7$, system size $n=700$, rcode(hex) $=3 \mathrm{~b} 46$ 9c0e e4f7 fa96 f93b 4d32 b09e d0e0. Cells are colored according to neighborhood look-up instead of the value. Space is across and time down the page. The basin of attraction field for this rule $(n=16)$ is shown figure 1.4. 


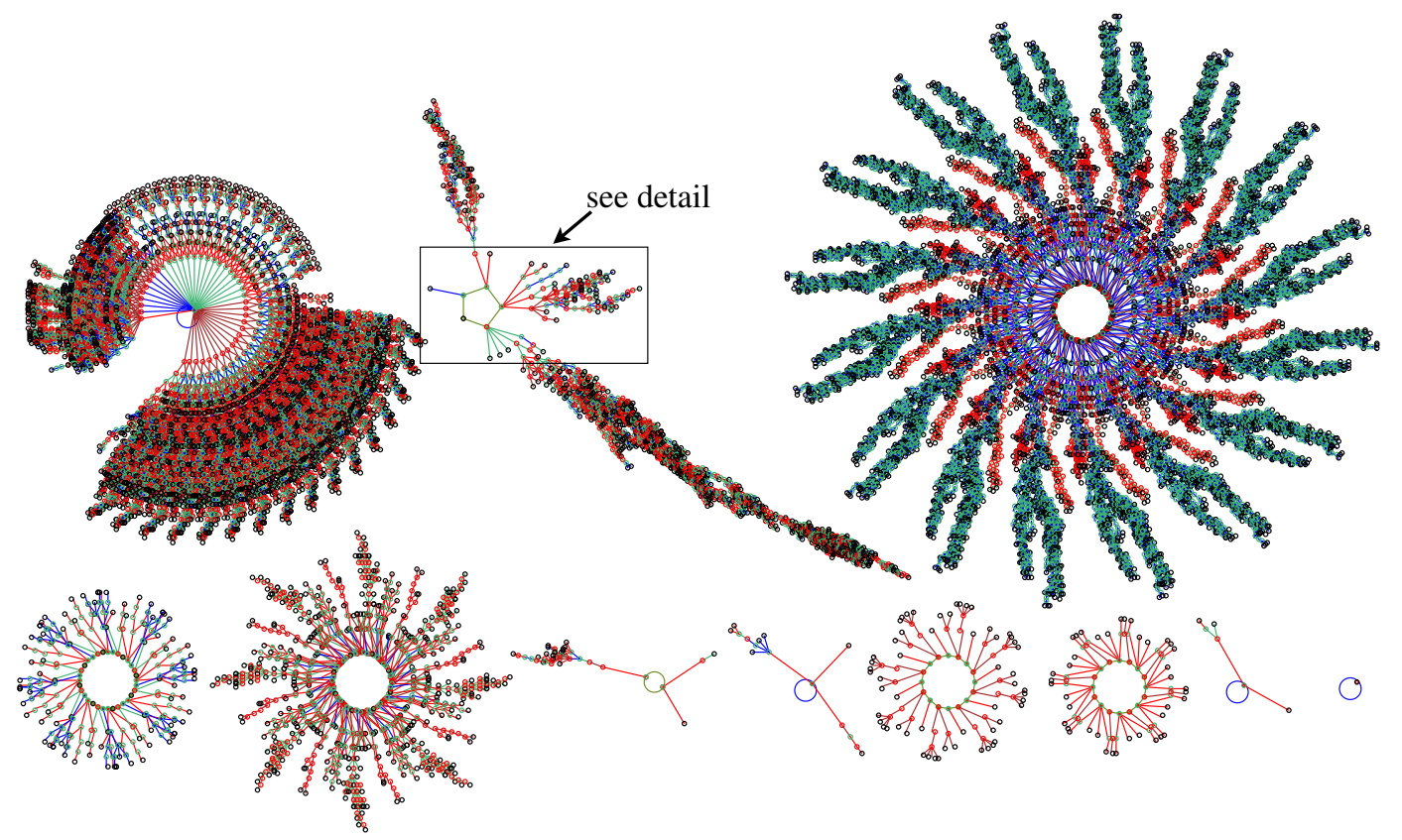

Figure 1.4: The basin of attraction field of a complex $v 2 k 7 \mathrm{CA}, n=16$, defined in figure 1.3, which shows an its space-time patterns. The $2^{16}=65536$ states in state space are connected into 89 basins of attraction. Only the 11 non-equivalent basins are shown, with symmetries characteristic of CA[25]. The period $(p)$, percentage of state space in each basin type $(s)$, and number of each type $(t)$, of the biggest three basins (top row), are as follows: (1) $p=1 s=15.7 \% t=1$. (2) $p=5 s=55.8 \% t=16$. (3) $p=192 s=22.9 \% t=1$. The field's $G$-density $=0.451, \lambda_{\text {ratio }}=0.938, Z=0.578$.

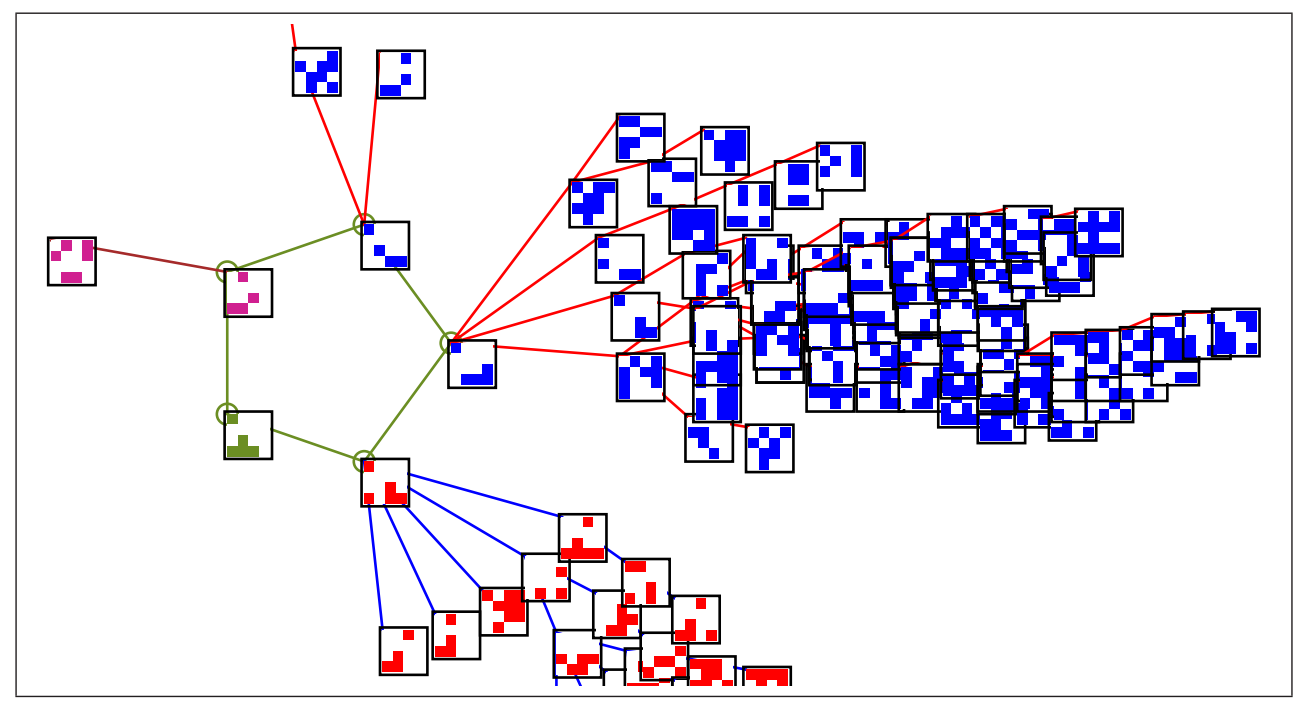

Figure 1.5: A detail of the 2 nd basin of attraction in figure 1.4. The states are shown as $4 \times 4$ bit patterns. 


\subsubsection{Totalistic rules - forwards only}

DDLab can be constrained to run "forwards only" for various types of totalistic rules which depend on just the totals of each value or color in a neighborhood, including outer-totalistic rules and reaction-diffusion rules. The rule-tables of totalistic rules (tcode and kcode) are much smaller than full rule-tables (rcode), so larger neighborhoods are possible $\left(k_{\text {Lim }}=25\right.$, instead of 13$)$ at the cost of disabling basin of attraction functions.

\subsection{Categorization}

It can be argued that attractor basins represent the network's "memory" by their hierarchical categorization of state-space [26, 27]. Each basin is categorized by its attractor and each subtree by its root. Learning/forgetting algorithms allow attaching/detaching sets of states as predecessors of a given state by automatically mutating rules or changing connections. This allows sculpting the basin of attraction field to approach a desired scheme of hierarchical categorization. More generally, preliminary "inverse problem" or "reverse engineering" algorithms are included to find the network that satisfies a given set of transitions.

\subsection{Network size limits}

Whereas large networks may be run forward to look at space-time patterns, or backward to look at subtrees, the size is limited when generating the entire basin of attraction field, given that state-space $S$ grows exponentially with system size $n\left(S=v^{n}\right.$, where $v$ is the value-range).

DDLab's upper limit for basin of attraction fields (listed in table 7.2) is $n=31$ for binary systems (lower for practical purposes). Otherwise the limit is $n=65025$, based on the maximum size of a $2 \mathrm{~d}$ network, $255 \times 255$, where $n$ can be represented by an unsigned short int. This applies to spacetime patterns (in 1d, $2 \mathrm{~d}$ and $3 \mathrm{~d}$ ), and to single basins or subtrees, though in practice much smaller sizes are appropriate for attractor basins (except in the case of subtrees for maximally chaotic CA "chain-rules"). Larger sizes may be tried, but may impose unacceptable time, memory or display constraints.

Networks with disordered (chaotic) dynamics, which have low in-degree or branchiness in their subtrees (such as CA chain-rules) allow backwards computation of much larger networks than for ordered dynamics which have high in-degree. For CA, rules giving chaotic dynamics have a high $Z$ parameter, rules giving ordered dynamics have a low $Z$ parameter[32].

For RBN and DDN, running backwards generally imposes a greater computational load than for $\mathrm{CA}$.

\subsection{Parameters and options}

DDLab is an applications program, it does not require writing code. The network's parameters, and the graphics display and presentation, can be very flexibly set, reviewed and altered from DDLab's graphical user interface.

Changes can be made on-the-fly, including changes to rules, connections, current state, scale, and alternative presentations highlighting different properties of space-time patterns. Networks of 
whatever dimension can be interchangeably represented in $1 \mathrm{~d}, 2 \mathrm{~d}$, and $3 \mathrm{~d}$. $2 \mathrm{~d}$ dynamics can be shown with a time dimension $(2 \mathrm{~d}+$ time $)$ in isometric projections.

The network architecture, states, data, and the screen image can be saved and loaded in a variety of tailor-made file formats, and most graphic output can be saved as vector PostScript files.

\subsection{Measures and data}

Various quantitative, statistical and analytical measures and data on both forward dynamics and attractor basin topology are available in DDLab, as well as various global parameters for rules and network architecture. The measures and data, shown graphically as well as numerically in most cases, include the following:

- Rule parameters: $\lambda, \mathrm{P}, \mathrm{Z}$.

- The frequency of canalyzing inputs. This can be set to any arbitrary level.

- Various measures on forward dynamics such as pattern density, frozen islands, damage spread between two networks, the Derrida plot, rule-table lookup frequency — which allows filtering, input entropy and its variability - which allows ordered, complex and chaotic rules to be classified automatically[32].

- Various global measures on the topology of attractor basins including garden-of-Eden density and in-degree frequency.

\subsection{Contents summary}

- Chapter 2 provides a descriptive summary of DDLab's functions.

- Chapter 3 describes how DDLab can be accessed, and information about the GPL license, copyright and registration.

- Chapter 4 describes DDLab's graphical user interface and gives some "quick start" examples. Its probably a good idea to try these right away to get the flavor of DDLab before reading on, or tackling the detailed reference manual.

- Chapters 5 to 36 contain the detailed reference manual.

For further background on the attractor basins of CA, RBN, DDN, and their implications, see publications [25]-[43], which are available at:

www.cogs.susx.ac.uk/users/andywu/publications 


\section{Chapter 2}

\section{Summary of DDLab functions}

This chapter provides a descriptive summary of DDLab's functions.

\subsection{DDLab's prompts}

DDLab's graphical user interface (chapter 5) allows setting, viewing and amending network parameters, and various presentation and analysis functions, by responding to prompts or accepting defaults. The prompts present themselves in a main sequence and also in a number of context dependent prompt windows. You can backtrack to previous prompts, and in some cases skip forward. A flashing cursor indicates the current prompt. "Return" (or the left mouse button) steps forward through the prompts, "q" (or the right mouse button) backtracks, or interrupts a running process (a run), such as space-time patterns or attractor basins being generated. There are also on-the-fly changes that can be made during a run.

\subsection{Initial choices}

Some initial choices in the prompt sequence set the stage for all subsequent DDLab operations, and cannot be amended later (or not easily) without backtracking. These include the following,

\subsubsection{Totalistic rules, forwards-only, TFO-mode}

There is a choice to constrain DDLab to run "forwards-only" for various types of totalistic rules and reaction diffusion rules (TFO-mode). This reduces memory load by cutting out full rule-tables and all attractor functions (prompts for these will not be displayed), and allows larger neighborhoods, $\left(k_{\text {Lim }}=25\right.$, instead of 13$)$. Choosing TFO-mode can only be made at the first prompt (chapter 6).

\subsubsection{Basin field or initial state}

If DDLab is not constrained in TFO-mode there is a further choice, to set either, FIELD-mode - for the basin of attraction field, which does not require a seed (initial state), or SEED-mode - for anything else (which does require a seed) - space-time patterns, a single basin of attraction, or a subtree. This choice can be amended in at a later stage, in section 30.4. 


\subsection{Setting the value-range}

The value-range $v$ can be set from 2 to 8 . If $v=2$ DDLab behaves as in the old binary version. Note that as $v$ is increased, $k_{\text {Lim }}$ will decrease (section 2.5), depending on whether TFO-mode was set in section 2.2.1.

The selection of the value-range can only be made at this early stage in the program.

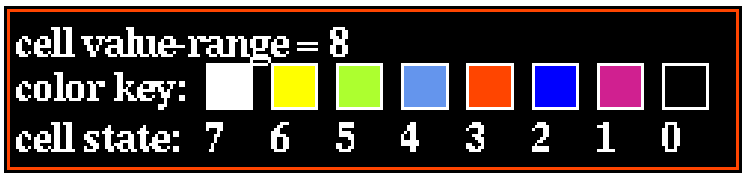

Figure 2.1: The cell value color key window (for a black background, $v=8$ ) that appears when the value-range is selected. The values themselves are indexed from 7 to 0 . See figure 7.1 for all color keys.

\subsection{Setting the network size}

The network size $n$ for $1 \mathrm{~d}$ is set early on in the prompt sequence, but this is superseded if a $2 \mathrm{~d}(i, j)$ or $3 \mathrm{~d}(i, j, h)$ network is selected in a subsequent prompt window. Although the size of $n$ for $1 \mathrm{~d}$ can be increased or decreased by one cell on-the-fly when drawing space-time patterns (section 32.3), in general the network size can only be changed at these early prompts.

For space-time patterns, the upper limit of network size $n_{\text {Lim }}=65025$, based on the maximum size of a $2 \mathrm{~d}$ network $(i, j)=255 \times 255$, where $n$ can be represented by an unsigned short int. This limit also applies for single basins and subtrees, though in practice much smaller sizes are appropriate, except when generating subtees for maximally chaotic CA chain-rules (section 16.11).

For basin of attraction fields, however, the upper limit of network size, $n_{\text {Lim }}$, is much smaller, and depends on the value-range $v$ as set out in section 7.3.

\subsection{The neighborhood $k$ or $k$-mix}

The size of the neighborhood $k$, the number of inputs each cell receives, can vary from 0 to $k_{\text {Lim }}$, which depends on the value-range $v$, and also on whether or not DDLab was constrained to run forwards-only for totalistic rules (TFO-mode, section 2.2.1). For example, for $v=8$ and unconstrained DDLab, $k_{\text {Lim }}=4$ to handle the large lookup-table whereas in TFO-mode $k_{\text {Lim }}=11$. $k_{\text {Lim }}$ for increasing value-range $v$, for both cases, is set out in section 7.2.

$k$ can be homogeneous, or there can be a mix of $k$-values in the network. The $k$-mix may be set and modified in a variety of ways, including defining the proportions of different $k$ 's to be allocated at random in the network, or a "scale-free" distribution, A $k$-mix may be saved/loaded from a file, but is also implicit in the wiring scheme (section 2.6). 


\subsection{Wiring}

The network's wiring scheme (i.e. its connections) has pre-defined layouts for local CA (for $1 \mathrm{~d}, 2 \mathrm{~d}$ and 3d) for each neighborhood size, $k=1$ to $k_{\text {Lim }}$ (chapter 10). Note, however, that for $3 \mathrm{~d} k_{\text {Lim }}=13$, because 3d neighborhoods greater than 13 have not yet been defined. Local $3 \mathrm{~d}$ wiring defines a cubic lattice with periodic boundary conditions. In $2 \mathrm{~d}$, the pre-defined neighborhood layouts define a toroidal lattice which can be either square or hexagonal. The square lattice includes the 5 cell von Neumann neighborhood and the 9 cell Moore neighborhood .

Wiring can also be set at random (nonlocal wiring), with a wide variety of constraints and biases, or by hand (chapter 12). The pre-defined neighborhoods in this case act as pseudo-neighborhoods to which the rule is applied.

A wiring scheme can be set and amended just for a predefined sub-network within the network, and may be saved/loaded from a file (chapter 19).

nonlocal wiring can be constrained in various ways (section 12.5) including confinement within a local patch of cells with a set diameter in $1 \mathrm{~d}, 2 \mathrm{~d}$ and $3 \mathrm{~d}$. Part of the network only can be designated to accept a particular type of wiring scheme, for example rows in $2 \mathrm{~d}$ and layers in $3 \mathrm{~d}$, and the wiring can be biased to connect designated rows or layers.

The network parameters can be displayed and amended in a $1 \mathrm{~d}, 2 \mathrm{~d}$ or $3 \mathrm{~d}$ graphic format, in a "spread sheet" (chapter 17), or as a network-graph which can be rearranged in various ways, including dragging nodes with the mouse (chapter 20).

\subsection{Rules}

The most general update logic or rule is expressed as a full rule-table (lookup-table), referred to as "rcode". There are useful subsets of the general case, two types of totalistic rules, "kcode" and "tcode". The simplest, tcode, depends on the sum of values in the neighborhood; kcode depends on the frequency of each value (color) in the neighborhood. If $k=2$ tcode and kcode are identical.

In addition, both types of totalistic rules can be made into outer-totalistic rules (also called semi-totalistic), where a different rule applies for each value of the central cell; the game-of-life is one such rule. Outer-totalistic rules also allow implementation of reaction-diffusion rules or excitable media[9].

As mentioned in section 2.2.1, DDLab can be constrained to run forwards only, for these various types of totalistic rules (TFO-mode), which allows greater $[v, k]$ networks than for rcode. Transformations and mutations would then apply to just the restricted rule-table, kcode or tcode.

If DDLab remains unconstrained, the totalistic rules, tcode and rcode (but not outer-totalistic rules) can still be selected, but they will be transformed into the full rule-table, rcode, which allows attractor basins. Transformations and mutations will apply to the rcode. Within rcode there are also subsets of rules that can be automatically selected at random, including isotropic rules, maximally chaotic chain-rules, Altenberg rules, and others, and the rules can be biased by various parameters, lambda, $Z$, and canalyzing inputs.

A network may have one homogeneous rule, as for CA, or a rulemix as for RBN and DDN. The rulemix can be confined to a subset of pre-selected rules. Rules may be set and modified in a wide variety of ways, in decimal, hex, as a rule-table bit pattern, at random or loaded from a file. 
A rule scheme can be set and amended just for a predefined sub-network within the network, and can be saved/loaded from a file (chapter 19). The "game-of-Life", "majority", and other predefined rules or rule biases can be selected (chapters 13, 14, 16).

Rules may be changed into their equivalents (by reflection and negative transformations), and transformed into equivalent rules with larger or smaller neighborhoods (chapter 18). Rules transformed to larger neighborhoods are useful to achieve finer mutations. Rule parameters $\lambda$ and $Z$, and the frequency of canalyzing inputs in a network can be set to any arbitrary level (chapter 15).

\subsection{Initial network state, seed}

An initial network state (a seed) is required to run a network forward and generate space-time patterns. A seed is also required to generate a single basin, by first running forward to find the attractor, then backward from each attractor state. A seed is required to generate a subtree, by simply running backwards from the seed. However, for most CA rules, most states in state-space have no predecessors (they are the leaves of a subtree, "garden-of-Eden" states), so from a random seed its usually necessary to run forwards by a few steps to penetrate the subtree before running backwards - this option provided (section 29.2).

A basin of attraction field does not require setting a seed, because appropriate seeds are automatically provided.

As in setting a rule, there are a wide variety of methods for defining the seed (chapter 21), in decimal or hex, as a bit pattern in $1 \mathrm{~d}, 2 \mathrm{~d}$ or $3 \mathrm{~d}$, at random (with various constraints or biases), or loaded from a file (see chapter 35 ).

The bit pattern method is a mini paint program, using the mouse and keyboard to draw colors. Figure 2.2 shows the method applied to draw a portrait, which is then transformed by applying a CA rule for three time-steps.

\subsection{Networks of sub-networks}

Its possible to create a system of independent or weakly coupled sub-networks within the base network, either directly, or by saving smaller networks to a file, then loading them at appropriate positions in the base network (chapter 19). Thus a $2 \mathrm{~d}$ network can be tiled with sub-networks, and $1 \mathrm{~d}, 2 \mathrm{~d}$ or $3 \mathrm{~d}$ sub-networks can be inserted into a $3 \mathrm{~d}$ base network.

The parameters of the sub-networks can be totally different from the base network, provided the base network is set up appropriately, with sufficient "memory" to accommodate the sub-network. For example, to load an DDN into a CA, the CA may need be set up as if it were an DDN. To load a mixed- $k$ sub-network into single- $k$ base network, $k$ in the base network needs to be at least as big as the biggest $k$ in the sub-network. Options are available to easily set up networks in this way. Once loaded, the wiring can be fine-tuned to interconnect the sub-networks.

A network can be automatically duplicated to create a total network made up of two identical sub-networks. This is useful to see the difference pattern (or damage spread) between two networks from similar initial states (section 31.5). 

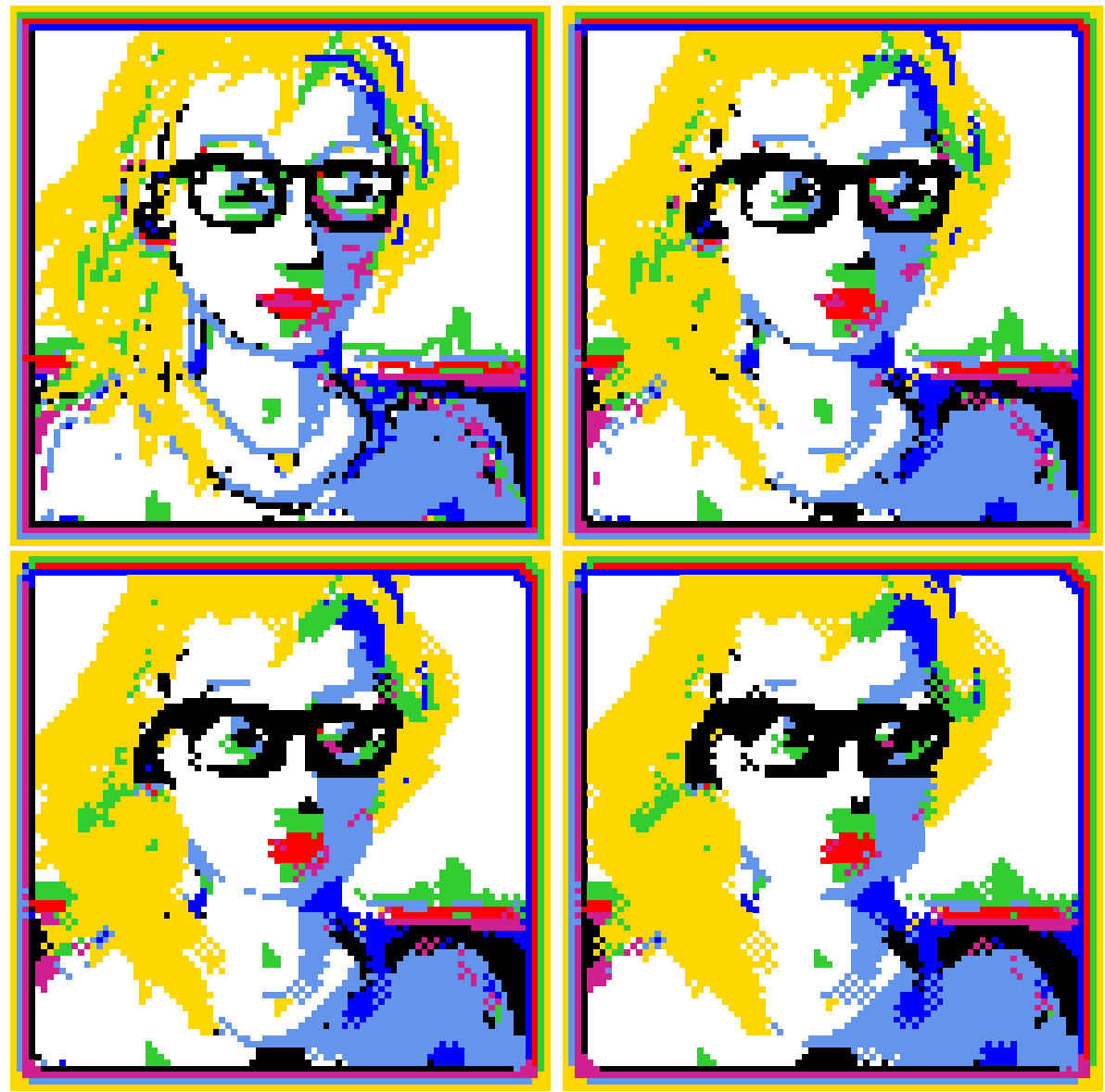

Figure 2.2: Drawing a $2 \mathrm{~d}$ seed, $88 \times 88, v=8$, with the mouse and keyboard, shown top-left. The seed was then transformed by applying a CA majority rule $(v 8 k 4)$ for three time-steps.

\subsection{Presentation options}

Many options are provided for the presentation of attractor basins and space-time patterns. Again, many of these settings can be changed "on-the-fly".

\subsubsection{Space-time patterns} chapters 31 and 32

A cell in a space-time pattern is colored according to its value, or alternatively according to a predefined color depending on its neighborhood at the previous time step, the entry in the ruletable that determined the cell's value. A key press will toggle between the two. Space-time patterns can be filtered to suppress cells that updated according to the most frequently occurring neighborhoods, thus exposing "gliders" and other structures (section 32.11.5). 

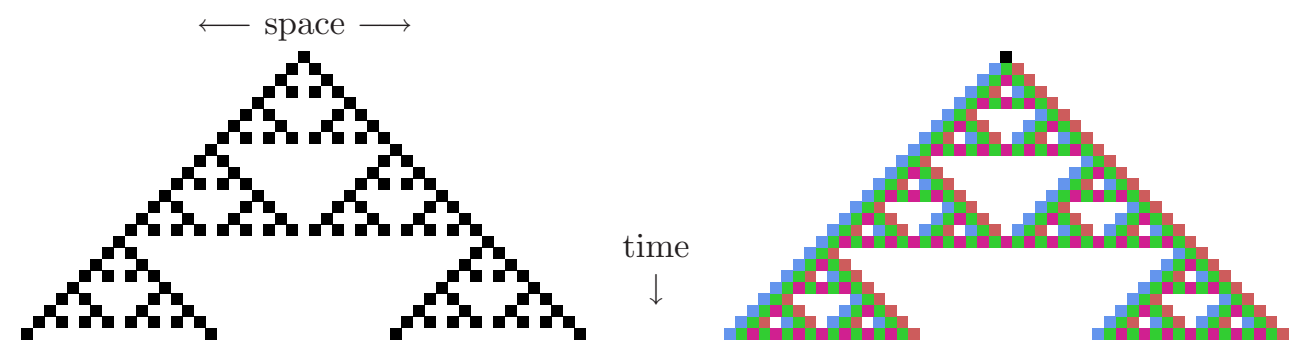

Figure 2.3: Space-time patterns of a $1 \mathrm{~d}$ CA, $v 2 k 3, n=51$, rcode (dec)90. 24 time-steps from an initial state with a single central 1 . Two alternative presentations are shown. Left: cells by value. Right: cells colored according to their look-up neighborhood.

The presentation can be set to highlight cells that have not changed in the previous $x$ generations, where $x$ can be set to any value (section 32.11.1). The emergence of such frozen elements is associated with "canalyzing inputs", and underlies Kauffman's RBN model of gene regulatory networks $[15,11]$.

$1 \mathrm{~d}$ space-time patterns are usual presented as successive time-steps scrolling vertically. $2 \mathrm{~d}$ networks are presented as a "movie" of successive time-steps, but can also be displayed with a time dimension $(2 \mathrm{~d}+$ time $)$ where successive time-steps are also scrolled, either vertically or diagonally, in isometric projections. 2d networks can be toggled between square and hexagonal layout. 3d networks are presented as a "movie" within a $3 \mathrm{~d}$ "cage". The presentation of space-time patterns can be switched "on the fly" between $1 \mathrm{~d}, 2 \mathrm{~d}, 2 \mathrm{~d}+$ time, and $3 \mathrm{~d}$, irrespective of their native dimensions. DDLab automatically unravels or bundles up the dimensions.

There are many other on-the-fly options, including changing the scale of space-time patterns, changing the seed, rule/s, wiring, and the size of $1 \mathrm{~d}$ networks (chapter 32).

Concurrently with these standard presentations, space-time patterns can be displayed in a separate window according to the network-graph layout. This can be rearranged in many ways, including various default layouts. For example a $1 \mathrm{~d}$ space-time pattern can be shown in a circular layout (section 32.19).

\subsubsection{Attractor basins} chapters 24 to 30

Options for attractor basins allow the selection of the basin of attraction field, a single basin (from a selected seed), or a subtree (also from a seed). Because a random seed is likely to be a garden-of-Eden state, to generate subtrees an option is offered to run the network forward a given number of steps to a new seed before running backward. This guarantees a subtree with at least that number of levels.

Options (and defaults) are provided for the layout of attractor basins, their size, position, spacing, and type of node display (as a spot, in decimal, hex or a $1 \mathrm{~d}$ or $2 \mathrm{~d}$ bit pattern, or none). Local $1 \mathrm{~d}$ and $2 \mathrm{~d}$ CA produce attractor basins where subtrees and basins are equivalent by rotational symmetry. This allows "compression" of basins (by default) into non-equivalent prototypes, though compression can be turned off. Attractor basins are generated for a given system size, or for a range of sizes. As attractor basins are generating, the reverse space-time pattern can be simultaneously displayed. 


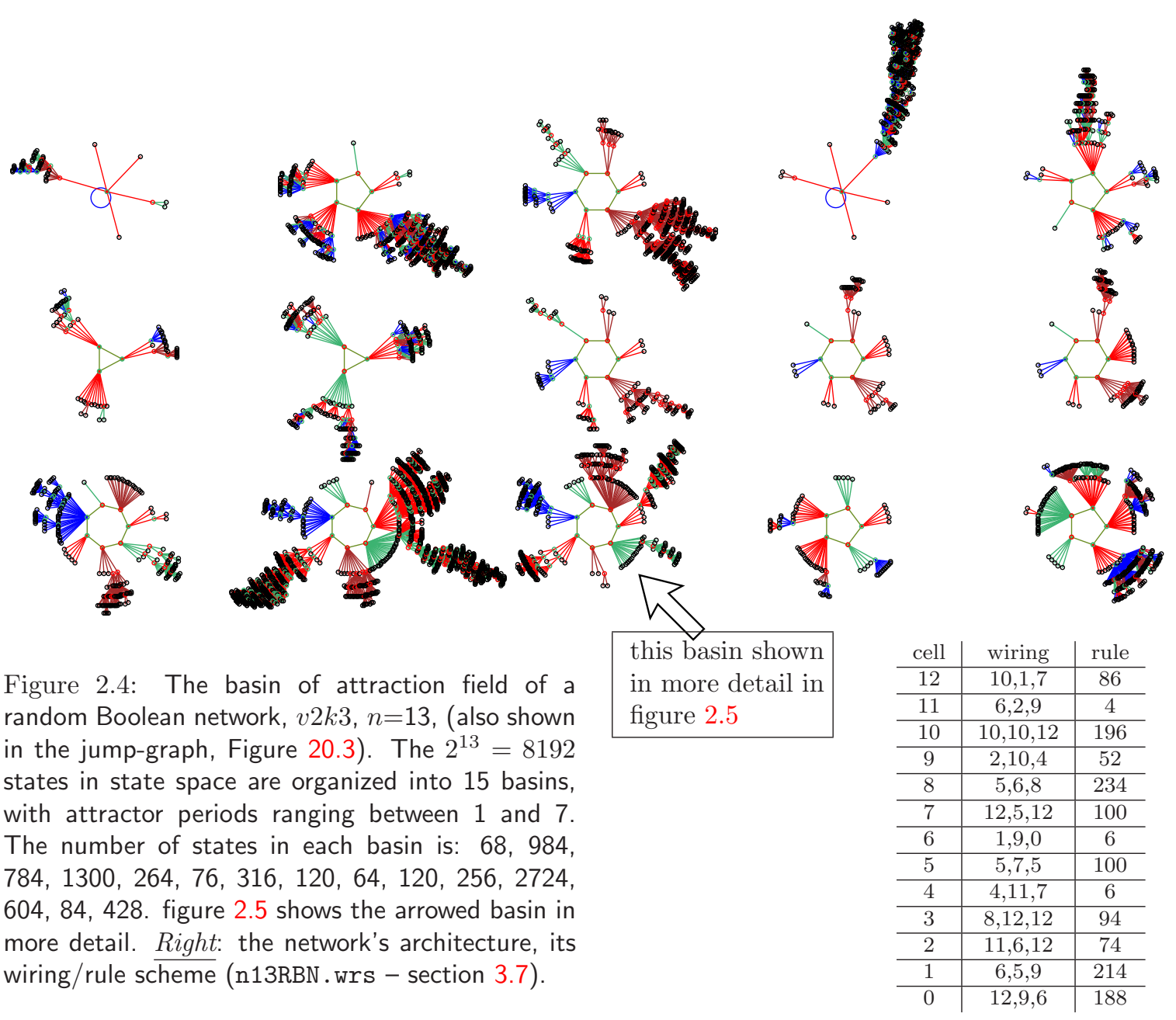

An attractor basin run can be set to pause to see data on each transient tree, each basin, or each field. Any combination of this data, including the complete list of states in basins and trees, can be saved to a file (chapter 27).

Normally a run will pause before the next "mutant" attractor basin, but this pause may be turned off to create a continuous demo of new attractor basins. A "screen-saver" demo option shows new basins continually growing at random positions (section 4.11, 24.8).

\subsubsection{Interrupting a run}

At any time, a space-time pattern or attractor basin run can be interrupted to pause, save or print the screen image, change various parameters, or backtrack through options (chapters 32 and 30). 


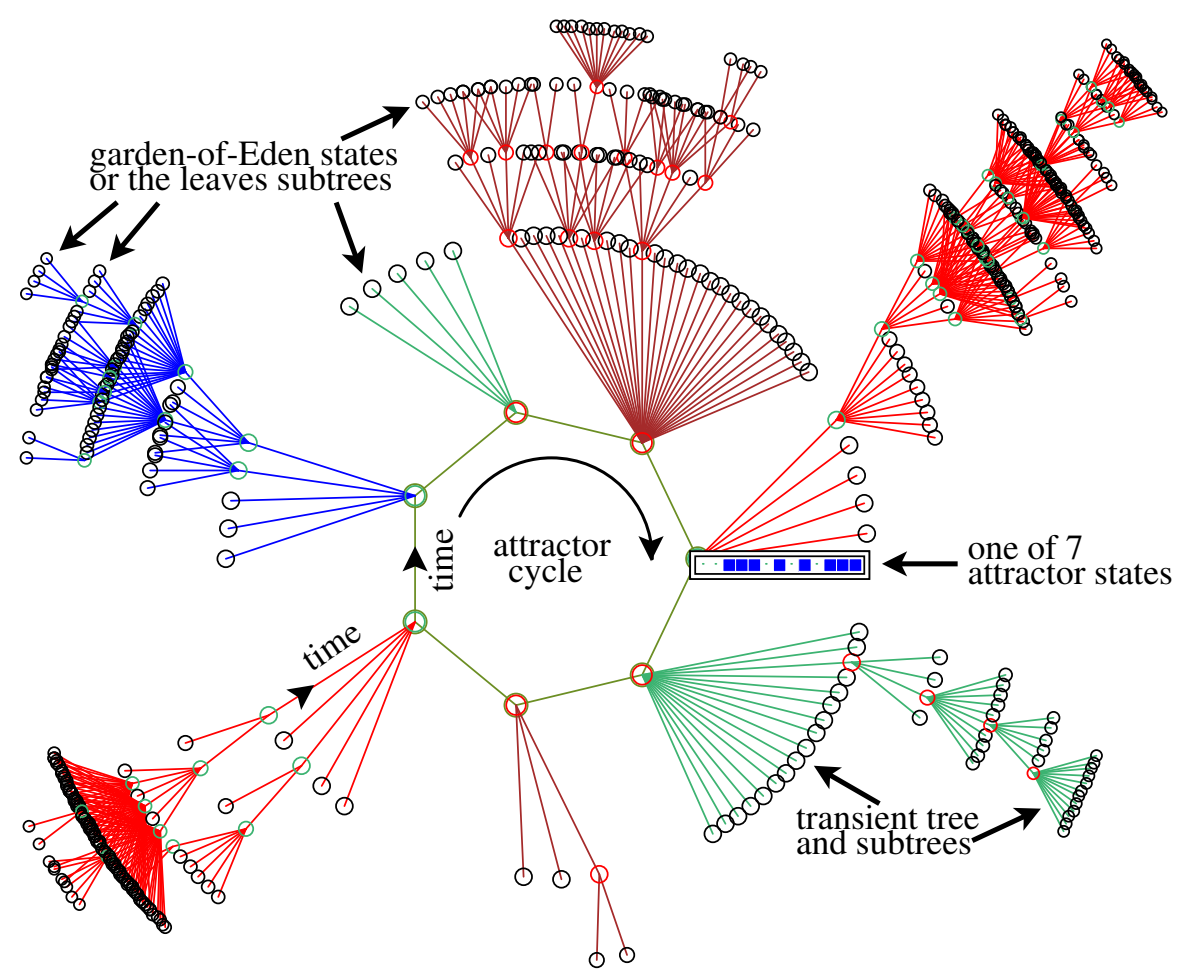

Figure 2.5: A basin of attraction (one of 15) of the random Boolean network, $v 2 k 3, n=13$, shown in figure 2.4. The basin links 604 states, of which 523 are garden-of-Eden states. The attractor period $=7$, and one of the attractor states is shown in detail as a bit pattern. The direction of time is inwards from garden-of-Eden states to the attractor, then clock-wise.

\subsection{Graphics}

In Linux-like versions, the DDLab screen starts up at $925 \times 694$ or a smaller size automatically set to comfortably fit on the monitor. This can be resized, moved and iconized in the usual way. In DOS the graphics will start up at a resolution of $640 \times 480$ (VGA) but can be reset to higher resolutions, or initially with a program parameter. The default background color is black, but can be reset to white either from within the program or with a program parameter. The text size and spacing is set automatically according to the screen resolution, but can be resized.

\subsection{Filing and Printing}

DDLab allows filing a wide range of file types, including network parameters, data, the screen image and vector PostScript files. (sections 19, 35). For compatibility with DOS, filenames follow the DOS format, so a filename has up to eight characters starting with a letter (but not "q") plus a 3 character extension. For example myfile25. dat. In DDLab, only the first part of the filename 
is selected — without the extension (or a default filename can be accepted), the extension is added automatically to identify the file type.

\subsubsection{Filing network parameters}

Network parameters and states can be saved and loaded for the following: $k$-mix, wiring schemes, rules, rule schemes, wiring/rule schemes, and network states (chapter 19).

\subsubsection{Filing data}

Data on attractor basins, at various levels of detail (chapter 27) can be automatically saved. A file of "exhaustive pairs", made up of each state and its successor, can be created (section 29.7).

Various data including mean entropy and entropy variance of space-time patterns can be automatically generated and saved (chapter 33), This allows sorted samples of CA rules to be created, discriminating between order, complexity and chaos (section 33.10), and complex rules, those featuring "gliders" or other large scale emergent structures, to be collected automatically, and some collections/samples of $1 \mathrm{~d}$ and 2d CA rules are provided with DDLab (sections 32.6.1, 33.10).

\subsection{Vector PostScript images}

Vector PostScript files can be generated for most DDLab graphics output: space-time patterns or snapshots (1d, 2d and 3d), attractor basins, the wiring graphic, the network-graph, and the attractor jump-graph. This is a new feature in DDLab described in chapter 36. Vector graphics is preferable for publication quality images. The methods work in both Linux-like systems and DOS. Images saved as vector PostScript files can be printed in GhostView, or converted to . pdf files and printed in Adobe (Acroread).

Previous bitmap methods, below, are still available. Most of the figures in this manual where produced as vector PostScript files, others as bitimage PostScript files.

\subsection{Bitmap images}

The screen image can saved and loaded using an efficient compressed format only applicable within DDLab (section 5.5). Alternatively, in Linux-like systems, a program such as XView can be used to grab the DDLab window or part of it, and to save the image in many standard bitmap formats.

In DOS, to save/print the image in a standard format, use a "stay resident screen grabber". A number of specialist screen grabbers are available, and others are part of "paint" programs. Alternatively run DDLab as a DOS application in Microsoft Windows and use their "paint" screen grabber.

\subsection{Printing the screen image}

Bitmap methods are still available to print the screen image directly from DDLab. In Linux-like systems, the screen can be printed within DDLab as a bitmap PostScript file to a laser printer (section5.6). Alternativly use XView to grab the bitmap image, in various formats. 


\subsection{Mutations}

A wide variety of network "mutations", as well as changes in presentation, can be be made, many on-the-fly while the simulation is running.

\subsubsection{Running Forward}

When running forward, key-press options allow changes to be made to the network and presentation on-the-fly (chapter 32). This includes "mutations" to wiring, rules, current state, and size. A number of $1 \mathrm{~d}$ "complex" rules (with glider interactions) can be set for $k=5,6$ and 7 (section 32.6.1).

\subsubsection{Running Backward}

When running backward, and attractor basins are complete, a key press will regenerate the attractor basin of a mutant network. Various mutation options can be pre-set (chapter 28) including random bit-flips in rules and random rewiring of a given number of wires. Sets of states can be specified and highlighted in the attractor basin to see how mutations affect their distribution (chapter 34).

\subsection{Quantitative, statistical and analytical measures}

Some of the measures and data on network dynamics available in DDLab are listed below. In most cases this information can be displayed graphically.

\subsubsection{Behavior parameters}

The following static parameters measured on rule look-up tables are available (section 16.19.1).

- The $\lambda$ parameter (sections 14.1.2, 16.3.1) and equivalent $P$ parameter.

- The Z-parameter[25, 32].

- The (weighted) average $\lambda$ and $Z$ for a mixed rule network (section 17.8.2).

- The frequency of canalyzing "genes" and inputs (chapter 15 for a rulemix network, section 18.6 for single rules).

- Post-function data (section 14.12).

$\lambda, Z$, and canalyzation can be set to any arbitrary level. 

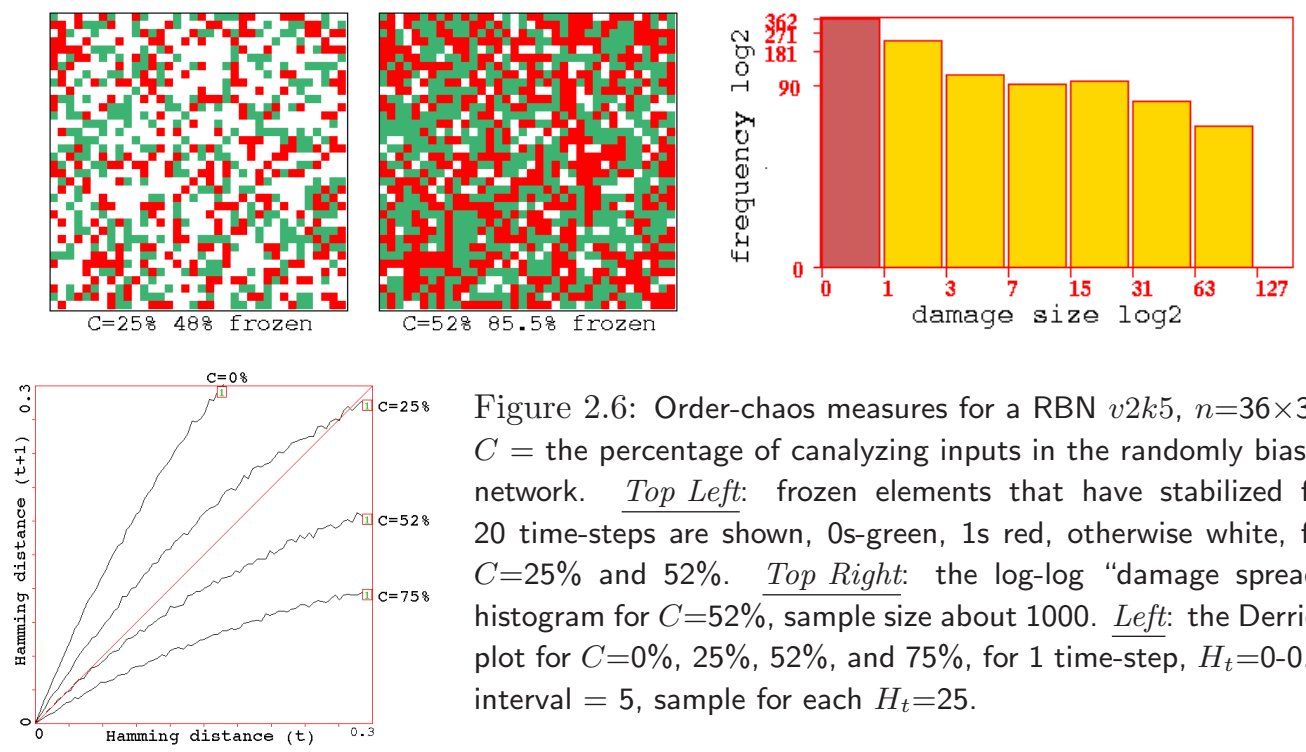

Figure 2.6: Order-chaos measures for a RBN $v 2 k 5, n=36 \times 36$. $C=$ the percentage of canalyzing inputs in the randomly biased network. Top Left: frozen elements that have stabilized for 20 time-steps are shown, 0s-green, 1s red, otherwise white, for $C=25 \%$ and $52 \%$. Top Right: the log-log "damage spread" histogram for $C=52 \%$, sample size about 1000 . Left: the Derrida plot for $C=0 \%, 25 \%, 52 \%$, and $75 \%$, for 1 time-step, $H_{t}=0-0.3$, interval $=5$, sample for each $H_{t}=25$.

\subsubsection{Network connectivity}

The following measures on network connectivity, i.e. the wiring, are available,

- Average $k$ (inputs), number of reciprocal links, and self links (section 17).

- Histograms of the frequency distribution of inputs, outputs, or both (all connections), in the network (section 17.8.13).

- The recursive inputs/outputs to/from a network element, whether direct or indirect, showing the "degrees of separation" between elements (sections 17.5.6, 17.5.7).

- The network-graph (section 20), where the wiring is analyzed in two ways, as an adjacency matrix or table, and derived from this, as a graph that can be analyzed in various ways, and rearranged and unraveled, including dragging vertices and defined components to new positions with "elastic band" edges.

\subsubsection{Measures on local dynamics}

The following measures on local dynamics, i.e. running the system forward from some initial state, its space-time patterns or trajectories, are available,

- A rule-table lookup frequency histogram, which can be toggled between $2 \mathrm{~d}$ and $3 \mathrm{~d}$ to include a time dimension (section 31.4).

- The entropy of the lookup frequency over time (section 32.12.4).

- The variance of the entropy, and an entropy/density scatter plot, where complex rules have their own distinctive signatures (section 32.12.5).

- A plot of mean entropy against entropy variability for an arbitrarily large sample of CA rules, which allows ordered, complex and chaotic rules to be classified automatically (chapter 33), 

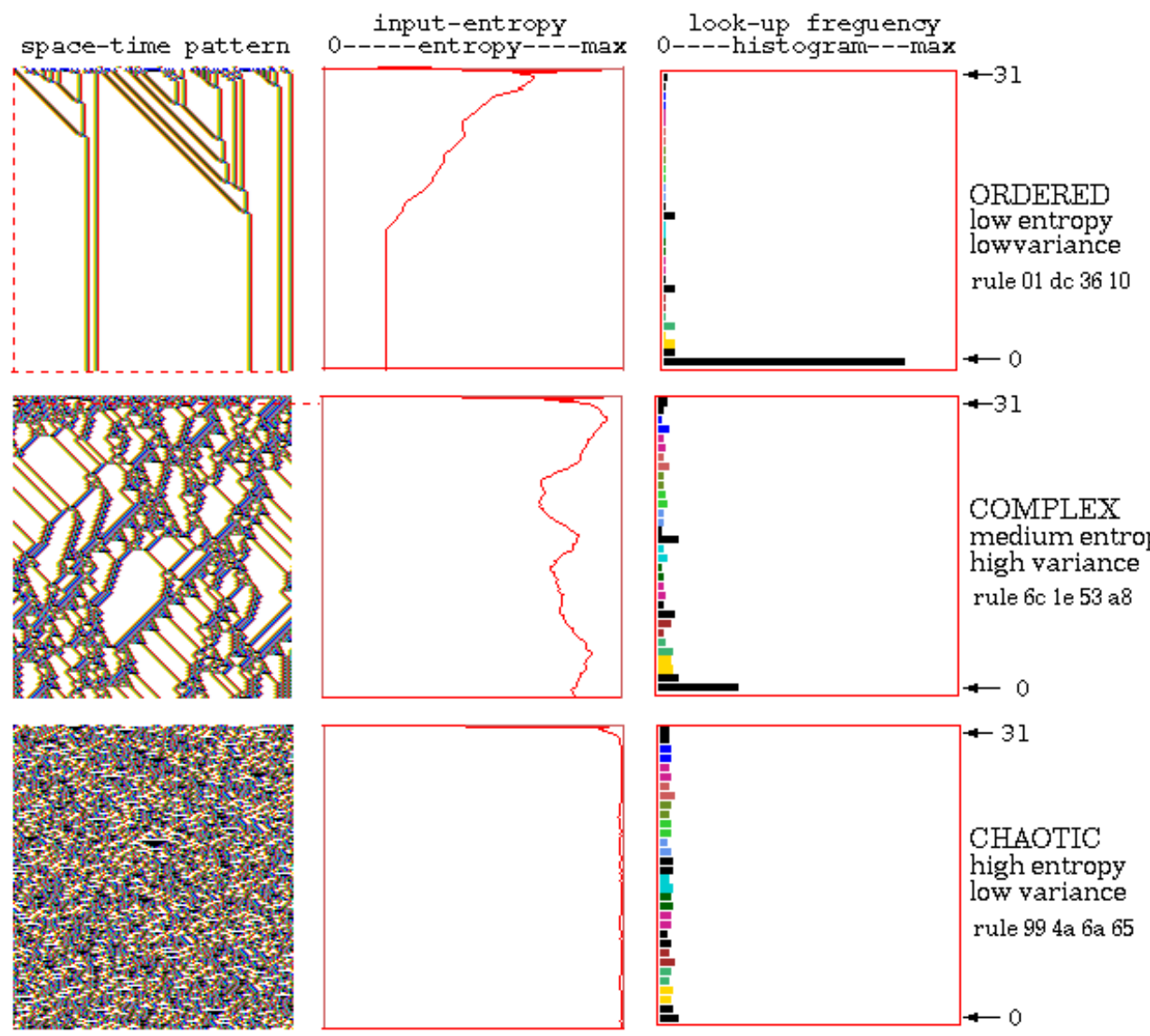

Figure 2.7: Typical 1d CA space-time patterns showing ordered, complex and chaotic dynamics, $n=150$, $v 2 k 5$ rcodes shown in hex. Alongside each space-time pattern is a plot of the input-entropy, where only complex dynamics exhibits high variability, caused by glider collisions.

also shown as a $2 \mathrm{~d}$ frequency histogram (section 33.6.2). Ordered, complex and chaotic dynamics are located in different regions allowing a statistical measure of their frequency. In addition the rules can be sorted by entropy variability allowing complex rules to be found automatically.

- The pattern density in a moving window of time-steps (section 31.4).

- The activity/stability of network elements. Frozen islands, the fraction of "genes" that have not changed over the last $x$ generations. For binary networks $v=2$ the fraction of frozen $0 \mathrm{~s}$ and 1s, and "genes" colored according the fraction of time they have been "on" (i.e. 1) in the same window of $x$ time-steps, falling into preset "frequency bins". This is also generalized for multi-value.

- The damage spread, or pattern difference, between two networks in $1 \mathrm{~d}$ or $2 \mathrm{~d}$. A histogram of damage spread can be automatically generated for identical networks with initial states differing by 1 bit (section 31.5). 
- The Derrida plot, and Derrida coefficient, analogous to the Liapunov exponent in continuous dynamical systems, measures how pairs of network trajectories diverge/converge in terms of their Hamming distance. This indicates if a random Boolean network is in the ordered or chaotic regime (chapter 22).

- A scatter plot of successive iterations in a 2d phase plane, the "return map", showing fractal structure, especially for chaotic rules (section 31.2.2.2).

\subsubsection{Measures on global dynamics}

The following measures on global dynamics, i.e. attractor basins, are available,

- Data on attractor basins. The number of basins in the basin of attraction field, their size, attractor period and branching structure of transient trees. Details of states belonging to different basins, subtrees, their distance from attractors or the subtree root, and their in-degree (chapter 27).

- A histogram of attractors showing the frequency of arriving at different attractors from random initial states. This provides statistical data on the basin of attraction field for large networks. The number of basins, their relative size, period, and the average run-in length can be measures statistically (section 31.6). An analogous method shows the frequency of arriving at different partly frozen patterns, fuzzy attractors called "skeletons" (section 31.7 ).

- Garden-of-Eden density plotted against the $\lambda$ and $Z$ parameters (section 24.9), and against network size (section 24.9.2).

- A histogram of the in-degree frequency of attractor basins or subtrees (section 24.6).

- The state-space matrix, a scatter-plot of the left half against the right half of each state bit string, using color to identify different basins, or attractor cycle states (section 24.5).

- The attractor jump-graph, an analysis of the basin of attraction field tracking where all possible 1-bit (or 1-value) flips to attractor states end up, whether to the same, or to which other, basin (section 20). The information is presented in two ways, as a jump-table: a matrix showing the jump probabilities between basins, and as a jump-graph: a graph with weighed vertices and edges giving a graphic representation of the jump-table. The jump-graph itself can be analyzed and manipulated in various ways, and rearranged and unraveled, including dragging vertices and defined components to new positions with "elastic band" edges.

\subsection{Reverse algorithms}

There are three different reverse algorithms for generating the pre-images of a network state.

- An algorithm for local 1d wiring[25] - 1d CA but rules can be heterogeneous.

- A general algorithm[26] for RBN, DDN, 2d or 3d CA, which also works for the above.

- An exhaustive algorithm that works for any "random mapping" including the two cases above. 


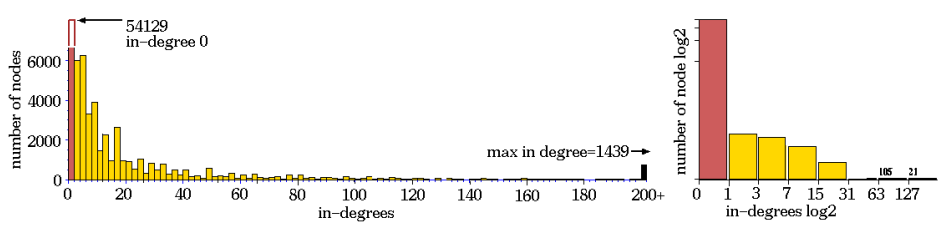

Ordered dynamics. Rule 01dc3610, $n=40, Z=0.5625, \lambda_{\text {ratio }}=0.668$. Above: in-degree histogram (normal and log-log). Right: complete subtree 7 levels deep, with 58153 nodes, $G$-density $=\overline{0.931}$.
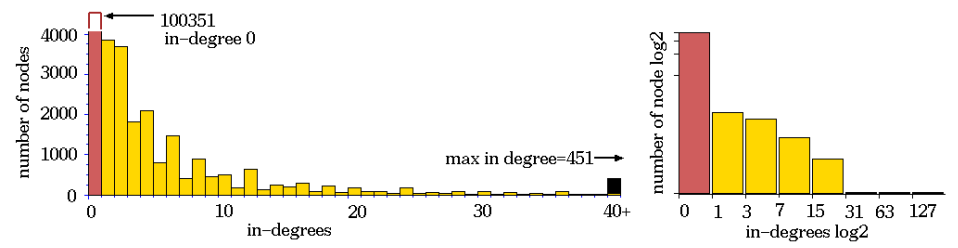

Complex dynamics. Rule 6c1e53a8, $n=50, Z=0.727, \lambda_{\text {ratio }}=0.938$. Above: in-degree histogram (normal and log-log). Right: subtree, stopped after 12 levels, with 144876 nodes, $G$-density $\overline{=0.692}$.
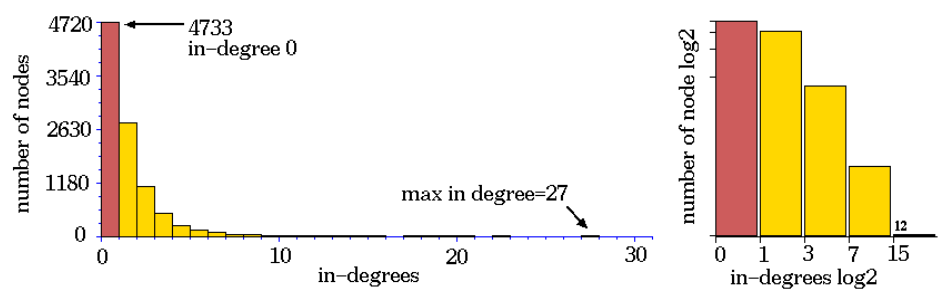

Chaotic dynamics. Rule 994a6a65, $n=50, Z=0.938, \lambda_{\text {ratio }}=0.938$. Above: in-degree histogram (normal and log-log). Right: subtree, stopped after about 75 levels, with 9446 nodes, $G$-density $=0.487$.

Figure 2.8: Subtrees of ordered-complex-chaotic CA. The rules and space-time patterns are shown in figure 2.7. The in-degree histogram of a typical subtree showing that convergence (bushiness of subtrees) is: ordered-high, complex-medium, chaotic-low.
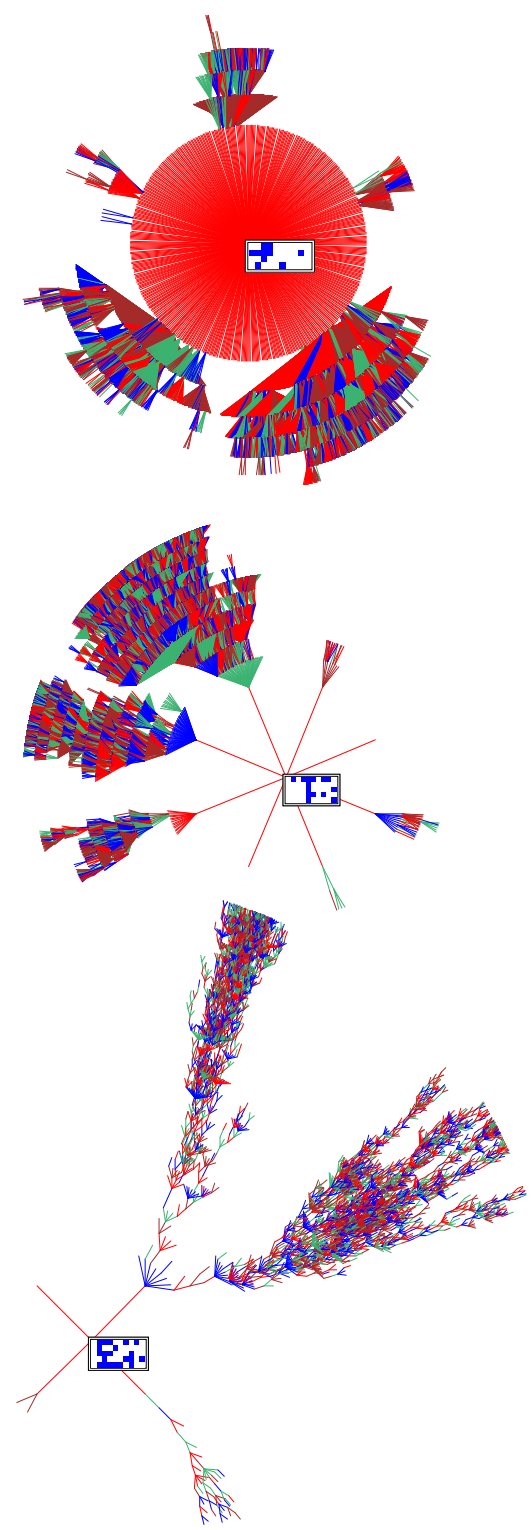

The first two reverse algorithms (section 29.6) generate the pre-images of a state directly; the speed of computation decreases with both neighborhood size $k$, and network size. The speed of the third exhaustive algorithm (section 29.7) is largely independent of $k$, but is especially sensitive to network size.

The method used to generate pre-images will be chosen automatically, but can be overridden. For example, a local 1d CA can be made to use either of the two other algorithms for benchmark purposes and for a reality check that all methods agree. The time taken to generate attractor basins is displayed in DDLab. For the basin of attraction field a progress bar indicates the proportion of states in state-space used up so far. 


\subsubsection{1d CA wiring reverse algorithm}

The CA reverse algorithm applies specifically for networks with 1d CA wiring (local wiring) and homogeneous $k$, such as $1 \mathrm{~d}$ CA, though the rules may be heterogeneous. This is the most efficient thus fastest algorithm, described in [25, 32]. Furthermore, compression of 1d CA attractor basins by rotation symmetry (section 26.1) speeds up the process.

\subsection{2 nonlocal wiring algorithm}

Any other network architecture, with nonlocal wiring, will be handled by a slower general reverse algorithm described in [26, 32]. A histogram revealing the inner workings of this algorithm can be displayed. Local $2 \mathrm{~d}$ or $3 \mathrm{~d}$ CA will also use this general reverse algorithm though in principle more efficient algorithms that take advantage of $2 \mathrm{~d}$ or $3 \mathrm{~d}$ local wiring could be devised. However, compression algorithms will come into play in $2 \mathrm{~d}$ to take advantage of the many rotation symmetries on the torus ${ }^{1}$.

\subsubsection{Exhaustive reverse algorithm}

A third, brute force, reverse algorithm first sets up a mapping, a list of "exhaustive pairs" of each state in state-space and its successor (this can be saved). The pre-images of states are generated by reference to this list. The exhaustive algorithm is restricted to small systems because the size of the mapping increases exponentially as $2^{n}$, and scanning the list for pre-images is slow compared to the direct reverse algorithms for CA and RBN. However, the method is not sensitive to increasing neighborhood size $k$, and is useful for small networks with large $k$. Exhaustive testing is also used for sequential updating (section 29.9).

\subsection{Random map}

The random mapping routine (section 29.8) creates a list of "exhaustive pairs", assigning a successor state at random to each state in state space, possibly with some bias (rules and wiring previously set are ignored). The attractor basins are reconstructed by reference to this random map with the exhaustive testing algorithm. The space of random maps for a given system size corresponds to the space of all possible basin of attraction fields and is the super-set of all other deterministic discrete dynamical systems.

\subsection{Sequential updating}

By default, network updating is synchronous, in parallel. DDLab also allows sequential updating, both for space-time patterns (section 31.3) and attractor basins (section 29.9). Default orders are forwards, backwards or a random order, but any specific order can be set out of the $n$ ! possible orders for a network of size $n$. The order can be saved/loaded from a file.

\footnotetext{
${ }^{1}$ Compression does not apply for a local $2 \mathrm{~d}$ CA with a hexagonal lattice, or for $3 \mathrm{~d} \mathrm{CA}$, as the algorithms to take account of these symmetries have not been resolved.
} 


\subsubsection{Neutral order components}

An algorithm in DDLab computes the neutral order components (section 29.10). These are sets of sequential orders with identical dynamics (section 29.10). DDLab treats these components as subtrees generated from a root order, and can generate a single component subtree, or the entire set of components subtrees making up sequence space (the neutral field) which are drawn in an analogous way to attractor basins.

\subsection{Sculpting attractor basins}

Learning and forgetting algorithms (section 34) allow attaching and detaching sets of states as predecessors of a given state by automatically mutating rules or wiring couplings. This allows "sculpting" the attractor basin to approach a desired scheme of hierarchical categorization. Because any such change, especially in a small network, usually has significant side effects, the methods are not good at designing categories from scratch, but might be useful for fine tuning a network which is already close to where its supposed to be.

When an attractor basin is complete, within the learning routine, a "target" state, together with a number of "aspiring pre-images" (predecessors) can, be selected. These states may be just highlighted in successive mutant attractor basins, or the learning/forgetting algorithms will attempt to attach/detach the aspiring pre-images to/from the target state, and can be set for either rule-table bit-flips or wire moves. In fact the bit-flip method cannot fail. New attractors can be created and subtrees transplanted. The result of learning/forgetting, including side effects, will be apparent in the new attractor basins. The algorithms, and their implications are described in[26].

More generally, a very preliminary method for reverse engineering a network, also known as the inverse problem, is included in DDLab, by reducing the connections in a fully connected network to satisfy an exhaustive map (for network sizes $n \leq 13$, section 18.7.4). The inverse problem is finding a minimal network that will satisfy a full or partial mapping (i.e. fragments of attractor basins such as trajectories). 


\section{Chapter 3}

\section{Accessing and running DDLab}

This chapter gives instructions for downloading, unpacking and running DDLab, and the various files available. Information is also given about the DDLab web site, manual, GPL license, copyright, and registration. The Linux, Cygwin, Mac, Unix, and Irix versions will be referred to as Linux-like, as opposed to the DOS version.

\subsection{The DDLab web site}

For the latest compiled versions, source code and manual, check the DDLab web site located at one of the following,

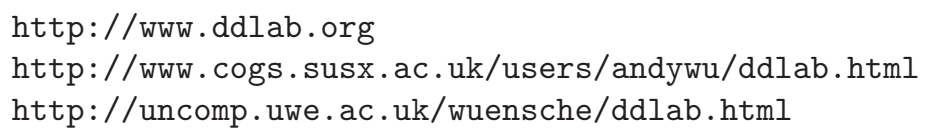

\subsection{DDLab at SourceForge}

DDLab compiled versions, source code and manual are also available at SourceForge.

http://sourceforge.net/projects/ddlab/files/

\subsection{Latest version of DDLab}

At the time of writing the latest release of DDLab was in May 2011, as follows.

version $\mathrm{m07}$, sub-directory ddlab_compiled_2011.

ddlab_linux_may_2011.tar.gz ... $\quad$ Linux/PC: compiled in Ubuntu 6.06.

ddlab_cygwin_may_2011.tar.gz ... Cygwin/Windows/PC: compiled with Cywin/X,

a Linux environment within Windows.

ddlab_mac_may_2011.tar.gz _. MAC OSX with X11: compiled in Mac OSX 10.4.10.

ddlab_dos_may_2011.tar.gz … DOS/PC: compiled with Watcom C version 11. 
dd_extra.tar.gz $\quad \ldots$ extra files for full functionality (section 3.7).

source code, sub-directory ddlab_code_2011.

ddlab_code_may_2011.tar.gz ... under the GNU General Public License, Version 3+.

the manual, sub-directory ddlab_manual_2011.

ddlabFeb2011.pdf _... corresponding exactly to this version of the software.

\subsection{Unzipping and running - Linux-like versions}

The Linux-like versions refer to Linux, Cygwin, Mac, Unix, and Irix, as opposed to the DOS version. Place the .tar.gz file in its own directory, called say, ddlab. To unzip and unpack follow the example below,

gunzip ddlab_linux_may_2011.tar.gz $\ldots$ to unzip the .gz file

tar-xvf ddlab_linux_may_2011.tar $\quad .$. to unpack the .tar file

This will give a readme file and the executable file ${ }^{1}$ ddlabm07 - we will use this filename in examples although the executable may be named ddlabm07_mac, ddlabm07_cygwin.exe etc.

To run the program enter

\section{.$/$ ddlabm07 \& (if dot is in your path ./ is not required)}

The \& retains control of the xterm window, where messages and data are sometimes shown. The default background is black - change to white with the program parameter -w,

$$
. / \mathrm{ddlabm07}-\mathrm{w} \&
$$

If working with source files, its preferable to run DDLab from a sub-directory (say ddlab/ddfiles) containing the ddextra.tar.gz files (section 3.7) - in that case enter,

$$
\text { . /ddlabm07 \& (two dots before the slash) }
$$

This ensures that files created within DDLab stay within the ddfiles subdirectory, and do not clutter up the ddlab directory.

\subsubsection{Unix library files}

DDLab for Unix is compiled with "static" set, so that missing library problems should not occur. However, the libraries libx11 and libsunmath need to be in your system - they usually are. If missing, they can be downloaded from the file unix_libs.tar.gz, which will unzip and unpack to give the following files, which should be installed in the same directory as DDLab.

$\operatorname{libX11.so.6.1}$

libsunmath.so.1

\footnotetext{
${ }^{1}$ If the executable permission is missing for some reason, it can be restored with the command chmod $+\mathrm{x}$ ddlabm07.
} 


\subsection{Unzipping and running - DOS}

The latest DOS version of DDLab, ddlab_dos_feb_2011.tar.gz can be unzipped with Winzip in earlier versions of Windows. For Windows Vista, open source "7zip" works to uncompress.

The DOS version may have some drawbacks; for better results install Cygwin/X, (a Linux environment inside Windows) and use the Cygwin version of DDLab.

The DOS version will unzip to give a readme file, two font files, and the following,

ddlabm07_dos.exe ... the program (for example).

dos4gw. exe ... the DOS extender, access to extended memory.

Keep all these files together in their own directory. For best results DDLab should be run in pure DOS (available in Windows98 and before), otherwise, prior to Vista, DDLab can be run from a DOS or "command line" window (some precautions will apply, sections 5.2.1, 5.2.2).

In Vista the DOS version does not work from the command line, but it works, but slowly, in "DOSBox", an open source MS-DOS emulator, intended for old PC games.

In pure DOS you can also add the following program parameters for a different graphics setup (this can also be changed later).

-w ... for a white background.

$-\mathrm{m} \ldots$ for $800 \times 600$ resolution.

$-\mathrm{h} \ldots$ for $1024 \times 768$ resolution.

For example, for a white background and 1024x768 enter ddlabm07 -w -h.

\subsection{The Quick Start Examples}

Chapter 4 gives brief "quick start" examples for a number of typical routines. Its a good idea to try these first to get the flavour of DDLab before reading the detailed manual.

\subsection{Extra data files}

The files in dd_extra.tar.gz, common to all platforms, contain data used by DDLab, though DDLab will run without the files. The files should be either in the same directory as the DDLab executable, or in a sub-directory and DDLab run from that sub-directory by prefixing . . / before the executable (section 3.4).

The lists below show some, but possibly not all, the files.

\subsubsection{Complex rule collections}

These are collections of complex rules for various combinations of $v, k$ and lattice dimensions, which can be loaded on-the-fly when running space-time patterns (section 32.6.1). Enter $\mathbf{g}$ for a random rule, or the next rule in the sequence, depending on the setup in section 31.2.8. 
$1 d$ complex rule collections, based on rcode

g_v2k5.r_s, g_v2k6.r_s, g_v2k7.r_s ..v 2 complex rules, as in binary DDLab.

g-v3k3.r_s, g_v4k2.r_s, g-v4k3.r.s, g_v5k2.r_s $\ldots v \geq 3$ complex rules.

$2 d$ complex rule collections, hexagonal lattice, based on kcode

g_v3k6.r_v, g_v3k7.r_v ...v=3 complex rules.

\subsubsection{Selected $2 \mathrm{~d}$ complex rules, $v=3$}

Selected $v=3$ complex $2 \mathrm{~d}$ rules based on kcode. These rules can be loaded individually while running space-time patterns. For $k=6$ and $k=7$, the default lattice is hexagonal, otherwise the default lattice is square. Some $k=6$ and $k=7$ rules also give interesting dynamics in $3 \mathrm{~d}$.

v3k4x1.vco ... see 4-way glider gun seed below.

v3k5x1. vco ... gliders bounce off static structures.

v3k6x1.vco ... the Beehive rule[39], hexagonal lattice.

v3k6x2.vco ... spirals overcome gliders, hexagonal lattice.

v3k6B1. vco ... burning paper or predator-prey.

v3k6n6. vco ... slow moving gliders, see 2-way glider-gun seed below.

v3k7w1.vco ... the spiral rule[40], hexagonal lattice

v3k7x1.vco ... gliders collisions make static structures.

v3k8x1.vco ... gliders move orthogonally and diagonally.

v3k9x1.vco ... gliders move orthogonally and diagonally.

\subsubsection{Selected seeds}

Selected 1d, 2d and 3d seeds (initial states) which can be loaded while running space-time patterns for interesting results for various rules.

pento.eed, Lgun_v2.eed, Lguns_v3.eed, Lguns_v8.eed ... game-of-Life seeds.

v3k4gun.eed ... seed for 4-way glider-gun (2d or $3 \mathrm{~d})-\mathrm{v} 3 \mathrm{k} 4 \mathrm{x} 1 . \mathrm{vco}$

v3k6n64.eed ... initial state for 2-way glider-gun - v3k6n6.vco

seeds for the 2d and $3 d$ Beehive-rule - v3k6x1.vco

Bcgun.eed, Bpuff.eed ... seeds for the 2d 6-way glider-gun, and the amazing puffer-train.

B3d_ggx.eed ... seed for the 3d 4-way glider-gun.

seeds for the 2d and 3d Spiral-rule - v3k\%w1.vco

ssg1.eed, sgg2.eed ... seeds for two types of 2 d 6 -way spiral glider-guns.

sgun3d.eed, sgun3d1.eed ... seeds for the 3d 4-way glider-guns.

\subsubsection{Sorted rule samples}

Samples of CA rule-space, sorted by input-entropy and its variability, classify rules between order, complexity and chaos[32]. The samples can be loaded and displayed as scatter plots (section 33.6), and rules can be selected on-the-fly (section 32.6.3) when running space-time patterns. The topic is described in "Classifying rule space" chapter 33. The following sample files are available, 


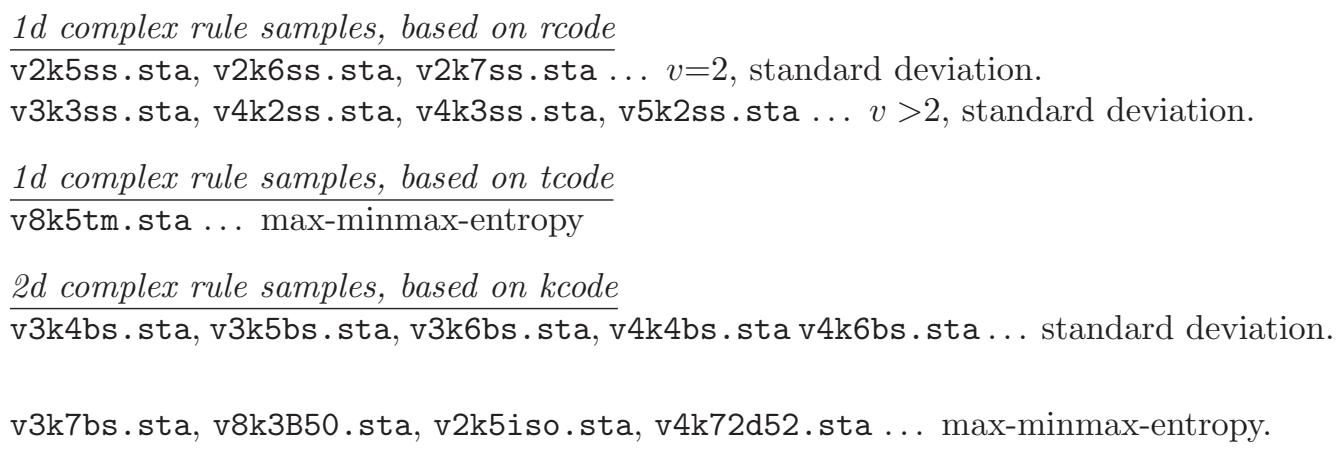

\subsubsection{Byl's self reproducing loop}

J.Byl's self reproducing loop[5], is a v6k5 2d CA, a simplification of Langton's loop.

v6k5_byl.rul ... Byl's self reproducing loop rule (rcode).

v6k5_byl.eed ... a seed to initiate Byl's loop.

\subsection{Copyright, License and Registration}

\section{Copyright}

DDLab is copyright (c) 1993-2011, Andrew Wuensche.

\section{$\underline{\text { License }}$}

DDLab is free software: you can redistribute it and/or modify it under the terms of the GNU General Public License (GPL) as published by the Free Software Foundation, either version 3 of the License, or any later version (www.gnu.org/licenses/gpl.html), which includes a Disclaimer as follows: This program is distributed in the hope that it will be useful, but without any warranty; without even the implied warranty of merchantability or fitness for a particular purpose.

\section{Registration}

By registering and paying a modest fee, the annoying UNREGISTERED banner (section 5.4) can be easily removed — www.ddlab.org/ddinc.html provides details.

\subsection{Previous versions of DDLab}

Previous versions of DDLab released at intervals since 1995 are still available, as are previous versions of the DDLab Manual - refer to www.ddlab.org. 


\section{Chapter 4}

\section{Quick Start Examples}

This chapter briefly describes the DDLab graphical user interface, and gives a number of examples of DDLab functions and routines. Try these examples first, to get the flavor of DDLab, before tackling the detailed program reference - chapter 5 onwards.

\subsection{The DDLab screen}

In Linux-like platforms, or Windows, DDLab occupies a window within the monitor screen, (in pure DOS the whole screen is occupied). For simplicity we will refer to the DDLab "screen", and various panels which appear within the screen as "windows" or "prompts". Their location within the screen is usually indicated, for example "top-right", "top-left", "bottom-left", etc.

If the executable filename is ddlabm07, to run DDLab, for DOS enter ddlabm07 at the DOS or command prompt. For Linux-like systems enter ./ddlabm07 \&) - always add a final \& to retain control of the terminal window, where various data may be displayed.

When DDLab is run with no program parameters, the screen appears with a black background. The program parameter -w gives a white background, i.e. ./ddlabm07 -w \& for Linux, ddlabm07 - w for DOS. Descriptions of colors assume a white background.

If an UNREGISTERED banner is displayed, enter return to continue ${ }^{1}$. A title bar is displayed across the bottom of the screen. A series of prompts are presented to set up the network, functions to be performed, and presentation. These prompts appear either in a main sequence for the most common settings, or in various windows that automatically open up.

The mouse cursor is used to set bits or values in rule-tables and network states, for "drawing" patterns, especially in $2 \mathrm{~d}$ networks, for dragging nodes in the network-graph and jump-graph, and for some other functions, but most user inputs are from the keyboard.

\subsubsection{User Input}

The flashing cursor (usually green) prompts for input. Enter appropriate input from the keyboard. To revise the input, press q, backspace, or the right mouse button. To accept the input, and move on to the next prompt or routine, press return or the left mouse button. If no input was entered, or if the input was inappropriate, a default input is automatically selected.

\footnotetext{
${ }^{1}$ Another program parameter turns off the UNREGISTERED banner for registered users.
} 


\subsubsection{Backtrack}

To backtrack to the preceding prompt, to revise, or interrupt a running routine such as space-time patterns or attractor basins, press $\mathbf{q}$, or right mouse button.

You can backtrack to any stage in the prompt sequence with $\mathbf{q}$ (or right mouse button), eventually to exit the program.

\subsubsection{Quitting DDLab}

To quit DDLab immediately (except in DOS) enter Ctlr-q at any prompt, followed by q. Otherwise backtrack with $\mathbf{q}$ to the start of the program, then enter $\mathbf{q}$ to exit.

\subsubsection{Skipping Forward}

At some points in the prompt sequence, its possible to skip forward, to avoid a succession of prompts for special settings. When the following top-center banner is visible,

accept defaults-d ... enter $\mathbf{d}$ to skip forward.

\subsubsection{The graphics setup - Linux-like systems}

Linux-like systems include Unix, Linux, Irix, Mac-X11 and Cygwin. The screen will start with a black background if no program parameters were set, and with a resolution automatically set to comfortably fit the monitor, but with an initial maximum of $925 \times 694$ pixels.

To change the graphics setup after DDLab has started, at the first prompt select $\mathbf{g}$ for graphics. A graphics setup screen will appear. Enter $\mathbf{b}$ to toggle the background between black and white. Other options allow changing the resolution, font size, text line spacing and cursor flash speed. The screen can be resized by dragging the corners (or edges) with the mouse in the usual way.

\subsubsection{The graphics setup - DOS, or Windows command line}

Although the DOS version of DDLab is still available, we recommend that you install Cygwin (a Linux environment inside Windows) and run the Cygwin version of DDLab.

If you are still running DDLab in DOS, or in a Windows command line, read on ...

Its recommended that you run DDLab in pure DOS mode, which is available in Windows98 and prior versions, where there is an option "Restart the computer in MS-DOS mode". Otherwise run DDLab in a DOS or command-line window where it should be run initially in low resolution only (the default). If you expand to full screen (toggle Alt-Enter) you may use higher resolutions, but the mouse cursor will probably not be visible. Be sure to revert to low resolution before changing from full screen back to the DOS/command-line window to avoid unforeseen consequences.

In DOS, the background can be changed between black and white, and the resolution between $640 \times 480,800 \times 600$, and $1024 \times 768$ pixels, given the necessary monitor, graphics card and driver.

The DDLab screen will start up at a resolution of $640 \times 480$ (VGA) with a black background, if no program parameters are set. Program parameters may be added for SVGA as follows: - $\mathrm{h}$ (for high, $1024 \times 768)$ and $-\mathrm{m}$ (for medium, $800 \times 600$ ), i.e. for high resolution start DDLab by entering ddlabm07 - h at the DOS prompt. For a white background enter ddlabm07 -w, or ddlabm05 - $\mathrm{h}-\mathrm{w}$ for both high resolution and a white background. 
To change the resolution or background after DDLab has started, at the first prompt select $\mathbf{g}$ for graphics. A graphics setup screen will appear. Enter $\mathbf{b}$ to toggle the background. Other options allow changing the resolution, font size and text line spacing and cursor flash speed.

\subsection{Basin of attraction fields}

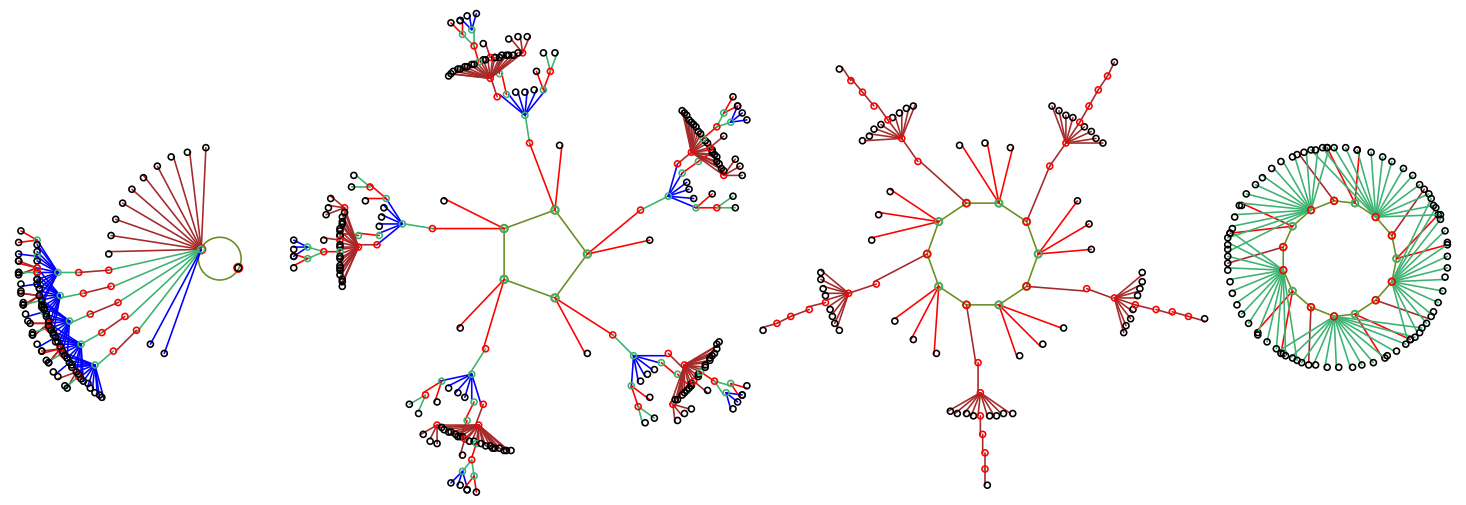

Figure 4.1: A basin of attraction field of a binary $1 \mathrm{~d}$ Cellular Automaton, $v 2 k 3, n=10$, decimal rule 9, with copies of equivalent basins suppressed.

To generate a basin of attraction field similar to figure 4.1, do the following:

1. From the first prompt keep accepting defaults with return or left mouse button (about 14 presses), until the top-center basin parameters banner appears, and a top-right window with a list of options starting with accept all basin defaults $-\mathbf{d}$. Enter $\mathbf{d}$ to skip these special options.

2. A final top-right prompt window appears, just before drawing basins. Enter return.

3. The basin of attraction field will be generated. Copies of equivalent basins are suppressed. The initial default setup is for a $1 \mathrm{~d} \mathrm{CA}$, network size $n=10$, value-range $v=2$, and neighborhood size $k=3$. If these parameters were changed they become the new defaults. The rule (chosen at random by default) appears in a window at bottom of the screen. A top-right window shows brief data on the field once it is generated. A progress bar below this window shows the proportion of state-space as it is used up. Vertical lines on this bar indicate the states used to seed the basins.

4. A prompt window appears top-left. Enter return for a new basin of attraction field, a one-bit "mutant" of the previous rule, with corresponding data. This process can continue indefinitely (it can also be set on automatic).

5. Enter q to interrupt and backtrack up the prompt sequence.

\subsubsection{Changing basin parameters}

Try the previous routine again, changing $v, n$ or $k$, which require backtracking to the main series of prompts. For example, to create figure 4.2 procede as follows,, 


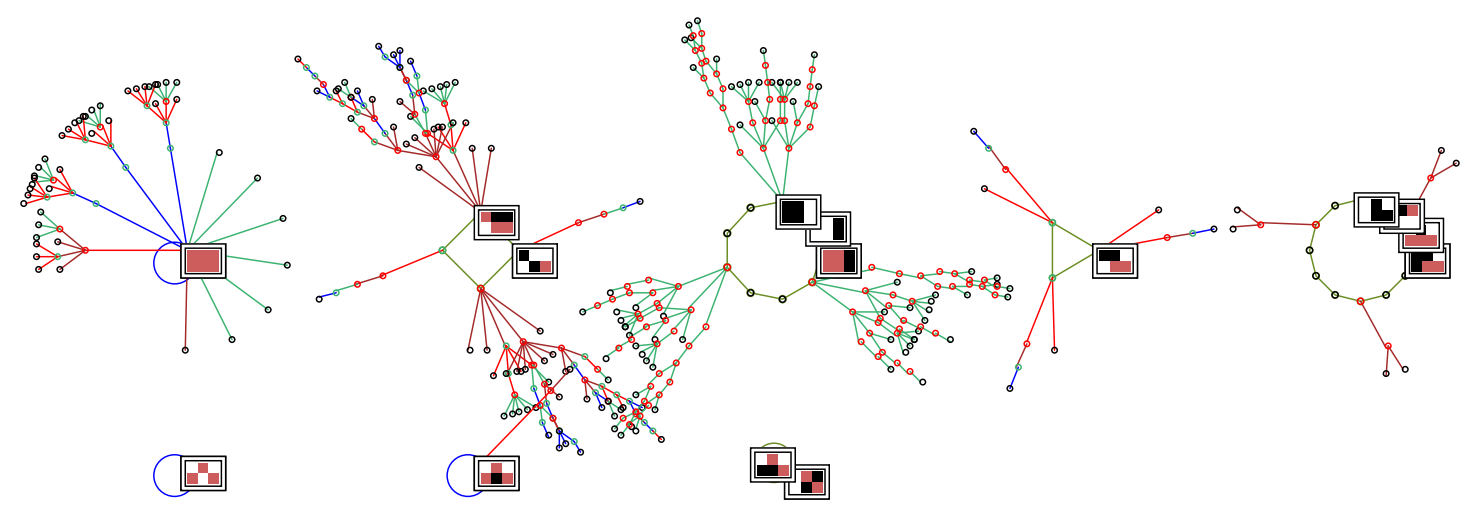

Figure 4.2: The basin of attraction field of multi-value $v 3 k 3 n=61 \mathrm{~d}$ CA. The lookup table is 120201201020211201022121111 (1886122584a655 in hex). Just the 8 nonequivalent basins are shown from a total of 23 , and attractor non-equivalent states are shown as $2 \mathrm{~d}$ patterns. Statespace $=v^{n}=3^{6}=729$. Note that the overlap can be fixed with layout options (chapter 25 or section 20.7).

1. To revise $v$ backtrack to the prompt Value range $\ldots$ : enter 3.

2. To revise $n$, at the prompt Network size ... : enter 6 .

3. To revise $k$, at the prompt Neighborhood size k: ... enter 3 .

4. At the prompt Select $\mathbf{v} 3 \mathrm{k} 3$ rule ... : enter $\mathbf{h}$ for the rule in hex, then enter 1886122584a655, then return to accept (for a random rule just enter return or $\mathbf{r}$ ).

The scale, position, node display etc. can be fine-tuned with the special basin parameters options, which can be accessed one by one, or by jumping directly to a category (chapter 31 ).

1. At the top-right revise from: options, enter $\mathbf{p}$ for the "display" category.

2. Enter return until the prompt highlight attractor ... : enter a for "all", then $\mathbf{d}$ twice to accept further defaults and start drawing basins.

3. Enter return for a mutant basin, or $\mathbf{q}$ to backtrack.

Note that increasing $v$ will reduce the maximum allowable $k$ and $n$ (sections 7.2,7.3), and as these values increase basins will take longer to generate.

\subsection{Backwards space-time patterns, and state-space matrix}

While the attractor basins are generating, various display settings, indicated in the bottom title bar, can be changed on-the-fly. However, basins may generate too fast to intervene on-the-fly. In this case, at the pause when a basin is complete, enter $\mathbf{s}$ for speed in a top-left window, and follow self-explanatory prompts to slow down. Alternatively, backtrack to slightly increase $n, v$ or $k$.

1. Enter s to toggle the "backwards" space-time pattern on-off, and see predecessors (preimages) being generated on the left of the screen (figure 4.3). Initially the attractor states will be displayed, then each state and its set of pre-images. Expand or contract the scale of the backwards space-time pattern with e and c. Toggle scrolling on/off with \#. 
Figure 4.3: Backwards space-time patterns relating to the basin of attraction field of the $v=2$ CA in figure 4.1 . Space across, time top down. The red and white bit patterns are the predecessors of black and white bit patterns.

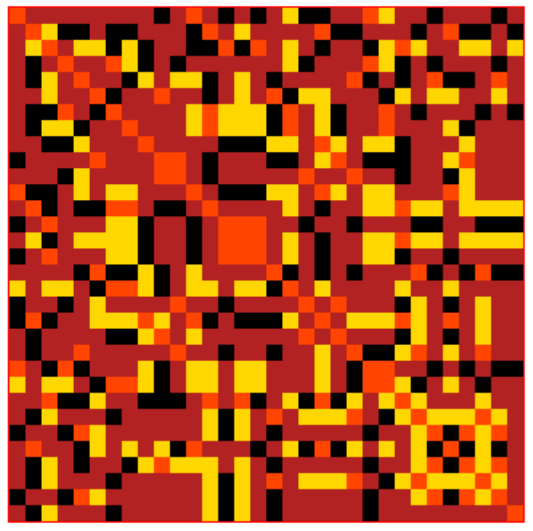

Figure 4.4: The state-apace matrix represents state-space, plotting the left half of each state bitstring against the right half. Colors represent different basins of attraction in figure 4.1.

2. Enter $\mathbf{m}$ to toggle the display of the state-space matrix in the lower right corner (figure 4.4). This reveals interesting symmetries. Different colors represent states in different basins.

3. Enter $<$ to incrementally slow down, or $>$ to restore maximum speed.

4. Enter $\mathbf{q}$ to interrupt and backtrack up the prompt sequence.

\subsection{Basin of attraction fields for a range of network sizes}

To produce output similar to pages in the "Atlas of Basin of Attraction Fields", Appendix 2 of the book "The global ynamics of Cellular Automata"[25], proceed as follows,

1. Backtrack with $\mathbf{q}$ (or right mouse button) to the start of the program.

2. At the third prompt, range of network size-r: enter $\mathbf{r}$.

3. Enter return until the top-center basin parameters banner appears, then a to restore all defaults, then $\mathbf{d}$ to skip further special options, then enter return to start the range of CA basin of attraction fields (with the same rule), for increasing sizes from 5 to 12 .

4. When complete, enter return for the next "mutant" CA rule.

5. Toggle the display of the "backwards" space-time patterns with $\mathbf{s}$ and the state-space matrix with $\mathbf{m}$ as described in 4.3 .

6. To slow down the generation of basins (probably required to intervene on-the-fly) enter $\mathbf{s}$ for speed at a pause in the top-left window, and follow self-explanatory prompts.

7. Enter $\mathbf{q}$ to interrupt and backtrack up the prompt sequence.

You may need to readjust the size of basins for everything to fit. To do this, backtrack to basin parameters and enter $\mathbf{l}$ for layout, then adust the size and spacing of basins with various self-explanatory prompts (chapter 25). 
5.

6.

7.

8.

9.

10 .
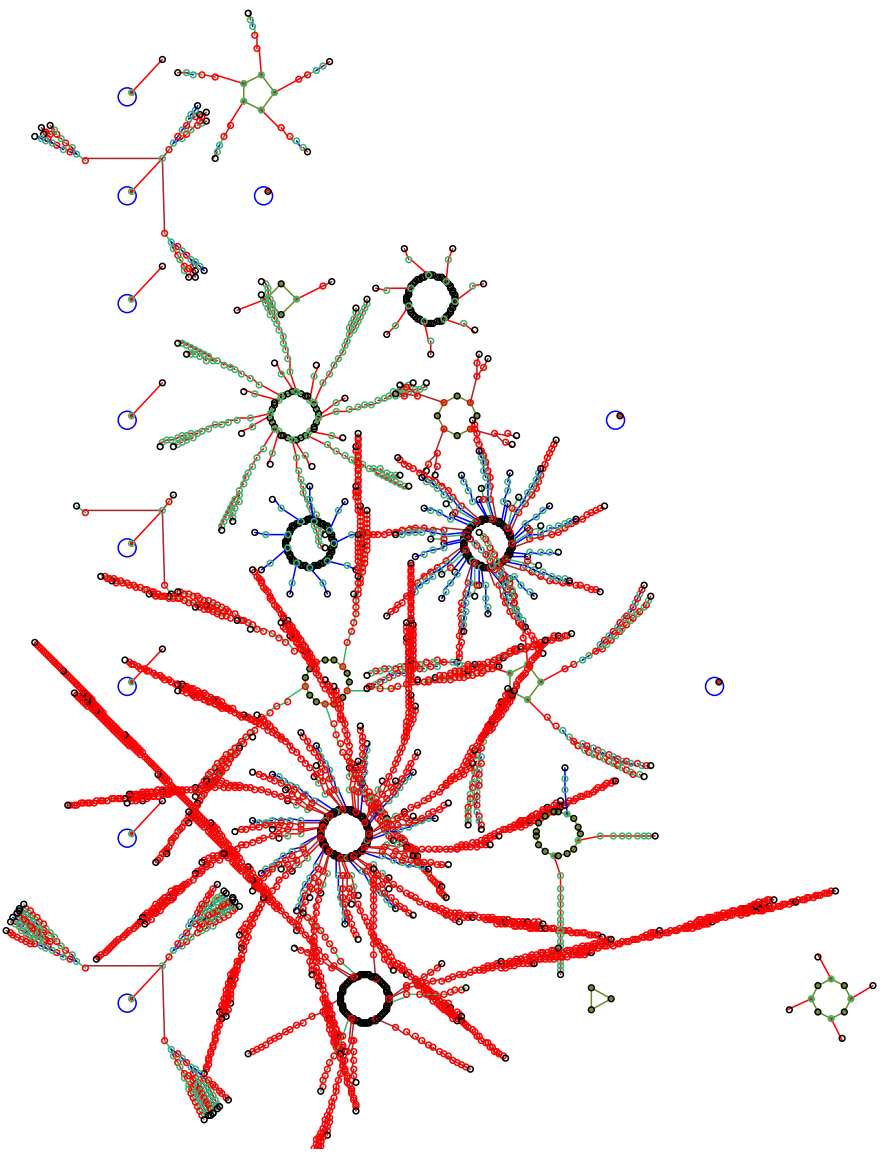

$0{ }_{0}$

11.

12.

Figure 4.5: Basin of attraction fields for a range of network size $n=5-12 . v 2 k 3$, rule $(\mathrm{dec})=30$

\subsection{A single basin of attraction}

Backtrack with $\mathbf{q}$ (or right mouse button) to the start of the program.

1. At the very first prompt enter $\mathbf{s}$.

2. At the Neighborhood size k: ... prompt, enter 4 .

3. Enter return until the top-center basin parameters banner, then a to restore all defaults, then $\mathbf{d}$ to skip further special options, then return in response to further prompts, to generate a singe basin for a CA, size 14 .

4. Enter return for the next mutant.

5. Toggle the display of the "backwards" space-time patterns with s and the state-space matrix with $\mathbf{m}$ as described in 4.3.

6. Enter q to interrupt and backtrack up the prompt sequence. 


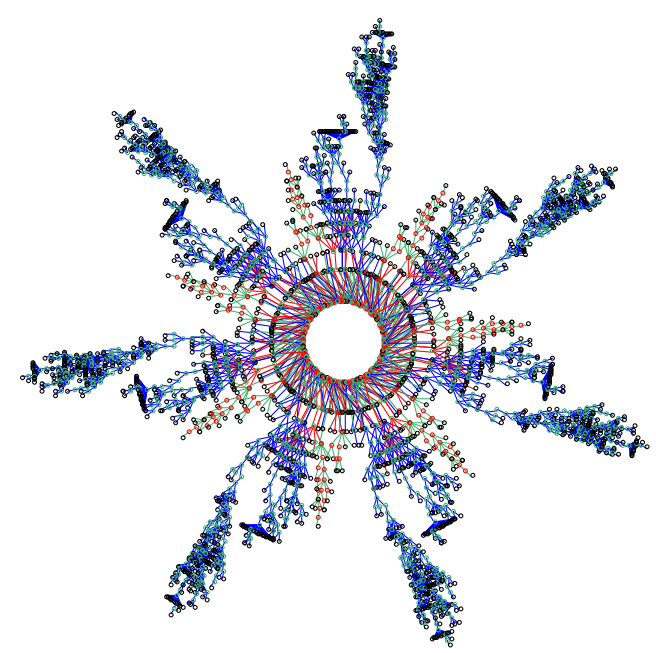

Figure 4.6: A single basin of attraction with 4333 states, and an attractor period of $140, v 2 k 4, n=14$, hex rcode $=76 \mathrm{~b} 5$, decimal seed $=3187$.

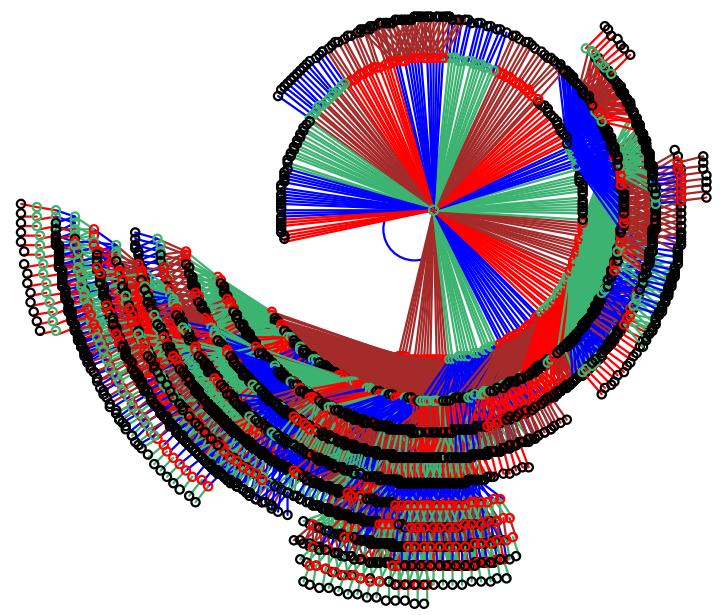

Figure 4.7: A single basin of attraction with 15541 states and an attractor period of one, a point attractor, $v 2 k 4, n=14$, hex $\operatorname{rcode}=\mathrm{ac} 88$, decimal seed $=3187$.

\subsection{A subtree}

Backtrack with $\mathbf{q}$ (or right mouse button) to the start of the program.

1. At the very first prompt enter $\mathbf{s}$.

2. Enter return in response to further prompts until the prompt Network size ... : select 27.

3. At the prompt Neighborhood size k: ... select 5 .

4. Enter return in response to further prompts until the Select SEED ... : prompt. Enter $\mathbf{r}$ for a "random seed" then a to set all cells at random.

5. Enter return until the top-center basin parameters banner, then a to restore all defaults, then $\mathbf{d}$ to skip further special options.

6. At the prompt backward for subtree-b, forward for basin-(def): select $\mathbf{b}$.

7. At the next prompt, forwards before backwards?

\section{how many steps (default 0): select 3 .}

This runs the CA forward by 3 time-steps (from the "seed"), before running backward from the state reached. The original randomly selected seed is likely to be a "garden-of Eden" state with no predecessors, so not much use as the root of a subtree.

8. Enter return in response to further prompts to generate the subtree. For a deeper subtree, enter a greater number of forward time-steps at the previous prompt. This might reach an attractor state, in which case the whole basin will be generated with the message subtree=basin in the top-right data window.

9. Enter return for the next mutant.

10. Enter $\mathbf{q}$ to interrupt and backtrack up the prompt sequence. 


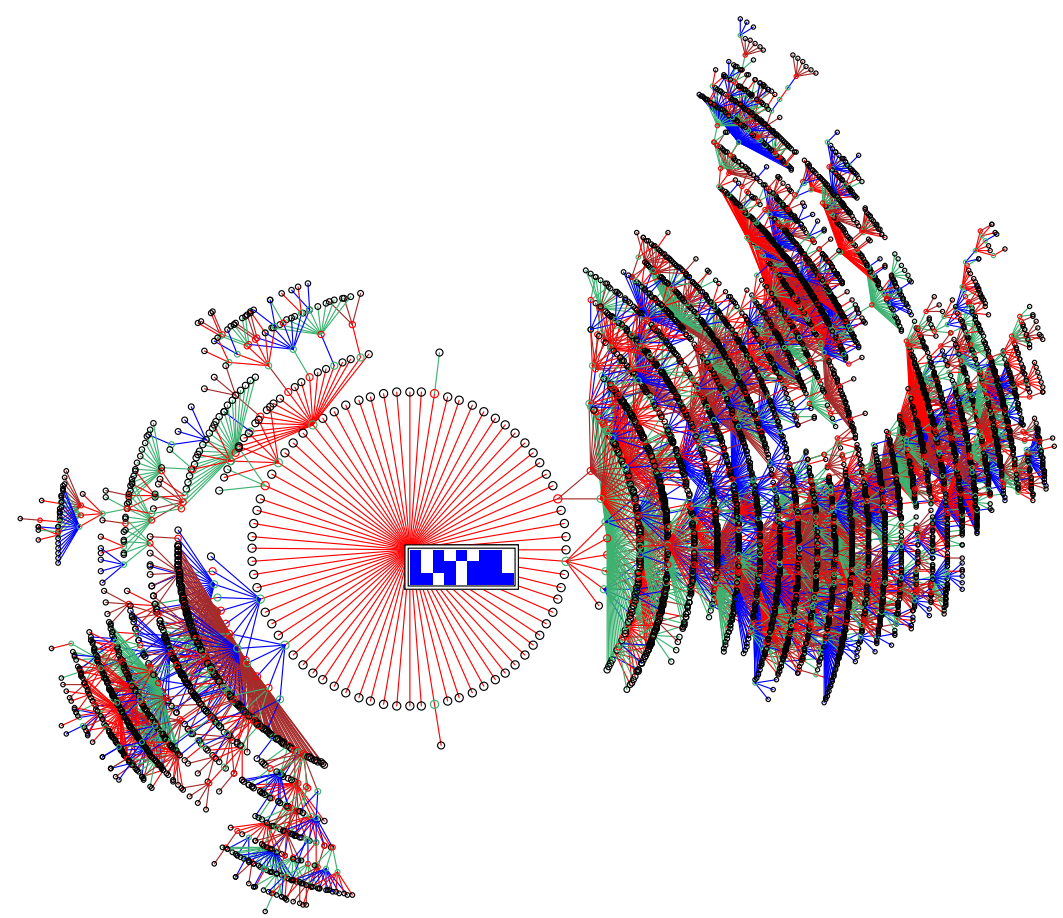

Figure 4.8: A subtree with 11324 states generated from the bit pattern at the center. $1 \mathrm{~d}$ CA, $v 2 k 5$, $n=27$ shown as $9 \times 3$, hex rcode 5 afbblae, hex seed 055addaf.

To highlight the "root" state as a bit pattern, backtrack to basin parameters, enter $\mathbf{p}$ for display, then return until the prompt highlight attractor (or subtree root ... : enter 1. There are various ways of displaying states or nodes in basins, described in section 26.2.

\subsection{Space-time patterns - SEED-mode or TFO-mode}

Space-time patterns can be run in either SEED-mode (based on rcode) which also allows single basins, or alternatively TFO-mode, where basin functions (and prompts) are disabled, so space-time patterns become the only possibility, and rules are restricted to totalistic rules expressed as tcode or kcode, with shorter rule-tables than rcode, allowing larger $[v, k]$. This is decided at the very first prompt (section 6.2.1), where initially FIELD-mode is active,

Exit-q, graphics setup-g, randseed-r, TFO:totalistic/forward only-t SEED:forward only/single basin/subtree-s (FIELD-def):

Enter $\mathbf{s}$ for SEED-mode - the main sequence prompts continue, or enter $\mathbf{t}$ for TFO-mode the first prompt changes to,

EXIT-q graphics setup-g randseed-r, disable TFO allow basins-b TFO:totalistic rules and forward only (def:) (this line in red) 
Enter return to accept TFO-mode and continue, or $\mathbf{b}$ to revert to FIELD-mode.

SEED-mode allows either single basins (the default) or space-time patterns. The choice is made after the main prompt sequence, at the basin parameters banner, and the prompt ... space-time pattern only-s.

Entering $\mathbf{s}$ changes the banner to space-time parameters and presents the space-time pattern options (section 31.1),

accept all space-time defaults-d

revise from: start/misc-ret updating-u

entropy-e damage-m attractors-a skeletons-s: (skeletons for $v=2$ only)

In TFO-mode, these prompts are presented directly, after the main prompt sequence.

\subsection{1d Space-time patterns}

Backtrack with $\mathbf{q}$ (or right mouse button) to the very first prompt.

1. At the very first prompt, enter $\mathbf{s}$ for SEED-mode.

2. At the prompt Network size ... : select 150 .

3. At the prompt Neighborhood size k: ... select 5 .

4. Enter return until the top-center basin parameters banner appears, then enter $\mathbf{s}$ for space-time pattern only - the top-center banner changes to space-time parameters

5. At the next prompt, accept all space-time pattern defaults-d, enter $\mathbf{d}$ to skip special options. The $1 \mathrm{~d}$ space-time pattern is generated, scrolling upwards, on the left of the screen. To the right is a histogram of the lookup frequency for each neighborhood relating to a window of 10 time-steps, and a plot of the entropy of this histogram, the "input-entropy". The on-the-fly key index on the right of the screen gives a complete list of immediate changes that can be made with a key hit (section 32.1). Some of these options are repeated on the right of the bottom title bar. Try the following (or any other) to see what happens:

g ... to change the rule to a "complex" rule, chosen at random from a database of complex rules in the file g_v2k5.r_s, available in the "ddextra" download.

3 ... to toggle cell color according to the lookup neighborhood or the value.

$\mathbf{u}$... to toggle the input-entropy - density plot.

4 ... for a new random initial state.

f ... to progressively "filter" the space-time pattern, and a to restore the unfiltered pattern.

$\mathbf{1}$... for a random "bit-flip" (mutate one output in the rule-table), and $\mathbf{2}$ to flip back.

If "bits" are flipped successively with key1, key $\mathbf{2}$ will flip them back in reverse order.

$\mathbf{e} / \mathbf{c} \ldots$ enter $\mathbf{e}$ or cto expand or contract the scale of the space-time pattern.

6. Enter $\mathbf{q}$ to pause, a top-right prompt appears with further options 32.16. For example, to select or revise a particular rule enter $\mathbf{v}$. A bottom-right prompt appears, enter return, then $\mathbf{h}$ for the new rule in hex, and enter the hex characters e9f6a 815 for the rcode in figure 4.9, then enter return until the space-time pattern continues from where it left off, but with the new rule.

7. Enter $\mathbf{q}$ to backtrack further. 

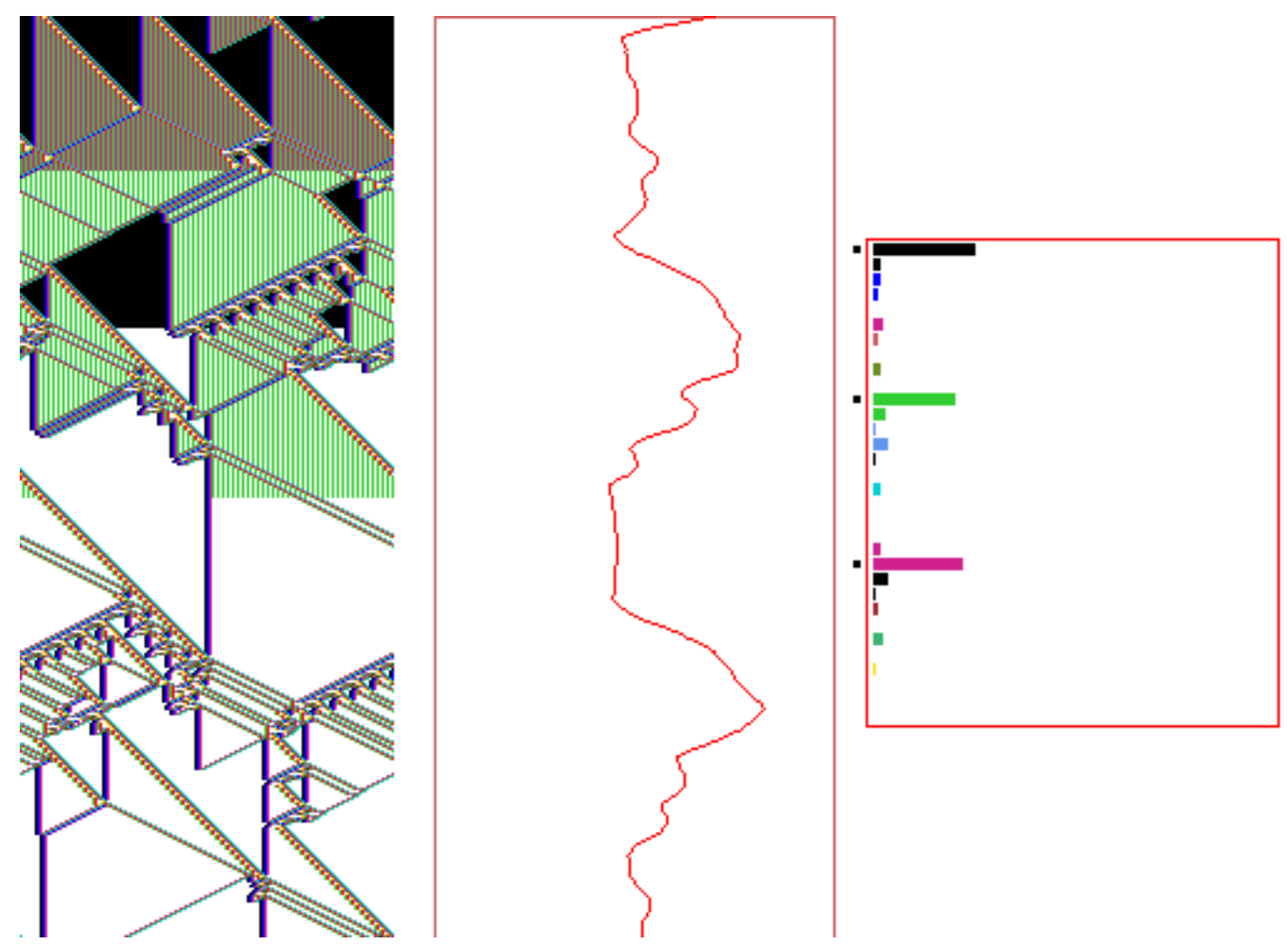

Figure 4.9: A space-time pattern of a $1 \mathrm{~d} v 2 k 5$ complex CA, rcode(hex) e9f6a815, $n=150$. About 360 time-steps, including some analysis shown by default. Left: the space-time pattern colored according to neighborhood, and progressively "filtered" at three times with key $\mathbf{f}$, suppressing the background domain to show up "gliders" more clearly. Center: the input-entropy/time plot. Right: the lookup frequency histogram for the last time step shown.

\subsubsection{1d ring of cells, and scrolling the ring}

A 1d CA has periodic boundaries, effectively a ring or circle of cells. Space-time patterns can be displayed as a movie of the changing patterns on this ring (section 32.19, figure 32.35), and the ring itself can be scrolled, making a scrolling tube (figure 4.10).

To display the ring alongside the normal space-time pattern (figure 4.9) proceed as follows,

1. Set up the normal space-time pattern as in section 4.8 .

2. Enter $\mathbf{q}$ for the interrupt prompts, then $\mathbf{g}$ to show the network as a graph, then return. A circle of cells will appear.

3. Enter $\mathbf{q}$ to exit the graph and continue. On-the-fly changes work on the circle as well as the normal space-time pattern.

\subsubsection{Scrolling the ring}

To display the ring and make it scroll, proceed as follows,

1. Set up the normal space-time pattern as above in section 4.8 . 
2. Enter $\mathbf{T}$ on-the-fly to toggle the display the $1 \mathrm{~d}$ space-time pattern to $2 \mathrm{~d}$.

3. Enter $\mathbf{q}$ for the interrupt prompts (section 32.16), then $\mathbf{g}$ to show the network as a graph, then return. A circle of cells will appear. The appearance/position of the ring can be altered (chapter 20) but the default setting will be fine.

4. Enter q to exit the graph and continue. Space-time patterns will play out as a movie on the ring.

5. Enter \# or \& to toggle scrolling the ring.

6. Enter $\mathbf{J}$ to toggle hiding the scrolling 2 d space-time pattern which may be also superimposed on the ring.

Initially the ring will move diagonally towards the bottom-right, then scroll diagonally upward, so that the present moment is the ring at the bottom-right.

Most on-the-fly options work as usual with the ring. Try the following key hits,

3 ... to toggle to cell color; according to the lookup neighborhood or the value.

@ ... to toggle the cell outline.

c ... to contract (or e expand) the spacing between successive rings (time-steps).

$4 \ldots$ for a new random initial state.

f ... to progressively filter, a to totally unfilter.

$4 \ldots$ for a new random initial state.

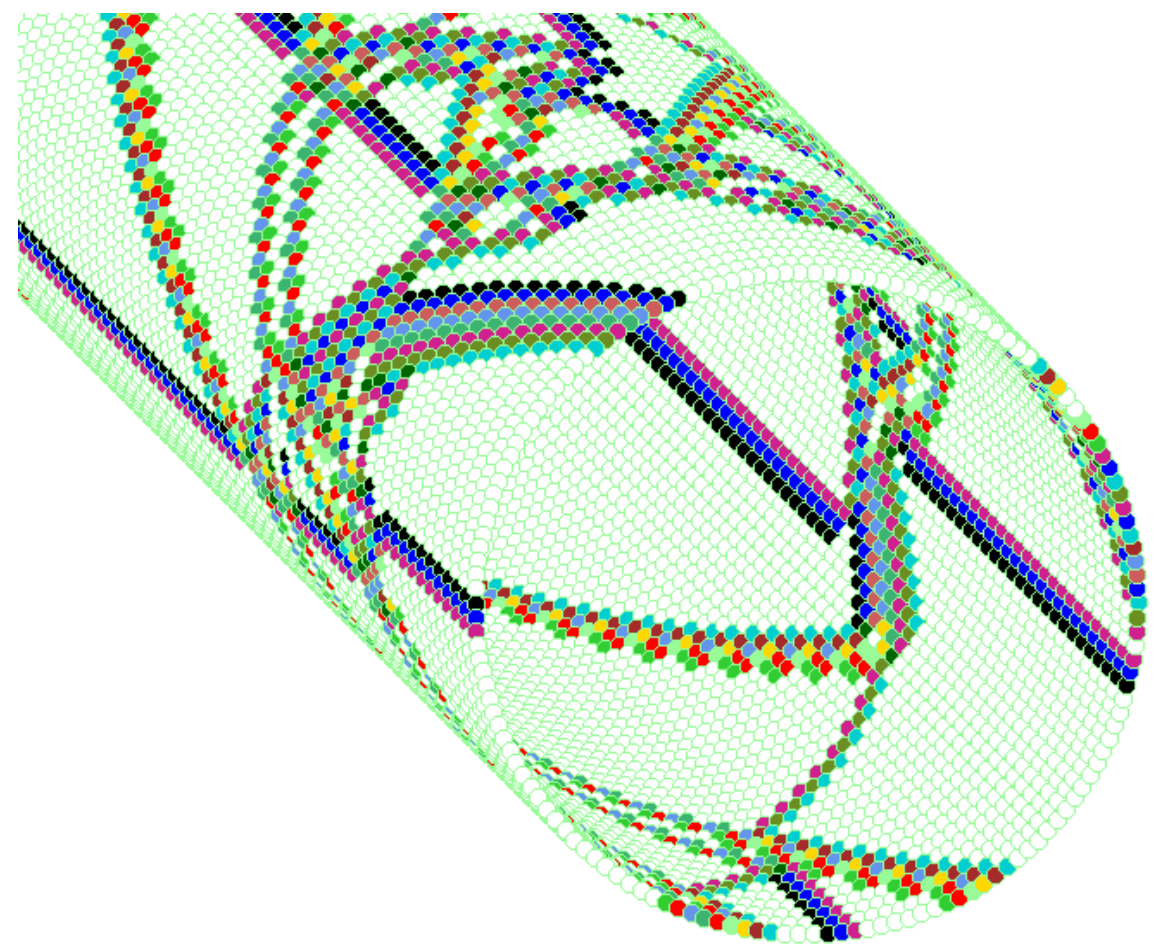

Figure 4.10: A 1d space-time pattern shown as a ring of cells scrolling diagonally upward - a scrolling tube. The present moment is the ring at the bottom-right - the closest ring. The space-time pattern is colored according to neighborhood, and has been filtered. 1d CA, $v 2 k 5$, rcode (hex) e9f6a815, $n=150$. 


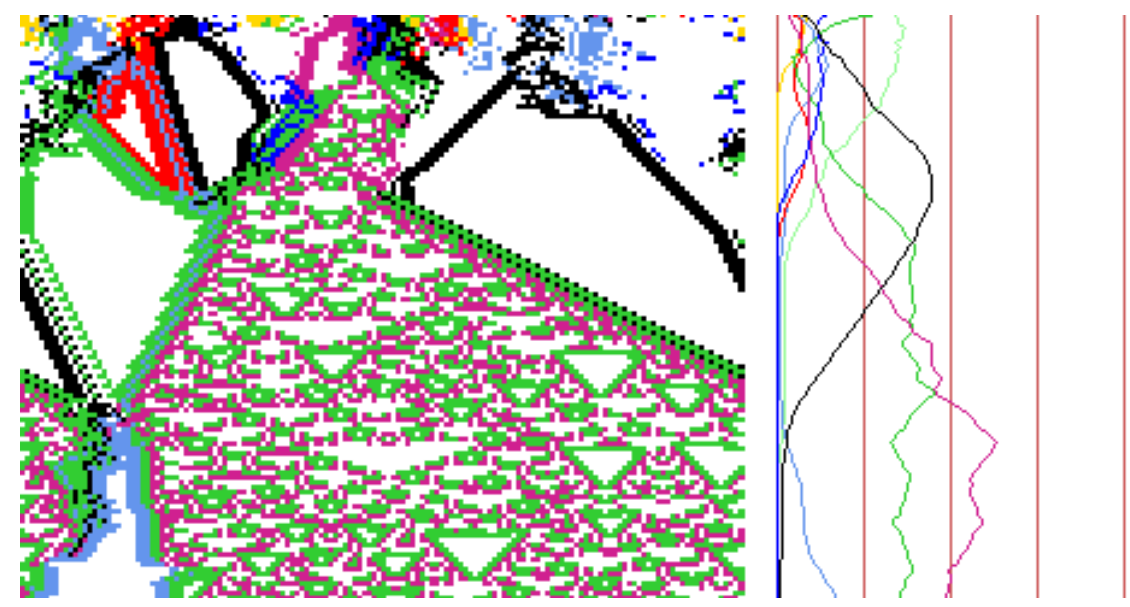

Figure 4.11: A 1d CA with a filtered Altenberg rule, kcode $v 8 k 7, n=150$, where the probability of a rule-table output depends on the fraction of colors in its neighborhood. On the right the color density is plotted for each of the 8 colors, relative to a moving window of 10 time-steps.

\subsubsection{Multi-value 1d space-time patterns in TFO-mode}

Set TFO-mode at the first prompt as described in section 4.7, then procede as follows,

1. At the second prompt, value-range ... : select 8, the current maximum in DDLab.

2. At the prompt Network size ... : select 150 .

3. At the prompt Neighborhood size k: ..., select 7 .

4. Enter return until a top-center space-time parameters banner appears, together with the top-right prompt accept all space-time pattern defaults-d - enter $\mathbf{d}$ to skip special options.

A space-time pattern is generated, scrolling upwards on the left of the screen, as in section 4.8. Because of high $[v, k]$, the randomly selected rule will appear extremely disordered, with high entropy. To set a rule with a much more ordered pattern, as in figure 4.11 Try the following on-the-fly key hits,

A ... for an totalistic "Altenberg" rule, where the output of each neighborhood relates to the fraction of colors in the neighborhood (section 16.9).

s ... to toggle between input-entropy and the density plot, the fraction of colors relating to a moving window of 10 time-steps.

$\mathbf{r}$... for a random rule, followed by $\mathbf{A}$ for another Altenberg rule, and $\mathbf{4}$ for a random initial state.

f ... to filter the space-time pattern repeatedly as required (unfilter with a). This highlights domain boundaries (figure 4.11). Filtering applies only if the input-entropy plot is active.

5 or $6 \ldots$ for a singleton seed, one random cell against $\mathbf{5}()$ a uniform background of 0 s, or 6 against a random but different uniform value (color). Note that the pattern symmetry is conserved however many times the totalistic rule is changed (try with $\mathbf{r}$ ) because totalistic rules are isotropic. 


\subsubsection{Noisy space-time patterns}

Its possible to introduce noise for any space-time pattern updating, in the $1 \mathrm{~d}$ examples above, and the $2 \mathrm{~d}$ and $3 \mathrm{~d}$ examples to follow. To do this:

While space-time patterns are running, enter one of the curly brackets on-the-fly, $\{$ for update probability, \} for output probability, which toggles between correct updating and $95 \%$ of cells updating at each time-step, or 95\% correct and 5\% updating randomly, where $95 \%$ is the initial default setting for both types of noise, which can be combined. Try this with "complex" rules (on-the-fly g, section 4.8), and with the "Altenberg" rules (on-the-fly A, section 4.8.3).

Changing the default settings is described in section (section 31.3).

\section{$4.92 \mathrm{~d}$ Space-time patterns}

The $2 \mathrm{~d}$ lattice is defined in the first top-right wiring prompts that appear after Neighborhood ... during the main prompt sequence, starting with,

WIRING: special-s load-1 random-r

local: 3d-3, 2d-2(hex+x square $+s)$, 1d-def:

Subsequent top-right prompts reset the network size $n$ and neighborhood size $k$ - previous main sequence settings are superseded.

\subsubsection{2d space-time patterns - game-of-Life}

Backtrack with $\mathbf{q}$ (or right mouse button) to the start of the program.

1. At the very first prompt, enter $\mathbf{s}$ for SEED-mode.

2. At the second prompt Value range ...: enter 2 .

3. Enter return until the top-right WIRING: prompt window appears, and enter $2 \mathrm{~s}$ for a hexagonal lattice.

4. At the next top-right prompt, 2d, enter width (def-40): enter 66, which is followed by depth (def 66): enter return, for a $66 \times 66$ square lattice.

5. At the next top-right prompt, Neighborhood size k: kmix-m, or enter 1-13 (def 3): enter 9.

6. Enter return until the main sequence prompt for rule selection appears,

Select v2k9 rcode $(\mathbf{S}=\mathbf{5 1 2})$ : . . . select life-L for the "game-of-Life". The lookup-table of the rule will be displayed as a bit pattern.

7. Enter return until the prompt Select SEED ( $\mathbf{2} \mathbf{2} \mathbf{2} \mathbf{i j}=\mathbf{6 6 , 6 6}) \ldots$ : enter return for a random block. Alternatively select empty-e to "empty" all cells to zero, then load-l to load a seed, then enter the filename "pento" at the LOAD SEED... prompt. This is the "r-pentomino" pattern that guarantees gliders. Alternatively, enter bits2d-b to draw the seed (section 21.4).

8. Enter return until the top-center basin parameters banner appears, then enter $\mathbf{s}$ for space-time pattern only.

9. The top-center banner changes to space-time parameters with the top-right prompt, accept all space-time pattern defaults-d, enter $\mathbf{d}$ to skip special options. 

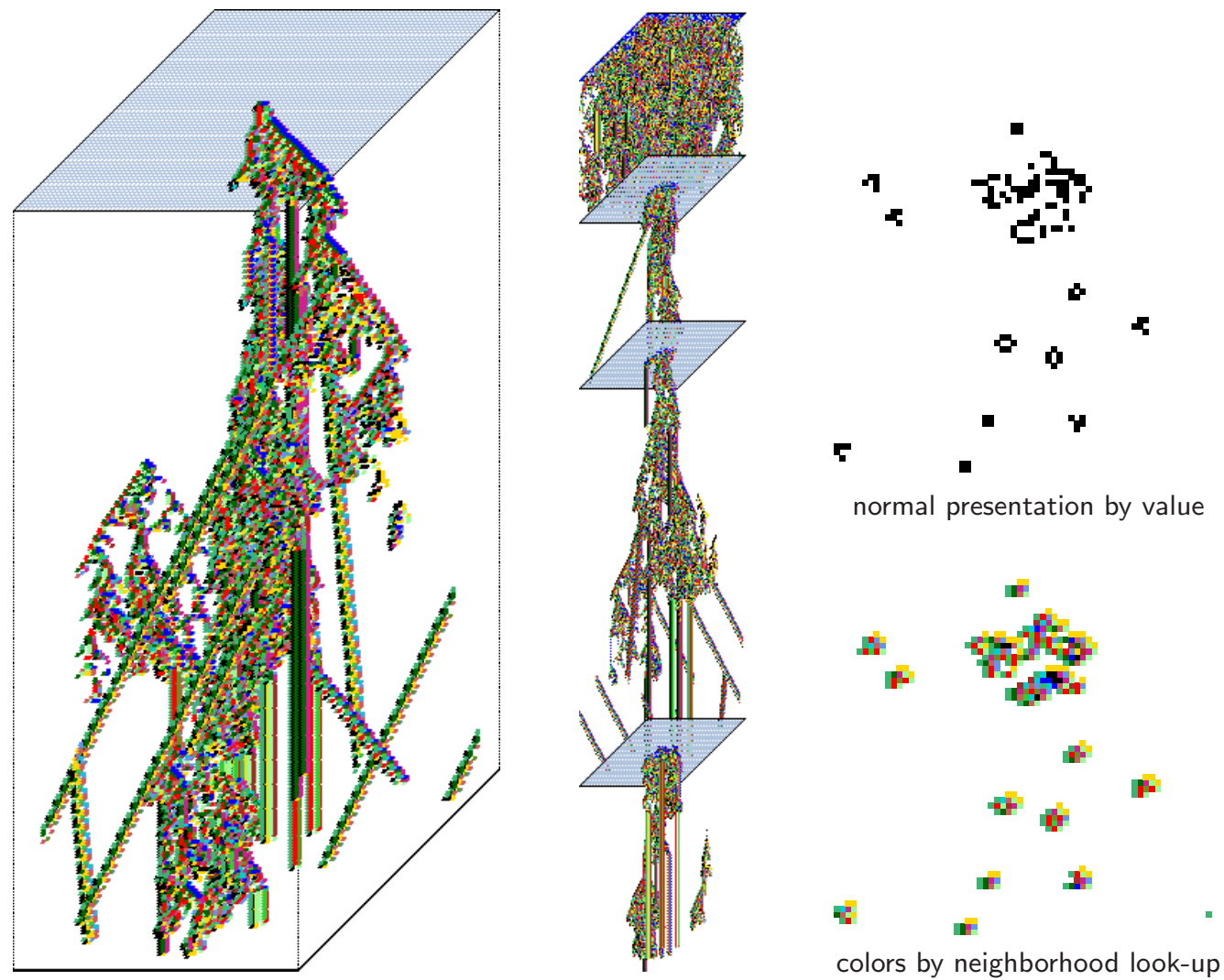

Figure 4.12: Space-time patterns of the $2 \mathrm{~d}$ game-of-Life, $(k=9, n=66 \times 66)$ with time-steps stacked below each other in a isometric projection scrolling upwards. Left: starting from the "r-pentomino" seed. Center: re-scaled to the smallest scale, with new seeds set on-the-fly at intervals.

Upper Right: The state at time-step 230. Lower Right: the same state colored according to the neighborhood look-up instead of the value.

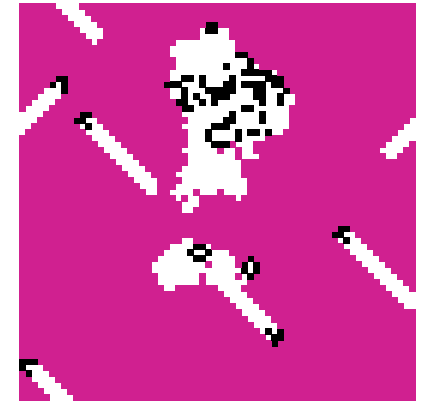

f1 - frozen purple, rest by value

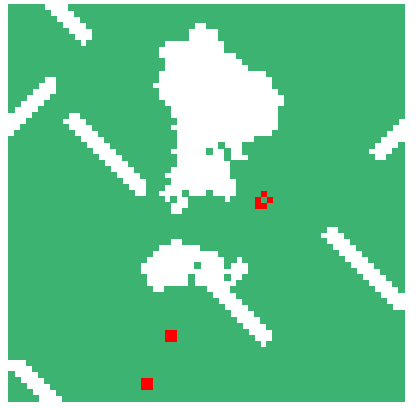

f2 - frozen 1s red, 0s green

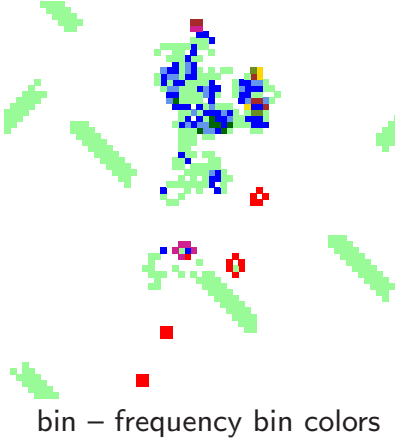

bin - frequency bin colors

Figure 4.13: Space-time snapshots of the game-of-Life $(k=9, n=66 \times 66)$, time-step 230 as in figure 4.12, but showing three alternative "frozen" presentations set on-the-fly (section 32.11.1). This produces a time-trail behind gliders. 


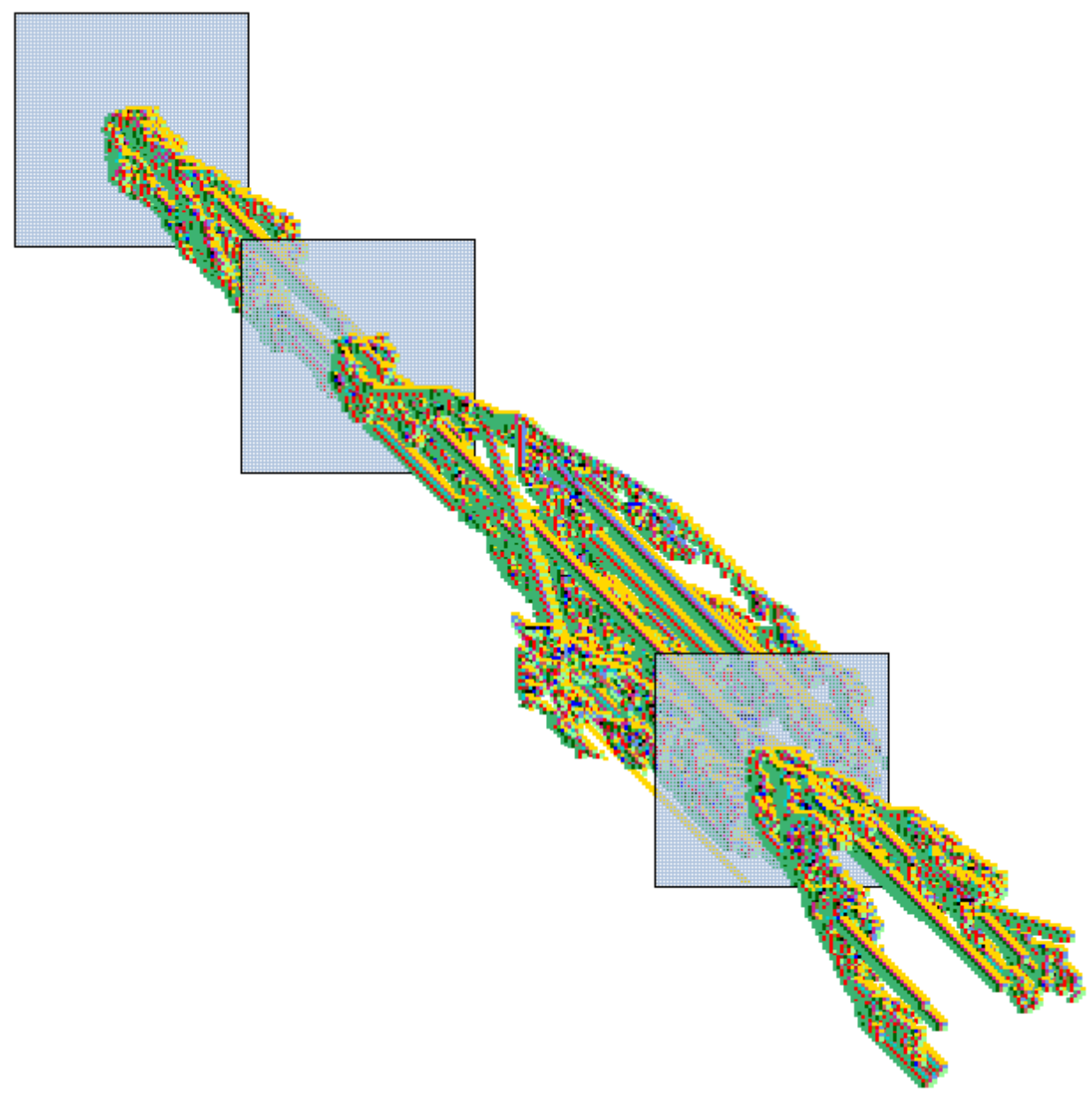

Figure 4.14: Space-time patterns of the game-of-Life $(k=9, n=66 \times 66)$ consisting of time-steps stacked in front of each other in a isometric projection scrolling diagonally upwards, with new seeds set at intervals, and alternate time-steps skipped.

The $2 \mathrm{~d}$ space-time pattern is generated in the top-left corner of the screen. The on-the-fly key index appears on the right of the screen. Try the following on-the-fly key hits (among others) to see what happens.

3 ... to toggle cell color - according to the lookup neighborhood or the value.

$\mathbf{h}$... to toggle three ways of displaying "frozen" regions — the stability of the pattern (figure 4.13).

o ... to restore the original seed.

t ... to toggle between the $2 \mathrm{~d}$ display and an isometric projection scrolling vertically upwards.

\# ... to toggle between the $2 \mathrm{~d}$ display and an isometric projection scrolling diagonally upwards.

$\mathbf{P}$... to toggle skipping alternate time-steps.

k ... for a new random central block. 4 for a fully random seed.

e/c ... to expand/contract the scale of the space-time pattern.

Enter $\mathbf{q}$ to interrupt and backtrack up the prompt sequence. 


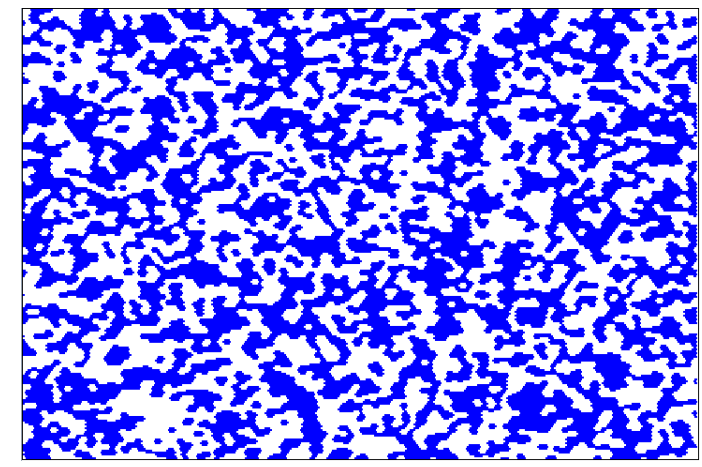

(a) $v 2 k 7$ majority rule tcode $=11110000$.

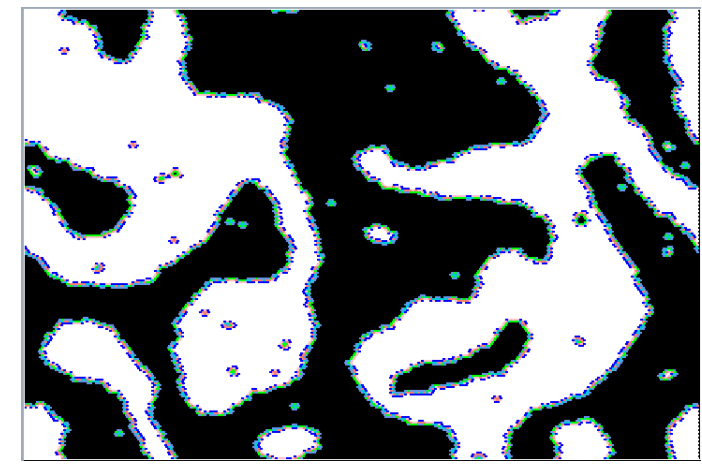

(b) $v 2 k 7$ modified majority rule tcode $=11101000$.

Figure 4.15: 2d space-time patterns of a $v 2 k 7$ CA on a hexagonal grid (240×240), from a random initial state showing aggregating behavior.

\subsubsection{2d Space-time patterns - binary totalistic rules}

Backtrack with $\mathbf{q}$ (or right mouse button) and follow steps 1 and 2 as for the game-of-Life (section 4.9.1).

1. Enter return until the top-right WIRING: prompt window appears, and enter $2 \mathrm{x}$ for a hexagonal lattice. Any previous network and neighborhood size settings will be superseded.

2. At the next top-right prompt, 2d, enter width (def-40): enter 240, which is followed by depth (def 240): enter return, for a $240 \times 240$ hexagonal lattice.

3. At the next top-right prompt, Neighborhood size k: kmix-m, or enter 1-13 (def 9): enter 7.

This results in a hexagonal $2 \mathrm{~d}$ lattice, where each cell has 6 nearest neighbors.

4. Enter return until the main sequence prompt totalistic: tcode-t...: enter $\mathbf{t}$.

5. At the prompt Select $\mathbf{v} \mathbf{2 k 7}$ rule $(\mathbf{S}=\mathbf{1 2 8}) \ldots$ : select select $m a j-\mathbf{m}$. The lookup-table of the majority rule will be displayed as a bit pattern with a flashing cursor on the left bit. Enter return to accept the majority rule 11110000 for figure 4.15(a), or first modify the bit pattern to 11101000 (enter 1 or 0, arrow keys to move) for figure 4.15(b).

6. Enter return until the prompt Select SEED $(\mathbf{v} \mathbf{2} \mathbf{2 d} \mathbf{i j}=\mathbf{6 6}, \mathbf{6 6}) \ldots$... enter $r n d-\mathbf{r}$ for a random seed, then all-a for "all" of the lattice, as opposed to a central block.

7. Enter return until the top-center basin parameters banner appears, then enter $\mathbf{s}$ for space-time pattern only.

8. The top-center banner changes to space-time parameters with the top-right prompt, accept all space-time pattern defaults-d, enter $\mathbf{d}$ to skip special options.

The $2 \mathrm{~d}$ space-time pattern is generated in the top-left corner of the screen. The on-the-fly key index appears on the right of the screen. Try the previously mentions key hits, or any others listed. 


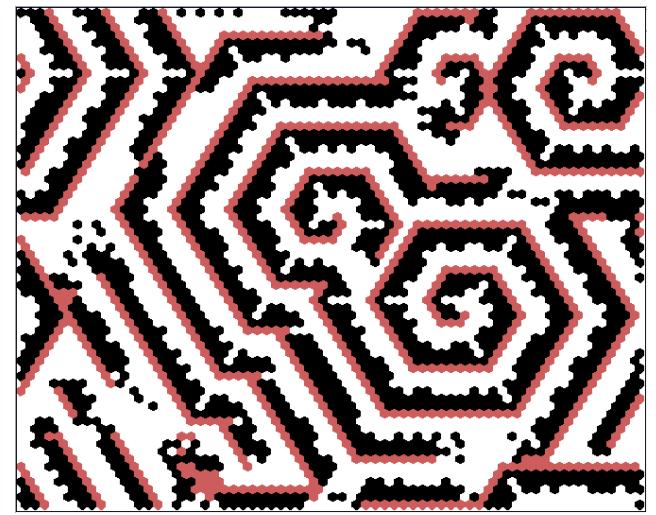

(a) $\mathrm{v} 3 \mathrm{k} 6 \mathrm{kcode}(\mathrm{hex})=0 \mathrm{a} 0282815 \mathrm{a} 0154$ giving spiral structures, from a random initial state.

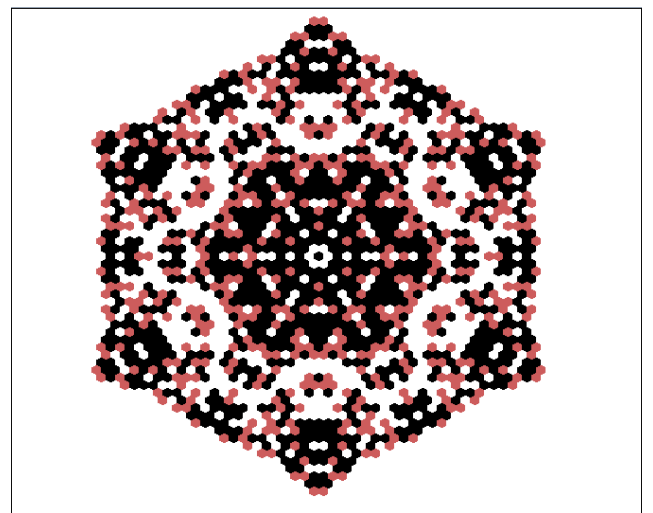

(b) v3k6 kcode(hex)=a0468298295220 (set at random) from a singleton seed.

Figure 4.16: 2d space-time patterns, v3k6 k-totalistic 2d CA on a hex lattice (240x240).

\subsubsection{Multi-value $2 \mathrm{~d}$ space-time patterns in TFO-mode}

Backtrack with $\mathbf{q}$ (or right mouse button) to the start of the program.

1. At the very first prompt, enter $\mathbf{t}$ for TFO-mode, if not already active.

2. At the second prompt Value range ...: enter 3 .

3. Enter return until a top-right WIRING prompt window appears, WIRING: special-s load-l random-r local: $\mathbf{3 d - 3}, \mathbf{2 d - 2}($ hex $+\mathrm{x})$, 1d-def: enter $2 \mathrm{x}$ for hexagonal $2 \mathrm{~d}$ wiring. Any previous network and neighborhood size settings will be superseded.

4. Enter return until the top-right prompt, 2d, enter width (def-40): enter 240, then at the continuation prompt depth (def 240): enter return, for a $240 \times 240$ lattice.

5. At the next top-right prompt, Neighborhood size k: kmix-m, or enter 1-25 (def 3): enter 6 .

This results in a hexagonal $2 \mathrm{~d}$ array, where each cell has 6 nearest neighbors.

6. Enter return until the main sequence prompt totalistic only: outertot-o/+o, tcode-t, (def-kcode): enter return for kcode.

7. At the prompt Select $\mathbf{v} 3 \mathbf{k} \mathbf{6}$ rule ... : enter $\mathbf{h}$ to specify the kcode in hex. Then enter 0a0282815a0154 for the kcode in figure 4.16(a). To correct an entry use backspace or the arrow keys.

8. Enter return until the prompt Select SEED ( $\mathbf{v} \mathbf{3} \mathbf{2 d} \mathbf{i j}=\mathbf{2 4 0 , 2 4 0 )}, \ldots$ : enter return for a random central block.

9. Enter return until the top-center space-time parameters banner appears, with the topright prompt, accept all space-time pattern defaults-d, enter $\mathbf{d}$ to skip special options.

The $2 \mathrm{~d}$ space-time pattern is generated in the top-left of the screen. The on-the-fly key index appears on the right of the screen. Try the previously mentions key hits, or any others listed.

Enter $\mathbf{q}$ to interrupt and backtrack up the prompt sequence. 


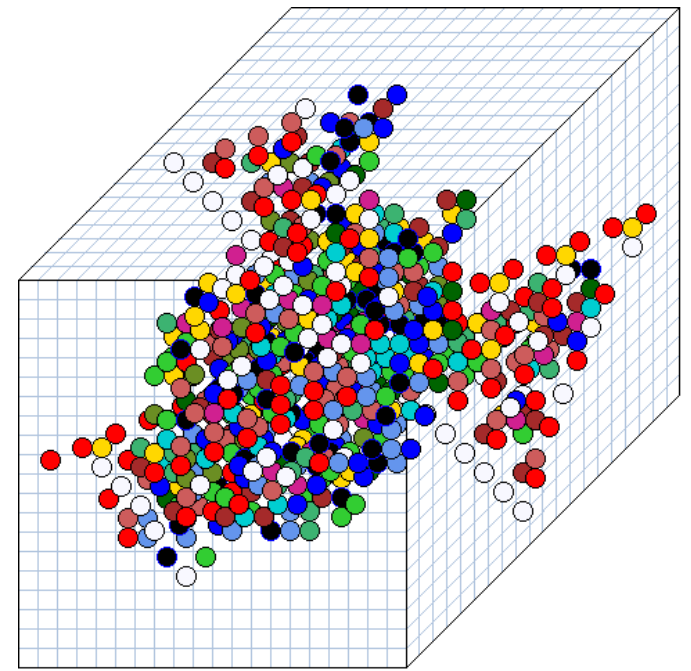

(a) $3 d$ CA $20 \times 20 \times 20$, random $v 2 k 7$ from a singleton seed

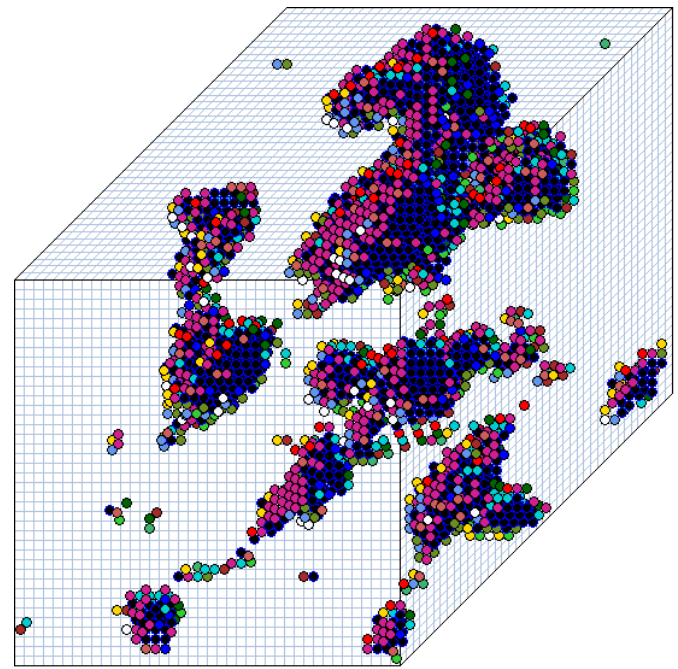

(b) $3 d$ CA $40 \times 40 \times 40$,

$v 2 k 7$ tcode $=11101000$

Figure 4.17: Examples of a $3 \mathrm{~d} v 2 k 7 \mathrm{CA}$, with a neighborhood arranged as a $3 \mathrm{~d}$ cross. The projection is isometric seen from below, as if looking up at the inside of a cage. Cells are shown colored according to neighborhood lookup for a clearer picture (instead of by value: 0,1 ).

(a) a snapshot $(20 \times 20 \times 20)$ with a randomly selected rule from a singleton seed.

(b) a snapshot $(40 \times 40 \times 40)$ - the maximum size DDLab supports, from an initial state set at random, but with a bias of $45 \% 1 \mathrm{~s}$. The rule is the same as in figure $4.15(\mathrm{~b})$ showing aggregating behavior.

\subsection{3d Space-time patterns}

The 3d lattice is defined in the first top-right wiring prompts that appear after Neighborhood ... during the main prompt sequence, starting with,

WIRING: special-s load-l random-r

local: 3d-3, 2d-2(hex+x square+s), 1d-def:

Subsequent top-right prompts reset the network size $n$ and neighborhood size $k$ - previous main sequence settings are superseded.

\subsubsection{3d Space-time patterns - examples}

Backtrack with $\mathbf{q}$ (or right mouse button) to the start of the program.

1. At the very first prompt, enter $\mathbf{s}$, enter $\mathbf{s}$ for SEED-mode, or $\mathbf{t}$ for TFO-mode.

2. Enter return until the top-right WIRING: prompt window appears, and enter 3 for a $3 \mathrm{~d}$ hexagonal lattice. 
3. At the next top-right prompt, enter the width, depth and height.

\section{3d, enter width (def-9): $\operatorname{depth}(\operatorname{def} 9)$ : height (def 9):}

The maximum cube would be $40 \times 40 \times 40$. The lattice has 3 -torus boundary conditions.

4. At the next top-right prompt,

Neighborhood size k: kmix-m, or enter 1-13 (def 6): enter 7.

The neighborhood is arranged as a $3 \mathrm{~d}$ cross.

5. Enter return to accept further defaults, including the rule and seed, until the top-center output parameters banner appears (for SEED-mode), then enter $\mathbf{s}$ for space-time pattern only. For TFO-mode this step is omitted.

6. The top-center banner changes to space-time parameters with the top-right prompt, accept all space-time pattern defaults-d, enter $\mathbf{d}$ to skip special options.

The $3 \mathrm{~d}$ space-time pattern is generated in the top-left of the screen. The on-the-fly key index appears on the right of the screen. Try the previously mentions key hits, or any others listed.

Enter $\mathbf{q}$ to interrupt and backtrack up the prompt sequence.

\subsection{1 "Screen-saver" demo}

The "screen-saver" demo ${ }^{2}$ provides a continuous show of single basins of attraction or subtrees popping up on the screen, generated at random positions, from random seeds, and according to the network architecture selected. For each successive basin (or subtree) the rules or wiring are mutated as specified in chapter 28 - the default is a random rule. For larger systems its unlikely that the same basin would ever repeat.

For a screen-saver demo of a CA similar to figure 4.18, backtrack with $\mathbf{q}$ (or right mouse button) to the start of the program.

1. At the very first prompt, enter $\mathbf{s}$ for SEED-mode.

2. At the second prompt Value-range $\mathbf{v}(\operatorname{def} 2, \max 8)$ : select 3 .

3. At the Network size ... prompt, select 10.

4. At the Neighborhood size k: ... prompt, select 3 .

5. Enter return until the top-center basin parameters banner appears, then enter all-a to restore all defaults, then return until the savescreen demo -s prompt; enter $\mathbf{s}$ to accept the option.

6. Then enter $\mathbf{d}$ to accept all further defaults, then return twice - the demo will start. The rule picked at random will be shown in the bottom rule-window.

While the demo is in progress, toggle the display of the "backwards" space-time pattern with key $\mathbf{s}$, and the state-space matrix with key $\mathbf{m}$ (section 4.3). Enter $\mathbf{q}$ to interrupt and backtrack up the prompt sequence. Try the savescreen demo with other setting of $v, n$, anf $k$.

\footnotetext{
${ }^{2}$ See also section 24.8
} 


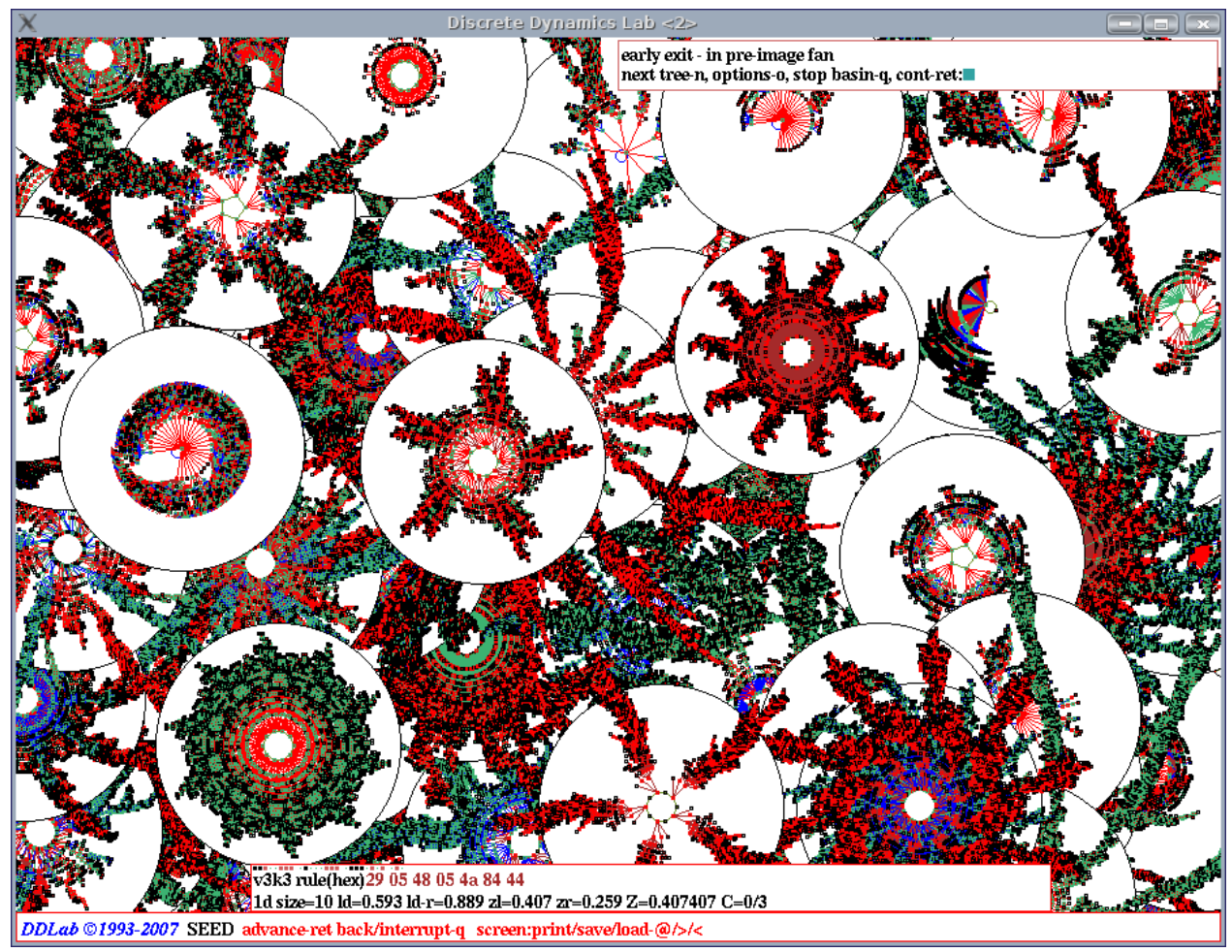

Figure 4.18: Screen-saver demo for Cellular Automata, $v=3, k=3, n=10$. Single basins with rules and initial states chosen at random are generated at random positions in the DDLab window.

\subsection{RBN and DDN}

Until now, the Quick Start Examples have dealt with cellular automata, which have a homogeneous local wiring template and a single rule throughout the network. A random Boolean network (RBN), or the more general Discrete Dynamical Network (DDN, where $v \geq 3$ ), departs from CA network architecture by allowing each cell in the network to have different nonlocal wiring, a different rule, and a different number of inputs, $k$, or any combination of the above. However, the value-range $v$ must be homogeneous. The network can be assigned a wiring scheme, rule scheme and $k$-mix in a variety of ways, including randomly (with or without biases).

- just a wiring scheme may be set, the rule and $k$ remain homogeneous, as in CA, by default.

- just a rule scheme may be set, the local wiring template and $k$ remain homogeneous, as in CA, by default.

- both a wiring scheme and rule scheme may be set, $k$ remains homogeneous by default. If $v=2$, this is what is usually understood as a random Boolean network (RBN).

- a $k$-mix may be set, which implies both a wiring scheme and a rule scheme, but the wiring scheme remains local by default.

- a network with a $k$-mix, a nonlocal wiring scheme and a rule scheme may be set. 
To generate attractor basins and space-time patterns for DDN, for any combination of parameters listed above, the preceding examples in sections $4.2-4.11$ can be adapted as described below.

Note that running backwards for networks with non-1d-CA wiring employs a different algorithm, with a greater computational load, than for 1d CA wiring (section 1.6), so when generating attractor basins for non-1d-CA wiring, $v, k$, and especially $n$, should be kept small, and $2 \mathrm{~d}$ or $3 \mathrm{~d}$ networks should be avoided.

1. For a random wiring (chapter 12), at the first top-right wiring prompt,

WIRING: special-s load-l random-r

local: 3d-3, 2d-2(hex $+x$ square $+s), 1 d-d e f:$

2. select $\mathbf{r}$ for $1 \mathrm{~d}$ random wiring - no further setting required.

3. select $\mathbf{s}$ for special wiring, which includes $2 \mathrm{~d}$ or $3 \mathrm{~d}$ random wiring, then follow further topright prompts as follows:

(a) 3d-3, 2d-2 (hex+x square+s), 1d-def: select the dimension, i.e. $2 x$.

(b) hand wire-h,

local 2d-2 (hex $+x$ square $+s)$, 1d-1, random-def:

enter return for random wiring.

(c) 2d, enter width ...: $\operatorname{depth~} \ldots$ : set the size.

(d) Neighborhood size k: $\mathbf{k m i x}-\mathbf{m}$... select the size of $k$, or enter $\mathbf{m}$ for mixed $k$, in which case there will be a series of further prompts to set the $k$-mix (section 9.3), but just enter return for a random $k$-mix.

(e) bias random wiring:... these further options (section 12.5) can be skipped with $\mathbf{d}$.

(f) Note: any new entries here for $n$ and $k$ will supersede earlier entries.

4. Whatever wiring is defined, local or random, in any dimension, the next top-right prompt (similar to below) allows the wiring to be examined in detail, and amended (section 17.1),

2d network (122x122), review/revise, wiring only - rule not set graph-g, matrix: revise-m view-M prtx-Mp graphic:1d+timestep-1 circle-c 2d-2:

5. After the totalistic:dots in the main sequence of prompts, a top-right window appears with options for a single rule or a rule-mix (chapter 14,

RULES: single rcode (def), load rulemix-1, list Post-P, nhood-matrix-a mix: no limit-n, or set limit up to 200:

Enter $\mathbf{n}$ for a rulemix, or return for a single rule.

6. Stepping back in the main prompt sequence, if a random $k$-mix is required, at the main sequence prompt,

Neighborhood size k: kmix-m, or enter 1-13 (def 3): enter m.

Various further options to set and bias the k-mix are presented (chapter 9). If in doubt enter return. For a random $k$-mix for a $2 \mathrm{~d}$ or $3 \mathrm{~d}$ network, first select the random wiring in $2 \mathrm{~d}$ or $3 \mathrm{~d}$ as above. 
7. Once rules have been set in the main prompt sequence, top-right options are presented to examine and amend the resulting network in detail, wiring and rules, similar to this (also applicable to CA),

$1 d$ network $(n=150)$, review/revise/learn, wiring and rcode graph-g, matrix: revise-m view-M prtx-Mp graphic: 1d+timestep-1 circle-c 2d-2:

- Enter return to skip and continue, $\mathbf{q}$ to backtrack.

- Enter 1, c, 2 (or $\mathbf{3}$ for $3 \mathrm{~d}$ ) to review the network as a wiring graphic, using the arrow keys to examine the wiring and rules of chosen elements (chapter 17).

- Enter $\mathbf{m}$ or $\mathbf{M}$ to review the network as a wiring matrix (section 17.2)

- Enter $\mathbf{g}$ to review the network as a graph, which can be rearranged by dragging nodes with the mouse (chapter 20).

- The options above have their own follow-up options described in the section listed.

Having set up the the RBN or DDN, follow the guidelines in previous sections in this chapter to generate attractor basins (for example figures 2.4, 2.5, 8.7), and space-time patterns (for example figures $2.6,16.8,32.19)$. 


\section{Chapter 5}

\section{Starting DDLab}

This and the following chapters provide the detailed program reference, listing and explaining all the various options and prompts with examples of DDLab's behavior. Before tackling this part of the manual, its a good idea to try some of the "Quick Start Examples" in chapter 4 to get a feeling and flavour of DDLab.

This chapter includes starting DDLab, the initial DDLab "screen", and some permanently available options to save, load and print the DDLab screen image.

In Linux-like systems run DDLab from a terminal (xterm) window rather than directly from an icon on the desktop, because messages and data are often shown in the terminal.

Before starting DDLab, make sure you are in the DDLab directory containing the DDLab executable file(s), or in the appropriate sub-directory (section 5.1). The executable file(s) downloaded from the DDLab website, are,

$$
\begin{aligned}
& \text { ddlabm07 } \ldots \text { for Linux-like operating systems } \\
& \text { ddlabm07.exe and dos4gw.exe } \ldots \text { for DOS. }
\end{aligned}
$$

The files in dd_extra.tar.gz are not essential for DDLab to run, but are required for some functions. They can be in the DDLab directory, or in a subdirectory which can be changed into within DDLab, or DDLab can be started from this subdirectory as shown in section 5.1 below. In either case the subdirectory becomes the default directory for filing (chapter 35).

\subsection{Running in Linux-like operating systems}

\section{refer also to section 4.1 .5}

For Linux-like systems (Unix, Linux, MacOSX, Irix, and Cygwin) enter,

$$
\begin{array}{rlll}
\text { ddlabm07 } & \& & \ldots & \text { if "dot" is in your path. } \\
. / \text { ddlabm07 } & \& & \ldots & \text { if "dot" is not in your path. } \\
\text {./ddlabm07 } & \& & \ldots & \text { from a subdirectory of the DDLab directory (containing the files in } \\
& & & \text { dd_extra.tar.gz). }
\end{array}
$$

i.e. the name of the executable followed by \&, to retain control of the terminal (or xterm) window, where information is often printed. 


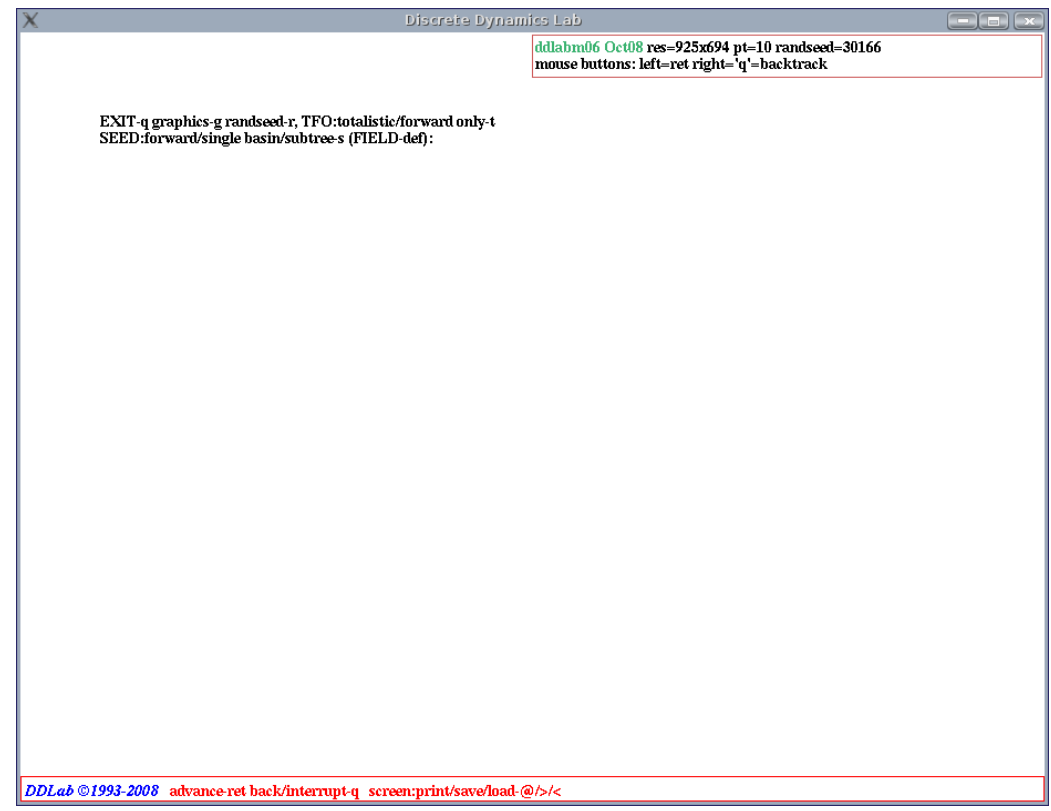

Figure 5.1: Typical graphical user interface when starting DDLab

The DDLab window will appear, similar to figure 5.1 with an initial resolution of $925 \times 694$. The message "Using backing store" will appear in the terminal window ${ }^{1}$, indicating that the DDLab window will be restored in the usual way when covered by other windows or moved off the screen. However, if DDLab is iconized, resized, or if the "desktop" (or "workspace") is changed, the immediate current contents in the screen will blank out ${ }^{2}$, but the contents will reappear as DDLab continues.

\subsection{Running in DOS or Windows}

\section{refer also to section 4.1 .6}

DDLab is still compiled for old fashioned DOS in the Watcom compiler, now open source and freely available. The DOS version of DDLab can be run in pure DOS (Window 98 or prior), or in a DOS or "command line" window in later editions of Windows prior to Vista. In Vista the DOS version does not work from the command line, but it works nicely (but slowly) in "DOSBox", an open source MS-DOS emulator, intended for old PC games.

Enter ddlabm07 or the name of the executable without the .exe extension. From a subdirectory of the DDLab directory (containing the files in dd_extra.tar.gz) enter . . dddlabm07. The DOS extender banner will briefly appear ${ }^{3}$.

Note that the Linux-like versions perform better, and are better supported, than the DOS version, so in a Windows environment its recommended you install Cygwin, which is a freely available Linux-like environment for Windows, and run the Cygwin version of DDLab within that.

\footnotetext{
${ }^{1}$ Refer to dd_linux_readme.txt for details about "backing store", and also possible missing fonts.

${ }^{2}$ In FVWM the contents remain when resizing or changing desktops, they only blank out when iconizing.

${ }^{3}$ To suppress this banner enter set dos4g=quiet at the DOS prompt before running DDLab.
} 


\subsubsection{Running in a pure DOS environment}

In a pure DOS environment, available in Windows 98 and prior, DDLab will start, occupying the whole screen. The resolution might need to be changed to correspond to the monitor and graphics driver, otherwise fonts might appear distorted (sections 5.7, 6.3.2).

To get into pure DOS from Windows98, from Start select Shut Down... then Restart the computer in MS-DOS mode.

\subsubsection{Running in Windows95, 98, and later editions}

In early versions of Windows, open a DOS window; in Windows95 or 98: Start - Programs MS-DOS Prompt; in WindowsME: Start - Run, and type command. In later versions of Windows prior to Vista ${ }^{4}$, open a "command line" or "command prompt" window, for example in WindowsXP: Start - All Programs - Accessories - Command Prompt.

From the DOS (or command line) window, DDLab will start either in the window, or in full screen (which is unpredictable) but whichever it is Alt+Enter toggles between the two. The initial resolution will be $640 \times 480$. Do not use the program parameter $-\mathrm{m}$ or $-\mathrm{h}$ unless in full screen.

In full screen the resolution should be changed to correspond to the monitor and graphics driver (otherwise fonts may appear distorted). However, before toggling back from a full screen to a DOS (or command line) window, the resolution must be changed back to "low" to avoid Windows warnings and unforeseen consequences. In higher resolutions than $640 \times 480$ the mouse cursor will probably not be visible.

DDLab is more stable and faster in pure DOS (section 5.2.1 above) or in full screen as above, than in a DOS (or command line) window.

\subsection{Command line arguments}

Command line arguments for various settings can entered after the executable name - first a space, then a dash (-), then the arguments, without spaces, in any order, for example,

./ddlabm07 -wt \& ... for Linux-like systems, where \& is the final entry.

The following command line arguments are available,

w $\quad$... white screen

b $\quad$... black screen (the default)

t $\quad .$. TFO-mode, totalistic rules and space-time patterns only - basins disabled

Additional commands for DOS only,

m $\quad$... medium resolution $800 \times 600$

h $\quad \ldots$ high resolution (VGA) $1024 \times 768$

These setting can also be reset once DDLab starts.

\footnotetext{
${ }^{4}$ In Vista the DOS version of DDLab will run in DOSBox, (sections 3.5 and 5.2).
} 


\subsection{The UNREGISTERED banner}

On start-up (and also before exiting) an unregistered version of DDLab will first display the following "UNREGISTERED" banner in the center of the screen. Press return to continue. By registering (section 3.8) this annoying banner can be easily removed.

UNREGISTERED, see www.ddlab.org, continue-ret:

\subsection{Title bar}

On start-up of a registered version (or if return is entered at the "UNREGISTERED" banner) DDLab's graphical user interface appears as described in figure 5.1

DDLab @1993-2009 advance-ret back/interrupt-q screen:print/save/load-@/->/<

A title bar is displayed across the foot of the screen, including a copyright reminder, the current mode if set (TFO, SEED, or FIELD), and some reminders of key presses which can be activated whenever the prompt cursor is flashing.

$$
\begin{aligned}
& \frac{\text { key press reminder }}{\text { advance-ret }} \ldots \frac{\text { what it means - permanently displayed }}{\text { enter return (or click the left mouse button) to register entries }} \\
& \text { to prompts (or accept defaults) and advance through the prompt } \\
& \text { sequence. } \\
& \text { back/interrupt-q ... enter } \mathbf{q} \text { (or click the right mouse button) to backtrack through } \\
& \text { prompt sequence or to interrupt a run. } \\
& \text { key press reminder ... what it means - displayed while the prompt cursor is flashing } \\
& \text { screen:print-@ ... enter @ to print the DDLab screen image, described in section 5.6.2. } \\
& \text { screen:save/load- }>/<\ldots \text { enter }>\text { to save, or }<\text { to load the screen image as a nat file, } \\
& \text { in DDLab's own format, described in section 5.6.1. }
\end{aligned}
$$

The title bar displays other on-the-fly options while generating attractor basins (section 30.3), or space-time patterns (section 32.2).

\subsection{Saving, Loading and Printing the DDLab screen}

The screen image can be saved and loaded within DDLab itself using its own file format. Alternatively the screen image can be saved and printed as a PostScript file for Linux-like systems. These options are permanently available while the prompt cursor is flashing. 


\subsubsection{Saving and Loading the screen in DDLab's own format}

DDLab has its own file format (.nat) for saving and loading the DDLab screen image, which works for both Linux-like and DOS versions. If $>$ or $<$ is entered at any time when the prompt cursor is flashing, the following top-right prompt is presented,

SAVE SCREEN-filename(no ext) .nat will be added (if $>$ is entered) or

LOAD SCREEN-filename(no ext) .nat will be added (if $<$ is entered) then list-?, quit-q, default myimage:

Filenames within DDLab (for all systems) follow the old DOS conventions: eight characters plus a three character extension. Enter the filename without the extension (.nat) which is added automatically, or accept the default filename myimage. There are further details on these filing options in chapter 35 . Note that a DDLab screen image loaded as above can subsequently be saved and printed as described in section 5.6.2 below for Linux-like systems.

\subsubsection{Saving and Printing the screen in Linux-like systems}

If @ is entered at any time when the prompt cursor is flashing, the DDLab screen can be printed to the default printer, or saved as a PostScript (.ps) file. In the DOS version, although this option to print (but not to save) still exists, it only works for some ancient printers, so is not recommended.

In Linux-like versions of DDLab, the following prompt is presented,

save/print: without frame $-\mathrm{s} / \mathrm{p}$, with frame $-\mathrm{S} / \mathrm{P}$, no greyscale $+\mathbf{n}$

then click mouse button in window, quit-q:

Enter $\mathbf{s}$ or $\mathbf{S}$ to just save, or $\mathbf{p}$ or $\mathbf{P}$ to save and print, were lowercase selects the DDLab screen without its frame.

Add $\mathbf{n}$ (i.e. sn) to save or print in black only, i.e. suppress greyscale or colors - some lighter colors will not be printed.

On pressing return the cursor becomes a diagonal cross-hair $X$. Make sure this cursor is within DDLab and click the left mouse button. The DDLab screen image will be saved as a bitmap PostScript file called temp_window_file.ps (size about 285k) in the current directory.

If printing was selected, the file will be sent to the default printer. While this is happening the message wait will appear in a top right window, followed by,

\section{done - press any key to continue:}

This reverts the program to the place before printing was selected. Note that the PostScript file temp_window_file.ps remains current until overwritten with another print instruction ${ }^{5}$.

The following messages might show up in the terminal window,

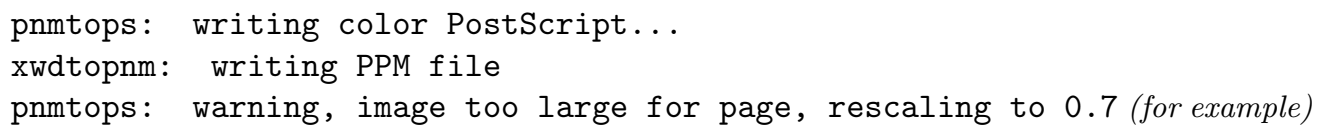

\footnotetext{
${ }^{5}$ To convert the PostScript to a PDF file, enter ps2pdf filename.ps in the terminal. The command to print a postscript file from the terminal is (for example) lpr -Pprintername filename.ps.
} 


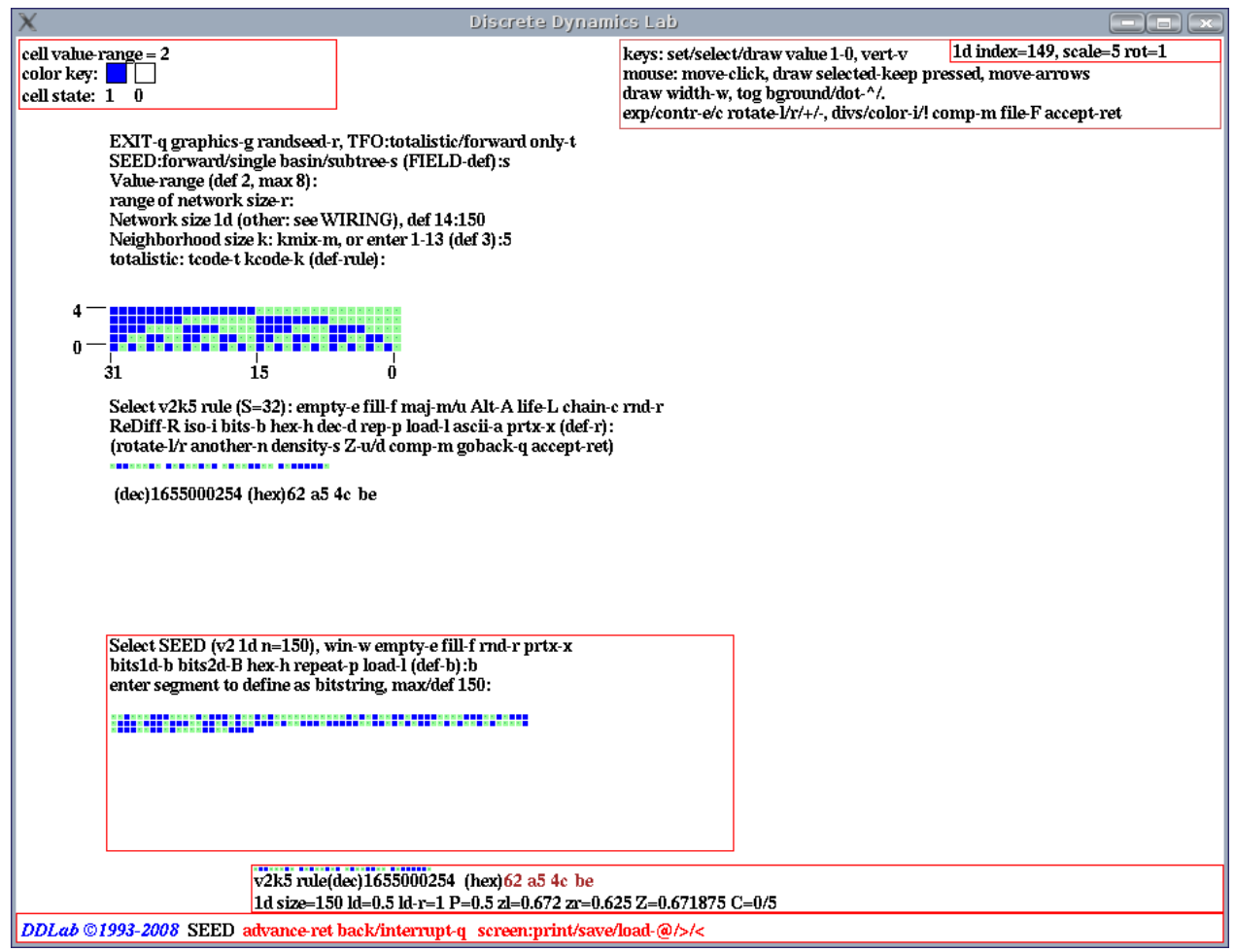

Figure 5.2: The main sequence of prompts, for the most commonly applicable 1d CA parameters, are presented down the left side of the screen, other prompts occur in various pop-up windows, for example in the top right hand corner.

The last indicates that the size of the DDLab window was too large for the printer paper size, so was automatically rescaled to fit. If the following error message is shown in the top-right window,

could not print -- xwdtopnm not found

or the following appears in the terminal window,

You need to set the environment variable " "XWDTOPNM_PATH" "
to the full path of your xwdtopnm to print in greyscale

... consult your systems manager.

A more versatile way to print the DDLab window, or a selected part of it, is to run XV (enter $\mathrm{xv} \&$ in the terminal), or another installed screen grabber. XV can grab, save, scale and print the image in one of the many file formats available.

\subsubsection{Printing the screen in DOS}

In DOS, the option to enter @ to print the DDLab screen is retained, but is largely redundant because it only works for some ancient printers (Epson MX-82 dot matrix, Cannon BJC 4000 bubble jet). The alternative is to use a DOS or Windows compatible screen grabber. 


\subsection{DDLab version and graphics info}

On startup, a top-right window appears similar to this,

Linux-like systems

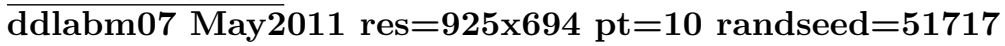

mouse buttons: left $=$ ret right $=$ ' $q$ '=backtrack

DOS - screen resolution is $640 \times 480,800 \times 600$ or $1024 \times 768$

ddlabm07 May2011 res $=640 \times 480$, randseed $=24081$

mouse found: buttons: left $=$ ret right $=$ 'q'=backtrack (if a mouse is detected)

or

mouse not found: ... (probably because of a missing DOS mouse driver)

This shows the following,

ddlabm07 ... the version of DDLab.

May2011 ... the release date.

res $=925 \times 694 \ldots$ DDLab screen resolution, which can be reset.

$\mathbf{p t}=\mathbf{1 0} \ldots$ the current text point size, which can be reset (also it weight).

randseed $=\mathbf{5 1 7 1 7} \ldots$ the random number "seed" which changes each time DDLab is run, and which can be reset, to exactly repeat default parameters.

mouse buttons ... a reminder that the left mouse button is equivalent to return, and the right mouse button is equivalent to $\mathbf{q}$ for backtracking through prompts.

In Linux-like systems the default DDLab screen resolution is automatically set to $925 \times 694$, but can be resized by dragging with the mouse in the usual way, or set to an exact size (section 6.3.1). In either case the text size will attempt to resize according to the screen width.

In DOS the default screen resolution is $640 \times 480$ pixels (VGA), but can be set higher within DDLab (section 6.3), or with a command line argument (section 5.3). In Windows, the resolution should be reset to higher (or lower) only when DDLab in full screen mode.

\subsection{The mouse cursor in DOS and Windows}

In DOS and Windows the mouse cursor behaves as it should in VGA resolution $(640 \times 480)$, and also in higher resolutions (SVGA) in Windows95 and prior. However, in Windows98 and above, at higher resolution only, the mouse is recognised and present (its position can be tracked), but it is not visible. This remains an unresolved problem.

\subsection{Memory (DOS only)}

DDLab allocates and frees memory as required. In DOS the RAM available is monitored, mainly for diagnostic purposes. A top-center "memory window" shows the amount of RAM available in bytes (including extended memory), for example $\mathbf{m e m}=\mathbf{3 4 9 0 4 9 3 1 2}$ which is frequently updated. Other information is also displayed in this window from time to time. 


\subsubsection{Virtual Memory}

skip this section unless your computer is antique

You may create a swap file on disk to augment RAM, using Watcom's "Virtual Memory Manager" (VMM), and use more memory than your computer actually has. When RAM is not sufficient, part of the program is swapped to the disc file until needed. The combination of the swap file and available RAM is the virtual memory.

To specify virtual memory, set the DOS environment variable, dos4gvm by entering the following at the DOS prompt,

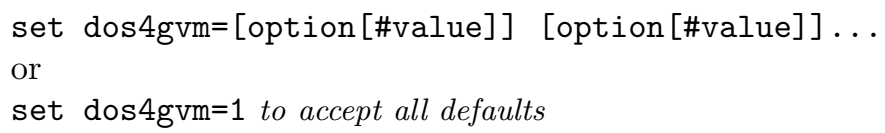

the VMM options and default values are listed below,

minmem ... the minimum amount of RAM managed by VMM, default 512KB.

maxmem ... the maximum amount of RAM managed by VMM, default 4MB.

swapname ... the swap filename, default dos4gvm. swp in the root directory.

virtualsize ... the size of the virtual memory space, default $16 \mathrm{MB}$.

For example, typing

set dos4gvm=maxmem\#8192 virtualsize\#32768 swapname\#c: \ddlab \ddlab.swp

... at the DOS prompt before running DDLab gives 32MB of virtual memory in a swap file called ddlab.swp in the ddlab directory. The virtual memory available will be displayed in the memory window (section 5.9).

\subsection{DDLab prompts, and backtrack}

On startup, the first of a series of prompts is displayed, described in chapter 6.2. A flashing cursor (usually green) prompts for input. Just enter return if in doubt, or the appropriate input from the keyboard. Press q, backspace (or the right mouse button) to revise, return (or the left mouse button) to accept and move on to the next prompt or routine. Just return (or left mouse button) automatically selects a default.

To backtrack to the preceding prompt, or up the prompt sequence, or to interrupt a running process such as space-time patterns or attractor basins, enter $\mathbf{q}$ (or right mouse button).

\subsubsection{Prompts: main sequence and pop-up windows}

Prompts occur in a main sequence for the most common 1d CA parameters, and also in various popup windows for RBN, $2 \mathrm{~d}$ and $3 \mathrm{~d}$ networks, network architecture graphics, analytical measures, data collection, presentation settings, and other special settings. Note that for $2 \mathrm{~d}$ and $3 \mathrm{~d}$ CA, and RBN, the main sequence prompts for the network "wiring" and neighborhood size are superseded in the first pop-up window, labelled "WIRING". 


\subsection{Exit DDLab}

To exit DDLab immediately for Linux-like systems, enter Ctlr-q at any prompt. For DOS or Windows, backtrack with q beyond the start of the program. The following central prompt will appear,

\section{EXIT DDLab-q, Restart-ret:}

Enter $\mathbf{q}$ to exit (or return to restart). On exiting, the following message should appear in the terminal, or at the DOS or command line prompt: DDLab clean exit. If not, a bug report would be appreciated. 


\section{Chapter 6}

\section{The first prompt in DDLab}

Prompts in DDLab are presented in a main sequence down the left side of the screen for the most commonly applicable parameters (including $1 \mathrm{~d} \mathrm{CA}$ ); other prompts are presented in various pop-up windows (figure 5.2).

This chapter describes the very first main sequence prompt (figure 5.1) which makes basic choices that set the stage for all subsequent DDLab operations: TFO-mode, SEED-mode, and FIELD-mode (the default). The first prompt also provides various options for graphics, filing, printing, and the random number seed, from pop-up windows.

\subsection{TFO-mode, SEED-mode, FIELD-mode}

- TFO-mode: constrains DDLab to run "forwards only" for space-time patterns, at the same time limiting the rules to various types of totalistic, outer-totalistic and reaction diffusion rules. Totalistic rule-tables (kcode and rcode) are much smaller than full rule-tables (rcode). All attractor functions, which depend on rcode (and their prompts) are disabled in TFO mode. These constraints reduce memory load, allowing larger neighborhoods (section 7.2). For binary $v=2, k_{\text {Lim }}=25$ instead of 13 . Selecting and deselecting TFO-mode can only be made at this first prompt. TFO-mode requires an initial state or seed, in the same way as SEED-mode (below).

- If DDLab is not constrained in TFO-mode (above), there is a further choice,

- FIELD-mode: to show the basin of attraction field, which does not require an initial state (the seed).

- SEED-mode: show anything else which does require an initial state or seed: running forward for space-time patterns, or generating a single basin of attraction, or a subtree. The TFO-mode also requires a seed.

The FIELD/SEED-mode setting can also be swapped when attractor basins are complete (section 30.4). Note that totalistic rules, kcode and tcode, can also be selected in FIELD and SEED-modes, but in this case the equivalent rcode will be applied. 


\subsection{The first prompt}

The first (main sequence) prompt in DDLab is as follows,

EXIT-q graphics setup-g randseed-r, TFO:totalistic/forwards onty-t SEED:forward/single basin/subtree-s (FIELD-def):

\subsubsection{TFO-mode: totalistic forwards only}

Enter $\mathbf{t}$ to select TFO-mode. The first prompt changes:

EXIT-q graphics setup-g randseed-r, disable TFO allow basins-b

TFO:totalistic rules and forward only (def:) (this line in red)

Enter return to continue in TFO-mode. Enter $\mathbf{b}$ to deselect TFO-mode, and restore the original prompt in section 6.2.

\subsubsection{FIELD, or a run requiring a SEED}

If TFO-mode is not selected in section 6.2 .1 above, a choice needs to be made between SEED and FIELD-modes.

Enter return at the first prompt in section 6.2 for a basin of attraction field. Generating a "basin of attraction field" (the default), does not require a seed because successive basins are seeded automatically, starting with the null state (all 0's).

Enter $\mathbf{s}$ at the first prompt in section 6.2 if you intend to generate any of the following, which do require a seed,

- forward space-time patterns, starting from a seed.

- single basin of attraction, containing a seed.

- subtree running backwards from a seed.

The seed will will be set at a later stages in the prompt sequence (chapter 21). Its easy to change between forward, single basin and subtree at later stages.

\subsubsection{Notice of the current mode}

A reminder of the current mode (TFO, SEED, or FIELD), once set will appear in the bottom title bar, for example,

DDLab @ 1993-2009 FIELD advance-ret back/interrupt-q screen:print/save/load-@/>/< 


\subsection{Graphics setup}

Enter $\mathrm{g}$ in section 6.2 or 6.2.1 to change the window size, background color, font size, line spacing or flashing cursor speed. A new graphics screen shows the current font and color palette. A top-right window gives prompts as below. When the graphics changes are complete the program will revert to the first prompt in section 6.2.

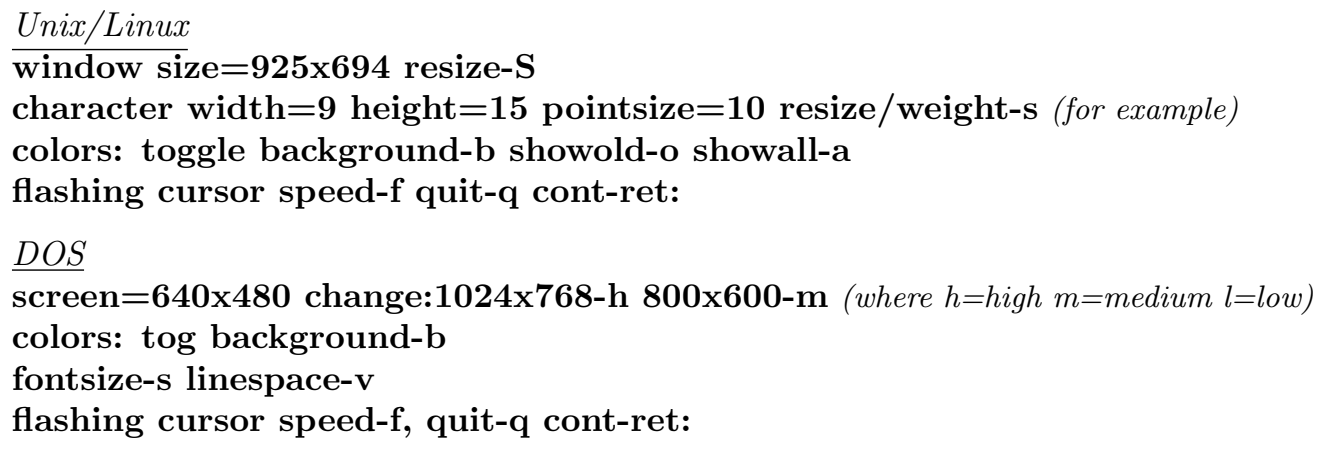

In Linux-like systems, the initial resolution of the DDLab window is set at $925 \times 694$, but can be resized with the mouse in the usual way, or a particular size can be set from these prompts.

In DOS there are three alternative resolutions, given an appropriate monitor and graphics driver.

\subsubsection{The DDLab window resolution, Linux-like systems}

Enter $\mathbf{S}$ in section 6.3 to resize the DDLab window to a particular size in pixels.

The following top-right prompt is presented,

new window size $(\min 300 \times 250)$

width (default 925): (for example)

height (default 694):

The smallest size allowed by this method is $300 \times 250$ shown in figure 6.1 . The window can also be resized to any size with the mouse by dragging a corner in the usual way. Note that when the window is resized the font size is automatically changed to an appropriate size.

\subsubsection{The DDLab window resolution in DOS}

Sections 5.2.1 and 5.2.2 describe the DDLab window in pure DOS, and in a DOS or command line window in various versions of Windows, which can be toggled to full screen with Alt+Enter. Once in full screen enter $\mathbf{l}, \mathbf{m}$ or $\mathbf{h}$ in section 6.3 above to change the resolution, where $\mathbf{l}=$ low: $6404 \times 480$, $\mathbf{m}=$ medium: $8004 \times 600$ and $\mathbf{h}=$ high: $1024 \times 768$. Take care to reset low resolution before toggling back to the DOS or command line window, to avoid bad things!

In pure DOS, the program parameters $-\mathrm{m}$ or $-\mathrm{h}$ can be entered, i.e. enter ddlabm06 -h to start in high resolution. 


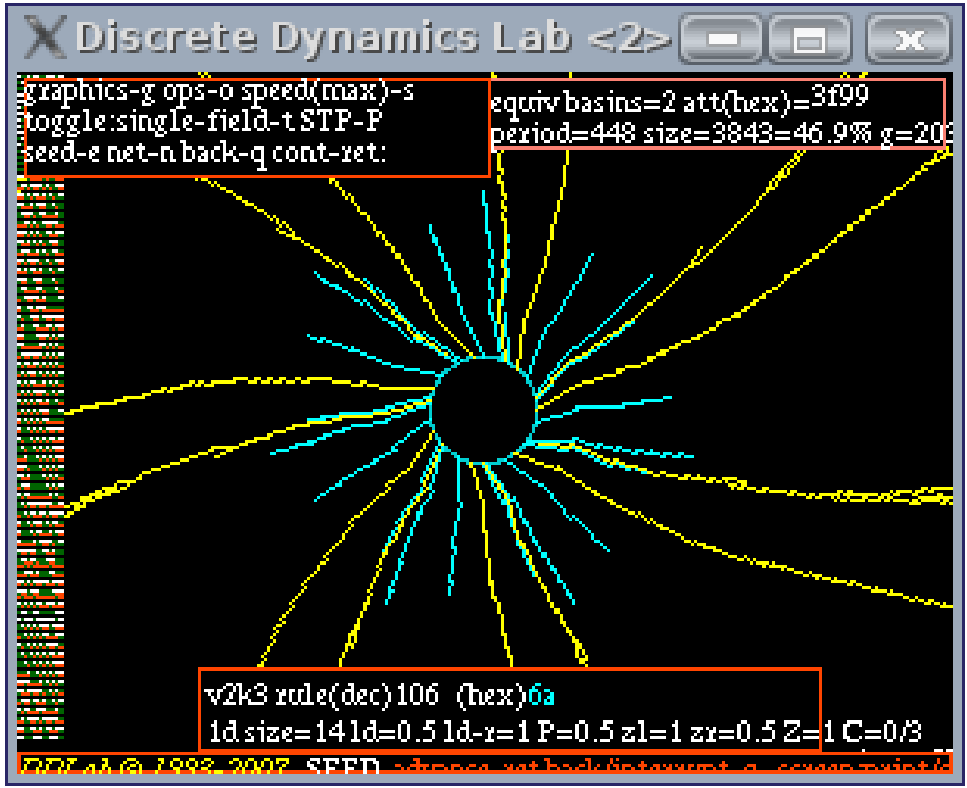

Figure 6.1: The smallest DDLab window in Linux, $300 \times 250$ pixels, that can be set from the graphics setup prompts. It is really too small to contain prompts and data. This example has the default black background.

\subsubsection{White or black background}

Select $\mathbf{b}$ in section 6.3 to toggle between a black and white background. In both cases the new color palette will be shown. A black background may look more elegant but is expensive in ink when printing. References to colors in this manual are based on a white background. Colors on a black background may be different. In DOS, toggling the background works in full screen, not in a DOS window.

\subsubsection{The color palette}

The graphics screen shows a color palette of the 16 colors (including black and white). In DOS just these colors are used. In UNIX these are the main colors, but more colors are also used, and can be seen by entering a (showall-a) in section 6.3, reverting to the 16 main colors with o (showold-o). This feature is intended mainly for program development.

\subsection{Changing the font size and line spacing}

The default font size and spacing between lines of text can both be changed. The methods differ between Linux-like systems and DOS.

\subsubsection{Font size and line spacing, Linux-like systems}

If $\mathbf{s}$ is entered in section 6.3, the following top-right prompts are presented in turn,

set new font size $(\min 7 \max 35$, now 10): weight, bold-2 medium-1 set new linespacing (now 15): (values shown are examples) 
Enter the new font size, followed by weight, followed by new linespacing, or enter return to accept defaults. The new settings will take effect immediately and appear in the Graphics setup prompts (section 6.3 where they can be readjusted.

\subsubsection{Font size, DOS}

If $\mathbf{s}$ is entered in section 6.3, a new screen appears showing some text in numbered fonts of various sizes, including a line segment font, and the following prompt,

font 5, to change enter font no: (values shown are examples)

Enter the font number corresponding to the new font.

\subsubsection{Line spacing, DOS}

If $\mathbf{v}$ is entered in section 6.3 , the following top-right prompt is presented,

set new linespace (now 20): (values shown are examples)

Enter the new line spacing.

\subsection{Changing the flashing cursor speed}

The speed at which the cursor flashes depends on the speed of your CPU and other factors. However the flash rate can be altered, enter $\mathbf{f}$ in section 6.3. The following top-right prompt is presented,

\section{change flashing cursor: restore-r or enter factor:}

Enter a factor to slow down or speed up the flash rate. For example to make it half as fast enter .5 , twice as fast enter 2 . To restore the default setting enter $\mathbf{r}$.

\subsection{Random number seed}

A new random number seed is generated by the computer's "timer" whenever DDLab is started. To change the random number seed, enter $\mathbf{r}$ at the first prompt in section 6.2. A specific random number seed can be set, or a random random number seed. The following prompt appears in a top-right window,

\section{enter random number seed 0-32767 (rnd-def):}

Using the same random number seed results in the same sequence of pseudo random numbers being produced by the random number generator. This allows identical multiple runs using default random settings of network parameters such as wiring, neighborhood mix, rules and initial state. Alternatively these setting can be loaded from previously saved files. 


\section{Chapter 7}

\section{Value-range $v$}

Figure 7.1: The cell value color key top-left window appears when the value-range is selected, and also at other times, with the colors indexed from $v$ - 1 to 0 .

Right: The color key windows for value-range $v=2$ to 8 , on a white background.

Below: Colors are different on a black background (section 6.3.3) - the $v=8$ black background color key.

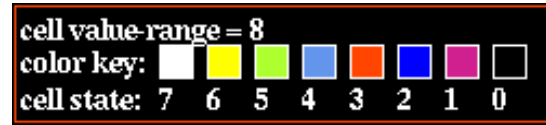

\begin{tabular}{|c|c|c|c|}
\hline $\begin{array}{l}\text { cell value range = } 2 \\
\text { color key: } \square \square \\
\text { cell state: } 1 \quad 0\end{array}$ & & & \\
\hline 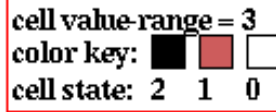 & & & \\
\hline $\begin{array}{l}\text { cell vahuerange }=4 \\
\text { color key: } \square \\
\text { cell state: } 3 \quad 21\end{array}$ & 0 & & \\
\hline $\begin{array}{l}\text { cell value range }=5 \\
\text { color key: } \\
\text { cell state: } \\
4\end{array}$ & 1 & 0 & \\
\hline $\begin{array}{l}\text { cell vahe-range }=6 \\
\text { color key: } \\
\text { cell state: } 5 \\
5\end{array}$ & 2 & 1 & 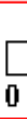 \\
\hline $\begin{array}{l}\text { cell vahe-range }=7 \\
\text { color key: } \\
\text { cell state: } 6 \\
6\end{array}$ & 3 & & 1 \\
\hline $\begin{array}{l}\text { cell vahe-range }=8 \\
\text { color key: } \\
\text { cell state: } 7 \\
\end{array}$ & 7 & $s$ & 2 \\
\hline
\end{tabular}

This chapter describes how to set the "value-range", $v$, available to a cell or network element, which can also be thought of as the number of its internal states, colors, or the size of its "alphabet". Older versions of DDLab were limited to binary (or Boolean) networks, where $v=2$.

Note that setting a high value-range, $v$, limits the maximum neighborhood size, $k_{\text {Lim }}$, (section 7.2) and also, for basin of attraction fields, the maximum network size, $n$ (section 7.3).

\subsection{The value-range prompt}

The value-range prompt, the second prompt in the main sequence, is as follows,

Value-range $\mathbf{v}(\operatorname{def} 2, \max 8): \quad(v=2$ on startup - then defaults to the last $v$ that was set)

Enter a new value-range (which becomes the new default) or enter return to accept the default. The selection of the value-range can only be made at this early stage in the program. 


\subsection{Value-range, limits on neighborhood size, $k_{\text {Lim }}$}

The upper limit of neighborhood size $k_{\text {Lim }}$ decreases with increasing value-range $v$ because ruletables may become too large. The size, $S$, of a full rule-table (rcode) is given by $S=v^{k}$, which larger than a $k$-totalistic rule-table (kcode) where $S=(v+k-1) ! /(k !(v-1) !)$, so $k_{L i m}$ depends on whether DDLab was constrained to run "forwards-only" for totalistic rules (TFO-mode). The $k_{\text {Lim }}$ for increasing value-range $v$, in both cases, is set out in table 7.1, and these limits are included in the prompts. The tables also show the size of the corresponding rule-tables, $S$.

Note that $k_{\text {Lim }}$ is different from $k_{\max }$ which is a deliberate selection of the maximum $k$ in a $k$-mix, a network with mixed neighborhood sizes, $k_{\max } \leq k_{\text {Lim }}$.

Unconstrained

\begin{tabular}{r|r|r}
$v$ & $k_{\text {Lim }}$ & table size $S$ \\
\hline 2 & 13 & 8162 \\
\hline 3 & 9 & 19683 \\
\hline 4 & 7 & 16484 \\
\hline 5 & 6 & 15629 \\
\hline 6 & 5 & 16807 \\
\hline 7 & 5 & 16807 \\
\hline 8 & 4 & 4096
\end{tabular}

TFO-mode

\begin{tabular}{r|r|r}
$v$ & $k_{\text {Lim }}$ & table size $S$ \\
\hline 2 & 25 & 26 \\
\hline 3 & 25 & 351 \\
\hline 4 & 25 & 3276 \\
\hline 5 & 25 & 23551 \\
\hline 6 & 17 & 26334 \\
\hline 7 & 13 & 27132 \\
\hline 8 & 11 & 31824
\end{tabular}

Table 7.1: The upper limit of $k, k_{\text {Lim }}$, for different value-ranges $v$. Left: FIELD-mode or SEED-mode with a full, unconstrained, rule-tables, capable of computing basins of attraction. Right: TFO-mode, for running totalistic rules, which have smaller rule-tables, forwards only, so the $\overline{k_{\text {Lim }}}$ can be larger. In both cases the sizes $S$ of the rule-tables for $v$ and $k_{L i m}$ are listed.

\subsection{Value-range, limits on network size, $n_{\text {Lim }}$}

For basin of attraction fields (FIELD-mode) the upper limits ${ }^{1}$ of network size $n_{\text {Lim }}$ decrease with the increasing value-range $v$, as set out in table 7.2, and these limits are included in the prompts. In practice much smaller sizes are advisable because of probable time/memory constraints.

\begin{tabular}{c|c|c}
$v$ & $n_{\text {Lim }}$ & state-space $v^{n} \approx$ billions \\
\hline 2 & 31 & $2147483648 \approx 2.15$ \\
\hline 3 & 20 & $3486784401 \approx 3.49$ \\
\hline 4 & 15 & $1073741824 \approx 1.07$ \\
\hline 5 & 13 & $1220703125 \approx 1.22$ \\
\hline 6 & 12 & $2176782336 \approx 2.18$ \\
\hline 7 & 11 & $1977326743 \approx 1.98$ \\
\hline 8 & 10 & $1073741824 \approx 1.07$
\end{tabular}

Table 7.2: For a basin of attraction field (FIELD-mode) the maximum network size, $n_{\text {Lim }}$, for different value-ranges $v$, and the corresponding maximum state-space $v^{n}$.

These limits also apply to the maximum number of most significant bits/values for the state-space matrix (section 31.2.2.1).

\footnotetext{
${ }^{1}$ These limits are required so that the size of state-space $v^{n}$ is within the maximum value of an unsigned long integer $\left(2^{32}-1=4294967295\right)$, a number used to index bits in a char array that identifies "used" states.
} 


\section{Chapter 8}

\section{1d network size $n$, or range- $n$}

This chapter describes setting the $1 \mathrm{~d}$ network size $n$, or a range of sizes. Setting the size of $2 \mathrm{~d}$ or $3 \mathrm{~d}$ networks, as well as other special options, is done at a later stage (section 11.6) in which case any $1 \mathrm{~d}$ size will be superseded and can be ignored by entering return.

$1 \mathrm{~d} n$ is set here in the main sequence of prompts (as is $k$ in section 9) to simplify setting up CA attractor basins, which are usually $1 \mathrm{~d}$, and to run $1 \mathrm{~d}$ CA forward for space-time patterns.

The chapter also looks at the upper limits of $n$ possible in DDLab, and the practical computational and speed limitations of combinations of $v, k$ and $n$ for attractor basins, with examples.

\subsection{Setting range of sizes, $1 \mathrm{~d}$}

It is possible to generate a series of basin of attraction fields for a range of $1 \mathrm{~d}$ network sizes (figure 8.1, with the network size printed on the left of the screen. The presentation is similar to the "Atlas" in "The Global Dynamics of Cellular Automata" [25]. Single basins or subtrees for a range of network sizes may also be generated.

If not in TFO-mode, the following main sequence prompt is presented,

range of network size-r, else-ret:

If $\mathbf{r}$ is selected the next two prompts are presented in sequence as follows, where the defaults for start and end depend on the value-range $v$,

range size: start (def 5): end (def 12): (for FIELD-mode, v=2)

Enter the start and end sizes, or enter return to accept the defaults, taking care to avoid excessively large sizes (section 8.3 below).

\subsection{Setting the size of one network, $1 \mathrm{~d}$}

If $\mathbf{r}$ was not selected in section 8.1 above, the $1 \mathrm{~d}$ network size is set with one of the following main sequence prompts, depending on the mode selected at the first prompt (section 6.2) FIELD, SEED, or TBO, and where default $n$ depends on the value-range $v$, 


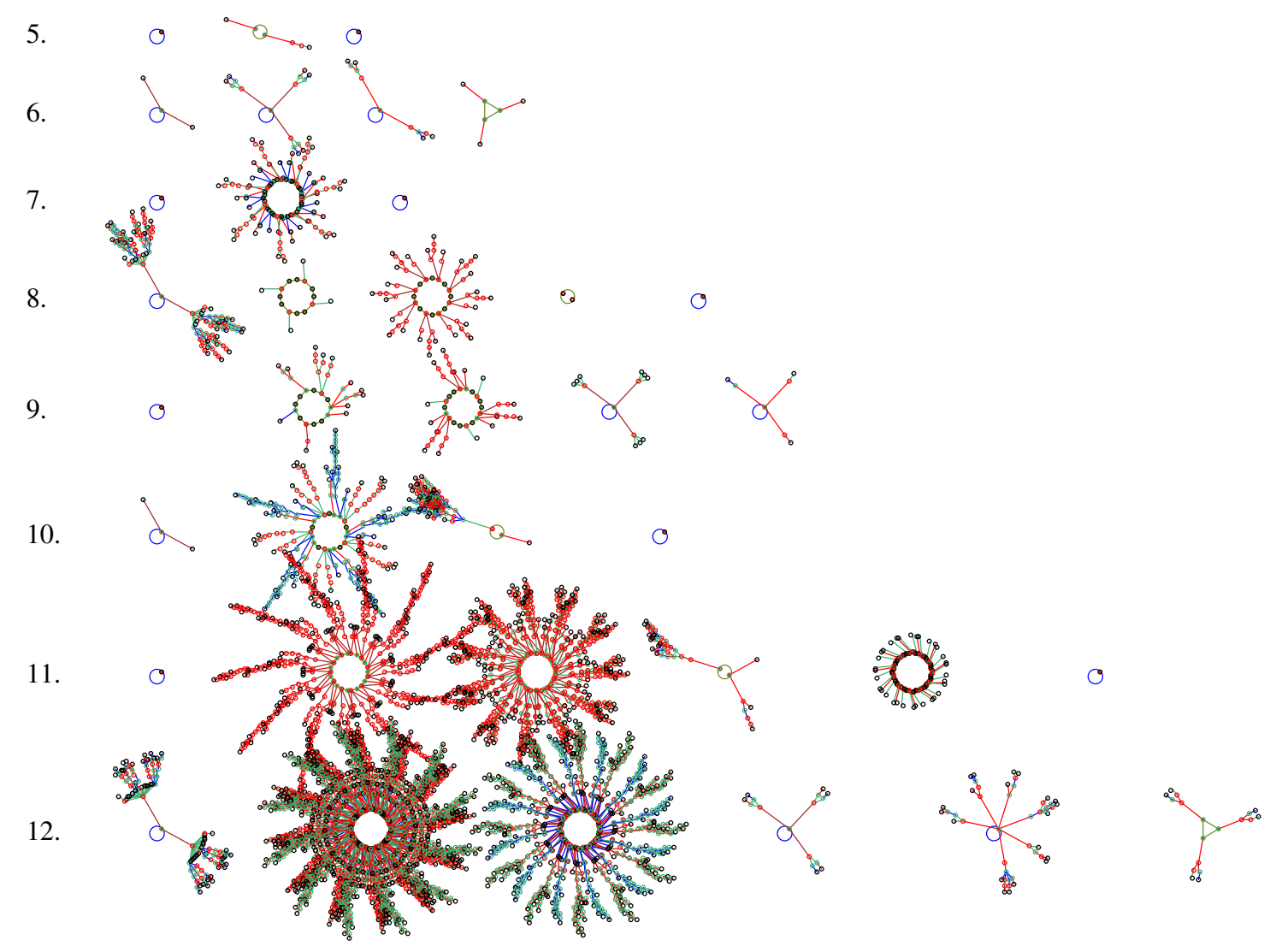

Figure 8.1: Basin of attraction fields for a range of network size 5-12. $v 2 k 4$ rcode(hex)=61a4.

for FIELD-mode

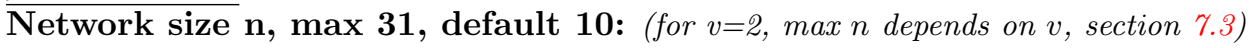

for SEED-mode

$\overline{\text { Network size }} \mathbf{n}, 1 \mathrm{~d}$ (other:see WIRING), def 14:

for TFO-mode

Network size n, 1d (other:see WIRING), def 150:

Enter the required $1 \mathrm{~d}$ network size, $n$, For $2 \mathrm{~d}$ or $3 \mathrm{~d}$ networks, the network size will be set as part of the wiring options (chapters $11-12$ ), so these prompts in sections 8.1- 8.2 may be ignored by pressing return.

The network size $n$ cannot be greater than $n_{\text {Lim }}$ (section 8.3). If a larger $n$ is selected, the default $n$ is applied with the following message,

$\ldots: 33$ too big! $\mathbf{n}=\mathbf{1 0}$ (in FIELD-mode, for example. $n_{\text {Lim }}$ is listed in table 7.2)

... :66000 too big! limit $=65025 ! \mathbf{n}=\mathbf{1 4}$ (in SEED-mode or TFO-mode, for example) 


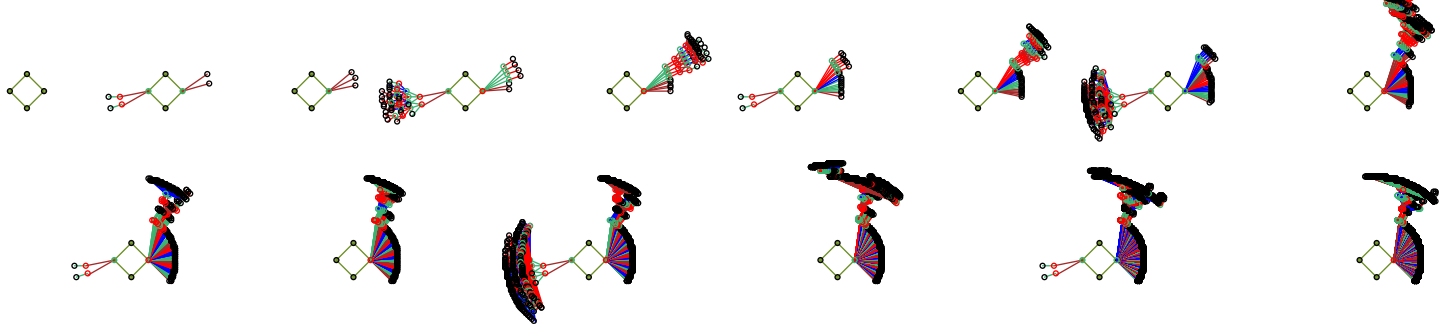

Figure 8.2: Single basins for a range of network sizes containing the state all $0 \mathrm{~s}, n=1$ to $15, v 4 k 2$ rcode $($ hex $)=a 7857 \mathrm{c} 0 \mathrm{~d}$.

\subsection{Network size limits}

Network size limits are also discussed in sections 1.6 and 7.3. To summarize, the upper limit of network size, $n_{\text {Lim }}$, supported by DDLab is as follows,

FIELD-mode

Basin of attraction fields: $n_{\text {Lim }}$ varies according to value-range $v$ (table 7.2) between $v=2: n_{\text {Lim }}=31$ (figure 8.6) and $v=8: n_{\text {Lim }}=10$. In practice $n$ should be well below these maximum limits.

\section{SEED-mode and TFO-mode}

Space-time patterns $(\mathbf{1 d}, \mathbf{2 d}$ or $\mathbf{3 d}): n_{\text {Lim }}=65025$, which corresponds to the maximum size of a square $2 \mathrm{~d}$ network, $255 \times 255$, and can be represented by an unsigned short ( 16 bits). The maximum size of a $3 \mathrm{~d}$ cube is $40 \times 40 \times 40$. The networks need not be square or cubic as long as $i \times j \leq n_{\text {Lim }}$, or $i \times j \times h \leq n_{\max }$, For $1 \mathrm{~d}$ networks shown in $1 \mathrm{~d}$, a much smaller size is preferable, say $n=150$ to 200 , to fit the screen and for a clear view of analytical graphic windows. However, $1 \mathrm{~d}$ networks can be shown in $2 \mathrm{~d}$ or $3 \mathrm{~d}$, allowing a better view of analytical graphic windows.

Subtrees: $n_{\text {Lim }}=65025$. In practice the size should be much smaller, say $n \leq 50$. For a practical size try $n=25$.

Single basins: $n_{\text {Lim }}=65025$. In practice the size should be smaller than for subtrees, say $n \leq 25$. For a practical size, try $n=18$.

\subsubsection{Computational and speed limitations for attractor basins}

In general, large networks (size $n$ ) may be run "forwards" to generate space-time patterns, and also find attractors for rules with low $Z$-parameter[32], but only networks with modest $n$ can be run "backwards" to generate attractor basins. Of these, a subtree allows the largest networks, and basin of attraction fields the smallest. If in doubt, small sizes should be tried first. Larger sizes might exhaust computer memory or take an excessive length of time to compute, especially for networks other than local 1d CA. 


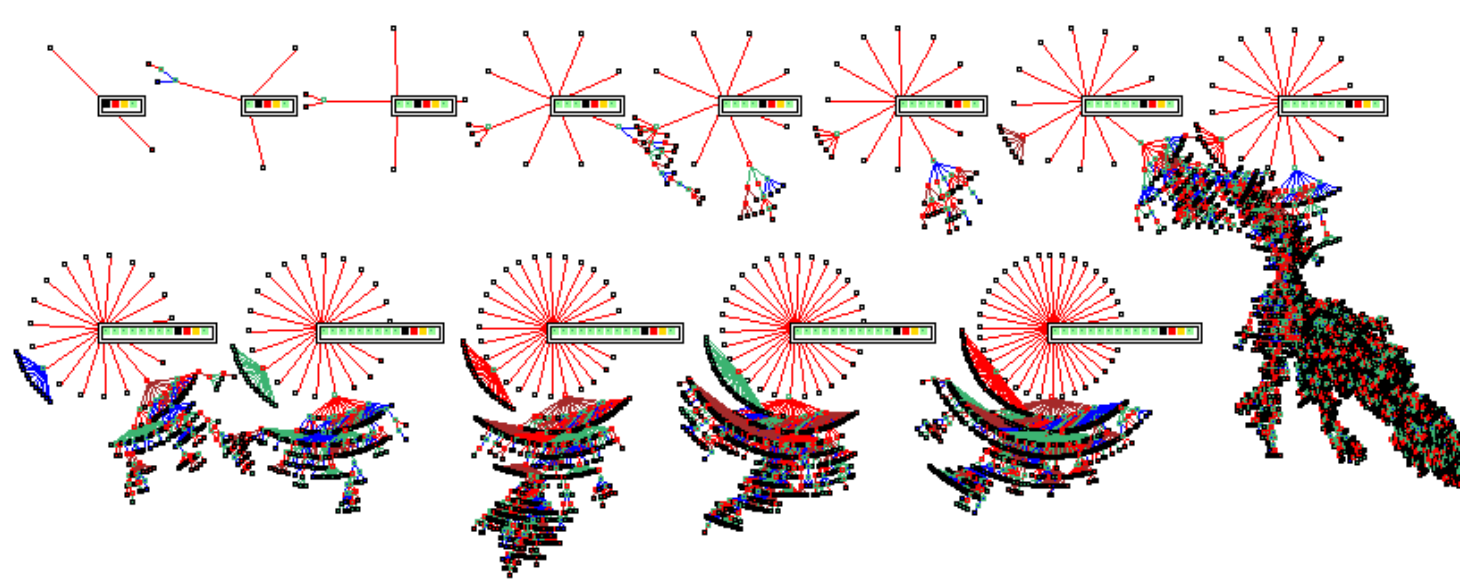

Figure 8.3: Subtrees for a range of network sizes from a root state containing from the string 3210, which is highlighted, $n=4$ to $15, v 4 k 3 \mathrm{rcode}($ hex $)=1 \mathrm{c} 49 \mathrm{a} 5 \mathrm{~b} 05 \mathrm{dbcdd} 148377635 \mathrm{fb} 0 \mathrm{~b} 60 \mathrm{~d} 84$.

It is not only $n$ that effects the computational and speed limitations for attractor basins. The complexity, thus speed, of computation for running backwards to find predecessors, and generate basin of attraction fields, single basins or subtrees, depends on a combination of $v, k$ and $n$. It also depends on the reverse algorithm, which is different and slower for random (nonlocal) wiring; if wiring is local $1 \mathrm{~d}$, the faster algorithm is applied, even if rules are mixed.

The speed also depends on the quality of the rule itself - how "branchy" are the resulting subtrees, the "in-degree". Typical in-degrees (number of predecessors of states) are predicted by a rule's $Z$ parameter[32] which varies between 0 and 1 , or the average $Z$ across the network for RBN and DDN. A rule with low $Z$ will have high "in-degree" (characteristic of "order"), thus short transients; excessive in-degree can overwhelm computer resources. On the other hand, a rule with high $Z$ will have low in-degree (characteristic of disorder or "chaos"), allowing subtrees for large $n$ to be computed, but transients and attractor periods tend to be extremely long, making basins of attraction difficult to reconstruct. The subset of chain-rules (section 16.11) have the lowest in-degree, usually just one, which does not increase with $n$; $1 \mathrm{~d}$ CA chain rules can therefore be run backwards for extremely large $n$, as in figure 8.4.

Finally, the speed will of course depend on the computer itself and what other programs are running.

\subsection{Times for basin of attraction fields}

The elapsed times to generate basin of attraction fields for a range of $v, k$ and $n$, for CA, RBN and DDN, on a 1.66GHz Laptop running Linux/Ubuntu $6.06^{1}$ are shown in tables 8.1- 8.4. below. These times are displayed as part of the data in a top-right window when the field is complete (section 27.2).

The system size $n$ that is appropriate for generating attractor basins in a reasonable time for combinations of $v$ and $k$ can be inferred from these examples. Large sizes may be tried, but may

\footnotetext{
${ }^{1}$ Lenovo 3000 N100 Laptop, Duo processor T2300e 1.66GHz, 1024 Mb RAM, running Linux Ubuntu 6.06.
} 


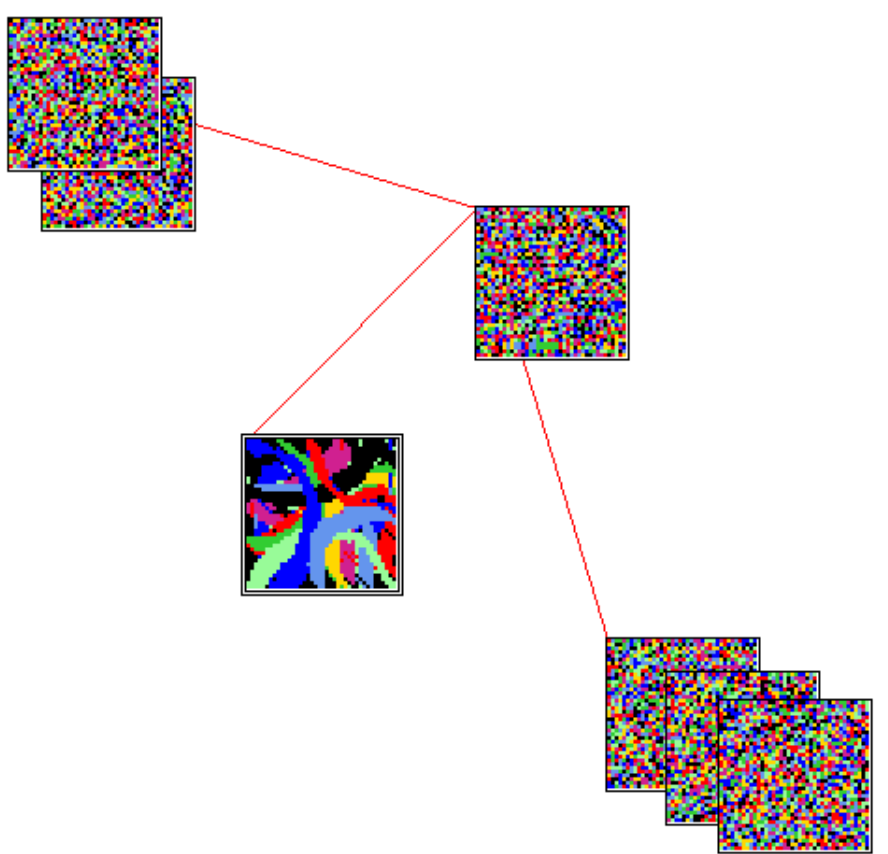

Figure 8.4: A subtree for a 1d CA chain-rule for a large network. $n=1600$ shown as $40 \times 40, v 8 k 4$. The root state of the subtree is located left of center.

impose unacceptable time, memory and display constraints. Single basins, and especially subtrees, may be generated for relatively much larger systems, especially for chain-rules (section 16.11), or close mutants of chain-rules, which have low in-degree.

\subsubsection{Examples for 1d CA}

Examples of the time needed to generate the basin of attraction field of some 1d CA rules for a range of $v, k$ and $n$, are shown in tables 8.1 and 8.2. The rules are specified in hex for $v=2$, and as the rule filenames for $v \geq 3$.

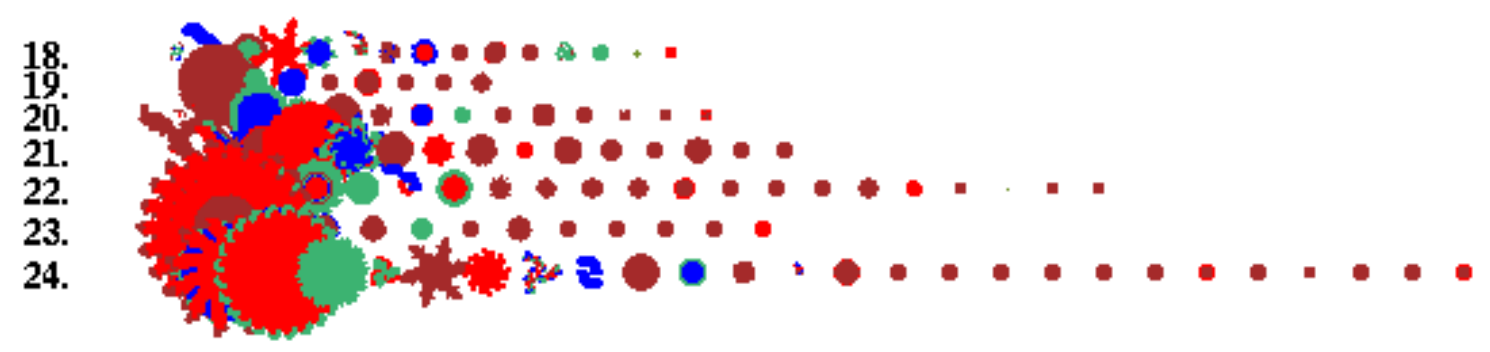

Figure 8.5: Basin of attraction fields for a range of network sizes, $v 2 k 5, n=18$ to 24 , to assemble part of the data for table 8.1 below. 


\begin{tabular}{c|r|r|r|r|r|r|r|l}
$n$ & 18 & 19 & 20 & 21 & 22 & 23 & 24 & $k$ and rule (hex) \\
\hline \multirow{3}{*}{ time } & $2.2 \mathrm{~s}$ & $4.6 \mathrm{~s}$ & $9.4 \mathrm{~s}$ & $19.4 \mathrm{~s}$ & $40.2 \mathrm{~s}$ & $1 \mathrm{~m} 23 \mathrm{~s}$ & $2 \mathrm{~m} 52 \mathrm{~s}$ & $k=3$ rule $\mathrm{c} 1(\mathrm{dec}) 193$ \\
\cline { 2 - 9 } & $3.8 \mathrm{~s}$ & $6.8 \mathrm{~s}$ & $13.9 \mathrm{~s}$ & $28.8 \mathrm{~s}$ & $58.0 \mathrm{~s}$ & $1 \mathrm{~m} 59 \mathrm{~s}$ & $4 \mathrm{~m} 10 \mathrm{~s}$ & $k=4$ rule e924 \\
\cline { 2 - 9 } & $9 \mathrm{~s}$ & $10 \mathrm{~s}$ & $20.9 \mathrm{~s}$ & $41.8 \mathrm{~s}$ & $1 \mathrm{~m} 26 \mathrm{~s}$ & $2 \mathrm{~m} 55 \mathrm{~s}$ & $6 \mathrm{~m} 0 \mathrm{~s}$ & $k=5$ rule b755d3d9
\end{tabular}

Table 8.1: Times for increasing $k$ and $n$ for $v=2$, basin of attraction fields, $1 \mathrm{~d}$ CA.

\begin{tabular}{|c|c|c|c|c|c|c|c|c|}
\hline$n$ & 12 & 13 & 14 & 15 & 16 & 17 & 18 & $v$ and rule file \\
\hline \multirow{3}{*}{ time } & $7.2 \mathrm{~s}$ & $18.5 \mathrm{~s}$ & $59 \mathrm{~s}$ & $3 \mathrm{~m} \mathrm{0s}$ & $84 \mathrm{~m} 54 \mathrm{~s}$ & $27 \mathrm{~m} \mathrm{55s}$ & $100 \mathrm{~m}$ & $v=3 \mathrm{~s}_{-} \mathrm{v} 3 \mathrm{k} 3 . \mathrm{rul}$ \\
\hline & $6 \mathrm{~m} 56 \mathrm{~s}$ & $20 \mathrm{~m} \mathrm{37s}$ & $109 \mathrm{~m}$ & & & & & $v=4 \mathrm{~s} \_\mathrm{v} 4 \mathrm{k} 3 . \mathrm{rul}$ \\
\hline & $132 \mathrm{~m} \mathrm{34ss}$ & & & & & & & $v=5$ s_v5k3.rul \\
\hline
\end{tabular}

Table 8.2: Times for increasing $v$ and $n$ for $k=3$, basin of attraction fields, $1 \mathrm{~d}$ CA.

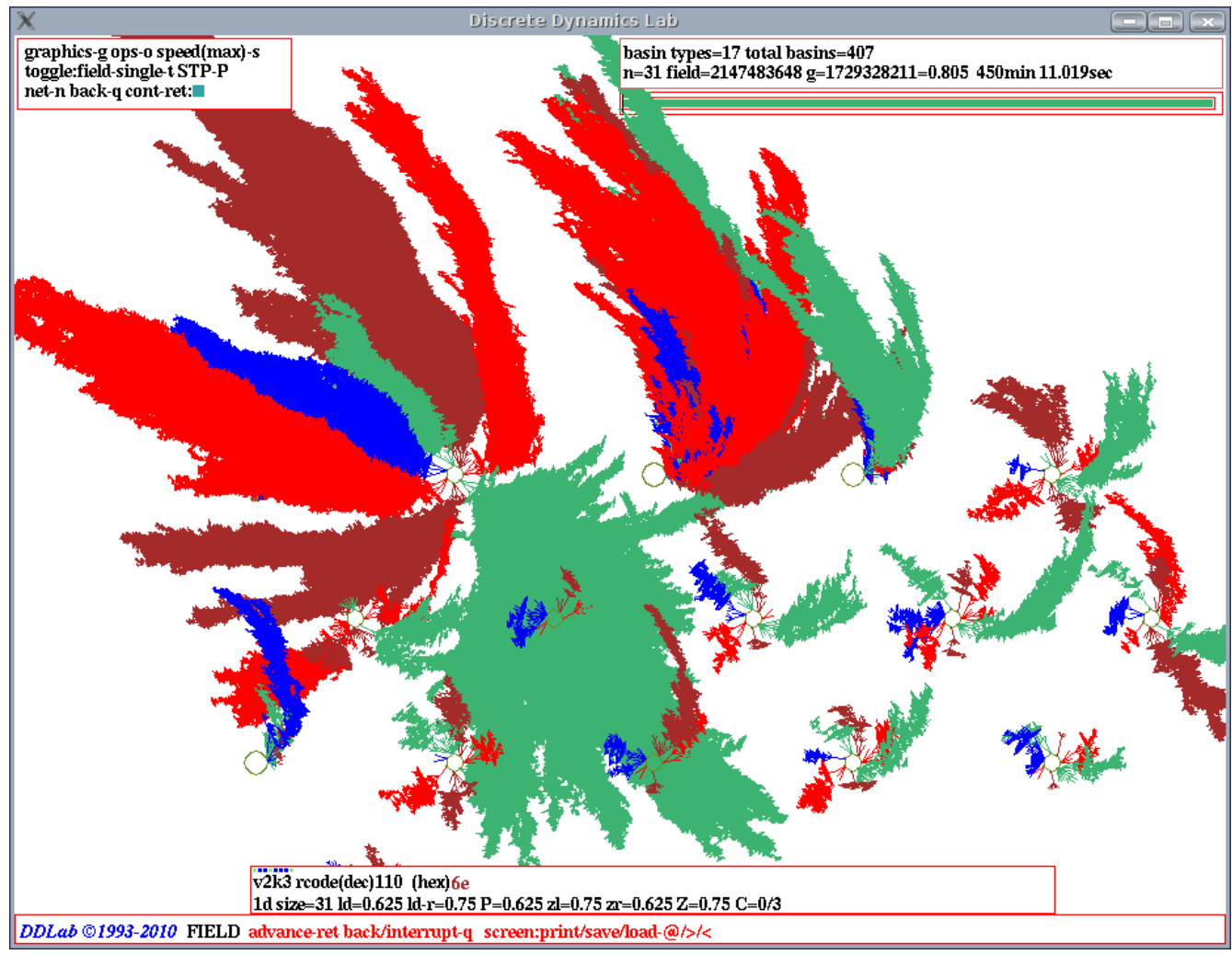

Figure 8.6: The basin of attraction field of $v 2 k 3$ rcode (dec) 110 , for $n=31$, which is the $n_{\text {Lim }}$ for $v=2$, elapsed times to generate 7.5 hours. The field is shown on the DDLab screen, with compression on (section 26.1), equivalent trees and subtrees suppressed (section 26.1.3), and nodes suppressed. (section 26.2). 


\subsubsection{Examples for RBN and DDN}

A similar exercise for RBN and DDN gave the timings shown in tables 8.3 and 8.4.

The same rules were used as for the CA in section 8.4.1, shown in hex for $v=2$, and as rule files for $v \geq 3$. However, the random wiring was not recorded. Note that it is the random wiring that requires a different and slower reverse algorithm, not the inhomogeneity of rules, so having one rule still provides a good indication of the speed, and allows a meaningful comparison between the various examples.

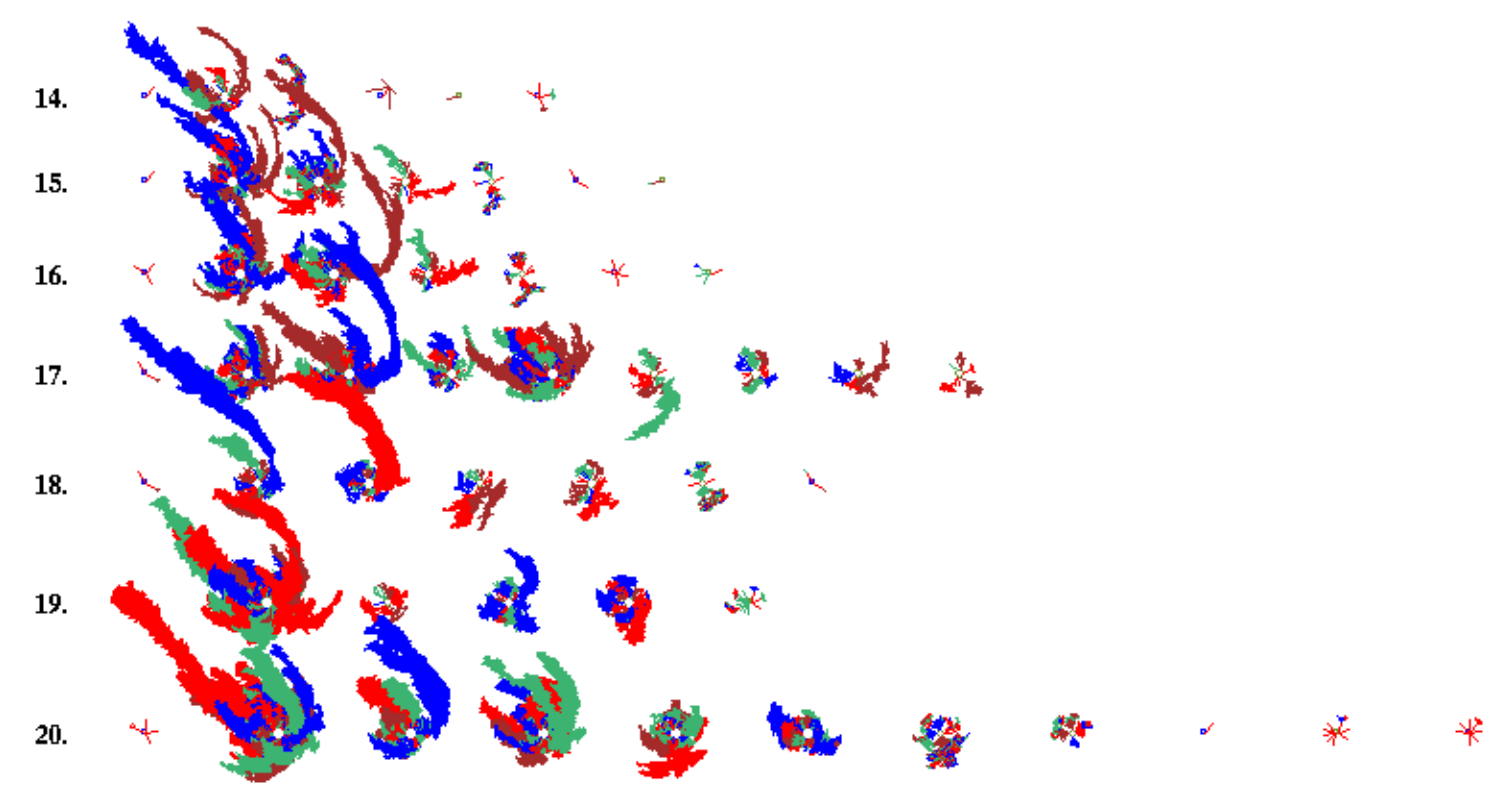

Figure 8.7: Basin of attraction fields for a range of network sizes, RBN, $v 2 k 3, n=14$ to 20 , to assemble part of the data for table 8.3 below.

\begin{tabular}{c|r|r|r|r|r|r|r|l}
$n$ & 14 & 15 & 16 & 17 & 18 & 19 & 20 & $k$ and rule (hex) \\
\hline \multirow{3}{*}{ time } & $0.81 \mathrm{~s}$ & $4.4 \mathrm{~s}$ & $8.4 \mathrm{~s}$ & $24.6 \mathrm{~s}$ & $39.2 \mathrm{~s}$ & $1 \mathrm{~m} 50 \mathrm{~s}$ & $4 \mathrm{~m} 54 \mathrm{ss}$ & $k=3$ rule c1 (dec: 193) \\
\cline { 2 - 9 } & $10.7 \mathrm{~s}$ & $25.7 \mathrm{~s}$ & $1 \mathrm{~m} 53 \mathrm{~s}$ & $4 \mathrm{~m} 19 \mathrm{~s}$ & $12 \mathrm{~m} 31 \mathrm{~s}$ & $26 \mathrm{~m} 46 \mathrm{~s}$ & $64 \mathrm{~m} 40 \mathrm{~s}$ & $k=4$ rule e924 \\
\cline { 2 - 9 } & $32.3 \mathrm{~s}$ & $2 \mathrm{~m} 50 \mathrm{~s}$ & $9 \mathrm{~m} 15 \mathrm{~s}$ & $15 \mathrm{~m} 24 \mathrm{~s}$ & $75 \mathrm{~m} 28 \mathrm{~s}$ & $143 \mathrm{~m}$ & $195 \mathrm{~m}$ & $k=5$ rule b755d3d9
\end{tabular}

Table 8.3: Times for increasing $k$ and $n$ for $v=2$, basin of attraction fields, RBN.

\begin{tabular}{c|r|r|r|r|r|r}
$n$ & 10 & 11 & 12 & 13 & 14 & $v$ and rule file \\
\hline \multirow{3}{*}{ time } & $8.0 \mathrm{~s}$ & $2 \mathrm{~m} 5 \mathrm{~s}$ & $9 \mathrm{~m} 20 \mathrm{~s}$ & $28 \mathrm{~m} 7 \mathrm{~s}$ & $152 \mathrm{~m}$ & $v=3$ s_v3k3.rul \\
\cline { 2 - 7 } & $9 \mathrm{~m} 2 \mathrm{~s}$ & $59 \mathrm{~m} 43 \mathrm{~s}$ & $268 \mathrm{~m}$ & & $v=4$ s_v4k3.rul \\
\cline { 2 - 6 } & $223 \mathrm{~m}$ & \multicolumn{1}{c}{} & & & $v=5$ s_v5k3.rul
\end{tabular}

Table 8.4: Times for increasing $v$ and $n$ for $k=3$, basin of attraction fields, DDN. 


\section{Chapter 9}

\section{Neighborhood $k$, or mixed- $k$}

This chapter describes setting a homogeneous 1d CA neighborhood $k$ with local wiring (nearest neighbour, next nearest etc.), or a $k$-mix, a mix of $k$ sizes, with the exact $k$-mix to be specified in a top-right window. Randomizing this wiring (making it nonlocal), $2 \mathrm{~d}$ or $3 \mathrm{~d}$, and other special wiring options come at a later stage (chapters 11,12 ) - in that any $1 \mathrm{~d} k$ entries in this chapter will be superseded, and can be ignored by entering return.

$1 \mathrm{~d} k$ is set here in the main sequence of prompts (as is $n$ in section 8) to simplify setting up CA attractor basins, which are usually $1 \mathrm{~d}$, and to run $1 \mathrm{~d}$ CA forward for space-time patterns.

\subsection{Selecting $k$, or a $k$-mix, for $1 \mathrm{~d}$ networks}

To set the neighborhood size $k$, or a $k$-mix for $1 \mathrm{~d}$ networks, the following main line prompt is presented, where the default $k$ and $k_{\text {Lim }}$ ( 3 and 13 in this example) depend on TFO-mode and $v$ as previously set (sections 6.1, 7.1),

Neighborhood size k: kmix-m, or enter 1-13 (def 3): (for example)

Enter the required value of $k$ (or $\mathbf{m}$ for a $k$-mix). If the $k$ entered is larger than $k_{\text {Lim }}$ (section 7.2) the default $k$ is applied with the following message,

... :14 too big! def set, cont-ret: (for example)

If $\mathbf{m}$ is entered for a $k$-mix, the actual mix will be set in section 9.3 below. For $2 \mathrm{~d}$ and $3 \mathrm{~d}$ networks, both the mix and size will be reset in the wiring options (chapters $11-12$ ), so the main sequence prompt can be ignored by entering return. The $k$ set becomes the new default in this option.

Once the network's rule or rules have been set (chapters 13-16), the network's $k$ or $k$-mix may be "neutrally" modified, both by increasing $k$ or reducing to effective $k$ (sections 18.7.1 - 18.7.3), for the network as a whole or for a particular cell.

\subsection{1 $k_{\text {Lim }}$ and minimum $k$}

The maximum number of input wires to a cell, $k_{\text {Lim }}$, currently supported in DDLab depends on TFO-mode and value-range $v$ as listed in section 7.2. For $v=2$, in TFO-mode $k_{\text {Lim }}=25$, and 
13 otherwise. Minimum $k=1$. Although $k=0$ is illegal, it can be created by setting effective $k=0$, described in section 9.2 below.

Note that $k_{\text {Lim }}$ is different from $k_{\max }$ which is a deliberate selection of the maximum $k$ in a $k$-mix, $k_{\max } \leq k_{\text {Lim }}$.

\section{$9.2 \quad$ Effective $k=0$}

Setting $k=0$ directly is illegal in DDLab. For effective $k=0$ you need single self-wiring and a neutral rule that conserves a cell's value. Set $k=1$ with the cell wired to itself (local wiring in $1 \mathrm{~d}, 2 \mathrm{~d}$ or $3 \mathrm{~d})$, and set its rule-table as follows,

\begin{tabular}{r|r|r|r}
$v$ & rule-table & hex & dec \\
\hline 2 & 10 & 02 & 2 \\
\hline 3 & 210 & 24 & 21 \\
\hline 4 & 3210 & $\mathrm{e} 4$ & 228 \\
\hline 5 & 43210 & 4688 & 2930 \\
\hline 6 & 543210 & $02 \mathrm{c} 688$ & 44790 \\
\hline 7 & 6543210 & 1 ac688 & 800667 \\
\hline 8 & 76543210 & fac688 & 16434824
\end{tabular}

Table 9.1: Effective $k=0$ neutral rules - the same for all rule types. $v=2$ to 8 , for single selfwiring, where $k=1$ is wired to itself. The rules can be entered in three alternative ways - as a bit/value rule-table, as the equivalent in hexadecimal (hex), and in decimal (dec).

Because $k=1$ these rule-tables are exactly the same for all rule types, rcode, kcode and tcode. Setting a neutral rule to a self-wired cell ensures that the cell is not receiving any inputs from other cells, and that it will conserve its current value. However, other cells may receive inputs from the effective $k=0$ cell. DDLab can identify any self-wired $k=1$ cells in a mixed- $k$ network, and gives an option to set the rule at these cells to make them effectively $k=0$ automatically (section 14.3), or this can be done by hand - the easiest way is to "kill a cell" from the $1 \mathrm{~d}$ wiring graphic (section 17.8.9).

Setting effective $k=0$ is useful for providing a fixed input signal to a network, or potentially for an arbitrary external input.

\subsection{Specifying the $k$-mix}

If $\mathbf{m}$ is selected in section 9.1 above, the following prompt appears in a top-right window,

set k-mix: load-l hand-h norm-n power/specify-s rnd-(def):

(norm-n only if $n \geq k_{\text {Lim }}$, i.e. not for very small networks)

The $k$-mix can be set in a variety of ways described below. Note that settings for particular cells in a $k$-mix can be changed later in the "wiring graphic" options (chapter 17).

\subsection{Loading a $k$-mix file}

Enter $\mathbf{l}$ in section 9.3 above to load the $k$-mix as a. $\operatorname{mix}$ file (section 35.2). The file would have been saved in sections 19.5 or 9.11.3. If the file is for a bigger network, as much $k$-mix data 
as will fit into the current network will be loaded, starting from cell index 0 . If on the other hand the file is for a smaller network, all the data will be loaded from cell index 0 , the excess cell indexes will be allocated a uniform background $k$-mix with the following top-right prompt,

k-mix loaded (10) less than net size (150) (values shown are examples) enter background k-mix 1-13, (default 5):

The $k$-mix encoding is described in section 19.3.1. The $k$-mix can be also be save/loaded in Network filing options - section 19.1 (see also section 17.8.12).

\subsection{Setting the $k$-mix by hand}

If $\mathbf{h}$ is selected in section 9.3, a series of prompts allow the $k$ at each cell index (starting from 0 ) to be set.

\section{set neighborhood size (1-13) at cell 0 (back-b restrnd-r):}

Enter the required $k$, or return for a random $k$ value between 1 and $k_{\text {Lim }}$. $\mathbf{b}$ allows backtracking to the previous cell index. Enter $\mathbf{b}$ to assign $k$ at random for all remaining cells.

\subsection{Setting a normal distribution}

Enter $\mathbf{n}$ to set a normal (Poisson) distribution of the frequencies of different $k$. This option does not apply for very small networks, when $n<k_{\text {Lim }}$. The distribution is achieved by assigning potential links at random to the network, up to a preset limit. However, every cell will be assigned at least one link. The following top-right prompts are presented in sequence,

set $\mathrm{k}$ upper bounds (def 13, limit 13):

set average $\mathbf{k}$ (def 2.60, $\max \mathbf{8 . 6 7})$ : (values depend on $k$ upper bounds)

Enter the upper bounds of $k$ for the distribution, followed by the target average $k$ in the network. An example of a normal distribution is shown in figure 9.1 generated in section 9.11.

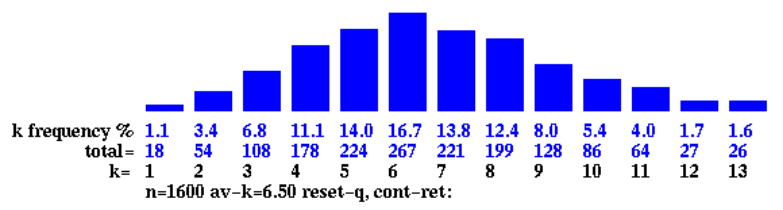

Figure 9.1: An example of a normal (Poisson) distribution of the frequencies of different $k . n=40 \times 40$, the range of $k=13$ and the average $k=6.5$

\subsection{Specify each $k$, or power-law distribution}

Enter $\mathbf{s}$ in section 9.3, to specify the distribution of different $k$, or to select a power distribution. The following top-right prompt is presented,

power-law-p, specify-(def):

These options are explained in sections 9.7.1 to 9.7.3 below. 


\subsubsection{Specify the percentage of different $k$}

If return is entered in section 9.7 above, the percentage of cells with different $k$ can be specified, and within those constraints the $k$-mix is assigned at random (i.e. shuffled). Alternatively, the $k$-mix need not be shuffled in which case it is assigned to the network in continuous blocks.

The following series of prompts are presented for each $k$ starting with $k=1$ (shown with example settings),

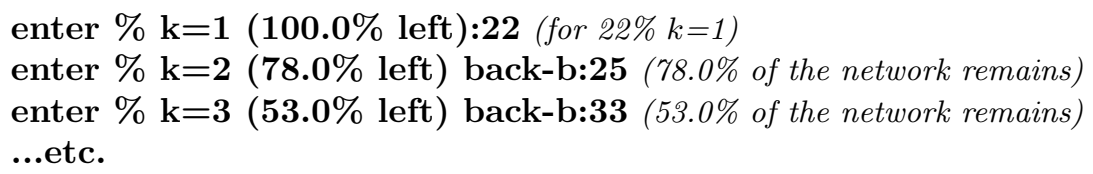

The prompts continue until none of the network remains, or until $k=k_{\text {Lim }}$. Return assigns zero of that particular $k$. An assignment that is too large for the network still remaining will be truncated. If on completion some of the network remains, this will be set with the last $k$ that was assigned. If none of the $k$ 's are assigned, the prompt reverts to section 9.3. At any stage from $k=2$ onwards it is possible to backtrack and revise with $\mathbf{b}$.

\subsubsection{Setting a power-law distribution of $k$}

Enter $\mathbf{p}$ at the prompt in section 9.7 to set a power-law distribution of the frequencies of different $k$. However, every cell will be assigned at least one input. The following top-right prompts are presented in sequence,

set $\mathbf{k}$ upper bounds (def 13, limit 13): (if $\left.k_{\text {limi }}=13\right)$

enter power-law exponent 1 to 3 (def 2.0):

Enter the upper bounds $k_{\max } \leq k_{\text {Lim }}$ for the distribution, followed by the target power-law exponent. An example of a power-law distribution is shown in figure 9.2. The output distribution can also be set to approximate a power-law (section 17.8.5, figure 17.18).

If both the inputs, $k$, and the outputs, follow a power-law, the network is said to be "scalefree", and characteristic of many natural and artificial networks, from metabolic networks [2] to the word-wide-web. The graph of a "scale-free" network is shown in figure 20.2.

\subsubsection{Showing the distribution}

When the $k$-distribution has been assigned, either by specifying the percentage of different $k$ in section 9.7.1, or by setting a power-law in section 9.7.2, data on the distribution, and the following prompt, are presented in a top-right window (for example, for a $2 \mathrm{~d} 40 \times 40$ network, and a power-law),

$$
\begin{aligned}
& \mathrm{k} 1=63.2 \%=1011 \mathrm{k} 2=15.6 \%=245 \mathrm{k} 3=7.15 \%=114 \mathrm{k} 4=4.0 \%=64 \mathrm{k} 5=2.6 \%=41 \\
& \mathrm{k} 6=1.8 \%=29 \mathrm{k} 7=1.4 \%=22 \mathrm{k} 8=1.1 \%=17 \mathrm{k} 9=0.8 \%=13 \mathrm{k} 10=0.7 \%=11 \\
& \mathrm{k} 11=0.6 \%=9 \mathrm{k} 12=0.5 \%=8 \mathrm{k} 13=0.4 \%=7 \\
& \text { shuffle: no-n yes- }(\mathrm{def}):
\end{aligned}
$$




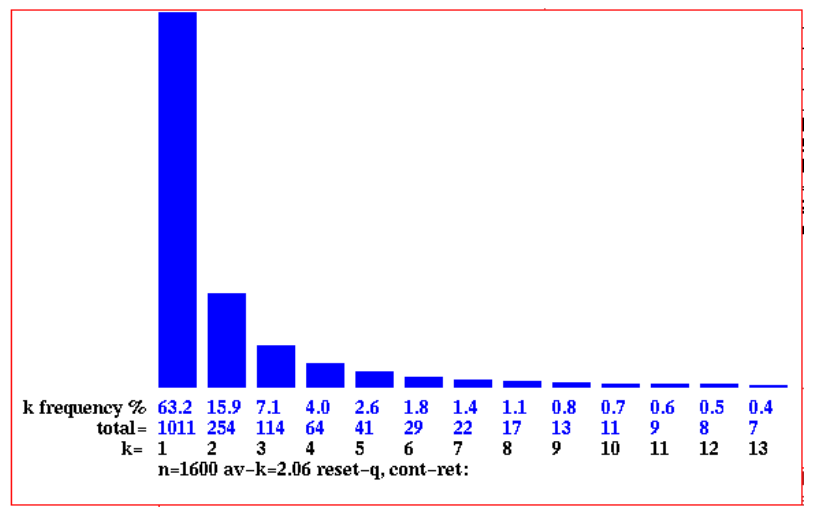

Figure 9.2: An example of a power-law distribution of the frequencies of different $k$. In this case $n=40 \times 40$, the range of $k=13$, the power-law exponent is 2 , and the average $k=2.05$.

This shows the percentage and number of different $k$ 's in the network. The $k$ 's are initially assigned in continuous blocks starting from the network cell 0. Enter return to randomly shuffle the assignment, or $\mathbf{n}$ not to shuffle and retain the blocks.

The distribution can be displayed as a histogram as in figures 9.1 and 9.2 generated in section 9.11 (and later in section 17.8.13).

\subsection{Setting the $k$-mix at random}

If return was entered in section 9.3, the default, a random $k$-mix is selected. A further prompt allows the $k$-mix be confined within upper and lower bounds before the random mix is assigned. In this example, $k_{\text {Lim }}=13$ (section 7.2),

$$
\text { set } \mathrm{k} \text { bounds 1-13: lower (def } 1) \text { : } \quad \text { upper (def 9, limit 13): (for example) }
$$

The upper bound is referred to as $k_{\max }$. The different $k$ 's will be assigned with equal probability.

\subsection{Setting a $k$-mix with uniform $k$}

Its sometimes useful to set up a $k$-mix network with homogeneous $k$, for example for a local $1 \mathrm{~d}$, 2d or 3d CA into which a DDN can be inserted (section 19.4), for example to provide a source of noise in the local network.

A $k$-mix with homogeneous $k$ is easily created in section 9.7 .1 , by setting the percentage of the required $k$ at $100 \%$, or in section 9.8 by setting equal values for the lower and upper bounds in a "random" the $k$-mix. The homogeneous $k$ network is treated as a $k$-mix network, and as such is also treated as a having nonlocal wiring and mixed rules even if the wiring is set as local $1 \mathrm{~d}$ (section 12.4.1), and there is just one rule (section 14.4.3). These issues relate to how memory is allocated to different types of network. 


\subsection{Increasing $k_{\max }$}

Options for changing $k$ for individual cells (or blocks of cells) occur at later stages in DDLab (sections 17.8.8, 18.7.1).

The default $k_{\max }$ is the upper bound set previously, for example in section 9.8. However $k_{\max }$ can be set to a higher value, up to the upper $k_{\text {Lim }}$ listed in section 7.2. The following top-right prompt is presented,

set greater max-k ( $\max 25, \operatorname{def} 9):\left(\right.$ if $k_{L i m}=25$ and $\left.k_{\max }=9\right)$

This enables the $k$ values in the network to be subsequently increased up the new $k_{\max }$. For example, if $100 \% k=5$ is set in section 9.7 .1 (a $k$-mix with uniform $k$ ), and $k_{\max }$ is set to say 9 , cells in the uniform $k$ network may later be reset to any value $\leq 9$.

\subsection{Reviewing the $k$-mix}

Finally, the $k$-mix is displayed in a top-right window, or a succession of windows for large networks. For example, figure 9.3 shows a randomly assigned $k$-mix between 1 and 25 for a network size 200 (in TBO mode), for each cell 199 to 0. To distinguish each (decimal) number, colors alternate between black and red. The percentage and number of different $k$ 's in the network is also displayed.

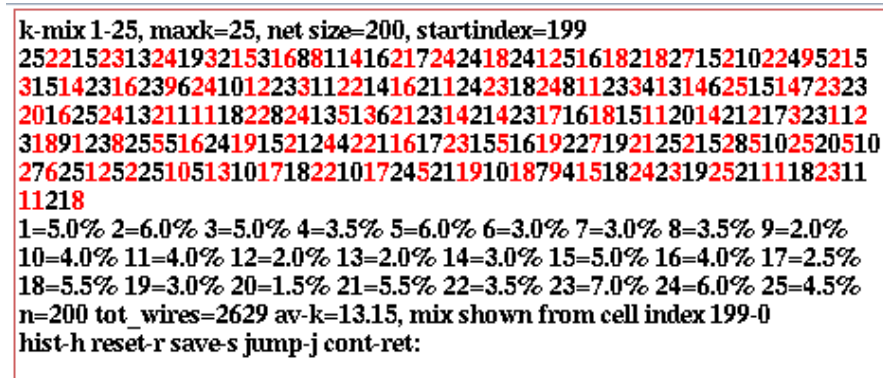

Figure 9.3: An example $k$-mix randomly assigned for $k=1-25, n=200$ (TBO-mode). To distinguish each (decimal) number, colors alternate between black and red.

Options are presented at the foot of the $k$-mix review window,

mix shown from cell index 244-0 (values shown are examples) hist-h reset-r save-s jump-j cont-ret:

Enter $\mathbf{h}$ to show the $k$ distribution histogram in a lower left window, as in figures 9.1 and 9.2. Enter $\mathbf{r}$ to revert to section 9.3 and reset the $k$-mix. Enter $\mathbf{s}$ to save the $k$-mix in a. $\operatorname{mix}$ file (section 35.2) which can be loaded as in section 9.4. Enter return to accept the $k$-mix. The other options are explained below. 


\subsubsection{Reviewing the $k$-mix in large networks}

Large networks may require several windows to display the $k$-mix. In this case the prompt above (section 9.11) includes an option more-m to see successive windows (for example),

mix shown from cell index 7011-4221 (values shown are examples) hist-h reset-r save-s more-m jump-j cont-ret:

\subsubsection{Jumping to a new cell index}

Alternatively, enter $\mathbf{j}$ in section 9.11.1 to jump to any cell index in the network, which becomes the first index in the new window. The following prompt is displayed,

jump to index (9800-0): (this example for a $2 d$ network, $99 \times 99$ )

\subsubsection{Saving the $k$-mix file}

Enter $\mathbf{s}$ in 9.11 to save the $k$-mix. A filing prompt box will allow saving a $\cdot$ mix file - the default filename is mymix.mix (section 35.2). The $k$-mix can also be saved in section 19.5, but can only be loaded in section 9.4. However, the $k$-mix in mixed- $k$ networks is implicit in the .w_s, .r_s and .wrs files (section 19.2), so does not usually need to be saved separately.

\subsection{Reviewing the $k$-mix within "network architecture"}

The $k$-mix may also be reviewed in Reviewing network architecture as described (chapter 17), where options allow changing $k$ (including "neutral" $k$ changes) up to $k_{\max }$ set in section 9.10, which may be greater than the maximum $k$ found in the network. This can be done for single cells, for predefined blocks, or for the whole network. 


\section{Chapter 10}

\section{The local neighborhood, and network geometry}

This chapter defines the CA neighborhood, the pseudo-neighborhood for RBN or DDN, the network geometry, and how these are indexed in DDLab.

\subsection{The CA neighborhood}

For CA, a cell updates according to the values of it local neighborhood, which usually includes the cell itself and its nearest neighbors. The relative position of the neighborhood cells in relation to the "target" cell is referred to as the "neighborhood template", or just the "neighborhood". For CA the neighborhood is homogeneous throughout the network, and thus requires periodic boundary conditions where each array edge wraps around to its opposite edge resulting in a ring of cells in $1 \mathrm{~d}$, the surface of a torus in $2 \mathrm{~d}$, and a 3 -torus in $3 \mathrm{~d}$. For $1 \mathrm{~d}$ and $2 \mathrm{~d}$ the neighborhood templates are defined in DDLab for $k=1$ to 25 ( $k \geq 14$ TFO-mode only), and $k=1$ to 13 for $3 \mathrm{~d}$.

The following sections describe the predefined neighborhoods and how they are indexed. Note that in $2 \mathrm{~d}$ these neighborhood templates can be square or hexagonal (sometimes both) to run on a square or hexagonal lattices, as shown in figure 10.3. Its also possible to construct any arbitrary neighborhood and assign it uniformly throughout the network (section 12.5.11).

\subsubsection{The pseudo-neighborhood, RBN and DDN}

For networks with nonlocal (random) wiring, RBN and DDN, DDLab provides methods (chapter 12) to specify how each "target" cell is connected to other cells with respect to its "pseudo-neighborhood", identical to the neighborhood in 1d, 2d and 3d CA. The set of cells that influence a given cell (its actual "neighborhood" to use the term loosely) may be scattered arbitrarily throughout the network. Each scattered cell is wired to one position in the target cell's pseudo-neighborhood. This allows a CA rule to be applied equally to local CA, RBN and DDN (chapter 13). 


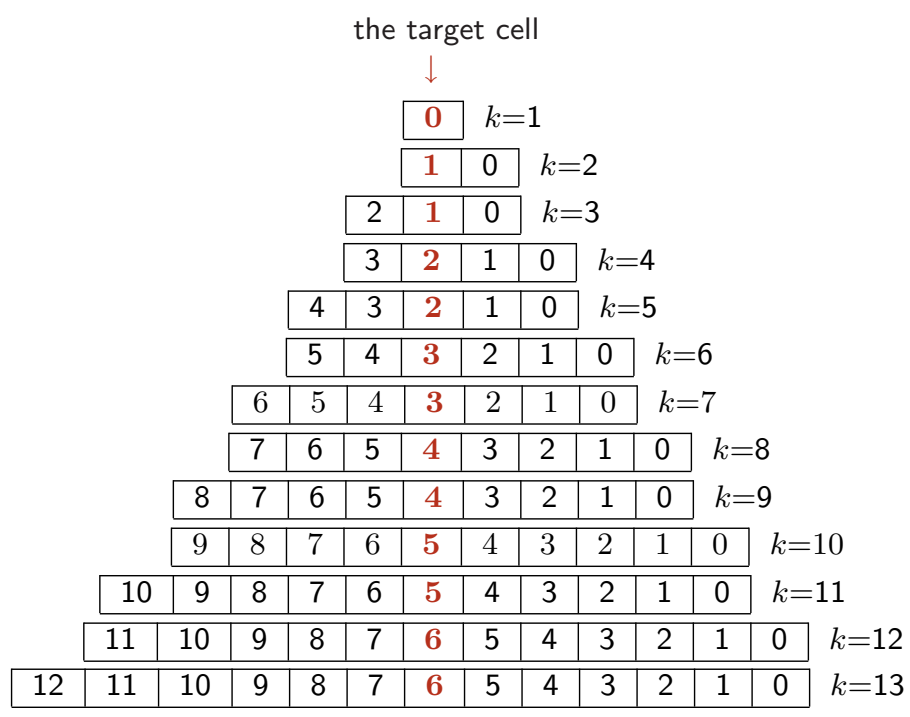

Figure 10.1: Neighborhood indexing $1 \mathrm{~d}, k=1$ to 13 . The central "target" cell is shown in red. The same principle applies for $k=14$ to 25 (TFO-mode only).

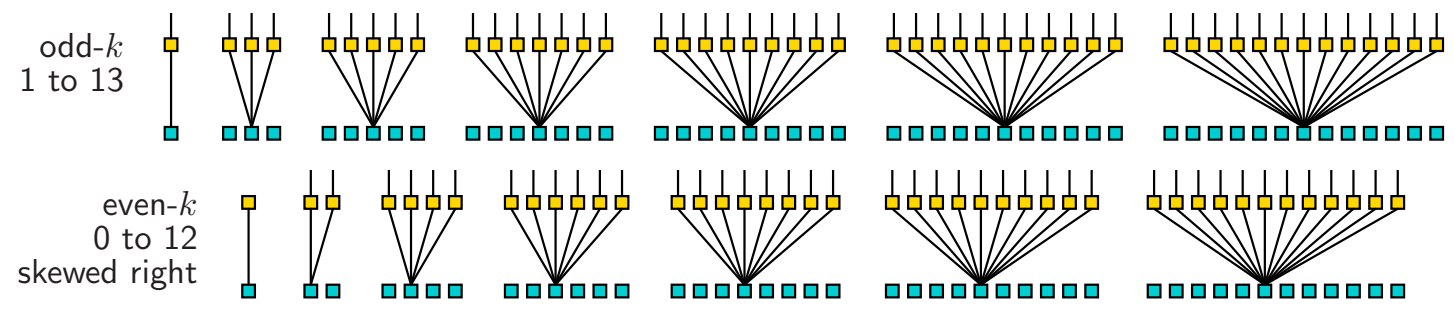

Figure 10.2: $1 \mathrm{~d}$ neighborhood templates as defined in DDLab, shown up to $k=13$ - the same principle applies for $k=14$ to 25 (TFO-mode only). Note that for even $k$ the neighborhood is asymmetric, skewed to the right. For effective $k=0$ see section 9.2.

\subsubsection{1d neighborhood}

The $1 \mathrm{~d}$ neighborhoods for $k=1$ to 25 are defined and indexed according to figures 10.2 and 10.1 . Cells in the neighborhoods are indexed in reverse order, $k-1, k-2, \ldots, 0$. For odd $k$ the target cell is centered, for even $k$ the neighborhood is asymmetric with an extra cell on the right.

\subsubsection{2d neighborhood}

The $2 \mathrm{~d}$ neighborhoods for $k=1$ to 25 are defined as shown in figure 10.3. To achieve maximum symmetry and for outer-totalistic rules (section 14.2) the target cell itself is sometimes not included in the neighborhood. Cells in the neighborhoods are indexed $k-1, k-2, \ldots, 0$, in reverse order in successive rows, from the top down, as in the example for $k=9$ in figure 10.4. Note that for a hexagonal lattice to work properly, the height of the network, $j$, should be even. 


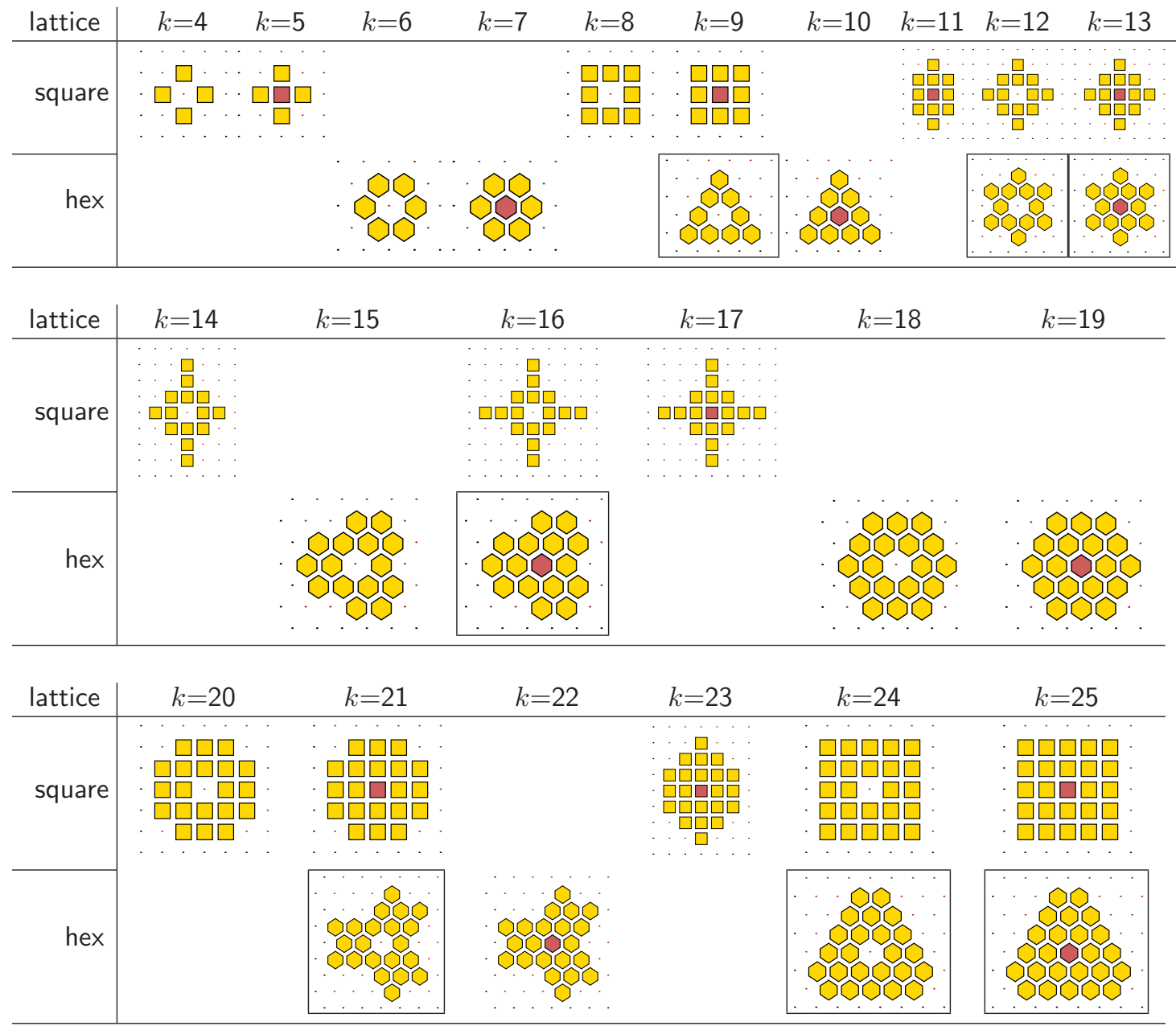

Figure 10.3: $2 \mathrm{~d}$ neighborhood templates as defined in DDLab, for $k=4$ to $25 . \quad k=1$ to 3 are the same as $1 \mathrm{~d}$, confined to the $i$ coordinate. $k \geq 14$ apply in TFO-mode only. Each $k$ has a template based on either a square or hexagonal layout, sometimes both, which is designed for best symmetry. If both, either hex or square can be selected - the neighborhoods shown without a black frame are the defaults. The templates may include or exclude the target cell. With a square layout, $k 5$ is known as the von Neuman neighborhood and $k 9$ as the Moore neighborhood — applied in the "game-of-Life" (section 16.10). With a hex layout, $k 6$ applies to the "beehive rule" [39] and $k 7$ to the "spiral rule" [40].

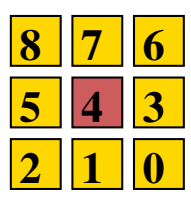

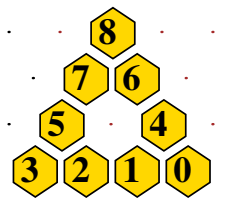

Figure 10.4: 2d neighborhood templates are indexed in reverse order starting with 0 bottom-left, as in these examples for $k=9$ for square and hexagonal neighborhoods. The index numbering is toggled on/off with index-i (section 17.4). 

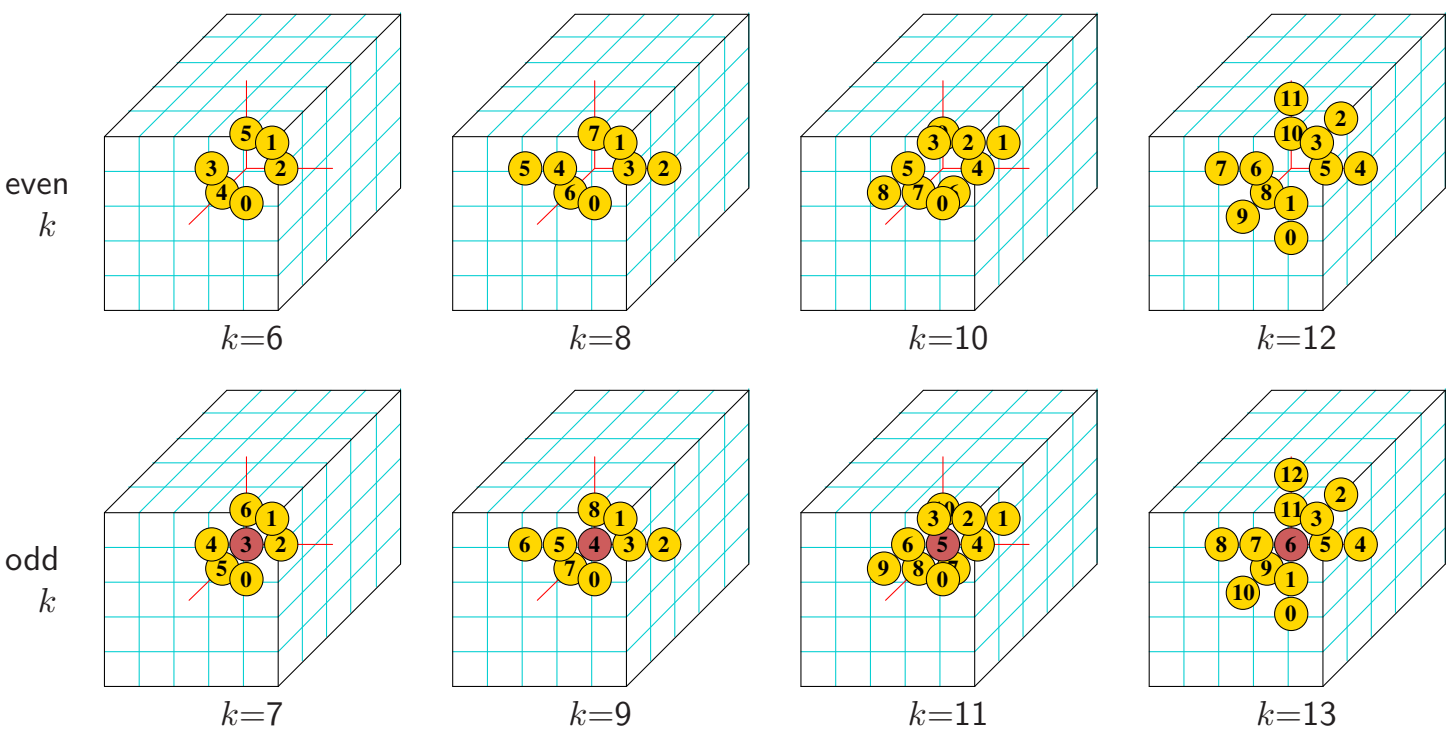

Figure 10.5: 3d neighborhood templates and indexing as defined in DDLab, $k=6$ to 13. For even $k$ the target cell itself is not included in the neighborhood. The view is isometric as if looking up into a cage. The $3 \mathrm{~d}$ neighborhood and network is simultaneously displayed in $2 \mathrm{~d}$ (section 17.7). The index numbering is toggled on/off with index-i (section 17.4).

\subsubsection{3d neighborhood}

The $3 \mathrm{~d}$ neighborhoods for $k=6$ to 13 are defined and indexed as shown in figure 10.5. To achieve maximum symmetry, for even $k$ the target cell itself is not included in the neighborhood.

In 3d networks, the neighborhoods define a cubic grid on the 3-torus. For $k \leq 5$ the neighborhoods are the same as for $2 \mathrm{~d}$, confined to horizontal $i, j$ layers - for $k \geq 6$ the neighborhood penetrates the vertical $h$ axis. Note that the only fully symmetrical $3 \mathrm{~d}$ neighborhoods are a $3 \mathrm{~d}$ nearest neighbor cross $-k=6$ and 7 , and a $3 \mathrm{~d}$ next-nearest neighbor cross $-k=12$ and 13 .

\subsection{Network geometry}

The cells in a network, size $n$, are indexed and arranged in a regular $1 \mathrm{~d}, 2 \mathrm{~d}$ or $3 \mathrm{~d}$ space or lattice, with axial dimensions $i$ for $1 \mathrm{~d}, i, j$ for $2 \mathrm{~d}$ and $i, j, h$ for $3 \mathrm{~d}$, as shown in figures 10.6, 10.7 and 10.8. These examples are from the network-graph presentation in chapter 20. This network "geometry" has real meaning for CA, or RBN/DDN where each cell's random inputs are confined close to the cell itself. For networks with fully random wiring the geometry simply allows convenient indexing and representation. The cells are indexed $n-1, \ldots, 0$, or according to their I,J,H coordinates.

CA have periodic boundary conditions, where opposite edges join, so CA (local) wiring in 1d creates a ring of cells. 2d CA wiring creates a regular square or hexagonal lattice on the torus. $3 \mathrm{~d}$ CA wiring creates a 3 -torus. RBN/DDN where inputs are confined close each target cell also have periodic boundary conditions by default, but this can be suppressed. 


\subsubsection{1d network indexing}

$1 \mathrm{~d}$ networks, size $n$, are indexed $n-1, \ldots, 0$, where $i=n$, and are usually laid out horizontally following figure 10.6 Left.

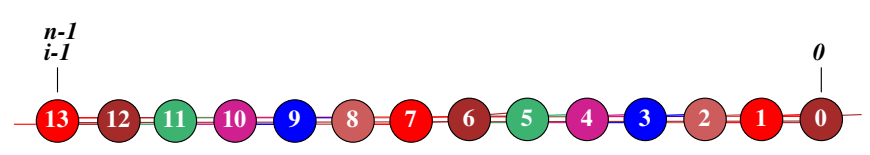

Figure 10.6: A 1d CA, $n=14 k=3$, indexed $13, \ldots, 0$, shown as a $1 \mathrm{~d}$ network-graph (chapter 20). Left: horizontal layout, and Right: circle layout.

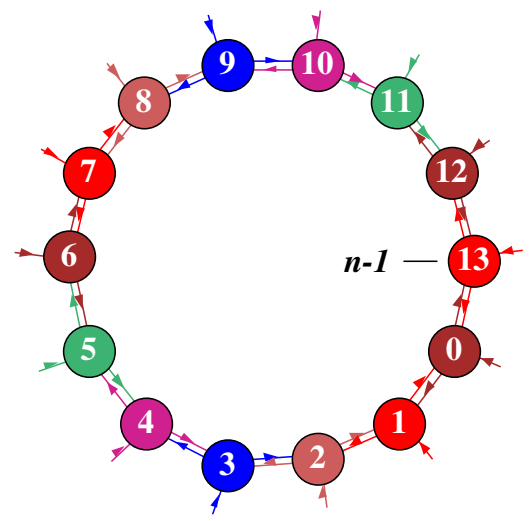

\subsubsection{2d network indexing}

$2 \mathrm{~d}$ networks, size $n$, are indexed $n-1, \ldots, 0$, with either square and hex layout as in figure 10.7. $i$ is the width (columns), $j$ is the depth (rows). Note that the cell indexes shown, $x$, are $1 \mathrm{~d}$ indexes.

For a $2 \mathrm{~d}$ network, size $i, j$, to convert the coordinates of a cell, $I, J$, to a $1 \mathrm{~d}$ index, $x=i J+I$.

Conversely, $I=x \bmod i, J=\left\lfloor\frac{x}{i}\right\rfloor$.

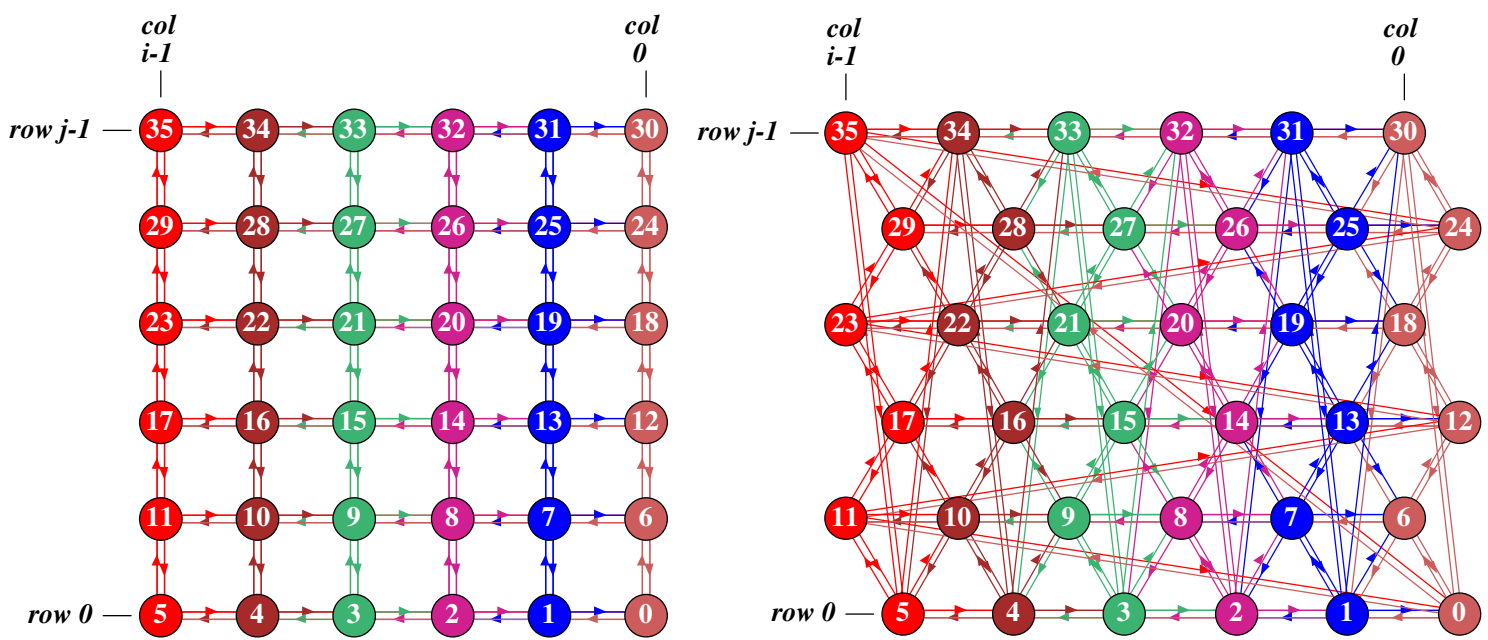

Figure 10.7: A 2d CA, $n=36$, where $i \times j=6 \times 6$, indexed $35, \ldots, 0$, shown as a $2 \mathrm{~d}$ network-graph (chapter 20). Left: square layout $(k=4)$. Right: hex layout $(k=6)$. In the network-graph prompt (section 20.2.1) key $2 d($ tog)-2 toggles between square and hex layout. 


\subsubsection{3d network indexing}

$3 \mathrm{~d}$ networks size $n$ are indexed $n-1, \ldots, 0$, as in figure 10.8. $i$ is the width (columns), $j$ is the depth (rows), and $h$ is the height (levels). Note that cell indexes shown, $x$, are $1 \mathrm{~d}$ indexes, For a $3 \mathrm{~d}$ network, size $i, j, h$, to convert the coordinates of a cell, $I, J, H$, to a $1 \mathrm{~d}$ index, $x=i j H+i J+I$. Conversely, $I=x \bmod i, J=\left\lfloor\frac{x \bmod i j}{i}\right\rfloor, H=\left\lfloor\frac{x}{i j}\right\rfloor$.

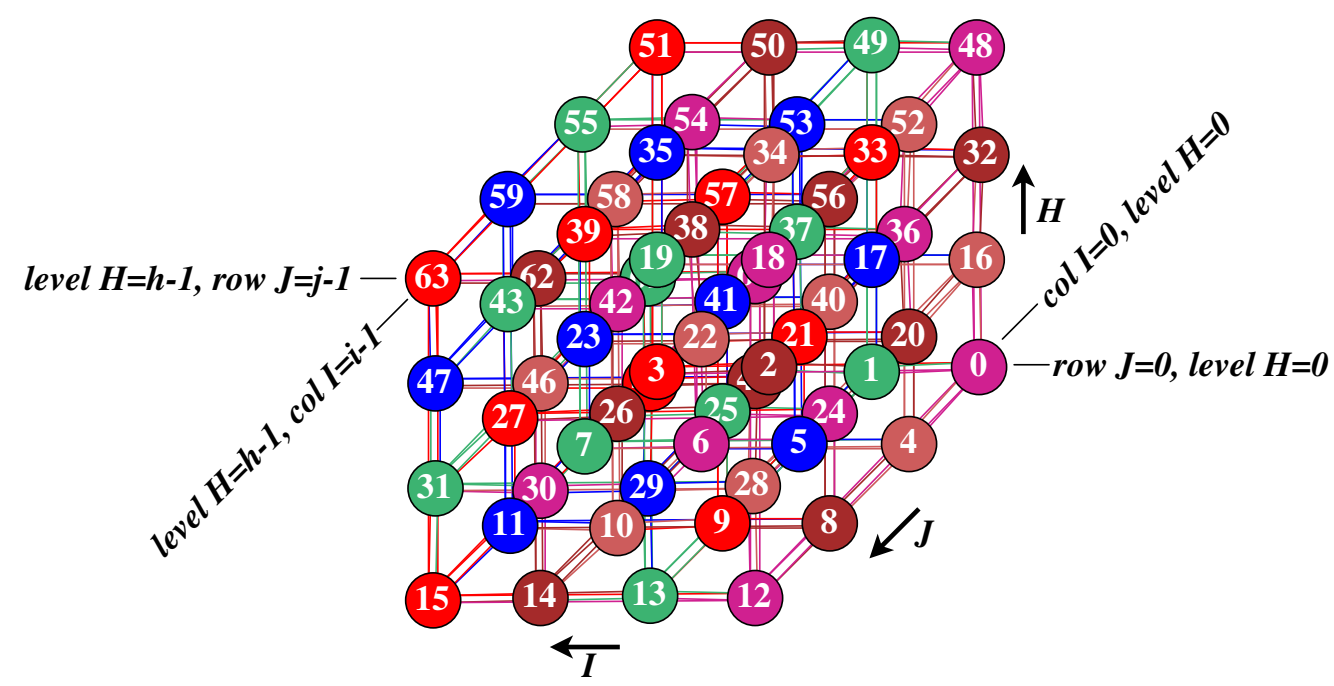

Figure 10.8: A 3d CA, $n=64,[i, j, h]=[4,4,4], k=6$, indexed $63, \ldots, 0$, shown as a $3 d$ network-graph, (chapter 20). The graph should be viewed as if looking up from below into a cage, were the bottom layer of cells are numbered $0-15$.
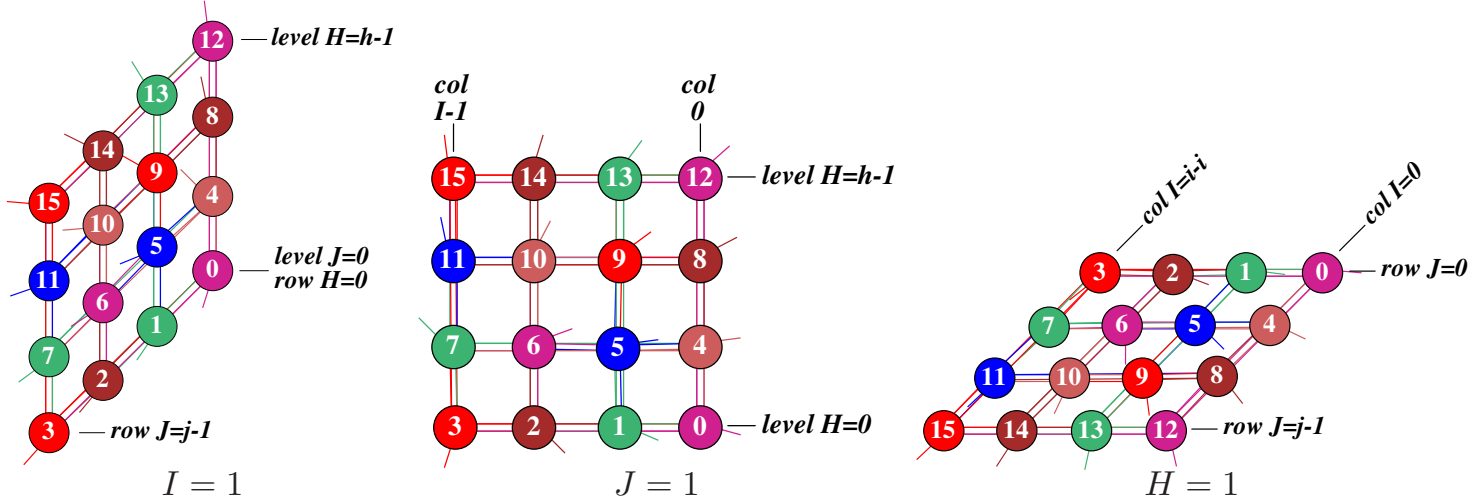

Figure 10.9: To clarify $3 \mathrm{~d}$ network indexing, the network-graphs above show three examples where the width (columns) $i$, depth (rows) $j$, and height (levels) $h$, are made to equal 1 in turn, so from left to right $[i, j, h]=[1,4,4],[4,1,4],[4,4,1] . \quad k=6$, cells numbered $0-15$. 


\section{Chapter 11}

\section{Setting the wiring, quick settings}

The main wiring prompts are displayed in context dependent top-right windows. The first wiring prompt gives options for quick wiring settings for CA in 1d, $2 \mathrm{~d}$ (hexagonal or square) or $3 \mathrm{~d}$, random wiring for just 1d, or loading a wiring file. Alternatively, special wiring allows more flexible wiring requiring further wiring prompts, described in chapter 12 .

\subsection{The first wiring prompt}

The first wiring prompt is as follows,

WIRING: special-s load-l random-r

regular 3d-3, 2d-2(hex+x square $+s)$, 1d-def:

\subsection{Special wiring}

If $\mathbf{s}$ is selected in section 11.1 above, prompts are presented for various special wiring options, including setting random wiring for $1 \mathrm{~d}, 2 \mathrm{~d}$ and $3 \mathrm{~d}$ networks (with various biases), described in chapter 12 .

\subsection{Loading the wiring scheme}

If $\mathbf{l}$ is selected in section 11.1, filing prompts will allow a . w_s file to be loaded, provised that $n$ in the file and base network are equal, and file $k_{\max } \leq$ base $k_{\max }$. (see also chapter 19).

\subsection{Random 1d wiring}

If $\mathbf{r}$ is selected in section 11.1, a wiring scheme will be assigned at random according to the previously selected neighborhood $k$ or $k$-mix settings. The next prompt allows a graphic 
representation to be displayed, where the wiring can be reviewed and altered (see chapter 17).

To assign random wiring for $2 \mathrm{~d}$ or $3 \mathrm{~d}$ networks, or bias the random wiring in various ways, $\mathbf{s}$ should be selected in section 11.1, and "special" wiring assigned as described in chapter 12.

\subsection{Local 1d wiring}

If return (the default) is selected in section 11.1, the wiring is set up as local 1d, with periodic boundary conditions. The network may be a $\mathrm{CA}$, or may have mixed $k$. The remaining wiring options will be skipped.

\subsection{Local $2 \mathrm{~d}$ or $3 \mathrm{~d}$ wiring}

If $\mathbf{2}$ or $\mathbf{3}$ is selected in section 11.1, the wiring will be set up as local $2 \mathrm{~d}$ or $3 \mathrm{~d}$ CA wiring according to the default neighborhoods in section 10.1.3. In both cases boundary conditions are periodic, i.e. the $2 \mathrm{~d}$ array can be imagined as drawn on the surface of a torus, and the $3 \mathrm{~d}$ array on a 3 -torus. The network can also have a $k$-mix with CA wiring for each neighborhood. Entering $2 \mathrm{x}$ or $\mathbf{2 s}$ will force the neighborhood, and the initial presentation of the lattice, to be hex or square, overriding the defaults in section 10.1.3. Just the hex/square presentation can be toggled later in the program.

As well as setting local wiring, further prompts will set $2 \mathrm{~d}$ or $3 \mathrm{~d}$ network size, and $k$ or a $k$-mix. Previous $n$ and $k$ settings from the main sequence of prompts will be superseded.

\subsubsection{Setting $2 d$ and $3 d$ network size}

The following option sets the $i \times j$ (width $\times$ depth) dimensions of 2 d networks, and the $i \times j \times h$ (width $\times$ depth $\times$ height) dimensions of 3 d networks.

\section{2d, enter width (def 40): $\quad \operatorname{depth}(\operatorname{def} 40)$ : or \\ 3d, enter width (def 9): $\quad \operatorname{depth}(\operatorname{def} 9): \quad$ height(def 9$)$ :}

Enter the width, depth (and height), keeping in mind the network size limits (section 8.3). For space-time patterns the maximum network size, $n_{\max }=65025$, corresponding to a square $2 \mathrm{~d}$ network, $255 \times 255$. The maximum $3 \mathrm{~d}$ cube is $40 \times 40 \times 40$. The network need not be square or cubic as long as $n \leq 65025$. The size limits apply so that network positions can be indexed by an unsigned short (16 bits).

If the dimensions selected for a $2 \mathrm{~d}$ or $3 \mathrm{~d}$ network exceed 65025 , a message such as the following is displayed,

$255 \times 256$ too big! max size $=65025$ cont-ret: $($ for $2 d$ )

or

40x40x41 too big! max size $=65025$ cont-ret: (for $3 d$, values shown are examples)

Pressing return restores the network size prompt.

If the intention is to generate attractor basins, the $2 \mathrm{~d}$ size should be kept small, for example $3 \times 3$ for quick results, $4 \times 4$ for slower results. 


\subsubsection{Set $k$, or $k$-mix}

If $2 \mathrm{~d}$ or $3 \mathrm{~d}$ was selected, the next top-right prompt resets $k$, or the $k$-mix, as described in chapter 9 .

Neighborhood size k: kmix-m, or enter 1-25 (def 9):

The upper bound, $k_{\text {Lim }}$, depends on the context - TFO-mode and $v$. Default $k$ depends on the previous selection. Enter a new value to reset $k$, or return to accept the default. Enter $\mathbf{m}$ for a $k$-mix and follow further instructions in chapter 9 .

\subsection{Networks too big for a basin field}

If FIELD-mode for a basin field was selected at the first prompt in chapter 6.2, and a network size $n$ in $2 \mathrm{~d}$ or $3 \mathrm{~d}$ is set larger than 31, FIELD-mode will be automatically changed to SEED-mode for a single basin or a space-time pattern — with the following message,

size too big for FIELD-mode $(\max 31)$

changed to SEED-mode! cont-ret

The title bar across the foot of the screen (section 5.5) is redrawn to reflect this change. The significance of FIELD-mode, SEED-mode (and TFO-mode) was described in chapter 6. 


\section{Chapter 12}

\section{Setting special wiring}

Selecting special wiring (enter $\mathbf{s}$ in section 11.1) allows a greater range of methods for wiring up the network (the wiring scheme). Special wiring is necessary to set random wiring for $2 \mathrm{~d}$ or $3 \mathrm{~d}$ networks. The network can be assigned local or random wiring in $1 \mathrm{~d}, 2 \mathrm{~d}$ (square or hex), 3d, or hypercube wiring, and the wiring can be "hand wired" and biased in a variety of ways.

Special wiring also allows the following functions, some of which may be restricted to predefined parts of the network (see also chapter 17).

- Confining random wiring within a set zone, from which some wires may be released.

- Suppressing periodic boundary conditions, selectively for the various axes in $2 \mathrm{~d}$ and $3 \mathrm{~d}$.

- Forcing or disallowing self-wiring.

- Forcing distinct wiring i.e. no duplication,

- Setting the same random wiring template for every cell in the network.

- There are additional functions for $3 \mathrm{~d}$ networks.

- Suppress links to particular layers of the network.

- Force direct links to specific layers.

- Apportion fractions of available random links between specified layers.

Once the special wiring is set, it can be amended by many flexible methods from the "wiring graphic" described in chapter 17.

\subsection{Setting up the network geometry}

Entering $\mathbf{s}$ in section 11.1 gives the first special wiring option, which allows the network geometry to be set as $1 \mathrm{~d}, 2 \mathrm{~d}$ (square or hex), 3d, and also as a hypercube for appropriate $n$ and $k$.

hypercube-h, 3d-3, 2d-2 (hex+x square+s), 1d-def

(hypercube-h only if $k=\log _{2} n$ or $\log _{2} n+1$, for example $k=3, n=8$ or 9 )

Sections below examine each option in detail. 


\subsection{Hypercube wiring}

In a fully connected $n$-dimensional hypercube, each state receives input from its $\log _{2} n$ oneHamming distance "neighbors", and may also receive input from itself. If $n=8$ and $k=\log _{2} n=3$, the hypercube is a simple cube with network states at the vertices and bi-directional links at the edges.

The hypercube wiring option is only active if the network size $n$ is a power of $2,(2,4,8,16, \ldots)$, and $k=\log _{2} n$, or $k=\log _{2} n+1$ where vertices also receive one additional input from themselves. For a simple cube, $n=8, k$ must equal 3 or 4 for the hypercube option to appear.

If $\mathbf{h}$ for a hypercube is selected in section 12.1 a further option allows degrading the hypercube "wiring", i.e. pruning uni-directional connections, according to some probability,

set \% prob (def 100):66 (for example)

For example, for an $n=8, k=4$ hypercube, if 66 is entered, wires are set with a probability of $66 \%$. A further prompt is presented,

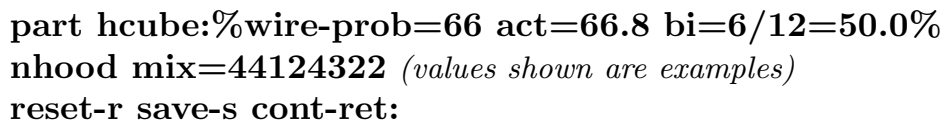

This indicates that the actual fraction of remaining wires and the number of and percentage of bi-directional links. Figure 12.1 gives 3d graph examples.

Enter $\mathbf{r}$ to reset the wiring probability, $\mathbf{s}$ to save the $k$-mix (see chapter 19), and return to accept. The resulting $k$-mix is given for the network as in section 9.11.
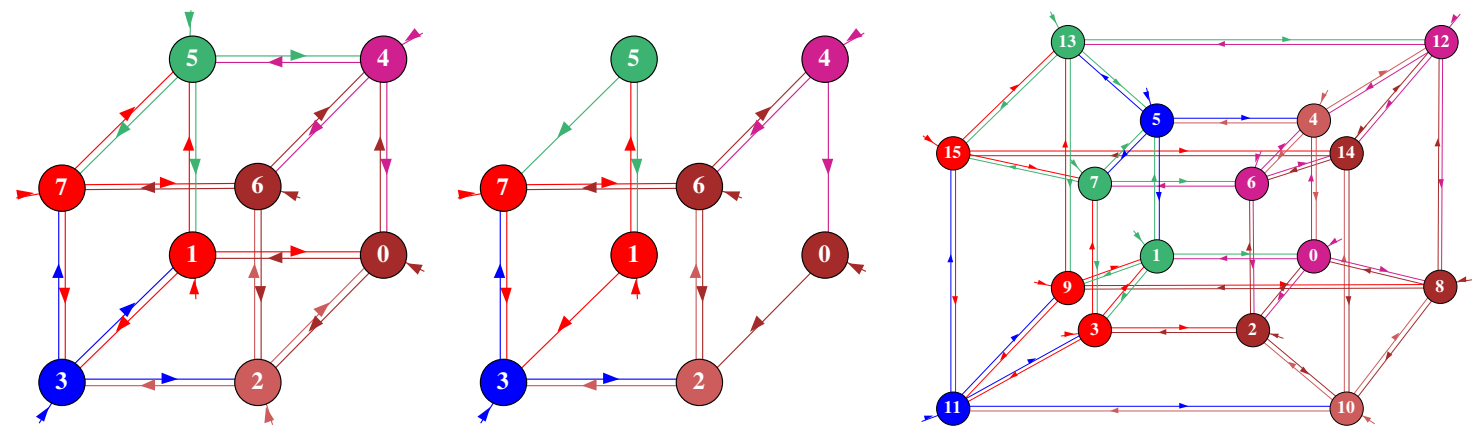

Figure 12.1: Hypercube wiring shown as a network-graph (section 20.4). Left: $n=8 \quad k=4$ hyprecube, which includes self-links. Center: a degraded version of the $n=8$ hypercube with some links missing. Right: $n=16 \quad k=5$ hyprecube. The $3 d-3$ network-graph option was used for the figures, and the graph was rearranged by dragging nodes (section 20.5). The figures should be viewed as if looking up from below. Output directed links are the same color as the source node. To regenerate these layouts automatically, enter file-f in section 20.4 and load the layout files hyp8.grh or hyp16.grh. 


\section{$12.31 \mathrm{~d}, 2 \mathrm{~d}$ and $3 \mathrm{~d}$ special wiring}

If return, $\mathbf{2}$ or $\mathbf{3}$ was entered in section 12.1, a 1d, $2 \mathrm{~d}$ or $3 \mathrm{~d}$ network will be selected to receive either local or random wiring, or "hand wiring" in a wiring matrix "spread sheet".

For local wiring, the wiring dimension would usually be set to match the network dimension, but its also possible to assign local $1 \mathrm{~d}$ wiring to a $2 \mathrm{~d}$ network, and local $1 \mathrm{~d}$ or $2 \mathrm{~d}$ wiring to a $3 \mathrm{~d}$ network.

Further network parameters, $i \times j(\times h$ for $3 \mathrm{~d}), k$ or the $k$-mix, and the wiring itself are set subsequently ${ }^{1}$. The following option is presented,

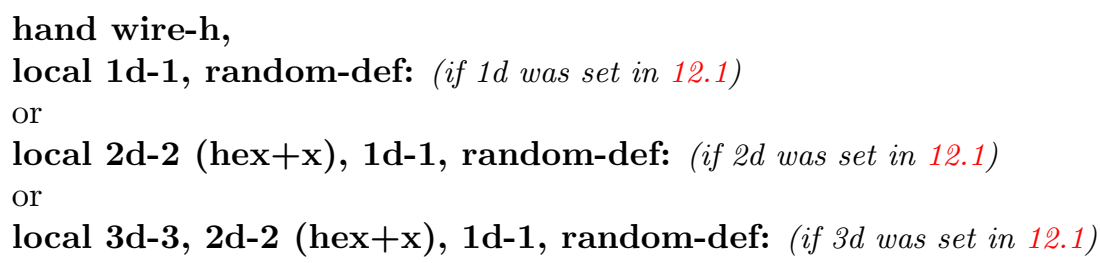

Once the network wiring has been set, the wiring of individual cells may be reviewed and altered in a "wiring matrix" or "wiring graphic" (chapter 17).

\subsection{Special wiring, local}

Enter $\mathbf{1}, \mathbf{2}, \mathbf{2} \mathbf{x}$ or $\mathbf{3}$ in section 12.3 for local $1 \mathrm{~d}, 2 \mathrm{~d}$ or $3 \mathrm{~d}(\mathrm{CA})$ wiring. Enter $\mathbf{2 x}$ to force a $2 \mathrm{~d}$ hexagonal layout if the default is square - this depends on $k$ (section 10.1.3).

Note that for local $1 \mathrm{~d}$ wiring in a $1 \mathrm{~d}$ network, the $n, k$ parameters set previously in chapters 8 and 9 remain valid. For local $2 \mathrm{~d}$ and $3 \mathrm{~d}$ wiring new prompts will be presented to reset these parameters.

\subsubsection{Local 1d treated as random}

For a $1 \mathrm{~d}$ network, if $\mathbf{1}$ for local $1 \mathrm{~d}$ wiring was selected in section 12.3, a subsequent option allows the local wiring to be treated as if it were "random" or nonlocal (always the case for $2 \mathrm{~d}$ and $3 \mathrm{~d}$ networks), allowing the local wiring to be altered in various ways, and also for wire moves to be allowed in "learning" (chapter 34). However, the "compression" of attractor basins (section 26.1) may be applied as long as the network retains local 1d wiring. The following prompt is presented,

\section{treat local 1d as random wiring-1, and compress-2:}

\subsubsection{Local $2 \mathrm{~d}$ and $3 \mathrm{~d}$}

For $2 \mathrm{~d}$ or $3 \mathrm{~d}$ networks, if $\mathbf{2}, \mathbf{2} \mathbf{x}$ or $\mathbf{3}$ for local $2 \mathrm{~d}$ or $3 \mathrm{~d}$ (CA) wiring was selected in section 12.3 , further prompts are presented to set the network size, $i \times j(\times h$ for $3 \mathrm{~d})$, as described in section 11.6.1,

\footnotetext{
${ }^{1}$ For $1 \mathrm{~d}, n, k$ or the $k$-mix, will remain as previously set in sections 8.2 and 9.1
} 
and neighborhood $k$, or the $k$-mix, as described in chapter 9 . Note that these settings override those previously set for $n$ and $k$ in chapters 8 and 9 .

Local $2 \mathrm{~d}$ and $3 \mathrm{~d}$ wiring is treated as if it were "random" or nonlocal, allowing it to be altered in any way. This is not the case by default for local 1d wiring (section 12.4.1 above).

\subsection{Special wiring, random}

If random wiring (the default) is selected in section 12.3 , (for $1 \mathrm{~d}, 2 \mathrm{~d}$ or $3 \mathrm{~d}$ ), and $n$, $k$, or the $k$-mix, have been set, a series of context dependent prompts are presented in sequence to allow a variety of biases to the random wiring (values shown are examples),

bias random wiring: confine to local zone $(\max =14 \mathrm{def}=14)$ : $\quad$ CA-c: release some wires from zone (def $0, \max 3)$ : (depending on $k$ )

suppress periodic boundary-s: $\quad$ i: $\quad$ j: $\quad$ h: $(i: j:$ for $2 d, i: j: h:$ for $3 d)$ exclude all selfwiring-2, selfwire center wire-1:

distinct wiring (no duplication)-n:

suppress links to layers (0-8), range1 low: high: range2 low: high: (3d only)

force direct link to a layer, enter (0-8): (depending on $h, 3 d$ only)

force random links to specific layers-y: (3d only)

same wiring everywhere-e:

DDLab will do its best to reconcile any contradictory settings. A small top-center window accept defaults-d is a reminder that at any time further prompts in this sequence can be skipped by entering $\mathbf{d}$. Enter $\mathbf{q}$ to backtrack. The options are explained below.

\subsubsection{Applying wiring biases to parts of the network}

At this early stage in DDLab's prompts the biases apply to the whole network, but it is important to note that the biases may be applied to just single cells or pre-defined parts of the network, as well as to the whole network, when these same options are accessed from the wiring graphic (section 17.3). In this case the biases only take effect when $\mathbf{r}$ (for random wiring) is selected in section 17.4 (see also 17.8.5).

To design particular wiring schemes, especially if the wiring needs to be tailored in specific ways between different parts of the network, its usually easier to set up a dummy wiring scheme at this stage, them revise it in chapter 17.

\subsubsection{Confining random wiring to a set zone}

The wiring may be confined within a periodic zone of a given diameter relative to each target cell. This diameter relates to the network geometry. Enter the local zone diameter at the prompt

\section{confine to local zone:}

in section 12.5. $1 \mathrm{~d}, 2 \mathrm{~d}$ and $3 \mathrm{~d}$ examples are shown in figures $12.2,12.3$ and 12.3 . which illustrate how network wiring is represented, explained more fully in chapter 17. 


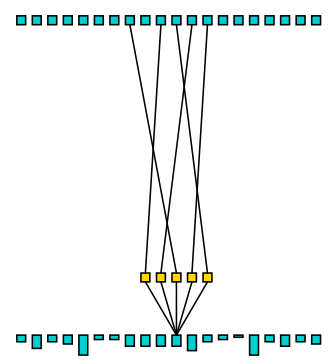

(a) random, zone $=7$

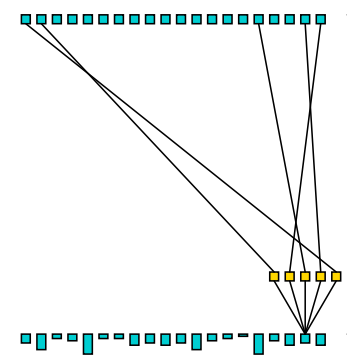

(b) random, zone $=7$

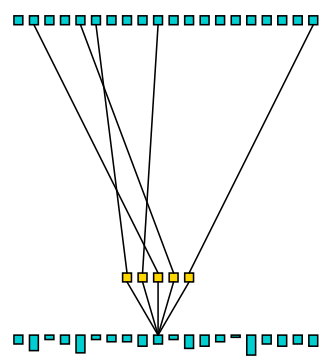

(c) random

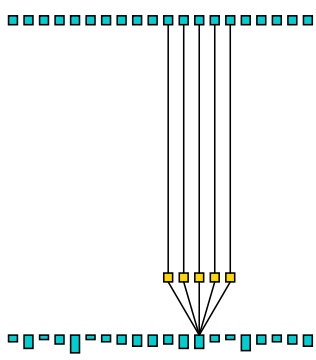

(d) CA wiring

Figure 12.2: Confining $1 \mathrm{~d} k=5$ random wiring within a local zone of a set diameter. $n=15$. (a) and (b) show random wiring within a 7 cell local zone, where (b) illustrates periodic boundary conditions. (c) shows fully random wiring, and (d) local, CA type, wiring for comparison. Wiring from the pseudoneighborhood is shown connected to to the previous time-step (see chapter 17)

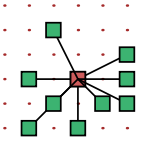

(b) a) random, zone $=5$

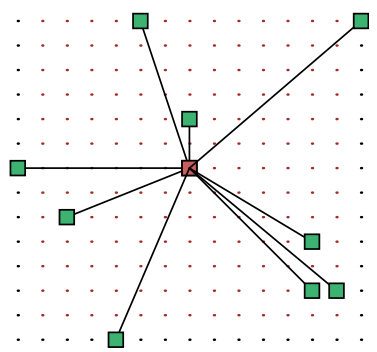

(b) fully random

Figure 12.3: Confining 2d random wiring within a 5 cell local zone. $n=15 \times 15$. (a) random wiring confined within a 5 cell zone. (c) fully random wiring.
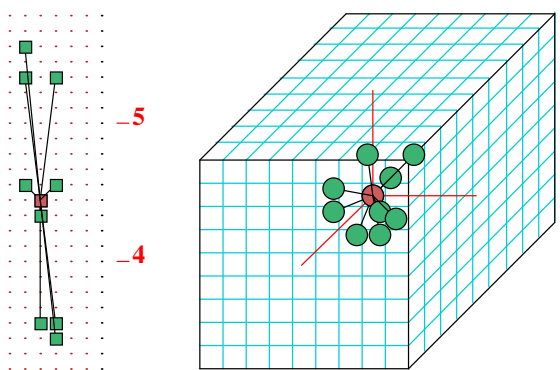

Figure 12.4: Confining $3 \mathrm{~d} \quad k=9$ random wiring within 3 cell local zone. $\quad n=9 \times 9 \times 9$. Left: $2 \mathrm{~d}$ showing successive layers. Right: $3 \mathrm{~d}$ isometric.

\subsubsection{Setting local wiring as random}

Enter $\mathbf{c}$ at the prompt CA-c: in section 12.5 to set local (CA) wiring as "random wiring". CA wiring in $1 \mathrm{~d}, 2 \mathrm{~d}$ or $3 \mathrm{~d}$ will be set depending on the native dimension of the network. This allows subsequent changes to be made to the CA wiring, for example, releasing some wires described below.

\subsubsection{Release wires from zone}

Some wire can be released from the local zone set in section 12.5.2 and from the CA wiring set in section 12.5.3. Enter the number of wires to be released at the prompt ...

release some wires from zone (def $0, \max 7)$ : (values shown are examples)

... in section 12.5. The number of wires specified will be chosen and reassigned at random to any position in the network, unless this is restricted by further biases. 


\subsubsection{Suppress periodic boundary conditions}

For a $1 \mathrm{~d}$ network, enter $\mathbf{s}$ at the prompt ...

suppress periodic boundary-s:

... in section 12.5 to suppress periodic boundary conditions.

For $2 \mathrm{~d}$ and $3 \mathrm{~d}$ networks further prompts appear,

$\mathbf{i}: \mathbf{j}$ : (and $\mathbf{h}$ : for $3 \mathrm{~d}$ ).

Periodic boundary conditions can be suppressed independently for each axis, $i, j$ and $h$. To do so, enter $\mathbf{s}$ in response to the prompts.

\subsubsection{Self-wiring}

Enter $\mathbf{2}$ or $\mathbf{1}$ at the prompt ...

exclude all selfwiring-2, selfwire center wire-1:

... in section 12.5 to exclude self-wiring, or to force target cells to wire to themselves. Self-wiring means that the cell will provide an input to its own pseudo-neighborhood, at the central position if available, or to a nearby position if not. chapter 10 describes the pseudo-neighborhoods.

\subsubsection{Distinct wiring}

Wiring may be made distinct to prevent two or more positions in the pseudo-neighborhood to be wired to the same cell. Enter $\mathbf{n}$ at the prompt...

\section{distinct wiring (no duplication)-n:}

... in section 12.5 for distinct wiring. DDLab will do its best to achieve distinct wiring, but this may conflict with a small "wiring zone" set in section 12.5.2 and with other wiring bias settings.

\subsubsection{Suppress links to 3d layers}

Links to horizontal layers in a 3d network can be suppressed. Two ranges of layers can be designated. Prompts are presented in sequence. Enter the levels to be suppressed starting with the lowest level. For example, the following entries would suppress links to layers $0,1,2,3$ and 6 ,

suppress links to layers (0-8), range1 low:0 high:3 range2 low:6 high:6

\subsubsection{Force a direct link to a $3 \mathrm{~d}$ layer}

Direct links can be "forced" to each cell in a designated 3d layer. A direct link means that cells at position $i, j$ link one wire to position $i, j$ in the designated layer. The wire originates from the pseudo-neighborhood index 0 (chapter 10).

For example, for a 9 layer $3 \mathrm{~d}$ network, enter the required layer index at the prompt ...

force direct link to a layer, enter (0-8):

... to which direct links will be forced. By default, if a layer is specified, all cells make a direct link, but this can be restricted to a specific cell, range of cells, or to a specific layer or range of layers from the wiring graphic (see chapter 17).

This option may be used to provide a constant input pattern to a $3 \mathrm{~d}$ network, for example to model inputs from a "retina". 


\subsubsection{Force random links to specific 3d layers}

Fractions of the available random links can be assigned to designated 3d layers. Previous biases to confine wiring to a set zone will be respected for the horizontal plane, but the vertical constraints will be overridden.

If $\mathbf{y}$ is entered at the prompt ...

\section{force random links to specific layers-y:}

$\ldots$ in section 12.5 , the following series of options are presented in a top-right window, for example in an 9 layer, $k=7,3 \mathrm{~d}$ network,

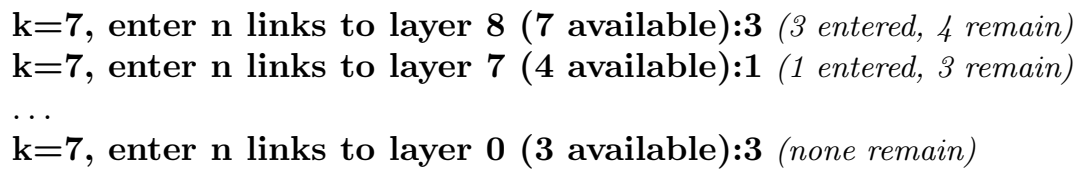

The prompts continue until layer 0, or until all links have been assigned and none remain. To miss a layer enter return or $\mathbf{0}$. If links remain at the end, they will be assigned at random, but still according to the biases specified in section 12.5 .

\subsubsection{Same random wiring everywhere}

The same random wiring "template" can be applied to every cell in the network to create a quasi-CA with periodic boundary conditions. To do this enter e at the prompt ...

\section{same wiring everywhere-e:}

... in section 12.5. Biases previously specified in section 12.5 and other options will be respected as much as possible. This can be restricted to just part of the network from the wiring graphic (chapter 17).

\subsection{Wiring by hand}

If $\mathbf{h}$ is selected in section 12.3, a blank wiring scheme is presented in the form of a matrix or "spread sheet", which may be filled in with the wiring positions for each cell's pseudo-neighborhood index. A completed wiring matrix can be amended in the same way (section 17.2.2).

Columns give the cell's pseudo-neighborhood index, $K(k-1, \ldots, 0)$, Rows give the cell network position, $N(n-1, \ldots, 0)$. The $0-0$ grid, or the 0 -minimum $n$ grid if the whole matrix does not fit within one window, is in the lower right hand corner. Each grid records the position in the network $x,(n-1, \ldots, 0)$, to which the $K^{\prime}$ th wire of the $N^{\prime}$ th cell is connected.

Note that positions $N$ and $x$ are $1 \mathrm{~d}$ indexes, even if the network is $2 \mathrm{~d}$ or $3 \mathrm{~d}$. Sections 10.2 .2 and 10.2.3 explain how to convert between $1 \mathrm{~d}$ and $2 \mathrm{~d}$ or $3 \mathrm{~d}$ coordinates.

Move around the matrix with the left/right/up/down arrow keys. Enter the new position at the flashing green cursor and complete the entry by moving to another grid with an arrow key or return. On a blank grid, or on zero, just return gives a random position. An entry outside the network limits will be ignored. Enter $\mathbf{q}$ to complete. Any undefined grids will be set to position 0 . While the wiring matrix is being set, the following reminder is displayed in a top-right window, 
4. 3. 2. 1 . 0 .

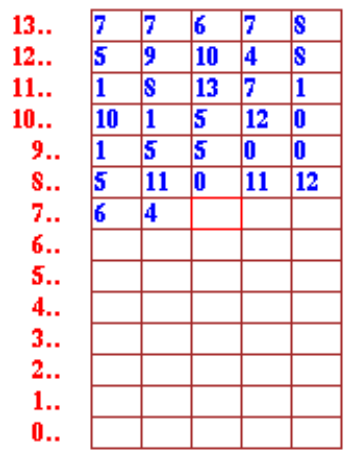

(a) $k=5, n=14$
10. 9. 8. 7. 6. 5. 4. 3. 2. 1. 0.

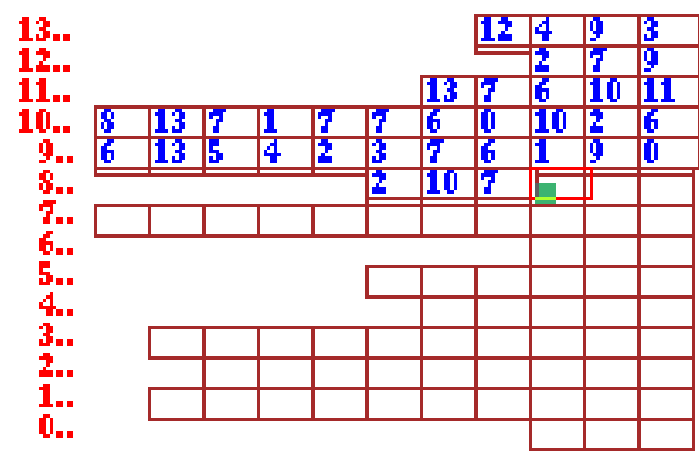

(b) mixed- $k=3$ to $11, n=14$

Figure 12.5: Setting wiring by hand on the blank wiring matrix, shown partly filled. Columns are indexed $k-1, \ldots, 0$, rows $n-1, \ldots, 0$. (a) shows an example matrix for a $k=5, n=14$ network. (b) shows an example matrix for a mixed- $k$ network, $k=3$ to $10, \mathrm{n}=14$.

\section{hand wire/revise: jump-j (values shown are examples) \\ enter wiring positions 0-144 (return on blank/0=random) \\ move-arrows more/complete-m layout-l font-f quit-q}

Enter $\mathbf{l}$ or $\mathbf{f}$ to alter the presentation of data and the amount visible in the matrix window. $\mathbf{f}$ is a 3-way toggle for the font size between normal, medium and small. $\mathbf{l}$ changes the matrix window width. The following top-right prompt is presented,

change window width $(\mathbf{9 2 2 - 2 3 1}$ now $=462)$ : (values shown are examples)

Enter the new width in pixels within the limits indicated.

Large networks may require several successive windows to display the matrix. Enter $\mathbf{m}$ to see the next window. Moving beyond the top or bottom row in the current window, with the arrow keys or return, also brings up the preceding or next window. Enter $\mathbf{j}$ to jump to a new cell index, the following prompt is presented,

jump to index (1599-0): (for a $2 d$ network, 40×40)

Enter the new cell index, which will become the first entry in the top row.

Enter $\mathbf{q}$ to accept the wiring (or return on the very last (bottom-right) entry) to conclude matrix entries, and go to the prompt in section 12.7 below. Any blank entries are assigned to position zero.

The wiring scheme can be reviewed and revised in the same wiring matrix format, or as a wiring graphic in $1 \mathrm{~d}, 2 \mathrm{~d}$ or $3 \mathrm{~d}$, as described in chapter 17.

\subsection{Reviewing wiring}

After the special wiring has been set as described in this chapter, various options allow the wiring to be reset, reviewed and amended from a wiring graphic or wiring matrix. A prompt similar to the following is presented, 
(for a $1 d$ network)

$1 d$ network $(n=150)$, review/revise, wiring only - rules not set graph-g, matrix: revise-m view-M prx-Mp

graphic: 1d:timesteps-1 circle-c 2d-2:

(for a $2 d$ network)

2d network (40x40), review/revise, wiring only - rules not set graph-g, matrix: revise-m view-M prxt-Mp

graphic: 1d:timesteps-1 circle-c 2d-2:

(for a $3 d$ network)

3d network (15x15x15), review/revise, wiring only - rules not set graph-g, matrix: revise-m view-M prxt-Mp

graphic: 1d:timesteps-1 circle-c $2 \mathrm{~d}+3 \mathrm{~d}-3$ :

These options are described in chapter 17. They provide very flexible methods to review and amend the wiring, and also the rules once set. It may be preferable to set up a suitable dummy network initially, then tailor it with these options.

If just quick wiring was set in chapter 11, these options do not appear at this point in the program, but they appear in any case after the rules have been set (chapters 13-16). 


\section{Chapter 13}

\section{Rule types}

Rules in DDLab can be set according to a number of different types (possibly overlapping) full rule-table (rcode), k-totalistic ${ }^{1}$ (kcode), t-totalistic (tcode), outer totalistic, and reactiondiffusion, so before a rule is actually selected in chapter 16, options are presented to select the type, and also whether a rulemix is required. These options are described below, and the chapter goes on to explain the rule types in detail. Further prompts in chapters 14 and 16 describe the rulemix options and the wide variety of rule sub-types within the chosen type.

\subsection{Selecting the rule type}

After the network wiring has been set or defaults accepted in chapters 11 and 12, the next prompt in the main sequence selects the type of rule. The prompt differs between rules based on full rule-tables (SEED-mode or FIELD-mode) which may be rcode, kcode or tcode, and just totalistic rules (TFO-mode) based on only the shorter kcode and tcode rule-table.

\subsubsection{Select full rule-tables, rcode}

In SEED-mode or FIELD-modes, all rule types rely on full rule-tables — rcode. Totalistic rules (kcode and tcode) and reaction-diffusion rules will be automatically transformed into rcode. The following prompt is presented,

totalistic: tcode-t kcode-k (def-rcode):

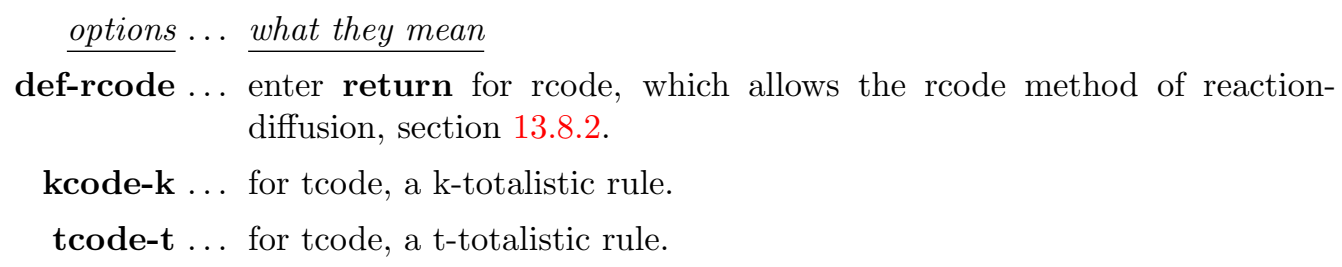

\footnotetext{
${ }^{1}$ Suggested by Antonio Lafusa[3].
} 


\subsubsection{Select kcode or tcode in TFO-mode}

In TFO-mode for kcode or tcode rule-tables, the following prompt is presented,

totalistic only: outertot-o/+o, tcode-t, (def-kcode):

(outertot-o/to does not apply for a k-mix, set in sections 9.1 or 11.6.2)

options.... what they mean

def-kcode ... enter return for kcode, a k-totalistic rule.

tcode-t ... for tcode, a t-totalistic rule.

outertot-o ... for outer-kcode (section 14.2 which allows the outer-kcode method of reaction-diffusion (sections 13.8.1, 14.2.1).

to ... for an outer tcode (section 14.2).

Note that for $v=2$, tcode and kcode lookup-tables and the resulting dynamics are identical.

\subsection{Rule types and combinations}

The rest of this chapter explains the various types of rule, or systems of logic, that act on the neighborhood, or pseudo-neighborhood, to determine a cell's output, and the conventions in DDLab for constructing and ordering rule-tables. The rules described first are Boolean functions as in older versions of DDLab, where the value-range $v=2$ consists of just $[0,1]$. Then the rules are generalized for multi-value logic, where the value-range (the number of colors) may be anything from $v=2$ to 8 .

In DDLab there are several types of rules, which are expressed as rule-tables (lookup-tables) as follows:

- full rule-tables: (not in TFO-mode) referred to as "rcode" listing the outputs of all possible neighborhood patterns (not in TFO-mode)

- k-totalistic rules: referred to as "kcode" — rule-tables listing the outputs for all possible combinations of totals (or frequencies) of the values in the neighborhood. kcode can be selected in TFO-mode, or in SEED-mode or FIELD-mode where the kcode is also expressed as rcode.

- t-totalistic rules: referred to as "tcode" - rule-tables listing the outputs of all possible totals, where the values in the neighborhood are simply added. tcode can be selected in TFO-mode, or in SEED-mode or FIELD-mode where the tcode is also expressed as rcode. For $v=2$, kcode and tcode are identical.

In addition DDLab offers combinations of rules as follows:

- rulemix: where each cell in the network can have a different rule, or where a restricted set of rules is distributed throughout the network, described in detail in chapter 14. A network with mixed- $k$ must have a rulemix.

- outer-totalistic rules: (TFO-mode only, and not for mixed-k) a number, $v$, totalistic rules (tcode or kcode) can be employed to create outer-totalistic rules, where a different rule applies according to the value of the center cell (section 13.7). For $v=2$ the game-of-Life can be set in this way, though it can also be set as rcode in section 16.10 . 
- reaction-diffusion rules: or excitable-media[9]. Cells may be either resting, excited, or refractory, and change according to thresholds and values (section 13.8). The resulting dynamics produces waves, spirals and related patterns that can resemble the BZ reaction and other types of excitable media. A reaction-diffusion rule can be set from rcode (section 13.8.2) or from an outer-kcode (section 14.2.1). Note that outer-kcode allows a greater range of $[v, k]$ than a rcode, but the latter allows a rulemix that can include more than one reaction-diffusion rule in the network.

\subsubsection{Rule-table size implications}

The lengths of rule-tables, $S$, depend on $v$ and $k$, where rcode $>$ kcode $>$ tcode,

$$
\begin{array}{ll}
\operatorname{rcode} \ldots & S=v^{k} \\
\operatorname{kcode} \ldots & S=(v+k-1) ! /(k ! \times(v-1) !) \\
\text { tcode } \ldots & S=k(v-1)+1
\end{array}
$$

This has implications on the $k_{\text {Lim }}$ (section 7.2). In SEED-mode or FIELD-mode, kcodes and tcodes will be implemented as the equivalent full rcode (the longest rule-table), whereas in TFO-mode the shorter kcode and tcode allow a greater $k_{\text {Lim }}$ for a given value-range $v$ (see also section 6.1).

Various subcategories of rcode and kcode can also be selected in chapter 16 and at a later stages in DDLab, including majority, Altemberg, chain, and isotropic. The same rule may apply to all cells in the network as for CA, or the network may have a mix of different rules.

\subsection{Binary full rule-table - rcode}

A full rule-table (rcode) for binary systems $(v=2)$ has $2^{k}$ entries. Table 13.1 shows how the size of the rule-table increases exponentially with $k$.

\begin{tabular}{c|c|c|c|c|c|c|c|c|c|c|c|c|c}
$k$ & 1 & 2 & 3 & 4 & 5 & 6 & 7 & 8 & 9 & 10 & 11 & 12 & 13 \\
\hline size of lookup-table $2^{k}$ & 2 & 4 & 8 & 16 & 32 & 64 & 128 & 256 & 512 & 1024 & 2048 & 4096 & 8192
\end{tabular}

Table 13.1: The size of rule-tables $k=1$ to 13

The convention in DDLab is to list neighborhood configurations from left to right in reverse Boolean value order ${ }^{2}$ following Wolfram[22], so that the all 1's neighborhood is on the left, as set out below. Each neighborhood is assigned an output $[0,1]$, and the resulting bitstring defines one of the $2^{2^{k}}$ possible rcodes. The most significant bit in the bitstring corresponds to the all 1 's neighborhood.

The rules may also be expressed in decimal (if applicable - section 16.6) or in hexadecimal. $k \geq 5$ rules are usually referred to by their hex rule numbers, $k \leq 3$ rules are usually referred to by their more familiar decimal rule numbers, for example, the $k=3$ binary rcode 60 below,

$$
\begin{array}{ccccccccc}
7 & 6 & 5 & 4 & 3 & 2 & 1 & 0 & - \text { Boolean value = rcode index } \\
111 & 110 & 101 & 100 & 011 & 010 & 001 & 000 & -\mathrm{k}=3 \text { neighborhood }
\end{array}
$$

${ }^{2}$ The rcode rule-table order can be inverted - section 18.3 
In DDLab the neighborhood configurations are rotated and displayed vertically for compactness making a so called "neighborhood matrix", showing also the $k$-index, as in these example for $k=3$, and $k=5$ below. A bit-string can be assigned in the corresponding order to make the rcode.

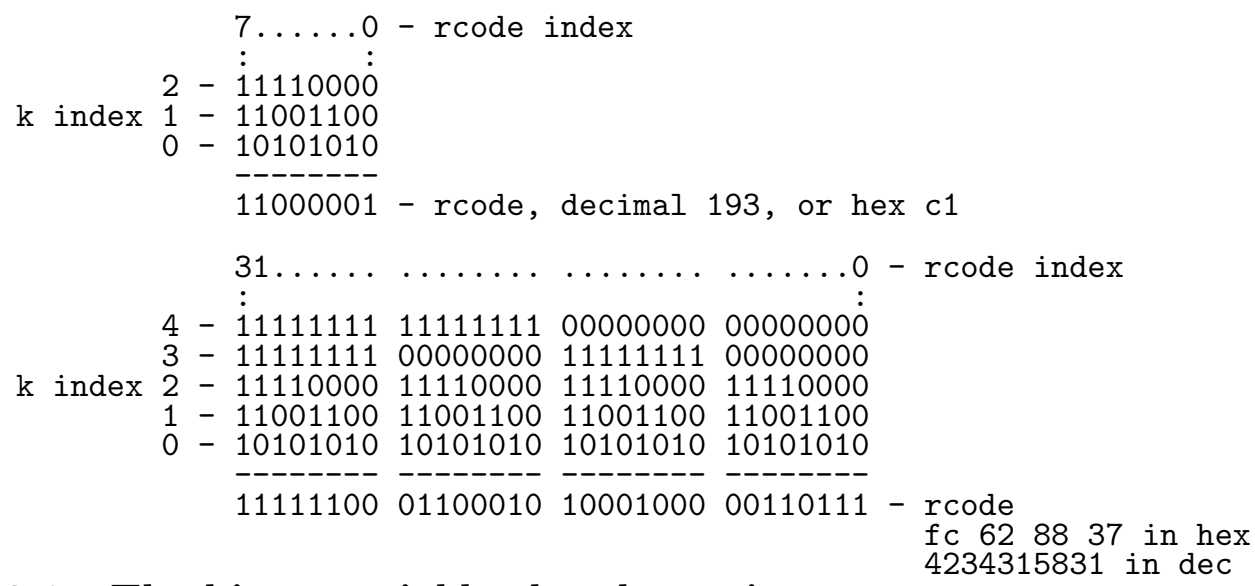

\subsubsection{The binary neighborhood matrix}

In the main sequence of prompts, a graphic of the binary neighborhood matrix is displayed (but not for mixed $k$ or in TFO-mode). Figure 13.1 gives examples for $k=1$ to 9 . The matrix can be rescaled and different parts shown (section 14.11).
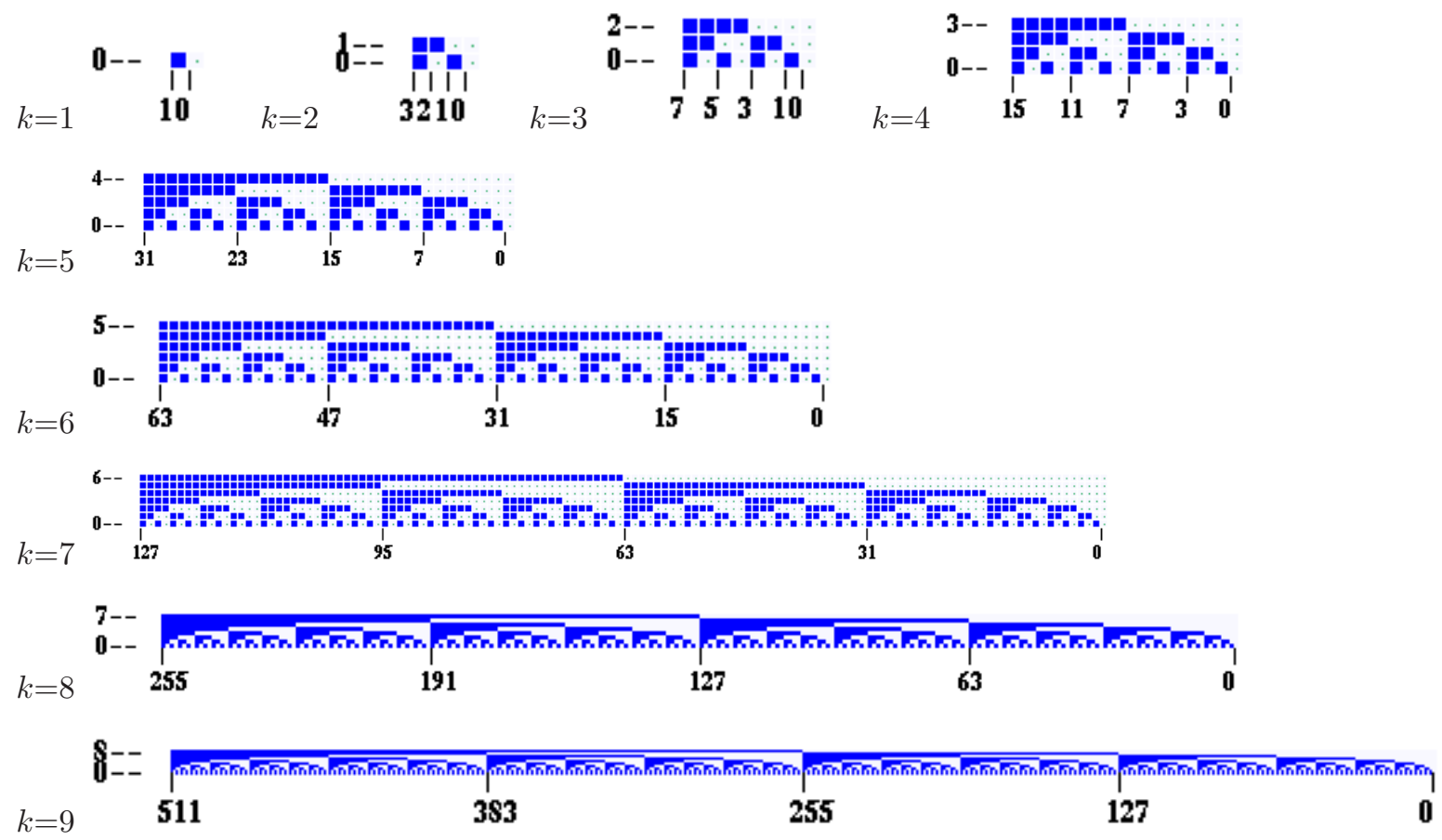

Figure 13.1: The binary neighborhood matrix, $k=1$ to 9 , as displayed in DDLab. 


\subsection{Binary totalistic rules}

For binary $(v=2)$ totalistic rules, a cell's value depends only on the sum of $1 \mathrm{~s}$ in its neighborhood — tcode and kcode are identical (for $v \geq 3$ they are different - section 13.6).

A tcode rule-table (tcode-table) has $k+1$ bits representing the possible totals, set out in reverse value order, from $k$ to 0 , where each total is assigned an output $[0,1]$. The resulting bit string defines one of the $2^{k+1}$ possible tcodes. If DDLab is not in TFO-mode, it automatically transforms the tcode into rcode (section 13.3). Here is an example for $k=5$,

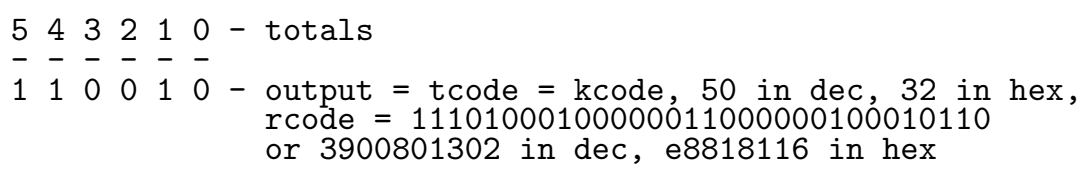

Totalistic codes are also useful for setting threshold functions, for example, the $k 5$ tcode-table 111000 is the majority rule.

\subsection{Multi-value full rule-table — rcode}

A full rule-table, rcode, for multi-value systems $>2\left(v=2\right.$ to 8 in DDLab), has $v^{k}$ entries. Table 13.1 shows how the size of the rule-table increases exponentially with $v$ and $k$, and also the limits of $k$ relative to $v$ allowed in DDLab - these limits are more generous for totalistic rules in TFO-mode (section 13.6).

\begin{tabular}{|c|c|c|c|c|c|c|c|c|c|}
\hline$k$ & 1 & 2 & 3 & 4 & 5 & 6 & 7 & 8 & 9 \\
\hline$v=3$ & 3 & 9 & 27 & 81 & 243 & 729 & 2187 & \multirow[t]{6}{*}{6561} & 19683 \\
\hline$v=4$ & 4 & 16 & 64 & 256 & 1024 & 4096 & \multirow[t]{5}{*}{16384} & & \\
\hline$v=5$ & 5 & 25 & 125 & 625 & 3125 & \multirow[t]{4}{*}{15629} & & & \\
\hline$v=6$ & 6 & 26 & 216 & 1296 & 7776 & & & & \\
\hline$v=7$ & 7 & 49 & 343 & 2401 & \multirow[t]{2}{*}{16807} & & & & \\
\hline$v=8$ & 8 & 64 & 512 & 4096 & & & & & \\
\hline
\end{tabular}

Table 13.2: The size of full rule-tables for $v=3$ to 8 , against $k$, showing the limits of $k$ in DDLab.

The convention in DDLab is to list neighborhood configurations from left to right in reverse $n$-ary value order ${ }^{3}$ so the all-max neighborhood is on the left. Each neighborhood is assigned an output $[0,1, \ldots, v-1]$, and the resulting $n$-string defines one of the $v^{v^{k}}$ possible rcodes. The rcodes may also be expressed in decimal (if applicable - section 16.6) or in hexadecimal. An example for a $v 3 k 3$ rcode is shown below, where the central row shows all possible neighborhoods, the top row their trinary values (the rcode index), and the bottom row the rcode itself,

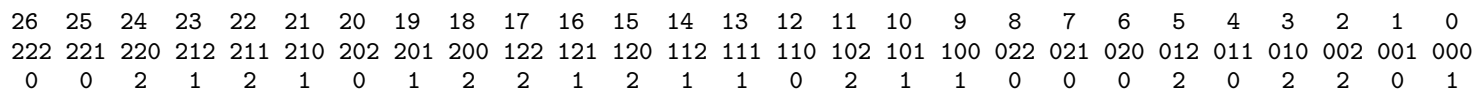

${ }^{3}$ The multi-value rcode rule-table can be inverted - section 18.3 
DDLab treats the $n$-string as a bitstring, assigning the minimum number of bits for each value; 1 bit if $v=2,2$ bits if $v=3$ or 4 , and 3 bits if $v=5$ to 8 . The resulting bitstring can be expressed in hexadecimal, in this case the rule is 026469949408a1.

In DDLab the neighborhood configurations are rotated and displayed vertically for compactness making a so called "neighborhood matrix", showing also the $k$-index. The $n$-ary string can be assigned in the corresponding order to make the rcode.

\subsubsection{The multi-value neighborhood matrix}

In the main sequence of prompts, a graphic of the multi-value neighborhood matrix is displayed (but not for mixed $k$ or in TFO-mode). figure 13.2 gives examples. The matrix can be rescaled and different parts shown (section 14.11).
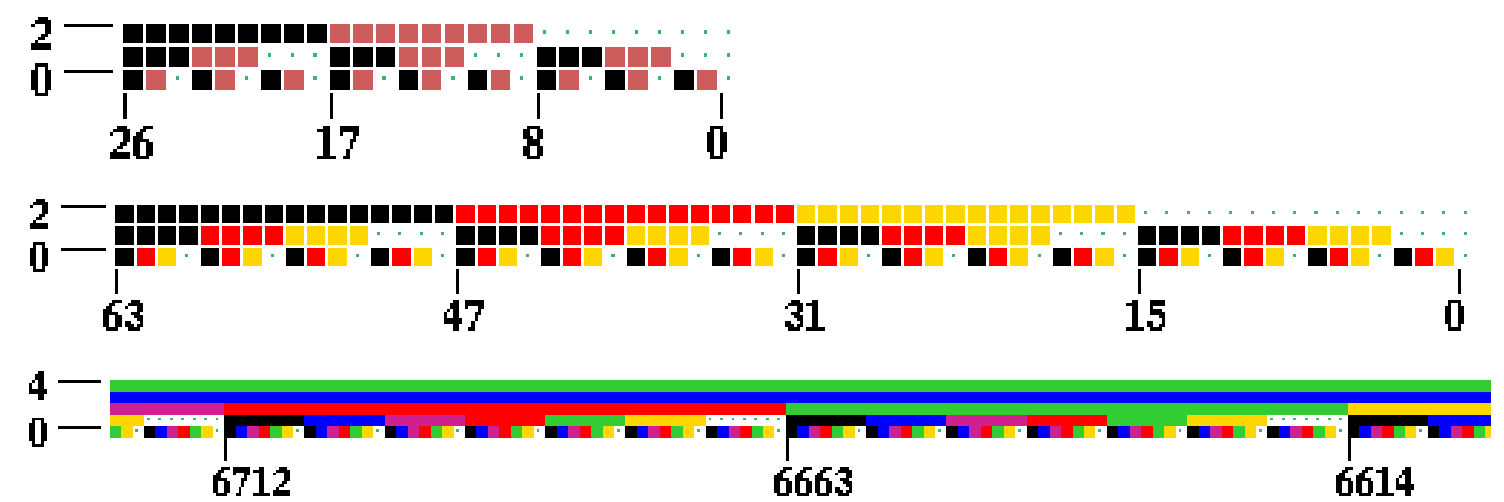

Figure 13.2: Examples of the multi-value neighborhood matrix, colored according to the value color key (section 7.1). Top: $v 3 k 3$, Center: $v 4 k 3, \underline{\text { Bottom: }} v 5 k 7$ central part only.

\subsection{Multi-value totalistic rules, tcode and kcode}

For multi-value systems, if $v \geq 3$, totalistic rules separate into two types - k-totalistic rules (kcode), and t-totalistic rules (tcode). For $v=2$, kcode and tcode are identical. 


\subsection{1 k-totalistic rules - kcode}

The kcode rule-table (kcode-table) is a list of the outputs for all possible combinations of valuefrequencies in the neighborhood. Each combinations is represented by a string of length $v$ (shown below vertically from $v$ - 1 down, for $v=3$,) giving the frequencies of the values $v$ - 1 to 0 , which must add up to $k$, so the last row of frequencies is redundant and could be omitted.

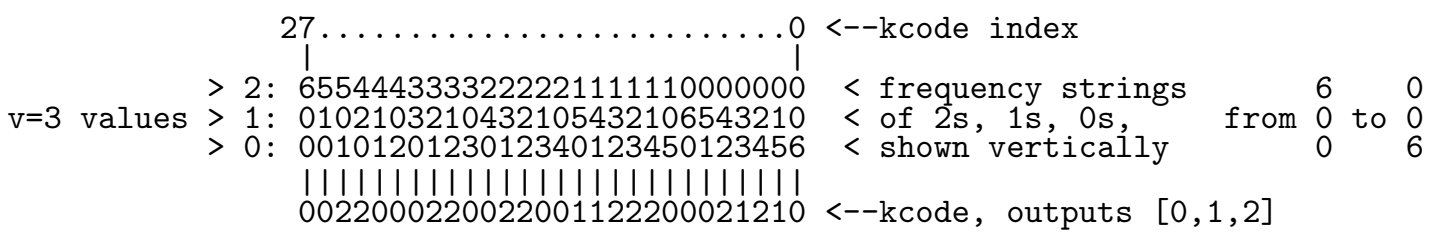

The ordering of the pattern strings themselves depend on their $v$-ary value, with the higher value on the left. This can be effectively inverted to allow kcode expressed in the opposite convention to run as intended (section 31.2.9).

In the example above for the $v 3 k 6$ "Beehive rule" [39] (also shown as a matrix in section 13.6.2) the kcode with outputs $[0,1,2]$ are listed in reverse order of the kcode index. The kcode may also be expressed in decimal (if applicable - section 16.6) or in hexadecimal. If DDLab is not in TFO-mode, it automatically transforms the kcode into the full rule-table, rcode (section 13.5).

Kcode rules are isotropic because they depend only on the frequency of each value (or color) in the neighborhood - the positions of values are irrelevant, so that symmetric space-time pattern must conserve their symmetry.

The size of a kcode-tables, $S=(v+k-1) ! /(k ! \times(v-1) !)$, is much shorter than a rcodetable. The values of $S$ for different $v$ and $k_{\text {Lim }}$ allowed in DDLab are shown in table 13.3 below. Table 13.4 shows all allowable values of $S$ for increasing with $v$ and $k$.

\begin{tabular}{r|r|r}
$v$ & $k_{\text {Lim }}$ & kcode size \\
\hline 2 & 25 & 26 \\
\hline 3 & 25 & 351 \\
\hline 4 & 25 & 3276 \\
\hline 5 & 25 & 23751 \\
\hline 6 & 17 & 26334 \\
\hline 7 & 13 & 27132 \\
\hline 8 & 11 & 31824
\end{tabular}

Table 13.3: kcode lookup-table size for $v$ and $k_{\text {Lim }}$ available in DDLab.

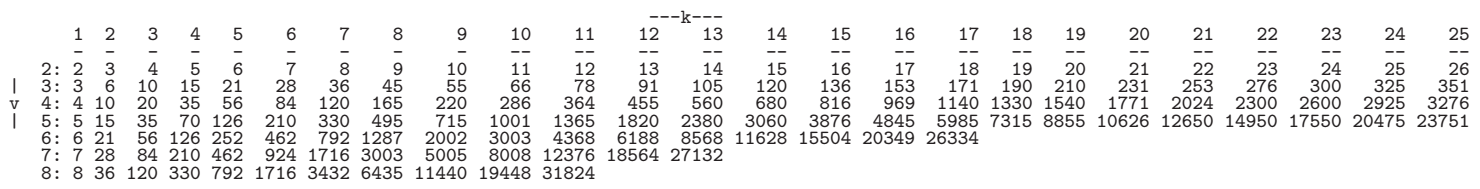

Table 13.4: The size of kcode-tables for $v=3$ to 8 , against $k$, showing the limits of $k$ in DDLab. 


\subsection{2 $\quad$ kcode $v=3$ matrix}

For ternary $(v=3)$ systems, kcode can usefully be show as a 2 d matrix[40], where the output state of each neighborhood is given by the row-index $i$ (the number of neighbors with value $=2$ ) and column-index $j$ (the number of neighbors with value $=1$ ). The number of neighbors with value $=0$ is given by $k-(i+j)$ so is not required. Two examples are given in table 13.5 for $k=6$ and $k=7$.

\begin{tabular}{cc|ccccccc} 
& & & & & & $j$ & & \\
& & 0 & 1 & 2 & 3 & 4 & 5 & 6 \\
\hline \multirow{4}{*}{$i$} & 0 & 0 & 1 & 2 & 1 & 2 & 0 & 0 \\
& 1 & 0 & 2 & 2 & 2 & 1 & 1 & \\
& 2 & 0 & 0 & 2 & 2 & 0 & & \\
& 3 & 0 & 2 & 2 & 0 & & & \\
& 4 & 0 & 0 & 2 & & & & \\
& 5 & 2 & 0 & & & & & \\
& 5 & 0 & & & & & &
\end{tabular}

\begin{tabular}{cc|cccccccc} 
& & & & \multicolumn{1}{c}{$j$} & $j$ & & & \\
& & 0 & 1 & 2 & 3 & 4 & 5 & 6 & 7 \\
\hline \multirow{4}{*}{$i$} & 0 & 0 & 1 & 2 & 1 & 2 & 2 & 2 & 2 \\
& 1 & 0 & 2 & 2 & 1 & 2 & 2 & 2 & \\
& 2 & 0 & 0 & 2 & 1 & 2 & 2 & & \\
& 3 & 0 & 2 & 2 & 1 & 2 & & & \\
& 4 & 0 & 0 & 2 & 1 & & & & \\
& 5 & 0 & 0 & 2 & & & & & \\
& 5 & 0 & 0 & & & & & &
\end{tabular}

Table 13.5: Matrix representations of $v=3$ k-totalistic rules. Left: $v 3 k 6$ Beehive rule[39] and Right: v3k7 Spiral rule[40], both on a 2d network with hexagonal tiling[39, 40].

\subsection{3 t-totalistic rules - tcode}

Tcode depends only to the sum of all the values in the neighborhood. The rules are not necessarily isotropic (as in kcode, section 13.6.1) because different sets of values can make up the same total.

The size of a tcode-tables, $S=k(v-1)+1$, is much shorter than a kcode (section 13.6.1), but the kcode $k_{\text {Lim }}$ is nonetheless set at the same level as for kcode. The values of $S$ for different $v$ and $k_{\text {Lim }}$ are shown in table 13.6 below,

\begin{tabular}{r|r|r}
$v$ & $k_{\text {Lim }}$ & kcode size \\
\hline 2 & 25 & 26 \\
\hline 3 & 25 & 51 \\
\hline 4 & 25 & 76 \\
\hline 5 & 25 & 101 \\
\hline 6 & 17 & 86 \\
\hline 7 & 13 & 29 \\
\hline 8 & 11 & 78
\end{tabular}

Table 13.6: tcode lookup-table size for $v$ and $k_{\text {Lim }}$ available in DDLab.

To construct tcode, each total, set out in reverse value order, $S-1$ to 0 , is assigned an output $[0,1, \ldots, v-1]$, which is the tcode value-string. Here is an example for $v 7 k 5$.

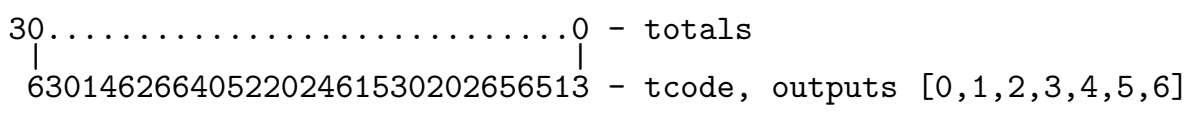

The tcode may also be expressed in decimal (if applicable - section 16.6) or in hexadecimal, as in section 13.5. If DDLab is not in TFO-mode, it automatically transforms the tcode into the full rule-table, rcode (section 13.3). 


\subsection{Outer-totalistic rules \\ TFO-mode only, and not for mixed-k}

Outer-totalistic rules apply in TFO-mode. The rules (kcode or tcode) depend on the value of the center cell, so a number of rules, $v$, need to be specified.

If $\mathbf{o}$ for kcode or to for tcode is entered at the prompt in section 13.1.2, the rules will subsequently be assigned in the same way as for a rulemix (chapter 14), but only to the first $v$ cells in the network (network index 0 to $v-1$ ) representing the values of the target cell, which will determine the rule to be applied. So this method just utilizes the rulemix functionality, it is not a proper rulemix.

The method works with any $k$, but makes most sense if the central cell is not wired to itself is empty in the neighborhood (section 10).

For the classic binary game-of-Life (section 14.2.2), two rules are required, and can be set "by hand" as shown below,

for $v=0$ : 000001000 - birth: exactly 3 live neighbors

for $v=1$ : 000001100 - survival: 2 or 3 live neighbors

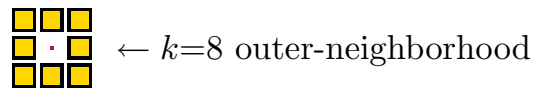

However, there are automatic methods for setting the game-of-Life and other Life-like rules, in both outer-kcode (section 14.2.2) and as a full rule-table, rcode (section 16.10).

\subsection{Reaction-Diffusion dynamics}

Reaction-Diffusion or excitable media dynamics[9], can be generated with a type of CA or DDN with 3 cell qualities: resting, excited, and refractory (or substrate, activator, and inhibitor). There is usually one resting type, one excited type, and one or more refractory types. In DDLab these correspond to the values 0,1 , and values $\geq 2$, which cycle between each other ${ }^{4}$.

A resting cell becomes excited if the number of excited cells in its neighborhood falls within the threshold interval (t). An excited cell type changes to refractory. A refractory cell type changes to the next refractory type (if there are more than one) and the final refractory type changes back to resting, completing the following cycle,

$$
\operatorname{resting}(0): \text { if within }(\mathrm{t}) \rightarrow \operatorname{excited}(1) \rightarrow \operatorname{refractory}(2 \rightarrow 3 \rightarrow \cdots \rightarrow v-1) \rightarrow \operatorname{resting}(0)
$$

In DDLab, reaction-diffusion can be set as outer-kcode in TFO-mode, which allows greater $[v, k]$, or as a full rule-table - rcode.

The resulting dynamics in $2 \mathrm{~d}$ can produce waves, spirals and related patterns that can resemble the $\mathrm{BZ}$ reaction and other types of excitable media. As well as the threshold interval, the dynamics is sensitive to the initial state and its density of non-resting types (non-zero values) - usually low for best results. This density ( $\lambda$ parameter) can be set in section 16.3.1. Interesting results can be achieved not only for $2 \mathrm{~d} \mathrm{CA}$, but also for $2 \mathrm{~d}$ DDN were the random wiring is confined to a tight local zone (section 12.5.2, figure 12.3a) as illustrated in figure 13.3.

\footnotetext{
${ }^{4}$ For binary networks $(v=2)$ the refractory type would be missing, so this would not be reaction-diffusion in its proper sense, though the option is still present in DDLab.
} 

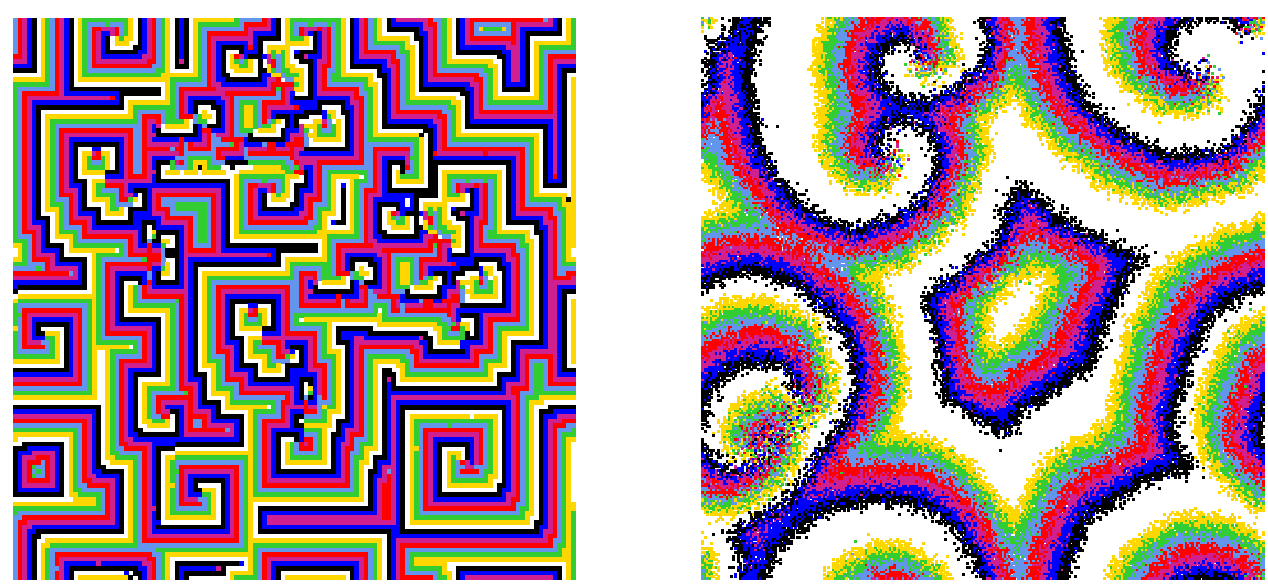

Figure 13.3: Reaction-Diffusion dynamics. Left: $v 8 k 8$, threshold interval is 1 to $6,122 \times 122$ square lattice. Right: $v 8 k 11$, threshold interval is 2 to $7,255 \times 255$ square lattice, random connections within a 24 diameter local zone.

\subsubsection{Reaction-Diffusion from outer-kcode}

Select outer-kcode (section 13.7) by entering o in section 13.1.2. Subsequent top-right prompts allow the selection of reaction-diffusion dynamics (section 14.2.1).

The required rules are automatically assigned to the first $v$ cells of the network (network index, 0 to $v-1$ ) in a quasi-outer-kcode ${ }^{5}$. The way this works is as follows, where resting $=0$, excited $=1$, refractory $\geq 2$ : $k$ code $(0)$ holds the threshold interval to change the cell from resting to excited $(0 \rightarrow 1)$. kcodes $(1,2,3, . ., \mathrm{v}-1)$ store the colors, so whatever the neighborhood, the cell cycles to the next color, then back to 0 , as described in section 13.8 .

\subsubsection{Reaction-Diffusion from a full rule-table — rcode}

Select rcode which implements any logic, by entering return in section 13.1.1. Then enter $R D-\mathbf{R}$ at the next main sequence prompt (section 16.1.1) for a reaction-diffusion rcode. After the threshold is set in section 13.8.3 below, the rule will be set automatically to emulate reaction-diffusion dynamics. Note that a reaction-diffusion rcode can be part of a rulemix.

\subsubsection{Selecting the threshold interval}

Once reaction-diffusion dynamics has been selected (in sections 13.8.1 or 13.8.2) a subsequent topright prompt sets the threshold interval. This example shows the threshold interval set between 1 and 6 in a $k=8$ network,

thresholds: lower (0-8):1 upper (1-8):6 (if $v=8$ )

This would make a resting cell change into an excited cell if there were between 1 and 6 excited cells in its outer neighborhood, otherwise it would remain at rest.

\footnotetext{
${ }^{5}$ As noted in section 13.7 , outer-totalistic rules are themselves a quasi-rulemix, using the rulemix functionality.
} 


\section{Chapter 14}

\section{Rulemix options}

All cells in a network may have the same rule as in classical CA, or cells may have different rules. This is referred to as a rulemix (or rule scheme), which may be a rcode-mix, kcode-mix or tcode-mix. If the network has heterogeneous neighborhoods sizes (a $k$-mix, chapter 9 and section 11.6.2) then there must be a rulemix (by default) - in this case section 14.1 below does not apply. For just one rule enter return at the prompt in section 14.1 and continue with chapter 16 .

This chapter describes the rulemix options, and also outer-totalistic rules in TFO-mode which share the same methods. Outer-kcode provides alternative methods for reaction-diffusion and Life-like dynamics ${ }^{1}$.

A rulemix can be assigned from ...

- the whole of rule-space (rule-spaces for a $k$-mix)

- a limited subset of rules to restrict the range of rules in the rulemix. The number of rules in the subset can be anywhere from 1 to $n$ (network size), but with an upper limit of 200 rules. This subset option does not apply to a $k$-mix, or to outer-totalistc rules in TFO-mode.

Rules in a rulemix or a limited subset can be set automatically at random with a pre-defined density-bias of non-zero outputs ( $\lambda$-parameter), and rules can be confined to various sub-categories. Alternatively, rules can be set individually "by hand" allowing almost any combination, and the rulemix can be loaded from a previously saved file.

This chapter also describes altering the presentation of the neighborhood matrix (section 14.11), and listing Post-functions, which are included in the initial rulemix prompt.

\subsection{Single rule or rulemix, and other options} not for a k-mix (unless all k's equal, section 14.6.7), or outer-totalistc rules in TFO-mode

After selecting a rule type in section 13.1, the next top-right prompt selects a single rule or a rulemix, and includes some other options. The top line of the prompt shows a reminder of the current mode, FIELD, SEED or TFO (section 6.1) and an option to preset the density-bias $(\lambda$-parameter). The prompt also differs somewhat according to the rule type,

\footnotetext{
${ }^{1}$ Reaction-diffusion rules or Life-like rules can also be set as a full rule-table (rcode), which allows inclusion in a rulemix.
} 
For rcode, kcode and tcode based on full rule-tables selected in section 13.1.1

FIELD-mode, bias-density (def 50.00\%)-s: (or SEED-mode, def $50.00 \%$ for $v=2$ )

RULES: single rcode (def), load rulemix-1, list Post-P, nhood-matrix-a

mix: no limit-n, or set limit up to 200: (or single tcode/kcode above)

In TFO-mode, based on short rule-tables selected in section 13.1.2

TFO-mode, bias-density (def 83.33\%)-s: (or def $83.33 \%$ - for $v=6$ )

KCODE: single kcode (def), load kcodemix-l (or TCODE: single tcode..)

mix: no limit-n, or set limit up to 200:

Enter return to skip all these rulemix options (and this chapter), and continue for a single rcode, kcode or tcode, in chapter 16. The rulemix options are summarized below.

\subsubsection{Summary of rulemix and other options}

A summary of the options in section 14.1 is as follows,

options.... what they mean

bias-density-s ... to change the density-bias - the fraction of non-zero values in the rule-table (section 14.1.2.

load rulemix-l ... to load rule scheme (rule-mix) - rcode-mix .r.s, kcode-mix .r_v, or tcode-mix .r_t. (section 35.2, and chapter 19).

list Post-P ... (not in TFO-mode) to list Post functions (section 14.12).

nhood-matrix-a ... (not in TFO-mode) to amend the presentation of the neighborhood matrix (section 14.11).

no limit-n ... to set an rcode-mix, kcode-mix or tcode-mix, taken from the whole of rule-space (section 14.4.1).

up to $200 \ldots$ enter a number, 1 to 200 (or 1 to $n$ if $n<200$ ) to set the size of a limited subset of rules (rcodes, kcodes or tcodes) from which the mix will be assigned to the network (section 14.4.2). The maximum size of the limited subset of rules (set limit) is 200, unless the network size $n<200$ - then the maximum set limit is $n$.

\subsubsection{Density-bias ( $\lambda$-parameter)}

Enter $\mathbf{s}$ in section 14.1 to change the density-bias - the fraction of non-zero values in the rule-table (the $\lambda$-parameter) but expressed as a percentage. This is applied probabilistically, so each rule (rcode, kcode or tcode) in a rulemix will not necessarily have exactly the given density-bias. The following top-right prompt is presented,

density-bias: enter \% (def 66.67\%): (for $v=3$, the default is an equal probability of each value)

Enter the new percentage density-bias - the prompt in section 14.1 will reappear with the revised setting. The density-bias will be applied when setting the following at random,

- single rules (section 16.3.1).

- a rulemix or subset of rules (sections 14.5, 14.4.2).

- a rulemix or subset of rules "by hand" (section 14.6).

Note that for single rules set at random, the density-bias can be reset, either exactly or probabilistically at later stages in section 16.3.1. 


\subsection{Outer-totalistic kcode or tcode TFO-mode only}

If outer-totalistic (kcode or tcode) was selected in TFO-mode in section 13.1.2, a quasi-rulemix with $v$ rules is the automatic choice, and a top-right reminder similar to this is presented,

TFO-mode,

OuterKcode: number of kcodes to be set $=$ value-range $=3$

cont-ret: (or OuterTcode/tcodes, values shown are examples)

The next prompt will be similar to setting a rule-subset (KcodeSet or TcodeSet) in section 14.4.2, but where the subset $\operatorname{size}=v$. The prompts are as follows,

for kcode (and $v=8$ )

OnterKcode(8): select by hand-h, RD-R Life-L maj-m/u Alt-A rnd-(def):

for tcode (and $v=4$ )

OuterTcode(4): select by hand-h, maj-m rnd-(def):

The rules will be allocated to the first $v$ cells in the network (index 0 to $v-1$ ) representing the values of the target cell, which determines the rule. The rules are set either at random with various biases, or by hand, as for a normal rulemix, but although the method utilizes the rulemix functionality, this is not a proper rulemix (section 13.7).

\subsubsection{Setting reaction-diffusion by outer-kcode}

for reaction-diffusion from rcode see section 13.8.2

Enter $\mathbf{R}$ in the OuterKcode... prompt in section 14.2 above to initiate a reaction-diffusion rule by outer-kcode (sections 13.8, 13.8.1 and figure 13.3). Then set the threshold interval as described in section 13.8.3. An alternative method for reaction-diffusion is from the full rule-table - rcode, which also allows reaction-diffusion rules to form part of a rulemix, (section 13.8.2).

\subsubsection{The game-of-Life and other Life-like rules by outer-kcode} for Life from a full rule-table (rcode) see section 16.10

If $k=8$ on a $2 \mathrm{~d}$ square lattice, giving this neighborhood 㗊 - John Conway's game-of-Life[6] can be set as an outer-kcode. Any other Life-like ${ }^{2}$ rule from the "Life family" can also be set, as well as Life-like rules with neighborhoods other than the Moore neighborhood, or with $v>2$. This can also be done from a full rule-table (rcode) in section 16.10, but the outer-kcode method provides a greater range of $v$ and $k$.

To set the classic game-of-life (but with possibly $v$ colors), enter $\mathbf{L}$ in section 14.2, the following top-right prompt (for $k=8$ ) appears,

\footnotetext{
${ }^{2}$ http://en.wikipedia.org/wiki/Life-like_cellular_automaton
} 


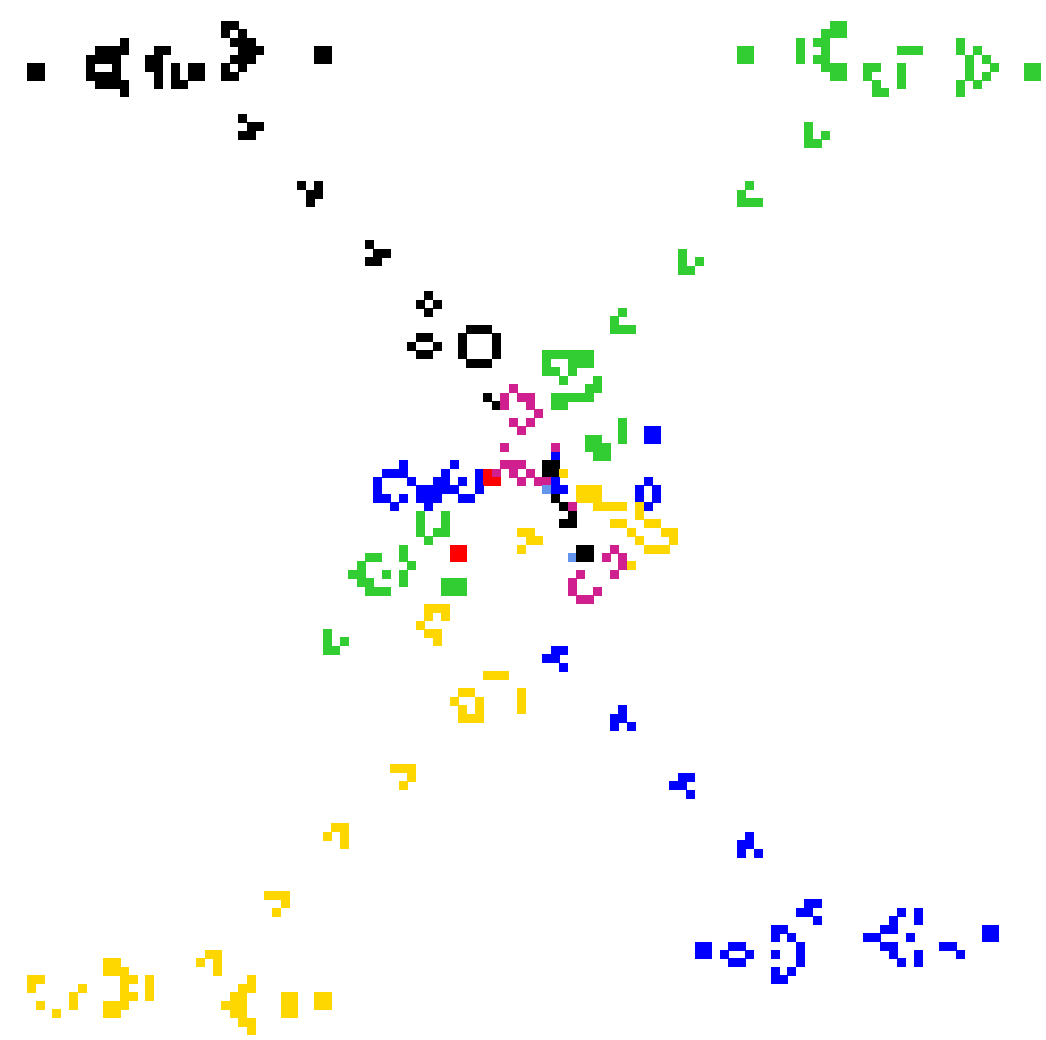

Figure 14.1: The game-of-Life (23/3) applied to a $v=8 \mathrm{CA}$. The algorithm in DDLab generates 8 outer kcodes giving the similar dynamics to classical binary Life, but including 7 colors + background. In this example 4 glider guns are located in each corner on a $122 \times 122$ lattice, shooting different colored gliders towards the center. The seed file is Lguns_v8.eed.

Life $\mathrm{k}=8$ (def: survival 2,3 , birth 3 ,) accept-ret amend-a:

Enter return to accept the default. If $\mathbf{a}$ is entered to amend, the following further prompts are presented,

accept-ret, or enter number + ret, $\max$ entries $=9$, $\max$ value $=8$ :

enter survival $(\mathbf{d e f}=\mathbf{2 . 3}$,$) : followed by ...$

enter birth $(\operatorname{def}=3$,$) :$

Enter the new values for survival, followed by the new values for birth. After each number enter return for the next number. There may be up to $k$ entries - their order, or repeats, are not significant. return without a number concludes the entries. q reverts to the first Life prompt, but with the defaults possible altered.

The Life option is available for $v \geq 2$, and any $k$ as well as the $k=8$ neighborhood, for $1 \mathrm{~d}$ and $3 \mathrm{~d}$ as well as $2 \mathrm{~d}$, and for a rulemix by hand. For $v>2$, for a (23/3) Life setting, the algorithm in DDLab generates an equivalent goop table giving the same dynamics as the classical binary Life, but including $v$-1 colors plus the background, as in figure 14.1. 
As well a using the prompts above, the game-of-Life as an outer totalistic rule, can also be set "by hand". For example, select $v=2, k=8$, and a 2 d square lattice. Then in section 13.1.2 select outer-totalistic (kcode or tcode). Two outer totalistic rules need to be set (because $v=2$ ), the first for a central cell of 0 , the second for a central cell of 1 . Select "by hand", then select $\mathbf{b}$ for bits in the single rule prompt, similar to prompt 14.6.2 or 16.1.2. Set each rule (kcode or tcode) in turn as below,

for $v=0$ : 000001000 - birth: exactly 3 live neighbours

for $v=1$ : 000001100 - survival: 2 or 3 live neighbours

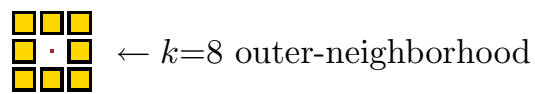

\subsection{Setting the neighborhood, $k=0$}

If a $k$-mix was selected in section 9.1 or 11.6 .2 , DDLab identifies any self-wired $k=1$ cells, and gives an option to set the rule (or kcode or tcode) at these cells to make them effectively $k=0$ (section 9.2). The following prompt is presented,

single self wirings $=4$, set rule for $\mathrm{k}=0$ equiv -0 :

(shows the number of $k=1$ self-wired rules found)

Enter $\mathbf{0}$ accept. Note that if the effective wiring to a cell is $k=0$, it will appear disconnected in the 1d wiring graphic (section 17.5), as illustrated in figure 14.2.

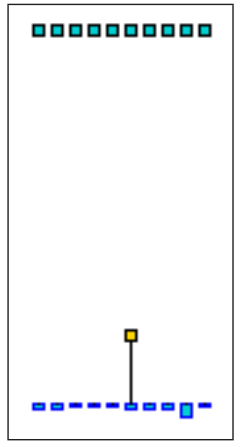

Figure 14.2: Effective neighborhood $k=0$, as shown in the $1 d$ wiring graphic (section 17.5) with no connections to the previous timestep.

\subsection{Methods for setting the rulemix or rule-subset}

A rulemix, rule-subset (or quasi-rulemix) will be set if,

- $\mathbf{n}$ for no limit was selected in section 14.1 - this sets a rulemix

- a number, 1 to $n$ (upper limit 200) for as rule-subset was selected in section 14.1 - this sets the subset size.

- the network has mixed- $k$ (set in chapter 9 or section 11.6.2) — this forces a rulemix.

- outer-totalistic was selected in section 13.1.2, followed by the outer-totalistic reminder in section 14.2 - this sets a quasi-rulemix of $v$ rules. 
There are two strategies for creating a simple rulemix.

- The direct strategy, the only possibility for a $k$-mix, otherwise enter $\mathbf{n}$ for no limit in section 14.1 - allows any rule from rule-space(s) to be included, so if randomly assigned, a repeat of the same rule is unlikely for a large rule-space.

- The indirect strategy (enter a number: 1 to $n$, upper limit 200). This first creates a limited subset of rules from which rules will be assigned at random to the network, so the final rulemix can be restricted to just a few rules - or even just one rule (section 14.4.3).

The options below, which differ only slightly between the direct and indirect strategies, allow rules to be set automatically at random (section 14.5) with various constraints depending on the type of rule, or individually (by hand) by the various methods described in chapter 16 .

\subsubsection{Setting a rulemix directly}

If $\mathbf{n}$ for no limit was selected in section 14.1, or for a $k$-mix, the rulemix will be assigned directly to the network with the following prompts, which depend on the type of rule.

For full rule-tables and rcode, the prompt is,

RcodeMix: select by hand-h, maj-m/u Alt-A chain-c iso-i Post-P canalyzing-C/+C, rnd-(def):

In TFO-mode, or if kcode or tcode was selected for full rule-tables (rcode) in section 13.1.1, the prompt is,

KcodeMix: select by hand-h maj-m/u Alt-A rnd-(def): (for kcode)

or

TcodeMix: select by hand-h rnd-(def): (for tcode)

\subsubsection{Setting a rulemix indirectly - specify a rule-subset} does not apply to a k-mix

A number ( 1 to 200), or ( 1 to $n$ if $n<200$ ), selected in section 14.1, specifies the size of the subset of rules. Rules from this subset will be automatically assigned at random to the network. The prompts are similar to section 14.4.1 but without maj and canalyzing, and Alt for tcode..

For rcode the prompt is,

RuleSet(22): select by hand-h (for a subset size 22)

Alt-A chain-c iso-i Post-P rnd-(def):

In TFO-mode, or if kcode or tcode was selected for full rule-tables (rcode) in section 13.1.1, the prompts are,

KcodeSet(5): select by hand-h, Alt-A rnd-(def): (for kcode, for subset size 5) or

TcodeSet(33): select by hand-h rnd-(def): (for tcode, for subset size 33) 


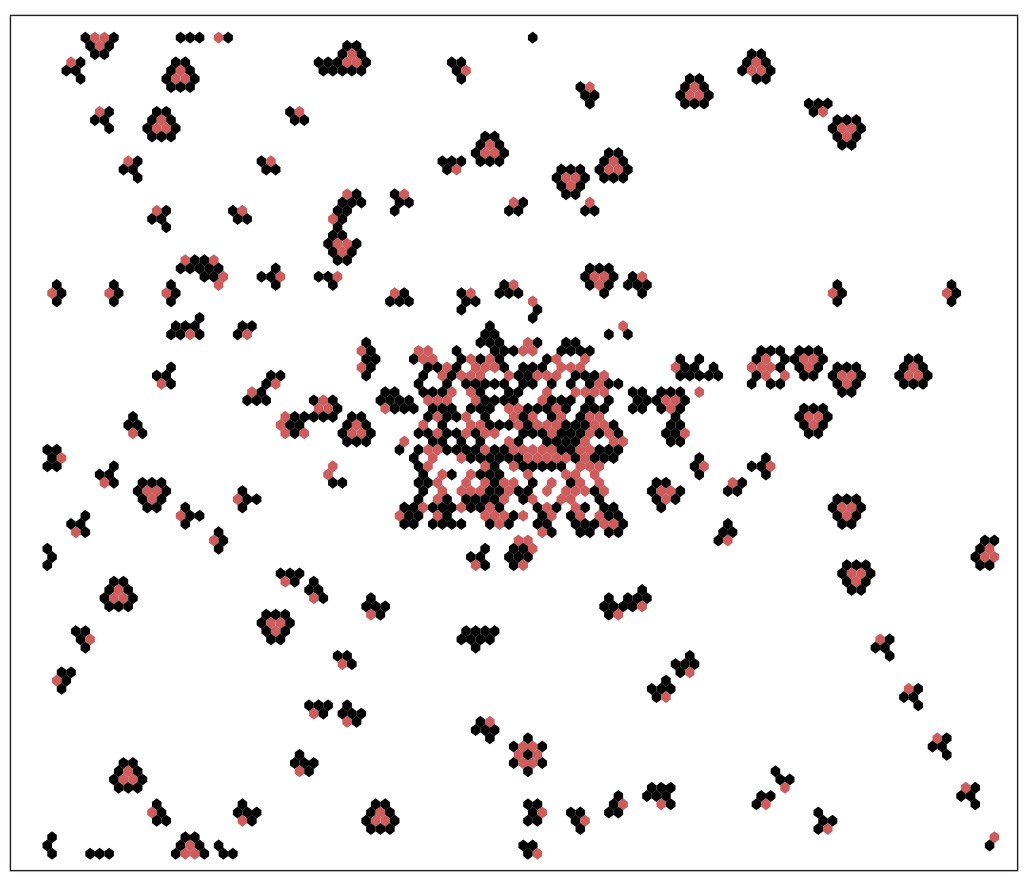

Figure 14.3: A $2 \mathrm{~d}$ CA set up with the spiral rule[40] $100 \times 100$ perturbed by a central $20 \times 20$ homogeneous block of chain rules that have been inserted. To do this first set up as a rulemix consisting of just one rule, then create a rule block (sections 17.6 .5 and 17.8.10).

\subsubsection{A rulemix with just one rule}

To set up any network (CA, RBN or DDN) with a homogeneous rule that allows later additions and revisions as if it had a rulemix (as in figure 14.3), select a rulemix with a limited subset of rules consisting of just one rule, i.e. enter 1 at the prompt in section 14.1.1, and set the rule by hand (section 14.6). This fools DDLab into assuming the network has a "rulemix" when in fact all the rules are the same.

\subsection{Rulemix - random}

The following options in section 14.4 assign rules either entirely at random, or at random from some subcategory of rules. The program continues with options to review network architecture described in chapter 17.

$$
\text { options } . . \text { what they mean }
$$

rnd-(def) ... enter return to assign rules entirely at random from the whole of rule-space, for rcode, kcode or tcode. The density-bias ( $\lambda$-parameter) can be preset in section 14.1.2

$\mathbf{m a j}-\mathbf{m} / \mathbf{u} \ldots$ enter $\mathbf{m}$ to assign majority (voting) rules (section 14.7). Enter $\mathbf{u}$ (not for tcode) to assign majority rules but with the outputs from uniform neighborhoods flipped or shifted (section 14.8). 
Alt-A ... (not for tcode) to assign rcode or kcodes at random from the subset of "Altenberg" rules, (section 16.9)

the options below for rcode only

chain-c ... to assign rcode at random from the subset of "chain" rules, (section 16.11)

iso-i ... to assign isotropic rcode at random, where rotated and reflected neighborhoods (in 1d, $2 \mathrm{~d}$ and $3 \mathrm{~d}$ ) have the same output.

Post-P ... to assign rcode at random from the subset of "Post functions" (section 14.12). A prompt to restrict the Post value will first be presented as in section 14.12.2.

canalyzing- $\mathbf{C} /+\mathbf{C} \ldots$ enter $\mathbf{C}$ to see and amend rcode canalyzing inputs, or enter another subcategory followed by $\mathbf{C}$ : $\mathbf{m C}, \mathbf{u C}, \mathbf{A C}, \mathbf{c C}, \mathbf{i C}$, or PC. Canalyzing inputs are described in chapter 15.

\subsection{Rulemix by hand}

A succession of rules can be set individually, by hand, to create a rulemix, rule-subset, or outertotalistic rules. Each rule is set just like a single rule in chapter 16 - all the relevant options apply depending on the rule type.

A top-right prompt gives the current rule index (starting with 0), and allows jumping to any other valid index, and changing the current default method of rule selection. The selected rule and other details (and options) for the rule will appear as for a single rule in chapter 16. At any stage, the remaining rules can be set at random with valid biases.

\subsubsection{By hand reminder}

If $\mathbf{h}$ is entered in 14.2 or 14.4 , a top-right context dependent reminder appears, for example,

for rcode

by hand: subset of 22 rcodes (or kcode, tcode)

for a totalistic rules

by hand: kcodemix of $\mathbf{1 5 0}$ kcodes (or rcodes, tcodes)

for outer-totalistic rules

by hand: outertot of $\mathbf{6}$ kcodes (or tcodes)

for a kmix - lower and upper values of $k$ are indicated

by hand: rcodemix of 150 rcodes (kmix 3-6) (or kcodemix, tcodemix)

\subsubsection{By hand single rule prompt}

At the same time as the "by hand" reminder above (section 14.6.1), the main sequence single rule prompt appears (heavily context dependent - see section 16.1) to accept or revise the default method of rule selection (and other options), for example,

Select v2k5 rcode $(S=32)$ : empty-e fill-f maj-m Alt-A life-L chain-c rnd-r ReDiff-R iso-i bits-b hex-h dec-d rep-p load-l values-v prtx-x (def-r): 
Enter return to accept the default rule selection method, or change it with any another valid entry, and set the rule for the current rule index. The rule selection methods are described in detail in chapter 16 and summarized in section 16.2.

\subsubsection{By hand options}

The rule at each cell (or subset, or outer totalistic) index is set in turn, starting from index 0 . A top-right prompt keeps track of the rule index, the current value of $k$, and the current selection method. For a $k$-mix, if a rule has already been set for the current $k$, the prompt start as follows,

index $=7 \mathrm{k}(3-9)$ (v3k4 rcodemix), same as last-s, all remaining-a: (for example)

Options s and a to copy previous rules are described in (section 14.6.6). Without a previous $k$ rule there are no copy options - the $k$-mix prompt is as follows, for example,

index $=7 \mathrm{k}(3-9)$ ( $\mathbf{v} \mathbf{k} 4$ rcodemix) (or kcodemix, tcodemix)

hand $=\mathbf{r}$, change-(any valid entry) or index-q: (if $\mathbf{r}$ is the current selection method)

For a homogeneous $k$ network, the prompt is as follows, for example,

$$
\text { index }=12 \text { (v3k4 rcodemix) (rnd all-a if hand }=r, m, A, c, i \text { only) }
$$

hand $=\mathbf{A}$, change-(any valid entry) or index-q, rnd all-a:

Opinion a is descibed in (section 14.6.5). Enter return to set the rule with the current selection method, possibly with intermediate steps. The methods are similar to setting a single rule (chapter 16). Once set, each rule (in bits/values and hex) may appear below the single rule prompt (section 14.6.2) and in the bottom "rule window" (section 16.19). Enter q to change the current selection method or index (section 14.6.4).

\subsubsection{Change the selection method or rule index}

To change the selection method, enter any valid option in sections 14.6.2 or 14.6.3.

If $\mathbf{q}$ is entered in prompt 14.6.3 the following top-right prompt appears, for example,

change: index(16)-i, start again-a, selection-s, cont-ret:

Enter a to restart, from index 0 .

If $\mathbf{i}$ is entered above, the following top-right prompt appears, for example,

this index $=16$, enter new index $0-16$ :

Enter a number ( $\leq$ the current index) to change the rule index.

If selection-s is entered above, the following top-right prompt appears, where the lower line gives a list of valid selection methods. The options included in the list depend on the rule type and $k$, and correspond to the main sequence single rule options (sections 14.6.2 and 16.1), for example,

for rcode

index $=16$ (v5k4), change selection method (now $\mathrm{r}$ ), abort-q as main sequence choices - $\mathrm{e} / \mathrm{f} / \mathrm{x} / \mathrm{m} / \mathrm{u} / \mathrm{A} / \mathrm{L} / \mathrm{c} / \mathrm{r} / \mathrm{i} / \mathrm{R} / \mathrm{b} / \mathrm{h} / \mathrm{d} / \mathrm{p} / \mathrm{l} / \mathrm{v}$ : 
the second line for kcode

as main sequence choices - e/f/x/m/u/A/r/b/h/d/p/l/v:

the second line for tcode

as main sequence choices - $\mathrm{e} / \mathrm{f} / \mathrm{x} / \mathrm{m} / \mathrm{r} / \mathrm{b} / \mathrm{h} / \mathrm{d} / \mathrm{p} / \mathrm{l} / \mathrm{v}$ :

Enter any one of the listed choices to change the selection method which becomes the new default. The selection method methods are described in detail in chapter 16 and summarized in section 16.2.

\subsubsection{Complete the rulemix automatically}

For a rulemix with homogeneous $k$, if the current selection method is setting rules at random, or at random with constraints (section 16.2), the following option is included in the prompt in section 14.6.3,

.. rnd all-a: (if hand $=r, m, A, c, i$, only)

Enter a to abandon setting rules by hand, and complete setting rules at random automatically for the rest of the network according to the current method and constraints, for example according to the density-bias $(\lambda$-parameter) if hand $=\mathbf{r}$ (section 16.3.1).

\subsubsection{Copy rules automatically for a $k$-mix}

While setting rules by hand for a $k$-mix, a rule set previously with a given $k$ can be copied. The following option is included in the prompt in section 14.6.3,

... same as last-s, all remaining-a: (if a rule for the current $k$ was set previously)

Enter $\mathbf{s}$ to copy to the current cell index, or a to also copy to all the remaining cells in the network with the same $k$ - these cell indexes will be skipped in the remaining rule assignment procedure. Enter return to ignore these options and continue.

\subsubsection{Mixed $k$ where all $k$ 's (and rules) are the same}

To create a $k$-mix network where all $k$ 's are the same ( $k=3$ in this example), at the prompt for setting the $k$-mix in section $9.3 \ldots$

set k-mix: load-l hand-h specify-s rnd-(def):

... select $\mathbf{s}$ to set the percentage of $k$ 's required (section 9.7.1), then enter return until $k=3$, then set $100 \%$,

enter $\% \mathrm{k}=3$ (100.0\% left) back-b:100

To make it possible in the future to increase $k$ for some cells, $k$-max can be set at some higher value (section 9.10) for example,

.. set greater max k-max (def 3 , limit 9):7 
After further options, DDLab will recognize if all $k$ 's are the same, and present the single rule or rulemix prompt (section 14.1) — for a single homogeneous rule enter $\mathbf{1}$.

\subsection{Rulemix - majority}

If $\mathbf{m}$ is selected, in section 14.4.1, a "majority" (voting) rule will be set at each cell. For $v=2$ the majority rule outputs 1 for a majority of $1 \mathrm{~s}$ in the neighborhood, 0 for a majority of $0 \mathrm{~s}$. In the case of even $k$, and a tie between 1 s and 0 s, the output is set according to the value of the central neighborhood index, as defined in chapter 10.

For $v>2$ the majority rule outputs the majority value in the neighborhood. In the case of a tie one of the most frequent values is chosen at random.

\subsection{Rulemix - majority with shifted uniform outputs not for tcode - see also section 16.8 for a single rule}

If $\mathbf{u}$ is selected in section 14.4.1, "majority" (voting) rules are set as in section 14.7 above, but the uniform (unanimous) neighborhoods have their outputs shifted by -1 , except for 0 which becomes $v$-1. For random wiring, this can result in some interesting bi-stable, tri-stable, $v$-stable, dynamics, as in figure 14.4. For $v=2$ this is the same as flipping the "end bits" (all-0s and all-1s), as before in binary DDLab (figure 16.8).

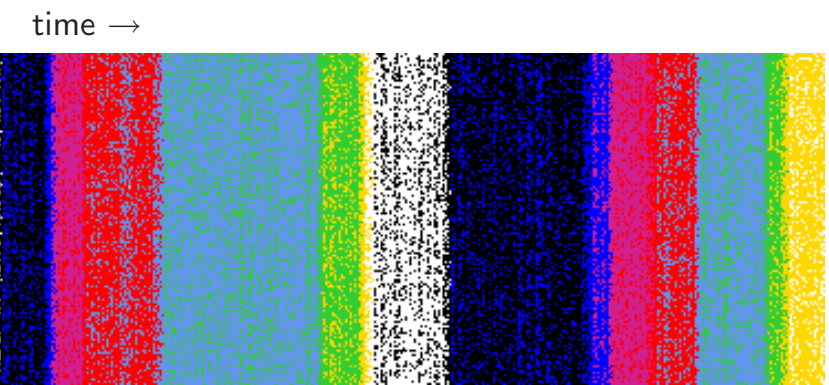

Figure 14.4: Shifted majority kcode-mix with random wiring, $v 8 k 11$, where uniform neighborhoods have their outputs shifted to a different color. The $1 \mathrm{~d}$ space-time pattern $(n=150)$ shows zones of color density that remain stable for an unpredictable number of time-steps before flipping to a different quasi-stable regime. The image shown is rotated by $90^{\circ}$ so the time flows from left to right.

\subsection{Rulemix for large networks, or large $k$}

Assigning the rulemix to large networks, especially for large $k$, may take some time. For $n \geq 5000$ or $(n \geq 500$ and $k \geq 10)$, the assignment is monitored by a progress bar near the top-right hand corner of the screen. When the assignment is complete, the program will continue with options to review network architecture described in chapter 17. 


\title{
14.10 The all 0s output
}

A rulemix set at random may be biased to have the "all 0s" neighborhood output in every rule reset to 0 - the default for rcode, or any rule may be allowed - the default for kcode and tcode. The following prompt is presented,

\author{
for rcode-mix \\ rcodemix: allow any rule-a, or force all 0s- $>0-($ def $)$ : \\ for kcode-mix or tcode-mix \\ kcodemix: force all $0 \mathrm{~s}->0-0$ :
}

For rcode, enter return to force all $0 \mathrm{~s}->0$, or a to leave the rulemix as is. For kcode or tcode, enter $\mathbf{0}$ to force all $0 \mathrm{~s}->0$, or return to leave rulemix as is.

In a locally wired network, forcing all $0 \mathrm{~s}->0$ ensures that a small zone of non-zero cells can grow at no more than the network's "speed of light", otherwise non-zero cells can appear at the first time-step throughout the network.

\subsection{Amending the neighborhood matrix}

for full rule-tables (rcode) only, not in TFO-mode

If $\mathbf{a}$ to amend the neighorhood matrix ( $k$-matrix) presentation (section 13.5.1) is selected in section 14.1 the following prompts are presented in sequence,

\section{nhood-matrix: exit-q, \% of index to show (def 100\%):33 \\ scale in pixels (def $2, \max 30): 8$ (values shown are examples)}

Enter the percentage to show just part of the matrix, which sets the maximum rule-table index on the left. Then set the scale in pixels. When the revised neighorhood matrix is displayed as in figure 14.5 below (or 13.2), the nhood-matrix:... prompt reappears for further amendment. Enter $\mathbf{q}$ to exit and continue.

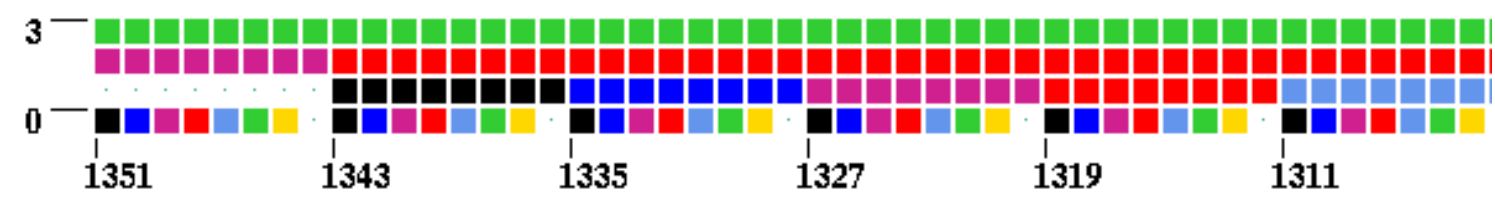

Figure 14.5: Part only of the multi-value neighborhood matrix, colored according to the value color key (section 7.1), this example for $v 8 k 4$, set at $33 \%$, and scale $=12$. 


\subsection{List Post functions}

for rcode only, not in TFO-mode

Post functions[21] are rules that belong to certain classes of Boolean functions that are closed under composition, and which play a role in the emergence of order in Boolean networks, a "softer" version of canalyzing functions. In DDLab, Post functions are generalized for multi-value, but as their theoretical relevance has only been demonstrated for Boolean functions, any multi-value results should be treated with caution.

Very briefly, if $u$ is the Post value from $[v-1, v-2, \ldots, 0]$ or just $[1,0]$ in the Boolean case, and the Post class is $[2,3,4, \ldots, i]$, where $i$ stands for all or "infinity", a rule-table (function) belongs (non-exclusively) to $A[u] 2, A[u] 3, \ldots, A[u] i$, if any $[2,3, \ldots, a l l]$ neighborhoods where the rule-table output $=u$ have a common $u$ component (DDLab only computes class 2,3 , and $i$ ).

In the literature the neighborhood (string) is the "vector", and the rule-table is the "function". By this definition, if a value $u$ occurs just once in the rule-table or not at all, the function belongs to Post class $A[u] i$. These classes are nested; $A[u] i \in A[u] 3 \in A[u] 2$. For Boolean functions the class $A[u] i$ must also be canalyzing. Note also that totalistic rules, kcode and tcode, are much more likely to include Post functions than rules in general.

\subsubsection{Initial Post-function prompt}

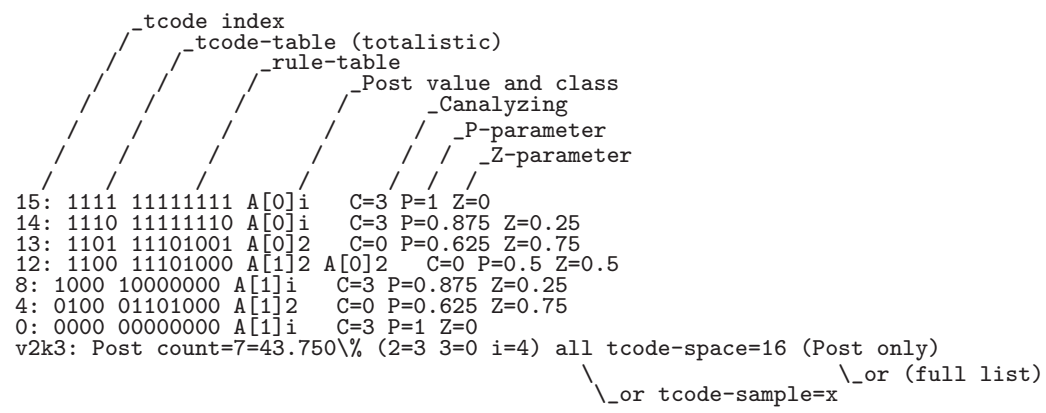

Table 14.1: Post function list and data output for tcode-space $v 2 k 3$, no restriction, with explanatory notes. Only rule-tables $\leq 128$ are displayed. The last line is the data summary.

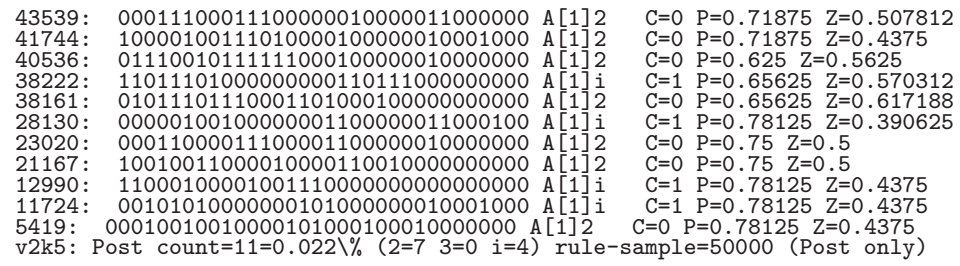

Table 14.2: Post function list and data output for a 50000 sample of $v 2 k 5$ rcode-space, restricted to Post value 1 , the sample index is on the left. For explanatory notes see table 14.1. 


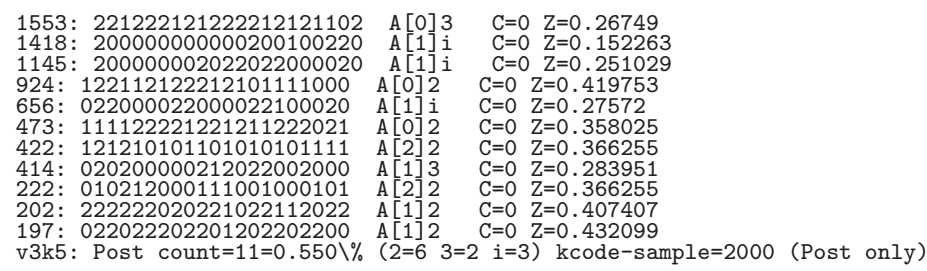

Table 14.3: Post function list (multi-value) and data output for a 2000 sample of $v 3 k 5$ kcode-space, unrestricted. Only the kcode-table is shown because the rule-table size $>128$. The sample index is on the left. For explanatory notes see table 14.1.

Enter $\mathbf{P}$ in section 14.1 to find and list all, or just samples, of Post functions in the terminal window (including data on canalyzing, and the $\mathrm{P}$ and $\mathrm{Z}$ parameters) and/or to save the list to a .dat file. A prompt similar to this, depending on the context, is presented,

Post functions: sample-s, rcode-space $\left(\mathbf{2}^{\wedge} \mathbf{8}=\mathbf{2 5 6}\right)$-p, all-a: (for rcode $v 2 k 8$ or kcode/tcodes-space, the option $\mathbf{- p}$ is removed if the table-size $>2^{32}$

Enter $\mathbf{p}$ for whole of rule-space, only possible if its size $\leq 2^{32}$ (then the size is shown in the prompt). Alternatively enter $\mathbf{s}$ for just a sample of rule-space. Add $\mathbf{a}$ in either case (i.e. pa or sa) to show all the functions, not just the Post functions, useful for finding parameter data on rules in general. q will exit the prompt, return has no effect.

\subsubsection{Restrict Post-functions}

If $\mathbf{a}$ for all functions was not selected in section 14.12.1, a top-right prompt to restrict the Post value is presented,

restrict Post value (0-1): (for Boolean functions. (0-2), (0-3) etc for multi value)

Enter the Post value $[v-1, v-2, \ldots, 0]$. Enter return for no restriction.

\subsubsection{Set Post-function sample size}

If $\mathbf{s}$ to select a sample was selected in section 14.12.1, a top-right prompt to set the sample size is presented,

\section{sample (def 10000):}

Enter the sample size or return to accept the default size.

\subsubsection{Final Post-function prompt}

The following top-right prompt, with exact wording depending on the context, is presented to print the data to the terminal, save it to a .dat file with (default filename post_data.dat), or both,

rule sample (2000) (Post only): print xterm-(def), save-s, both-b:

possible alternatives: all rules/kcode/tcodes, rule/kcode/tcode sample, (Post only)/(full list)

To cut short a lengthy computation in progress enter q. When complete, the program returns to section 14.12.1. Enter q to exit Post-functions, or start a new listing. 


\section{Chapter 15}

\section{Setting Canalyzation in a random rule-mix}

rcode only - not TFO-mode.

The dynamics on random Boolean networks can be induced towards order by biasing the rules (rcode only) with increasing proportions of "canalyzing" inputs, with applications in modelling genetic regulatory networks[11, 30].

A canalyzing input may be defined as follows: if a particular value $(0,1, \ldots, v-1)$ on an input to a cell (referred to as a "gene" in this context) determines the gene's output irrespective of its other inputs, that input is said to be canalyzing. A given gene may have from zero to $k$ canalyzing inputs, but the outputs of all of these must be the same. As $k$ increases, the fraction of rule-space with canalyzing rules (having at least one canalyzing input) deceases exponentially (section 24.9.5), so the probability of setting such a rule at random deceases accordingly.

Traditionally, canalyzation applies to Boolean rules, but the same definition is generalized for multi-value networks and applied in multi-value DDLab. However, the examples in this chapter remain focused on Boolean rules.

The methods bias the randomly assigned rules by varying or tuning the fraction of canalyzing rules, or inputs, in the network. The algorithms for tuning canalyzation in DDLab are designed to minimize secondary biases in the distribution of canalyzing inputs. Rule-tables will be amended at random for the required degree of canalyzation.

\subsection{Selecting Canalyzing}

Canalyzing can be selected at various points in DDLab.

\subsubsection{Selecting canalyzing from the rcode-mix}

When setting the rcode-mix directly in section 14.4.1, at the top-right prompt,

RcodeMix: select by hand-h, maj-m Alt-A chain-c iso-i Post-P canalyzing-C/+C, rnd(def): (canalyzing- $C /+C$ not for $k$ code or tcode)

Enter $\mathbf{C}$ to apply canalyzing to random rules, or $\mathbf{m C}, \mathbf{A C}, \mathbf{c C}, \mathbf{i C}$ (but not $\mathbf{P C}$ ), to apply canalyzing to biased random rules. The (biased) rules will be set first, then modified by cumulative random mutation to achieve the required canalyzation. 


\subsubsection{Selecting canalyzing from wiring graphic - transform rule}

Once the rulemix has been set, canalyzing can be reached from the wiring graphic options (section 17.4). At the wiring graphic option,

... rule: Save/rev/trans-S/v/t ...

... enter $\mathbf{t}$ for the transform rule options (section 18.1). A top-right prompt similar to the one below will be presented,

transform rule: solid-o invert-v comp-c neg-n ref-r canal-C (all+a)

equiv $>\mathrm{k}$ (4-7), max k-m, eff k: all-K this-k, save-s:

Enter Ca to select canalyzing for the whole network, described in this chapter, or just $\mathbf{C}$ to set canalyzing for a single rule described in section 18.6. The transform rule prompt also appears automaticaly after a single rcode is set in the main prompt sequence, allowing canalyzing to be set for that rule.

\subsubsection{Selecting canalyzing from the Derrida plot}

Canalyzing can be selected from the Derrida plot (chapter 22). The following top-right prompt appears once the Derrida plot is complete (section 22.5),

Derrida plot complete

reset-r canalyzing-C:

Enter $\mathbf{C}$ to reset the canalyzing for a new Derrida plot.

\subsection{The first canalyzing prompt}

A series of top-right prompts allow the canalyzation to be set and reviewed. The first canalyzing prompt is as follows,

set canalyzing genes-g inputs-(def):

Enter $\mathbf{g}$ to specify canalyzing genes, the fraction of network elements with at least one canalyzing input. Enter return (the default) to specify the fraction of canalyzing inputs (wires), where the total number of wires is $n \times k$ for homogeneous $k$, or the sum of all $k$ 's for a mixed $k$ network.

\subsection{Canalyzing percentage or number}

For a network with homogeneous $k$, according to whether "genes" or "inputs" were selected in section 15.2 above, the next prompt is as follows,

c-inputs: redo-q number-n \%-(def): (or c-genes)

or

mixed $k$, c-inputs: redo-q number-n \%-(def): ( or c-genes, for mixed $k$ networks) 

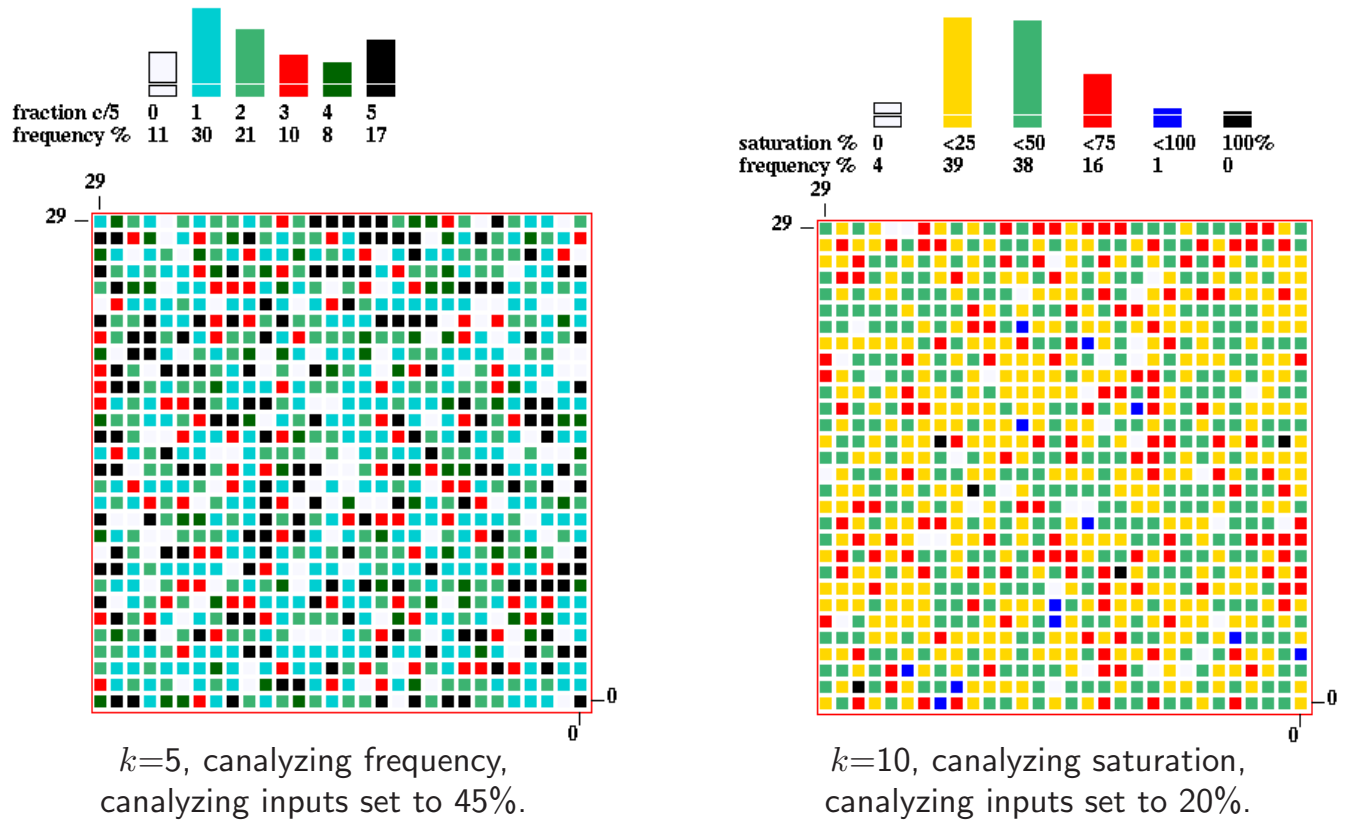

Figure 15.1: Canalyzing frequency/saturation for homogeneous $k$ networks, $n=30 \times 30$, showing the degree of canalyzation of each gene according to a color code, and a histogram of canalyzation in the network - the colors in the network and histogram correspond. Above Left: canalyzing frequency histogram. Above Right: canalyzing saturation histogram (because $k \geq 10$ ). The colors in the network and histogram correspond.

Enter return (the default) to set a percentage of the total genes/inputs as canalyzing, or enter $\mathbf{n}$ for a specific number. From this point on, the options for networks with homogeneous $k$, and mixed $k$, are somewhat different.

\subsection{Canalyzing - homogeneous $k$}

The next prompt gives the fraction and percentage of the current canalyzing genes and inputs, and asks for the required canalyzing settings, for example,

c-inputs $=\mathbf{0} / \mathbf{4 5 0 0}=\mathbf{0 . 0 \%}$ genes $=\mathbf{0} / \mathbf{9 0 0}=\mathbf{0 . 0 \%}$ (for a $2 d$ network, $k=5,30 \times 30$ ) set new \% c-inputs, redo-q accept-ret: (values depend on the earlier choices)

If, say, 45 (for $45 \%$ ) is entered. The prompt will reappear with updated information, c-inputs $=2025 / 4500=45.0 \%$ genes $=793 / 900=88.1 \%$ set new \% canalyzing inputs, redo-q accept-ret:

Enter a new canalyzing setting, or return to accept - the program will continue with options to review network architecture described in chapter 17 , or enter $\mathbf{q}$ to revert to the first canalyzing prompt (section 15.2). 


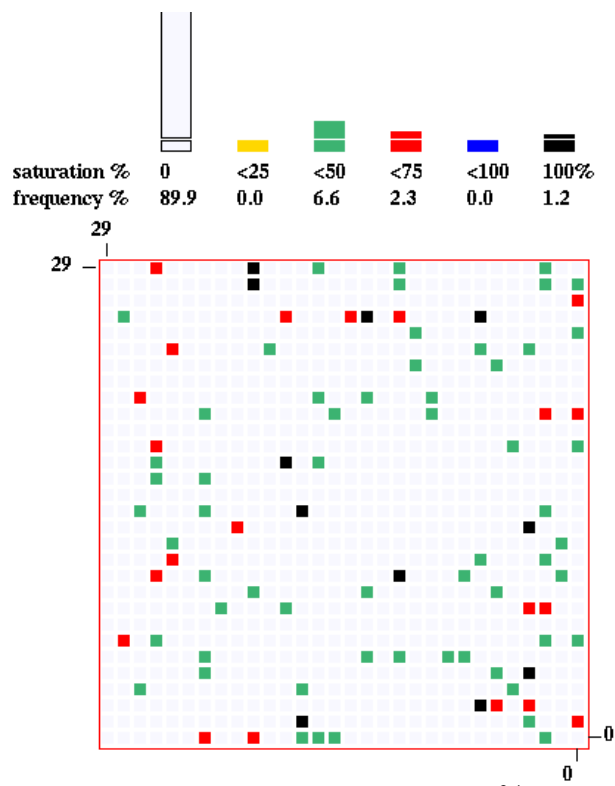

Initial canalyzing inputs, 3.0\%.

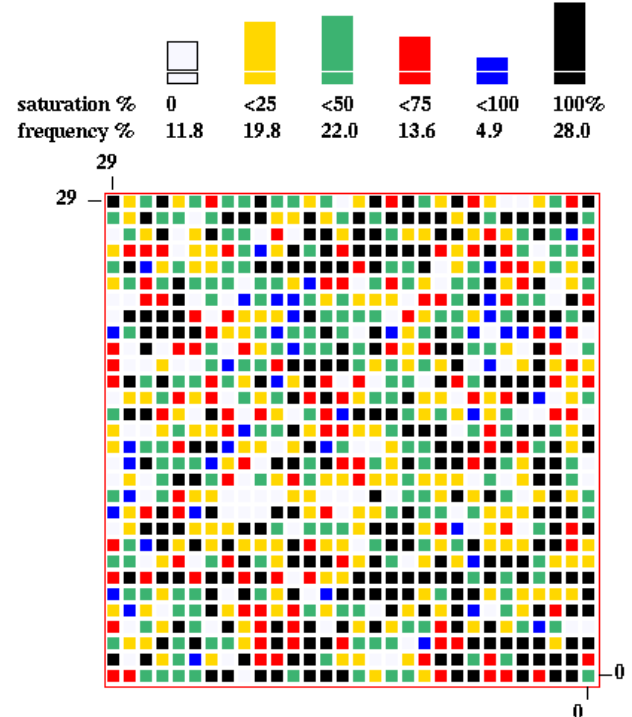

Canalyzing inputs set to $45 \%$.

Figure 15.2: Canalyzing saturation for two networks with the same (roughly even) $k$-mix 3-7, with the canalyzing saturation histograms above, relating to section 15.5. $n=30 \times 30$. Left: The network showing the initial, expected, canalyzing. Right: The canalyzing inputs set to $45 \%$. The colors in the network and histogram correspond.

At the same time as the prompt appears, a window in the lower right hand corner of the screen gives a $2 \mathrm{~d}$ graphical display of of the degree of canalyzation of each gene, irrespective of the network's native dimensions (figure 15.1).

Above this is a histogram of canalyzation in the network. For $k \leq 9$ the "canalyzing frequency" histogram shows the frequency of each degree of canalyzation, 0 to $k$ (figure 15.1 Above Left). For $k \geq 10$ or for mixed- $k$ (section 15.5) a different histogram, "canalyzing saturation" shows the frequency of different percentages of canalyzation in six columns, for $0 \%, 100 \%$, and intervals of $25 \%$ in between, i.e. $0 \%,<25 \%,<50 \%,<75 \%,<100 \%, 100 \%$ (figure 15.2 Above Right).

\subsection{Canalyzing - mixed $k$}

For a network with mixed $k$, following the prompts in sections 15.2 and 15.3 , the next prompt shows the percentage of different $k$ 's (see also section 9.7.1), and offers an option to reset the canalyzation for just a subset of the network having a particular $k$, or for the whole network,

$\% \mathrm{k}: 3=19.94=19.75=19.16=21.0 \quad 7=20.3$

enter $\mathbf{k}$ for just one nhood, all-(def) (genes/inputs depends on the earlier choice) 


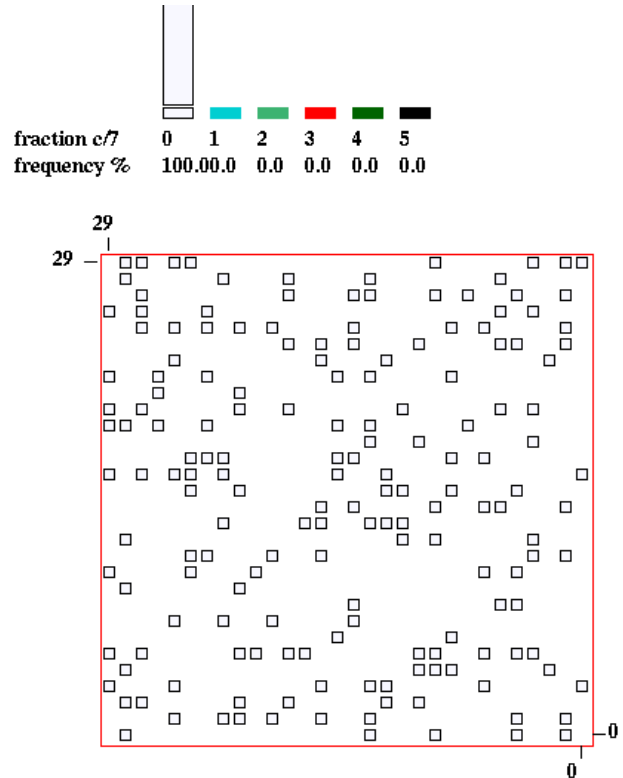

The initial $k=5$ genes with no canalyzing inputs, as expected.
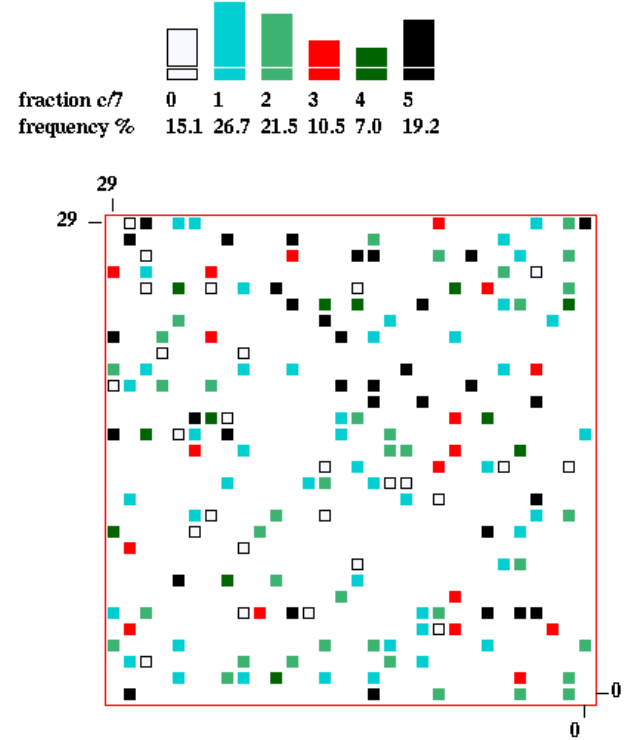

Canalyzing inputs to the $k=5$ genes set to $45 \%$.

Figure 15.3: Just one $k$ in a mixed $k$ network. Canalyzing saturation for two networks with the same (roughly even) $k$-mix, 3-7, as shown in section 15.5. $n=30 \times 30$. Only the selected $k=5$ genes are shown. An empty outline indicates no canalyzing inputs and gaps are genes where $k \neq 5$. Left: The initial network showing $k=5$ genes with no canalyzing inputs, as expected. Right: Canalyzing inputs of the $k=5$ genes set to $45 \%$. The colors in the network and histogram correspond.

\subsubsection{Canalyzing for the whole network - mixed $k$}

Enter return to set canalyzing for the whole mixed- $k$ network. The next prompt gives the fraction and percentage of the current canalyzing genes and inputs, and asks for the required canalyzing settings. For example, the prompt may appear as follows, in this case for a $30 \times 30$ network, with a $k$-mix of 3 to 7 (figure 15.2),

c-inputs $=\mathbf{1 3 4} / \mathbf{4 5 3 0}=\mathbf{3 . 0 \%}$ genes $=\mathbf{9 1} / \mathbf{9 0 0}=\mathbf{1 0 . 1 \%}(2 d$ network, $30 \times 30)$

set new \% c-inputs, redo-q accept-ret: (values depend on the earlier choices)

The reason for the initial canalyzing degree (figure 15.3 Left) is that about $23.5 \%$ of inputs in randomly assigned $k=3$ rules are likely to be canalyzing. For $k=4$ rules the figure is about $1.5 \%$ (section 24.9.5).

If, say, $\mathbf{4 5}$ (for $45 \%$ ) is entered. The prompt will reappear with updated information,

$$
\text { c-inputs }=\mathbf{2 0 3 4} / \mathbf{4 5 3 0}=\mathbf{4 5 . 0 \%} \text { genes }=\mathbf{7 9 4} / \mathbf{9 0 0}=\mathbf{8 8 . 2 \%}(2 d \text { network, } 30 \times 30)
$$

set new \% c-inputs, redo-q accept-ret: (values depend on the earlier choices)

Enter a new canalyzing setting, or enter return to accept, the program will continue with options to review network architecture described in chapter 17 , or enter $\mathbf{q}$ to revert to the first canalyzing prompt (section 15.2). 


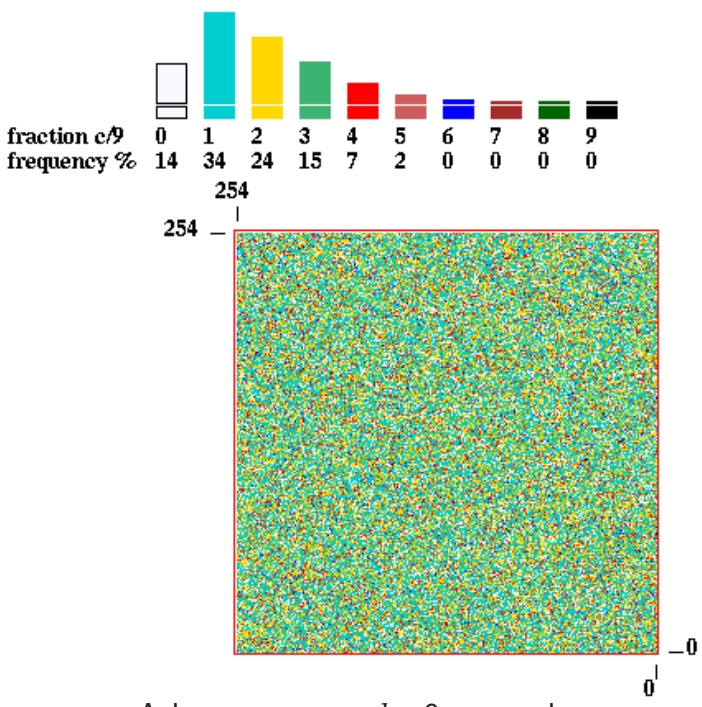

A homogeneous $k=9$ network.

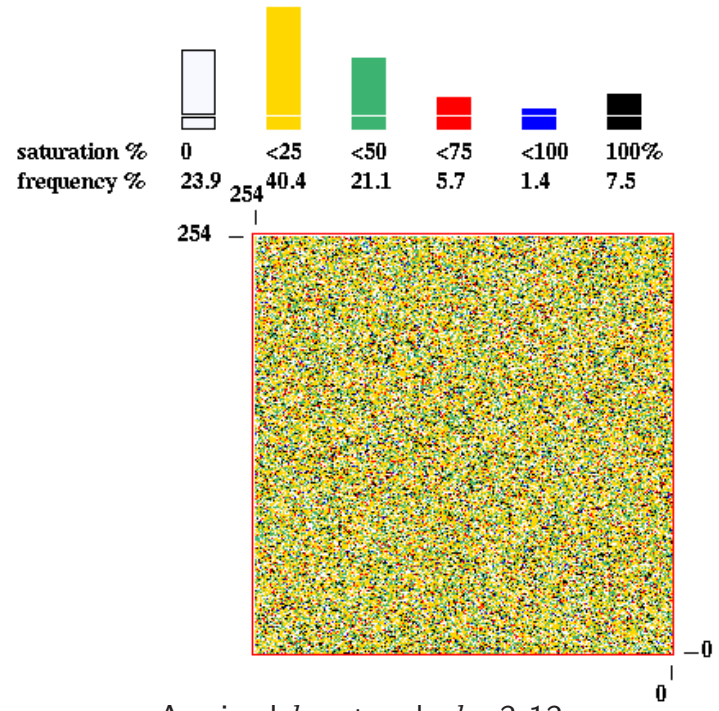

A mixed $k$ network, $k=3-13$.

Figure 15.4: Examples of two large networks, $n=255 \times 255$, with large $k$. The canalyzing inputs were set to $20 \%$. Several tries with small increments were required to reach this setting.

At the same time as the prompt appears, a window in the lower right hand corner of the screen gives a $2 \mathrm{~d}$ graphical display of the degree of canalyzation of each gene, irrespective of the network's native dimensions (figure 15.3). Above this is a histogram of canalyzing saturation for the mixed- $k$ network showing the frequency of different percentages of canalyzation in six columns, for $0 \%, 100 \%$, and intervals of $25 \%$ in between, i.e. $0 \%,<25 \%,<50 \%,<75 \%,<100 \%, 100 \%$ (figure 15.2 Above).

\subsubsection{Canalyzing for a particular $k$ in a mixed $k$ network}

To set canalyzing for genes with one specific $k$ only in a mixed- $k$ network, ignoring the rest, enter the required value of $k$ at the prompt ...

enter $\mathrm{k}$ for just one nhood, all-(def):

$\ldots$ in section 15.5. It must be a valid value that exists in the $k$-mix. For example, if $k=5$ is selected (for the network shown in figure 15.3 Left) the following prompt will appear,

$$
\begin{aligned}
& \mathrm{k}=5(19.1 \%) \mathrm{c}-\mathrm{inputs}=0 / 860=0.0 \% \text { genes }=0 / 172=0.0 \%) \\
& \text { set new \% c-inputs, redo-q accept-ret: }
\end{aligned}
$$

This indicates that $19.1 \%$ of the network consists of $k=5$ genes, and that none of the inputs to those genes are canalyzing, which is to be expected.

If, say, 45 (for $45 \%$ ) is entered. The prompt below will reappear with updated information, and the graphic will update - (figure 15.3 Right),

$\mathrm{k}=5(19.1 \%) \mathrm{c}$-inputs $=387 / 860=45.0 \%$ genes $=146 / 172=84.9 \%)$

set new \% c-inputs, redo-q accept-ret: 
Enter a new canalyzing setting, or enter return to accept - the program will continue with options to review network architecture described in chapter 17 , or enter $\mathbf{q}$ to revert to the first canalyzing prompt (15.2).

At the same time as the prompt appears, a window in the lower right hand corner of the screen gives a $2 \mathrm{~d}$ graphical display of the degree of canalyzation (figure 15.3) showing just the $k=5$ genes selected. An empty outline indicates no canalyzing inputs and gaps are genes where $k \neq 5$.

Above this is the "canalyzing frequency" histogram for just the $k=5$ genes (figure 15.3 Above). This format is employed rather than "canalyzing saturation" because in this example we are dealing with just one $k \leq 9$.

\subsection{Canalyzing for large networks, or large $k$}

For large networks, or large $k$, as in figure 15.4, the algorithm for assigning canalyzing may take some time. Also, the degree of canalyzation achieved may be less than requested. In this case more tries should be made (in sections 15.4, 15.5.1, 15.5.2) to reach the required setting, increasing by small increments.

A top-center window monitors the percentage of canalyzing inputs set so far. A progress bar near the top-right hand corner of the screen also monitors the assignment of rules to the network, as in section 14.9. 


\section{Chapter 16}

\section{Setting a singe rule}

This chapter describes the alternative methods of setting a single rule: rcode, kcode or tcode. The methods also apply to setting rules "by hand" for a rulemix in section 14.6.

\subsection{The first single rule prompt}

If one of the following selections was made at previous prompts,

return ... (the default) for a single rcode, kcode or tcode in section 14.1,

$\mathbf{h}$... for by hand-h for various kinds of rulemix: (see also section 14.6)

- setting a rulemix directly (section 14.4.1),

- setting a rule-subset (section 14.4.2),

- outer-totalistic kcode or reaction-diffusion (section 14.2).

then a main sequence prompt (sections 16.1.1 or 16.1.2) is displayed to accept or revise the default method of rule selection (and other options). The prompt shows a reminder of the value-range $v$, the neighborhood size $k$, the rule type (rcode, kcode or tcode), the size of the corresponding rule-table $S$, and the list of options which are context dependent and may vary considerably.

\subsubsection{Single rcode prompt examples}

For a full rule-table, rcode (not TFO-mode or a $k$-mix) a graphic of the binary neighborhood matrix is displayed (sections 13.3.1, 13.5.1), and the prompt is presented as follows, for example,

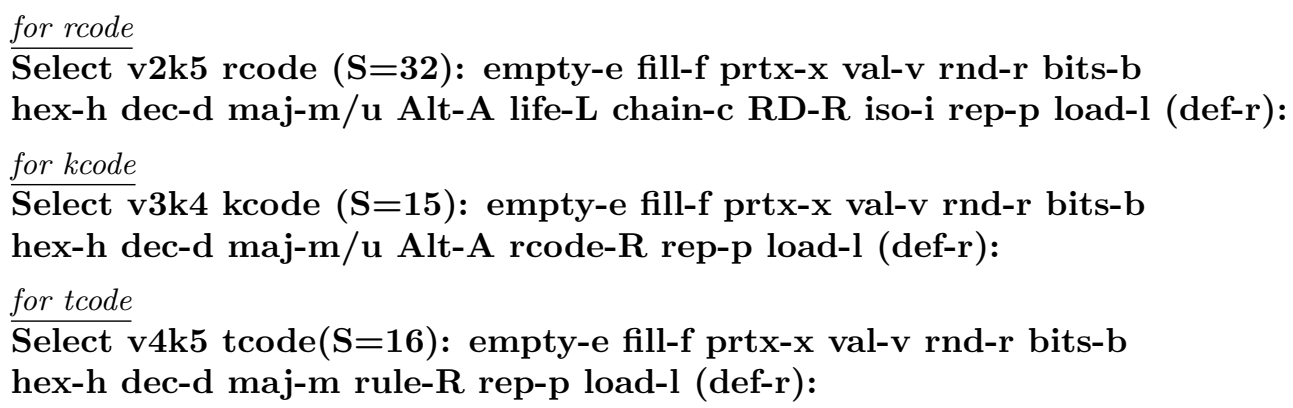




\title{
16.1.2 TFO-mode single rule prompt examples
}

\author{
for kcode \\ Select v3k4 kcode ( $\mathrm{S}=15)$ : empty-e fill-f $\mathrm{k}+-\mathrm{k}$ prtx/swap-x val-v rnd-r bits-b \\ hex-h dec-d maj-m/u Alt-A rep-p load-l (def-r): \\ for tcode \\ Select v4k5 tcode $(S=16)$ : empty-e fill-f prtx-x val-v rnd-r bits-b \\ hex-h dec-d maj-m rep-p load-l (def-r):
}

\subsection{Methods for setting a rule}

The meaning of the prompts for setting a rule ${ }^{1}$ in section 16.1 are summarized below. More details are given in the rest of this chapter. These options also apply to a rule in a rulemix set "by hand" in section 14.6, where the selected method stays as the default unless changed in section 14.6.4. Some options do not apply to particular cases as noted. rnd-r is the initial default in the main sequence of prompts, bits-b is the default if a rule has already been selected, or if revising a rule at later stages (sections 30.5.1, 32.16.1).

options .... what they mean

empty-e ... to reset all rule-table entries to 0 .

fill-f ... to fill the lookup-table with any valid value with the top-right prompt,

fill with value $0-4(\operatorname{def} 4)$ : (if $v=4)$

This allows a clean slate for setting bits or values in section 16.4.

k+-k ... (only for kcode in TFO-mode) to create and save a kcode with an increased neighborhood $k+$ by inserting a random string of the correct length within the current kcode (section 16.17).

prtx-x ... show the rule in the terminal (section 16.18).

prtx/swap-x ... (for kcode only) as above, but also allows swapping output values to make an equivalent kcode (section 16.18.5).

val-v ... to save or load the rule(*.tbl), as an ASCII string. The following topright prompt is presented (for example),

ASCII table $(\mathrm{v}=4 \mathrm{k}=3 \mathrm{~S}=55)$ as ascii string, load/save-l/s:

note... After the prompts above the program reverts to the first single prompt in section 16.1. The prompts below set a rule and the program continues.

rnd-r ... to set the rule at random, for a given density bias or $\lambda$ parameter (section 16.3).

bits-b ... to set the rule as bits or values — "draw" the rule-table on a $1 \mathrm{~d}$ or $2 \mathrm{~d}$ graphic array, using the mouse or keyboard (section 16.4).

hex-h ... to set the rule in hexadecimal, in a mini "spread sheet" (section 16.5).

\footnotetext{
${ }^{1}$ Some of these methods are similar to setting a seed in section 21.2.
} 
dec-d ... (if applicable) to set the rule in decimal (section 16.6).

$\mathbf{m a j}-\mathbf{m} / \mathbf{u} \ldots$ Enter $\mathbf{m}$ to set a "majority" (voting) rule (section 16.7).

Enter $\mathbf{u}$ (not for tcode) to assign a majority rule but with the outputs from uniform neighborhoods flipped or shifted (section 16.8).

Alt-A ... (not for tcode) to set an "Altenberg" rule, where the output of each neighborhood is set probabilistically according to the frequency of the values in that neighborhood (section 16.9).

Life-L ... (for rcode only, and if $k \geq 5$ ) to set to the "game-of-Life" or quasi "Life" (section 16.10).

chain-c... (for rcode only) to set a maximally chaotic rule, where $Z_{\text {left }}=1$ or $Z_{\text {right }}=1$, but not both. For binary, In addition both $Z_{\text {left }}$ and $Z_{\text {right }}$ are greater than 0.5 (section 16.11).

RD-R ... (for rcode only) to select a reaction-diffusion rule see section 13.8.2.

rcode-R ... (for tcode or kcode and full rule-table - not TFO-mode) to show a tcode or kcode as a full rule-table (rcode) which can then be modified.

iso-i ... (for rcode only) to set the rule at random but as an isotropic rule, where rotated and reflected neighborhoods (in $1 \mathrm{~d}, 2 \mathrm{~d}$ and $3 \mathrm{~d}$ ) have the same output.

rep-p ... to repeat the last rule. (16.13).

load-l ... to load a rule. from a .rul, .vco, or .tco, file. (section 35.2). $[v, k]$ in the file must be the same as in the base.

Once a single rule have been set, except after rnd-r or in a rulemix "by hand" (section 14.6), the bits-b option is activated automatically. Once accepted the rule is reconfirmed as a bit/value pattern (in decimal if applicable) and in hex, and simultaneously displayed in the rule window (section 16.19). At any stage enter $\mathbf{q}$ to backtrack and revise.

\subsection{Setting the rule at random}

A rule set at random is unbiased by default, but biases can be applied by two methods, firstly the proportion of non-zero values ( $\lambda$-parameter), secondly a more explicit bias - the proportions of different values in the rule-table, either as percentages, or as actual numbers of each value. An uneven distribution of values in the rule-table, by either method, is more likely to result in complex dynamics than an unbiased rule-table (figure 33.16).

Enter $\mathbf{r}$ in section 16.1, to generate a random rule, displayed as a rule-table bit/value string, Density-bias is simultaneously displayed in a lower top-right density window (section 16.3.1). Initially there is an equal probability of each value, unless this was reset.

In addition to random biases, various changes and transformations ${ }^{2}$ can be made (repeatedly) until return or $\mathbf{q}$ is entered - to accept the rule. The following subsequent reminder is shown,

\section{tog gaps-g, rotate-l/r another-n density-s/v Z-u/d comp-m back-q accept-ret}

(Z-u/d for rcode only)

\footnotetext{
${ }^{2}$ These options are similar to setting a random initial state or seed in section 21.3.
} 
These options are summarized below — with more detail in the sections indicated.

$$
\begin{aligned}
& \text { options ... what they mean } \\
& \text { tog gaps-g ... to toggle gaps between successive blocks of } 8 \text { bits/values. This is the } \\
& \text { default for binary rules (i.e. between chars) as in figure 16.10. } \\
& \text { rotate-l/r . . enter } \mathbf{l} \text { or } \mathbf{r} \text { to rotate rule-table left or right by one output, with the edge } \\
& \text { outputs wrapping around. } \\
& \text { another-n ... for another random rule with the same density bias }-\lambda \text { parameter. } \\
& \text { density-s } / \mathbf{v} \ldots \text { enter } \mathbf{s} \text { to change the density bias }-\lambda \text { parameter (section 16.3.1). Enter } \mathbf{v}
\end{aligned}
$$

Some of these options are described in greater detail below.

\subsubsection{Random rule density-bias ( $\lambda$ parameter)}

Enter $\mathbf{s}$ in section 16.3 to change the density-bias ${ }^{3}$ - the fraction of non-zero values in the ruletable (the $\lambda$-parameter) but expressed as a percentage. The initial default is an equal probability of each value, but this may have been reset in section 14.1.2. A lower top-right density window shows information about the density, in this example for rcode $v 5 k 6$,

density $(4-1$ s)exact $=12500 / 15625=80.000 \%$, bias- $80.000 \%$

where "density (4-1s)" indicates the non-zero values, "prob" the current method of setting the density as opposed to "exact", 15625 is the size of the rcode-table, then the actual density, and the bias requested.

There are two alternative methods to change the density-bias, exact or prob (exact is the initial default). The following two stage top-right prompt is presented, for example,

\footnotetext{
${ }^{3}$ The methods for rule density-bias are similar to seed density-bias (section 21.3.1).
} 
for $v=2,22 \%$ entered, with prob as the default method

bias-density: enter \% (def 50.000\% prob):22 exact-e: (enter e to change to exact)

for $v=8$, 33\% entered, with exact as the default method

bias-density: enter \% (def $\mathbf{8 7 . 5 0 0 \%}$ exact):33 prob-p: (enter $\mathbf{p}$ to change to prob)

Enter the new density-bias as a percentage - the result updates the density window. For binary this is the probability of setting 1s. For $v>2$ it is the probability of setting non-zero values - set without bias. The new density-bias becomes the new default. If the prob method is active the density-bias requested is applied as a probability, whereas the exact method will apply the requested bias as closely as possible. To change the default method between prob to exact, enter $\mathbf{e}$ or $\mathbf{p}$ as indicated. The density-bias and method will be maintained for further random rules generated with another-n in section 16.3 updating the density window, and when re-setting random rules for space-time patterns with on-the-fly key $\mathbf{r}$ (section 32.5.1). This is also one of the method to bias a random rule sample for classifying rule space (section 33.2.1).

\subsubsection{Random rule value-bias}

Enter $\mathbf{v}$ in section 16.3 to fix the proportions of different values in the rule-table, either as percentages, or as actual numbers of each value if the rule-table size $S \leq 255$. The following series of prompts are presented in a top-right window,

\section{if $S \leq 255$ \\ bias random rule, \%/value-p/v:}

if $S>255$

bias random rule, value-p:

Enter $\mathbf{p}$ for percentages or $\mathbf{v}$ for numbers of each value, or return to abandon value-bias. The prompt continues as follows (these example for kcode $v=8$, where the rule-table size $S=120$ ),

if $\mathbf{p}$ was selected

0(100:45 1(55:4 2(51:4 3(47:4 4(43:9 5(34:9 6(25:18

if $\mathbf{v}$ was selected

0(120:56 1(64:7 2(57:7 3(50:7 4(43:12 5(31:12 6(19:15

For each value, from 0 to $v$-2, enter the percentage or number - the value for $v$ - 1 is set automatically. The total available for successive each value is shown with a bracket, for example at 3(50: (for $v=3$ ) enter a number $\leq 50$. If exactly 50 is entered the allocation will halt as complete. Entering a number $>50$ or return results in allocating 50 divided by the number of remaining values, so entering return repeatedly gives an even distribution.

A lower top-right window shows the resulting value-bias distribution for the example above, where "7-0" indicates the order of values,

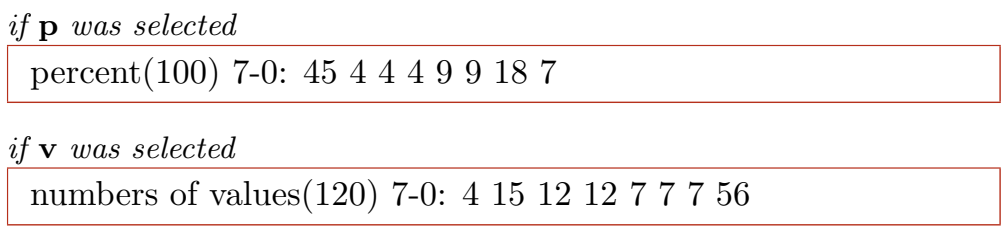


If set, the value-bias will be maintained for further random rules generated with another-n in section 16.3 updating the density window, and when re-setting random rules for space-time patterns with on-the-fly key $\mathbf{R}$ (section 32.5.1). This is also one of the method to bias a random rule sample for classifying rule-space (section 33.2.1).

\subsubsection{Random rule parameters} not applicable in TFO-mode

For each alternative rule created in section 16.3, current rule parameters (as finally appear in the rule window - section 16.19) are shown in an upper top-right rule parameters window. This example is for a $v 2 k 5$ rcode,

$\mathrm{C}=1 / 5=* 3^{* * *} \mathrm{zl}=0.1875 \mathrm{zr}=0.1875$

$\mathrm{ld}=0.09375 \mathrm{ld}-\mathrm{r}=0.1875 \mathrm{P}=0.90625 \mathrm{Z}=0.1875$

abbreviations ... what they mean

$\mathrm{C}=1 / 5 \ldots$ the number of canalyzing inputs[11], in this case 1 out a possible $5(k=5)$.

$* 3 * * * \ldots$ if there are canalyzing inputs, this shows which are canalyzing, in this case one input at index 3 (from a possible 0 to 4 , see chapter 10).

$\mathrm{zl} \ldots Z_{\text {left }}$.

$\mathrm{zr} \ldots Z_{\text {right }}$.

ld ... the $\lambda$ parameter[17].

ld-r ... the $\lambda$ ratio[25].

$P$... the $P$ parameter[11].

$\mathrm{Z}$... the $Z$ parameter[25, 32].

\subsection{Setting the rule as bits or values}

If $\mathbf{b}$ is selected in section 16.1, a bit or value pattern representing the current rule-table (initially just 0s) is displayed. This consists of a single row or multiple rows for longer rule-tables. For $v=2$ the default has gaps between successive blocks of 8 bits (i.e. between chars) but this can be toggled. The default scale of the pattern depends on the size of the rule-table, S, but can be changed. The maximum rule-table index, $S$-1, is in the top-left hand corner, the zero index in the lower right hand corner of the pattern. The current position on the pattern is indicated by a small flashing cursor (initially top-left as in figures 16.1-16.2), and also in a top-right inset window.

The colors correspond to values (chapter 7) but 0s are initially colored light green. If a rule is already current, it can be reset to all-0s with empty-e or to any uniform value with fill-f in sections 16.1.1 or 16.1.2 to provide a clean slate for setting other bits/values. Alternatively, use the bit/value setting method for fine adjustments to rule-tables set by other methods. Figure. This also illustrates alternative presentations which can be changed on-the-fly.

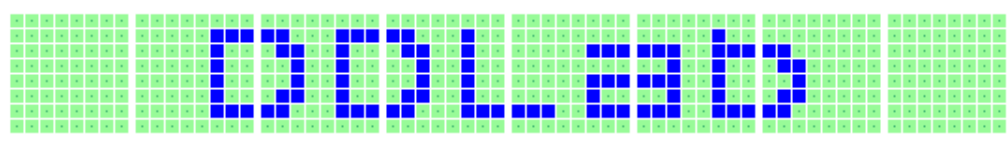

Figure 16.1: Setting bits starting with all 0s, with default gaps and white divisions. $v 2 k 9$, $S=512$. 

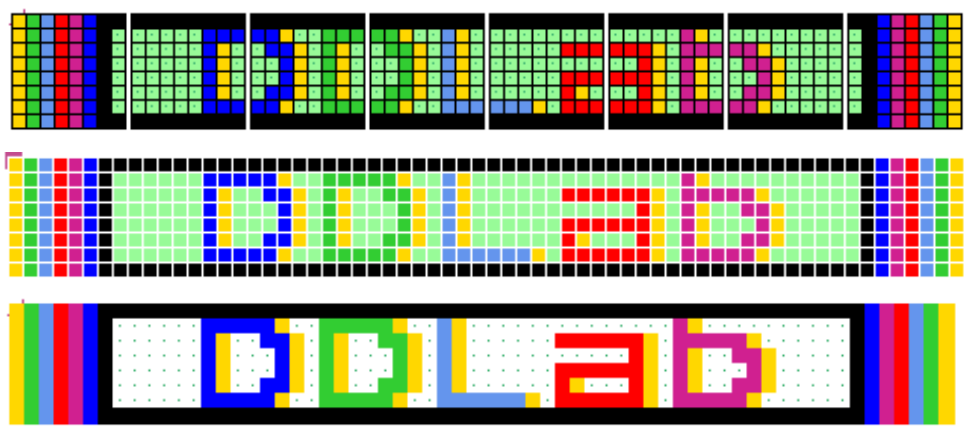

Figure 16.2: Setting values alternative presentations, $v 8 k 3$, $\mathrm{S}=512$

Top: gaps, black divisions, 0s light green with dots.

Center: no gaps, white divisions, Os light green, no dots.

Bottom: no gaps, no divisions, Os white with dots.

\subsubsection{Rules: bits/values reminder window}

Bits or values in the rule-table are set with the mouse and keyboard. Similar methods apply for the seed in section 21.4.1. During the procedure, a top-right window, and inset display, gives reminders of various options and current settings (summarized below), this example for a $v 5 k 6$ kcode where the rule-table size is 210 ,

$$
\begin{aligned}
& \text { the inset while drawing with the mouse } \rightarrow \text { left button: draw 1s width=1 } \\
& \text { the inset with current settings }
\end{aligned}
$$

$$
\text { options.... what they mean }
$$

keys: select/draw 4-0 ... enter a valid number to select the value/color, and keep the key pressed to draw a horizontal line.

vert-v... enter $\mathbf{v}$ for a vertical line, and keep $\mathbf{v}$ pressed to draw a vertical line downwards in the selected value/color.

mouse: move-click ... left click on the rule pattern to reposition the small cursor and activate drawing - sometimes the right button also needs to be clicked (or right-left a few times) to activate.

draw-drag ... draw the selected value/color by dragging the cursor with the left mouse button pressed - release the button to stop. The right mouse button draws the complementary value/color (section 16.20) in the same way.

width-w ... enter $\mathbf{w}$ to change the width (initially 1 ) of the line to be drawn, where the max width is the number of rows. The following topright prompt is presented (for example),

\section{reset line width (now 1) $\max 4$ :}

The width is shown in the drawing inset, and stays for the current drawing session until revised. 
move-arrows ... all four arrow keys move the cursor around the seed rule-table (up/down arrows for multilpe rows).

PScript-P ... to save the rule bit/value pattern as a vector PostScript image (section 16.4.3).

file-F ... to save the rule pattern as a $1 \mathrm{~d}$ seed file (section 16.4.3).

tog: gaps-g ... enter $\mathrm{g}$ to toggle gaps between sucessive blocks of 8 bits/values (figure 16.3).

tog: 0color $-\wedge \ldots$ to toggle the zero value color between light green and white.

tog: dot-. ... enter a dot to toggle a dot at the center of each zero cell (figure 16.2).

tog: divs/divcolor-i/! . . enter $\mathbf{i}$ to toggle division lines between the cells. Enter ! to toggle the division line color between white and black (figure 16.2).

$\exp /$ contrect-e/c ... enter e to expand, $\mathbf{c}$ to contract the scale by one pixel.

rotate- $\mathbf{l} / \mathbf{r} /+/-\ldots$ enter $\mathbf{l}$ or $\mathbf{r}$ to rotate the rule-table left or right by the rotation interval (initialy 1) with the edge outputs wrapping around. Enter + or - to increace or decreace the rotation interval shown in the current settings inset.

flip-f ... to flip (reflect) the rule-table.

comp-m ... to toggle the rule to its complement. For binary this swaps 1 s and 0s. For $v>2$ the values are shifted; each output $a$ changes to $a_{m}$ by subtraction from the maximum value $v-1$, so $a_{m}=(v-1)-a$, and the maximum value changes to zero (section 16.20).

back/accept-q/ret ... enter ret to accept the rule, q to backtrack.

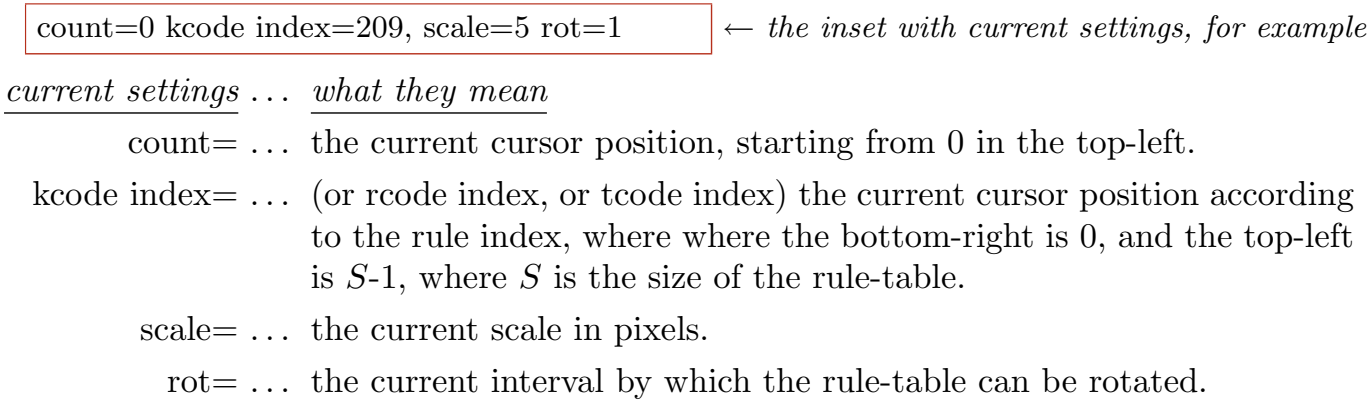

\subsubsection{Rules: setting bits/values with the keyboard and mouse}

The active position (to be updated) in the rule-table is highlighted by a small flashing cursor initially in the top-left. Its position is displayed in the top-right inset window. The flashing cursor is repositioned with the mouse by clicking either the left or right button on the new position ${ }^{4}$, or moved with the left/right/up/down arrow keys.

\footnotetext{
${ }^{4}$ Mouse behavior differs slightly between Linux-like systems and DOS. In Linux the mouse cursor changes direction within the bit/value rule-value pattern, pointing north-west instead of north-east, and within the pattern, left or right mouse clicks reposition the flashing cursor. In DOS the mouse cursor is confined within the pattern and clicking the left or right buttons sets values as well as repositioning the flashing cursor.
} 


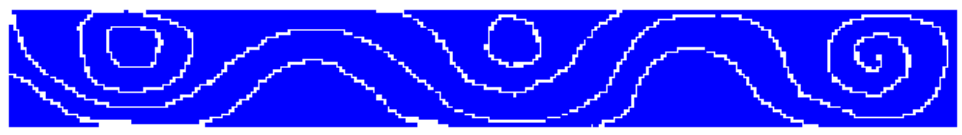

Figure 16.3: Drawing bits or values on the rcode pattern by dragging the mouse cursor.

Top: drawing value 0 with line width $=1$ on an all-1s bit pattern,

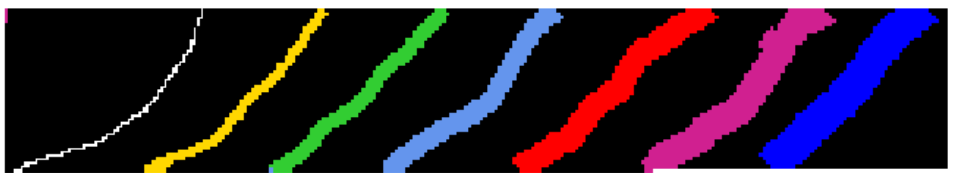
$v 2 k 13$ rcode, $\mathrm{S}=8192$.

Bottom: drawing values $0,1,2,3,4,5,6$, with line width $1,2,3,4,5,6,7$, on an all-7s value pattern, $v 8 k 9$ kcode, $\mathrm{S}=11440$.

Setting or drawing values on the rule-table pattern is done with the keyboard or mouse (figures 16.1,16.3). To set (and activate) a value at the cursor position, press a valid number key (without return) - the cursor will then advance to the right by one unit. To draw a horizontal line towards the right keep the key pressed; eventually the line will continue to the next row or jump back to the top-left. To draw a vertical line downwards with the active value, press $\mathbf{v}$.

To draw the active value with the mouse, drag the cursor anywhere over the rule-table pattern with the left button pressed - release to stop. To draw the complement of the active value (section 16.20) drag with the right button pressed. While a mouse button is pressed, the inset in the reminder window changes to show which button, the current active value, and the current width of the line, for example,

left button: draw $3 \mathrm{~s}$ width $=2$ or right button: draw $4 \mathrm{~s}$ width $=3$

Initially the left button draws the value $v-1$ and the right draws 0 , so for binary 1 and 0 . To activate drawing with the mouse it is sometimes necessary to click the left and right button alternately. To accept the rule-table enter return. Once accepted, the rule is also displayed in decimal (if applicable), in hex, and in the rule window (section 16.19).

\subsubsection{Rules: save as 1d seed or PostScript}

The rule-table can be saved as a vector PostScript image, or as a 1d seed file, by selecting PScript--P or file-F in section 16.4.1 — both options follow the same methods as the $1 \mathrm{~d}$ seed options in section 21.4.7. The following prompts appear in a top-right window,

for PostScript, PScript-P

PostScript RCODE: save all-a, save patch-p: (or $k C O D E, T C O D E$ )

for a $1 d$ seed file, file-F

KCODE as 1d SEED: save all-a, save patch-p: (or RCODE, TCODE)

Enter $\mathbf{a}$ for the whole rule-table. If $\mathbf{p}$ is entered, successive prompts are presented in a topright window to define a patch - the default is set by the last two mouse clicks on the rule-table. The mouse click index shows up in the inset panel in section 16.4.1. This patch prompt example is for $v 8 k 3$ rcode,

1d: $\max \mathrm{i}=511$, revise coords-ret, accept patch 71-278-p: (patch = last 2 mouse clicks) start i: end i: (... if ret was entered above)

Enter $\mathbf{p}$ to accept the default patch. Enter return to set the start/end values manually - the defaults are the mouse click coordinates. 


\subsubsection{Rules: PostScript prompt}

When creating a PostScript image of a rule-table, there are a variety of presentation possibilities as illustrated in this chapter, and in section 21.4.9 for a seed where similar methods apply.

Enter $\mathbf{a}$ (or $\mathbf{p}$ for part of the rule-table) at the prompt in section 16.4.3 to save the rule-table image as a vector PostScript (*.ps) file (default filename my_sPS.ps). Various top-right options will be presented for the exact appearance of the image. This example is for $v=8$ RCODE,

\section{create a PostScript image for $1 \mathrm{~d}, 64 \times 8$ \\ symbols-s greyscale-g color-c exit-q (def-c):}

These options are summarized below (then subsequent options continue),

options.... what they mean

symbols-s ... to show values as symbols (as in figures $21.7,21.8$ for a seed).

greyscale-g ... to show values in greyscale (as in figures 21.7 for a seed).

color-c ... (the default) to show values in color.

Once these options have been selected, the prompt to amend other settings is presented,

cellscale $=\mathbf{5 . 0 0} \operatorname{dots}($ on $)=\mathbf{0 . 7 0} \operatorname{divs}(\mathrm{off})$, amend settings-a: (for example)

Enter return to accept the defaults, or enter a for the following prompts presented in sequence,

change: cellscale: togdots-x: dotscale: $\operatorname{divs}(0,1,2)$ :

Enter changes required or return to accept defaults, which follow the current bits/values presentation. The options are summarized below,

$$
\begin{aligned}
\frac{\text { options }}{\text { cellscale }} \ldots & \frac{\text { what they mean }}{\text { enter a new cell width in pixels. }} \\
\text { togdots- } \mathbf{x} \ldots & \text { to toggle zero dots on/off. } \\
\text { dotscale } \ldots & \text { (if dots are on) enter a new width for zero dots in pixels, which can be a } \\
& \text { decimal number. } \\
\operatorname{divs}(\mathbf{0 , 1 , 2}) \ldots & \text { the initial default division (color) depends on the bits/values presentation, } \\
& \text { and is shown in the prompt: (none, } \mathbf{1}, \mathbf{2} \text { ) where none means that there } \\
& \text { is no division and adjoining cells touch. To change, enter } \mathbf{0} \text { for none, } \mathbf{1} \text { for } \\
& \text { black, and } \mathbf{2} \text { for white. The new designation becomes the default. }
\end{aligned}
$$

Cells with value zero (0color) are initially colored light green in the bits/values presentation (section 16.4), which can be togged to white with 0color- $\wedge$ in section 16.4.1 - the PostScript file will follow the current 0color.

Once these choices are complete, the *.ps file is saved from the filing prompt (section 35.2). The default filename is my_sPS.ps, the same as for a 1d space-time pattern. section 36.1 explains how to view, edit, and crop the PostScript image. 


\subsection{Setting the rule in hex}

If $\mathbf{h}$ is selected in section 16, the rule is defined in hexadecimal (hex), which is a shorter way of denoting a rule than the rule-table itself. The method is the same as setting a seed in hex in section 21.5. The hex expression of the current rule (initially just $0 \mathrm{~s}$ ) is displayed. Each hex character $(0-9$ and $\mathrm{a}-\mathrm{f})$ shows the value of 4 bits, and the characters are displayed in one byte pairs. During the hex setting procedure, a top-right reminder window displays the following,

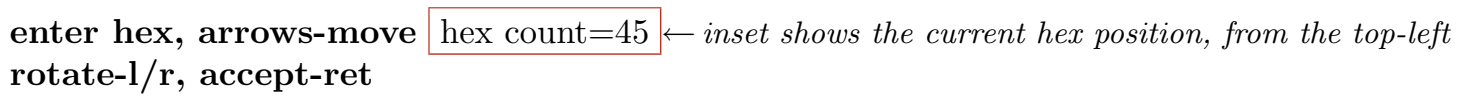

The hex character to be updated is highlighted by a flashing cursor, initially in the top-left. Its position is displayed in the top-right inset window. The flashing cursor is moved with the arrow keys, left/right and up/down for more than one hex line. To overwrite, enter a hex character from the keyboard, without return. This automatically moves the cursor one position to the right. Hex characters entered which exceed the current value-range will be automatically corrected downwards to the maximum value after the hex string has been accepted. Enter $\mathbf{l}$ or $\mathbf{r}$ to rotate the rule-table by one bit or value as in sections 16.3 and 16.4.1, which will be immediately reflected in the hex presentation. To accept the rule as expressed in hex, enter return, whereupon the rule will be presented as bits/values (section 16.4), where it can be reconfirmed or amended.

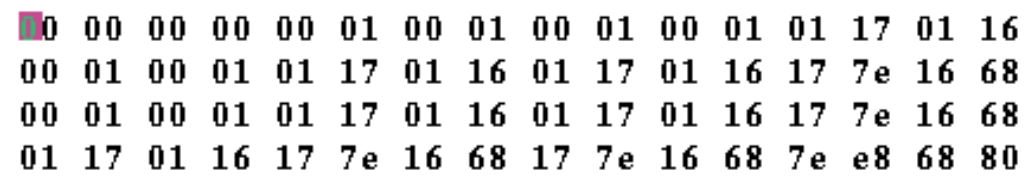

Figure 16.4: Setting rcode in hex, showing the "game-of-Life", $v 2 k 9$.

\subsection{Setting the rule in decimal}

applicable only for a limited range of $v$ and rule-table size $S$

The decimal option is useful for binary rules with small neighborhoods, such as the $v 2 k 3$ "elementary rules" with their well known decimal rule numbers[22, 25] or for $v=2$ tolalistic rules. However the decimal option remains valid so long as the rule-table (rcode, kcode or vcode) is within the limits ${ }^{5}$ in table 16.1 which lists the maximum length of the bit/value string $S$ and the corresponding maximum decimal rule number, for each value-range $v$. If $\mathbf{d}$ is an available option and is selected in section 16.1, the rule can be specified by its decimal equivalent. The following prompt is displayed,

$$
\text { k=3 rule, enter 0-255 (def-rnd-dec): (for example) }
$$

Enter a decimal number, or return for a random number which will be displayed. If the number entered is outside the permitted range, the program presents the message,

\footnotetext{
${ }^{5}$ The same limits apply when setting a seed in decimal (table 21.1 section 21.6).
} 


\begin{tabular}{c|c|c}
$v$ & max- $S$ & max decimal \\
\hline 2 & 32 & 4294967295 \\
\hline 3 & 20 & 3486784400 \\
\hline 4 & 16 & 4294967295 \\
\hline 5 & 13 & 1220703124 \\
\hline 6 & 12 & 2176782335 \\
\hline 7 & 11 & 1977326742 \\
\hline 8 & 10 & 1073741823
\end{tabular}

Table 16.1: Rules in decimal - rule-table size limilations - the maximum bit/value string $S$ for each value-range $v$, and the corresponding maximum decimal number.

k=3 rule, enter 0-255 (def-rnd-dec):333 - too big! back-ret: (for example)

Enter return to revert to the first single rule prompt (section 16.1). Once successfully selected, the decimal rule is presented again as bits/values (section 16.4), where it can be reconfirmed or amended.

\subsection{Setting a majority rule}

If $\mathbf{m}$ is selected, in section 16.1, the "majority" (voting) rule will be set — an example was shown in see figure 2.2. The algorithm differs between rcode, kcode and tcode, and also between $v=2$ and $v>2$. For rcode or kcode the majority value in the neighborhood becomes the output.

In case of a tie,

- for rcode and $v=2$ the central cell wins, as before in the old binary version of DDLab.

- otherwise for kcode or $v>2$ one of the majority values is picked at random (see figure 16.6).

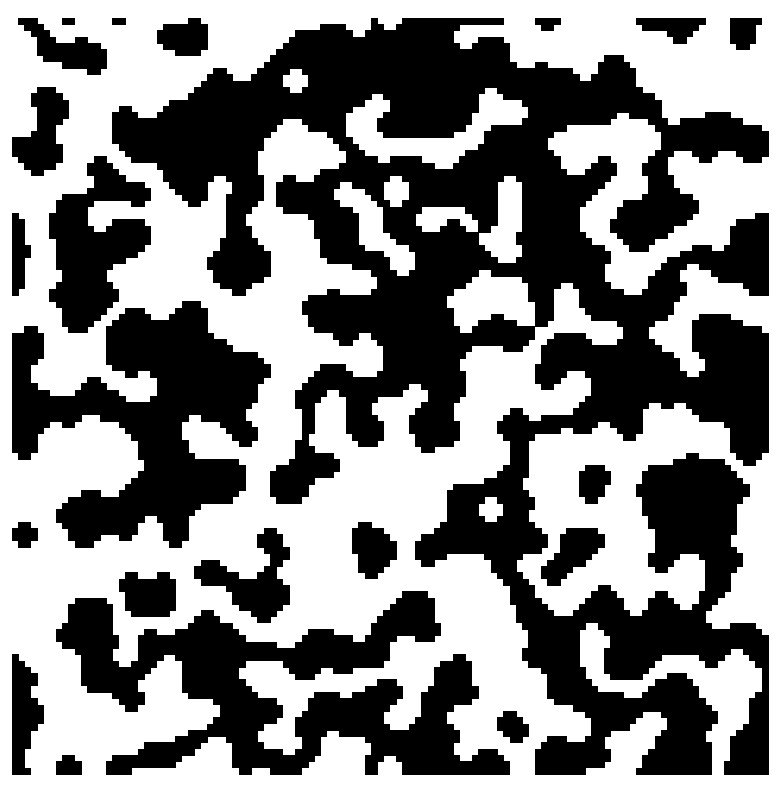

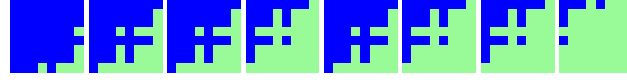

Figure 16.5:

Above: Majority rcode $v 2 k 9$, shown as a bit pattern of $S=512$ bits.

Left: Space-time snapshot from a random initial state on a $120 \times 120$ square lattice, where the dynamics has stabilized on an attractor. 


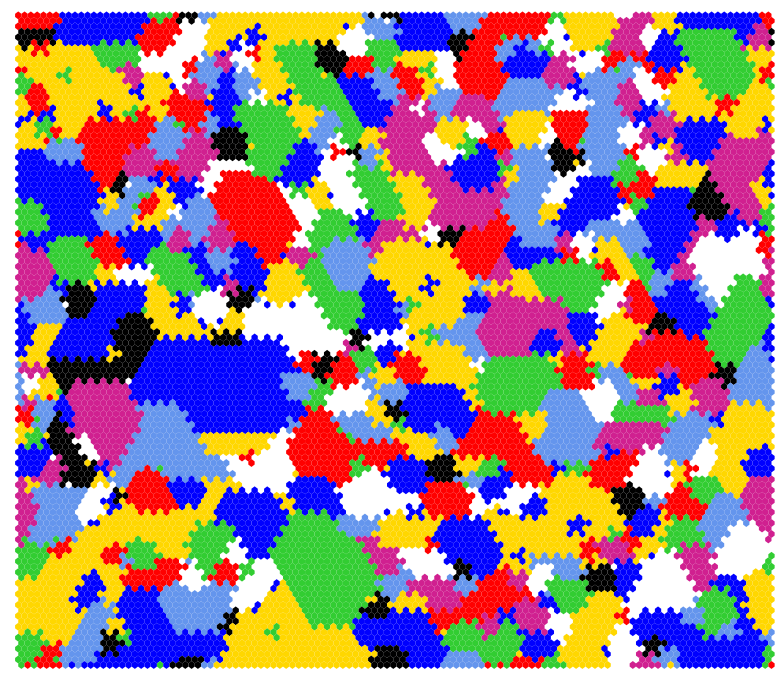

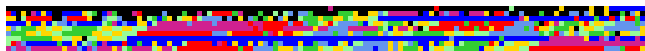

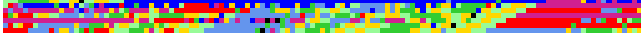

Figure 16.6:

Above: Majority kcode $v 8 k 6$ shown as a value pattern of $S=1716$ values.

Left: Space-time snapshot from a random initial state on a $120 \times 120$ hexagonal lattice, where the dynamics has stabilized on an attractor.

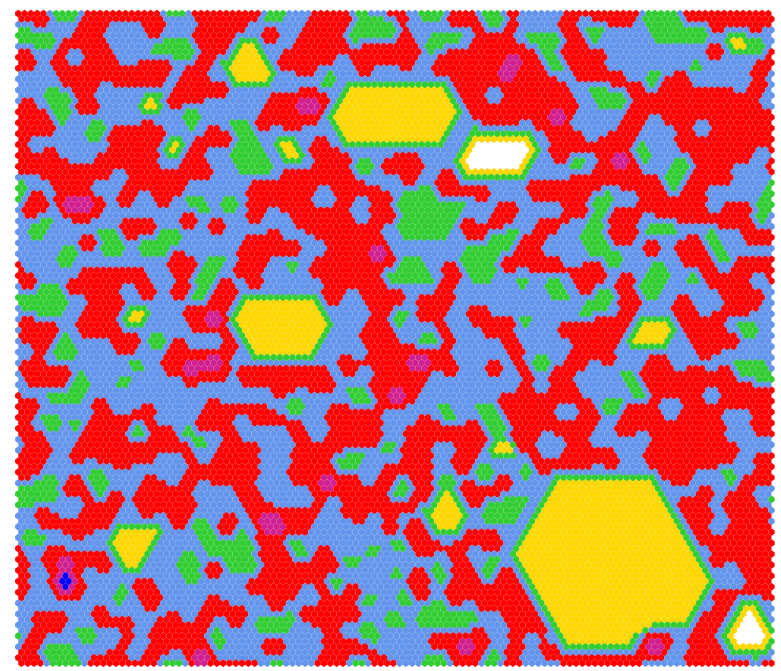

Figure 16.7:

Above: Majority tcode $v 8 k 7$ shown as a value pattern of $S=50$ values.

Left: Space-time snapshot from a random initial state on a $120 \times 120$ hexagonal lattice, where the dynamics evolves into competing patches.

For tcode, the tcode-table is divided into $v$ approximately equal sectors, and the values are allocated to the sectors in decending order from the left (see figure 16.7). Once selected, the majority rule will be presented again as bits/values (section 16.4), where it can be reconfirmed or amended.

Figures $16.5-16.7$, and 4.15. show examples of the majority rule bit/value pattern, and evolved snapshots of $2 \mathrm{~d}$ spacetime patterns, for rcode, kcode and tcode. 


\subsection{Majority with shifted uniform outputs}

not for tcode - see also section 14.8 for a rulemix

If $\mathbf{u}$ is selected in section 16.1 a "majority" (voting) rule is set as in section 16.7 above, but the uniform (unanimous) neighborhoods have their outputs shifted by -1 , except for 0 which becomes $v$-1. For $v=2$ this is the same as flipping the "end bits", as before in binary DDLab, so that unanimity gives the opposite vote, otherwise the majority wins - the tcode is 011001 .

For random wiring, this can result in some interesting bi-stable, tri-stable, $v$-stable, dynamics, for example in figure 16.8 for $v=2$, and for a rulemix of kcode majority rules, $v=8$, in figure 14.4.

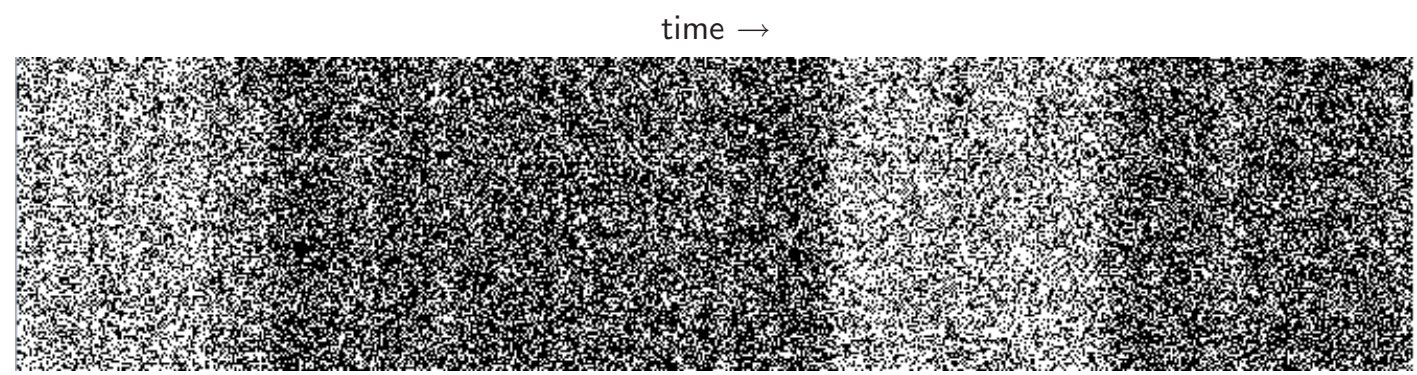

Figure 16.8: Flipped (shifted) majority rcode with random wiring, $v 2 k 5$, where uniform neighborhoods (all-0s and all-1s) have their outputs flipped to the opposite color. A 1d space-time pattern $(n=150)$ shows bi-stable pattern density, where the duration of the two density regimes is unpredictable. The image shown is rotated by $90^{\circ}$ so the time flows from left to right.

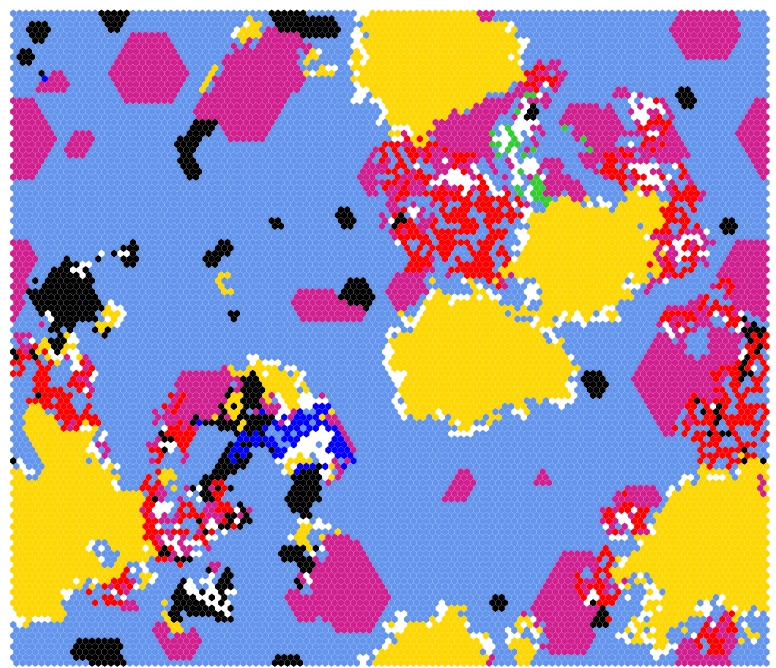

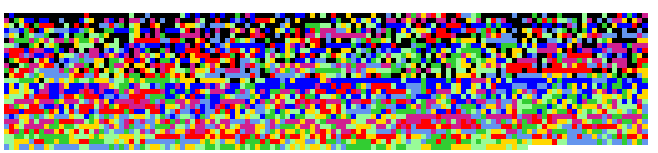

Figure 16.9:

Above: Altenberg kcode example $v 8 k 7$, shown as a value pattern of $S=3432$ values.

Left: Evolved space-time snapshot from a random initial state on a $120 \times 120$ hexagonal lattice. 


\subsection{Setting Altenberg rules not applicable to tcode}

Enter A to set an "Altenberg" rule (suggested by Lee Altenberg) where the output of each neighborhood is set probabilistically according to the frequency of the values in that neighborhood. This is a sort of probabilistic majority rule, and results in mobile ordered zones in the dynamics. An example kcode and $2 \mathrm{~d}$ snapshot is shown in figure 16.9, and a $1 \mathrm{~d}$ space-time pattern in figure 4.11.

\subsection{The game-of-Life and other Life-like rules — rcode for rcode and $k \geq 5$ - (for Life in outer-kcode, TFO-mode, see section 14.2.2)}

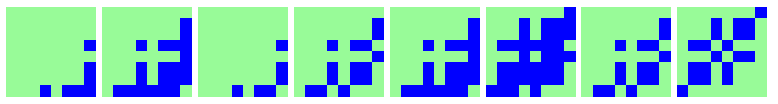

Figure 16.10: Conway's game-of-Life (rule 23/3) shown as a 512 bit rcode.

For $v 2 k 9$ on a square lattice, and the Moore neighborhood 㗊 - John Conway's game-of-Life[6] can be set - see figures 4.12 and 4.14 for examples of space-time patterns. Alternatively any other Life-like rule from the "Life family" can also be set, with different values of $v$ and $k$.

The Life-like rules can also be set in outer-kcode in TFO-mode (section 14.2.2 and figure 14.1), which allows a greater range of neighborhoods sizes, up to $k=25$ in $1 \mathrm{~d}$ and $2 \mathrm{~d}$, but this section describes the method for the full rule-table, rcode.

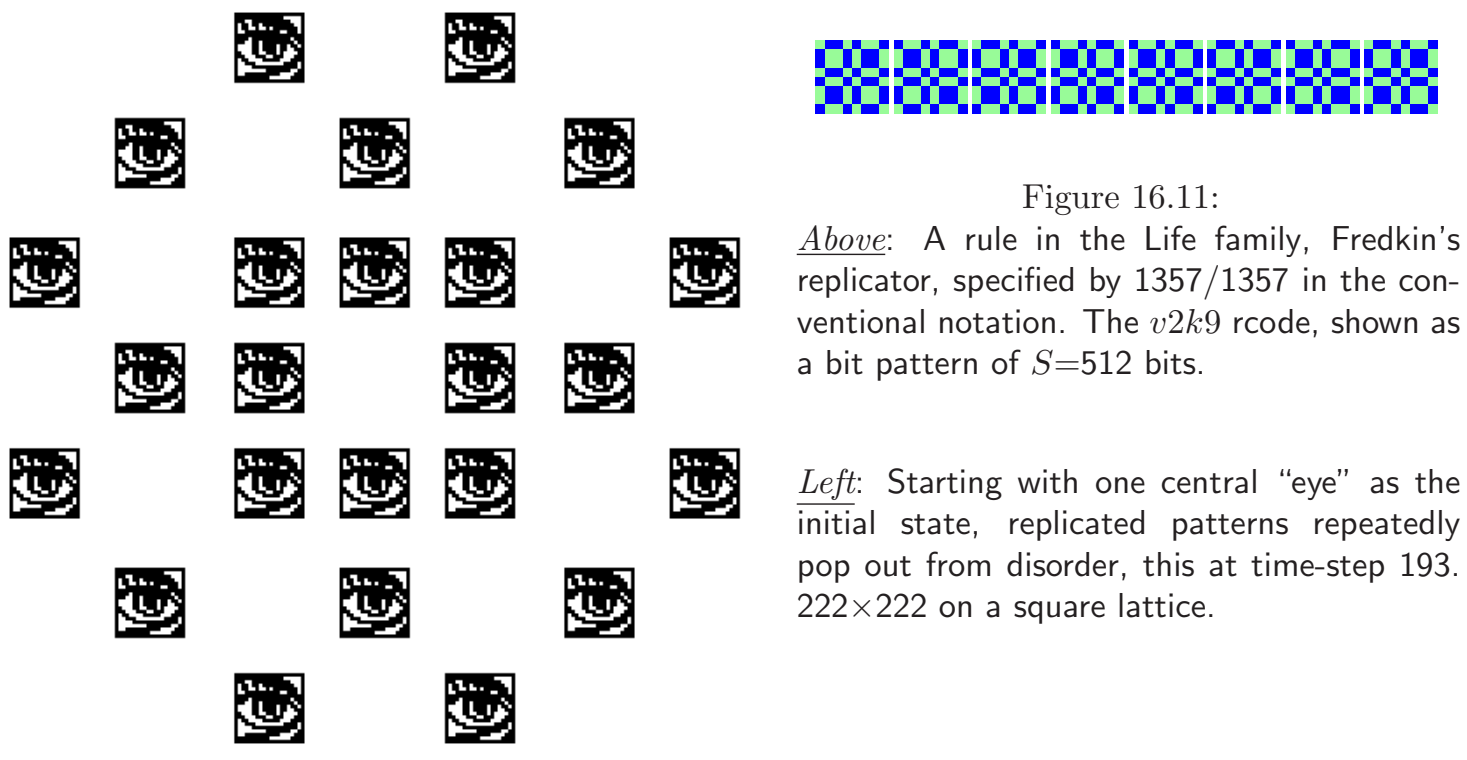


Classical Life is specified as (23/3) in Mirek's Cellebration notation ${ }^{6}$. A cell is either alive (1) or dead/empty(0). The first part of $(23 / 3)$ defines the survival of a cell, requiring 2 or 3 live neighbors, the second part of $(23 / 3)$ defines birth, requiring 3 live neighbors, otherwise the cell is dead (by overcrowding or exposure). This notation, when entered in DDLab, is automatically translated into the full lookup-table, rcode (figure 16.10). Any other Life-like rule can be specified in this notation, for example 1357/1357 for Fredkin's replicator in figure 16.11.

\subsubsection{Setting Life-like rules — rcode}

When $\mathbf{L}$ is entered in section 16.1 the following top-right prompt is presented,

Life $\mathbf{k}=\mathbf{9}$ (def: survival 2,3, birth 3,) accept-ret amend-a: for $k=9$

Enter return to accept the default. If $\mathbf{a}$ is entered to amend, the following further prompts are presented,

accept-ret, or enter number + ret, $\max$ entries $=9$, $\max$ value $=8$ :

enter survival $(\mathbf{d e f}=\mathbf{2 . 3}$,$) : followed by ...$

enter birth $(\operatorname{def}=3$,$) :$

Enter the new values for survival, followed by the new values for birth. After each number enter return for the next number. There may be up to $k$ entries - their order, or repeats, are not significant. return without a number concludes the entries. q reverts to the first Life prompt, but with the defaults possibly altered.

The Life option is available for $v \geq 2$, and any $k \geq 5$ as well as the $k=9$ neighborhood, for $1 \mathrm{~d}$ and $3 \mathrm{~d}$ as well as $2 \mathrm{~d}$, and for a rulemix by hand. For $v>2$, for a given Life setting, the algorithm in DDLab generates an equivalent rcode giving the same dynamics as binary Life, but including $v$ colors. For example, $v=3$ and $(23 / 3)$ gives the same game-of-Life dynamics but with two colors plus the background, as in figure 16.12 .

To make changes to particular bits/values in Life-like rule-tables, amend bits or values as in section 16.4.

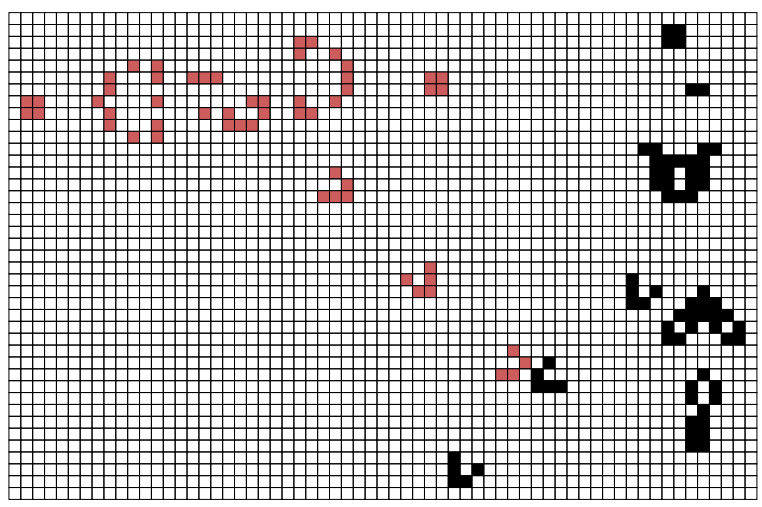

${ }^{6}$ http://en.wikipedia.org/wiki/Life-like_cellular_automaton
Figure 16.12:

The game-of-Life $(23 / 3)$ appled to a $v=3$ CA. The algorithm in DDLab generates an equivalent rcode giving similar dynamics to classical binary Life, but including 2 colors + background. In this example two glider guns have been constructed, one shooting black gliders SE, the other shooting red gliders SW. The seed file is Lguns_v3. eed. 


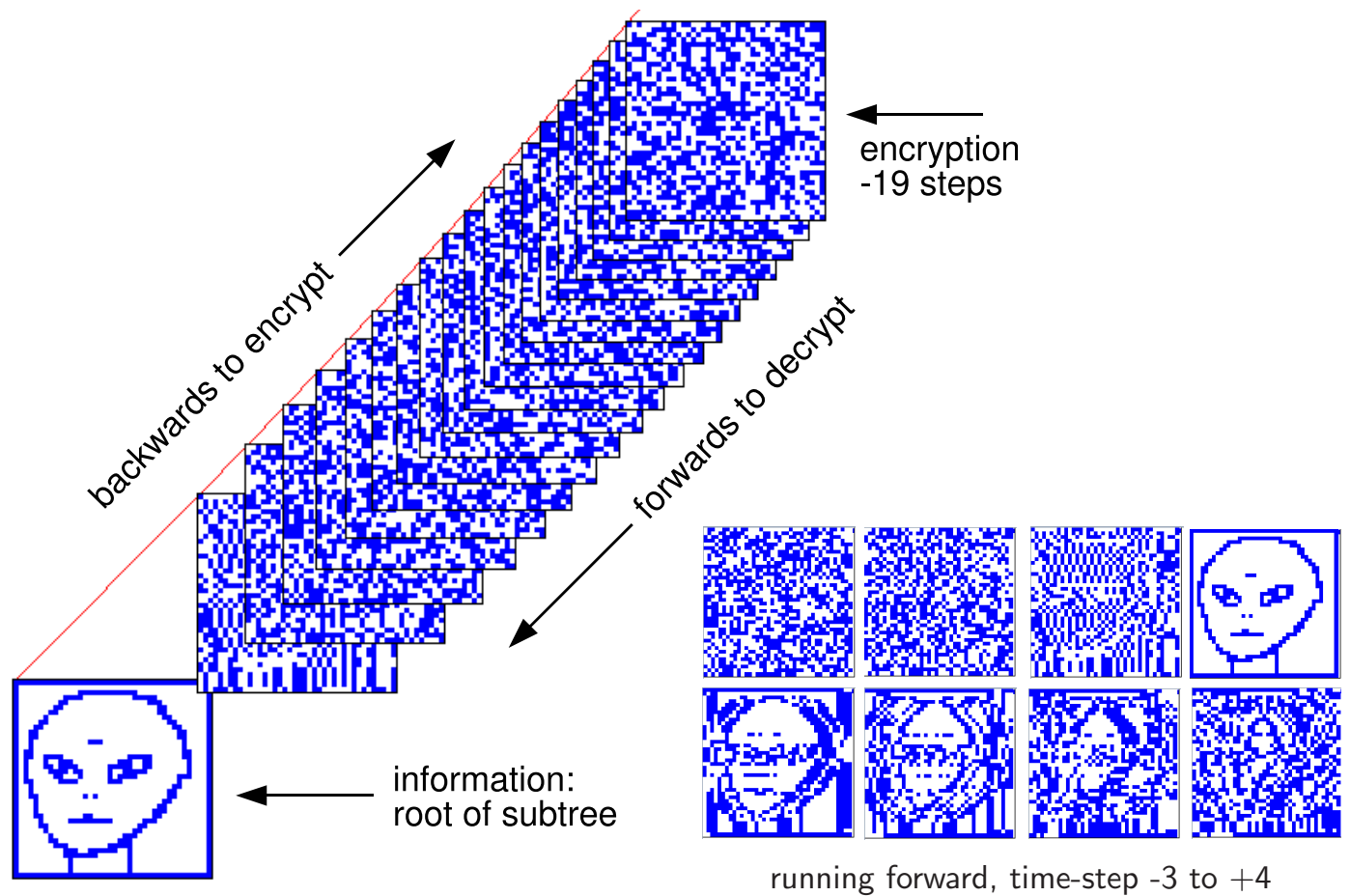

Figure 16.13: Left: a subtree applied to encrypt information[41] at its root state (the "alien" seed), set

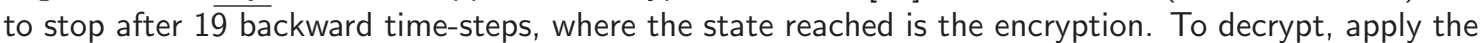
same rule to run forwards by the same number of time-steps. Right: time-steps before and after the alien suddenly pops out, then merges back into chaos. The root state is a $1 \mathrm{~d}$ bit-pattern, here displayed in $2 \mathrm{~d}(n=1600,40 \times 40)$. The alien seed was drawn with the drawing function in DDLab (section 21.4). The seed could also be an ASCII file, or any other form of information. A $v 2 k 7$ chain-rule (rcode) was set at random, and the subtree was generated with the CA reverse algorithm. Note that the subtree has not branched - branching is highly unlikely because of the large system size.

\subsection{Setting a chain-rule}

rcode (full rule-table) only

Enter $\mathbf{c}$ in section 16.1 to set a chain-rule.

Chain-rules are maximally chaotic rules, where $Z_{\text {left }}=1$ or $Z_{\text {right }}=1$, but not both. The global dynamics of chain-rules exhibit extremely low convergence in subtrees. For larger systems, states usually have just one pre-image, so subtrees form a "chain". Garden-of-Eden density becomes order zero with increasing system size. Chain-rules comprise approximately the square root of rule-space (figure 24.10), and the CA reverse algorithm is especially efficient for generating their subtrees. These characteristics make chain-rules suitable for encryption[41], for example, by running "information" backwards to encrypt, forwards to decrypt as in figure 16.13. The methods apply equally for $v>2$. 


\subsection{Setting reaction-diffusion - rcode for reaction-diffusion by by outer-kcode see section 14.2.1}

Enter $\mathbf{R}$ in secton 16.1 above to initiate a reaction-diffusion rule with the full rule-table - rcode (sections 13.8, 13.8.2 and figure 13.3). Then set the threshold interval as described in section 13.8.3. Section 14.2.1 describes an alternative method for reaction-diffusion from outer-kcode, which allows greater $[v, k]$.

\subsection{Repeating the last rule}

If $\mathbf{p}$ is selected in section 16 (for a single rule - not part of a rulemix), the last rule that was set for the given $[v, k]$ is repeated by automatically loading the "last rule" file with the same $v, k$ and rule type parameters (section 16.15). The file would have been automatically saved when the last rcode, kcode or tcode was selected. Once selected, the repeated rule is presented again as bits/values (section 16.4), where it can be reconfirmed or amended.

In a rulemix, where a sequence of rules is entered by hand (see section 14.6), the "last rule" file is not relevant - selecting $\mathbf{p}$ repeats the previously entered rule from RAM. For a $k$-mix the last rule with the current $k$ is repeated.

\subsection{Loading a single rule}

If $\mathbf{l}$ is selected in section 16, a top-right filing prompt (section 35.2) is presented allowing a single rule to be loaded from a file. The defaults are myrul_v $v$.rul for rcode, myvco_v $v$ vco for kcode, and mytco_v $v$.tco for tcode - for example myrul_v2.rul, if the value-range, $v=2$. If successfully loaded, the rule is presented again as a bit/value pattern (section 16.4) where it can be reconfirmed or amended.

Note that both the value-range $v$ and neighborhood size $k$ in the file must be the same as in the single rule current (base) setup. If not, error messages will appear as described in section 35.3. However, a single rule file can be loaded at a give position in mixed rule network provided file- $k$ $\leq$ base- $k$ - enter $\mathbf{v}$ from the wiring graphic, section 17.4.

\subsection{Automatic saving of last rule}

After a single rule has been set, it will be automatically saved as the "last rule" of that rule type, with a different filename for rcode, kcode or tcode, and $[v, k]$. The "last rule" filename has the following format: $1_{-} \mathrm{v} x \mathrm{k} y$. ext where the extension is .rul for rcode, . vco for kcode and .tco for tcode, for example, 1_v4k5.rul. 


\subsection{Single rule file encoding}

The binary file defining a single rule is encoded ${ }^{7}$ starting with 2 leading bytes as follows: Byte 0 $=$ value-range $v$, byte $1=$ neighborhood $k$. The rest of the rule-table size $S$ (from 0 to $S$-1) is set as bits in successive bytes.

$S$ depends on the rule type, $v$ and $k$ as follows:

$$
\begin{aligned}
& \operatorname{rcode} \ldots \quad S=v^{k} \\
& \operatorname{kcode} \ldots \quad S=(v+k-1) ! /(k !(v-1) !) \\
& \operatorname{kcode} \ldots \quad S=v \times k
\end{aligned}
$$

The number of bits required for each rule-table output $\left(v_{b i t s}\right)$ is as follows:

$$
\begin{aligned}
& v=2 \ldots 1 \text { bit }\left(v_{b i t s}=1\right) \text { as in the old binary version of DDLab. } \\
& v=3 \text { or } 4 \ldots 2 \text { bits }\left(v_{\text {bits }}=2\right) \\
& v=5,6,7,8 \ldots 3 \text { bits }\left(v_{\text {bits }}=3\right)
\end{aligned}
$$

A single rule encoding therefore requires 2 leading bytes plus the rule-table bytes $R$, where $R=\left\lceil\frac{S \times v_{b i t s}}{8}\right\rceil$ bytes, the upper absolute value, minimum 1 byte.

\subsection{Create a similar kcode with increased $k$} only for kcode in TFO-mode

Enter $\mathbf{k}$ in section 16.1.2 to create and save a kcode with an increased neigborhood $(k+)$ by inserting a random string of the correct length centrally within the current kcode. This may preserve some aspects of the original dynamics, as in figure 16.14. The following top-right prompt is presented,

create similar to $\mathbf{k} 6 \mathbf{k c o d e}$ and save, enter greater $\mathbf{k}(\mathbf{7 - 2 5})$ : (if the current $k=6$ )

The two rules are printed in the terminal as shown below - the inserted random string of length 8 is indicated.

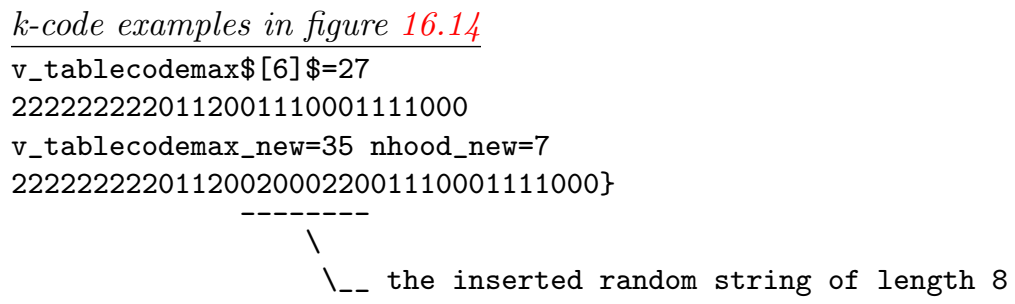

\footnotetext{
${ }^{7}$ In the binary version of DDLab, the old style encoding started with the neighborhood $k$ at byte 0 . In the new style encoding byte 0 is reserved for the value-range $v$, and subsequent bytes are displaced by one. Old style files are nevertheless compatible for loading in the present version of DDLab. For wiring+rulemix encoding see section 19.3 and for seed encoding see section 21.9 .
} 


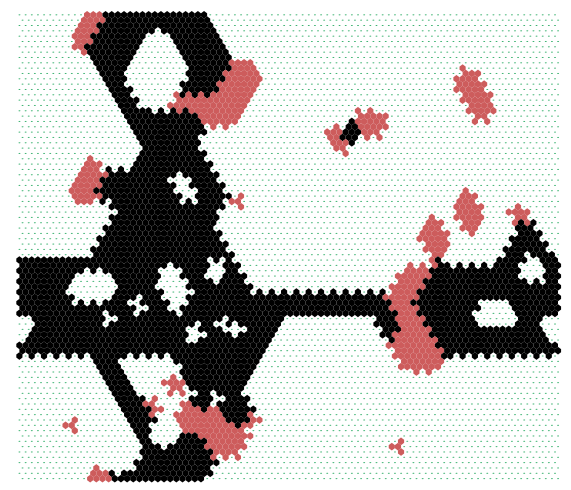

example for $v 3 k 6$ majority kcode

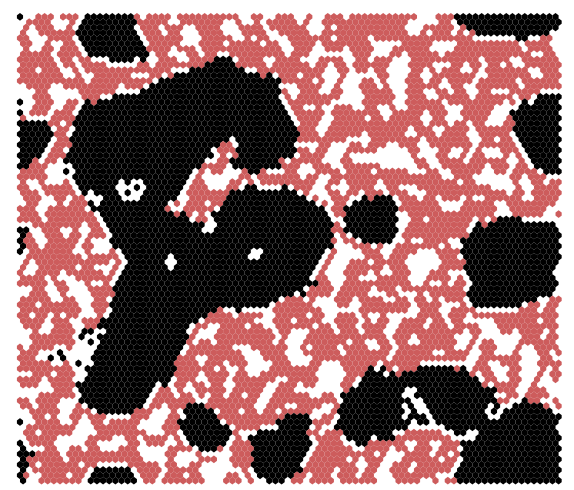

example for analogous $v 3 k 7$ kcode

Figure 16.14: The effect of inserting a random string within a $v 3 k 6$ kcode majority rule to create an analogous $v 3 k 7$ kcode. The figures show space-time pattern snapshots from random initial states on a $88 \times 88$ hexagonal lattice. Some aspects of the majority behavior are retained in $k 7$. Note that there are many possibilities for both examples - the actual kcodes are as shown in this section.

The new string is automatically saved as the last kcode (1_v $x \mathrm{k} y \cdot \mathrm{vco})$, for example $1 \_\mathrm{v} 3 \mathrm{k} 7 . \mathrm{vco}$, which can be loaded with repeat-p after backtracking and re-selecting the new $k$. The program reverts to the prompt in section 16.1 .

\subsection{Show the rule in the terminal}

Select $\mathbf{x}$ in section 16.1 to show the rule data immediately in the terminal, and to present a top-right prompt (section 16.18.3) for the rule data in more detail. For kcode, this includes a further option to swap values (colors) to make an equivalent kcode (section 16.18.5).

\subsubsection{Immediate rule data}

Immediate rule data will appear in the terminal as in table 16.2 (including the cell index for a rulemix),

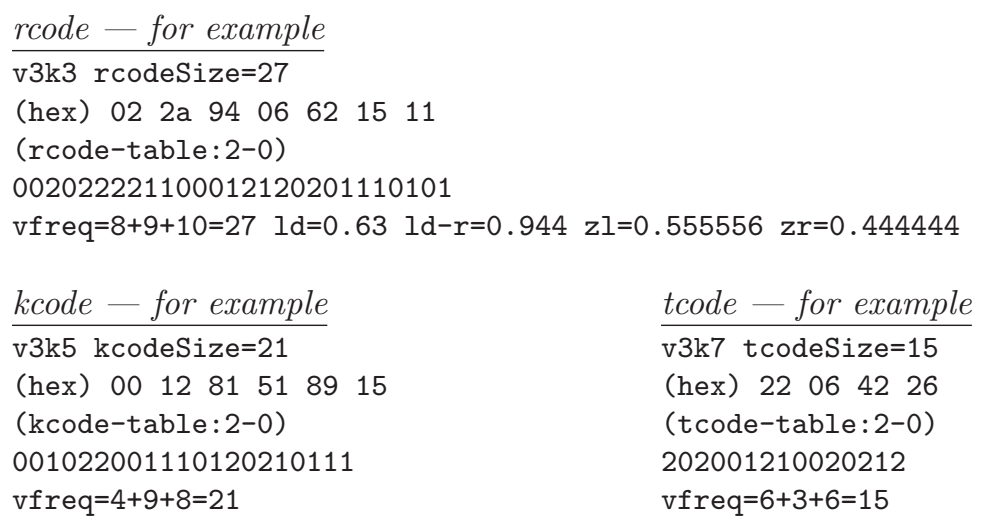

tcode - for example
v3k7 tcodeSize $=15$
(hex) 22064226
(tcode-table:2-0)
202001210020212
vfreq $=6+3+6=15$

Table 16.2: Examples of the immediate rule data in the terminal (xterm) for rcode, kcode and tcode. 


\subsubsection{Immediate rule data for a rulemix by hand}

If a rulemix is being set by hand in section 14.6 (including a rule-subset, or outer-totalistic), enter $\mathbf{x}$ in section 14.6.3 to show the immediate rule data for the last rule defined. The cell index will be added to the data as in the example below,

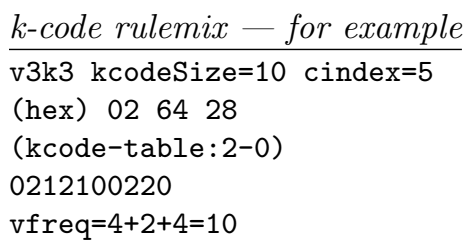

\subsubsection{Rule data in more detail - vertical layout}

Simultaneously with the immediate rule data, a prompt is presented for more detail, showing in particular how the neighborhoods and the neighborhood index relates to the rule-table outputs in a vertical layout. For kcode there are additional options for a horizontal layout, and for a matrix layout if the value-range $v=3$.

$$
\begin{aligned}
& \text { for rcode or tcode }- \text { for example } \\
& \text { print rcode-table(S=19) to xterm (vert)-v: (or tcode-table) } \\
& \text { for } k \text { code - matrix- } m \text { if } v=3 \text { only - for example } \\
& \text { swap values-s, print kcode-table(S-21) to xterm (vert } / \text { horiz/matrix)-v } / \mathbf{h} / \mathbf{m} \text { : }
\end{aligned}
$$

Enter $\mathbf{v}$ to show the rule-table details with a vertical layout - the same rules as in table 16.2.

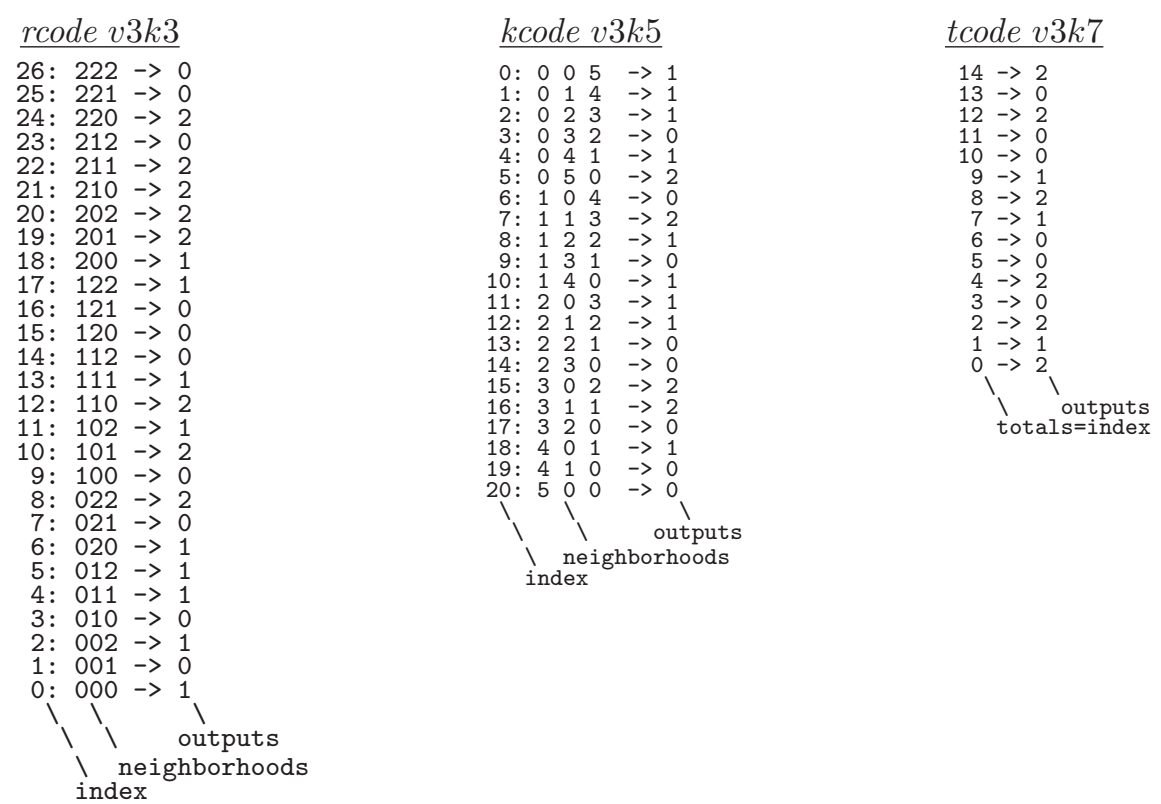

Table 16.3: Examples of the vertical layout for rcode, kcode and tcode, with explanatory notes, for the same rules as table 16.2. 

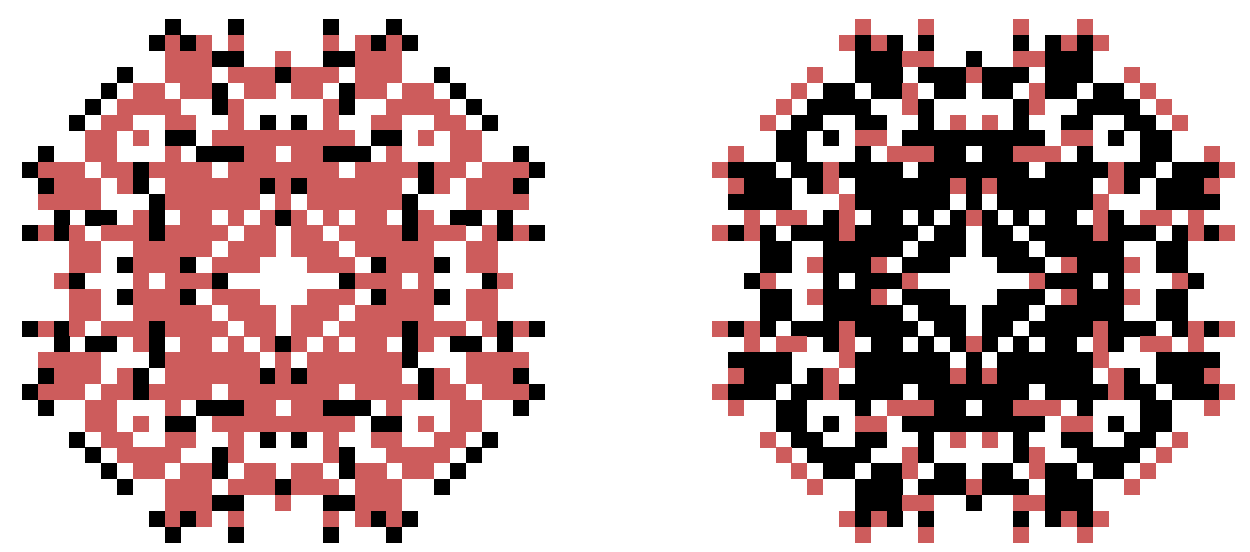

Figure 16.15: Space-time snapshots of $2 \mathrm{~d} C \mathrm{CA}$, with two equivalent $v 3 k 6$ kcodes, with swapped values 1 and 2 (red and black). The initial state was a single central cell on a $40 \times 40$ square lattice. The snapshot was taken at time-step 54.

as rules are transformed or mutated. Examples of rule windows and their data are shown in figure 16.16, and decoded in section 16.19.1.

full rule-table

v2 23 rcode(dec) 110 (hex) $6 \mathrm{e}$

$1 \mathrm{~d}$ size $=14 \mathrm{ld}=0.625 \mathrm{ld}-\mathrm{r}=0.75 \mathrm{P}=0.625 \mathrm{zl}=0.75 \mathrm{zr}=0.625 \mathrm{Z}=0.75 \mathrm{C}=0 / 3$

full rule-table setting kcode, so both rcode and kcode are shown

v4k5 rcode(hex) 470345 f4 030922 da $45224 c 63$ f4 da 632 d 030922 da 0939 al 5422 al 06 9a da 549 a 8 a $45224 c 6322$ al $069 a$ kcode(hex) $=47$ 0d $424 a$ cd e4 46 ab 54 fa a6 80 de $6 \mathrm{c}$

1d size $=14 \mathrm{ld}=0.773 \mathrm{ld}-\mathrm{r}=0.826 \mathrm{zl}=0.458 \mathrm{zr}=0.458 \mathrm{Z}=0.458008 \mathrm{C}=0 / 5$

kcode in TFO-mode and $2 d$

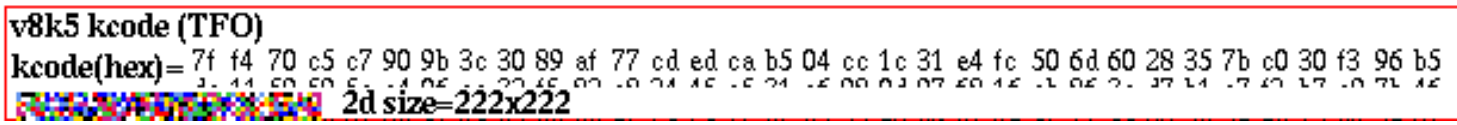

Figure 16.16: Rule window examples, images grabbed from the screen.

\subsubsection{Decoding the rule window}

The abbreviated headings in this example of the rule window are explained below,

-1.- (the rcode - look-up table shown as a bit pattern)

$v 2 k 3$ rcode(dec) 186 (hex)ba (values shown are examples)

$1 \mathrm{~d}$ size $=14 \mathrm{ld}=0.625 \mathrm{ld}-\mathrm{r}=0.75 \mathrm{P}=0.625 \mathrm{zl}=0.75 \mathrm{zr}=0.25 \mathrm{Z}=0.75 \mathrm{C}=1 / 3 * * 0$ Post $=\mathrm{A}[0] \mathrm{i}$ 


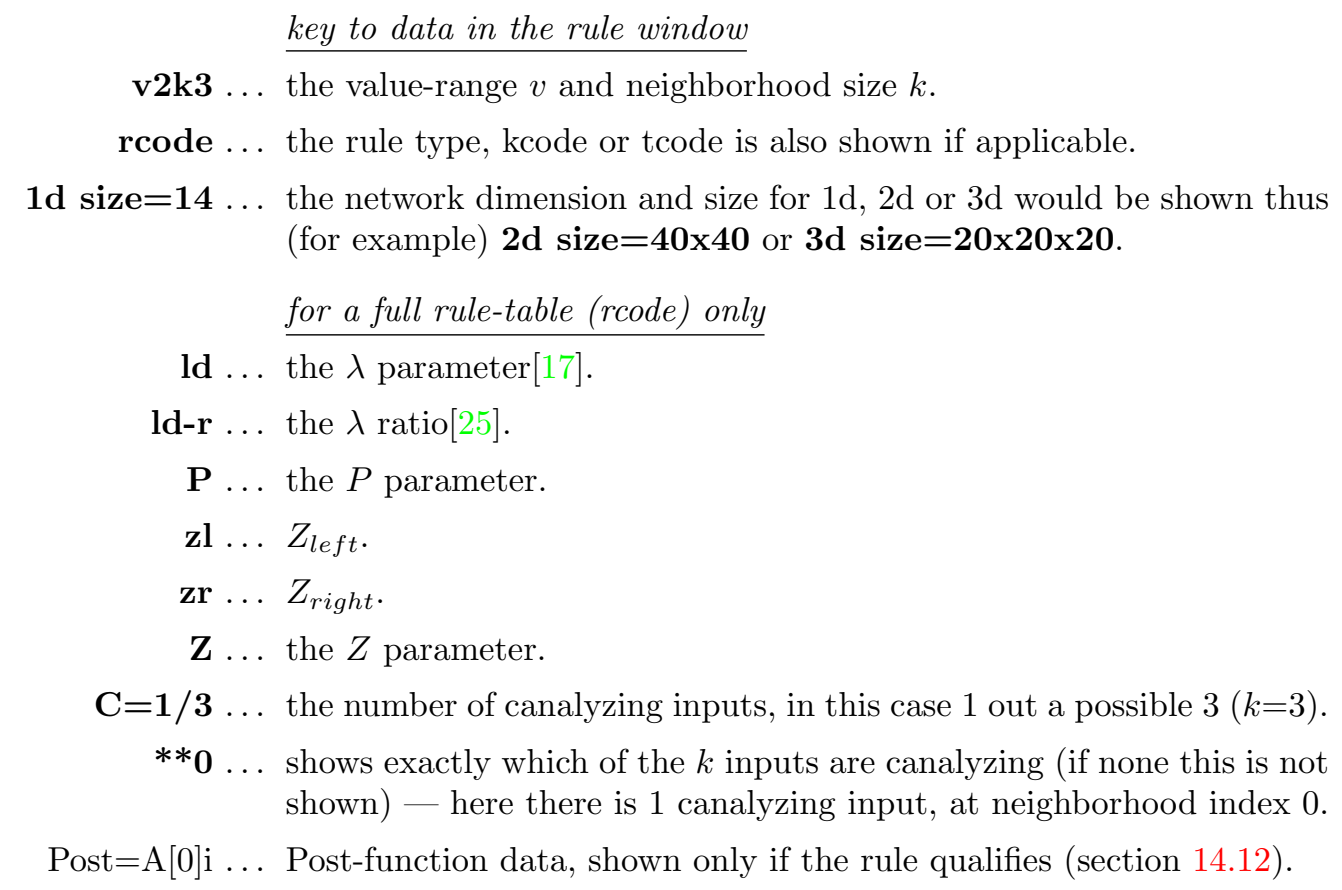

\subsection{Complementary values}

Various rule and seed setting methods include an option to complement values, for example comp-m in sections 16.3, 16.4, 18.5.1 and 21.4.

Transforming a value into its complement is simple for binary $(v=2)$ - change 1 s to 0 s and vice versa. For any value-range $v$ the "complement" of a value in DDLab is defined as follows: each value $a$ is changed to its complement $a_{c}$ by subtracting $a$ from the maximum value $v-1$, so $a_{c}=(v-1)-a$. This satisfies binary, and for any $v$ the maximum value is the complement of zero. For odd $v$ this means that the "central" value does not change.

The values are therefore swapped as follows,

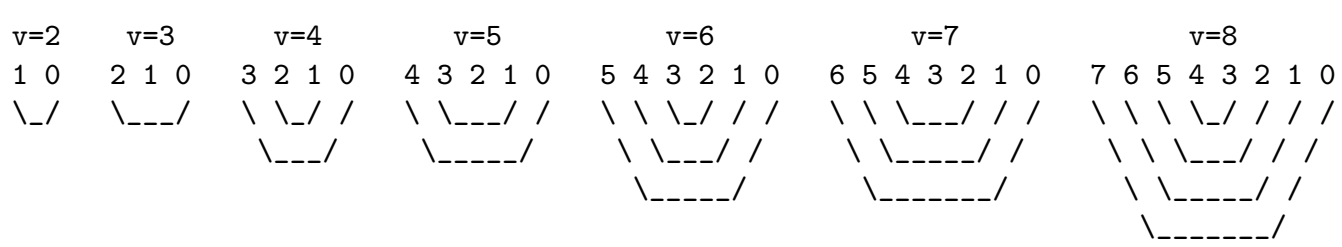

Complementary values as defined apply to seed options (section 21.4.2) as well as rules. 


\subsection{Transforming the single rule}

full rule-tables (rcode) only - not TFO-mode

For full rule-tables (rcode) only, including kcode and tcode expressed as rcode, single rules that have been set can be transformed in various ways. Here is an example of the top-right prompt, though it will vary according to context, presented once a single rule has been selected,

transform rcode: solid-o invert-v comp-m neg-n ref-r, canal-C:

equiv greater $\mathbf{k}(3)$ : (4-13), eff $\mathbf{k - k}$ : (if $k=3$ )

save/prtx: rcode/kcode-s/S/x/X

The various methods for transforming rules are described in chapter 18, and the prompt may also be accessed from the wiring graphic (chapter 17), space-time pattern interrupt options (section 32.16.1) and attractor basin complete options (section 30.5). 


\section{Chapter 17}

\section{Reviewing network architecture}

This chapter describes ways of reviewing the network architecture by the following methods,

$$
\begin{aligned}
& \text { wiring matrix .... } \text { shows the wiring (and rules if set) in a table or spread- } \\
& \text { sheet format, where the wiring can be changed. }
\end{aligned}
$$

1d, 2d or 3d wiring graphic ... the most powerful method for tailoring the wiring and rules, and also seeing the details of the network.

Alternatively the network can be reviewed as a graph, described in chapter 20. The graph option does not allow changes to the underlying network, but the graph can be unravelled by dragging nodes and components, and rotated, rescaled, and many other manipulations performed. Default presentations include: circle, spiral, 1d, 2d and 3d.

The methods described in this chapter, the wiring matrix and wiring graphic, allow wiring and rules, set in chapters $10-16$, to be examined, changed, and tailored to requirements, including biased random settings to pre-defined parts of the network. These are very flexible methods, and for RBN/DDN its usually easier to set up a suitable dummy network initially, then tailor it here.

Although there are some differences in the methods for amending the network between the $1 \mathrm{~d}$, $2 \mathrm{~d}$ and $3 \mathrm{~d}$ wiring graphic formats, in most cases they are the same or similar. The $1 \mathrm{~d}$ graphic has two alternatives. Wiring can be shown between successive time-steps, or between cells arranged in a circle. Both $1 \mathrm{~d}$ methods apply whatever the native dimensions of the network. The $2 \mathrm{~d}$ graphic can be applied to both $1 \mathrm{~d}$ and $2 \mathrm{~d}$ networks. For a $3 \mathrm{~d}$ network, the wiring graphic shows a simultaneous display in both $2 \mathrm{~d}$ and $3 \mathrm{~d}(2 \mathrm{~d}+3 \mathrm{~d})$, where the $2 \mathrm{~d}$ graphic shows horizontal slices (levels) through the $3 \mathrm{~d}$ network stacked above each other.

\subsection{The network architecture prompt see also "Reviewing wiring" section 12.7}

Once special wiring (chapter 12), or both the wiring and rules, have been set, a top-right network architecture prompt is presented. The exact wording and options offered depend on other settings. The prompt can also be activated at later stages in DDLab, while drawing space-time patterns (section 32.16) or attractor basins (sections 30.4, 30.5, 30.5.1). 
The prompt starts as follows, where the network sizes shown are examples,

$1 d$ network $(\mathbf{n}=\mathbf{1 5 0}), \ldots$ (for a 1 d network)

2d network (40x40), ... (for a $2 d$ network)

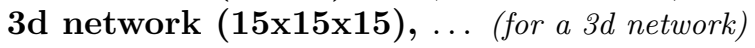

The first line of the prompt continues,

... wiring only - rules not set (if rules have not been set)

... review/revise/learn, wiring and rules (if rules were set)

The prompt continues as follows,

graph-g, matrix: revise-m view-M prtx-Mp

graphic: 1d+timesteps-1 circle-c 2d-2: (for a $1 d$ or $2 d$ network)

or

graphic: 1d+timesteps-1 circle-c 2d+3d-3: (for a $3 d$ network)

For example, for a 1d network with rcode set, the prompt appears as follows,

1d network $(n=150)$, review/revise/learn, wiring and rcode graph-g, matrix: revise-m view-M prtx-Mp

graphic: 1d:timesteps-1 circle-c 2d-2:

options.... what they mean

graph-g ... for the network-graph (chapter 20), where network nodes and connections can be rearranged and unravelled.

matrix: ... the "wiring matrix" section 17.2

revise-m ... for the network architecture in a spread-sheet type format, the wiring matrix, where the wiring can be changed.

view-M ... to view the wiring matrix and save matrix as a vector PostScript file 17.2.1.

prtx-Mp ... to view as above, but also to print the wiring matrix in the terminal.

graphic: ... the "wiring graphic" section 17.3

1d:timesteps-1 . . enter 1 for a $1 \mathrm{~d}$ wiring graphic where wiring is shown between successive time-steps, section 17.5, applies also to $2 \mathrm{~d}$ and $3 \mathrm{~d}$.

circle-c ... enter $\mathbf{c}$ for a $1 \mathrm{~d}$ wiring graphic arranged in a circle, section 17.5, applies also to $2 \mathrm{~d}$ and $3 \mathrm{~d}$.

2d-2 ... enter 2 for a $2 \mathrm{~d}$ wiring graphic, section 17.6, applies also to $1 \mathrm{~d}$.

$\mathbf{2 d}+\mathbf{3 d} \mathbf{d}-\mathbf{3} \ldots$ enter $\mathbf{3}$ for the wiring graphic in $3 \mathrm{~d}$, and simultaneously in $2 \mathrm{~d}$ in successive horizontal slices, section 17.7. 


\subsection{The wiring matrix}

Enter $\mathbf{m}$ or $\mathbf{M}$ in section 17.1 to show the network architecture in a spread-sheet type format, the wiring matrix; $\mathbf{M}$ to see the matrix or save it as a vector PostScript file; $\mathbf{m}$ to change the wiring. The rules in a rulemix (if set) are also shown, as much as will fit; the wiring matrix window, and fonts, can be re-sized. Examples are shown in figure 17.1. Rules cannot be changed in the wiring matrix; this can be done in the wiring graphic (section 17.3).

As described in section 12.6, columns give the cell's pseudo-neighborhood index, $K(k-1, \ldots, 0)$. Rows give the cell network position, $N(n-1, \ldots, 0)$. The $0-0$ grid (or the 0 -minimum $n$ grid if the whole matrix does not fit within one window) is in the lower right hand corner. Each grid records the position in the network $x,(n-1, \ldots, 0)$, to which the $K$ 'th wire of the $N$ 'th cell is connected.

Note that positions $N$ and $x$ are $1 \mathrm{~d}$ indexes, even if the network is $2 \mathrm{~d}$ or $3 \mathrm{~d}$ - sections 10.2.2 and 10.2.3 explain how to convert $x$ to $2 \mathrm{~d}$ or $3 \mathrm{~d}$ coordinates, and vice versa. A column to the left of the cell index shows the out-degree and out degree histogram of each cell.

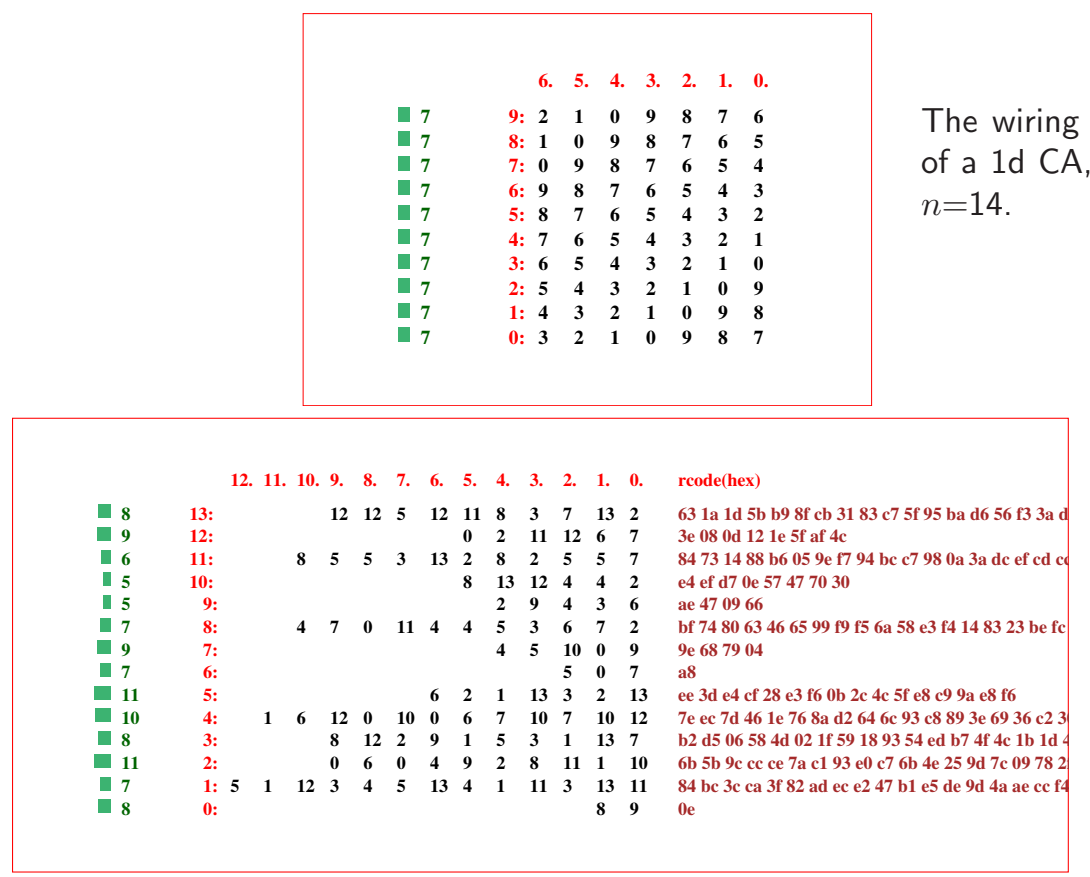

The wiring matrix for a $v=2$ k-mix $(k=2$ to 13) with random wiring, showing the rcode in hex for each cell, as much as will fit.

Figure 17.1: Wiring matrix examples. $k-1, \ldots, 0$ indexes columns, $n-1, \ldots, 0$ indexes rows. The column on the left shows the "out-degree" of each cell, the number of wires leaving it, also shown as a histogram. For a rulemix only (if set), rules are shown in hex, as much as will fit, in the right column. For $k \leq 3$, decimal rules are added. If the matrix was set with $\mathbf{m}$ in section 17.1 , the wiring can be set by hand as in a spread-sheet (section 12.6) and a rule in the active cell also appears in the rule window (section 16.19). If the matrix was set with $\mathbf{M}$ in section 17.1 , the matrix cannot be amended, but there is an option to create a vector PostScript file, as in these figures. 


\subsubsection{Viewing the wiring matrix and creating vector PostScript}

If $\mathbf{M}$ was selected in section 17.1, the wiring matrix is just displayed (enter $\mathbf{m}$ in section 17.2.2 to amend), together with the following top-right prompt,

if the matrix fits one window

PScipt-P layout-l font-f jump- $(\mathrm{j}=13-0)$ quit-q:

for a large matrix requiring a succession of windows, this example for a 2d network, $40 \times 40$

more-ret PScipt-P layout-l font-f jump- $(\mathrm{j}=1599-0)$ quit-q:

options ... what they means

more-ret ... for a large network that requires more than one window, enter return for the next section of the list, otherwise enter return to continue.

PScipt-P . . enter $\mathbf{P}$ to save the matrix as seen in the matrix window to a vector PostScript file. The default filename is my_mxPS.ps (see chapter 36).

layout-l ... enter $\mathbf{l}$ to change the matrix window width. The following top-right prompt is presented, for example,

change window width $(922-231$ now $=462)$ :

Enter the new width in pixels within the limits indicated.

font-f ... enter $\mathbf{f}$ to change the font size, a 3 -way toggle between normal, medium and small.

jump-j(1599-0) ... enter a number within the limits indicated to jump directly to a given cell index, which will become the first entry in the top row.

quit-q ... enter q to revert to the network architecture prompt in section 17.1.

For DOS there is a further option: enter $\mathbf{p}$ to print the current matrix window, with printer limitations as noted in section 5.6.3.

\subsubsection{Amending the matrix see also "Wiring by hand" section 12.6}

Enter $\mathbf{m}$ in section 17.1 , or $\mathbf{h}$ in in section 12.3, to display the wiring as a matrix or "spread sheet", which allows filling in the wiring positions for each cell's pseudo-neighborhood index. If $\mathbf{m}$, the wiring will have already been set in chapters 11 and 12, so all positions will be filled but can be amended. If $\mathbf{h}$, the matrix will start as a blank grid, to be set "by hand" (section 12.6) as in figure 12.5. In both cases, the following top-right reminder is presented,

hand wire/revise:jump-j (for a $2 d$ network, 40×40)

enter wiring positions 0-1599 (return on blank/0=random

move-arrows more/complete-m layout-l font-f quit-q

These options are also described in section 12.6 "Wiring by hand", but this section assumes a wiring matrix that has already been set - selected with $\mathbf{m}$ in section 17.1. Move around the matrix with the left/right/up/down arrow keys. Enter the new position at the flashing green cursor and complete the entry by moving to another grid with an arrow key or return. On zero, just return gives a random position. A rectangle is drawn around any position covered. An entry outside the network limits will be ignored. 
Large networks may require several successive windows to display the matrix. Enter $\mathbf{m}$ to see the next window. Moving beyond the top or bottom row in the current window with the arrow keys or return also brings up the preceding or next window. return on the very last (bottom right) entry makes the program continue. Enter $\mathbf{j}$ to jump to a new cell index, the following prompt is presented,

jump to index (1599-0): (for a $2 d$ network, 40×40)

Enter the new cell index, which will become the first entry in the top row. Options $\mathbf{l}$ or $\mathbf{f}$ to alter the presentation of data and the amount visible in the matrix window are described in section 17.2.1 above. Enter $\mathbf{q}$ to accept the wiring and revert to the prompt in section 17.1.

\subsection{The wiring graphic}

The wiring graphic is a diagram where the wiring and rules can be viewed, amended and reset by flexible methods described in the rest of this chapter.

Enter 1, c, $\mathbf{2}$ or $\mathbf{3}$ (for 1d time-steps, 1d circle, 2d or 3d) in section 17.1.

$$
\text { options ... what they mean }
$$

1d:timesteps-1 ... for a $2 \mathrm{~d}$ wiring graphic shown between successive time-steps. circle-c ... for a $2 \mathrm{~d}$ wiring graphic shown between cells arranged in a circle.

2d-2 ... for a $2 \mathrm{~d}$ wiring graphic, which may also be selected for a $1 \mathrm{~d}$ network. 2d+3d-3 . . for a $3 d$ wiring graphic which only applies to a $3 \mathrm{~d}$ network.

Note the native dimensions of the network can differ from the dimensions chosen for the wiring graphic. Both $1 \mathrm{~d}$ methods apply whatever the native dimensions of the network. The $2 \mathrm{~d}$ graphic can be applied to both $1 \mathrm{~d}$ and $2 \mathrm{~d}$ networks. However the $3 \mathrm{~d}$ graphic only applies to a $3 \mathrm{~d}$ network. The display and amendments apply to the "active cell" or to a pre-defined block of cells.

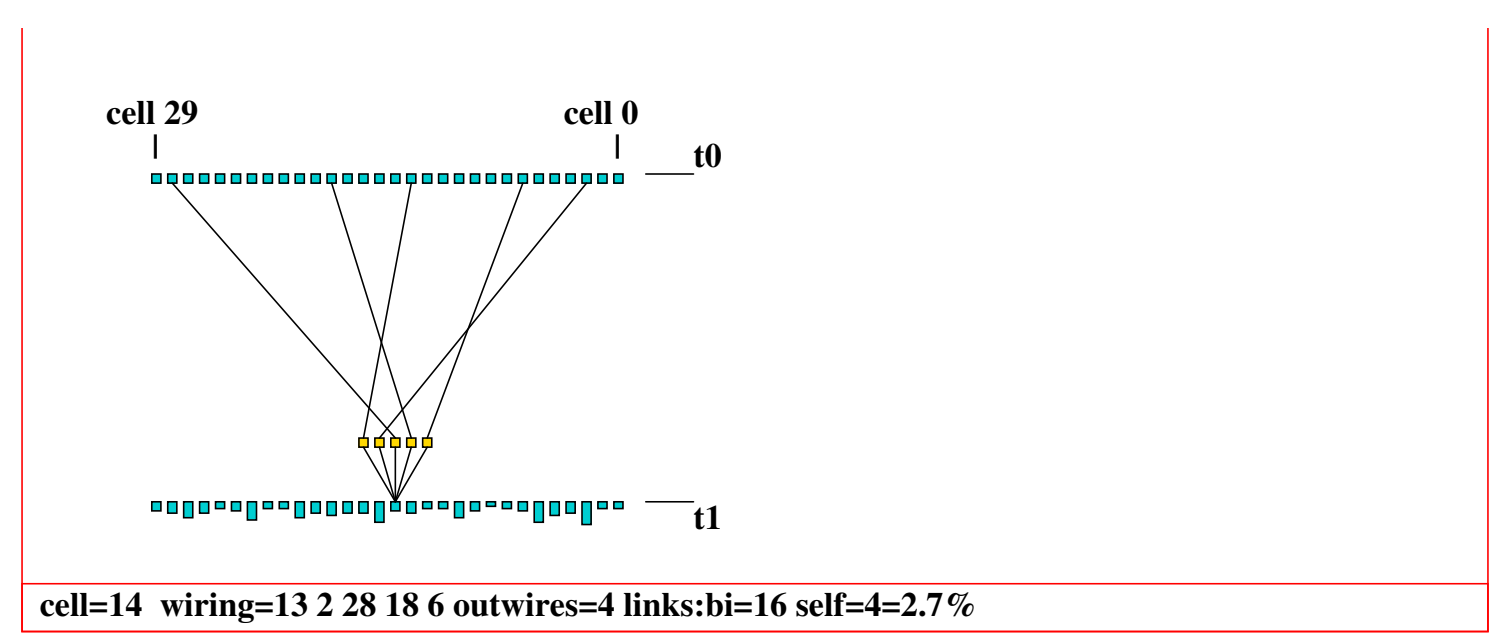

Figure 17.2: The $1 \mathrm{~d}$ wiring graphic showing the wiring between successive time-steps, $k=5, n=30$. Each cell's "outwires" are represented by the height of the lower cells, at time-step $t_{1}$. 


\subsection{The wiring graphic reminder}

A wiring graphic reminder of the options available appears in a top-right window at the same time as the wiring graphic. The options vary between the $1 \mathrm{~d}, 2 \mathrm{~d}$, and $3 \mathrm{~d}$ wiring graphic but there are also many similarities. Various context dependent options are presented for analyzing the network, and amending the wiring or rule/s for a single cell, a group of cells - a "block", or for the network as a whole ${ }^{1}$.

The first line of the reminder shows the network dimensions and size. If the rules have not been set, the first line of the prompt reads wiring only instead of wiring/rules. If a block is active, its bottom right and top-left coordinates are shown instead of the coordinates of the active cell, i.e. rewire $2 \mathrm{~d}$ block 3,3-8,8: instead of rewire $2 \mathrm{~d}$ cell 26,24:.

Examples of the 1d, 2d, and $3 \mathrm{~d}$ wiring graphic reminders are shown below.

$1 d$ time-step and circle wiring graphic

1d ( $n=150)$ wiring/rules: move-arrows, jump-j

one cell: right/left arrows, 15 cells: up/down arrows, redraw-T PScript-P

block-b tog:all-g pseudo-p, in/out-i/o avZ-z highlight/Learn-l/L

rewire 1d cell 74: untangle-u hand-h $\mathrm{rnd}-\mathrm{r} / \mathrm{w} / \mathrm{R}$ special-s local:1d-1

change $\mathrm{k}=8$ (max 13)-k, kill-K del-d, rule: Save/rev/trans-S/v/t, Derrida plot-D file/data-f hist:In(k)/Out/Both-I/O/B, reset-q cont-ret:

2d wiring graphic

2d (40x40) wiring/rules: move-arrows, jump-j exp/contr-e/c up/down-u/d redraw-T PScript-P toghex-X

block-b tog:block-g links-n pseudo-p index-i avZ-z highlight/Learn-l/L rewire $2 d$ cell 26,24: hand-h rnd-r/w/ $R$ special-s local:1d-1 2d-2

change $k=8(\max 13)-k$, kill-K, rule: Save/rev/trans-S/v/t, Derrida-D file/data-f hist:In(k)/O/B-I/O/B, reset-q cont-ret:

$3 d$ wiring graphic

3d (20x20x20) wiring/rules: move-arrows, levels up/down-[/], jump-j $\exp /$ contr-e/c/E/C up/down-u/d redraw-T PScript-P

block-b tog:block-g links-n pseudo-p index-i avZ-z highlight/Learn-l/L rewire 3d cell 5,5,5: hand-h rnd-r/w/ $R$ special-s local:1d-1 2d-2 3d-3 change $k=6(\max 13)-k$, kill-K, rule: Sace/rev/trans-S/v/t, Derrida-D file/data-f hist:In(k)/Out/Both-I/O/B, reset-q cont-ret:

\subsubsection{Wiring graphic options summary}

The wiring graphic options options activate as soon as the key is pressed, without pressing return - some have secondary prompts. Below is just a brief summary following the order in which the options are listed in the reminders. More details are given in further sections in this chapter.

\footnotetext{
${ }^{1}$ Note that while interrupting incomplete attractor basins, though the wiring graphic can be accessed, any options for changing wiring or rule/s are deactivated and do not appear in the prompt.
} 
$\underline{\text { presentation options } . . . ~ w h a t ~ t h e y ~ m e a n ~}$

move-arrows, jump-j ... use the arrow keys to move around the network or enter $\mathbf{j}$ to jump to a particular cell index. For 1d (section 17.5.2). For $2 \mathrm{~d}$ (section 17.6.2).

levels up/down-[/] ... (3d graphic only) move with left/right/up/down arrows within each level (or jump) as for $2 \mathrm{~d}$ above, and use the square braces, "[" for up, "]" for down, to move between levels (section 17.7.2).

exp/contr-e/c... (2d and 3d graphic only) to expand or contract a $2 \mathrm{~d}$ wiring graphic (section 17.6.7), or the $2 \mathrm{~d}$ version of the $3 \mathrm{~d}$ wiring graphic (section 17.7.7).

$\exp /$ contr-E/C ... (3d graphic only) to expand or contract the $3 \mathrm{~d}$ version of the $3 \mathrm{~d}$ wiring graphic (section 17.7.7).

up/down-u/d ... (2d and $3 d$ graphic only) to shift the $2 \mathrm{~d}$ version of the $3 \mathrm{~d}$ wiring graphic to see the relevant part (section 17.7.8). Also works for the $2 \mathrm{~d}$ wiring graphic (17.6.8).

redraw-T ... to redraw the current wiring graphic.

*PScript-P ... to redraw and create a vector PostScript file of the current wiring graphic (section 17.8.14).

toghex-x ... (2d graphic only) to toggle between square and hex layout.

block-b ... define a block of cells in $1 \mathrm{~d}, 2 \mathrm{~d}$ or $3 \mathrm{~d}$, for resetting the wiring or rules (sections 17.5.3, 17.6.5, 17.7.5).

tog: toggle options ...

all-g ... toggle for the active cell, showing the active cell on its own, or the active cell against a background of the block (if set) or all network connections (if a block is not set).

links-n ... (2d and 3d graphic only) a 5-way toggle for alternative presentations of wiring relative to either the active cell block. For the active cell these options may be used with pseudo-p below. (sections 17.6.3, 17.7.3).

pseudo-p ... toggle showing/omitting the pseudo-neighborhood.

index-i ... (2d and $3 d$ graphic only) Toggle the index numbers in the pseudo-neighborhood. The numbers shown up if the scale is large enough.

miscellaneous options...

in/out-i/o ... (1d time-steps and circle only) show the indirect inputs and outputs of past time-steps, the "degrees of separation" between cells (sections 17.5.6, 17.5.7).

*avZ-z ... (rcode-mix and/or $k$-mix) to calculate the average and weighted average $Z$ and $\lambda$ parameters (section 17.8.2).

*highlight/learn-l/L . . (rcode only) enter $\mathbf{L}$ to implement the learning, forgetting, highlighting functions. Enter $\mathbf{l}$ for only highlighting nodes in attractor basins (chapter 34). For space-time patterns, highlighting does not apply and the option $\mathbf{l}$ is absent. 
rewire: options ... shows the coordinates of the cell, or block if active.

untangle-u ... (1d time-steps and circle only) "untangle" the wiring of the cell, block, or the whole network, and reset the rules for equivalent dynamics (section 17.5.8).

*hand-h ... rewire the active cell by hand (section 17.8.4).

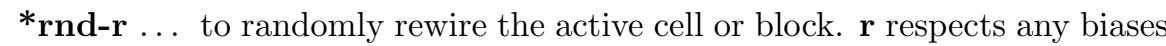
set in special-s below, with a local zone equal to the shortest axis for $2 \mathrm{~d}$ and $3 \mathrm{~d}$ ).

*rnd-w ... (if a block is active) to randomly rewire cells outside the active block, with biases as rnd-r above.

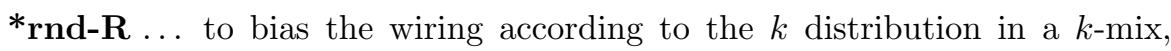
allowing a power-law distribution of outputs as well as inputs (section 9.7.2). For homogeneous $k, \mathbf{R}$ gives totally unbiased random wiring.

special-s ... set "special" wiring biases, which can then be applied to the active cell or block with $\mathbf{r}$ above (section 17.8.6).

*local:1d-1 2d-2 3d-3 ... set local CA wiring for the cell or block (section 17.8.7).

${ }^{*}$ change $\mathbf{k}=\mathbf{8}(\max 13)-\mathbf{k} \ldots$ (rcode-mix set) change $k$ up or down for the cell or block, up to $k_{\text {Lim }}$ (section 7.2).

*kill-K ... (rcode-mix set) kill or neutralize a cell by cutting all its links, both inputs and outputs, except for one input to itself. The rcode is also set for effective $k=0$ (section 17.8.9).

del-d ... (1d networks and wiring graphics, k-mix, rcode set, main prompt sequence, not in TFO-mode) delete a cell from the network, and shorten the network by one (section 17.5.9).

rule: options ...

*Save-S ... save the rule for a cell,

*rev-v ... revise or load the rule for a cell, and copy to a block (section 17.8.10).

*trans-t ... transform the rule or rules, set canalyzing inputs (section 17.8.11).

more miscellaneous options...

*Derrida plot-D ... draw the Derrida plot for the network, described in chapter 22 .

*file/data-f ... filing, save and load the network architecture.

*hist:In(k)/Out/Both-I/O/B ... show a histogram of the frequency distribution of inputs (i.e. $k$ ), outputs, or both (i.e all connections) in the network.

*reset-q .. backtrack to redisplay the network (section 17.1).

Options marked with an asterisk such as * rnd-r apply jointly for $1 \mathrm{~d}, 2 \mathrm{~d}$ and $3 \mathrm{~d}$ networks most of these options are described further in section 17.8. Other options apply more specifically, or uniquely, to a particular dimension - these options are described further in sections 17.5 for $1 \mathrm{~d}$, 17.6 for $2 \mathrm{~d}$, and 17.7 for $3 \mathrm{~d}$. 


\subsection{Wiring graphic, $1 \mathrm{~d}$}

Enter $\mathbf{1}$ or $\mathbf{c}$ in section 17.1 for a 1d graphic, which shows how a particular cell (the "active cell"), or a pre-defined $1 \mathrm{~d}$ block of cells, is wired in a $1 \mathrm{~d}, 2 \mathrm{~d}$ or $3 \mathrm{~d}$ network.

If $\mathbf{1}$ is entered, the wiring is shown between two time-steps, from time-step $t_{0}$ to $t_{1}$, as in figure 17.2 and elsewhere. Network cells at $t_{0}$ are shown in a row above cells at $t_{1}$, and the out-degree of each cell is indicated by the height of the cell's representation at $t_{0}$ (the cell wiring out-degree histogram).

If $\mathbf{c}$ is entered, the wiring is shown between cells, represented as nodes or radial lines, arranged in a circle, as in 17.3 and elsewhere. The zero index is due east and increases clockwise. The cell index is shown within each disk for networks smaller than about $n=40$. For networks greater than about $n=162$, cells are shown as short radial lines. Each cell's "outwires" are represented by the length of radial lines outside each cell (the cell wiring out-degree histogram).

In both presentations, an option allows the pseudo-neighborhood to be omitted, showing just direct links. The active cell is moved around the network with the arrow keys, and its possible to "jump" to a new location. The rule for the active cell appears in the rule window described in section 16.19 .

Sections 17.5.1-17.5.9 below explain some of the options specifically for $1 \mathrm{~d}$ in more detail that were summarized in section 17.4.1.
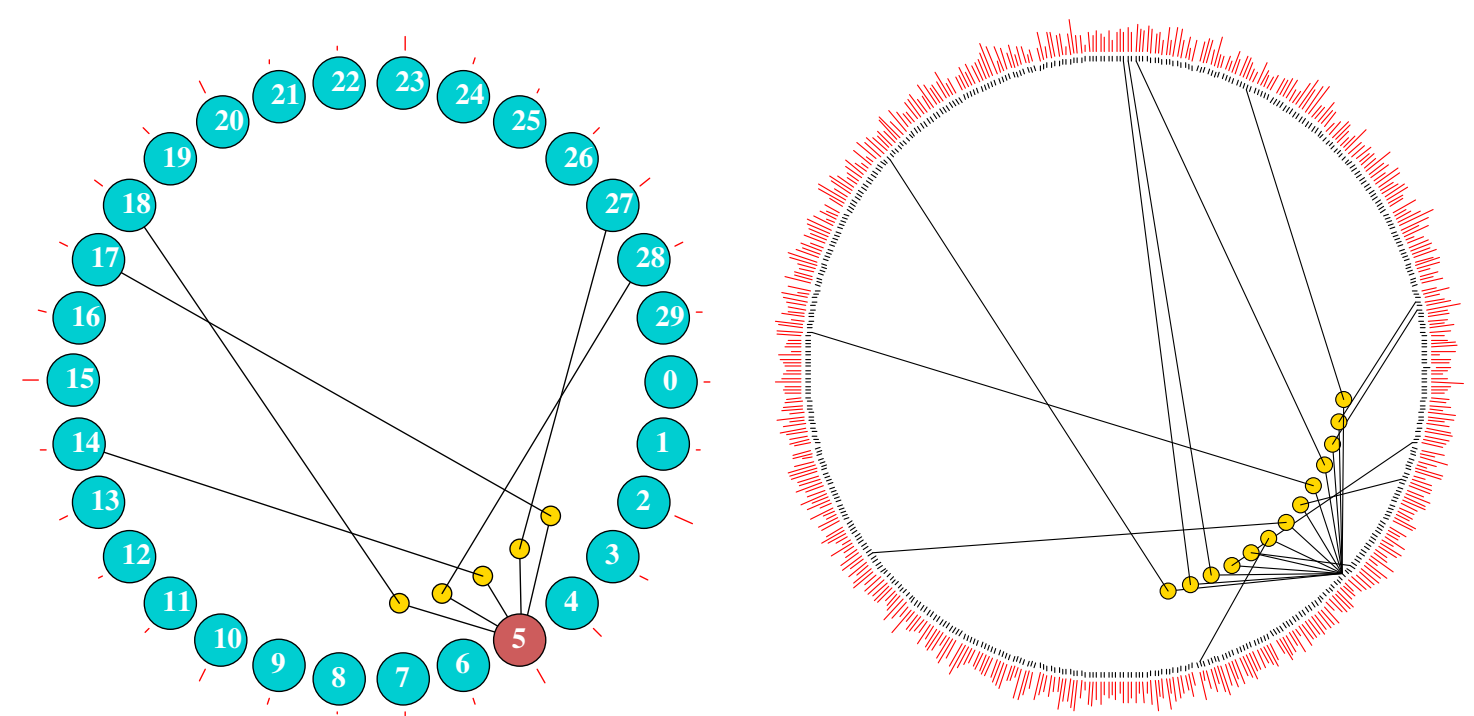

Figure 17.3: Examples of the 1d circle wiring graphic. Left: the same network as in figure 17.2, $k=5$, $n=30$. Right: $k=13, n=500$. The "active" cell is moved by the arrow keys. Each cell's "outwires" are represented by the length of the red radial lines outside the circle. The cell index is shown for networks smaller than about $n=40$. For networks greater than about $n=162$, the nodes themselves are not shown, but just the radial lines. 

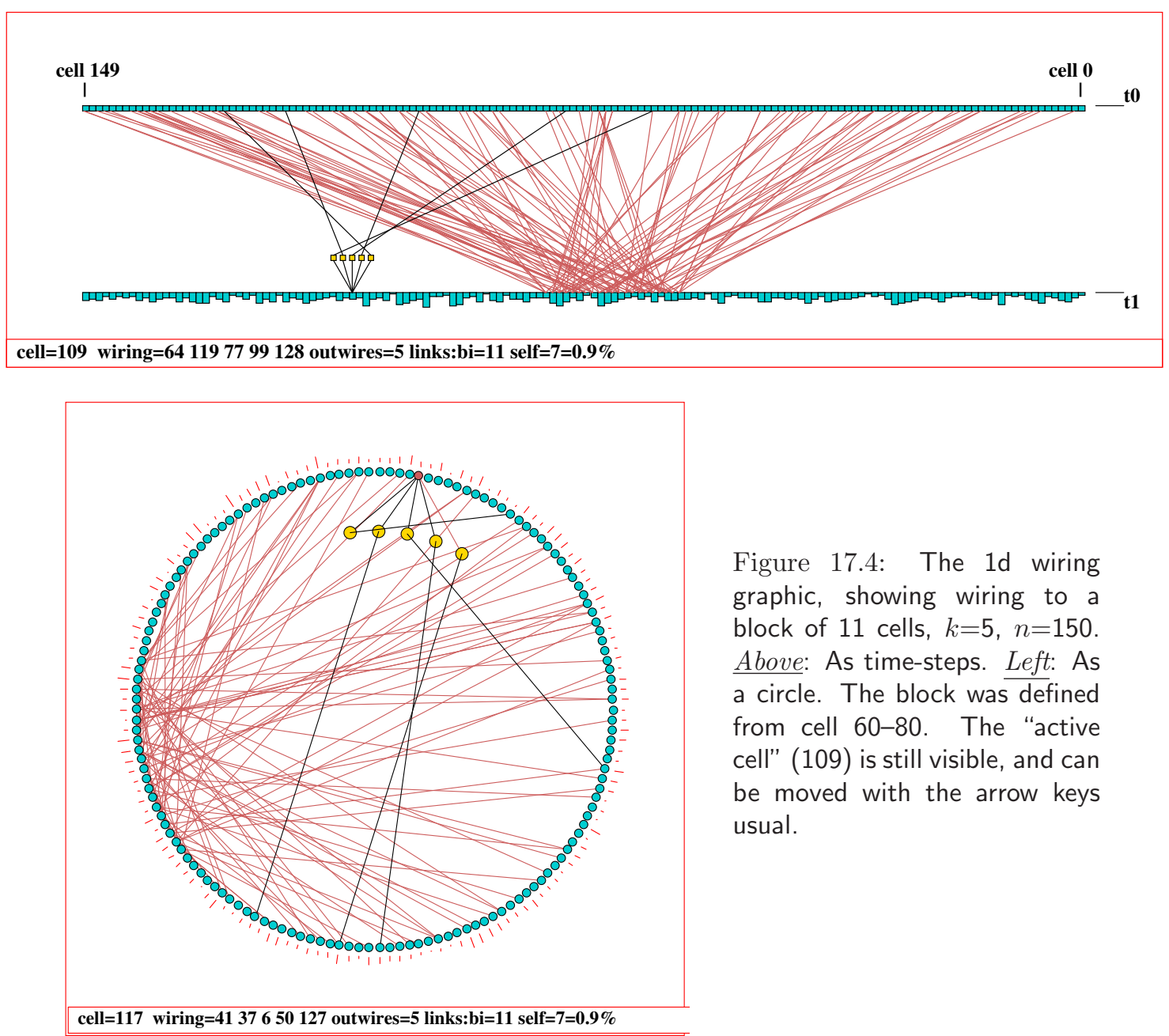

Figure 17.4: The 1d wiring graphic, showing wiring to a block of 11 cells, $k=5, n=150$. Above: As time-steps. Left: As a circle. The block was defined from cell 60-80. The "active cell" (109) is still visible, and can be moved with the arrow keys usual.

\subsubsection{Data - 1d wiring graphic}

Various data about the active cell's wiring are shown below the 1d graphic. For example, in figure 17.2 , a $k=5$ network, with mixed rules and nonlocal wiring, the data is as follows,

\section{cell=14 wiring=13 228186 outwires=4 links:bi=16 self=4=2.7\%}

See section 17.8.1 to decode this data. The rule and rule data for the active cell are also shown (if the rule/s have been set) in the rule window described in section 16.19. 

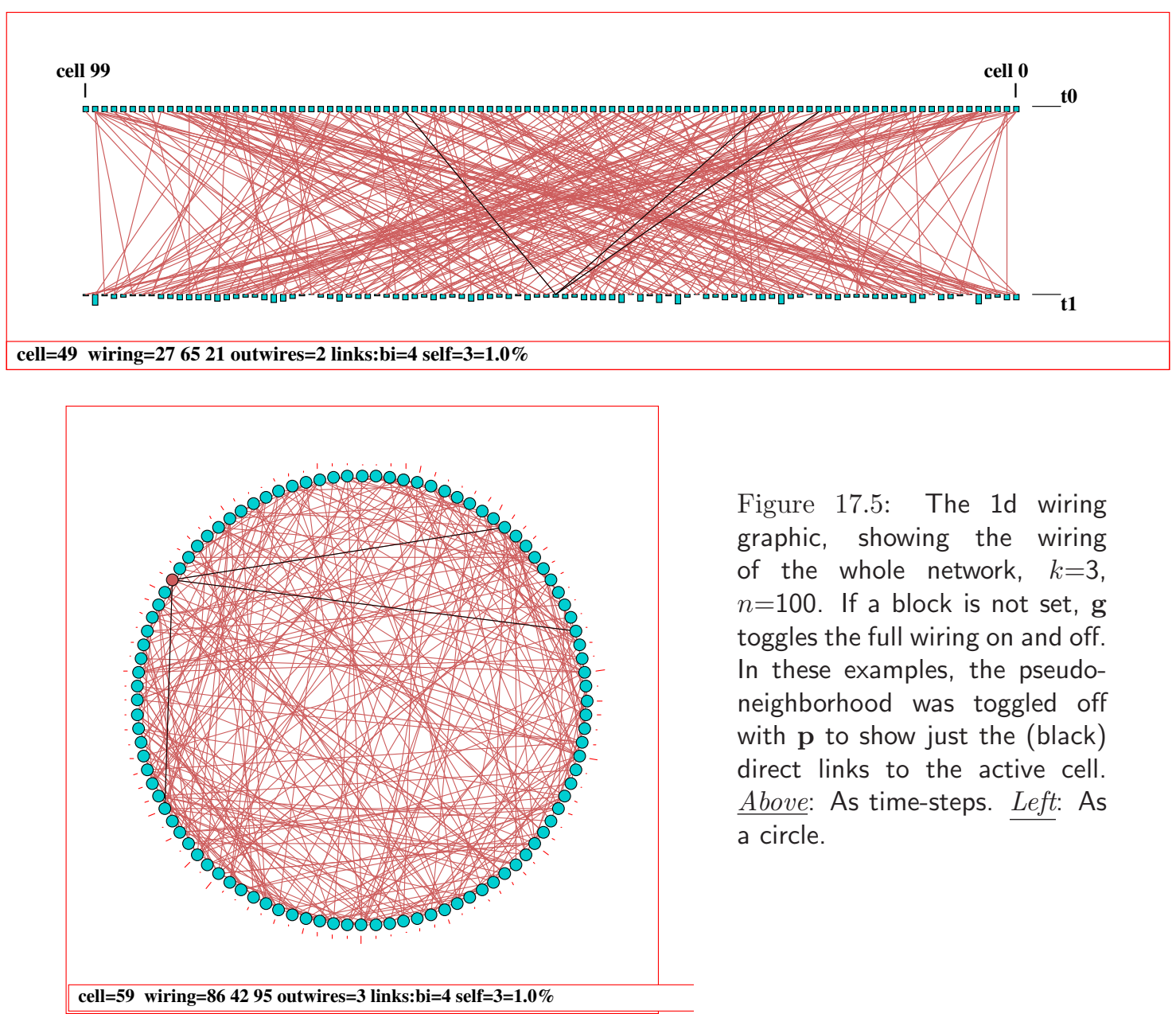

Figure 17.5: The 1d wiring graphic, showing the wiring of the whole network, $k=3$, $n=100$. If a block is not set, $\mathrm{g}$ toggles the full wiring on and off. In these examples, the pseudoneighborhood was toggled off with $\mathbf{p}$ to show just the (black) direct links to the active cell. Above: As time-steps. Left: As a circle.

\subsubsection{Moving or jumping between cells, 1d}

To move the active cell, use the arrow keys. The left and right arrow keys move by one cell, the up and down arrow keys move by by $n / 10$ cells for $n>20$ or by 2 cells otherwise. Alternatively, enter $\mathbf{j}$ to jump to a specific cell index. The following prompt is displayed,

jump to index (1295-0): (for example)

\subsubsection{Defining a block, $1 \mathrm{~d}$}

Enter $\mathbf{b}$ in section 17.4 to define a block of cells. The following top-right prompt is presented,

1d block-1, all-a single cell-def:

(then, if $\mathbf{1}$ is selected ...)

1d block (0-49, def 102), low: high: 

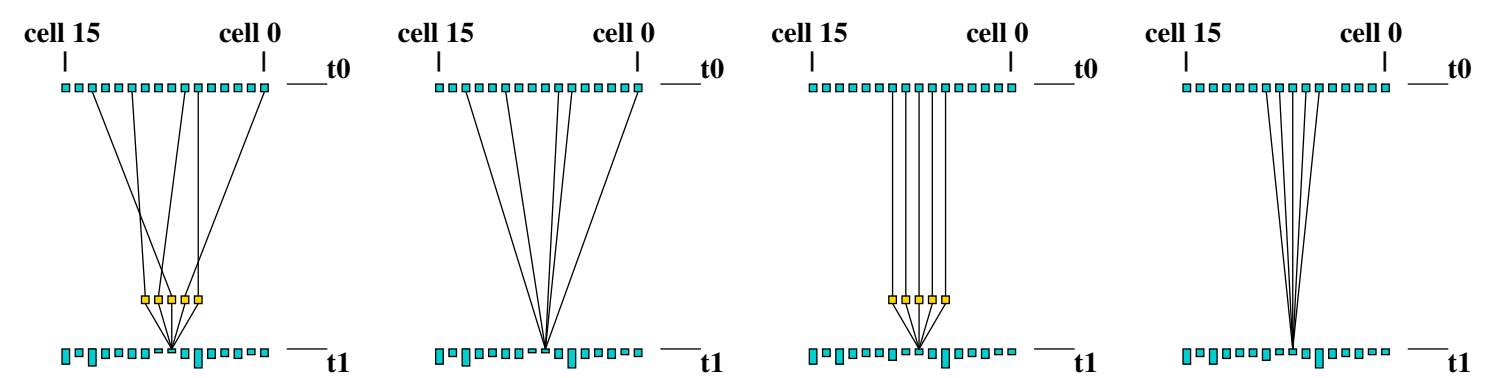

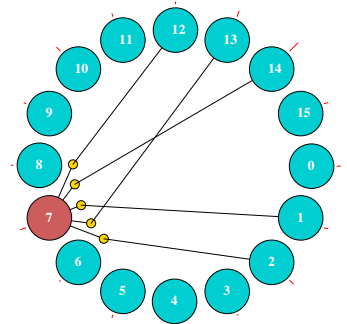

a. random wiring pseudo-n'hood

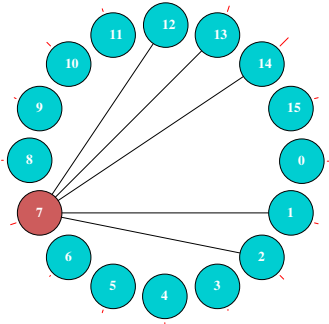

b. random wiring direct

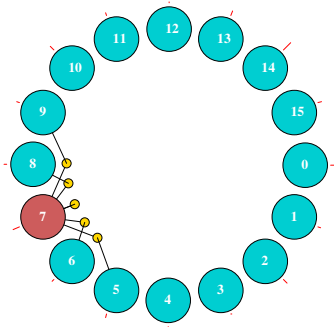

c. CA wiring pseudo-n'hood

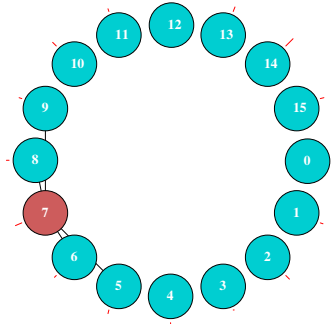

d. CA wiring direct

Figure 17.6: Examples of the 1d wiring graphic, $k=5, n=20$. (a) and (c) include the pseudoneighborhood, (b) and (d) show just direct wiring, toggle between with p. (a) and (b) have have random wiring, (c) and (d) have their wiring changed to 1d CA by entering 1 . The same wiring inputs to cell 7 are shown: Top Row: as time-steps, Bottom Row: as a circle.

Enter 1 to define the block, a to define the whole network as a block. If $\mathbf{1}$ is entered, a subsequent prompt is presented for the low, then high, cell indexes delimiting the block.

The wiring of all the cells in the block will be shown in the graphic as direct links, colored light red. The active cell will still be visible and can be moved as usual. For a 1d network shown in 2d, the prompt is as described in section 17.6.5

An active block is indicated in the wiring graphic reminder (section 17.4) for example,

rewire 1d block 22-33: ...

To deactivate the block enter $\mathbf{b}$ in section 17.4, then return. If the block is active and visible the following rewire options ...

\section{.. untangle-u ... rnd-r/w/R special-s local:1d-1 \\ change $\mathbf{k}=\mathbf{8}(\max 13)-\mathrm{k} \ldots$ (for example)}

... will apply to just the block, not to the active cell if it is outside the block.

The following rule options apply to the active cell, but if the block is active and visible the new rule can then be copied to the block.

... rule:Save/rev/trans-S/v/t 


\subsubsection{Toggling the block, $1 \mathrm{~d}$}

Enter $\mathbf{g}$ in section 17.4 to toggle the active block, making it either visible or invisible, without deactivating it. Note that options in section 17.5.3 affect a block only if it is both active and visible. If no block is active, toggling with $\mathrm{g}$ just shows the wiring in the whole network.

\subsubsection{Include the pseudo-neighborhood, or direct wiring only, 1d}

The default wiring representation for the active cell includes the pseudo-neighborhood. Alternatively just the direct connections may be shown, always the case for a block. Enter p to toggle between the two (figure 17.6).

\subsubsection{Recursive inputs to a cell, $1 \mathrm{~d}$}

Enter $\mathbf{i}$ in section 17.3 to show all recursive inputs to a cell, whether direct or indirect, showing the "input degrees of separation" between cells. This will include inputs to inputs, and so on, relative to past time-steps, showing the potential upstream influence on the cell from past network dynamics. This is shown in the direct wiring format (section 17.5.5).

Initially, just the wiring from the previous time-step appears, with the following prompt displayed in the top-left hand corner of the wiring window,

step 1, inputs $=\mathbf{2} / \mathbf{1 0 0}$, step-s all-def: (for a $k=2, n=100$ network)

Enter return to generate all inputs in one go. Enter $\mathbf{s}$ to show the inputs in steps, by entering return, or enter q to quit early. In both cases, inputs that relate to different past time-steps are shown in different colors (cycling through about 7 colors). A small insert in the top-right hand corner of the wiring window graphically indicates the fraction of cells connected by inputs so far.

Intermediate prompts show the number of inputs so far, for example,

step 4 , inputs $\mathbf{2 7} / \mathbf{1 0 0}=\mathbf{2 7 \%}$ cont-ret: (values shown are examples)

Once all possible input cells have been found, the following prompt appears in the top-left hand corner of the wiring window,

step $\mathbf{1 2}$, inputs $\mathbf{8 0} / \mathbf{1 0 0}=\mathbf{8 0 \%}$ complete (values shown are examples)

Enter return to reactivate the main wiring reminder (section 17.4) and revert to the normal wiring graphic functions.

\subsubsection{Recursive outputs from a cell, $1 \mathrm{~d}$}

Enter $\mathbf{o}$ in section 17.4 to show all recursive outputs from a cell, whether direct or indirect, showing the "output degrees of separation" between cells, the converse of of recursive inputs in section 17.5.6. This will include outputs from outputs, and so on, relative to future time-steps, showing the potential influence from the cell on future network dynamics downstream.

Initially just the immediate wiring outputs (if any) will be shown from $t_{0}$ to $t_{1}$. The number of these outputs may be less than that shown as "outwires" in the $1 \mathrm{~d}$ wiring window data (section 17.5.1) because there may be duplicate output wires which are only counted once. The following prompt is displayed in the top-left hand corner of the wiring window, 

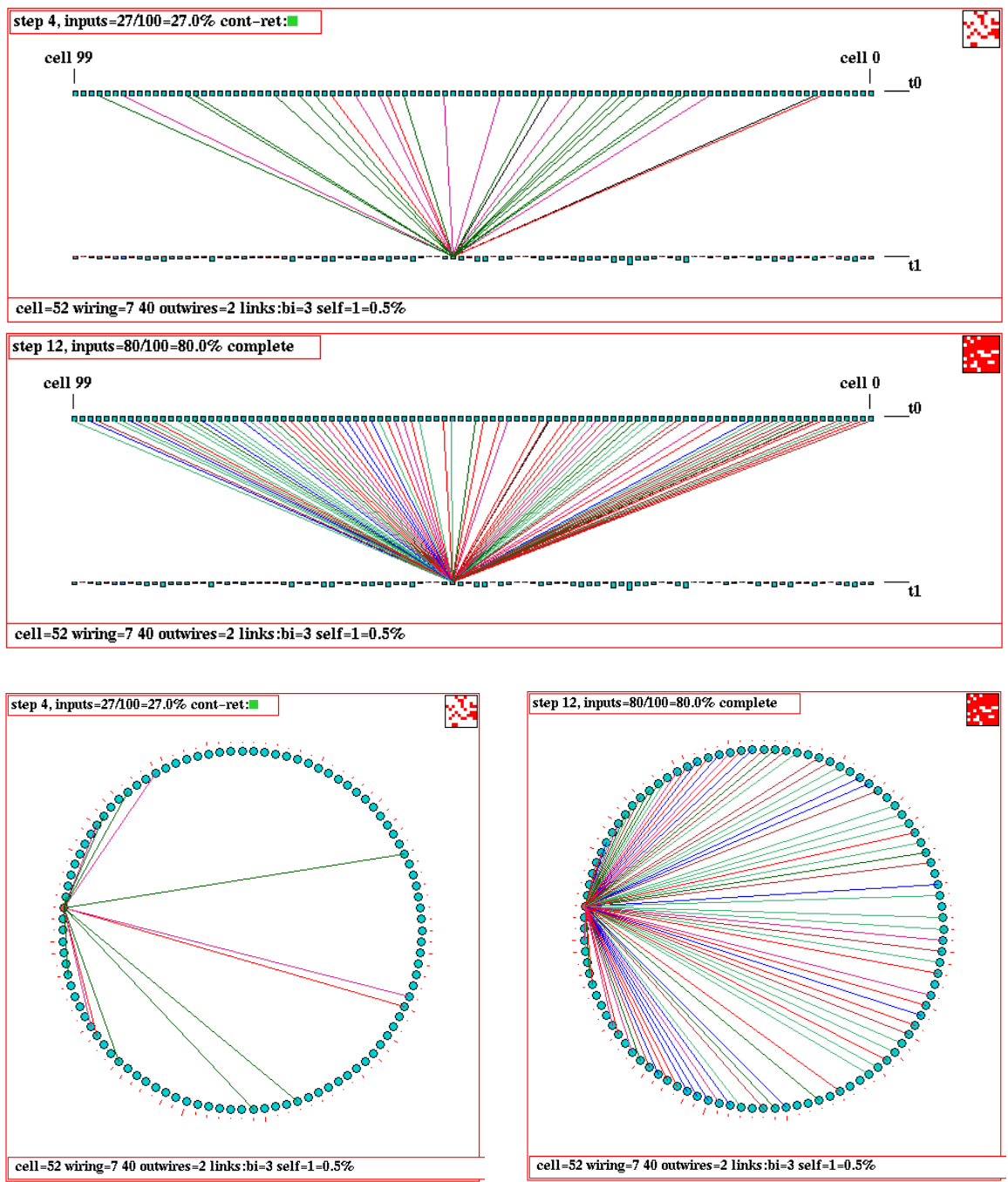

Figure 17.7: Recursive inputs (direct and indirect) to a given cell for a $k=2, n=100$ RBN . Above: As time-steps. Below: As a circle. Firstly, inputs are shown after 4 backward steps with 27 cells reached. Secondly, the complete inputs are shown after 12 backward steps with 80 cells reached. A small $2 \mathrm{~d}$ insert in the top-right hand corner of the wiring window graphically indicates the fraction of cells connected so far.

step $\mathbf{1}$, outputs $=\mathbf{2} / \mathbf{1 0 0}$, step-s all-def: (for a $k=2, n=100$ network)

Enter return to generate all outputs in one go. Enter $\mathbf{s}$ to show the outputs in steps, by entering return, or enter $\mathbf{q}$ to quit early. In both cases, outputs that relate to different future time-steps are shown in different colors (cycling through about 7 colors). A small insert in the top-right hand corner of the wiring window graphically indicates the fraction of cells connected by outputs so far.

Intermediate prompts show the number of outputs so far, for example, 

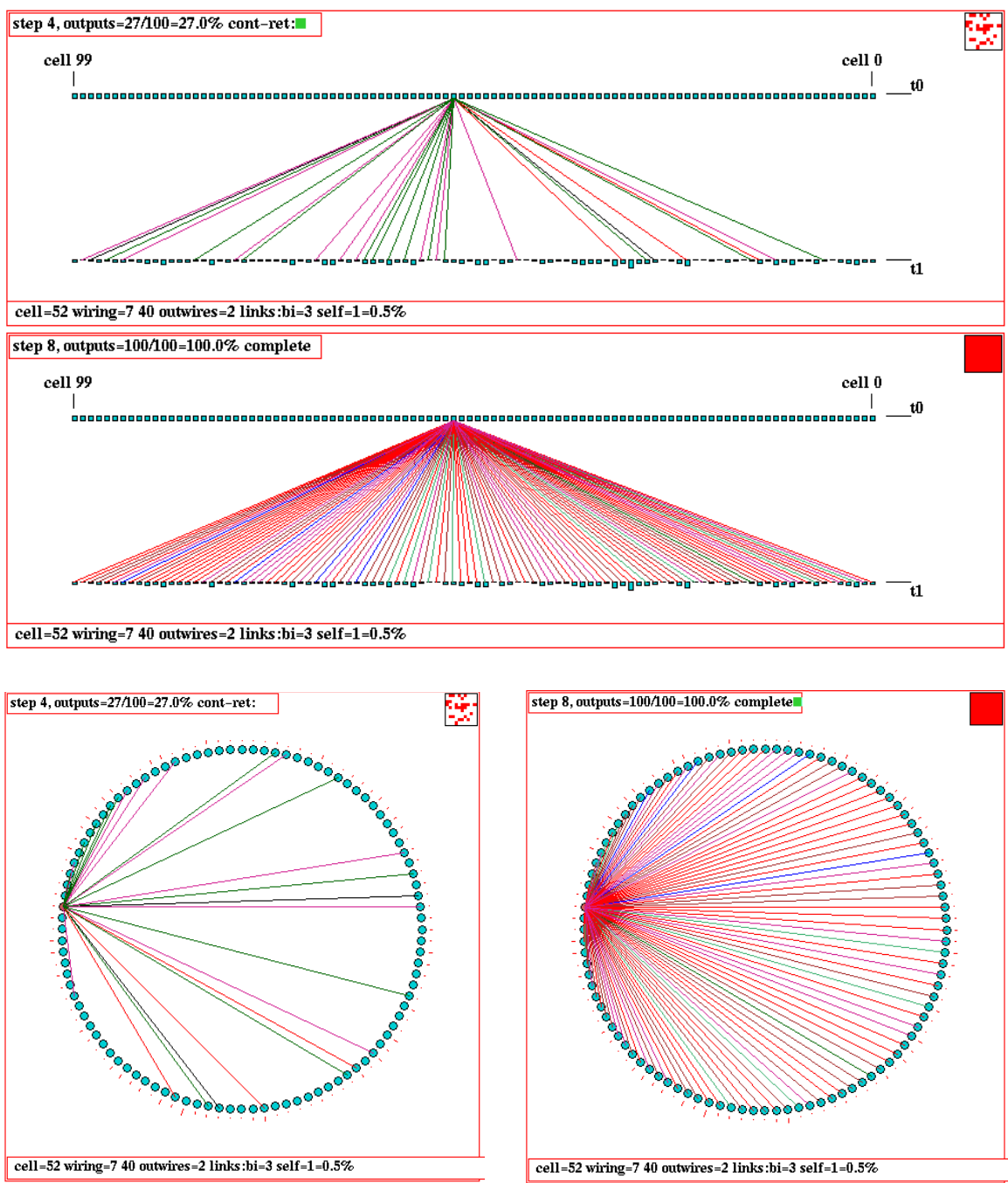

Figure 17.8: Recursive outputs (direct and indirect) from a given cell for a $k=2, n=100$ RBN. Above: As time-steps. Below: As a circle. Firstly, outputs are shown after 4 forward steps with 27 cells reached. Secondly, the complete outputs are shown after 8 forward steps with all 100 cells reached. A small $2 \mathrm{~d}$ insert in the top-right hand corner of the wiring window graphically indicates the fraction of cells connected so far.

step 4 , outputs $=\mathbf{2 7} / \mathbf{1 0 0}=\mathbf{2 7 \%}$ cont-ret: (values shown are examples)

Once all possible output cells have been found, the following prompt appears in the top-left hand corner of the wiring window,

step $\mathbf{8}$, outputs $=\mathbf{1 0 0} / \mathbf{1 0 0}=\mathbf{1 0 0 \%}$ complete (values shown are examples)

Enter return to reactivate the wiring graphic reminder (section 17.4) and revert to the normal wiring graphic functions. 


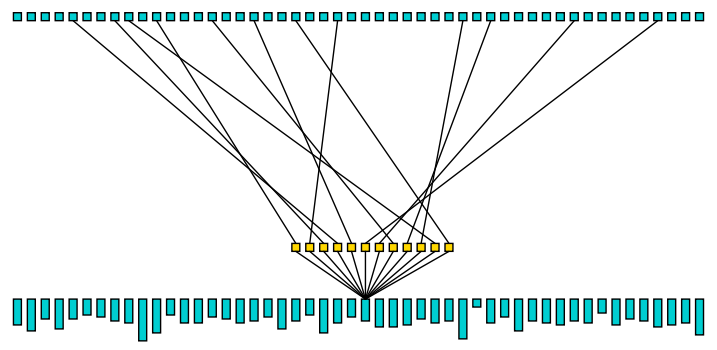

random wiring, wires cross

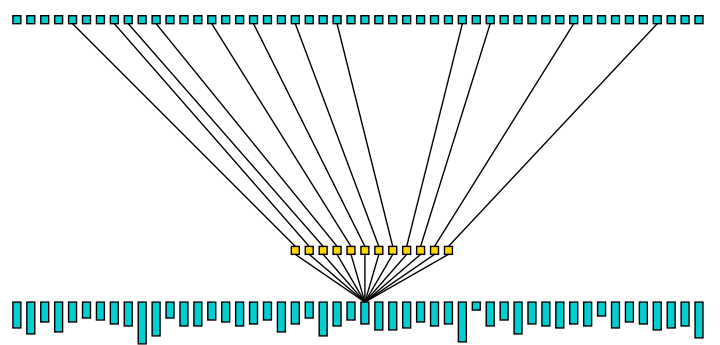

untangled random wiring

Figure 17.9: Untangling the wiring: wire connection points to the $1 \mathrm{~d}$ pseudo-neighborhood for the active cell are rearranged so that the wires do not cross, and the rcode (if set as part of a rulemix) is automatically transformed to give equivalent behavior. In TFO-mode there is no need to transform the rule. In this example $k=12, n=50$.

\subsubsection{Untangling the wiring}

Enter $\mathbf{u}$ in section 17.4 to "untangle" the wiring for the active cell, for the defined block if visible, or for all cells in the network, so that wire connection points to the pseudo-neighborhood (when shown in 1d) do not cross each other (figure 17.9) — the wiring positions in the network itself are unchanged. This option applies in the $1 \mathrm{~d}$ wiring graphic (for $2 \mathrm{~d}$ and $3 \mathrm{~d}$ networks as well as $1 \mathrm{~d}$ ) but the effect is best seen in 1d time-steps rather than the alternative circle presentation.

The following top-right prompt is presented,

untangle 1d wiring: all-a, single cell-(def): (for the active cell)

or if a block is active and visible

untangle 1d wiring: block 11-22, all-a, single cell-(def): (for example)

If an rcode-mix was set, rules are transformed for equivalent dynamics ${ }^{2}$. For a single rcode network (section 14.1) the wiring will be untangled, but the rcode will remain unchanged - to get around this, set the rcode-mix where all the rules are the same (section 14.4.3).

Note that in TFO-mode there is no need to transform rules when untangling because changing the connection points of wires to the pseudo-neighborhood has no effect on dynamics.

\subsubsection{Deleting a cell}

(1d networks and wiring graphics, $k$-mix, rcode set, main prompt sequence, not in TFO-mode)

Enter $\mathbf{d}$ in section 17.4 to delete a cell from the network and shorten the network by one cell. This option is only available in the main prompt sequence, not while interrupting space-time patterns or attractor basins. Backtrack to the main prompt sequence if necessary. In addition, the base network itself must be $1 \mathrm{~d}$ and have mixed- $k$, with the rcodes set (so not in TFO-mode). When a cell is deleted, all links, both inputs and outputs, between the cell and other cells will be cut, and cell indexes greater than the deleted cell will be reduced by one.

\footnotetext{
${ }^{2}$ If the order of the $k$ inputs in the pseudo-neighborhood is changed, the rcode is transformed to achieve identical behavior. Thus there are $k$ ! equivalent pseudo-neighborhood orders and corresponding rcode.
} 
19

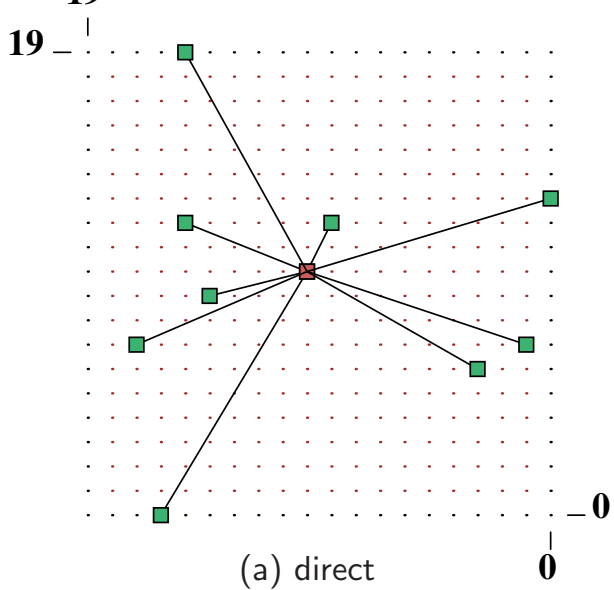

19

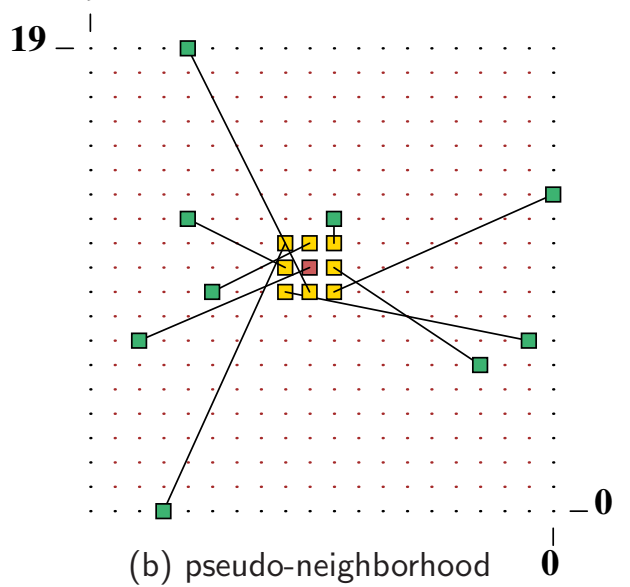

Figure 17.10: The $2 \mathrm{~d}$ wiring graphic, $n=20 \times 20 \mathrm{RBN}, k=9$, which can be toggled between (a) direct wiring - the default for random wiring, and (b) wiring to the pseudo-neighborhood - the default for local wiring.

\subsection{Wiring graphic, $2 \mathrm{~d}$}

Enter 2 in section 17.1, for the $2 \mathrm{~d}$ graphic. This shows how a particular cell (the "active cell"), or a pre-defined $2 \mathrm{~d}$ block of cells, is wired in the $2 \mathrm{~d}$ network (or in a $1 \mathrm{~d}$ network presented in $2 \mathrm{~d}$ ). The $2 \mathrm{~d}$ graphic can be expanded and contracted, and shifted up and down if necessary to see the relevant part. The active cell is moved around the network with the arrow keys, and its possible to "jump" to a new location. The rule for the active cell appears in the rule window described in section 16.19.

For $1 \mathrm{~d}$ networks shown in $2 \mathrm{~d}$, the most reasonable $i, j$ dimensions are automatically computed, with $i \geq j$ (for $n$ prime $i=n, j=1$ ).

The display can be toggled between "direct wiring" and wiring to the pseudo-neighborhood (section 17.6.4) as in figure 17.10. By default, "direct wiring" is displayed if the wiring is nonlocal, and the pseudo-neighborhood for local CA wiring. Enter $\mathbf{p}$ to toggle between the two. There is also a 5-way toggle (enter $\mathbf{n}$ ) to alter the presentation of connections, and the cells connected, which is especially useful in visualizing blocks.

The current cell is colored red, its pseudo-neighborhood yellow, the "actual neighborhood" cells are colored green (see examples in figure 12.3).

Sections 17.6.1-17.6.8 below explain some of the options specifically for $2 \mathrm{~d}$ in more detail that were summarized in section 17.4.1.

\subsubsection{Data - 2d wiring graphic}

As for the 1d wiring graphic, various data about the active cell's wiring are shown at the foot of the $2 \mathrm{~d}$ wiring graphic. For example, for figure 17.10 the data is as follows, 
$2 \mathrm{~d}$ cell=10,10=210 wiring=16,0 14,9 9,12 15,12 17,7 3,6 1,7 15,19 0,13 outwires=7 links:bi=37 self=10=0.3\%

See section 17.8.1 to decode this data. The current cell, and cell input positions, are shown as $I, J$ coordinates. The rule and rule data are shown in the rule window (section 16.19).

\subsubsection{Moving or jumping between cells, 2d}

To move the active cell use the arrow keys. Alternatively, enter $\mathbf{j}$ to jump to a specific cell. The $2 \mathrm{~d}$ cell position is specified by its $I, J$ coordinates. The following prompts are presented,

\section{jump to coord I,J \\ enter I(19-0): $\quad$ enter $\mathbf{J}(\mathbf{1 9 - 0})$ : (for a $2 d$ network size $20 \times 20$ )}

\subsubsection{Alternative wiring presentation, $2 \mathrm{~d}$}

A 5-way toggle allows alternative presentations of the active cell, or an active block. Enter links-n to toggle between the five alternatives, as shown in figure 17.11. For the active cell, the 5 -way toggle works both with direct wiring and with the pseudo-neighborhood (pseudo-p) in section 17.7.4.

For a $2 \mathrm{~d}$ block, the alternatives are shown in in figure 17.12.

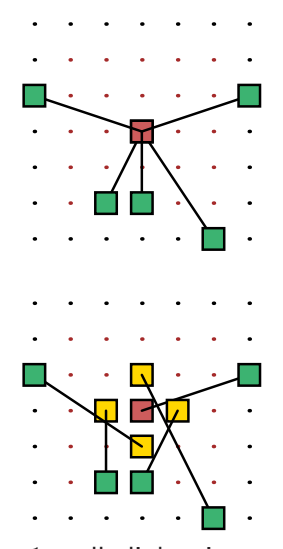

1. cell+links+inputs

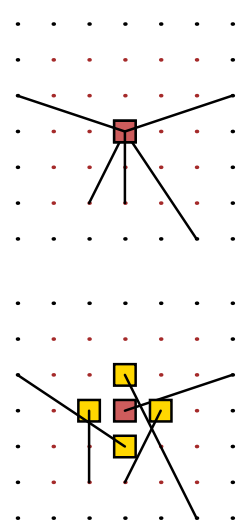

2. cell+links

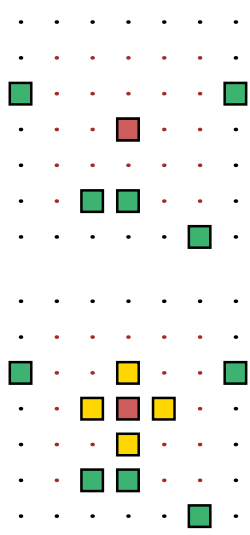

3. cell+inputs only

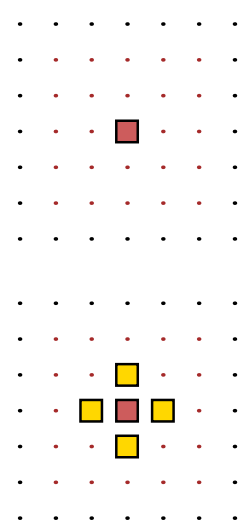

4. cell only
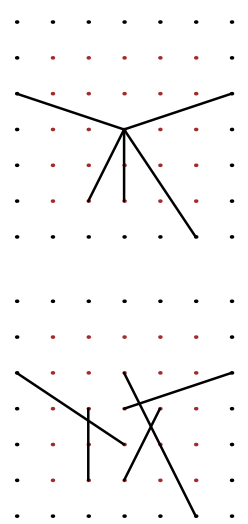

5. links only

Figure 17.11: Toggling between 5 alternative ways of showing a cell and its connections in $2 \mathrm{~d}$ with links-n. Top Row: shows direct wiring. Bottom Row: shows the pseudo-neighborhood toggled on (with pseudo-p, section 17.6.4). $n=7 \times 7$ random (nonlocal) wiring, $k=5$.

\subsubsection{Include the pseudo-neighborhood, or direct wiring only, 2d}

By default, "direct wiring" is displayed if the wiring is nonlocal, and the pseudo-neighborhood is displayed for local CA wiring. Enter $\mathbf{p}$ to toggle between the two, as between the top and bottom rows of figure 17.11. 


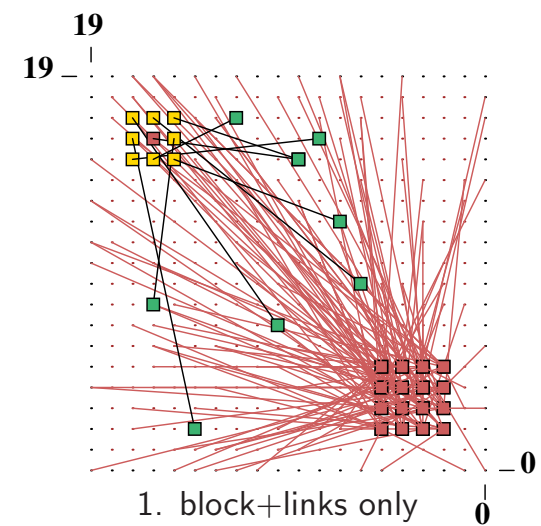

Left: The 2d wiring graphic, just after a block was set - defined by the lower right $(2,2)$ and upper left $(5,5)$ coordinates. While visible, the presentation of the block is subject to the 5-way toggle (with $\mathbf{n}$ ). The active cell behaves as usual, apart from not responding to $\mathbf{n}$, but if the block is made invisible by toggling with $\mathrm{g}$, the 5 -way toggle applies to the active cell (as in figure 17.11) instead of the block.

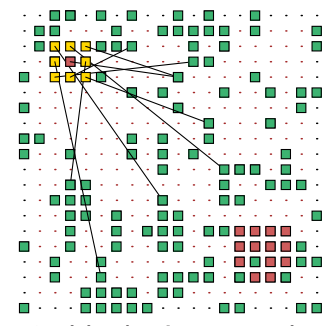

2. block+inputs only

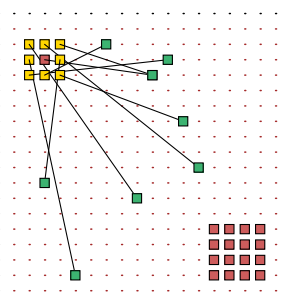

3. block only

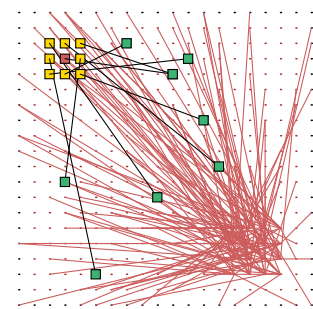

4. links only

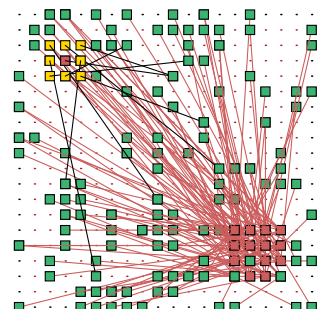

5. block+input+links

Figure 17.12: Toggling between 5 alternative presentations of a $2 \mathrm{~d}$ block (with $\mathbf{n}$ ), starting with the initial block presentation (1. block+links only). $n=20 \times 20 \mathrm{RBN}, k=9$.

\subsubsection{Defining a block, 2d}

Enter $\mathbf{b}$ to define a $2 \mathrm{~d}$ block of cells. The following top-right prompt is presented,

2d block-2, all-a single cell-def: (then, if $\mathbf{2}$ is selected ...)

2d block (this=16,16 max 19,99) (for a $20 \times 20$ )

low corner (def 0,16$)$ I: J:

high corner $(\operatorname{def} 0,16)$ I: $\mathrm{J}$ :

Enter $\mathbf{2}$ to define a block, a to define the whole network as a block. If $\mathbf{2}$ was entered, enter the low and high cell coordinates defining the block. The wiring of all the cells in the block will be shown in the graphic as direct links colored light red, as in figure 17.12. The active cell will still be visible and can be moved, and its pseudo-neighborhood toggled with $\mathbf{p}$, as usual. As long as a block is active and visible (toggle with $\mathbf{g}$ ), the 5 -way toggle $\mathbf{n}$ applies to the block.

For a $2 \mathrm{~d}$ network shown in $1 \mathrm{~d}$, the prompt is as shown in section 17.5.3

An active block is indicated in the wiring graphic reminder (section 17.4),

rewire 2d block 2,2-5,5: ...

To deactivate the block enter $\mathbf{b}$ in section 17.4, then return. If the block is active and visible the following rewire options...

... rnd-r/w/R special-s local:1d-1 2d-2

change $\mathbf{k}=\mathbf{9}(\max 13)-\mathbf{k} \ldots$ (for example) 
... will apply to just the block, not to the active cell if it is outside the block.

The following rule options apply to the active cell, but if the block is active and visible the new rule can then be copied to the block.

... rule:Save/rev/trans-S/v/t

\subsubsection{Toggling the block, $2 \mathrm{~d}$}

If a block is active (section 17.6.5), enter $\mathbf{g}$ to toggle the block, making it either visible or invisible, without deactivating it.

\subsubsection{Expand/Contract the scale, 2d}

The scale of the $2 \mathrm{~d}$ wiring graphic is set automatically to fit within its window. Enter e to expand, c to contract this default scale. The minimum contraction is one screen pixel for each network cell.

\subsubsection{Shifting the $2 \mathrm{~d}$ graphic up and down}

If only part of the graphic fits within the display area, it can be shifted up and down to see the relevant part. This may be necessary for larger $2 \mathrm{~d}$ networks, especially if they have been expanded in section 17.6.7. Enter $\mathbf{u}$ or $\mathbf{d}$ to move the graphic up or down by $1 / 5$ of its vertical dimension.

\subsection{Wiring graphic, 3d}

Enter 3 in section 17.1 , for the $2 \mathrm{~d}+3 \mathrm{~d}$ graphic, where the network is shown simultaneously in $2 \mathrm{~d}$ and $3 \mathrm{~d}$ (see figure 17.13). As in $2 \mathrm{~d}$, this shows how a particular cell (the "active cell"), or a pre-defined $3 \mathrm{~d}$ block of cells, is wired in the $3 \mathrm{~d}$ network. The $2 \mathrm{~d}$ and $3 \mathrm{~d}$ versions of the $2 \mathrm{~d}+3 \mathrm{~d}$ graphic can be independently expanded and contracted.

The $2 \mathrm{~d}$ version shows successive horizontal slices (levels), stacked above each other, with the levels indicated. For larger networks the $2 \mathrm{~d}$ version can be shifted up and down to see the relevant part. Otherwise the $2 \mathrm{~d}$ version behaves very much as the $2 \mathrm{~d}$ graphic described in section 17.6.

The $3 \mathrm{~d}$ version shows an isometric projection seen from below, as if looking up into a cage. The active cell (for $2 \mathrm{~d}+3 \mathrm{~d}$ together) is moved around one level with the arrow keys, the square bracket keys [ and ] move up and down between levels, and its possible to "jump" to a new location. The rule for the active cell appears in the rule window described in section 16.19. As in the $2 \mathrm{~d}$ wiring graphic (section 17.6), the display can be toggled between "direct wiring" and wiring to the pseudo-neighborhood (as in figure 17.13). By default direct wiring is displayed if the wiring is nonlocal, and to the pseudo-neighborhood for local CA type wiring. There is also a 5-way toggle to alter the presentation of connections, and the cells connected, which is especially useful in visualizing blocks. The current cell is colored red, its pseudo-neighborhood yellow, the "actual neighborhood" cells are colored green (figure 17.14).

Sections 17.7.1 - 17.7.8 below explain some of the options specifically for $3 \mathrm{~d}$ in more detail that were summarized in section 17.4.1. 


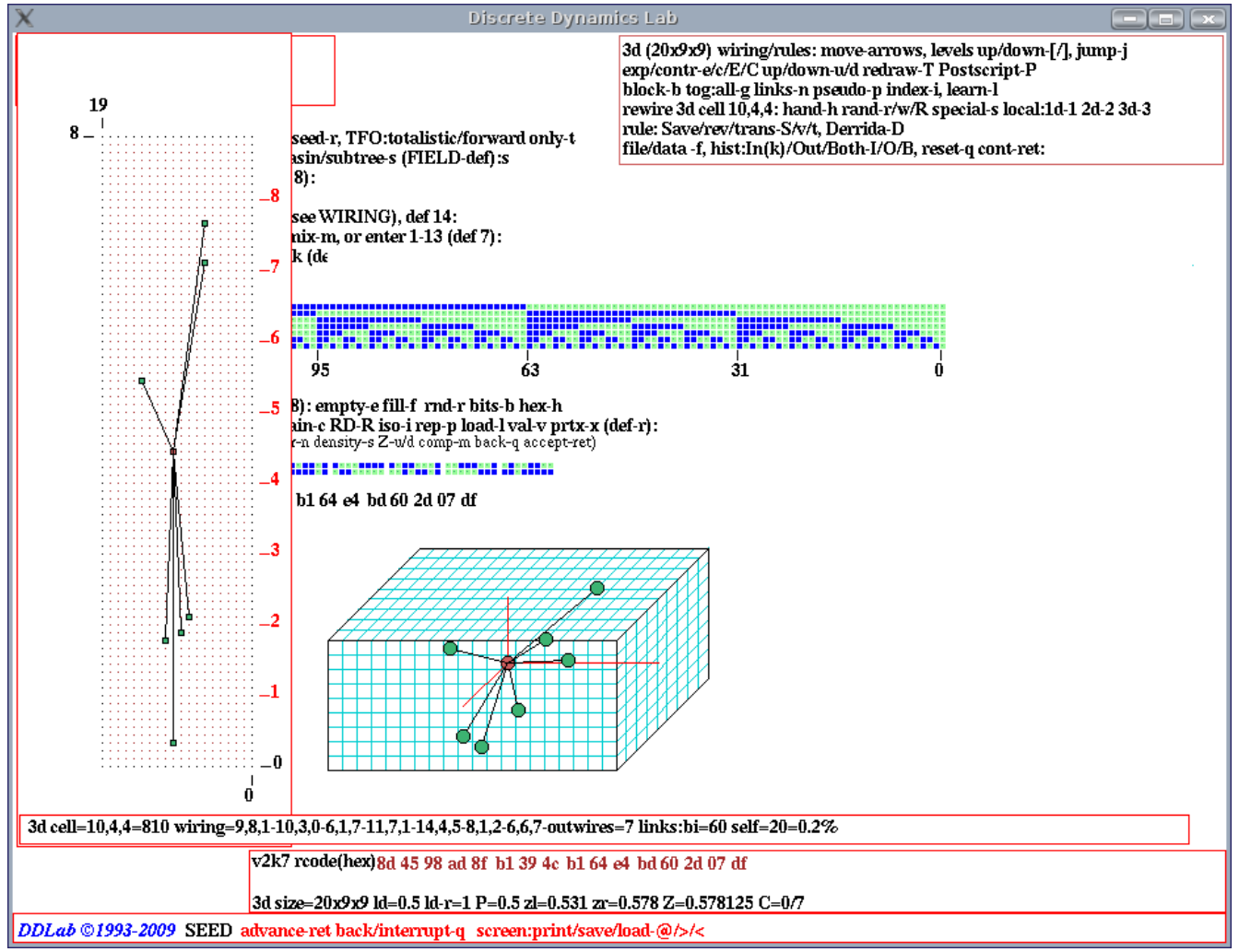

Figure 17.13: A 3d wiring graphic as it appears on the DDLab screen after the rules have been set. $[i, j, h]=[20 \times 9 \times 9]$ RBN, $k=7$. Left: the network in 2 d showing successive levels 0 to 9 stacked above each other. Right: the network in $3 \mathrm{~d}$, as if looking up into a cage.

\subsubsection{Data - 3d wiring graphic}

As for the $1 \mathrm{~d}$ and $2 \mathrm{~d}$ wiring graphics, various data about the current cell's wiring are shown at the foot of the $3 \mathrm{~d}$ wiring graphic as in figure 17.14. See section 17.8.1 to decode this data. The current cell position, and the actual neighborhood positions, are shown as $i, j, h$ coordinates. The rule and rule data are shown in the rule window (section 16.19).

\subsubsection{Moving or jumping between cells, 3d}

To move the active cell use the arrow keys to move around one level. The square bracket keys [, ], move up and down between levels, Alternatively, enter $\mathbf{j}$ to jump to a specific cell. The $3 \mathrm{~d}$ cell position is specified according to the $i, j, h$ coordinates. The following prompts are presented,

jump to index $\mathbf{I}, \mathbf{J}, \mathbf{H}$ (this example for a $3 d$ network size $20 \times 9 \times 9$ )

enter I(19-0): $\quad$ enter $J(8-0)$ : $\quad$ enter $\mathbf{H}(8-0)$ : 


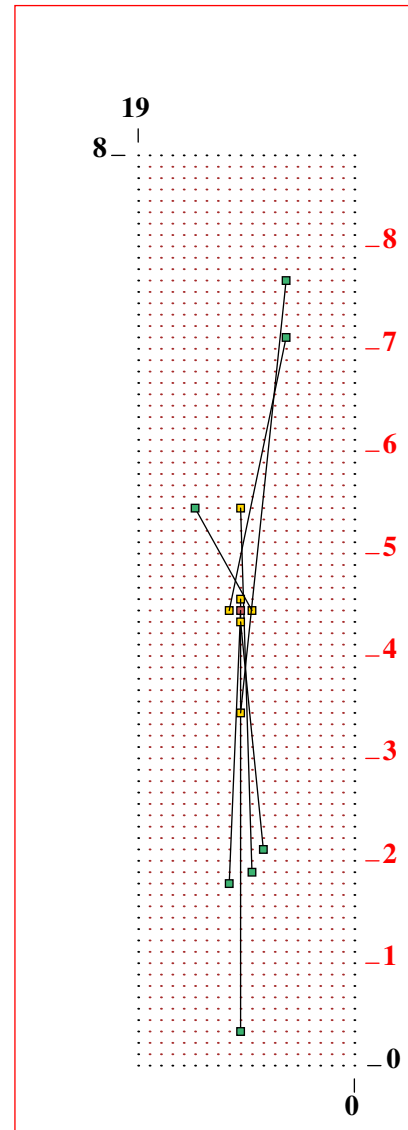

Figure 17.14: 3d wiring graphic: as figure 17.13 but toggled (with $\mathbf{p}$ ) to show the pseudo-neighborhood. $[i, j, h]=[20 \times 9 \times 9]$ RBN, $k=7$. Left: the network in $2 \mathrm{~d}$ showing successive levels 0 to $\overline{9}$ stacked above each other (expand/contract with e/c). Right: the network in $3 d$, as if looking up into a cage (expand/contract with $\mathbf{E} / \mathbf{C}$ ), where you see the ceiling, back wall and right side wall. Bottom: wiring data.

$3 d$ cell $=10,4,4=810$ wiring $=9,8,1-10,3,0-6,1,7-11,7,1-14,4,5-8,1,2-6,6,7-o u t w i r e s=7$ links:bi=60 self $=20=0.2 \%$

\subsubsection{Alternative wiring presentation, 3d}

A 5-way toggle allows alternative presentations of the active cell, or an active block. Enter $\mathbf{n}$ to toggle between the five alternatives, as shown in $3 \mathrm{~d}$ in figure 17.15 - alternatives also appear in the $2 \mathrm{~d}$ version of the $3 \mathrm{~d}$ wiring graphic in a similar way as in figure 17.12. For the active cell, the 5-way toggle works both with direct wiring and with the pseudo-neighborhood (pseudo-p) in section 17.7.4.

For a $3 \mathrm{~d}$ block, the alternatives are shown in in figure 17.16 .

\subsubsection{Include the pseudo-neighborhood, or direct wiring only, 3d}

As in 2d, by default, "direct wiring" is displayed if the wiring is nonlocal, and the pseudoneighborhood for local CA wiring. Enter $\mathbf{p}$ to toggle between the two, as between the top and bottom rows of figure 17.15.

\subsubsection{Defining a block, 3d}

Enter $\mathbf{b}$ to define a $3 \mathrm{~d}$ block of cells. The following top-right prompt is presented, 

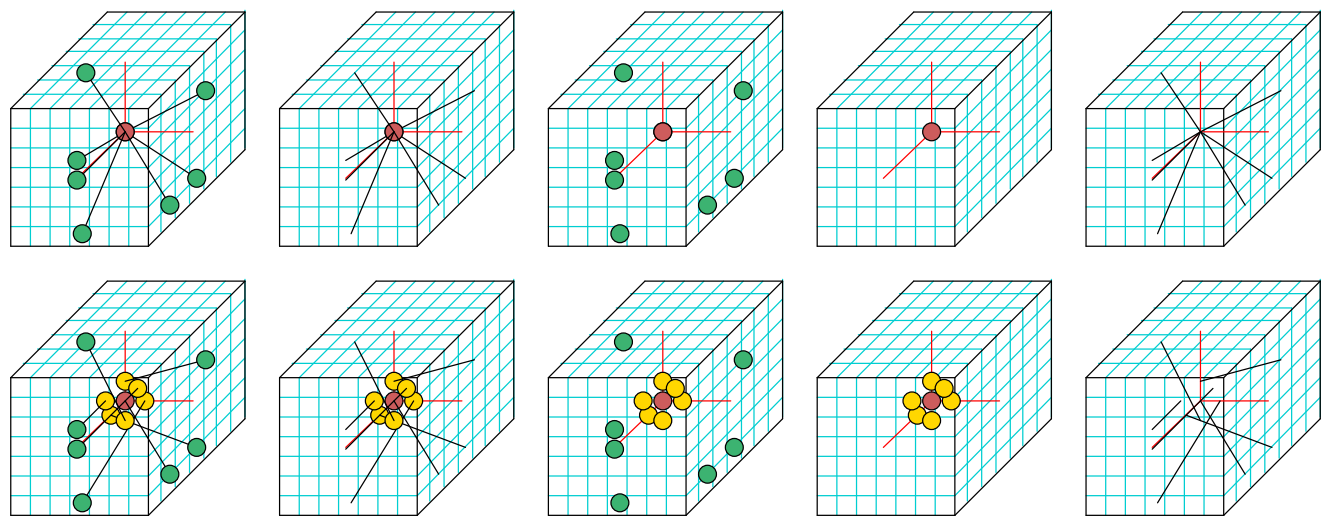

3. cell+inputs

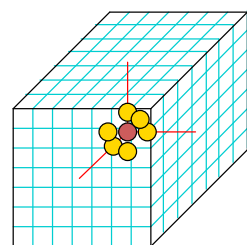

4. cell+only

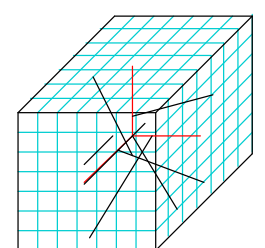

5. links only

Figure 17.15: Toggling between 5 alternative ways of showing a cell and its connections in $3 \mathrm{~d}$ with n. Top Row: with direct wiring. Bottom Row: with the pseudo-neighborhood set (toggle with $\mathbf{p}$, section 17.7.4). $n=7 \times 7 \times 7$ RBN, $k=7$. The $2 \mathrm{~d}$ version of the $3 \mathrm{~d}$ wiring graphic (as in figure 17.14) presents the same information.

3d range-3, all-a single cell-def: (then, if $\mathbf{3}$ is selected ...)

3d block (this $=\mathbf{7 , 7 , 7} \max \mathbf{1 4 , 1 4 , 1 4 )}$ (for a $40 \times 40$ )

low corner $(\operatorname{def} 0,0,7)$ I: $\mathrm{J}: \quad \mathrm{H}$ :

high corner $($ def 0,24$)$ I: $\quad$ : $\quad H:$

Enter 3 to define the block, a to define the whole network as a block. If $\mathbf{3}$ was entered, enter the low and high cell indexes defining the block. The wiring of all the cells in the block will be shown in the graphic as direct links, colored light red. The active cell will still be visible and can be moved as usual.

For a $3 \mathrm{~d}$ network shown in $1 \mathrm{~d}$, the prompt is as shown in section 17.5.3

An active block is indicated in the wiring graphic reminder (section 17.4),

rewire 3d block 9,9,9-15,15,15: ...

To deactivate the block enter $\mathbf{b}$ in section 17.4 , then return. If the block is active and visible the following rewire options ...

\section{... hand-h rnd-r/w/R special-s local:1d-1 2d-2 3d-3 \\ change $\mathbf{k}=\mathbf{7}(\max 13)-\mathbf{k} \ldots$ (for example)}

... will apply to just the block, not to the active cell if it is outside the block.

The following rule options apply to the active cell, but if the block is active and visible the new rule can then be copied to the block.

\section{... rule:Save/rev/trans-S/v/t}

\subsubsection{Toggling the block, $3 \mathrm{~d}$}

If a block is active (see 17.7.5 above), enter $\mathbf{g}$ in section to toggle the block, making it either visible or invisible, without deactivating it. 


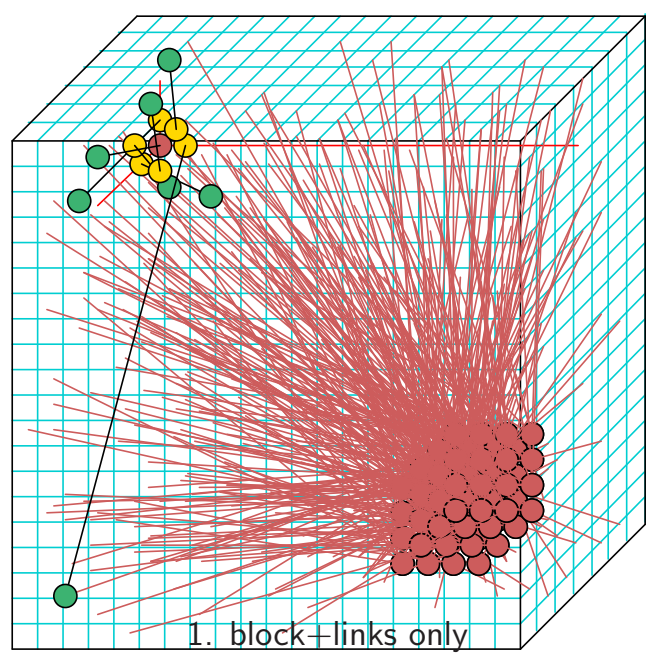

Left: The 3d wiring graphic, just after a block was set - defined by the lower right $(2,2,2)$ and upper left $(5,5,5)$ coordinates. While visible, the presentation of the block is subject to the 5-way toggle (with $\mathbf{n}$ ). The active cell behaves as usual, apart from not responding to $\mathbf{n}$, but if the block is made invisible by toggling (with $\mathrm{g}$ ), the 5-way toggle applies to the active cell (as in figure 17.11) instead of the block.

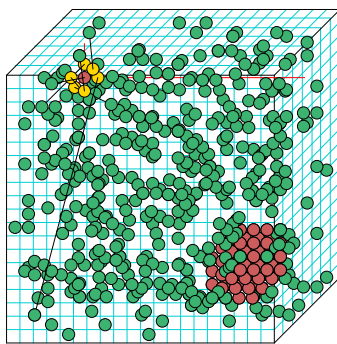

2. block+inputs only

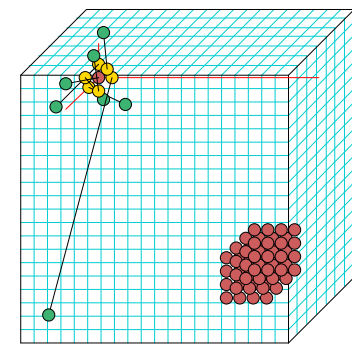

3. block only

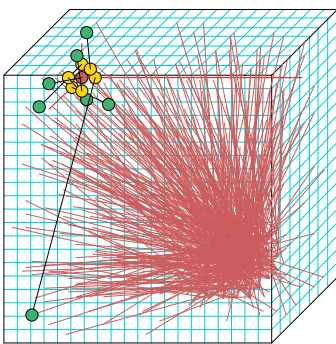

4. links only

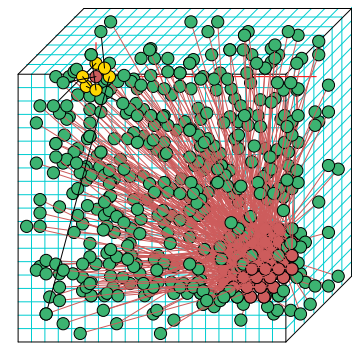

5. block+input+links

Figure 17.16: Toggling between 5 alternative presentations of a $3 \mathrm{~d}$ block (with $\mathbf{n}$ ), starting with the initial block presentation (1. block+links only). $n=20 \times 7 \times 20 \mathrm{RBN}, k=7$. The $2 \mathrm{~d}$ version of the $3 \mathrm{~d}$ wiring graphic (as in figure 17.14) presents the same information.

\subsubsection{Expand/Contract the scale, 3d}

The scale of the $2 \mathrm{~d}$ and $3 \mathrm{~d}$ versions of the $3 \mathrm{~d}$ wiring graphic can be independently expanded and contracted. Enter e to expand, $\mathbf{c}$ to contract the $2 \mathrm{~d}$ version. Enter $\mathbf{E}$ to expand, $\mathbf{C}$ to contract the $3 \mathrm{~d}$ version. The minimum contraction in both cases is one screen pixel for each network cell.

\subsubsection{Shifting the 3d graphic up and down}

If only part of the $2 \mathrm{~d}$ version of the $3 \mathrm{~d}$ wiring graphic fits within the display area, it can be shifted up and down to see the relevant part. This is necessary for larger $3 \mathrm{~d}$ networks, especially if they have been expanded in section 17.7.7.

Enter $\mathbf{u}$ or $\mathbf{d}$ to move the graphic up or down by about one level. Enter $\mathbf{s}$ to restore the default start position. 


\subsection{Further options for the 1d, 2d and 3d wiring graphics}

The following options which were marked with a asterisk in section 17.4 apply jointly to $1 \mathrm{~d}, 2 \mathrm{~d}$ and $3 \mathrm{~d}$ networks, and are described in the remainder of this chapter.sections 17.8.2-17.8.12 below,

\subsubsection{Decoding wiring graphic data - 1d, 2d and 3d}

Various data about the active cell's wiring are shown at the foot of the $1 \mathrm{~d}, 2 \mathrm{~d}$ and $3 \mathrm{~d}$ wiring graphic. For example,

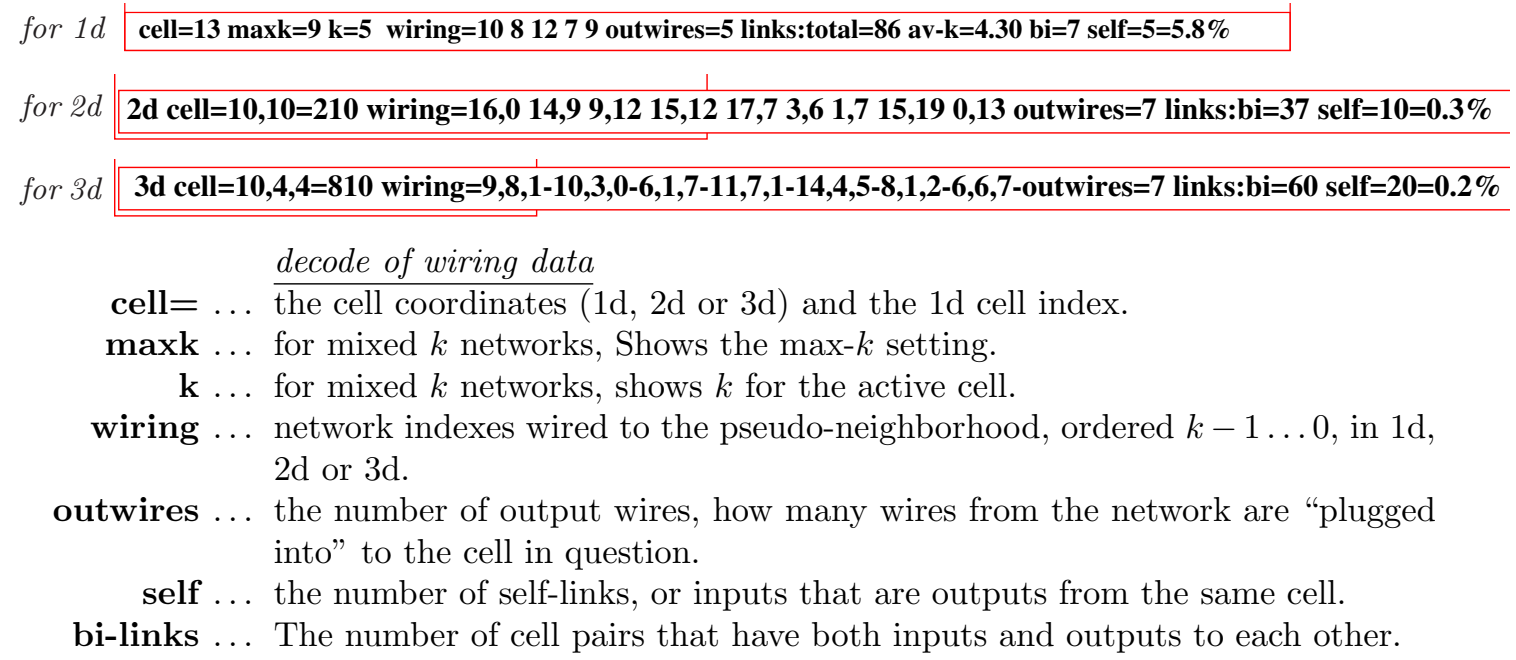

The active cell's rule with other rule details (updated as the active cell is changed) are displayed in the lower rule window at the foot the DDLab screen (section 16.19).

\subsubsection{Computing the (weighted) average $\lambda$ and $Z$ parameters} rcode-mix and/or k-mix

This option applies once rcode-mix has been set in chapter 14 - rcode-mix is set automatically for a $k$-mix.

Enter $\mathbf{z}$ in section 17.4 to calculate and display the average, and weighted average, $\lambda_{r}$ and $Z$ parameters of the rules making up the rulemix.

The data is displayed in a top-right window, for example, for a $1 \mathrm{~d}$ network $n=150, k=3-7$, and randomly assigned rules,

av:ld-r $=0.839063 \mathrm{Z}=\mathbf{0 . 6 3 6 5 3}$

wt.av:ld-r $=0.826438 \mathrm{Z}=0.626692$ cont-ret:

For random wiring and/or mixed $k$ networks, the weighted averages take account of the influence that each cell has on the network according to its proportion of the network's out-wires, represented graphically by the height of cells at $t_{0}$ in a $1 \mathrm{~d}$ wiring graphic, for example in figure 17.4.1. In networks with local wiring and no rulemix the weighted average equals the average. 


\subsubsection{Options for learning pre-images \\ rcode only - not in TFO-mode.}

Enter $\mathbf{L}$ in section 17.4 to start the "Learning, forgetting, and highlighting" functions (chapter 34). Learning/forgetting involves attaching/detaching sets of states as pre-images of a target state. For attractor basins, enter $\mathbf{l}$ to just highlighting selected states.

Learning/forgetting usually requires both nonlocal wiring and a rule-mix. Algorithms automatically adapt the network's wiring and/or rules to achieve the required transitions between states. The results and side affects of learning can be seen most clearly in a basin of attraction field, but the methods (allowing larger networks) also apply to a single basin, a subtree, or simply to a space-time pattern running forward. For further details refer to chapter 34 .

\subsubsection{Hand rewiring}

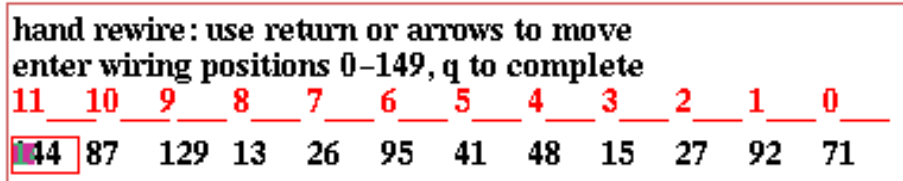

Figure 17.17: Hand wiring a single cell from the 1d wiring graphic, $n=150, k=13$

Enter $\mathbf{h}$ in section 17.4 to rewire individual wires for just the active cell by hand, which is rewired in a way similar to that described in sections 12.6 and 17.2.2, but for the active cell only. A topright window displays the pseudo-neighborhood wiring connections as a 1d "spread sheet" with $k$ entries (figure 17.17), including the following reminder, for example,

\section{hand rewire: use return or arrows to move enter wiring position 0-149, q to complete (this example for a $1 d$ network, size 150)}

Note that the wiring position $x$ is a $1 \mathrm{~d}$ index, even if the network is $2 \mathrm{~d}$ or $3 \mathrm{~d}$. To convert between the index and coordinates see sections 10.2.2 and 10.2.3.

\subsubsection{Random rewiring}

Enter $\mathbf{r}$ in section 17.4 to randomly reset the wiring of the active cell, or of the block if defined and visible. Enter $\mathbf{w}$ to randomly resets the wiring of the cells outside an active block, if the active block is visible.

Both $\mathbf{r}$ and $\mathbf{w}$ will respect the biases on random wiring (which include local CA wiring) set in section 17.8.6 (see also section 12.5). Note that for non-square $2 \mathrm{~d}$ networks and non-cubic $3 \mathrm{~d}$ networks, the random wiring is limited to a local zone equal to the shortest axis. In this case unbiased random wiring (for homogeneous $k$ ) can be set with $\mathbf{R}$.

For a $k$-mix network, if $\mathbf{R}$ is entered, the wiring will be randomly reset, but biased by the $k$ distribution. That is, the probability of plugging wires into a cell with $k$ inputs will be given by $P=k / T$, where $T$ is the total number of links in the network. so that cells with most inputs also end up with the most outputs and vice versa. If a power-law distribution of the $k$-mix was set for the network in section 9.7.2, entering $\mathbf{R}$ will rewire to give an approximate power-law distribution 
of outputs (figure 17.18), creating a "scale free" network, said to be characteristic of many natural and artificial networks, from metabolic networks to the world-wide-web[2]. The graph of such a network is shown in figure 20.2 .

For homogeneous $k, \mathbf{R}$ gives totally unbiased random wiring.

\subsubsection{Biased random rewiring}

If $\mathbf{s}$ (for special) is selected in section 17.4 a series of options are presented in a top-right window that allow a variety of restrictions or biases to the random wiring before it is set in section 17.8.5 above. These biases apply to the active cell, or to the block if defined and visible.

The special wiring options are fully described in section 12.5 (Special wiring, random) - they differ to some degree between the $1 \mathrm{~d}, 2 \mathrm{~d}$ or $3 \mathrm{~d}$ wiring graphics.

\subsubsection{Local 1d, $2 \mathrm{~d}$ or $3 \mathrm{~d}$ wiring}

If $\mathbf{1}, \mathbf{2}$ or $\mathbf{3}$ is selected in section 17.4 the wiring of the active cell, or block if defined and visible, will be reset to local 1d, 2d or 3d CA-like wiring to the local neighborhood, with periodic boundary conditions.

For 3d networks, 1d, 2d or 3d local CA wiring applies. For 2d networks, 1d, 2d local wiring CA applies. For 1d networks, only 1d local CA wiring applies.

Note that CA wiring can also be set as "random wiring" in section 17.8.6 above, allowing various biases to the $\mathrm{CA}$ wiring as described in section 12.5 .

\subsubsection{Changing the neighborhood size, $k$} rcode-mix set only - not in TFO-mode

Given a rcode-mix (section 14.1), and irrespective of $k$-mix, enterk in section 17.4 to change the neighborhood size $k$ of the active cell, or of the block if active and visible. The following additional prompt appears,

$$
\text { reset nhood size k (1-13): (if } \left.k_{L i m}=13\right)
$$

The maximum $k$ allowed is $k_{\text {Lim }}$, which may be greater than the actual max- $k$ in the network (section 9.10 ). $k_{\text {Lim }}=13$ for binary rcode. The new wiring will preserve as much as possible of the old neighborhood wiring. If $k$ is increased, the wiring at the extra pseudo-neighborhood indexes is set as local 1d, 2d or 3d (depending on the network dimensions) with periodic boundary conditions.

For a homogeneous $k$ network, an individual cell or block may only have its $k$ setting reduced. The network will from then on be treated as a $k$-mix with max-k equal to the original value of $k$.

Changing $k$ effects the highest pseudo-neighborhood indexes, which are either removed or added. The original rcode (or part of it if $k$ was reduced) are preserved. Any excess rule-table entries are set to 0 .

Note that $k$ may also be increased from the transformation options (sections 17.8.11, 18.7.1) giving a neutral transformation with equivalent dynamics. 


\subsubsection{Kill a cell}

rcode-mix set only - not in TFO-mode

Enter $\mathbf{K}$ in section 17.4 to kill or neutralize the active cell, or the block if active and visible. by cutting all links, both inputs and outputs. The cell retains one input to itself, $k=1$, and the rcode is set so the cell's value stays constant, with effective $k=0$ (section 9.2). For $v=2$ the decimal rcode is 2 . The cell then has has no influence on the network, which can be applied to model gene knockout. Alternatively, the cell can serve as a constant unchanging input to other cells if they rewire into it.

For 1d networks, a cell can also be deleted entirely (section 17.5.9).

\subsubsection{Revising and copying the rule}

if rules are set

Enter $\mathbf{v}$ in section 17.4 to revise the rule of the active cell. Once the rule is revised, it can be copied to a block. This option is intended for networks with mixed rules. For a single rule network (set in section 14.1), i.e. without a rulemix, any revision applies to the whole network. Note that a rulemix where all the rule are the same can be set up as described in section 14.4.3.

A secondary window is presented in the lower right hand corner of the screen, with rule selection prompts. The various methods for revising and re-selecting rules are the same as in Setting a singe rule described in chapter 16 .

If a block is active and visible, the rule can be automatically copied to all cells in the block which have the same $k$ as the cell in question. The following prompt is presented in a top-right window, showing the block coordinates and their 1d equivalent indexes in brackets (section 17.2),

for a 1d wiring graphic (values shown are examples)

Copy rule to 33-55 -c:

for a $2 d$ wiring graphic, $40 \times 40$

Copy rule to $2 \mathrm{~d}$ range $0,22-39,33$ (880-1359)-c:

for a $3 d$ wiring graphic, $20 \times 20 \times 20$

Copy rule to $2 d$ range 0,0,8-19,19,12 (3200-5159)-c:

Enter $\mathbf{c}$ to copy the rule within the block, to all cells with matching $k$. Once set (and copied), the new rule is displayed in the rule window (section 16.19).

To change a rule to one with a different neighborhood size $k$, first change $k$ in the rewiring window (section17.8.8), then change the rule.

\subsubsection{Transforming the rule}

rcode set only - more options rcode-mix - more options $k$-mix - not in TFO-mode

If $\mathbf{t}$ is selected in section 17.4, the rcode for just the active cell may be transformed in the various ways described in detail in Transforming network rules, chapter 18. This option is intended for networks with rcode-mix or $k$-mix. For networks with homogeneous rcode any revision applies to the whole network.

A top-right window is presented with the transformation prompts. The rule may be transformed to equivalent or related rules. For example the rule may be complemented, or transformed to an equivalent rule by negative or reflection transformations. Canalyzing inputs may be set or amended 
for the active cell or the whole network as described in section 15. A $k$-mix allows more options - neutral transformations to rules with greater $k$, or $k$ reduced to "effective $k$ " for the particular cell or for the whole network.

The network may be "reverse engineered" by loading an exhaustive mapping of transitions (section 18.7.4) and automatically generating the minimal mixed- $k$ network that satisfies the mapping, (i.e. reduced to effective- $k$ ), one solution to the "inverse problem".

\subsubsection{Filing, from the wiring graphic}

Enter $\mathbf{f}$ in section 17.4 for the network filing options described in chapter 19, Save/load/print network architecture. To load a network file, first set up a similar "dummy" network. The whole network or just the wiring or rulemix can be saved or loaded to a file. The options vary according to a $k$-mix, rules set, and other constraints.

If a network file smaller than the base network is loaded, it will be automatically be defined as a block in $1 \mathrm{~d}, 2 \mathrm{~d}$ or $3 \mathrm{~d}$. A prompt first locates the block, for example in a $2 \mathrm{~d}$ network,

\section{$2 \mathrm{~d}: \mathrm{i}, \mathrm{j}=40,40$, file: $\mathrm{i}, \mathrm{j}=11,11$, enter start coords (def 14,14 , max 29,29 , rnd-r) I: $\mathbf{J}:$}

Enter the lower coordinates of the block, $\mathbf{r}$ for a random position, or return for a central position. Once loaded, the block is shown in the wiring graphic of the base network as in figures 17.4.1, 17.12 and 17.16. Any number of sub-networks can be loaded into the base network.

A network description can also be printed to a file, to the xterm window for Linux-like systems, or to a printer for DOS. For further details see chapter 19, Save/load/print network architecture.

\subsubsection{The histogram of the network's $k$ and output distribution}

Enter I, $\mathbf{O}$ or $\mathbf{B}$ in section 17.4 to show a histogram of link frequency distribution. I gives the $k$ distribution - of inputs, $\mathbf{O}$ gives the output distribution, and $\mathbf{B}$ gives both the input and output, i.e of all connections in the network. The $k$ distribution can also be displayed from section 9.11. Examples of power-law distributions are shown in figures 17.18. Random wiring without bias would give a Poisson distribution as in figure 9.1.

The histogram plots each $k$ or output ( $x$ axis), against its frequency in the network as a percentage ( $y$ axis). The actual frequency, as a percentage and total, are shown under each histogram bar. The following information and prompt is also shown,

$$
\mathbf{n}=\mathbf{1 0 0} \text { av-k=2.02 save/load-s/l cont-ret: (for example) }
$$

$\mathbf{n}$ is the network size which should equal the sum of all the frequency totals. av-k, av-out or av-in-out is the average $k$, out-degree, or all links to a node.

Enter $\mathbf{s}$ or $\mathbf{l}$ save or load the histogram data as a .his file (section 35.2). In both cases the data appear in the xterm window (not for DOS), for example for figure 17.18 Top Left,

histogram: $1+13$ columns

$\begin{array}{llllllllllllll}0 & 1015 & 254 & 113 & 64 & 41 & 29 & 21 & 16 & 13 & 11 & 9 & 8 & 6\end{array}$ 


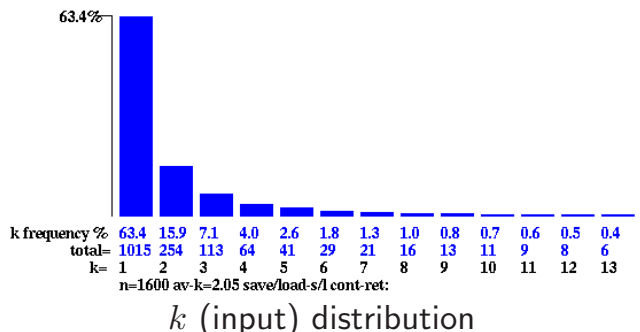

$k$ (input) distribution

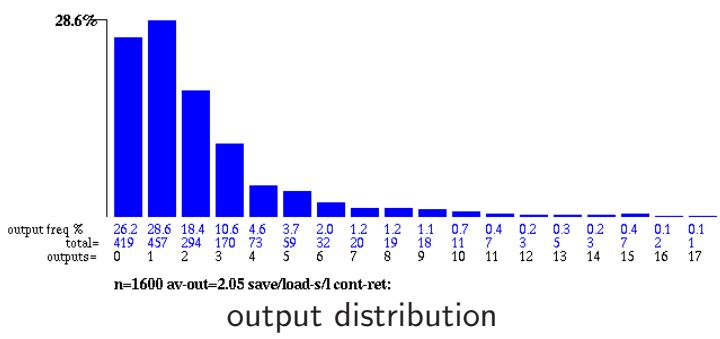

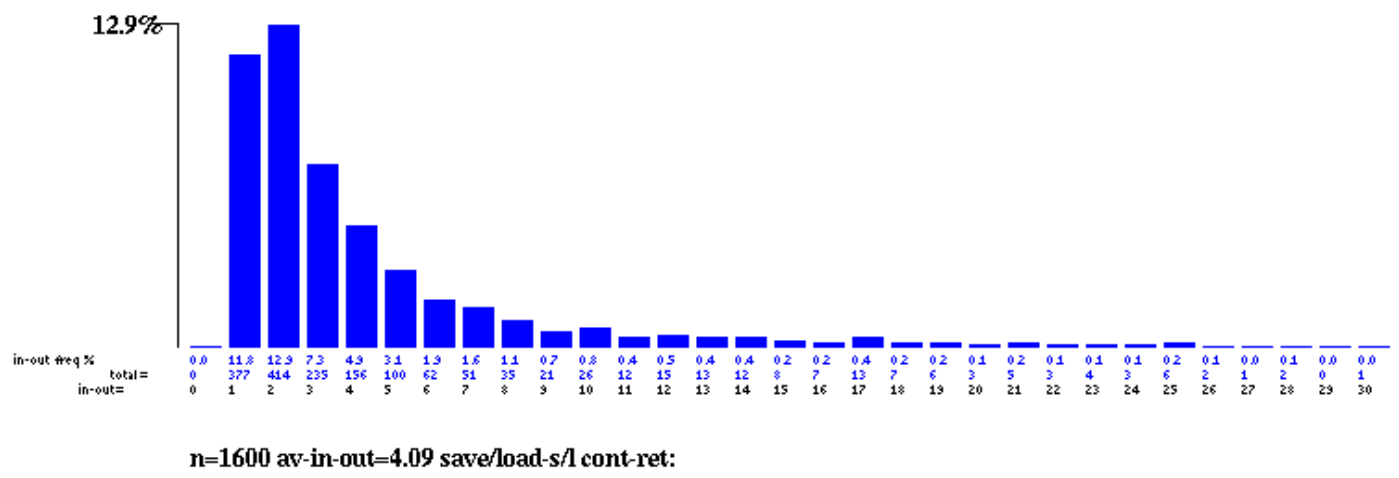

both $k$ and output distribution

Figure 17.18: Histograms of a power-law distribution of network links, $(k)$ inputs, outputs, and both combined. The power-law exponent was set to 2.0 in section 9.7 .2 for a mixed $=k$ network $40 \times 40$. Random wiring was reset for the whole network with $\mathbf{R}$ (section 17.8 .5 ), where outputs are preferentially allocated according to $k$.

\subsubsection{Creating a vector PostScript file of the wiring graphic}

Enter $\mathbf{P}$ in section 17.4 to save the wiring graphic image (1d, 2d or $3 \mathrm{~d}$ ) as a vector PostScript file following follow the presentation exactly as on the screen, including text data. The following top-right prompt appears,

\section{save wiring graphic to PostScript: greyscale-P color-p:}

Select greyscale or color, then the filename - default my_wgPS.ps. The complete wiring graphic will be redrawn as the file is being created, for example, figures 17.4.1, 17.10 and 17.14 in this chapter. Note that a vector PostScript image can be cropped (section 36.1). 


\section{Chapter 18}

\section{Transforming rules}

rcode only - not TFO-mode

If not in TFO-mode, rule transformation options are automatically presented after a single rcode, kcode or tcode has been set (section 16.21), and can also be selected from the following options,

- the wiring graphic, section 17.8.11

- space-time pattern interrupt options, section 32.16.1.

- attractor basin complete options, section 30.5

For totalistic rules the equivalent rcode expression of the kcode or tcode will be transformed. If there is not a rulemix, transformations apply to the whole network. For a rulemix from the wiring graphic, transformations apply to the active cell. When interrupting space-time pattern or attractor basin, the required cell index is first selected with an upper-right prompt, similar to the examples below,

enter cell index, 1599-0: (this example for $2 d$ space-time patterns ${ }^{1}, 40 \times 40$ )

enter cell index, 11-0: (this example for an attractor basin, $n=12$ )

The rcode may be saved at any stage as a *.rul file (chapter 19), and the transformed rcode is displayed in the lower rule window. The transformation options include,

- inverting the rcode between two conventions for listing neighborhoods (section 18.3).

- various manipulations of the rcode table, including "solidifying" (section 18.4) and negative and reflection transformations within equivalence classes and rule clusters[25] (section 18.5).

- setting or amending canalyzing inputs ( $v=2$ only) (section 18.6).

- transforming the neighborhood ${ }^{2}$, equivalent rcode with greater $k$, or with smaller "effective- $k$ " (section 18.7).

- creating a network to satisfy an exhaustive mapping of transitions ( $v=2$ only) (section 18.7.4).

\footnotetext{
${ }^{1}$ Note that the cell index $x$ is expressed as $1 \mathrm{~d}$, even if the network is $2 \mathrm{~d}$ or $3 \mathrm{~d}$ - sections 10.2 .2 and 10.2.3 explain how to convert $x$ to $2 \mathrm{~d}$ or $3 \mathrm{~d}$ coordinates, and vice versa.

${ }^{2}$ Transforming the neighborhood does not apply when interrupting space-time patterns (section 32.16.1) or attractor basins.
} 


\subsection{Options for transforming rules}

Examples of the transformation options are show below. They vary according to the context ${ }^{3}$ depending on factors such as the rulemix, $k$-mix, and $k_{\text {max }}$. Any number of transformations may be made cumulatively.

\subsubsection{Transform options, single rule}

For a single rule network, transformations apply to all cells in the network.

transform rcode: invert-v solid-o comp-m neg-n ref-r canal-C

equiv $>\mathbf{k}(3)$ :(4-13), eff $\mathbf{k}($ if $k=3$ )

save/prtx: rcode-s/x

or if totalistic, kcode (or tcode)

save/prtx: rcode/kcode-s/S/x/X

\subsubsection{Transform options, mixed $k$}

For a mixed- $k$ network the transformation options are invoked from the wiring graphic in section 17.8.11. Then first two lines of the prompt are ...

transform rule: invert-v solid-o comp-m neg-n ref-r canal-C (all+a) equiv $>\mathbf{k}(3):(4-7)$, max $\mathbf{k}-\mathbf{M}$, eff $\mathbf{k}$ : all-K this-k (if $k=3$ and $k_{\max }=7$ )

If $k=k_{\max }$ equiv $>\mathbf{k} \ldots$ is omitted.

For $v=2$ only, and $k_{\max }=n$ and $n \leq 13$, there is an additional option to load an exhaustive map (.exh) file (see also section 29.8.1), which would have been saved previously in section 29.7.2, so the second line of the prompt would start with ...

exh map-e, ...

\subsubsection{Transform options, mixed rule, homogeneous $k$}

For a mixed rule network with homogeneous $k$ network, the "greater $k$ " and "effective $k$ " transformations do not apply.

transform rule: invert-v solid-o comp-m neg-n ref-r canal-C (all+a) save-s:

Note that a mixed- $k$ network may be set up where all rules and $k$ 's are the same, allowing the full scope of the transformations.

\footnotetext{
${ }^{3}$ Transformations which change the size of $k$ are omitted while interrupting space-time patterns, and the transformation prompt is deactivated altogether while interrupting incomplete attractor basins.
} 


\subsubsection{Transform all cells in a mixed rule network}

For a single rule network, transformations described in sections 18.3 to 18.6 apply to all cells, whereas for a mixed rule or mixed- $k$ network, just the selected cell in the wiring graphic (section 17.8.11) can be transformed, or all cells in the network.

To transform all cells, add a to the selection, i.e. enter va in section 18.3, oa in section 18.4, ma in section 18.5.1, na in section 18.5.2, ra in section 18.5.3 and Ca in section18.6. Entering Ca allows canalyzing inputs for the whole network to be reset as described in chapter 15 .

\subsection{Saving or printing the transformed rule}

The transformed rule (rcode) is displayed in the rule window. Enter $\mathbf{s}$ at prompt in section 18.1.1 to save the rule (section 35.2), or $\mathbf{x}$ to print the rule in the terminal (xterm) for Linux-like systems. If a totalistic rule was selected, $\mathbf{S}$ will save, and $\mathbf{X}$ will print, the totalistic version of the rule the kcode or tcode.

\subsection{Inverting the rcode}

The rcode-table (or any rule-table) can be expressed according to two alternative conventions, where the output of the all-0s neighborhood is either on the left or right. In DDLab the all-0s output is on the right (the least significant bit/value) and the all-max-value output is on the left (sections 13.3, 13.5), so that a rule-table string can be expressed as a decimal number following Wolfram convention[22] (if within the limits in section 16.6). Other conventions (e.g. [13]) follow the opposite order.

Enter $\mathbf{v}$ (or va) at the prompt in section 18.1 to invert the rcode/s between the two conventions. Effectively, the outputs of complementary neighborhood pairs in the rule-table are swapped. For example (for $k=5$ ) the outputs of 00000 and 11111, or 00111 and 11000, etc. The example in figure 18.1 shows a $1 \mathrm{~d} v 2 k 7$ "density classification" CA rcode[13] inverted to the convention in DDLab.

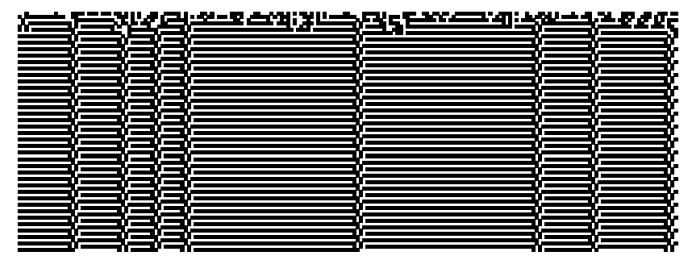

$0504058705000 f 77037755837 \mathrm{bffb} 77 \mathrm{f}$

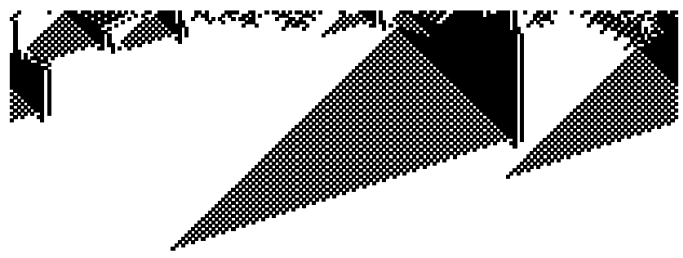

feedffde c1aaeec0 eef000a0 ela020a0

Figure 18.1: Transforming rcode: Left: the space-time patterns of a $1 \mathrm{~d}$ density classification rule from[13] does not appear as intended because the order convention is the opposite of DDLab's.

Right: after the rule is inverted to DDLab's convention the density rule behaves correctly. $n=199$, $\overline{v 2 k 7}$. The same random initial state is used in both cases. The rule-tables are shown in hexadecimal. 


\subsection{Solidifying the rule}

If $\mathbf{o}$ (or oa) is entered at the prompt in section 18.1, the rcode/s will be changed to achieve the following behavior. For binary $(v=2)$ "on" (i.e. "1") cells will remain on. For $v \geq 3$ a cell with the highest value $(v-1)$ will remain on.

\subsection{Equivalence classes and rule clusters}

As described in [25], a binary $1 \mathrm{~d}$ rcode has equivalent rcodes by three symmetry transformations, negative, reflection, and negative+reflection in either order (figure 18.2), described in sections 18.5 .2 and 18.5.3 below. The transformations make potentially 4 equivalent rcodes there may be fewer because a transformation may lead to identity. For example, the rule-space of $256 v 2 k 3$ elementary rules[22] collapse into 88 equivalence classes, so only 88 prototype rules are required to fully describe rule-space behavior[25]. The rcodes are equivalent in the sense that a transformed initial state will make equivalent transformed space-time patterns, and attractor basins states are equivalent in the same way - their topology is identical.

Complementary transformations (section 18.5.1) will further group the rcodes into 48 rule clusters[25] which share a number or properties, including in-degree, $g$-density and the $Z$-parameter. For $v \geq 3$, and greater dimensions, there will be more equivalent rules in an equivalence class, by permutations of values and more reflections.

\subsubsection{Complementary transformation}

If $\mathbf{m}$ (or $\mathbf{m a}$ ) is entered at the prompt in section 18.1, the rcode/s will transformed to its "complement". For binary $(v=2)$ this is done by flipping all entries in the rcode-table, 1 to 0 and 0 to 1 . For $v \geq 3$ each entry $a$ is changed to its complement $a_{c}=(v-1)-a$. See section 16.20 for a further explanation. A complementary rcode is usually not equivalent to the original.

\subsubsection{Equivalent rcode by the Negative transformation}

If $\mathbf{n}$ (or na) is entered at the prompt in section 18.1, the rule will be transformed into its "negative" equivalent rule, by swapping the outputs of complementary neighborhood pairs in the rule-table, then transforming the resultant rule-table into its complement. A negative rule and the start rule have equivalent dynamics, but with all state configurations in the space-time pattern complemented.

Note that a negative transformation followed by a reflection transformation (section 18.5.3), or vice versa (the order is equivalent) produces another equivalent rule, making 4 in all. For certain rules the transformations result in identity.

\subsubsection{Equivalent rcode by the Reflection transformation}

If $\mathbf{r}$ (or ra) is entered at the prompt in section 18.1, the rule (or rules) will be transformed into its equivalent rule by "reflection" (by swapping the outputs of pairs of asymmetric reflected neighborhoods in the rule-table). A reflected rule and the start rule have equivalent, but reflected dynamics. 


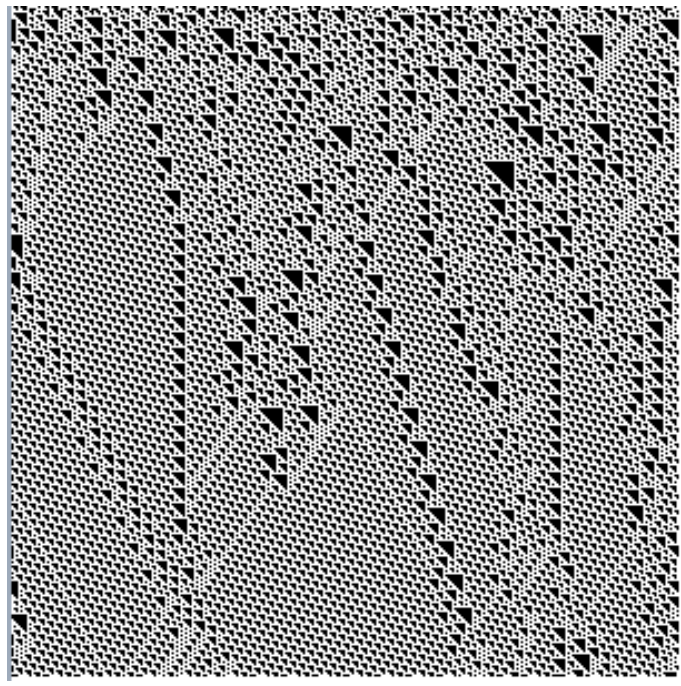

rule 193

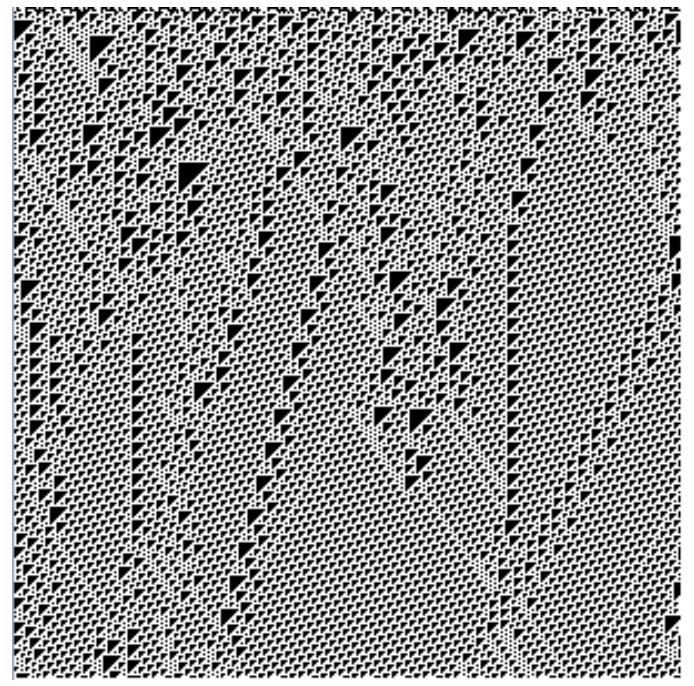

rule $137\left(193_{r}\right)$

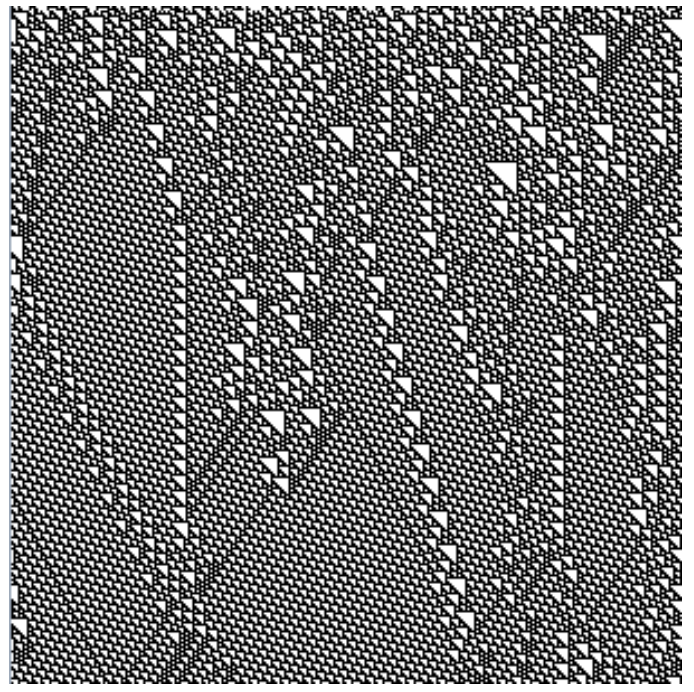

rule $124\left(193_{n}\right)$

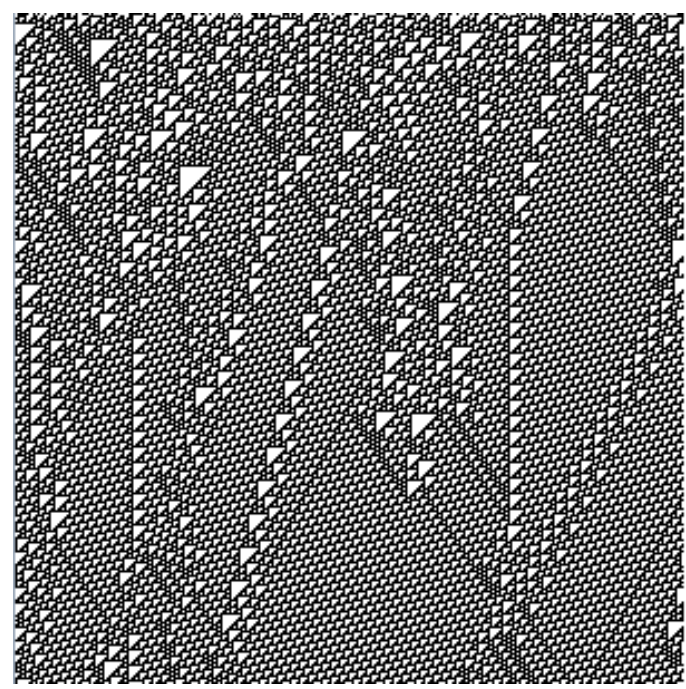

rule $110\left(193_{n r}\right)$

Figure 18.2: Equivalent transformations of the elementary rule $v 2 k 3$ rcode (dec)193, giving (negative) rule 124, (reflected) rule 137, and (negative+reflected) rule 110 (in either order). The initial state $E$ has also been transformed to $E_{n}, E_{r}$, and $E_{n r}$ to produce equivalent space-time patterns - this is done in section 21.4.1, where comp-m gives the complement or negative transformation for a binary state, and flip-h (horizontal flip) gives the reflection.

Note that a reflection transformation followed by a negative transformation in section 18.5.2, (or vice versa - the order is equivalent) produces another equivalent rule, making 4 in all[25] (figure 18.2). For certain rules the transformations result in identity. 


\subsection{Setting canalyzing inputs, single rcode} see also chapter 15

If $\mathbf{C a}$ is entered at the prompt in section 18.1 for a mixed rcode network, canalyzing inputs can be randomly biased for the whole network as described in chapter 15

If just $\mathbf{C}$ is entered, canalyzing inputs may be amended for a single rcode, or for the active cell in a mixed rcode network. A given number of the rcode's $k$ input wires may be set to be canalyzing, either explicitly or at random. The following top-right prompt is presented.

\section{canalyzing inputs: explicitly-e random-n:}

\subsubsection{Canalyzing inputs at random, single rcode}

Enter $\mathbf{n}$ to set a given number of inputs as canalyzing at random (which can be reduced as well as increased). The following prompt appears, enter the required number,

canalyzing inputs $=\mathbf{0}$, set new number $(0-5): \quad($ for $k=5)$

Once set, the prompt in section 18.6 will reappear.

\subsubsection{Canalyzing inputs explicitly, single rcode}

Enter e to set the canalyzing inputs explicitly, the following prompts appear in sequence,

$$
\text { wire (0-5): } \quad \text { input value }(\mathbf{1 , 0}) \text { : } \quad \text { output value(1,0): (for } v 2 k 5)
$$

Enter the neighborhood index (wire) to be set as canalyzing, and the input and output canalyzing values, $v-1$ to 0 ( 1 or 0 for binary). Once set, the prompt in section 18.6 will reappear. Other inputs wires may be explicitly set as canalyzing, but the output must be the same as before to preserve previously set canalyzing inputs (chapter 15).

\subsection{Neutral transformations of the neighborhood $k$} not applicable when interrupting space-time patterns (section 32.16.1) or attractor basins

Options for neutral transformations of the neighborhood $k$ allow $k$ to be increased, or decreased to find effective $k$, and rules are adjusted to conserve dynamics. These options apply from the main series of prompts at the start, not when interrupting space-time patterns or attractor basins.

\subsubsection{Equivalent rules with greater $k$}

A rule with a given neighborhood size $k$ has equivalent rules with any greater value of $k$. If $k<k_{\text {Lim }}$ (section 7.2), or $k<k_{\max }$ for a mixed $k$ network, then the options in section 18.1 includes a prompt such as equiv $>\mathbf{k}$ (4-13), ... (for example) 
Enter a greater value of $k$, which will produce a new rcode - a neutral transformation for $1 \mathrm{~d}$ networks ${ }^{4}$. Increasing $k$, for example from $k 3$ to $k 6$ or $k 6$, results in rcode giving identical dynamics to that of the original, but mutations of the larger rcode are finer grained, so the transformation is useful for looking at close mutations of a given rcode, as in figures 28.3 and 28.4. Filtering spacetime patterns with very complicated background domains may also require neutrally increasing $k$, for example the $k 3$ rcodes 110 and 54 to $k 5$ (section 32.11.5, figures 32.26-32.28).

For networks with a homogeneous rule the transformation applies to the whole network. In mixed $k$ networks, only the chosen cell in the network is transformed. For mixed rule, homogeneous $k$ networks, the option does not apply.

An extra wire added to an even- $k$ pseudo-neighborhood is added on the left, at the new $k-1$ index. An extra wire added to odd- $k$ is added at index 0 (on the right), the original connections are retained and their indexes increased by one. Thus the original connections remain in the enlarged pseudo-neighborhood. If more than one wire is added these steps are repeated. Rcodes are transformed so that the added wires are effectively redundant and play no role in the dynamics ${ }^{5}$. However, if the rcode is mutated the extra wires would come into play. Extra wires are connected within the network as they would be in a $1 \mathrm{~d}$ cellular automata.

\subsubsection{Reducing $k_{\max }$ to the maximum $k$ in the network}

For mixed $k$ networks, $k_{\max }$ may be larger than the maximum $k$ in the network. This may have been deliberately set in section 9.10, or a network may have been loaded with a smaller $k_{\max }$ than the base network (section 19.4.4). Enter $\mathbf{M}$ to reduce $k_{\max }$ to the maximum $k$ found in the network.

\subsubsection{Effective $k$}

The pattern of values in a rule-table may be such that some wires are redundant, playing no role in the dynamics. An example is a rule that has been neutrally transformed with greater $k$, in section 18.7.1 above. If $\mathbf{k}$ or $\mathbf{K}$ is entered at the prompt in section 18 and redundant wires exist, the rule will be neutrally transformed to a rule with decreased $k$ (to a minimum of $k=1$ ) by pruning redundant connections and transforming the reduced rule-table appropriately.

For a single rule network, the effective $k$ transformation applies to the whole network. For mixed $k$ networks, if lower-case $\mathbf{k}$ is entered only the chosen cell in the network is transformed, whereas if upper-case $\mathbf{K}$ is entered all cells will have their rules reduced to each effective $k$ (to a minimum of $k=1$ ). To make the effective $k=0$, see section 14.3.

If a mixed $k$ network was set up where $k_{\max }=n$, an option is presented to load an exhaustive mapping of transitions (section 35.2) and "reverse engineer" the network. An algorithm automatically transforms the network to satisfy the mapping. The effective- $k$ transformation may then be applied to reduce the network back to its minimal $k$-mix. A further option allows $k_{\max }$ to be reduced to the maximum $k$ found in the network (section 9.10).

\subsubsection{Reverse engineering - loading an exhaustive map}

For a network were $k_{\max }=n$ and $n \leq 13$, an exhaustive list of transitions, a random map, that was previously created (section 29.8) may be loaded to "reverse engineer" the network by transforming

\footnotetext{
${ }^{4}$ The algorithm is designed for $1 \mathrm{~d}$ networks; for $2 \mathrm{~d}$ and $3 \mathrm{~d}$ the transformation is not neutral.

${ }^{5}$ Added wires can be neutrally reduced to effective $k$ in section 18.7.3 to revert to the original wiring and rcode.
} 
it to satisfy the mapping. This is a rudimentary solution to the inverse problem. At present it is restricted to the case where all transitions are specified, and where $k_{\max }=n$ (the network size), so $n \leq 13$ because for rcode $k_{\max }=13$ in DDLab.

The resulting dynamics, and attractor basins, will correspond to the mapping. The method works in three stages, firstly the rules and wiring are transformed for a fully wired network where $k=n$ for all cells, then the effective $k$ option (section 18.7.3) is implemented, followed by the option, if required, to reduce $k_{\max }$ to the actual maximum $k$ found in the network (section 18.7.2).

1. Enter e at the prompt in section 18 to load the mapping file and transform the network to a fully wired network, which can be seen by pressing return and reverting to "Reviewing network architecture, wiring and rules" (section 17).

2. Enter $\mathbf{K}$ to reduce the wiring to effective $k$ (section 18.7.3). There will be a pause while this is being computed.

3. Enter $\mathbf{M}$ to reduce $k_{\max }$ to the maximum $k$ found in the network (section 18.7.2).

The result may be seen by reverting to "Reviewing network architecture, wiring and rules" (section 17), and by generating attractor basins (section 30) to check that they are the same as the basins for the network that was used to generate the exhaustive map. 


\section{Chapter 19}

\section{File/print network architecture}

While the wiring graphic (section 17.4) is visible, the filing option $\mathbf{f}$ is available to save, load or print network architecture, described in this chapter. The $k$-mix, rule scheme and wiring scheme may be treated in isolation, or combined. Various compatibility issues apply for loading files into a "base" network depending on the base properties. Broadly, a file network must fit into the base network and $k_{\max , \text { file }} \leq k_{\max , \text { base }}$ - if so, networks with different dimensions can be loaded into each other. When successfully loaded, a file network is automatically defined as a block (sections $17.5 .3,17.6 .5$ or 17.7.5), with its wiring visible in the wiring graphic, where the block can be separately manipulated. Any number of sub-networks can be loaded into the base network.

The ASCII data can also be saved, and printed in the terminal (xterm) for Linux-like systems.

\subsection{Network filing options}

While the wiring graphic is visible, Hit f (without return) in the "wiring graphic reminder" (section 17.4) for the top-right network filing prompt. The prompt differs between homogeneous- $k$ ( $k$-hom) and mixed- $k(k$-mix), and also depends on the context, where $\mathbf{n} / \mathbf{a}$ signifies nonapplicable - two examples are shown below,

for homogeneous- $k$ and mixed-rule networks

k-hom, rulemix, save/load/print:

wiring-only: print-pw, load-lw, save-sw

rulemix-only: print-pr, load-lr, save-sr

rulemix+wiring: print-pb, load-lb, save-sb:

for mixed-k networks - mixed-rules by default

k-mix, save/load/print: save kmix-k (just k-mix can be saved here, but not loaded ${ }^{1}$ )

wiring-only: print-pw, save-sw

rulemix-only: $\mathbf{n} / \mathbf{a}$ (rulemix+wiring applies instead)

rulemix + wiring: print-pb, load-lb, save-sb:

\footnotetext{
${ }^{1}$ The $k$-mix information is held as part of the wiring scheme and so is loaded with rulemix +wiring. Just the $k$-mix may be saved by entering $\mathbf{k}$, but the $k$-mix in isolation may only be loaded earlier in the program sequence (section 9.4)
} 
The following additional variations apply to the prompt,

- If rules were not yet set, no rules appears in the top line - rules-only and rulemix + wiring are non-applicable.

- For a single rule network, no rulemix appears in the top line - rulemix-only and rulemix + wiring are non-applicable. To avoid this, the single rule can be set as a rulemix (section 14.4.3).

- For a $k$-mix, rulemix-only is non-applicable - rulemix+wiring apples instead.

Subject to these constraints, to save, load or print the network architecture enter two key strokes as follows,

$$
\begin{aligned}
\text { first option } & \text { second option } \\
\mathbf{p}-\text { to print } & \mathbf{w} \text { - for wiring } \\
\mathbf{l}-\text { to load } & \mathbf{r} \text { - for rules } \\
\mathbf{s}-\text { to save } & \mathbf{b} \text { - for both wiring and rules }
\end{aligned}
$$

For example, enter sw to save a wiring-only only file (.wso), sr for a rulemix-only file (.rso), or enter lb to load a rulemix+wiring file (.wrs) which combines both wiring and rule scheme. The $k$-mix is implicit in the wiring encoding (section 19.3.1).

\subsection{Wiring/rulemix filenames}

The binary files defining the wiring scheme (wiring-only), rule scheme (rulemix-only), or wiring and rule scheme combined (rulemix+wiring) have the following filename extensions and default filenames, where $x$ is 2 to 8 , the value-range $v$.

$$
\begin{aligned}
& \text { wiring-only ... } \frac{\text { extension }}{\text {.w_s }} \frac{\text { default filename }}{\text { my_wso.w_s }} \\
& \text { rulemix-only ... .r.s my_rso_x.r.s for rcode } \\
& \text {.r_v my_rso_ } x \text {. v_s for kcode } \\
& \text {.r_t my_rso_x.t_s for tcode } \\
& \text { rulemix+wiring } \ldots \text {.wrs my_wrs_ } x \text {.wrs for rcode } \\
& \text {.wrv my_wrs_ } x \text {.wrv for kcode } \\
& \text {.wrt my_wrs } \_x \text {. wrt for tcode }
\end{aligned}
$$

\subsection{Wiring/rulemix encoding}

The encoding for all three file types above (section 19.2) starts with 6 bytes as follows ${ }^{2}$, where $k_{\max }=$ the maximum neighborhood size in a mixed- $k$ network (for homogeneous- $k, k_{\max }=k$ ), $n$ is the network size, $(i, j)$ the dimensions of a $2 \mathrm{~d}$ network, and $(i, j, h)$ for $3 \mathrm{~d}$,

\footnotetext{
${ }^{2}$ In the binary version of DDLab, the old style encoding started with 5 bytes. In the new style encoding byte 0 is reserved for the value-range $v$, and subsequent bytes are displaced by one. Old style files are nevertheless compatible for loading in the present version of DDLab. For single rule encoding see section 16.16 and for seed encoding see section 21.9 .
} 
byte $0 \ldots$ value-range $v$, if byte $0=0$ then old style encoding is recognized.

byte $1 \ldots$ the lower 7 bits $=k$ or $\max -k$ for a $k$-mix. The highest bit=1 indicates a $k$-mix, the highest bit $=0$ indicates homogeneous- $k$.

byte $2,3 \ldots$ network size, $n$.

byte $4,5 \ldots i, j$. If $i=0$ and $j=0$ the network is 1 d. If both $i \geq 1$ and $j \geq 1$ the network can be either $2 \mathrm{~d}$ or $3 \mathrm{~d}$. $h=\frac{n}{i \times j}$. If $h>1$ the network is $3 \mathrm{~d}$, otherwise $2 \mathrm{~d}$.

The encoding continues as follows, for each successive cell index from $0 \ldots(n-1)$, where the number of bytes encoding a rule-table, $R$, depends on the rule type, the value-range $v$ and the neighborhood $k$ as specified in "Single rule file encoding" section 16.16 but without the 2 leading bytes,

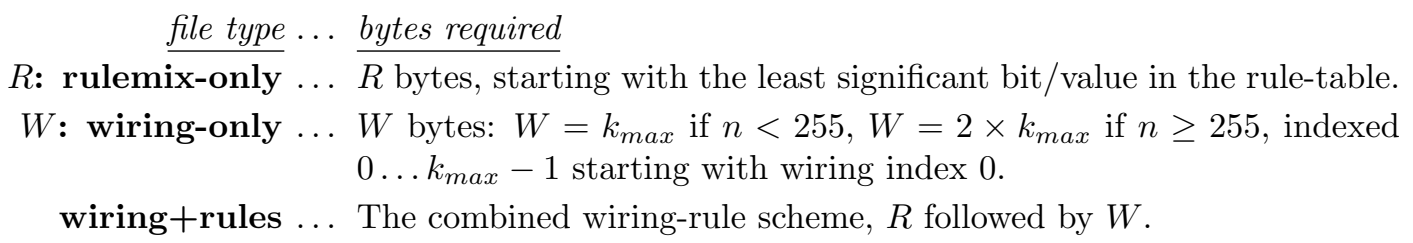

\subsubsection{Mixed- $k$ encoding}

For mixed- $k$ networks, the $k$-mix is implicit in the wiring encoding. Where the actual $k$ of a cell is smaller than $k_{\max }$, there will be excess bytes for both the rules and wiring in the encoding in section 19.3 above. These excess bytes are filled as follows,

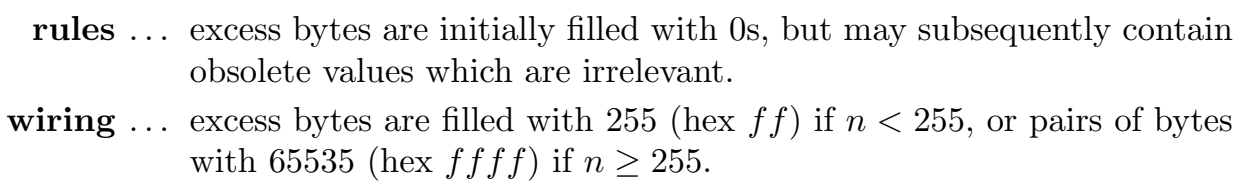

Counting the number of bytes not containing $f f$ or $f f f f$ is how the file keeps track of the $k$-mix. Note that $k=0$ is illegal, but an effective $k=0$ can be set (section 9.2 and 14.3).

The $k$-mix can also be saved separately in a .mix file (section 19.5).

\subsection{Loading networks and sub-networks}

Once a base network is set up and visible as a wiring graphic (section 17.3), previously saved network files, types wiring-only, rulemix-only and rulemix + wiring, can be loaded, either to replace the base completely or to insert a sub-network positioned somewhere within the base network. If successfully loaded the file network is automatically defined as a block (sections 17.5.3, 17.6.5 or 17.7.5), with its wiring visible in the wiring graphic - the block can be separately manipulated. Initially the block's wiring, whether local or random, will be self-contained, but can be wired-in to the base network as in figure 19.4. Any number of sub-networks can be loaded into the base. 


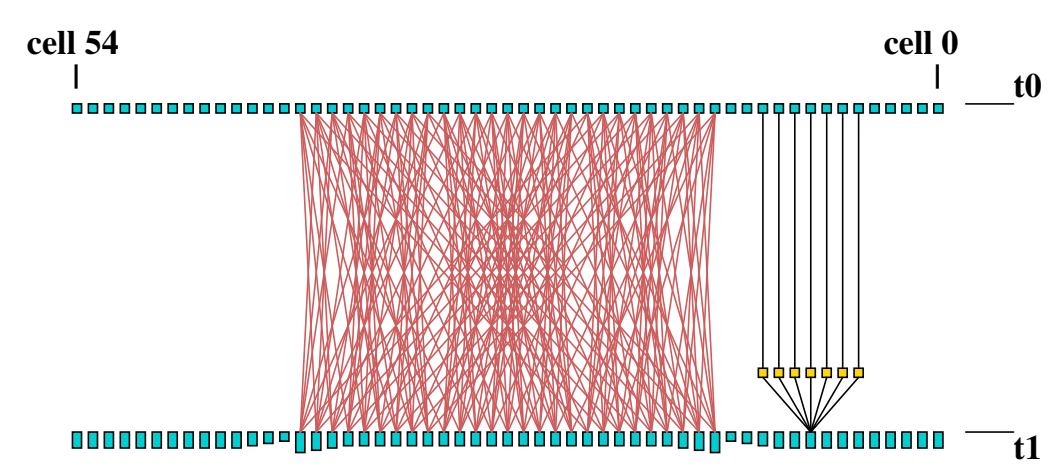

Figure 19.1: Loading a $3 \mathrm{~d}$ wiring-only file $(3 \times 3 \times 3, k=6)$ into $1 \mathrm{~d}$ CA base network $(n=55, k=7)$. The file appears in the $1 \mathrm{~d}$ wiring graphic as a bock (section 17.5.3).

\subsection{1 loading networks - compatibility}

A valid file type in section 19.1 may be still turn out to be incompatible with the base network which becomes clear once the file is selected. The rules for compatibility between the base and file network relate to value-range $v$, neighborhood $k$, network size $n$, and with edge sizes $i, j, h$ in $2 \mathrm{~d}$ and 3d. The rules, exceptions, and other issues are described below (sections 19.4.1.1 to 19.4.1.4).

\subsubsection{Compatibility with $v$}

For compatibility with value-range, $v, v_{\text {file }}=v_{\text {base }}$ is required. Otherwise a top-right error message appears,

$$
\text { file-v(2) != base-v(3), can't load, cont-ret: (for example) }
$$

An exception applies if loading wiring-only from a wiring graphic before rules have been set, when $v$ is irrelevant.

\subsubsection{Compatibility with $k$}

For compatibility with neighborhood $k, k_{\max , \text { file }} \leq k_{\max , \text { base }}$ is required. Otherwise a top-right error message appears,

file-kmax $(7)>$ base-kmax(6), can't load, cont-ret: (for example)

If a wiring-only or a wiring+rules file with mixed- $k$ is loaded into a homogeneous- $k$ base, the base will automatically be redefined as having mixed- $k$. Loading wiring-only into a homogeneous- $k$ base with rules set will truncate rule-tables where $k_{\text {file }}<k_{\text {base }}$.

An exception to $k_{\text {max, file }} \leq k_{\text {max,base }}$ applies if loading rules-only; in this case $k_{\text {file }}=k_{\text {base }}$ (both with homogeneous $k$ ) is recommended. Loading a mixed- $k$ file, or a file where $k_{\text {file }}<k_{\text {base }}$ is risky because $k_{\text {base }}$ will remain unchanged - a smaller rule-table will simply overwrite the start of a larger base rule-table giving unexpected results. Attempting this results in a top-right warning message,

rulemix-only: loading kmix-file is risky, load anyway-l, abort-ret: (or, for example)

rulemix-only: loading file-k(3) < base-k(5) is crazy, load anyway-l, abort-ret: 


\subsubsection{Compatibility with $n$}

For compatibility with network size $n, n_{\text {file }} \leq n_{\text {base }}$ is required. Otherwise a top-right error message appears,

file-n(150) > base netsize(100), can't load, cont-ret: (for example)

An exception applies if loading a rulemix-only file, where both file and base have homogeneous- $k$ and $k_{\text {file }}=k_{\text {base }}$. In this case $n_{\text {file }}>n_{\text {base }}$ will load as may rules as will fit.

\subsubsection{Compatibility with edge sizes and dimensions}

For compatibility with edge sizes $i, j, h$ in $2 \mathrm{~d}$ or $3 \mathrm{~d}$, its recommended that file coordinates fit within the base coordinates - if so, as well as loading a file into a base with the same dimensions, lower dimensions can be loaded into higher - the file coordinates are first reallocated with the higher dimension, for example a $1 \mathrm{~d}$ file network $n=30$ is treated as $2 \mathrm{~d} i, j=30 \times 1$ or $3 \mathrm{~d} i, j, h=30 \times 1 \times 1$, a $2 \mathrm{~d}$ file network $i, j=30 \times 40$ is treated as $3 \mathrm{D}$ network $i, j, h=30 \times 40 \times 1$. A top-right warning message will show incompatible dimensions,

\section{file-j(40) > base-j(30), load anyway-l, abort-ret: (for example)}

If $\mathbf{l}$ is entered to load anyway, the start position will be set in section 19.4.3 as if the base were 1d. This is also the case if loading higher dimensions into lower, which can be done as long as $n_{\text {file }} \leq n_{\text {base }}$. Loading in these ways results in a consecutive block, not the usual $2 \mathrm{~d}$ or $3 \mathrm{~d}$ block in a $2 \mathrm{~d}$ or $3 \mathrm{~d}$ base, but the block can still be independently manipulated, as in figure 19.2.
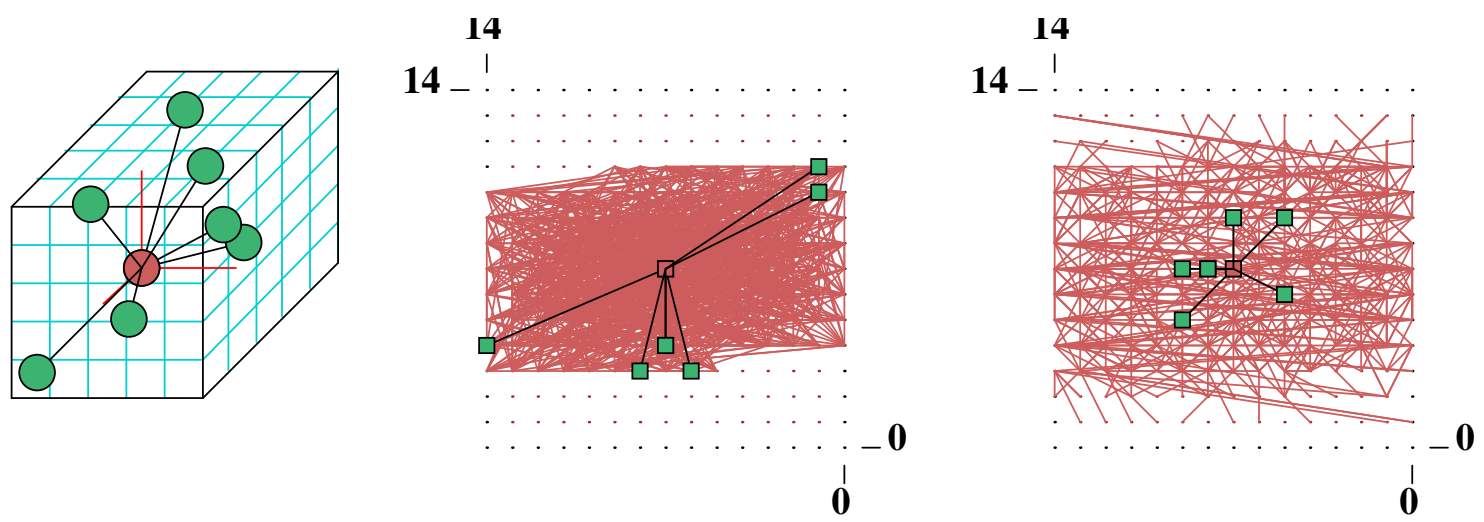

Figure 19.2: Left: a small 3d DDN $(i, j, h=5 \times 5 \times 5, k=7)$ was saved as a rulemix+wiring file. Center: the $3 \overline{\mathrm{d} D D N}$ was loaded as a sub-network into the center of a $2 \mathrm{~d} \operatorname{DDN}(i, j=15 \times 15, k=9)$, with random wiring confined to a 5 cell local zone (section 12.5.2). The sub-network is automatically defined and visible as a block, but because the sub-network dimension is higher than the base, the block consists of consecutive cells. When first loaded the sub-network is self-contained with its original boundaries - the dynamics within the sub-network would be independent of the rest of the network, but not vice versa.

Right: The result of resetting the sub-network block with confined random wiring by pressing $\mathbf{r}$ in the

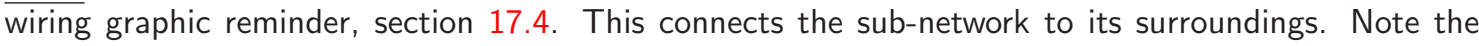
active cell, which can be moved anywhere. 


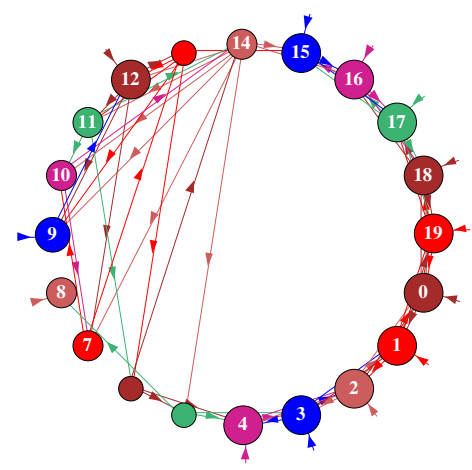

Figure 19.3: Loading a complicated subnetwork $(n=10)$ with a rulemix, $k$-mix and nonlocal wiring into a simple $1 \mathrm{~d}$ CA $(n=20)$. To do this the CA is set up to have a rulemix with just one rule. The figure shows the resulting network as a network-graph (chapter 20).

\subsubsection{Loading a complicated network into a CA and vice versa}

In general, to load a file network with a rulemix, the base also requires a rulemix. but this can be confined to a limited number of rules, or just one rule. What if a complicated file network with a rulemix, $k$-mix, and random wiring, (RBN or DDN) needs to be loaded as a sub-network into a simple CA base network? In such a case (figure 19.3) the CA base network can easily be set up where its single rule is treated as a rulemix (section 14.4.3). Uniform $k$ can also be treated as mixed- $k$ (section 9.9), and local wiring as nonlocal (section 12.4.1), but neither is necessary.

Conversely, if a simple CA needs to be loaded as a sub-network into a complicated base network with mixed rules, the CA should be set up and saved as a rulemix with one rule. To load a CA into another CA, where their rules or $k$ differ, both the base and the file should be have a rulemix with one rule.

\subsubsection{Loading sub-networks in a set position}

A file sub-network that is smaller than the base network, which can fit into the base network as specified in section 19.4.1.4 can be loaded in a set position even if the dimensions of the file and base network are not the same, as long as $n_{\text {file }} \leq n_{\text {base }}$.

If the dimensions and edge sizes are equal, the file will be loaded without further ado, otherwise prompts allow the file sub-network to be positioned in the base network (the default is a central position).

For a 1d base, or if edge sizes do not fit, or higher dimensions are being loaded into lower and load anyway-l is selected in section 19.4.1.4, prompt is as follows,

for $1 d$ networks

1d: array length $=150$, length file $=14$, enter start pos (for example)

(def 68, max 136, rnd-r)):

For a $2 \mathrm{~d}$ or $3 \mathrm{~d}$ base, if edge sizes fit, the prompt is as follows,

for $2 d$ networks

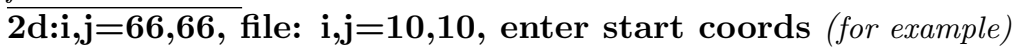

$($ def 28,28, $\max 56,56$, rnd-r)) i: $\quad$ j:

for $3 d$ networks

$\overline{3 d: i, j, h=40,40}, 40$, file:i,j, $h=\mathbf{9}, \mathbf{9}, \mathbf{9}$, enter start coords (for example)

$($ def 15,15,15 max 31,31,31, rnd-r)) i: j: h: 


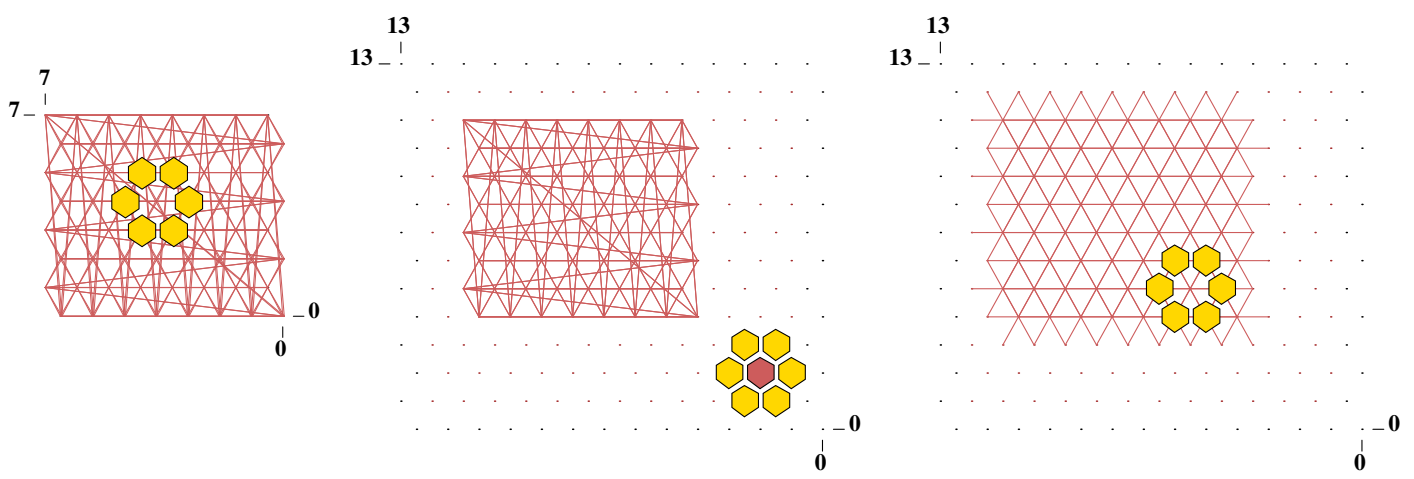

Figure 19.4: Left: a small 2d hex CA $(i, j=8 \times 8, k=6)$ was saved as a rulemix+wiring file.

Center: the CA was loaded as a sub-network into a larger $2 \mathrm{~d}$ hex CA $k=7(i, j=14 \times 14, k=7)$, positioned at coordinates $I, J=4,4$ and automatically defined and visible as a block in the wiring graphic. Because the CAs are both hexagonal, for correct wiring, $j$ in both networks, and the position coordinate $J$ should be even. When first loaded the sub-network CA is self-contained with its original periodic boundaries - the dynamics within the sub-network would be independent of the rest of the network, but not vice versa.

Right: The result of resetting the sub-network block as $2 \mathrm{~d}$ by pressing $\mathbf{2}$ in the wiring graphic reminder section 17.4. This connects the sub-network (the block) to its surroundings - removing its periodic boundaries. Note the active cell, which can be moved anywhere, showing its neighborhood.
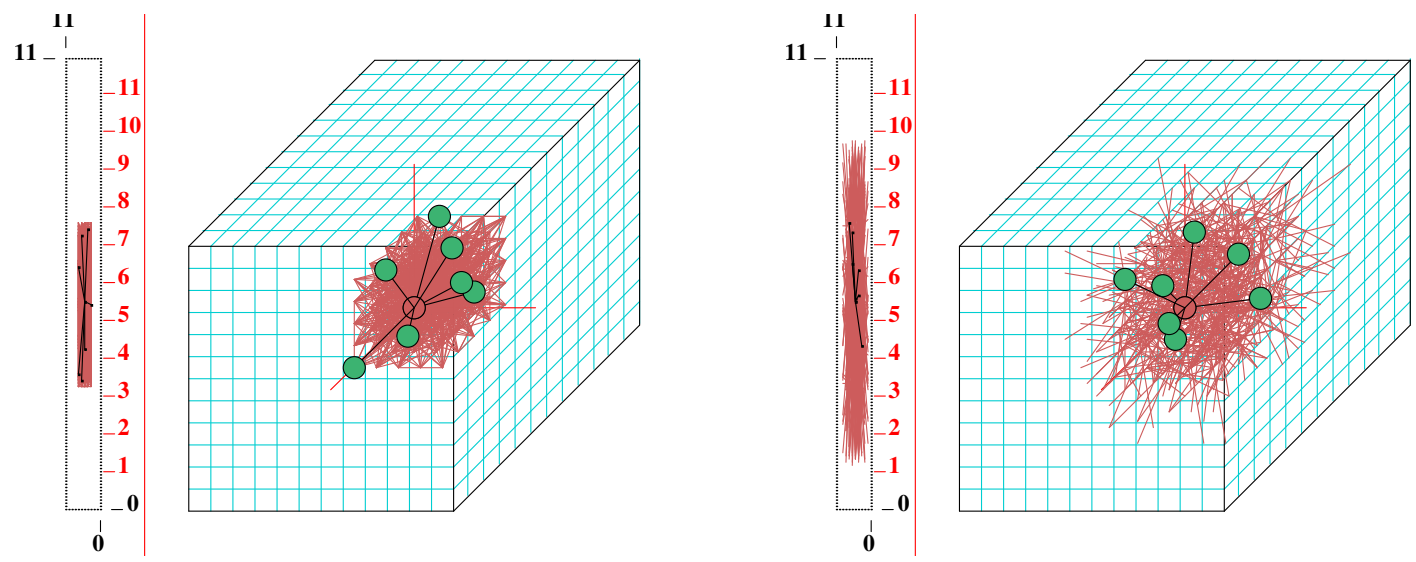

Figure 19.5: (showing the $2 \mathrm{~d}+3 \mathrm{~d}$ wiring graphic) Left: A small $3 \mathrm{~d}$ DDN $(i, j, h=5 \times 5 \times 5, k=7$ ) (the same as in figure 19.2) was saved as a rulemix + wiring file, then loaded as a sub-network into the center of a larger $3 \mathrm{~d}$ DDN $(i, j, h=12 \times 12 \times 12, k=7)$. When first loaded the sub-network is automatically defined and visible as a self-contained block within its original boundaries - the dynamics within the sub-network would be independent of the rest of the network, but not vice versa.

Right: The random wiring for the block was first confined to a 5 cell local zone (section 12.5.2), then its wiring was randomized by pressing $\mathbf{r}$ in the wiring graphic reminder, section 17.4. This connects the sub-network to its surroundings. 


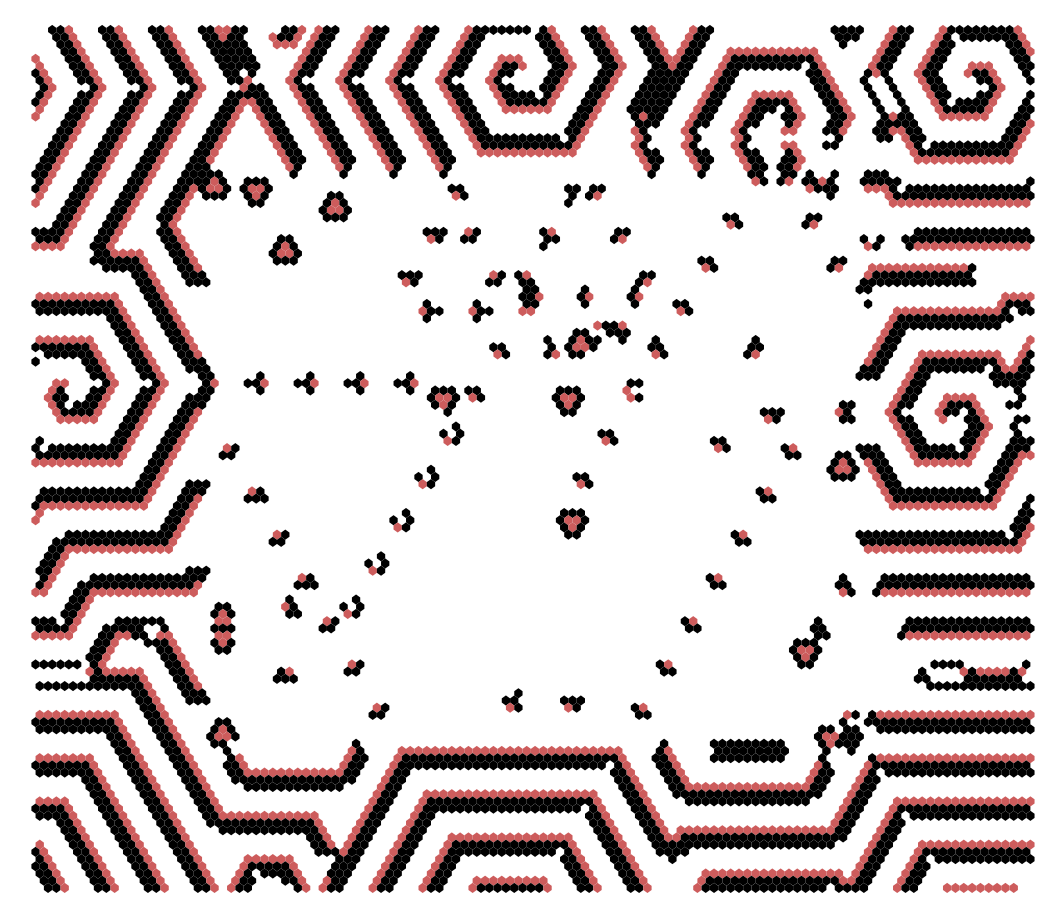

Figure 19.6: Space-time snapshot of a 2d CA inside another 2d CA with different $k$. The $2 d(80 \times 80)$ $k=7$ CA spiral-rule[40] ( $v 3 k 7$ kcode(hex) 020609a2982a68aa64), was loaded into the 2d $(120 \times 120)$ $k=6$ complex CA which generates spiral structures (v3k6 kcode(hex) 020282815a0254). See also figure 32.25 .

Enter the start coordinates for positioning index 0 of the file sub-network, return for the default central position, or $\mathbf{r}$ for a permissible random position.

Any number of independent sub-networks can be loaded consecutively. The sub-network block will be visible and active in the base wiring graphic, where it can be be wired-in or otherwise manipulated, Deactivate/reactivate the block with $\mathbf{g}$, cancel the block with $\mathbf{b}$ then return, in the wiring graphic reminder (section 17.4). Sub-network can be wired into other sub-networks or other parts of the network as described in chapter 17.

\subsubsection{Loading $k$-mix networks}

A $k$-mix network file can be loaded into a homogeneous $k$ ( $k$-hom) or a $k$-mix base network provided that the base max- $k$ is at least as big as the file max- $k$ (the precise base $k$-mix is not relevant). A base max- $k$ smaller than the file max- $k$ results in the following error message,

$$
\text { file-kmax(5) > base-kmax(4), can't load, cont-ret: (for example) }
$$

The max- $k$ in a $k$-mix network file may be smaller than max- $k$ in the base network. Max- $k$ can be reduced to the actual maximum $k$ found in the network as described in section 18.7.2.

Note that only the combined network file, wiring and rules, can be loaded into a base network with mixed $k$. The combined file need not be a mixed $k$ file. To load "just rules" or "just wiring" in section 19.1, the base network must have homogeneous- $k$. 


\subsection{Saving just the $k$-mix}

Enter $\mathbf{k}$ in section 19.1 to save just the $k$-mix (see also section 9.11.3). A top-right filing prompt appears to save a .mix file (default filename mymix.mix), which is encoded in $n$ bytes, recording the neighborhood size $k$ for each cell (index 0 to $n$-1) in each byte. Information on the $k$-mix is also implicit in the wiring-only (.w_s) and rulemix+wiring (.wrs) files (section 19.3.1), so usually the $k$-mix does not need to be saved separately. Loading just the $k$-mix from a .mix file is only possible towards the start of DDLab (section 9.4).

\subsection{Printing network data to the terminal or file}

If $\mathbf{p}$ (followed by $\mathbf{w}, \mathbf{r}$ or $\mathbf{b}$ as applicable) is selected in section 19.1, the following prompt is presented,

\section{for Linux-like systems \\ network text data: to xterm-p, file-f both-b: \\ for DOS \\ network text data: to printer-p, file-f both-b:}

Enter $\mathbf{p}$ to print the data to the terminal( xterm) in Linux-like systems, or to a printer in DOS. Enter $\mathbf{f}$ to save the ASCII data to a .dat file, or $\mathbf{b}$ to print and save simultaneously. Before saving, a top-right window will prompt for the file name (default my_net.dat — section 35.2).

The way the data is presented depends on the selections $\mathbf{w}, \mathbf{r}$ or $\mathbf{b}$ in section 19.1 , mixed- $k$ or homogeneous- $k$, the type of rule, and if in TFO-mode. The data starts with two introductory lines. The first shows the value-range, $k$ or the $k$-mix, and the network dimensions $i, j, h$ and size $n$, for example,

$\mathrm{v} 2 \mathrm{k} 5,1 \mathrm{~d} \mathrm{n}=7 \quad \ldots$ for $v=2$, homogeneous $-k, 1 d$ network

$\operatorname{v3kmix}(4-1), 2 d 55 \mathrm{n}=25 \quad \ldots$ for $v=3, k$-mix, $2 d$ network $5 \times 5$

$\mathrm{v} 2 \mathrm{k} 4,3 \mathrm{~d} 6 \times 6 \times 6 \mathrm{n}=276 \quad \ldots$ for $v=2$, homogeneous $-k$, 3d network $6 \times 6 \times 6$

The second line is a reminder that labels the data and gives the data order, for example,

cell. wiring(3-0), rcode(hex) ld ld-r Z C A...for rcode

cell. wiring(13-0), kcode(hex) rcode(hex) ld ld-r Z C A... for kcode+rcode

cell. wiring(9-0), tcode(hex) rcode(hex) ld ld-r Z C A... for tcode+rcode

cell. wiring (5-0), kcode(hex)... for kcode in TFO-mode (or tcode)

label ... what they means

cell ... 1d index of the cell.

$\mathrm{k}$... for a $k$-mix only, the neighborhood size $k$ for the cell.

wiring (3-0) ... wiring scheme of the pseudo-neighborhood in this case for 3,2,1,0 for $k=4$.

kcode ... kcode in hex (if applicable).

tcode ... tcode in hex (if applicable). 
rcode ... rcode in hex (if applicable) - kcode or tcode will be shown before the rcode if a totalistc rule was selected in section 13.1.1.

ld ... $\lambda$ parameter.

$l d-r \ldots \lambda$ ratio.

$\mathrm{Z} \ldots Z$ parameter.

C ... canalyzing shown in two parts (for $k=3$ ) firstly the fraction of canalyzing inputs $(0 / 3,1 / 3$ dotts $)$, then which of the $k$ inputs are canalyzing, i.e. ${ }^{*} 1^{*}-$ input 1 is canalyzing, $2^{* *}$ - input $2,{ }^{*} 10$ - both inputs 1 and 0 . If there are no canalyzing inputs the second part is not shown.

A ... The Post function class, e.g. A[1]i (section 14.12). 0 is shown if there are no Post functions.

The examples of network data below as shown in the terminal (xterm) are for small networks, though the data can be very lengthy for larger $v, k$ and $n$.

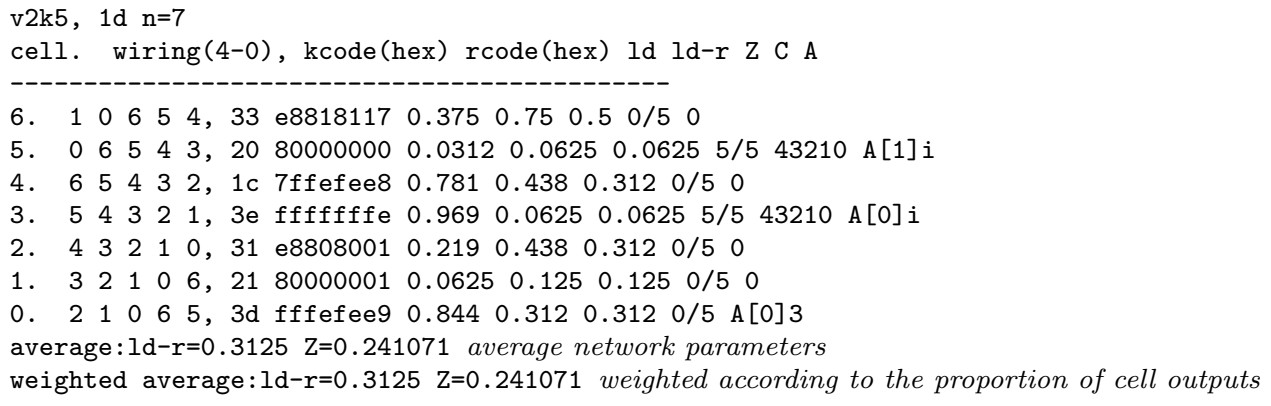

The example above is for kcode in the context of full rule-tables (not TFO-mode) mode so both kcode and rcode are listed. The rule parameters ( $l d ~ l d-r ~ Z C ~ A)$ follow the rcode. The data ends with a summary of average network parameters and the weighted average according to the proportion of cell outputs.

The example below is a $k$-mix and kcode in TFO-mode. Rule parameters ( $1 \mathrm{~d} l d-r$ Z C A) do not apply in TFO-mode.

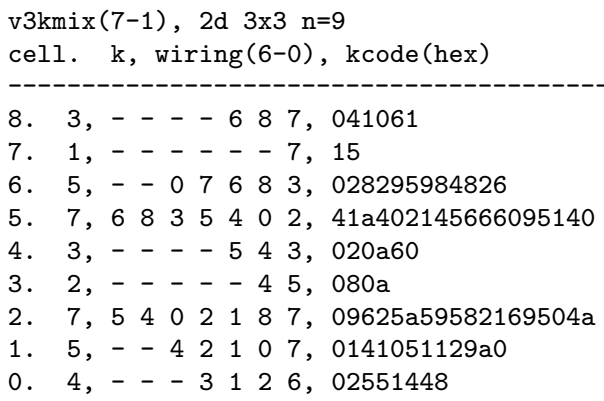




\section{Chapter 20}

\section{The network-graph, and attractor jump-graph}

DDLab includes powerful graph tools which have two distinct applications. Firstly, to represent the CA, RBN or discrete dynamical network itself - the network-graph, how the network elements are connected by their directed links, their wiring scheme. Secondly, to represent the stability of basins of attraction, the probability of jumping between basins due to perturbations to attractor states - the jump-graph of the basin of attraction field, where nodes represent basins of attraction, and edges represent jump probability, including jumps from a basin back to itself.

The underlying graph data can also be shown as a table, called the "network-table" or "adjacency-matrix" for the network-graph, and the "jump-table" for the jump-graph. As the graph manipulation options are the same for the network-graph and the jump-graph, they are described together in this chapter. The attractor jump-graph also applies to the attractor histogram (section 31.6.8, figure 31.19) where basin properties are found statistically by running a network forwards from many random initial states. As well as their standard presentation, space-time patterns can be run simultaneously within a network-graph allowing arbitrary layouts (section 32.19).

The network-graph does not allow changes to the underlying network (see chapter 17 to do this), but for both the network-graph and jump-graph, flexible methods are available for rearranging and unravelling the graph, including dragging vertices and defined components to new positions with elastic links, rescaling nodes and links, and changing links just within the graph.

Both the network-graph and jump graph can be shown without links (layout only) which is applied to present space-time patterns or basins of attraction in arbitrary layouts. The layouts can be saved/loaded (*.grh file) to restore a particular graph. The graphs, including basins of attraction positioned according to graph nodes, can be saved as vector PostScript files.

\subsection{Unravelling the jump-graph and the network-graph}

The layout of the graphs can be rearranged and unravelled to reveal the topology. Single nodes, connected fragments, or whole components, can be dragged with the mouse, according to inputs and/or outputs. The distance of fragment links from a node can be restricted, i.e. dragging the 


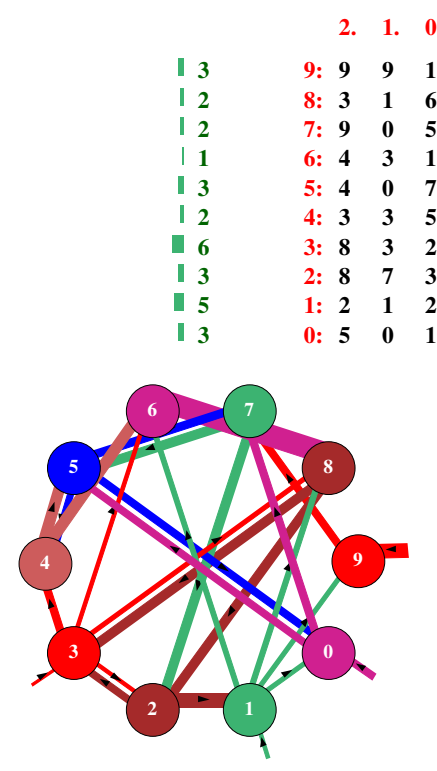

(a) default circle layout rcode(hex/dec)

$\begin{array}{ll}\mathbf{8 1} & \mathbf{1 2 9} \\ \mathbf{9 e} & \mathbf{1 5 8} \\ \mathbf{7 b} & \mathbf{1 2 3} \\ \mathbf{1 f} & \mathbf{3 1} \\ \mathbf{2 1} & \mathbf{3 3} \\ \mathbf{7 7} & \mathbf{1 1 9} \\ \text { c7 } & \mathbf{1 9 9} \\ \mathbf{4 7} & \mathbf{7 1} \\ \text { c7 } & \mathbf{1 9 9} \\ \text { c8 } & \mathbf{2 0 0}\end{array}$

Table 20.1: The network "matrix" of the RBN $v 2 k 3, n=10$, used in some of the examples: the network-graph (figure 20.1), the adjacency-matrix (table 20.2), the jump-graphs (figures 20.4, 20.12), "ant hits" (figure 20.5.3), and the jump-table (table 20.3). section 17.2 describes how a network matrix is generated.

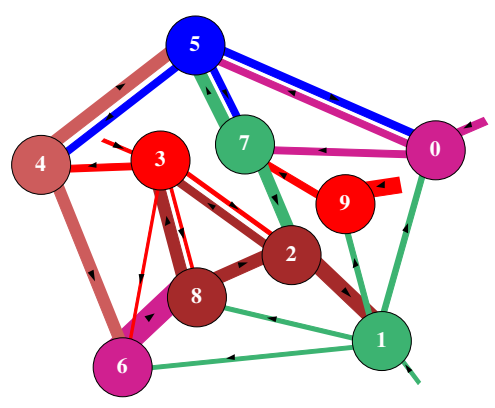

(b) nodes rearranged

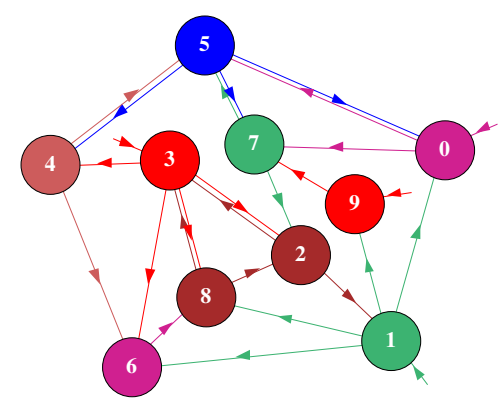

(c) links reduced to simple lines

Figure 20.1: Network-graphs of the same RBN $v 2 k 3, n=10$, defined in table 20.1 showing alternative presentations (a) The default circle layout where edge width reflects the nodes's outputs, and node diameter reflects $k$, the node's inputs, all equal in this example. (b) Layout rearranged by dragging nodes with the mouse. (c) Directed links shown as thin lines with arrows.

node + its immediate links (step 1), the node + immediate links + their immediate links (step 2), etc. Arbitrary 1d, 2d and 3d blocks can also be defined and dragged. Nodes with the fewest links can be automatically moved to the outer edges.

The pre-programmed graph layouts available are a circle of nodes, a spiral, 1d, $2 \mathrm{~d}$ or $3 \mathrm{~d}$, and random. Any layout can be "shaken" to randomly reposition nodes close to their current position. The graph can be rotated, expanded, contracted, and flipped. The graph is displayed in a large window across the lower part of the screen, smaller for the jump-graph to allow space to show the basin of attraction field above the window.

The default presentation for the jump-graph or $1 \mathrm{~d}$ network-graph is a circle layout (figures 20.1(a), 20.2). The default network-graph layout for a $2 \mathrm{~d}$ or $3 \mathrm{~d}$ network is $2 \mathrm{~d}$ or $3 \mathrm{~d}$ (figures 20.7 - 20.9). The default sizes of nodes are scaled according to the neighborhood size $k$ for the network-graph, or basin volume for the jump-graph. The width of links/edges can be scaled according to both node size and the proportion of available outputs comprising the link. If a node has connections to itself, this is represented by a short stub projecting from the node, also scaled as above. Proportional link/edge width is the initial default for the jump-graph, and uniformly thin lines with arrows for the network-graph, but this can be toggled. The nodes can also be toggled between proportional and a uniform size, and node numbering can also be toggled.

The nodes are numbered (if big enough) according to the cell order, 0 to $n$ - 1 for the networkgraph, 1 to the number of basins for the jump-graph. Successive nodes are assigned different colors cycling through six colors. Outgoing edges are colored according to the parent node, and aligned asymmetrically clockwise relative to the parent, which ensures that outgoing and incoming edges do not overlap if two nodes link to each other; the directed edges are separated by a central gap. 


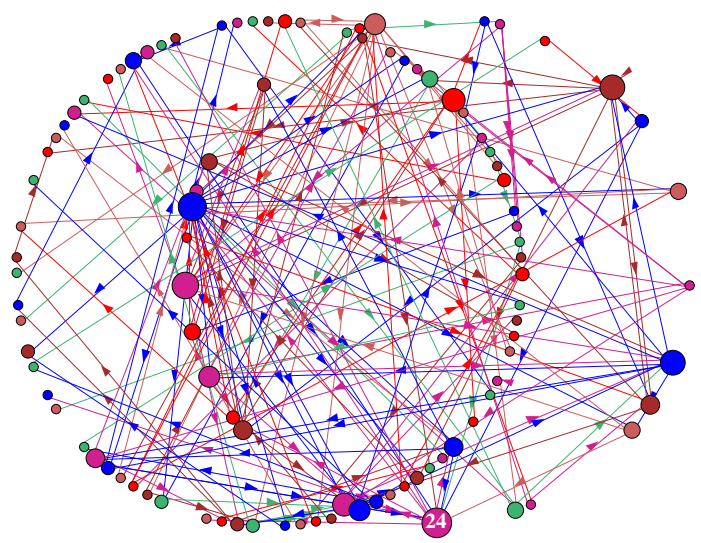

(a) circle layout, one node dragged with its 2-step inputs

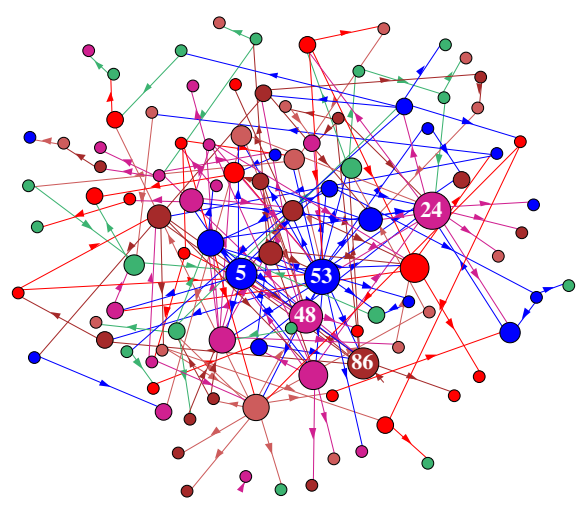

(b) unscrambled

Figure 20.2: Two versions of the network-graph for the same network with a power-law wiring distribution, both inputs ( $k=1$ to 10 ) and outputs, $n=100$. To set up power-law wiring see sections 9.7 .2 and 17.8.5. Nodes are scaled according to $k$. (a) A circle layout, but with one node dragged, together with its 2-step inputs. (b) the same network unscrambled - nodes rearranged as described in figure 20.6.

\subsection{The network-graph}

The network-graph is selected from the network architecture prompt (section 17.1), or from the space-time pattern pause prompt (section 32.16). The network architecture prompt itself is displayed at various stages in DDLab, firstly after special wiring is set (chapter 12), or after both the wiring and rules have been set in the main sequence of prompts, and at later stages. If $\mathbf{g}$ is entered a the network architecture prompt the following preliminary prompt appears,

no links $-\mathrm{N}$, show links -def:

If $\mathbf{g}$ is entered at the space-time pattern pause prompt, the preliminary prompt has the opposite default,

show links -L (caution for large networks), layout only -def:

Showing the network-graph in "layout only", without links/edges, is useful for an alternative presentation of space-time patterns (section 32.19), which can be in any. If "layout only" is selected The network-graph options differ accordingly.

\subsubsection{The network-graph reminder}

The top-right network-graph reminder of the first set of options available is presented as follows,

NETWORK-graph: drag-(def) PScript-P ant-a unscram-u tog:win-w rank-k settings-S rotate-x/X flip-h/v exp/contr:nodes-e/c links-E/C both-B/b

Unreach-U tog:table-t/T nodes-n/N links-l arrows-A $/</>$ layout:file-f circle/spiral-o/O $1 \mathrm{~d} / 2 \mathrm{~d}(\operatorname{tog}) / 3 \mathrm{~d}-1 / 2 / 3 \mathrm{rnd}-\mathrm{r} / \mathrm{R}$ quit-q: 
For "layout only" (no links) some options in the reminder are omitted,

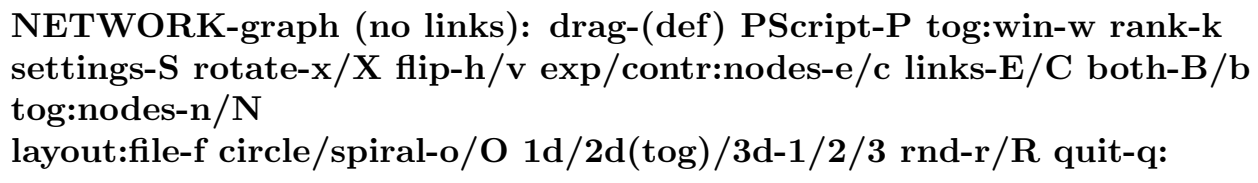

For large network-graphs (with links) the following top-right message may be displayed first, with an inset window that monitors progress such as $\mathbf{5 5 \%}$,

computing network-graph, 1600 nodes, quit-q, or wait ...

The network-graph is shown in a large window occupying most of the screen. These options are summarized in section 20.4. section 20.5 covers options for dragging nodes and fragments.

\subsection{The jump-graph of the basin of attraction field}

Perturbations due to noise or external signals are most likely to take affect once a system has relaxed to its attractor, because that is where the dynamics spends the most time. Taking single-bit or single-value perturbations to attractor states as the simplest case, the jump-graph represents the probabilities of jumping between basins, and gives some insight into the stability and adaptability of the dynamics, which is especially relevant in attractor models of memory in neural networks and of cell differentiation in genetic networks.

The jump-graph algorithm analyzes the basin of attraction field to track where all possible single-bit/value flips to attractor states end up, whether to the same, or to which other basin. The information is presented in two ways: as a graph with weighed vertices and edges (figures 20.3 - 20.5), and as a jump-table - a matrix showing the jump probabilities between basins (section 20.12). The attractor jump-graph algorithm applies to any network (with either synchronous or sequential updating), for CA (with compression suppressed), for RBN or DDN, and also for random maps, and to any reverse algorithm including exhaustive testing.

An alternative use of the jump-graph is a method for just laying out attractor basins in any arbitrary position. For this, jump edges are unnecessary - if omitted (layout only) attractor nodes can be dragged more efficiently and CA compression can be retained.

As well as the complete basin of attraction field, the jump-graph can also be computed for the attractor histogram (section 31.6), which gathers data on attractors and their relative sizes by statistical methods. This is done by running a network forwards from many random initial states, identifying different attractors, and creating a histogram of the frequency of falling into each. The method can be applied to large networks, especially RBN/DDN - too large to generate the basin of attraction field (section 1.6 for network size limits).

\subsubsection{Selecting the jump-graph}

FIELD-mode only - see also section 24.3

The jump-graph prompt is labelled jump-j in the "basin parameters" sequence of prompts (section 24.1). To go directly to the jump-graph prompt enter $\mathbf{j}$ at the first output parameter prompt, or arrive there by viewing the output parameters in sequence. The following top-right prompt is presented, 


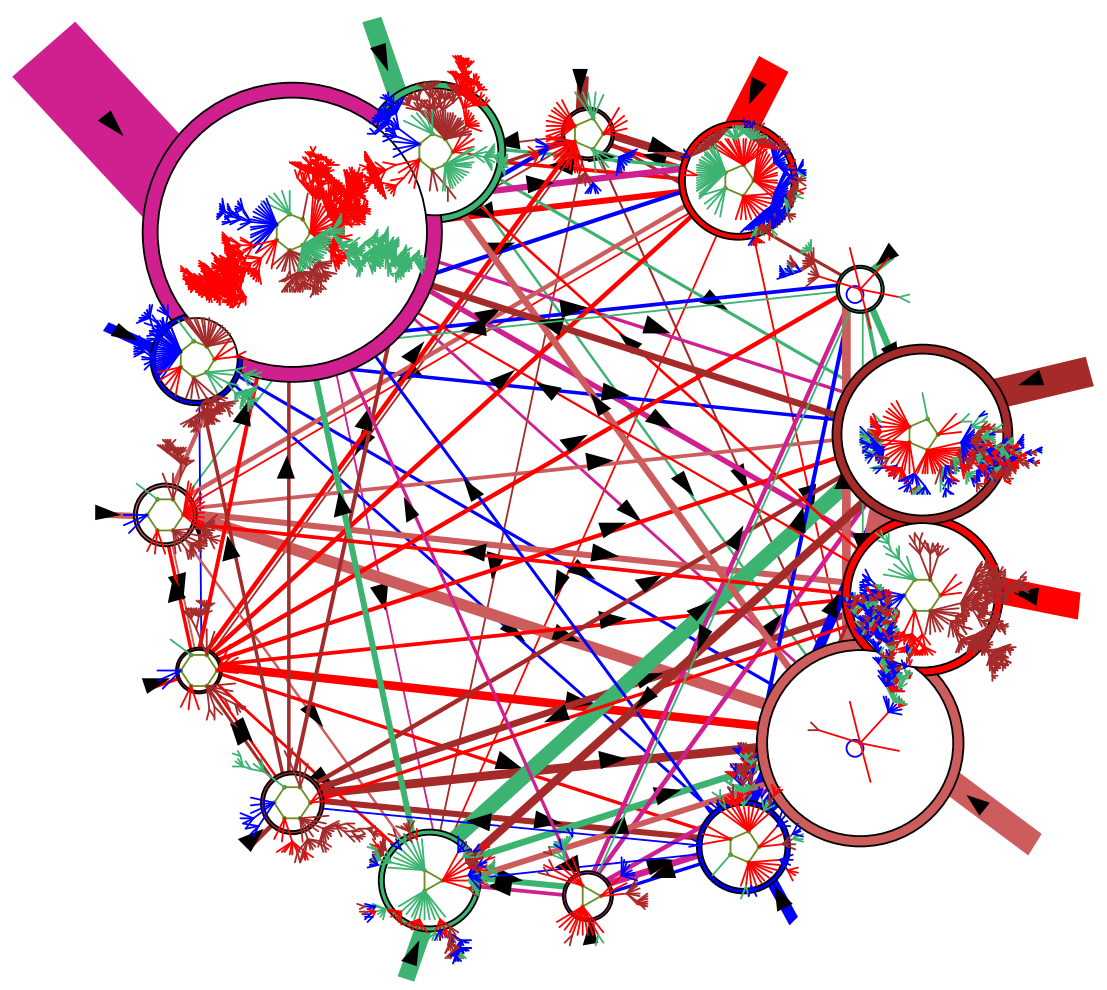

Figure 20.3: The jump-graph, with basins redrawn within its nodes (RBN $v 2 k 3, n=13$, as in figure. 2.4). The jump-graph shows the probability of jumping between basins due to single bit-flips to attractor states. Nodes representing basins are scaled according to the number of states in the basin (basin volume), and can be rearranged and dragged. Links are scaled according to both basin volume and the jump probability. Arrows indicate the direction of jumps. Short stubs are self-jumps. When jump-graph links are eliminated this becomes a layout-graph, providing a method for arbitrarily arranging the basin of attraction field.

\section{jump: attractor jump-graph $-\mathrm{j}$, no edges layout only $+\mathrm{L}$ :}

Enter $\mathbf{j}$ to show the jump-graph, or $\mathbf{j} \mathbf{L}$ for "layout only" - the jump-graph without edges is applied for just laying out basins in a basin of attraction field in any arbitrary position. If the jump-graph (with edges) is selected, "compression" for CA will be turned off automatically, with the following message,

\section{... - compression OFF: (if compression was on, Enter return to continue)}

\subsubsection{The jump-graph reminder}

If selected in section 20.3.1 (once the basin of attraction field is complete) the jump-graph is automatically generated, and the top-right jump-graph reminder of the first set of options available is presented as follows,

\footnotetext{
${ }^{1}$ The jump-graph can also be selected in section 31.6 .8 for the attractor histogram.
} 


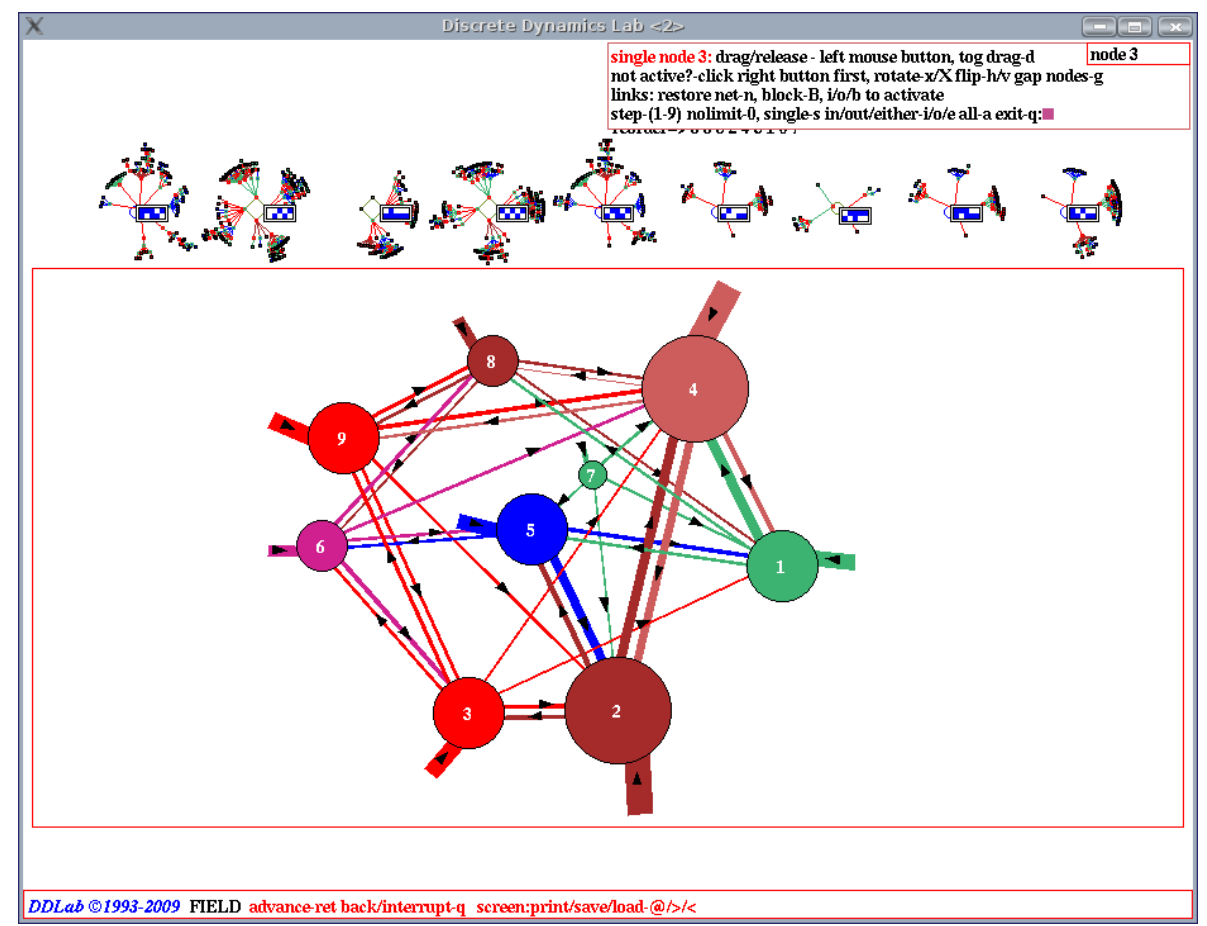

Figure 20.4: A screen shot of the basin of attraction field at the top of the screen and its jump-graph in a large window across the lower part of the screen. The jump-graph was adjusted and nodes were dragged to new positions with the left mouse button. Drag options are shown in the top-right "drag reminder" (section 20.5.1). This is the RBN $v 2 k 3, n=10$, defined in table 20.1. Basins were set to a small scale and repositioned near the top of the screen (chapter 25) so as not to be concealed behind the jump-graph window, though this can be togged to show hidden basins.

JUMP-graph: drag-(def) PScript-P ant-a unscram-u tog:win rank-k settings-S rotate- $\mathrm{x} / \mathrm{X}$ flip-h/v exp/contr:nodes-e/c links-E/C both-B/b basins-i/I/s Unreach-U tog:table-t/T nodes-n/N links-l arrows-A/ $</>$ layout:file-f circle/spiral-o/O 1d/2d(tog)/3d-1/2/3 rnd-r/R quit-q:

For "layout only" (no edges) some options in the prompt are omitted,

JUMP-graph (no edges): drag-(def) PScript-P tog:win rank-k settings-S rotate-x/X flip-h/v exp/contr:nodes-e/c links-E/C both-B/b basins-i/I/s tog:nodes-n/N layout:file-f circle/spiral-o/O $1 \mathrm{~d} / 2 \mathrm{~d}(\mathrm{tog}) / 3 \mathrm{~d}-1 / 2 / 3$ rnd-r/R quit-q:

Jump-graphs (with edges) may take some time to compute if the basin of attraction field (or attractor histogram) has many long period attractors. The following top-right message may be 

nodes-n/N ... enter $\mathbf{n}$ to toggle between scaled and unscaled nodes. Enter $\mathbf{N}$ to toggle node numbers. Numbers only appear on those node that are big enough - about .7 of the current text height. The numbering is 0 to $n$ - 1 for the network-graph, 1 to the number of basins for the jump-graph.

links-1 ... to toggle between scaled links/edges and thin lines (see figure 20.1).

arrows-A $/</>\ldots$ enter $\mathbf{A}$ to toggle showing arrows. Enter $<$ or $>$ to decrease or increase the arrow size by a default factor $(0.07)$, or a revised factor.

layout: options ...

file-f ... to save or load the current graph layout of node positions, a .grh file (section 35.2).

circle/spiral-o/O ... enter $\mathbf{o}$ or $\mathbf{O}$ to show the graph as a circle or spiral. Circle layout is the default (except for the $2 \mathrm{~d}$ or $3 \mathrm{~d}$ network-graph). Spiral layout requires at least 30 nodes to be effective

$\mathbf{1 d} / \mathbf{2 d}(\operatorname{tog}) / \mathbf{3 d - 1 / 2 / 3} \ldots$ enter $\mathbf{1}, \mathbf{2}$ or $\mathbf{3}$ to show the graph arranged in $1 \mathrm{~d}, 2 \mathrm{~d}$ or $3 \mathrm{~d}$. This is especially relevant for a network-graph where the underlying network is $2 \mathrm{~d}$ or $3 \mathrm{~d}$ - then the graph in $2 \mathrm{~d}$ or $3 \mathrm{~d}$ is the default. The initial $2 \mathrm{~d}$ network-graph is either square or hexagonal according to the underlying network - thereafter key 2d(tog)-2 toggles between square and hexagonal (examples in figures 10.6 - 10.8).

$\mathbf{r n d}-\mathbf{r} / \mathbf{R}$... enter $\mathbf{r}$ to "shake" the layout, repositioning nodes randomly nearby their current position. Enter $\mathbf{R}$ for a completely random layout.

quit-q ... enter $\mathbf{q}$ to quit the graph. For a network-graph this returns to the network architecture prompt in section 17.1. For a jump-graph this returns to the "Attractor basin complete prompt" section 30.4 - enter return for the next mutant.

\subsection{Dragging nodes or fragments}

Enter return, or click the left or right mouse button at the network-graph or jump-graph reminders (sections 20.2.1, 20.3.2) to enable dragging nodes or fragments with the mouse, and other features; the "drag reminder" replaces the top-right network-graph or jump-graph reminders - enter q to revert. Click on a node to activate it first in the drag options.

The initial setup allows single nodes to be dragged. A node is activated and dragged with elastic links by clicking and holding down the left mouse button, releasing the node in a new position. Alternatively, elastic links can be suppressed and the active node dragged leaving a trail - on release the links (and fragment nodes if any) snap into position - for large graphs this is a more efficient method. Sometimes a node will not activate, if so, click on it alternately with the right then left mouse button. When a node is activated its number appears in the drag prompt, node 5, for example. 


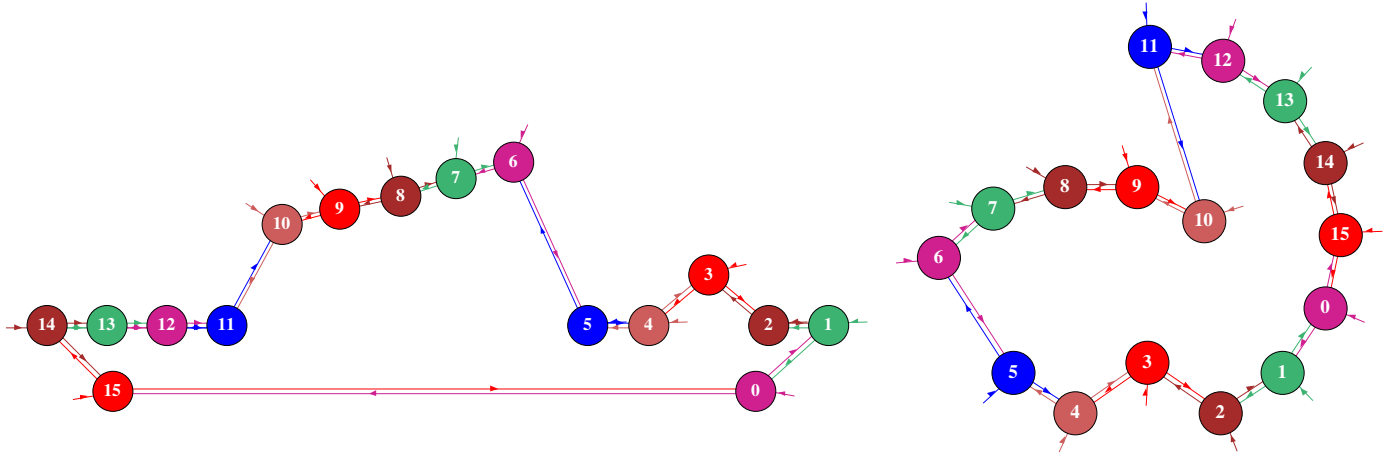

Figure 20.7: Dragging network-graph nodes and fragments of a $1 \mathrm{~d}$ CA $n=16, k=3$, starting with Left: a network-graph with 1d layout and Right: circle layout. Single nodes have been dragged, and in $\overline{\text { both }}$ cases node 8 and its 2 -step neighbors have been dragged and rotated.
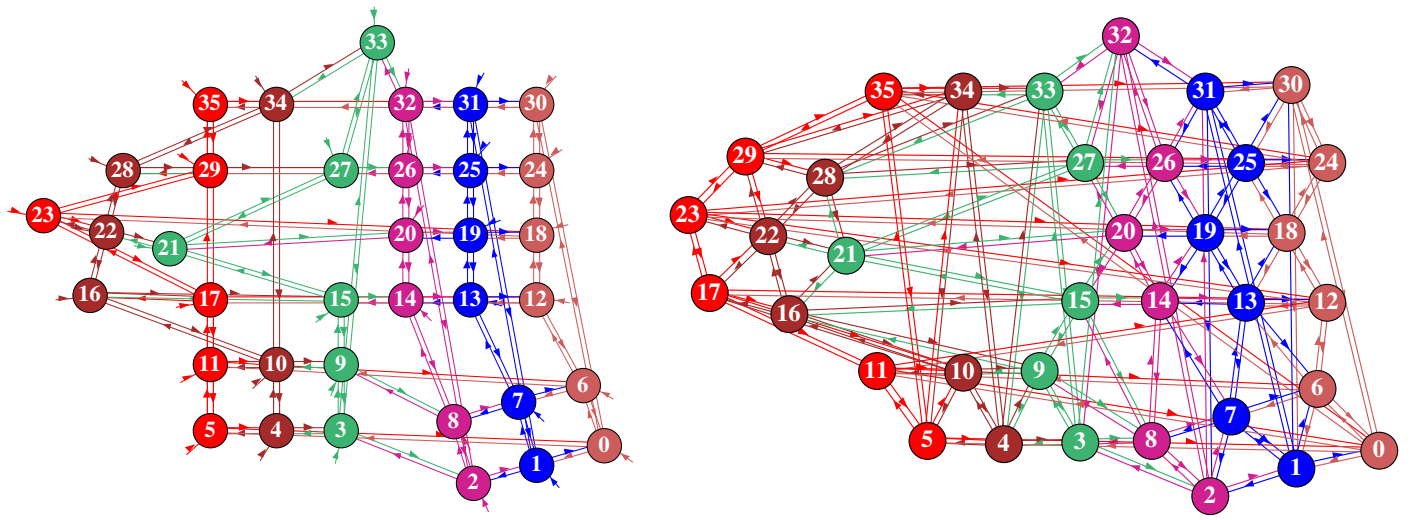

Figure 20.8: Dragging network-graph nodes and fragments starting with Left: a regular 2d networkgraph of a $2 \mathrm{~d}$ CA $6 \times 6$, square $k=5$, and Right: hexagonal $k=6$ network. In both cases, a node has been dragged from the top row, and node $\overline{22}$ and its 1 -step neighbors have been dragged and rotated, and a fragment $(0-8)$ has been dragged and rotated from the lower right hand corner.

As well as dragging a single node, there are options for dragging connected fragments or arbitrary blocks. The fragments are defined as being at a given distance from a node according to input links, output links, or either inputs or outputs. Fragments can be separately dragged, rotated and flipped. Single nodes can have their inputs and/or outputs links cut. Directed links between pairs of nodes can be cut or added. So called "gap nodes", which have only inputs, or only outputs, can be disconnected from the graph, showing the effective components, because gap nodes cannot transmit information. Note that breaking links and creating new links affects only the graph, not the original underlying links which can be restored.

\subsubsection{The drag reminder}

There are 4 types of interchangeable drag situations/reminders, listed below together with their main functions and how to select one from another, 


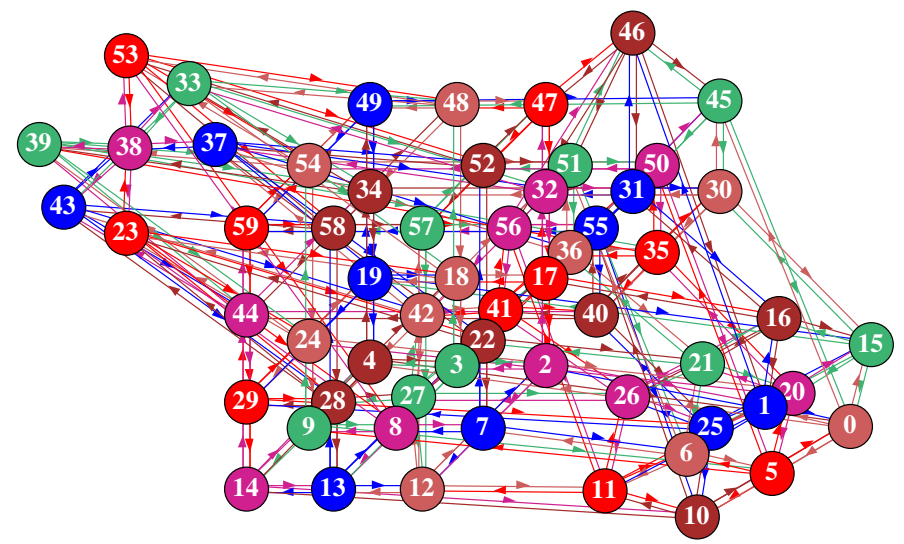

Figure 20.9: Dragging network-graph nodes and fragments starting with a regular $3 \mathrm{~d}$ network-graph $5 \times 3 \times 4, k=6$. Node 46 was dragged from the top level, node 38 and its 1-step neighbors were dragged and rotated, and a fragment $(0-26)$ was dragged and rotated from the lower right hand corner.

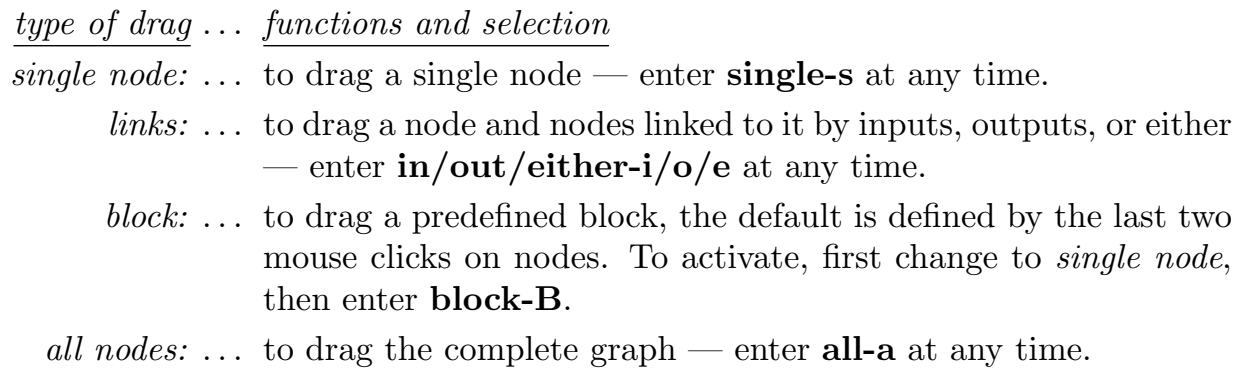

Enter return, or click the left or right mouse button in the network-graph or jump-graph prompts (sections 20.2.1, 20.3.2) for the initial single node drag reminder - clicking on a node will select that node, clicking in empty space gives node $\mathbf{0}$ (exit-q to revert at any time),

single node: drag reminder

node 0, single: drag - left button, tog drag-d (node 1 for the jump-graph)

not active?-click right button first, rotate-x/X flip-h/v gap-g

links: restore net-n, block-B

step-(1-9) nolimit-0 single-s in/out/either-i/o/e all-a exit-q:

The prompt changes if $\mathbf{i}, \mathbf{o}$ or $\mathbf{e}$ (for inputs, outputs, or either) is entered, for example, entering $\mathbf{i}$, and clicking node 4 , then node 7 , gave the following,

links: drag reminder (inputs, outputs, either) - step=nolimit or 1-9

node 7, inputs, step=nolimit: drag - left button, tog drag-d (or outputs or either)

not active?-click right button first, rotate- $\mathrm{x} / \mathrm{X}$ flip-h/v gap-g

links 7: cut/restore/net-c/r/n, link 7-4: cut/add/restore-C/A/R

step-(1-9) nolimit-0, single-s in/out/either-i/o/e all-a exit-q:

As the last node clicked was 7 , links to that node can be cut. The one-but-last node clicked was 4, so link 7-4 appears in the prompt — links between node 7 and 4 can be cut or added. 
When adding an input link its direction will point from 4 to 7 . When adding an output link its direction will point from 7 to 4 .

$\mathbf{s t e p}=$ nolimit signifies that dragging a node will also drag the connected component, indirectly linked by either inputs, outputs, or either, along with it. This can be changed by entering a number step-(1-9), to limit the size of the linked fragment. For example, entering 1 results in step=1 in the top line, and only the nodes immediately linked to the active node will be dragged.

Enter $\mathbf{s}$ to revert to dragging single nodes. The option block-B, to define an arbitrary block to be dragged, is only available if single or Block is active - which appears in the top line. If $\mathbf{B}$ is entered, the block is first defined in $1 \mathrm{~d}, 2 \mathrm{~d}$ or $3 \mathrm{~d}$ (section 20.5.3). The default block is the block between the last 2 mouse clicks. Then the drag reminder changes to look like this (for example),

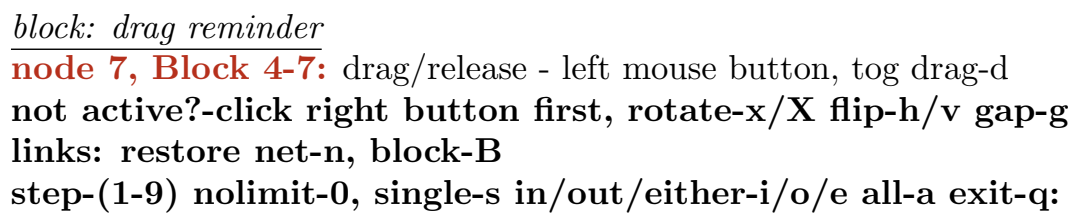

The defined block can the be independently dragged from any node in the block, but dragging any single node will also drag an active block. Enter $\mathbf{B}$ for a new block.

\subsubsection{Drag graph options}

The following is a summary of the drag options, which take effect as soon as the key is hit, without entering return. "fragment" refers to a connected component or defined block.

options... what they mean

drag - left button ... click the left mouse on a node to activate it and hold it down to drag the node or fragment - then release in a new position. Initially, to activate a node it may be necessary to click the right button first, then the left, possibly a few times. Hence the reminder,

\section{not active?-click right button first}

An active mouse cursor points North West instead of North East.

tog drag-d ... enter $\mathbf{d}$ to toggle between two methods of dragging nodes or fragments. By default, dragging will move a node or fragment with elastic links/edges. Alternatively, elastic links can be suppressed and the active node dragged leaving a trail — on release the links (and fragment nodes if any) snap into position and the trail disappears. For large graphs this is a more efficient method. Enter $\mathbf{d}$ to toggle between the two methods.

rotate- $\mathbf{x} / \mathbf{X} \ldots$ enter $\mathbf{x}$ or $\mathbf{X}$ to rotate the graph or fragment by 15 degrees clockwise, or anticlockwise. Rotation is centered on the active node. The default rotation angle can be revised in section 20.10 .

flip-h/v ... enter $\mathbf{h}$ or $\mathbf{v}$ to flip the graph or fragment horizontally or vertically.

gap-g ... enter g to cut all links to "gap nodes". Gap nodes have only inputs, or only outputs. Disconnecting them from the graph will show the effective components, because gap nodes cannot transmit information. 


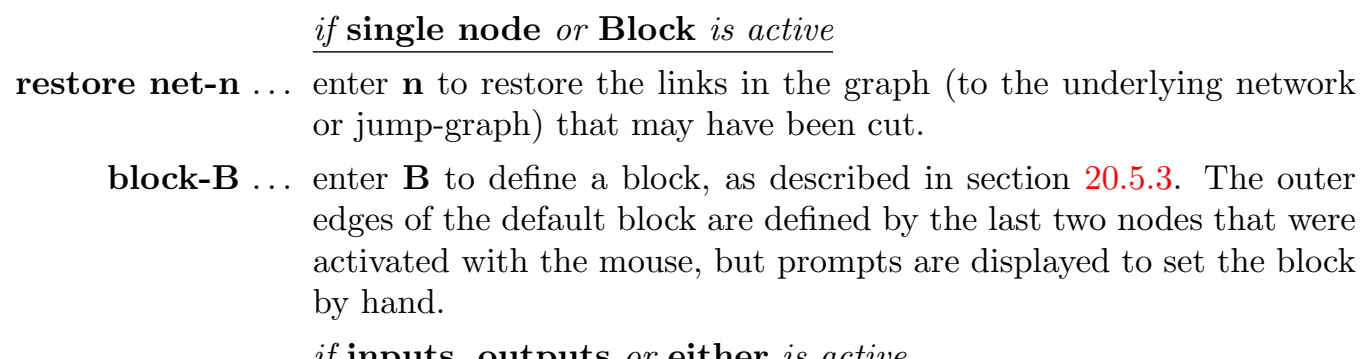

links 23: cut/restore/net-c/r/n ... for example

enter $\mathbf{c}$ to cut (disconnect), $\mathbf{r}$ to restore, the active node from/to the network by cutting/reconnecting all its input, output, or any link, depending on which option, inputs, outputs or either is active. Enter $\mathbf{n}$ to restore the graph to the underlying network or jump-graph.

link 23-19: cut/add/restore-C/A/R ... for example

cut- $\mathbf{C}$... enter $\mathbf{C}$ to cut, $\mathbf{A}$ to add, $\mathbf{R}$ to restore, links between the active node and the previously active node. The node numbers pair of nodes appear in the prompt. The links that are cut/added/restored are either input, output, or any link depending on which option, inputs, outputs or either is active.

in all cases

step-(1-9) ... enter a number between $\mathbf{1}$ and $\mathbf{9}$ to limit a fragment by the distance from the active node. This relates to a continuous chain of directed edges - inputs, outputs, or any link, depending on which option, inputs, outputs or either is active. For example, enter $\mathbf{1}$ to limit the fragment to immediate links, 2 to include indirect links 2 steps away, etc., (up to 9 steps). The current status is shown in the drag reminder, for example $\mathbf{s t e p}=\mathbf{1}$

nolimit-0 ... enter $\mathbf{0}$ to define a fragment by a continuous chain of inputs, outputs, or either inputs and outputs, however indirectly, relative to the active node, depending on which option, inputs, outputs or either is active. Gap nodes would interrupt such a chain. $\mathbf{s t e p}=$ nolimit is shown in the drag reminder.

single-s ... enter $\mathbf{s}$ to restore dragging single nodes. The heading changes to single node 23: (for example).

in/out/either-i/o/b ... enter i, o or e to define the type of link — inputs, outputs, or either (i.e. any link), for dragging fragments or cutting links. The drag reminder will show the active status - inputs, outputs or either.

all-a ... enter a to drag all nodes, the whole graph. The prompt heading changes to all nodes:.

exit-q ... Enter q to exit the drag reminder and return to the network-graph or jump-graph reminder (sections 20.2.1, 20.3.2). Its easy to flip between the two reminders to implement alternative functions. 


\subsubsection{Defining a block}

If single node or Block is active in the drag reminder (section 20.5.1), enter $\mathbf{B}$ to define a block ${ }^{2}$ in $1 \mathrm{~d}, 2 \mathrm{~d}$ or $3 \mathrm{~d}$. For a $2 \mathrm{~d}$ or $3 \mathrm{~d}$ network-graph, a block can be defined according to its 2 outer corners. For a jump-graph or a 1d network-graph, the block is simply a range of nodes between an upper and lower limit (as in section 17.5.3). The easiest way to set the block is to click (activate) the outer corners to set the defaults. Make sure these are really activated by checking that the node number appears in the drag reminder, for example node 301. Any pair of opposite corners can be defined by clicking in any order, but this will be converted automatically to the lower right and upper left corners.

One of the following top-right prompts is presented to accept defaults or set the block by hand, depending if the type of graph,

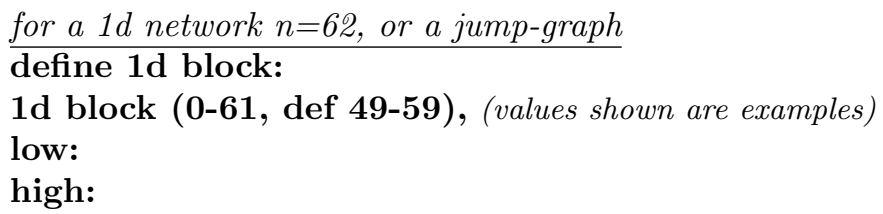

for a 2d network, 22x22

2d network: define block: 1d-1, 2d(def):

2d block (this $=\mathbf{5}, \mathbf{2} \max =\mathbf{2 1}, \mathbf{2 1}$ ), (values shown are examples)

low corner $(\operatorname{def} 5,2)$ i: j:

high corner $(\operatorname{def} 7,5)$ i: $\mathrm{j}$ :

for a $3 d$ network, $6 x 6 x 6$

define 3d network 1d-1 3d-(def):

3d block (this $=\mathbf{0 , 3}, \mathbf{0} \max =\mathbf{5 , 5 , 5}$ ), (values shown are examples)

low corner $(\operatorname{def} 0,3,0)$ i: $\mathrm{j}$ : h:

high corner $(\operatorname{def} 5,3,5)$ i: $\quad \mathrm{j}: \quad$ h:

A $1 \mathrm{~d}$ block is defined by entering the low and high node index. This also applies for a $2 \mathrm{~d}$ or $3 \mathrm{~d}$ network if $\mathbf{1 d - 1}$ is selected first, otherwise enter the coordinates of the opposite (low and high) corners to define the block, or accept the defaults - the last two nodes that were activated with the mouse, which are shown in the prompt. Enter return to accept each default. Once the block is defined, dragging any node (whether inside or outside the block) will also drag the block, and rotate-x/X, flip-h/v, will apply just to the block.

\subsection{Probabilistic "ant"}

Enter a in section 20.4 to launch a projectile or probabilistic "ant" in the graph. The ant has a red body and leaves a black trail on the center line between nodes. The default path starts at a randomly selected node and traces a Markov chain, a path that takes the next link according to the output probabilities. The "ant" keeps track of the frequency of visiting/hitting vertices for an

\footnotetext{
${ }^{2}$ The methods are similar to defining a block in chapter 17 "Reviewing network architecture" (sections 17.5.3, 17.6.5, 17.7.5).
} 
arbitrary sample ${ }^{3}$ of ant hits. While active, a number of ant options are presented in a top right prompt as follows,

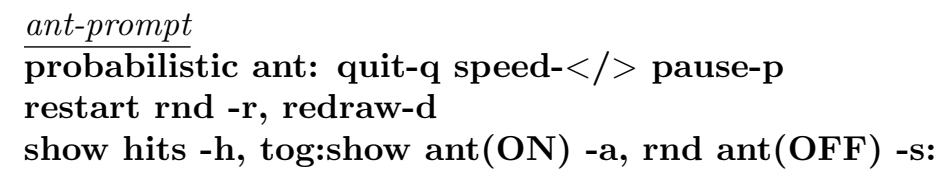

The meaning of these prompts are listed below,

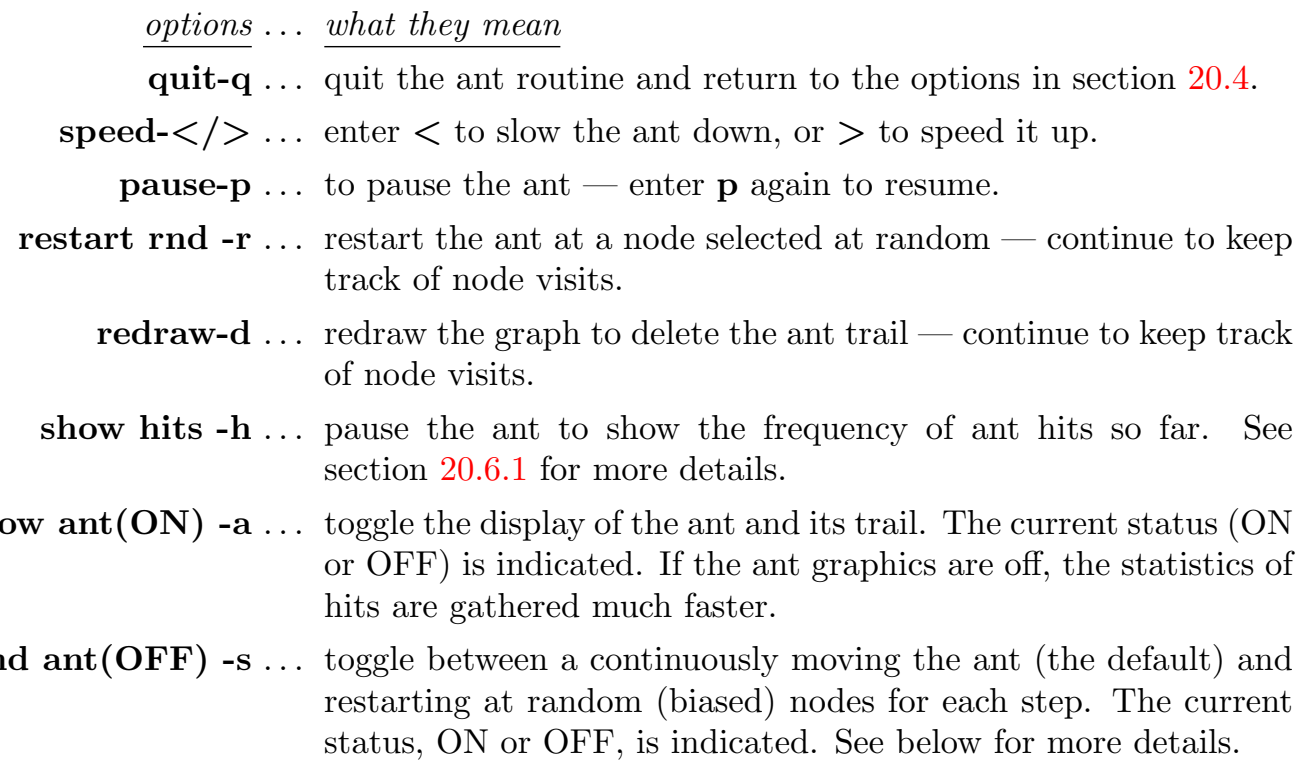

The rnd ant( $\mathbf{O N})$ produces a different kind of sample; at each step the ant is re-started at a random node, biased by $k$ (network-graph), or by the relative size of the basin of attraction that the node represents (jump-graph). Thus the ant can hit all reachable nodes even if they occur in separate components (ergodic sets) of the jump-graph.

By contrast, the default rnd ant(OFF) starts at a random node and follows a Markov chain, a continuous path according to the output probabilities in the graph. Some graphs are fully interlinked, but in a graph consisting of distinct components, if the path starts within such a component, it will be trapped inside. Note that the eigenvectors of the jump table of a component (which may be the whole graph) should be the same as the list of hit frequencies of the component.

\subsubsection{Show ant hits}

Enter $\mathbf{h}$ while the probabilistic ant is active to show the number of hits so far and the percentage at each node. The nodes will be redraw and scaled according to hit frequency - edges, and nodes with zero hits are not shown. The following top-right options are presented, for example,

\footnotetext{
${ }^{3}$ The maximum sample of ant hits is the maximum size of an unsigned long int $-1=4294967295$. If this is reached "Show ant hits" (section 20.6.1) is activated automatically.
} 


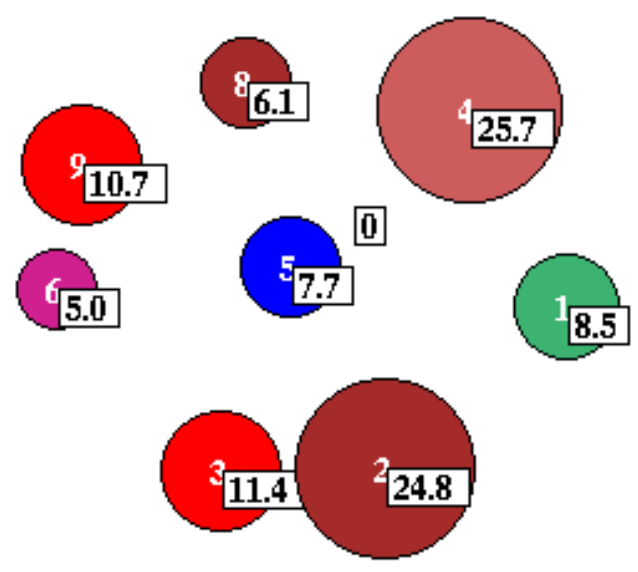

Figure 20.10: Probabilistic ant hits, or node visits (enter $\mathbf{h}$ followed $\mathbf{n}$ in section 20.6). This example is for the RBN $v 2 k 3, n=10$, defined in table 20.1 . 60 million jumps took a few seconds. The percentage of hits/visits to each basin is shown, and can be compared with basin volume in figures 20.4, 20.12 and table 20.3. Node 7 is unreachable from other nodes so has zero hits.

ant hit-prompt

showing \% hits on each basin (or each node for a network-graph) total hits so far $=1453697$

show $\%$ frequency $-n$ noNodes-N, quit-q cont-ret:

\begin{tabular}{|c|c|}
\hline options & what they mean \\
\hline low $\%$ frequency $-n$ & $\begin{array}{l}\text { enter } \mathbf{n} \text { to add the frequencies of hits (as a percentage of total } \\
\text { hits so far) to the scaled nodes (as in figure 20.5.3). }\end{array}$ \\
\hline noNodes-N & $\begin{array}{l}\text { enter } \mathbf{N} \text { to show just the frequencies without showing the nodes, } \\
\text { which might be necessary if large nodes obscure the data. }\end{array}$ \\
\hline quit-q & $\begin{array}{l}\text { enter } \mathbf{q} \text { to quit the ant/hit-options and return to the graph } \\
\text { reminders (section } 20.2 .1 \text { or 20.3.2). }\end{array}$ \\
\hline cont-ret & $\begin{array}{l}\text { enter return to continue accumulating the ant-hits, returning to } \\
\text { the ant-prompts (section } 20.6 \text { ). }\end{array}$ \\
\hline
\end{tabular}

\subsection{Redraw basins at jump-graph nodes}

When drawing the basin of attraction field, the number of basins, their attractor periods, transient lengths, in-degree - so the sizes and shapes of basins - are hard to predict. A compact layout without overlaps can be somewhat difficult using the methods in chapter 25, which describes the layout in the main window. An alternative flexible layout method is to select the jump-graph (with or without edges). As illustrated in figure 20.11, a jump-graph without edges allows a compressed basin of attraction field showing just the prototype basins (section 26.1).

Once the basin of attraction field has been drawn in the main window, revealing the size/shape information, the jump-graph can be rearranged for an appropriate layout, and basins can be redrawn at the jump-graph nodes. The scale of basins can be changed just for this procedure. The basin of attraction field with its new scale and layout will be saved as a PostScript file if this option was set in section 24.2. 


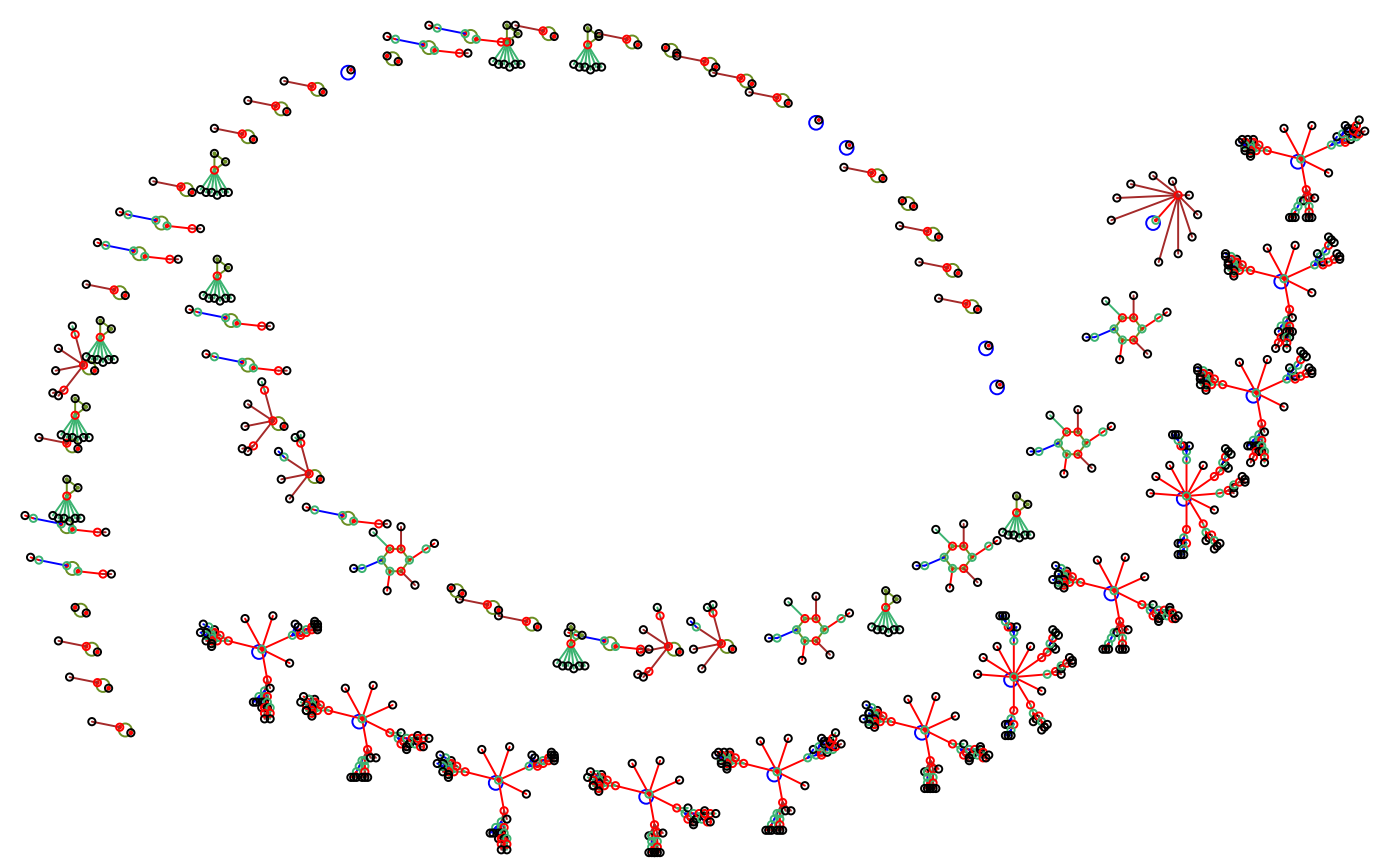

Above: all 73 basins - nodes and fragments were dragged starting from a circle layout. Below: the 11 prototype basins - starting from a square layout.

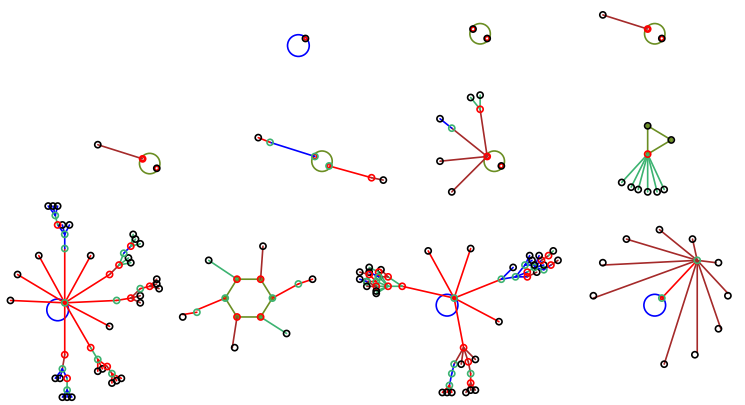

Figure 20.11: Inserting basins in the jumpgraph for a $1 \mathrm{~d}$ CA $v 2 k 3, n=10$, rule (dec)133. For a $1 \mathrm{~d}$ or $2 \mathrm{~d} C A$, if edges were omitted in the jump-graph (section 20.3.1), the basin of attraction field will be compressed by default (section 26.1) to include just the prototype (non-equivalent) basins. Otherwise all the basins in state-space are shown.

Enter $\mathbf{i}$ in section 20.3.2 to redraw the basins centered at the jump-graph nodes positions, but without the jump-graph itself - suppressing nodes and/or edges, as in figure 20.11. Enter I to keep the jump-graph, drawing basins within the nodes as in figure 20.12.

Enter s in section 20.3.2 to first rescale the basins to be redrawn in the jump-graph, the following top-right prompt is presented,

\section{change basin scale for graph}

rad main window $=27.7$, in graph $=9.0$

select attractor $\mathrm{rad}(\min 0.24 \operatorname{def} 9)$ :

Enter the new attractor radius which can be estimated in relation to the basins in the main window. Enter return to revert to the options in section 20.4 and redraw the jump-graph. 


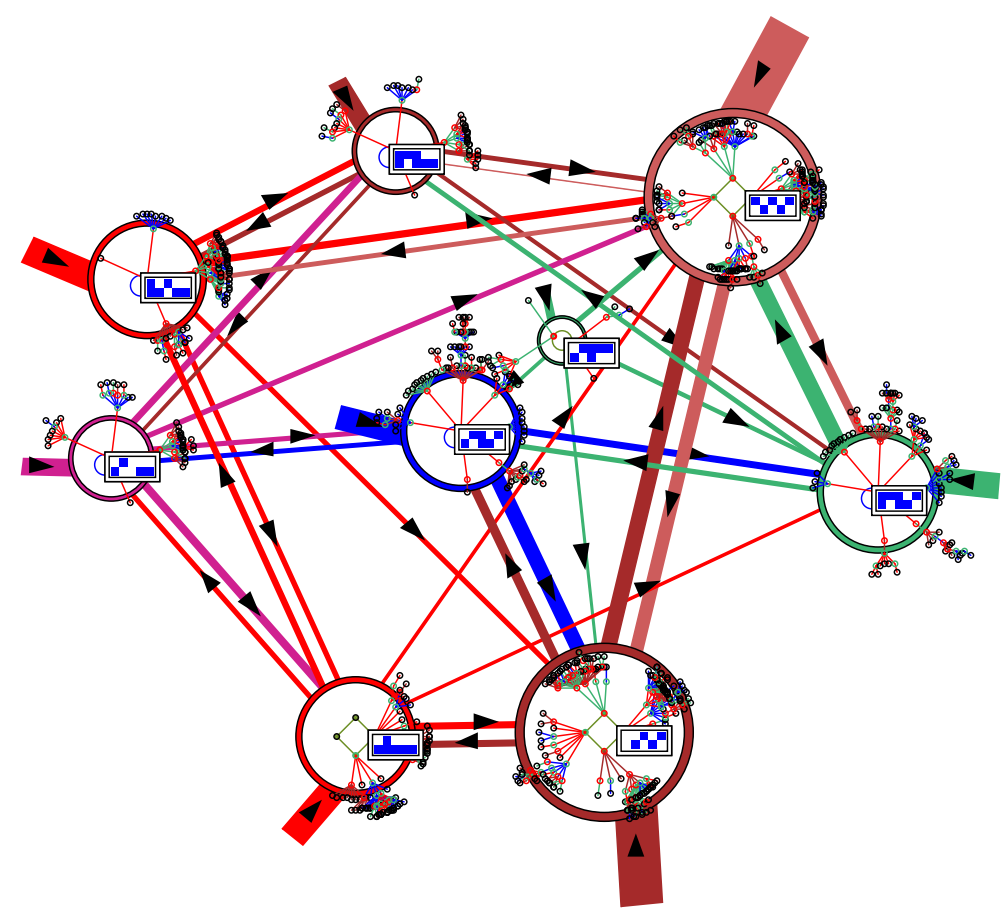

Figure 20.12: Basins of attraction redrawn at the jump-graph nodes, retaining the jump-graph itself. In this example one state in each attractor is also displayed. To create this figure as a vector PostScript file, the separate jump-graph and basin PostScript files were combined. This example is for the RBN $v 2 k 3, n=10$, defined in table 20.1 .

\subsubsection{PostScript of jump-graph basins}

Creating a vector PostScript file of attractor basins, whether drawn in the main windows or in the jump-graph, is activated in the "basin parameters" sequence of prompts, labelled PScript-P in section 24.1. To go directly to the PostScript prompt enter $\mathbf{P}$ at the first output parameter prompt, or arrive there by viewing the basin parameters in sequence.

The following top-right prompt is presented (see also section 24.2),

if saving to PostScript is currently active

save basin to postScipt (now p): greyscale-P color-p cancel-0: (for example)

if saving to PostScript is currently inactive

save basin to postScipt (now OFF): greyscale-P color-p: (for example)

Enter $\mathbf{0}$ to deactivate, $\mathbf{P}$ or $\mathbf{p}$ to activate - a filename prompt will be presented (section 35.2). If saving to PostScript is active, a new file will be created and overwritten to the selected filename each time an attractor basin is drawn (default filename my_bPS.ps). To conserve the file of the 
basins in the jump-graph, the selected filename should be renamed with utilities ${ }^{4}$ outside DDLab before a new attractor basin is generated.

The PostScript files of the attractor basin and the jump-graph (section 20.8) are two separate ASCII files, but can be combined (as in figure 20.12) by appending the basin file (without headers) at the end of the jump-graph file in an external editor. Alternatively, concatenate the files in the terminal - "cat my_jgPS.ps my_bPS.ps > combined_file.ps" — for example. .

\subsection{PostScript of the network-graph or jump-graph}

Enter $\mathbf{P}$ at the network-graph or jump-graph reminders (sections 20.2.1, 20.3.2) to save the graph as a vector PostScript file. The following top-right prompt is presented,

\section{PostScript: greyscale-P color-p:}

Enter $\mathbf{P}$ or $\mathbf{p}$ for greyscale or color. A filename prompt will be presented (section 35.2). The default filename is my_ngPS .ps for a network-graph and my_jgPS .ps for a jump-graph. Once saving is complete, the program reverts to the graph reminders.

\subsection{Graph layout file}

It is sometimes useful to save a graph layout where time and effort have gone into a particular arrangement such as an unravelled network-graph or a jump-graph designed for redrawing basins at node positions (section 20.7).

Enter file-f at the network-graph or jump-graph reminders (sections 20.2.1, 20.3.2) to save the current graph layout, or load a layout where the number of nodes in the file and current graph must correspond. The following top-right prompt is presented,

\section{graph layout file: save-s load-l:}

Enter $\mathbf{s}$ or $\mathbf{l}$ to save or load. A filename prompt will be presented (section 35.2). The default filename is my_grh.grh. When loading, if the number of nodes in the file and current graph do not correspond, the following top-right message is shown,

can't load: file nodes $=\mathbf{1 0}$, graph nodes $=17$ : (for example)

If the file is successfully loaded, the current graph will snap into the new layout. The program reverts to the graph reminders.

\subsection{Revise settings}

Enter $\mathbf{S}$ in section 20.4 to change the default settings for the rotation angle, the expand/contract factors for both nodes and links, and the arrow size for links shown as thin lines with arrows as in figure 20.1. The following top-right prompts are presented in sequence,

\footnotetext{
${ }^{4}$ For example, rename in a terminal, look at the PostScript file in "GhostView".
} 
settings:rotation angle (start $=15)$, now 15.00 degrees:

expand/contract nodes: factor $(1$ to 2 , start $=1.2)$, now 1.20 :

expand/contract links: factor $(1$ to 2 , start $=1.1)$, now 1.10 :

change arrow size: $(0$ to .3 , start $=0.07)$, now 0.07 :

If required, enter the new setting, which may be decimal. The allowed expand/contract factor ranges from 1.0 to 2.0. The arrow size range is from 0 to 0.3 , where 0 will suppress arrows entirely, but arrows can also be suppressed and sizes changed from initial graph option in section 20.4, arrows- $\mathbf{A} /</\rangle$. Revised setting become the defaults, start= shows the initial settings.

\subsection{Unreachable nodes}

Enter $\mathbf{U}$ in section 20.4 to show up unreachable or hard to reach nodes in a network-graph or basins in a jump-graph by disconnecting and/or isolating them from the rest of the graph. A node/basin is unreachable if it has no incoming links from other basins - in the adjacency-matrix or jump-table (section 20.12) there would be a blank column.

An unreachability threshold of zero is the default, but this can be reset to a higher value, so that a node/basin is isolated if it has $x$ or fewer incoming links. The following top-right prompts are presented in sequence,
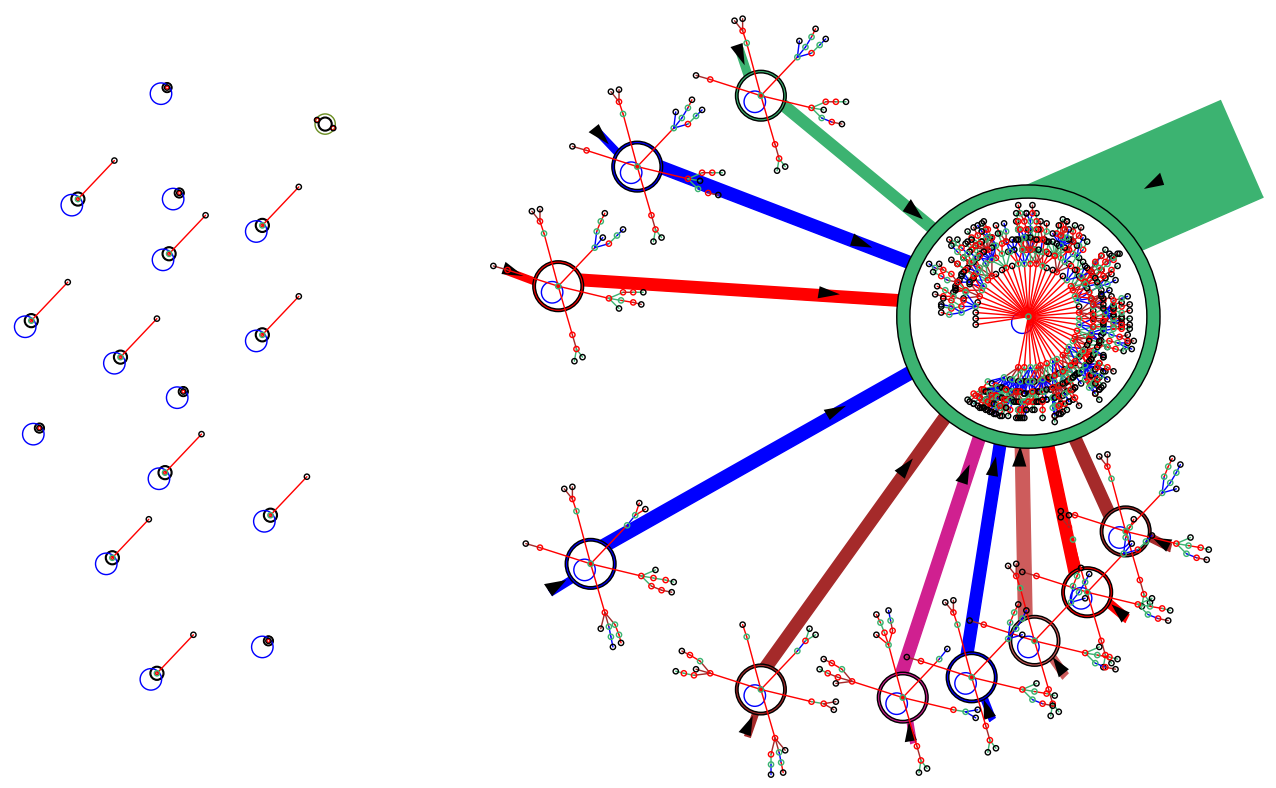

Figure 20.13: Disconnecting and isolating unreachable nodes. The isolated nodes are repositioned randomly on the far left side of the graph window. in this case with the default unreachability threshold of zero. This example is for a jump-graph of a $1 \mathrm{~d}$ CA, $v 2 \mathrm{k} 3$ rcode $104, n=10$. The basins of attraction were redrawn at the nodes (section 20.7). 
links from unreachable nodes: suppress u: restore-(ret):

if $\mathbf{u}$ is entered, the following further prompts are presented

isolate unreachable $-\mathrm{i}$ :

change unreachable threshold (now 0):

Enter $\mathbf{u}$ above to suppress outgoing links to nodes/basins whose incoming links are at or below the current threshold, or return to restore these links. If $\mathbf{u}$ is entered, at the next prompt enter $\mathbf{i}$ to isolate the unlinked nodes, which will be repositioned randomly on the left side of the window. At the next prompt enter a new threshold if required.

\subsection{The adjacency-matrix and jump-table}

Enter $\mathbf{t}$ or $\mathbf{T}$ in section 20.4 to show a table of outputs from each node. This is called the "adjacency-matrix" for the network-graph, and the "jump-table" for the jump-graph. The table can be presented either according to the number of outputs (enter $\mathbf{t}$ ), or the fraction of the total outputs from each node (enter $\mathbf{T}$ ). The table is presented in the graph window - enter return to revert back to the graph.

In an adjacency-matrix (table 20.2), the rows (0 to $n$ - 1 ) show the number (or fraction) of output wires from each network element (cell) to each network element (columns 0 to $n$-1). Two further columns headed out and $\mathbf{k}$ show the total outputs and inputs from/to each network element.

$\begin{array}{lllllllllllll} & 0 & 1 & 2 & 3 & 4 & 5 & 6 & 7 & 8 & 9 & \text { out } & \mathrm{k} \\ 0: & 1 & . & . & . & . & 1 & . & 1 & . & . & 3 & 3 \\ 1: & 1 & 1 & . & . & . & . & 1 & . & 1 & 1 & 5 & 3 \\ 2: & . & 2 & . & 1 & . & . & . & . & . & . & 3 & 3 \\ 3: & . & . & 1 & 1 & 2 & . & 1 & . & 1 & . & 6 & 3 \\ 4: & . & . & . & . & . & 1 & 1 & . & . & . & 2 & 3 \\ 5: & 1 & . & . & . & 1 & . & . & 1 & . & . & 3 & 3 \\ 6: & . & . & . & . & . & . & . & . & 1 & . & 1 & 3 \\ 7: & . & . & 1 & . & . & 1 & . & . & . & . & 2 & 3 \\ 8: & . & . & 1 & 1 & . & . & . & . & . & . & 2 & 3 \\ 9: & . & . & . & . & . & . & . & 1 & . & 2 & 3 & 3\end{array}$

Table 20.2: The adjacency-matrix of the RBN $v 2 k 3, n=10$, defined in table 20.1. The rows ( 0 to $n-1)$ show the number of output wires from each cell to other cells (columns 0 to $n$ - 1 ). This can also be shown as fractions of the total node output. Zero values are show as dots. The last two columns show the total outputs and inputs $(k)$ for each cell.

In a jump-table (table 20.3), successive rows are labelled 1 to $N$, were $N$ is the number of basins in the basin of attraction field. For each basin, the columns, also labelled 1 to $N$, show the number (or fraction of total) jumps to each basin. The meaning of the four further columns labelled $\mathbf{P}, \mathbf{J}$, Volume and Self are as follows,

$\underline{\text { label }} \ldots \underline{\text { what it means }}$

$\mathbf{P} \ldots$ the period of the attractor.

J ... the total number of possible 1-bit flips or jumps, which equals the network size multiplied by the attractor period, $n \times P$.

Volume ... the total number of states in the basin of attraction, and its percentage of state-space, for example $\mathbf{1 1 0}=\mathbf{1 0 . 7 4 \%}$.

Self ... the percentage of total jumps $\mathbf{J}$ that are self-jumps. 


\begin{tabular}{lrrrrrrrrrrrrrr} 
& 1 & 2 & 3 & 4 & 5 & 6 & 7 & 8 & 9 & $\mathrm{P}$ & $\mathrm{J}$ & \multicolumn{2}{c}{ Volume } & Self \\
$1:$ & 5 &. &. & 3 & 1 &. &. & 1 &. & 1 & 10 & $110=10.74 \%$ & $50.00 \%$ \\
$2:$ &. & 24 & 4 & 8 & 4 &. &. &. &. & 4 & 40 & $235=22.95 \%$ & $60.00 \%$ \\
$3:$ & 1 & 5 & 24 & 2 &. & 4 &. &. & 4 & 4 & 40 & $106=10.35 \%$ & $60.00 \%$ \\
$4:$ & 4 & 8 &. & 24 &. &. &. & 1 & 3 & 4 & 40 & $233=22.75 \%$ & $60.00 \%$ \\
$5:$ & 1 & 3 &. &. & 5 & 1 &. &. &. & 1 & 10 & $108=10.55 \%$ & $50.00 \%$ \\
$6:$ &. &. & 2 & 1 & 1 & 5 &. & 1 &. & 1 & 10 & $54=5.27 \%$ & $50.00 \%$ \\
$7:$ & 3 & 3 &. & 3 & 4 &. & 7 &. &. & 2 & 20 & $18=1.76 \%$ & $35.00 \%$ \\
$8:$ & 1 &. &. & 1 &. & 1 &. & 5 & 2 & 1 & 10 & $56=5.47 \%$ & $50.00 \%$ \\
$9:$ &. & 1 & 1 & 1 &. &. &. & 1 & 6 & 1 & 10 & $104=10.16 \%$ & $60.00 \%$
\end{tabular}

Table 20.3: The jump-table of the jump-graph in figure 20.12 showing the number of jumps from each basin (rows) to each basin (columns). This example is for the RBN $v 2 k 3, n=10$, defined in table 20.1. Zero values are show as dots.

\subsubsection{Printing and scanning tables}

When the jump-table or adjacency-matrix is visible, the following top-right options allow the table to be printed in the terminal (not for DOS), and scanned to see all parts of a large table where the data does not all fit inside the window.

\section{scan table: exit-ret xterm-x jump-j reset-s down-d up-u right-r left-l}

The meaning of these prompts is listed below,

xterm-x ... (not for DOS) to print the table in the terminal. ${ }^{5}$

exit-ret ... exit the table and return to the jump-graph and prompts in section 20.4.

jump-j ... jump to place a given row and column in the top-left position of the table. The following top-right prompt is presented,

jump: row: column:

reset-s ... redraw the default table starting with the first row and column.

down-d ... move down, increase the first row index.

up-u ... move up, decrease the first row index.

right-r ... move right, increase the first column index.

left-l ... move left, decrease the first column index.

\subsection{Space-time patterns within the network-graph}

Space-time patterns can be shown running within the network-graph layout simultaneously to the normal $2 \mathrm{~d}$ presentation described in chapter 32 . Enter $\mathrm{g}$ at the space-time pattern interrupt/pause prompt (section 32.16) to set up the network-graph while space-time patterns are running - section 32.19 gives further details.

This allows enormous flexibility, as the network-graph has various default layouts: circle, spiral, 1d, 2d (square or hex), 3d, as well as allowing dragging nodes and components, and many other options for rearranging the graph (figures $32.35-32.36$ ).

\footnotetext{
${ }^{5}$ Displaying the table in the terminal (together with the graph) can also be selected from sections 20.3 .1 and 20.2 .
} 
Once the network-graph space-time patterns are running, and the normal space-time presentation is in $2 \mathrm{~d}$, diagonal scrolling can be set with on-the-fly key \#. To produce a scrolling image as in figure 20.14 or 4.10, suppress normal space-time patterns with on-the-fly key $\mathbf{J}$.

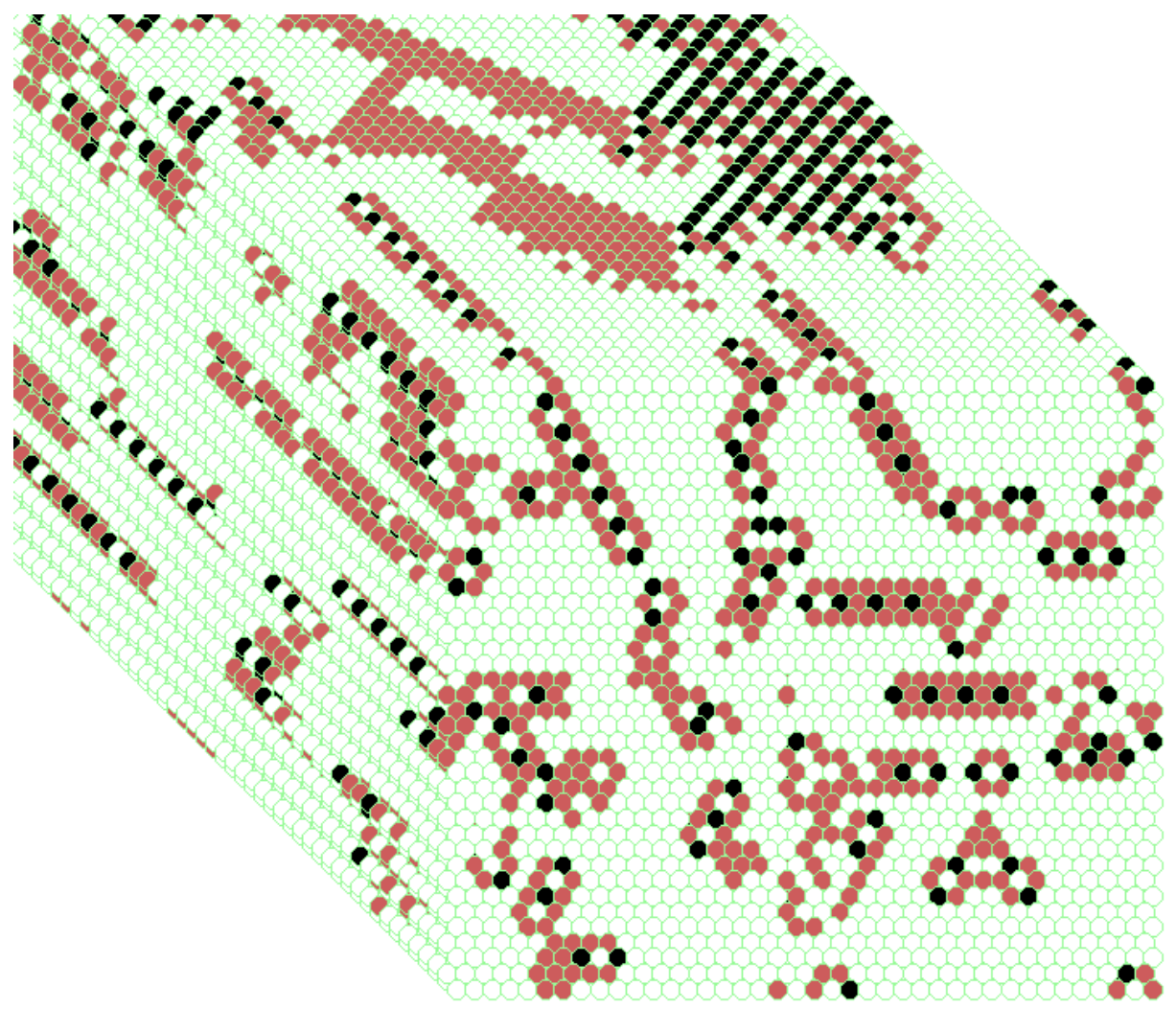

Figure 20.14: A 2d space-time pattern shown shown running within the network-graph layout, which has also been set to scroll diagonally. The present moment is the $2 \mathrm{~d}$ pattern at the bottom-right. 2d CA 40×40, v3k6, kcode(hex) 0a502804958100. 


\section{Chapter 21}

\section{The Seed or initial state}

To run a network forwards, or to generate a single basin or subtree (i.e. if $\mathbf{s}$ was selected in section 6.2.2), or if in TFO-mode, an initial state or "seed" is required. This chapter describes how the seed is specified and amended, its presentation, saving and loading, and vector PostScript. Note that a seed is not required to generate a basin of attraction field.

Section 10.2 described how the network is indexed and arranged in a regular $1 \mathrm{~d}, 2 \mathrm{~d}$ or $3 \mathrm{~d}$ lattice (figures 10.6 - 10.8). The seed is indexed in the same way, as illustrated in figures $21.1-21.3$, and these figures indicate the presentation while setting the seed at random in section 21.3 or as bits/values in section 21.4. Specifying a seed will assign a value from 0 to $v$ - 1 to each cell in the lattice, colored according to the default colors (chapter 7). The methods are similar to "setting a single rule" in chapter 16 .

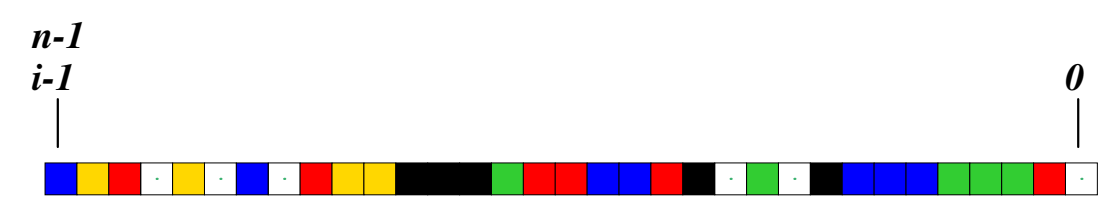

Figure 21.1: A 1d seed (initial state) is indexed as in network indexing (section 10.2.1). This example is for a random seed, $n=33, v=6$.

\subsection{The seed prompt}

To set the seed a lower left window is displayed in the main prompt sequence. When resetting a seed while generating space-time patterns or basins, the window appears in the lower centerright. The size of the window varies according to previous parameters, but can be resized. The exact wording of the prompt depends on the context (see examples below). In general, when first setting the seed the default is rnd-r - useful for a random seed or block with a given density (section 21.3). If a seed was previously set, the default is bits-b - useful for amending or drawing the seed (section 21.4), and loading seeds or sub-seeds (section 21.7). 


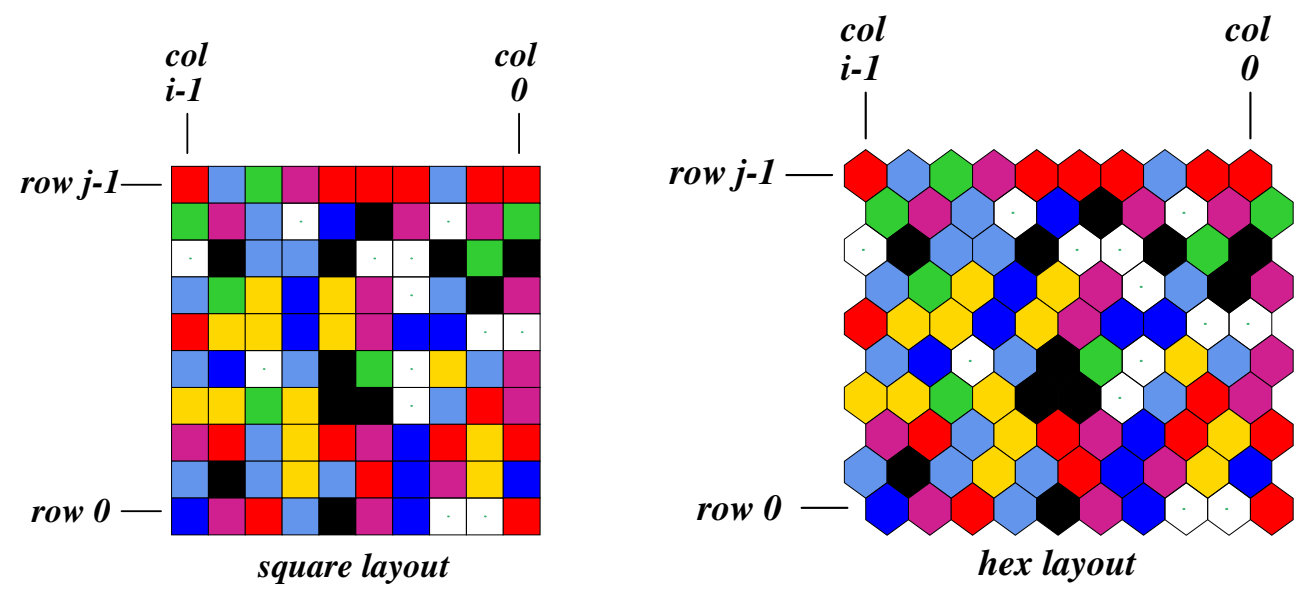

Figure 21.2: A 2d seed (initial state) is indexed as in network indexing (section 10.2.2), showing both square and hex layouts. This example is for a random seed $[i, j]=[20,20], v=8$.
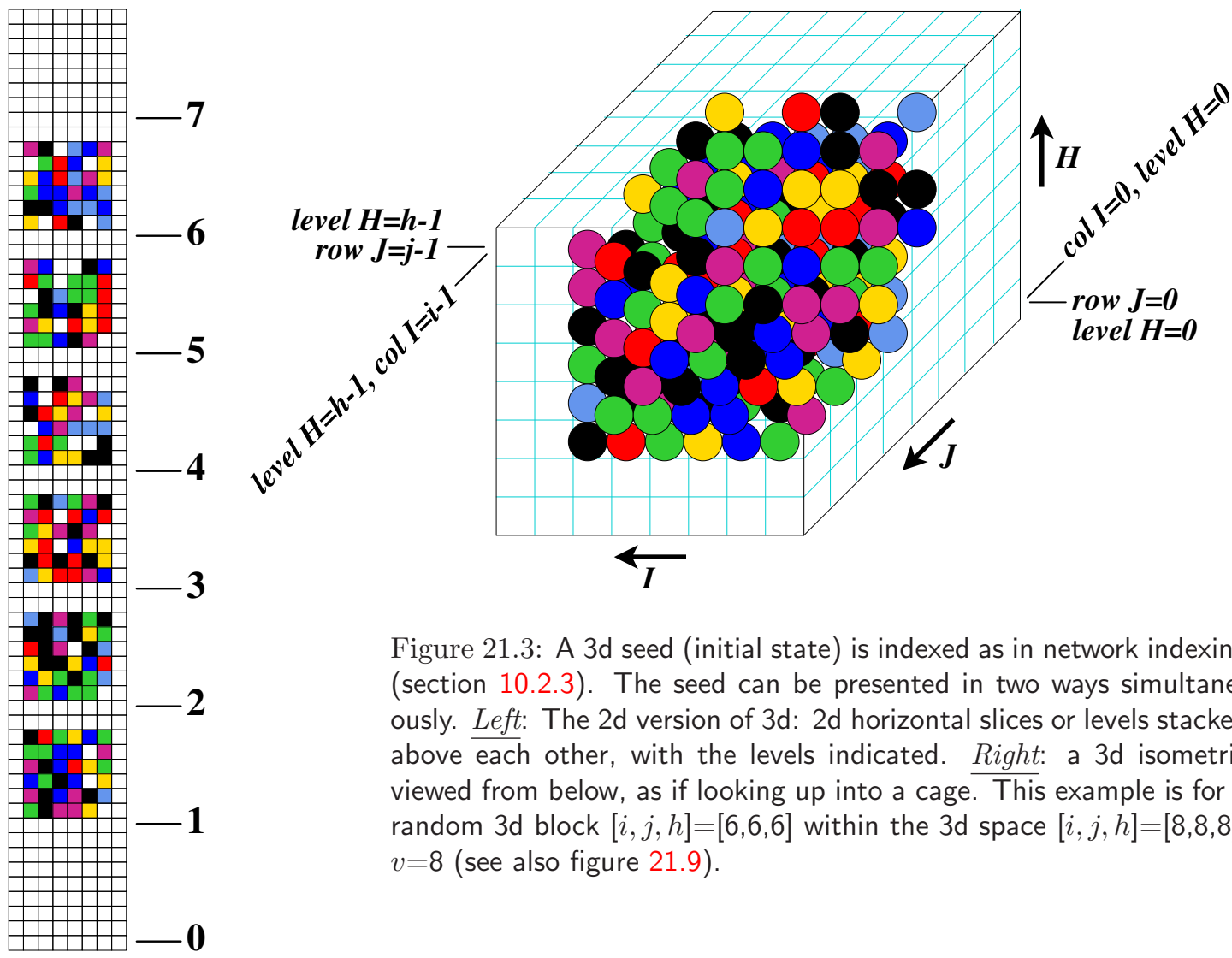

Figure 21.3: A 3d seed (initial state) is indexed as in network indexing (section 10.2.3). The seed can be presented in two ways simultaneously. Left: The $2 \mathrm{~d}$ version of $3 \mathrm{~d}$ : $2 \mathrm{~d}$ horizontal slices or levels stacked above each other, with the levels indicated. Right: a $3 \mathrm{~d}$ isometric viewed from below, as if looking up into a cage. This example is for a random $3 \mathrm{~d}$ block $[i, j, h]=[6,6,6]$ within the $3 \mathrm{~d}$ space $[i, j, h]=[8,8,8]$, $v=8$ (see also figure 21.9). 
example for a $1 d$ network

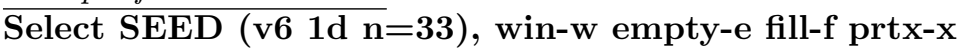

rnd-r bits1d-b bits2d-B hex-h dec-d repeat-p load-l (def-r):

example for a $2 d$ network

Select SEED (v8 $\mathrm{ij}=\mathbf{2 0}, 20$ ), win-w empty-e fill-f prtx- $\mathrm{x}$

rnd-r bits2d-b hex-h repeat-p load-l (def-r):

example for a $3 d$ network

Select SEED (v8 3d ijh=8,8,8), win-w empty-e fill-f prtx-x

rnd-r bits2d+3d-b hex-h repeat-p load-l (def-r):

\subsection{Methods for setting a seed}

The meaning of the prompts for setting a seed ${ }^{1}$ in section 21.1 are summarized below. Further details are provided in the rest of this chapter.

options ... what they mean

win-w ... to resize the seed window, which may be too small for effectively setting the seed in bits or hex, subsequent prompts are presented, for example,

change window size (max-m), width (now 480): height (now 550):

Enter the new window width and height (minimum $50 \times 50$ ), or enter $\mathbf{m}$ for the maximum in each case to fit the screen.

empty-e ... to reset all cells to 0 .

fill-f ... to fill the seed with any valid value with the top-right prompt,

\section{fill with value 0-4 (def 4$)$ : (if $v=4)$}

This allows a clean slate for setting bits or values in section 21.4.

prtx-x... to show the seed in the terminal, both in hexadecimal and as a string of values.

note... After the prompts above the program reverts to the seed prompt in section 21.1. The prompts below set a seed and the program continues.

dec-d ... (if applicable) to set the seed in decimal (section 21.6).

rnd-r ... to set the seed at random, for a given density of a central block — which is displayed in the density window (section 21.3).

bits*-b ... to set the seed as bits or values (section 21.4) by "drawing" using the mouse or keyboard as follows,

bits1d: on a 1d graphic array for 1d networks. Subsequent prompts allows just a segment to be set (section 21.4.5).

bits2d: on a $2 \mathrm{~d}$ graphic array for $2 \mathrm{~d}$ networks.

bits2d+3d: on a $2 \mathrm{~d}$ graphic version of the $3 \mathrm{~d}$ array (shown simultaneously) for $3 \mathrm{~d}$ networks, as in figure 21.3.

\footnotetext{
${ }^{1}$ These methods are similar to setting a rule in section 16.2 .
} 
bits2d-B ... (for $1 d$ networks) to set the seed as bits or values as above, but on a $2 \mathrm{~d}$ graphic.

A subsequent prompt allows changing $i, j$ (section 21.4.6).

hex-h ... to set the seed in hexadecimal, in a mini "spread sheet" (section 21.5).

dec-d ... (if applicable) to set the seed in decimal (section 21.6).

repeat-p ... a repeat of the last seed that was set in the current session..

load-1 ... loaded from a .eed file (section 21.7).

Enter one of the responses above at the prompt in 21.1 or accept the default. Some of these methods are described in more detail in the rest of this chapter.

\subsection{Setting the seed at random}

If $\mathbf{r}$ is selected in section 21.1, a seed, or just a central block, which applies if $i \geq 20$ for $1 \mathrm{~d}$, and in all cases for $2 \mathrm{~d}$ and $3 \mathrm{~d}$, can be set at random, or with some bias. A subsequent prompt is first presented to set the block size,

central 1d block (def diam 30) all-a ( $\max 150)$ : (for a 1 d network, $n=150)$

central 2d block (def diam 14) all-a (max 44): (for a 2 d network, $88 \times 44$ )

central 3d block (def diam 8), all-a ( $\max 25)$ : (for a 3 d network, $60 \times 25 \times 40)$

The default block size is about $i / 5$ for $1 \mathrm{~d}$. For $2 \mathrm{~d}$ or $3 \mathrm{~d}$ the block is square or cubic, with a default block size $1 / 3$ of the network's smallest axis. Enter the size of the random block or accept the default, or enter a for a random seed across the whole network.

The pattern is created at random according to the current density-bias of non-zero values ${ }^{2}$ which appears in a top-right window - initially there is an equal probability of each value, but this may be reset in section 21.3.1 . An alternative "value-bias", the proportions of different values in seed (disregarding any block) can also be set, either as percentages, or as actual numbers of each value - the method is the same as for a rule (section 16.3.2) so will not be repeated here.

The seed is displayed as a bit/value pattern in $1 \mathrm{~d}$ or $2 \mathrm{~d}$ as in figure 21.4. The seed is also shown in decimal (if applicable - section 21.6).

In addition to random biases, various changes and transformations ${ }^{4}$ can be made (repeatedly) until return or $\mathbf{q}$ is entered to accept the seed. The following subsequent reminder is shown,

rotate-l/r another-n density-s/v comp-c goback-q accept-ret (for 1d)

or

rotate-l/r/u/d another-n density-s/v comp-c goback-q accept-ret (2d and $3 d$ )

These options are explained below.

\footnotetext{
${ }^{2}$ For a block size $\leq 3$ the density bias does not apply.

${ }^{3}$ The method is similar to setting the density-bias ( $\lambda$ parameter) for a rule in section 16.3.1

${ }^{4}$ These option are similar to setting a rule at random (section 16.3).
} 


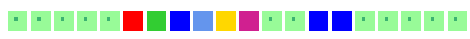

1d $n=20$, block $=10$

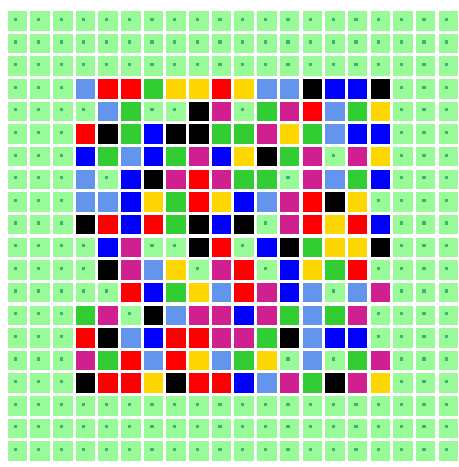

$2 \mathrm{~d} 20 \times 20$, block $=14 \times 14$

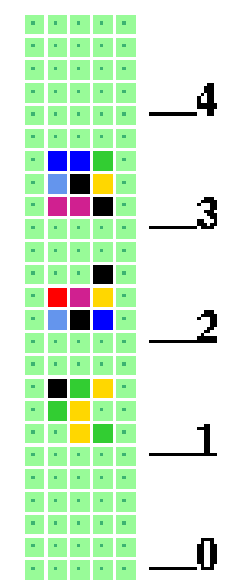

3d $5 \times 5 \times 5$, block $=3 \times 3 \times 3$

Figure 21.4: Examples of the graphic output when setting a seed at random (section 21.3), in this case a central random block, for $1 \mathrm{~d}, 2 \mathrm{~d}$, and $3 \mathrm{~d}$ - which is a $2 \mathrm{~d}$ version of $3 \mathrm{~d}$ similar to figure 21.3 Left. Note that the "random" presentation is less versatile than "bits or values" (section 21.4).

options.... what they mean

tog gaps-g ... to toggle gaps between successive blocks of 8 bits/values.

rotate-l/r/ $\mathbf{~} / \mathbf{d} \ldots$ enter $\mathbf{l}$ or $\mathbf{r}$ to rotate the seed left or right by one cell, or $\mathbf{u}$ or $\mathbf{d}$ to rotate the seed up or down by one cell - for $2 \mathrm{~d}$ and $3 \mathrm{~d}$ only. Rotation is subject to periodic boundaries, a ring of cells in $1 \mathrm{~d}$, a torus in $2 \mathrm{~d}$.

another-n ... for another random seed or block with the same density bias.

density-s/v . . enter $\mathbf{s}$ to change the density bias (for a block size $>3$, section 21.3.1). Enter $\mathbf{v}$ to set the value-bias, the proportions of different values in the seed (disregarding any block) either as percentages, or as actual numbers of each value if the seed size $n \leq 255$ - the method is the same as for a rule in (section 16.3.2) so not be repeated here.

comp-m ... to toggle/transform the seed into its complement. For binary this changes 1 s to $0 \mathrm{~s}$ and vice versa. For $v>2$ each value $a$ is changed to its complement $a_{c}=(v-1)-a$ (as in section 16.20 for rules).

back-q . . . to backtrack to the first seed prompt (section (21.1) — the latest seed is remembered, and can be amended with bits-b or hex-h.

accept-ret ... to accept the rule. Once accepted, the seed can be saved in section 21.8.

\subsubsection{Non-zero seed density bias}

The density of non-zero values in the random seed or block (expressed as a percentage) is displayed in a top-right density window. The default is an equal probability of each value, so $50 \% 1 \mathrm{~s}$ for binary. 


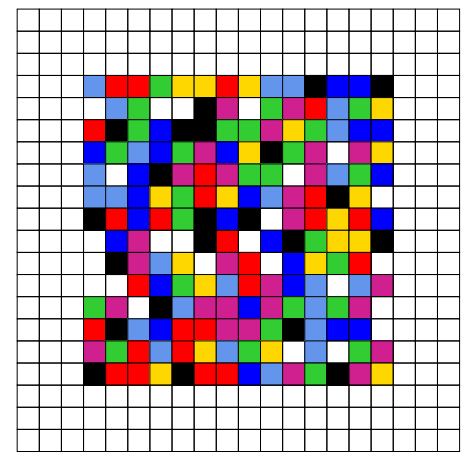

(a) default density bias $=87.245 \%$

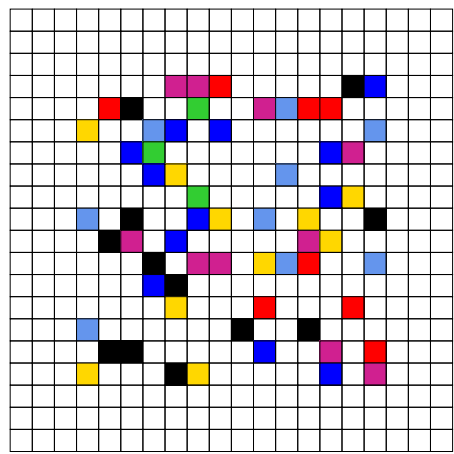

(b) reset density bias $=33 \%$

Figure 21.5: Setting the $v=8$ density bias for a central block $14 \times 14$ within a $2 \mathrm{~d}$ seed $i, j=20 \times 20$, showing the default and reset density. The graphic output would appear as in figure 21.4 , but is presented here from "Setting the seed as bits or values" (section 21.4).

The following density window example relates to figure 21.5(a),

$$
\text { density }(7-1 \mathrm{~s}) \text { exact }=171 / 400=42.750 \% \text {, block }=171 / 196=87.245 \% \text {, bias- } 87.500 \%
$$

where "density (7-1s)" indicates the non-zero values, "exact" the current method of setting the density as opposed to "prob", then the actual density of the seed and the block (if defined), and the default bias or the bias requested. The bias applies to a block if defined in section 21.3, otherwise to whole seed. Enter $\mathbf{s}$ in section 21.3 to change the density-bias ${ }^{5}$ There are two alternative methods, exact or prob (exact is the initial default). The following two stage top-right prompt is presented, for example,

for $v=2,22 \%$ entered, with prob as the default method

bias-density: enter \% (def 50.000\% prob):22 exact-e: (enter e to change to exact)

for $v=8$, 33\% entered, with exact as the default method

bias-density: enter \% (def $\mathbf{8 7 . 5 0 0 \%}$ exact):33 prob-p: (enter $\mathbf{p}$ to change to prob)

Enter the new density-bias as a percentage - the result updates the density window - this example relates to figure $21.5(\mathrm{~b})$.

density $(7-1 \mathrm{~s})$ exact $=64 / 400=16.000 \%$, block $=64 / 196=32.653 \%$, bias $=33.000 \%$

For binary this is the probability of setting $1 \mathrm{~s}$. For $v>2$ it is the probability of setting non-zero values - set without bias. The new density-bias becomes the new default. If the prob method is active the density bias requested is applied as a probability, whereas the exact method will apply the requested bias as closely as possible. To change the default method between prob to exact, enter $\mathbf{e}$ or $\mathbf{p}$ as indicated. The density-bias and method will be maintained for further random seeds or blocks generated with another-n in section 21.3, updating the density window, and when re-setting random seeds for space-time patterns on-the-fly (sections 32.3 ).

\footnotetext{
${ }^{5}$ The methods for seed density-bias are similar to rule density-bias (section 16.3.1).
} 


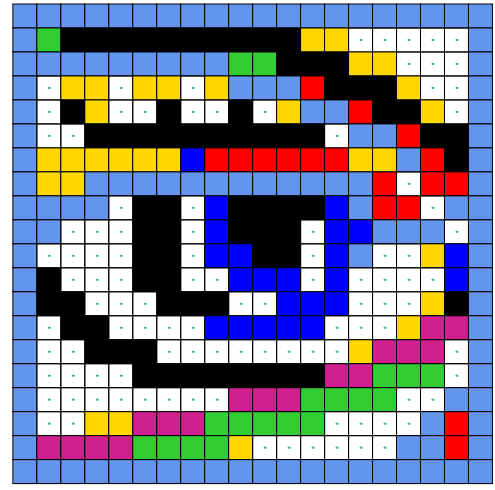

$v=8$ revised presentation

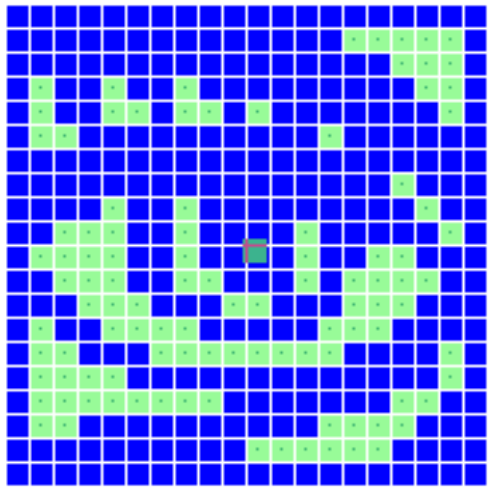

$v=2$ default presentation

Figure 21.6: Drawing bits or values on a $2 \mathrm{~d}$ seed $[i, j]=[20,20]$. Left: The $v=8$ "eye" was drawn with the mouse and keyboard. Right: the default presentation of the eye for $v=2$; light green 0 s and white divisions; the flashing cursor was moved to the center. The $v=8$ eye was loaded into a $v=2$ base seed.

\subsection{Setting the seed as bits or values}

If $\mathbf{b}$ or $\mathbf{B}$ is selected in section 21.1 the seed can be set as bits/values in $1 \mathrm{~d}, 2 \mathrm{~d}$ or $2 \mathrm{~d}+3 \mathrm{~d}$. $1 \mathrm{~d}$ networks can be displayed in both $1 \mathrm{~d}$ (enter b) and $2 \mathrm{~d}$ (enter B). Bits/values are set with the keyboard or drawn with the mouse on the seed graphic, which could be $1 \mathrm{~d}, 2 \mathrm{~d}$, or $3 \mathrm{~d}$, similar to figures 21.1 - 21.3. The default scale of the bit/value pattern varies according to the dimension and size of the network, but can be changed. The current position on the grid is indicated by a small flashing cursor (initially top-left as in figure $21.6 \mathrm{Left}$ ) and also in a top-right inset window.

The colors correspond to the value colors (chapter 7 ) but 0 s are initially colored light green. If a rule is already current, it can be reset to all-0s with empty-e or to any uniform value with fill-f in section 21.1 to provide a clean slate for setting bits/values. Alternatively, use this bit/value setting method for fine adjustments to a seed set by other methods.

\subsubsection{Seed: bits/values reminder window}

Bits or values in the seed are set with the mouse and keyboard. Similar methods apply for the rule in section 16.4. During the procedure, a top-right reminder window, and inset, display reminders of various options and current settings (summarized below) which are context dependent and differ between $1 \mathrm{~d}, 2 \mathrm{~d}$ and $3 \mathrm{~d}$ seeds, for example,

1d seed, $v=2, n=150$

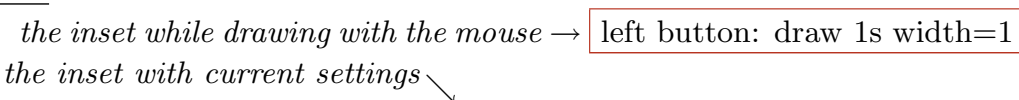

keys: set/select/draw value 1-0, vert-v $\operatorname{count}=01 \mathrm{~d} \mathrm{I}=149$, scale $=5$ rot $=1$ mouse: move-click draw-drag width-w move-arrows, PScript-P file-F tog: gaps/0color/dots/divs/divcolor-g/ $\wedge / . / \mathrm{i} /$ ! $\exp /$ contr-e/c rotate-l/r/+/- flip-h comp-m back/accept-q/ret 
$2 \mathrm{~d}$ seed, $v=3,2 \mathrm{~d}$ seed $i, j=20 \times 20$

keys: set/select/draw value 2-0, vert-v count $=02 \mathrm{~d} \mathrm{IJ}=19 \mathrm{x} 19$, scale $=5$ rot $=1$ mouse: move-click draw-drag width-w move-arrows, PScript-P file-F tog: gaps/0color/dots/divs/divcolor-g/ $\wedge / . / \mathrm{i} /$ ! U/D exp/contr-e/c rotate-l/r/u/d/+/- spin-s flip-h/V comp-m toghex-x back/accept-q/ret

3d seed, $v=8, i, j, h=15 \times 15 \times 15$

keys: set/select/draw value $7-0$, vert-v $\operatorname{count}=03 \mathrm{~d} I J H=14 \times 14 \times 14$, scale $=5$ rot $=1$ mouse: move-click draw-drag width-w move-arrows, PScript-P file-F tog: gaps/0color/dots/divs/divcolor-g/^/./i/! U/D exp/contr-e/c/E/C rotate-l/r/b/f/u/d/+/- spin-s flip-h/V comp-m update3d-t back/accept-q/ret

\subsubsection{Seed: bits/values options summary}

options ... what they mean

keys: select/draw 7-0 ... enter a valid number to select the value/color, and keep the key pressed to draw a horizontal line.

vert-v... enter $\mathbf{v}$ for a vertical line, and keep $\mathbf{v}$ pressed to draw a vertical line downwards in the selected value/color.

mouse: move-click ... left click on the bits/values pattern to reposition the small cursor and activate drawing - sometimes the right button also needs to be clicked (or right-left a few times) to activate.

draw-drag ... draw the selected value/color by dragging the cursor with the left mouse button pressed - release the button to stop. The right mouse button draws the complementary value/color (section 16.20) in the same way.

width-w ... enter $\mathbf{w}$ to change the width (initially 1 ) of the line to be drawn, where the max width is the number of rows for $1 \mathrm{~d}$, or the smallest axis for $2 \mathrm{~d}$, including $1 \mathrm{~d}$ shown as $2 \mathrm{~d}$, and the $2 \mathrm{~d}$ version of $3 \mathrm{~d}$. The following top-right prompt is presented (for example),

\section{reset line width (now 1) max 15:}

The width is shown in the drawing inset, and stays for the current drawing session until revised (figure 16.3, for rule patterns, gives examples).

move-arrows ... all four arrow keys move the cursor around the seed (up/down arrows for multiple rows).

PScript-P . . to save the seed as a vector PostScript image (sections 21.4.7, 21.4.10).

file-F ... to load/save a seed (section 21.4.7).

tog: gaps-g ... enter $\mathrm{g}$ to toggle gaps between successive blocks of 8 bits/values (as in figure 16.2 for rules) .

tog: 0color- $\wedge \ldots$ to toggle the zero value color between light green and white (figure 21.6).

tog: dot-. ... enter a dot to toggle a dot at the center of each zero cell (figure 21.8). 
tog: $\operatorname{divs} /$ divcolor-i/! . . enter $\mathbf{i}$ to toggle division lines between the cells. Enter ! to toggle the division line color between white and black (figure 21.8).

U/D ... (for 2d and 3d only) to pan a 2d seed graphic up and down. 2d will pan by 1 row. A $2 \mathrm{~d}$ version of $3 \mathrm{~d}$ (figure 21.3) will pan by 1 level. This allows different parts of large seeds to be brought into focus for setting bits.

$\exp /$ contrect-e/c/E/C ... enter e to expand, $\mathbf{c}$ to contract the scale by one pixel for $1 \mathrm{~d}$ and $2 \mathrm{~d}$. Similarly, for $3 \mathrm{~d} \mathbf{E} / \mathbf{C}$ for just the $3 \mathrm{~d}$ presentation, and $\mathbf{e} / \mathbf{c}$ for the $2 \mathrm{~d}$ version of $3 \mathrm{~d}$, which are treated separately.

rotate-l/r/b/f/u/d/+/- ... The seed can be "rotated" about the periodic axes in $1 \mathrm{~d}, 2 \mathrm{~d}$ and $3 \mathrm{~d}$ by one cell. $\mathbf{l} / \mathbf{r}$ rotates left and right on the $i$ axis, For $2 \mathrm{~d} \mathbf{u} / \mathbf{d}$ rotates up and down on the $j$ axis. For $3 \mathrm{~d} \mathbf{u} / \mathbf{d}$ rotates levels up and down on the $h$ axis, and $\mathbf{b} / \mathbf{f}$ rotates backwards and forwards on the $j$ axis. Enter + or - to increase or decrease the rotation interval - shown in the current settings inset.

spin-s ... (for $2 d$ and $3 d$ only) For $2 \mathrm{~d}$ where $i=j$ the seed is spun clockwise by $90^{\circ}$ so 4 spins returns to the start. Similarly for $3 \mathrm{~d}$ where $i=j$, each level $h$ is spun clockwise by $90^{\circ}$. If $i \neq j$ a square part including $[I, J]=[0,0]$ will be spun. For $2 \mathrm{~d}$ hexagonal layout there may be some distortion.

flip-h/V . . enter $\mathbf{h}$ for a horizontal flip or reflection. For $1 \mathrm{~d}$ this reflects the $i$ axis (figure 18.2), $2 \mathrm{~d}$ and $3 \mathrm{~d}$ each $i$ axis is reflected. For $2 \mathrm{~d}$ and $3 \mathrm{~d}$ enter $\mathbf{V}$ for a vertical flip of each $j$ axis.

comp-m ... to toggle the seed into its complement. For binary this swaps $1 \mathrm{~s}$ and $0 \mathrm{~s}$, a negative transformation (figure 18.2). For $v>2$ the values are shifted; each output $a$ changes to $a_{m}$ by subtraction from the maximum value $v-1$, so $a_{m}=(v-1)-a$, and the maximum value changes to zero (section 16.20).

update3d-t ... (for $3 d$ only) to update the $3 \mathrm{~d}$ view after drawing bits/values on the $2 \mathrm{~d}$ version of $3 \mathrm{~d}$.

back/accept-q/ret ... enter ret to accept the rule, $\mathbf{q}$ to backtrack.

\subsubsection{Seed: bits/values current settings inset}

count $=03 \mathrm{~d} \mathrm{IJH}=14 \mathrm{x} 14 \mathrm{x} 14$, scale $=5 \mathrm{rot}=1 \leftarrow$ the inset with current settings, for example

current settings ... what they mean

count $=\ldots$ the current cursor position, starting from 0 in the top-left.

IJH ... the current cursor coordinates.

scale $=\ldots$ the current scale of cells in pixels, which can be expanded and contracted.

rot $=\ldots$ the current interval by which the rule-table can be rotated. 


\subsubsection{Seed: setting bits/values with the keyboard and mouse}

The active position (to be updated) in the seed graphic is highlighted by a small flashing cursor initially in the top-left. For $3 \mathrm{~d}$, the flashing appears only on the $2 \mathrm{~d}$ version of $3 \mathrm{~d}$. Its position is displayed in the top-right inset window. The flashing cursor is repositioned with the mouse by clicking either the left or right button on the new position ${ }^{6}$, or moved with the left/right/up/down arrow keys.

Setting or drawing values on the seed graphic is done with the keyboard or mouse. This applies to the $2 \mathrm{~d}$ version of $3 \mathrm{~d}$ - the $3 \mathrm{~d}$ view can be updated by pressing $\mathbf{t}$. To set (and activate) a value at the cursor position, press a valid number key (without return) - the cursor will then advance to the right by one unit. To draw a horizontal line towards the right keep the key pressed. While the key is pressed the line will continue to the next row or jump back to the top-left. To draw a vertical line downwards with the active value, press $\mathbf{v}$.

To draw the active value with the mouse, drag the cursor anywhere over the seed with the left button pressed - release to stop. To draw the complement of the active value (section 16.20) drag with the right button pressed. While a mouse button is pressed, the inset in the reminder window changes to show which button, the current active value, and the current width of the line, for example,

left button: draw $2 \mathrm{~s}$ width $=3$ or right button: draw $8 \mathrm{~s}$ width $=1$

Initially the left button draws the value $v-1$ and the right draws 0 , so for binary 1 and 0 . To activate drawing with the mouse it is sometimes necessary to click the left and right button alternately. To accept the seed enter return. Once accepted, the seed is also displayed in decimal (if applicable - section 21.6).

\subsection{5 setting bits/values: $1 \mathrm{~d}$ segments} for $1 d$ seeds only

For a 1d network, if $\mathbf{b}$ is selected at the seed prompt (section 21.1), the following prompts allows just a segment of the seed to be defined,

segment: enter length $(\max / \mathbf{d e f}=\mathbf{2 0 0}):($ for $n=200)$

Enter return for the whole seed, or enter the segment size whereupon a further prompt positions the segment within the network,

seglength 5: enter start position from left 0-149, def centered: (for example)

Enter return for a centered segment, or a position from the left. A bit pattern representing just the segment is displayed for setting bits/values. There is no limit to adding and overwriting segments.

\footnotetext{
${ }^{6}$ Mouse behavior differs slightly between Linux-like systems and DOS. In Linux the mouse cursor changes direction within the bit/value pattern, pointing north-west instead of north-east, and within the pattern, left or right mouse clicks reposition the flashing cursor. In DOS the mouse cursor is confined within the pattern and clicking the left or right buttons sets values as well as repositioning the flashing cursor.
} 


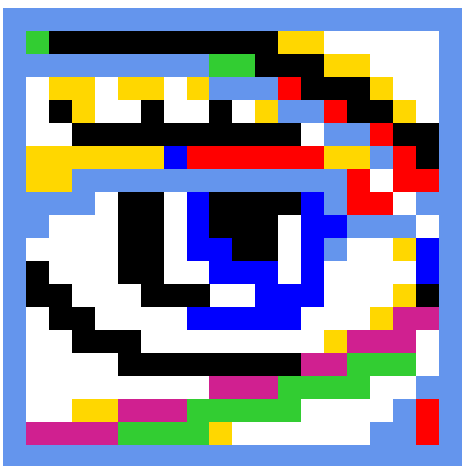

S1. no divs, no dots

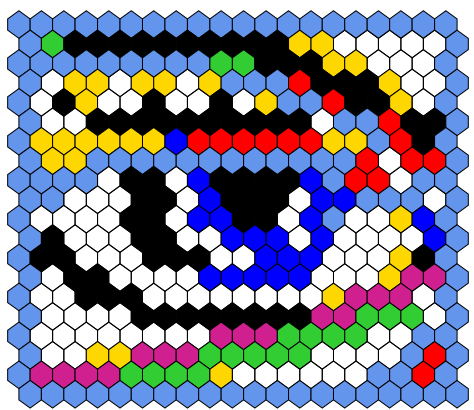

H1. black divs, no dots

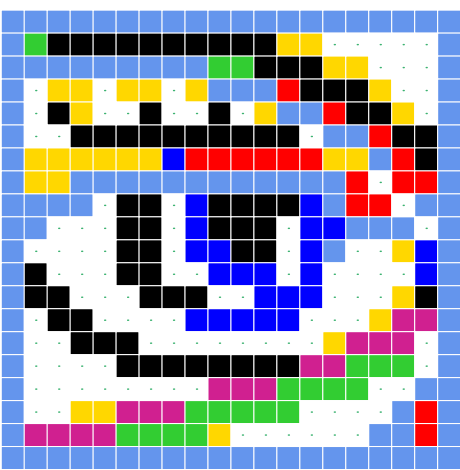

S2. white divs, small dots

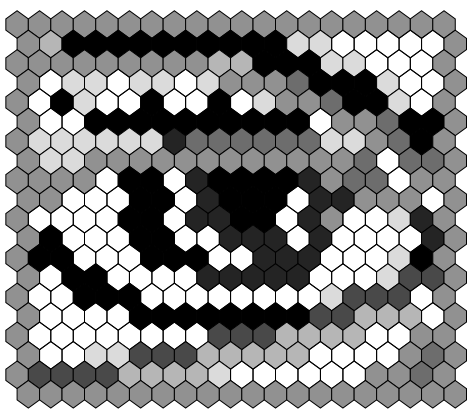

H2. greyscale, black divs, no dots

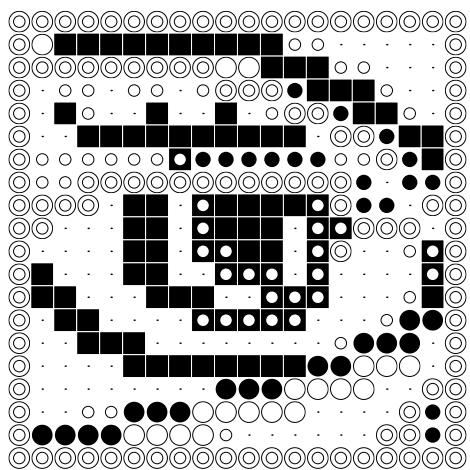

S3. symbols, dots, white divs

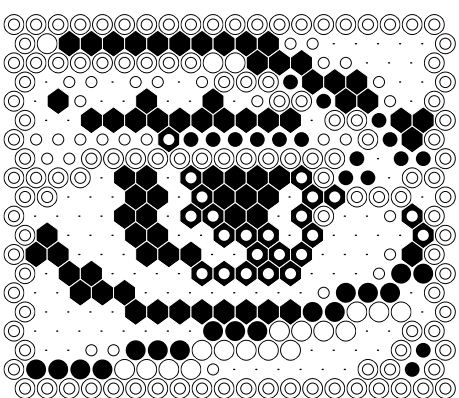

H3. S3. symbols, dots, white divs

Figure 21.7: Examples of PostScript 2d seed output, the "eye" as in figure 21.6, $[i, j]=[20,20], v=8$. A variety of options are available: color, greyscale, symbols, hex, square, black or white divisions between cells or none, variable size zero dots or none. Top Row: square layout. Bottom Row: hex layout.

\subsection{6 setting bits/values: $1 \mathrm{~d}$ shown as $2 \mathrm{~d}$ for 1d seeds only}

For a network, $1 \mathrm{~d}$, if $\mathbf{B}$ is selected at the seed prompt (section 21.1), the $1 \mathrm{~d}$ seed is shown in $2 \mathrm{~d}$ as a square or rectangular bit/value pattern, $i \times j$. Default $j$ (the number of rows) is set automatically as the highest factor of $n \leq \sqrt{n}$. The following prompt allows the default $i$ to be revised,

now 20 x 10, reset i-axis: (for $n=200$ )

The $1 \mathrm{~d}$ seed is broken up into successive rows starting with the maximum cell index in the top-left. If $i$ is not a factor of $n$, some cells will be missing from the bottom row. The $2 \mathrm{~d}$ arrangement can be saved as a $2 \mathrm{~d}$ PostScript or seed file, and loaded back into another $2 \mathrm{~d}$ seed file (section 21.4.7).

\subsubsection{Setting bits/values: filing and PostScript}

The seed can be saved as a vector PostScript image, or as a $1 \mathrm{~d}, 2 \mathrm{~d}$ or $3 \mathrm{~d}$ seed file, by selecting PScript--P or file-F in section 21.4.1 - both options follow similar methods, and are similar to the rule options in section 16.4.3. File extensions and default filenames (chapter 35) are as follows, 
PostScript files ...*.ps files.

for $1 \mathrm{~d}, 2 \mathrm{~d}$, and the $2 \mathrm{~d}$ version of $3 \mathrm{~d}$ (as in figure 21.3 Left)

- default filename my_sPS.ps.

for the $3 \mathrm{~d}$ image only (as in figure 21.3 Right)

- default filename my_3dPS.ps.

seed files $\ldots *$.eed - default filename mysee_v $v$.eed where $v=$ value-range.

One of the following prompts appears in a top-right window,

for PostScript, PScript-P

PostScript 1d: save all-a, save patch-p: (for 1d)

PostScript 2d: save all-a, save patch-p: (for 2d)

PostScript 3d: save all 2d-a, save patch 2d-p, only 3d-3: (for $3 d$ )

for a seed file, file-F

SEED: load-l, save all-a, save patch-p: (for $1 d, 2 d$ or $3 d$ )

for a 1d seed which can be saved as a $2 d$ seed file, file-F

SEED: load-l, save1d $>2 d-x$, save all-a, save patch-p:

If bits2d-B was selected at the $1 \mathrm{~d}$ seed prompt (section 21.1) to display the seed in $2 \mathrm{~d}$, there is an extra option save1d $>\mathbf{2 d} \mathbf{d}-\mathbf{x}$ to save the $1 \mathrm{~d}$ as a $2 \mathrm{~d}$ seed. The $i, j$ coordinates selected might give an incomplete rectangle; this is acceptable for both the $2 \mathrm{~d}$ seed and PostScript.

These options are summarized below,

load-l ... load a seed file (section 21.7). As many seed files as required can be loaded into the current seed. Different $v\left(v_{\text {file }} \leq v_{\text {base }}\right)$, dimensions and sizes are possible subject to the constraints listed in section 21.7.1.

save-a ... save the seed, as a seed file or PostScript. For PostScript and 3d, save-a applies to the $2 \mathrm{~d}$ version of the $3 \mathrm{~d}$ image, as in figure 21.3.

save patch-p ... save part of the seed as a seed or PostScript file, first defining the limiting coordinates of the patch (in $1 \mathrm{~d}, 2 \mathrm{~d}$ or $3 \mathrm{~d}$ ) in section 21.4.8.

only 3d-3 ... (for PostScript of $3 d$ only) save a PostScript file of the seed 3d graphic (section 21.4.10).

save1d $>\mathbf{2 d}-\mathbf{x} \ldots$ (for $1 d$ seed file) save a $1 \mathrm{~d}$ seed as a $2 \mathrm{~d}$ seed. The seed must be displayed with bits2d-B in section 21.1.

A top-right prompt gives various options for the PostScript presentation (section 21.4.9). Files are loaded and saved from the filing prompt (section 35.2).

\subsubsection{Setting bits/values: saving a patch}

Part only of the seed (in 1d, 2d, or 3d) can be saved, as either a seed file or as a vector PostScript image file. The limiting coordinates of the patch are set by the last two mouse clicks on the bit/value seed graphic, or by entering coordinates.

If $\mathbf{p}$ is selected in section 21.4.7, one of the following set of prompts are shown in sequence in a top-right window, 
example for a $1 d$ network

1d:max $\mathrm{i}=149$, revise coords-ret, accept patch $55-37-\mathrm{p}$ : start i: end i:

example for a 2 d network

2d:max i,j=39,39, revise coords-ret, accept patch 26,23-14,14 -p: file:i,j=6,6 start i: end i: start $\mathrm{j}$ : end $\mathrm{j}$ :

example for a $3 d$ network

3d:max $\mathrm{i}, \mathrm{j}, \mathrm{h}=8,8,8$, revise coords-ret, accept patch $6,6,5-2,1,3-\mathrm{p}$ :

start i: end i: start $\mathrm{j}$ : end $\mathrm{j}$ : start h: end h:

Enter $\mathbf{p}$ to accept the default coordinates according to the last two mouse clicks, or return to set the coordinates manually on the second prompt line, which otherwise does not appear. return at a manual setting gives the default mouse click coordinates. The patch seed file can then be loaded back at any position within the same or another seed (section 21.7). The facility to save and load a patch is useful to set up special configurations such as glider collisions.

\subsubsection{Setting bits/values: PostScript prompt}

for 1d, 2d, and the 2d version of $3 d$ as in figure 21.3 Right

When creating a PostScript image of a seed there are a variety of presentation possibilities as illustrated in the figures in this chapter $(21.1-21.10)$ and other figures in the manual. Note that the following options do not apply to a PostScript 3d image as in figure 21.3 Right, which follows its own methods (section 21.4.10).

Enter $\mathbf{a}$ (or $\mathbf{p}$ for part of the seed) at the prompt in section 21.4.7 to save the seed as a vector PostScript (*.ps) file (default filename my_sPS.ps). The following top-right prompt is displayed, where direct-d applies only when interrupting space-time patterns ${ }^{7}$, for example,

create PostScript image for $2 \mathrm{~d}, \mathbf{2 0 \times 2 0}$ (or $1 \mathrm{~d}, \mathbf{3 d}$ )

symbols-s greyscale-g color-c direct-d exit-q (def-c):

These options are summarized below (then subsequent options continue),

options... what they mean

symbols-s ... to show values as symbols (figures $21.7,21.8$ ).

greyscale-g ... to show values in greyscale (figures 21.7).

color-c... (the default) to show values in color.

direct-d ... (only when interrupting space-time patterns, section 32.16) to scan cell colors directly from a $1 \mathrm{~d}$ space-time pattern, or a snapshot of $2 \mathrm{~d}$, or the $2 \mathrm{~d}$ version of $3 \mathrm{~d}$. If direct-d is selected the second line is replaced with,

direct: greyscale-g color-c (def-c): (symbols-s does not apply)

\footnotetext{
${ }^{7}$ From the space-time pattern pause/interrupt prompt (section 32.16 ) enter e to revise the original, last, or current state which gives the seed prompt in section 21.1. Alternatively enter $\mathbf{P}$ for an immediate PostScript prompt similar to section 21.4.9. For 1d space-time patterns, $\mathbf{P}$ gives the direct option only.
} 


\begin{tabular}{|c|c|c|c|c|}
\hline$v$ & values & color hex & symbols hex & symbols square \\
\hline 2 & 10 & & & \\
\hline 3 & 210 & & & \\
\hline 4 & 3210 & & & \\
\hline 5 & 43210 & & & \\
\hline 6 & 543210 & & & \\
\hline 7 & 6543210 & & & \\
\hline 8 & 76543210 & & & \\
\hline
\end{tabular}

Figure 21.8: Symbols for PostScript seed output for $v=2$ to 8. Enter $\mathbf{s}$ in the PostScript prompt (section 21.4.9) to show values as symbols replacing colors. Symbols come in the two versions shown, hex and square, depending on the bits/values display in section 21.4 .2 which is toggled with $\mathbf{x}$. The value zero is shown here as a dot, but this can be blank or the dot size changed.

For interrupted 1d space-time patterns only, if direct-d was selected above, the next two prompts limit the part of the image to be saved (section 32.18)

1d size $(\max / \operatorname{def}=150): \quad$ time-steps $(\max / \mathrm{def}=669)$ :

Once these options are complete, the prompt to amend other settings is presented, for example,

cellscale $=5.00 \operatorname{dots}($ on $)=0.70 \operatorname{divs}(2)$, amend settings-a:

Enter return to accept the defaults, or enter a for the following prompts presented in sequence,

change: cellscale: togdots-x: dotscale: $\operatorname{divs}(0,1,2)$ :

Enter changes required or return to accept defaults, which follow the current bits/values presentation. The options are summarized below,

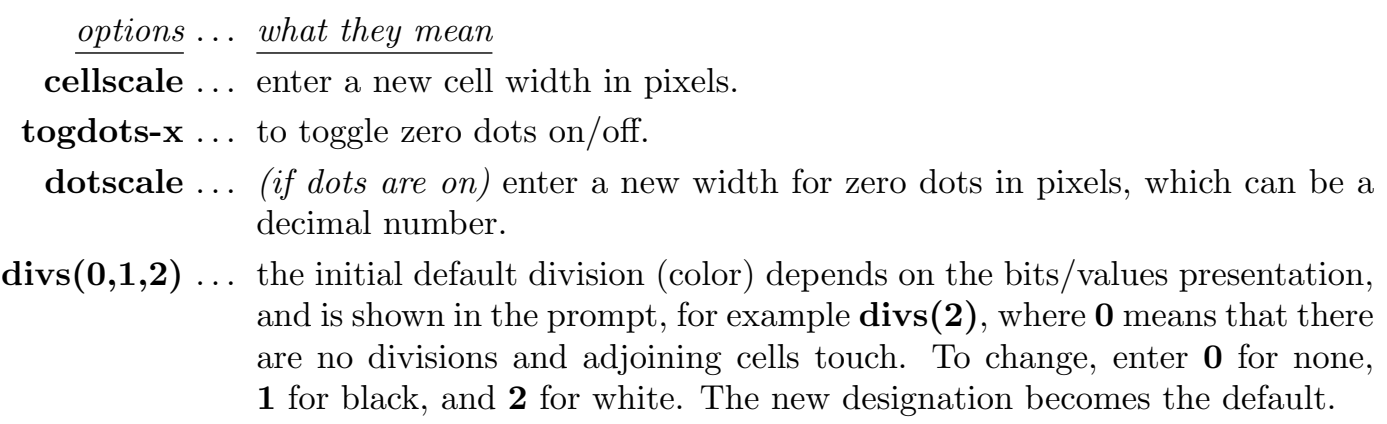


Cells with value zero (0color) are initially colored light green in the bits/values presentation (section 21.4), which can be togged to white with 0color-^ in section 21.4.1 - the PostScript file will follow the current 0color.

Once these choices are complete, the *.ps file is saved from the filing prompt (section 35.2). section 36.1 explains how to view, edit, and crop the PostScript image.

\subsubsection{Setting bits/values: PostScript 3d image} for the $3 d$ image as in figure 21.3 Right

For a 3d network (or space-time pattern) if PScript--P is selected in section 21.4.7 the following top-right prompt is presented

PostScript 3d: save all 2d-a, save patch 2d-p, only 3d-3: (for $3 d$ )

Th save all or part of the $2 \mathrm{~d}$ version of the $3 \mathrm{~d}$ seed

If save all $2 d$-a or save patch $2 d$-p is selected for a vector PostScript file of the $2 d$ version of the $3 \mathrm{~d}$ seed, prompts are presented described in section 21.4.9.

Enter only $3 d-\mathbf{3}$ to save the $3 \mathrm{~d}$ image to a vector PostScript file at the same scale as the $3 \mathrm{~d}$ seed image. - the following top-right prompt is presented,

\section{3d to PostScript 3d: greyscale-P color-def:}

Enter $\mathbf{P}$ for greyscale, or return for the same colors as the $3 \mathrm{~d}$ seed image. The top-right filing prompt (section 35.2 is then presented to the vector PostScript*.ps file (default filename my_3dP.ps). section 36.1 explains how to view, edit, and crop the PostScript image.

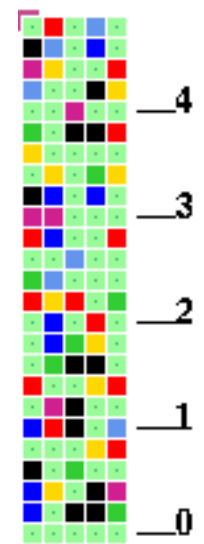

$2 d$ version of $3 d$

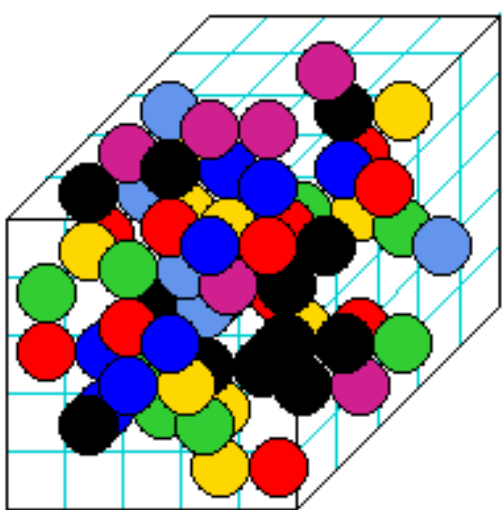

$3 d$ isometric viewed from below

Figure 21.9: An example of a 3d bit/value seed as it appears with the prompt in section 21.5. The seed is shown a $2 d$ version of $3 d$, and a $3 d$ isometric. Drawing bits/value applies only to the $2 d$ version - but both $2 \mathrm{~d}$ and $3 \mathrm{~d}$ can be rescaled independently. This figure is bitmap graphics, not vector PostScript, but the $2 \mathrm{~d}$ and $3 \mathrm{~d}$ images can be captured separately in vector PostScript; for $3 \mathrm{~d}$ in section 21.4.10 (as in figure 32.18 Left); for $2 \mathrm{~d}$ in sections 21.4 .7 or 21.4.10. $v=8, n=5 \times 5 \times 5$ (see also figure 21.3). 


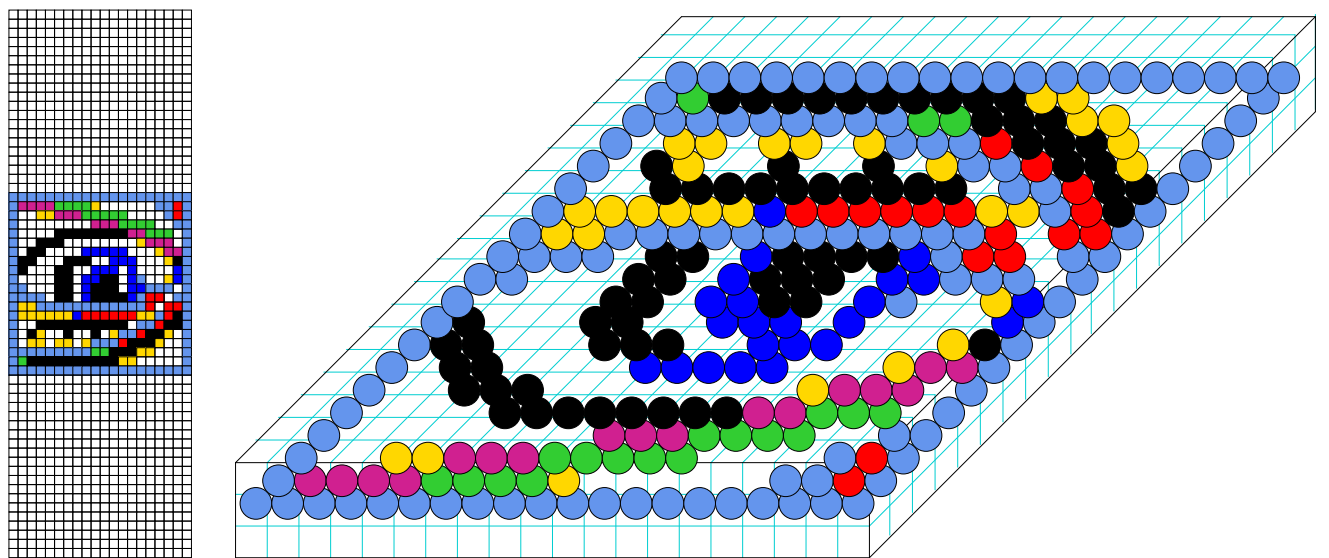

Figure 21.10: The $v=82 \mathrm{~d} 20 \times 20$ "eye" in figure 21.6 was loaded into a $3 \mathrm{~d}$ seed $20 \times 20 \times 3$, then flipped upside-down with $\mathbf{V}$. The result is shown as it appears simultaneously in the $2 \mathrm{~d}$ version of $3 \mathrm{~d}$, and in the $3 \mathrm{~d}$ isometric.

\subsection{Setting the seed in hex}

If $\mathbf{h}$ is selected in section 21.1, the seed is defined in hexadecimal (hex) which can be useful for creating repetitive patterns. The method is the same as setting a rule in hex in section 16.5. The hex expression of the current seed (initially just 0s) is displayed. Each hex character $(0-9$ then $\mathrm{a}-\mathrm{f})$ shows the value of $4 \mathrm{bits}$, and the characters are displayed in one byte pairs. During the hex setting procedure, a top-right reminder window displays the following,

enter hex, arrows-move hex count $=14 \leftarrow$ inset shows the current hex position, from the top-left rotate-l/r, accept-ret

The hex character to be updated is highlighted by a flashing cursor, initially in the top-left. Its position is displayed in the top-right inset window. The flashing cursor is moved with the arrow keys, left/right and up/down for more than one hex line. To overwrite, enter a hex character from the keyboard, without return. This automatically moves the cursor one position to the right. Hex characters entered which exceed the current value-range will be automatically corrected downwards to the maximum value after the hex string has been accepted. Enter $\mathbf{l}$ or $\mathbf{r}$ to rotate the underlying seed by one cell as in sections 21.3 and 21.4, which will be immediately reflected in the hex presentation. To accept the hex seed enter return, whereupon the seed will be presented as bits/values displayed in $1 \mathrm{~d}, 2 \mathrm{~d}$ or $2 \mathrm{~d}+3 \mathrm{~d}$ (section 21.4 ), where it can be reconfirmed or amended.

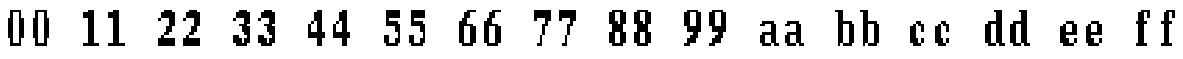

Figure 21.11: In this example $(v=8, n=42)$ the seed was first set in hex from the possible hex characters $(0-9$ and $a-f)$. The figure shows the result once accepted, where the bits/values option (section 21.4) activates below the hex presentation, allowing the seed to be reconfirmed or amended. 


\subsection{Setting the seed in decimal}

applicable only for a limited range of $v$ and system size $n$

The decimal option remains valid as long as the decimal equivalent of the bit/value string representing the seed is within the limits ${ }^{8}$ in table 21.1.

\begin{tabular}{c|c|c}
$v$ & max- $n$ & max decimal \\
\hline 2 & 32 & 4294967295 \\
\hline 3 & 20 & 3486784400 \\
\hline 4 & 16 & 4294967295 \\
\hline 5 & 13 & 1220703124 \\
\hline 6 & 12 & 2176782335 \\
\hline 7 & 11 & 1977326742 \\
\hline 8 & 10 & 1073741823
\end{tabular}

Table 21.1: Seeds in decimal - network size limitations - the maximum size of a seed $n$ for each value-range $v$, and the corresponding maximum decimal number.

If $\mathbf{d}$ is an available option and is selected in the seed prompt (section 21.1), the seed can be specified by its decimal equivalent. The following prompt is displayed,

decimal seed, enter 0-4782968 (def-rnd-dec): (example for $n=14, v=3$ )

Enter a decimal number, or enter return for a random number within the permitted range, which will be displayed. If the number entered is outside the permitted range, the program presents an error message, for example,

decimal seed, enter 0-4782968 (def-rnd):5555555 - too big! cont-ret:

Enter return to revert to the seed prompt. Once successfully selected, the decimal seed is presented again as bits/values (section 16.4), where it can be reconfirmed or amended.

\subsection{Loading a seed}

Enter $\mathbf{l}$ in section 21.1, or $\mathbf{F}$ followed by $\mathbf{l}$ in section 21.4 .7 to load a seed (.eed file) ${ }^{9}$. After loading (from the top-right filing prompt, section 35.2) DDLab will know the file parameters: value-range $v$, dimensions $1 \mathrm{~d}, 2 \mathrm{~d}$, or $3 \mathrm{~d}$, and the size of the coordinates $[i, j, h]$. File-seeds that differ from the base-seed in any of these parameters can still be loaded, but a top-right window warns of different $v$, or prompts to position a small file-seed (of any dimension) that fits within a larger base-seed. A bigger file-seed dimension is reduced to the base-seed dimension. If any file-coordinate $[i, j, h]$ is bigger than the corresponding base-coordinate, a part of the file is loaded automatically without further prompts. section 21.7 .1 below lists these constraints in more detail.

\footnotetext{
${ }^{8}$ The same limits apply when setting a rule in decimal (table 16.1 section 16.6).

${ }^{9}$ Saving a seed was described in sections 21.4.7 and 21.8 - saving just part of a seed, a patch, in section 21.4.8.
} 


\subsubsection{Constraints for loading a seed}

all parameters equal ... if all file-parameters are the same as all base-parameters, the file is loaded without further ado.

value-range $v \ldots$ if file- $v$ is different from base- $v$, the values loaded will be adapted to correspond to base- $v$. The following top-right warning is presented, for example)

\section{file-v(2) != base-v(8), abandon-q, load anyway-ret:}

Any file- $v>$ base- $v$ will be changed to the maximum base- $v$. Note that base colors will conform to the base color key (section 7.1).

file-dim < base-dim ... a smaller file-seed dimension is increases to the base-seed dimension, by adding coordinates of size 1 . For file-2d, $h$ is set to 1 for base-3d. For file- $1 \mathrm{~d}, j$ is set to 1 for base- $2 \mathrm{~d}$, and $[j, h]$ is set to $[1,1]$ for base-3d.

file-dim > base-dim ... a bigger file-seed dimension is reduced to the base-seed dimension. For file-3d, its $2 \mathrm{~d}$ version will load correctly if the base- $2 \mathrm{~d}$ has the corresponding $[i, j]$ coordinates. Likewise a file- $2 \mathrm{~d}$ or file-3d will appear correctly in a 1d-base shown in $2 \mathrm{~d}$ with corresponding $[i, j]$ coordinates.

file- $i, j, h \leq$ base- $i, j, h \ldots$ if file- $[i, j, h]$ fits inside base- $[i, j, h]$, then if any file-axis is smaller than the base-axis, a prompt is presented in a top-right window to locate the file-seed within the base ${ }^{10}$, for example,

example for a $1 d$ network

1d: $i=100$, file size $=20$, enter start pos

(def 40, max 80, rnd-r):

example for a $2 d$ network

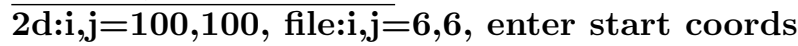

(def 47,47, $\max 94,94$, rnd-r) i: $\quad$ j:

example for a $3 d$ network

3d:i,j,h=40,40,40, file:i,j, $h=15,15,15$, enter start coords

(def 12,12,12 max 25,25,25, rnd-r) i: j: h:

Enter the coordinates in the base-seed to locate the file-seed's lower right cell, return for the default central position, or $\mathbf{r}$ for a random coordinate. Any number of file seeds can be loaded.

file- $i, j, h>$ base- $i, j, h \ldots$ for a $2 \mathrm{~d}$ or $3 \mathrm{~d}$ base, if any file-coordinate $[i, j, h]$ is bigger than the corresponding base-coordinate, the larger coordinate is automatically centered on the smaller base - any smaller axis starts at index 0 . For a $1 \mathrm{~d}$ base the file-seed starts at index 0 .

\footnotetext{
${ }^{10}$ This is similar to loading a sub-network in section 19.4.3.
} 


\subsection{Saving a seed}

Once the seed has been accepted, a top-right window shows the density of non-zero bits/values (section 21.3.1) and gives another chance to revise or save the seed,

density $3-1 \mathrm{~s}=\mathbf{3 0 3} / \mathbf{4 0 0}=\mathbf{7 5 . 7 5 0 \%}$, bias $=\mathbf{7 5 . 0 0 0 \%}$ (example for $v=4$ )

SEED: revise-q save-s cont-ret

Enter $\mathbf{q}$ to revise, or $\mathbf{s}$ to save the seed (as a .eed file ${ }^{11}$ ) from the top-right filing prompt (section 35.2). If just return is entered, the seed will be saved automatically with the default filename mysee_v $v$.eed where $v$ is the value-range. The seed could also have been saved from the reminder window when setting bits/values (section 21.4, enter $\mathbf{F}$ followed by $\mathbf{s}$ ).

\subsection{Seed file encoding}

The .eed seed file is encoded ${ }^{12}$ starting with 5 leading bytes as follows, where $n$ is the network size, $(i, j)$ the axes of a $2 \mathrm{~d}$ network, and $(i, j, h)$ the axes of a $3 \mathrm{~d}$ network,

byte $0 \ldots$ value-range $v$.

byte $1,2 \ldots$ network size, $n$.

byte $3,4 \ldots[i, j]$ which gives the dimensions and axis sizes, If $i=0$ and $j=0$ the seed is $1 \mathrm{~d}$. If both $i>1$ and $j>1$ the seed can be either $2 \mathrm{~d}$ or $3 \mathrm{~d}$. $h=n /(i \times j)$. If $h>1$ the seed is $3 \mathrm{~d}$, otherwise $2 \mathrm{~d}$.

The rest of the file consists of the seed values from 0 to $n-1$, where the number of bits required for each cell $\left(v_{b i t s}\right)$ is as follows:

$$
\begin{aligned}
& v=2 \ldots 1 \text { bit }\left(v_{\text {bits }}=1\right) \\
& v=3 \text { or } 4 \ldots 2 \text { bits }\left(v_{b i t s}=2\right) \\
& v=5,6,7,8 \ldots 3 \text { bits }\left(v_{b i t s}=3\right)
\end{aligned}
$$

A seed encoding requires 5 leading bytes plus the bytes for the seed itself $R$, where $R=\left\lceil\frac{n \times v_{b i t s}}{8}\right\rceil$ bytes, the upper absolute value, minimum 1 byte.

\footnotetext{
${ }^{11}$ Seed files saved in this multi-value version of DDLab are not compatible with the old binary version of DDLab, and vice versa.

${ }^{12}$ The seed encoding can be compared with single rule encoding (section 16.16) and wiring+rulemix encoding (section 19.3).
} 


\section{Chapter 22}

\section{The Derrida plot}

Once the rule or rules have been set, the Derrida plot[8] (and Derrida coefficient) can be selected from the wiring graphic in section 17.4 - enter Derrida-D. Any type of network may be plotted, a CA, RBN or DDN, in $1 \mathrm{~d}, 2 \mathrm{~d}$, or $3 \mathrm{~d}$, in normal and TFO-mode.

The main application of the Derrida plot has been as an order-chaos measure for large random Boolean networks in the context of models of genetic regulatory networks[11, 15], where the canalyzing inputs can be tuned to move the dynamics between order and chaos (section 15). The Derrida plot provides a statistical measure of the divergence/convergence of network dynamics in terms of "Hamming distance" $(H)$. $H$ between two binary states of equal size, $n$, is the number of bits that differ. The normalized Hamming distance is $H / n$. For multi-value, $H$ is the number of values that differ, but exactly the same principles apply.

The Derrida plot is generated as follows

1. Randomly select a pair of initial states, $I_{1}, I_{2}$, separated by a small Hamming distance of $H_{0}$, at time-step $t_{0}$.

2. Iterate each state forward independently, according to the network architecture, by usually one, or more time-steps, $i$ ( $i$ must be 1 to compute the Derrida coefficient).

3. Measure the Hamming distance, $H_{i}$, at time-step $i$, between the pair of final states, $F_{1}, F_{2}$.

4. Repeat the above for a sample of pairs of initial states with the same initial Hamming distance $H_{0}$, and plot normalized $H_{0}$ (x-axis) against the mean normalized value of the $H_{i}$ 's, $(y$-axis).

5. Repeat the whole procedure for a range of increasing initial Hamming distances. The increase (Ham steps) must be 1 to compute the Derrida coefficient, but may be set to larger intervals otherwise.

\subsection{Selecting the Derrida plot}

To select the Derrida plot, first display the network as a wiring graphic after rules are set (section 17.1). One of the top-right wiring graphic options (section 17.4) is the following,

... Derrida plot-D: (enter $\mathbf{D}$ to select) 

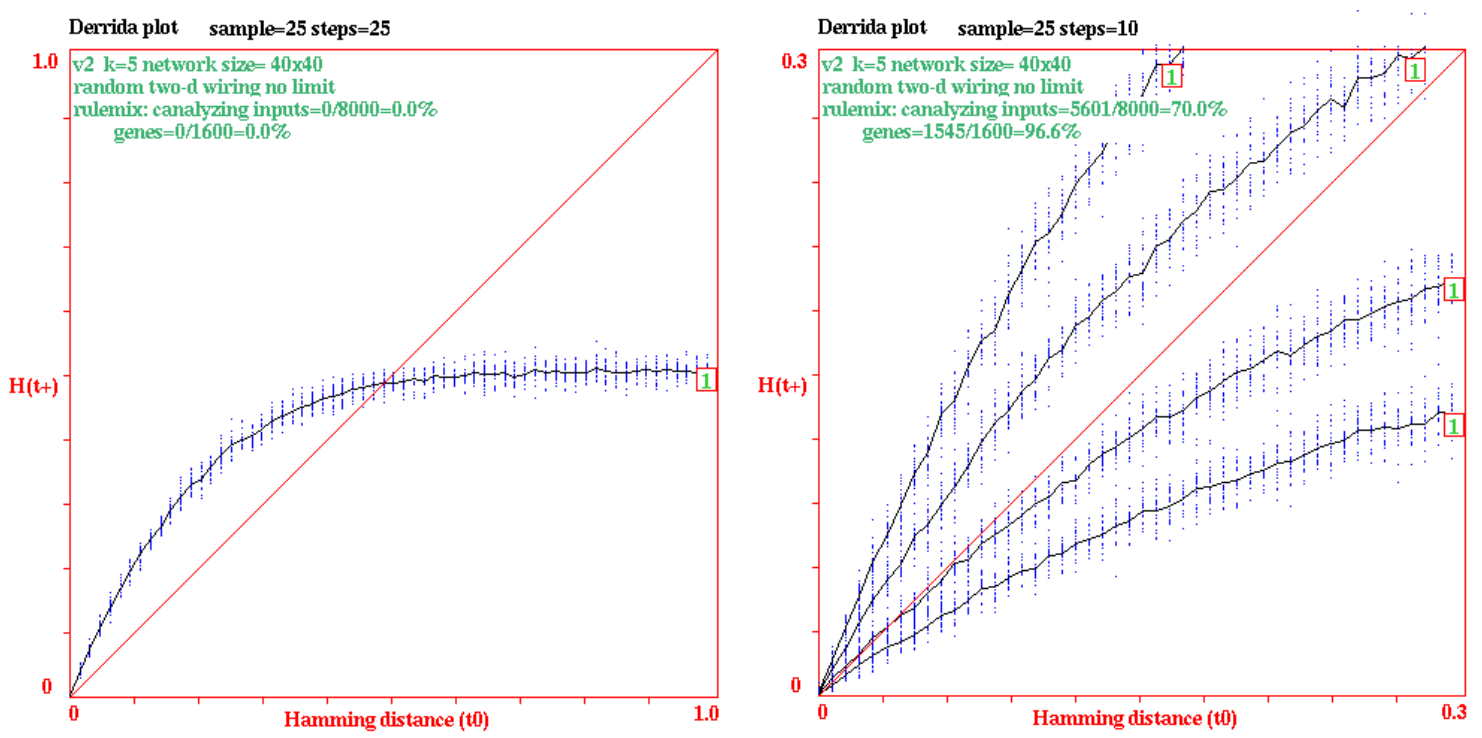

Figure 22.1: Examples of Derrida plots for a $2 d$ random Boolean network, $k=5, n=40 \times 40$. Data about the network are shown in the top-left hand corner of the graph (section 22.3), and Derrida data above the top (sample and steps). The Derrida coefficient (section 22.6) would also be shown, but is not in this case because steps $>1$. The number of iterations is shown at the end of each curve.

Left: $\operatorname{Max} H=1$, showing the full range of Hamming distance, sample $=25$, steps $=25$.

Right: 4 superimposed plots for increasing canalyzing settings (0\%, 20\%,50\% and $70 \%)$, giving progressivly lower slopes. $\mathrm{MaxH}=0.3$, so zooming in for the most significant first part of the graph, sample $=25$, steps $=10$. Canalyzing can be changed before a new plot, and previous plots kept.

\subsection{Derrida plot options}

If $\mathbf{D}$ is selected in section 22.1, the following series of prompts are presented in turn in a top-right window to change the Derrida plot parameters or accept the defaults,

for a $40 \times 40 R B N$, values shown are examples

Derrida plot: quit-q, Dc sample (now 15, max 320):

maxH (def 1.000): sample (def 25) tog spread (ON)-s: details-d

iterations (def 1): Ham steps $(\operatorname{def} 1$ net=1600): keep-k:

Revised parameters generally become the new defaults. The parameters are described in section 22.2.1 below. 


\subsubsection{Derrida plot parameters}

The meaning of the parameters in the Derrida plot options (section 22.2) are given below, where values in brackets are initial defaults or examples.

$$
\frac{\text { parameters }}{\text { quit-q }} \cdots \frac{\text { what they mean }}{\text { quit the Derrida plot. }}
$$

Dc sample (now 15, max 320): ... the number of initial points that will form the basis of the Derrida coefficient, derived from the initial slope (section 22.6). The initial default depends on the size of the network, but is 15 for large networks.. Generally, between 10 and 20 points are appropriate. Enter return to accept the default or enter a new value which becomes the new default.

maxH (def 1.00) ... the maximum normalized Hamming distance of the plot defining the extent of the $x y$ axis (maximum 1). A small $\max H$ gives a detail of just the left hand corner of the plot, which is perhaps the most significant. Only a small initial part of the plot is required to calculate the Derrida coefficient, enough to contain the Dc sample (section 22.6). Enter return to accept the default (initially 1) or enter a new decimal value $(0<\max H \leq 1)$ which becomes the new default.

sample (def 25) ... the sample size for each initial Hamming distance. A smaller sample gives a quicker plot. A larger sample provides a more accurate measure. Enter return to accept the default (initially 25) or enter a new value which becomes the new default.

tog spread (ON)-s: or (OFF) ... to toggle plotting the spread of each sample. Enter return to accept the default (initially $(\mathbf{O N})$ ) or enter $\mathbf{s}$ to toggle this becomes the new default.

details-d ... enter $\mathbf{d}$ to pause at each sample pair and show detailed data in a top-right window (mainly for debugging and diagnostic purposes), for example,

$$
\text { sample }=\mathbf{4} \mathbf{H}(\mathbf{t 0})=\mathbf{2 1} \mathbf{H}(\mathbf{t} 1)=\mathbf{1 6} \text { q-quit: (for example) }
$$

In this example, at the 4th sample point, the initial Hamming distance is $H_{t 0}=21$ (not normalized), the final Hamming distance, is $H_{t 1}=16$, at time-step 1 . For system size $n \leq 40$ the bit/value pattern of the initial and final pairs of states are also shown above the prompt. Enter return at the prompt above for the next sample pair, or $\mathbf{q}$ to disable the pause and continue without interruption.

iterations (def 1) ... the number of forward time-steps required. Enter return to accept the default (initially 1) or enter a new value which becomes the new default. Iterations must be 1 to compute the Derrida coefficient.

Ham steps (def 1 net=1600) ... the interval between successive initial Hamming distances (not normalized). The minimum and default is 1. A small interval provides a more accurate plot. A larger interval provides a quick and sketchy plot. Enter return to accept the default (initially 1 ) or enter a new value which becomes the new default. The system size $n$ is 
shown as a reminder as it will be subdivided by the number of Ham steps. Ham steps must be 1 to compute the Derrida coefficient.

keep-k ... enter $\mathbf{k}$ to keep a previous plot and superimpose a new plot, as in figure 22.1 Right. The new plot may have revised network parameters.

\subsection{Data within the Derrida plot}

Within the Derrida plot, some data is shown relating to both the network, and to Derrida parameters, as in figures 22.1 and 22.2, described below.

\subsubsection{Network data}

The top-left hand corner of the graph will show context dependent information as follows.

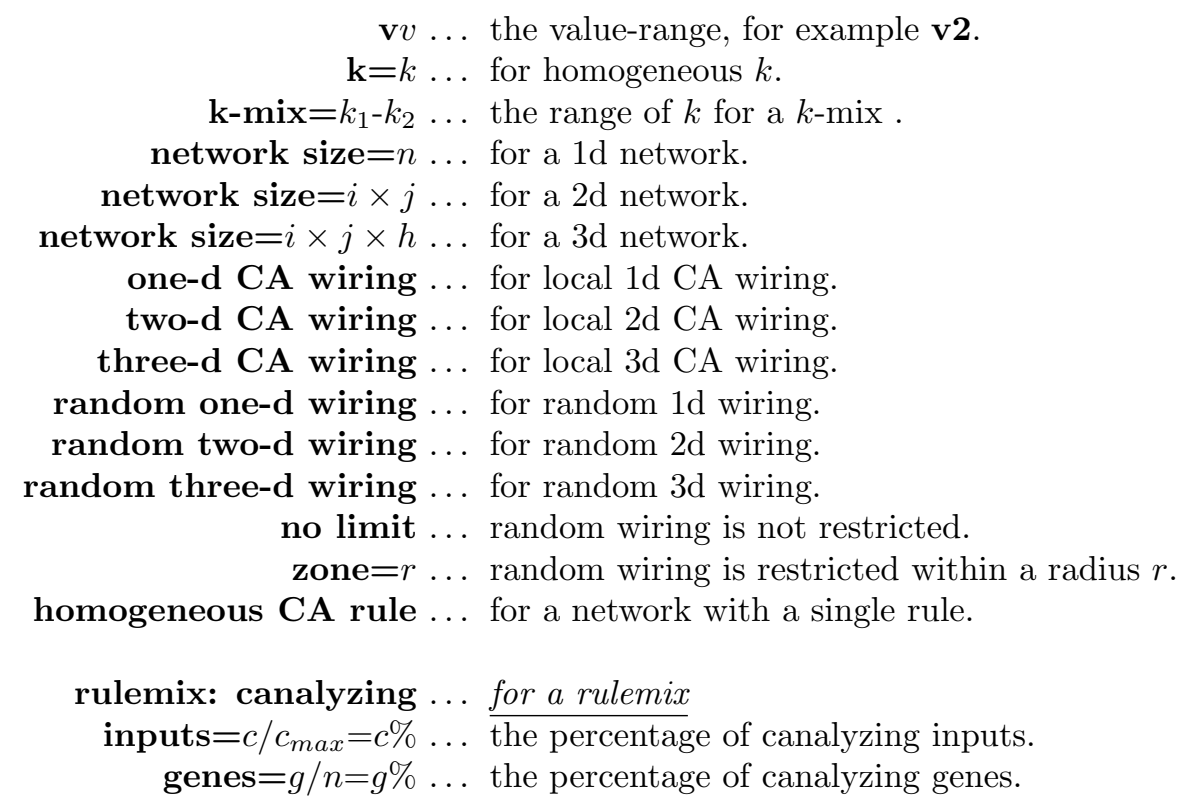

\subsubsection{Derrida data}

Some Derrida data is shown at the top of the graph, for example,

$$
\text { Derrida plot } \quad \text { sample }=55 \text { slope }=46.4 \mathrm{deg} \mathrm{Dc}=0.072(15 \mathrm{H})
$$

sample is explained in section 22.2.1. slope, Dc and $(\mathbf{1 5} \mathbf{H})$ relate to the Derrida coefficient, described in section 22.6, and appear only if iterations=1 and Ham steps=1 in section 22.2. The number or iterations is shown at the end of each curve, in a small box. 


\subsection{Interrupting the Derrida plot}

While the Derrida plot is in progress, the following top-right message is displayed,

plotting Derrida, sample $=25$, iteration $=1$ step $=1$

quit/pause-q (values shown are examples)

If the plot is interrupted with $\mathbf{q}$, the same parameters as in section 22.2 (except Dc sample and $\operatorname{maxH}$ ) can be changed in mid-plot. The following series of prompts are presented in turn in a top-right window,

interrupted at Hamm $=0.297$, quit now-q, cont-ret:

sample (now 25): $\quad$ tog $\operatorname{spread}(\mathrm{ON})$ : details-d (values shown are examples)

iterations (now 1): Ham steps $($ now $=1$ net $=1600)$ : keep-k:

Enter $\mathbf{q}$ to stop the plot at the point reached, return to continue the plot, or revise parameters before continuing. Note that changing iterations will have drastic effects.

\subsection{Completing the Derrida plot}

When the Derrida plot is complete, or if interrupted and stopped in section 22.4, the number of iterations is displayed at the end of the curve, and the following top-right prompt appears,

\section{Derrida plot complete reset-r canalyzing-C:}

Enter $\mathbf{r}$ to reset the plot. The options in section 22.2 are presented, but with an additional option redraw (no spread)-r - for a quick redraw of the plot from memory without the sample spread. Enter $\mathbf{C}$ in section to revise the network's canalyzing settings, described in chapter 15 for a rule-mix, and section 18.6. for a single rule network. Previous plots can be retained (enter $\mathbf{k}$ in section 22.2) to produce a multiple plot.

\subsection{The Derrida coefficient}

The Derrida coefficient $(D c)$, analogous to the Liapunov exponent in continuous dynamical systems, is derived from the initial slope of the Derrida plot, a tangent to the curve at the origin, which indicates whether the network dynamics is convergent (ordered) or divergent (chaotic) and to what extent.

A slope of $45^{\circ}(D c=0)$ indicates the dynamics is balanced between order and chaos. A slope above the $45^{\circ}$ diagonal (positive $D c$ ) is in the chaotic regime and correlates with increasing chaos. A slope below $45^{\circ}$ (negative $D c$ ) is in the ordered regime and correlates with increasing order. The initial slope is based on the first few points of the Derrida plot. The Derrida coefficient is only calculated if iterations $=\mathbf{1}$ and Ham $\mathbf{s t e p s}=\mathbf{1}$ in section 22.2. 

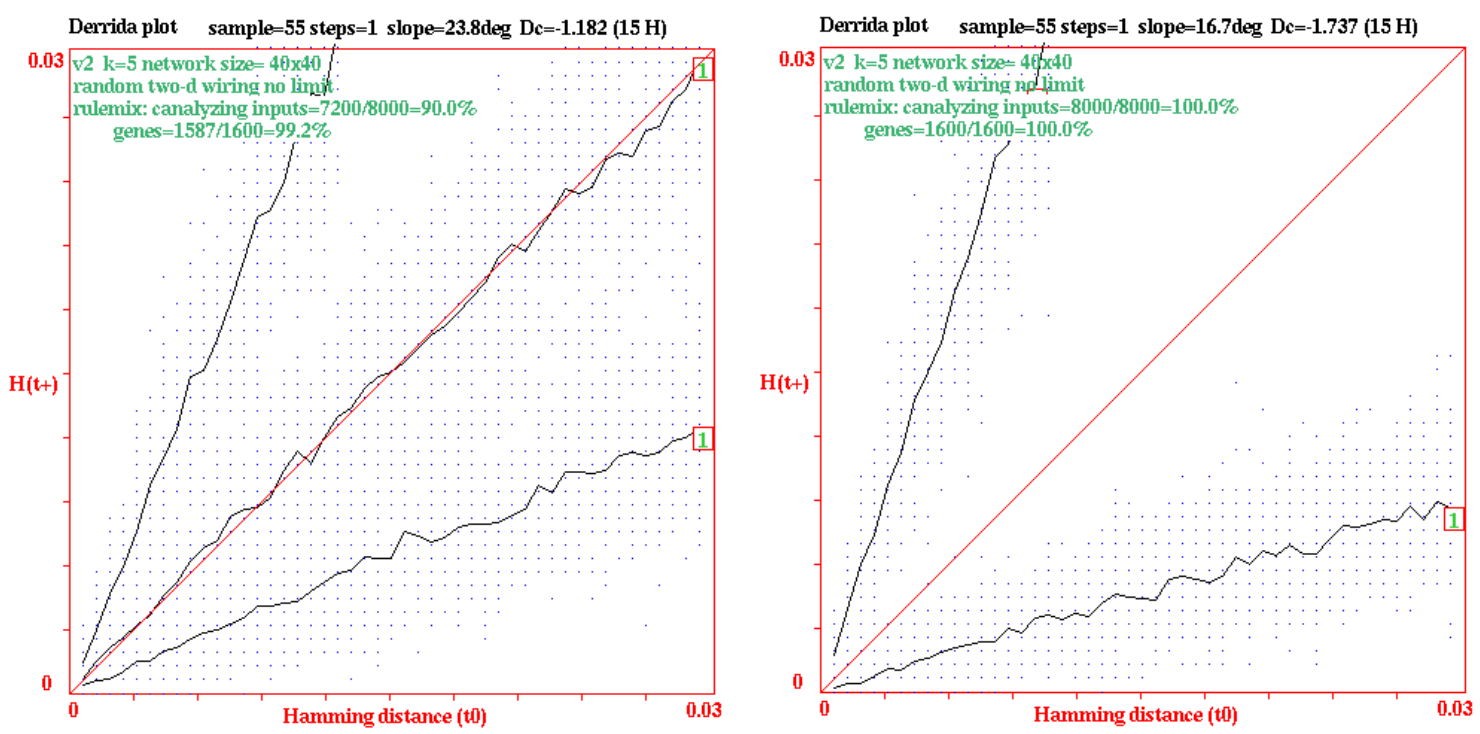

Figure 22.2: Examples of Derrida plots and their Derrida coefficients $(D c)$ for a $2 \mathrm{~d}$ RBN, $k=5$, $n=40 \times 40$ with various canalyzing, settings $C \%$, changed in section 22.5 for a range of behavior. Other parameters were as follows: $\mathrm{Dc}$ sample $=15, \mathrm{MaxH}=0.03$, sample $=55$, iterations $=1$, steps $=1$. The average slope, $\delta^{\circ}$, and the Derrida coefficient, $D c$, for the plots are given below.

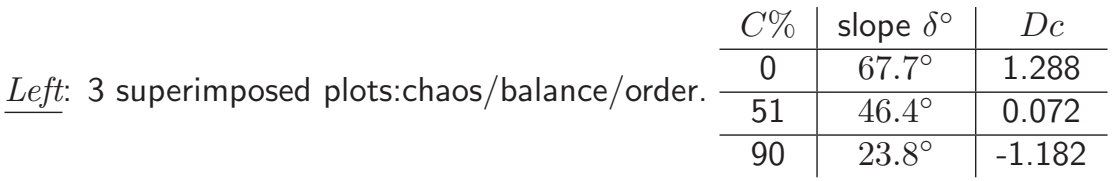

Right: 2 superimposed plots:extreme chaos (chain-rules) and order. \begin{tabular}{c|c|c}
$C \%$ & slope $\delta^{\circ}$ & $D c$ \\
\hline 0 & $71.4^{\circ}$ & 1.572 \\
\hline 100 & $16.7^{\circ}$ & -1.737
\end{tabular}

The number of points to determine the slope at the origin (the $D c$ sample) is selected to lie approximately on a straight line - the default is 15 for large networks. The average slope between these points and the origin is taken as the initial slope. If the initial slope $=\delta^{\circ}$, then the Derrida coefficient, $D c=\log _{2}\left(\tan \left(\delta^{\circ}\right)\right)$.

Figure 22.2 gives examples for a $k 5 \mathrm{RBN}$, with varying degrees of canalyzing (section 15 ) to demonstrate a range of behavior. 


\title{
Chapter 23
}

\section{Graphic conventions for attractor basins}

\author{
not in TFO-mode
}

Figure 23.1 and section 23.1 explain the idea of basins of attraction in discrete dynamical networks. Attractor basins are represented by state transition graphs, where nodes - network states, are linked by directed edges - state transitions. States in deterministic networks have one successor but possibly a number of predecessors (pre-images), so trajectories occur within trees. In a finite network a trajectory must encounter a repeat state, defining its attractor. Attractor basins are thus made up of transient trees rooted on attractor cycles.

This chapter describes the graphic conventions for drawing the state transition graphs of attractor basins and subtrees, illustrated in figures $23.2-23.5$, and in other figures in the manual.

\subsection{Basins of Attraction - the idea}

Given an invariant network architecture and the absence of noise, the dynamics on a discrete dynamical network is deterministic, and follows a unique trajectory from any initial state. When a state that occurred previously is re-visited, which must happen in a finite state-space, the dynamics become trapped in a perpetual cycle of repetitions defining the attractor (state cycle) and its period (minimum one, a stable point). The approach is analogous to Poincaré's "phase portrait" in continuous dynamics.

These systems are dissipative. A state may have multiple "pre-images" (predecessors), or none, but just one successor. The number of pre-images is the state's "in-degree". In-degrees greater than one require that transient states exist outside the attractor. Tracing connections backwards to successive pre-images of transient states reveals a tree-like topology where the "leaves" are states without pre-images, known as garden-of-Eden states. Conversely, the flow in state-space is convergent. The set of transient trees and the attractor on which they are rooted make up the basin of attraction. Local dynamics connects state-space into a number of basins, the basin of attraction field, representing the system's global dynamics. 


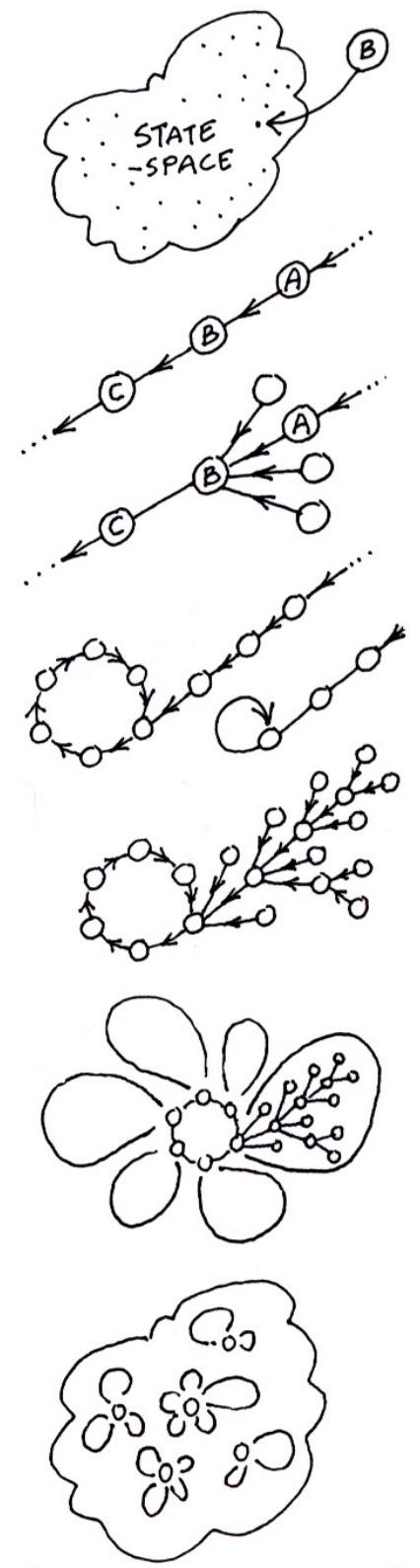

For a binary network size $n$, an example of one of its states $B$ might be $1010 \ldots 0110$. State-space is made up of all $S=2^{n}$ states $\left(S=v^{n}\right.$ for multi-value) - the space of all possible bitstrings or patterns.

Part of a trajectory in state-space, where $C$ is a successor of $B$, and $A$ is a pre-image of $B$, according to the dynamics of the network.

The state $B$ may have other pre-images besides $A$, the total number is the in-degree. The pre-image states may have their own pre-images or none. States without pre-images are known as garden-of-Eden states.

Any trajectory must sooner or later encounter a state that occurred previously - it has entered an attractor cycle. The trajectory leading to the attractor is a transient. The period of the attractor is the number of states in its cycle, which may be just one - a point attractor.

Take a state on the attractor, find its pre-images (excluding the preimage on the attractor). Now find the pre-images of each pre-image, and so on, until all garden-of-Eden states are reached. The graph of linked states is a transient tree rooted on the attractor state. Part of the transient tree is a subtree defined by its root.

Construct each transient tree (if any) from each attractor state. The complete graph is the basin of attraction. Some basins of attraction have no transient trees, just the bare "attractor".

Now find every attractor cycle in state-space and construct its basin of attraction. This is the basin of attraction field containing all $2^{n}$ states in state-space, but now linked according to the dynamics of the network. Each discrete dynamical network imposes a particular basin of attraction field on state-space.

Figure 23.1: The idea of basins of attraction in discrete dynamical networks (section 23.1).

\subsection{Network states, nodes}

Network states in attractor basins are usually represented by circular nodes, but may also be shown as a bit/value pattern in $1 \mathrm{~d}$ or $2 \mathrm{~d}$, or the decimal or hex value of the state, or they may not be shown (section 26.2). 
○
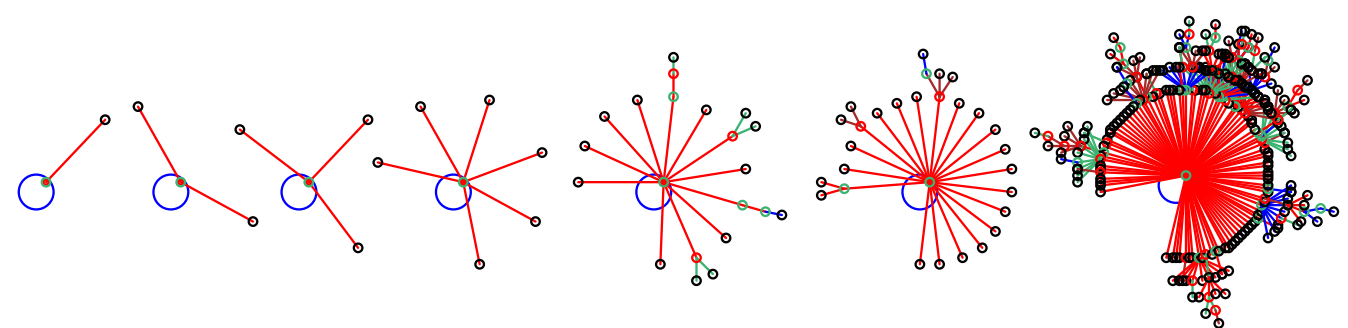

Figure 23.2: A point attractor (period=1) is represented as a node cycling to itself. The single node and the center line of the pre-image fan (level 1 ) are set at a default angle of $45^{\circ}$. The examples above show point attractors with increasing fan size, from 0 upwards (from $v 2 k 3$ rcode(dec) $77, n=12$ ).

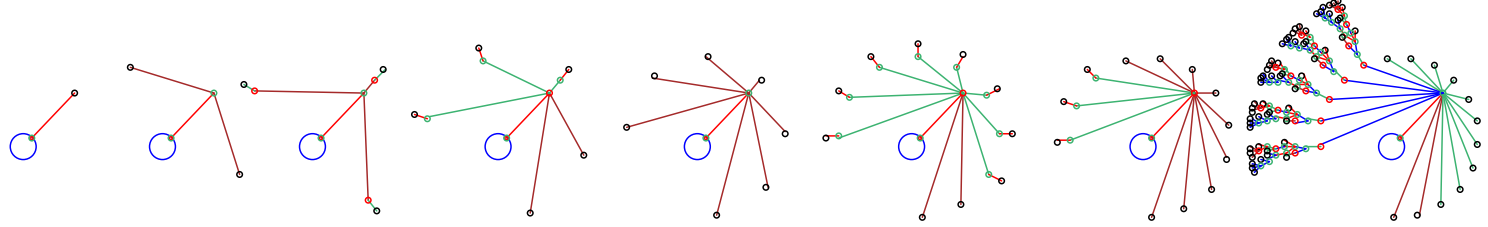

Figure 23.3: The pre-image fan (level 2) of a single pre-image (level 1) of a point attractor. The examples show increasing fan sizes, from 0 upwards (from $v 2 k 3 \operatorname{rcode}(\mathrm{dec}) 110, n=(1-3)$ and $n=(6-10))$. In this case both the point attractor and its pre-image are uniform states (all0s and all1s) so the pre-image fan and attached trees are organized into equivalent groups (section 23.4.2).
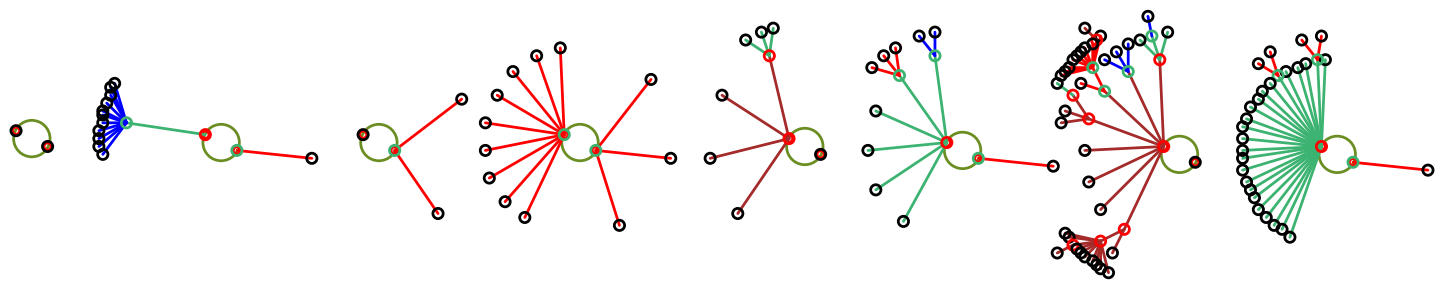

Figure 23.4: Period 2 attractors which cycle to each other. The two nodes are set at a default angle of $-15^{\circ}$. Varying sizes of the pre-image fan to both attractor states are shown, from 0 upwards. A fan at level 1 is slightly angled to indicate that the direction of time is clockwise in the attractor - the center lines of subsequent fans are radial to the center of the attractor cycle (taken from rcode $v 2 k 3$ $\operatorname{rcode}(\mathrm{dec}) 33, n=13)$.

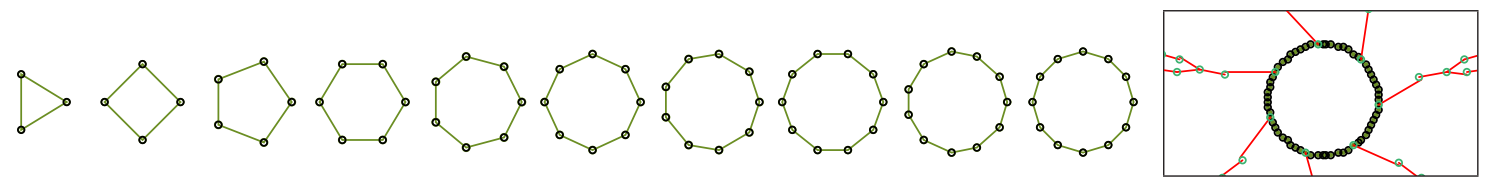

Figure 23.5: Attractors with periods $\geq 3$ are represented by polygons whose diameter approaches an upper limit with increasing period. The first 10 examples from the left have periods of 3 to 12 (from rcode (dec) $248, n=(3-12))$. The framed example on the right is a fragment of a basin from $v 2 k 3$ rcode(dec) $30, n=14$, with period 63 . A single edge or a fan at level 1 is slightly angled to indicate that the direction of time is clockwise in the attractor - the center lines of subsequent fans are radial to the center of the attractor cycle - see figure 2.5 for a clearer example. 


\subsection{Attractor cycles}

Where three or more states make up an attractor cycle, this is represented as a regular polygon with nodes at the vertices (figure 23.5). The direction of time is clockwise. The "last" vertex, from which the first subtree is generated, is shown due east, so the first vertex at which the cycle is entered is one node clockwise from due east. A point attractor is shown as a node (positioned north-east) on a circle, indicating that the node cycles to itself (figure 23.2). A two state attractor is shown as two nodes on a circle (positioned south-east and north-west), where the south-east node is the "last" node. The diameter of attractor cycles increases asymptotically with system size $n$, up to the maximum selected, which also determines the scale of the entire basin.

\subsection{Transient trees}

The pre-images or predecessors (if any) of the "last" attractor state are computed first. The preimage fan angle is set according to the in-degree and increases asymptotically to a maximum value with increasing in-degree. In general the center line of the fan angle is radial to the attractor cycle center point, but for fans rooted on the attractor, the fan angle is tilted slightly to indicate that the direction of time around the cycle is clockwise (figure 2.5).

The pre-image fan is computed and drawn for each node at each successive level in the tree. The endpoints of the fan where the nodes are drawn are positioned on notional concentric circles around the attractor cycle corresponding to successive levels in the tree. The distance between each successive concentric circle, and thus between tree levels away from the root, decreases asymptotically for 90 levels, thereafter remains constant.

The tree will grow away from the attractor, backwards in time. Once the tree is complete with all its garden-of-Eden states reached, the next tree (anti-clockwise on the attractor) is computed. Note that with compression on (section 26.1), equivalent trees will be computed and drawn simultaneously).

\subsubsection{Transient tree colors}

A cycle of four colors is used to draw transition edges. The color scheme of the attractor basin depends on the start edge color which can be changed, resulting in four alternative color schemes for both edges and transition nodes (section 26.3.3).

The edges in the same pre-image fan are drawn in the same color (except for the uniform states - section 23.4 .2 below). If the attractor period is 5 or less, successive fans are assigned a different color so that a given transient tree may have a mix of colors. For attractor periods of 6 or more, all fans in the same tree are assigned the same color, and the color is changed for the next non-equivalent tree. Note that with compression on (section 26.1) equivalent trees will be colored identically. Conventions for bit pattern node colors are described in section 26.2.1. 


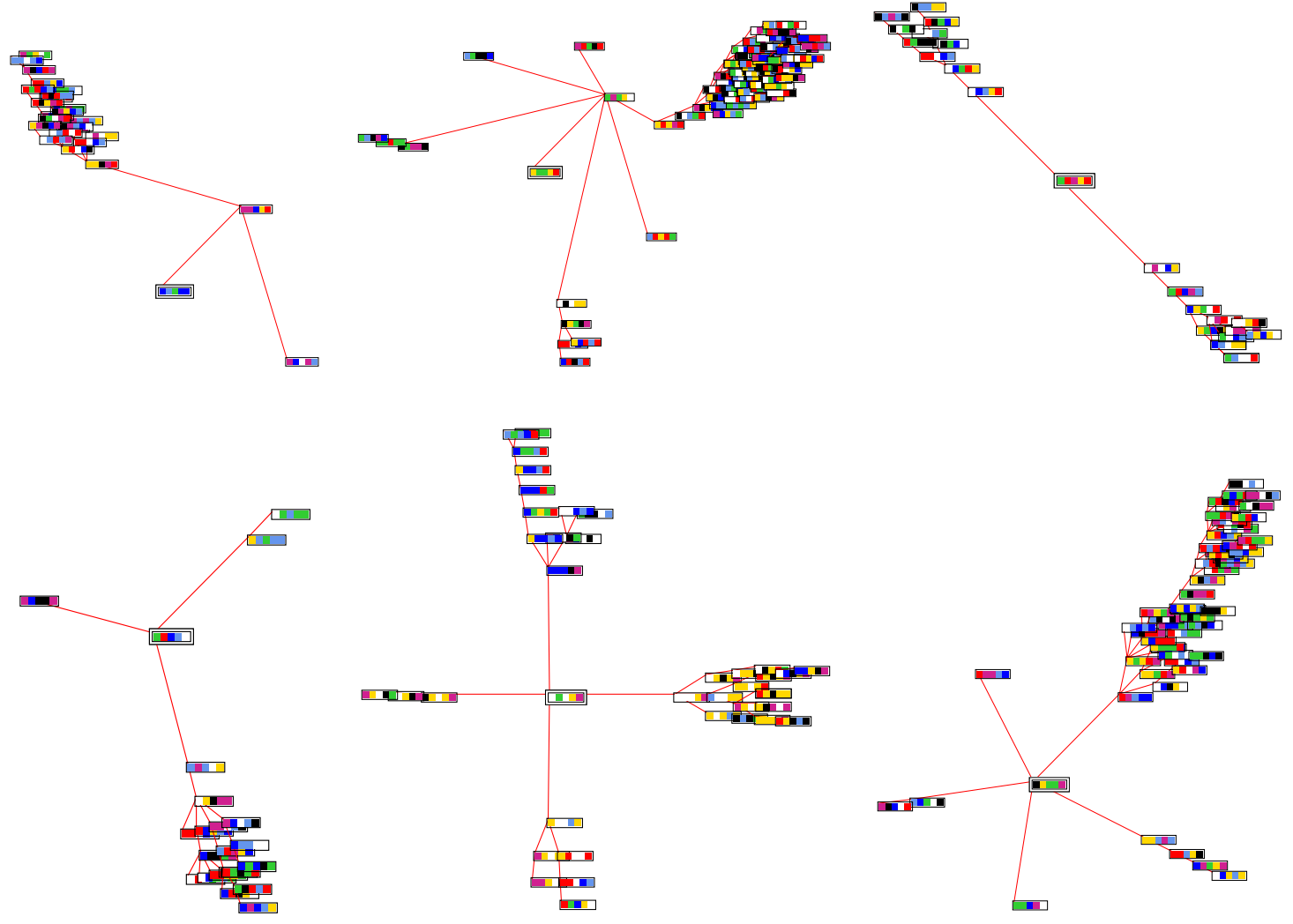

Figure 23.6: Examples of subtrees from root states with in-degrees (at level 1) of 1 to 5 . These examples are for $v 8 k 3 \mathrm{CA}, n=5$ for various rules, and random initial states, running forward by about 3 time-steps before running backward. States are shown as value patterns - the root state is highlighted by default inside a double outline. For in-degree 1 at level 1 (the two subtrees top-left) similar layout principles apply as in figure 23.3.

\subsubsection{Transient trees for uniform states} for $1 d$ and $2 d C A$

If CA compression is set, a special algorithm is employed to speed up computation of the subtree of the "uniform" states, where all cells have the same value. When uniform states are on the attractor, the attractor period cannot exceed $v$ ( 2 for binary). If a given state is a pre-image of a uniform state, then that state's rotation equivalents[25] must also be pre-images, and may be computed simultaneously by an appropriate rotational transformation.

The first level of a uniform state's tree is organized into groups of equivalents (shown in different colors). The subtree of each representative state in each group at this first level is computed in turn. Each subtree will be completed before the next is started. Successive pre-image fans in each non-equivalent subtree are assigned a different color. Equivalent subtrees will be computed and drawn simultaneously, and will be colored identically (figure 26.3). 


\subsubsection{Subtree only}

A subtree only may be selected, running backward from a given state, or from a state a specified number of time-steps forward from a given state. Running forward before running backward is usually necessary because most states have no pre-images - they are leaves, garden-or-Eden states. The first pre-image fan is evenly spread around a notional circle with a diameter equal to the current maximum attractor diameter. Successive pre-image fans are computed and drawn for each node at each successive level in the subtree.

If the subtree seed has just one pre-image, the pre-image fan at level 2 will be spread out as shown in figure 23.6 (the two subtrees top-left) following similar layout principles as figure 23.3. 


\section{Chapter 24}

\section{Output parameters for attractor basins}

not in TFO-mode.

There are many parameter options and sub-options relating to attractor basins. Each can be looked at in turn, but for convenience they are divided into seven categories to allow jumping directly to the category where options need to be changed from the current default settings. All options have defaults so the remainder can be skipped at any time - accept defaults-d.

The parameter categories (and relevant chapters) are,

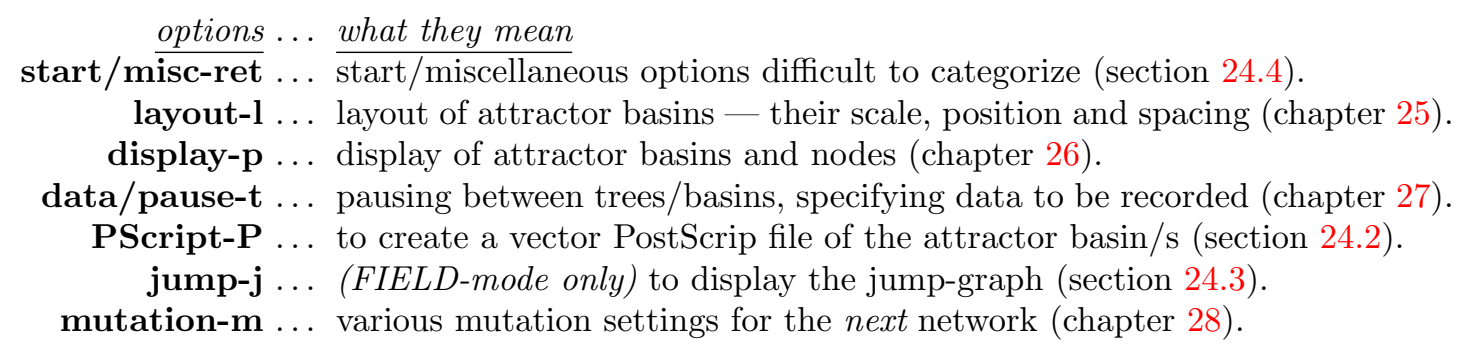

\subsection{The first output parameter prompt for attractor basins}

The first output parameter prompt is presented in a top-right window, and differs between SEED and FIELD-modes. At the same time basin parameters appears in a top-center window.

SEED-mode

accept all basin defaults-d, space-time patterns only-s

restore defaults: all-a, layout only-L

revise from: start/misc-ret layout-l display-p

FIELD-mode data/pause-t PScript-P mutation-m:

accept all basin defaults-d

restore defaults: all-a, layout only-L

revise from: start/misc-ret layout-l display-p

data/pause-t PScript-P jump-j mutation-m: 


\subsubsection{Output parameter prompt for attractor basins - options summary}

The meaning of the options in section 24.1 are summarized below, and described in detail in this chapter and chapters 25 to 28.

As soon as a "revise from" option is selected, the reminder accept defaults-d appears in a top-center window - enter $\mathbf{d}$ (at any time) to accept all remaining defaults, and skip further output parameter prompts.

options ... what they mean

accept all basin default-d ... to accept all current output parameter defaults, and skip further prompts. This can be done at any stage in the prompt sequence. New settings generally become the new defaults, but can be reset to the original. The next prompt for a basin of attraction field will be presented in section 29.4, or for a subtree or single basin in section 29.1.

space-time patterns only-s... (SEED-mode only) enter $\mathbf{s}$ to skip all attractor basin options and go directly to the output parameters for space-time patterns (chapter 31). Note that a final chance to select space-time patterns is also given in section 29.1.

restore defaults: ... to restore defaults to their original settings

all-a ... to restore all defaults .

layout only-L ... to restore just the layout defaults — described in chapter 25 .

revise from: ... jumping directly to an option category

At any point enter $\mathbf{q}$ (or $\mathbf{q}$ more than once) to backtrack to the first output parameter prompt (section 24.1).

start/misc-ret ... enter return to start miscellaneous (hard to categorize) options, presented in sequence in a top-right window (section 24.4).

layout-1 ... for a sequence of prompts to set the scale, position and spacing of attractor basins - described in chapter 25 .

display-p ... for a sequence of display prompts, the compression of equivalent basins and trees, types of nodes, orientation, and in-degree angle — described in chapter 26.

data/pause-t ... for a sequence of prompts for pausing attractor basins to re-adjust the layout on-the-fly, and to show/print data at various levels of detail - described in chapter 27.

PScript-P ... to create a vector PostScript file of the attractor basin (section 24.2).

jump-j ... (FIELD-mode only) to activate the jump-graph (section 24.3).

mutation-m ... for a sequence of prompts to set the type of mutation (to wiring or rules) to be applied to the next network generating the next attractor basin/s with the same parameters — described in chapter 28. 


\subsection{PostScript of attractor basins}

Enter PScript-P at the first output parameter prompt (section 24.1), or arrive there by viewing the output parameters in sequence, to create a vector PostScript file of the basin of attraction field, single basin, or subtree, or a range of the above. The following top-left prompt is presented,

if PostScript is currently OFF, the initial case

PScript: save basin to PostScript (now OFF): greyscale-P color-p:

if PostScript is currently ON, either greyscale-P or color-p

PScript: save basin to PostScript (now p): greyscale-P color-p cancel-0:

Enter $\mathbf{0}$ to deactivate, $\mathbf{P}$ or $\mathbf{p}$ to activate - a filename prompt will be presented (section 35.2). If saving to PostScript is active, a new file will be created and overwritten to the selected filename each time an attractor basin is drawn (default filename my_bPS.ps). To conserve the file, the selected filename should be renamed with utilities ${ }^{1}$ outside DDLab before a new attractor basin is generated. Examples of vector PostScript images of basins are to be found throughout this manual, for example in chapters 25 and 26. For PostScript files of jump-graph basins refer to section 20.7.1.

\subsection{Activate the jump-graph}

FIELD-mode only - see also section 20.3.1

Enter jump-j at the first output parameter prompt (section 24.1) to go directly to the jump-graph prompt, or arrive there by viewing the output parameters in sequence. The following prompt is presented,

\section{jump: attractor jump-graph $-\mathbf{j}$, no edges layout only $+\mathbf{L}$ :}

Enter $\mathbf{j}$ to show the jump-graph (with edges), or $\mathbf{j} \mathbf{L}$ for "layout only" — where the jump-graph methods are applied without edges for just laying out attractors in any arbitrary position.

If the genuine jump-graph (with edges) is selected, "compression" for CA will be turned off automatically, with the following message,

\section{... - compression OFF: (if compression on, enter return to continue)}

The jump-graph (section 20.3) represents the probabilities of jumping between basins due to single-bit or single-value perturbations to attractor states, and gives some insight into the stability and adaptability of the dynamics. An alternative use of the jump-graph is a method for just laying out the basins in a basin of attraction field in any arbitrary position. A complete description of the jump-graph and its functions is provided in chapter 20.

\footnotetext{
${ }^{1}$ For example, rename in a terminal, look at the PostScript file in "GhostView".
} 


\subsection{Miscellaneous (hard to categorize) options}

The rest of this chapter describes the first category of miscellaneous (hard to categorize) options,
options.... what they mean
state-space matrix ... an additional graphical method for representing states in attractor basins (section 24.5) which can also be activated/activated on-the-fly (section 30.3).

in-degree frequency ... the frequency of different in-degrees in a subtree, basin or field shown as a histogram (section 24.6).

show/count majority ... highlight and count states in basins or subtrees with a majority of a given value. For $v=2$ this provides an alternative measure of fitness in the "density classification problem" [7, 13] (section 24.7).

screensave demo ... (SEED-mode only) a continuous demo of single basins or subtrees for different or mutant rules (sections 4.11, 24.8).

$G$-density, $Z$ and $\lambda \ldots$ graphs of the density of garden-of-Eden (leaf) states against system size, and how various rule parameters are distributed in rule-space (section 24.9).

backwards space-time patterns ... to show space-time patterns being computed "backwards" as attractor basins are being drawn (section 24.10) which can also be activated/deactivated on-the-fly (section 30.3).

basin speed ... to slow down drawing attractor basins and backwards space-time patterns or revert to full speed (section 24.11), which can also be done on-the-fly (section 30.3), while interrupting (section 30.2), and at the "attractor basin complete" prompt (section 30.4).

Enter return at the first output parameter prompt (section 24.1) to access these options in sequence.

\subsection{State-space matrix}

The state-space matrix plots each state in state-space on a $2 \mathrm{~d}$ grid in the lower right corner of the screen, plotting the left half against the right half of each state bit/value string. The $x$-axis represents the left $n / 2 \mathrm{bits} /$ values, the $y$-axis represents the right $n / 2$ bits/values. If $n$ is odd, the extra bit/value is included on the left, and the grid is a flat rectangle as in figure 24.2 (bottom), otherwise the grid is square. The scale of the matrix is set automatically according to $n$, though this can be changed.

For a single basin, attractor cycle states and transient states are shown in different colors. For a basin of attraction field, each basin is assigned a different color (cycling through 15 colors). The start color for the color cycle may be reset to produce different color schemes. If the grid size is big enough, the decimal equivalent of the states are also printed (figure 24.1). 

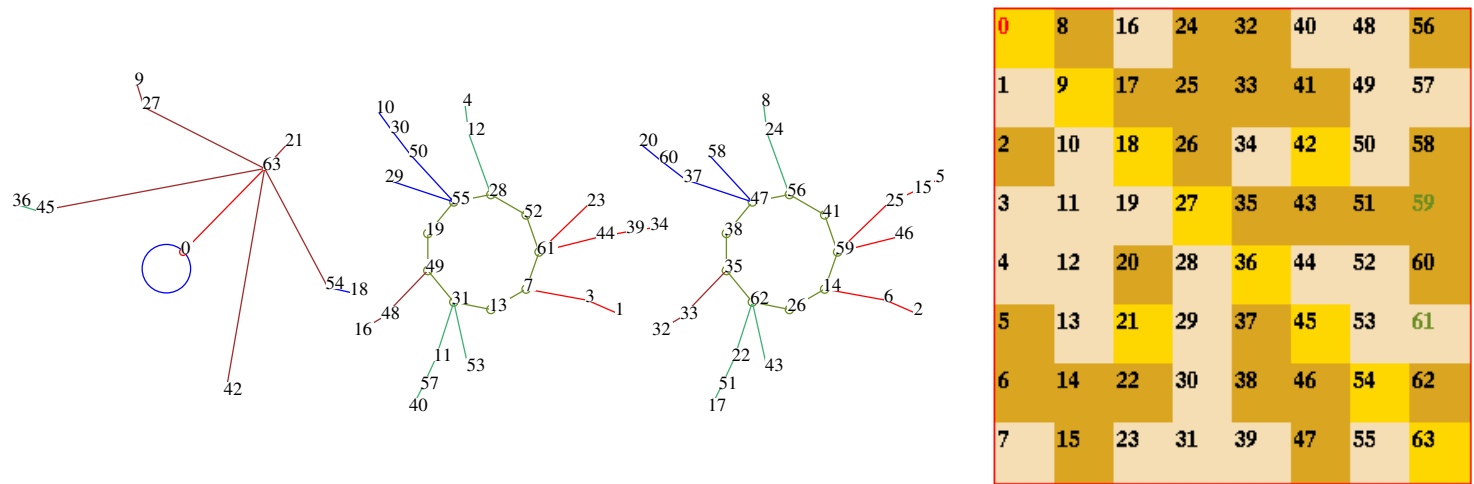

Figure 24.1: The state-space matrix of the basin of attraction field of a cellular automaton, $v 2 k 3$ rcode (dec)110, $n=6$. Left: the uncompressed state transition graph. decimal numbers representing states. Right: The state-space matrix, where the 3 basins are represented by 3 colors. The decimal equivalents of states are shown in both cases.

Enter return at the first output parameter prompt (section 24.1) for the first miscellaneous (start/misc:) option (section 24.4) for the state-space matrix,

start/misc: state-space matrix: all-states-m, attractor only-a

show and change: matrix size-s, start color-c:

\subsubsection{State-space matrix — options summery}

options.... what they mean

all-states-m ... to display the matrix, including all states in the subtree, single basin or field. For a single basin, attractor cycle states and transient states are shown in different colors. For a basin of attraction field, each basin is assigned a different color (cycling through 15 colors).

attractor only-a ... to display the matrix showing just attractor states in the single basin or field. For a basin of attraction field, each basin is assigned a different color (cycling through 15 colors).

matrix size-s ... to change the matrix size from the default. The following prompt is presented,

\section{change matrix size: $\%$ of $1 / 2$ screen(default $63 \%$ ):}

Enter the new size as a percentage of half the screen width. If the matrix size is big enough, the decimal equivalent of states will be shown on the matrix.

start color-c ... to change the start color in the color cycle of 15 colors, and produce different color schemes. The following prompt is presented,

change start color (now 10): enter 1-15: 

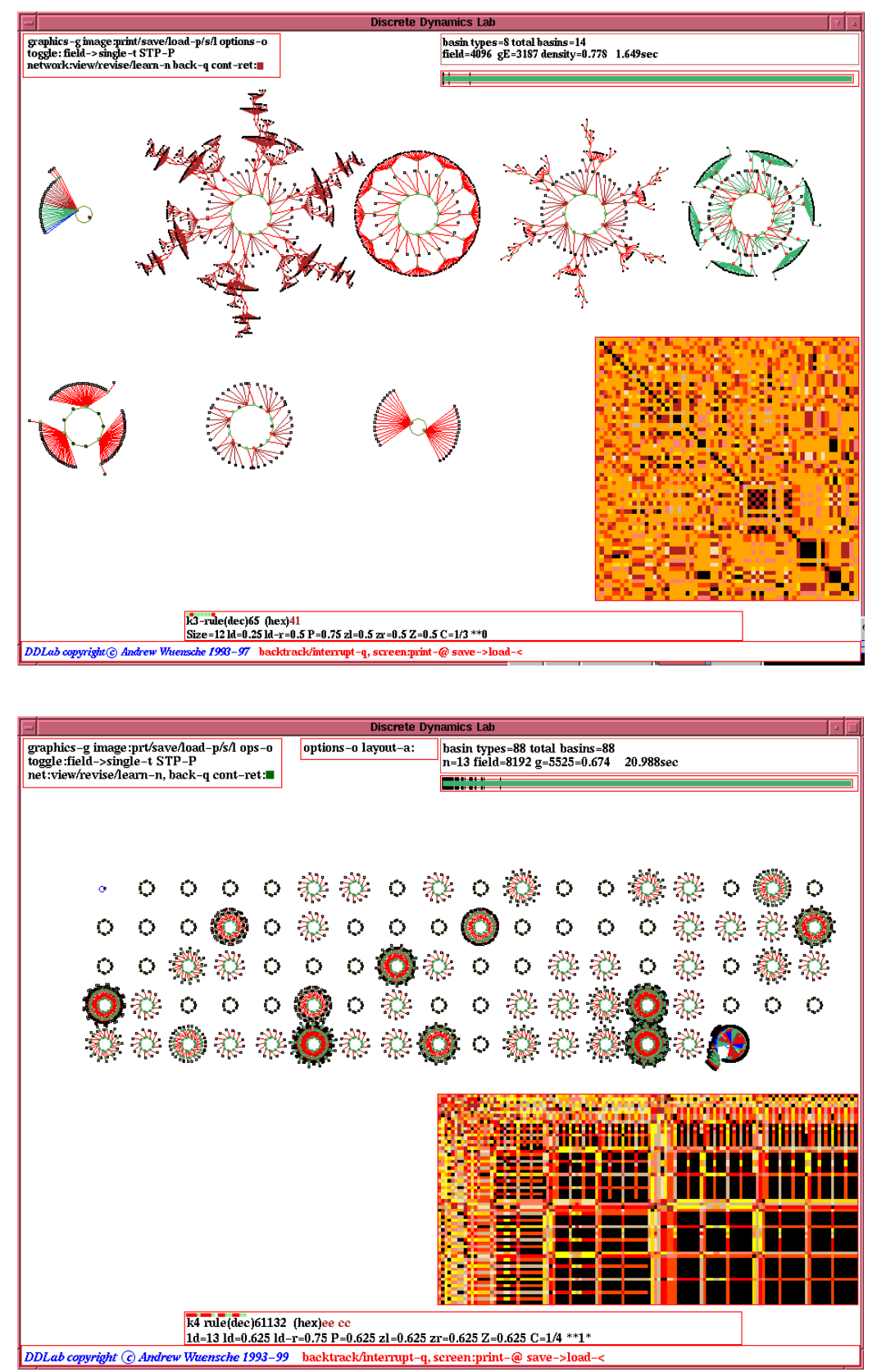

Figure 24.2: The state-space matrix (lower right on each screen) of the basin of attraction field of a CA, with compression off. Top: $v 2 k 3$ rcode (dec)65, $n=12$, the 8 basins types are represented by 8 colors in the matrix. Bottom: $v 2 k 4$ rcode (hex)eecc, $n=13$. Because $n$ is odd, and the extra bit is included on the left, the grid is a flat rectangle. The 88 basins are represented by cycling through 15 colors. 


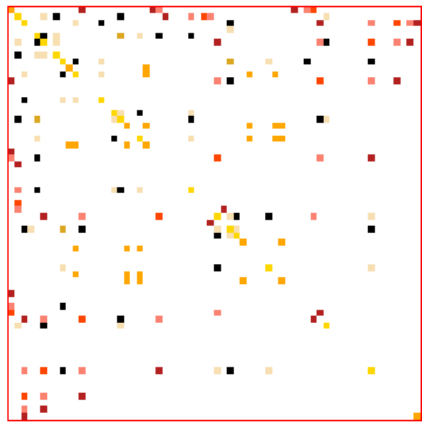

(a) attractor states only

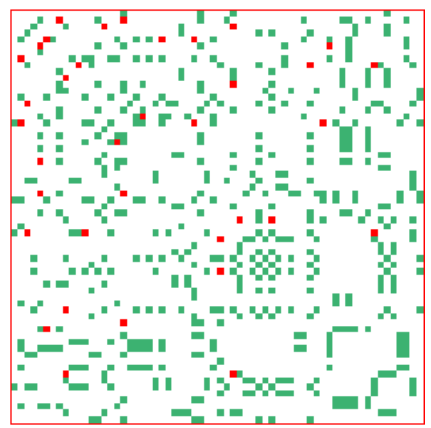

(b) a single basin

Figure 24.3: The state-space matrix of the same CA in figure 24.2 top, $v 2 k 3$ rcode (dec)65, $n=12$.

(a) just the attractor states of the of the 8 basin types represented by 8 colors.

(b) just the states in the 5th basin type (top-right in figure 24.2 top). The attractor and transient states are represented by 2 different colors.

\subsubsection{Toggle the matrix on-the-fly}

Whether set or not in section 24.5, the state-space matrix can be toggled on/off while attractor basins are being drawn. The following reminder appears in the bottom title bar (section 5.5),

matrix-m STP-s scroll-\# exp-e contr-c

Enter $\mathbf{m}$ to toggle the state-space matrix on-the-fly. This can also be done for space-time patterns (section 31.2.2.1). Options \#, e and $\mathbf{c}$ are described in section 24.12.

\subsection{In-degree frequency histogram}

DDLab can record the frequency of different in-degrees (the number of pre-images or predecessors) that occur in a subtree, basin or field. If this option is selected, the in-degree information is displayed as a histogram when the attractor basin is complete, or during a temporary pause (section 30.2). The following top-right prompt is presented in section 24.4,

\section{in-degree frequency histogram-h:}

If $\mathbf{h}$ is entered, the histogram setting remains active for all further attractor basins. It may be deactivated by not entering $\mathbf{h}$ at this prompt, by restoring all "output parameter" defaults (section 24.1), or by backtracking through the main prompt sequence beyond section 9 .

\subsubsection{In-degree frequency cut-off}

If $\mathbf{h}$ is entered above, the following prompt allows setting an upper threshold (or cut-off) to the in-degrees recorded, to limit the size of the histogram. Any cut-off in the range of 50 to 5000 may be set, the default is 200 , or the current setting. The occurrence of in-degrees equal to or above the cut-off value will be added to the cut-off frequency "bin" in the histogram.

select cut-off in-degree bin (50-5000, default 200): 


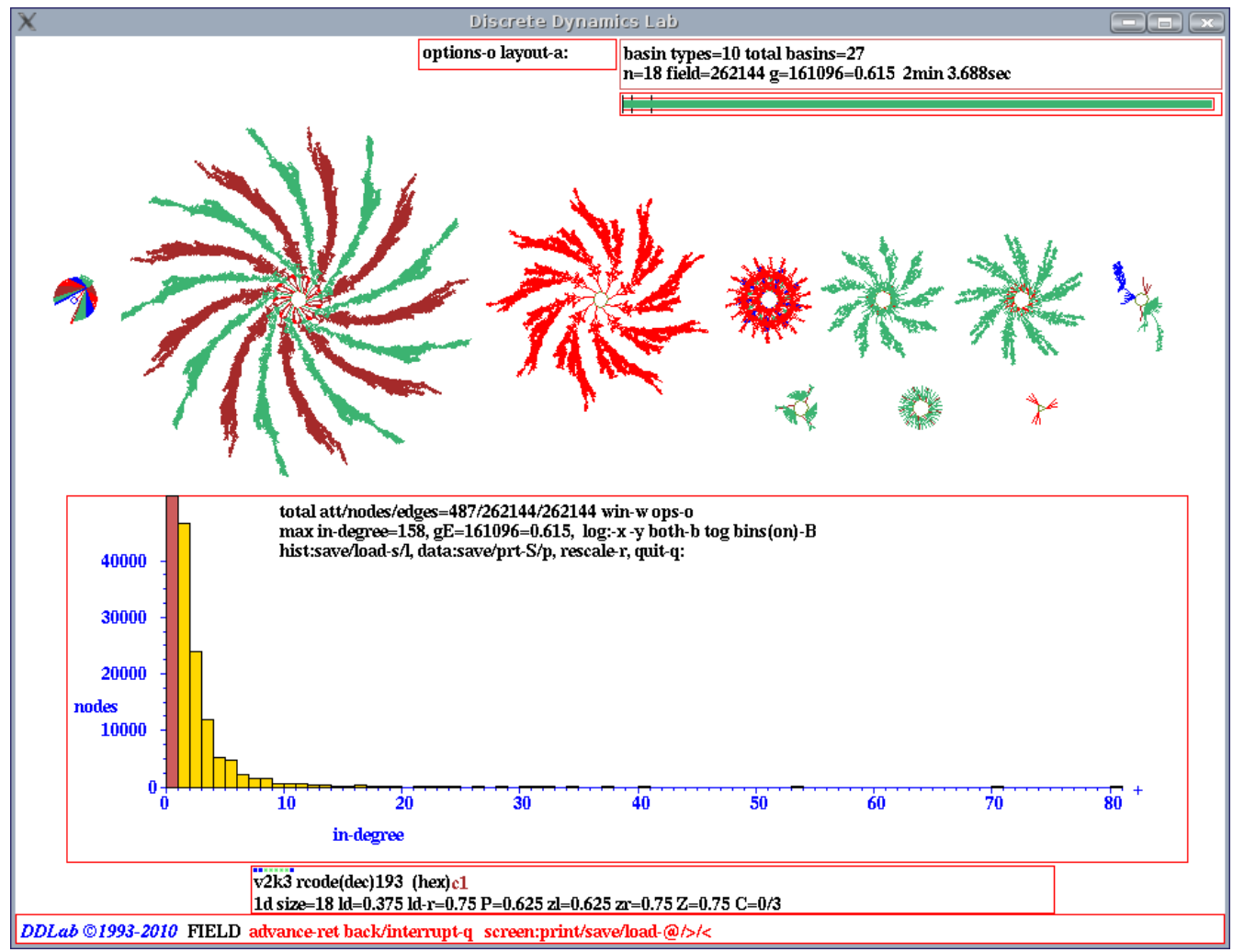

Figure 24.4: In-degree histogram which has been rescaled from the default settings, of a basin of attraction field of a CA, $v 2 k 3$, rcode (dec)193, $n=18$. The attractor basins are shown at the top.

\subsubsection{Drawing the in-degree histogram}

While the in-degree histogram is active, Attractor basins are drawn in the usual way, and in-degree information is recorded. The histogram and data will be displayed in a window in the lower section of the screen once the attractor basin is complete, or can be seen at any time if paused on-the-fly (enter $\mathbf{q}$ ), in which case a top-right prompt similar to the following is presented (variations and other pause options are described in section 30.2),

early exit - in pre-image fan (if within a pre-image fan)

inhist-h, next-tree/basin-n options-o stopfield-q speed-s cont-ret: (for a basin field)

During a pause, enter $\mathbf{h}$ to see the histogram and data corresponding to the attractor basin generated so far. The $x$-axis represents the range of in-degrees, from in-degree zero (garden-ofEden states) upward. The $y$-axis represents the number of states having a given in-degree. The last, black, frequency column (if applicable) represents combined in-degrees equal to the cut-off value and above. The garden-of-Eden column is colored red, the other columns yellow. The $x$ and $y$ axis are automatically re-scaled according to the range of in-degrees encountered, but can also be manually re-scaled or shown in log form for a clearer view of the spread of frequencies or to amplify smaller in-degrees. 

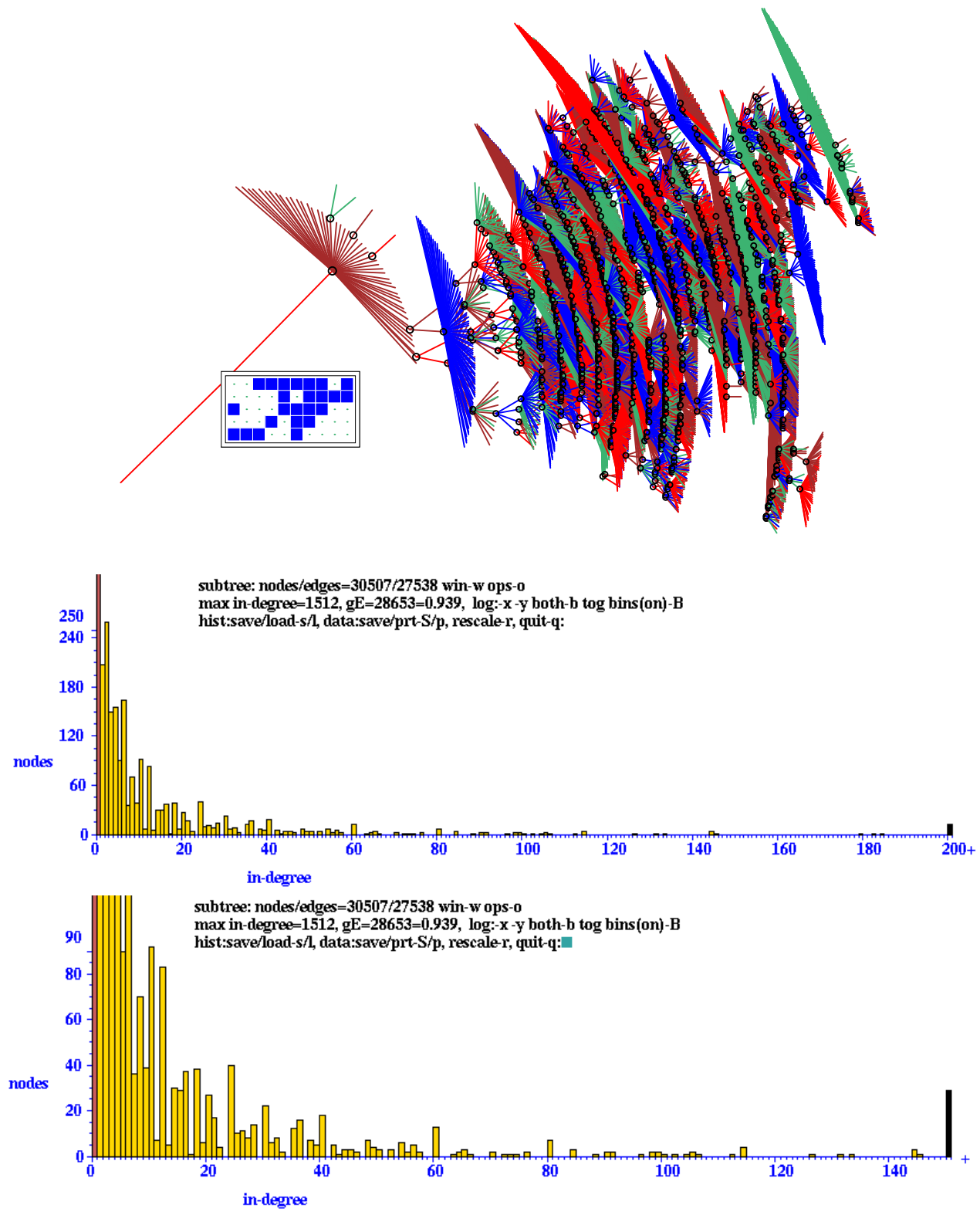

Figure 24.5: Top: A subtree of a CA, $v 2 k 3$ rcode $(\mathrm{dec}) 193$, with the $n=501 \mathrm{~d}$ root state shown in $2 \mathrm{~d} 10 \times 5)$, leaf nodes suppressed and the STG rotated by $90^{\circ}$. Below: Two versions of the in-degree histogram, with increasing rescaling ( $x$ and $y$ axis) for a closer view. Excess in-degrees (indicated by + ) are included in the last black column on the right. Zero in-degrees (leaf states) are in the first (red) column on the left. 


\subsubsection{In-degree data and prompts}

An example of the data displayed in the window, and initial prompts (figure 24.4) for the basin of attraction field of a $v 2 k 3 \mathrm{CA}$, rcode(dec) $193, n=18)$ is shown below,

total att $/$ nodes $/$ edges $=487 / 262144 / 262144$ win-w ops-o

max in-degree $=158, \mathrm{gE}=161096=0.615$, log:- $\mathrm{x}-\mathrm{y}$ both- $\mathrm{b}$ tog bins(on)-B

hist:save/load-s/l, data:save/prt-S/p, rescale-r, quit-q:

For a subtree the prompt starts with subtree: nodes/edges $=\ldots$

\subsection{4 in-degree window — data decode}

The data shown in in-degree frequency window (section 24.6.2) signifies the following,

total att ... the number of states on attractor cycles (does not apply to subtrees).

nodes ... the number of nodes whose pre-images have been computed.

edges ... the total number of edges computed so far.

max in-degree ... the largest in-degree found, possibly more than the cut-off limit.

$\mathbf{g E} \ldots$ the frequency and fraction of garden-of-Eden states (in-degree 0), usually

the greatest frequency.

Note that for a complete basin (or field), nodes=edges, for a complete subtree, nodes=edges +1 , and for an incomplete attractor basin, (i.e. if interrupted), nodes $<$ edges.

\subsection{5 in-degree window - options summery}

win-w ... to toggle the in-degree window itself, in case its hiding attractor basins.

ops-o ... for further options described in section 30.4.

log:-x-y both-b ... to transform to a log plot on either axis or both (section 24.6.6).

tog bins(on) ... for a binned or simple log plot (section 24.6.6).

hist:save/load-s/1 ... to save histogram data as DDLab binary file, (default filename my_prh.prh), or load the data file and regenerate a new histogram.

data:save/prt-S/p ... to save histogram data as an ASCII file, (default filename my_data.dat), or print the ASCII data to the terminal (not for DOS). The following example is for CA, $v 2 k 3$ rule 193, $n=10$ (basin field shown below).

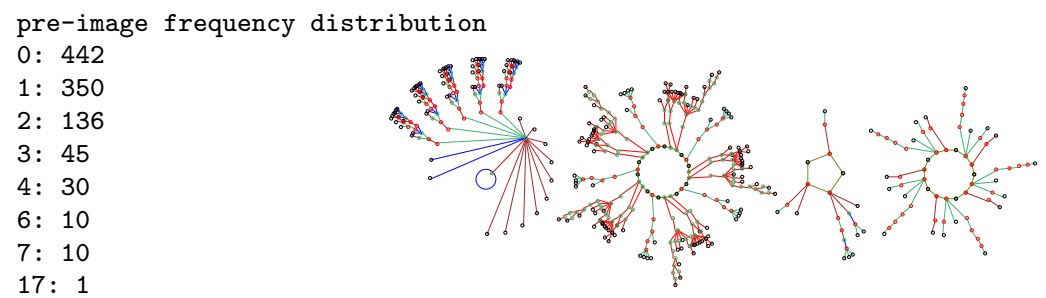

rescale-r ... to rescale the histogram (section 24.6.7). 

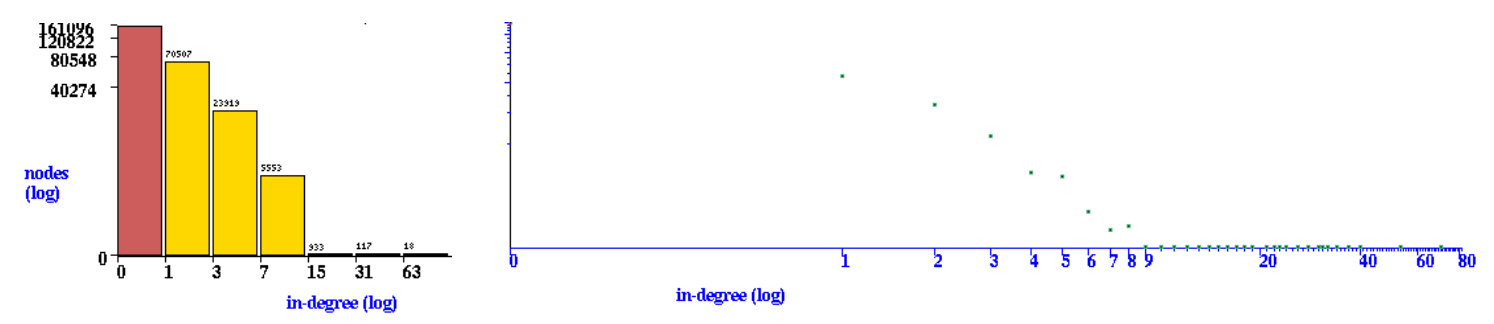

Figure 24.6: The in-degree histogram as a log-log plot can be implemented in two ways on the $x$-axis, Left: preserving a histogram (the default) with bins that capture an increasing range of in-degrees, $0,1-2,3-7,8-15$, etc. Right: a simple log-log plot. The $x$ and $y$ axis can also be treated separately. These plots are for the $\overline{\mathrm{CA}}, v 2 k 3$ rcode (dec) 193 , in figure 24.5 .

\subsubsection{In-degree log plot}

The in-degree histogram can be transformed to a log-log plot base 2 . There are two ways of treating the $x$-axis. The default (binned) method preserves a histogram with equal width bars, where each bar combines a range of in-degrees, $0,1-2,3-7,8-15$, etc. The alternative method is a simple $\log -\log$ plot. Both are illustrated in figure 24.6. Enter B to toggle between the two methods the in-degree histogram changes color, yellow for binned, otherwise green.

Enter $\mathbf{x}, \mathbf{y}$ or $\mathbf{b}$ in section 24.6.3 to replot the histogram according to $\log 2$ scale for just the $x$-axis, just the $y$-axis, or both axes.

\subsubsection{Rescaling the $\mathrm{x} / \mathrm{y}$-axis}

Enter $\mathbf{r}$ in section 24.6 .3 to rescale the $x$ and $y$ axes. The following additional prompts are presented, depending on $\mathrm{x}$-max, the max in-degree found,

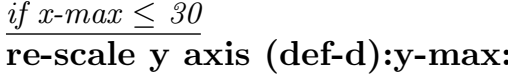 \\ if $x$-max $>30$, prompts in turn) \\ re-scale $\mathrm{x} / \mathrm{y}$ axis (def-d):x-max (30-158): $\quad \mathrm{y}$-max:}

Enter the rescaled settings, or $\mathbf{d}$ to restore the originals - the histogram will be redraw accordingly, as in figure 24.5.

The $x$-axis is initially scaled to show the spread of in-degrees from zero to the maximum found (or to the cut-off value). If the cut-off value is shown, + is added at the end of the $x$-axis indicating that in-degrees equal to or greater than the cut-off value have occurred.

If the maximum in-degree found is greater than 30 , the $x$-axis can be rescaled by entering a new maximum in-degree. Excess in-degrees (off the scale) will be shown as a black column on top of the maximum in-degree column, and + will be added to the $x$-axis.

The $y$-axis is initially scaled to contain the highest in-degree frequency column in the histogram (usually column 0 , leaf states). It may be rescaled to any value, for instance to show smaller in-degree frequencies at a larger scale. 

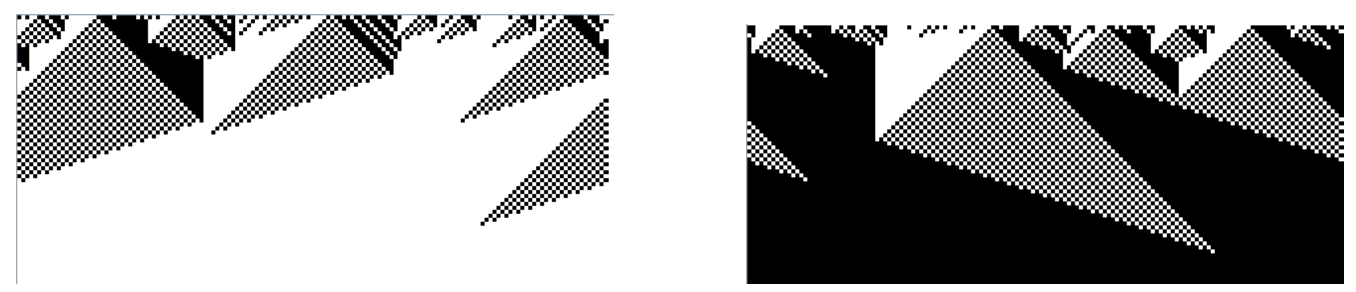

Figure 24.7: The density classification problem in emergent computation seeks to evolve a rule that is able to categorize states between a majority $0 \mathrm{~s}$ and $1 \mathrm{~s}$. In this example of typical space-time patterns ( $n=149$ ), $v 2 k 7$ rcode (hex)(ff f0 8c f0 ff e0 $00 \mathrm{f0} \mathrm{ff} f 000 \mathrm{f0}$ ef e0 00 e0), evolved by Raja Das[7].

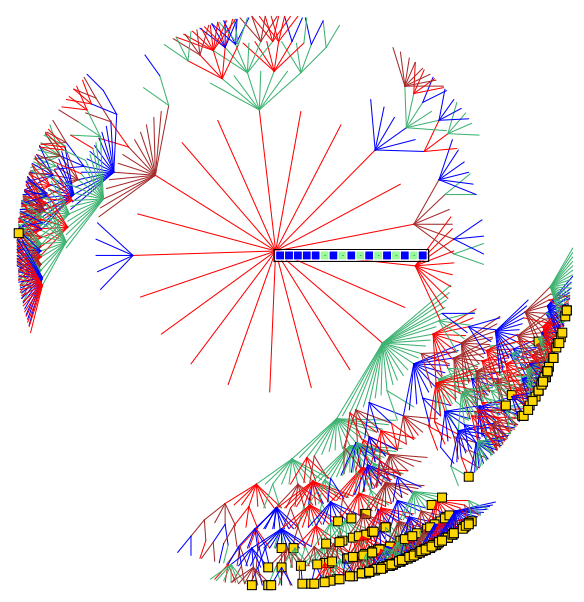

Figure 24.8: A subtree showing exceptions to density classification. Using the Raja Das[7] rule (as in figure 24.7), a subtree was generated from an initial state with 111 's out of $n=17$. Most states in the subtree also have a majority of $1 \mathrm{~s}$, but there are $9.63 \%$ exceptions. indicated in the "Data on subtrees" window (section 27.2.6) shown below, and by yellow squares in the subtree.

subtree $=1901$ maj0 $=183=0.0963 \operatorname{root}($ hex $)=01$ i5 55 $\mathrm{g}=1510=0.794 \mathrm{ml}=9 \mathrm{mp}=26$

\subsection{Density classification problem — attractor basins}

The "density classification problem" is a favourite exercise in emergent computation[7, 13] which seeks to evolve CA rules able classify states between a majority 0 s and $1 \mathrm{~s}$ - the fitness is measured by running forward from many random initial states (implemented in section 31.6.1).

This section introduces an alternative method of measuring fitness, by generating basins or subtrees, and highlighting and counting the exceptions to the intended classification. A good density rule will have two point attractors, the all 0 state attracting the majority- 0 states, and the all-1 state attracting the majority-1 states (figures 24.7, 24.9). Likewise, a subtree with a given majority should attract the same majority value (figure 24.8). DDLab is able to test for a majority of any value ( 0 to 7 ) in basins or subtrees, but the examples in this section relate just to the majority of $1 \mathrm{~s}$ and $0 \mathrm{~s}$. The following top-right prompt is presented in section 24.4,

\section{show/count states with majority, enter $(0-1):($ for $v=2)$}

Enter $\mathbf{1}$ or $\mathbf{0}$ to highlight the majority states, which are displayed as small yellow squares with a black border (figure 24.8). The number and percentage of the value selected is also displayed in the data window when a basin or subtree is complete (sections 27.2.3, 27.2.6) as in figure 24.8. 

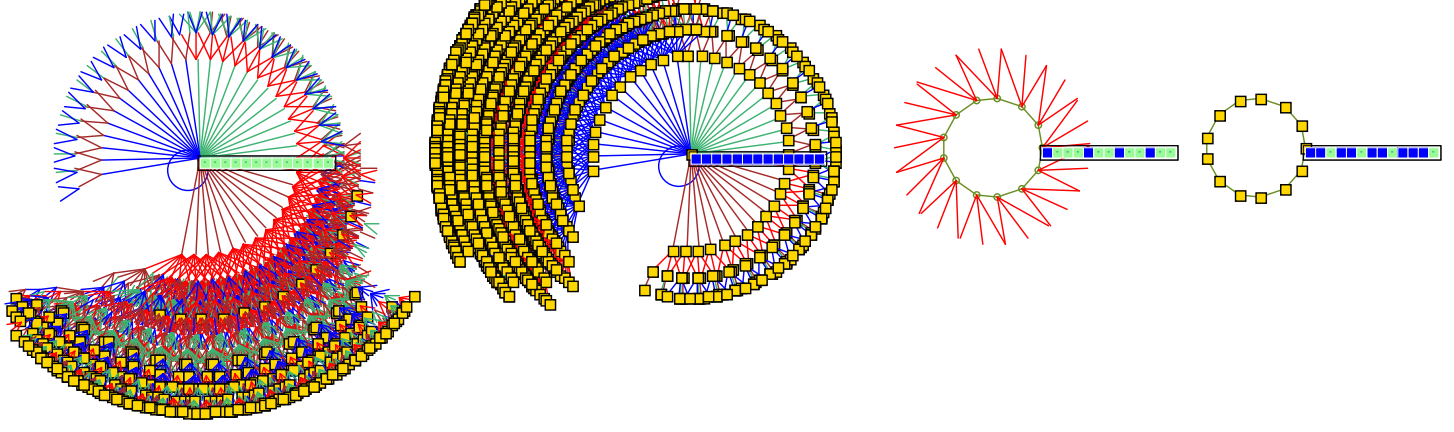

Figure 24.9: A basin of attraction field showing states with a majority of $1 \mathrm{~s}$ as yellow squares. $n=13$, $v 2 k 7$, same rule as in figure 24.8. Nearly all states belong to the all 0's and all 1's point attractors. Data on the relative volume of these basins and (density of majority-1s) within each: $51.9 \%(7.34 \%)$ and $47.3 \%(97.3 \%)$ - taken from the basin data window (section 27.2.3) at a pause (section 27.1.2).

\subsection{Screen-saver demo}

If a single basin or subtree was selected at the first prompt in section 6.2 , an option is presented for a continuously changing display where basins or subtrees are generated randomly in overlapping bubbles on the screen (figure 4.18). The following top-right prompt is presented in section 24.4,

\section{screensave demo -s:}

Enter $\mathbf{s}$ to select the demo. An example is shown in figure 4.18. The backwards space-time patterns and state-space matrix may also be set to work at the same time, or toggled on-the-fly (sections 24.5.2, 24.10). Most setting, (network, layout, display, mutation) apply to the screensaver demo. For a quick-start example see section 4.11.

\section{$24.9 \quad G$-density, $Z$ and $\lambda$}

The $G$-density - of Garden-of-Eden (leaf) states — those without predecessors — in a subtree, basin or field, is a measure of convergence in network dynamics, which in turn relates to the quality of typical space-time patterns in CA, ordered-complex-chaotic[32]. A number of static parameters from the rcode-table itself also predict convergence, to varying degrees. Although these parameters are generalized for multi-value $v \geq 3$, the options involving parameters (sections 24.9.3 - 24.9.5) apply only to binary $(v=2)$ rules. If $S$ is the rcode size, the binary parameters can be defined as follows,

$\lambda$-parameter ... the fraction of 1 s in $S[17]$.

$\lambda_{\text {ratio }} \ldots$ the total of the lesser value (1s or 0s) divided by $S / 2$.

$Z$-parameter ... the probability that the next unknown cell in the CA reverse algorithm is determined[25, 32, 42]. 


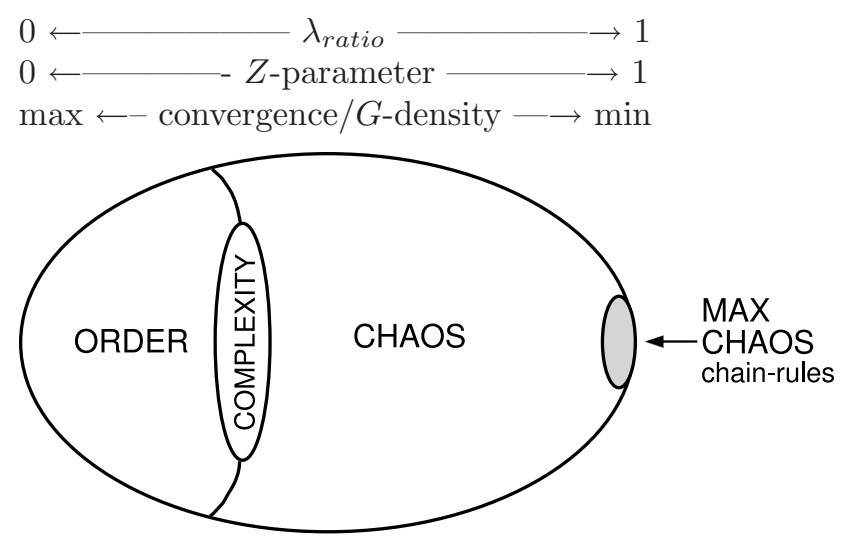

Figure 24.10: A view of rule-space (after Langton[17]). Tuning the $Z$-parameter from 0 to 1 shifts the dynamics from maximum to minimum convergence, from order to chaos, traversing a phase transition where complexity lurks. The chain-rules (about the square root of rule-space) on the right are maximally chaotic and have the very least convergence, decreasing with system size, making them suitable for dynamical encryption (section 16.11).

figure 24.10 is a schematic view of rule-space illustrating how convergence and $G$-density relate to order/chaos - predicted by the $Z$-parameter and to a lesser extent by $\lambda_{\text {ratio }}$ - only $Z$ is able to identify the maximally chaotic chain-rules (section 16.11). Complex rules occur at the order/chaos phase transition, but are difficult to identify by these static parameters ${ }^{2}$.

$\lambda_{\text {ratio }}$ is utilised instead of the $\lambda$-parameter because it can be compared directly to $Z-$ both vary between 0 and 1 - where a low value indicates order and high convergence, and a high value indicates chaos and low convergence. $\lambda_{\text {ratio }}$ approximates $Z$, which depends on the distribution as well as the proportions of values in the rcode $-Z$ predicts behavior more accurately (figure 24.13). To test these relationships, this section describes how graphs of $G$-density against $Z$ and/or $\lambda_{\text {ratio }}$ can be plotted for predefined CA rules.

Plots can be made of $Z$ against $\lambda_{\text {ratio }}$ (and $Z_{\text {left }}$ against $Z_{\text {rigt }}$ ) to relate parameters for predefined CA rule samples. The proportions of rules in rule-space with various levels of canalyzing inputs (chapter 15), $\lambda_{\text {ratio }}$, and the $P$-parameter ${ }^{3}$ may be computed and displayed (figures 24.1, 24.2 ) - these measures are of interest in predicting convergence and stability in random Boolean networks.

Plots of the variation of $G$-density with network size are also available, another useful measure of the order-chaos spectrum of behavior applied mainly to CA[27, 41] (figure 24.12).

The following top-right prompt is presented in section 24.4 ,

$$
\begin{aligned}
& \text { if } v=2 \\
& \text { G-density plot: Zpara-1, + Lambda ratio-2, size range-3 } \\
& \text { parameter plot: Zpara-Lda ratio (C-P data), Zleft-Zright -L: }
\end{aligned}
$$

if $v \geq 3$ the G-density graph only

\section{G-density plot: size range-3}

\footnotetext{
${ }^{2}$ Chapter 33 describes alternative methods for automatically classifying rule-space including complex rules.

${ }^{3}$ The $P$-parameter[11] is the fraction of $0 \mathrm{~s}$ or $1 \mathrm{~s}$ in the rule-table (whichever is more), $P$ varies from 1 (order) to .5 (chaos). The $P$-parameter is favoured in theoretical biology over the equivalent $\lambda$-parameter or $\lambda_{\text {ratio }}$.
} 


\subsubsection{G-density, $Z$ and $\lambda$ - options summery}

options... what they mean

G-density plot: ... plots involving $G$-density, for predefined rules ...

Zpara-1 ... enter 1 for a plot of $Z$ against $G$-density (sections 24.9.3-24.9.3.2).

+Lambda ratio-2 ... enter 2 for two simultaneous plots, $Z$ against $G$-density, and $\lambda_{\text {ratio }}$ against $G$-density (sections 24.9.3 - 24.9.3.2).

size range-3 ... enter 3 to plot $G$-density against network size $n$. (section 24.9.2).

parameter graph : ... plots between parameters enter $\mathbf{L}$ for a predefined rule sample ...

Zpara-Lda ... to plot $Z$ against $\lambda_{\text {ratio }}$ (sections 24.9.4-24.9.4.3 .

Zleft-Zright $\ldots$ to plot $Z_{\text {left }}$ against $Z_{\text {right }}$ (sections 24.9.4-24.9.4.3).

The $Z$-parameter is the greater of the two.

(C-P data) $\ldots$ to see the proportions of rules in rule-space $-\lambda_{\text {ratio }}, P$-parameter, and canalyzing inputs (section 24.9.5). This can be activated once the $Z-\lambda_{\text {ratio }}$ or $Z_{\text {left }}$ against $Z_{\text {right }}$ plots (above) are complete.

\subsubsection{G-density plotted against network size}

for to multi-value, $v \geq 3$, as well as binary $v=2$

The rate of increase of $G$-density with network size (system size) $n$ is another order-chaos indicator in CA. For the vast majority of rules, $G$-density increases with $n$, with ordered dynamics showing the highest rate[32]. Very chaotic rules may approximate a zero rate, but only the maximally chaotic chain-rules show $G$-density decreasing with increasing $n$ (figure 24.12).

Enter 3 in section 24.9 to plot $G$-density against a range of $n$. If required, this can be done for a number of selected rules on the same plot. The following prompt is presented,

\section{G-density - size range: \\ start size $(\mathbf{d e f}=5): \quad$ end size $(\max =\mathbf{3 1}): \quad$ (end size $<20$ is recommended)}

Enter the start and end values of $n$. Note that if the end size is set high, computation time may be inordinately long.

Enter $\mathbf{d}$ at the next prompt (optional) which normally skips all further options, but in this case skips to mutation (chapter 28). The plot will be drawn in the lower right of the screen. The basin of attraction fields (or single basins, or subtrees) will be draw (at a small scale by default) in a window in the upper part of the screen (figure 24.11). When each attractor basin is complete the $G$-density ( $y$-axis) is plotted against the network size $n$, and the plot will continue automatically for successive values of $n$ (figure 24.12). Once complete, the following prompt appears in the top-right corner of the data window (section 27.2),

\section{... quit-q rule-r mut-(def) keep+k:}

Enter $\mathbf{r}$ to select a new rule (set in a lower-right window, chapter 16). To superimpose a new plot on an old plot enter or add $\mathbf{k}$, - i.e. enter $\mathbf{r k}$ for a new rule, or just $\mathbf{k}$ for the next mutant. Otherwise enter return for the next mutant - the rule will change according to the type of mutation set in section 28.1. Enter $\mathbf{q}$ to quit the plot and backtrack. 


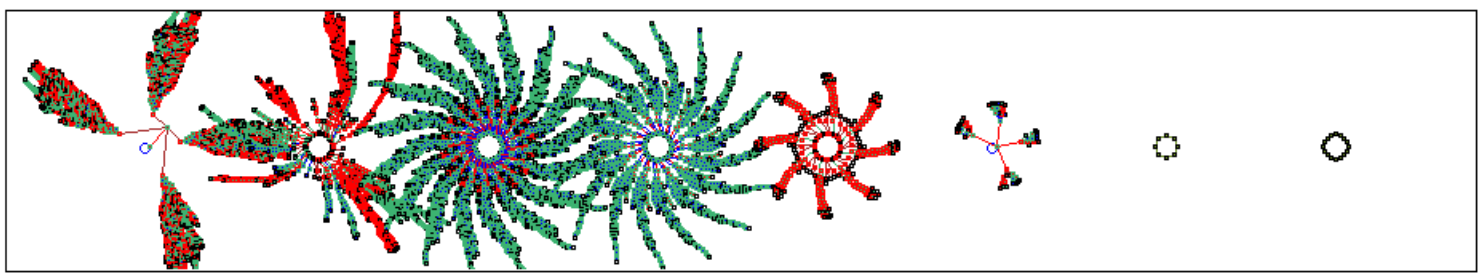

Figure 24.11: As the plot of $G$-density against network size $n$ is in progress, and also the plots against $\lambda_{\text {ratio }}$ or $Z$ (figure 24.13), the attractor basins are drawn in an upper window at a default scale and layout (which can be changed in chapter 25). This example shows the basin of attraction field for 1d CA, $v 2 k 5$ rcode 9569a999, $n=16$, in figure 24.12 .

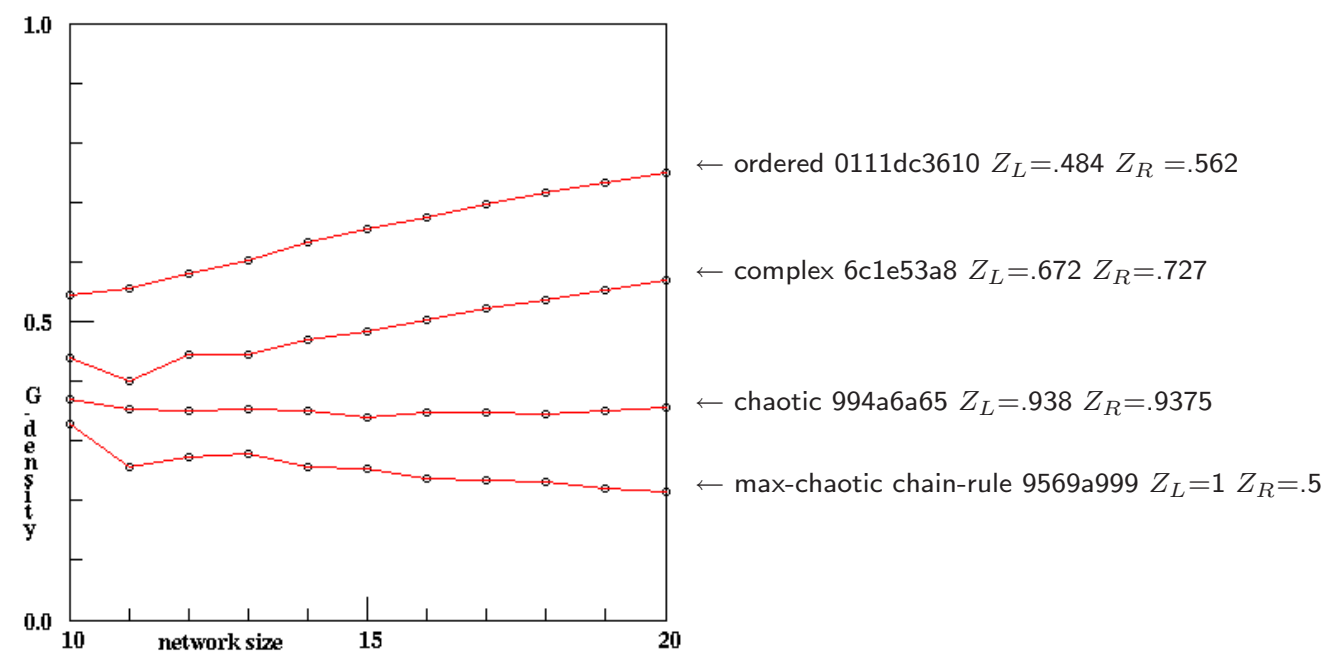

Figure 24.12: Plotting $G$-density against a range of $n$ for ordered, complex and chaotic $1 \mathrm{~d}$ CA, $v 2 k 5$ (rcode indicated), where the ordered rule has the highest rate of increase of $G$-density. Very chaotic rules may approximate a zero rate; only chain-rules show $G$-density decreasing.

24.9.3 G-density against $Z$ and/or $\lambda_{\text {ratio }}$ for binary, $v=2$, rcode or tcode only - not for kcode

Enter $\mathbf{1}$ in section 24.9 for a graph of $G$-density against $Z$, or $\mathbf{2}$ for an additional graph of $G$-density against $\lambda_{\text {ratio }}$ drawn simultaneously.

Enter $\mathbf{d}$ at the next prompt (optional) to skip further options. The $Z$ graph will be drawn in the lower right of the screen, the $\lambda_{\text {ratio }}$ graph beside it on the left. A subtree, basin or field for each of the set of rules specified (in reverse decimal order) will be drawn (at a small scale by default) in a window in the upper part of the screen, as in figure 24.11. When each attractor basin is complete the $G$-density ( $y$-axis) is plotted against the rule's $Z$ and/or $\lambda_{\text {ratio }}$ on the $x$-axis. 

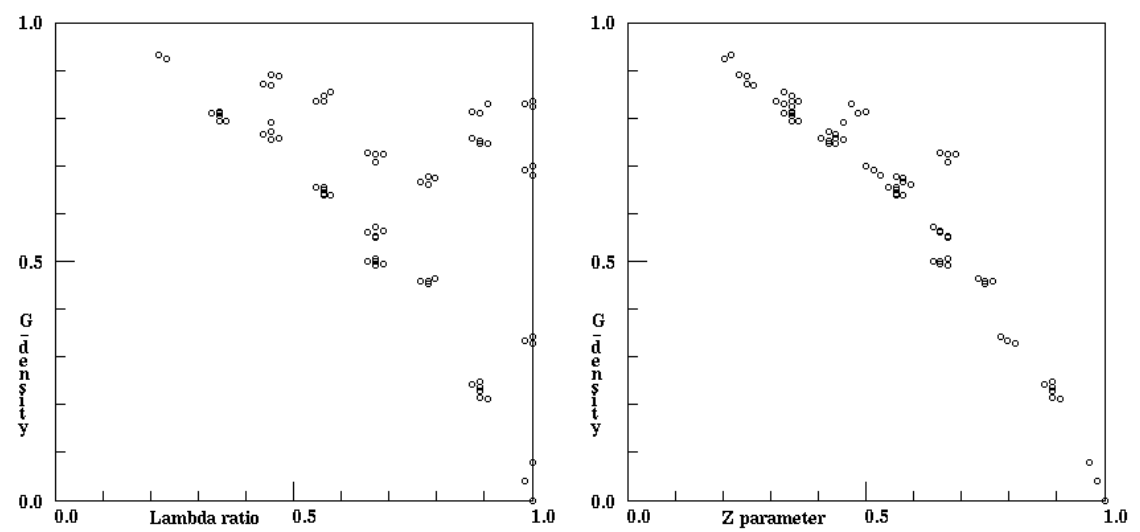

Figure 24.13: $G$-density plotted against Left: $\lambda_{\text {ratio }}$, and Right: the $Z$ parameter. $n=12$, for the set of all $v 2 k 7$ totalistic rules (tcode). The complete basin of attraction field was generated for each tcode and the garden-of-Eden states counted. $Z$ provides a tighter prediction of $G$-density than $\lambda_{\text {ratio }}$.

\subsubsection{1 $G$-density for tcode, or rcode $k \leq 3$}

For tcode, or rcode if $k \leq 3$, the rules included in the plot consist of a countdown from the currently active rcode or tcode as a decimal number. To include the entire rule-space, the maximum rule should be selected. An easy way to do this is to enter fill-f in section 16.1.1, which sets all bits to 1 .

The range of $Z$ for the plot may be restricted, with a lower and upper limit. Note that low values of $Z$ (especially $Z=0$ ) should be avoided except for small $n$, because in-degree (and $G$-density) will be very high. Very high in-degrees can exhaust computer memory or take a long time to compute. The following prompts are presented, firstly to set the lower limit, then the upper.

$\min Z(\operatorname{def} 0.2)$ : $\quad$ set $\max Z(\operatorname{def} 1)$ :

\subsubsection{G-density for $k>3$ rcode}

For rcode where $k>3$, the following prompt is presented,

\section{G-density plot: number of rules (def 1250):}

Enter the number of rules to be plotted or accept the default. The rules will be selected at random, but with a bias to include a representative distribution over values of $Z$.

\subsubsection{Z - $\lambda_{\text {ratio }}$ plots}

for binary, $v=2$, rcode or tcode only - not for kcode

Enter $\mathbf{L}$ in section 24.9 to plot of the $Z$-parameter ( $x$-axis) against $\lambda_{\text {ratio }}$, or $Z_{\text {left }}$ against $Z_{\text {right }}$, for a predefined set of rules. The plot is located in the lower right part of the screen. In addition, the proportions of rules in the rule sample with different levels of $\lambda_{\text {ratio }}$, the $P$ parameter, and canalyzing inputs may be displayed once the graph is complete (section 24.9.5). The measures are computed directly from rule-tables. The following prompt is presented,

plot: Zleft-Zright-1, Zpara - Lda ratio and C-P data-(def): 

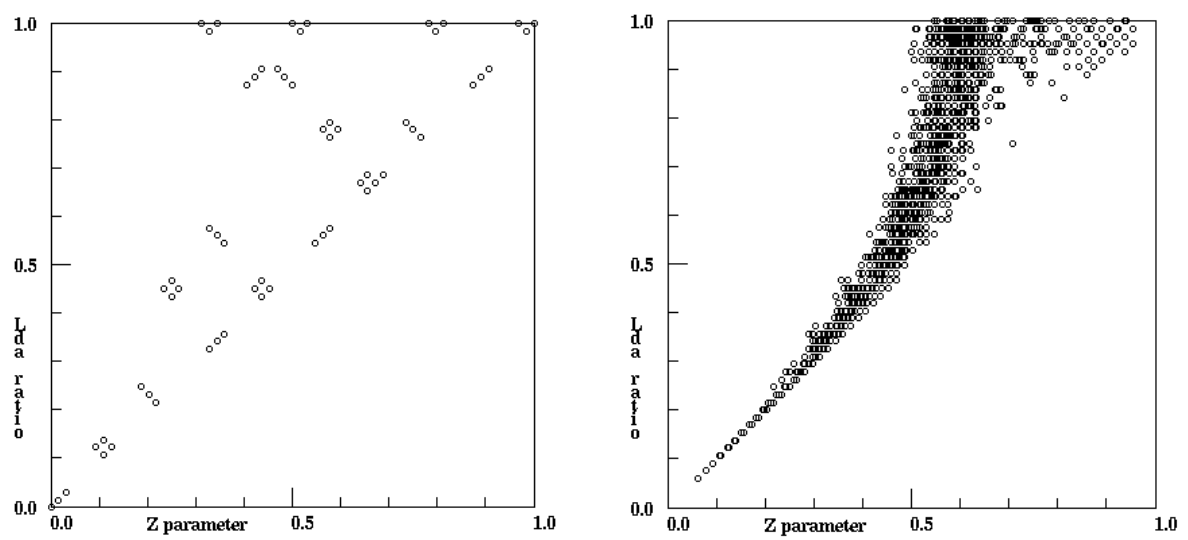

Figure 24.14: $\lambda_{\text {ratio }}$ plotted against $Z$. Left: all $256 v 2 k 7$ tcodes in 72 clusters. Right: a random sample of $2200 v 2 k 7$ rcodes, but biased for a representative distribution over $\lambda_{\text {ratio }} \overline{\text { and } Z \text {. }}$.

$Z_{\text {left }}$ and $Z_{\text {right }}$ are components of the $Z$ parameter. $Z$ itself is greater of the two[25]. Whichever plot is selected, the prompts that follow are similar. Enter return for a $Z-\lambda_{\text {ratio }}$ plot, or 1 for a $Z_{\text {left }}-Z_{\text {right }}$ plot.

24.9.4.1 Z - $\lambda_{\text {ratio }}$ or $Z_{\text {left }}-Z_{\text {right }}$

There are two methods of predefining the rule set,

countdown ... from the (start) rule selected in section 16.1.1. If the start rule is all 1s (or the maximum decimal rule number $2^{k}-1$ ) the entire rule-space will be included, but computation can be lengthy for large $k$. For tcode, and rcode $k \leq 3$, this is the only method. For rcode $k \leq 3$ the plot will proceed without further options. Countdown is also available for $k=4$ and $k=5$ rcode.

sample ... a random sample of rules which can be biased (or not) to cover a representative spread of $\lambda_{\text {ratio }}$ and $Z$ - for rcodes $k \geq 4$.

24.9.4.2 $Z-\lambda_{\text {ratio }}$ or $Z_{\text {left }}-Z_{\text {right }}$ for $k \geq 4$ rcode

For rcode where $k=4$ or $k=5$, the following prompt allows the rule sample size to be specified, as well as the countdown method,

lambda-Z graph: countdown-C, number of rules (def 2200):

or

Zleft-Zright graph: countdown-C, number of rules (def 2200):

Enter $\mathbf{C}$ for countdown as described in section 24.9.4.1 - this may take some time for $k=5$ where rule-space $=2^{32}=4294967296$. For $k \geq 6$ the countdown option is omitted because rule-space becomes too big.

Enter a number to set the sample size. By default the rules will be selected at random, but with a bias to include a representative distribution over values of $\lambda_{\text {ratio }}$ and $Z$. The following further option allows an unbiased random sample.

random rule sample will have lambda bias, no bias-n: 


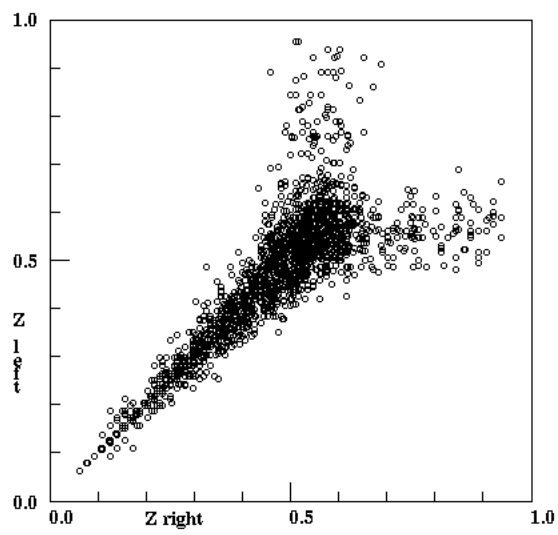

Figure 24.15: $Z_{\text {left }}$ plotted against $Z_{\text {right }}$ for $2200 v 2 k 7$ rcodes selected at random but with a bias to cover a representative spread of $Z$.

An unbiased sample is useful in estimating the proportion of rule-space with different measure of the $\lambda_{\text {ratio }}, P$-parameter and canalyzing inputs. Once the plot is complete, a lower window prompts for these details, described in section 24.9.5.

\subsubsection{3 $Z-\lambda_{\text {ratio }}$ or $Z_{\text {left }}-Z_{\text {right }}$ for tcode}

For tcode only, The following prompt is presented, allowing "equivalent" tcode to be skipped,

show only equivs-e all-a (def clusters): (tcode only)

All these alternatives perform a decimal countdown of tcodes from the start tcode selected in section 16.1.1. Because each tcode must be transformed to rcode, computation can be lengthy for large $k$. These options allow skipping (and seeing) equivalent rules.

Enter a to compute all rules (without skipping). Enter e to show one representative tcode from each set of equivalent tcodes. Enter return for the default - one tcode from each cluster ${ }^{4}$.

A further prompt allows a pause to see the equivalents of members of the tcode cluster,

\section{pause to show codes-p:}

If $\mathbf{p}$ is entered above, after each point is plotted, data about equivalent tcodes and clusters will be displayed in a top-right window, for example, for $k=7$ tcodes and countdown from the maximum tcode 255 ,

\section{v2k7-tcode: $233=10422=151$ equiv $=2$ clust $=4$ tot $=22$ cont-ret:}

This signifies that tcodes 233 and 104 are equivalent, as are their complements, 22 and 151 . These 4 tcodes make up a cluster. In this example, the number of clusters plotted so far is 22 .

Once the plot is complete, a top-right window gives information about the frequency of canalyzing levels, for example for $v 2 k 3$ rcode (elementary rule-space),

\section{c-frequency:136 782418 total rules $=256$ last $=256$}

This shows the frequency of rules with the 4 possible canalising levels $(0,1,2,3)$ - because $k=3$. A lower window prompts for more detailed parameter frequency information, described in section 24.9.5.

\footnotetext{
${ }^{4}$ There are at most 2 equivalent tcodes, the start tcode and its negative (the reflection of a tcode $=$ identity), and at most 4 tcodes in a cluster which includes the complements of both equivalents[25]. Rules in a rule cluster have the same measures of $Z, \lambda_{\text {ratio }}$ (and also $G$-density) so the resulting plots will be identical.
} 


\subsubsection{Proportions of canalyzing inputs and $\lambda_{\text {ratio }}-P$}

Once the $Z-\lambda_{\text {ratio }}$ or $Z_{\text {left }}-Z_{\text {right }}$ plot in section 24.9 .4 is complete, data on the proportions of rules in the rule sample with different frequencies of canalyzing levels $(0-k)$, and parameters $\lambda_{\text {ratio }}$ and $P$ (which are equivalent), can be displayed. The following prompt is presented in a small window to the left of the plot,

\section{tot plotted $=\mathbf{2 0 0 0 0}$ (for example) \\ C-P data-d, +print-p: (+print-p not for $D O S$ )}

Enter $\mathbf{d}$ to see the data table displayed across the top of the screen, or $\mathbf{p}$ to also print the data to the xterm window (not for DOS) as in tables 24.1 and 24.2.

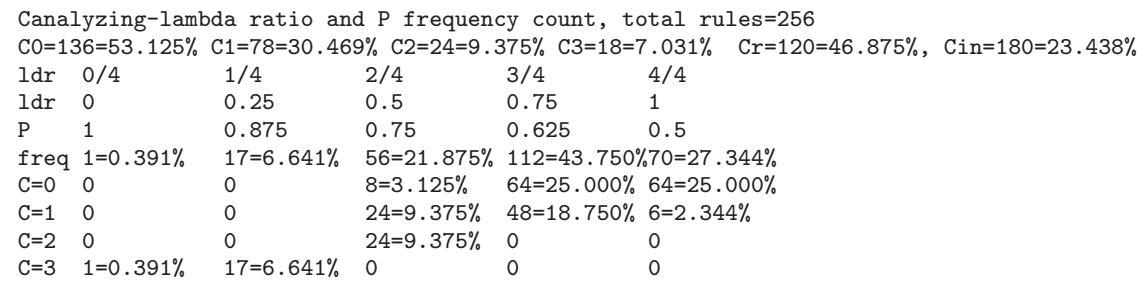

Table 24.1: Canalyzing, $\lambda_{\text {ratio }}$ and $P$ data for $v 2 k 3$ rcode (elementary rule-space) with 256 rules.

Canalyzing-lambda ratio and P frequency count, total
$\begin{aligned} & \text { rules=65536 } \\ & \text { C0=94.638\% }\end{aligned}$ C1=3080=4.700\%

Table 24.2: Canalyzing, $\lambda_{\text {ratio }}$ and $P$ data for $v 2 k 44$ rcode rule-space, 65536 rules.

The key to data in tables 24.1 and 24.2 is as follows,

\section{data heading ... what it means}

$\mathrm{C} 0=, \mathrm{C} 1=, \mathrm{C} 3=, \ldots \ldots$ the number and percentage of the $k+1(0-k)$ possible canalyzing levels found - numbers shown if less than 9999.

$\mathrm{Cr}$... the number and percentage of rules with at least 1 canalyzing input.

Cin ... the number and percentage of canalyzing inputs in the rule sample.

$\operatorname{ldr}$ (first line) ... the $2^{k-1}+1$ possible values of $\lambda_{\text {ratio }}$ as a fraction.

ldr (second line) ... as above shown in decimal. $\lambda_{\text {ratio }}=$ the fraction $0 \mathrm{~s}$ or $1 \mathrm{~s}$ (whichever is less) relative to half rule-table size, $\lambda_{\text {ratio }}$ varies from 0 (order) to 1 (chaos).

$\mathrm{P} \ldots$ as above showing the equivalent values of the $P$-parameter. $P$ is the fraction of $0 \mathrm{~s}$ or $1 \mathrm{~s}$ in the rule-table (whichever is more), $P$ varies from 1 (order) to .5 (chaos), and is related to $\lambda_{\text {ratio }}$ as follows, $P=1-\left(\lambda_{\text {ratio }} / 2\right)$ 
freq ... the number and percentage of rules that were found in each $P$ or $\lambda_{\text {ratio }}$ category above.

$\mathrm{C}=0, \mathrm{C}=1, \mathrm{C}=2, \ldots$ the number and percentage of each canalyzing level that was found in each $P$ or $\lambda_{\text {ratio }}$ category above - numbers shown if less than 999.

\subsection{0 "Backwards" space-time patterns}

At the same time that attractor basins are being drawn, "backwards" space-time patterns may be displayed on the left of the screen, if selected in section 24.4. Figure 24.16 shows examples. The sequence of events reflect the way basins and subtrees are computed. For basins of attraction, there will be three distinct phases in the space-time patterns,

1. The run-in from the initial seed state, which includes the transient and attractor cycle, disclosed from the first repeat.

2. The attractor cycle itself, which is redrawn as it is being recorded.

3. Then the space-time patterns for drawing each transient tree, starting from the "root" and back through successive levels of the tree. After a gap in the pattern, the root of each tree (or subtree) is shown, followed by its immediate pre-images. Trees are computed from each attractor root state in turn (omitting the pre-image on the attractor), cycling anti-clockwise. The set of pre-images of attractor states must exclude the pre-image on the attractor itself to avoid infinite regress.

For a subtree, phase 1 and 3 are similar, but here the run-in phase is replaced by an optional forward-run (section 29.2) and there is no attractor. A forward-run for a given number of timesteps is usually necessary, other than for maximally chaotic "chain" rules (section 16.11), because most of state-space consists of leaves (garden-of Eden states) - a leaf state has no pre-images thus no subtree.

The initial default is not to show the backward space-time patterns - the following top-right prompt is presented in section 24.4 to toggle the current setting (enter $\mathbf{s}$ ).

\section{backwards space-time patterns (now OFF) tog-s: (or now ON)}

\subsubsection{Scroll space-time patterns}

By default, 1d space-time patterns (both forward and backward) will scroll vertically. The pattern can also be presented in successive vertical sweeps, restarting at the top when the screen is full, which is somewhat faster than scrolling. If space-time patterns were set in section 24.10, the following prompt is presented,

\section{sweep backwards space-time pattern-s, scroll-def:}

Enter $\mathbf{s}$ to sweep, or return to scroll. Scrolling can also be toggled on/off on-the-fly (section 24.12). 

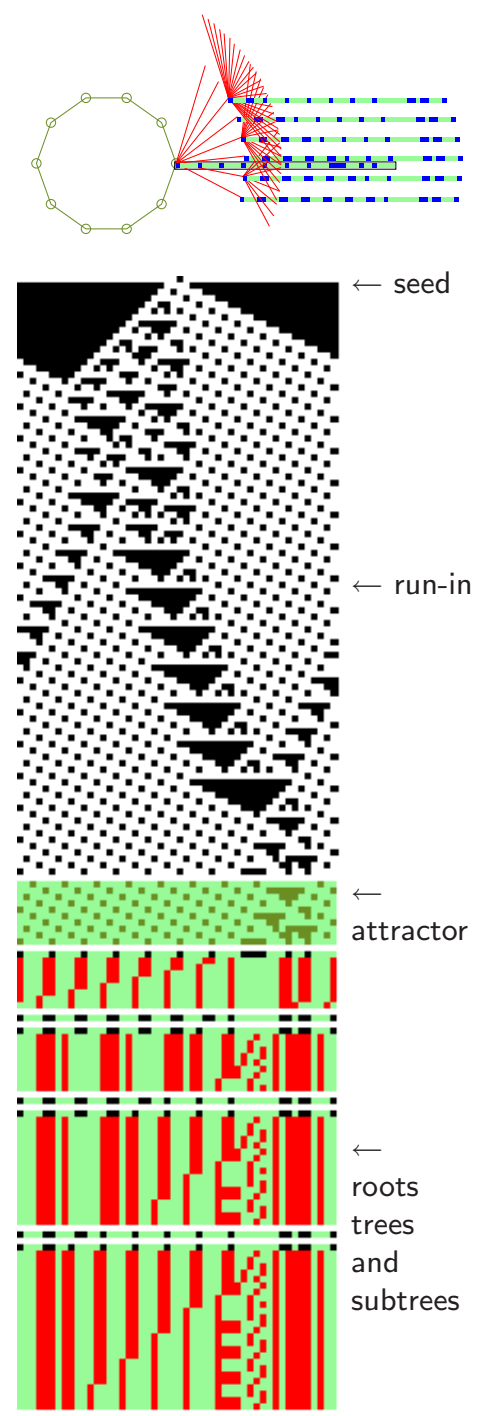

(a) basin of attraction $v 2 k 5$
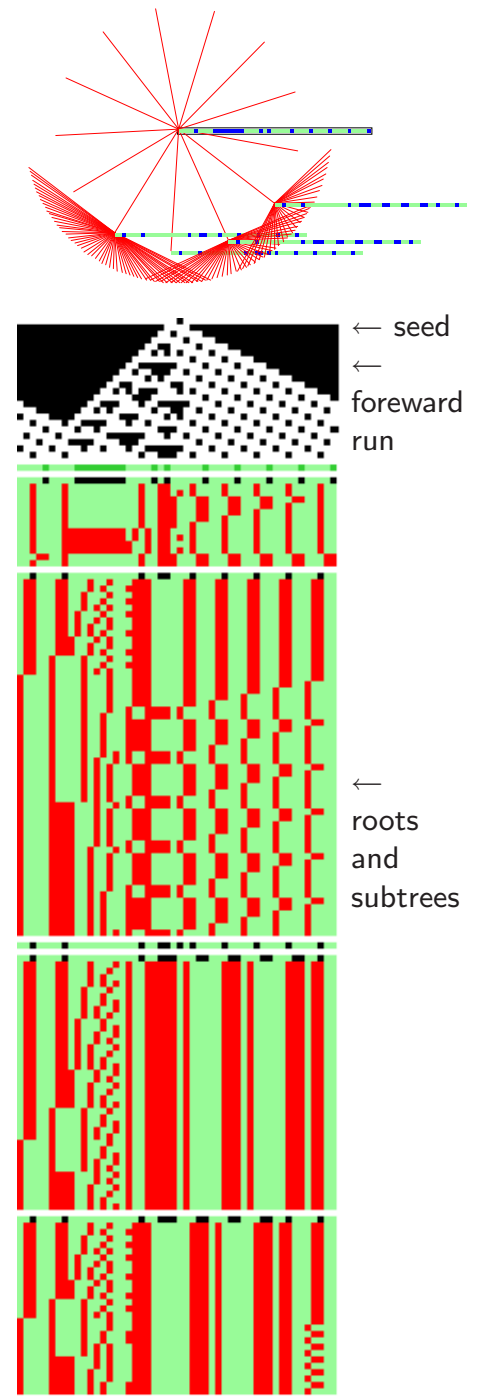

(b) subtree $v 2 k 5$

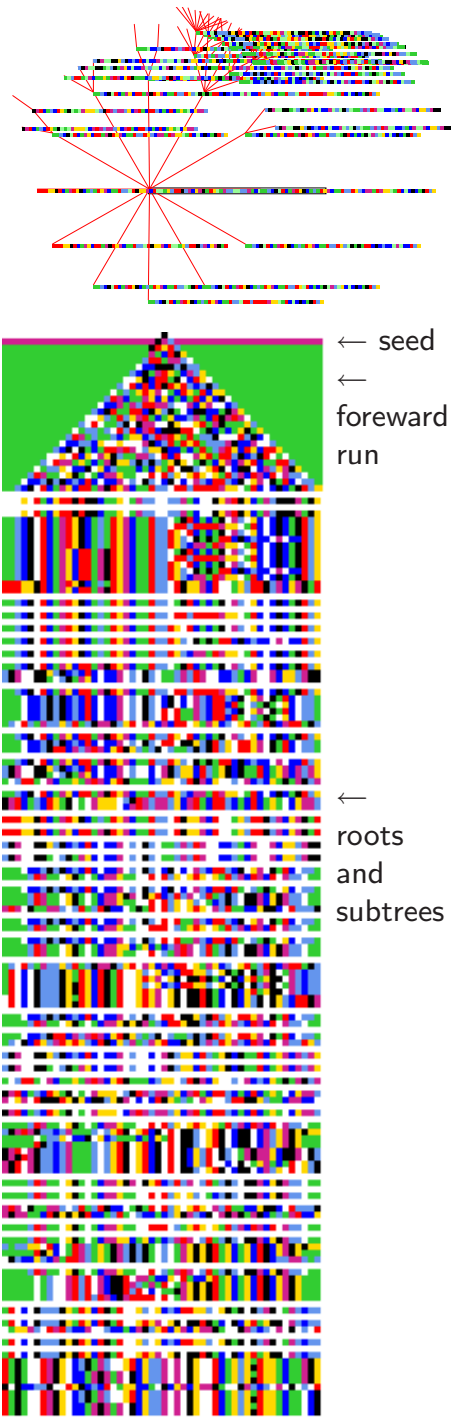

(c) subtree $v 8 k 3$

Figure 24.16: "Backwards" space-time patterns $(n=50)$ from a singleton seed, also showing (at top) the basin or subtree state transition graph with the bit/value patterns at nodes. (a,b) v2k5 rcode (hex)e0897801. (a) shows a basin of attraction, (b) a subtree from a state 22 time-steps forward from the original seed. (c) $v 8 k 3$ modified chain rule (rcode), showing a subtree from a state 25 time-steps forward from the original seed. For $v=2$ only, colors distinguish distinct phases: run-in or forward run, attractor, subtree roots, and their preimages. The figures show just the start of the backward run, which may continue for an indeterminate length of time. 


\subsection{Basin speed}

Drawing drawing basins and backwards space-time patterns may generate too fast for some purposes, such as the key hit on-the-fly options in section 24.12 where slowing down is done with the $<$ key - so slowing down may need to be preset.

The "basin speed" option in section 24.4 has the following top-right prompt,

basin speed: max-(def), or slowmotion (try 300):

Enter a number to slow down, the higher the slower. Maximum speed can be restored on-the-fly with the $>$ key. The same slowdown option can be reached while interrupting (section 30.2), and from the "attractor basin complete" prompt (section 30.4). An addition/alternative to slowmotion is to pause backward iteration after each tree or each pre-image fan (section 27.1.2).

\subsection{Basin on-the-fly options}

refer also to section 30.3

While attractor basins and backwards space-time patterns are being drawn, a number of settings can be changed on-the-fly. A reminder similar to this will appear in the bottom title bar (section 5.5),

matrix-m STP:tog/scroll/exp/contr-s/\#/e/c slow/max- $</>$

DDLab (C)1993-2011 advance-ret back/interrupt-q matrix-m STP:tog/scroll/exp/contr-s/\#/e/c slow/max-</ $>$

Changes take effect as soon as the key is pressed, without return, and apply whether or not preset in the relevant sections in this chapter. Section 30.3 describes the options. 


\section{Chapter 25}

\section{Layout of attractor basins}

not in TFO-mode.

Basins of attraction are drawn either singly, or in groups - for the basin of attraction field, for a range of network size $n$, or for mutant single basins. Groups of basins are drawn in successive rows starting from a top-left position. Before drawing starts, the following layout parameters may be altered (or defaults accepted): their scale, position, angle, horizontal and vertical spacing, rows sraggered, spacing increase (for a range of network size $n$ ), and the right border before the next row starts. A basin of attraction field may be displayed as successive single basins as well as in a group. Initial default layout values depend on the type of attractor basin selected. Once revised, the new values generally become defaults, but may be reset to the original values.

If $\mathbf{l}$ is selected at the first "basin parameter" prompt in section 24.1 (or if the basin parameters prompts are viewed in sequence) a layout preview for attractor basins will appear, together with the first layout prompt described below (section 25.2). Some layout parameters may also be varied on-the-fly, after each subtree or basin is drawn (section 25.3).

The basin of attraction field can also be redraw within the attractor jump-graph window, according to the jump-graph layout (section 20.7) providing many additional layout possibilities to those described in this chapter.

\subsection{The layout preview}

The layout preview shows the spacing and scale of attractor basins as they would appear on the screen according to the current default settings, and appears in the top-left quadrant of the screen, in a window representing the screen at $1 / 2$ size. Polar coordinates are used to scale and position basins, and these are indicated. $x$ and $y$ axes divide the screen with coordinates 0,0 at the center. At the left of the screen $x=-100$, at the right $x=+100$. At the top of the screen $y=+100$, at the bottom $y=-100$.

Basins are represented by concentric circles, where the inner circle is the maximum attractor diameter. Successive circles indicate successive levels. The first basin representation shows the first 90 levels where the distance between successive levels decreases asymptotically (thereafter the distance between levels remains constant). In the first basin every 20th circle is highlighted, as well the attractor circle itself and the first 3 levels. Other basin representations show the attractor circle and the first 3 levels only. If a single basin or subtree was selected (without a range of network size $n$ ) only one preview basin is shown. Otherwise a representative number of basins are shown to anticipate the layout (figures $25.1-25.2$ ). 

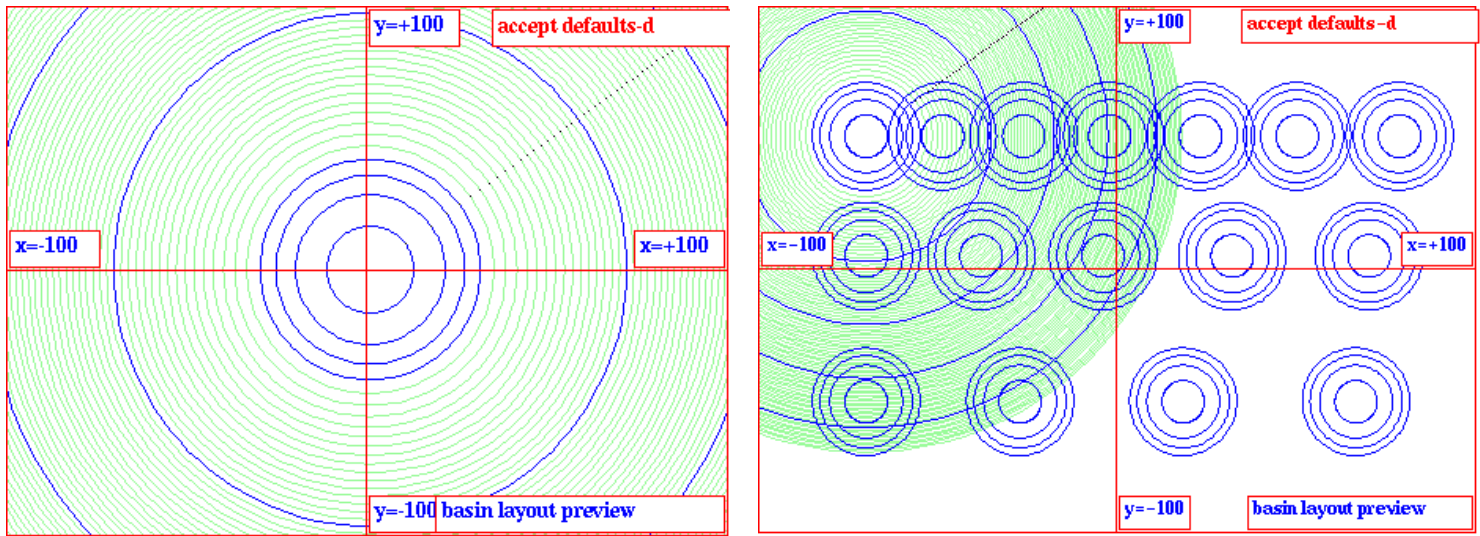

Figure 25.1: The default layout previews, Left: for a single basin or subtree, and Right: for a range of single basins.
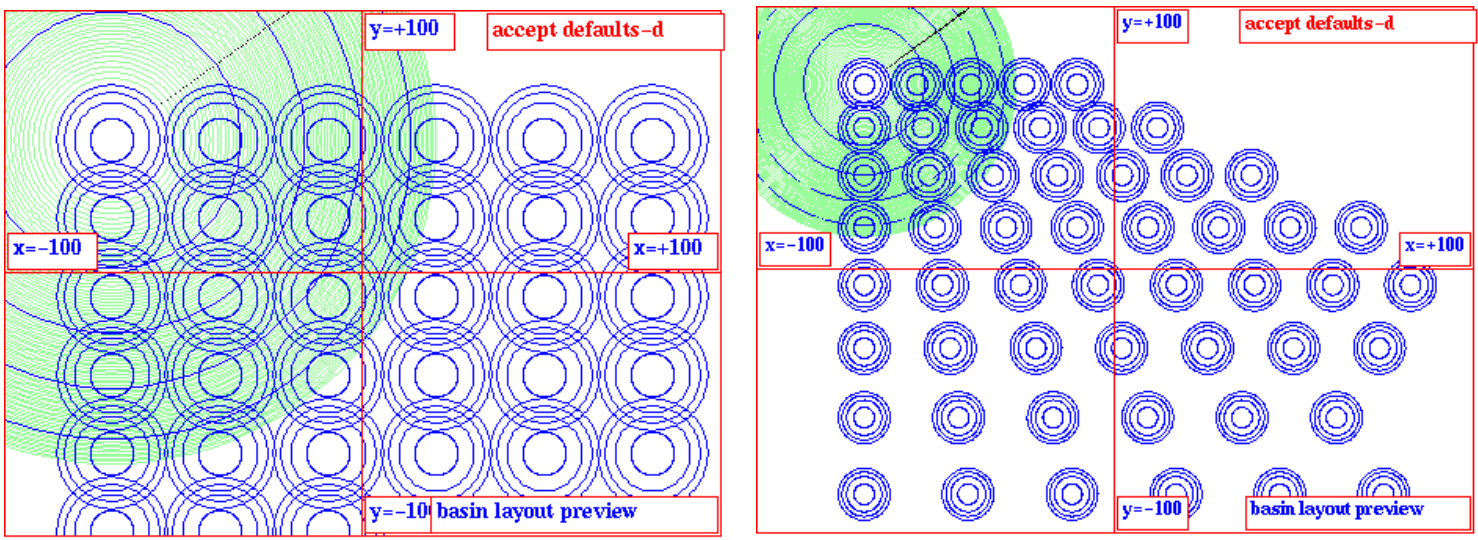

Figure 25.2: The default layout previews, Left: for a basin of attraction field, and Right: for a range of basin of attraction fields.

\subsection{The first layout prompt}

Enter $\mathbf{l}$ at the first output parameter prompt in section 24.1 to skip directly to the preview for the layout of attractor basins (section 25.1). Alternatively these options can be viewed in sequence.

The layout preview for attractor basins will appear (described above, section 25.1), together with the following (top-right) first layout prompt ,

layout: reset defaults: all-r mutants-M ( $\mathbf{M}$ for single basins only) select att rad, \% of $1 / 2$ screen, $x$-axis (min 0.24 def 6 ):

(min depends on screen resolution, the default depends on previous selections) 


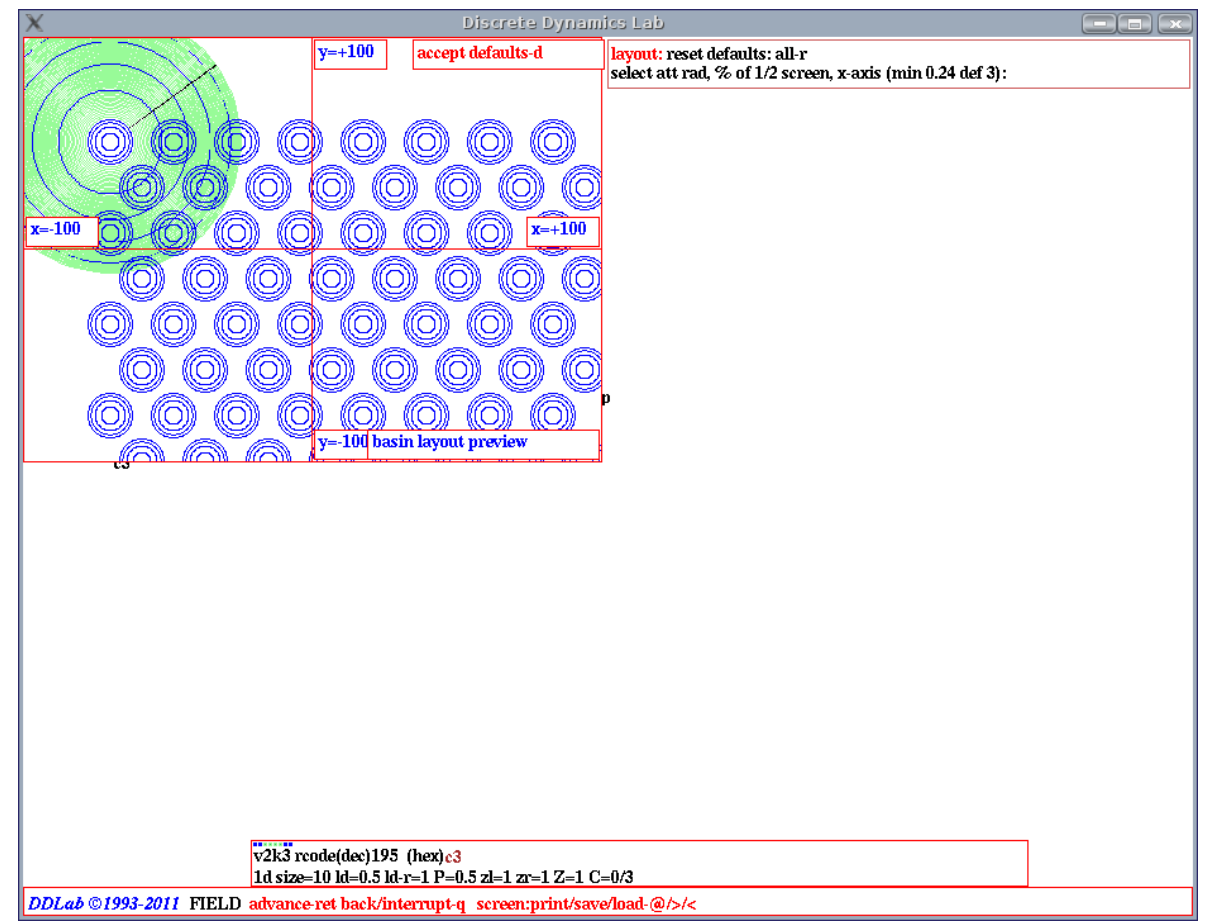

Figure 25.3: The layout preview as it appears on the DDLab screen. This examle is for a basin of attraction field, rad=3, start position: $x=-70, y=50$, spacing: $x, y$, and right border $=22$, with staggered rows.

\subsubsection{Reset all layout defaults}

Enter $\mathbf{r}$ in section 25.2 to restore all layout prompts to their original settings. These default settings depend on the type of attractor basin selected. A revised layout preview will be displayed.

\subsubsection{Mutants for single basins on one screen}

For single basins only, enter $\mathbf{M}$ in section 25.2 for a special default layout appropriate for displaying successive mutants on one screen - for example to display the set of one bit mutants of a rule, with the original basin shown by itself in the top row (section 28.3.1, figure 28.3).

\subsubsection{Basin scale, attractor radius}

The first layout prompt (section 25.2) gives the current attractor radius, scaled according to the $x$-axis. In the example, ( $\operatorname{def} 6)$ means $6 \%$ of $1 / 2$ of the screen width. To alter the default basin radius controlling the scale of attractor basins, enter a new radius - allowing up to two places after the decimal point. The minimum radius allowed depends on the screen resolution and will correspond to a scale of one pixel. A small scale is somtimes required for basins with very long transients. 


\subsubsection{Basin start position}

To alter the default position of the first basin, enter return in section 25.2. The following top-right prompt is presented,

start position, \% offset from screen center

$\mathbf{x}(\mathbf{d e f}-\mathbf{7 0}): \quad \mathbf{y}(\mathbf{d e f} \mathbf{5 0}): \quad$ (values shown are examples)

Enter the new polar coordinates for the first basin, first $x$, then $y$. As shown in figure 25.1, the screen center is at 0,0 and the edges of the screen are as follows, left $x=-100$, right $x=+100$, top $y=+100$, bottom $y=-100$. A position outside the screen limits is permitted ${ }^{1}$, allowing, for example, the relevant part of a large basin to be displayed.

\subsubsection{Show the field as successive basins FIELD-mode only}

In FIELD-mode (but not for a range of $n$ in section 8.1), a further option is presented (in the same window as section 25.2.4) to display each basin in the basin of attraction field one by one successive single basins at the same start position.

\section{show field as successive basins-s, and pause-p:}

Enter $\mathbf{s}$ to show successive basins without pausing. Enter $\mathbf{p}$ to pause after each basin - data on each basin (and other options) is will be displayed (section 27.2.3) - enter return will generate the next basin.

\subsubsection{Basin spacing and stagger rows FIELD-mode only}

To alter the default spacing between basins in a basin of attraction field (see figure 25.2 left), the following prompts are presented in sequence:

\section{basin spacing, $\%$ of $1 / 2$ screen (defaults depend on the radius set in section 25.2.3) $\mathrm{x}$ spacing (def 30): y spacing(def 30) tog stagger rows (now off)-s:}

Enter the new spacing according to the polar coordinate scale, first $x$, then $y$. Remember that the screen is 200 units wide and 200 units high. Lastly, enter $\mathbf{s}$ to toggle the staggering of alternate rows, as in figure 25.3

\subsubsection{Select minimum right border width FIELD-mode only}

To alter the default minimum right border width, enter the new border width as a percentage of the current $x$-spacing. If the distance between the center of the next basin and the right border is less than this setting, the basin will be drawn at the start of the next row. Negative values for the right border are permitted. The following top-right prompt is presented,

select right border as \% of $\mathrm{x}$ spacing (default $\mathbf{4 5 . 0 \% ) :}$

(the default right border depends on the spacing set in section 25.2.6)

\footnotetext{
${ }^{1}$ The center of attractors can be located up to one screen height below the bottom screen edge. Any attractors that would be located below this limit are repositiond on the limit, and their basin transients are not drawn.
} 


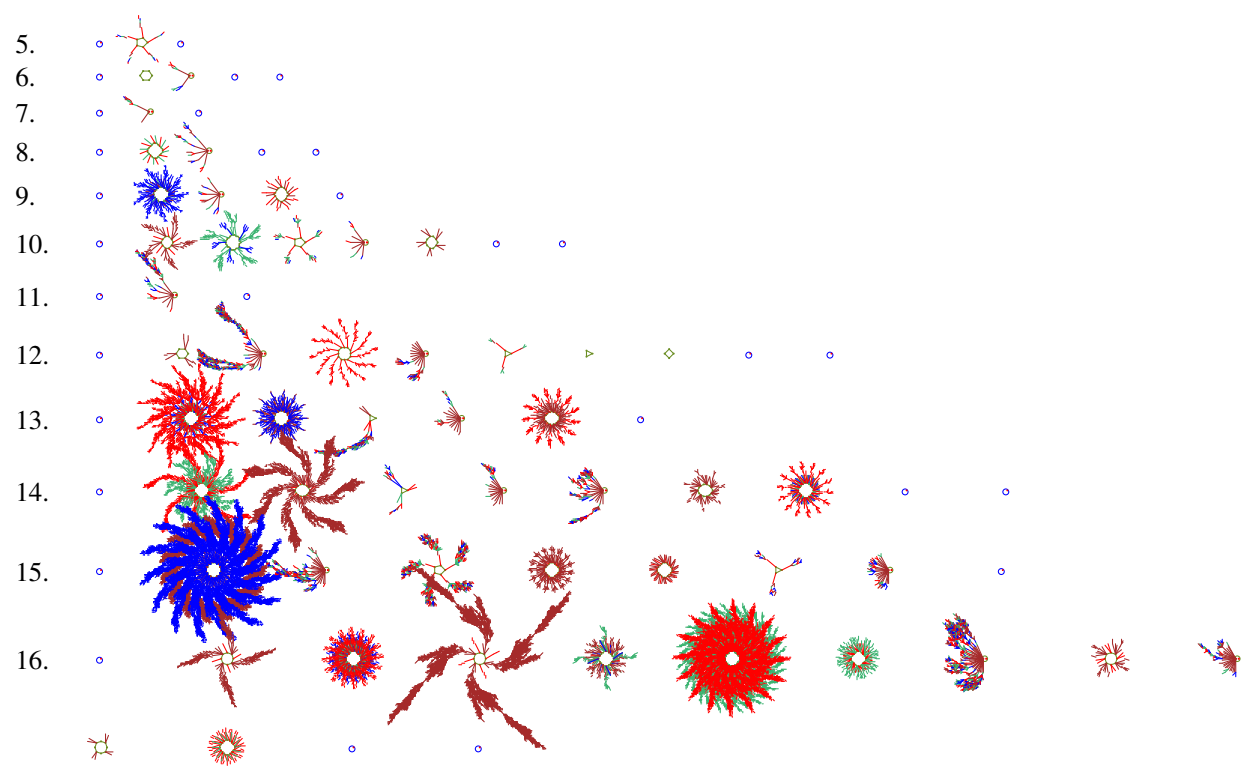

Figure 25.4: The basin of attracion field for a range of network size, $n=5-16$, shown on the left, $v 2 k 4$ rcode (hex)8b26. The spacing between basins increases at a set rate as larger basins are expected for greater $n$. In this example, the basin scale is 1.2 (section 25.2.3), the $x, y$ start position is $-90,70$ (section 25.2.4), the initial $x, y$ spacing belween basins is 6,6 (section 25.2.6), the right border width is $45 \%$ (section 25.2.7), and the $x, y$ spacing increase is set at $12 \%$.

\subsubsection{Amend the spacing increase for a range of $n$}

If a range of network size $n$ was selected in 8.1, the spacing between successive basins (and fields) is set to increases by a default percentage which may be amended (see figure 25.2 right). The increase is usually required because the size of basins tends to increase with greater $n$. The following prompt is presented,

$\%$ increase in spacing for successive network size,

$\mathrm{x}$ increace (def 10\%): y increase (def 10\%): (values shown are examples)

Enter the new percentage increase, first for $x$ (for the horizontal spacing), followed by $y$ (for the vertical spacing). This becomes the new default. An example of a range of basin of attraction fields is shown in figure 25.4 where the default setting were slightly amended, and in figure 4.5.

\subsubsection{Accept or revise layout parameters}

Finally, the layout preview showing the current settings (as amended) is displayed, with the following prompt to revise or accept,

\section{layout: revise-q, accept-ret:}

Enter $\mathbf{q}$ to revert to the first layout prompt (section 25.2) and re-adjust settings, or return to accept the layout and continue. 


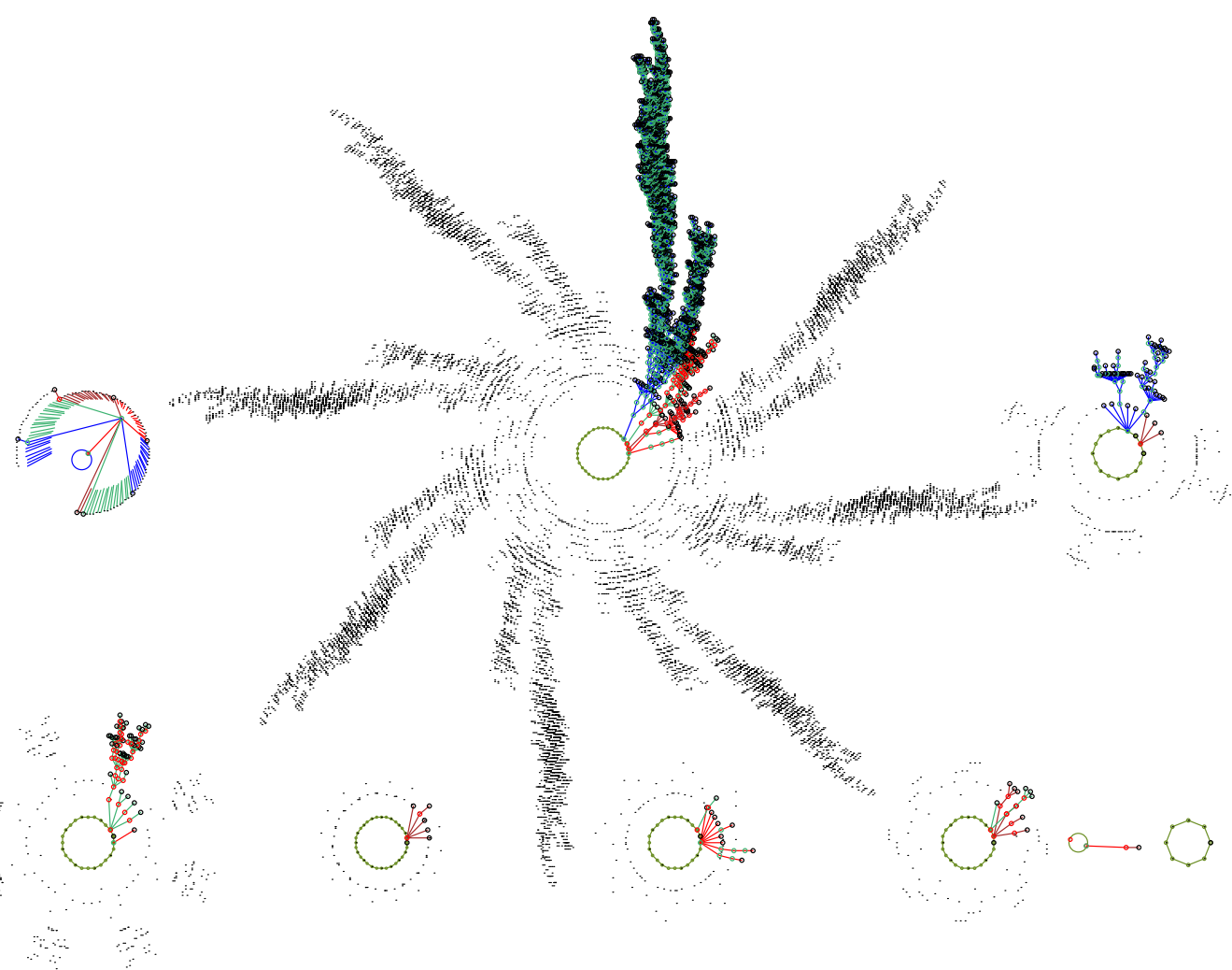

Figure 25.5: Amending the layout of a basin of attraction field on-the-fly, for a CA $v 2 k 3$, rcode (dec)110, $n=16$. The field is shown with copies of equivalent trees suppressed exept for garden-of-Eden nodes shown as dots (section 26.1.3). Layout parameters were set as follows: basin scale 3.8 (section 25.2.3), $x, y$ start position $-75,13$ (section 25.2.4), initial $x, y$ spacing belween basins is 65,73 (section 25.2.6), right border width 15\%, (section 25.2.7). A pause after each basin was set (section 27.1.2) allowing the $x$ spacing to be changed - after the 4th basin to 40 , after the 7 th basin to 15 .

\subsection{Amend the layout during pause}

If one of various "pause" options is selected in section 27.1.2, for a basin of attraction field or range of fields, layout options become available after each basins (or field) is draw, including the angular orientation, subtree fan angle, and the spacing and position of each successive basin. At the pause, data will be displayed in a top-right window (chapter 27), and one of the following prompts will be presented in a small top-center window (section 27.1.3) as follows,

next field-ret nopause-q ops-o layout-a: (for a field-pause)

next basin-ret nopause-q ops-o layout-a: (for a basin-pause)

Enter a for the layout options (for others see section 27.1.3) - the following top-right prompt, amend angles-a next pos-p just spacing-ret:

These options are described in sections 25.3.1 to 25.3.4 below. 


\subsubsection{Amend the orientation and fan angle during pause}

Enter a in section 25.3 to amend the basin orientation, and the "fan angle" — the spread of in-degree edges at nodes. Further prompts will be presented in turn,

change basin orientation (now 0), enter angle:

change pre-image fan angle(now 1.00), enter factor: (values shown are examples)

See section 26.3 for further details about these settings.

\subsubsection{Amend the spacing and right border during pause}

If return is entered in section 25.3 to amend the spacing (and right border). Prompts are presented as described in section 25.2.6 and 25.2.7 above. Figures 25.5 and 27.1 give examples.

\subsubsection{Amend the next position during pause}

Enter $\mathbf{p}$ in section 25.3 to amend the next position (as well as the spacing). Prompts are presented similar to those described in section 25.2.4 above,

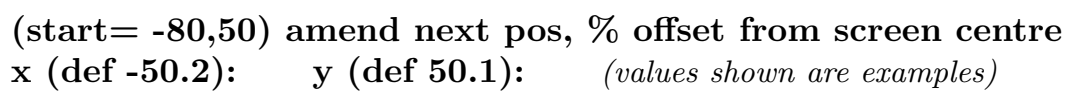

The last start position is shown as a reference. Enter the next position's polar co-ordinates, first $x$, then $y$. Remember that the screen center is at 0,0 and the edges of the screen are as follows, left $x=-100$, right $x=+100$, top $y=+100$, bottom $y=-100$. A position outside the screen limits is permitted. See section 25.2.4 for further details about these settings. Figures 25.5 and 27.1 give examples.

After the new position is set the new $x$ co-ordinate becomes the new default $x$ start position for future basins, the default $y$ co-ordinate is not affected.

When this option is complete, the spacing options 25.2.6 and 25.2.7 are presented.

\subsubsection{Amend the spacing increase during pause}

If a range of network size $n$ was selected in 8.1, the spacing increase between successive basins can be amended as described in section 25.2.8. 


\section{Chapter 26}

\section{Display of attractor basins}

not in TFO-mode.

This chapter describes various options to change the default display of attractor basins. Enter $\mathbf{p}$ at the first "output parameter" prompt in section 25.2 to jump directly to the display prompts, or reach them by viewing the output parameter in sequence. For local $1 \mathrm{~d}$ or $2 \mathrm{~d} \mathrm{CA}$, attractor basins can be "compressed" (section 26.1). In this case the first prompt is in section 26.1.1. Otherwise the first prompt is in section 26.2.

\subsection{Compression of equivalent CA dynamics}

If the network is a local $1 \mathrm{~d}$ or $2 \mathrm{~d}$ CA (i.e. CA wiring and just one rule), rotational symmetries of the periodic array result in equivalent dynamics [25]. A given network state (periodic pattern) $A$ is embedded in a particular past and future made up of other connected network states. If $A$ is translated around its circle (1d) or torus (2d) by an arbitrary number of moves, and transformed to state $B$, the states in $B$ 's past and future must be identical transformations of the states in $A$ 's past and future. The two sets of states are rotational equivalents, and their connection topology is identical.

A complication arises because some states are made up of repeating pattern segments, for example 011011011011 has 4 repeating segments 011 . If the repeating segment size $=s$, there will be only $s$ rotational equivalent states irrespective of the network size $n$, whereas with no repeating segments (always the case if $n$ is prime), the number of rotational equivalents is $n$. This becomes more complicated for a $2 \mathrm{~d}$ torus were repeating segments exist in two directions, and errors may occur in the algorithm in this case. Compression in $3 \mathrm{~d}$ has not been implemented.

Note also that states with a given repeating segment size $s$, cannot be upstream of a state with greater $s$. In an attractor cycle the value of $s$ for all the states must be equal. The uniform states where all values are equal (all 0 s and all 1 s for binary) with the shortest repeating segment size $(s=1)$ are often powerful attractors.

For local 1d and 2d CA, "compression" algorithms automatically come into play (unless disabled in section 26.1.1) to compute equivalent basins, transient trees, and subtrees from the uniform states, from each prototype. This considerably speeds up computation. For equivalent basins, only the prototype is shown. The display of equivalent trees and subtrees may also be suppressed to varying degrees. 


\subsubsection{Suppress compression}

The first prompt for local 1d or 2d CA allows compression (section 26.1) to be suppressed (or reinstated if turned off). The top-right prompt gives a reminder of the $v, k$ and $n$ (or $i \times j$ for $2 \mathrm{~d}$ ), and is presented as follows,

display: local 1d CA, v2k3 size=10 (for example)

suppress compression in basins/fields is ON, off-s: (or ... is OFF, on-s)

Enter $\mathbf{s}$ to suppress (or reinstate) the compression algorithms.

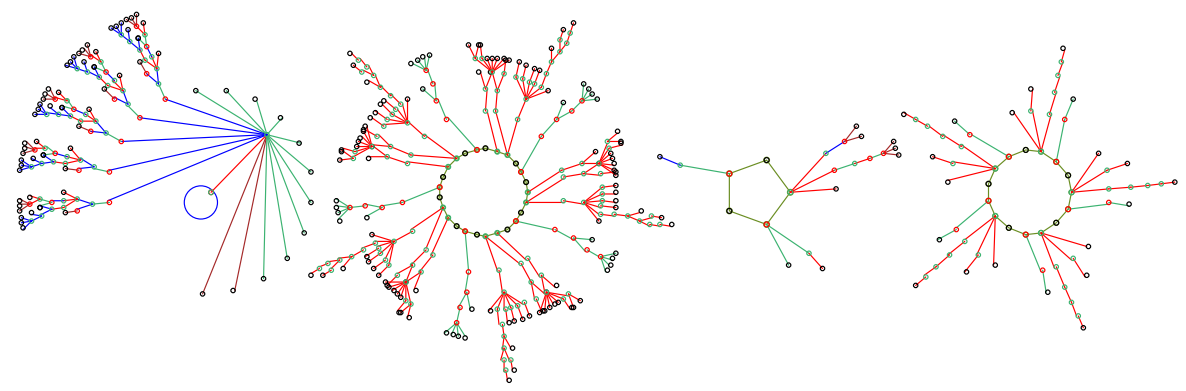

Figure 26.1: A basin of attraction field with compression of rotational equivalent dynamics, $v 2 k 3$ rcode $(\operatorname{dec}) 110, n=10$. Only one prototype of each set of equivalent basins is drawn. In addition, equivalent trees on attractor cycles (2nd basin), and subtrees from a uniform states (1st basin) are copied and rotated, thus further speeding up computation as a reverse algorithm need not be re-applied.

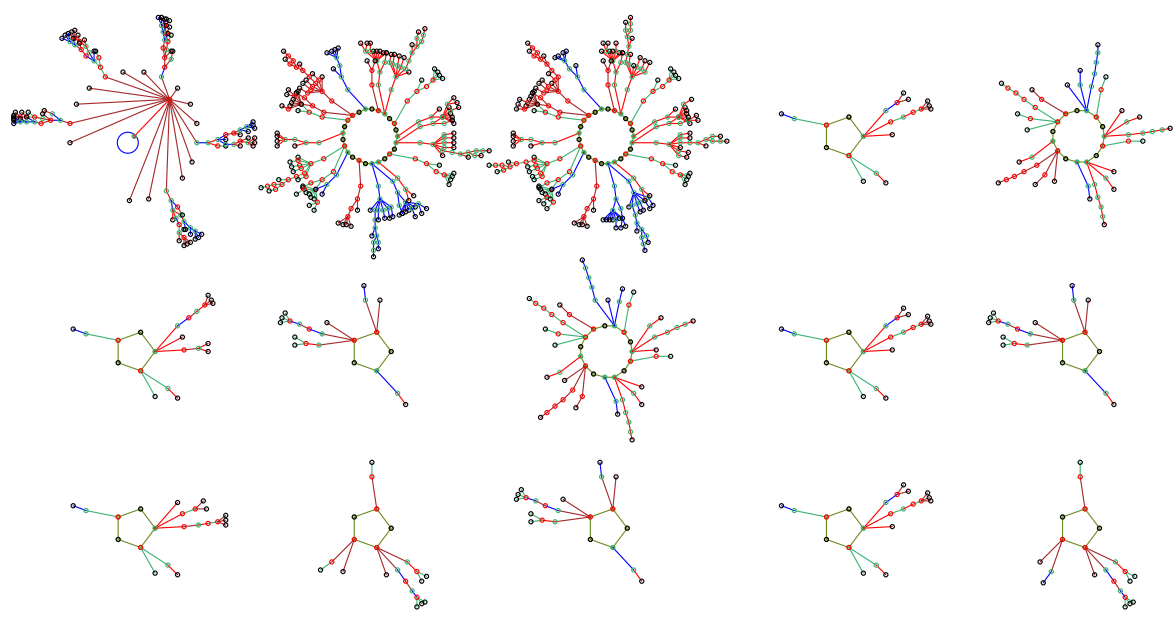

Figure 26.2: The same basin of attraction field as in figure 26.1 without compression of equivalent dynamics, so that all components of the state transition graph are computed from scratch with a reverse algorithm, not copied and rotated as in figure 26.1. All states in state space are represented. 

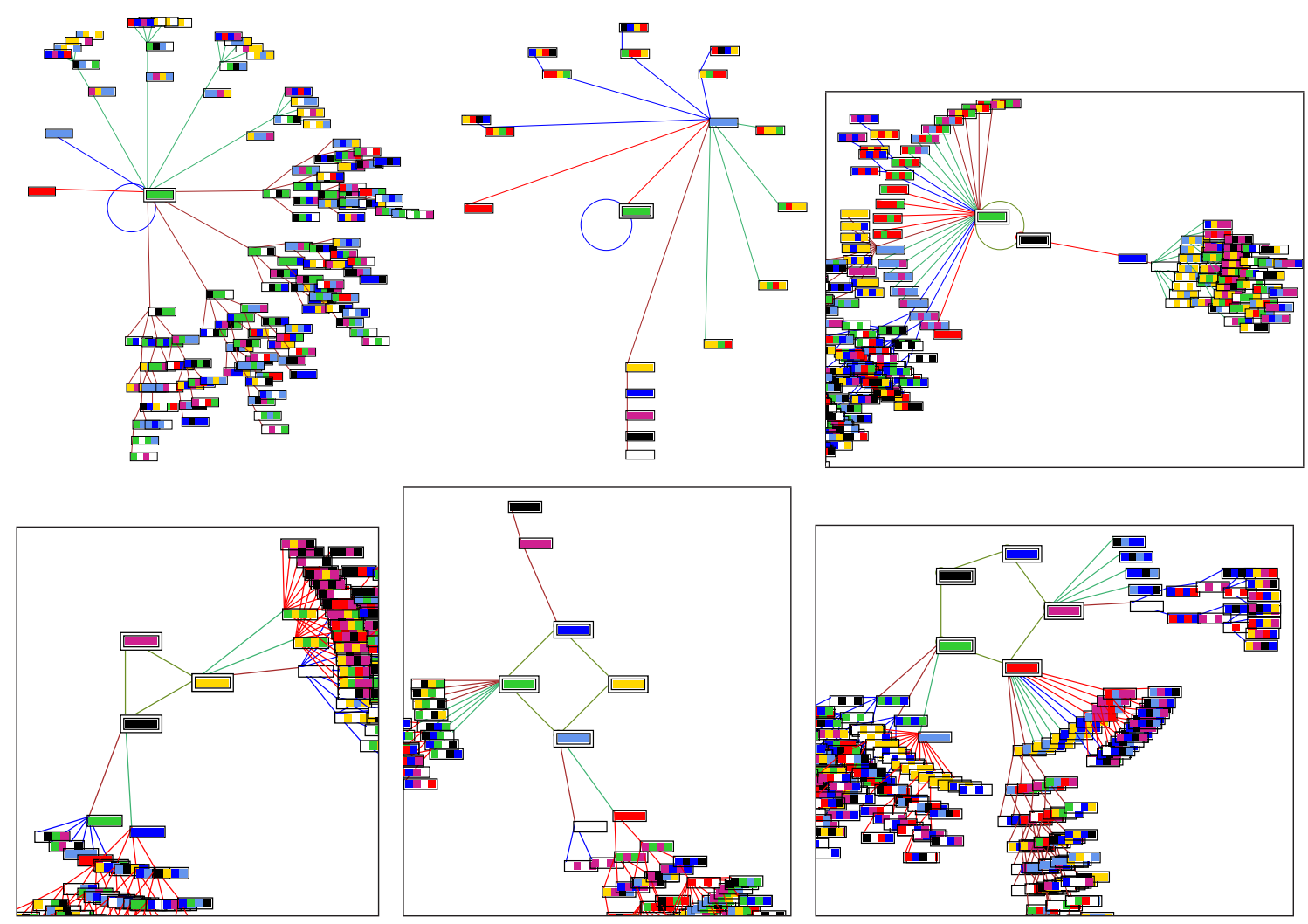

Figure 26.3: The special properties of CA uniform states, which lie on or very close to attractors, allow speeding up computation of their pre-image fan and attached trees. Each (sub)tree type only needs to be computed once, the equivalent (sub)trees simply have their states rotated. The pre-image fan is organized into equivalent groups. This is illustrated here by some $1 \mathrm{~d}$ CA $v 8 k 2$ rcodes $n=4$, but the method applies equally to binary, $v=2$, or any value-range $v$. States are shown as $v=8$ value patterns - attractor states are highlighted by default inside a double outline. The attractor periods shown range from 1 to 5 . The framed basins are fragments centered on the attractor.

\subsubsection{Pre-images of uniform states}

The uniform states, where all cell values are equal, enjoy a privileged status in the dynamics of CA - they have maximum rotational symmetry (RS) so lie on or very close to attractors. Uniform states can only be pre-images of each other ${ }^{1}$. States with higher RS must be downstream of states with lower RS - all states in an attractor must have equal RS[25]. Take any pre-image of a uniform state - all its rotations must also be valid pre-images, so this property allows a method to speed up computation (if "compression" is on, section 26.1) as well as organizing the pre-image fan and attached trees into equivalent groups. At present the method applies to uniform states on the attractor, or to a uniform state that is the unique pre-image of a point attractor (figures 26.3, 26.8). In principle this could be extended to any uniform state, and also to states with repeating segments in general - this is not implemented in DDLab at present.

\footnotetext{
${ }^{1}$ The property that uniform states can only be pre-images of each other applies to any system with a homogeneous rule - RBN and DDN as well as CA. However, the uniform state equivalent tree method only applies to CA.
} 


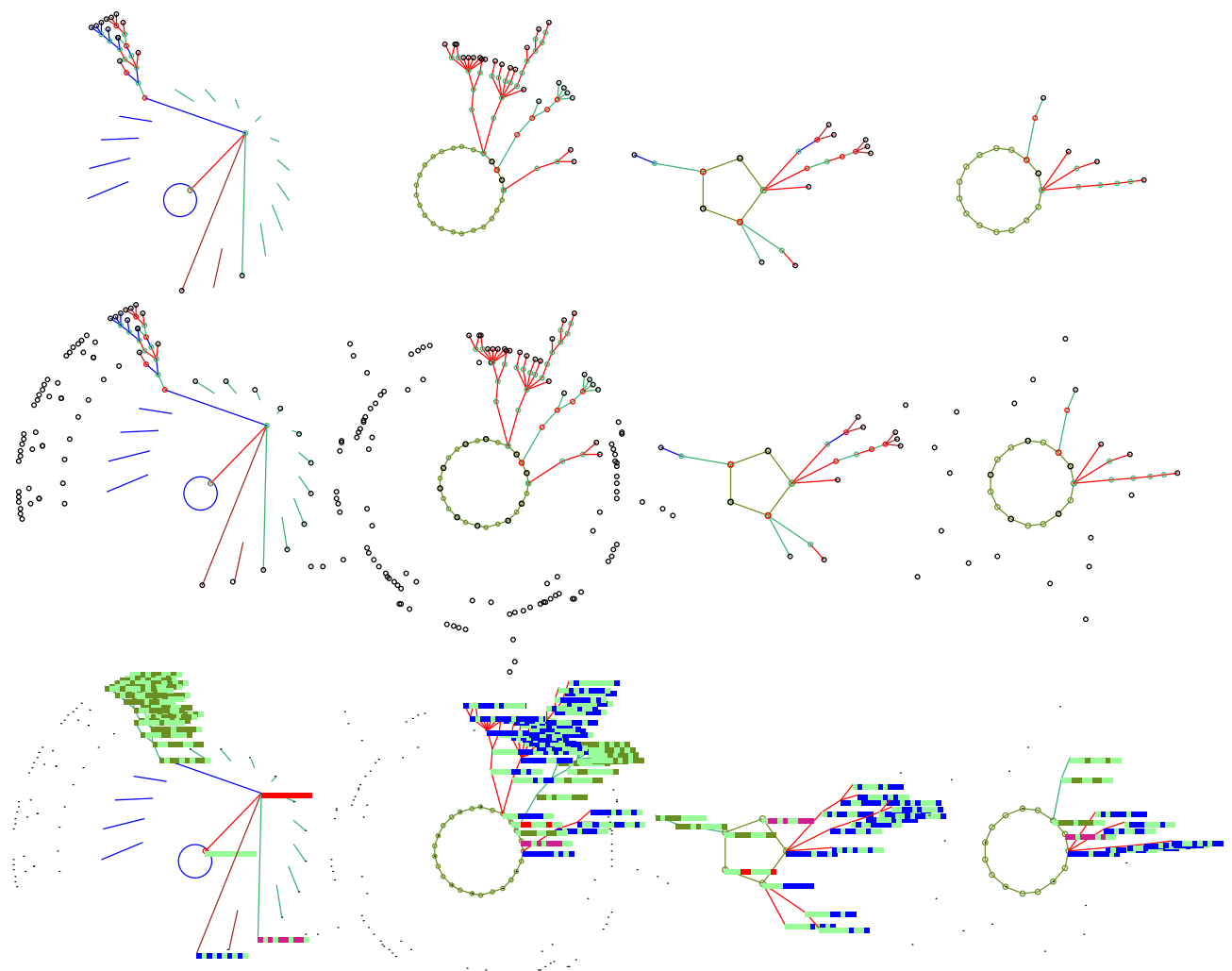

Figure 26.4: Suppressing equivalent subtrees in a basin of attraction field, $v 2 k 3 \operatorname{rcode}(\mathrm{dec}) 110, n=10$, (as in figure 26.1). Top: all equivalent subtrees suppressed, however, short edges are still shown from a uniform state (1st basin). Center: leaf states in the suppressed subtrees shown as spots, and Bottom: as one pixel dots. Nodes in the prototype trees are shown as selected in section $26.2-$ in the latter case as a $1 d$ bit pattern.

\subsubsection{Suppress copies of trees (and subtrees)}

If compression remains set, a further option is offered to suppress copies of transient trees from attractor cycles, and subtrees from uniform states. However, the garden-of-Eden (g-of-E) or leaf nodes can be retained as spots/dots to indicate the footprints of the suppressed transients (figures $25.5,26.4$ ). The prototype tree/subtree is drawn in full with whatever node display set in section 26.2. The following top-right prompt is presented,

\section{copies of trees (\& subtrees from uniform states) suppress: all-3, show only g-of-E nodes: normal-2 small-1:}

Enter 3 to suppress all copies of equivalent trees or subtrees. This results in a clearer picture of crowded basins. Alternatively, to retain just the leaf nodes in the equivalent trees or subtrees, showing just their footprints, enter $\mathbf{2}$ for bold footprints, or $\mathbf{1}$ for subdued footprints where each leaf node is shown as one pixel. 

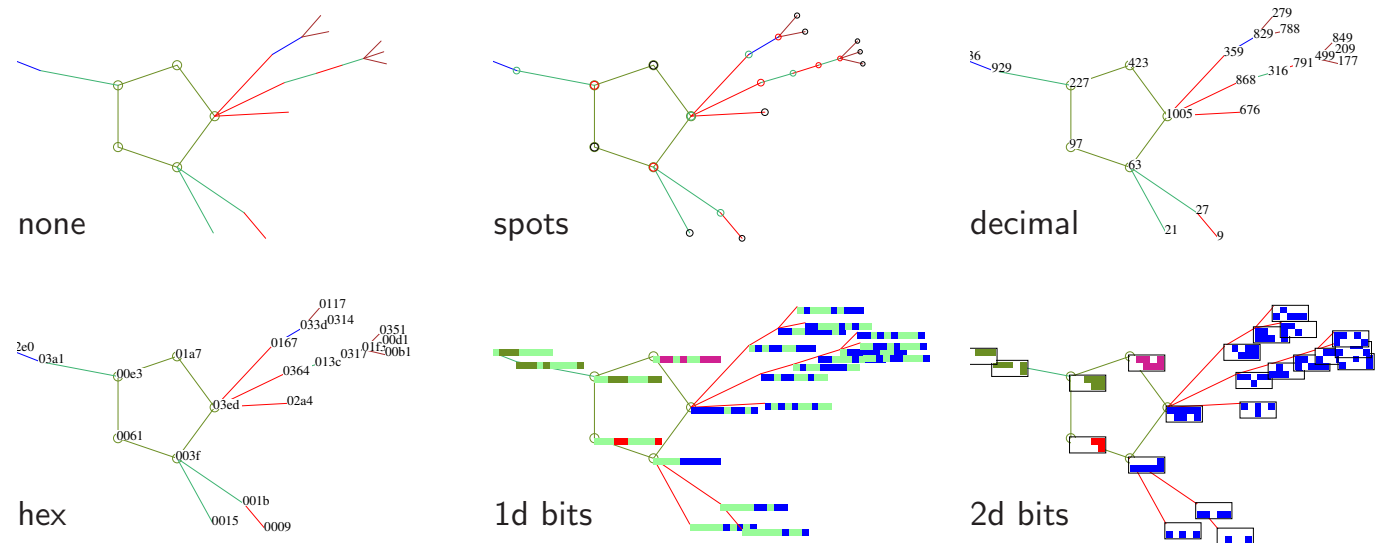

Figure 26.5: Examples of alternative node display. from the Top-Left: none (except on the attractor): spots, decimal, hex, $1 \mathrm{~d}$ bit/value pattern, and $2 \mathrm{~d}$ bit/value pattern, where the $i \times j$ dimensions can be preset. This is the small basin in figures $26.1-26.4, v 2 k 3$ rcode $(\mathrm{dec}) 110, n=10$.

\subsection{Node display}

The nodes in attractor basins may be displayed in a variety of ways (summarized below) as small circles or spots, as scalable bit/value patterns in $1 \mathrm{~d}$ or $2 \mathrm{~d}$, as the hex or decimal value of the pattern, or nodes need not be shown. Whatever the selection, node display can be restricted to just rotationally symmetric states (figure 26.6), just leaf states, or states other than leaf states.

As well as the primary method of node display, attractor nodes can be independently highlighted as bit/value patterns. Pre-defined sets of nodes can also be highlighted in the "learning" routines described in chapter 34. A top-right prompt to set the node display (similar to the following) is presented, with options summarized below. The default depends on previous selections,

nodes: 2d-B 1d-b hex-h dec-c spot-s (dec-c if applicable, 2d-b for a 2d network)

just Sym-nodes $+\mathrm{S}$, just g-nodes $+\mathrm{g}$, no g-nodes $+\mathrm{G}$, none-n (def-s):

(def- shows the current setting)

For networks other than local $1 \mathrm{~d}$ or $2 \mathrm{~d} \mathrm{CA}$, this is the first prompt in the display sequence, preceded by the title display: - the prompts in sections 26.1.1 and 26.1.3 are omitted.

options $\cdots$ type of node

2d-B ... as a $2 \mathrm{~d}$ bit/value patterns for $1 \mathrm{~d}$ (or $3 \mathrm{~d}$ ) networks).

$\mathbf{2 d - b} \ldots$ as a $2 \mathrm{~d}$ bit/value patterns for $2 \mathrm{~d}$ networks.

$\mathbf{1 d}-\mathbf{b} \ldots$ as $1 \mathrm{~d}$ bit/value patterns for (1d or $3 \mathrm{~d}$ networks).

hex-h ... in hex (hexadecimal).

dec-c ... in decimal ((if applicable - section 21.6).

spot-s ... as small circles or spots, cycling through 4 colors to contrast with transient edges colors, except garden-of-Eden states which are colored white. 


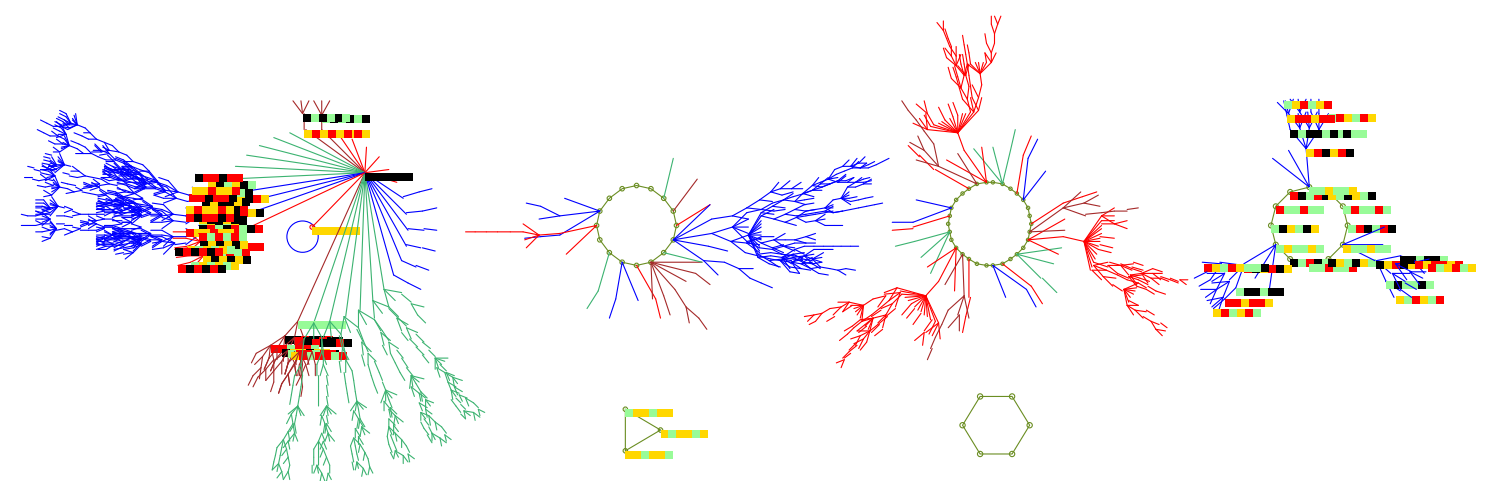

Figure 26.6: Symmetrical states only, those made up of repeating segments (including the uniform states) are shown as value-pattern nodes in a basin of attraction field - other nodes are suppressed. Symmetric states occur in basins 1, 4 and 5 .

$v 4 k 3$ CA, $n=6$, rcode(hex) 4d3f8c86143ffca77a0ed4b5d172e7c3.
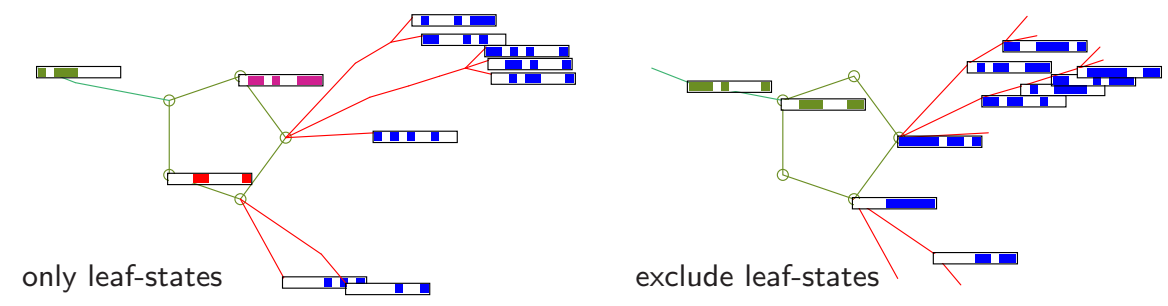

Figure 26.7: Showing just leaf-states or excluding leaf-states, $v 2 k 3$ rcode(dec)110, $n=10$, (as in figure 26.4). Left: only leaf-states and attractor states without transients are shown. Right: leaf-states and attractor states without transients, are excluded. The nodes are shown as $2 \mathrm{~d}$ bit patterns $10 \times 1$.

\section{+options ... to restrict node display}

just Sym-node $+\mathbf{S} \ldots$ add $\mathbf{S}$ for just rotationally symmetric states consisting of repeating segments, which include the uniform states. For example enter bS for figure 26.6 .

just g-nodes $+\mathrm{g} \ldots$ add $\mathbf{g}$ for just leaf states (and attractor states without transients). For example enter $\mathbf{B g}$ for figure $26.7 \mathrm{Left}$.

no g-nodes $+\mathbf{G} \ldots$ add $\mathbf{G}$ to exclude leaf states (and attractor states without transients). For example enter BG for figure 26.7 Right.

none-n ... to omit nodes altogether.

The options for node display can be combined with any other node options - compression (sections 26.1.1 - 26.1.3) and highlighting attractor and subtree root nodes (section 26.2.2).

The colors of spots cycle through four colors to contrast with transient edge colors (section 23.4.1). Decimal and hex numbers are shown black. For $v \geq 3$ nodes displayed as $1 \mathrm{~d}$ and $2 \mathrm{~d}$ value-patterns follow the default value colors (chapter 7 ), but for binary systems the colors of 1s vary to distinguish trees — described below (section 26.2.1). 


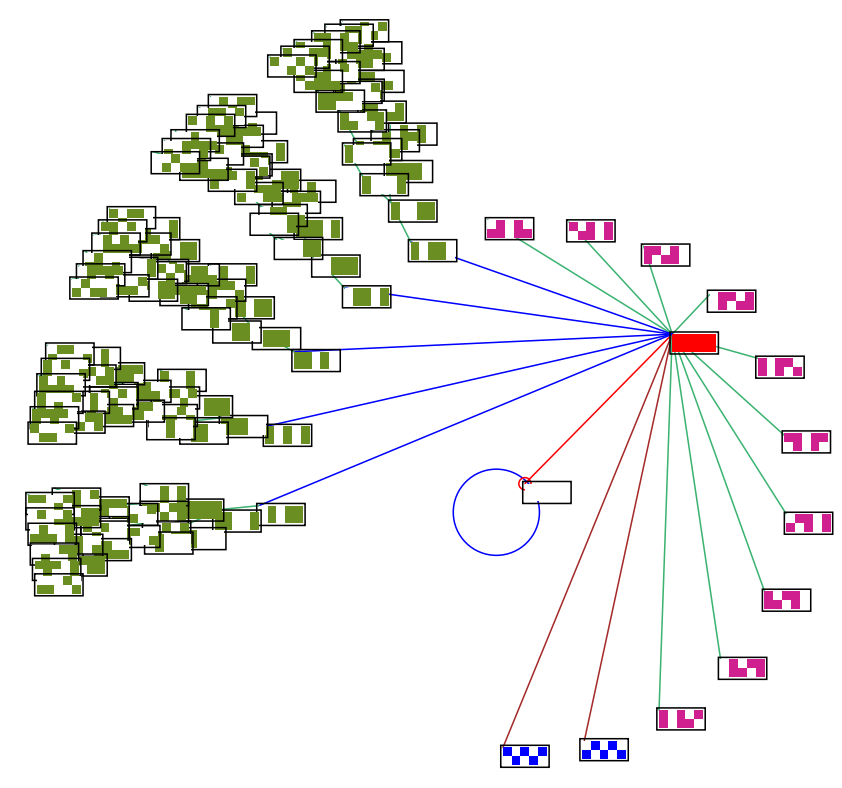

Figure 26.8: The pre-images of a uniform state's tree are organized into groups of equivalents. For binary systems, the bit patterns of these states, and states in their subtrees are shown in the same color, cycling through four colors. Nodes are shown as $2 \mathrm{~d}$ bit patterns $5 \times 2$. This is the first basin in figure 26.1, v2k3 rcode(dec) $110, n=10$.

\subsubsection{Node colors}

When nodes are displayed as spots (the default), the colors of transient nodes are set to one of 4 colors contrasting with the colors of transition edges (section 23.4.1) where a cycle of four colors distinguishes transient trees for attractor periods of 6 or more. This is also the case for the bit patterns color of $1 \mathrm{~s}$ in binary systems, whereas for $v \geq 3$ the default value colors apply (chapter 7 ). Leaf (garden-of-Eden) nodes as spots are black. 2d bit patterns have an outer border, $1 \mathrm{~d}$ bit patterns do not.

In binary systems, nodes in the same pre-image fan are drawn in the same color (except for the uniform states, figure 26.8). If the attractor period is 5 or less, successive fans are assigned a different color so that a given transient tree may have a mix of colors. For attractor periods of 6 or more, all nodes in the same tree are assigned the same color, and the color is changed for the next non-equivalent tree. Note that with compression on (section 26.1) equivalent trees will be colored identically, and the pre-images of a uniform state's tree are organized into groups of equivalents. These states, and states in their subtrees, are shown in the same color, cycling through four colors.

\subsubsection{Highlight attractor, or subtree root, in $2 \mathrm{~d}$}

One or all attractor states, or the root state in a subtree, can be highlighted as a bit/value pattern in $2 \mathrm{~d}$, irrespective of the overall node display set in section 26.2. The following top-right prompt is presented,

\section{highlight attractor (or subtree root) in $2 \mathrm{~d}$ : one-1 all-a:}

Enter 1 to highlight one attractor state - this state is the last attractor state reached in the initial forward run, and is positioned due east on the attractor cycle (figure 26.13). 


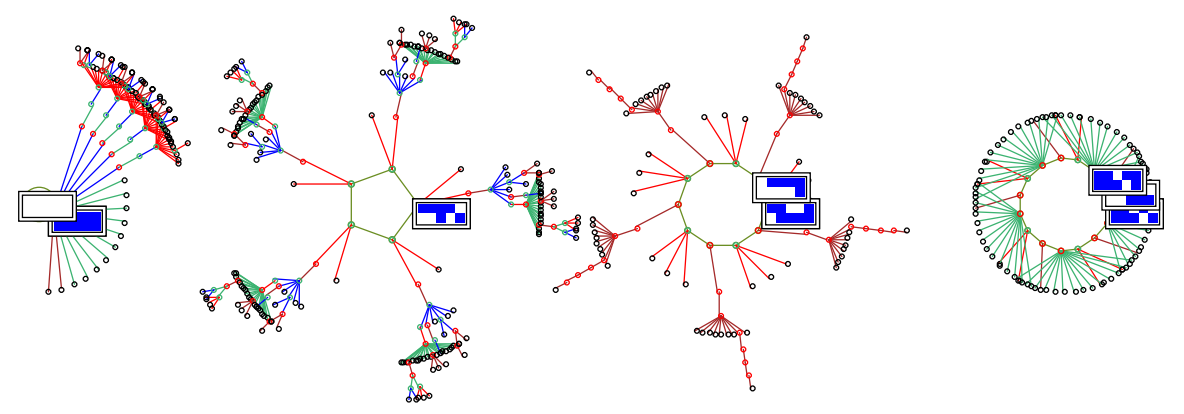

Figure 26.9: Highlighting all non-equivalent attractor states as a $2 \mathrm{~d}$ bit pattern for the basin of attraction field of a $1 \mathrm{~d}$ CA, $v 2 k 3 \operatorname{rcode}(\mathrm{dec}) 111, n=10$. Other node are shown as spots.
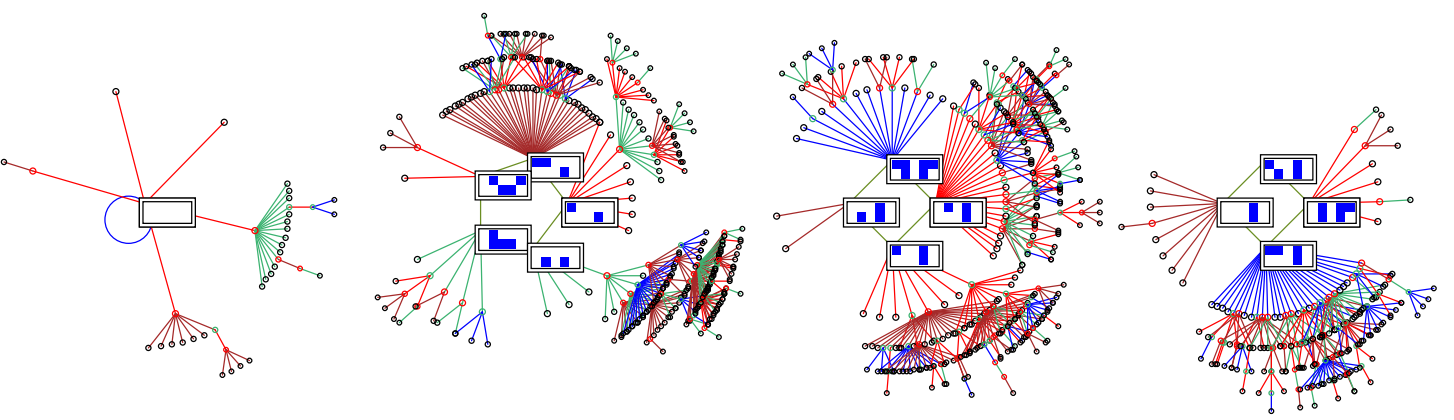

Figure 26.10: Highlighting all attractor states in a RBN basin of attraction field, $v 2 k 4, n=10$. Other node are shown as spots.

Enter a to highlight all non-equivalent attractor states for local 1d or 2d CA (figure 26.9) - if compression is set in section 26.1.1. Otherwise if $\mathbf{a}$ is entered all attractor states will be highlighted, as in figure 26.10 for a RBN. If either $\mathbf{1}$ or $\mathbf{a}$ is entered, and running backwards for a subtree is subsequently selected in section 29.1, the subtree root will be highlighted as a bit pattern as in figure 26.12

\subsubsection{Change the $2 \mathrm{~d}$ node $i, j$}

$2 \mathrm{~d}$ bit/value patterns at all or some nodes are selected by entering,

2d-B ... for $2 \mathrm{~d}$ bit/value patterns for $1 \mathrm{~d}$ ( or 3d) networks (section 26.2).

$\mathbf{2 d - b} \ldots$ for $2 \mathrm{~d}$ bit/value patterns for $2 \mathrm{~d}$ networks (section 26.2).

$\mathbf{1}$ or $\mathbf{a}$... to highlight all attractor states, just one, or a subtree root (section 26.2.2).

A 2 d network retains its $i, j$ (a 3 d network its $i$ ) as the default, set in sections 11.6.1 or 12.3. For $1 \mathrm{~d}$ networks a default $i, j$ is chosen automatically, where $j$ (the number of rows) is the highest whole factor of $n$, where $j \leq \sqrt{n}$ (see also section 21.4.6). For any dimension, the following top-right prompt allows the default $i$ to be revised,

2d node ij now $7 \times 2$ : reset i $(\max 14)$ : (example for $1 d n=14)$ 
The network is broken up into successive rows starting with the maximum cell index in the top-left hand corner. If the $i$ selected is not a whole factor of $n$, some cells will be missing from the bottom row, which is evident if dots are activated in section 26.2.4.

\subsubsection{Alter scale, divisions and dots - node as bits/values}

If nodes are set as bits/values either in $1 \mathrm{~d}$ or $2 \mathrm{~d}$ (section 26.2) or if the attractor or subtree root nodes are highlighted in $2 \mathrm{~d}$ (section 26.2.2), divisions between cells, dots at zero values, or both, can be added to the basic presentation, illustrated in table 26.1 below,

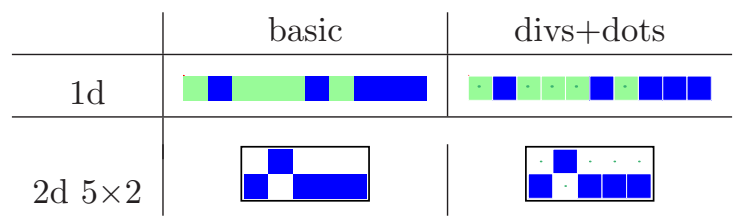

Table 26.1: Alternative presentations for bit/value nodes, $v=2, n=10$

For a cell scale in pixels $p \leq 5$ the presentation without divisions or dots is the default. The scale can be changed, and divisions/dots toggled, with the following top-right prompts, which are presented in turn, for example,

\section{alter cell scale, now 5 pixels: tog divs (now OFF)-i: tog dots (now OFF)-t:}

If required, enter the new cell scale in pixels, then toggle the divisions, then the dots, from their present settings (ON or OFF). These new setting become the new defaults, and will also be applied in a PostScript image (section 24.2). Note that divisions and dots will not apply if the cell scale in pixels $p \leq 3$. The cell scale in "backwards" space-time patterns (section 24.10) and the cell scale of basin nodes as bit/value patterns described in this section, will be the same. Both can be changed on-the-fly as basin are being drawn (section 30.3).

\subsubsection{Alter decimal or hex node scale}

If nodes were set as decimal or hexadecimal in section 26.2 the default node label size is set automatically depending on the basin scale (section 25.2.3). This default can be altered by a given factor. The following top-right prompt is presented, for example,,

dec/hex node scale (now 10), enter factor (now 1.0), original-o:

\subsection{Change orientation, fan angle, edge color}

The next three prompts allow the default orientation of attractor basins (section 23.3), the default pre-image fan angle, and the default edge colors, to the changed. The following top-right prompts are presented in turn,

change basin orientation (now 0), enter angle:

change pre-image fan angle (now 1.00), enter factor:

change start edge color (now 0 ) $-0-1-2-3-3$ : 

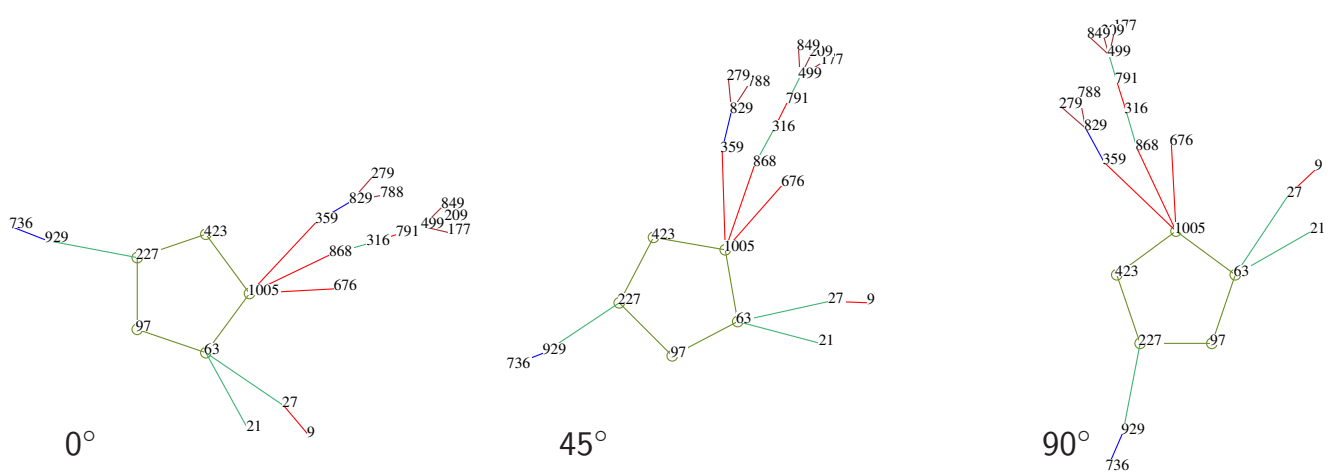

Figure 26.11: Changing the orientation of a single basin of attraction, starting with $0^{\circ}$, then setting $45^{\circ}$, and finally $90^{\circ}$. Labels are in decimal with a seed of $63, v 2 k 3$ rcode(dec) $110, n=10$.

\subsubsection{Orientation}

Changing the orientation allows attractor basins to be rotated anti-clockwise by the angle that is entered at the prompt - change basin orientation (now 0), enter angle: - in section 26.3. The default is $0^{\circ}$ due east. figure 26.11 gives examples. Note that for a single basin of attraction, with the orientation at $0^{\circ}$, the seed state is positioned one step clockwise from east. Changing the orientation works for all types of attractor basins. The orientation of individual basins in a field can also be changed during a pause as fields are drawn (section 30.2).

\subsubsection{Pre-image fan angle}

The pre-image fan-angle is the angle containing all the pre-image edges converging on a node - set automatically depending on the number of edges. It is sometimes useful to decrease the angle for denser branching occurring in large networks or ordered rules (figure 26.12), or to increase the angle for chaotic rules with low branching (figure 26.13). This is done by entering a multiplication factor at the prompt - change pre-image fan angle (now 1.00), enter factor: - in section 26.3. The fan-angle can also be changed during a pause as fields are drawn (section 30.2).
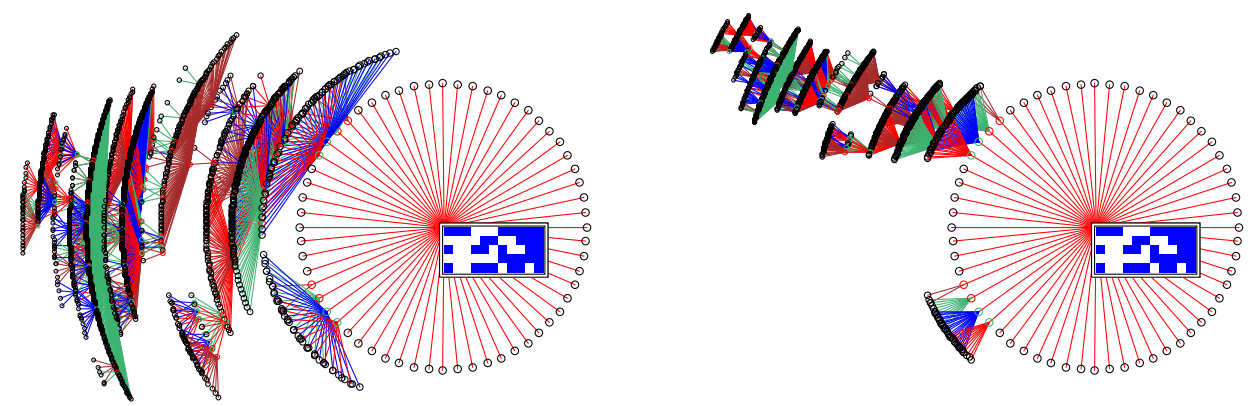

Figure 26.12: Decreasing the pre-image fan-angle of a subtree of a $1 \mathrm{~d}$ CA $v 2 k 3$ rcode(dec) $110, n=55$. The subtree root, (hex)71f0cf34c0fced, is highlighted as a bit pattern. Other nodes are show as spots. Left: default fan-angle (factor $=1)$. Right: reduced fan-angle $($ factor $=0.3)$. Note that the fan converging on the root of a subtree is always $360^{\circ}$, and is not affected. 

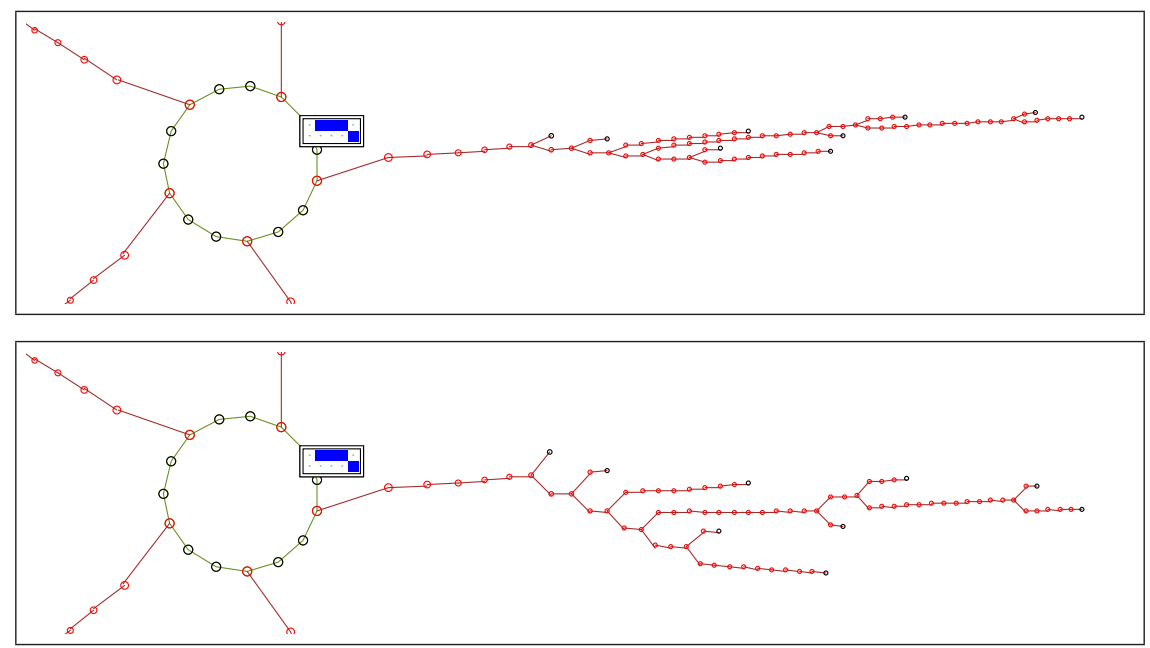

Figure 26.13: Increasing the pre-image fan-angle of a single basin of attraction of a $1 \mathrm{~d} \mathrm{CA}, v 2 k 3$ rcode $(\mathrm{dec}) 30 n=10$. This is a chain-rule with very low in-degree, characteristic of chaos. In each case, part of the basin including the attractor and one complete tree is shown. One attractor state is highlighted as a bit pattern. Other nodes are show as spots. Top: default fan-angle (factor $=1)$. Bottom: increased fan-angle (factor $=3)$. The basin orientation was also changed to $34^{\circ}$ (section 26.3.1).

\subsubsection{Edge color}

As described in section 23.4.1, a cycle of four colors is used to draw transition edges, and this also determines node colors (section 26.2.1). The resultant attractor basin color scheme ${ }^{2}$ depends on the start edge color (red is the default) which can be changed with this prompt in section 26.3, which also indicates the current color cycle,

change start edge color (now 0 ) $-0-1-2-3$ :
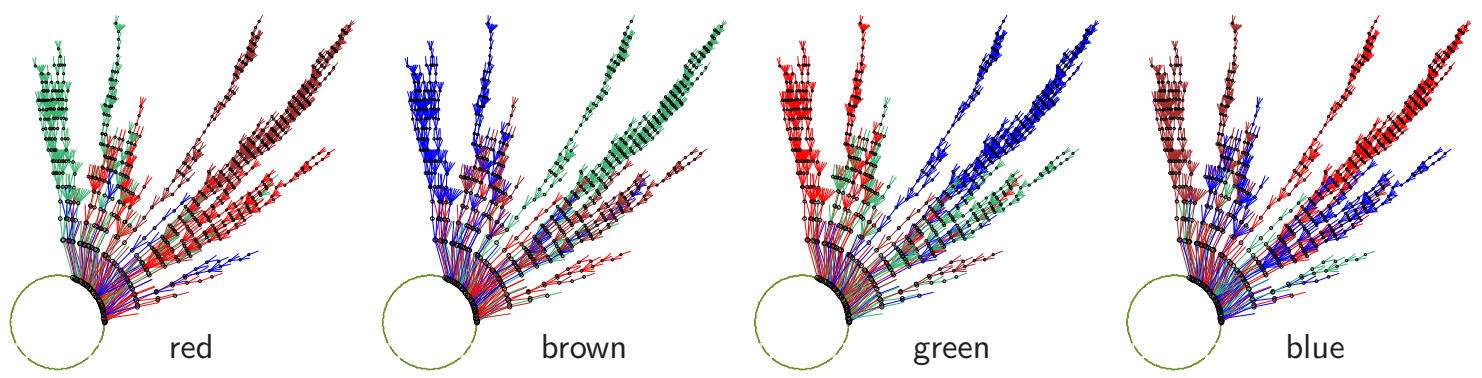

Figure 26.14: Alternative edge start colors (indicated) result in different basin color schemes. This is a single basin with copies of trees suppressed (section 26.1.3), the fan angle reduced by 0.5 (section 26.3.2) and leaf nodes excluded (section 26.2). Transient nodes are shown as spots. $v 2 k 3$ rcode(dec) $110, n=15$, seed(hex)660d.

\footnotetext{
${ }^{2}$ Colors are different on a black background (section 6.3.3).
} 


\section{Chapter 27}

\section{Pausing attractor basins, and data}

not in TFO-mode.

This chapter describes methods for pausing attractor basins according to a hierarchy of stages during drawing (section 27.1), to show/save/print data at each stage (section 27.1.1), and activate other options including amending the layout (section 27.1.3). The level of data detail can be set in section 27.3, including a complete sorted list of all the states (section 27.5). Printing/saving the data can be done with or without pausing. The data is generated as attractor basins are drawn. The methods apply to any attractor basin type - a basin of attraction field, a single basin or a subtree, or a range $n$ of the above.

To jump directly to the pause/data category of prompts, enter $\mathbf{t}$ at the first "output parameter" prompt in section 25.2, or arrive there by viewing the output parameters in sequence. When attractor basins are complete, a data summery is displayed in any case, but setting a pause allows intermediate data during the drawing itself.

\subsection{Pause stages hierarchy}

The top down hierarchy of stages of pause/data, from least to most detail, is as follows,

1. pause after each basin of attraction field in a range of network size $n$ (section 8.1).

2. pause after each basin of attraction in a basin of attraction field, or after each single basin or each single subtree for a range of network size $n$.

3. pause after each tree (or subtree from the uniform states) in a basin of attraction.

4. pause after each fan - the set of pre-images of each state in a tree.

From the selected pause/data stage, relevant upper stages will also apply. For example, if stage 3 is selected for a pause after each tree, a data pause will also occur at stages 2 and 1 . Each stage gives a specific top-center pause prompt described in section 27.1.3. 


\subsubsection{Pause after each field for a range of fields}

If a basin of attraction field for range of $n$ was specified in section 8.1, the following prompt is displayed,

pause/data: field range 5-12, pause after each field-f: (for example)

Enter $\mathbf{f}$ to pause after each successive field. A top-center prompt is also shown, described in section 27.1.3. If $\mathbf{f}$ was entered, the prompt in section 27.1.2 is presented for further stages of detail.

\subsubsection{Pause after each basin, tree, or pre-image fan}

A pause can be selected for a basin of attraction field, a single basin, or a subtree (including a range $n$ set in section 8.1) at various stages of detail. The following context dependent top-right prompt is presented, (enter 1, $\mathbf{2}$ or $\mathbf{3}$ as required, or return not to pause),

(for a basin of attraction field, including a range $n$ set in section 8.1)

pause/data: pause for data: fan-3 tree-2, basin-1, none-def:

(for a single basin, or a subtree)

pause/data: pause for data: fan-3 tree-2, none-def:

\subsubsection{The pause prompt}

At at each pause, as well as basin data in a top-right window, a prompt is presented in a top-center window depending on the stage reached in the pause hierarchy (section 27.1) as follows,

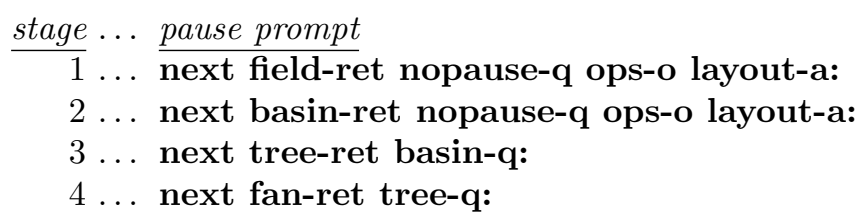

-a ... for the layout options, to amend the angular orientation, subtree fan angle, and the spacing and/or position of each successive basin, described in section 25.3.

\subsection{Attractor basin complete - data window}

When an attractor basin is complete (or abandoned), data are displayed in a top-right window. Examples and decoding are shown below for a CA with compression on (section 26.1). More detailed data, including a sorted list of states, may also also printed or saved to a file (sections 27.3, 27.5). 


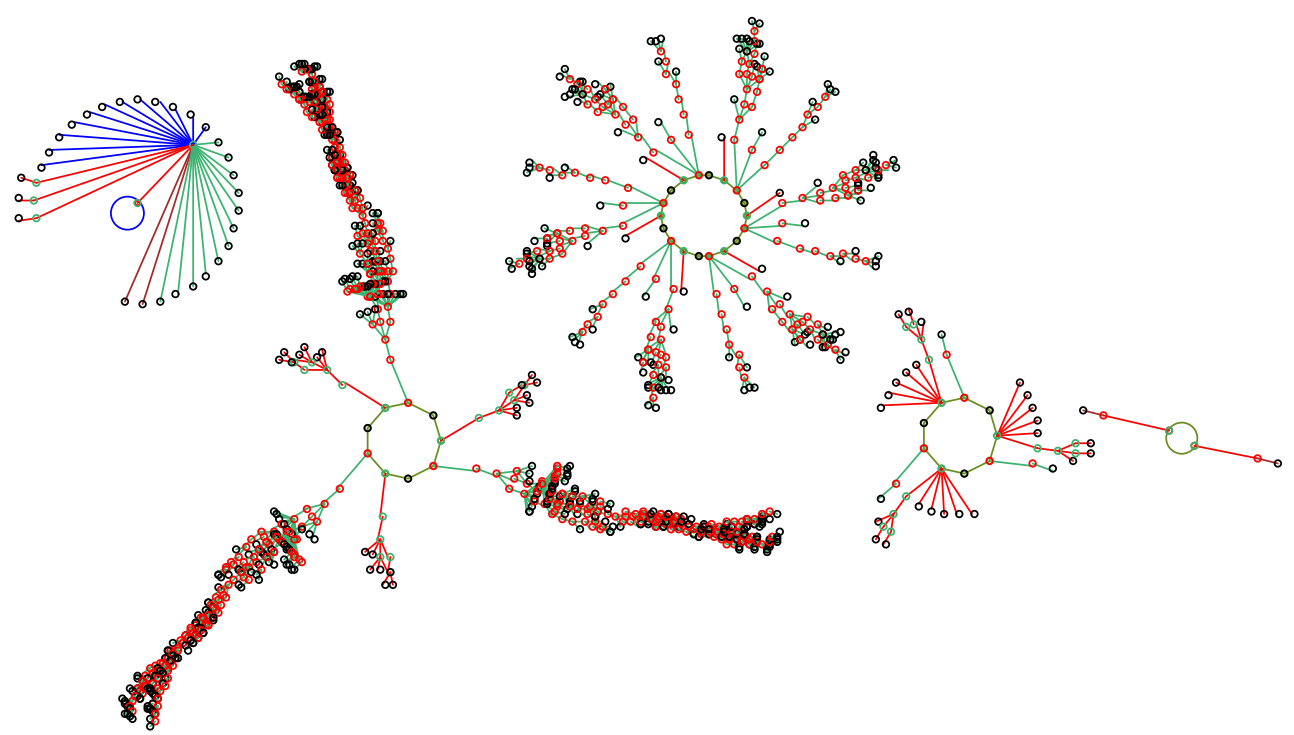

Figure 27.1: A basin of attraction field of a CA, $v 2 k 3$ rcode (dec) $110, n=12$, relating to various data outputs in section 27.2 , and saved in sections 27.4 .1 to 27.4 .4 . In this example the default layout was amended in the jump-graph with no edges (section 20.3).

\subsubsection{Data on basin of attraction fields}

By default, when a basin of attraction field is complete, unless a range of fields was specified (sections 8.1, 27.1.1), data on the field is presented in a top-right window. An example of data on a field (for $v 2 k 3$ rcode (dec) $110, n=12$ ) as in figure 27.1 is shown below,

\section{for a single field, or if pausing in a range of fields \\ basin types $=5$ total basins $=11$ \\ $\mathrm{n}=12$ field $=4096 \mathrm{~g}=1971=0.4810 .109 \mathrm{sec}$}

If the basin of attraction field is interrupted ${ }^{1}$ (by entering $\mathbf{q}$ twice, see section 30.2), the data on the incomplete field appears as in the example below, depending on the point of interruption,

EARLY EXIT basin types $=3$ total basins $=5$

$\mathrm{n}=12$ sspace $=4096$ field $=3358 \mathrm{~g}=1637=0.487$ (computation slowed)

$$
\text { data type } . . \text { decode of data }
$$

EARLY EXIT ... indicates that the field was interrupted.

basin types $=\ldots$ the number of basin prototypes displayed.

total basins $=\ldots$ the total number of basins in the field.

$\mathbf{n}=\ldots$ the network size.

\footnotetext{
${ }^{1}$ In this and other examples, the computation was slowed (section 24.11) to give enough time to interrupt.
} 
field $=\ldots$ the total number of states in the field, which should equal the size of state-space, $v^{n}$, unless interrupted. If the computed field $\neq v^{n}$, the size of state-spaces $(\mathbf{s s p a c e}=)$ will be displayed as well as the incorrect size of the field (field $=$ ). If the field was not interrupted this would indicate an error (section 27.2.2).

$\mathbf{g}=x=y \ldots$ the number and density of garden-of-Eden states in the field.

$x \mathbf{m i n} y \mathbf{s e c} . .$. the time taken to draw the field.

\subsubsection{Errors in basin of attraction fields}

Inconsistencies and errors in computing attractor basins can be caused by parameter changes while the basin is in the process of being drawn. One such change is abandoning a tree and continuing with the next tree (section 30.2.1). Another is to change some aspect of the network itself, which is possible while pausing or interrupting (section 30.2).

If such an error occurs in a basin of attraction field, the progress bar (section 30.1) is likely to go off its scale, the size of state-space as well as the field will be indicated in the data window, for example sspace=1024 field=1486. A message will also appear below the progress bar, for example, ERROR excess states $=\mathbf{4 6 2}$ indicating that more states were computed than the size of state-space. section 30.2.2 gives further details.

Note that similar errors can occur in single basins or subtrees, but these will not be indicated.

\subsubsection{Data on basins}

An example of data on a basin (for $v 2 k 3$ rcode (dec) $110, n=12$ ) is shown below ${ }^{2}$.

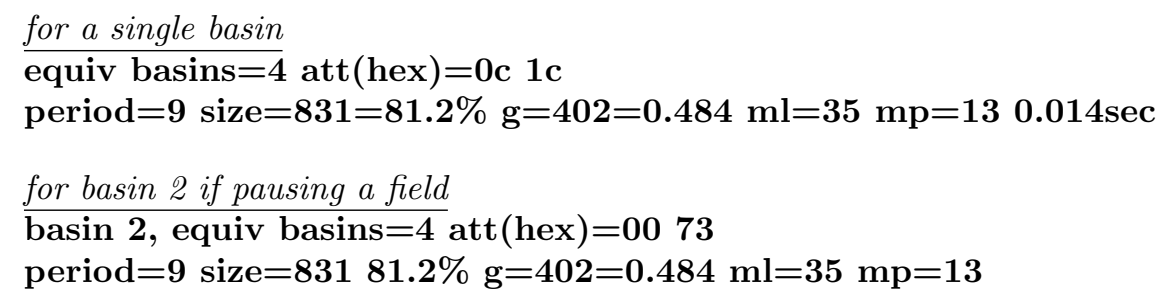

If the single basin is interrupted (by entering q twice, see section 30.2), the data on the incomplete basin appears as in the example below,

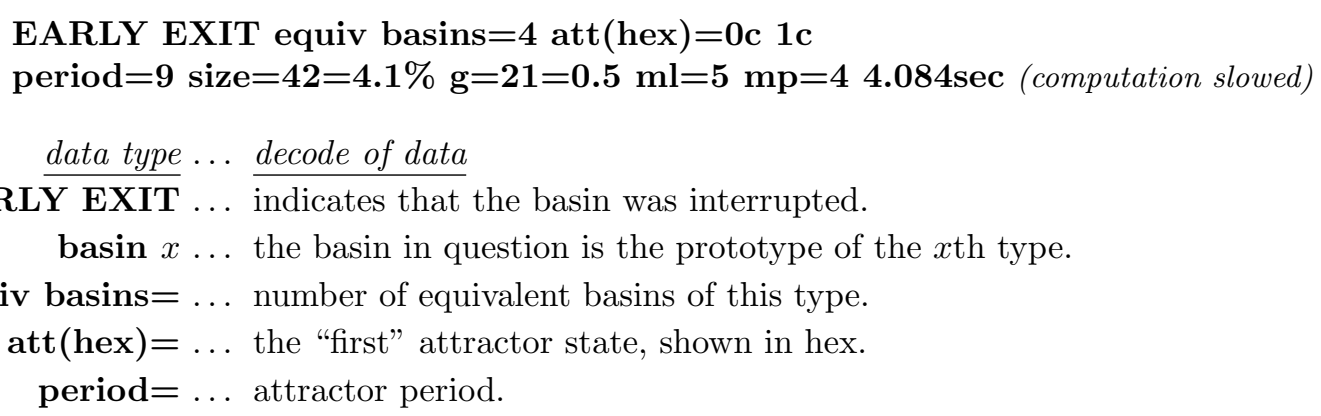

\footnotetext{
${ }^{2}$ If a count of states with a majority of a given value is active, this data will also be displayed in the basin (or subtree) data window, for example $\mathbf{m a j} \mathbf{1}=\mathbf{5 7 2}=\mathbf{0 . 1 2 6}$ (section 24.7).
} 
$\operatorname{size}=x=y \% \ldots$ size of the basin, and the percentage of state-space made up by the basin and its equivalents.

$\mathbf{g}=x=y \ldots$ the number and density of garden-of-Eden states in the basin.

$\mathbf{m l}=\ldots$ the maximum number of levels in the basin outside the attractor - the length of the longest transient.

$\mathbf{m p}=\ldots$ the maximum in-degree found in the basin.

$x \mathbf{m i n} y \mathbf{s e c} \ldots$ the time taken to draw the basin.

\subsubsection{Data on trees}

An example of data on a tree in basin 2 (as shown in figure 27.1),

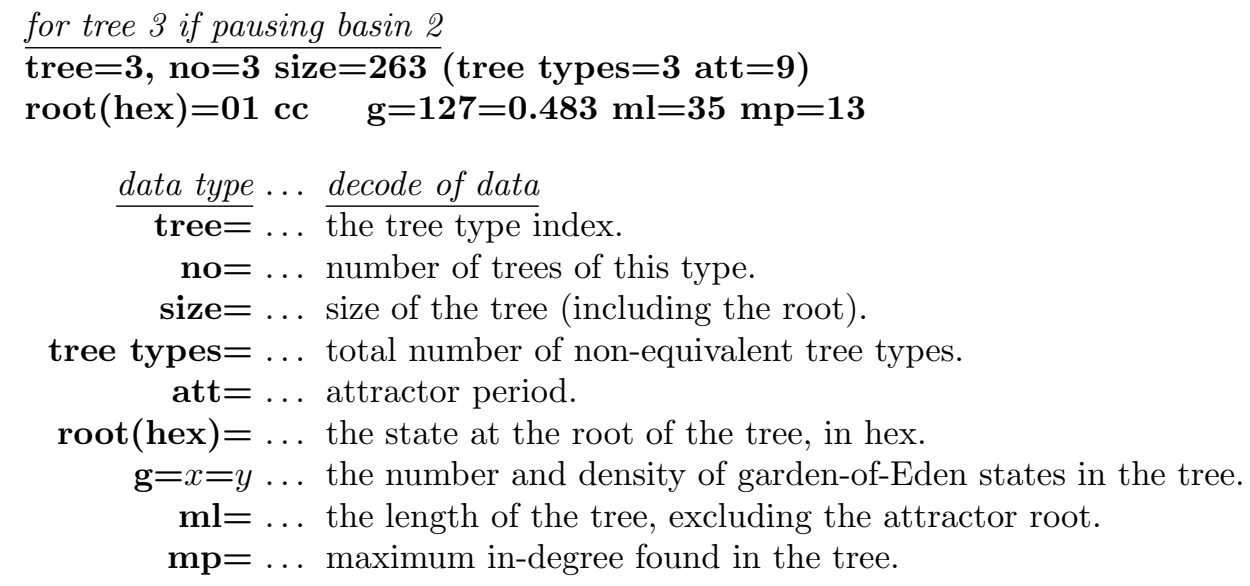

\subsubsection{Data on pre-image fans}

An example of data on a pre-image fan, in the 2nd tree in basin 2 (as shown in figure 27.1),

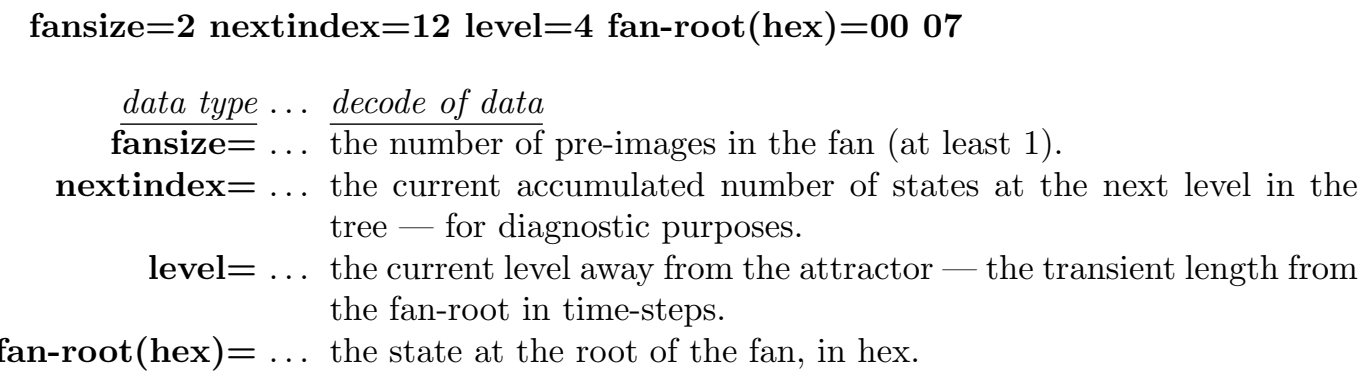

\subsubsection{Data on subtrees}

If generating just one subtree from an arbitrary state, on completion, data will be displayed in a top-right window. The first example (figure 27.2) is for a large CA, $n=150$. A seed state chosen at random was iterated forward by 3 steps and the subtree was generated from the state reached - the subtree root. Running forwards by some steps before running backward (section 29.2) is usually necessary because a random state is very likely to be a garden-of-Eden state. 


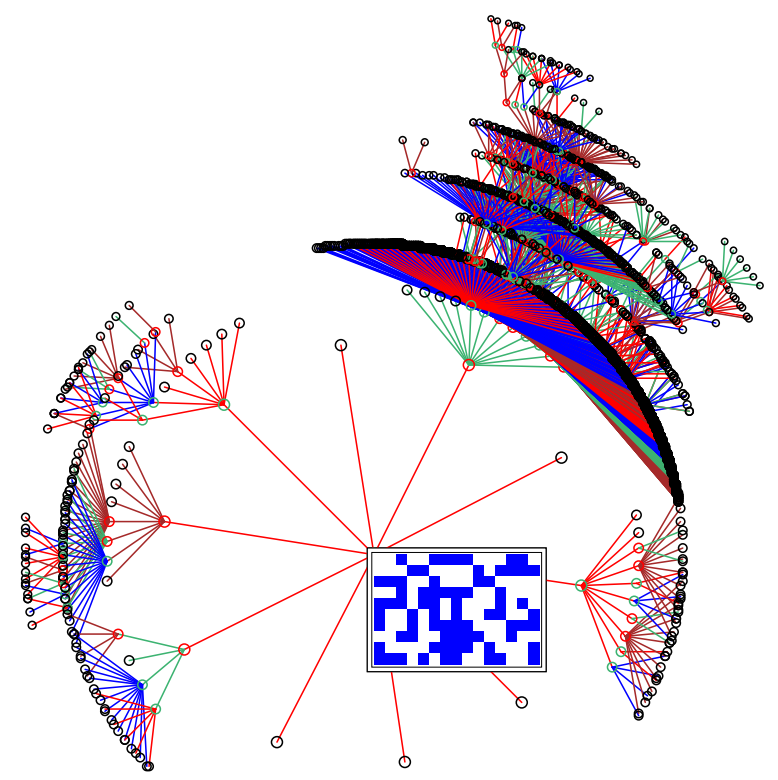

Figure 27.2: A subtree for a $1 \mathrm{~d}$ CA, from a root state shown as a $2 \mathrm{~d} 15 \times 10$ bit pattern. The root was reached by iterated 3 steps forward from the seed state, which was chosen at random. $n=150$, $v 2 k 5$ rcode(hex)aa5566a1.

For such a large network the root is not shown in the data window - they are only shown if $n \leq 56$. This limit does not apply when data is printed or saved (section 27.4.7).

$$
\begin{aligned}
& \text { subtree }=4335 \\
& \mathrm{~g}=4030=0.93 \mathrm{ml}=11 \mathrm{mp}=12535.292 \mathrm{sec}
\end{aligned}
$$

If the subtree is interrupted (by entering $\mathbf{q}$ twice, see section 30.2), the data on the incomplete subtree appears as in the example below,

\section{EARLY EXIT part subtree $=946$$$
\mathrm{g}=7=0.0074 \mathrm{ml}=2 \mathrm{mp}=8502.500 \mathrm{sec}
$$

The second example (figure 27.3) is for a small CA $n=14$ with the same rcode, where a random seed was iterated forward by 33 steps before generating the subtree. However, the state reached (the root of the subtree) turned out to be on the attractor, so all the states in the basin were generated. This is indicated by subtree=basin. DDLab keeps track of a repeat to prevent the subtree running backwards for ever around the attractor and its trees.

$$
\begin{aligned}
& \text { subtree }=\text { basin }=6895 \operatorname{root}(\text { hex })=10 \mathrm{f} 1 \\
& \mathrm{~g}=2505=0.363 \mathrm{ml}=84 \mathrm{mp}=626.014 \mathrm{sec}
\end{aligned}
$$

$$
\text { data type } . . . \text { decode of data }
$$

EARLY EXIT ... indicates that the subtree was interrupted.

subtree $=\ldots$ size of the subtree.

part subtree $=\ldots$ size of an interrupted subtree. 


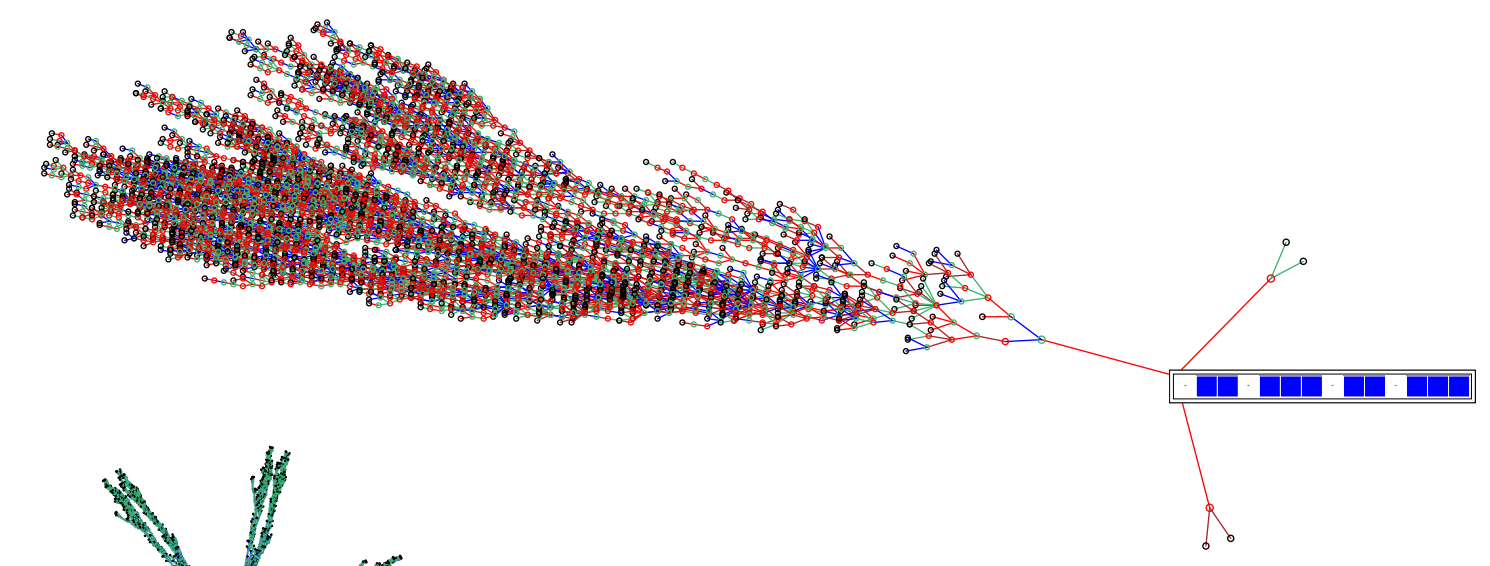

Figure 27.3: Above: a "subtree" where the root happens to lie on the attractor, so all the states in the basin are shown - running backwards for ever is prevented. Left: The whole basin (with compression set) as it would normally appear, shown at a smaller scale. This example is a CA, $n=14, v 2 k 5$ rcode (hex)aa5566a1.

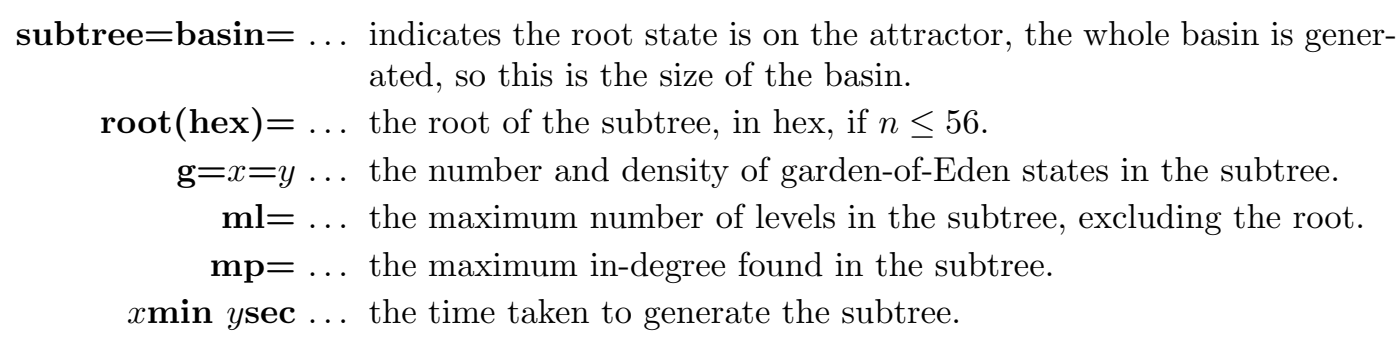

\subsubsection{Data on subtrees from a uniform state}

If a subtree pause is selected for a CA in section 27.1.2 when generating the tree from a uniform state (where all values are the same), with compression set the pause will occur after each equivalent set of subtrees, and data will be displayed on each subtree prototype. An example of the data on the 4 th prototype subtree in figure 27.4 is shown below,

$$
\begin{aligned}
& \text { seg-fan }=211(17 \text { segs }) \text { seg-tree }=4 \text { no }=14 \text { size }=59 \\
& \operatorname{seg}-\operatorname{root}(\text { hex })=0042 \mathrm{~g}=51=0.864 \mathrm{ml}=8 \mathrm{mp}=49 \\
& \text { data type... decode of data } \\
& \overline{\mathbf{s e g}-\mathrm{fan}=\ldots} \text { the total number of pre-images of the uniform state. } \\
& (x \text { seg) ... made up of } \mathrm{x} \text { segments or groups of rotation equivalent states. } \\
& \text { seg-tree }=\ldots \text { the subtree prototype number. } \\
& \text { no }=\ldots \text { number of subtrees of this type. } \\
& \operatorname{size}=\ldots \text { size of the subtree including its root. }
\end{aligned}
$$




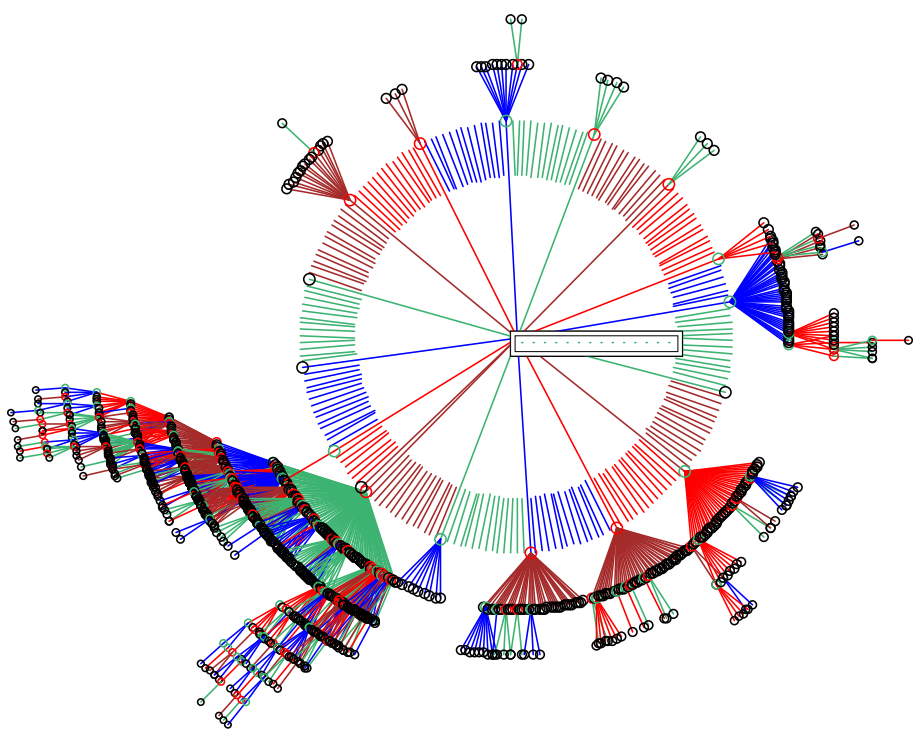

Figure 27.4: Prototype subtrees from a uniform state, all $0 \mathrm{~s}$, for a CA, $v 2 k 3$ rcode (dec) $96, n=14$. Copies of the prototypes have been suppressed in section 26.1.3, and the default fan angle reduced by a factor of 0.3 in section 26.3.2.

$\operatorname{seg}-\operatorname{root}($ hex $)=\ldots$ the state at the root of the subtree, in hex.

$\mathbf{g}=x=y \ldots$ the number and density of garden-of-Eden states in the subtree.

$\mathbf{m l}=\ldots$ maximum number of levels in the subtree including its root.

$\mathbf{m p}=\ldots$ maximum in-degree found in the subtree.

Once all prototype subtrees have been generated, data on the complete tree is presented as follows (decode as in section 27.2.6),

subtree $=$ basin $=16333 \operatorname{root}($ hex $)=0000$

$\mathrm{g}=13363=0.818 \mathrm{ml}=9 \mathrm{mp}=2129.266 \mathrm{sec}$

\subsection{Print or save data}

printing data in the terminal is for Linux-like systems only

As attractor basins are drawn, data similar to those described above (sections 27.2.1 - 27.2.7), and also a list of states in different formats (section 27.5) may be printed in the terminal from which DDLab was launched (for Linux-like systems only) and/or output to a . dat file.

All the states or just the attractor states can be listed, with or without extra details (section 27.5). States are shown as value strings. The extra details are the state's basin number, level, and the number of its pre-images.

The following prompts, which have the same options, are presented in turn, firstly to print, then to save the data,

for printing data in the terminal - Linux-like systems only

print data: basin data only-1, and tree data-2

include states: details-s/+s no-details-S/+S att-only-a/+a: 
for saving data as an ascii file

save data: basin data only-1, and tree data-2

include states: details-s/+s no-details-S $/+\mathrm{S}$ att-only-a/+a:

Enter a combination of the following,

$$
\text { options.... what they mean }
$$

basin data only-1 ... for basin data (section 27.2.3) or subtree data (section 27.4.7).

and tree data-2 ... for basin and tree data (section 27.2.6).

include states: ... show just the states, or add states to selections above (section 27.5).

details-s $/+$ s ... list all states including details.

no-details-S $/+\mathbf{S} \ldots$ list all states without details.

att-only-a/+a ... list only attractor states states including details.

For example, enter,

1a ... for basin data, combined with a list of attractor state including details.

$2 \mathrm{~s}$... for basin and tree data, combined with a list of all states including details.

S ... for a list all states without details.

If the save data options were selected, further prompts will appear to set the file name as a . dat file (section 35.2) - the default filename is my_data.dat. The format of the data printed in the terminal, or in the ascii data file, is shown in the examples in section 27.4, together with the decoding of the data.

\subsection{Data format}

Examples of the data format, printed in the terminal (Linux-like systems only) or saved to a . dat ascii file (section 27.3) are described and decoded below.

\subsubsection{Network parameters data}

If $\mathbf{1}$ or $\mathbf{2}$ is selected in section 27.3, the following network parameters data are given first, including the rule, network dimension and size, and various rule parameters.

This example relates to the figure 27.1 .

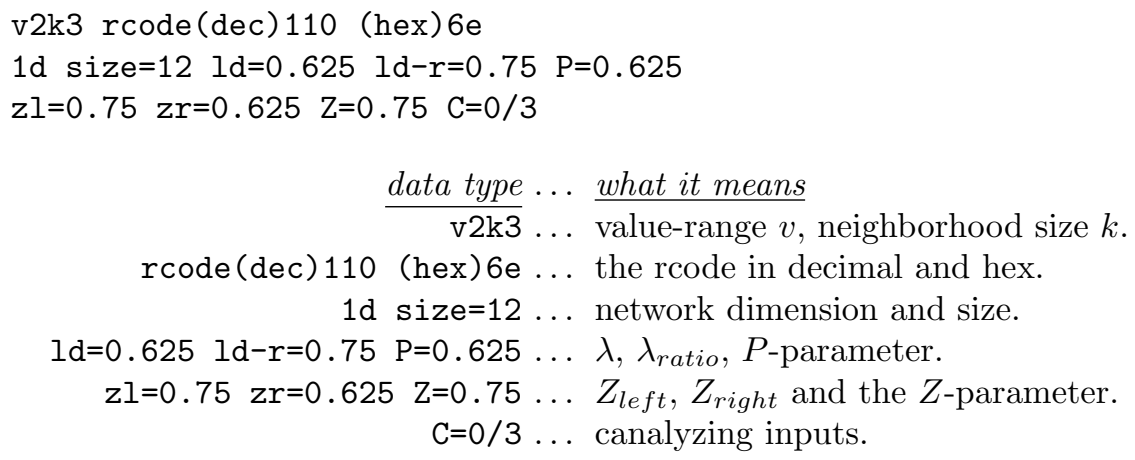


This initial network parameters data are only given for single rule networks. For mixed rule (and mixed $k$ ) networks, the complete network parameters can be printed and saved (also to a .dat ASCII file) as described in section 19.6.

\subsubsection{Basin field data}

An example of the complete data for the basin of attraction field in figure 27.1 (enter $\mathbf{1}$ in section 27.3),

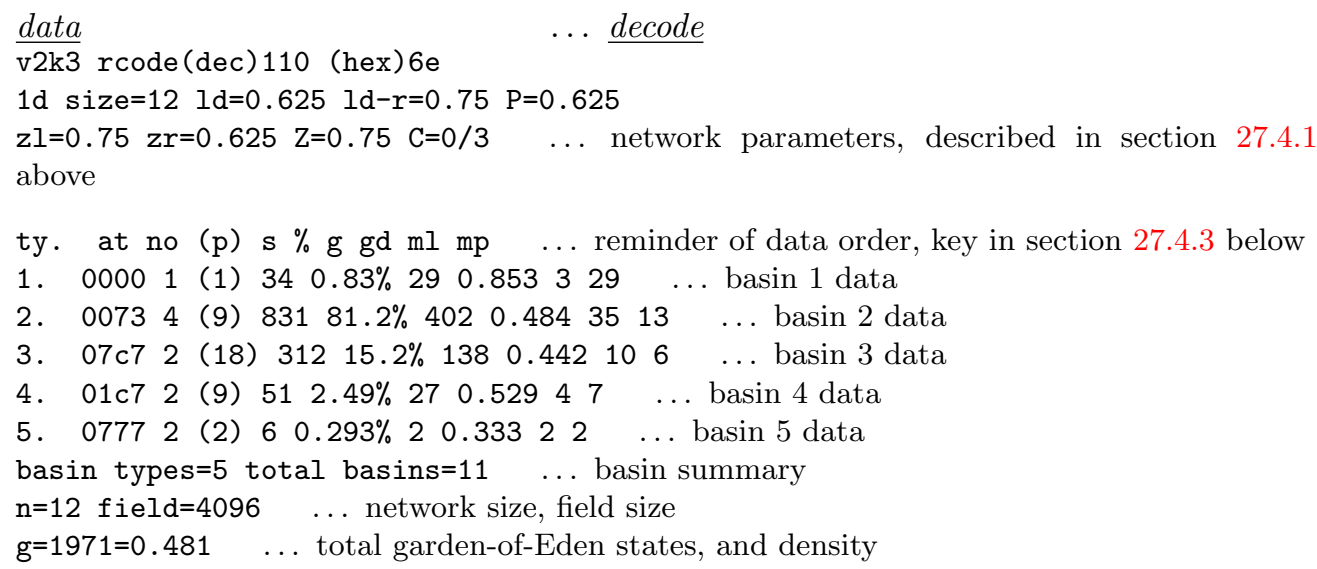

\subsubsection{Key to basin data order}

The key to data on basins is set out below, where the labels are shown as a reminder.

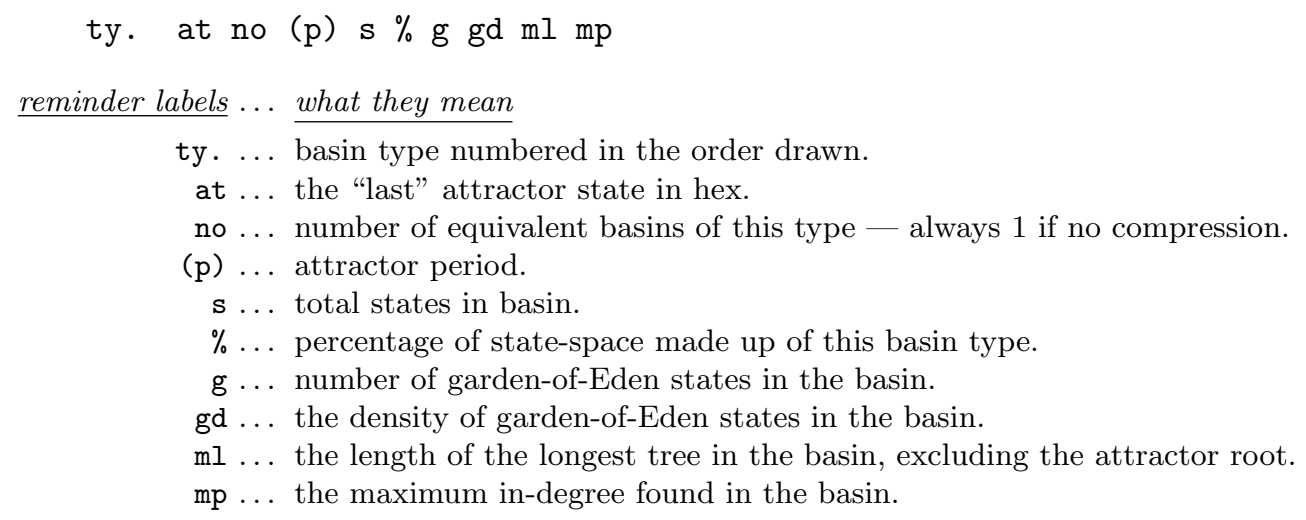

If CA compression is suppressed (section 26.1), or for a non-CA network where there are no equivalent basins — data on every basin will be shown.

\subsubsection{Tree data}

An example of the same data as for the basin of attraction field in section 27.4.2 above (figure 27.1), but also including tree (and subtree) data, which precedes the basin data summary is given below (enter 2 in section 27.3). For CA, subtree data is given for each subtree type rooted on the pre-images of a uniform attractor state (all values equal) in section 27.2.7. 


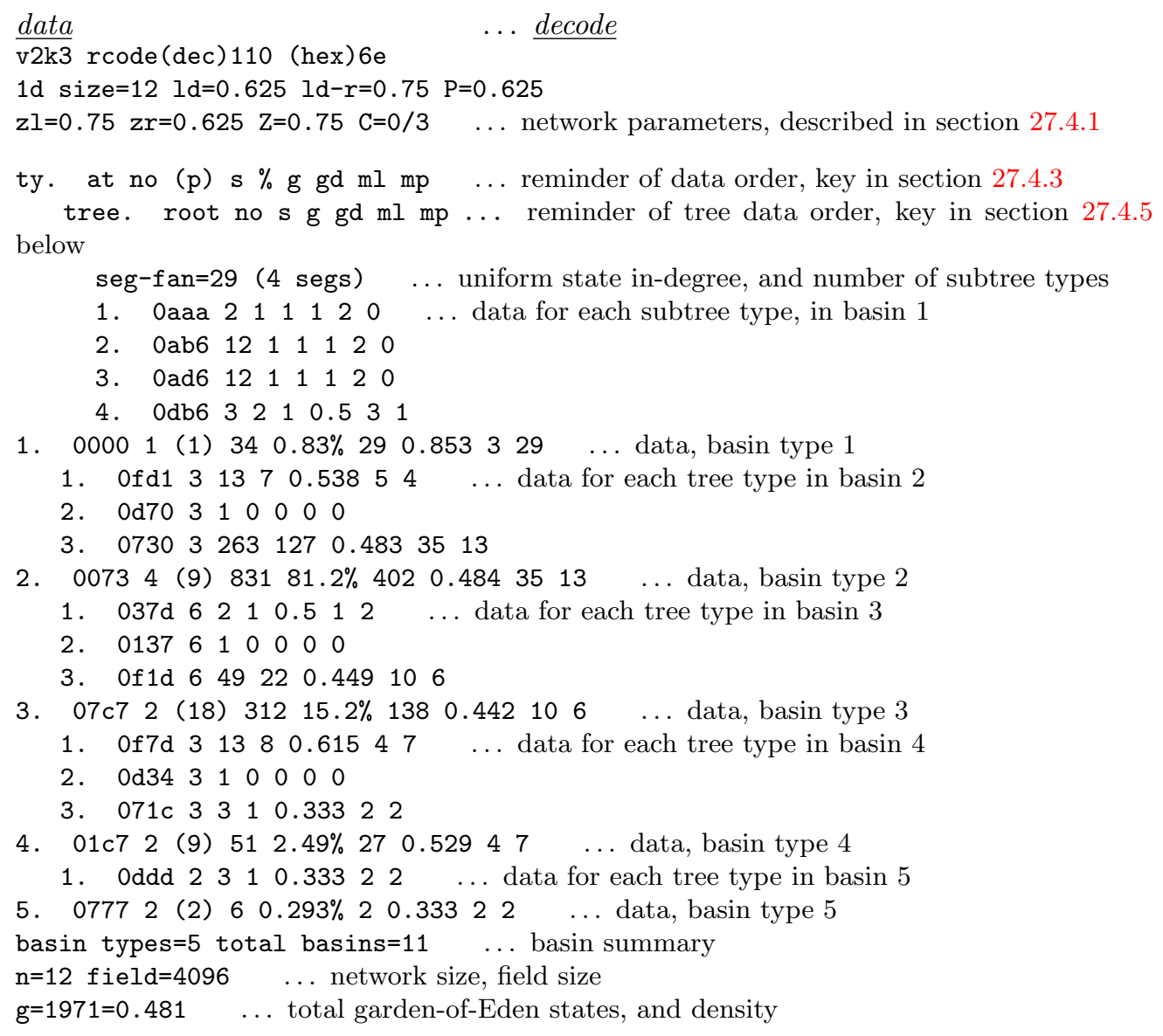

\subsubsection{Key to tree data order}

The key to data on trees is set out below, where the labels are shown as a reminder.

tree. root no $\mathrm{s} g \mathrm{gd} \mathrm{ml} \mathrm{mp}$

reminder labels... what they mean

tree. ... tree (or subtree) type numbered in the order drawn.

root ... the state at the root of the tree, in hex.

no ... number of equivalent trees of this type (always 1 if compression suppressed).

s ... the size of the tree including the root.

g ... number of garden-of Eden states in the tree.

gd ... the density of garden-of-Eden states in the tree.

$\mathrm{ml}$... the maximum number of levels in the tree, excluding its root.

$\mathrm{mp}$... the maximum in-degree found in the tree.

Note that for CA with compression active (section 26.1), data is also given on subtrees from a uniform state. If CA compression is suppressed, or for a non-CA network where there are no equivalent trees — data on every tree will be shown. 


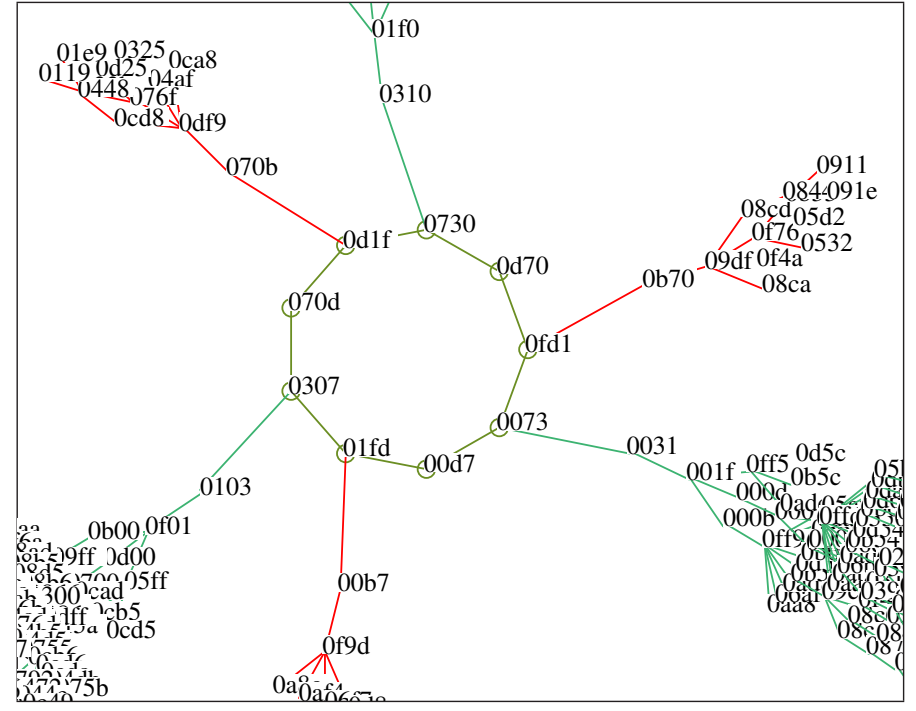

Figure 27.5: A detail of the second basin in figure 27.1 with nodes shown in hex. CA $v 2 k 3$ rcode(dec) 110 , $n=12$. To reproduce this basin and the data in section 27.4.6. generate this single basin with the seed (hex)0073.

\subsubsection{Single basin data}

An example of the data for a single basin, with tree data, is given below (enter $\mathbf{2}$ in section 27.3). This is the second basin in figure 27.1. A detail with nodes in hex (figure 27.4.5) shows how this data corresponds to the states on the attractor.

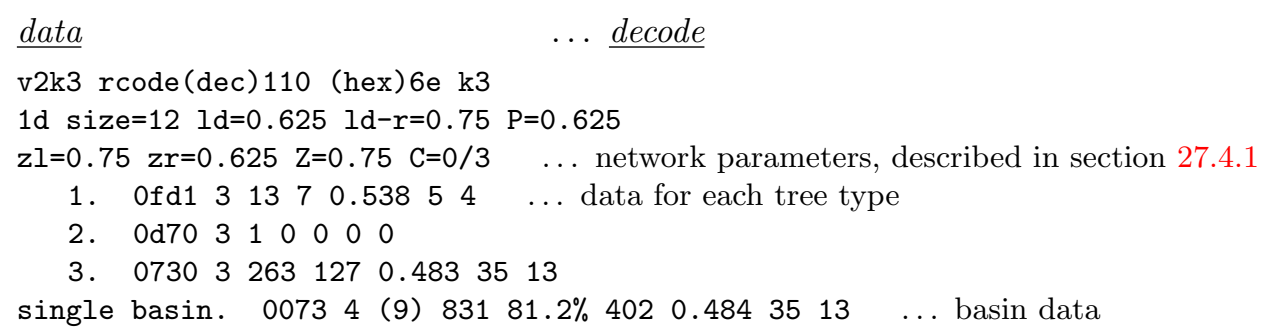

The tree data order is as in section 27.4.5, and the basin data order is in section 27.4.3, but the reminders of the data orders are not shown.

\subsubsection{Subtree data}

An example of the data for the subtree $(n=150)$ shown in section 27.2 and figure 27.2 , is given below (enter 2 in section 27.3).

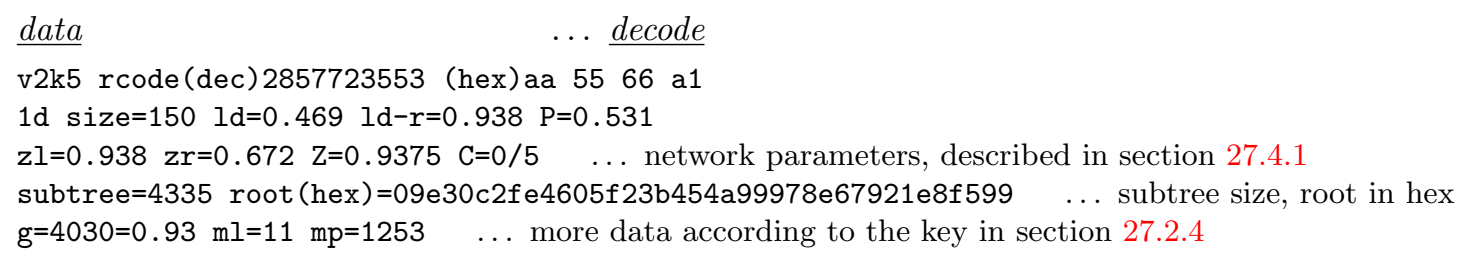



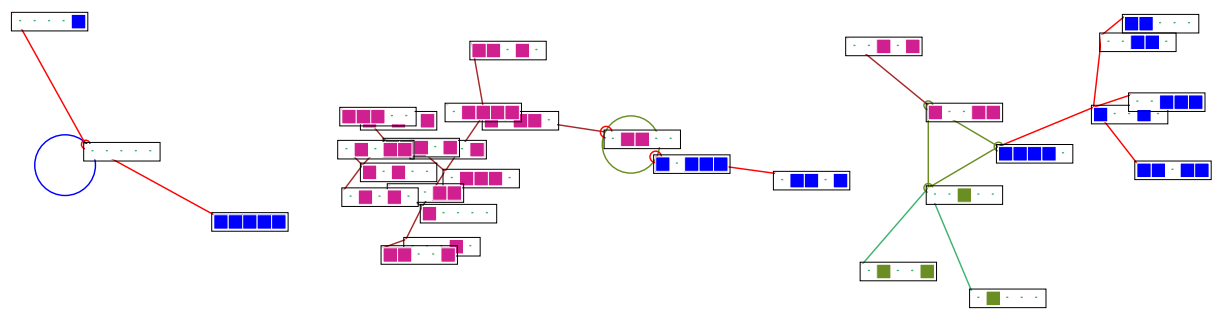

2.1. 0 .

4: 412

3: 3444

2: 0304

1: $2 \begin{array}{lll}2 & 4\end{array}$

0: 122

Figure 27.6: The basin of attraction field of an RBN, with states listed in section 27.5. The RBN has a homogeneous rule, $v 2 k 3$ rcode $(\mathrm{dec}) 110, n=5$. Nodes are displayed as a $2 \mathrm{~d}$ bit pattern (section 26.2). Right: The wiring matrix (section 17.2).

\subsection{List of states}

Examples of the list of states and data (with explanatory notes) are shown in sections 27.5.1 to 27.5.3 below, for a basin of attraction field of a small $\mathrm{RBN}^{3}$ defined in figure 27.6. The list of states can be very lengthy so a small network, $n=5$, is applied in these examples.

As well as showing just the list of states and data, the list can also be broken up into sections giving basin data, or in addition tree data, by entering $1 \mathbf{s}$ or $2 \mathrm{~s}$ in section 27.3. Both of these options show network parameters, a reminder of the basin data (and tree data) order at the top, and a summary of data for the basin of attraction field at the bottom, as in section 27.4. Compare these lists with the basin of attraction field and its nodes shown in figure 27.6.

\subsubsection{Just the list of states}

The example below shows just the list of states and three items of extra data about the states. Enter $\mathbf{s}$ in section 27.3, or $\mathbf{S}$ to exclude the extra data. The first column is the state bit-string (or value-string for multi-value). The next three columns give extra information about each string as follows,

the basin number ... as drawn in sequence. For a CA, if compression has not been suppressed (section 26.1) this is the basin prototype number. For a subtree or single basin the number is always 1 .

level ... the number of steps (levels) of the state away from the attractor for a basin of attraction, or from the subtree root for a subtree. If this is 0 then the state is on the attractor, or is itself the subtree root.

pre-images ... the number of the state's immediate predecessors (pre-images). If this is 0 then the state is a garden-of-Eden (leaf) state.

\footnotetext{
${ }^{3}$ Note that in an RBN compression does not apply (section 26.1).
} 


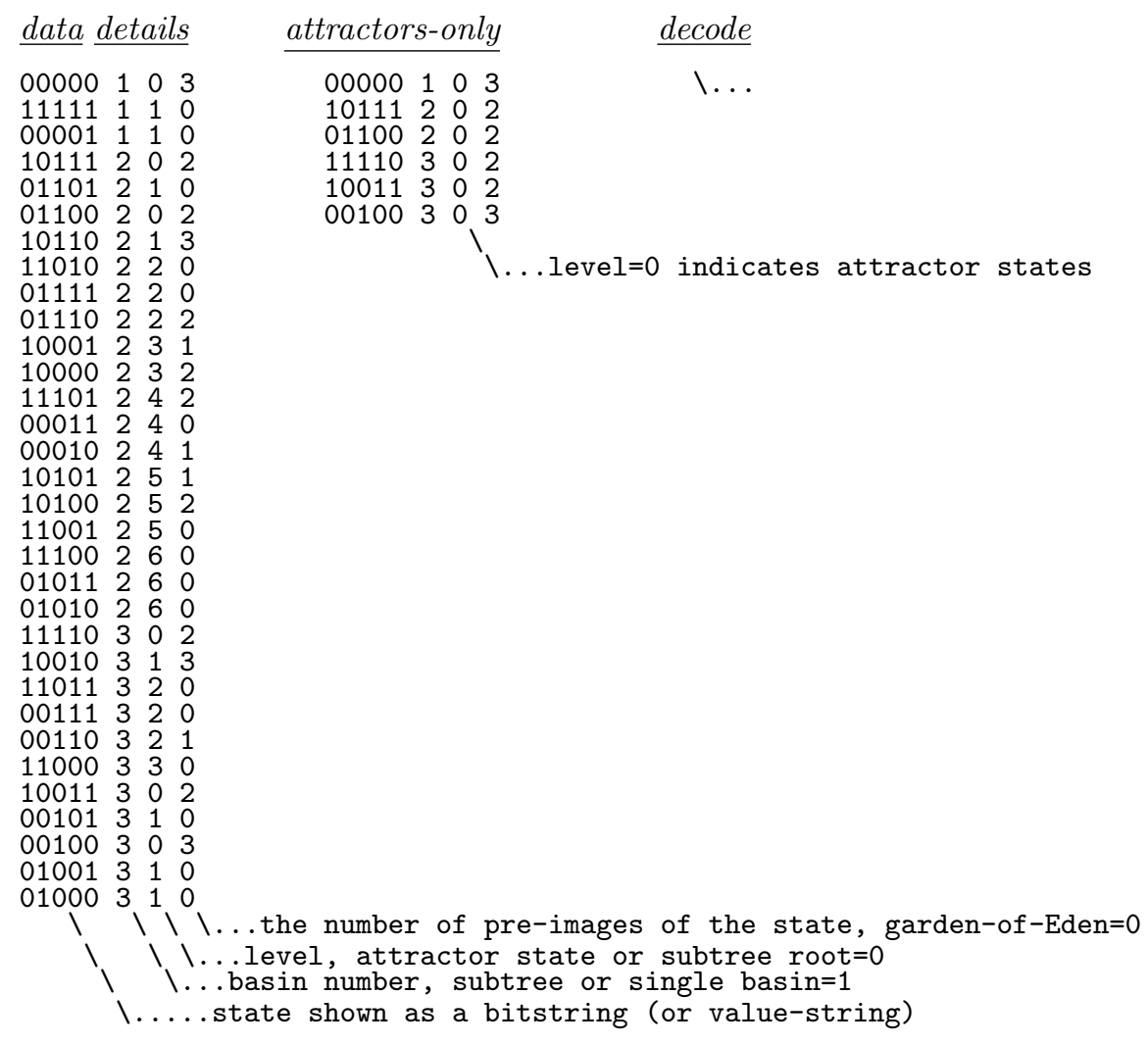

\subsubsection{The list of states with basin data}

This example shows the same list as in section 27.5.1 together with basin data. Enter 1s in section 27.3,

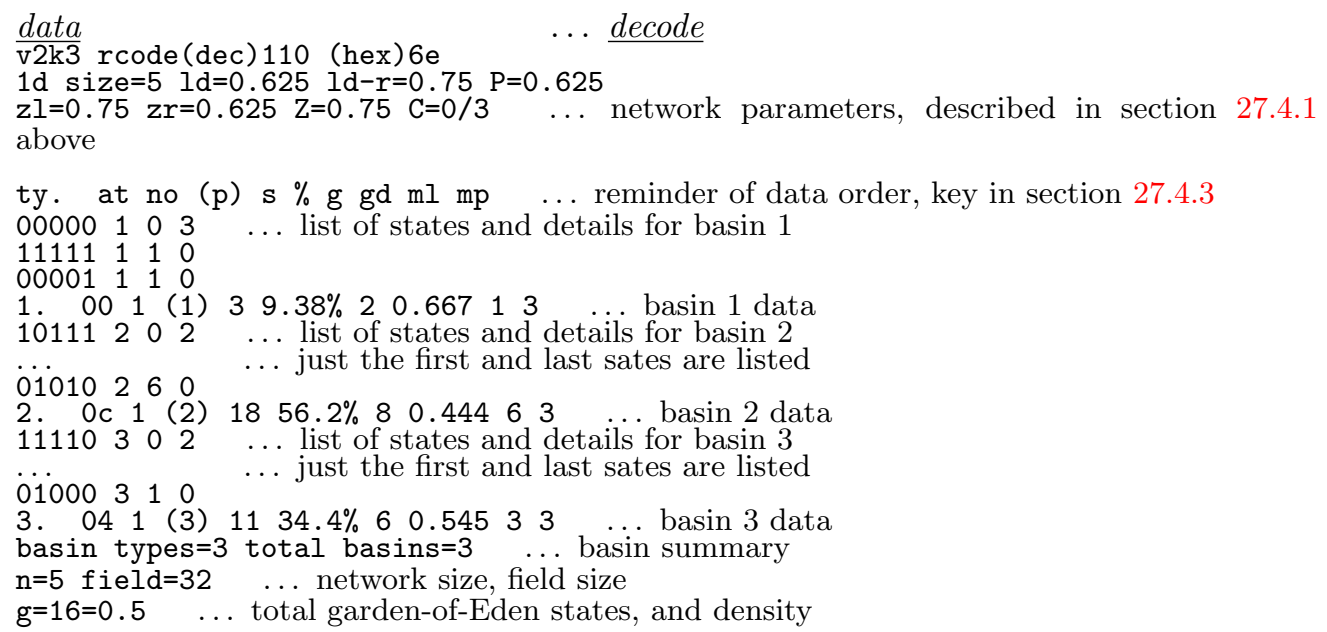




\subsubsection{The list of states with basin and tree data}

This example shows the same (complete) list as in sections 27.5.1 and 27.5.2 together with basin data and tree data (decode notes are omitted). Enter $2 \mathrm{~s}$ in section 27.3.

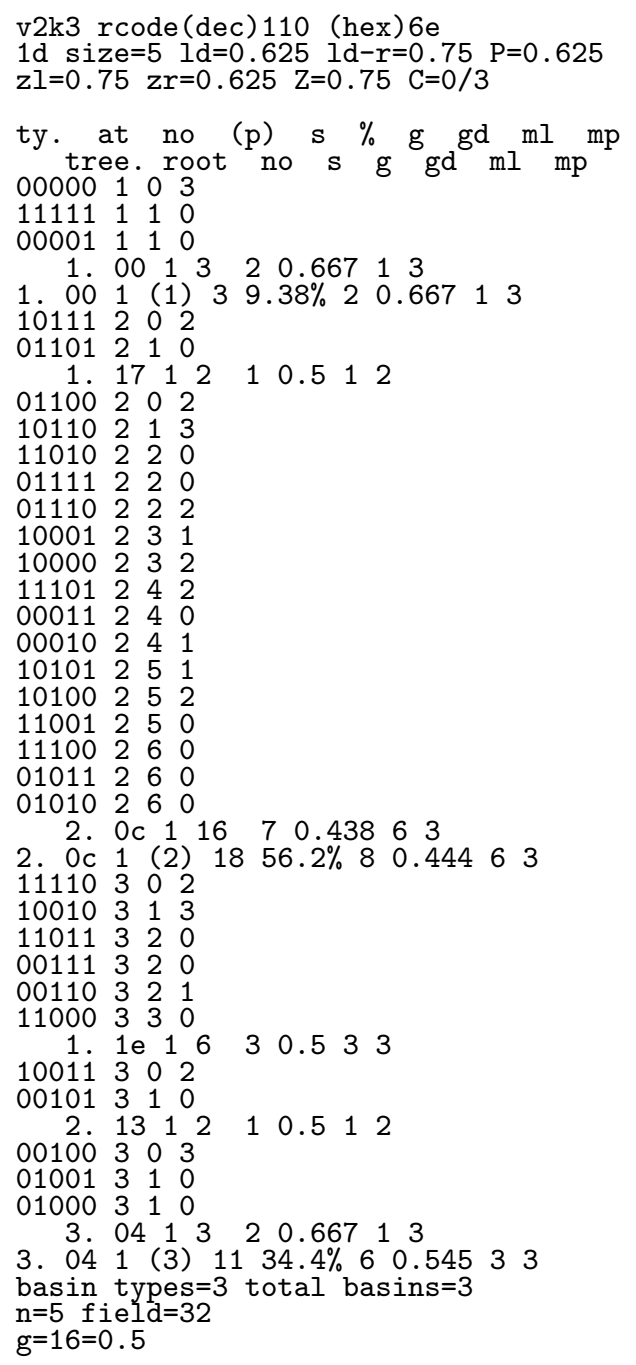

The format and keys of network parameters, basin data and tree data are described in sections 27.4.1 to 27.4.5. 


\section{Chapter 28}

\section{Mutation of attractor basins}

not in TFO-mode.

When the attractor basin has been drawn for a given network, a new attractor basin may be immediately generated with the same presentation settings, but with a change, or mutation, to some aspect of the network. This process can be repeated indefinitely and automatically. Mutations can be cumulative or relate back to the starting network, and may comprise just one bit/value in a rule-table or just one wire move in a RBN/DDN. A whole spectrum of mutations can be preset from small to large, from all possible one bit/value mutants, a countdown of the decimal rule number, up to fully random rules and/or wiring. This chapter describes the various mutation options. The methods apply to RBN/DDN as well as CA, rulemix and $k$-mix, and to all the rule types, rcode, kcode and tcode.

Mutants of single basins can be grouped on one screen (section 25.2.2) - especially useful to show the set of one bit/value mutants of a rule (section 28.3.1).

\subsection{The first mutation prompt}

If $\mathbf{m}$ is selected at the first "output parameter" prompt (section 24.1), or if the output parameter prompts are viewed in sequence, the first top-right mutation prompt is as follows,

mutation: (for next) chain-c Post-P tcode-t kcode-k rcode-r none-n (Post-P $v=2$ only) wiring-w/+w, rule: special-s, set bits/values-b, single bit/value-1-(def):

\subsubsection{The first mutation prompt - options summary}

The options are summarised below, some are expanded in greater detail in later sections.

options ... what they mean

chain-c ... for a random chain-rules (section 16.11). The rules results in sup-trees with very low in-degree, suitable for encryption[41].

Post-P ... (value-range $v=2$ only) for random Post-functions (section 14.12). If $k \leq 5$ a Post-function is found at random from rcode-space. For $k \geq 6$ a Post-function if found at random from kcode-space where Post-function are more abundant — otherwise the search would take too long. 


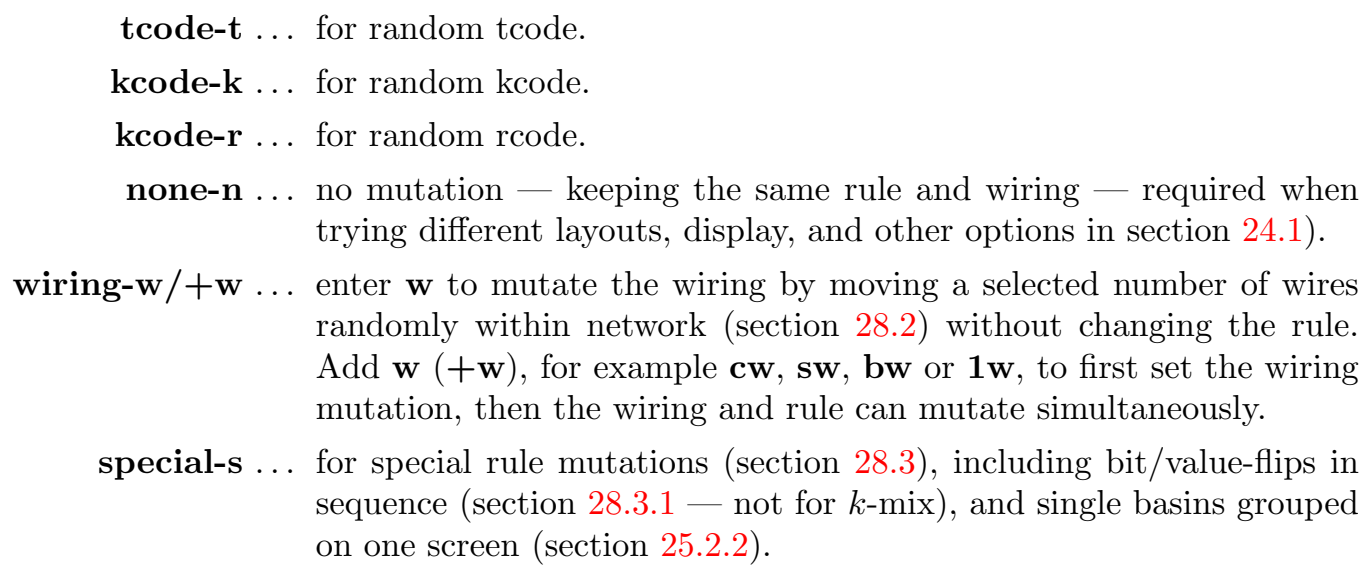

set bits/values-b ... to set the number of bits/values to flip in rcode (section 28.4).

single bit/value-1 ... (the default) to flip one bit/value at random (section 28.4).

For a rulemix (including a $k$-mix), these option generally apply at all cell locations simultaneously unless noted otherwise.

\subsection{Mutate wiring}

Enter $\mathbf{w}$ at the first mutation prompt (section 28.1), or another option followed by $\mathbf{w}$, to move one or more wires in the network to random positions for each new attractor basin. A top-right prompt similar to the following is presented,

wires to move $=\mathbf{1} / \mathbf{3 0}=\mathbf{3 . 3 3 \%}(\mathrm{def})$ : all-a number-n \%-p: (values shown are examples) local-L, from original wiring-w/ $+\mathrm{w}$ :

In this example, $n=10$ and $k=3$, so the total number of wires is 30 . In a mixed $k$ network the total number of wires equals the sum of the $n$ neighborhoods.

The default is to move just one wire. The wiring mutations are either cumulative, or can restart from the original wiring with wiring-w/ $+\mathbf{w}$ below. The wire moves are made by picking a random wire of a random cell for each wire move. Note that a local CA will be re-assigned as a randomly wired (nonlocal) network if wiring mutation is chosen, so compression in basins (section 26.1) will be deactivated.

The options below activate the wiring change at each mutation,

$\frac{\text { options }}{\text { return }} \ldots \frac{\text { what they mean }}{\text { to move one random wire in the network, the default. }}$

all-a ... for new random wiring for the whole network.

number-n ... to specify the number of wires to move, selected at random, with the following subsequent prompt, ... number of wires:

\%-p ... to specify the percentage of total wires to move, selected at random, with the following subsequent prompt, ... \% of total wires: 


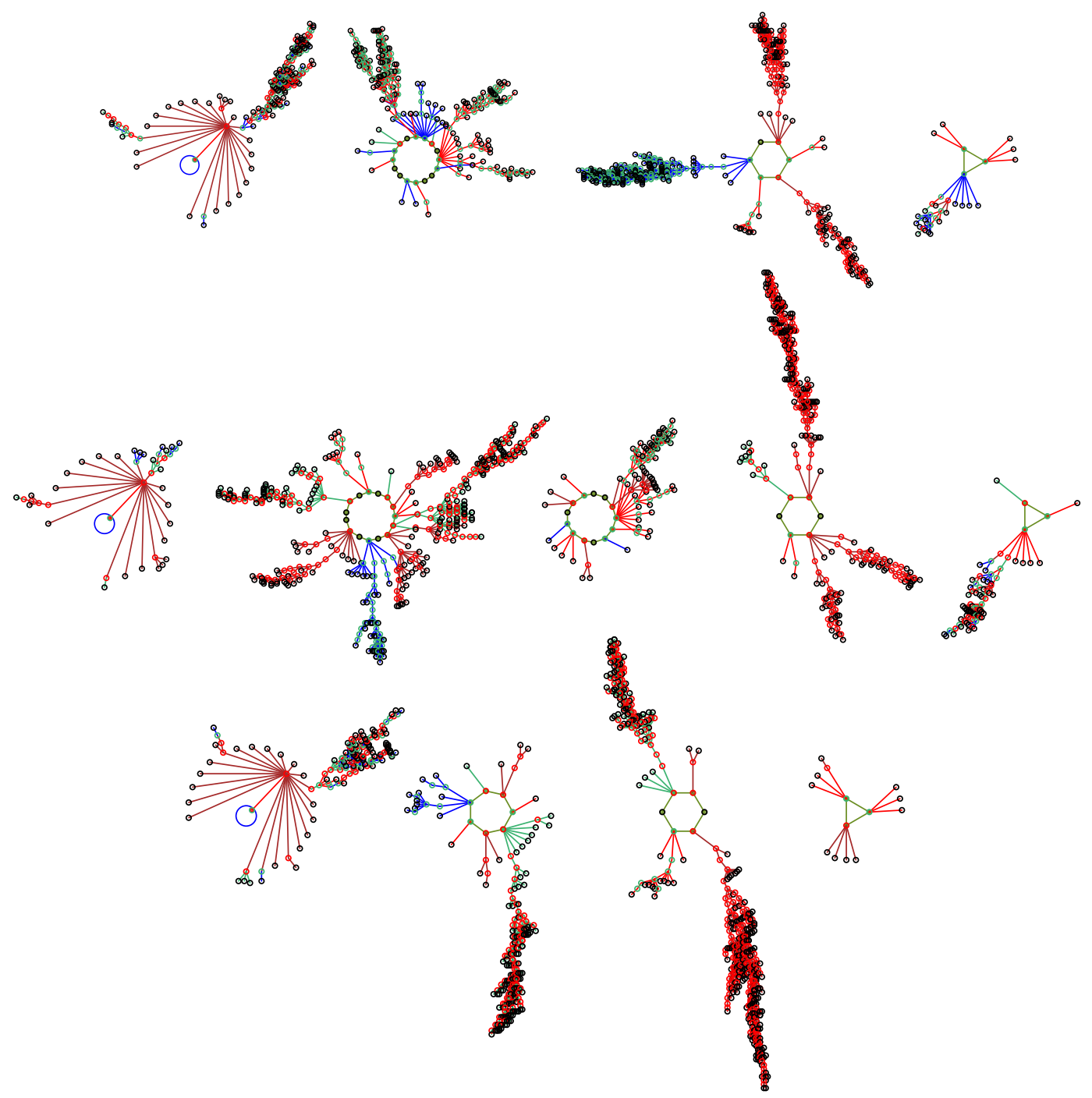

Figure 28.1: Starting with CA $v 2 k 3$ rule (dec) $110, n=10$, as shown in figure 26.2, three mutant basin fields are shown where one randomly chosen wire was moved at random to a new position. The actual moves were as follows: Top: cell 9, 098 $\rightarrow 398$. Center: cell 3, 432 $\rightarrow 452$. Bottom: cell 5, 654 $\rightarrow 634$.

local-L ... to restore local wiring (for $1 \mathrm{~d}, 2 \mathrm{~d}$ or $3 \mathrm{~d}$ ). For $1 \mathrm{~d}$ and a single rule, compression in basins (section 26.1) will be restored, and the program will revert to the first mutation prompt (section 28.1).

wiring-w/+w ... enter $\mathbf{w}$ for the default single wire move to start from the original wiring at each mutation, otherwise mutations are cumulative. For noncumulative mutations of several wires, enter the option followed by $\mathbf{w}$, for example aw or pw. 


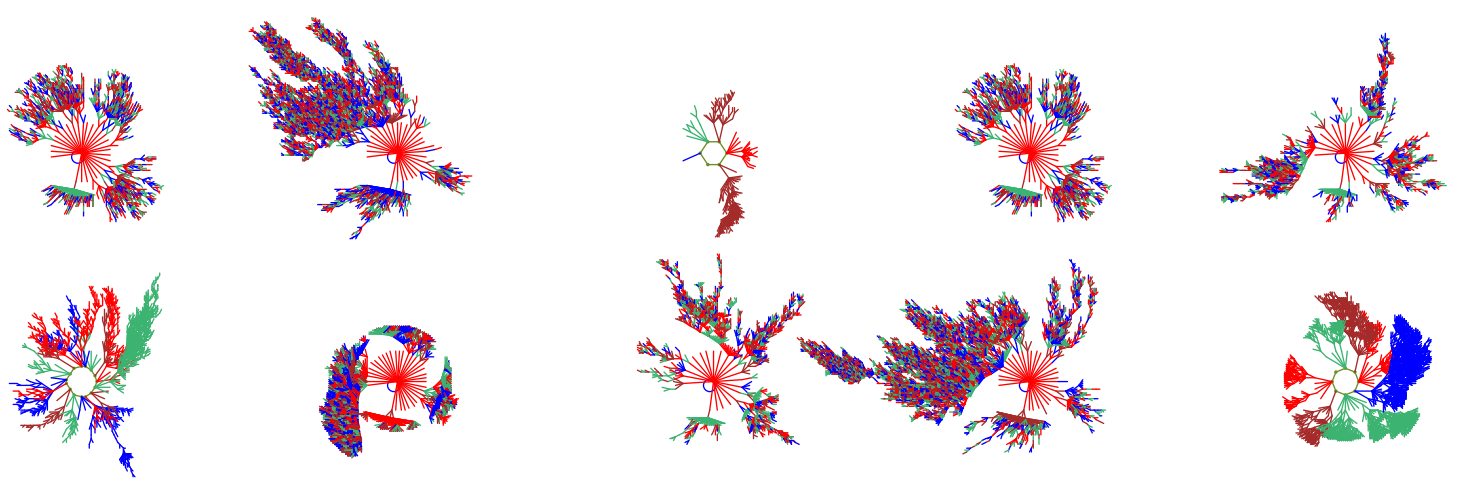

Figure 28.2: Mutant basins of attraction shown on one screen - selected with mutants-M in section 25.2.2. The original basin Top left: is a CA $v 4 k 2, n=7$, kcode(hex)027e60, seed(hex)cdca (as in figure 28.5 but without compression). The other basins are one wire move mutants from the original. As there are many possible single wire moves, this exact set of mutants would be hard to reproduce.

\subsection{Special mutation options}

Special mutation options take into account the different rule types (rcode, kcode or tcode) selected in section 13.1.1. For single basins or subtrees, successive mutants can be generated automatically and grouped on one screen (pausing at each full screen) if mutants-M is selected in section 25.2.2. Otherwise there would be a pause with the "attractor basin complete" prompt (section 30.4) after each mutant is complete.

The following kinds of changes/mutations are included in special mutations,

1. A countdown by decimal rule number, provided the rule-table is within the decimal limits (table 16.1). For a rulemix or $k$-mix, the rule-number at each cell is decreased by one.

2. A succession of random single bit/value flips. For a rulemix or $k$-mix one cell is first selected at random for the flip.

3. Flipping successive bits or values in the rule-table from left to right, either cumulatively or singly - from the original string at each flip. For a rulemix this applies to all rule-tables simultaneously. This option does not apply for a $k$-mix.

Enter $\mathbf{s}$ at the first mutation prompt (section 28.1) for the special rule mutation options, which depend on whether kcode, tcode, or neither, are active, and whether the rule-table is within the decimal limits. The following is an example of a special mutation prompt in a top-right window,

(kcode active, and both kcode and rcode within decimal limits)

specify rcode or kcode mutation kcodeno-5 kcodeflip-4 rcodeno-3 rcodeflip-2 (def-4) (Mutant Layout Set) bit/value flip from left: cumulative-1 single-0:

The options, and the wording subject to the context, are described below, 


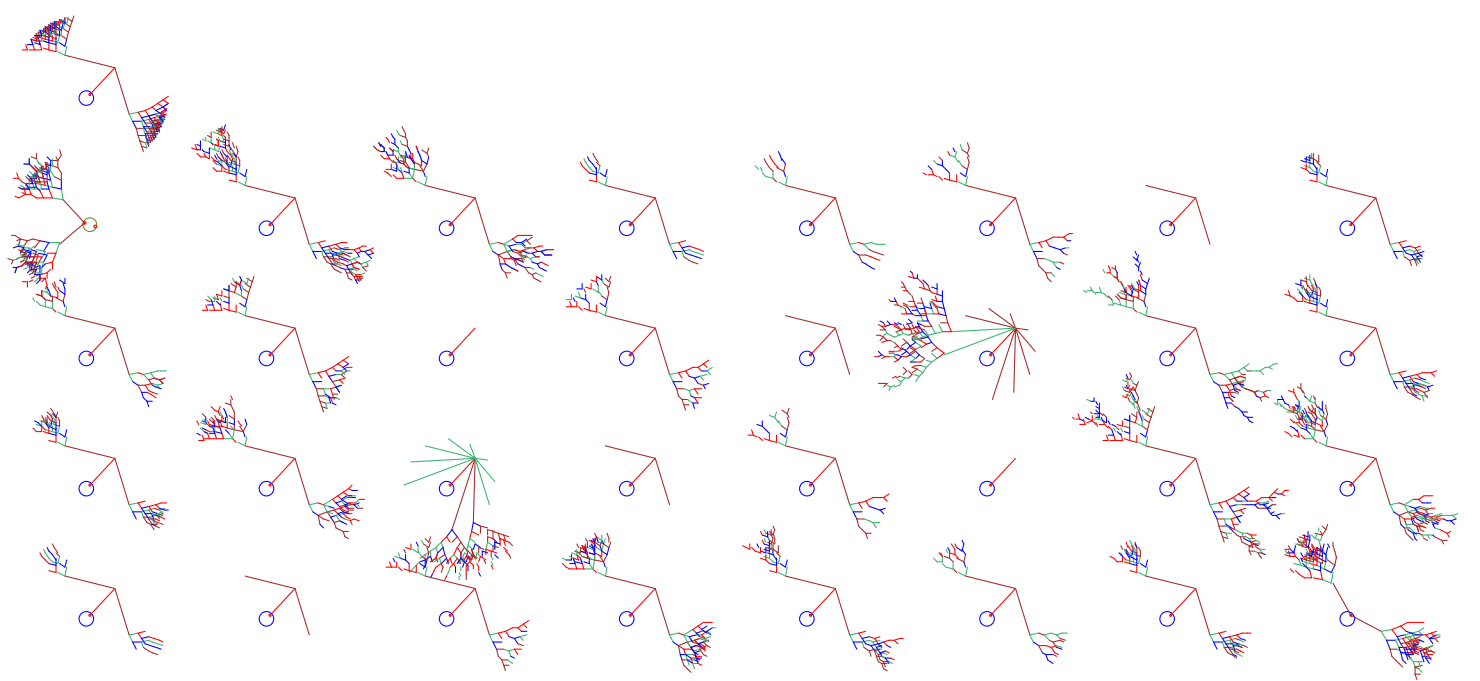

Figure 28.3: 32 mutant one-bit basins of attraction shown on one screen selected with mutants-M in section 25.2.2. The original rule Top left: is a 1d CA $v 3 k 3, n=8$, rcode 60 , seed all 0s. where all states belong to one very regular basin. The rule was first transformed to its equivalent $k=5$ rcode (hex)f00ff00f, with 32 bits in its rule-table. Below: All 32 one-bit (non-cumulative) mutant basins are shown. If the rule is the genotype, the basin of attraction can be seen as the phenotype.

info and options... what they mean

specify rcode... ... always appears - the rcode options apply even if kcode or tcode is active.

... or kcode... ... (if kcode active) otherwise ... or tcode..., or neither, appears.

kcodeno-5 ... (if kcode active and within decimal limits) for a countdown from the initial decimal kcode number.

tcodeno-5 ... (if tcode active and within decimal limits) for a countdown from the initial decimal tcode number.

kcodeflip-4 ... (if kcode active) for a random bit/value flip in kcode.

tcodeflip-4 ... (if tcode active) for a random bit/value flip in tcode.

rcodeno-3 ... (if rcode within decimal limits) for a countdown from the initial decimal rcode number.

rcodeflip-2 ... for a random bit/value flip in the rcode.

(def-4) ... enter return for the default - kcodeflip-4 or tcodeflip-4 is the default if either is active, otherwise the default is rcodeflip-2.

(Mutant Layout Set) ... (for single basins, and if mutants-M was selected in section 25.2.2). bit/value flip from left: ... (not for $k$-mix) Successive flips from left to right (cumulative or single - below). section 28.3.1 provides further details.

cumulative-1 ... keep previous flips. single-0 ... restore previous flips — so single bit/value flip from the original rule. 


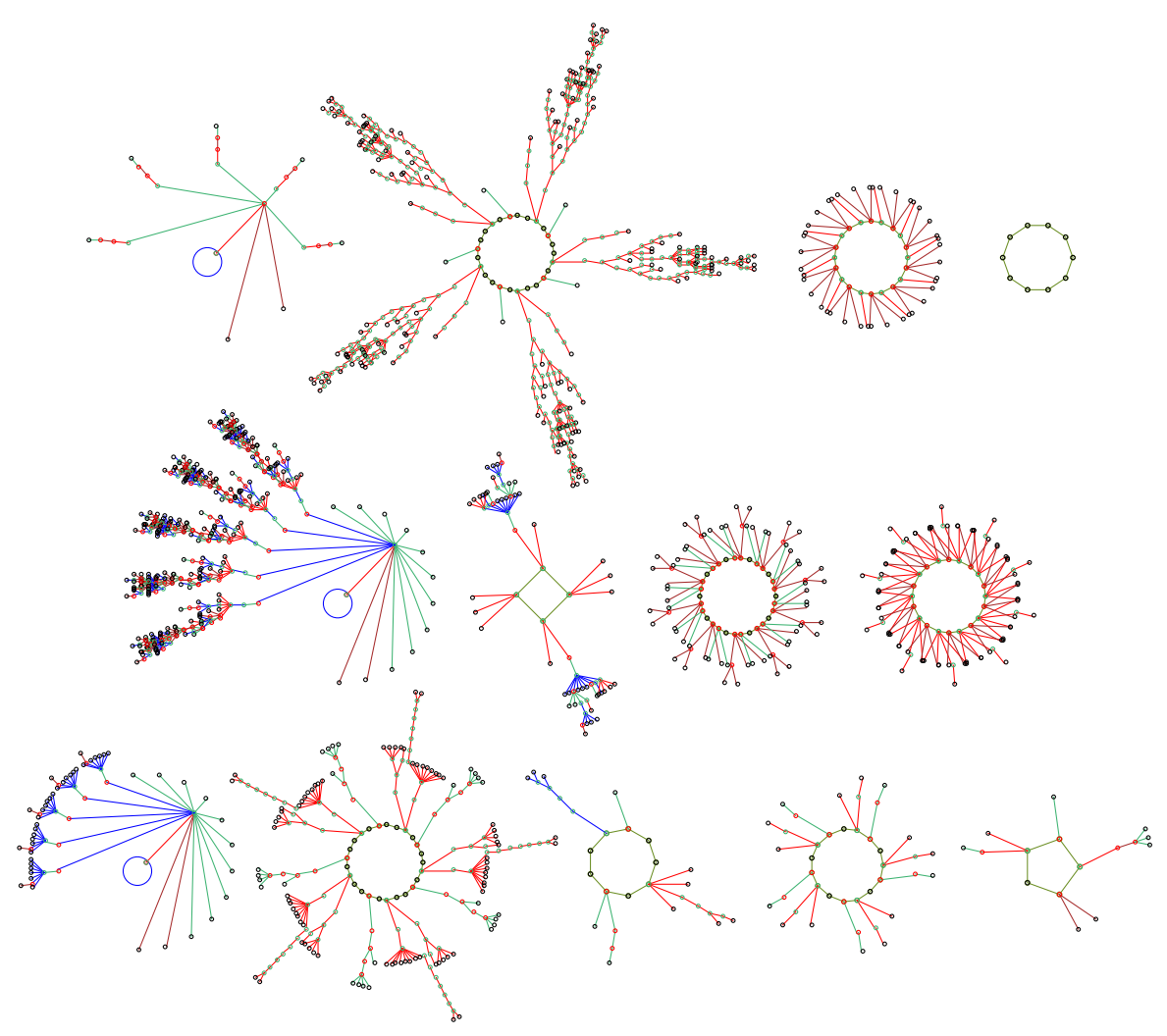

Figure 28.4: Mutation of the basin of attraction field by one bit-flips. The $v 2 k 3$ rcode (dec) 110 was first transformed to the equivalent $k 5$ rcode (hex) $3 \mathrm{cfc} 3 \mathrm{cfc}$ (section 18.7.1). The CA, $n=10$ (basin field shown in figure 26.1), was then mutated in sequence by a single bit from the left (non-cumulatively). Three mutant basin fields are shown (from the top) for the 5th, 8th and 14th bit-flip The three rules (hex) are $34 f \mathrm{fc} 3 \mathrm{cfc}, 3 \mathrm{dfc} 3 \mathrm{cfc}, 3 \mathrm{cf} 83 \mathrm{cfc}$.

\subsubsection{Bit-flip or value-flip in sequence}

not for mixed-k

If $\mathbf{1}$ or $\mathbf{0}$ is entered in section 28.3, at each mutation, successive bits in the rule-table (tcode, kcode or rcode) will be flipped in sequence from left to right. The difference is that for cumulative-1 the previous flip remains unchanged, whereas for single-0 the previous flip will be restored to its former state. The single-flip results in a set of rules 1 Hamming distance from the start rule the cumulative-flip results in a steady increase in the Hamming distance.

The start and end position of flips can be altered from the default at the extreme ends of the rule-table. The following top-right prompt is presented, this example for $v 2 k 3$ rcode,

\section{rcode: specify start - end position of bit/value flip (countdown) start position (def 31$)$ : $\quad$ end position (def 0 ):}

Enter the new start position, followed by the new end position. Once the end position is reached, the next mutation will revert to the start. 

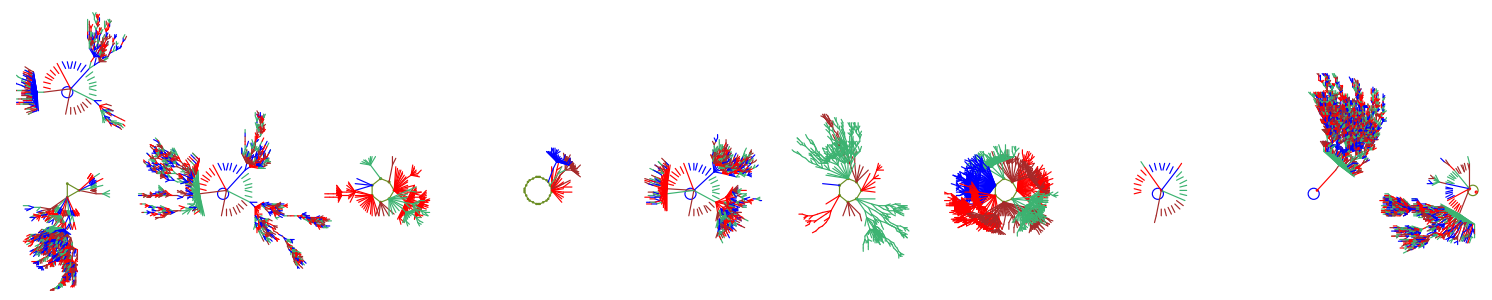

Figure 28.5: Mutant basins of attraction shown on one screen - selected with mutants-M in section 25.2.2. The original basin Top left: is a CA $v 4 k 2, n=7$, kcode (hex)027e60, seed (hex)cdca (as in figure 28.2 but without CA compression active). Lower row: 10 one-value (non-cumulative) mutant basins. As $v=4$ and the value-flip is random, this exact set of mutants would be hard to reproduce. In this example, both the nodes (section 26.2), and equivalent trees (and subtrees) (section 26.1.3), are suppressed.

For a rulemix the mutation applies to the rule at each cell simultaneously.

Note that finer mutations can be made to rcode if first transformed to an equivalent rcode with greater $k$ (section 18.7.1). For single basins only, the mutant basins can be grouped on one screen with the original basin by itself in the top row - by selecting mutants-M in the layout (section 25.2.2). For a bit/value-flip in sequence, if the set of mutants fits on one screen the program will pause once the set is complete (or when the screen is full). Figures 28.5 and 28.3 give examples.

\subsection{Flip bits or values}

Enter return at the first mutation prompt (section 28.1) for the default one bit or value flip, or enter $\mathbf{b}$ to first alter the number of bits or values to be flipped at random in kcode or tcode if active, or in rcode. The total number of bits/values $T$, the maximum that may be flipped, depends on the network - if $S$ is the rule-table size (section 13.2.1), $n$ the network size, and $i$ the network index, $T$ is given as follows,

$$
\begin{aligned}
\text { single rule } \ldots T & =S \\
\text { rulemix } \ldots T & =S \times n \\
k \text {-mix } \ldots T & =\sum_{i=0}^{n-1} S_{i}
\end{aligned}
$$

$T$ appears in the prompt and is the basis for the percentage setting. Examples of the top-right prompt are as follows,

(single rcode $v 4 k 3, T=v^{k}=64$ )

rcode: bits/values to flip $=1 / 64=1.56 \%$ : all-a number-n $\%$-p

(homogeneous $k$, mixed rcode $\left.v 4 k 3 n=10, T=n\left(v^{k}\right)=640\right)$

rcode: bits/values to flip $=1 / 640=0.156 \%$ : all-a number-n $\%$-p

Mutations are made by picking a random bit or value of a random cell's rule-table for each bit/value flip. The options are described below, 
options.... what they mean

$\overline{\text { return }}$... to flip one bit or value, the default.

all-a ... for a new random rule or rules.

number-n ... to specify the number of random flips, with the following subsequent prompt, ... number of bits/values:

\%-p ... to specify the percentage of total bits/values to randomly flip, with the following subsequent prompt, ... \% of total bits/values:

\subsection{No pause before next mutant}

When an attractor basin is complete, the program will pause ${ }^{1}$ and wait for return to be entered for the next mutant attractor basin. To suppress the pause and produce a continuous display of mutant attractor basins, the following top-right prompt is presented,

\section{don't pause after mutant-y:}

Enter $\mathbf{y}$ to suppress the pause. There will be a short delay to see the complete attractor basin before the next mutant is generated. The default delay can be changed - the following subsequent prompt is presented if $\mathbf{y}$ was selected above,

\section{change delay, enter factor (now 1.00 max 10):}

Enter a factor to change the delay. For example to make it half as long enter .5, twice as long enter 2. To restore the default setting enter 1. This option works for all types of attractor basins, basin fields, single basins, and subtrees, including a range of sizes set in section 8.1. To interrupt the continuous display enter $\mathbf{q}$.

\footnotetext{
${ }^{1}$ The "attractor basin complete" prompt (section 30.4) provides many other options, including methods to examine the current network.
} 


\section{Chapter 29}

\section{Final options for attractor basins}

not in TFO-mode.

This chapter describes a number of final options before attractor basins are drawn, which depend on the parameters set previously in chapters 24 to 28 .

If $\mathbf{s}$ for a "single basin/subtree" was selected at the first DDLab prompt in section 6.2 (and a seed in chapter 21) then a choice will need to be made between a subtree and a single basin of attraction, or to opt for space-time patterns only (as in section 24.1).

For a subtree, its possible (and usually advisable) to run forward by a given number of time-steps from the selected seed, and generate the subtree from the state reached. The number of backward steps in the subtree, or in trees in a single basin, can be limited to see just the core behavior of large networks.

One of two different reverse algorithms that compute pre-images directly are applied automatically by default for either local or nonlocal wiring. However, the nonlocal algorithm can be applied to local wiring as a reality check. The inner workings of both algorithms can be observed.

As a further reality check, an exhaustive algorithm can be selected. There are further options for attractor basins of networks with sequential instead of synchronous updating, and for (biased) random maps, which rely on the exhaustive algorithm. Finally, there is an option to generate neutral order components utilising DDLab's subtree methods, showing how the space of sequential orders is partitioned into sets of orders with identical dynamics.

\subsection{Subtree or single basin}

If a single basin or subtree was selected at the first DDLab prompt in section 6.2 (and a seed in chapter 21) a top-right prompt allows either running backward to generate a subtree, or running forward to determine the attractor cycle and generate a single basin of attraction,

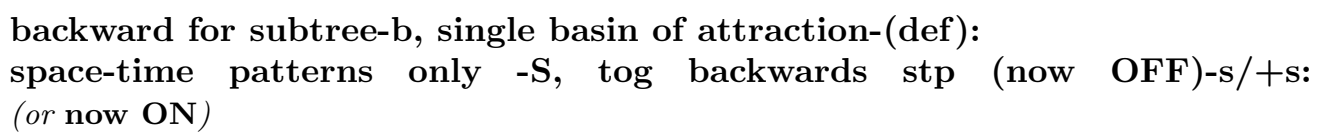

Enter return or $\mathbf{s}$ for a single basin of attraction. Enter $\mathbf{b}$ or $\mathbf{b s}$ for a subtree ${ }^{1} \cdot \mathbf{s} /+\mathbf{s}$ toggles the current setting for showing backward space-time patterns (section 24.10).

\footnotetext{
${ }^{1}$ Select a subtree if "neutral order components" are to be generated (section 29.10).
} 
Enter $\mathbf{S}$ to abandon further options in this chapter and proceed with the options for just space-time patterns (chapter 31).

\subsection{Subtree: Run forward before running backwards}

If $\mathbf{b}$ is entered for a subtree in section 29.1, a further top-right prompt is presented to first run the network forward from the seed state by a specified number of time-steps before running backwards,

\section{forwards before backwards? how many steps (def 0 ):}

The state reached on the forward run becomes the root of the subtree. Running forwards before running backwards is usually necessary ${ }^{2}$, other than for very chaotic networks such as CA with "chain" rules (section 16.11) - for chain rules large network sizes $\left(n_{\text {Lim }}=65025\right.$, section 8.3) are permissible. For most other networks, a seed selected at random is very likely to be a gardenof-Eden state which has no pre-images thus no subtree, and a much smaller size is required (section 8.3).

Enter the number of forward steps, or accept the default, 0. A final prompt will be presented (section 29.4) before the subtree is drawn.

\subsection{Single basin of attraction}

If a single basin of attraction was selected in section 29.1 a final prompt will be presented (section 29.4) before the basin is drawn. For a single basin of attraction (or subtree) although $n_{\text {Lim }}=65025$ (section 8.3) large network sizes should be applied with extreme caution.

\subsubsection{Single basin history limit}

To generate a single basin of attraction, the network must first be run forward from the initial state (seed) to find the attractor. States at each successive time-step are added to a history array. The state at each new time-step is checked against this history looking for a repeat which would define the attractor cycle. The maximum number of steps in the history array (the history limit) is 65535 (the upper limit of an unsigned short int). If the number of iterations exceeds the history limit, no more time-steps will be added to the history array, but the checks of new time-steps against the history array will continue - for a large state-space and chaotic rules (especially chain-rules section 16.11) the attractor may therefore not be found.

\subsubsection{Interrupting while looking for attractor}

If the dynamics is interrupted (with q) while the network is still iterating forward looking for the attractor cycle, the following top-right message is presented,

\section{still looking for attractor cycle, 1247 iterations (for example) exit-q, cont-ret:}

\footnotetext{
${ }^{2}$ However, enter return for zero forward steps if "neutral order components" are to be generated (section 29.10).
} 
Enter return to continue looking, or $\mathbf{q}$ to give up - the top-right attractor basin complete prompt will appear (section 30.4).

\subsection{Final attractor basin prompt}

Before drawing attractor basins, a final top-right prompt is presented as a reminder of the type of wiring (local or nonlocal) and the type of attractor basin (subtree, single basin, or basin field), and giving further special options. Enter return to skip these options and generate the attractor basin. The wording of the prompt depends on the network parameters, and for example,

for a subtree with nonlocal wiring
subtree (nonlocal) $\mathbf{n}=\mathbf{8}$, local-x exh-e rmap-r seq-s nto-o
view ppstack- $1+$ pause-2, reorder(on) tog- $\mathrm{R} /+\mathbf{R}$, limit backsteps-b/+b:

for a single basin with nonlocal wiring single basin (local) $\mathrm{n}=14$, nonlocal-x exh-e rmap-r seq-s view ppstack-1, limit backsteps $+\mathrm{b}$ :

for basin of attraction field with local wiring basin field (local) $n=10$, nonlocal-x exh-e rmap-r seq-s nto-o view ppstack-1:

\subsubsection{Final attractor basin prompt — conditions and reminders}

The special options must meet the following conditions to be included in the final attractor basin prompt (section 29.4).

exh-e rmap-r seq-s . . state-space, $v^{n}$, must be within the limits in table 29.1.

nto-o ... for $v=2$ and $n \leq 12$ only, and a basin field, or a subtree with zero forward steps (section 29.2).

The first line of the final attractor basin prompt includes the following reminders,

subtree, single basin, basin field ... the type of attractor basin.

$\mathbf{n}=\mathbf{1 0}, \mathbf{n}=\mathbf{5 - 1 2} \ldots$ the network size $n$, or the start and finish for a range of $n$ (section 8.1).

(local), (nonlocal) ... refers to the wiring - whether local as in 1d CA, or nonlocal as in RBN, DDN, or for any other network with nonlocal wiring, including $2 \mathrm{~d}$ and $3 \mathrm{~d} \mathrm{CA}^{3}$. Local/nonlocal is toggled with $\mathbf{x}$ (section 29.4.2).

\subsubsection{Final attractor basin prompt — options summary}

Enter return to generate the attractor basin with the default reverse algorithm for local or nonlocal wiring. Other options are summarized below, and described in detail in the rest of this chapter,

\footnotetext{
${ }^{3} 2 \mathrm{~d}$ and $3 \mathrm{~d}$ CA "local" wiring is treated as nonlocal in DDLab's algorithms.
} 
options ... what they mean

nonlocal-x ... for 1d local wiring, enter $\mathbf{x}$ to redefine the wiring as nonlocal and use the nonlocal algorithm.

local-x ... for nonlocal wiring (except mixed- $k$ ), enter $\mathbf{x}$ to restore $1 \mathrm{~d}$ local wiring (if changed to nonlocal above), or to rewire any nonlocally wired network with 1d local wiring - the nonlocal wiring will be forgotten, but it could be saved and reloaded (chapter 19).

exh-e ... ( $v^{n}$ within the limits in table 29.1 only) for attractor basins of the current network using the exhaustive algorithm. Optionally save/print the exhaustive pairs. (section 29.7).

rmap-r... ( $v^{n}$ within the limits in table 29.1 only) for attractor basins of a random map (uses the exhaustive algorithm) consisting of exhaustive pairs (optionally with Hamming bias). Optionally load/save/print the exhaustive pairs. (section 29.8)

seq-s ... ( $v^{n}$ within the limits in table 29.1 only) for attractor basins of the current network, but with sequential updating (uses the exhaustive algorithm). (section 29.9)

nto-o ... (subject to the conditions in section 29.4.1) to find the neutral order components in sequential updating and show the components as if they were subtrees. (section 29.10).

view ppstack-1 ... to view the changing partial pre-image stack as a histogram, indicating the inner workings of the reverse algorithms (section 29.6) for either local wiring (section 29.6.1), or nonlocal wiring (section 29.6.2).

+pause-2 ... (for nonlocal wiring only) to view the partial pre-image histogram, as above, and also pause after each set of valid pre-images (section 29.6.2).

reorder(on)-R/+R ... (for nonlocal wiring only) the reverse algorithm reorders the cells for more efficient computation. The reordering can be toggled off/on by entering $\mathbf{R}$, or another entry followed by $\mathbf{R}$, for example $\mathbf{1 R}$ (section 29.6.3).

limit backsteps-b/+b ... (for a single basin or subtree only) to restrict the number of backward steps, enter $\mathbf{b}$ or another entry followed by $\mathbf{b}$ (section 29.5).

\subsection{Limit backward steps}

Its possible to limit the number of backward steps (or levels) in subtrees (from the root state), or in the trees of single basins (from the attractor), to see just the core behavior. If $\mathbf{b}$, or another entry followed by $\mathbf{b}$, is entered in section 29.4 , the following top-right prompt is presented,

\section{enter max backward steps (def no limit):}

Enter the number of backward steps. Trees and subtree will stop generating further pre-images when the number is reached. Enter return not to impose a limit. 

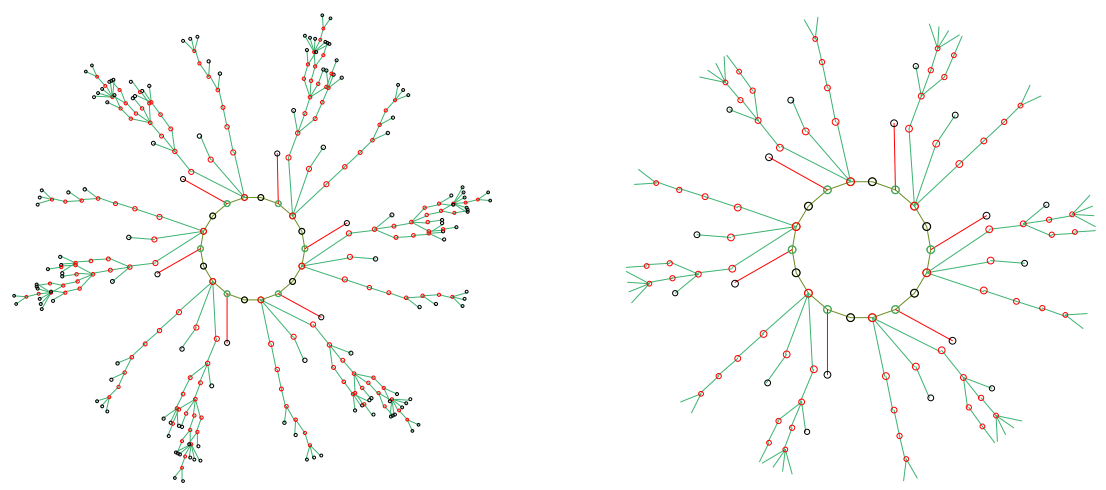

Figure 29.1: Limiting the number of backward steps from the attractor in the trees of a single basins. Left: the complete basin. Right: trees limited to 5 backward steps. $v 2 k 3$ rcode(dec)110, $n=12$, seed (hex) $08 \mathrm{e} 0$.

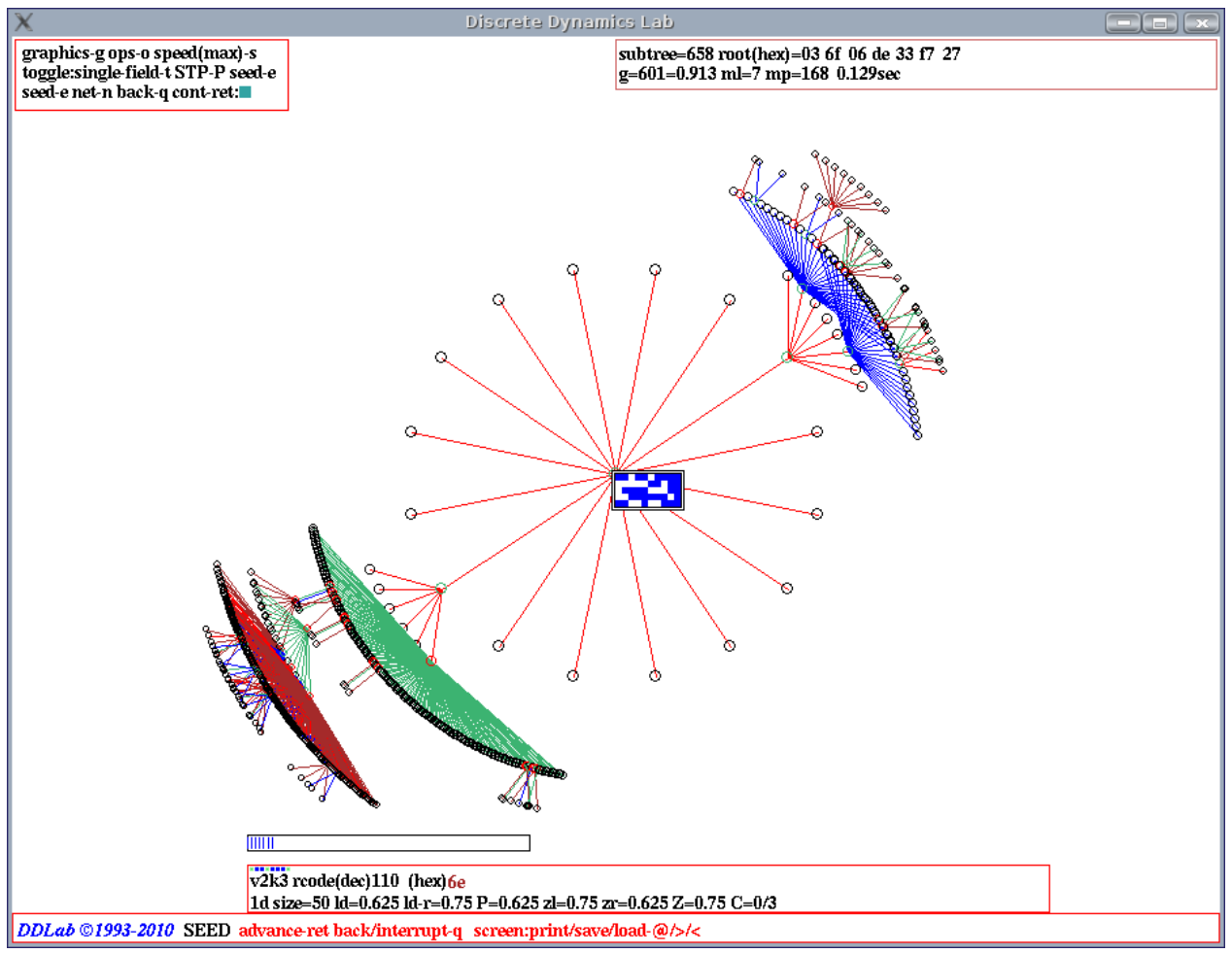

Figure 29.2: A 1d local CA subtree, showing the partial pre-image stack, $v 2 k 6 \operatorname{rcode}(\mathrm{dec}) 110, n=50$. The partial pre-image stack is represented by a horizontal bar extending from left to right within a rectangle near the bottom of the screen - its length (in pixels) shows the varying size as the partial pre-image stack grows and shrinks, leaving a trace of its maximum extent (shown in this example). It may be necessary to slow computation (sections $24.11,30.4$ ) to see the bar. 


\subsection{Viewing the partial pre-image stack}

For networks with synchronous updating (the default in DDLab) two distinct reverse algorithms apply to generate pre-images[25, 26, 31] — for either local or nonlocal wiring. The local algorithm is for homogeneous- $k$ networks with 1d CA (nearest neighbor) wiring, but with possibly mixed rules. The nonlocal algorithm is for any other type of wiring (RBN, DDN, mixed- $k, 2 \mathrm{~d}$ or $3 \mathrm{~d}$ networks). The nonlocal algorithm in general is considerably slower. Both algorithms work by generating a set of partly computed pre-images, "partial pre-images", held in a stack until either completed or rejected as invalid. Each partial pre-image can give rise to more partial pre-images.

Some inner workings of these algorithm may be observed by viewing the partial pre-image stack as it initially grows, then shrinks, both for the local wiring algorithm (section 29.6.1), and for nonlocal wiring algorithm (section 29.6.2).

\subsubsection{Partial pre-image stack, local wiring}

For local 1d wiring, enter 1 in section 29.4, to see how the partial pre-image stack varies in size for the local $1 \mathrm{~d}$ reverse algorithm. This is shown as a horizontal bar near the bottom of the screen, whose length (in pixels) corresponds to the varying size of the partial pre-image stack (figure 29.2).

\subsubsection{Partial pre-image stack, nonlocal wiring}

For nonlocal wiring, enter $\mathbf{1}$ or $\mathbf{2}$ in section 29.4 for a view of the inner workings of the nonlocal reverse algorithm. A histogram will appear in the lower half of the screen with $n$ columns (figure 29.2) showing the changing partial pre-image stack for each successive cell in the network starting from the left. When the attractor basin is complete (or abandoned), a final histogram gives the average over all histograms to date.

If $\mathbf{1}$ is entered in section 29.4, the histogram changes continually, and the following top-right prompt is presented,

\section{partial pre-image histogram: start pause-p:}

If $\mathbf{2}$ is entered in section 29.4, or if $\mathbf{p}$ is entered above, there will be a pause just before each pre-image fan is drawn — but no pause for garden-of Eden states. The following top-right prompt is presented, explained below,

pre $=81$ (wild $=9$ ) abandon pause-p, early exit-q, see next-def:

$\frac{\text { info or option }}{\text { pre }=\mathbf{8 1}} \ldots \frac{\text { what it means }}{\ldots \text { the size of the pre-image fan. }}$

(wild=9) ... (if wildcards exist) the number of pre-images resulting from wildcards.

abandon pause-p ... to abandon pausing.

early exit-q ... to abandon the single basin or subtree (section 30.2.3), giving the

"Attractor basin complete" prompt (section 30.4).

see next-def ... for the next pre-image fan and pause. 

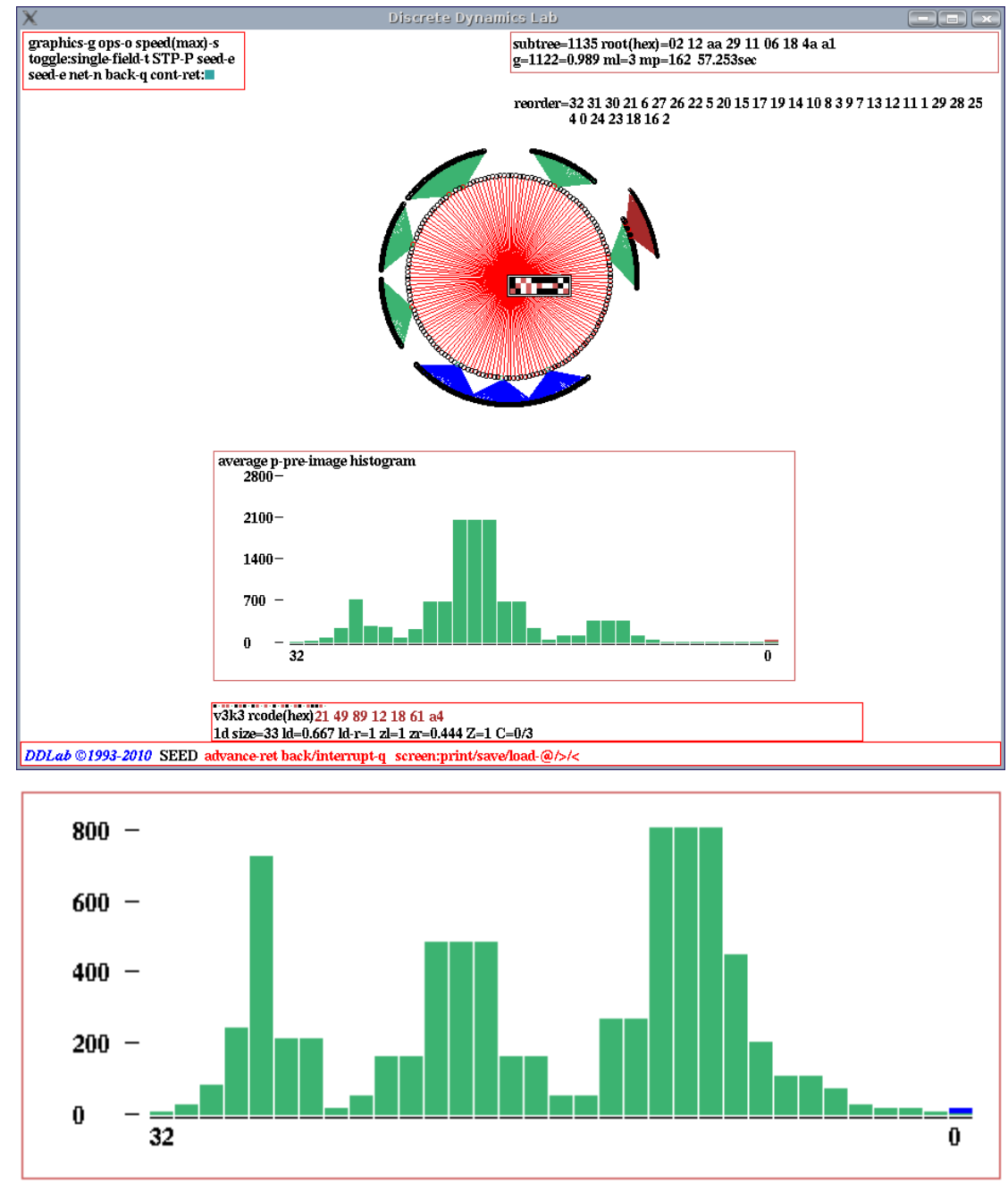

Figure 29.3: A 1d nonlocal CA subtree ( $v 3 k 3, n=33$ - defined below). The changing histogram shows the varying size of the partial pre-image stack for each next cell. The final bar height represents the number of pre-images of a particular state. The numbers below the top-right window show how cells were reordered to optimize the nonlocal 1d reverse algorithm (section 29.6.3).

Top: The DDLab screen showing the complete subtree and the final histogram giving the average over all histograms to date. $\underline{\text { Above: }}$. The histogram of the first subtree at level 1 . The network: $n=33, v 3 k 3$ rcode(hex) $214989121861 \mathrm{a} 4$ (chain rule) and random wiring defined in file v3n33alg.wso, 2 steps forward from the initial state (hex) 010a154814556a4201 before running backwards.

Successive values in the histogram tend to increase then decrease - if the value reduces to zero at any time the state is a garden-of-Eden state. The value of the last green column represents the final set of pre-images. However these pre-images may contain "wild-cards" cells, which have no outwires. An additional blue column (red for the final histogram) on top of the last green column shows additional pre-images that result from filling in these wild-cards. 


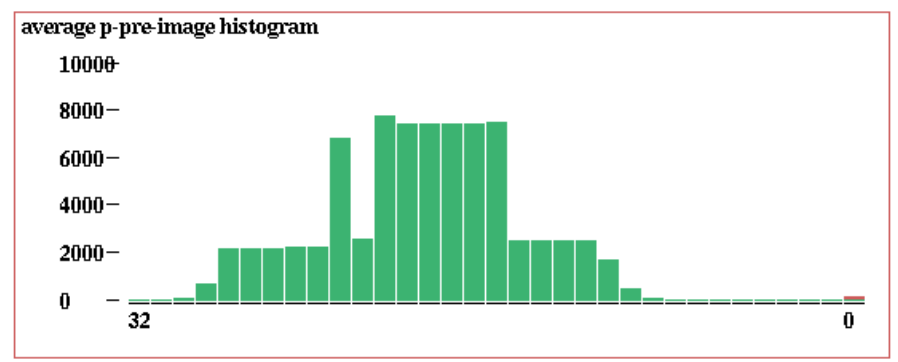

Figure 29.4: The final partial pre-image histogram, with the reordering algorithm suppressed, for the same network as in figure 29.3 Top. An approximate comparison of the time $t$, and the maximum average stack height $h$, with the reordering algorithm active is as follows, active: $t=57 \mathrm{sec}, h=2000$, inactive: $t=5 \mathrm{~min} 51 \mathrm{sec}, h=8000$.

\subsubsection{Reorder to optimize the nonlocal reverse algorithm}

The algorithm to reorder cells for more efficient computation is implemented by default, however it can be toggled off/on by entering $\mathbf{R}$, or another entry followed by $\mathbf{R}$, for example $\mathbf{1} \mathbf{R}$ in section 29.4. Toggling reordering off implements the reverse algorithm from left to right.

The nonlocal reverse algorithm[26, 29] builds a stack of partial (i.e. incomplete) pre-images by taking each cell in turn and filling in valid entries taken from the rule-table of that cell according to the cell's wiring. Any conflict between successive entries disqualifies the partial pre-image. The partial pre-image stack will grow, then shrink as conflicts mount (figure 29.3).

The algorithm works for cells taken in any order. However, to reduce the growth of the partial pre-image stack, the cells are reordered to ensure there is wiring overlap of successive cells (if possible), to allow conflicts to happen early. For fully random wiring, this reduces the growth of the partial pre-image stack considerably compared to a left to right (or some arbitrary) order. A left to right order is of course very efficient for 1d CA wiring. Memory load can be reduced and computation speed increased. The new order (if applied) is shown below the top-right prompt window (figure 29.3). The reordering reduced the time (and memory) to complete this subtree by a factor of about 6 (figure 29.4).

The new order is local within the reverse algorithm and makes a small difference to the presentation of attractor basins. The partial pre-image histogram in section 29.6.2 (if active) will appear according to the new order.

\subsection{Exhaustive algorithm}

$v^{n}$ within the limits for the exhaustive algorithm in table 29.1

Enter e in section 29.4 to use the exhaustive algorithm, instead of either of the default reverse algorithms (sections 29.6, 2.18). The following exhaustive pairs prompt (below the top-right) is presented, and options summarized below.

exhaustive pairs: compute-ret, and save-s, and prt-p/+p: (prt-p/+p not for DOS) 


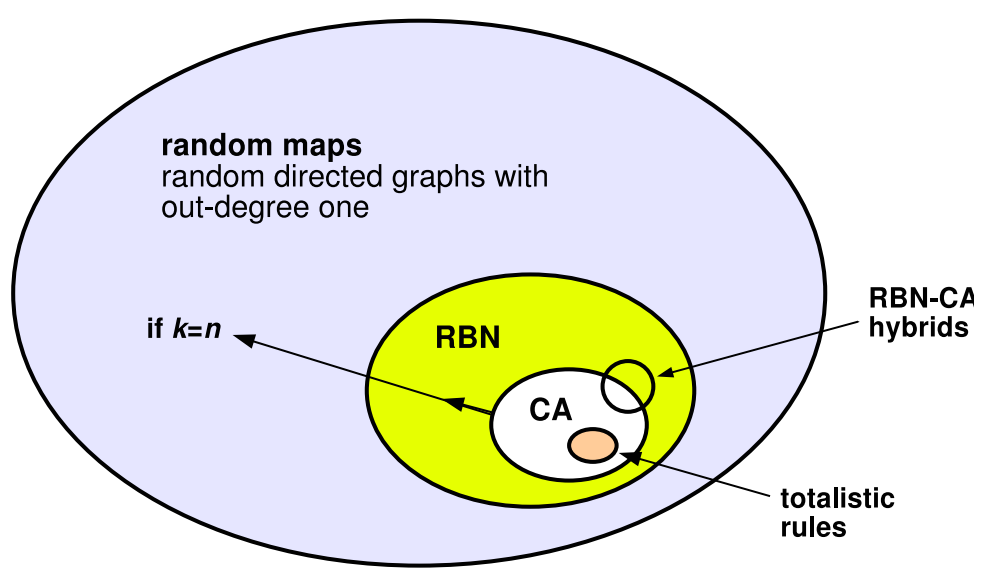

Figure 29.5: CA, DDN/RBN, and random maps, are systems that form a hierarchy of subsets, with random maps the most general. This is reflected by the reverse algorithms, for (a) 1d local wiring, (b) nonlocal wiring but including (a), and (c) exhaustive testing which includes (a) and (b) as well as random maps and asynchronous updating.

After the attractor basin is drawn, the exhaustive algorithms remains active and the prompt reappears before the next mutant (chapter 28) — enter $\mathbf{q}$ to exit.

options ... what they mean

return ... to compute the list according to the current network architecture.

and save-s ... to compute the list as above, and save it to a .exh file, which can be loaded as a random map (sections 29.8 and 29.8.1).

and prt-p/+p ... (not for DOS) enter $\mathbf{p}$ to compute the list as above, and print to the terminal (section 29.7.3), or or $\mathbf{l p}$ or sp to load or save the list and print it at the same time.

Although the exhaustive algorithm is limited to small networks the method applies to CA, $\mathrm{DDN} / \mathrm{RBN}$, and random maps, systems that form a hierarchy of subsets with random maps as the most general (figure 29.5). The exhaustive algorithm also applies to asynchronous updating (section 29.9).

The exhaustive algorithm is efficient for basin of attraction fields, or large single basins/subtrees that use up a good proportion of state-space, and additionally is not sensitive to neighborhood size, $k$. However the method is highly sensitive to increasing network size $n$. Every state in statespace must be iterated forward by one step according to the network architecture, and the list of $v^{n}$ "exhaustive pairs", each state and its successor (a non-random map) held in an array. The maximum network size $n_{\text {exhL }}$ is listed in table 29.1 for each value-range $v$. In practice $n$ is limited to much smaller networks to compute the complete list in a reasonable time. However, even with the maximum size it should be possible to see the start of the list being printed in the terminal (with prt-p/+p).

Once complete, attractor basins are generated by scanning the list of successors for pre-images of a given state - if the given state is not found it is a garden-of-Eden (leaf) state. 


\begin{tabular}{c|c|c}
$v$ & $n_{\text {exhL }}$ & state-space $v^{n} \approx$ millions \\
\hline 2 & 28 & $268435455 \approx 268.4$ \\
\hline 3 & 18 & $387420488 \approx 387.4$ \\
\hline 4 & 14 & $268435455 \approx 268.4$ \\
\hline 5 & 12 & $244140624 \approx 244.1$ \\
\hline 6 & 11 & $362797055 \approx 262.8$ \\
\hline 7 & 10 & $282475248 \approx 282.5$ \\
\hline 8 & 9 & $134217727 \approx 134.2$
\end{tabular}

Table 29.1: Maximum network size for the exhaustive testing algorithm for different value-ranges $v$, and the corresponding maximum state-space $v^{n}$.

\subsubsection{Generating the exhaustive list}

Enter return or $\mathbf{p}$ in section 29.7 to compute the exhaustive list. A horizontal red progress bar near the top-right of the screen will monitor the proportion of state-space completed so far, together with the following message,

\section{setting up exhaustive pairs, interrupt-q:}

If $\mathbf{q}$ is entered to interrupt, which may be necessary if you lose patience with a large network, the following prompt is presented,

exhaustive pairs $25 \%$ complete; stop-q options-o cont-ret:

Enter $\mathbf{q}$ to abandon the exhaustive pairs computation, enter o for the "options" prompt in section 30.4 , or return to continue.

\subsubsection{Saving the exhaustive pairs}

Enter $\mathbf{s}$ in section 29.7 to compute and then save the exhaustive pairs. Once the list is generated the following prompt is presented below the red progress bar,

\section{SAVE exhaustive pairs-s:}

Enter $\mathbf{s}$ to save the list as an .exh file (chapter 35). The list is essentially a random map (section 29.8) but is not random, and the file can only be loaded from the random map prompt (section 29.8, 29.8.1).

\subsubsection{Printing the exhaustive pairs in the terminal}

Enter $\mathbf{p}$ to compute and print the list of exhaustive pairs to the terminal, or sp to save the list and print it at the same time. The list is presented in four columns. The left two are the consecutive states in state-space ordered by the decimal equivalents of the rcode, first the state in decimal, then its bit/value string. The right two columns show the corresponding successor states, first state bit/value string, then in decimal - tables 29.2 and 29.3 give examples. 


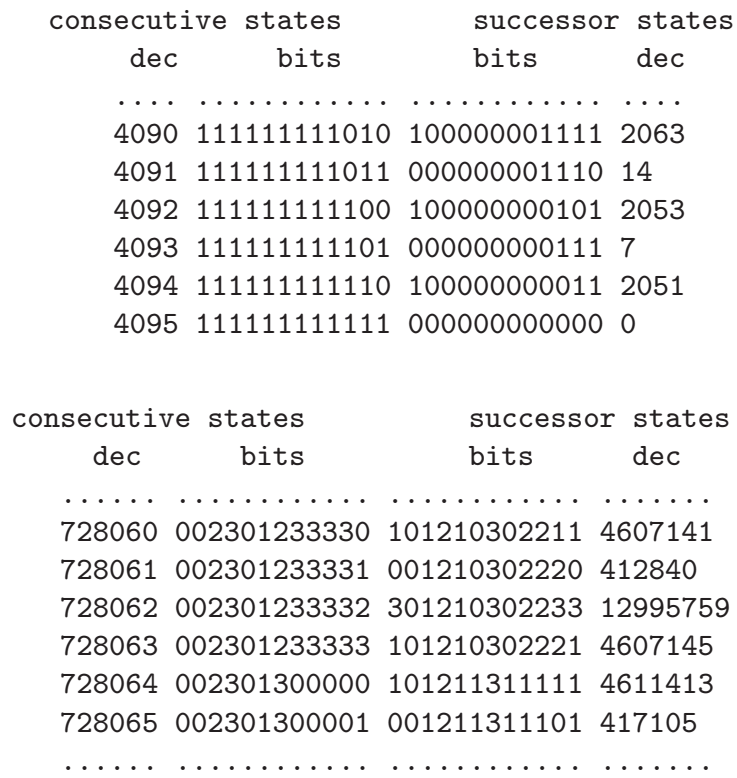

Table 29.2: Printing exhaustive pairs, the final six items of the list, for the $1 \mathrm{~d}$ CA $v 2 k 3$ rule (dec) 110 , $n=12$, as in figure 27.1 .

Table 29.3: Printing exhaustive pairs, for a $v 4 k 3$ rule selected at random, $n=12$. A six item sample from the list after 60 seconds, The complete list of 16777215 (16.7 million) items took about 3 minutes (without printing).

\subsection{Random map}

$v^{n}$ within the limits for the exhaustive algorithm in table 29.1

Enter $\mathbf{r}$ in section 29.4 to generate a random map, which also consists of exhaustive pairs but created at random (but possibly with a Hamming distance bias), instead of by some dynamical rule. The following prompt below the top-right is presented, with some options similar to "exhaustive pairs" (section 29.7) — the same size constraints apply (table 29.1),

$$
\begin{array}{r}
\text { random map: load-l, rnd-(def), set Ham-h, and save-s/+s, and prt-p/+p: } \\
(\text { prt-p/+p not for DOS) }
\end{array}
$$

After the attractor basin is drawn, the random map function remains active and the prompt reappears before the next (new) random map - enter q to exit. However, if Hamming bias was set, the prompt changes as follows,

random map: load-l, Ham1-(def), reset Ham-h, and save-s/+s, and prt-p/+p:

(Ham $x$ shows the current Hamming bias)

options... what they mean

load-1 ... to load an .exh file of a random map (section 29.8.1).

rnd-(def) ... enter return to create a random map, which is a list of random states just like exhaustive pairs. While the (random) exhaustive pairs are being assigned, a horizontal red progress bar near the top-right of the screen will monitor the proportion of state-space completed so far, together with prompts to interrupt etc. as described in section 29.7.1. 


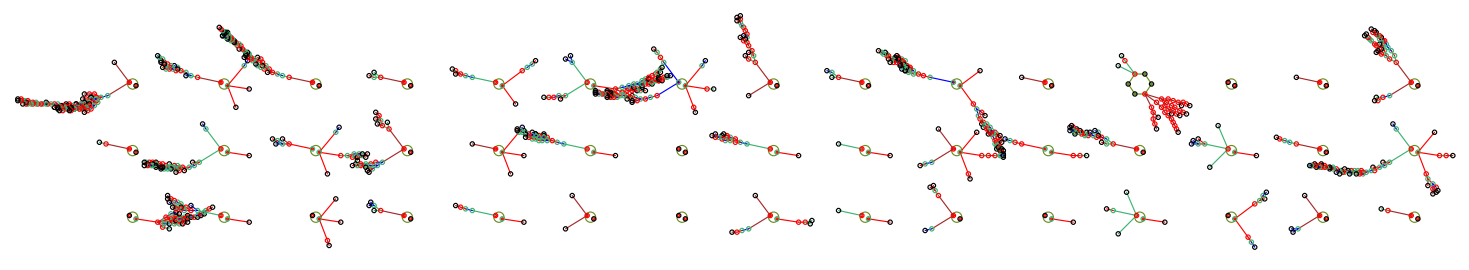

$H=1$
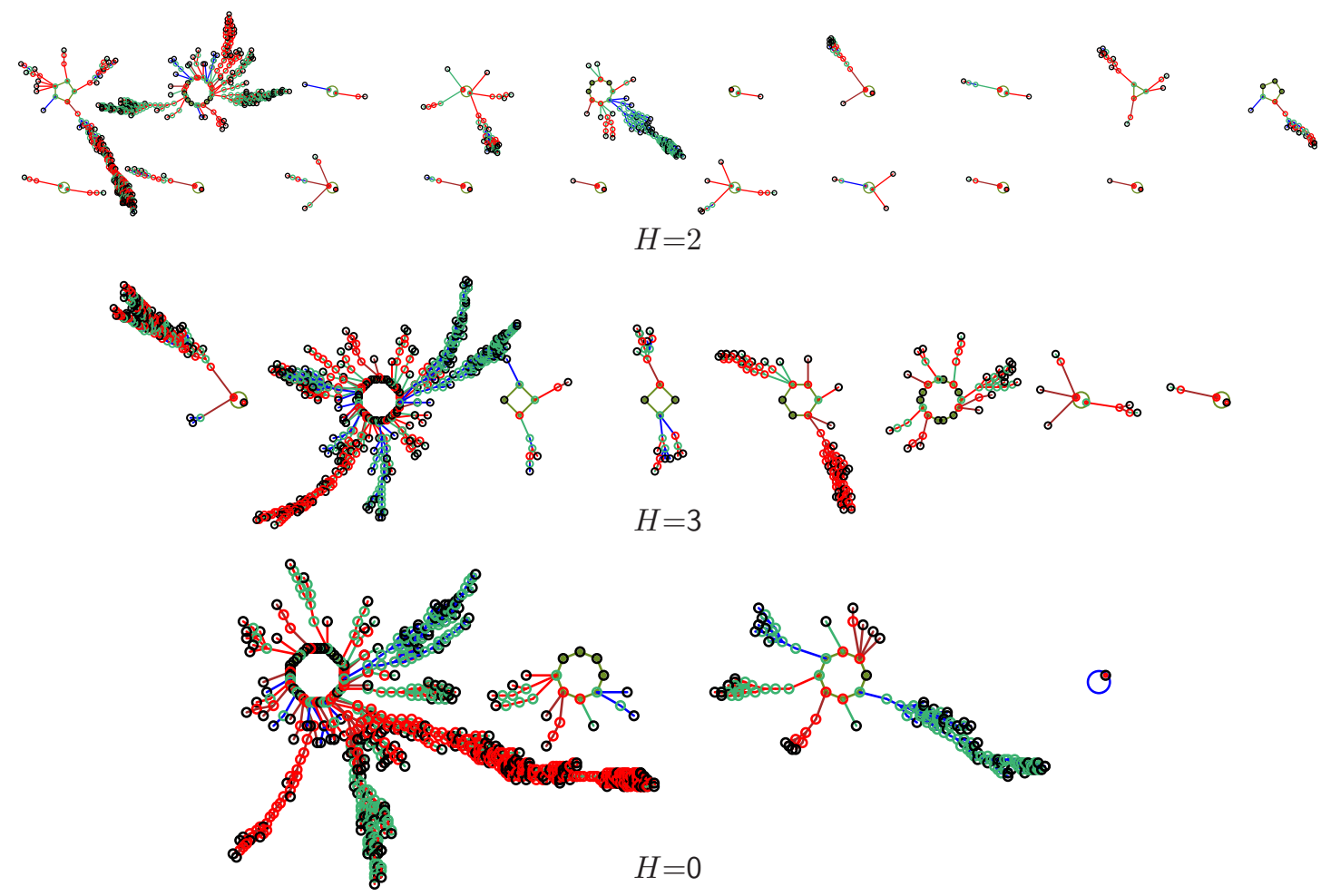

Figure 29.6: Typical random map graphs (basin of attraction fields) with different Hamming bias $H$. From the top, $H=1, H=2, H=3$, and without bias $H=0$, so fully random. $H=1$ is the most fragmented, $H=0$ tends to have a characteristic giant component $[29$, ch.3].

set Ham1-(def) ... enter return to create a random map with the current Hamming distance bias, shown as $\operatorname{Ham} x$, i.e. Ham2, Ham3 etc.

set Ham-h ... (or reset Ham-h) to create a random map, but biased according to Hamming distance (section 29.8.2).

and save-s $/+\mathbf{s} \ldots$ enter $\mathbf{s}$, to create a random map as above, and save it to a .exh file, or hs to reset the Hamming bias and save. A prompt will appear to SAVE exhaustive pairs-s:, the same as in section 29.7.2.

and prt-p $/+\mathbf{p} \ldots$ (not for $D O S$ ) enter $\mathbf{p}$ to create a random map as above,and print to the terminal (section 29.7.3), or lp or sp, to load or save the list and print it at the same time, or hsp to reset the Hamming bias, save, and also print. 
In DDLab, a random map is a directed graph with out-degree one, representing an exhaustive list of transitions, and is arguably the most general discrete dynamical system. The set of all random maps contains the subset of all DDN/RBN, which in turn contains the subset of all CA.

Provided that the size of the map is a power of the value-range $v$, the function to set up a random map is implicit in the exhaustive testing algorithm (section 29.7). This algorithm creates a list of $v^{n}$ pairs of states, each state and its successor, by iterating each state in state-space forward by one step according to the network dynamics. A random map, on the other hand, assigns the successors at random (or with some bias). Assigning successors according to the dynamics of a particular class of network is just a special case of such a random assignment.

Some randomly assigned successors states are likely to occur more than once, so other states will not occur at all (there will be no room left in the list). These are the states without pre-images, "garden-of-Eden" states. A random map has all the properties of a dynamical system, trajectories, attractors, etc. though this may be irreducible to a sparsely connected network. A fully connected network, however, where $k=n$, where each cell receives inputs from all cells (including from itself), can implement any random map. The space of all such fully connected networks, and all random maps (of the Boolean hypercube of length $n$ ) is $\left(2^{n}\right)^{2^{n}}$, which is also the upper limit for the space of all possible binary basin of attraction fields.

\subsubsection{Loading the random map or exhaustive pairs}

Enter $\mathbf{l}$ in section 29.8 to load a previously saved list of exhaustive pairs from an .exh file (chapter 35), and draw the attractor basins. Note that the current values of $v, n$ must match the file value of $v, n$. If not, a top-right warning ${ }^{4}$ such as the following will be displayed,

file $\mathbf{n}=\mathbf{7} !=$ network $\mathbf{n}=\mathbf{8}$ (wrong file) cont-ret: (values shown are examples)

The default filename for a random map (or exhaustive pairs) file is myexh_v $x$.exh where $x$ is the current value-range $v$. The (binary) encoding is described in section 35.1 (EXHAUSTIVE).

\subsubsection{Biasing the random map by Hamming distance}

If $\mathbf{h}$ was entered in section 29.8 the random map can be biased (or the current bias reset) according to Hamming distance. The following prompt near the top-right is presented,

$$
\text { set Hamming distance (0-10): (for } n=10)
$$

Enter the Hamming distance $H(0-n)$. 0 signifies no bias, a fully random map. If biased, the exhaustive pairs will differ by $H$ bits or values, i.e. between each bit/value string and its assigned successor. figure 29.6 shows random map graphs (basin of attraction fields) with a range of Hamming bias $H$ from $H=1$ to no bias, a fully random map. $H=1$ is the most fragmented, $H=0$ tends to have a characteristic giant component[29, ch.3].

While the exhaustive pairs are being assigned, a horizontal red progress bar near the top-right of the screen will monitor the proportion of state-space completed so far, together with prompts to interrupt etc. as described in section 29.7.1.

\footnotetext{
${ }^{4} \mathrm{~A}$ mismatch in $v$ will usually be detected because the file size will be incorrect, but this will be reported as a mismatch in $n$.
} 


\subsubsection{Random map data}

In addition to the normal data shown in a top-right window when a subtree, basin, or the basin of attraction field is complete (chapter 27), for a complete random map (basin of attraction field), additional data shows the frequency of different cycle periods in a bottom-center window, which is of some interest in graph theory[29, ch.3]. This example (from figure 29.6 $H=2$ ) shows that there are 14 cycles with period 2, 1 with period 3, etc. The last column $(10+)$ gives the frequency of period 10 or more.

$\begin{array}{llllllllllll}\text { Random Map, v2 } \mathrm{n}=10 & \text { cycle period: } & 1 & 2 & 3 & 4 & 5 & 6 & 7 & 8 & 9 & 10+ \\ \text { Ham bias=2 } & \text { cycle frequency: } & 0 & 14 & 1 & 0 & 2 & 0 & 0 & 1 & 0 & 1\end{array}$

For a single basin or subtree, the window just gives the message,

Random Map, v2 $\mathbf{n}=\mathbf{1 0}$ (for example)

\subsection{Sequential updating}

$v^{n}$ within table 29.1 - this limit does not apply for space-time patterns (section 31.3.2)

By default, network updating is synchronous, in parallel. Alternatively, the updating can be sequential ${ }^{5}$. The way that cell indexes are listed from left to right is the sequential order, and corresponds to an update-tag (from 0 to $n$-1), for example,

$\begin{array}{llllllllllllll}0 & 1 & 2 & 3 & 4 & 5 & 6 & 7 & 8 & 9 & 10 & 11 & -\end{array}$ the update-tag for $n=12$

$\begin{array}{llllllllllllll}2 & 8 & 10 & 5 & 6 & 9 & 7 & 4 & 11 & 0 & 3 & 1 & - & \text { a possible sequential order }\end{array}$

There are $n$ ! (factorial $n$ ) possible sequential orders in a network of size $n$. DDLab provides simple default orders, left to right (e.g. 9876543210 for $n=10$ ), right to left (e.g. 0123456789 for $n=10$ ), and a random order. A specific order can also be set by hand. The order can be saved and loaded from a ord file. For $n \leq 12$, all possible orders can be listed - identified by an "order-index" from 0 (left to right) to $n$ !-1 (right to left), and an order can be chosen from this list.

Enter $\mathbf{s}$ in section 29.4 for sequential updating. with the following top-right prompt,

sequential update: >-(def) <-b rnd-r set-s all-S: (all-S if $n \leq 12)$

At the same time a top-center reminder backtrack sequential-q remains in place while sequential selection is in progress. The options are summarized below.

options.... what they mean

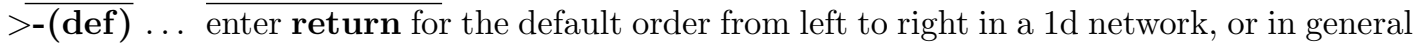
counting down the cell index $n-1$ to 0 (section 10.2).

$<-\mathbf{b}$... for the opposite order, from right to left, cell index 0 to $n-1$.

rnd-r ... for a random order (section 29.9.1).

set-s ... to set a specific order by hand (section 29.9.2).

all-S ... (if $n \leq 12$ ) to list all $n$ ! possible orders and select a specific order from the list (section 29.9.3).

\footnotetext{
${ }^{5}$ This section is also relevant to sequential updating for space-time patters (section 31.3 .2 )
} 

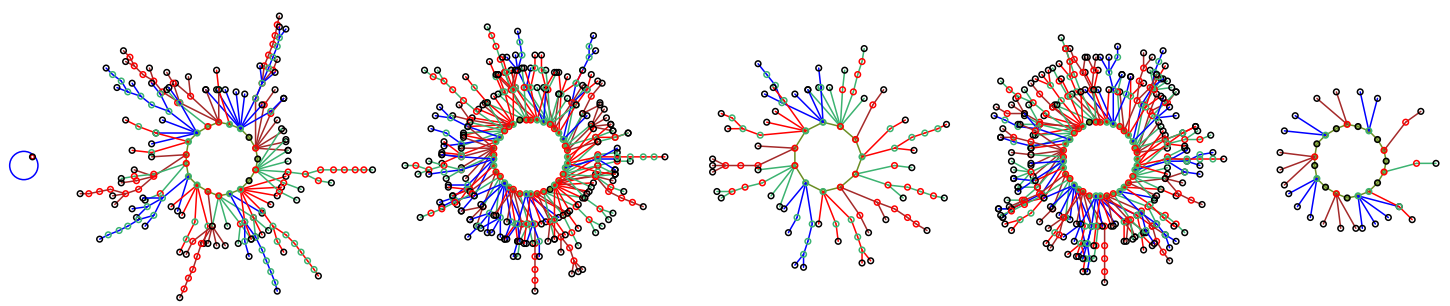

(a) order $=$ right $\rightarrow$ left, 0123456789

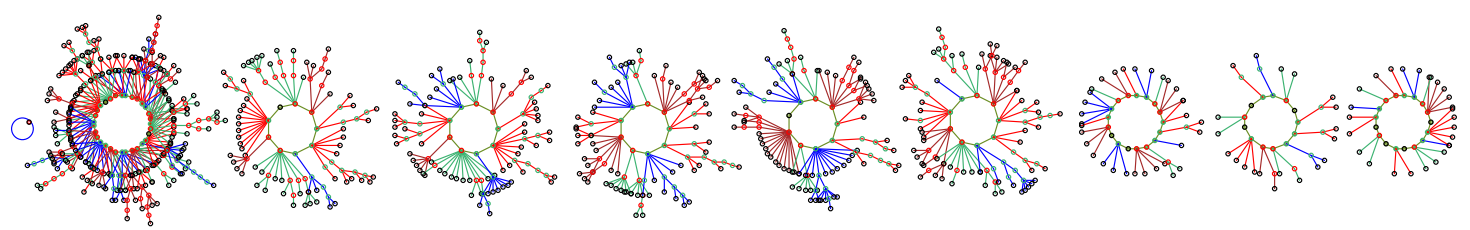

(b) randomly ordered, order $=4536780129$

Figure 29.7: A basin of attraction field for a 1d CA with sequential updating, $v 2 k 3$ rule (dec)110, $n=10$. (a) sequential left to right, (b) randomly sequential. The order is shown in the lower rule window.

\subsubsection{Random order}

Enter $\mathbf{r}$ in section 29.9 (or in section 31.3 .2 for space-time patterns ${ }^{6}$ ) to set a random order. A top-right prompt shows the order (which can be revised), together with the order-index in decimal and hex if $n \leq 12$, for example,

random order, $\mathrm{n}=12$

50131094811726 (dec)=199658374 (hex)=be68b84 (for $n=14)$

update-tags shown from $0-11$

save/load-s/l $>-f<-b$ re-order-r cont-ret:

In the example above, cell index 5 (update-tag 0 ) is the first to be updated, and cell index 6 (update-tag 13) is the last to be updated. The info and options are described below,

info and options... what they mean

$\mathbf{n}=\mathbf{1 2} \ldots$ the system size.

$\begin{array}{lllllll}5 & 0 & 1 & \ldots & 6 & \ldots\end{array}$ the random order as a list of cell indexes.

$(\mathbf{d e c})=\ldots \quad$ (if $n \leq 12)$ the order-index in decimal, a number defining the order.

$($ hex $)=\ldots \quad$ if $n \leq 12)$ the order-index in hexadecimal.

update-tag shown from $\mathbf{0 - 1 1} \ldots$ reminder of the update-tags.

save-s ... to save the order to a .ord file (section 35.2).

load-1 ... to load an order.

$>$-f ... for a left to right order, as in section 29.9.

$<-\mathbf{b} \ldots$... for a right to left order, as in section 29.9.

re-order-r ... for a new random order.

cont-ret ... to accept the order and continue.

\footnotetext{
${ }^{6}$ For space-time patterns (section 31.3.2) - for large networks there are two extra options: more-m to see the next window of the update order, and jump-j to jump to a selected update-tag.
} 


\subsubsection{Set specific order}

Enter $\mathbf{s}$ in section 29.9 to set a specific order by hand. The sequence of cell indexes is set with the following top-right prompt,

$$
\begin{aligned}
& \text { enter cell index (no repeats) } 0-11 \text { or rnd-(def) back-b } \\
& \text { update 4: } \quad 48103 * * * * * * * * \text { (for } n=12 \text {, for example) }
\end{aligned}
$$

In this example, update-tags $0-3$ have been entered as cell indexes $4,8,10,3$, and the cell index for update-tag 4 is requested. A prompt for each update-tag from 0 to $n$ - 1 is presented in turn. Stars $(*)$ indicate unallocated update-tags, otherwise the allocated cell indexes are shown. Enter a unique cell index (repeats will be rejected) for each successive update-tag. return gives a valid random cell index. Enter $\mathbf{b}$ to backtrack to the previous update-tag.

Once the selected sequence is complete, the following top-right prompt is presented,

sequence complete 0-11 (for $n=12$ )

48103091116527 (dec)=188097014 (hex)=b3621f6 save/load-s/l reselect-r cont-ret:

info and options ... what they mean

0-11 ... the range of cell indexes, and update-tags for $n=12$.

$\begin{array}{lllllll}4 & 8 & 10 & 3 & \ldots & 7 \ldots & \ldots\end{array}$ the sequential order as a list of cell indexes.

$(\mathbf{d e c})=(\mathbf{h e x})=\ldots \quad($ if $n \leq 12)$ the order-index in decimal and hex, as in section 29.9.1.

save/load-s/1 . . . to save or load the order to a .ord file as in section 29.9.1.

reselect-r ... to start again — set a new order.

cont-ret ... to accept the order and continue.

\subsubsection{List all orders}

$n \leq 12$ only

Enter $\mathbf{S}$ in section 29.9 to list all $n$ ! possible orders and select a specific order. As many orders as will fit will be displayed in a window on the right of the screen, followed by further options. For example, for $n=12$ (the maximum list size permitted) the first two orders and last two orders in the list are shown below,

all possible orders $n=12,0-479001599$

decindex - order - hexindex

0 - $01323456789 \mathrm{ab}-0$ (this is the right to left order)

$1-01323456789 \mathrm{ba}-1$

... (the list continues, the last two order are shown below)

$$
\begin{aligned}
& 479001598 \text { - ba987654b3201 - 1c8cfbfe } \\
& 479001599 \text { - dcba9876543210 - 1c8cfbff (this is the left to right order) } \\
& \text { jump/copy-j orderseed-s accept-a more-ret: (or top-ret if on the last page) }
\end{aligned}
$$

The center column is the sequential order, with cell indexes shown in hex for compactness. The left and right columns show the order-index ( 0 to $n$ !) which specifies and codes for each order. The left column is in decimal, the right in hex.

The prompts on the bottom line are explained below, 


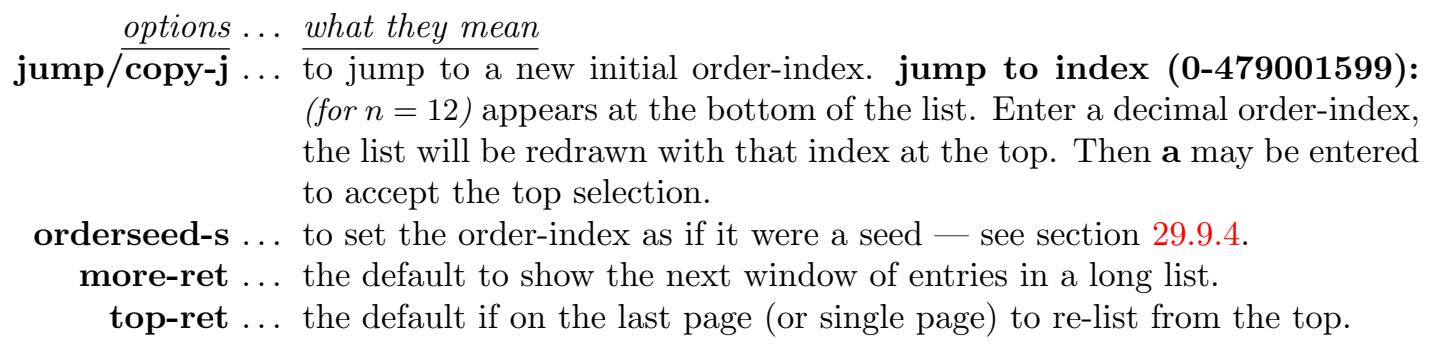

\subsubsection{List all orders — set order seed}

Enter $\mathbf{s}$ in section 29.9.3 to set the order-index as if it were a seed (chapter 21). A lower left order seed prompt appears with similar options ${ }^{7}$ to the seed prompt (sections 21.1,21.2),

Select order seed, $\max =(\operatorname{dec}) 479001598=($ hex $) 1 \mathrm{c} 8 \mathrm{cfbff}$ prtx- $\mathrm{x}$ rnd-r bits1d-b bits2d-B hex-h dec-d (def-d): (for $n=12)$

These options are fully described in section 21.2 and further sections in chapter 21, and are briefly summarized below,

$$
\begin{aligned}
\text { options } & \cdots \\
\text { dec-d } & \ldots \text { what they mean } \\
& \text { is greater than max, too big! back-ret will appear to re-enter. }
\end{aligned}
$$

rnd-r bits1d-b bits2d-B hex-h ... any of these methods also apply for the order seed. If the entry exceeds max, the following message appears re-enter,

order index $55889>\max (40319)$ cont-ret: (for $n=8$ )

As soon as the list (section 29.9.3) and its prompts reactivate, enter a to accept the selection.

\subsubsection{Drawing the attractor basin with sequential updating}

Once the sequential order has been set, the exhaustive pairs prompt will be shown (section 29.7). - enter return to compute ${ }^{8}$ while displaying the prompt in section 29.7.1. Once the exhaustive pairs are complete, the attractor basin will be drawn. At the same time, the "rule window" will be displayed at the bottom of the screen, showing the rule (or the rule at index 0 for an RBN), and other information (section 16.19), and in addition the updating order is shown in alternating red-black colors. For $n \leq 12$ the sequential order is shown in hex, and the order-index is also shown in decimal and hex. For $n>12$ just the sequential order is shown in decimal.

\section{sequpdate: 4536780129 dec $=1630512$ hex $=18 \mathrm{e} 130$ v $2 \mathrm{k} 3$ rcode(dec) 110 (hex) $6 \mathrm{e}$ $1 \mathrm{~d}$ size $=10 \mathrm{ld}=0.625 \mathrm{ld}-\mathrm{r}=0.75 \mathrm{P}=0.625 \mathrm{zl}=0.75 \mathrm{zr}=0.625 \mathrm{Z}=0.75 \mathrm{C}=0 / 3$}

Figure 29.8: The rule window for sequential updating. This example is for $v 2 k 3 \operatorname{rcode}(\mathrm{dec}) 110, n=10$, and a sequential order as in figure 29.7 (b).

\footnotetext{
${ }^{7}$ Note that some of the sub-options in section 29.9 .4 may be irrelevant in the context of the order seed.

${ }^{8}$ This may take some time for larger networks, as noted in section 29.7)
} 


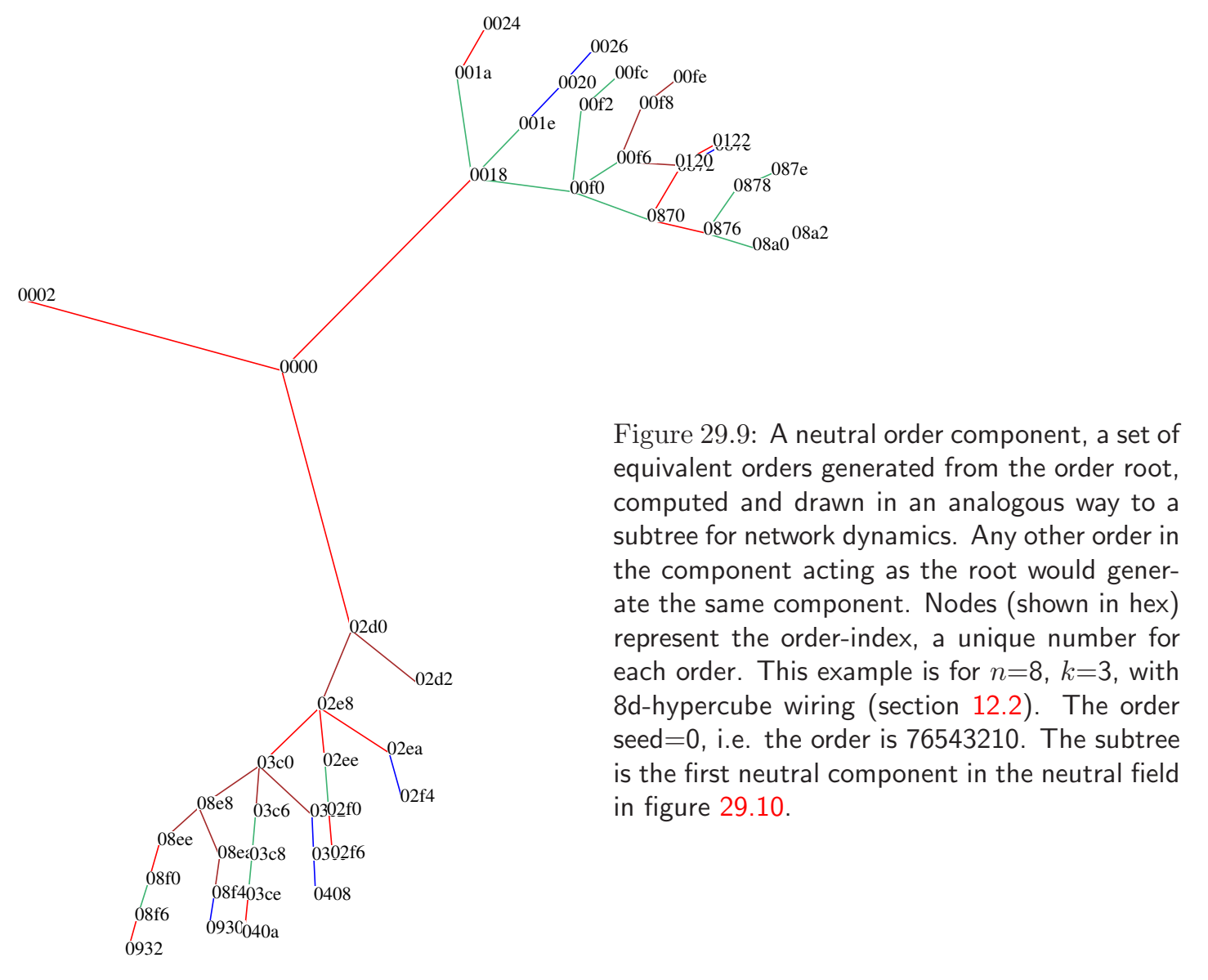

\subsection{Neutral order components}

for $v=2$ and $n \leq 12$ only, and a basin field, or a subtree with zero forward steps (section 29.2)

In sequential updating, different orders may produce equivalent dynamics. Its of some interest from a mathematical perspective to compute the neutral order components, how sequence space is divided up into sets of orders with identical dynamics. This turns out to depend on the network's wiring scheme. The algorithm in DDLab that computes neutral order components (limited to network size $n \leq 12$ ), does so by starting with a particular order (the order seed), and generating its equivalent orders by swapping the order of pairs of neighboring cells that have no links to each other, neutral pairs. Valid swaps within the first order are treated as "pre-images", and these pre-images may have pre-images in turn (repeat orders are discounted). When no valid swaps remain the neutral component is complete.

DDLab treats these components as subtrees. A single component subtree (figure 29.8), or the entire set of components subtrees (figure 29.10) making up sequence space (the neutral field) can be computed and drawn in an analogous way to a single subtree or basin of attraction field for network dynamics. The layout and node label options described in chapter 26 apply. 


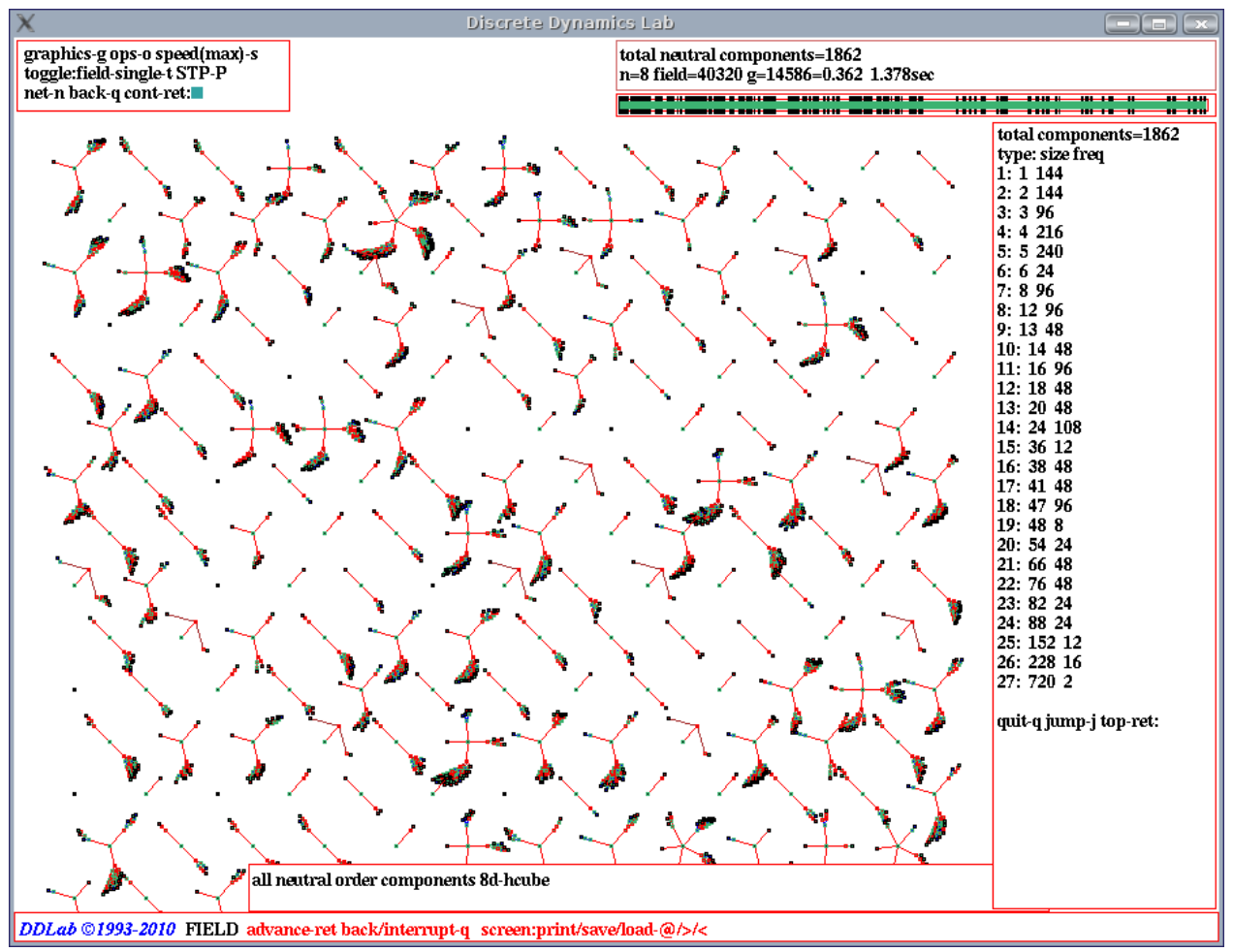

Figure 29.10: The neutral field, the subtrees making up sequence space - the first 175 or so, out of a total of 1862, are shown - computed and drawn in an analogous way to a basin of attraction field for network dynamics. A list of component sizes and the frequency of different sizes is shown in a window on the right once the neutral field is complete. This example is for $n=8, k=3$, with $8 \mathrm{~d}$-hypercube wiring (section 12.2). The first subtree (top right, order seed=0) is shown in detail in figure 29.9.5

Nodes are labelled according to the order-index (a unique number, 0 to $n$ !-1, section 29.9.3), in decimal, hex, as a bitstring, etc as previously selected in section 26.2. The default is as a spot.

Enter nto-o at the prompt in section 29.4 to generate the neutral subtree (section 29.10.1) or neutral field (section 29.10.2), depending on whether a subtree or basin of attraction field is currently selected ${ }^{9}$. Note that the rule or rule scheme, or a subtree's initial state, selected previously, plays no role in computing neutral order components.

\subsubsection{Neutral subtree}

The generate a neutral subtree, select backward for subtree-b in section 29.1, then return in section 29.2 to ensure zero steps for forwards before backwards?. Then enter nto-o in section 29.4 for a neutral subtree. A lower center window prompts for the neutral order seed, or subtree root,

\footnotetext{
${ }^{9}$ The neutral order prompt nto-o is not activated if a single basin of attraction was selected in section 29.1.
} 
Select order seed, $\max =(\mathrm{dec}) 40319=($ hex $) 9 \mathrm{~d} 7 \mathrm{f}$ prtx $-\mathrm{x}$

rnd-r bits1d-b birs2d-B hex-h dec-d (def-d): (for $n=8)$

Enter an order-index, 0 to $n !-1$ as the seed, in decimal, hex, as a bitstring, etc. (as in section 29.9.4). The selection works in the same way as selecting a network seed, described at length in chapter 21. A selection greater than $n !-1$ will be rejected, with the message,

order-index $44444>\max (4318)$ cont-ret: (for $n=8)$, for example)

The following examples relate to figure 29.9.5, for $n=8, k=3$, with 8d-hypercube wiring, (section 12.2) As the subtree is dawn, a message appears in a window at the bottom of the screen,

one neutral component 8d-hypercube

When the subtree is complete, a top-right window gives some data on the subtree as follows,

neutral component $\operatorname{size}=48$ root $($ hex $)=0000$
$\mathrm{~g}=16=0.333 \mathrm{ml}=8 \mathrm{mp}=3 \quad 0.006 \mathrm{sec}$

\subsubsection{Neutral field}

Enter $n$ to-o in section 29.4 for a neutral field. All neutral order components making up sequence space will be generated ${ }^{10}$ (figure 29.10). The order components are drawn as a series of "subtrees" (figure 29.9.5), and the data on each subtree appears in a top-right window while the next subtree is being drawn (section 29.10.1 describes the data). In order to pause to see the data for each subtree, select a pause in section 27.1.2.

Once the neutral field is complete, a top-right window shows data on the last subtree, and component types are listed on the right of the screen. The list gives the type according to size, the size itself, and the frequency of different sizes. As many component types as will fit will be listed, followed by further options. For example, for $n=8, k=3$, with $8 \mathrm{~d}$-hypercube wiring, shown in figure 29.10, the list is as follows,

total components $=1862$

type: size freq

1: 1144

2: 2144

3: 396

4: 4216

... (the list continues, the last three types are shown below)

25: 15212

26: 22816

27: 7202

quit-q jump-j top-ret: (top-ret if on the last page)

or

quit-q jump-j more-ret: (more-ret if more than one window is required)

\footnotetext{
${ }^{10}$ Although all neutral order component subtrees are generated, you only see those that fit within the DDLab screen, which depends on the layout and scale set in chapter 25.
} 
The prompts on the bottom line are explained below,

$$
\begin{aligned}
& \text { options .... what they mean } \\
& \text { quit-q ... Enter q to backtrack and show a summery of the neutral field in a top-right }
\end{aligned}
$$

$$
\begin{aligned}
& \text { total neutral components }=1862 \\
& \mathrm{n}=8 \text { field }=40320 \mathrm{~g}=14585=0.362 \quad 1.345 \mathrm{sec}
\end{aligned}
$$

The neutral field list will remain visible but inactive.

jump-j ... to jump to a new type index (useful for a long list),

$$
\text { jump to index (0-100): (for example) }
$$

appears at the bottom of the list. Enter a type index, the list will be redrawn with that index at the top.

more-ret ... the default to show the next window of entries in a long list.

top-ret ... the default if on the last page (or single page) to re-list from the top.

Its a good idea to drastically reduce the scale of the subtrees in section 25.2 as there are usually many small neutral order components. To reserve a vacant space for the list window and not to hide some subtrees, set the minimum right border at a high value in section 25.2.7. 


\section{Chapter 30}

\section{Drawing attractor basins, and changes on-the-fly}

not in TFO-mode.

Following the final options described in section 29.4, the drawing of attractor basins will commence, according to the current presentation, layout and other settings. During the drawing, some of these settings can be reset, either by pausing the drawing and responding to further prompts, or immediately on-the-fly by various key hits. Many network parameters may also be reset without the need to backtrack to the early prompts. This chapter describes these options.

Note also that if one of various "pause" options was selected in section 27.1.2, the angular orientation, spacing and/or position of each successive basin, tree or subtree can be amended during the pause (section 25.3).

\subsection{The progress bar for basin of attraction fields}

For basin of attraction fields only, a horizontal green progress bar below the data window (section 27.2) indicates how much of state-space has been used up so far. A bar that over-shoots or under-shoots indicates an error. The vertical bars on the horizontal bar indicate the seeds (by their decimal equivalent distance along the bar) for successive basins, starting with 0 on the left.

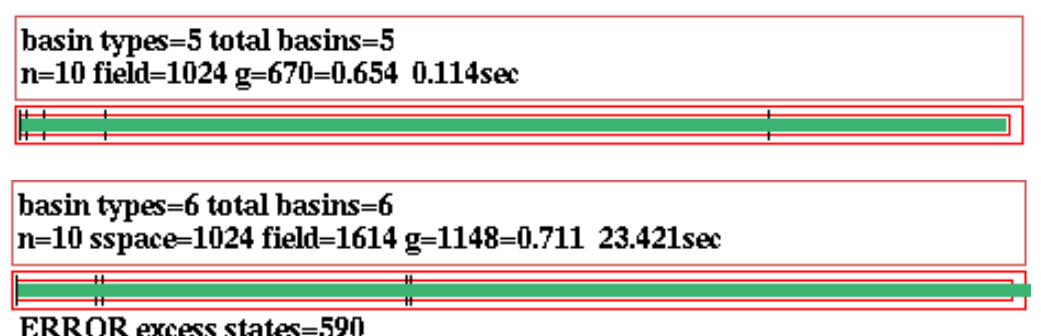

ERROR excess states $=590$

Figure 30.1: Examples of the basin of attraction field progress bar and the data window, for an RBN. Top: For a normal run without error. Bottom: An example where the wiring was changed during a pause, leading to inconsistent data where the size of the field is greater than state-space. The progress bar has gone off its scale on the right. 


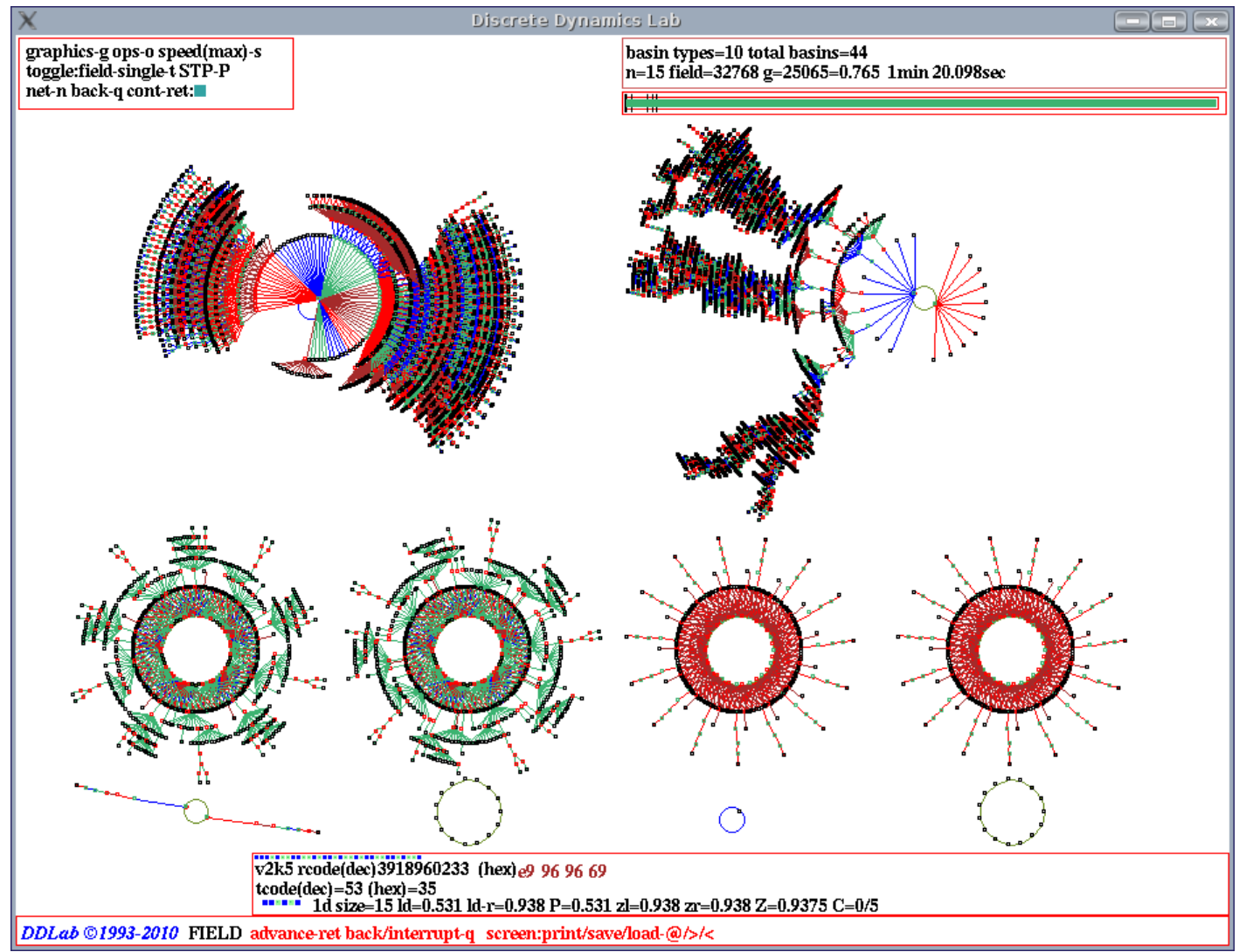

Figure 30.2: The DDLab screen showing a basin of attraction field. This example is for a $1 \mathrm{~d} C \mathrm{~A}, n=15$, $v 2 k 5$ tcode (dec)53. To achieve this layout, a pause was selected after each basin in section 27.1.2, and the position and spacing of basins were amended as described in section 25.3. Various typical indications on the screen are as follows,

top-left: ... the "attractor basin complete" options window (section 30.4).

top-right: ... the data window (section 27.2).

below top-right: ... the progress bar (section 30.1).

bottom-center: ... the rule window (section 16.19.

foot of screen: ... the title bar (section 5.5). While attractor basins are being drawn, this also shows reminders about on-the-fly options (section 30.3).

\subsection{Attractor basins, interrupting and changing}

Enter $\mathbf{q}$ to interrupt attractor basins with a top right prompt. If the interrupt occurred between successive pre-images within a pre-image fan (as opposed to between successive pre-image fans) this is indicated in an extra first line,

early exit - in pre-image fan 
Then one of the following prompts is presented, depending on whether the attractor basin is a subtree, single basin or a basin of attraction field,

for a subtree

options-o stopsubtree-q speed-s cont-ret:

for a single basin

next-tree-n options-o stopbasin-q speed-s cont-ret:

for a basin of attraction field

next-tree/basin-n options-o stopfield-q speed-s cont-ret:

If the in-degree histogram was set in section 24.6, a further option inhist-h is offered to view the histogram as computed so far, for example,

inhist-h, options-o, stop subtree-q, cont-ret: (for a subtree)

There options are summarized below, with some described in more detail in the rest of this section,

options... what they mean

inhist-h ... to view the in-degree histogram (section 24.6).

next-tree-n ... to abandon the tree that is currently being generated and start the next tree. This will create errors in a basin of attraction field (section 30.2.1).

options-o ... to show a truncated version of the top-left "attractor basin complete" options (section 30.4).

speed-s ... to slow down drawing attractor basins and backwards space-time patterns or revert to full speed, as in section 24.11. Slow motion can also be invoked on-the-fly (section 30.3), from the "attractor basin complete" prompt (section 30.4), and beforehand from section 24.4.

stopfield-q ... (or stopbasin-q, or stopsubtree-q) to abandon the attractor basin that was interrupted (section 30.2.3).

cont-ret ... enter return to continue the attractor basin from the point of interrupt.

When the implementation of these options is finished, the attractor basin will either continue from the point at which it was interrupted, or if certain parameters were changed, including a new rule or seed, a new attractor basin will be started.

\subsubsection{Abandoning a tree and continuing with the next tree}

Enter $\mathbf{n}$ in section 30.2 , to abandon the tree that is currently being generated and start the next tree. This also applies to a subtree from the uniform states (section 26.1.2). If compression (section 26.1) was not suppressed in section 26.1.1 all the equivalent trees (or subtrees from the uniform states) will be abandoned, and the next set of equivalent trees started.

Abandoning a subtree before it is complete will result in errors in attractor basin data (section 27.2). For a basin of attraction field, the progress bar is likely to go off its scale, with a message such as ERROR excess states $=\mathbf{1 9 1 2 4}$ below it. This indicates that more states were computed than the size of state-space. 


\subsubsection{Errors in attractor basins}

Its important to note that any changes to the network parameters made during a pause or interrupt will inevitably result in errors/inconsistencies in data and attractor basin structure. The view/revise/learn-n option allows the network to be changed. If changes are made during a pause, and the attractor basin is then continued, the result will be inconsistent with any given network. For a basin of attraction field, the progress bar is likely to go off its scale. The size of state-space as well as the field will be indicated in the data window. For example,

$$
\ldots \text { sspace }=1024 \text { field }=1486
$$

A message will also appear below the progress bar. For example,

ERROR excess states $=462$

This indicates that more states were computed than the size of state-space.

Note also that although the wiring in a 1d CA may be examined and changed during the pause, the change is only temporary, and will be automatically restored to allow the attractor basin of the $1 \mathrm{~d}$ CA to continue using the $1 \mathrm{~d}$ CA reverse algorithm. For other networks, RBN and $2 \mathrm{~d}$ or $3 \mathrm{~d}$ CA this does not apply, and the wiring can be changed during a pause, but of course this will lead to inconsistencies and errors as described earlier. Rule changes during a pause will remain in effect for any network, again leading to inconsistencies and errors.

\subsubsection{Abandon the attractor basin}

Enter $\mathbf{q}$ in section 30.2 to abandon the attractor basin that was interrupted. The data on the incomplete attractor basin is shown in a top-right window (section 27.2), preceded by the words "EARLY EXIT" to indicate that the data applies to an incomplete attractor basin. For example,

EARLY EXIT basin types $=7$ totalbasins $=76$

$\mathrm{n}=15$ sspace $=32768$ field $=23222 \mathrm{~g}=16711=0.72$

At the same time the "attractor basin complete" prompt is presented in a top-left window (section 30.4).

\subsection{Attractor basin options, on-the-fly}

A number of key hits allow changing the presentation of attractor basins and backwards space-time patterns on-the-fly - the following reminder is shown in the bottom title bar (section 5.5), depending on the type of system selected in section 29.4,

for nonlocal wiring, which includes the full set of options matrix-m STP:tog/scroll/exp/contr-s/\#/e/c ppstack:tog-P slow/max $</>$

DDLab (C) 1993-2011 advance-ret back/interrupt-q matrix-m STP:tog/scroll/exp/contr-s/\#/e/c slow/max-</>

The option ppstack:tog-P applies only for the nonlocal wiring reverse algorithm (section 29.6.2). The option matrix-m does not apply for neutral order components (section 29.10). The key hits are described below, 
on-the-fly key hits ... what they means

matrix-m ... Toggle the state-space matrix (section 24.5) on and off.

tog/scroll/exp/contr-s/\#/e/c... Enter s to toggle backwards space-time patterns on and off (section 24.10), \# to toggle between scrolling (the default) and sweeping the patterns, e/c to expand or contract the scale of both the backwards space-time patterns and the nodes of the basins when shown as bit/value patterns (section 26.2).

ppstack:tog-P ... (for the nonlocal reverse algorithm) enter $\mathbf{P}$ to toggle showing the partial pre-image histogram (section 29.6.2). A top-right prompt is presented,

partial pre-image histogram: start pause-p:

Enter $\mathbf{p}$ to pause the histogram — section 29.6.2 gives further details.

slow $/ \max :</>\ldots$ slow down with $<$ which halves computation speed each time its hit. Enter $>$ to revert to maximum speed.

These changes take effect as soon as the key is pressed, without return, but basins may be drawn too fast to hit a key in time. To allow for this, computation may be slowed at various other points - during an interrupt (section 30.2), at the "attractor basin complete" prompt (section 30.4), or beforehand (sections 24.11 or 24.4).

\subsection{Attractor basin complete prompt}

When the attractor basin is complete, or if abandoned (section 30.2.3), the following options are presented in a top-left window,

graphics-g ops-o speed(max)-s (or speed(slow)-s if not max)

tog: single-field-t STP-P (or field-single-t whichever is active)

seed-e net-n, back-q cont-ret: (seed-e for single basin or subtree)

Enter $\mathbf{q}$ to backtrack to previous prompts. Enter return to generate another attractor basin, for a mutated network according to the mutations set in chapter 28, all other parameters and settings remaining unchanged.

If o was entered when interrupting in section 30.2, the prompt is truncated with fewer options as follows,

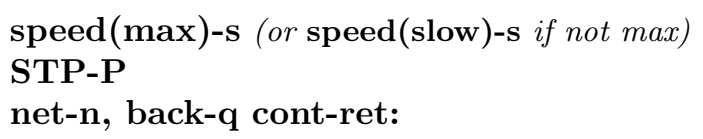

Enter $\mathbf{q}$ or return to revert to the interrupt prompt (section 30.2).

The other options are summarized below, 


\begin{abstract}
options... what they mean
graphics-g ... to alter the graphics setup described in section 6.3.

ops-o ... to open up a range of further rule, network and seed options, which are presented in a top-right window (section 30.5).

$\operatorname{speed}(\max )-\mathbf{s} \ldots$ the current speed status (max) or (slow). Enter $\mathbf{s}$ to slow down drawing attractor basins and backwards space-time patterns or revert to full speed, as in section 24.11. Slow motion can also be invoked on-the-fly (section 30.3), while interrupting (section 30.2), and from output parameters (section 24.4).

single-field-t ... (or field-single-t) to toggle between a single basin and the basin of attraction field. When changing to a single basin the prompt sequence restarts at the seed prompt (chapter 21). When changing to a field the prompt sequence restarts at the first output parameters prompt (section 24.1). Note that the "layout" defaults (chapter 25) may need to be reset.

STP-P ... to toggle between showing and not showing "backwards" space-time patterns (section 24.10).

seed-e ... (for a single basin or subtree) to revise the seed for a single basin or subtree. The seed options described in chapter 21 are presented in a lower central window. This option does not appear for a basin of attraction field.

net-n ... for the many network architecture options described in chapter 17, including viewing, revising and filing, the Derrida plot (chapter 22), and learning (chapter 34), a repeat of the option in section 30.4 .
\end{abstract}

\title{
30.5 Further attractor basin complete options
}

Enter $\mathbf{o}$ in section 30.4 above to display a range of further (context dependent) options for amending the rule, the network, or seed (for a single basin or subtree only). A top-right prompt similar to the following is presented,

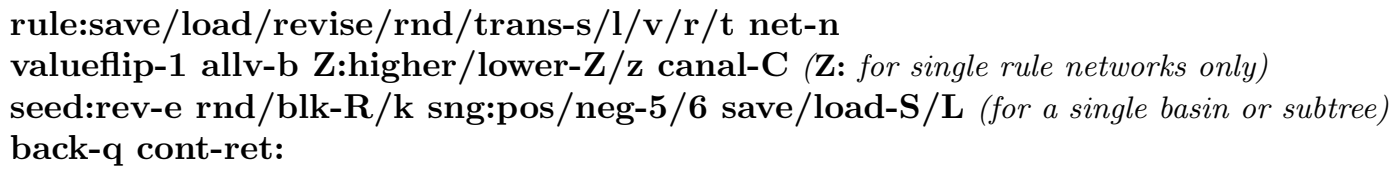

Enter $\mathbf{q}$ to backtrack to previous prompts in section 30.4. Enter return to generate another attractor basin, for a mutated network according to the mutations set (chapter 28). Mutations are only put into effect if the rule and seed parameters were not revised (below).

The remaining options are listed in two categories, revising the rule (section 30.5.1), or the seed (section 30.5.2) for single basins or subtrees only. Changes to rules or seeds generally result in new attractor basins being started. However, changes made during an interrupt when attractor basins are incomplete, and continue from the point of interrupt, will result in errors as described in sections 30.2.2. 


\subsubsection{Attractor basin — revising rule/s}

Part of the prompt (section 30.5) for revising the rule, or rules in mixed rule networks, is as follows, rule:save/load/revise/rnd/trans-s/l/v/r/t net-n valueflip-1 allv-b Z:higher/lower-Z/z canal-C (Z: for single rule networks only)

The meaning of the options are summarized below,

$$
\text { options … what they mean }
$$

save/load-s/l ... enter $\mathbf{s}$ or $\mathbf{l}$ to save or load the rule (35.2). For mixed rule networks this applies to the rule at cell index 0 only.

revise-v ... to review/revise the rule in a lower right window as described in chapter 16. For mixed rule networks the following prompt is presented below the top-right window to select the cell index,

enter cell index, 9-0: (for example)

rnd-r ... to reset a new random rule, or all rules at random in a rulemix.

trans-t ... to transform the rule as described in chapter 18. For mixed rule networks the following prompt is presented below the top-right window to select the cell index,

enter cell index, 9-0: (for example)

net-n ... for the many network architecture options described in chapter 17, including viewing, revising, transforming, filing, the Derrida plot (chapter 22), and learning (chapter 34).

valueflip-1 ... for a random bit/value-flip in the rule-table, or for all rule-tables in a rulemix.

allv-b ... to flip outputs of uniform neighborhoods to the neighborhood value, all0s $\rightarrow 0$, all1s $\rightarrow 1$, all2s $\rightarrow 2$ etc. - in a single rule, or for all rules in a rulemix.

Z:higher/lower-Z/z ... (for single rule network only) enter $\mathbf{Z}$ to progressively force the $Z$-parameter higher, or enter $\mathbf{z}$ to force it lower, as in section 16.3.

canal-C ... to reset canalyzing inputs in one rule (as in section 18.6), or for all rules in a rulemix (as in chapter 15).

Once the rule is revised it will be shown in the lower rule window described in section 16.19.

\subsubsection{Attractor basin - revising the seed}

for a single basin or subtree only

Part of the prompt (section 30.5) for revising the seed, which applies to a single basin or subtree only, not for a basin of attraction field, is as follows,

$$
\text { seed:rev-e rnd/blk-R/k sng:pos/neg-5/6 save/load-S/L }
$$

The meaning of the options are summarized below, 


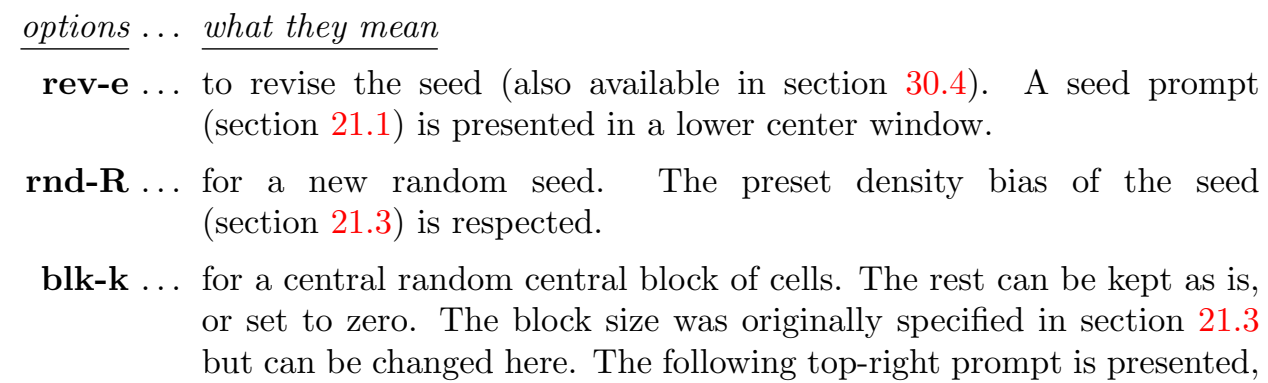

rnd block: change size (now 4), rest: keep-k, all 0s-(def):

If a new block size is entered the prompt reappears showing the new size. Enter $\mathbf{k}$ to keep the rest of the seed, return for the new block on a zero background. The preset density bias (section 21.3.1) of the block is are respected.

sng:pos/neg-5/6 . . for $v=2$ : enter 5 for a positive singleton seed, a central cell set to 1 , the remainder set to 0 . Enter $\mathbf{6}$ for a negative singleton seed.

For $v \geq 3$ : enter $\mathbf{5}$ for a central cell set to any random value except zero against a zero background. Enter $\mathbf{6}$ for a central cell with any random value against a different uniform background.

save-S ... to save the original seed, or the current state - the latest state in the attractor basin. - the following top-right prompt is presented,

save seed: original-o, current-c:

The seed is saved as a .eed file (section 35.2).

load-L ... to load a new seed, a .eed file (section 35.2), subject to the constraints listed in section 21.7.1. 


\section{Chapter 31}

\section{Output parameters for space-time patterns}

The output parameters for space-time pattern allow various default settings to be revised before space-time patterns start. Many of these settings, and some extra ones, can also be invoked onthe-fly - while space-time patterns are running (chapter 32), and from the interrupt window (section 32.16). Space-time patterns (running forward) apply in the following circumstances:

- in TFO-mode (section 6.2.1): the first output parameter appears after setting the seed (chapter 21).

- in SEED-mode (section 6.2.2): the first output parameter prompt appears ...

- if space-time patterns only-s was selected at the "first output parameter prompt for attractor basins" (section 24.1),

- or alternatively at a final stage, if space-time patterns only-S was selected at the "subtree or single basin" prompt (section 29.1).

Its possible to jump directly to a particular category of options, namely updating, entropy, damage, attractors, and skeletons, apart from the first set of start/misc miscellaneous options. Although there are many output parameter options and sub-options relating to space-time patterns, all have defaults - any remaining options can be skipped at any time by entering $\mathbf{d}$.

\subsection{The first output parameter prompt for space-time patterns}

The first output parameter prompt for space-time patterns is presented in a top-right window. At the same time space-time parameters appears in a top-center window.

accept all space-time defaults-d

revise from: start/misc-ret updating-u

entropy-e damage-m attractors-a skeletons-s: (skeletons for $v=2$ only) 


\subsubsection{Output parameter prompt for space-time patterns — options summary}

The output parameter options may be looked at in turn (up to damage-m), but they are divided into categories to allow jumping directly to the category where options need to be set. The categories in (section 31.1) are summarised below, together with subcategories where applicable - and described in detail in the rest of this chapter.

As soon as a "revise from" option is selected, the reminder accept defaults-d appears in a top-center window - enter $\mathbf{d}$ (at any time) to accept all remaining defaults, and skip further output parameter prompts. Enter $\mathbf{q}$ to backtrack. Options marked with an asterisk $(*)$ can also be modified on-the-fly (chapter 32 ) and with a carat $\left({ }^{\wedge}\right)$ from the interrupt/pause prompt (section 32.16).

\section{categories.... what they mean, and section in this chapter}

start/misc-ret ... enter return to access all the options in turn, (except attractors-a and skeletons-s) starting with the miscellaneous options 31.2.

*31.2.1 . . cell color by neighborhood or value.

*31.2.2 . . plotting the state-space matrix and return map.

*31.2.3 . . . the threshold for showing "frozen" cells.

$* 31.2 .4 \ldots$ cell-scale.

*31.2.5 . . displaying space-time patterns in other than "native" dimensions $-1 \mathrm{~d}, 2 \mathrm{~d}$, or $3 \mathrm{~d}$.

*31.2.6 . . sweeping or scrolling space-time patterns.

${ }^{*} 31.2 .7 \ldots$ options for pausing or stepping through space-time patterns.

31.2.8 ... (if the files are accessible) select sequential or random order for loading lists of glider rules on-the-fly .

$31.2 .9 \ldots$ (TFO-mode only) inverting the kcode.

updating-u ... jump directly to options for asynchronous or noisy ways to update the network (section 31.3).

*31.3.1 ... probabilistic updating.

*31.3.2 ... sequential updating.

*31.3.3 ... partial order updating.

entropy-e ... jump directly to methods for analyzing space-time patterns (section 31.4 ).

*31.4 ... input-entropy and pattern density.

31.4.1 ... single cell input-entropy and pattern density.

*31.4.2 . . generation size for input-entropy and pattern density - of the moving window of trailing time-steps.

damage-m ... jump directly to damage spread from perturbations - the difference between two networks (section 31.5):

31.5.2 . . a histogram of damage spread for a sample of initial states.

attractors-a ... jump directly (the only way) to find attractors by running forwards and creating a histogram of attractor types (section 31.6).

skeletons-s ... (for binary systems, $v=2$, only) jump directly (the only way) to find "skeletons" by running forwards and creating a histogram of skeleton types. Skeletons are fuzzy attractors where a predefined proportion of the network has stabilized (section 31.7). 


\subsection{Miscellaneous options - space-time patterns}

Enter return at the first output parameter prompt (section 31.1) for the first category of miscellaneous (hard to categorize) options, starting with section 31.2.1 below. All the output parameter options in all categories could be accessed in turn if required.

\subsubsection{Color cells by value or neighborhood}

By default each cell is colored according to its value ${ }^{1}$ (chapter 7 ). Alternatively cells may be colored according to the rule-table index that was "looked up" to determine the cell's value, in other words the cell's neighborhood at the previous time-step. These colors are assigned from a palette of 16 or 256 (section 6.3.4). The option applies to the full value-range but is mainly intended for binary systems, $v=2$. Color by value or neighborhood can be toggled on-the-fly (key 3, section 32.9.5), but for color by neighborhood from the start the following top-right prompt is presented,

start/misc: cellcolor: nhood-n value-(def):

Figures 31.1, 31.2 and 31.3 show the difference between the value and neighborhood display of $v 2$ space-time patterns, for $1 \mathrm{~d}, 2 \mathrm{~d}$ and $3 \mathrm{~d}^{2}$ - these were all generated from a single central 1 seed.
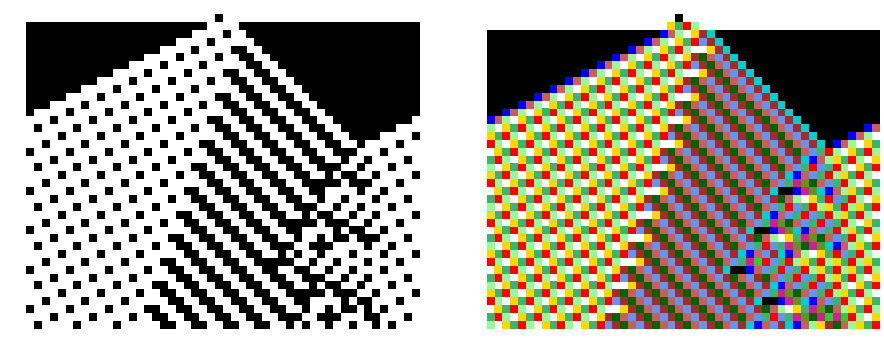

Figure 31.1: 1d space-time pattern, 40 time-steps for a CA $n=50, v 2 k 5$, rule $8226 \mathrm{dc} 23$. Space is across, time is down the page.

Cell color: Left: value.

Right: neighborhood.
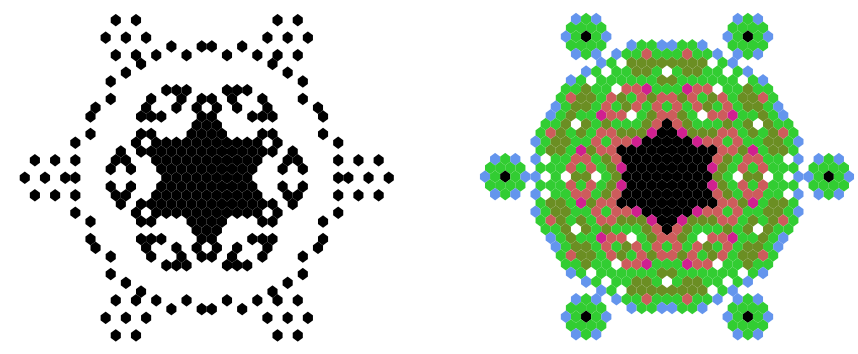

Figure 31.2: 2d space-time pattern. The 19th time-step for a CA $n=38 \times 38$ hex lattice, $v 2 k 6$ tcode (dec)114.

Cell color: Left: value.

Right: neighborhood.

\footnotetext{
${ }^{1}$ For $v=2$ the color of $1 \mathrm{~s}$ starts black but changes between black and blue as various on-the-fly changes are activated (section 32), and black-blue can be toggled (section 32.9.6).

${ }^{2}$ In $3 \mathrm{~d}$ or $2 \mathrm{~d}+$ time (section 32.10 .2 ), cells with zero value are not shown — although the neighborhood rule-table index may be positive. This allows a somewhat clearer view of the space-time structure which would otherwise be a solid mass.
} 

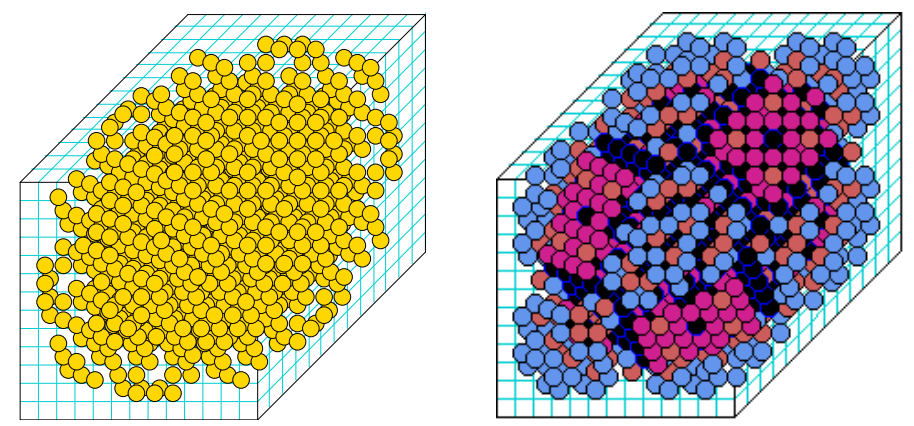

Figure 31.3: 3d space-time pattern. The 90th time-step for a CA $n=13 \times 13 \times 13, v 2 k 6$ tcode $(\mathrm{dec}) 114$. Cell color: Left: value.

Right: neighborhood.

Note that cells with zero value are not shown in either case to avoid a solid mass.

\subsubsection{State-space matrix and return map}

The "state-space matrix" and "return map" are two methods for representing states as dots on a scatter plot $^{3}$. These plots provide a kind of signature of the dynamics. To speed up the plots considerably, toggle the space-time pattern display off (on-the-fly $\mathbf{S}$, section 32.9 .8 ). The following prompt is presented,

\section{show state-space matrix-y, return map-D:}

Enter $\mathbf{y}$ to show the state-space matrix, $\mathbf{D}$ to show the return map. Both options can also be toggled on-the-fly (key $\mathbf{y}$ or $\mathbf{D}$, sections 32.12 .8 and 32.12.7 ).
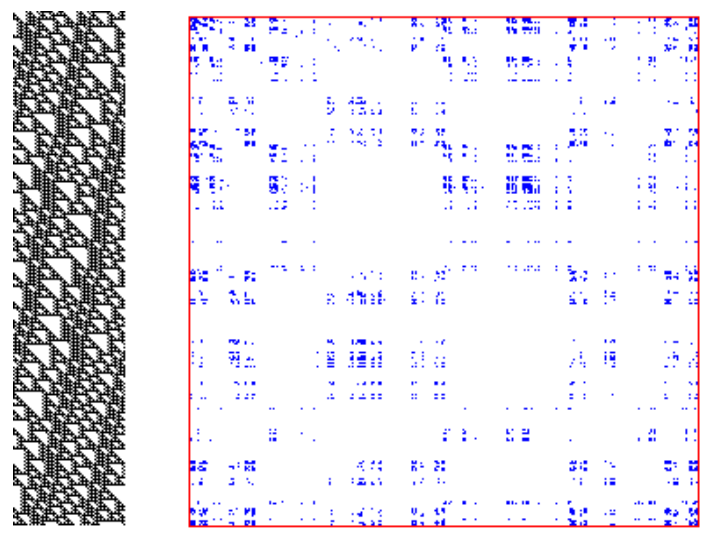

Figure 31.4: The state-space matrix for $v 2 k 5$ rcode $473 c c f 18$, $n=62$, plotting the left half against the right half of each state's bit string - an attractor was reached after about 1200 time-steps.

Left: an example of the spacetime pattern.

Right: the state-space matrix.

\subsubsection{State-space matrix}

As in section 24.5 for attractor basins, the state-space matrix plots each state in the space-time pattern on a $2 \mathrm{~d}$ grid near the bottom of the screen, plotting decimal values representing the left half of the bit/value string ( $x$-axis) against the right half (y-axis). If $n$ is odd, the extra

\footnotetext{
${ }^{3}$ Other types of state-space scatter-plots are the "entropy-density plot" (section 32.12.3), and the "Derrida plot" (chapter 22).
} 
bit/value is included on the left, and the grid is a flat rectangle, otherwise the grid is square. Although the state-space matrix will give an output for any network size and value-range, only a limited number, $X$, of the most significant bits/values are included from each half of the string to find its decimal value (an unsigned long int). $X$ for different value-range $v$ corresponds to table 7.2.

\subsubsection{Return map}

The return map in a 2d-phase plane plots each state against its successor. For a binary network, the state (bitstring) $B_{0}, B_{1}, B_{2}, B_{3} \ldots B_{n-1}$ is converted into a decimal number $M(0-2)$ as follows,

$$
M=B_{0}+B_{1} / 2+B_{2} / 4+B_{3} / 8+\cdots+B_{n-1} / 2^{n-1}
$$

For a mutli-value network, the bits are replaced by values, and each term is divided by $v-1$ for an equivalent result. As the network is iterated, $M_{t}$ at time-step $t$ (x-axis) is plotted against $M_{t+1}$ at time-step $t+1$ (y-axis) in the bottom-center of the screen. From a classical dynamical systems viewpoint, note the fractal structure of the resulting trajectories and attractors. An example is shown in figure 31.5 .
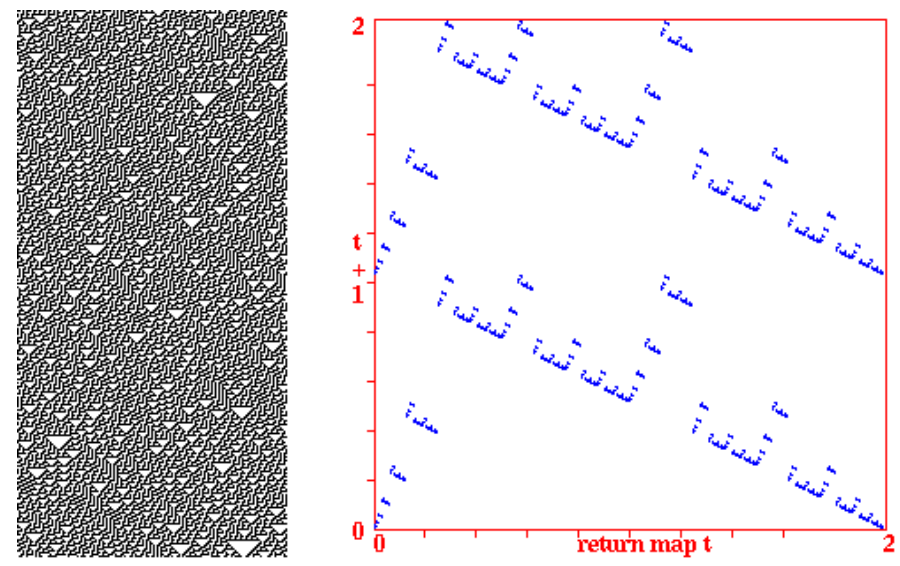

Figure 31.5: The return map for $v 2 k 3$ rcode $30, n=150$, plotting the state at each time-step, converted into a decimal number 0 -2, against its successor, for about 50,000 timesteps.

Left: an example of the space-time pattern.

Right: the return map, note the $\overline{\text { fractal }}$ structure characteristic of chaotic dynamics.

\subsubsection{Frozen generation size}

Once running, the space-time pattern presentation can be reset on-the-fly to highlight "frozen" cells - that have not changed in the previous $x$ time-steps, or that fall into preset "frequency bins" (enter h, a 4-way toggle, section 32.11.1). The number of time-steps, or generations, making up the frozen threshold may be reset from the initial default of 20 . The new setting becomes the default. The following prompt is presented,

\section{enter new 'frozen' generation size (now 20):}

The frozen generation size (and frequency bins) can also be reset on-the-fly (key $\mathbf{H}$, section 32.11.2). The current sizes are shown in the "on-the-fly key index". 


\subsubsection{Cell scale}

The cell scale of space-time patterns is set automatically for different network sizes and dimensions, but this can be altered. The following top-right prompt is presented,

cellscale (now 1 pixel): for a $1 d$ network $n=150$

Enter the new cell scale in whole pixels. This can also be changed with on-the-fly keys e or $\mathbf{c}$ to expand or contract (section 32.9.11).

\subsubsection{Space-time patters in other than the native dimension}

Space-time pattern can be displayed in a dimension other than the "native" dimension. A 1d network can be displayed in $2 \mathrm{~d}$ or $3 \mathrm{~d}$, a $2 \mathrm{~d}$ in $1 \mathrm{~d}$ or $3 \mathrm{~d}$, and $3 \mathrm{~d}$ in $1 \mathrm{~d}$ or $2 \mathrm{~d}$. This can also be changed on-the-fly (key T a 3-way toggle, in section 32.10.1). One of the following top-right prompt is presented,

1d network, show STP in 1d-1 2d-2 3d-3 (def 1d): (for 1d)

2d network, show STP in 1d-1 2d-2 3d-3 (def 2d): (for 2d)

3d network, show STP in 1d-1 2d-2 3d-3 (def 3d): (for $3 d$ )

If not the native dimension, the $2 \mathrm{~d}$ and $3 \mathrm{~d}$ axes $i, j,(h)$ are set automatically by factorizing $n$. A native $3 \mathrm{~d}$ network will be displayed in $2 \mathrm{~d}$ as a stack of horizontal $2 \mathrm{~d}$ slices as in figure 32.17 . Note that if $n$ is prime, $i=n$, and both $j$ and $h$ will equal 1 . Then if $2 \mathrm{~d}$ or $3 \mathrm{~d}$ is selected, a single row of cells will be displayed across the top of the screen in the $2 \mathrm{~d}$ or $3 \mathrm{~d}$ format.

\subsubsection{Scrolling 1d space-time patterns}

1d space-time patterns will scroll vertically by default on reaching the bottom of the screen. Alternatively the patterns can be made to sweep instead of scroll - restart from the top on reaching the bottom of the screen. For 1d space-time patterns presented in 1d, the following top-right prompt is presented,

\section{sweep space-time pattern-s, scroll-def:}

Enter $\mathbf{s}$ to sweep. The two methods can also be toggled on-the-fly ${ }^{4}$ (enter \#, section 32.13.3). If the input-entropy or pattern density is set (section 31.4) these plots will also scroll or sweep.

\subsubsection{Pause and step}

DDLab offers various methods of scanning space-time patterns, some of which are also available on-the-fly (section 32.13.4), and from the interrupt/pause prompt (section 32.13.4). From the output parameters, options are offered to make single time-steps, to pause after a given number of time-steps, or to always pause when $1 \mathrm{~d}$ (or $2 \mathrm{~d}$ isometric) space-time patterns reach the foot of the screen. No pause is the default. The following top-right prompt is presented,

\footnotetext{
${ }^{4} 2 \mathrm{~d}$ presentations of space-time patterns can also be scrolled on-the-fly, either vertically (section 32.10.2), or diagonally (section 32.13.3) — which can also include scrolling the network-graph (section 32.19 and figure 32.14).
} 
step-s, pause screen full-p, or enter time-step (no pause-def):

\author{
options ... what they mean \\ step-s ... to show each next iteration by pressing return. This can also be set on-the- \\ fly (key + , section 32.13.4). \\ screen full-p ... to pause when the screen is full. This can also be set from the interrupt/pause \\ prompt (key N, section 32.13.4). \\ time-step ... enter a number to specify a run of time-steps before a pause. At each pause, \\ enter $\mathbf{x}$ in the interrupt/pause prompt (section 32.16) for the next run of \\ time-steps, or return to stop pausing and continue as normal. The default \\ run equals the "frozen generation" size (default 20 time-steps, section 31.2.3).
}

\title{
31.2.8 Glider rule order
}

if a list of complex rules is accessible

Collections of complex (or "glider") rules — that produce emergent space-time structures including interacting gliders are available with DDLab (section 3.7.1) for some combinations of rule type, $v, k$, and lattice dimensions ${ }^{5}$. A rule from such a numbered collection can be loaded on-the-fly (key g) while space-time patterns are running (section 32.6.1). If a collection is detected for the current $v, k$, the following top-right prompt is presented for either a random or consecutive number from the list,

\section{v3k6 complex rules (select on-the-fly with "g") \\ consecutive enter start index 1-54, rnd-def: (for example)}

Enter return for a rule picked randomly from the list, or a mumber to start consecutive selections, to be loaded later on-the-fly. Consecutive list numbers will cycle back to one on reaching the maximum number. In the example above there are 55 rules in TFO-mode, for kcode $v 3 k 6$, intended for $2 \mathrm{~d}$. This option is also available from the interrupt/pause prompt (key $\mathbf{G}$, section 32.16.1).

\subsubsection{Inverting the kcode in TFO-mode}

for kcode in TFO-mode only

The kcode-table can be expressed according to two alternative conventions. In DDLab the highest $v$-ary value of value-frequencies in the neighborhood is on the left (section 13.6.1) and the lowest on the right. To allow kcode with the opposite convention to run as intended, the following top-right prompt is presented,

\section{invert kcode value order -i:}

Enter $\mathbf{i}$ to run the kcode according to the inverted convention, or return for the standard convention. The kcode itself is not changed, just the way it is accessed while running forward. This is analogous to inverting the rcode in section 18.3 but in that case the rcode itself is changed.

\footnotetext{
${ }^{5}$ Although the complex rules relate to specific dimensions, they often result in interesting dynamics in other than the intended dimensions.
} 


\subsection{Asynchronous and noisy updating}

By default, updating the next time-step is deterministic and synchronous, but can be made noisy and asynchronous.

Enter $\mathbf{u}$ at the first output parameter prompt (section 31.1) to skip directly to the "update" category of options for probabilistic, sequential and partial order methods of updating, or arrive there by viewing the output parameters in sequence. The following top-right prompt is presented,

updating: sequential-s, partial-order-p, probabilistic-def:

Enter return to moving directly to the prompt for probabilistic updating (section 31.3.1) which is also reached after sequential or partial-order prompts, or enter $\mathbf{s}$ or $\mathbf{p}$ for the prompts to set various types of sequential or partial-order updating (sections 31.3.2 and 31.3.3), There can be any combination of these settings, and they can also be toggled on-the-fly (section 32.4).

\subsubsection{Probabilistic updating}

There are two types of probabilistic or noisy updating methods, where the probability, by default $100 \%$, can be reset to a lower value,

update probability ... where each cell updates with a given probability at each time-step. This is very similar to partial order updating (section 31.3.3). If this probability is set to $P$, there is a $1-P$ chance that a cell is not updated but stays the same.

output probability ... where each cell updates correctly with a given probability at each time-step. If the probability is set to $P$, there is a $1-P$ chance that a cell's value will be randomly assigned instead of respecting the dynamical rule, which introduces noise..

Enter return at the updating prompt (section 31.3), for the following top-right prompt, or arrive there by viewing the output parameters in sequence,

update prob (def $95.00 \%)$ 0-100:

output prob update (def 95.00\%) 0-100:

First set the required update probability, then the output probability. These are set as a percentage, which may be a decimal number. If both probabilities are set to less than $100 \%$, then the update probability will be implemented first, and only cells that were updated will be subject to the output probability. figure 31.6 gives examples.

Both probabilities can be toggled on-the-fly (section 32.4). If a probability is set it will be active when space time-patterns start. 


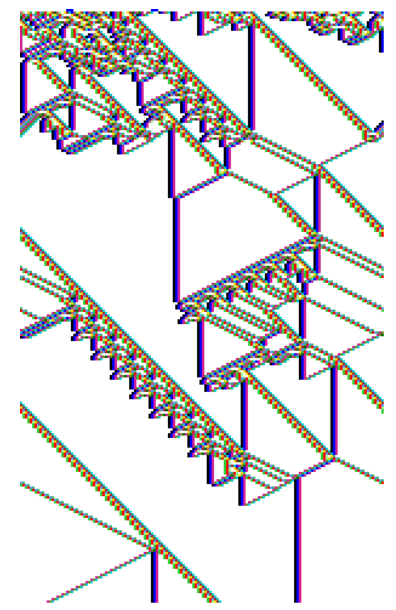

default prob $=100 \%$

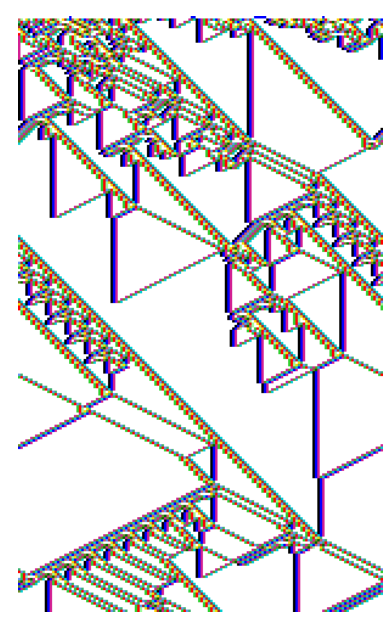

update $\mathrm{prob}=99.8 \%$

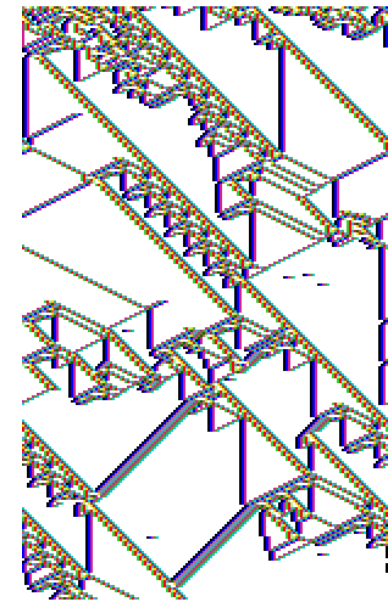

output prob $=99.8 \%$

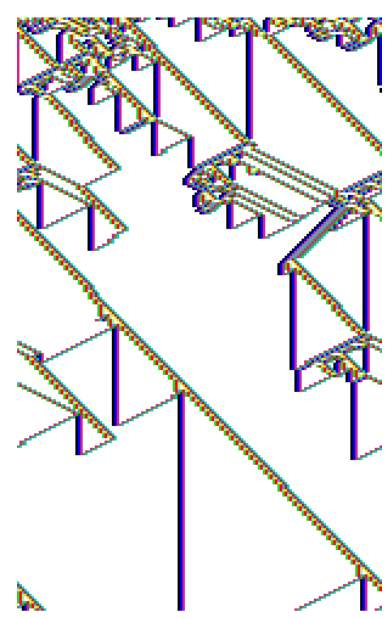

update prob $=98 \%$

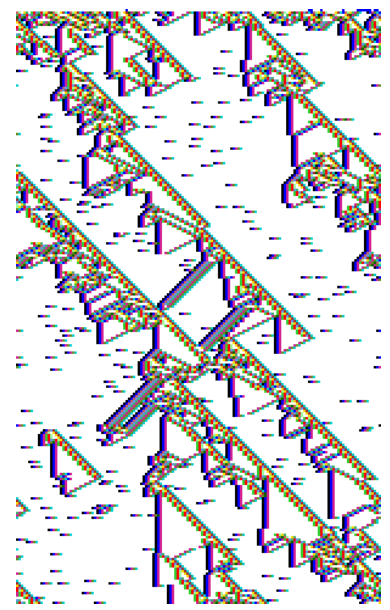

output prob $=98 \%$

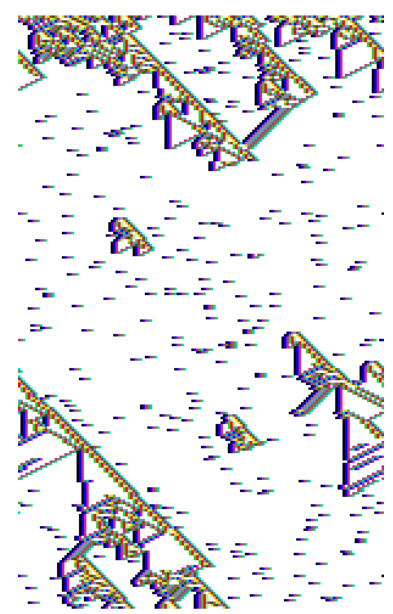

output + update $=98 \%$

Figure 31.6: Probabilistic updating: the "update" probability that a cell will be updated at all, and the "output" probability that updating obeys the rule-table, otherwise update randomly (section 31.3.1). For $v 2 k 5$ CA rcode(hex)e9f6a815, $n=150,245$ time-steps from the same initial state. Cells colors are by neighborhood (section 31.2.1) and filtered (section 32.11) to emphasize gliders.

\subsection{2 sequential updating - space-time patterns}

In sequential updating, each cell is updated in turn in some arbitrary order - then the "state" is the configuration when all $n$ updates are complete. There are $n$ ! possible sequential updating orders in a network of size $n$. As for attractor basins (section 29.9), DDLab provides simple default orders, forward or backward along the network index, a random order (figure 31.7a), and a specific order set by hand. A random order can also be set to change at each iteration. The order can be saved and loaded from a .ord file. All possible orders can be listed (as in section 29.9.3) but this applies for $n \leq 12$ only. 


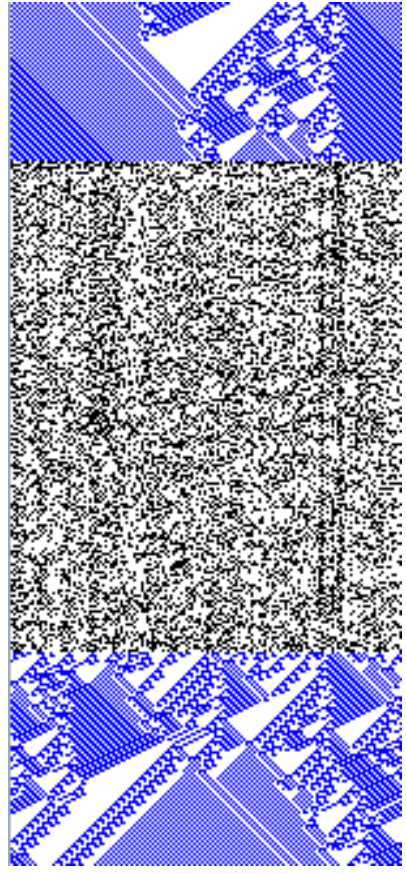

(a) random sequential order

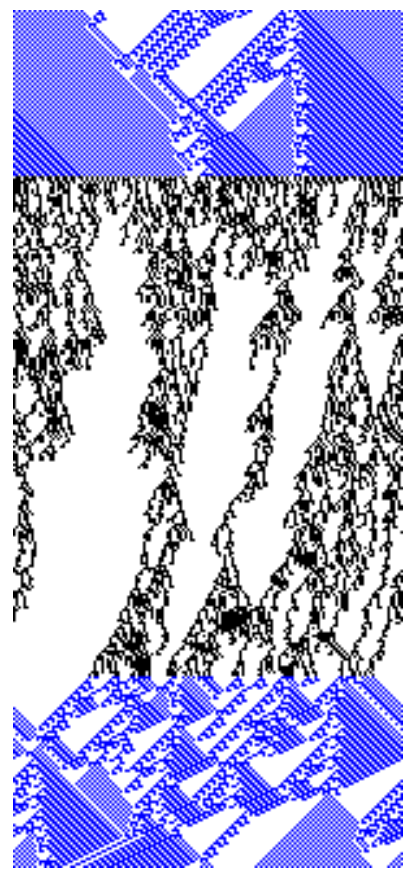

(b) partial order 100-120

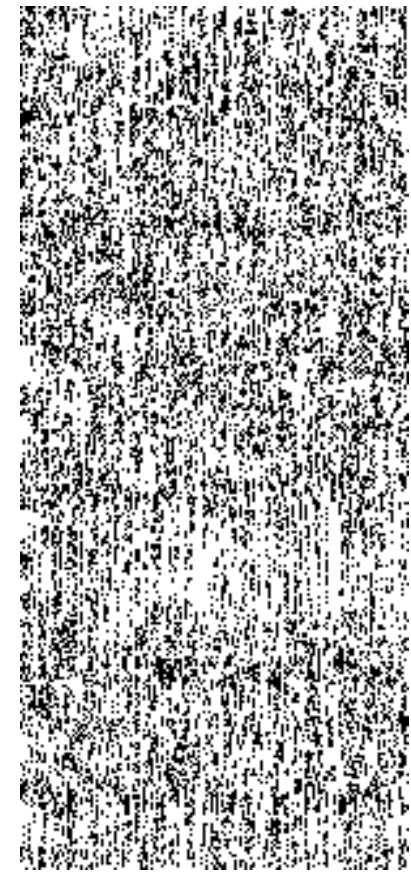

(c) sequential+partial order

Figure 31.7: Examples of updating with sequential, partial order, and both orders combined, for a 1d CA $v 2 k 5$ rcode(hex)bc82271c, $n=150$. In case (a) and (b) parallel updating was switched to the new order on-the-fly, for about 180 time-steps, then back to parallel. In case (c) about 310 time-steps are shown with combined sequential and partial order updating

These options are essentially the same as for setting sequential updating for attractor basins, except that the limits on $n$ in table 29.1 do not apply. Synchronous/sequential updating (whatever order was set) can be toggled on-the-fly (section 32.4) as in figure 31.7. If sequential updating was not selected, toggling will give the default left to right order.

Enter $\mathbf{s}$ at the updating prompt (section 31.3) for the following top-right sequential updating prompt $^{6}$,

sequential update: >-(def) <-b rnd-r rernd-R set-s all-S: (all-S if $n \leq 12$ )

These options are summarized below — the detailed descriptions are to be found in section 29.9.

options ... what they mean

$>$-(def) ... enter return for the default order from left to right in a 1d network, or in general counting down the cell index $n-1$ to 0 (section 10.2).

$<-\mathbf{b}$... for the opposite order, from right to left, cell index 0 to $n-1$.

rnd-r ... for a random order presented in a top-right window including saving and loading the order (section 29.9.1).

\footnotetext{
${ }^{6}$ The sequential updating prompt for space-time patterns is similar to the sequential updating prompt for attractor basins (section 29.9).
} 
rernd-R ... for a new random order at each iteration.

set-s ... to set a specific order by hand, including saving and loading the order (section 29.9.2). $n \leq 630$ normally fits into the "by hand" window.

all-S ... (if $n \leq 12$ ) to list all $n$ ! possible orders and select a specific order from the list (section 29.9.3).

\subsection{3 partial order updating — space-time patterns}

Partial order updating is when a subset of cells update in parallel, followed by the next subset - then the "state" is the configuration after each updated subset. Lower and upper limits define a size window or "update subset" - the default is the largest possible window, between 1 and network size $n$. At each time-step, a random size is set between these limits, and applied randomly to positions in the network - only these are updated. This is very similar to "update probability" (section 31.3.1) but the partial order method guarantees a fixed range of the update subset, including just one constant value if lower and upper limits are the same ( $\min =\max )$.

Enter $\mathbf{p}$ in section 31.3 to set partial order updating. The following top-right prompt for the minimum and maximum size limits of the update subset are presented in turn (for $n=150$ ),

\section{partial order updating: update subset limits (def 1:150): min: 100 max:120}

In this example (figure 31.7b), an update subset $s$ of between 100 and 120 ( $s$ set randomly) will be assigned at random to the network. Only these cells are updated to make the next state, and the process is repeated at each iteration.

Synchronous/partial order updating can be toggled on-the-fly (section 32.4) — if partial order updating was not defined, toggling gives the default update subset. Sequential updating (section 31.3.2) can be active at the same time as partial order updating (figure 31.7c) - in this case the partial order subset will be updated sequentially at each iteration to make the next state.

\subsection{Input-entropy and pattern density}

Measures on space-time patterns, taken over a window of time-steps and shown graphically, provide some useful insights into dynamics and rule-space[27, 39, 32]. The measures are as follows,

input-frequency ... the frequency of rule-table lookups, or neighborhood size blocks provides the basis for entropy measures and filtering space-time patterns. To increase block size, a rule can be transformed to an equivalent rule with a larger neighborhood (section 18.7.1).

input-entropy ... the Shannon entropy of the input-frequency distribution — provides the basis for classifying rule-space (chapter 33).

pattern density ... the fractions of different values - provides an entropy-density scatter plot (figure 32.32) - a characteristic signature of each complex CA.

The measures apply either to the whole network or to one selected cell. Although input-entropy is intended for a homogeneous-rule network, it works for a rulemix provided $k$ is homogeneous. For a $k$-mix a single position in the network must be selected (for both entropy and density) but this can be done in all cases if required. 

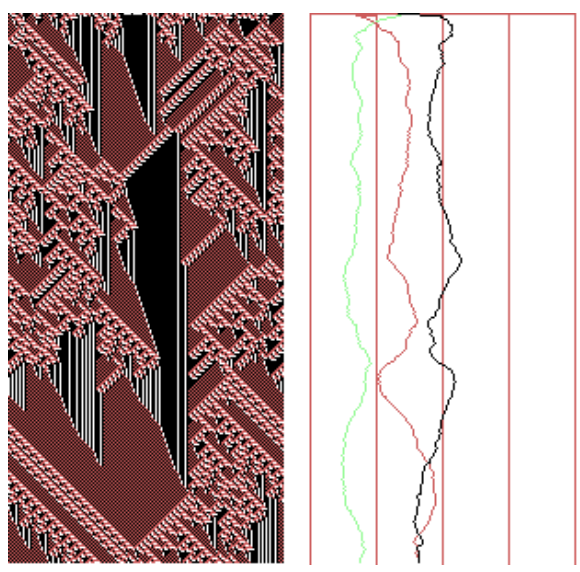

Pattern density plots of each value, alongside space-time patterns in value colors

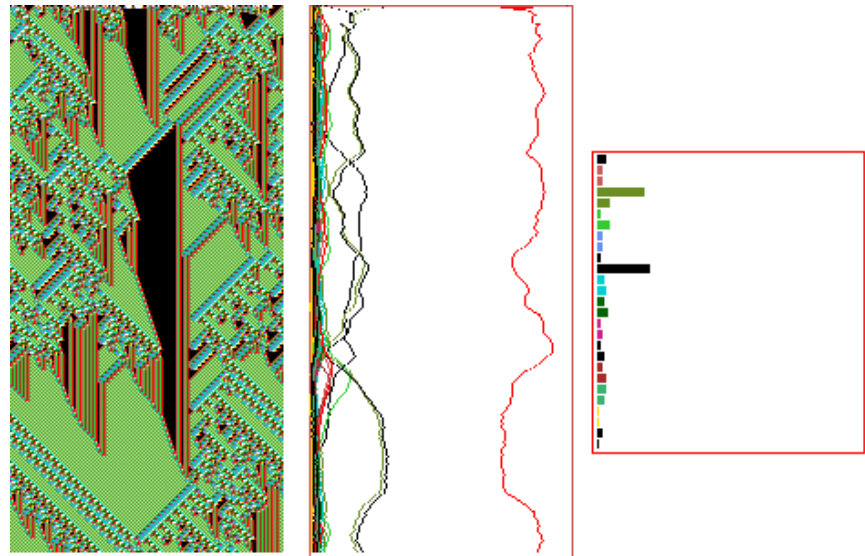

Input-Entropy plot and input-frequency histogram (and plots), alongside space-time patterns in neighborhood colors.

Figure 31.8: The pattern density and input-entropy plots, for the same $v 3 k 31 \mathrm{~d}$ CA, rcode (hex)2698a14246a466, $n=150,300$ time-steps from the same random initial state. Space is across and time down as in figure 2.3. Measures were averaged over a moving window of 10 time-steps.

Left: the pattern density alongside space-time patterns in value colors - the density of each value is plotted on the $\mathrm{x}$-axis which has 4 divisions. The density plots correspond to value colors except zero which is colored light green.

Right: the input-entropy (in red) alongside space-time patterns shown in neighborhood colors - the entropy is on the $x$-axis, with zero entropy on the left and maximum entropy on the right. The input frequency histogram with 27 bars is on the extreme right showing the frequency of each neighborhood, with the zero neighborhood at the bottom. In this example the input frequencies are also plotted on the input-entropy graph, toggled on-the-fly (section 32.12.4).

Note that the entropy and density both become active if either is selected, but interactive plots of the selected measure will appear first, alongside space-time patterns - thereafter the two can be toggled on-the-fly (section 32.12.3).

Enter e in section 31.1 to skip directly to the "entropy" category of options, or arrive there by viewing the output parameters in sequence. The following top-right prompt is presented,

\section{for native $1 d$ networks with homogeneous $k$ analysis: none-n, pattern-density -p input-frequency/entropy-(def): \\ for native $2 d$ or $3 d$ networks, or mixed- $k$ analysis: none-(def) pattern-density -p input-frequency/entropy-e:}

Input-frequency and pattern-density can be toggled on-the-fly, as well as other aspects of the presentation (section 31.4.3). 


\subsubsection{Single cell input-entropy and pattern density}

If either input-entropy or pattern density was set, for homogeneous $k$ the next prompt allows a single cell to be selected,

$$
\text { single-s all-(def): (for homogeneous } k \text { ) }
$$

If a single cell was selected above, or for a mixed- $k$ network, the next prompt selects the position of the cell in the network,

$$
\begin{aligned}
& \text { for a } 1 d \text { network } n=150 \\
& \text { single: enter index (max } 149 \text { def } 0) \text { : } \\
& \text { for a } 2 d \text { network } 40 \times 0 \\
& \text { single 2d: 2d index (max } 39,39 \text {, now } 0,0) \\
& \text { enter } \mathrm{i} \text { : enter } \mathrm{j} \text { : }
\end{aligned}
$$

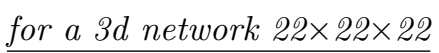

$$
\begin{aligned}
& \text { single 3d: 3d index }(\max 21,21,21 \text {, now } 0,0,0) \\
& \text { enter } \mathrm{i} \text { enter } \mathrm{j} \text { : enter } \mathrm{h} \text { : }
\end{aligned}
$$

This can be amended on-the-fly (section 31.4.3).

\subsubsection{Generation size - moving window of time-steps}

The next prompt sets the analysis generation size, the size of the moving window of trailing time-steps over which the measures are averaged. For input-entropy these are past time-steps, for pattern-density the present time-step is also included. The initial default is 10 for the whole network, and 500 for a single cell - the new setting becomes the default,

enter new 'analysis' generation size (now 10): (for the whole network)

This can be amended on-the-fly (section 31.4.3).

\subsubsection{On-the-fly changes to input-entropy and pattern density}

Once space-time patterns are running, and either input-entropy and pattern density was activated, on-the-fly options (section 32.3) apply to make changes as follows,

$$
\begin{aligned}
& \underline{\text { key }} \cdots \underline{\text { on-the-fly option }} \\
& \text { s ... toggle between input-entropy and pattern density. } \\
& \text { G . . change the current analysis generation size (section 32.12.6). which is displayed in } \\
& \text { If a single cell was set above, its position is also shown and can be changed, for } \\
& \text { example, } \\
& \text { G..a-gens }=10 \text { pos }=9 \ldots \text { for } 1 \mathrm{~d} \\
& \text { G..a-gens }=10 \text { pos }=\mathbf{5 , 6} \ldots \text { for } 2 \mathrm{~d}
\end{aligned}
$$

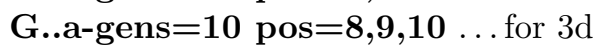$$
\text { the on-the-fly key index (sections } 32.1 \text { and 32.3), for example, G..a-gens=10. }
$$ 
if input-entropy is displayed

$\mathbf{0}$... toggle input-frequency histogram bars between normal width and 1 pixel — useful for large rule-tables.

$\%$... show the input-frequency histogram with a time dimension - if the number of bars $\leq 64$ (figure 32.31 and section 32.12.1).

)/( . . amplify the input-frequency histogram,and restore default.

j ... 3-way toggle between input-entropy, input-frequencies as a set of graphs, and both together (section 32.12.4).

u ... tog entropy-density scatter plot, where rules have characteristic signatures (figure 32.32 and section 32.12.5).

E ... (while the entropy-density scatter plot is active) a plot of mean entropy against entropy variability ${ }^{7}$ for a sample of rules, giving a $3 \mathrm{~d}$ histogram that separates out order, complexity and chaos (chapter 33).

\subsection{Damage, the difference between two networks}

$1 d$ and $2 d$ networks only - not $3 d$

Its possible to graphically represent the difference, or the spread of "damage", between the spacetime patterns of two networks. Typically the networks are identical except for a 1 bit/value difference in their initial state, and the damage is shown as a "purple stain" on a third space-time pattern, spreading from the different bit/value. This option applies to $1 \mathrm{~d}$ and $2 \mathrm{~d}$ networks only.

Damaged cells can be defined in two ways. Once damaged, keep the damage irrespective of future dynamics, or simply the instantaneous difference at each time-step - this can be toggled on-the-fly (key "=" section 32.12.9).

Methods are also provided for automatically gathering statistical data on the sizes of damage spread from many random initial states that differ by one bit/value, shown as a histogram. This is one method of characterizing order and chaos in network dynamics, especially in random Boolean networks applied as idealized models of genetic regulatory networks[11, 30].

Enter $\mathbf{m}$ at the first output parameter prompt (section 31.1) to skip directly to the "damage" category of options, or arrive there by viewing the output parameters in sequence. The following top-right prompt is presented,

\section{damage: show difference between 2 networks keep damage-f, don't keep-F, histograms: actual-a bins-b:}

Enter $\mathbf{a}$ or $\mathbf{b}$ to shown a histogram of damage spread for a sample of initial states generated automatically (section 31.5.2) - a gives the actual damage sizes, $\mathbf{b}$ gives damage falling into a number of preset bins. Keeping the damage applies - once damaged, a cell stays damaged.

Otherwise, for a manual version of damage spread (without a sample or histogram) enter $\mathbf{f}$ to keep the damage, or $\mathbf{F}$ for the instantaneous difference at each time-step.

\footnotetext{
${ }^{7}$ Entropy variability is either a min-max measure — the maximum entropy up-slope found, or standard deviation.
} 

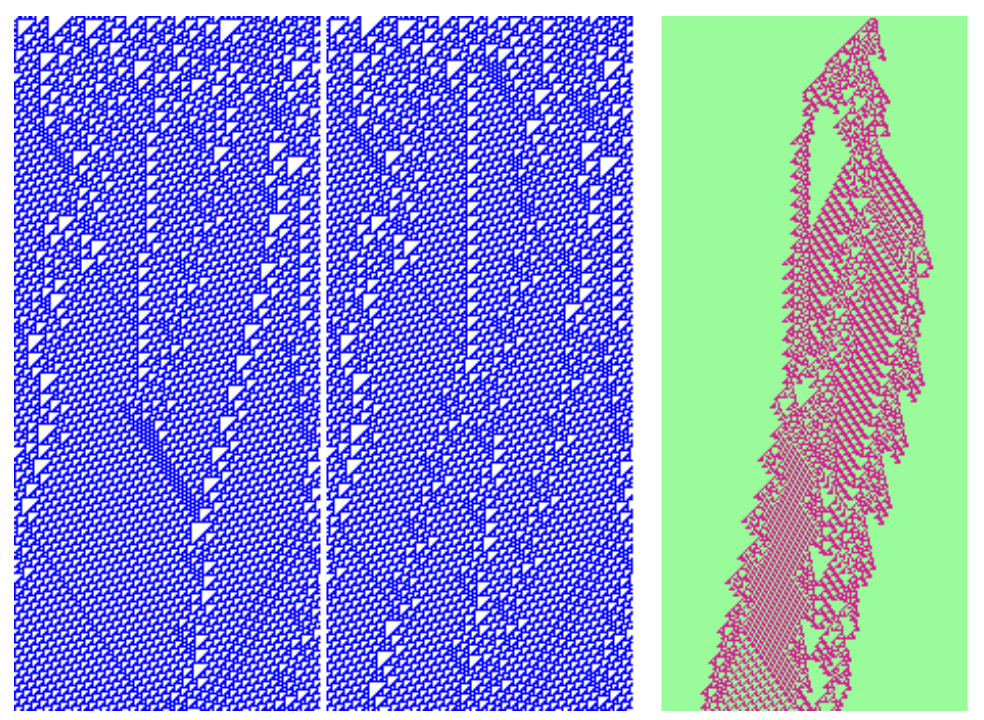

Figure 31.9: The difference in the dynamics between two 1d CA. Left: the space-time patterns of two identical CA (color by value), except that the random initial state differs by one bit. Right: the instantaneous difference between the two at each time-step shown as a third space-time pattern. $v 2 k 3$ rcode $(\mathrm{dec}) 110, n=150+150$.

\subsubsection{Duplicate the network and seed}

A subsequent option, normally selected together with the "damage" option (section 31.5) above, duplicates both the network and the initial state. This actually doubles the size of the original network, making a network containing two identical sub-networks, each with the same initial state. The following top-right prompt is presented,

\section{duplicate network and seed-y:}

Enter $\mathbf{y}$ to duplicate the network. Though normally used together, the "damage" option (section 31.5) and duplicating the network can be used independently ${ }^{8}$. If "damage" was selected and the network is not duplicated, the original network and its initial state will simply be notionally divided in two, and the "damage" displayed will be between the two halves of the network. However, the network can only be divided if $n$ for $1 \mathrm{~d}$, or $i$ for $2 \mathrm{~d}$, is even, otherwise the following top-right message is displayed,

\section{can't divide odd width network, cont-ret:}

Once the network is duplicated, prompts are presented in turn to look at and possibly modify the duplicated wiring (section 17.1), and the duplicated seed ((section 21.1). Figures 31.5.1 and 21.1 give examples. When the network is run with these option set, a third space-time pattern shows the difference between the two sub-networks as in figures 31.9 and 31.5.2.

\footnotetext{
${ }^{8}$ This can be repeated to re-double the network making 4,8 , etc independent networks, but the total cell count cannot exceed $n_{\text {Lim }}=65025$ (section 8.3)
} 

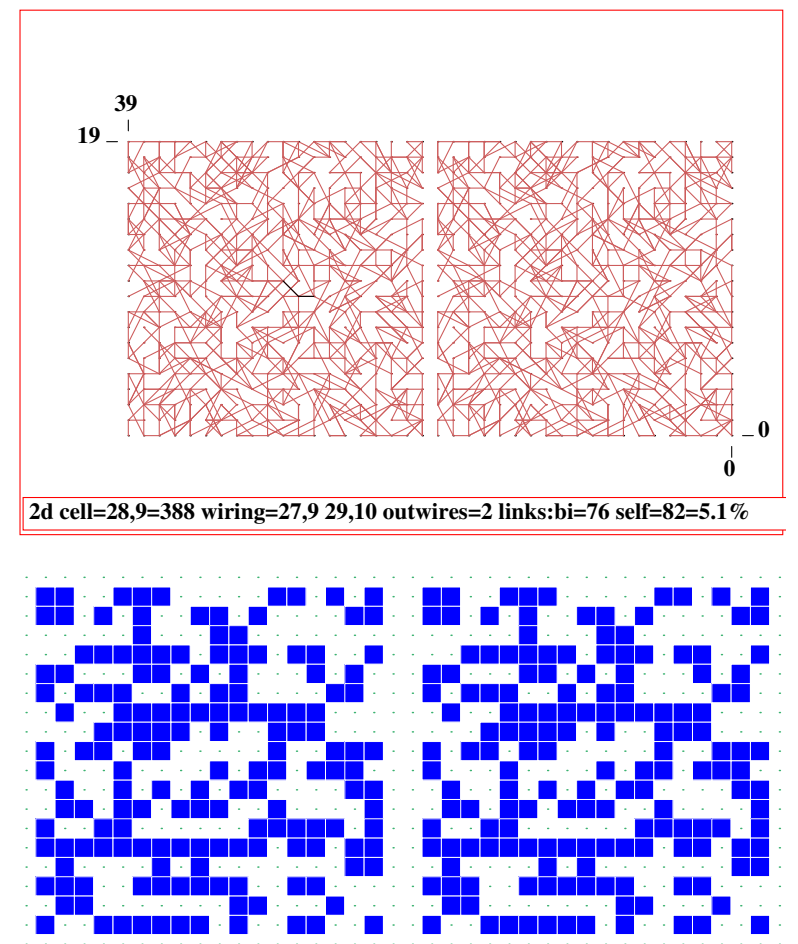

Figure 31.10: A duplicated 2d RBN, $40 \times 20, v 2 k 2$, showing just links, made up of two identical $20 \times 20$ subnetworks. The original $20 \times 20$ network was set with random wiring confined to a 5 cell local zone (section 12.5.2) and with periodic boundaries suppressed (section 12.5.5).

The display shown here is achieved by first defining a block equal to the whole network (enter a, section 17.6.5), then applying a 5-way toggle to show links only (enter $\mathbf{n}$, section 17.6 .3 and figure $17.11(5))$.

Figure 31.11: A duplicated $2 \mathrm{~d}$ seed, $40 \times 20$ consisting of two identical $20 \times 20$ sub-seeds

Note that if $\mathbf{f}$ or $\mathbf{F}$, for a manual version of damage spread, was selected in section 31.5, and the network was duplicated, then there will be no difference in the initial states, thus no damage. To introduce a difference, a random bit/value flip can be made on-the-fly (key 1 , section 32.8.2). A new random initial state (the same for each sub-network) can be set on-the-fly (key 4, section 32.8.1).

\subsubsection{The Damage Histogram}

$1 d$ and $2 d$ networks only - not $3 d$

Enter $\mathbf{a}$ for actual, or $\mathbf{b}$ for bins, in section 31.5, to generate a histogram of damage spread for a sample of initial states generated automatically. The prompt to duplicate the network (section 31.5.1) is presented. Do not duplicate if this was done previously, otherwise the duplicated network will be reduplicated, making 4 copies of the original.

Top-right prompts are presented in sequence as follows to set the histogram parameters. The second line differs between actual or bins. The defaults may vary and some can also be reset later during a histogram pause (section 31.5.4). This example is for two $20 \times 20$ sub-networks.

\section{Damage Spread Histogram}

cut-off size (min 20, def 400): (for actual damage, a entered in section 31.5)

delay damage, time-steps before damage start (def 0 ):

define damage, time-steps same damage (def 5):

(for damage bins, the second line is as follows, $\mathbf{b}$ entered in section 31.5)

no of bins (min 10, def 100): 

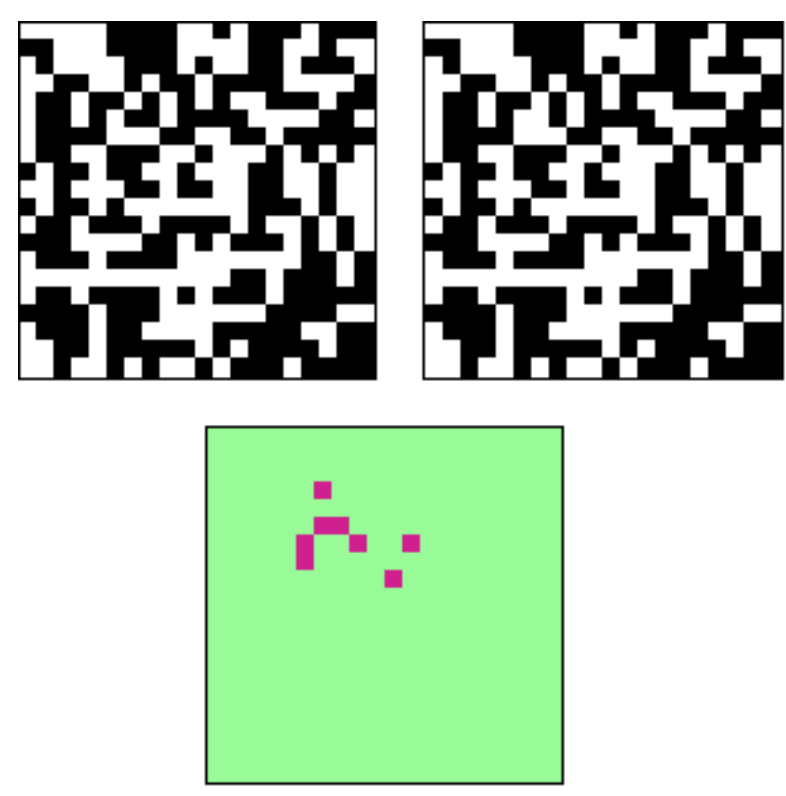

Figure 31.12: An example of $2 \mathrm{~d}$ "damage". The difference in dynamics between two 2d RBN. Above: the space-time patterns of two identical $20 \times 20$ RBN (color by value), except that one bit was flipped in the initial state. The RBN has $k=2$, with connections as in in figure 31.5.1. Below: the "damage spread" between the two, which has stabilized after a number of time-steps, shown as a third space-time pattern. In this example keep-f was selected in section 31.5 , so that once a cell is "damaged" - it stays "damaged".

These options are described below,

options ... what they mean

cut-off size ... (for actual damage only) set the cut-off or accept the default, where any damage above the cut-off is lumped together in one excess bin.

no of bins... (for damage bins only) set the number of bins or accept the default.

delay damage ... set the number of time-steps before the two sub-networks are compared and the measure of the damage starts - the default is 0 .

define damage ... to define at what point the damage is deemed to have stopped spreading. By default, if the damage has not changed for 5 consecutive time-steps, it is considered to be complete - to have stabilized, but this number can be reset.

\subsubsection{Drawing the damage histogram}

The histogram is drawn in a window in the lower part of the screen, as in figure 31.13 and 31.14. At each update, a new unbiased random initial state is assigned to the sub-networks, differing by one bit/value at a random position. When the damage stops spreading (see "define damage" in section 31.5.2), the histogram is updated - plotting the damage size ( $x$ axis) against the frequency of each size ( $y$ axis). The $y$ axis is rescaled automatically to allow the highest histogram bar to fit. A top-right data window keeps track of the current damage, for example damage $=11 / 400=2.8 \%$. To considerably speed up computation for generating these histograms, the display of space-time pattern graphics can be toggled off on-the-fly (key $\mathbf{S}$, section 32.9). 


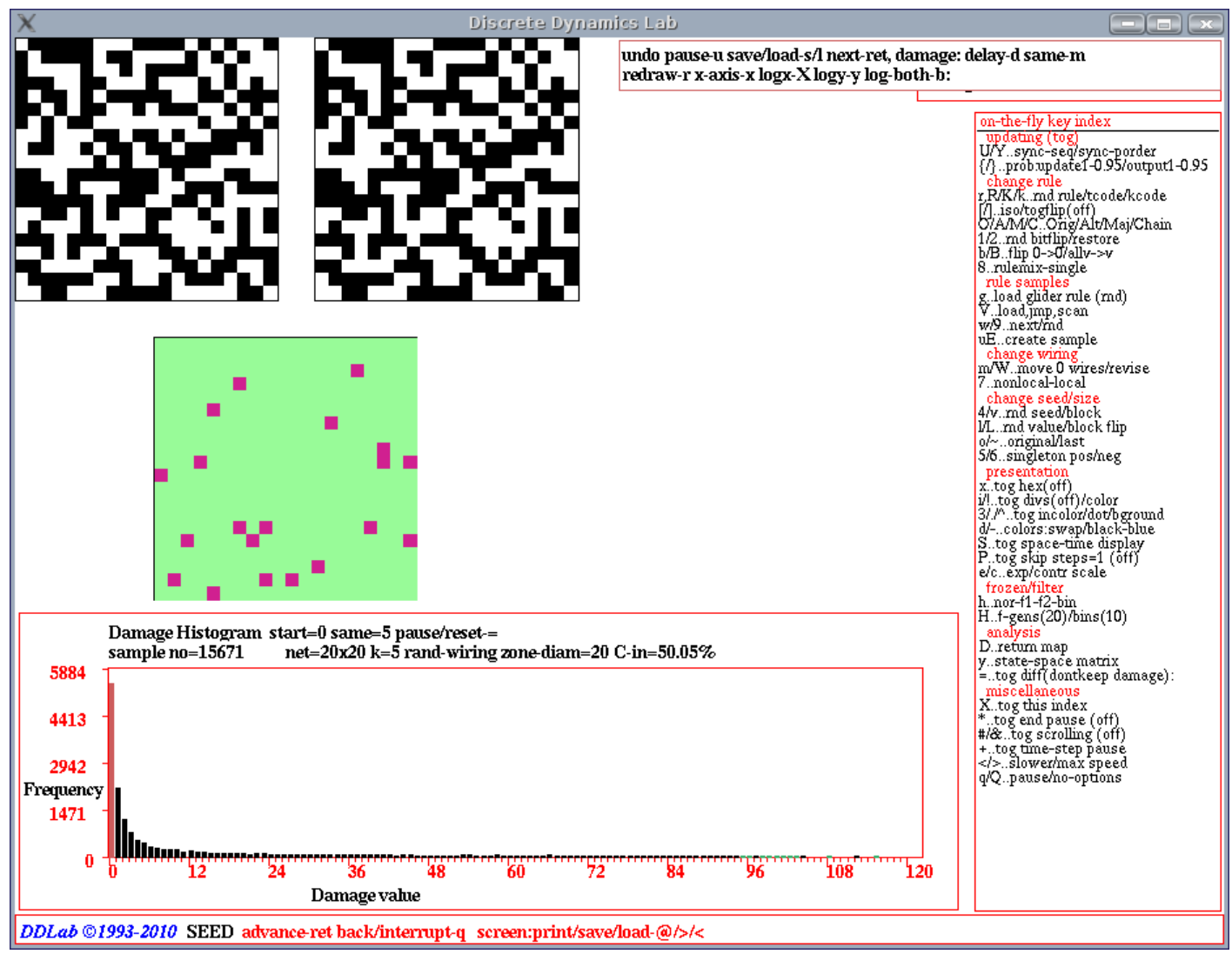

Figure 31.13: The DDLab screen, showing the automatic process for gathering statistics on (actual) damage. This example is for two $20 \times 20 \mathrm{RBN}, k=5$, with canalyzing inputs set to $50 \%$, (chapter 15 ). At each pass, a new random initial state is assigned to the sub-networks, differing by one bit at a random position. When the damage stops spreading, the histogram of the damage size ( $x$ axis) against the frequency of damage size ( $y$ axis), is updated. The process was paused with the $=$ key.

Information and reminders shown within the histogram window, are described below,

info/reminders ... what they mean (values shown are examples) start $=\mathbf{0} \ldots$ number of time-steps before the measure of damage begins.

same $=\mathbf{5} \ldots$ number of time-steps defining damage as having stabilized.

pause $/$ reset $-\ldots$ enter $=$ (the equals sign $)$ to pause and reset the histogram parameters.

sample no $=\mathbf{1 5 6 7 1} \ldots$ the size of the sample so far.

net $=20 \times 20 \ldots$ network size.

$\mathbf{k}=5 \ldots$ neighborhood size $k$, or " $\mathrm{k}=3$ to $6 "$ for mixed- $k$.

local wiring ... local wiring, as in CA.

rand-wiring ... random wiring, as in $\mathrm{RBN}$.

zone diam $=9 \ldots$ the size of the local zone confining random wiring (section 12.5.2).

$\mathbf{C - i n}=\mathbf{5 0 . 0 0 \%} \ldots$ the percentage of canalyzing inputs. 

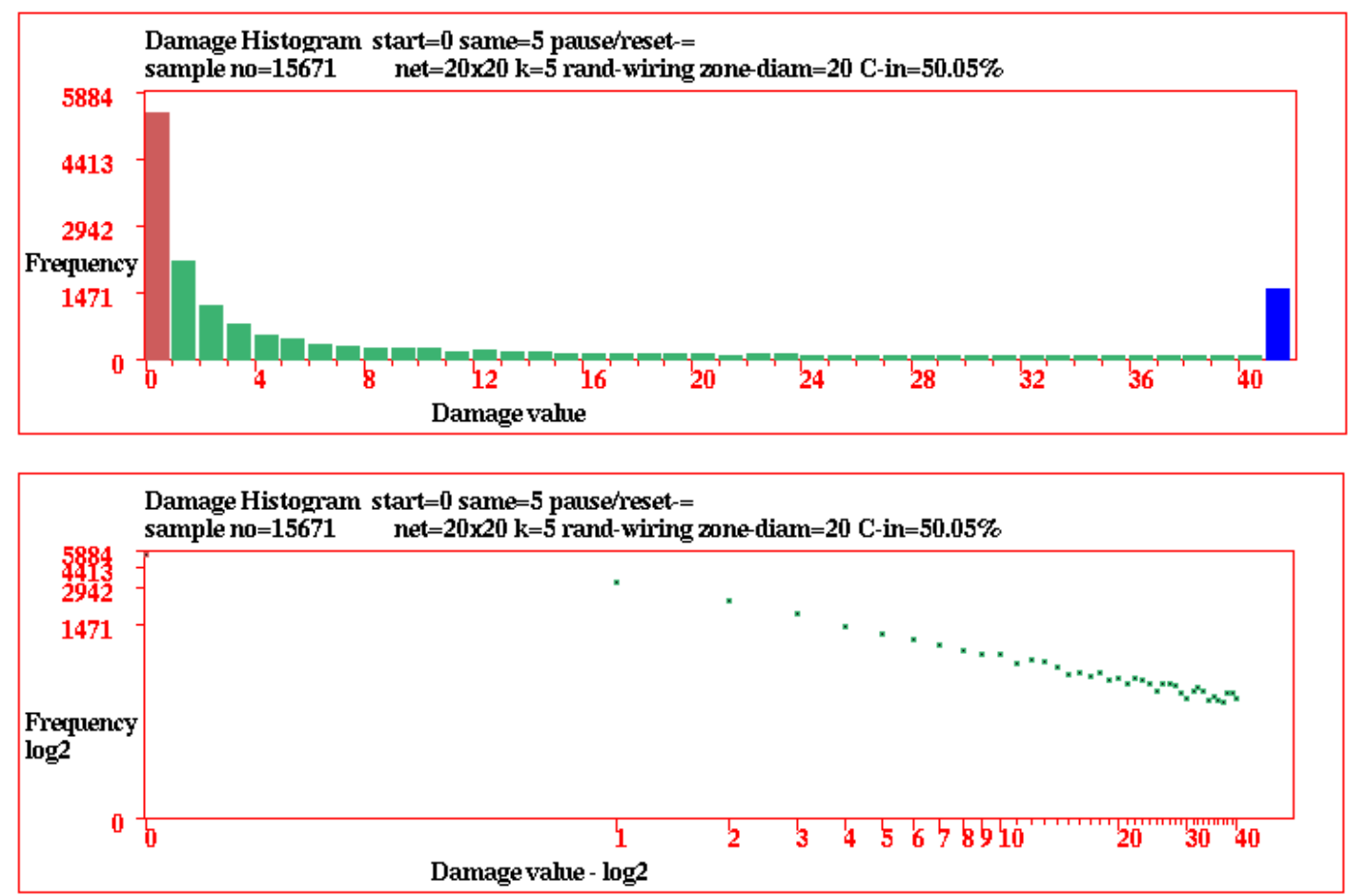

Figure 31.14: The damage histogram showing actual damage, for the same network as in figure 31.13. Top: with the damage cut-off set at 40 , the frequency of damage of 41 and above is represented by the blue bar on the extreme right. Bottom: the damage histogram as a log-log plot.

Enter $=($ the equals sign) to pause the histogram and pause options (section 31.5.4). Alternatively, pause by entering $\mathbf{q}$ for the general pause options for space-time patterns (section 32.14). Most on-the-fly options (chapter 32) can be activated during the histogram routine. To speed the damage histogram, toggle the space-time graphics off with the on-the-fly option $\mathbf{S}$ (section 32.9.8), which also helps to avoid a graphics clash in $1 \mathrm{~d}$.

\subsubsection{Pausing the damage histogram}

While the histogram is being drawn (section 31.5.3), enter $=$ (the equals sign) to pause, the following top-right prompt is presented to amend parameters and presentation,

stp-q undo-pause-u save/load-s/l next-ret, damage: delay-d same-m redraw-r xcut-off-X logx-x logy-y log-both-b: (xcut-off-x for actual damage only) 


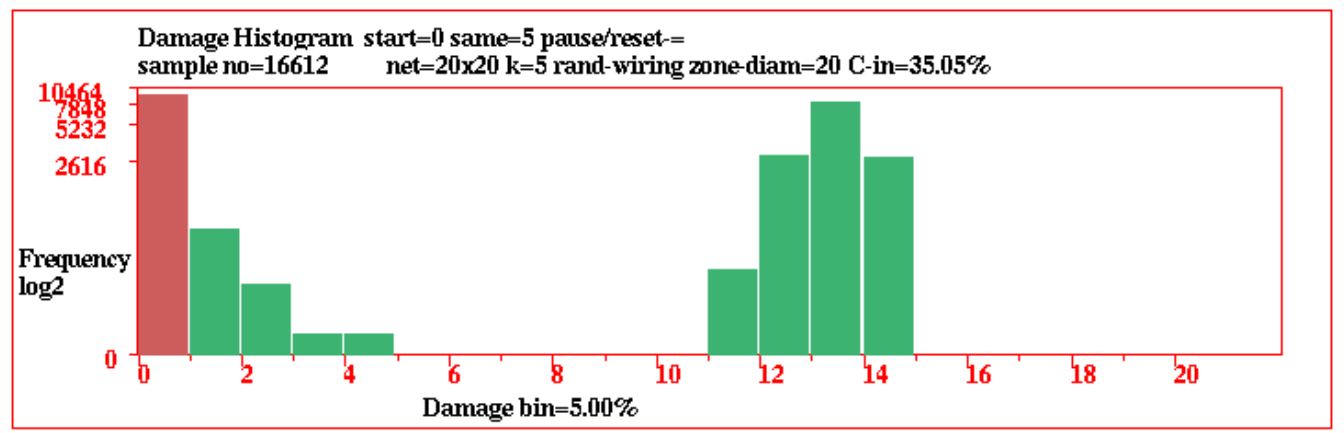

Figure 31.15: The damage histogram with the damage frequency allocated to 20 equal size bins, show with the $y$ axis in log form. This is a similar network as in figure 31.13 , but with canalyzing inputs set to $35 \%$, resulting in less ordered dynamics and a bimodal distribution of damage frequency.

These options are explained below,

$$
\begin{aligned}
\frac{\text { options }}{\mathbf{s t p}-\mathbf{q}} \cdots & \frac{\text { what they mean }}{\text { for the space-time pause prompt with many options (section } 32.14 \text {. Enter } \mathbf{q}} \\
& \text { again to backtrack, or return to the histogram pause prompt. }
\end{aligned}
$$

undo pause-u ... to continue the histogram without pause.

save/load-1/s . . enter $\mathbf{s}$ to save the histogram data as a . his file (section 35.2 ) - the file holds the current frequencies ( 0 to max $x$-axis) in successive pairs of bytes. Enter $\mathbf{l}$ to load and print the data in the terminal (not for DOS).

next-ret ... enter return to continue the histogram, but pausing after each sample.

delay-d ... to alter the damage delay (section 31.5.2).

same-m ... to redefine damage, the number of time-steps defining damage as having stabilized (section 31.5.2).

redraw-r ... to redraw the histogram, which may have been overwritten by other graphics. xcut-off-X ... (for actual damage only) to re-scale the x-axis, the following top-right prompt is presented,

revise x-cut-off, now 400 ( $\min 20$, def 400): (for example)

enter the new cut-off. Any damage above the cut-off is lumped together in one excess bin.

$\log \mathrm{x}-\mathrm{x}, \log \mathrm{x}-\mathrm{y} \ldots$ to show the $x$-axis or $y$-axis separately in $\log$ form, with the following top-right reminder,

$\mathrm{x}$-axis $\log 2$, cont-ret: or $\mathrm{y}$ axis $\log 2$, cont-ret:

log-both-b ... to show both the $x$ and $y$-axis in $\log$ form, a log-log plot (as in figure 31.14 Bottom), with the following top-right reminder,

xy axes $\log 2$, cont-ret: 


\subsection{Attractor histogram}

A basin of attraction field cannot be computed for networks larger than the limits in table 7.3. Even within these limits, larger sizes may be not be practical because of probable time/memory constraints. However, statistical data on the range of attractors and their relative sizes for networks well beyond the limits in table 7.3 can be obtained by automatically running a network forwards from many random initial states, identifying different attractors and creating a histogram of the frequency of each type. The largest basins will be found the most frequently — small basins may be missed.

Attractors are identified by comparing and recording attractor states, and the data gathered includes the period and the average transient length - the number of time-steps to reach the attractor. This method is suitable for ordered networks - that have short transients and attractor cycles where the attractor can actually be reached and disclosed in a reasonable time, so is not practical if the dynamics are too chaotic - in that case the "skeleton" histogram is potentially an alternative method (section 31.7).

The method has been applied in RBN models of genetic regulatory networks[11, 29] with a critical degree of canalyzing functions (chapter 15) as disclosed by the Derrida plot (chapter 22). For CA, the method is also useful for ordered and complex (glider) rules ${ }^{9}$ with very high in-degree — which is a limitation on reverse algorithm methods for generating attractor basins (section 2.18).

Enter a at the first output parameter prompt (section 31.1) to skip directly to the "attractor" category of options (the only way to arrive there) to generate the attractor histogram. The following top-right reminder prompt is presented,

\section{attractors: histogram, cancel-q cont-ret:}

Enter return - for $v \geq 3$ the histogram will commence, for $v=2$ there is a further option (section 31.6.1, below).

\subsubsection{Density classification problem - attractor histogram} for binary systems $(v=2)$ only

For binary systems $(v=2)$ only, there is a further top-right prompt for the inclusion of data on initial state densities that fall into the all 0 s or all 1 s point attractors,

\section{include seed density data for all $=0 / 1$ s point attractors-a:}

Enter a to include this data, which is intended mainly to check the fitness of rules that have been evolved to solve the "density classification problem", a favourite exercise in emergent computation[7, 13], where initial states with densities $d<0.5$ are evolved to fall into the all 0s point attractor, and $d>0.5$ to the all 1 s point attractor $(d=0.5$ for even $n$ are also included in DDLab). An alternative method for density classification based on attractor basins is described in section 24.7.

\footnotetext{
${ }^{9}$ For example, ordered rules such as majority rules (section 16.7) or Altenberg rules (section 16.9), complex (glider) rules (section 3.7.1), or the game-of-Life (sections 16.10 and 14.2.2).
} 


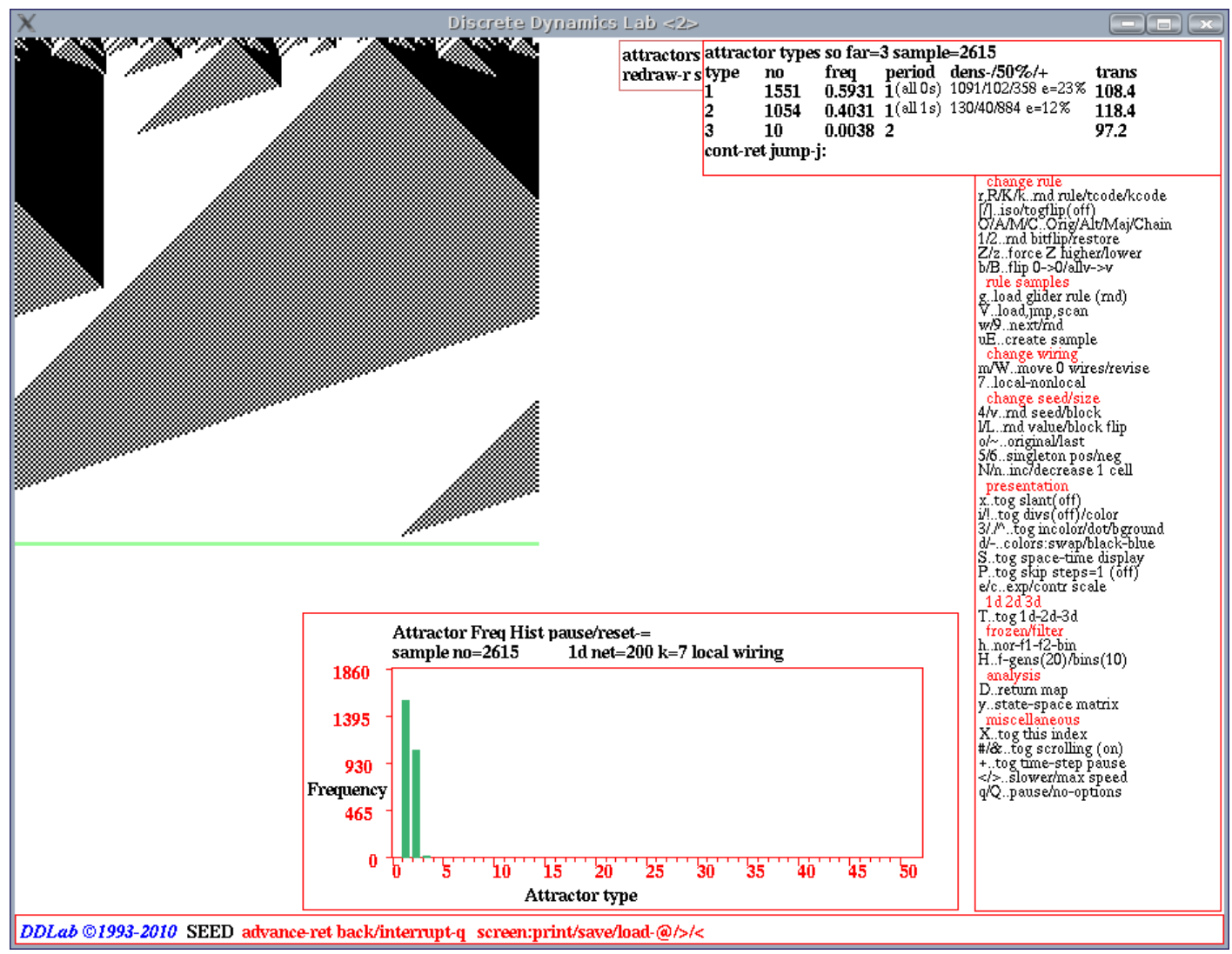

Figure 31.16: A snapshot of the DDLab screen, showing the attractor histogram for density classification, applying the Raja Das rule (as in figure 24.7) to a 1d CA $v 2 k 7, n=200$. Top left: a sample space-time pattern (scale enlarged) leading to the all-0s point attractor (repeated in green). Top Right: a window showing data on the three attractors found, giving the fraction densities $(d<0 . \overline{5}, d=0.5$, $d>0.5)$ arriving at the all $0 \mathrm{~s}$ and all $1 \mathrm{~s}$ point attractors - the data is described in section 31.6.5.3. Bottom center: the attractor histogram for a sample of 2615. As $n$ is even, a third (infrequent) 2-state attractor, consisting of alternating values $0101 \ldots$ and $1010 \ldots$, is able to exist.

\subsubsection{Drawing the attractor histogram}

The histogram is drawn in a lower-center window. Space-time patterns (in 1d or 2d) appear in the top-left as usual, from each new initial state. The histogram is continuously updated (in black), showing the attractor type ( $x$ axis) against the frequency of arriving at that type ( $y$ axis), which indicates the relative size of the basin of attraction. The axes rescale automatically (and columns turn green) on reaching the current scale limits.

At the same time, a top-right data window gives current data: the current total of attractors found, the current type, its period and average transient length, for example,

$$
\text { types }=44 \text { this }=8 \text { attperiod }=12 \text { avtrans }=14.0
$$




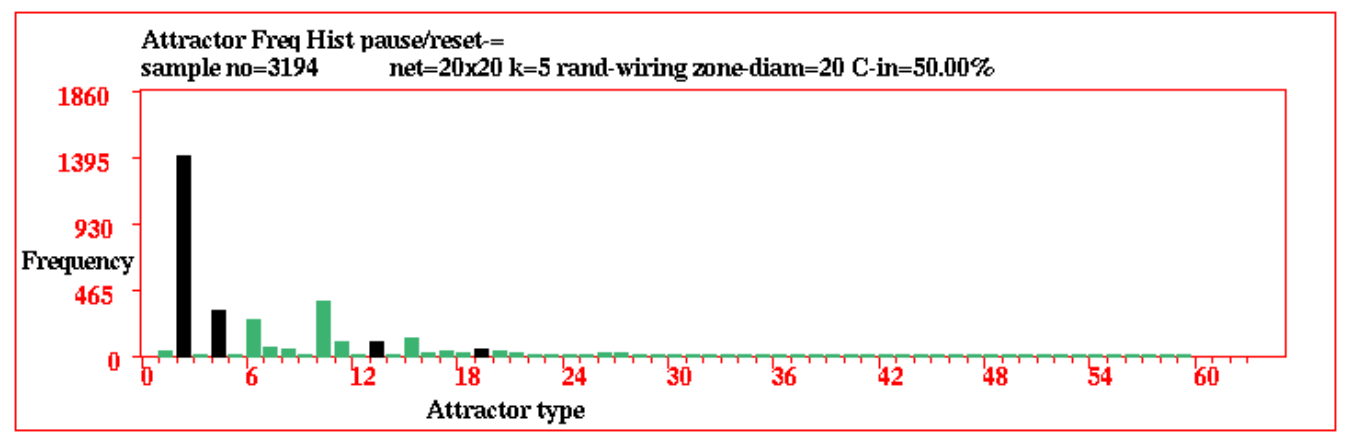

unsorted histogram, for a sample of 3194

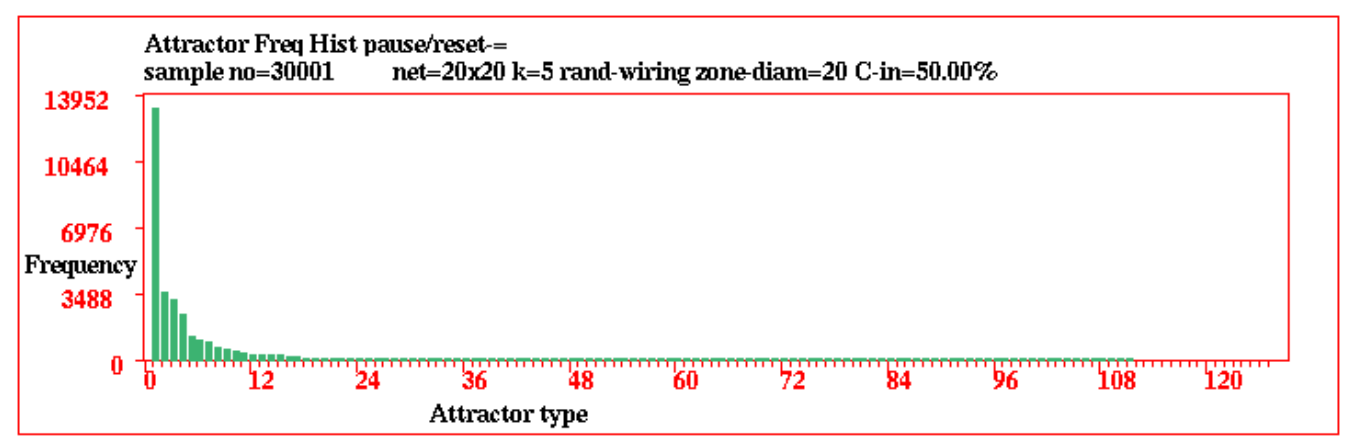

sorted histogram, according to frequency, for a sample of 30001, with 111 attractors

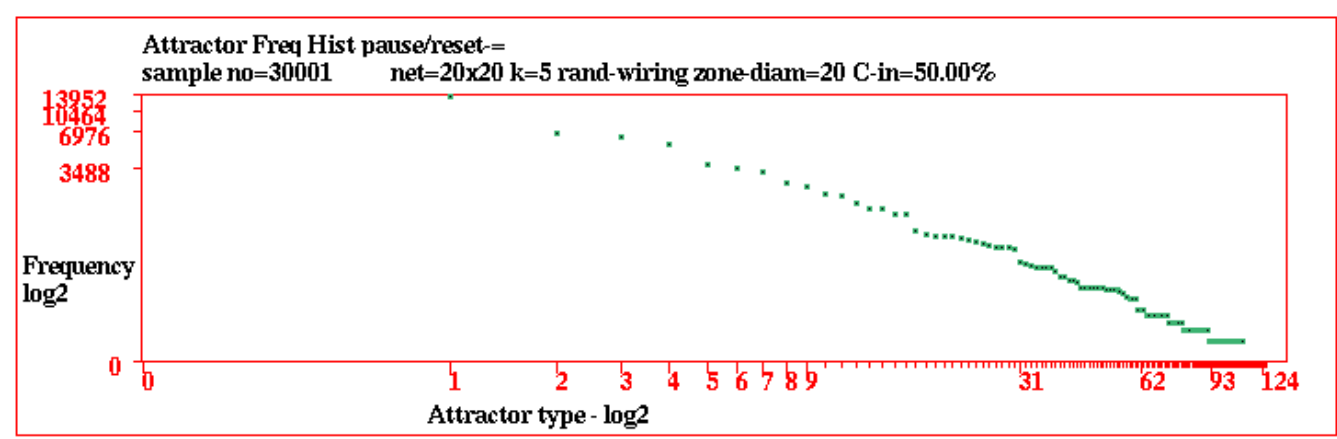

The sorted histogram, shown as a log-log plot

Figure 31.17: The attractor frequency histogram, for a $20 \times 20 \mathrm{RBN}, v 2 k 5$, with unbiased random wiring, and canalyzing inputs set to $50 \%$ (chapter 15 ). Each sample starts with a new unbiased random initial state, and the attractor type into which the transient falls is identified, either as an existing type, or a new type which is added to the list of existing types. The histogram columns are continuously updated, in black, showing the attractor type ( $x$-axis) against the frequency of arriving at that type (y-axis). Given a large enough sample, bar height indicates the relative size of the basin of attraction. The axes rescale automatically on reaching the current scale limits. Columns turn green when rescaled, redrawn, or sorted. 


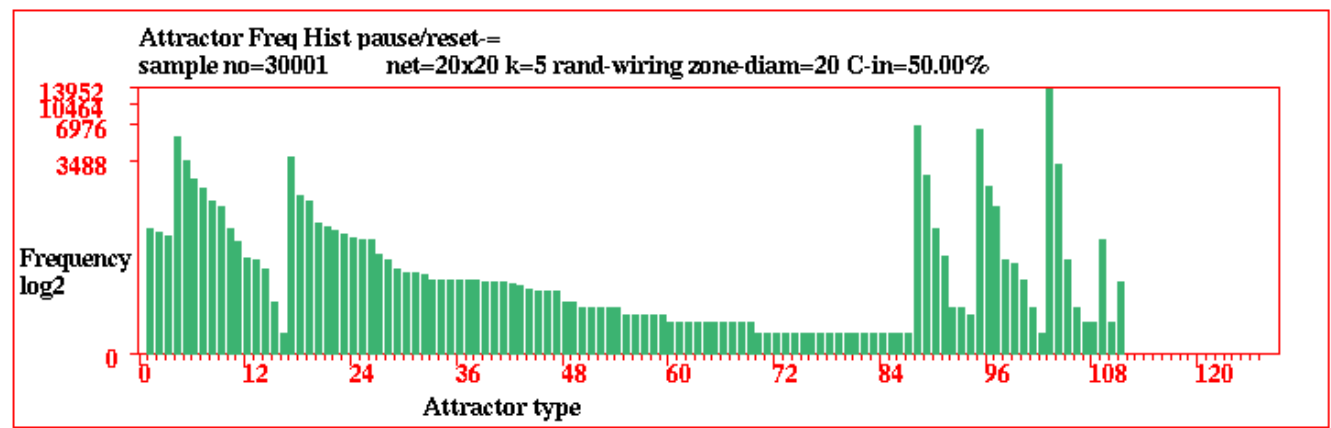

first sorted by frequency (figure 31.17), center) then by period

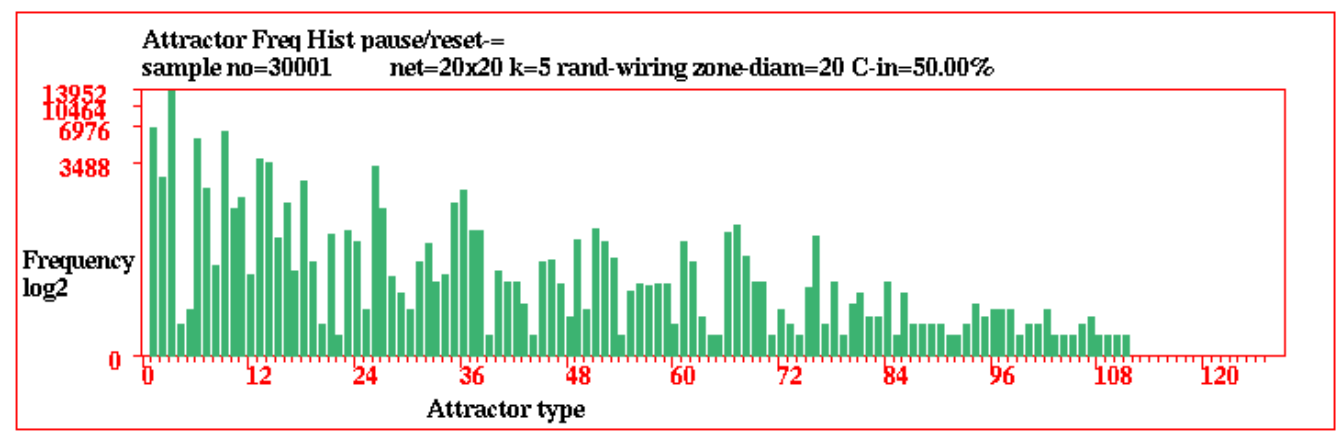

first sorted by frequency (figure 31.17), center) then by average transient

Figure 31.18: The attractor histogram for a sample of 30001 and 111 attractors, sorted by attractor period and average transient length, with log $y$-axis. (section 31.6.4).

Most space-time pattern on-the-fly options work during the histogram routine. To speed the attractor histogram, toggle space-time graphics off with on-the-fly option $\mathbf{S}$ (section 32.9.8.

\subsubsection{Histogram window information}

Information is also displayed at the top of the histogram window (figure 31.18), including some reminders about the network, decoded as follows (values shown are examples),

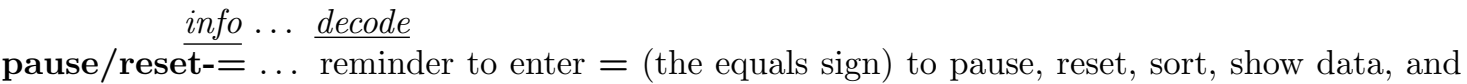
other options (section 31.6.3).

sample no $=\mathbf{3 9 1 4} \ldots$ the size of the sample so far.

net $=\mathbf{2 0} \times \mathbf{2 0} \ldots$ network size for $2 \mathrm{~d}$, or $\mathbf{n e t}=\mathbf{2 0 0}$ for $1 \mathrm{~d}$.

$\mathbf{k}=5 \ldots$ neighborhood size, $k$, or $\mathbf{k}=\mathbf{3 - 5}$ for a mixed- $k$.

local wiring ... for local wiring, as in CA.

rand-wiring ... for random wiring, as in RBN.

zone $\operatorname{diam}=\mathbf{2 0} \ldots$ the size of the relative local zone within which random wiring is confined, see section 12.5.2.

C-in $=\mathbf{5 0 . 0 0 \%} \ldots$ the percentage of canalyzing inputs (section 15$)$. 


\subsubsection{Pausing the attractor histogram}

The space-time pattern on-the-fly pause (key q, section 32.14) applies while drawing the histogram.

However, there is a special pause for the histogram itself, enter $=$ (the equals sign) for the following top-right prompt which provides a range of histogram options,

\section{attractors histogram: hist-h stp-q, data:show-d print-p save-S redraw-r sort-s data-d clear-c undo-pause-u next-ret jump-graph-j:}

These options are summarized below, some described further in the sections indicated ${ }^{10}$,

$$
\underline{\text { options } . . . ~ w h a t ~ t h e y ~ m e a n ~}
$$

hist-h . . f further histogram options: to save/load, rescale, and log-plot (section 31.6.4).

stp-q ... for the space-time pause prompt with many options (section 32.14. Enter $\mathbf{q}$ again to backtrack, or return to the histogram pause prompt.

data: ... attractor data - for $1 \mathrm{~d}$ networks print/save includes listing attractor states. show-d ... show attractor data in the DDLab screen (section 31.6.5). print-p ... print the attractor data in the terminal (section 31.6.6). save-S ... save the attractor states as a . dat file (section 31.6.6).

redraw-r ... to redraw the histogram, which may have been overwritten by other graphics.

sort-s ... sort the histogram by frequency and other measures (section 31.6.7).

clear-c ... clear the histogram and resume from scratch with the same or an amended network. The space-time pattern pause prompt (section 32.14) is first presented where wiring/rules can be changed - enter return to continue with the original network.

undo-pause-u ... to continue the histogram without pausing.

next-ret ... enter return to continue the histogram, but pausing after each sample.

jump-graph-j ... to construct a jump-graph from the histogram data (section 31.6.8).

\subsubsection{Rescaling the attractor histogram}

If $\mathbf{h}$ for further histogram options is entered in section 31.7.3, or after sorting (enter $\mathbf{s}$ ), the following top right prompts are presented,

\section{save/load-l/s undo-pause-u cont-ret $\mathrm{x}$-axis-x logx-X logy-y log-both-b:}

These options are explained below,

\footnotetext{
${ }^{10}$ These option are the same or similar to the skeletons histogram options in section 31.7.3.
} 


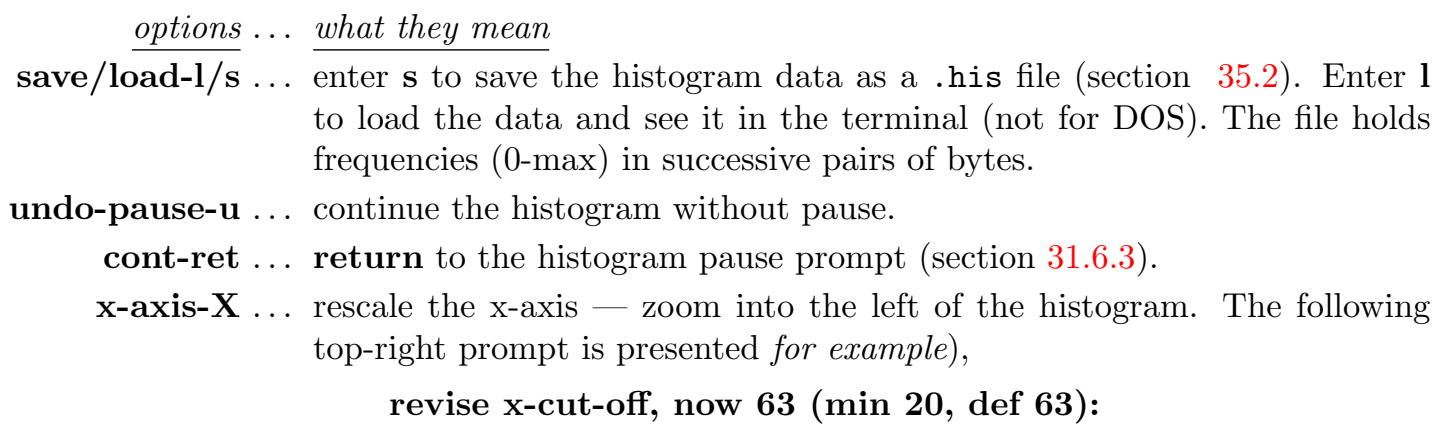

$\log \mathrm{x}-\mathrm{x}, \log \mathrm{x}-\mathrm{y} \ldots$ to show the $x$-axis or $y$-axis separately in $\log$ form, with the following top-right reminder,

$\mathrm{x}$-axis $\log 2$, cont-ret: or $\mathrm{y}$ axis $\log 2$, cont-ret:

$\log$-both-b ... show both the $x$ and $y$-axis in log form (as in figure 31.17, bottom), with the following top-right reminder,

xy axis log2, cont-ret:

\subsubsection{Attractor histogram data}

Enter $\mathbf{d}, \mathbf{p}$ or $\mathbf{s}$ in section 31.6 .3 to show the data in the DDLab screen (section 31.6.5.1), or print to the terminal (DOS only) or save to file 31.6.6. The data gives a summery for each attractor according to the histogram order, so sorting the histogram beforehand is advisable. For $1 \mathrm{~d}$ networks, print/save data also includes a list of the states in each attractor.

\subsubsection{Attractor histogram screen data}

Enter $\mathbf{d}$ in section 31.6.3 to show a list of attractor types, as many as will fit, displayed in a window on the right of the screen, followed by further options. The list order depends on whether the list was sorted in section 31.6.7, if not, the list is ranked in the order that attractor types were found.

The following example is for the sorted histogram in figure 31.17,

\begin{tabular}{|c|c|c|c|c|}
\hline $\begin{array}{l}\text { attrac } \\
\text { type }\end{array}$ & $\begin{array}{l}\text { tor typ } \\
\text { no }\end{array}$ & $\begin{array}{l}\text { s so f } \\
\text { freq }\end{array}$ & period & $\operatorname{trans}$ \\
\hline 1 & 13041 & 0.4347 & 4 & 54.1 \\
\hline 2 & 3610 & 0.1203 & 8 & 77.0 \\
\hline 3 & 3095 & 0.1032 & 6 & 40.0 \\
\hline \multicolumn{5}{|c|}{ (the list continues) } \\
\hline 39 & 22 & 0.0007 & 12 & 33.3 \\
\hline 40 & 21 & 0.0007 & 12 & 24.8 \\
\hline 41 & 20 & 0.0007 & 16 & 27.4 \\
\hline
\end{tabular}

Enter $\mathbf{q}$ to quit the data, $\mathbf{j}$ to jump to a new type index - with the prompt index (111-1): or return to see more of a long list or to quit the data if the end of the list is visible. 


\subsubsection{Attractor histogram data decode}

The column headings in section 31.6.5.1 and the labels in section 31.6.6 denote the following,

$$
\begin{aligned}
& \text { type } \ldots \text { the attractor type index. } \\
& \text { no } \ldots \text { the number of this attractor type found. } \\
& \text { freq } \ldots \text { the frequency of this type. } \\
& \text { period } \ldots \text { the attractor period. } \\
& \text { trans } \ldots \text { the average transient (or run-in) length. }
\end{aligned}
$$

\subsubsection{Histogram data for density rules} for binary systems $(v=2)$ only

Enter a in section 31.6.1 to include extra data on initial state densities that fall into the all 0s or

\begin{tabular}{|c|c|c|c|c|c|}
\hline type & no & freq & period & den- $/ 50 \% /+$ & trans \\
\hline 1 & 1551 & 0.5931 & $\mathbf{1}($ all $0 \mathrm{~s})$ & $1091 / 102 / 358 \mathrm{e}=23 \%$ & 108.4 \\
\hline 2 & 1054 & 0.4031 & $\mathbf{1}$ (all 1s) & $130 / 40 / 884 \mathrm{e}=12 \%$ & 118.4 \\
\hline 3 & 10 & 0.0038 & 2 & & 97.2 \\
\hline
\end{tabular}
all 1s point attractors,

An additional column, headed den- $/ \mathbf{5 0 \%} /+$, shows the percentage of initial states with densities $(d<0.5, d=0.5, d>0.5)$ arriving at the all 0 s and all 1 s point attractors, and $\mathbf{e}=$ the $\%$ error in density classification. For example, the data in figure 31.16 is as follows,

\subsubsection{Print/Save attractor state data}

Enter $\mathbf{p}$ or $\mathbf{S}$ in section 31.6.3 to print to the terminal, or to save, attractor state data. If saving (to a .dat ASCII file), further prompts will appear to set the file name (section 35.2).

For 1d networks, the data includes a list of states for each attractor. For example, below is some of the data for the sorted histogram in figure 31.19 showing heading information, a summery for each attractor, and the attractor list.

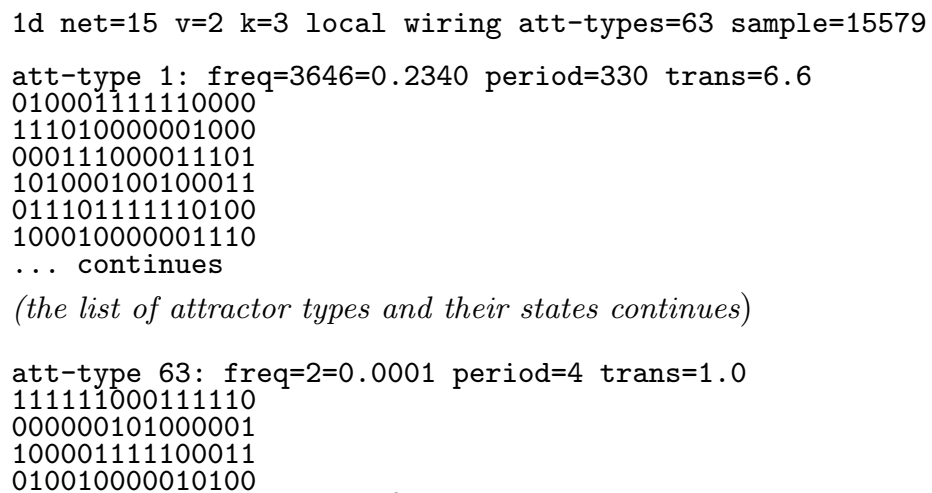

For $2 \mathrm{~d}$ network the list of attractor states is omitted - the data consists of just of the heading information and a summery for each attractor. For example, below is the start of the data for the sorted histogram in figure 31.17, 

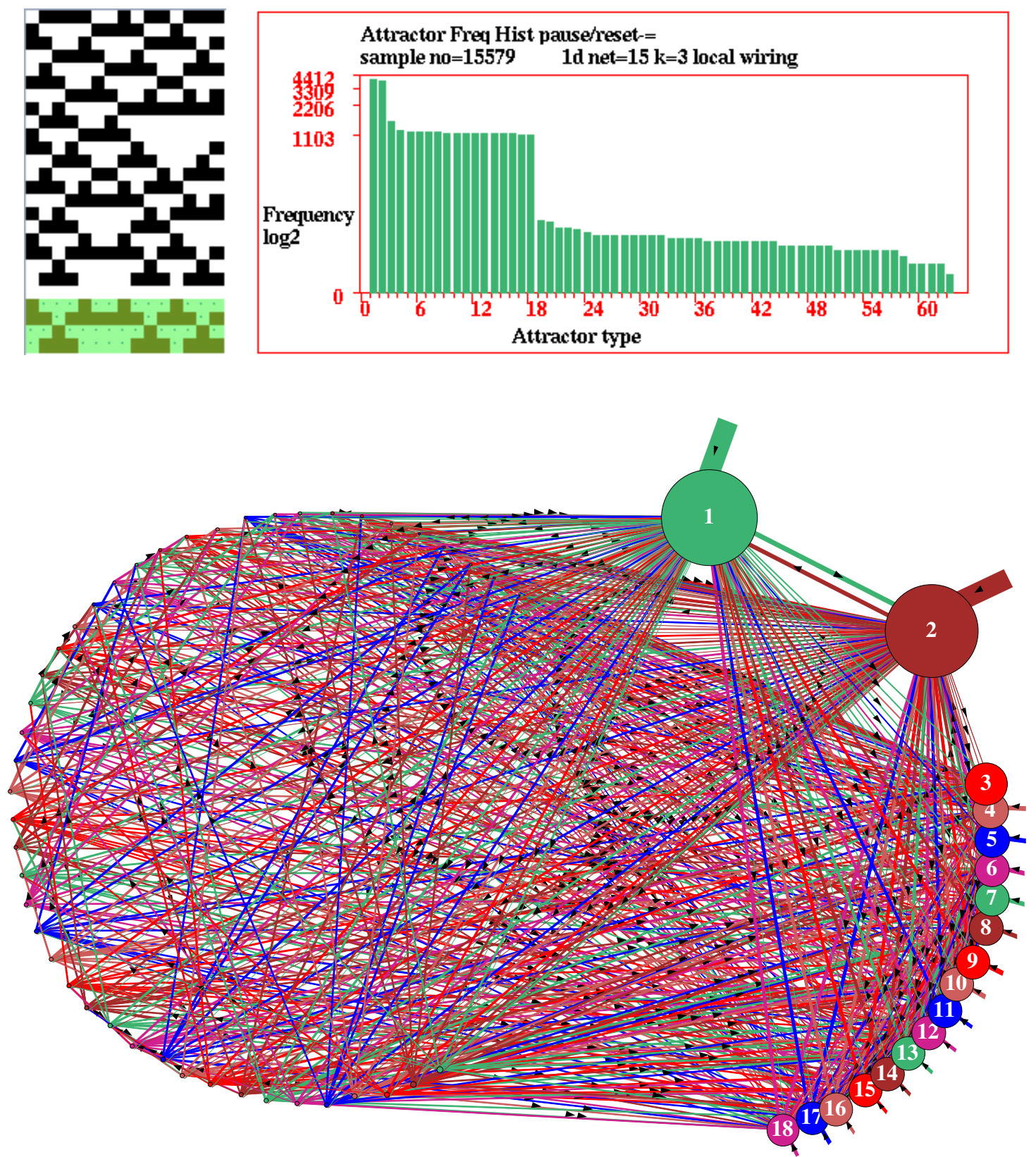

Figure 31.19: The attractor histogram jump-graph, for a 1d CA, v3k3 rcode(dec)54, $n=15$. Top left: a sample space-time pattern showing the attractor repeated. Top Right: The attractor histogram with $\log y$-axis, all 63 attractors were found after a sample of 15579. Above: the jump-graph (chapter 20.3) - nodes and links scaled according to attractor frequency which reflects basin volume, and the graph rearranged 20.5 starting from a circle layout. 


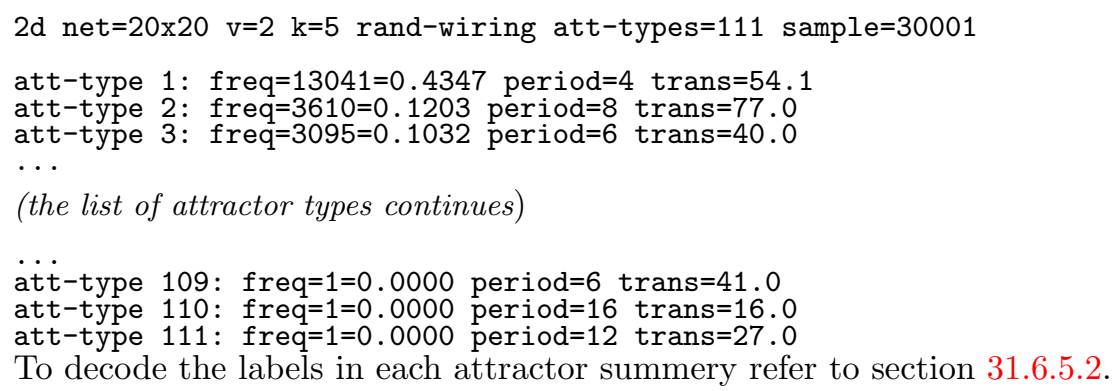

To decode the labels in each attractor summery refer to section 31.6.5.2.

\subsubsection{Sorting the attractor histogram}

Enter $\mathbf{s}$ in section 31.6.3 to sort the histogram. The following top-right prompt is presented,

$$
\text { sort by: attperiod-a avtrans-t (frequency-def): }
$$

Initially the attractor types are ranked in the order they were found, as in figure 31.17 (top). Enter return to sort by frequency. An example is shown in figure 31.17 (center). This corresponds to sorting according to the size of the basin of attraction, as the probability of falling into a basin depends on its size.

Enter a to sort by attractor period, $\mathbf{t}$ to sort by average transient (run-in) length. The result of the new sorting depends on how the list is currently sorted. Having first sorted by frequency, the examples in figure 31.18 show the data sorted first by the attractor period, then by the average transient.

\subsubsection{Attractor histogram jump-graph}

The jump-graph (section 20.3) can be applied to the attractor histogram just as to the complete basin of attraction field (section 24.3).

Enter $\mathbf{j}$ in section 31.6.3 to show the jump-graph of the attractor histogram.

Although the example in figure 31.19 took just a few seconds to compute, a jump-graph with many long period attractors would take a longer time. While jump-graph is being computed the following top-right message is shown, including an inset box on the right giving the percentage complete (enter $\mathbf{q}$ to abandon),

$$
\text { computing jump-graph, quit-q, or wait... } 35 \%
$$

Once the jump-graph is drawn, it can be rearranged, unravelled, rescaled, including dragging vertices and defined components to new positions with elastic links - all jump-graph functions in chapter 20 apply except redrawing basins at jump-graph nodes 20.7. 


\subsection{Skeleton (fuzzy attractor) histogram (for binary systems, $v=2$, only)}

The dynamics on large RBN where $k \geq 3$ typically result in extremely (astronomically) long transients and attractor periods, making it impractical to find attractors by the attractor histogram method (section 31.6), even if a significant fraction of the network is stabilized (frozen) by tuning canalyzing inputs (chapter 15), because the rest of the dynamics remains chaotic. To overcome this problem in RBN models of genetic regulatory networks ${ }^{11}$ each different frozen sub-pattern is treated as a fuzzy attractor, called the "skeleton"[11, 29]. In this context network elements are referred to as "genes" - though the method can also be applied to CA.

The degree of stability of a frozen sub-pattern to qualify as a skeleton is first defined. The network is run forwards from many random initial states. If the stability threshold is reached, the particular pattern of frozen genes is identified as either a new or previously found skeleton type, and a histogram is built up of the frequency of each type. As for the attractor histogram (section 31.6), various data are recorded (section 31.7.4), including the average transient length.

Enter $\mathbf{s}$ at the first output parameter prompt (section 31.1) to skip directly to the "skeletons" category of options (the only way to arrive there) to generate the skeleton histogram. The following top-right prompt is presented,

skeletons: histogram, cancel-q cont-ret:

Enter return to continue and set the parameters to define a skeleton (section 31.7.1).

\subsubsection{Skeleton parameters prompt}

Five parameters are required to define a skeleton. A series of prompts are presented in turn in a top-right window to reset the parameters or accept defaults, as follows,

frozen skeletons: 0s-0 1s-def both-b:

no of steps frozen: gene (def-20): skeleton (def-20):

$\%$ frozen (def-100): \% same type (def-100):

$$
\text { options .... what they mean }
$$

0s-0 1s-def both-b: ... The frozen pattern value. Enter return for 1s only, $\mathbf{0}$ for 0 s only, or $\mathbf{b}$ for both, which is not a good choice if the entire pattern freezes, as in a majority rule.

gene $(\mathbf{d e f}-20) \ldots(\mathrm{g})$ The number of time-steps that a gene must remain unchanged to be considered frozen. This is the same parameter as frozen generation size (sections 31.2.3 and 32.11.2).

\footnotetext{
${ }^{11}$ In RBN models of genetic regulatory networks in eukaryotic cells, the different patterns of active genes making cell types in multi-cellular organisms are interpreted as dynamical attractors, [15, 20, 30, 35]. RBN attractor skeletons represent the most stable sub-patterns of active genes. The strong overlap between skeletons parallel overlaps in gene expression between eukaryotic cell types[11].
} 


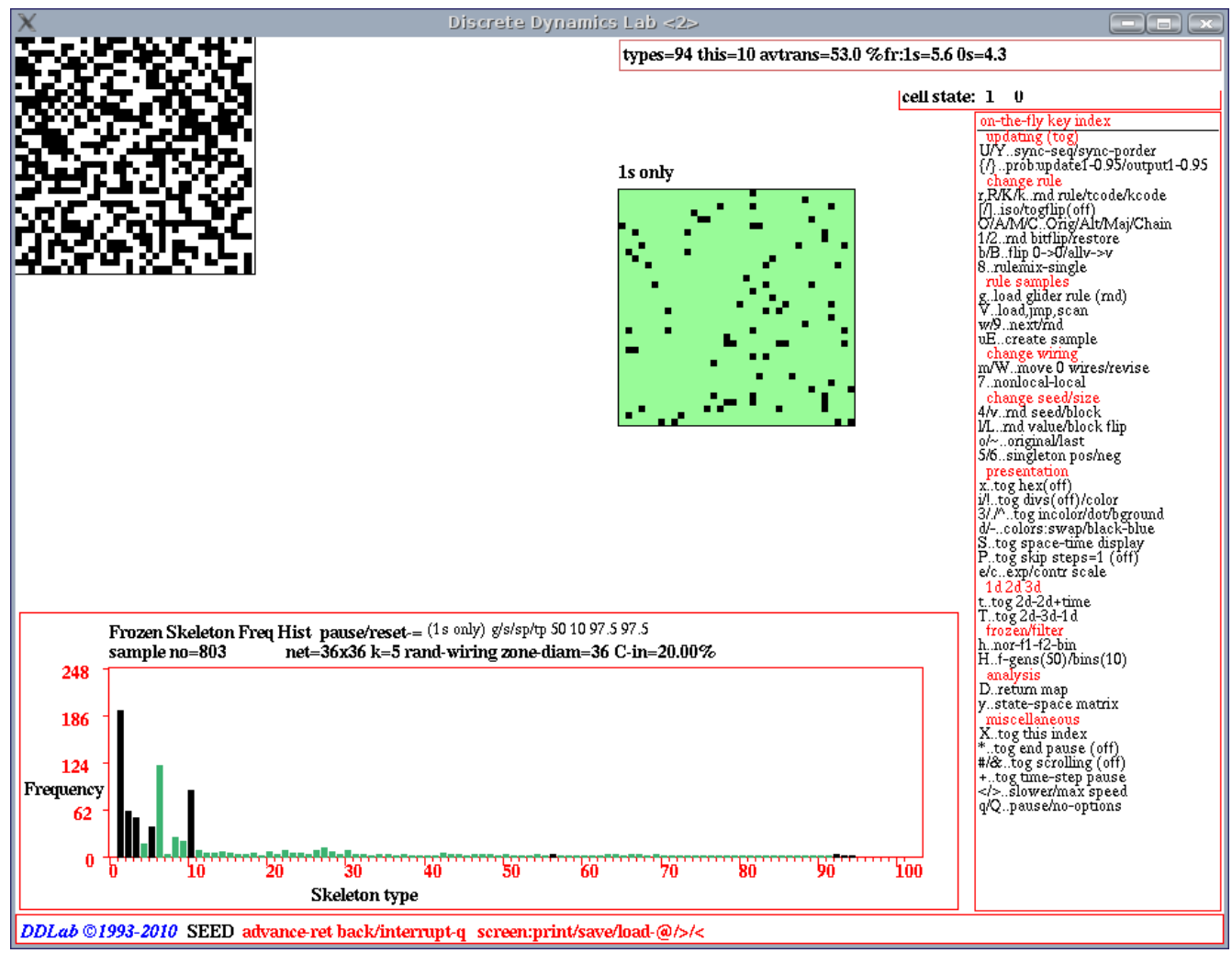

Figure 31.20: A snapshot of the DDLab screen, showing the skeleton histogram at sample 803 with 94 skeletons found.. The network is a $36 \times 36 v 2 k 5$ RBN with unbiased random wiring and $20 \%$ canalyzing inputs (chapter 15). The parameters in section 31.7 (1s only), labelled $\mathbf{g} / \mathrm{s} / \mathrm{sp} / \mathrm{tp}$, are 50, 10, 97.5\%, $97.5 \%$. Each sample starts with a new random initial state, and the skeleton into which the transient falls is identified (if any). A skeleton that cannot be matched with a pre-existing type is added as a new type. The histogram columns are continuously updated (in black), showing the skeleton type ( $x$ axis) against the frequency of arriving at that type ( $y$ axis). The axes rescale automatically (and columns turn green) on reaching the current scale limits. Top left: a snapshot of the space-time pattern. Top center: the skeleton, pattern of frozen 1s. $\overline{\text { Bottom: }}$ the unsorted histogram.

The snapshot was grabbed without pausing, but by slowing down updating with on-the-fly option < (section 32.2).
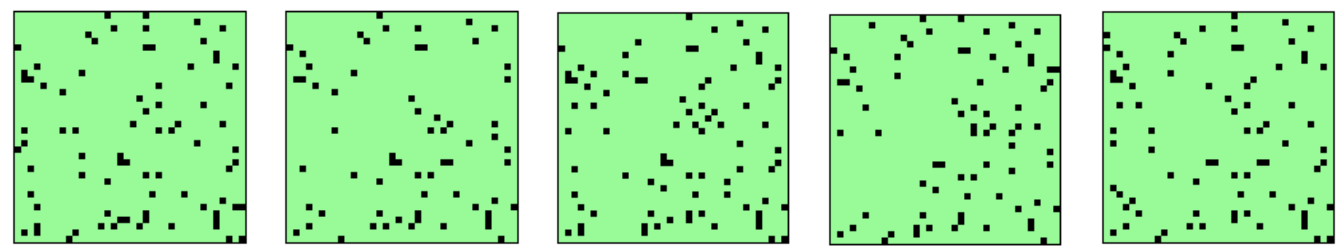

Figure 31.21: Examples of different skeletons, consisting of frozen 1s, from the histogram in figure 31.20. The current skeleton is shown in the top-center of the screen as the histogram is updated. 


\section{skeleton (def-20) ... (s) The number of time-steps the sub-pattern of frozen genes must remain unchanged (within a set Hamming distance) to be consid- ered a frozen skeleton. \\ \% frozen (def-100) ... (sp) The skeleton percentage Hamming distance limits in (s) above. \\ \% same type (def-100) ... (tp) How close (within a percentage Hamming distance) must a skeleton type be to a pre-established type to qualify as that type, otherwise a new type is established.}

The frozen pattern value $(0 \mathrm{~s}, 1 \mathrm{~s}$ or both) and the other parameters, labelled "g/s/sp/tp" as noted above, are also shown in the histogram window (section 31.7.2).

\subsubsection{Drawing the Skeleton histogram}

Just like the attractor histogram (section 31.6.2), the skeleton histogram is drawn in a lower-center window. Space-time patterns (in 1d or $2 \mathrm{~d}$ ) appear in the top-left as usual, from each new initial state. The histogram is continuously updated (in black), showing the attractor type ( $x$ axis) against the frequency of arriving at that type ( $y$ axis), which indicates the relative size of the basin of the quasi attractor. The axes rescale automatically (and columns turn green) on reaching the current scale limits.

As the histogram is updated, for each sample a representation of the skeleton is shown in the top-center of the screen, noting if the skeleton consists of frozen 0s, 1s, or both (figures 31.20 and 31.21). At the same time, a top-right data window gives current data, the current total of skeletons found, the current type, and average transient length, and the percentage of frozen $1 \mathrm{~s}$ and $0 \mathrm{~s}$, for example,

$$
\text { types }=83 \text { this }=4 \text { avtrans }=52.9 \% \mathrm{fr}: 1 \mathrm{~s}=5.40 \mathrm{~s}=4.8
$$

Just as for the attractor histogram, information is displayed at the top of the skeleton histogram window (figure 31.22, decoded in section 31.6.2.1. Skeletons include the following extra items,

$$
\begin{aligned}
& \text { info ... decode }
\end{aligned}
$$

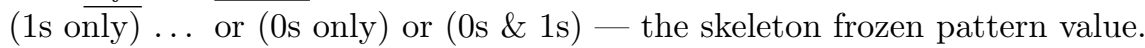

$$
\begin{aligned}
& \mathrm{g} / \mathrm{s} / \mathrm{sp} / \mathrm{tp} \ldots \text { other skeleton parameters, as listed in section 31.7. }
\end{aligned}
$$

Most space-time pattern on-the-fly options work during the histogram routine. To speed the skeleton histogram, toggle space-time graphics off with on-the-fly option $\mathbf{S}$ (section 32.9.8.

\subsubsection{Pausing the skeleton histogram}

The space-time pattern on-the-fly pause (key q, section 32.14) applies while drawing the histogram.

However, there is a special pause for the histogram itself, enter $=$ (the equals sign) for the following top-right prompt which provides a range of histogram options,

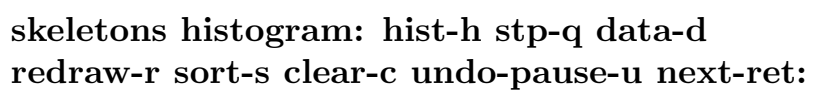


These options are summarized below, most are the same as for the attractors histogram (section 31.6.3) — some are described further in the sections indicated,

options.... what they mean

hist-h ... further histogram options: to save/load, rescale, and log-plot — the same as in "Rescaling the attractor histogram" (section 31.6.4).

stp-q ... for the space-time pause prompt with many options (section 32.14. Enter q again to backtrack, or return to the histogram pause prompt.

data-d ... show attractor data in the DDLab screen (section 31.7.4.

redraw-r... to redraw the histogram, which may have been overwritten by other graphics.

sort-s ... sort the histogram by frequency and other measures (section 31.7.5).

clear-c ... clear the histogram and resume from scratch with the same or an amended network. The space-time pattern pause prompt (section 32.14) is first presented where wiring/rules can be changed - enter return to continue with the original network.

undo-pause-u ... to continue the histogram without pausing.

next-ret ... enter return to continue the histogram, but pausing after each sample.

\subsubsection{Skeleton histogram data}

Enter $\mathbf{d}$ in section 31.7.3 to show a list of skeleton types, as many as will fit, displayed in a window on the right of the screen, followed by further options. The list order depends on whether the list was sorted (as in section 31.7.5), if not, the list is ranked in the order that attractor types were found.

The following example is for the sorted histogram in figure 31.17,

\begin{tabular}{|c|c|c|c|c|c|}
\hline 1s sk & ton & pes so & $r=$ & san & $e=$ \\
\hline type & no & freq & $\% \mathrm{fr}$ & s-1s & trans \\
\hline 1 & 1302 & 0.4340 & 5.5 & 5.9 & 53.0 \\
\hline 2 & 385 & 0.1283 & 5.0 & 5.1 & 53.1 \\
\hline 3 & 157 & 0.0557 & 4.8 & 5.5 & 53.0 \\
\hline$\ldots(t)$ & list co & tinues) & & & \\
\hline 179 & 1 & 0.0003 & 5.1 & 5.2 & 52.0 \\
\hline 180 & 1 & 0.0003 & 4.5 & 5.6 & 52.0 \\
\hline 181 & 1 & 0.0003 & 4.3 & 5.5 & 52.0 \\
\hline $\begin{array}{l}\text { mor } \\
\text { (or, } \\
\text { cont }\end{array}$ & 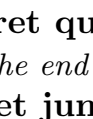 & $\begin{array}{l}\mathbf{q} \text { jump } \\
\text { the list } \\
\text {;. }\end{array}$ & & & \\
\hline
\end{tabular}

Enter $\mathbf{q}$ to quit the data, $\mathbf{j}$ to jump to a new type index — with the prompt index (181-1): or return to see more of a long list or to quit the data if the end of the list is visible. 


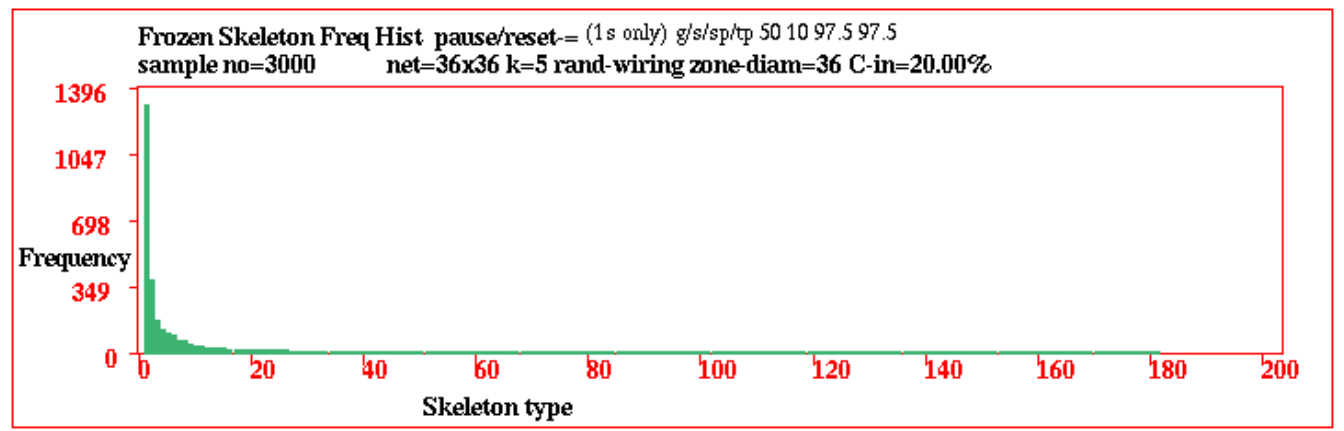

sorted according to frequency

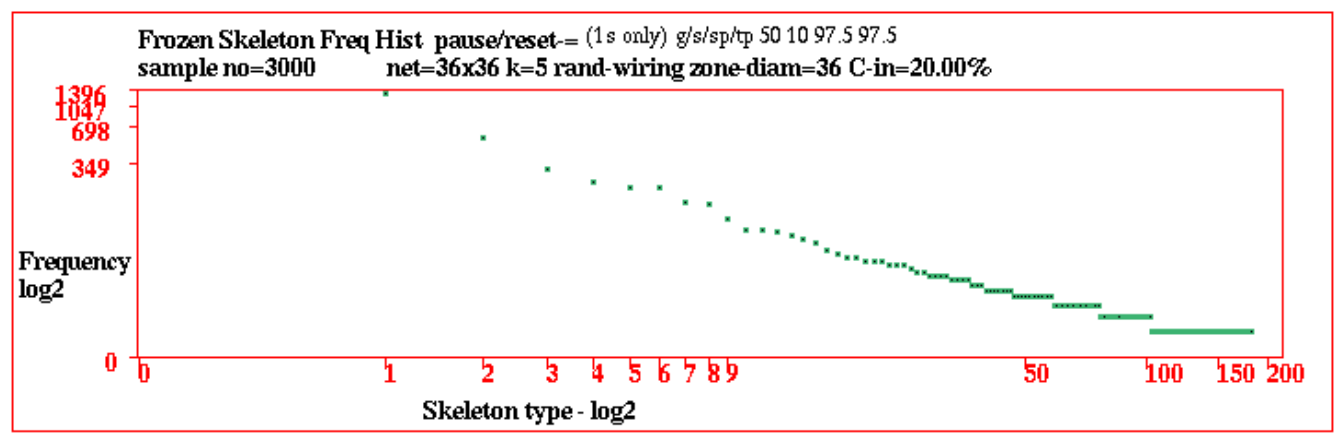

shown as log-log

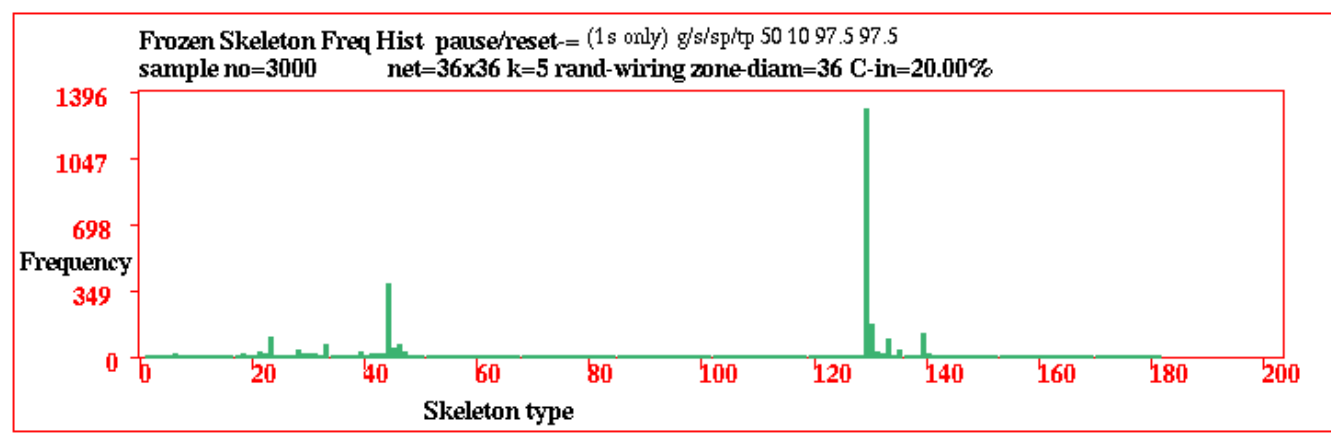

sorted according to average transient

Figure 31.22: The skeleton histogram for the same network as in figure 31.20 but paused at sample 3000 with 181 skeletons found, showning different presentations.

\subsubsection{Skeleton histogram data decode}

The column headings in section 31.7.4 denote the following,

type ... the skeleton type index.

no ... the number of this skeleton type found.

freq .... the frequency of this type.

$\%$ fr- 0 s- 1 s ... the percentage of frozen 0 s and 1s making up the skeleton.

trans ... the average transient (or run-in) length. 


\subsubsection{Sorting the skeleton histogram}

Enter $\mathbf{s}$ in section 31.7.3 to sort the histogram. The following top-right prompt is presented,

\section{sort by: frozen-0-1-b avtrans-t frequency-def:}

Initialy the skeleton types are ranked in the order they were found, as in figure 31.20 . Enter return to sort by frequency. as in in figure 31.22. This corresponds to sorting according to the fraction of state-space "drained" by the skeleton. Enter $\mathbf{t}$ to sort by average transient (run-in) length. The histogram can also be sorted by the number of frozen $0 \mathrm{~s}, 1 \mathrm{~s}$ or both $0 \mathrm{~s}$ and $1 \mathrm{~s}$, enter $\mathbf{0}, \mathbf{1}$ or $\mathbf{b}$. The result of the new sorting depends on how the list is currently sorted. Having first sorted by frequency, the examples in figure 31.22 show the data sorted first by the attractor period, then by the average transient. 


\section{Chapter 32}

\section{Drawing space-time patterns, and changes on-the-fly}

Following the output parameters in chapter 31 , space-time patterns ${ }^{1}$. will start in the top-left of the DDLab screen. This chapter describes parameters and options (including many from chapter 31 ) that can be reset or activated while space-time patterns are running, either immediately on-the-fly by various key hits, or by pausing the drawing and responding to further prompts.

On-the-fly options include changes to updating, rules, wiring, seed, scale, dimensions, presentation, "frozen" regions, filtering, and various methods of analysis. There are also options for loading glider rules, and automatically classifying rule-space (chapter 33).

If space-time patterns are paused/interrupted (section 32.16), pause options include rules (chapter 16), network architecture (chapter 17), vector PostScript (chapter 36), canalyzing (chapter 15), the state (chapter 21), and the network-graph (chapter 20) - to run a concurrent space-time pattern according to the network-graph presentation. On-the-fly options can also be activated during the pause. Many relevant changes and functions in the DDLab manual can therefore be implemented without backtracking.

\subsection{On-the-fly key index}

An "on-the-fly key index" window is usually displayed while space-time patterns are running, as a reminder of the various options which are activated directly by key hits. The window appears on the right of the screen and can be toggled on-off the $\mathbf{X}$ key.

The items listed are context dependent - an example is shown in figure 32.1, and in DDLab screen snapshots in chapters 31, 32 and 33. The options are divided into categories, with subheadings in red, and may vary for different types of network and current settings. These include the dimensions of the underlying network, and STP-dimensions - the dimensions of the spacetime pattern presentation, which are not necessarily the same. On-the-fly options are summarized in section 32.3 and explained in greater detail in sections $32.4-32.13 .6$.

Note that the same options can be activated during a pause (sections 32.16, 32.17).

\footnotetext{
${ }^{1}$ In TFO-mode, running forward to draw space-time patterns is the only possibility, otherwise this needs to be selected, because the default is to draw attractor basins. If not in TFO-mode, the following steps are required: Enter $\mathbf{s}$ in section 6.2 for SEED-mode. Then for "space-time patters only", enter $\mathbf{s}$ in section 24.1, or $\mathbf{S}$ in section 29.1
} 
Figure 32.1: An example of the "on-the-fly key index" for space-time patterns - a reminder of the options which can be activated directly by key hits.

The window appears on the right of the DDLab screen and can be toggled on-off with the $\mathbf{X}$ key.

In TFO-mode the top heading reads

on-the-fly key index: TFO kcode

otherwise the heading in SEED-mode is as shown.

The options are divided into subcategories, with subheadings in red. They are context dependent, and may vary for different types of network, current settings, network dimensions, and STP-dimensions, so some options may be missing or different in an actual run of space-time patterns. This example is for SEED-mode, $v=2$, a $1 \mathrm{~d}$ network with local wiring, presented in 1d (1d-STP), with input-entropy active. Because of this setup, the options below are not included.

change rule

8..rulemix/single (if mixed-rule network)

presentation

@..tog cell outline (if network-graph is active)

d/-..tog shuffle colors/restore (for $v \geq 3$ )

1d $2 \mathrm{~d} 3 \mathrm{~d}$

t..tog $2 \mathrm{~d}-2 \mathrm{~d}+$ time (if $2 d-S T P$ )

$\mathrm{p} /$ I..plane/balls (if $2 d+$ time only)

$\mathrm{p} / \mathrm{I} / \mathrm{J}$..plane/balls/invisible (for 2d-STP scrolling diagonally)

analysis

$=$. tog $\operatorname{diff}(\mathrm{keep})$ (if "damage" is active, section 31.5)

miscellaneous

\$..make sound (for DOS only) on-the-fly key index

updating (tog)

U/Y..sync-seq/sync-porder

$\{/\}$..prob:update1-0.95/output1-0.95

change rule

$\mathrm{r}, \mathrm{R} / \mathrm{K} / \mathrm{k}$..rnd/rnd/tcode/kcode

[/]..iso/togflip(off)

$\mathrm{O} / \mathrm{A} / \mathrm{M} / \mathrm{C}$..Orig/Alt/Maj/Chain

$1 / 2$..rnd bitflip/restore

$\mathrm{Z} / \mathrm{z}$..force $\mathrm{Z}$ higher/lower

b/B..flip allos->0/allVs->V

rule samples

g..load glider rule (rnd)

V..load,jmp,scan

$\mathrm{w} /: / 9$..next/prev/rnd

uE..create sample

change wiring

$\mathrm{m} / \mathrm{W}$..move1 wire/revise

7..nonlocal-local

change seed/size

4/v...rnd seed/block

1/L..rnd value/block flip

$\mathrm{o} /$. .original/last

5/6..singleton pos/neg

$\mathrm{N} / \mathrm{n}$..inc/decrease 1 cell

presentation

x..tog slant(off)

i/!..tog $\operatorname{divs}($ off $) /$ color

$3 / . / \wedge$..tog incolor/dot/bground

d/-..colors:swap/black-blue

S..tog space-time display

P..tog skip steps=1 (off)

e/c..exp/contr scale

1d $2 \mathrm{~d} 3 \mathrm{~d}$

T..tog $1 d-2 d-3 d$

frozen/filter

h..nor-fi-f2-bin

H..f-gens(20)/bins(10)

f/F/a..filter/undo/all

analysis

0/\%..tog lookhist:1-2/1-time

)/(..lookhist: amplify/restore

s..tog entropy-density

j..tog ent-in-both

u..entropy/density plot

G..a-gens (now 10)

D..return map

y..state-space matrix

miscellaneous

$\mathrm{X} . . \operatorname{tog}$ this index

\#/\&.. tog scrolling (on)

+ .. tog time-step pause

$</>$..slower/max speed

q/Q..pause/no-options 


\subsection{On-the-fly prompts — bottom title bar}

The following on-the-fly prompts appear in the bottom title bar (section 5.5) whenever space-time patterns are running - they are also displayed among the prompts in the on-the-fly key index (figure 32.1, section 32.3).

STP:tog/exp/contr-S/e/c, tog index-X, slow/max speed: $</>$

DDLab (C) 1993-2011 advance-ret back/interrupt-q STP:tog/scroll/exp/contr-S/e/c, tog index-X, slow/max- $</>$

The key hits are described below,

on-the-fly key hits... what they means

STP:tog-S... to toggle space-time patterns on and off. If space-time patterns are off they continue to run in the background - this considerably speeds up the "damage", "attractor" and "skeleton" histograms (sections 31.5.2, $31.6,31.7$ ), and "classifying rule space" (chapter 33).

STP: $-\mathbf{e} / \mathbf{c} \ldots$ to expand or contract the scale of space-time patterns.

slow $/ \max :</>\ldots$ slow down with $<$ which halves computation speed each time its hit. Enter $>$ to revert to maximum speed.

\subsection{Summary of on-the-fly options for space-time patterns}

On-the-fly key index options (section 32.1) are summarized below, divided into categories as in figure 32.1. Further details are given in the sections indicated,

updating (tog) - section 32.4

U/Y..sync-seq/sync-porder ... sequential and partial order updating (sections 31.3.2 - 31.3.3).

U ... toggle synchronous-sequential updating (section 32.4.1)

Y ... toggle synchronous-partial order updating (section 32.4.2).

\{/\}..prob:update1-0.95/output1-0.95 ... noisy updating with probability settings (section 31.3.1).

$\{\ldots$ toggle deterministic-update probability (section 32.4 ).

\}... toggle deterministic-output probability (section 32.4). 
change rule - section 32.5

r,R/K/k..rnd rule/tcode/kcode ... new random rule/s — preset density bias ( $\lambda$ parameter for rcode) is respected (section 16.3.1)

$\mathbf{r}$... for a random rule biased according to the $\lambda$ parameter, (section 16.3.1), but with the all-0s neighborhood output set to zero for a stable background.

$\mathbf{R}$... for a random rule according to value-bias section 16.3.2.

K ... (SEED-mode only) random tcode expressed as rcode (t-rcode).

k... (SEED-mode only) random kcode expressed as rcode (k-rcode).

[/]..iso/togflip(on) ... (rcode only) new isotropic rcode (section 32.5.2).

[... set new isotropic rcode.

] ... tog isotropic bit/value flip (on)/(off). If togflip(on) isotropic dynamics is conserved when using on-the-fly $\mathbf{1 / 2 . . b i t f l i p / r e s t o r e ~ ( s e c t i o n ~ 3 2 . 5 . 4 ) , ~}$ whereas togflip(off) does not.

O/A/M/C..Orig/Alt/Maj/Chain ... new rule/s (section 32.5.3).

O ... restore original rule.

A ... random Altenberg rule.

M ... random majority rule.

C ... random chain rule.

1/2..rnd bitflip/restore ... mutate/restore rule/s (section 32.5.4).

1 ... mutate rule-table by 1 random bit.

2 ... restore mutated bits in reverse order.

$\mathbf{Z} /$ z..force $\mathbf{Z}$ higher/lower ... (for single rcode only) change rule's Z-parameter (section 32.5.5).

$\mathbf{Z}$... force $Z$ higher.

z ... force $Z$ lower.

b/B..flip all0s->0/allVs->V ... change the output of uniform neighborhoods (the "hot bits" [19]) to the neighborhood value (section 32.5.6).

b ... flip output of all the all-0s neighborhood to 0 .

B ... flip the output of all uniform neighborhoods to the neighborhood value.

8..rulemix-single ... (mixed rule network only, but not $k$-mix) toggle rulemix/single rule at cell 0 (section 32.5.7).

rule samples - section 32.6

g..load glider rule (rnd) ... load a glider rule from a collection (if available), either randomly (rnd) or sequentially (seq) (section 32.6.1).

V..load,jmp,scan ... load rule sample, select start, and type of scan. (section 32.6.2 and chapter 33). 
w/:/9..next/prev/rnd ... scan rules from an active the sample.

w ... select the next index in the sample.

:... (colon) select the previous index in the sample.

9 ... jump to a random sample index.

$\mathbf{u E . . c r e a t e ~ s a m p l e ~ . . . ~ e n t e r ~} \mathbf{u}$ followed by $\mathbf{E}$ to create a sample of classified rule-space (section 33.2 and chapter 33). $\mathbf{u}$ initiates an entropy/density scatter plot (section 32.12.5) — if this is already current, the on-the-fly prompt is E..create sample.

\section{change wiring - section 32.7}

$\mathbf{m} / \mathbf{W}$..move 1 wires ... move $x$ wires, the current $x$ is shown (section 32.7.1).

$\mathbf{m}$... randomly move (rewire) one or more wires.

W ... change the number of wires to move with $\mathbf{m}$, or set local (CA) wiring, $(1 \mathrm{~d}, 2 \mathrm{~d}$, or $3 \mathrm{~d})$ depending on native network dimensions.

7..nonlocal-local ... toggle nonlocal/local wiring (section 32.7.2). This does not change any original nonlocal wiring. The current status is given by the prompt order, i.e. 7..local-nonlocal indicates that the network is currently being run as if it has local wiring.

change seed/size - section 32.8

4/v..rnd seed/block ... random seed or block (section 32.8.1) — the preset density bias (section 21.3.1) of the seed or block, and the size of the block (section 21.3) are respected.

4 ... random seed.

k... random block.

1/L..rnd value/block flip ... random value or block (section 32.8.2).

1 ... mutate the current state by one random bit/value flip - one cell value randomly changed at a random position.

L . . flip a central block randomly in the current state. The block size (section 21.3) and pre-set density bias (section 21.3.1) are respected.

o/ ..original/last ... restore previous seeds (section 32.8.3).

o ... restore the original seed (chapter 21).

$\sim \ldots$ restore the latest seed that was changed on-the-fly.

5/6..singleton pos/neg ... (for binary $v=2$ ) set a singleton seed, a central cell against a uniform background. (section 32.8.4).

5 ... positive singleton seed, a one against zeros.

6 ... negative singleton seed. a zero against ones. 
5/6..singleton zero/rnd ... (for $v \geq 3$ ) set a singleton seed, a central cell against a uniform background. (section 32.8.5).

5 ... a random value against a uniform background of zeros.

6 ... a random value against a uniform background of a different value.

$\mathbf{N} / \mathbf{n} .$. inc/decrease 1 cell ... (for $1 d$ single-rule networks presented in 1d) change network size (section 32.8.6).

$\mathbf{N}$... increase network size by 1 cell.

n ... decrease network size by 1 cell.

presentation - section 32.9

x..tog slant(off) ... (for 1d) a 3-way toggle to slant time-steps (off)-(on-right)-(on-left) - to make asymmetric space-time patterns appear symmetric (section 32.9.1).

x..tog hex(off) ... (for 2d) toggle between a square and hexagonal lattice (section 32.9.2).

@..tog cell outline ... (if the network-graph layout of STP active, section 32.19) toggle the cell presentation with and without an outline (section 32.9.4).

i/!..tog divs(off)/color ... (for $1 d$ and $2 d S T P$ - takes effect if the cell scale $\geq 4$ ) to change division lines between cells (section 32.9.3).

i ... toggle divisions on-off.

!... toggles division colors black-white.

3/./^..incolor/dot/bground ... for colors by neighborhood, and the presentation of zero cells (section 32.9.5).

3 ... toggles between cell color by value and by neighborhood.

.... (full stop) (for 1d and 2d STP - takes effect if the cell scale $\geq 4$ ) toggles having a dot on zero cells.

$\wedge \ldots$ (for 1d and 2d STP, and value colors) toggles between white and light green for zero cells.

d/-..colors:swap/black-blue ... (for binary, $v=2$ ) to swap or change color (section 32.9.6).

d ... swaps the color of $0 / 1$ cells between white/black.

- ... (minus sign) toggles colors of 1s between black and blue.

d/-..tog shuffle colors/restore ... (for $v \geq 3$ ) to shuffle or restore colors (section 32.9.7).

d ... shuffle the colors at random.

- ... (minus sign) restore the default colors.

S..tog space-time display ... toggle the display of space-time graphics on/off (section 32.9.8).

P..tog skip steps=1 (off) ... toggle skipping time-steps (section 32.9.9).

\$..tog sound ... (DOS only) toggle sound generated by space-time patterns (section 32.9.10). 
e/c..expand/contr scale ... for normal space-time patterns: expand/contract the cell scale. For diagonally scrolling network-graph space-time patterns (figures 32.14, 32.16): expand/contract the spacing between time-steps (section 32.9.11).

e... expand cell scale (or the spacing) by 1 pixel.

c ... contract cell scale (or the spacing) by 1 pixel.

1d 2d 3d- section 32.10

T..tog 1d-2d-3d ... a 3-way toggle between 1d-2d-3d STP - the order changes to show which is current and which is next in the toggle sequence, e.g. T..tog 2d-3d-1d. (section 32.10.1).

t..tog $\mathbf{2 d - 2 d + t i m e ~ . . . ~ ( f o r ~} 2 d S T P$ ) toggle between $2 \mathrm{~d}$ and $2 \mathrm{~d}+$ time the order changes to show which is current, e.g. t..tog 2d+time-2d (section 32.10.2).

I..tog balls ... (for $3 d S T P$, 2d+time) toggle between balls and parallelograms (section 32.10.3).

p/I..plane/balls ... (for 2d+time STP)

p ... draw 2d plane - parallelogram grid (section 32.10.4).

I ... toggle cells between balls and parallelograms.

p/I/J..plane/balls/invisible ... (for 2d STP diagonal scrolling)

p ... draw 2d plane - square or hex grid (section 32.10.4).

I ... toggle cells between balls and squares or hexagons (section 32.10.3).

J ... toggle the visibility of $2 \mathrm{~d}$ diagonal scrolling STP to see just the scrolling network-graph (sections 32.10.5, 32.13.3.3, figure 32.14).

frozen/filter - section 32.11 (if input-entropy is active) filter (otherwise)

h..nor-f1-f2-bin ... 4-way toggle showing "frozen" cells in 3 different ways (section 32.11.1).

H..f-gens (now 20)/bins(10) ... change "frozen" parameters (section 32.11.2).

f/F/a..filter/undo/all ... (if input-entropy active) (section 32.11.5)

f ... progressively filter.

F ... progressively unfilter in reverse order.

a ... restore all filtering.

analysis - section 32.12

s..tog entropy-density ... (if input-entropy is active) toggle input-entropy/pattern density (section 32.12.3). 
0/\%..tog lookuphist:1-2/1-time ... (if input-entropy is active - section 32.12.1) the presentation of the input-frequency histogram.

0 ... ((zero key) toggle between normal and 1 pixel width bars.

$\%$... show with the histogram with a time dimension - if the number of bars $\leq 64$.

)/(..lookhist: amplify/restore ... (if input-entropy is active section 32.12.3) amplify the input-frequency histogram.

) ... double the length of bars each time ")" is pressed.

( ... restore the default length.

s..tog entropy-density ... toggle showing the input-entropy or pattern densities (as a set of $v$ value-density graphs) instead of the input-entropy (section 32.12.3).

j..tog ent-in-both ... (if input-entropy is active section 32.12.3) a 3 -way toggle to show the input-entropy, the input-frequencies as a set of graphs, or both together (section 32.12.4) (section 32.12.5).

u..entropy/density plot ... (if input-entropy is active section 32.12.3) toggle showing a scatter plot of entropy-density where rules have characteristic signatures. (figure 32.32, section 32.12.5).

G..a-gens (now 10) ... change analysis generations (section 32.12.6).

D..return map ... toggle the return map (section 32.12.7).

y..state-space matrix ... toggle state-space matrix (section 32.12.8).

=..tog diff (keep damage) ... (if the "damage" is active, section 31.5), toggle between two definitions of damage, (keep) - once damaged, keep the damage, and (inst) - the instantaneous difference at each time-step.

miscellaneous - section 32.13

X..tog this index ... toggle the on-the-fly key index (section 32.13.1).

*..tog end pause (on) ... toggle the end pause on/off (section 32.13.2).

\#/\&..tog scrolling ... toggle the scrolling on/off with either \# or \& (section 32.13.3).

+..tog time-step pause ... pause after each time-step (section 32.13.4).

$</>$..slow/max speed ... speed (section 32.13.5).

$<\ldots$ slow down the space-time patterns.

$>$... restore full speed.

q/Q..pause/no-options ... (section 32.13.6).

q ... pause space-time patterns and display data/options.

Q ... turn off data/options when pausing. 


\subsection{Updating (tog)}

These on-the-fly options toggle between synchronous and either sequential or partial order updating, and between deterministic and two kinds of noisy updating. Any combination of settings is permissible.

\subsubsection{U/Y..sync-seq/sync-porder}

The current status of sequential and partial order updating, as opposed to synchronous, is given by the prompt order - [sync-seq or seq-sync] and [sync-porder or porder-sync].

The following on-the-fly key hits toggle the current status,

U ... toggles between synchronous and sequential updating (defined in section 31.3.2).

Y . . toggles between synchronous and partial order updating (defined in section 31.3.3).

\subsection{2 \{/\}..prob:update1-0.95/output1-0.95}

The values of the update and output probabilities (section 31.3.1) are shown in the prompt. The current status is given by the prompt order, so update0.95-1 would mean update probability (0.95) is active.

$\{$...to toggle between deterministic updating and update probability.

\} ... to toggle between deterministic updating and output probability.

\subsection{Change rule}

The following on-the-fly key hits change the current rule, including random rules with or without special biases, and mutations to the current rule by bit/value flips - which can be restored. A top-right window with the appropriate label (figure 32.2) shows the new rule. For mixed-rule networks, the random rules or mutations apply to all the cells/rules in the network, but the top-right information will show the rule at network index zero only.
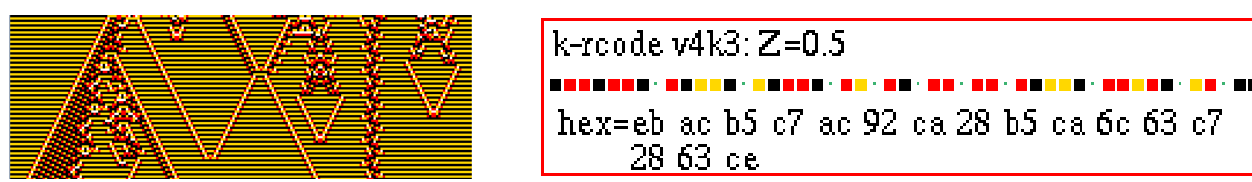

Figure 32.2: Setting a random k-rcode on-the-fly with key $k$, showing Left: about 70 time-steps of the $1 \mathrm{~d} n=200$ space-time pattern, and Right: information on the new rule that appears in a top-right window when the key is hit. The label "k-rcode" indicates this is kcode expressed as rcode. If not in TFO-mode, the $Z$-parameter is included. The rule-table is shown as a value-string and in hex, as much as will fit in both cases. The same format is applied for other rule types. Other possible labels are: rcode, tcode, kcode, k-rcode, t-rcode, iso rcode, Alt rcode, Alt kcode, Maj rcode, Maj kcode, Maj tcode, or chain rcode - as noted in sections 32.5.1 - 32.5.3. 


\subsection{1 r,R/K/k..rnd rule/tcode/kcode}

Set a new random rule (or rules for a rulemix) where the density bias set in section 16.3.1 is respected. Information about the new rule appears in a top-right window similar to figure 32.2, with possible labels: rcode, tcode, kcode, k-rcode, or t-rcode.

$\mathbf{r}$... for a random rule biased according to the $\lambda$ parameter, (section 16.3.1), but with the all-0s neighborhood output set to zero for a stable background.

$\mathbf{R}$... for a random rule according to value-bias section 16.3.2.

$\mathbf{K}$... (if not in TFO-mode) random tcode expressed as rcode (t-rcode).

$\mathbf{k}$... (if not in TFO-mode) random kcode expressed as rcode (k-rcode).

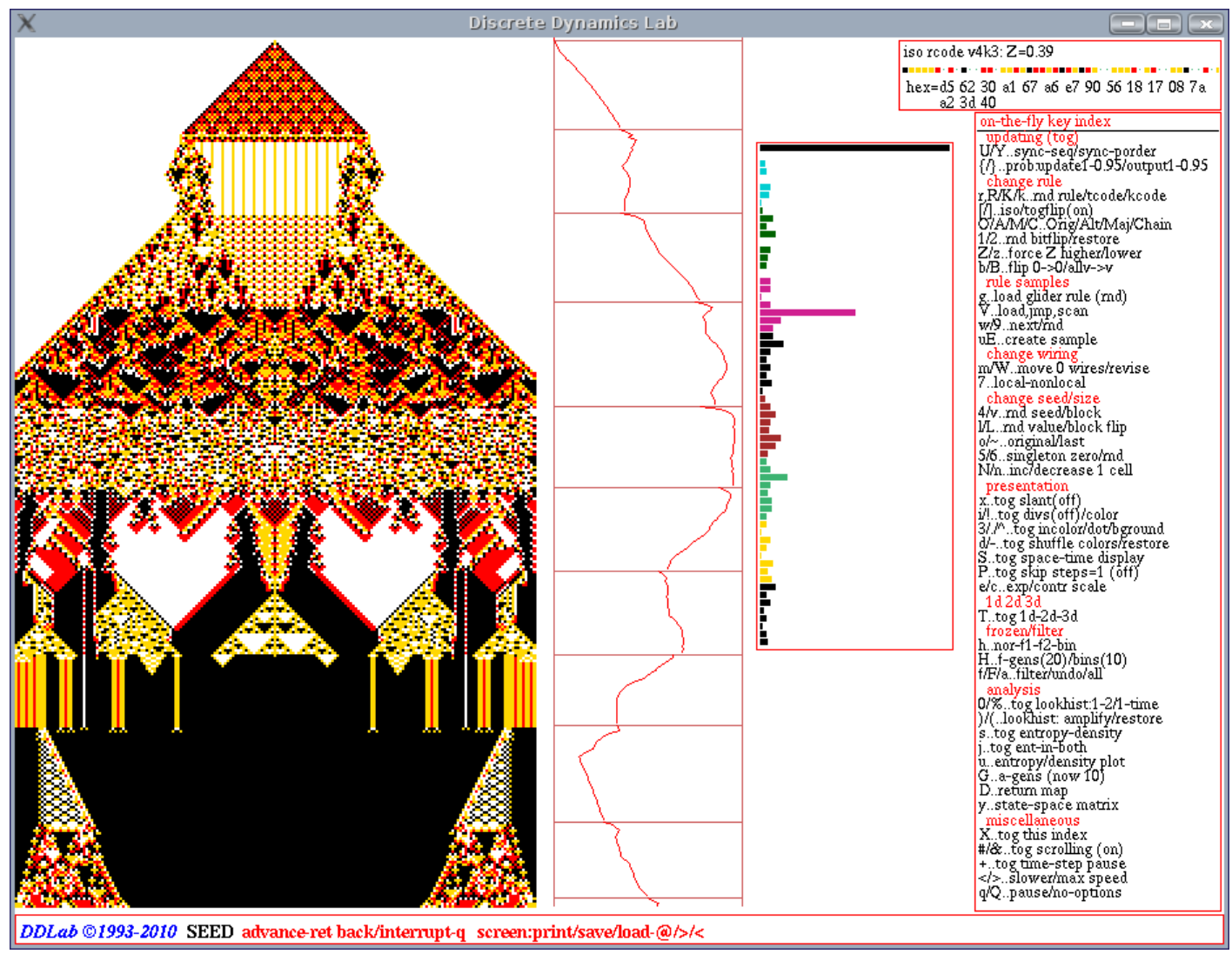

Figure 32.3: A snapshot of the DDLab screen, showing a $1 \mathrm{~d} n=200$ space-time pattern made up of a series of $v 4 k 3$ random isotropic rcodes, where reflected neighborhoods have the same output, set on-the-fly with key [ (left square bracket). Note that the symmetry is preserved. The start of each new rule, which picks up the current state from the previous rule, is indicated by a horizontal line on the entropy plot to the right of the space-time pattern. The last rule set is shown in a top-right window. 


\subsection{2 [/]..iso/togflip(on)}

(not in TFO-mode because totalistic rules are already isotropic)

Set a new random isotropic rcode (or rcodes for a rulemix) where rotated and reflected neighborhoods (in $1 \mathrm{~d}, 2 \mathrm{~d}$ and $3 \mathrm{~d}$ ) have the same output (figure 32.3. Information about a new single rule appears in a top-right window similar to figure 32.2 , labelled "iso rcode".

[ ... (the left square bracket) sets a new random isotropic rcode (iso rcode).

] ... (the right square bracket) toggles between (on) and (off) for isotropic mutation. "togflip(on)" in the on-the-fly key index (figure 32.1) indicates that the "bitflip/restore" on-the-fly function (section 32.5.4) will also flip/restore other relevant bits/values to preserve isotropic rcode. "togflip(off)" indicates a normal single flip, which does not preserve isotropic rcode.

\subsubsection{O/A/M/C..Orig/Alt/Maj/Chain}

Reset the original rule, or set a new random rule (or rules for a rulemix) with special biases. Information about the new rule appears in a top-right window similar to figure 32.2, with possible labels: Orig rcode, Orig kcode, Orig tcode, Alt rcode, Alt tcode, Maj rcode, Maj kcode, Maj tcode, or chain rcode.

O ...to restore the rule that was originally selected (or reset) in chapter 16. For mixed-rule networks the rule is assigned to cell index 0 only (Orig rcode, Orig kcode, Orig tcode).

A ... (not for tcode) set a random Altenberg rule (Alt rcode, Alt tcode) (section 16.9).

M ... set a random majority rule (Maj rcode, Maj kcode, Maj tcode) (section 16.7).

C ... (not in TFO-mode) set random chain rule (chain rcode) (section 16.11).

\subsubsection{1/2..rnd bitflip/restore}

Enter 1 on-the-fly to mutate the rule-table by a random by one bit/value flip, or $\mathbf{2}$ to flip back (repeatedly) to restore the mutation. For isotropic rcode (section 32.5.2), if togflip(on), both flip and flipback conserve isotropic dynamics by also changing other relevant bits/values.

For a rulemix the function only applies to the rule at network index zero. Information about the rule appears in a top-right window (figure 32.4).

kcode $v 4 k 3:$ flip 98, pos 16
hex $=5$ d 9 c 699738

kcode $v 4 k 3:$ flipback 97, pos 16
hex $=5 f 9 c 699738$

Figure 32.4: Mutating on-the-fly by a random bit/value flip with key $\mathbf{1}$, restore (flipback) with key 2 - both can be done repeatedly so after multiple flips the original rule can be restored with multiple flipbacks. A top-right window gives information in both cases - this example is for $v 4 k 3 \mathrm{kcode}$, Left: mutation - showing the flip number and its position in the rule-table. Right: undoing the mutation - showing the flipback number and its position in the rule-table. 


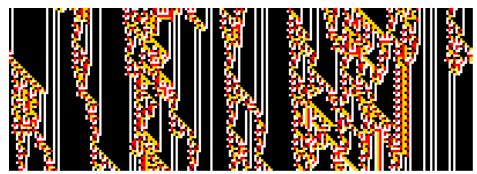

rcode $v 4 \mathrm{k} 3: \mathrm{zl}=0.31 \mathrm{zr}=0.44$

hex $=c 3$ bb 0.532451 a 4400528 e $7 f$ d8 eb

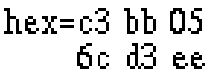

Figure 32.5: Info when changing the $Z$-parameter up and down on-the-fly with keys $Z$ and $z$. Initial dynamics with high $Z$ was progressively changed towards order by repeated hits with key $z$ - ordered domains emerged. Left: about 70 time-steps of the $1 \mathrm{~d} n=200$ space-time pattern, and Right: information on the new rule that appears in a top-right window when the key is hit, where $\mathrm{zl}$ is $\overline{z_{\text {left }}}$ and $\mathrm{zr}$ is $z_{\text {right }}-$ the $Z$-parameter is the greater of the two[25, 42].

\subsubsection{Z/z..force $\mathrm{Z}$ higher/lower}

for single rcode only

The current rule can be adapted by tuning the $Z$-parameter, progressively forcing $Z$ higher (towards chaos) or lower (towards order), by selectively mutating the rcode. This is done onthe-fly with the following key hits,

\section{$\mathbf{Z}$... (upper case) to force $Z$ higher.}

z ... (lower case) to force $Z$ lower.

Information about the rule appears in a top-right window (figure 32.5). The algorithm is the same as in section 16.3 - flipping bits/values at random positions, and only retaining the flips that produce the desired change in $Z$. The algorithm for forcing $Z$ lower can get stuck, but can usually be unstuck with a random bit/value flip (section 32.5.4).

\subsection{6 b/B..flip all0s- $>0 /$ allVs- $>V$}

The "hot bits" in a $v=2$ rule-table, the outputs of the all- 0 s and all-1s neighborhoods, are especially important in the dynamics[19]. This notion is extended for $v \geq 3$, and the following on-the-fly key hits flip all-0s to 0 , or flip the outputs of all uniform neighborhoods to the neighborhood value, which provides a stable background for singleton seeds (section 32.8.4).

b ... flip output of all-0s to 0 .

B ... flip the output of all uniform neighborhoods to the neighborhood value.

Information about the new rule appears in a top-right window similar to figure 32.2 , with possible labels: rcode, kcode, tcode. All the rules in a rulemix are modified.

\subsubsection{8..rulemix-single}

mixed rule network only, but not $k$-mix

Enter 8 to toggle on-the-fly between running the network according to the its rulemix, or according to the single rule at cell index 0 . The current status is given by the prompt order, i.e. 8..single-rulemix indicates that the single rule is current. 


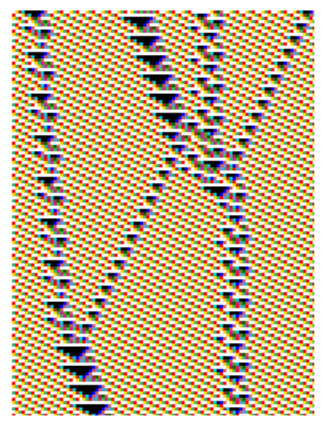

a) $e 0897801$

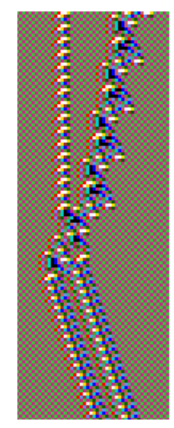

b) $7 e 8696 \mathrm{de}$

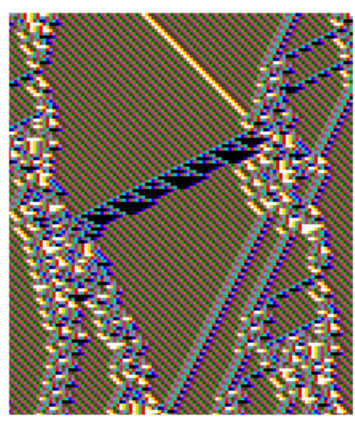

c) $\operatorname{ad} 9 \mathrm{c} 7232$

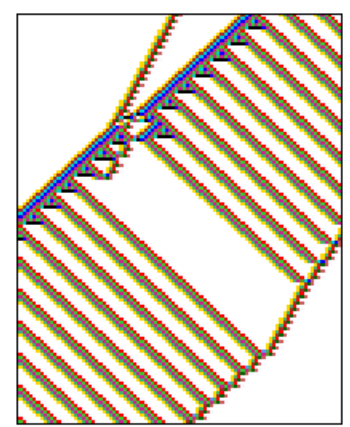

e) $1 \mathrm{c2a} 4798$

Figure 32.6: Examples of $v 2 k 5$ complex space-time patterns with interacting gliders[27] from a sample of glider rules (g_v2k5.r_s) included with DDLab, which can be loaded on-the-fly while space-time patterns are being drawn (cells colored by neighborhood, section 31.2.1). The rcodes are shown in hex.

\subsection{Rule samples}

These on-the-fly options relate to glider rule collections (section 32.6.1), and to automatically classifying rule-space[32] (sections 32.6.2 - 32.6.4), including creating, sorting, analysing and probing the sample. Automatically classifying rule-space is a larger topic described and illustrated in detail in "Classifying Rule Space" chapter 33.

\subsection{1 g..load glider rule (rnd)}

if a rcode or kcode glider file is detected

Collections of complex (or "glider") rules are available for some combinations of $[v, k]$ and lattice dimensions $^{2}$. The available files are listed in section 3.7.1. If the current $[v, k]$ corresponds to one of these files, and if the directory/file is accessible (section 35.5), on-the-fly key g loads a rule from the collection while space-time patterns are running (figure 32.8), either randomly (rnd) or in sequence (seq), depending on the setup in section 31.2.8 - this can also be togged from the pause prompt (section 32.16). hitting on-the-fly key $\mathbf{g}$ loads a rule, and also Rule information is simultaneously shown in a top-right window, including the collection size and sample number, in a top-right window (figure 32.9).

If a rulemix is active (but not a $k$-mix) the rule is loaded at cell index 0 - this can be applied to the whole network by toggling with key 8..rulemix-single (section 32.5.7).

\subsubsection{1 creating a glider rule collection}

Glider rule collections are ordinary "rulemix-only" files (sections 19.4, 19.2, 19.3) but with a special type of filename, where $x$ is the value-range, and $y$ the neighborhood size.

g_v $x$ k $y$.r_s ... for rcode, for example g_v3k3.r_s

g_v $x \mathrm{k} y . r_{-} \mathrm{v} \ldots$ for kcode, for example g_v3k6.r_v

\footnotetext{
${ }^{2}$ Although the complex rules relate to specific dimensions, they often result in interesting dynamics in other than the intended dimensions.
} 


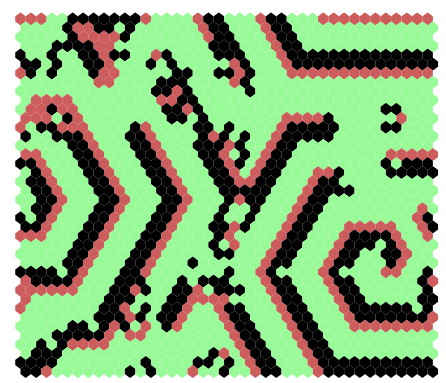

4: 0002000220022001112200021110

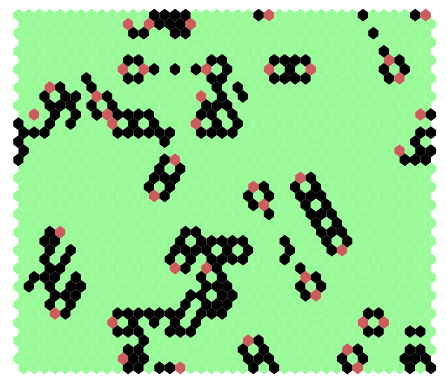

31: 0122220200020200000200120210

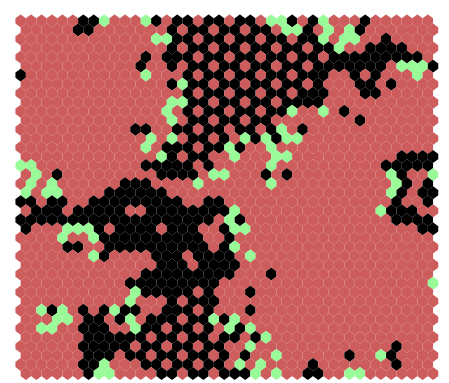

9: 1202022101201121110121112101

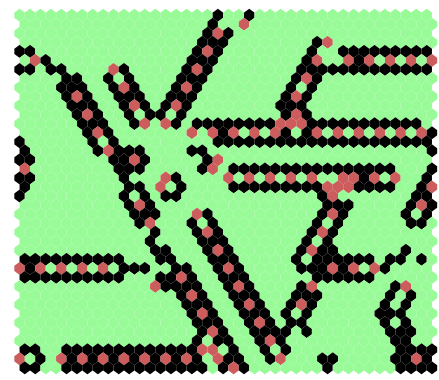

32: 0121220020210200110200021210

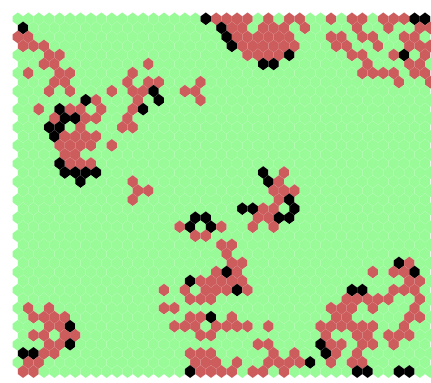

16: 0001001121001121111200001000

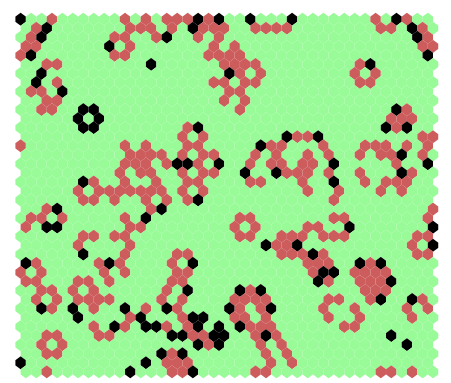

54: 1202022101201121110121110101

Figure 32.7: Examples of $2 \mathrm{~d}$ space-time snapshots of complex kcode on a $2 \mathrm{~d} 40 \times 40$ lattice, from a sample of glider rules (g_v2k6.r_v) included with DDLab, which can be loaded on-the-fly. The sample number and kcode table are shown for each rule. The color of zero (background) values were toggled to light-green with on-the-fly key $\wedge$ (circumflex).

The special name is recognized by DDLab. An available file for the current $[v, k]$ is automatically loaded after the neighborhood $k$ is set in chapter 9, also revealing the number of rules in the collection,. If a collection is not available for the current $[v, k]$, on-the-fly key $\mathbf{g}$ (section 32.6) will have no effect. New collections can be created, extended and modified by loading single rule files into a rulemix-only file, or combining two rulemix-only files ${ }^{3}$. This is best done in a $1 \mathrm{~d}$ wiring graphic (section 19.4).

\subsubsection{V..load, jmp, scan}

Automatically classified samples of rule-space (chapter 33), sorted by input-entropy, and its variability[32] (either standard deviation or a min-max measure (section 33.1), can separate ordered, complex and chaotic rules and show their distribution in rule-space.

Some samples files are available for various combinations of $[v, k]$ and lattice dimensions, listed in section 3.7.1. The files have the extension .sta. If a sample is not currently active, on-the-fly key $\mathbf{V}$ will load, then display, the sample (section 33.5). The sample can also be loaded during a pause (section 32.16.2). If a sample is currently active (i.e.loaded), entering on-the-fly key $\mathbf{V}$ results in the following top-right prompt,

rule sample: load new-n, rule index/scan 1-15834: (kcode file v3k6bs.sta)

\footnotetext{
${ }^{3}$ The rule at index 0 in a glider file is not referenced - this needs to be taken into account when combining two rulemix-only files to make a larger glider file.
} 


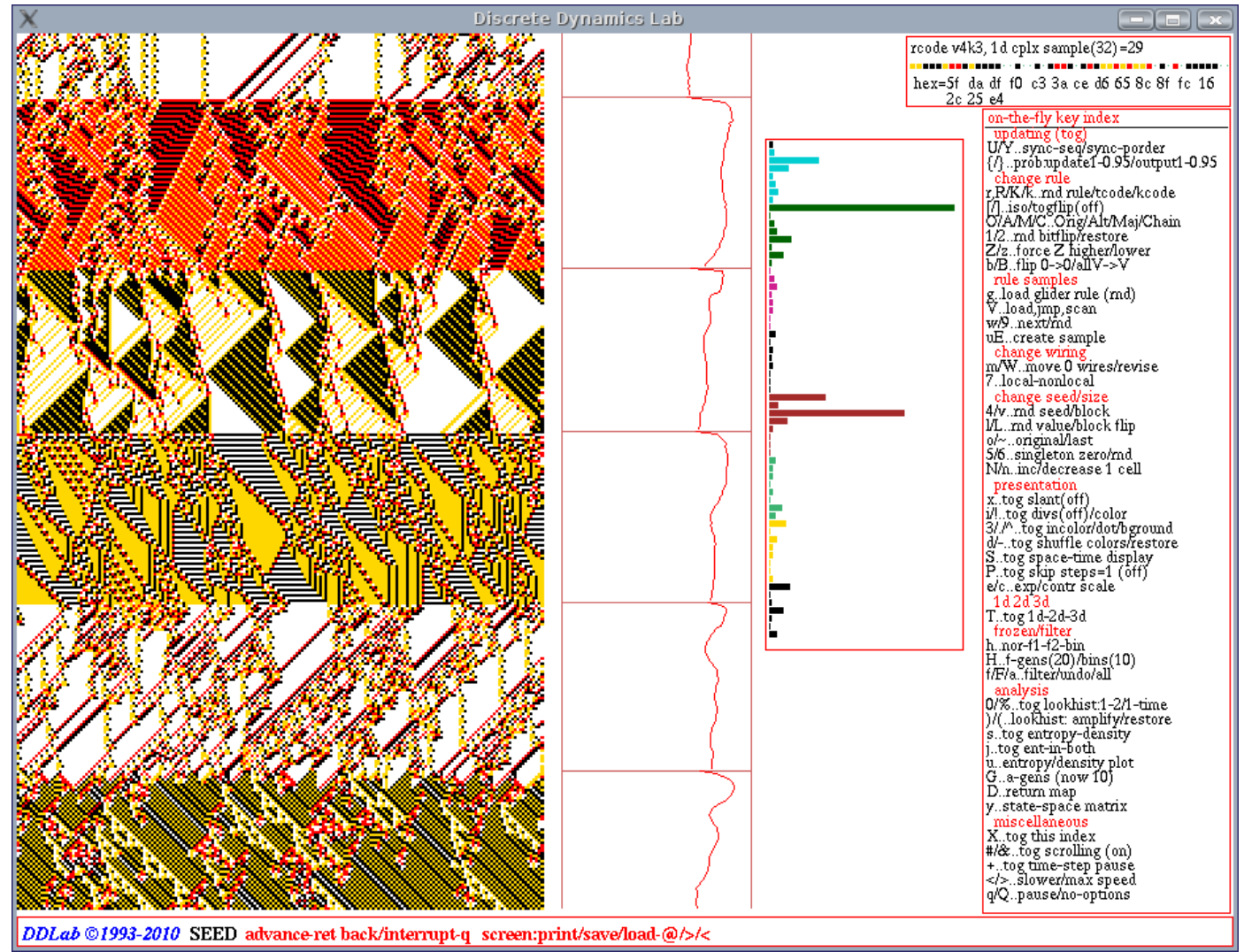

Figure 32.8: A snapshot of the DDLab screen, showing $1 \mathrm{~d} n=200$ space-time patterns, a series of "glider" rules from the $v 4 k 3$ complex rcode collection loaded on-the-fly with key $\mathrm{g}$. The start of each new rule, which preserves the current state, is indicated by a horizontal line on the entropy plot to the right of the space-time pattern. The last rule set is shown in a top-right window, including its sample number.
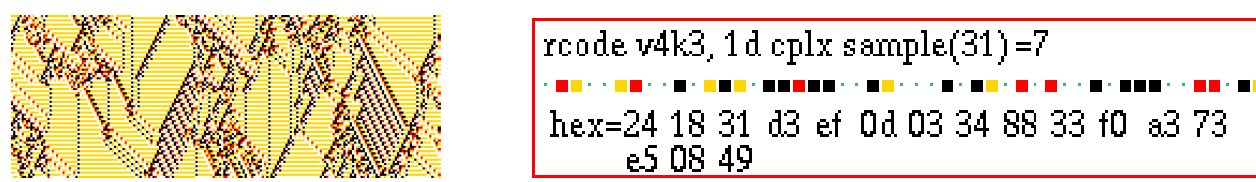

Figure 32.9: Info when loading a complex rule on-the-fly with key $g$. Left: about 70 time-steps of the $1 \mathrm{~d} n=200$ space-time pattern, and Right: information on the new rule that appears in a top-right window when the key is hit. " $1 \mathrm{~d}$ cplx sample $(31)=7$ " means that rule number 7 from 31 rules in the $v 4 k 3$ complex rcode collection is currently selected.

Enter $\mathbf{n}$ for a new sample, otherwise the space-time patterns of successive rules can be scanned, from rule index 1 (the default, enter return) or from any rule index (enter a number) - described in sections 33.7, 33.7.2. For further details see chapter 33. 


\subsection{3 w/:/9..next/prev/rnd}

Once a rule sample is active (i.e. loaded, section 32.6.2 above), the sample can be scanned with the following key hits, to select and immediately apply individual rules,

w ...for the next rule index in the sample, starting with index 1 or another index set in section 32.6.2.

: ....(colon) for the previous index.

9 ... to jump to a random sample index.

Section 33.7 and 33.7.2 give further explanations. Each time a rule is changed, a top-right window shows the sample index, the rule's mean-entropy and variability (standard-deviation or min-max), and the rule itself in hex, as in figure 33.15. For further details see chapter 33.

\subsection{4 uE..create sample}

Enter $\mathbf{u}$ followed by $\mathbf{E}$ to create a sample of automatically classified rule-space. Prompts are presented as described in sections 33.2. The initial $\mathbf{u}$ initiates an entropy/density scatter plot (section 32.12.5). If this plot is current, the on-the-fly prompt is E..create sample, so enter just E to create a sample. For further details see chapter 33.

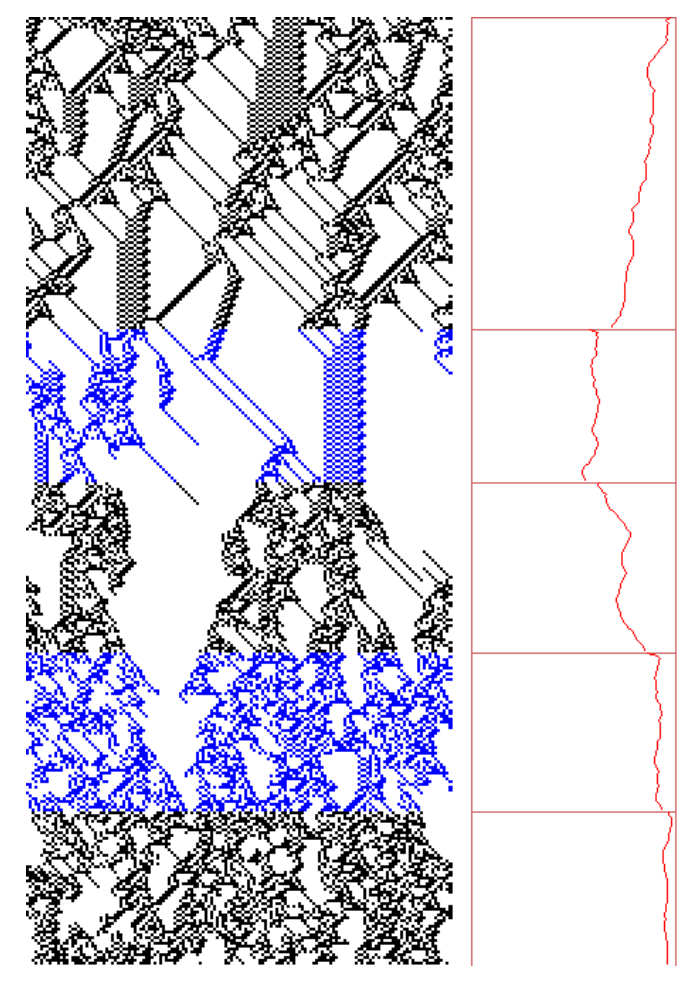

Figure 32.10: Randomizing the wiring of a $1 \mathrm{~d}$ CA by stages, for $v 2 k 5$ rcode(hex)6c1e53a8, $n=150$. The wire moves were first revised to $2 \%$ (15 out of 750 wires) with on-the-fly option $\mathbf{W}$, then the wiring was cumulatively randomized with on-the-fly with option $\mathbf{m}$ (section 32.7.1), at the points indicated by color changes, and horizontal lines in the input-entropy plot. Note that glider structure is progressively degraded. 


\subsection{Change wiring}

These on-the-fly options either change (mutate) the wiring (section 32.7.1), or toggle between local and nonlocal dynamics in a nonlocal network (section 32.7.2).

\subsection{1 $\mathrm{m} / \mathrm{W}$..move 1 wires}

Enter $\mathbf{m}$ to randomly move one wire or a preset number of wires (selected at random), which can be repeated for cumulative rewiring (figure 32.6.4).

Enter $\mathbf{W}$ to reset the number of wires to be randomly moved with $\mathbf{m}$, or to localize the wiring. Prompts similar to "mutate wiring" (section 28.2) will be presented in a top-right window, for example,

wires to move $=1 / 9600=0.0104 \%$ : all-a number-n \%-p:

local-L: (for a $2 d 40 \times 40 k=6$ network)

options ... what they mean

all-a ... to select all wires, i.e. randomly rewiring the whole network when $\mathbf{m}$ is pressed.

number-n ... to reset a given number of wires to move, selected with the following subsequent prompt, ... number of wires: - the new setting will show up in the on-thefly prompt, for example,

m/W..move 25 wires

\%-p ... to specify the percentage of total wires to move, selected with the following subsequent prompt, ... \% of total wires:

local-L ... to restore local $(\mathrm{CA})$ wiring $(1 \mathrm{~d}, 2 \mathrm{~d}$ or $3 \mathrm{~d})$ depending on native network dimensions. For mixed- $k$ each cell is assigned the relevant local neighborhood.

\subsubsection{7..nonlocal-local}

Enter 7 on-the-fly to toggle between running a network with nonlocal wiring, and running the network as if the wiring was local $(1 \mathrm{~d}, 2 \mathrm{~d}$ or $3 \mathrm{~d})$ depending on native network dimensions - where local (CA) wiring is defined in chapter 10.

The original nonlocal wiring held in memory remains intact when local wiring is toggled. The current status is given by the prompt order, i.e. 7..local-nonlocal indicates that the network is currently being run as if it has local wiring. If the wiring held in memory is itself local, there will be no difference between local and nonlocal. However, wire moves that perturb local wiring with $\mathbf{m}$ in section 32.7 .1 will only show up if nonlocal wiring is toggled.

For mixed- $k$ with random wiring, toggling to local wiring treats each cell as having the relevant local neighborhood. 


\subsection{Change seed/size}

These on-the-fly options amend (mutate) the current state or seed, and also (for $1 \mathrm{~d}$ networks displayed in 1d) the of size of the network .

\subsubsection{4/v..rnd seed/block}

When setting a random seed or block on-the-fly, the preset density biases (section 21.3.1), and the size of the block (section 21.3) are respected. Enter hey hits as follows,

$4 \ldots$ to reset the current network state at random.

$\mathbf{v}$... to reset the network state as a central random block (in $1 \mathrm{~d}, 2 \mathrm{~d}$ or $3 \mathrm{~d}$ ). The size of the block was set in section 21.3.

\subsection{2 l/L..rnd value/block}

To mutate or perturb the current state, enter on-the-fly key hits as follows,

$1 \ldots$ to flip/change one bit/value randomly at a random position.

L ... to flip a central block randomly in the current state. The block size (section 21.3) and pre-set density bias (section 21.3.1) are respected.
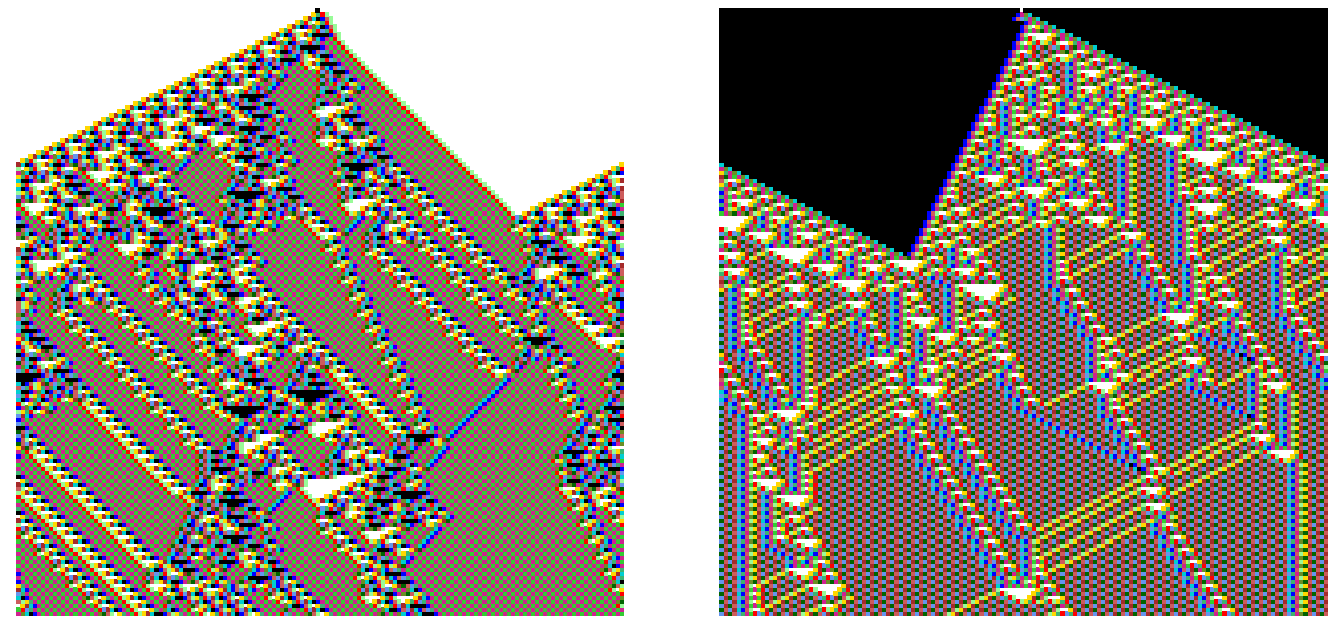

Figure 32.11: Left: a positive singleton seed, a central 1 surrounded by 0 s, and Right: a negative singleton seed, a central 0 surrounded by 1 s, set on-the-fly with key hits $\mathbf{5}$ and $\mathbf{6}$ (section 32.8.4). Left: $v 2 k 5$ rcode(hex) 968c4f76, Right: $v 2 k 5$ rcode(hex) rule d28f022c. $n=150,150$ about time-steps,

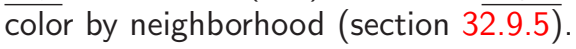




\subsection{3 o/ ..original/last}

To restore previous seeds/states, enter on-the-fly hits as follows,

o ... to restore the original seed (chapter 21).

$\sim$.. to restore the latest seed that was changed on-the-fly by any method in sections 32.8 .

\subsubsection{5/6..singleton pos/neg}

for binary $v=2$

Enter 5 or $\mathbf{6}$ on-the-fly to reset the seed as a positive or negative singleton seed, a central 1 surrounded by 0 s, or a central 0 surrounded by 1 s (figure 32.11 ).

\subsubsection{5/6..singleton zero/rnd}

for $v \geq 3$

Enter $\mathbf{5}$ or $\mathbf{6}$ on-the-fly to reset the seed as a singleton seed, $\mathbf{5}$ for a random value against a uniform background of zeros, $\mathbf{5}$ for a random value against a uniform background of a different value.

\subsubsection{N/n..inc/decrease 1 cell}

only for $1 d$ networks shown in $1 d$

Enter $\mathbf{N}$ or $\mathbf{n}$ on-the-fly to increase or decrease the size of the network by 1 cell. The original network is preserved as far as possible. The extra cell is assigned local wiring for networks that were originally defined as local, otherwise random wiring is assigned. For a mixed rule, homogeneous- $k$, network, one of the rules from the current set is assigned to the extra cell. For a mixed- $k$ network, a random $k$ in the current range is assigned, and a random rule. The change in size is indicated in a top-right window, for example,

$\mathrm{n}$ increased $166->167$ or $\mathrm{n}$ decreased $167->166$.

\subsection{Presentation}

These on-the-fly options amend various aspects of space-time pattern the presentation, including color and scale.

\subsection{1 x..tog slant(off)}

for $1 d$ networks only, shown in $1 d$

Enter $\mathbf{x}$ for a 3-way toggle to slant time-steps (off)-(on-right)-(on-left) — shown in the on-the-fly index. This can make asymmetric space-time patterns appear symmetric. If the slant is on, successive time-steps are shifted by half a cell-width either right or left as in figure $32.12^{4}$.

\footnotetext{
${ }^{4}$ For the vector PostScript image of slanted space-time patterns, an odd number of time-steps should be selected to avoid distortion.
} 


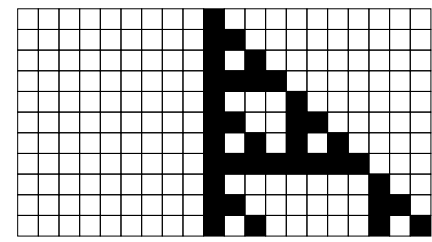

slant(off)

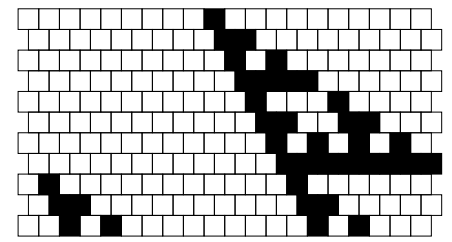

slant(on-right)

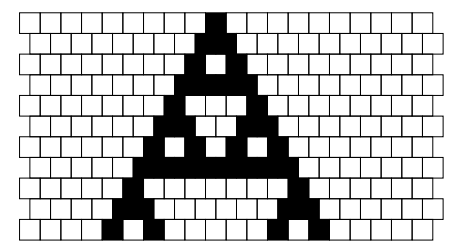

slant(on-left)

Figure 32.12: Slanting 1d space-time patterns with key $\mathbf{x}$, a 3-way toggle (section 32.9.1). If the slant is on, successive time-steps are shifted by half a cell-width either right or left. In this example, left slant produces symmetry - for the elementary CA $v 2 k 3$ rcode(dec) 60 .

\subsection{2 x..tog hex(off)}

for $2 d$ networks only, and $2 d S T P$

Enter $\mathbf{x}$ on-the-fly to toggle between a square or hexagonal lattice (figure 32.13). An even number of rows is required for a hexagonal lattice to avoid an inconsistent presentation. The on-the-fly index gives the current status: hex(off) or hex(on).
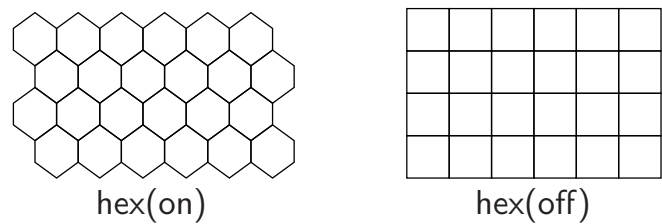

Figure 32.13: Toggling between a hex or square $2 \mathrm{~d}$ lattice with key $\mathbf{x}$ (section 32.9.2). A proper hex presentation requires an even number of rows

\subsection{3 i/!.tog divs(off)}

for $1 d$ and $2 d S T P$ - takes effect if the cell scale $\geq 4$

To set division lines between cells, or change division line color, enter on-the-fly key hits as follows,

i ... to toggle divisions on-off. The on-the-fly index gives the current status $\operatorname{divs}($ off $)$ or $\operatorname{divs}($ on).

! ... to toggle division colors between black and white.

\subsection{4 @..tog cell outline}

if the network-graph layout of STP is active, section 32.19

Enter @ on-the-fly to toggle the cell presentation with and without an outline, giving a different appearance to network-graph layout space-time patterns (figure 32.14)

\subsection{5 $3 / . / \wedge$..incolor/dot/bground}

On-the-fly key hits for cell colors by neighborhood, and the presentation of zero (background) cells, are as follows, 


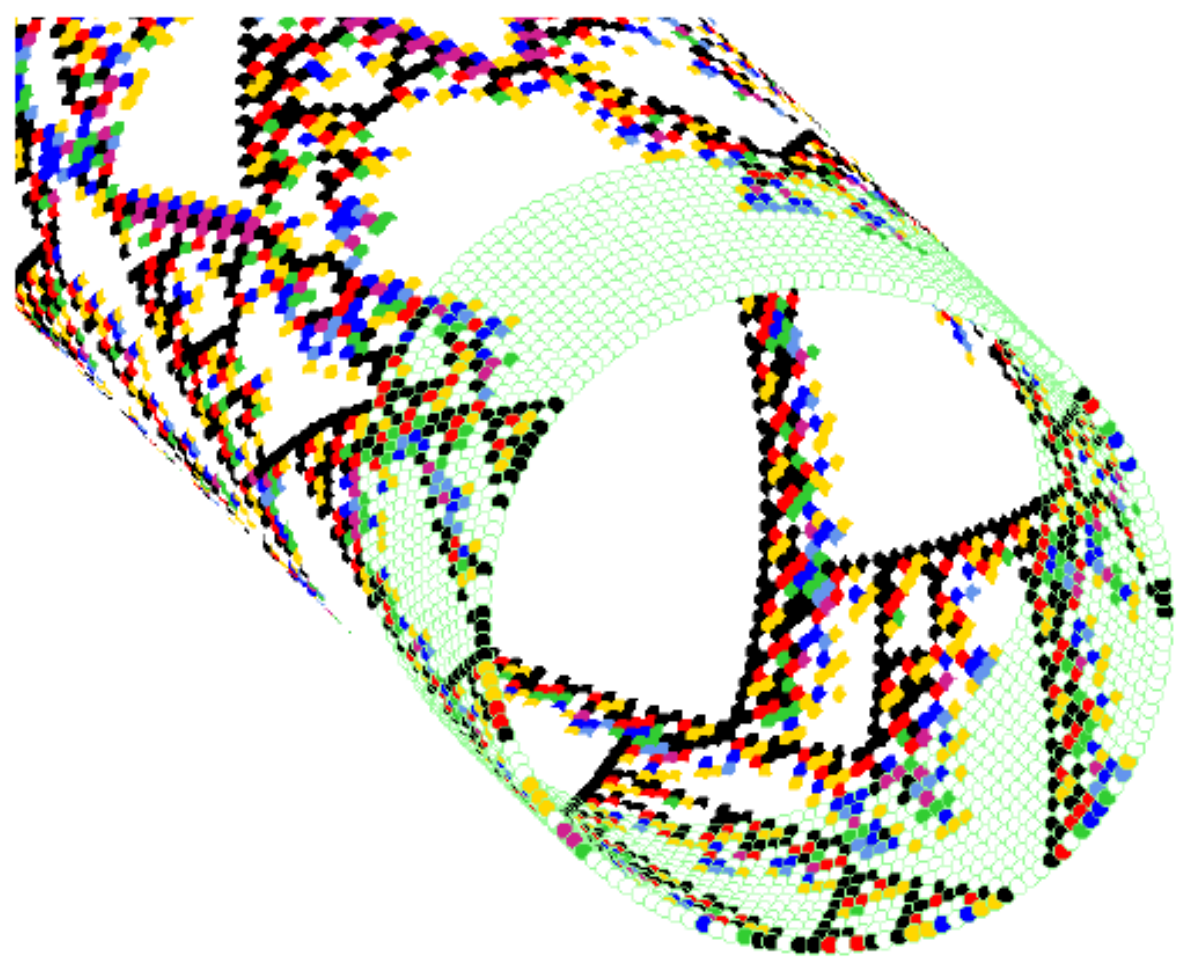

Figure 32.14: Toggling the cell outline in network-graph space-time-patterns (section 20.13) with onthe-fly key @. This example is a 1d (scrolling) ring of cells (as figure 4.10). The cell outline is active for the leading 14 time-steps only, showing up the zero-value cells in particular. $n=150, v 8 k 3 \mathrm{kcode}$, index 7 from the rule sample in figure 33.5. Figure 32.35 shows the circle layout without scrolling.

3 ...to toggle between cell colors according to the neighborhood "looked up" and the actual cell value. This can also be preset in section 31.2.1. Examples are shown in figure 32.19 (a,b), and figures 31.1 to 31.3 for $1 \mathrm{~d}, 2 \mathrm{~d}$ and $3 \mathrm{~d}$ networks.

....(for 1d and 2d STP) (full stop) toggles putting a dot on zero cells. Dots appear for the cell scale $\geq 4$ pixels.

$\wedge \ldots$ (for $1 d$ and $2 d S T P$, and value colors) to toggle between white and light green for zero cells. This is sometime necessary to show up the background.

\subsection{6 d/-..colors:swap/black-blue}

for binary, $v=2$

To swap or change color, enter on-the-fly key hits as follows, The color key as in figure 7.1 is displayed top-right.

d ... to swap colors of $0 / 1$ cells between white and black.

- ... (minus sign) to toggles the color of 1s between black and blue. 


\subsection{7 d/-..tog shuffle colors/restore}

for $v \geq 3$

To shuffle or restore colors, enter on-the-fly key hits as follows, The color key as in figure 7.1 is displayed top-right.

d ... to randomly shuffle the colors assigned to different values.

- ... (minus sign) to restore the default colors.

\subsubsection{S..tog space-time display}

Enter $\mathbf{S}$ on-the-fly to toggle the display of space-time graphics on/off. Not showing space-time patterns, which continue to run in the background, speeds up other processes, for example creating a sample of automatically classified rule-space (chapter 33), histograms of "damage" (section 31.5.2), and histograms of attractors (section 31.6).

\subsubsection{P..tog skip steps $=1$ (off)}

Enter $\mathbf{P}$ on-the-fly to toggle skipping on/off. The number of time-steps to be skipped can be changed in section 32.16.8. The default is 1, i.e. showing every second time-step. The current number of time-steps, and whether skipping is currently on or off, is indicated in the prompt, for example, P..tog skip steps $=\mathbf{3}$ (on).

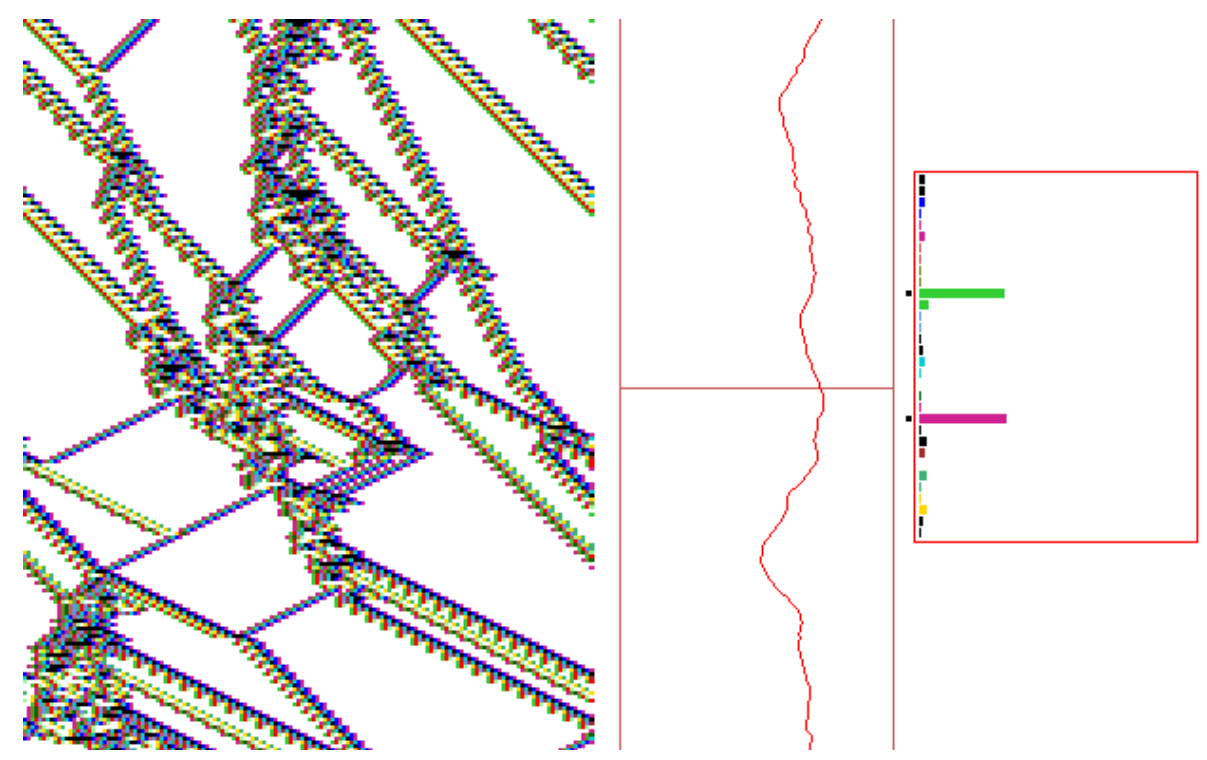

Figure 32.15: Skipping time-steps in a 1d CA with the default steps=1 (section 32.9.9), activated on-the-fly with key hit $\mathbf{P}$ at about the mid point of the space-time pattern (after about 100 time-steps) — indicated by a horizontal line in the input-entropy plot — so skipping alternate time-steps thereafter. The space-time pattern is shown according to neighborhood color, and is also filtered - indicated by square dots in the input-frequency histogram. $v 2 k 5$ rcode(hex)968c4f76, $n=150$. 


\subsubsection{0 \$..tog sound DOS only}

Enter $\$$ on-the-fly to toggle sound generated by space-time patterns. Both the pitch and delay are generated by 8 bits near the center of the network.

\subsubsection{1 e/c..expand/contr scale}

Enter e on-the-fly to expand the scale of cells in the space-time pattern by 1 pixel, enter $\mathbf{c}$ to contract the scale by 1 pixel. This changes the scale of the space-time pattern in $1 \mathrm{~d}, 2 \mathrm{~d}$ or $3 \mathrm{~d}$. The initial scale can be preset in section 31.2.4.

Keys e/c have a different effect for diagonally scrolling network-graph space-time patterns (section 20.13), as in figure 32.14 - the spacing between time-steps is expanded or contracted by one pixel as illustrated below.
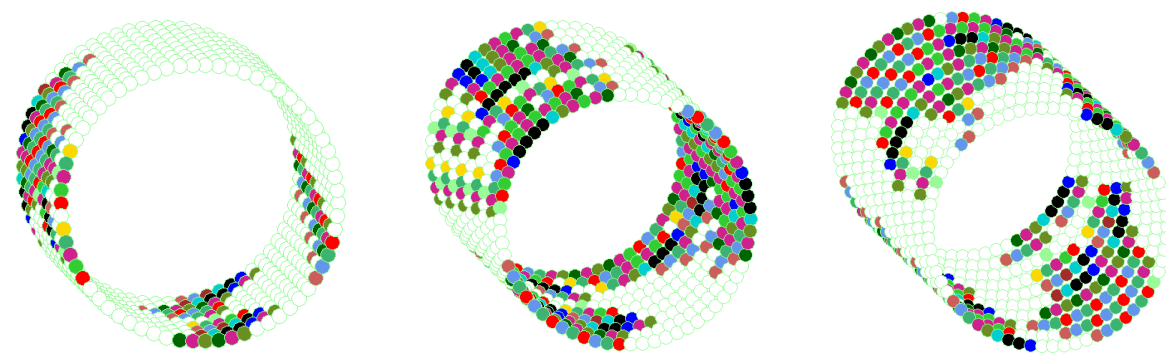

Figure 32.16: For diagonally scrolling network-graph space-time patterns, as in figure 32.14 , key hits e/c expand/contract the spacing between time-steps by one pixel. These examples each have the same number of time-steps, but with expanded spacing - shown in $1 \mathrm{~d}$ circle layout, $n=66,10$ time-steps.

\section{$32.101 d 2 d 3 d$}

Space-time patterns (STP) can be displayed in a dimension other than the "native" dimension. A $1 \mathrm{~d}$ native network can be displayed in $2 \mathrm{~d}$ or $3 \mathrm{~d}$, a $2 \mathrm{~d}$ native in $1 \mathrm{~d}$ or $3 \mathrm{~d}$, and a $3 \mathrm{~d}$ native in $1 \mathrm{~d}$ or $2 \mathrm{~d}$. The STP dimension can be preset to something other than the native dimension in section 31.2.5, or changes can be toggled on-the-fly, described here.

In addition, 2d STP can be toggled to $2 \mathrm{~d}+$ time, or scrolled diagonally - this and $3 \mathrm{~d}$ STP have additional on-the-fly options. A $3 \mathrm{~d}$ native network when toggled to $2 \mathrm{~d}$ STP results a stack of $2 \mathrm{~d}$ horizontal slices (figure 32.17 ) following the method in figure $21.3 \mathrm{Left}$.

\subsubsection{T..tog 1d-2d-3d}

if $2 d+$ time is not active (section 32.10.2)

$\mathbf{T}$ is a 3-way toggle between 1d-2d-3d STP - the order changes to show which is current and which is next in the toggle sequence, i.e. if $2 \mathrm{~d}$ is active the prompt is $\mathbf{2} \mathbf{d}-\mathbf{3 d} \mathbf{d} \mathbf{- 1 d}$, if $3 \mathrm{~d}$ is active the prompt is $\mathbf{3 d - 1} \mathbf{d}-\mathbf{2 d}$. Note that $1 \mathrm{~d}$ and $2 \mathrm{~d}$ STP can be scrolled (section 32.13.3).

On-the-fly methods for converting between STP dimensions are as follows: 


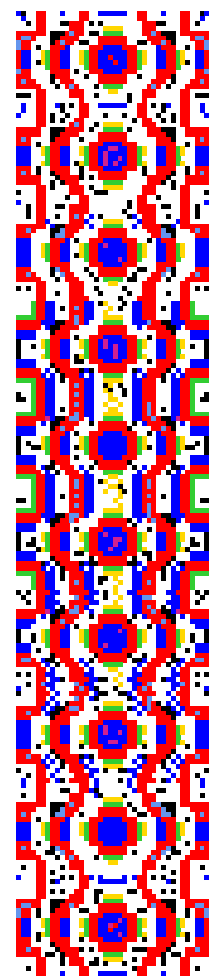

Figure 32.17: A $3 \mathrm{~d}$ network shown in $2 \mathrm{~d}$ by toggling $3 \mathrm{~d}$ to $1 \mathrm{~d}$ to $2 \mathrm{~d}$ on-the-fly. Below: The 3d space-pattern, $40 \times 20 \times 10$. The projection is isometric seen from below, as if looking up into a cage. Left: the $2 \mathrm{~d}$ representation consists of 10 horizontal slices, one below the other, starting at the top, i.e. a series of horizontal cross-sections through the $3 \mathrm{~d}$ volume. $v 8 k 6$ majority kcode but with shifted uniform outputs (section 16.8). The seed was a random central block which settled to this semi-stable pattern.

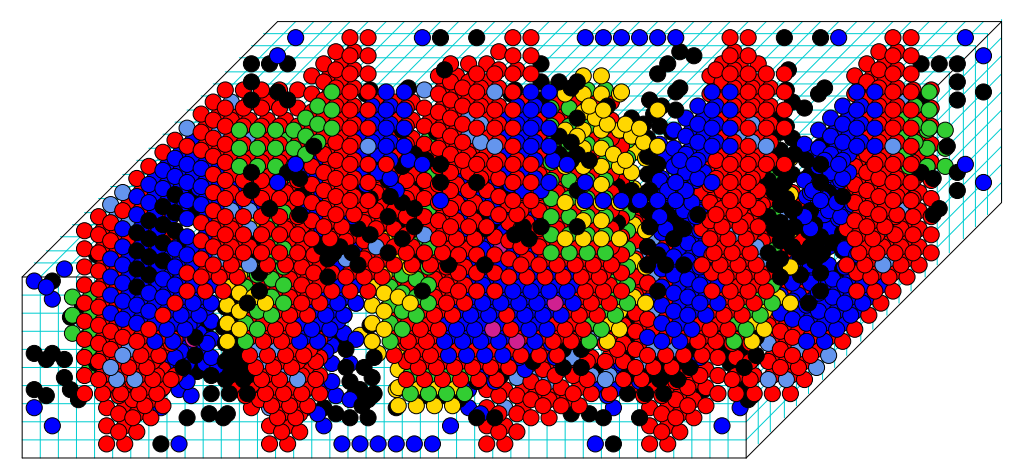

$1 \mathrm{~d}$ to $2 \mathrm{~d}: i=$ the smallest factor $\geq \sqrt{n}, j=n / i$

$1 \mathrm{~d}$ or $2 \mathrm{~d}$ to $3 \mathrm{~d}: i=$ the smallest factor $\geq \sqrt[3]{n}, j=$ the smallest factor $\geq \sqrt{n / i}, h=n /(i \times j)$ For $1 \mathrm{~d}$ prime $n$, the $2 \mathrm{~d}$ and $3 \mathrm{~d}$ representation will be a long string length $n$.

3d to $2 \mathrm{~d}$ : The $2 \mathrm{~d}$ representation consists of $h$ horizontal slices (levels), $i \times j$, stacked one below the other starting from the top, i.e. a series of horizontal cross-sections through the $3 \mathrm{~d}$ volume (figures 32.17, $21.3 \mathrm{Left}$ ).

$2 \mathrm{~d}$ or $3 \mathrm{~d}$ to $1 \mathrm{~d}$ : This will be represented as normal $1 \mathrm{~d}$ space-time patterns, were the cells are indexed $n-1$ to 0 from left to right. The cell scale is automatically reduced to 1 pixel.

\subsection{2 t..tog $2 \mathrm{~d}-2 \mathrm{~d}+$ time for $2 d S T P$}

Enter $\mathbf{t}$ to toggle between $2 \mathrm{~d}$ STP, and $2 \mathrm{~d}$ plus a time dimension, drawn as a $3 \mathrm{~d}$ isometric projection (figure 4.12). For binary $(v=2)$ networks, this is best seen when cells are colored according to the neighborhood (section 32.9.5). The current status is shown by the prompt sequence, i.e. if $2 \mathrm{~d}+$ time is active the prompt is t..2d+time-2d. By default 2d+time will "sweep" - restart at the top on reaching the bottom of the screen. Enter \# or bf \& to scroll $2 \mathrm{~d}+$ time (section 32.13.3). 


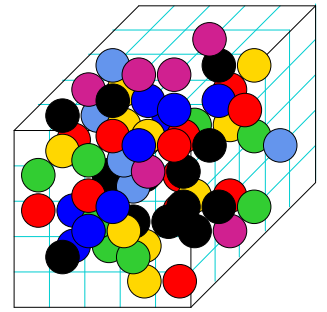

cells as balls

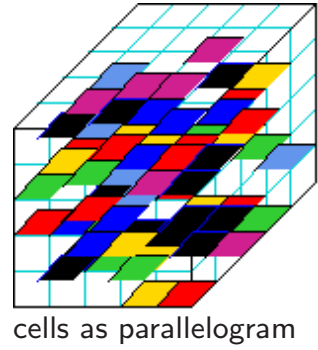

Figure 32.18: Toggling on-the-fly between showing $3 \mathrm{~d}$ cells, Left: as balls or circles, or Right: as flat parallelograms. $v=8, \overline{n=5 \times 5} \times 5$. Only the balls presentation can be captured as vector PostScript (section 21.4.10).

\subsubsection{I..tog balls}

if $3 d S T P, 2 d+$ time

Enter I on-the-fly to toggle between showing 3d cells as balls (circles) or as flat parallelograms (figure 32.18) - also applies to $2 \mathrm{~d}+$ time (section 32.10 .2 ).

\subsection{4 p..plane}

for $2 d+$ time or diagonal scrolling

Enter $\mathbf{p}$ on-the-fly to draw a plane grid within the space-time patterns at the current time-step to highlight the geometry. This plane is also drawn whenever the seed, rule/s or wiring are changed. Figures 4.12 and 4.14 give examples.

\subsubsection{J..invisible}

if $2 d$ diagonal scrolling is active (section 32.13.3.2)

Enter $\mathbf{J}$ on-the-fly to toggle (make invisible) normal diagonally scrolling space-time patterns. The purpose of this option is to see just the scrolling network-graph, if set (section 32.13.3.3 and figure 32.14).

\subsection{Frozen/Filter}

filter - if input-entropy is active (section 31.4)

"Frozen" options allow visualizing the activity/stability of cells. "Filter" options allow progressively filtering categories of cells according to neighborhood frequency (starting with the highest) to show up interacting configurations (gliders) more clearly. Note the frozen and filter options can be used together, and work for $1 \mathrm{~d}, 2 \mathrm{~d}$ and $3 \mathrm{~d}$ (including RBN and DDN), but filtering the relies on data from the input-entropy histogram, so the input-entropy option must be active, the default for $1 \mathrm{~d}$ networks. For $2 \mathrm{~d}$ and $3 \mathrm{~d}$ networks this option would need to be set in section 31.4.

\subsection{1 h..nor-f1-f2-bin}

Enter $\mathbf{h}$ on-the-fly to toggle through the following four ways of of displaying cells in space-time patterns, the normal display, two ways of showing "frozen" cells, and frequency bins (figure 32.19 b,c,d,e). The method can also identify gliders by their time-trails (figure 4.13). The current status is given by the prompt order, i.e. 7..f1-f2-bin-nor indicates that the f1 presentation is current. 


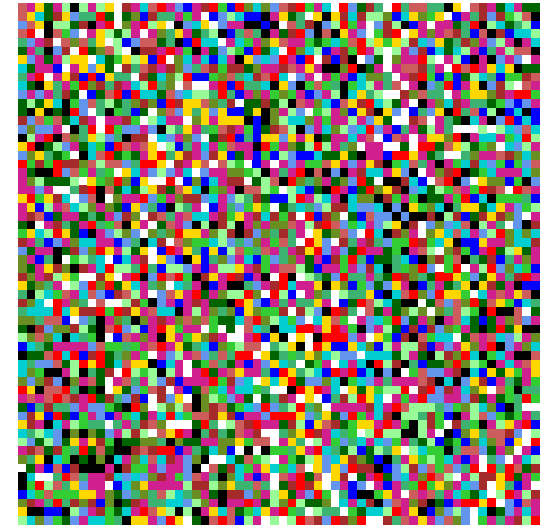

(a) cells by neighborhood

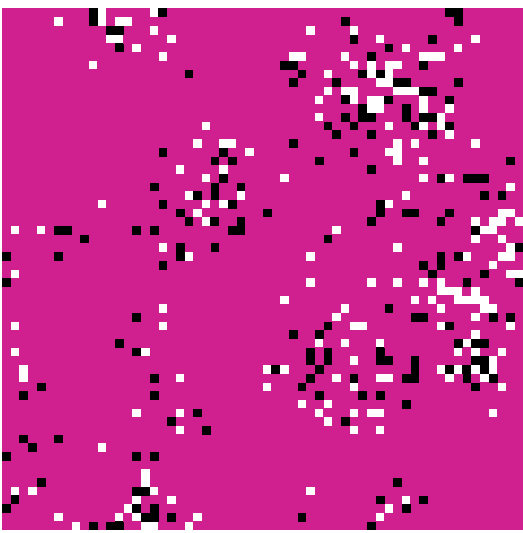

(c) f1 - frozen purple, otherwise value colors

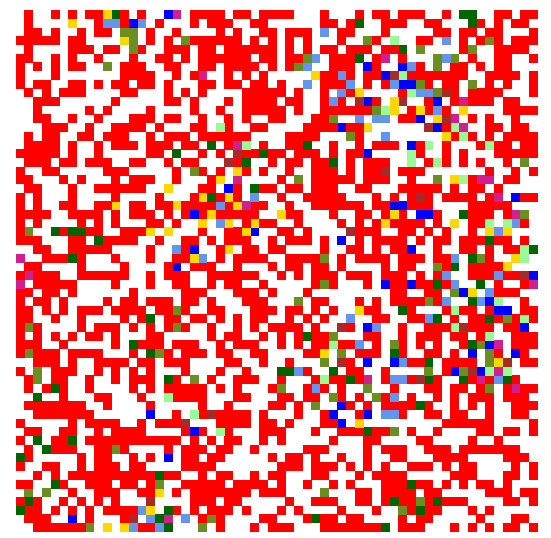

(e) bin - frequency bin colors

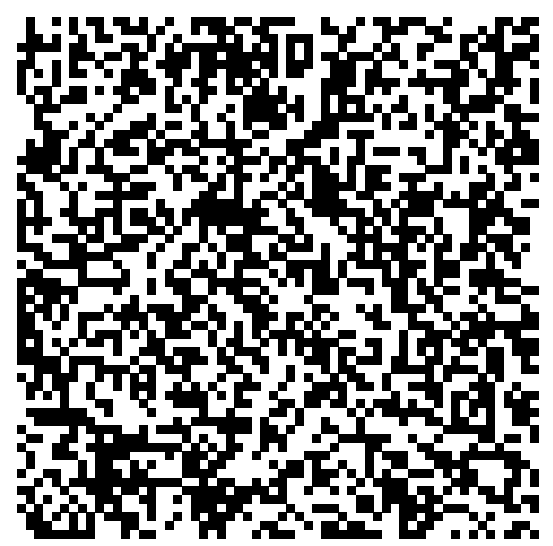

(b) cells by value

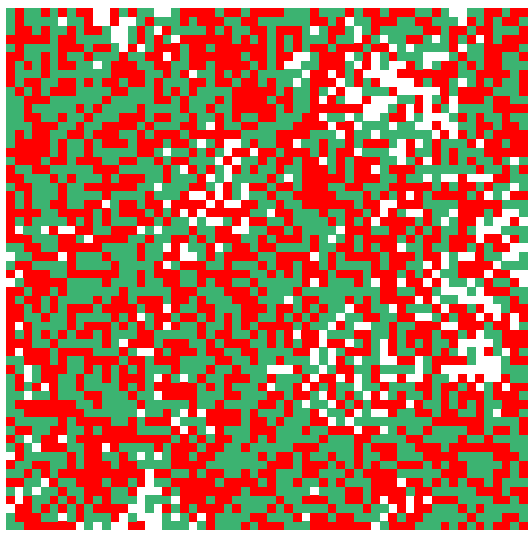

(d) f1 - frozen 1s red, 0s green, otherwise white

Figure 32.19: Toggling between $(\mathrm{a} / \mathrm{b})$ - cells by value and neighborhood (section 32.9.5), and between (b/c/d/e) - cells by value, two different "frozen" presentations, and frequency bins (section 32.11.1). $60 \times 602 \mathrm{~d}$ RBN, $v 2 k 5$ with wiring restricted to an 8 cell radius, mixed rules with canalyzing inputs set to $51 \%$. The 5 alternatives are snapshots of the same stabilized dynamics. 
On-the-fly key $\mathbf{h}$ toggles to the next frozen/bin presentation (f1, f2, bin) then back to "normal". At the same time a top-right window gives the relevant color key and other information figures 32.20 - 32.23 below. To qualify as "frozen" a cell's value remains unchanged according to parameters in section 32.11.3. Frequency bins are defined in section 32.11.4. Figure 32.19 gives space-time pattern examples.

nor ... "normal" colors. If the 4-way toggle returns to nor, cells are shown according to their actual cell value, even if color by neighborhood "looked up" was previously active, and the following color key reminder is shown in a top-right window,

\section{cell vahuerange $=2$ \\ color key: \\ cell state: $1 \quad 0$}

Figure 32.20:

$v=2$

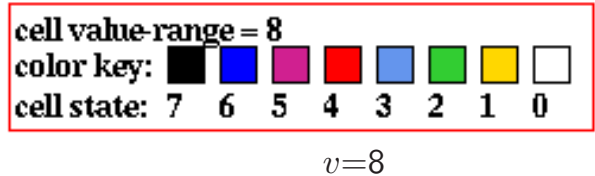

$v=8$

f1 ... "frozen" (method 1): frozen cells are colored purple. Dynamic, unfrozen cells, are show as "normal" (figure $32.19 \mathrm{c}$ ). The following color key reminder is shown in a top-right window,

frozen cells for at least 20 time-steps

color key: frozen $1-0 s=$

cells not frozen shown according to value

Figure 32.21: $\quad v=2$

frozen cells for at least 20 time-steps

color key: frozen $7-0 s=$

cells not frozen shown according to value

$v=8$

f2 ... "frozen" (method 2): for binary, $v=2$ : frozen 1 s are colored red, frozen 0s green. For $v \geq 3$ : frozen $(v-1)$ 's are colored red, frozen ( $v-2$ to 0$)$ 's green. Dynamic, unfrozen, cells are white (figure $32.19 \mathrm{~d}$ ). The following color key reminder, which also shows an updating percentage of reds, greens, and the total frozen, is shown in a top-right window,

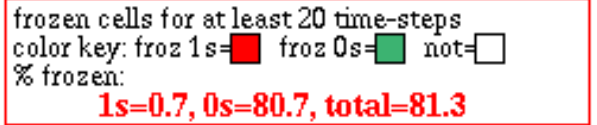

Figure 32.22:

$v=2$ frozen cells for at least 20 time-steps color key: froz $7 \mathrm{~s}=\square$ froz $6-0 \mathrm{~s}=\square$ not $=\square$ \% frozen: $7 \mathrm{~s}=2.76-0 \mathrm{~s}=2.0$ total $=4.7$

bin ... colors are assigned from a spectrum representing the fraction of time each cell have been "on" in a (frozen generation) time window of $x$ time-steps, so falling into a preset number of frequency bins, and bin boundaries (figure 32.19 e). For binary, $v=2$, "on" signifies 1 . For $v \geq 3$, "on" signifies the maximum value $(v-1)$. The following top-right reminder shows the bin color scheme, and bin boundaries defined by their upper limits (these parameters can be reset in section 32.11.2,

frequency of $1 \mathrm{~s}$ in 20 time-step window color key: $\square \square \square \square \square \square \square \square \square \square \square$
10 bins: 2

Figure 32.23: $v=2$ frequency of $7 \mathrm{~s}$ in 20 time-step window color key: $\square \square \square \square \square \square \square \square \square \square \square \square$

$$
v=8
$$




\subsubsection{H..f-gens (now 20)/bins (10)}

Enter $\mathbf{H}$ on-the-fly to change "frozen" parameters, the default number of frozen generations, or the number of bins and bin boundaries, related to section 32.11.1 above. The following top-right prompts are presented in turn (showing the initial defaults),

enter new 'frozen' Generation size (now 20):

reset bin boundaries, enter new total $(\max 20$, now 10$)$ :

\subsection{3 frozen generations}

The "frozen" generation size is the number of time-steps that a cell must remain unchanged to qualify as "frozen", which can be reset with the following prompt,

enter new 'frozen' Generation size (now 20):

Enter a new frozen generation size, which also sets the maximum number of bins. The current frozen generation size is shown in the on-the-fly index and becomes the new default.

\subsection{4 frequency bins}

To change the number of bins, and the bin boundaries, the following top-right prompt is presented after the frozen generation prompt in section 32.11.3,

reset bin boundaries, enter new total (max 20, now 10):

The current number of bins is shown in both the on-the-fly window and in this prompt. First enter the new bin number, which cannot be greater than the frozen generation size (otherwise it is reduced automatically) - a subsequent top-right prompt sets the bin boundaries,

\section{unequal bins-u, equal-def:}

Enter return to set equal size bins (or as equal as possible) automatically. Otherwise enter $\mathbf{u}$ to set the bin boundaries by hand (example in figure 32.24). A frequency bin is the interval above the previous bin and its own upper limit. To define the bins, enter successive upper limits - each entry should be greater than the last. The following top-right prompts are presented in sequence,

enter bin boundary 1 (def 2$)$ :

enter bin boundary 2 (def 4$)$ :

etc... invalid entries are corrected, by may also result in the following top-right warning

invalid bin boundaries, cont-ret: (enter return to backtrack)

frequency of $1 \mathrm{~s}$ in 100 time-step window color key: $\square \square \square \square \square$
Figure 32.24: Frequency bins set by hand shown - the top-right bin window (see figure 32.23). Frozen generation size $=100$, no of bins $=5$, bin boundaries: $1,5,20,100$. 


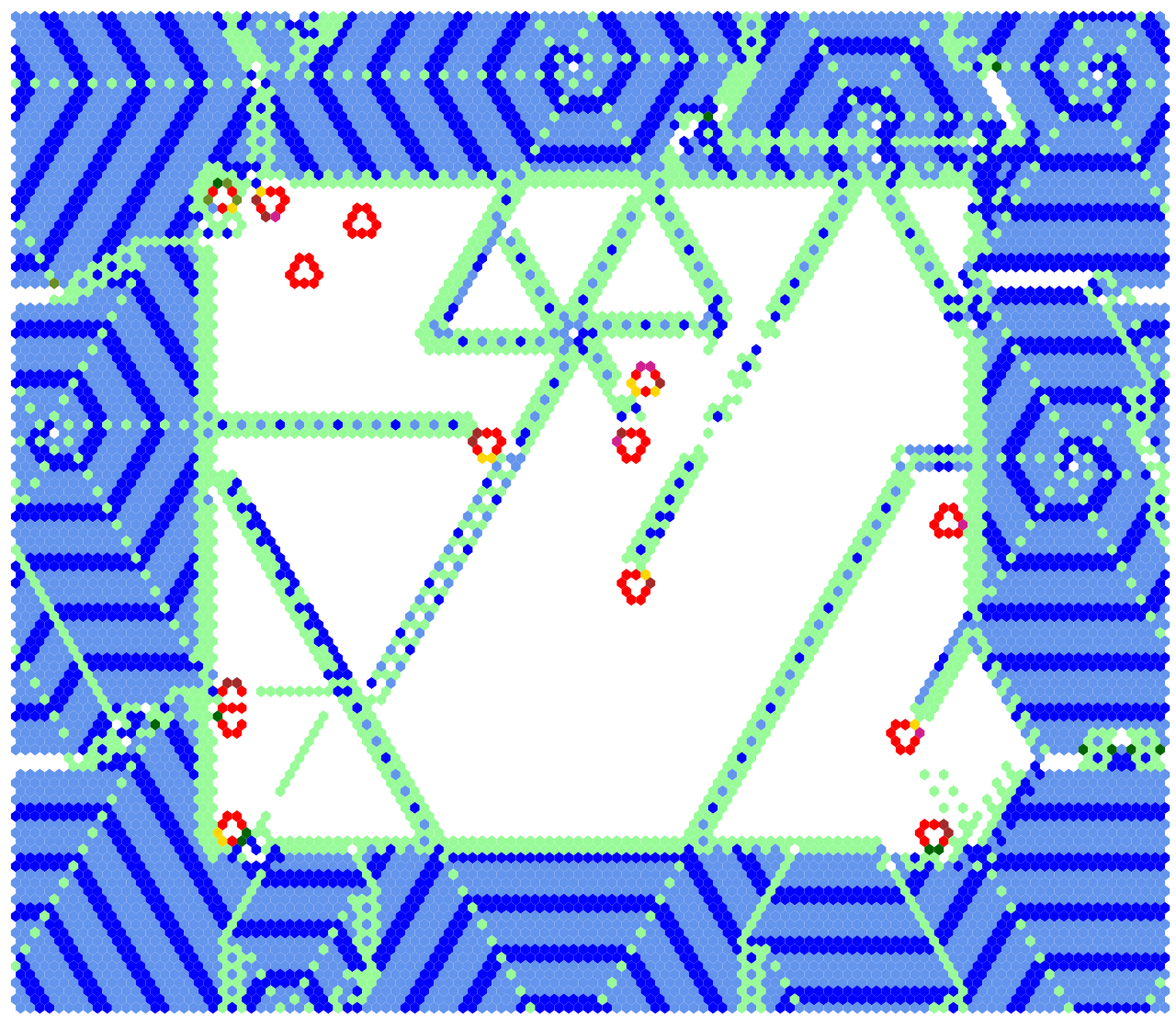

Figure 32.25: Space-time snapshot of a 2d CA inside another 2d CA with different $k$, the same spacetime pattern as figure 19.6 (where rule details are given) but colored according to (default) frequency bins (section 32.11.4). The $2 \mathrm{~d}(80 \times 80) v 3 k 7 \mathrm{CA}$ spiral-rule[40] was loaded into a $2 \mathrm{~d}(120 \times 120) v 3 k 6$ complex CA which generates spiral structures.

\subsection{5 f/F/a..filter/undo/all}

if input-entropy is active (section 31.4)

On-the-fly filtering algorithms take streaming data from the input-frequency histogram (section 31.4), then suppress the printing of cells of the most frequent (unsuppressed) neighborhood. This suppresses the background domains first, being the most frequent. Gliders and other rare structures are left until last, so can be isolated and seen more clearly (figures 4.9, 32.26 32.28). The method works for any type of network (1d, $2 \mathrm{~d}$ or $3 \mathrm{~d}$ ) provided that the input-entropy is active.

Filtering is implemented on-the-fly with the following key hits,

f ... to progressively filter space-time patterns.

$\mathbf{F}$... to unfilter in reverse order.

a ...to immediately restore the unfiltered pattern. 

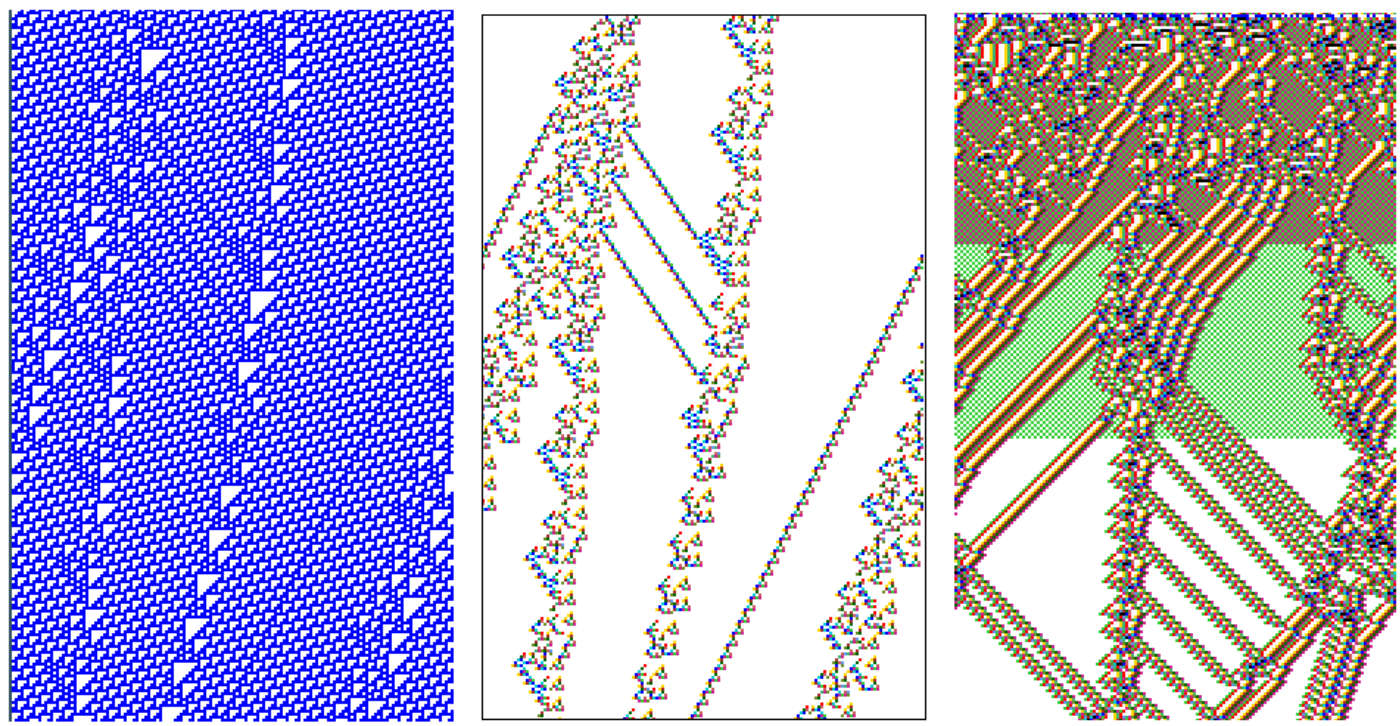

Figure 32.26: Examples of filtering space-time patterns to show up gliders more clearly $(n=150$, about 200 time-steps). Left: unfiltered space-time patterns of $v 2 k 3$ rcode 110 , transformed to the equivalent $v 2 k 5$ rcode $3 \mathrm{cfc} 3 \mathrm{cfc}, n=150$ (cells by value). Centre: the same space-time patterns - filtered (cells by neighborhood lookup). Right: space-time patterns of the $v 2 k 5$ rcode $360 a 96 f 9$. Cells are shown by neighborhood lookup, and are progressively filtered in 2 stages.

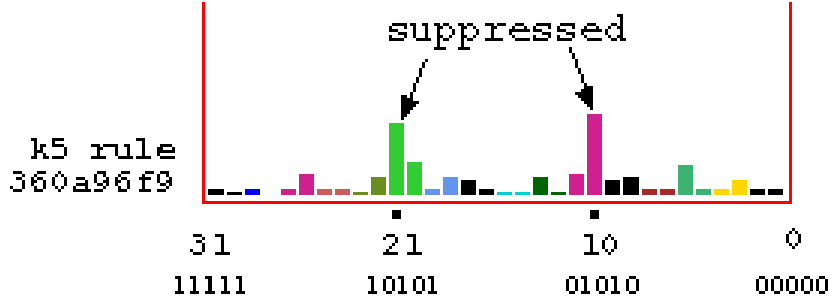

k5 rule $3 e f a j e f e$ ( $\mathrm{k} 3110$ )

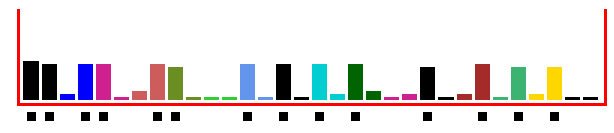

Figure 32.27: The lookup-frequency histogram relating to figure 32.26 . Left Above: v2k5 rcode(hex)360a96f9. Left Below: $v 2 k 3$ rcode(dec)110 transformed to $v 2 k 5$ rcode(hex)3cfc3cfc.

Suppressed neighborhoods are indicated with a dot. Note that the lookup frequency histogram is shown vertically in DDLab.

A dot is shown alongside the lookup-frequency histogram indicating which neighborhoods are currently suppressed (figure 32.27). As well as (and in addition to) progressively filtering/unfiltering on-the-fly, specific selected neighborhoods can be filtered in isolation (section 32.16.7).

For most glider rules, only a few neighborhoods need to be suppressed to filter domains. Rules with very complicated background domains, such as the $v 2 k 3$ rcode(dec) 54 and 110, must first be transformed to equivalent rules with greater $k(k=5$ in this case) for successful filtering, which requires suppressing a number of the $k=5$ neighborhoods (figures 32.28).

The method can also filter chaotic domains, and reveal discontinuities within or between chaotic domains (figure 32.29). 

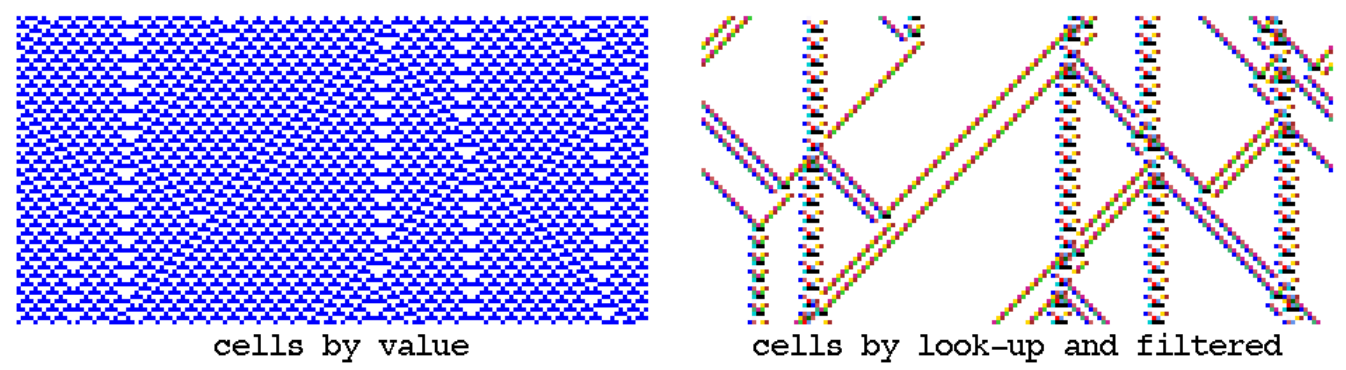

Figure 32.28: Space-time patterns from the same initial state showing interacting gliders, which are embedded in a complicated background. Left: unfiltered, cells by value. Right: cells by neighborhood lookup, with the background domain filtered. The $k=3$ rule 54 was transformed to its equivalent $v 2 k 5=5$ rcode(hex) $0 f 3 c 0 f 3 c, n=150$.
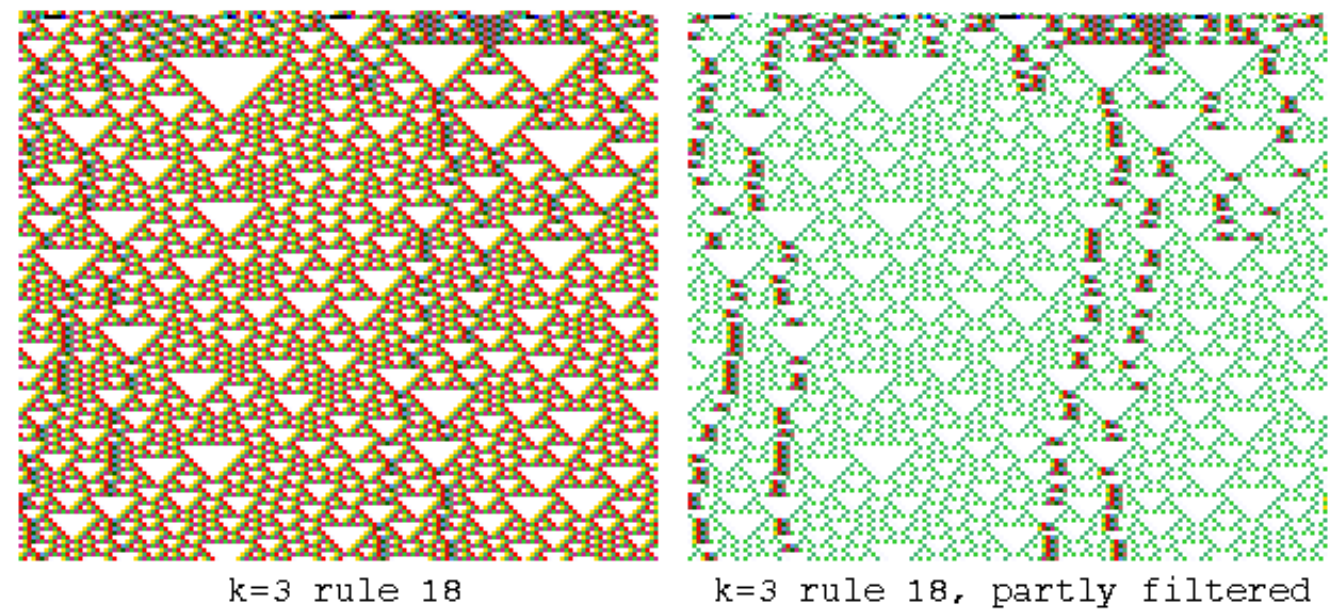

Figure 32.29: Unfiltered and partly filtered space-time patterns of $v 2 k 3$ rule 18 , transformed to $v 2 k 5$ rcode(hex) 030c030c. $n=150$, about 130 time-steps from the same random initial state, showing discontinuities within the chaotic domain.

\subsection{Analysis}

These on-the-fly options relate to various methods of real-time analysis of space-time patterns, including the input-frequency histogram, input-entropy, pattern density, the entropy-density plot, return map, state-space matrix, and alternative presentations of the above. 


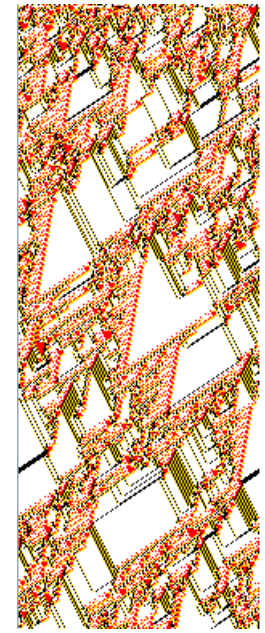

(a) STP

filter $\times 4$

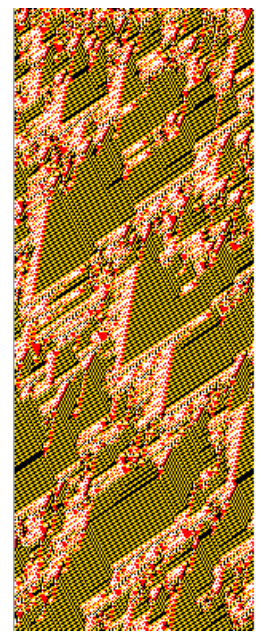

(b) STP

unfiltered

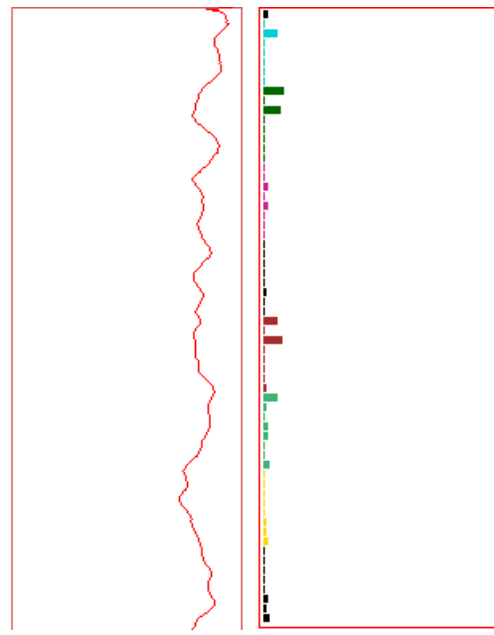

(c) input-entropy

(d) input-hist default (b)-(c)-(d): The default display of $1 \mathrm{~d}$ space-time patterns (STP) showing (b) the STP scrolling (value colors) alongside (c) the input-entropy plot, alongside (d) the default input frequency histogram - unfiltered and not amplified - in this case with 27 bars showing the frequency of each neighborhood, with the zero neighborhood at the bottom.

(a) shows the same STP filtered four times.

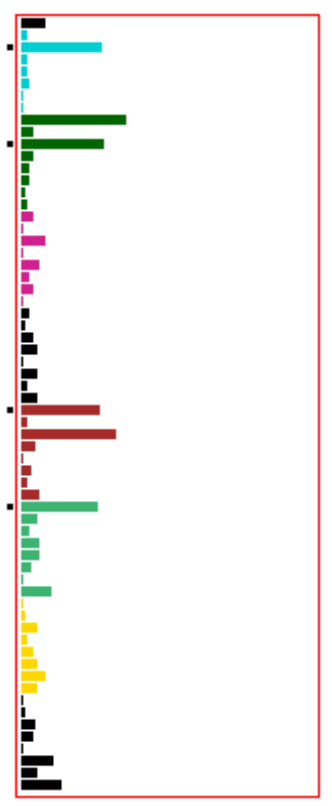

(e) input-hist amplified $\times 2$, filter $\times 4$

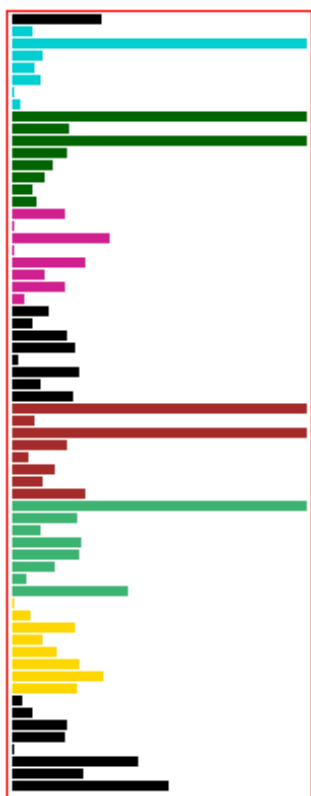

(f) input-hist amplified $\times 4$

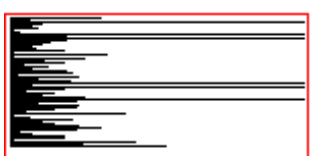

The input frequency histogram (IFH) as (d) above, with 27 bars - the length of the rule-table. (e) and (f) show thick bars, in colors that match the STP plot. Rule-tables greater than 128 are presented in multiple columns. An alternative IFH presentation, useful for long rule-tables, is $(\mathrm{g})$ where bars are thin black lines. Thick/thin IFH presentations are toggled with on-the-fly key "0", and the bars can be amplified (repeatedly) with ")" and restored with "(". (d) is the default, (e) has been amplified twice, and (f) and (g) 4 times. In the thick IFH presentation, filtered bars are indicated by a square dot as in (e) which is filtered 4 times.

Figure 32.30: Input frequency histogram (IFH) presentation while running space-time patterns, showing the amplification of bars and which bars are filtered in space-time patterns. $v 4 k 31 \mathrm{~d}$ CA, rcode (hex)f519751e8ea60489ff3ccaec94849968, $n=150.588$ time-steps from the same random initial state. 


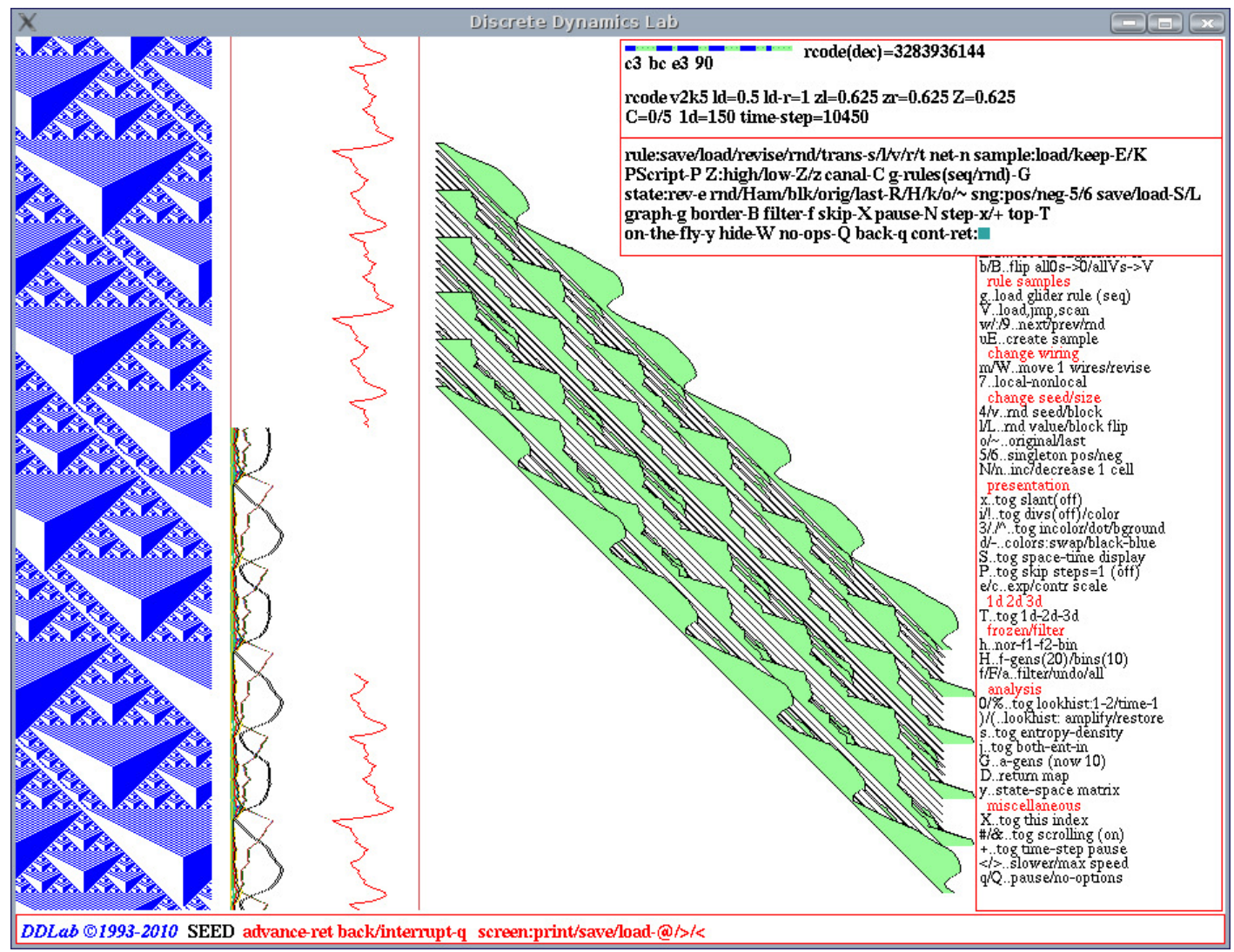

Figure 32.31: A snapshot of the DDLab screen, showing the input frequency histogram in $3 \mathrm{~d}$ with an added time dimension. Various sections of the screen show the following: Far Left: a scrolling spacetime pattern of a rule by Wentian Li,[18] $v 2 k 5$ rcode(hex) c3bce390, $n=150$. Near Left: three alternative scrolling graphs (toggled with $\mathbf{j}$, section 32.12.4) - input-entropy - the lookup frequency of each neighborhood - both simultaneously. Center: the input-frequency histogram in 3d (section 32.12.1). Top Right: the interrupt/pause prompt (section 32.16). Far Right: on-the-fly key index (section 32.1).

\subsubsection{0/\%..tog lookuphist:1-2/1-time}

if input-entropy is active (section 31.4)

On-the-fly key hits to change the presentation of the input-frequency histogram are as follows,

0 ... (zero key) to toggle between normal and 1 pixel width bars (figure $32.30 \mathrm{~g}$ ).

$\%$... to toggle between the usual $2 \mathrm{~d}$ histogram, expanded to $3 \mathrm{~d}$ with a diagonal time axis if the number of bars $\leq 64$ (figure 32.31

\subsection{2 )/(..lookhist: amplify/restore}

if input-entropy is active (section 31.4)

Enter on-the-fly key ")" to double the length of bars (can be repeated) - enter "(" to restore the default length (figure $32.30 \mathrm{~d}, \mathrm{e}, \mathrm{f}$ ). 


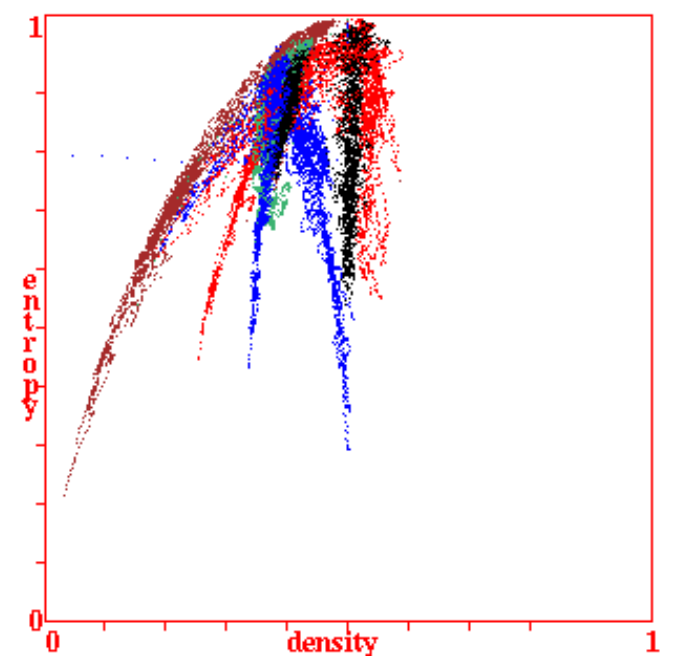

$161 \mathrm{~d}$ CA $v 2 k 5$ complex rcode, $n=150$

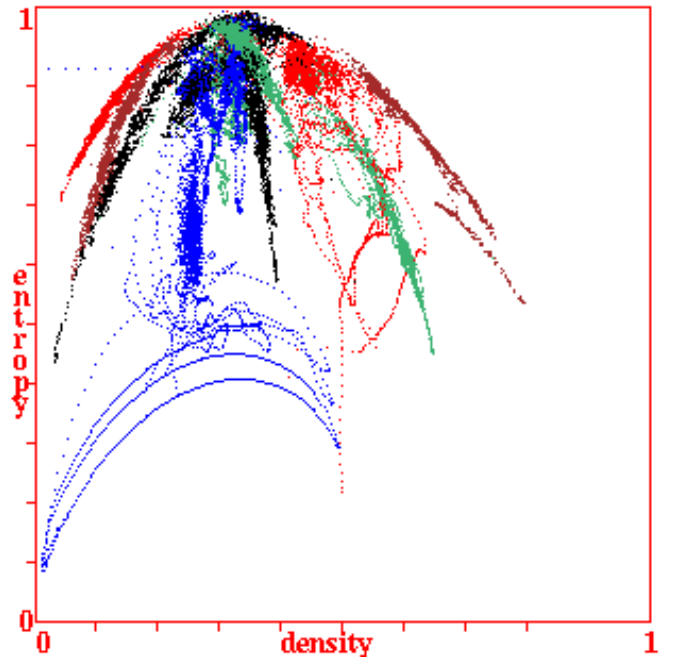

$161 d$ CA $v 3 k 3$ complex rcode, $n=150$

Figure 32.32: Entropy/density scatter plot[32]. The density is defined as follows: for binary, $v=2$, the fraction of $1 \mathrm{~s}$; for $v \geq 3$, the fraction of non-zero values. Input-entropy is plotted against the density relative to a moving window of time-steps, which can be reset in section 32.12.6. Plots for the first 16 rcodes in the complex rule collections (section 32.6.1) are show superimposed - each has its own distinctive signature, with high input-entropy variability. About 1000 time-steps are plotted from several random initial states.

\subsection{3 s..tog entropy-density}

if either input-entropy or pattern density is active (section 31.4)

Enter s on-the-fly to toggle between showing the input-entropy, or the pattern densities as a set of $v$ value-density graphs (section 31.4 and figure 31.8). The current status is given by the prompt order, i.e. density-entropy would indicates that density is current. These measures relate to a moving window of time-steps (default 10) that can be reset in section 32.12.6. For 1d networks the input-entropy and pattern density is the default, but $2 \mathrm{~d}$ and $3 \mathrm{~d}$ networks it needs to be set in section 31.8 to be active.

\subsection{4 j..tog ent-in-both}

if input-entropy is active (section 31.4)

On-the-fly key hit $\mathbf{j}$ is a 3-way toggle to show the input-entropy, or the input-frequencies as a set of graphs, or both together (figures $31.8,32.31$ ). The current status is given by the prompt order,

ent-in-both ... just the input-entropy graph.

in-both-entropy ... the input-frequency of each neighborhood as a set of superimposed graphs. The colors of the graphs are the same as the colors of the bars in the inputfrequency histogram described in section 31.4.

both-ent-in ... showing both of the above simultaneously. 


\subsection{5 u..tog entropy/density plot}

if input-entropy is active (section 31.4)

Enter $\mathbf{u}$ on-the-fly to toggle showing the density/entropy scatter plot (figure 32.32) in a lower central window, where the entropy ( $y$-axis) is plotted against the density ( $x$-axis), relative to a moving window of time-steps (default 10), which can be reset in section 32.12.6).

Complex rules have distinctive scatter signatures, with high input-entropy variability, which lie within a parabolic envelope because high entropy is most probable at medium density, low entropy at either low or high density. Chaotic rules, on the other hand, give a compact scatter at high entropy (at the top of the parabola). For ordered rules the entropy rapidly falls off with very few data points because the system moves rapidly to an attractor.

\subsubsection{G..a-gens (now 10)}

if input-entropy is active (section 31.4)

Enter $\mathbf{G}$ on-the-fly to change the default number of analysis generations, the size of the moving window of time-steps relating to input-entropy and pattern density (sections 32.12.3, 32.12.5).

The following top-right prompt is presented,

\section{enter new 'analysis' Generation size (now 10):}

The current analysis generations is shown in both the on-the-fly window and in this prompt. The initial default is 10 time-steps.

\subsubsection{D..return map}

Enter D on-the-fly to toggle the "return map" in a 2d-phase plane, a scatter plot described in section 31.2.2.2 and figure 31.5, providing characteristic fractal signatures for chaotic rules.

\subsection{8 y..state-space}

Enter $\mathbf{y}$ on-the-fly to toggle the "state-space matrix" which plots each state in the space-time pattern on a $2 \mathrm{~d}$ grid plotting the left half of the bit string against the right half, described in section 24.5 and figure 31.4, providing a kind of signature of the dynamics.

\subsection{9 =..tog diff (keep damage)}

if the "damage" is active (section 31.5)

The method to graphically represent and analyse the difference, or the spread of "damage", between the space-time patterns of two networks is described in section 31.5.

Enter "=" (the equals sign) on-the-fly to toggle between two definitions of damage, (keep damage) - once damaged keep the damage, and (dontkeep damage) - the instantaneous difference at each time-step. The on-the-fly index shows the current status. 


\subsection{Miscellaneous}

These final miscellaneous on-the-fly options include pausing, scrolling, stepping, and changing the speed of space-time patterns.

\subsubsection{X...index display}

Enter $\mathbf{X}$ on-the-fly to toggle the on-the-fly key index itself.

\subsection{2 *..tog end pause (on)}

for $1 d$ or $2 d+$ time space-time patterns

Enter * (the star key) on-the-fly to toggle the end pause on/off. If scrolling is not active, 1d space-time patterns (and $2 \mathrm{~d}+$ time) are displayed in successive vertical sweeps. If the end pause is on, when the iterations reach the foot of the screen the interrupt/pause options (section 32.16) are presented in a top-right window. If the end pause is off, the sweeps continue indefinitely. The current status is shown in the prompt by (on) or (off).

\subsection{3 \#/\&..tog scrolling}

for $1 d$, 2d, or $2 d+$ time space-time patterns

Enter \# (the hash key) or $\&^{5}$ on-the-fly to toggle space-time pattern scrolling on/off, for $1 \mathrm{~d}, 2 \mathrm{~d}$ (including the network-graph), or $2 \mathrm{~d}+$ time. These dimensions can be different from the network's native dimension (section 32.10.1).

\subsubsection{1d scrolling}

Toggle a 1d space-time presentation between scrolling upwards or making successive vertical sweeps (section 31.2.6). If set, the input-entropy or pattern density plots (sections 32.12 .3 and 31.4) will also scroll or sweep.

\subsubsection{2d diagonal scrolling}

Toggle a $2 \mathrm{~d}$ space-time presentation between a $2 \mathrm{~d}$ movie and scrolling diagonally upwards. When scrolling is set, successive $2 \mathrm{~d}$ snapshots will move diagonally towards the bottom-right, then scroll diagonally upward, so that the present moment is the movie at the bottom-right (figure 4.14).

An active network-graph will scroll simultaneously (section 32.13.3.3 below).

\subsubsection{3 network-graph scrolling}

If normal 2d space-time patterns (STP) are toggled to scroll diagonally upwards (section 32.13.3.2), an active network-graph (section 32.19) will also scroll simultaneously. Scrolling of both starts once normal 2d STP reaches the foot of the DDLab screen, with the present moment of both at the bottom-right. Enter J on-the-fly (section 32.10.5) to make the original 2d invisible and see just the scrolling network-graph (figures 4.10, 20.14).

\footnotetext{
${ }^{5} \&$ is provided as an alternative key hit for scrolling because \# is apparently not available on some Mac systems.
} 


\subsubsection{2d+time scrolling}

Toggle a $2 \mathrm{~d}+$ time presentation (section 32.10 .2 ) between successive vertical sweeps and scrolling.

\subsection{4 +..tog time-step pause}

Enter + (the plus key) on-the-fly to pause after each time-step and other options (also available from the pause prompt, section 32.16.8). A top-right prompt is presented similar to the following,

time-step 6828, next-ret, reset count-r, save current-s: stepback $\mathrm{x}$ timesteps $(\max =$ frazen-gens $=19$

on-the-fly/interrupt options $=\mathbf{y} / \mathbf{i}$, end pause-q: (values shown are examples)

Enter return for the next time-step. The prompt will reappear until end pause-q is entered. The full list of options is described below,

$$
\begin{aligned}
& \text { options and info } \ldots \frac{\text { what they means }}{\text { time-step } 6828} \ldots \text { the current time-step (for example). } \\
& \text { next-ret } \ldots \text { enter return for the next time-step. } \\
& \text { reset count-r } \ldots \text { to reset the count to } 1 . \\
& \text { save current-s ... to save the current space-time pattern. }
\end{aligned}
$$

stepback $x$ time-steps $(\max =$ frozen-gens $=19 \ldots$ enter the number of time-steps to stepback and restart from a previous time-step. The maximum stepback is the frozen generation size, preset in section 31.2.3, or reset on-the-fly in section 32.11.2.

on-the-fly/interrupt options $=\mathbf{y} / \mathbf{i} \ldots$ enter $\mathbf{y}$ to select any on-the-fly option presented in a small top-right window. Enter $\mathbf{i}$ to reselect the interrupt/pause prompt (section 32.16).

end pause-q ... to exit the prompt and continue.

\subsection{5 $<$..slow $/$ max speed}

Enter $<$ (left arrow key) on-the-fly to slow down the iteration speed of space-time patterns by half (eventually iteration will stop). Enter $>$ to restore iteration back to full speed.

\subsection{6 q/Q..pause/no-options}

Enter $\mathbf{q}$ on-the-fly to pause space-time patterns. The top-right interrupt/pause window will appear (section 32.16). To avoid showing the window which may cover required parts of the screen ${ }^{6}$, enter Q. Thereafter $\mathbf{q}$ will pause but no longer display the window - the following bottom-right prompt is presented instead,

\section{ops-Q cont-ret:}

Enter $\mathbf{Q}$ to restore the original pause method, or return to continue space-time patterns.

\footnotetext{
${ }^{6}$ The interrupt/pause window can toggle itself (option $\mathbf{W}$ ) to reveal what is beneath.
} 


\subsection{Interrupting space-time patterns}

If the space-time pattern iteration is interrupted on-the-fly with $\mathbf{q}$, or if "end pause" is set in section 32.13.2 and space-time patterns reach the foot of the DDLab screen, the program will pause - a large top-right pause window is then displayed ${ }^{7}$ in two sections; the upper section shows network, time-step and rule data - described in section 32.15; the lower section presents a number of interrupt/pause options — described in sections 32.16 to 32.16.9.

\subsection{Rule details for space-time patterns}

On pausing space-time patterns (on-the-fly q) the upper section of the top-right pause window shows network and time-step data, and data ${ }^{8}$ about the current single rule (or the rule at cell index 0 for a mixed-rule network). An example of the data for $v 2 k 5$ rcode is shown below,

a1 $1 \mathrm{~b}$ c1 26 - $\quad \operatorname{rcode}(\mathrm{dec})=\mathbf{2 7 0 2 9 5 0 6 9 4}$ (the rule)

rcode $\mathrm{v} 2 \mathrm{k} 5 \mathrm{ld}=0.406 \mathrm{ld}-\mathrm{r}=0.812 \mathrm{zl}=0.617 \mathrm{zr}=0.578 \mathrm{Z}=0.617188$

$\mathrm{C}=0 / 5 \mathrm{~d}=150$ time-step $=1597$

network and time-step data

$1 d=150 \ldots$ the network dimension and size for $1 \mathrm{~d}$, or the equivalent for $2 \mathrm{~d}$ or $3 \mathrm{~d}$, for example, $2 \mathrm{~d}=\mathbf{4 0 \times 4 0}$ or $\mathbf{3 d}=\mathbf{2 0 \times 2 0 \times 2 0}$.

time-step ... the current time-step number.

key to rule data

(the rule) ... its bit/value pattern (as much as will fit), in decimal (for small tables), and in hex (as much as will fit).

rcode ... or kcode or tcode - the rule type.

$\mathbf{v} \mathbf{2 k 5} \ldots$ the value-range $v$ and neighborhood size $k$. A $k$-mix will be indicated, for example v2k(2-7).

$\mathbf{l d}, \mathbf{l d}-\mathbf{r} \ldots \frac{\text { for a full rule-table (rcode) only }}{\lambda \text { parameter[17], } \lambda \text { ratio[25]. }}$

zl, zr, Z ... $Z_{\text {left }}, Z_{\text {right }}$, the $Z$ parameter[25, 32].

$\mathbf{C}=\mathbf{0} / \mathbf{5} \ldots$ the number of canalyzing inputs, in this case none, out a possible $5(k=5)$.

$* 3 * * * \ldots$ shows exactly which of the $k$ inputs are canalyzing (if none this is not shown)

— for example, 1 canalyzing input, at neighborhood index 3.

For a mixed- $k$ network, rule date is omitted, so an example of the remaining data would be similar to the following,

rcode $\mathrm{v} 2 \mathrm{k}(1-9) 1 \mathrm{~d}-150$ time-step $=256$

\footnotetext{
${ }^{7}$ The display of the pause window can be suppressed in section 32.13.6.

${ }^{8}$ This data is similar to the data in the rule window (section 16.19).
} 


\subsection{Space-time pattern interrupt/pause prompt}

On pausing space-time patterns (on-the-fly q) the lower section of the top-right pause window ${ }^{9}$ provides a range of context dependent interrupt/pause options ${ }^{10}$ for amending/saving/loading the rule and state, and other functions including displaying the network, network-graph and generating a PostScript file, as well as any on-the-fly options from section 32.3.

The following is presented in a top-right window,

rule:save/load/revise/rnd/trans-s/l/v/r/t net-n sample:load/keep-E/K

PScript-P Z:high/low-Z/z canal-C g-rules(rnd/seq)-G state:rev-e rnd/Ham/blk/orig/last-R/H/k/o/ sng:pos/neg-5/6 save/load-S/L graph-g/p, border-B filter-f skip-X pause-N step-x/+ top-T on-the-fly-y hide-W no-ops-Q back-q cont-ret:

Note that some of the options depend on the context, and only appear as follows,

$$
\begin{aligned}
& \text { options ... context } \\
& \text { trans-t ... not in TFO-mode. } \\
& \text { Z:high/low-Z/z ... single rule networks, and not in TFO-mode. } \\
& \text { canal-C ... not in TFO-mode. } \\
& \text { g-rules(rnd/seq)-G ... if a complex rule collection is detected for the current rule } \\
& \text { type, } v \text { and } k \text { (section 32.6.1). }
\end{aligned}
$$

Enter q to backtrack (initially to section 32.17). Enter return to continue space-time patterns, including any changes. The remaining options are listed in the following categories,

$$
\begin{aligned}
& \text { category } \cdots \text { section } \\
& \text { revising rule/net ... 32.16.1 } \\
& \text { classified samples of rule-space ... } 32.16 .2
\end{aligned}
$$

capturing space-time patterns as a vector PostScript file ... 32.16.3

$$
\text { revising the seed ... 32.16.4 }
$$

space-time patterns on the network-graph ... 32.16.5

$$
\text { network border ... } 32.16 .6
$$

finer control of filtering ... 32.16 .7

skip, pause and step ... 32.16.8

miscellaneous options ... 32.16 .9

\footnotetext{
${ }^{9}$ Showing the interrupt/pause window can be suppressed (section 32.13.6), or the window can be toggled to uncover the hidden area with option hide-W.

${ }^{10}$ Some of these options are similar to "further attractor basin complete" options in section 30.5.
} 


\subsubsection{Revising rule/net}

The following rule and network options in section 32.16, are explained below,

rule:save/load/revise/rnd/trans-s/l/v/r/t net-n ... (trans-t not in TFO-mode)

canal-C ... (not in TFO-mode)

$\mathbf{Z}:$ higher $/$ lower-Z/z ... (single rule networks only, and not in TFO-mode)

$\underline{\text { options } . . . ~ w h a t ~ t h e y ~ m e a n ~}$

save/load-s/l . . enter $\mathbf{s}$ or $\mathbf{l}$ to save or load the rule (section 35.2). For mixed rule networks this applies to the rule at cell index 0 only.

revise-v ... to review/revise the rule in a lower-right window as described in chapter 16. For mixed rule networks the following prompt is presented below the top-right window to select the cell index,

enter cell index, 149-0: (for example)

rnd-r ... to reset a new random rule, or all rules at random in a rulemix.

trans-t ... (rcode only) to transform the rule as described in chapter 18. For mixed rule networks the following prompt is presented below the top-right window to select the cell index,

enter cell index, 149-0: (for example)

Note that the transform options allow canalyzing inputs to be set for the whole network as well as for the selected rule (chapter 15).

net-n ... for the many network architecture options described in chapter 17, including viewing, revising, transforming, filing, rewiring, the Derrida plot (chapter 22), and learning (chapter 34).

canal-C ... (not in TFO-mode) to change or set canalyzing inputs. For a single rule network the canalyzing prompts comply with section 18.6 but are presented in a lower-right window. For a mixed rule network the canalyzing prompts are described in chapter 15.

g-rules(rnd/consec)-G ... (if a collections of complex rules is detected) to choose either a random or consecutive rule from the collection (loaded on-the-fly with $\mathrm{g}$ - section 32.6.1). The current status is shown in the prompt order (i.e. consec/rnd). The same can be set in section 31.2.8.

$\mathbf{Z}:$ higher/lower-Z/z ... (single rule networks only, and not in TFO-mode) enter $\mathbf{Z}$ to progressively force the $Z$-parameter higher, or enter $\mathbf{z}$ to force it lower, as in section 32.5.5 and 16.3. 


\subsubsection{Classified samples of rule-space - load/keep}

The following options for loading/displaying samples of automatically classified rule-space (chapter 33) are explained below.

sample:load/keep-E/K

$\underline{\text { options } . . . ~ w h a t ~ t h e y ~ m e a n ~}$

load-E ... to load, then display, a new sample (section 33.5).

keep-K ... to keep, then display, a sample that is already loaded (section 33.5).

The samples can also be loaded on-the-fly (section 32.6.2), and scanned (section 32.6.3).

\subsubsection{Directly scanning for PostScript}

option ... what it means

PScript-P ... (for 1d STP, or a snapshot of $2 d$ or the $2 d$ version of $3 d$ ) enter $\mathbf{P}$ in section 32.16 to directly capture 1d space-time patterns (STP), or a snapshot of $2 \mathrm{~d}$ STP, as a vector PostScript file, by directly scanning the DDLab image irrespective of the native dimensions. The method is described in section 32.18 .

An advantage of direct scanning is that the PostScript colors will be the same as the screen image, according to the many alternative on-the-fly presentations. For a native PostScript snapshot (1d, 2d or 3d) see section 32.16.4.

\subsubsection{Revising the seed and native PostScript}

The following network state (seed) options in section 32.16, are explained below,

state: rev-e rnd/Ham/blk/orig/last-R/H/k/o/ sng:pos/neg-5/6 save/load-S/L $\begin{aligned} & \text { options } \cdots \text { what they mean } \\ & \text { rev-e } \cdots \text { to review/revise/save/load the state (seed) and many oth } \\ &\text { are presented in a lower center window (section } 21.1) . W \\ & \text { the following top-right prompt gives a choice of three } \\ & \text { follows, } \\ & \quad \text { revise/reset seed: original-o last-l current-c: }\end{aligned}$

Enter o for the original seed, set or reset (here) in section 21.1. Enter l for the latest seed that was changed, either on-the-fly with any key hit in section 32.8, or in section 21.1. Enter $\mathbf{c}$ for the current state, at the time-step of the pause (section 32.16).

These options include vector PostScript capture (1d, 2d and $3 \mathrm{~d})$ according to native dimensions - the only method for the $3 \mathrm{~d}$ isometric as in figure 32.18 Left (section 21.4.10). 
rnd-R ... for a new random seed. The preset density bias (section 21.3.1) of the seed (section 21.3) is respected — the same as on-the-fly key hit 4 (section 32.8.1).

Ham-H ... to change the values of a percentage of cells in the current state distributed at random. For binary networks $(v=2)$, the number of changed, $H$, is known as the Hamming distance. For $v \geq 3$ the $H$ cells will be assigned values at random that differ from the original values. The following topright prompt is presented,

\section{enter \% ramdom Hamminig change to current state:}

blk-k ... for a central random central block of cells. The rest can be kept as is, or set to zero. The block size was originally specified in section 21.3 but can be changed here, and this will remain the block size for on-the-fly blocks set with $\mathbf{v}$ (section 32.8.1). The following top-right prompt is presented,

rnd block: change size (now 30), rest: keep-k, all 0s-(def):

If a new block size is entered the prompt reappears showing the new size. Enter $\mathbf{k}$ to keep the rest of the state, return for the new block on a zero background. The preset density bias (section 21.3.1) block is are respected.

orig-o ... to restore the original seed set (or reset) in section 21.1.

last- $\sim$.. to restore the latest seed that was changed, either on-the-fly with any key hit in section 32.8, or in section 21.1.

sng:pos/neg-5/6 . . for $v=2$ : enter $\mathbf{5}$ for a positive singleton seed, a central cell set to 1 , the remainder set to 0. Enter 6 for a negative singleton seed.

For $v \geq 3$ : enter $\mathbf{5}$ for a central cell set to any random value except zero against a zero background. Enter $\mathbf{6}$ for a central cell with any random value against a different uniform background.

save-S ... to enter the state (seed). When $\mathbf{S}$ is entered, the following top-right prompt gives a choice of three possibilities (as for rev-e) as follows,

save seed: original-o last-l current-c:

Enter o for the original seed, set or reset in section 21.1. Enter $\mathbf{l}$ for the latest seed that was changed, either on-the-fly with any key hit in section 32.8, or in section 21.1. Enter c for the current state, at the time-step of the pause (section 32.16).

Then save the state/seed - . eed file (section 35.2).

load-L ... to load a new seed - . eed file, (section 35.2).

\subsubsection{Space-time patterns on the network-graph}

graph-g/p ... (p if the network-graph is active)

Enter g to set up a network-graph (chapter 20), then run space-time patterns within it according to the graph layout and node sizes. This allows considerable presentation flexibility. section 32.19 gives further 
details. The network-graph can be scrolled on-the-fly (section 32.13.3.3). Normal space-time patterns will continue to run simultaneously in the usual way unless disabled with on-the-fly (key hits $\mathbf{S}$, or $\mathbf{J}$ if scrolling the network-graph). Enter $\mathbf{g}$ to deactivate graph space-time patterns if active.

Enter $\mathbf{p}$ to capture a snapshot of the current network-graph as a vector PostScript file (section 32.19.2 and chapter 36).

\subsubsection{Network border}

border-B ... By default, networks in 1d, 2d and 3d have periodic boundaries, but this can be effectively eliminated if required, creating "falling off the edge" boundaries by fixing the value of border (edge) cells. This can be useful to absorb glider and other propagating structure which would otherwise "wrap around" and disturb the central space-time pattern.

If $\mathbf{B}$ in section 32.16, the following consecutive prompts are presented to set the border width and value, in a top-right window,

fixed border: width(0-9, now 1): (for example)

border value: $(0-2)$, now 0$)$ : (for $v=3$ )

\subsubsection{Finer control of filtering}

if input-entropy is active (section 31.4)

filter-f . . Enter $\mathbf{f}$ in section 32.16 for finer control of filtering than in section 32.11.5. The following top-right prompt is presented (for example, for the $v 2 k 5 \mathrm{CA}$ in figures 32.26 and 32.27 ),

filtered: 2110 (if unfiltered none shown here)

filter/undo enter +/- k-index (31-0), max-m reset-r:

filtered: 2110 show the neighborhood indexes that are currently filtered, otherwise none is shown. Specific neighborhoods can be filtered and unfiltered. For example, to filter neighborhood index 26 enter $\mathbf{+ 2 6}$, to unfilter neighborhood index 21 enter -21. Enter $\mathbf{m}$ to filter the current most frequent unsuppressed neighborhood. Enter $\mathbf{r}$ to reset, i.e. unfilter all neighborhoods. Any change is immediately indicated in the top line of the prompt. Enter return to accept. On resuming space-time patterns the changes will be applied, and filtered neighborhoods will be indicated by dots alongside the bars in the lookup-frequency histogram.

\subsubsection{Space-time - skip, pause and step}

The following options to skip, pause, backtrack and step through time-steps, in section 32.16, are explained below, (see also section 31.2.7),

skip-X pause-N step-x/+ 


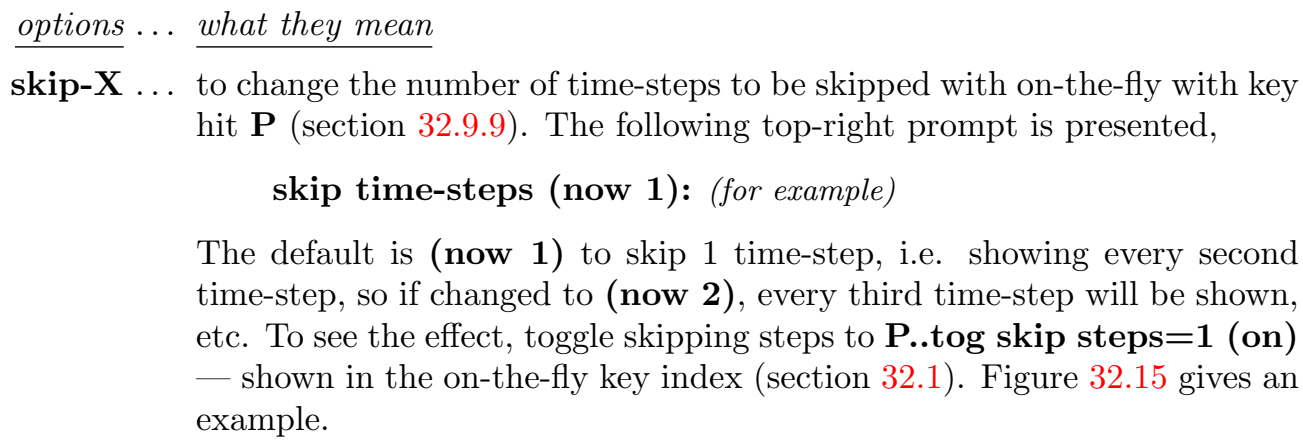

The default is (now 1) to skip 1 time-step, i.e. showing every second time-step, so if changed to (now 2), every third time-step will be shown, etc. To see the effect, toggle skipping steps to P..tog skip steps=1 (on) — shown in the on-the-fly key index (section 32.1). Figure 32.15 gives an example.

pause-N . . . to set a pause in the space-time pattern after a preset number time-steps. The following top-right prompt is presented,

pause: screen full-p, after f-gens(20)-f or enter time-step (no pause-def):

Enter $\mathbf{p}$ to set the end pause on, so a pause always occurs when the screen is full, as in section 32.13 .2 (takes effect only for $1 \mathrm{~d}$ and $2 \mathrm{~d}+$ time space-time if not scrolling), or return set the end pause off.

Enter $\mathbf{f}$ for a one time pause after the frozen generation number of timesteps set in section 32.11.2 (default 20), or enter a number for a one time pause after that number of time-steps.

step-x ... to step through space-time patterns in blocks of time-steps separated by a pause. The block size is the number of time-steps that were set with pause-N above (default 20).

step- $+\ldots$ to pause after each time-step and other options. A top-right prompt is presented similar to the following,

\title{
time-step 66, next-ret, reset count-r, save current-s stepback $\mathrm{x}$ time-steps $(\max =$ frozen-gens $=19$ on-the-fly/interrupt options $=y / i$, end pause-q:
}

Enter return for the next time-step. The prompt will reappear until end pause-q is entered. This is also an on-the-fly prompt (section 32.13.4) where the full list of options is described.

\subsubsection{Miscellaneous options}

\author{
options ... what they mean \\ top-T ... to continue $1 \mathrm{~d}$ (and $2 \mathrm{~d}+$ time) space-time patterns starting at the top of \\ the screen. This also resets the time-step count. \\ on-the-fly-y ... to activate any on-the-fly option during a pause. \\ The following top-right prompt is presented in a short window, \\ enter on-the-fly option:
}

Enter any key listed in the current on-the-fly key index (as in figure 32.1). The pause prompt (section 32.16) will reappear to make other changes if 
required - enter return to continue the space-time pattern, where the on-the-fly option selected will take effect.

hide-W ... to toggle the whole pause/interrupt window and reveal what's hidden beneath - enter $\mathbf{W}$ again to restore the window.

no-ops- $\mathbf{Q}$... enter $\mathbf{Q}$ to pause without displaying the pause/interrupt window so as not to hide that area of the screen (an alternative to hide-W above. When space-time patterns are resumed with return, pausing with $\mathbf{q}$ gives a bottom-right title-bar prompt, instead of the usual pause/interrupt window,

ops-Q cont-ret:

Enter return to continue - pausing with q gives the title-bar prompt.

Enter $\mathbf{Q}$ to continue but cancel the title-bar pause - pausing with $\mathbf{q}$ resumes the pause/interrupt window.

back-q ... to backtrack form space-time patterns, initially giving top-left options described in section 32.17 .

cont-ret ... resume space-time patterns from before the pause, with changes taking effect.

\subsection{Quit and further options}

Enter $\mathbf{q}$ in section 32.16 to quit space-time patterns and backtrack. The following top-left prompt is first presented,

\section{graphics-g speed(max)-s \\ seed-e \\ net-n back-q cont-ret:}

Enter $\mathbf{q}$ to backtrack to previous options, return to continue the space-time pattern, or select an option below,

$$
\text { options... what they mean }
$$

graphics-g ... to alter the graphics setup described in section 6.3.

$\operatorname{speed}(\max )-\mathbf{s} \ldots$ to reduce the speed of space-time pattern iteration, or to restore full speed. The following top-right prompt is presented,

basin speed: max-(def), or slowmotion (try 300):

Enter return for full speed, or a number to slow down - the greater the slower.

seed-e ... to revise the seed. The seed options described in chapter 21 are presented in a lower central window, a repeat of rev-e in section 32.16.4.

net-n ... for the many network architecture options described in chapter 17, including viewing, revising and filing, the Derrida plot (chapter 22), and learning (chapter 34), a repeat of net-n in section 32.16.1. 

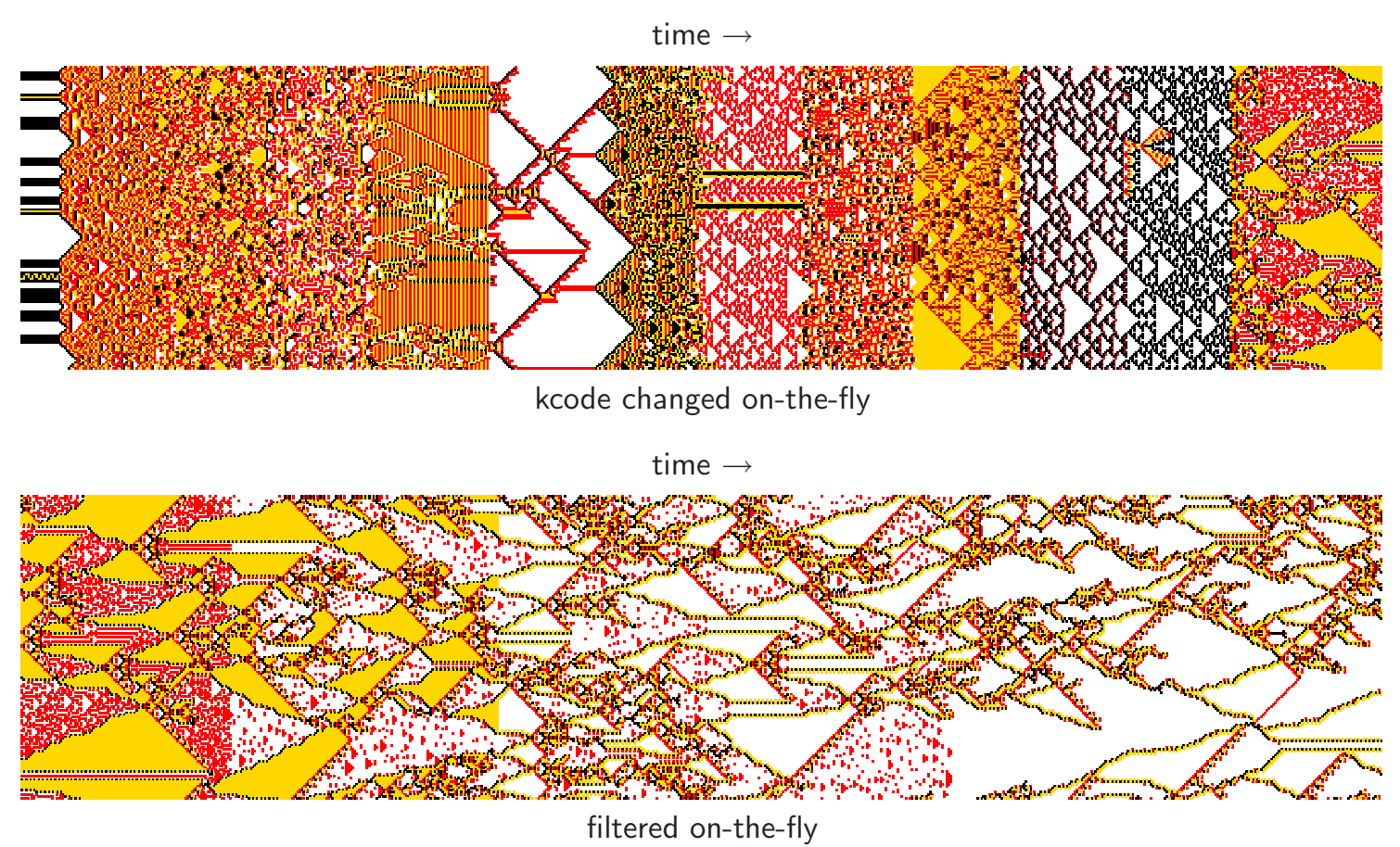

Figure 32.33: Directly scanned 1d space-time patterns for vector PostScript, $v 4 k 3 \mathrm{kcode}, n=150$, 669 time-steps. The images shown are rotated by $90^{\circ}$ so time flows from left to right.

Top: the rule was changed randomly on-the-fly (section 32.5.1) several times as the space-time pattern scrolled. Each new rule picked up the current state as its initial state.

Bottom: the space-time pattern was allowed to run on keeping the last rule, $v 4 k 3$ kcode (hex) 89d17b2278, and the space-time pattern was filtered 4 times on-the-fly (section 32.11).

\subsection{Directly scanning space-time patterns for vector PostScript}

$1 d S T P$, or a snapshot of $2 d$ or the $2 d$ version of $3 d$

The space-time image on the screen can be directly scanned to generate a vector PostScript (*.ps) file (default filename my_sPS.ps). This is the only method available for $1 \mathrm{~d}$ space-time patterns (i.e. including time-steps), and one of the possible methods for a snapshot of $2 \mathrm{~d}$ or the $2 \mathrm{~d}$ version of $3 \mathrm{~d}$. An of advantage of direct scanning as opposed to using information about a state held in memory ${ }^{11}$ is that the PostScript colors will be exactly as presented at that moment, according to the many alternative on-the-fly presentations options, such as color by neighborhood, filtering, and frozen cells. For 1d there could be new seeds and rules within one space-time pattern (figure 32.33).

Direct scanning still allows resetting the scale, divisions between cells, dots on zero cells, and selecting greyscale or color, and follows the current screen dimensions (section 32.10) irrespective

\footnotetext{
${ }^{11}$ For a PostScript snapshot of the native space-time pattern held in memory $(1 \mathrm{~d}, 2 \mathrm{~d}$ or $3 \mathrm{~d})$, enter state:rev-e in section 32.16, select the state required (original, last, or current), then follow the seed methods in sections 21.4 .7 and 21.4.10.
} 
of the native dimensions.
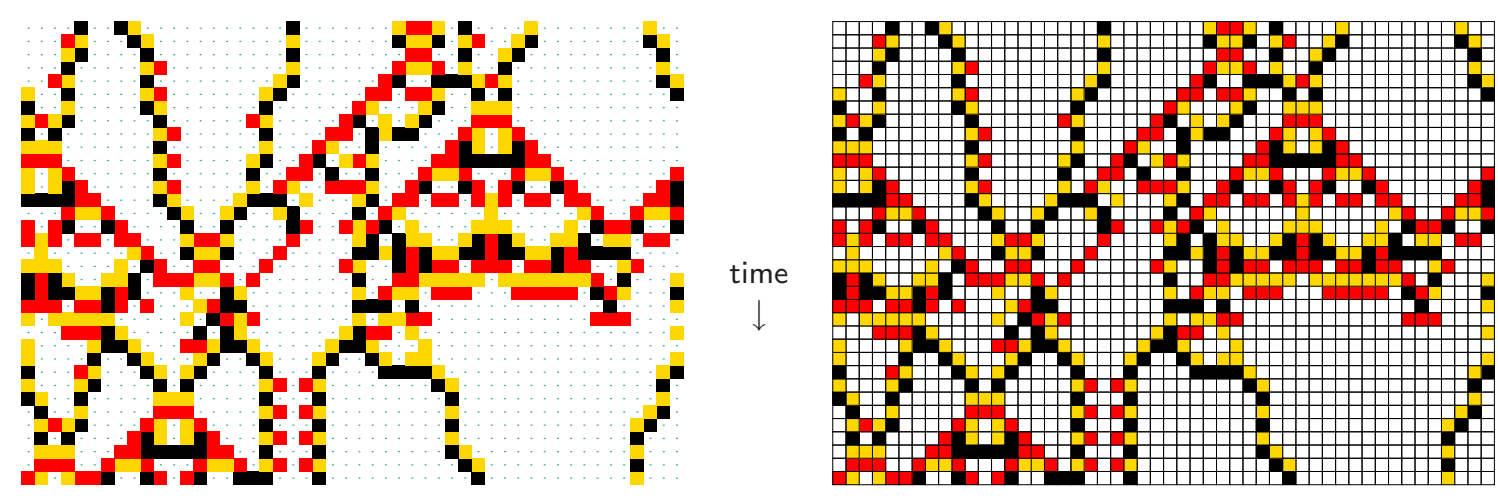

Figure 32.34: PostScript images from a scanned 1d space-time pattern, for the same $v 4 k 3$ kcode as in figure 32.33, $n=50,35$ time-steps. Left: dots in zero cells. Right: black divisions between cells.

Enter $\mathbf{P}$ in section 32.16 for the top-right PostScript options.

For 1d space-time patterns, direct scanning is the only method, and just part of the space-time pattern can be scanned - the prompt starts as follows,

create PostScript image for 1d space-time patterns, exit-q

$1 \mathrm{~d}$ size $(\max / \mathbf{d e f}=150)$ : time-steps $(\max / \mathbf{d e f}=669)$ : (values shown are examples)

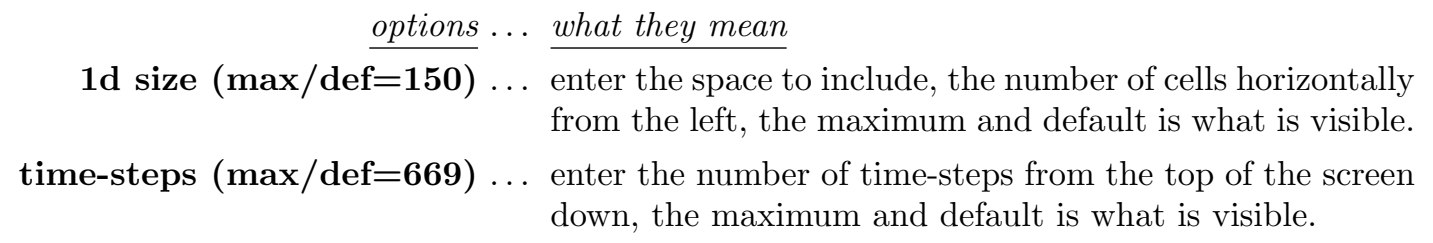

For a 2d snapshot (figures 32.7, 32.19, 32.25), direct scanning (direct-d) is just one of several possible methods, the others are described in section 21.4 .9 , - the prompt starts as follows,

create PostScript image for $\mathbf{2 d}, \mathbf{4 0 x} 40$ (values shown are examples)

symbols-s greyscale-g color-c direct-d exit-q (def-c):

Enter $\mathbf{d}$ for direct scanning.

For both $1 \mathrm{~d}$ space-time patterns, and a $2 \mathrm{~d}$ snapshot, the top-right prompts continue as follows,

greyscale-g color-c (def-c): (the default follows the last selection)

$$
\begin{aligned}
& \text { options ... what they mean } \\
& \text { greyscale-g: ... for greyscale instead of color. } \\
& \text { color-c (def-c): ... for color instead of greyscale. }
\end{aligned}
$$

This is followed by the prompt to amend other settings, 
cellscale $=1.00 \operatorname{dots}(\mathrm{off})=0.70 \operatorname{divs}(\mathrm{off})$, amend settings-a:

Enter return to accept the defaults, or enter a for the following prompts presented in sequence, change: cellscale: togdots-x: dotscale: $\operatorname{divs}(0,1,2)$ :

Enter changes required or return to accept defaults. The options are summarized below,

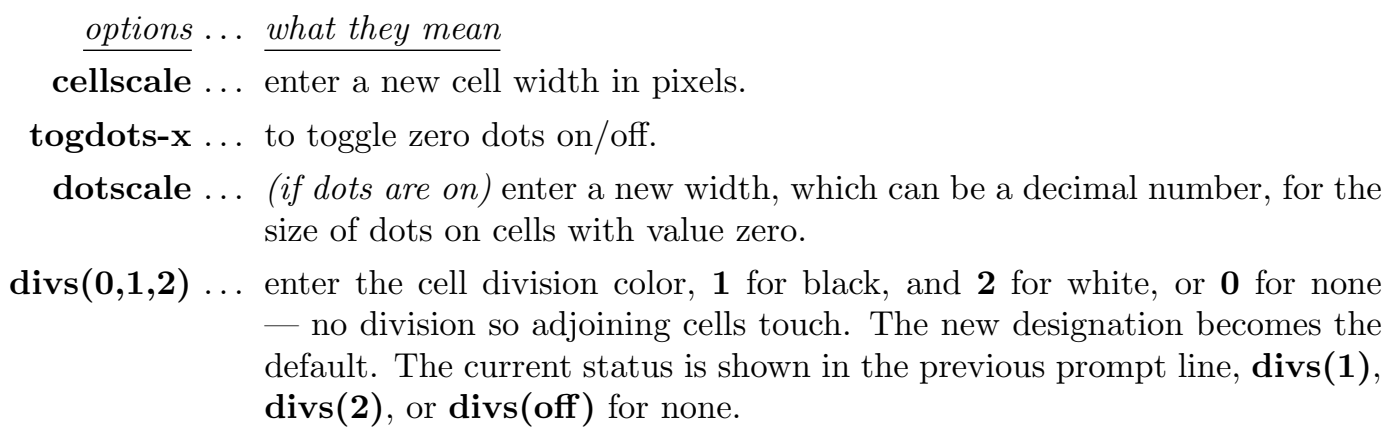

Once these choices are complete, the *.ps file is saved from the filing prompt (section 35.2). section 36.1 explains how to view, edit, and crop the PostScript image.

\subsection{Network-graph layout of space-time patterns}

Space-time patterns can be shown running within the network-graph layout (chapter 20) in addition to the normal presentation described in this chapter and chapter 31. This allows enormous flexibility, as the network-graph has a number of default layouts: circle, spiral, 1d, 2d (square or hex), $3 \mathrm{~d}$, as well as allowing dragging nodes and components, and many other options for rearranging the graph.

Enter $\mathbf{g}$ in section 32.16. The following top-right option is first displayed,

\section{show links-L (avoid for large networks), layout only-def:}

Enter return for the layout only, without links, which is much faster and more economical with memory, and may be essential for large (2d or $3 \mathrm{~d}$ ) networks. This option only appears if space-time patterns are interrupted. However, the links can be shown in the normal way by entering $\mathbf{L}$. The network-graph appears in a large central window, but for a layout only, the options in sections 20.2, 20.4, and 20.5 are abbreviated to omit link options.

In this case the initial top-right options (compare with section 20.2) are as follows,

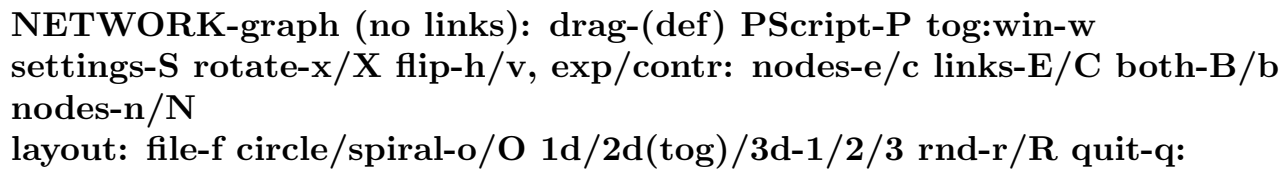

The top-right options for dragging nodes and fragments (compare with section 20.5) are as follows (for example), 


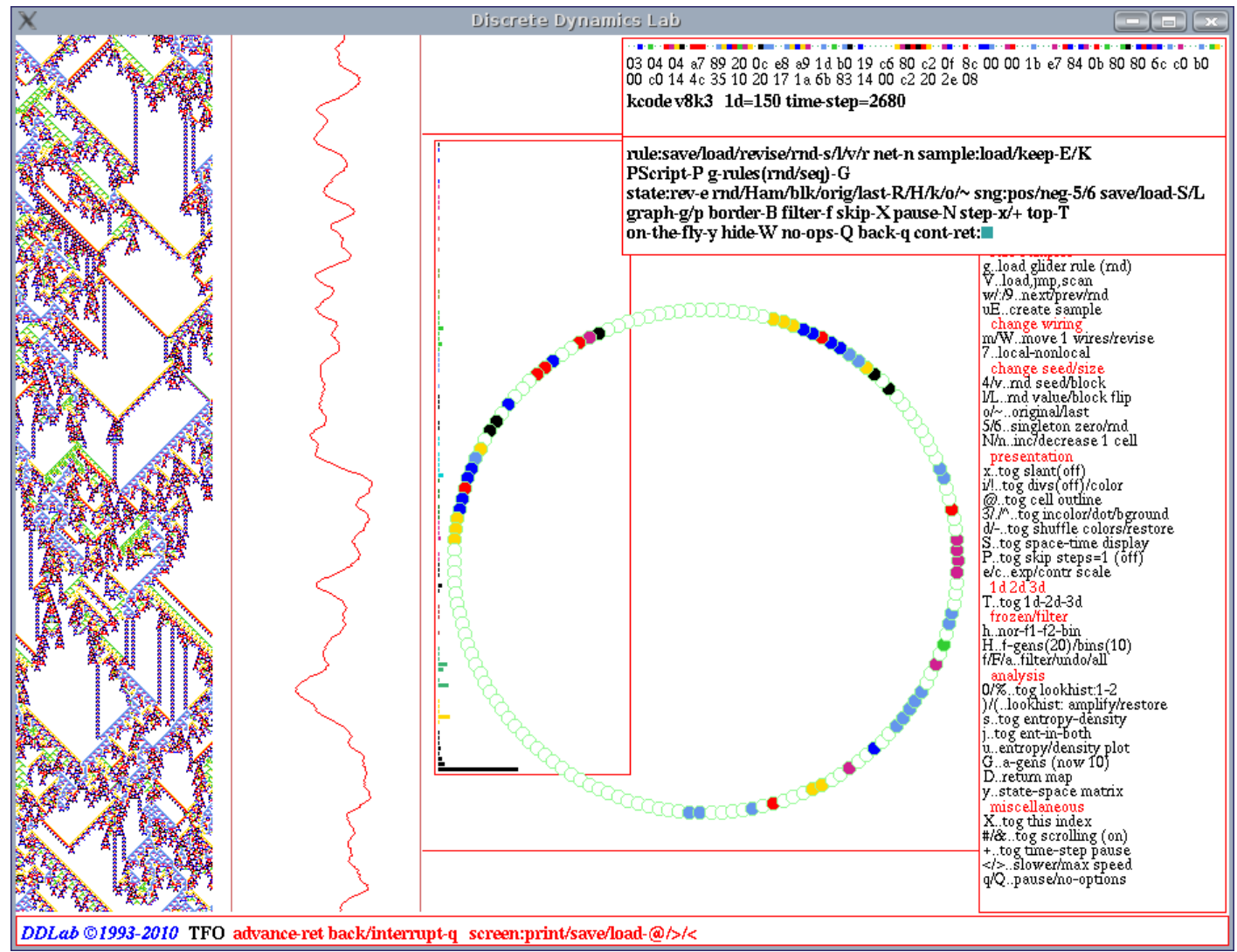

Figure 32.35: A snapshot of the DDLab screen, showing 1d CA space-time patterns (STP) according to the (default) circle network-graph layout, which reflects the actual geometry of a 1d CA with periodic boundary conditions. $n=150, v 8 k 3$ kcode, index 8 from the rule sample in figure 33.5. The normal space-time patterns are on the left of the screen. Many on-the-fly options apply simultaneously to the normal and network-graph layout STP.

node 205, single: drag/release - left mouse button, tog drag-d

not active?-click right button first, rotate-x/X flip-h/v

block-B

single-s all-a exit-q:

Rearrange the graph and the node sizes by the methods described in chapter 20. On exiting the graph routine, space-time patterns will be shown according to the final network-graph layout in a large central window, simultaneously with the normal space-time patterns.

\subsubsection{On-the-fly options within the network-graph}

Most current on-the-fly options (listed in figure 32.1) have the same simultaneous effect on both normal space-time patterns and space-time patterns within the network-graph, including colors and filtering. However some key hits have no effect or have special functions, listed as follows, 


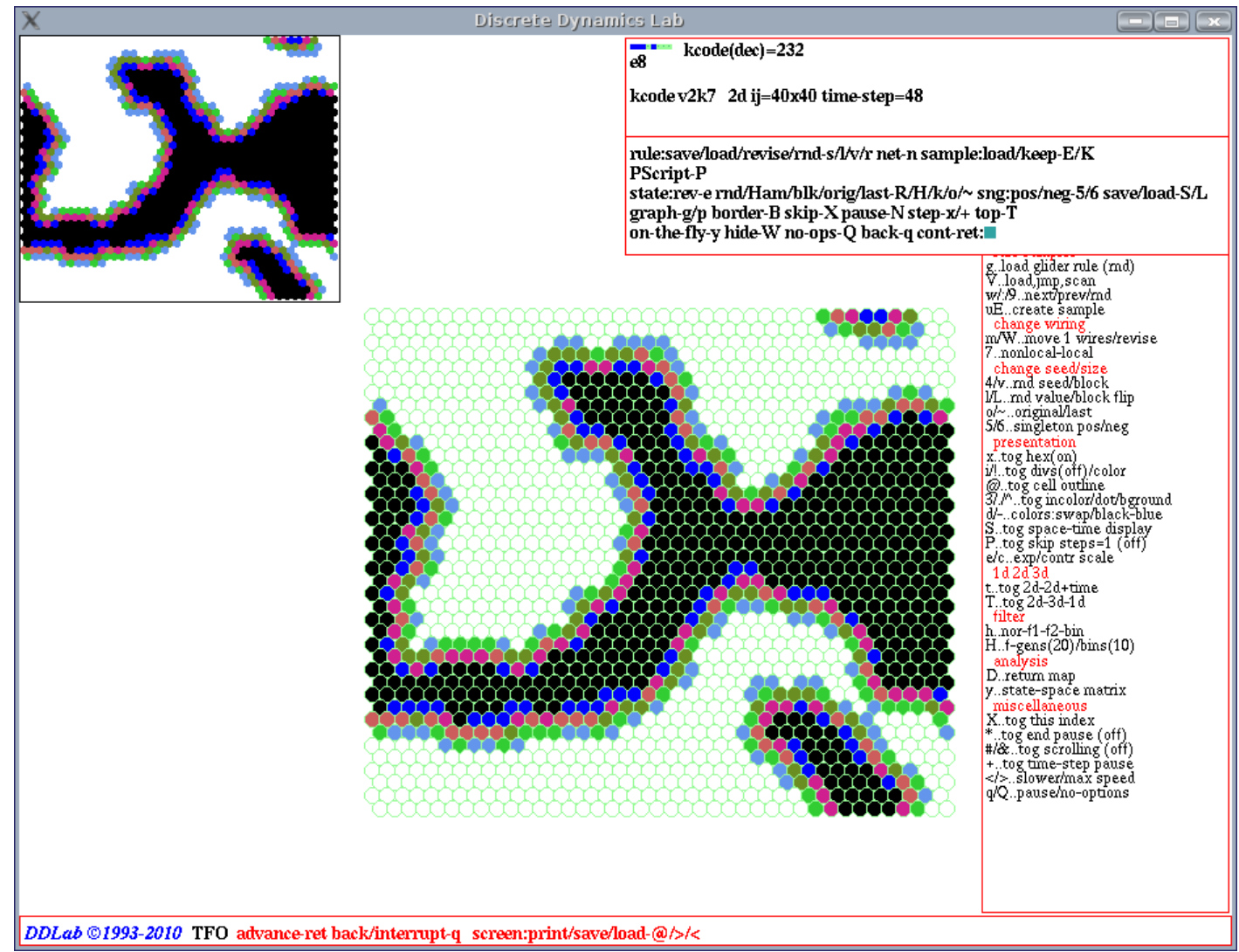

Figure 32.36: A snapshot of the DDLab screen, showing Center: 2d CA space-time patterns within the network-graph. Top-Left: the normal $2 \mathrm{~d}$ space-time patterns continue at the top-left of the screen. Most on-the-fly options apply to both normal and network-graph space-time patterns. $v 2 k 7$ tcode(dec) 232 (a modified majority rule) $n=40 \times 40$ with hexagonal layout and color by neighborhood.

on-the-fly category .... key hits with no effect, or special functions in the network-graph

change seed/size ... $\mathbf{N} / \mathbf{n} .$. inc/decrease $\mathbf{1}$ cell — turns off the network-graph.

presentation ... x..tog slant(off) or x..tog hex(off) - no effect.

@..tog cell outline - applies only to the network-graph (section 32.19).

i/!..tog $\operatorname{divs}($ off $) /$ color - no effect.

3/./^..incolor/dot/bground - only the "." (dot) has no effect.

S..tog space-time display - no effect.

e/c..expand/contr scale — no effect unless diagonally scrolling the network-graph — then $\mathbf{e} / \mathbf{c}$ also expands/contracts the spacing between network-graph time-steps (figure 32.16).

$1 d 2 d 3 d \ldots$ - no effect by any on-the-fly key hits.

analysis ... - no effect by any on-the-fly key hits.

miscellaneous... - \#/\&..tog scrolling - if 2d STP is active, scrolls both 2d STP and the network-graph simultaneously (section32.13.3) — otherwise no effect. 


\subsubsection{Network-graph space-time pattern as vector PostScript}

If the network-graph space-time pattern is active, the current time-step can be saved to a vector PostScript file. Enter $\mathbf{p}$ in the pause options (section 32.16). The following top-right prompt is displayed,

\section{save network-graph snapshot to PScript: abort-q greyscale-P color-def:}

Enter return for color, $\mathbf{P}$ for greyscale (to toggle the cell outline enter @ on-the-fly beforehand). The top-right filing prompt (section 35.2) will be displayed to select the filename (default my_sgPS.ps).

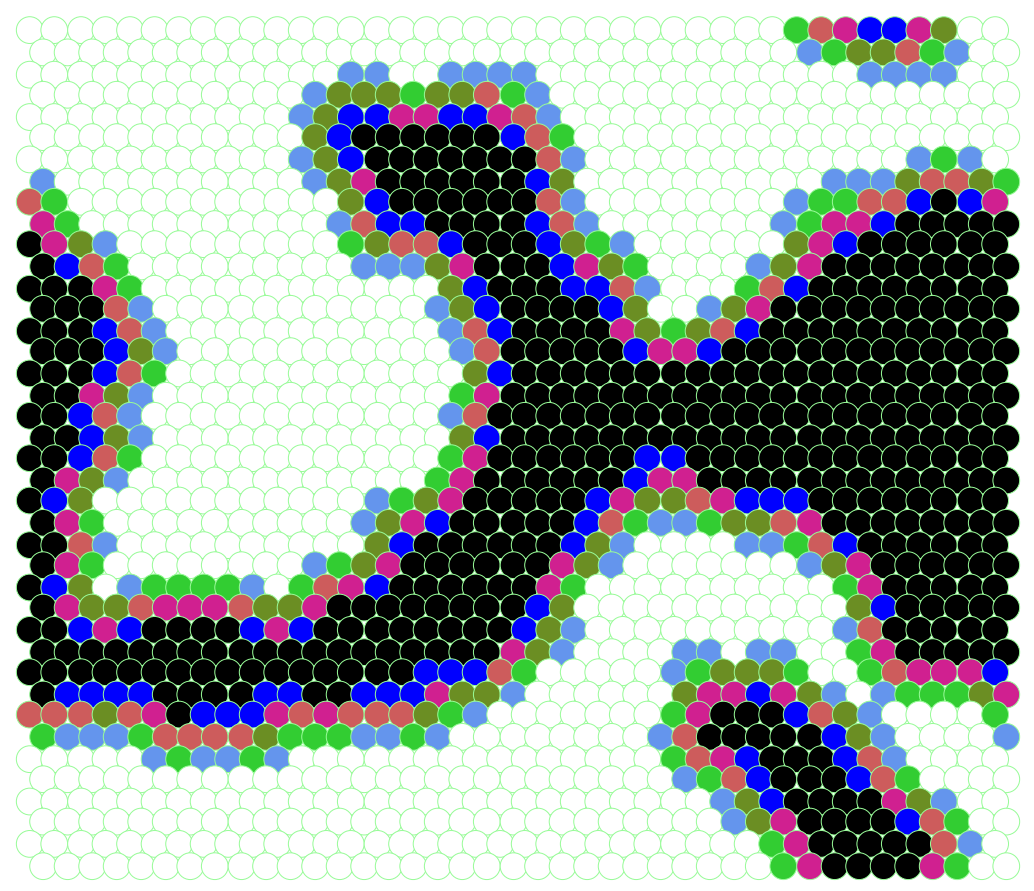

Figure 32.37: A vector PostScript image of network-graph in figure 32.36 - the same 2d CA spacetime-step. 


\section{Chapter 33}

\section{Classifying rule space}

This chapter describes methods to automatically classify samples of CA rule-space by inputentropy variability[29, 32, 38, 39], to distinguish between ordered, complex and chaotic rules as in figures 2.7, 33.1, and elsewhere, and to estimate the distribution of rule classes in rule-space, including creating, sorting, plotting, analysing and probing the sample. Originally applied to $1 \mathrm{~d}$ binary rcode[29, 32], the methods now apply equally to $v \geq 3$, for kcode and tcode in TFO-mode, and in $2 \mathrm{~d}$ and $3 \mathrm{~d}$ as well as $1 \mathrm{~d}$. Sample files for some combinations of $[v, k]$ and lattice dimensions illustrate classification results, and are available with DDLab (section 3.7.1). The collections of complex (or "glider") rules (section 32.6.1) were mostly picked out from these sample files.

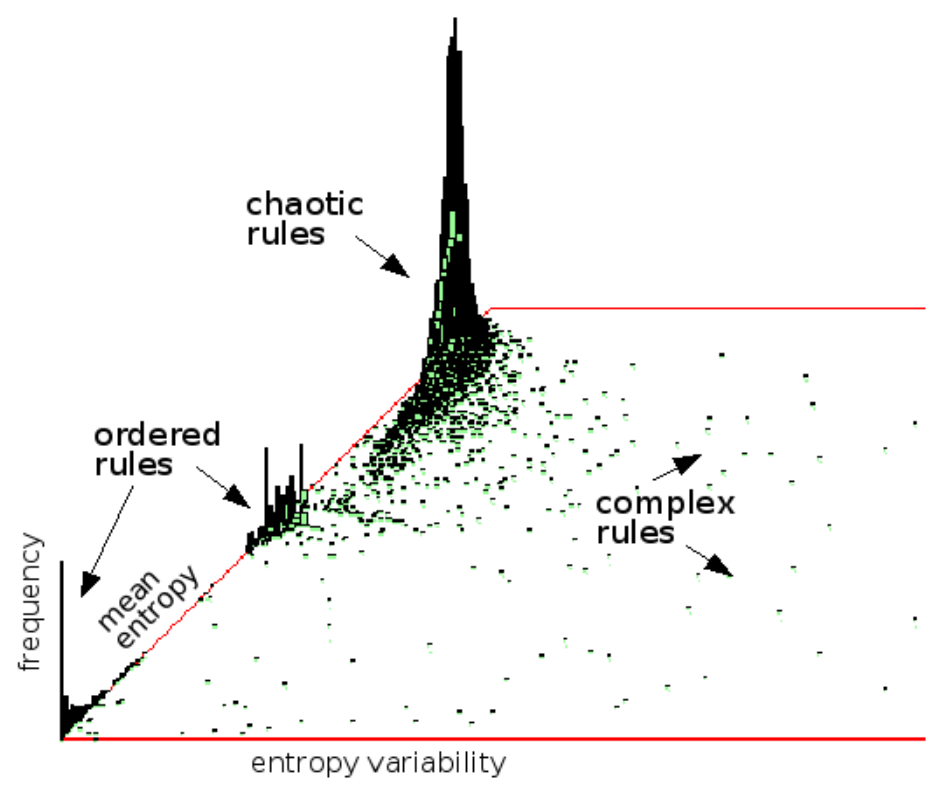

Figure 33.1: A 2d histogram for a sample of 15800 2d hexagonal unbiased CA kcodes $v 3 k 6$ (file v3k6bs.sta), plotting mean entropy against entropy variability (standard deviation). The method automatically classifies rules between ordered, complex and chaotic dynamics[38, 39]. The vertical axis shows the frequency of rules on a $256 \times 256$ grid - most are chaotic. 

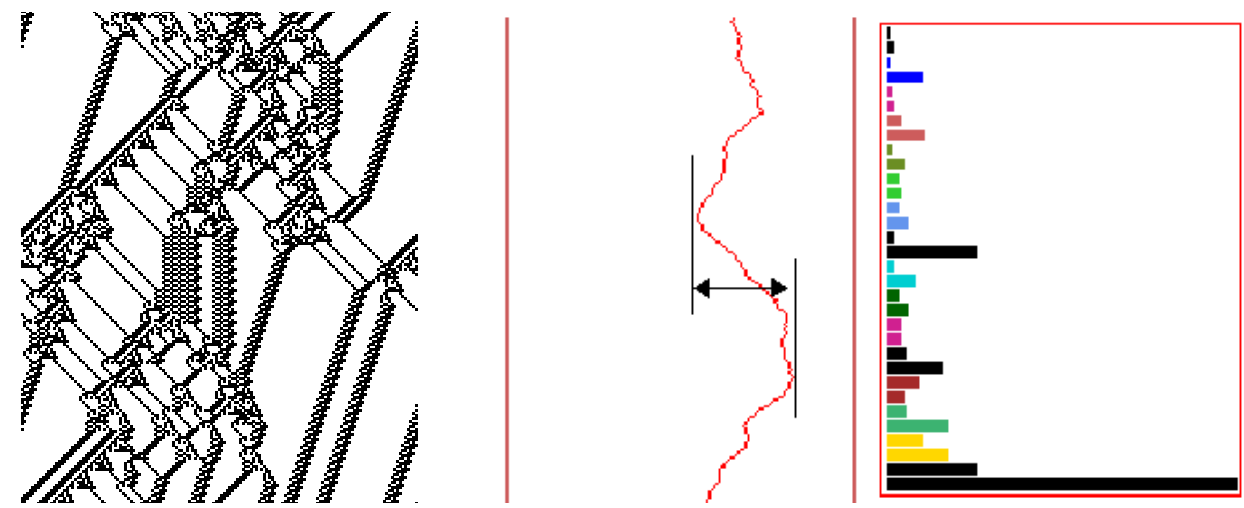

Figure 33.2: Left: the space-time patterns of a 1D complex CA with interacting particles, $v 2 k 5$ rcode (hex)6cle53a8, $n=150$ about 200 time-steps. Right: a snapshot of the input frequency histogram measured over a moving window of 10 time-steps. Center: the changing entropy of the histogram; its variability, either min-max (maximum up-slope as indicated) or standard deviation (sdev), provides a non-subjective measure to separate complexity from either order or chaos - high variability implies complex dynamics.

Rules can be generated at random or with some bias. Biases include isotropic rules, fixing the $\lambda$ parameter, or fixing the proportions of different values in the rule-table. In addition, the rule-table can be adapted so that the all-zero neighborhood outputs zero, or more generally all- $V$ neighborhoods output $V$. These biases make complex dynamics more probable.

For each successive rule, the space-time pattern is run from a set of random initial states. For each initial state, after a delay to allow the CA to settle into its typical behavior, the variability of the input-entropy and the mean entropy are recorded - these measure are described in section 33.1. Then the average results from the set of initial states are plotted - the entropy variability ( $x$ axis) against the mean entropy ( $y$-axis), and the data for each rule is appended to a . sta file. The results of an automatic classification can be interpreted as follows,

\begin{tabular}{r|c|c|c} 
& order & complexity & chaos \\
\hline mean entropy & low & medium & high \\
\hline entropy variability & low & high & low
\end{tabular}

High variability implies large scale structural interactions, often produced by particles colliding, because collisions create local chaos raising the entropy, from which particles re-emerge lowering entropy. The basic argument is that if the entropy continues to vary sufficiently in settled dynamics, moving both up and down, then some kind of self-organizing collective behavior must be unfolding. This might include competing zones of order and chaos, or different types of competing chaos, as well as glider dynamics.

So complexity can be separated by its high variability. Both order and chaos have low variability, but can be separated from each other by entropy (figure 33.1), though the probability of order diminishes with increased $v$ and $k$ in an unbiased sample. Once the sample is sorted, by variability followed by mean entropy, rule dynamics can be scanned by decreasing variability, from the highest at index 1 or any other starting point. The most interesting rules can be saved or added to collections of glider rules (section 32.6.1). 


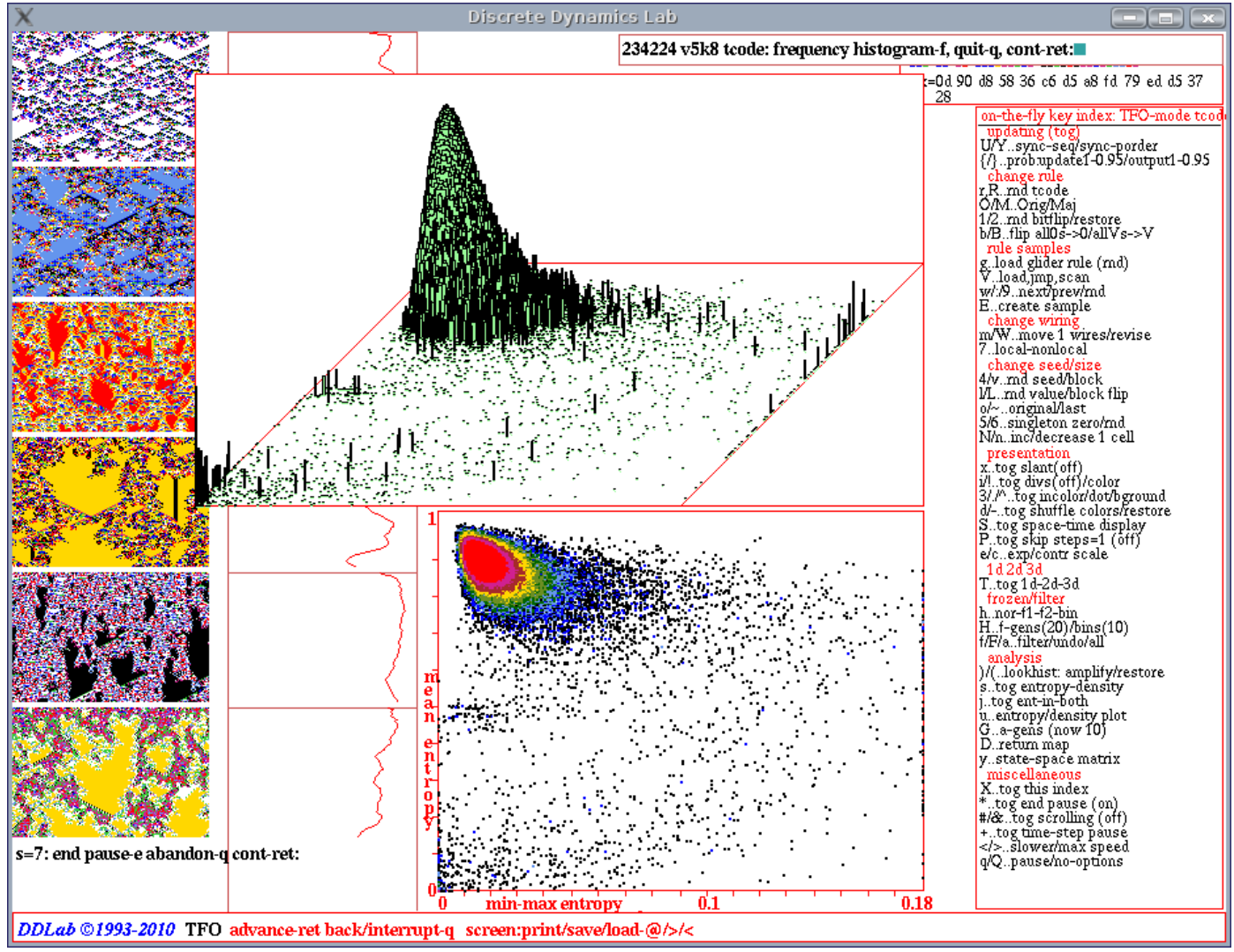

Figure 33.3: A snapshot of the DDLab screen, showing a 1d tcode unbiased sample (TFO-mode) with 234224 tcodes (file v8k5tm.sta), sorted by min-max entropy. Left: space-time pattern blocks of 100 time-steps showing sequential examples, pausing at sample 7 (section 33.7.2). Bottom Center: at the pause above, enter $\mathbf{q}$ then $\mathbf{K}$ to show the scatter plot, based on the min-max entropy (the maximum up-slope), where the frequency of points falling on a $266 \times 256$ grid is indicated by colors (section 33.6). Top Center: at the prompt in section 33.6, enter $\mathbf{q}$ then $\mathbf{f}$ for the $2 \mathrm{~d}$ histogram, with the frequency of points in each grid square represented on the $z$-axis, in this case in log form (section 33.6.2).
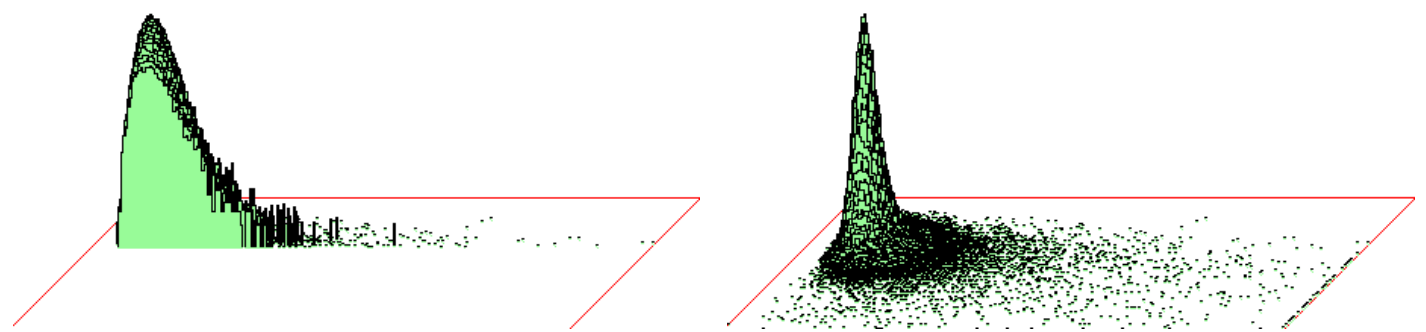

Figure 33.4: The 2d histogram in figure 33.3 can be presented according to Left: successive $y$-axis (mean entropy) slices - paused at $y=45$. and Right: a normal $z$-axis (frequency of rules) - the default. 


\subsection{Input entropy and variability (min-max or sdev)}

The input-frequency histogram tracks how frequently the different entries in a rule-table are actually looked up and is usually averaged over a moving window of time-steps. The input-entropy is the Shannon entropy ${ }^{1}$ of this input-frequency histogram.

One of two measures of variability of the input-entropy can be selected, either standard deviation $^{2}$ or a min-max measure - the maximum up-slope found so far - the rise in entropy following a minimum value (figure 33.2). When trawling for complex rules, min-max is preferable because standard deviation would give a high value to monotonic entropy decrease, characteristic of a foreground pattern gradually dying out, which would contaminate a sorted sample of complex rules. Dying out dynamics give low min-max so this problem is avoided.

\subsection{Creating a rule sample — initial prompts}

To automatically create a rule sample, or run a test, first set up any CA with the desired parameters: - SEED-mode or TFO-mode, $v, k$, and system size - 1d, $2 \mathrm{~d}$ or $3 \mathrm{~d}$. The size should be relatively small (say $n=150$ for $1 \mathrm{~d}, 40 \times 40$ for $2 \mathrm{~d}$ ) to amplify variability in complex dynamics because local order and chaos tend to balance each other out in a large network. Then run the space-time patterns (chapter 32). For 1d, scrolling should be set off (for speed) in section 32.13.3. The inputentropy plot should be active; this is the default for $1 \mathrm{~d}$, but for $2 \mathrm{~d}$ or $3 \mathrm{~d}$ it needs to be activated (sections 31.4, 32.12.3).

While the space-time patterns are iterating, select on-the-fly option $\mathbf{u E . . c r e a t e ~ s a m p l e , ~ e n t e r ~}$ $\mathbf{u}$ followed by $\mathbf{E}$. The initial $\mathbf{u}$ initiates an entropy-density scatter plot (section 32.12 .5 ). If this plot is current, the on-the-fly prompt is E..create sample, so just $\mathbf{E}$.

Alternatively, enter $\mathbf{q}$ for the space-time pattern pause interrupt (section 32.16), and enter $\mathbf{y}$ giving a small top-right prompt (which allows any on-the-fly option) enter on-the-fly option: enter $\mathbf{u}$, then $\mathbf{y}$ again followed by $\mathbf{E}$.

The following prompts are presented in turn in a top-right window (enter $\mathbf{q}$ to backtrack or exit),

entropy sample, automatic-a test-(def): sdev-s min-max-(def): record time-steps, start (def 30): end (def 430):

sample for each rule (def 5): bias rnd rules -b:

These options are summarized below — with more detail in the sections indicated.

\footnotetext{
${ }^{1}$ The Shannon entropy of the input-frequency histogram, the input-entropy $H$, at time-step $t$, for one time-step $(w=1)$, is given by $H^{t}=-\sum_{i=0}^{S-1}\left(\frac{Q_{i}^{t}}{n} \times \log _{2}\left(\frac{Q_{i}^{t}}{n}\right)\right)$, where $Q_{i}^{t}$ is the lookup frequency of neighborhood $i$ at time t. $S$ is the rule-table size (section 13.2.1), and $n$ is the CA size. $H$ is usually averaged over a moving window of (say $w=10$ ) time-steps.

${ }^{2}$ The standard deviation is given by $\sigma=\sqrt{\sum \frac{x_{i}^{2}}{n}}$ where $x_{i}$ is deviation from mean of input-entropy at time-step $i$, and $n$ is the number of time-steps measured.
} 


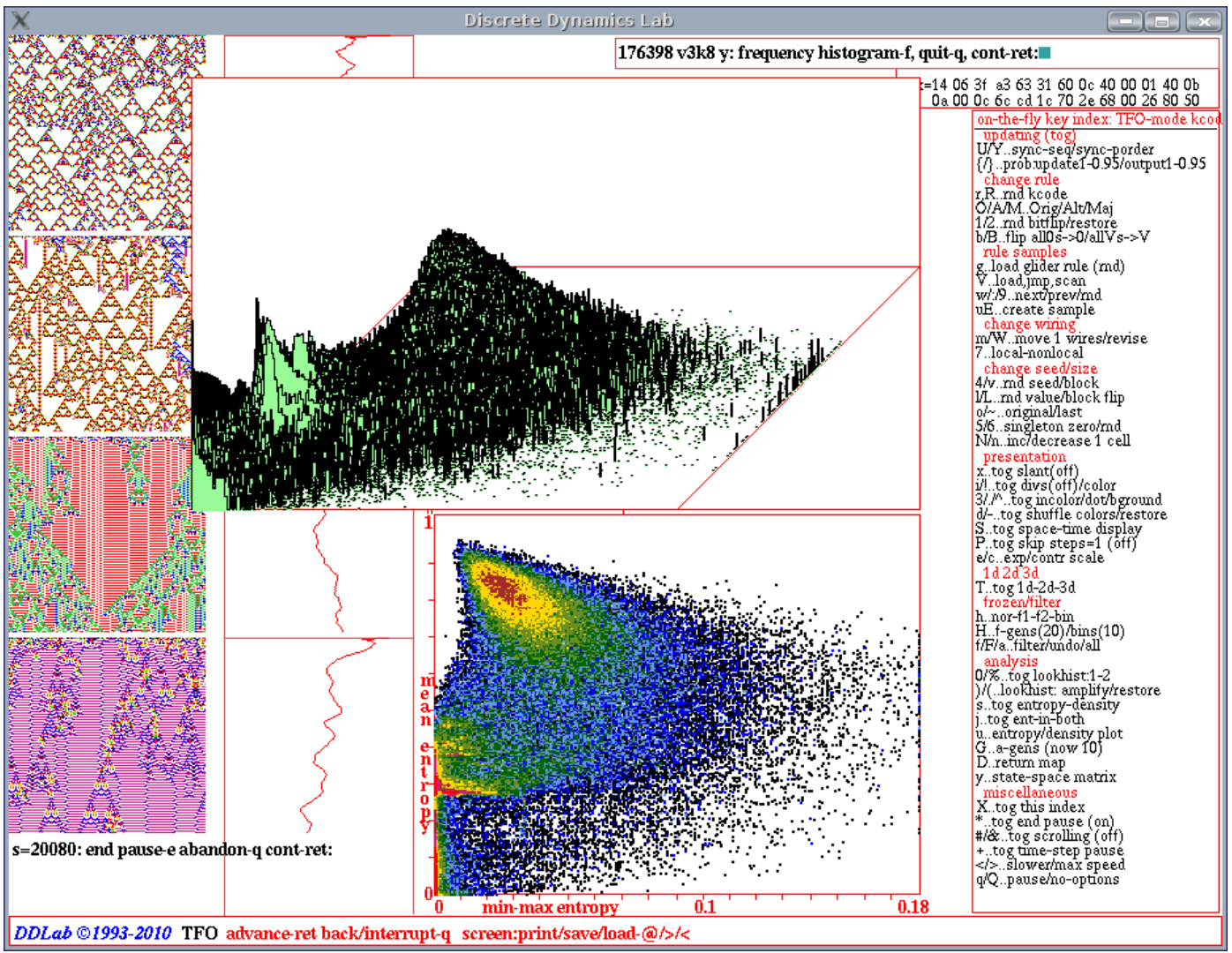

Figure 33.5: A snapshot of the DDLab screen, showing a 1d kcode sample (TFO-mode) with 176398 rules (file v8k3B50.sta), sorted by min-max entropy. The random rules were biased by setting the fraction of non-zero values in the rule-table (the $\lambda$-parameter) to $50 \%$ in section 16.3.1. An uneven distribution of values in the rule-table is more likely to result in complex dynamics than an unbiased rule-table (contrast with figure 33.4). This is borne out by the extensive spread of rules into the high variability area of the plot. About $14 \%$ of this rule-space, with the highest entropy variability, display particle interaction and other complex structures in space-time patterns. Left: space-time patterns in blocks of 150 time-steps showing sequential examples, pausing at sample 20081 . Bottom Center: the scatter plot with min-max entropy. Top Center: the $2 \mathrm{~d}$ histogram with the $z$-axis in log form.

\section{$\underline{\text { options } . . . ~ w h a t ~ t h e y ~ m e a n ~}$}

automatic-a ... to create an automatic sample (section 33.4).

test-(def): ... enter return for a test (section 33.3), showing the measures and the entropy/density scatter plot for successive rules.

sdev-s min-max-(def): . . enter return for the min-max measure of variability, or $\mathbf{s}$ for standard deviation (section 33.1).

start (def 30): ... enter the time-step when measures should start. By not including the initial time-steps, the dynamics can be allowed to settle into typical behavior before measures are recorded. 
end (def 430): ... enter time-step when space-time patterns and measures stop.

sample for each rule (def 5): ... enter the number of runs from different random initial seeds, from which measures are averaged.

bias rnd rules -b: ... to bias the random rules (section 33.2.1).

\subsubsection{Biasing random rules}

A rule bias that was applied when setting a single rule in section 16.3, either a density-bias (section 16.3.1), or a value-bias (section 16.3.2), will carry over to the random rule sample. A density-bias (the proportion of non-zero values in the rule-table $-\lambda$-parameter) is always present in the background, but if not deliberately altered gives an equal probability of each value - effectively no bias. An active value-bias (the proportions of each value in the rule-table) will override the density-bias.

Enter $\mathbf{b}$ in section 33.2.1 to reset the value-bias, and other biases - isotropic rules, and adapting the rule-table so that the all-zero neighborhood outputs zero, or more generally all- $V$ neighborhoods output $V$. A top-right prompt is presented, which varies according to context. The first bias option (not in TFO-mode) is to set an isotropic rule where rotated and reflected neighborhoods (in 1d, $2 \mathrm{~d}$ and $3 \mathrm{~d}$ ) have the same output. Rules in TFO-mode are totalistic, so isotropic by definition.

iso-i: (not for TFO-mode because totalistic rules are isotropic)

If the isotropic option was not selected, options for the value-bias follow (if in TFO-mode these are the first options). The options depend on whether a value-bias is active or not,

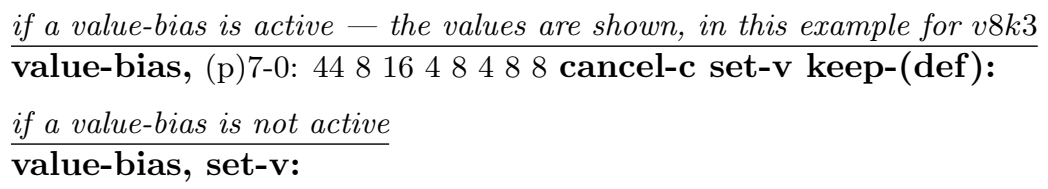

For an active value-bias, enter c to cancel (deactivate), or return to keep the existing valuebias.

Enter $\mathbf{v}$ to set (or reset) the value-bias - this results in a series of prompts, firstly to set the value bias either as percentages, or as actual numbers of each value (if the rule-table size $S \leq 255$ ) with the prompt \%/value-p/v: or \%/value-p:. If $\mathbf{p}$ (or $\mathbf{v}$ if applicable) is entered, prompts will follow to set the percentage (or the number) of each value from 0 to $v-2$; the value for $v-1$ is set automatically. The method is described in greater detail in section 16.3.2.

Following the value-bias, or if value-bias is cancelled, kept, or not set/reset, two final successive prompts are presented as follows,

\section{all0s-0: allVs-v:}

Enter $\mathbf{0}$ to set the output of the all-0s neighborhood to 0. Enter $\mathbf{v}$ to set the outputs of all uniform neighborhoods to the neighborhood value (the same can be done on-the-fly, section 32.5.6). These options, which provide a stable background domain, apply on top of previous biases. If $\mathbf{q}$ is entered, the prompts revert to the first bias option - this can be useful to reconfirm the value-bias. 


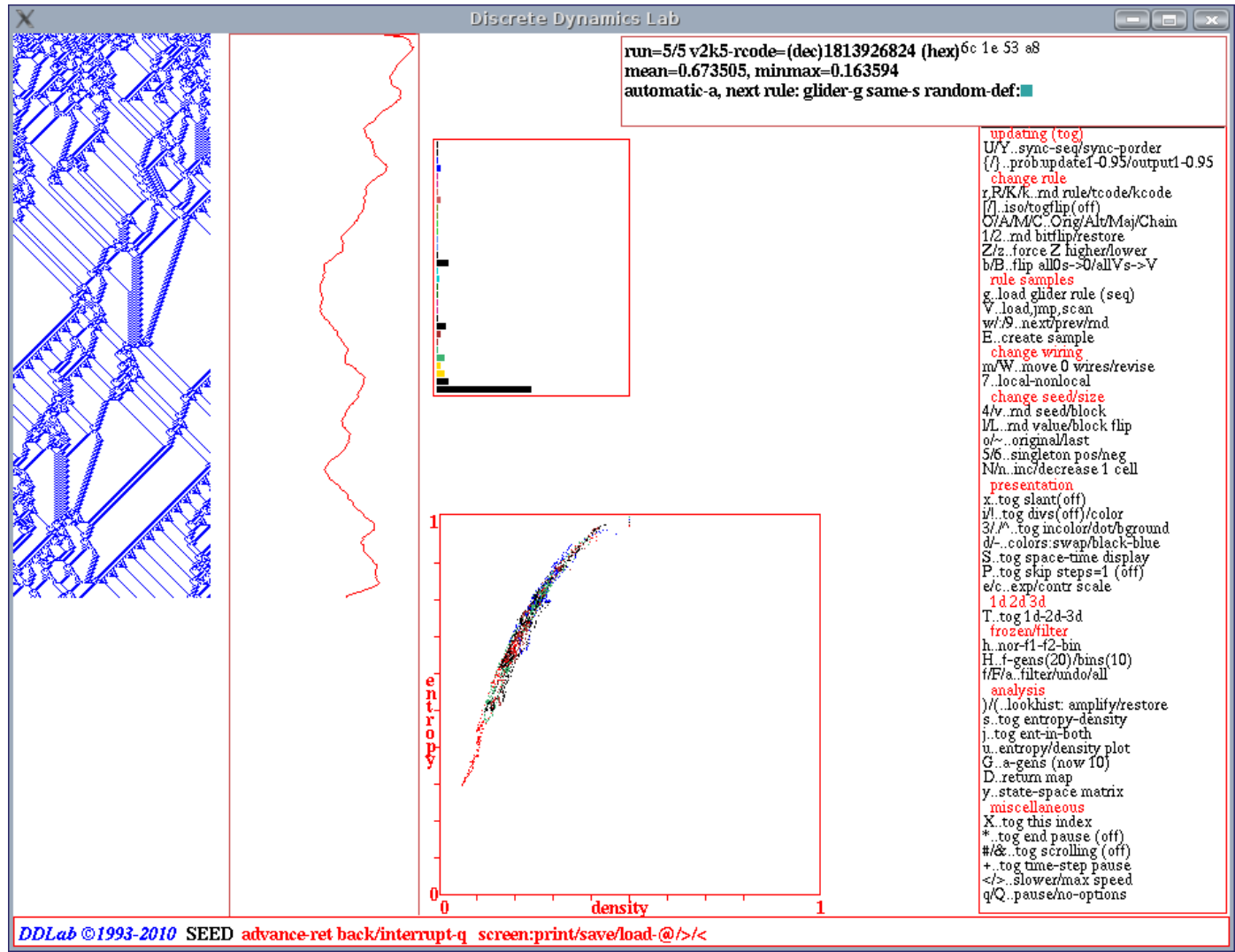

Figure 33.6: A snapshot of the DDLab screen while running a test, showing mean entropy and min-max entropy in a top-right window for a sample of random initial states. This example for a $1 \mathrm{~d} n=150 v 2 k 5$ CA, rcode 6c1e53a8 from the glider rule collection (section 3.7.1). As space-time patterns (including the input-frequency histogram and input-entropy plot, section 31.4) are being drawn, the entropy/density scatter plot (section 32.12.5) is drawn in a lower-center window. The plot shows distinctive signatures for complex rules, and is drawn in alternating colors for successive runs. The default parameters in section 33.2 (start 30 , end 430 , size 5 ) were used, meaning the measures were started at time-step 30 and ended at 430 , i.e. they were taken over 401 time-steps, and the number of runs from different random initial states was 5 .

\subsection{Running a test}

Enter return at the first prompt in section 33.2 to run a test, then enter return to accept other parameter defaults, or change any of these if required.

Space-time patterns will run from different random initial states for the set sample of each same active rule (without pause until the sample is complete), and for the set number of time-steps. Initially, the active rule will be the current rule, thereafter a new random rule can be applied according to any biases described in section 33.2.1, or the same rule can be repeated, or a rule from a glider rule collection (section 3.7.1) can be set. 
After each run from an initial state in the sample, the following top-right window with data on the rule and measures is presented (for example),

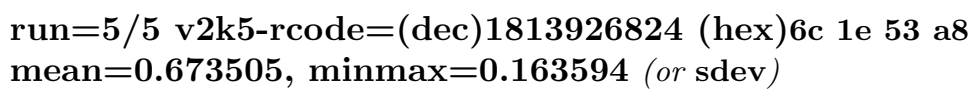

This remains unchanged until the next sample is complete.

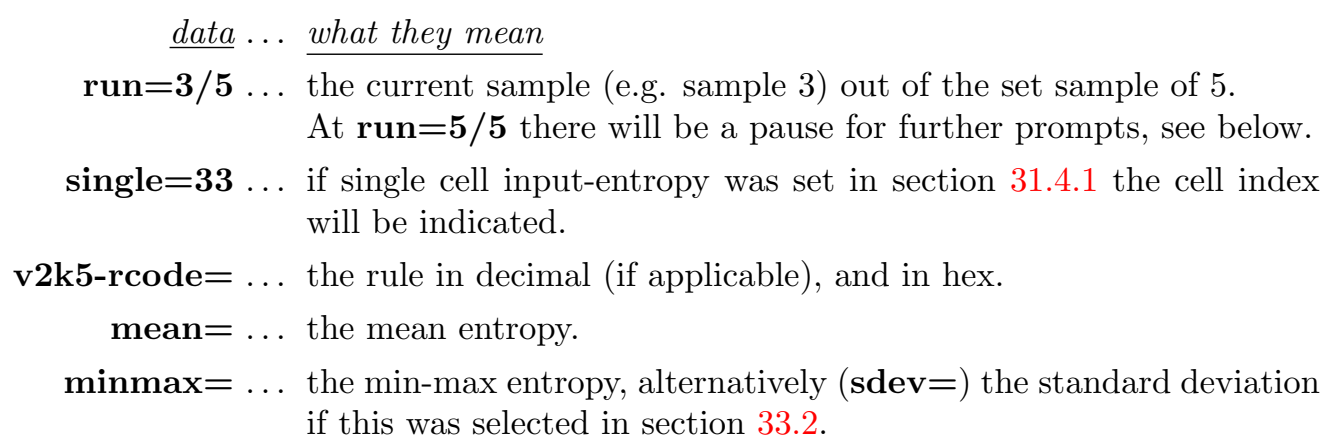

While a run is in progress, the entropy/density scatter plot (section 32.12.5) is drawn in a lower center window. The plot shows distinctive signatures for complex rules, and is drawn in alternating colors for successive sample runs.

When the sample runs are complete (i.e. $\mathbf{r u n = 5 / 5}$, figure 33.6), the top-right window will pause to include the following options,

automatic-a, next rule: glider-g same-s random-def:

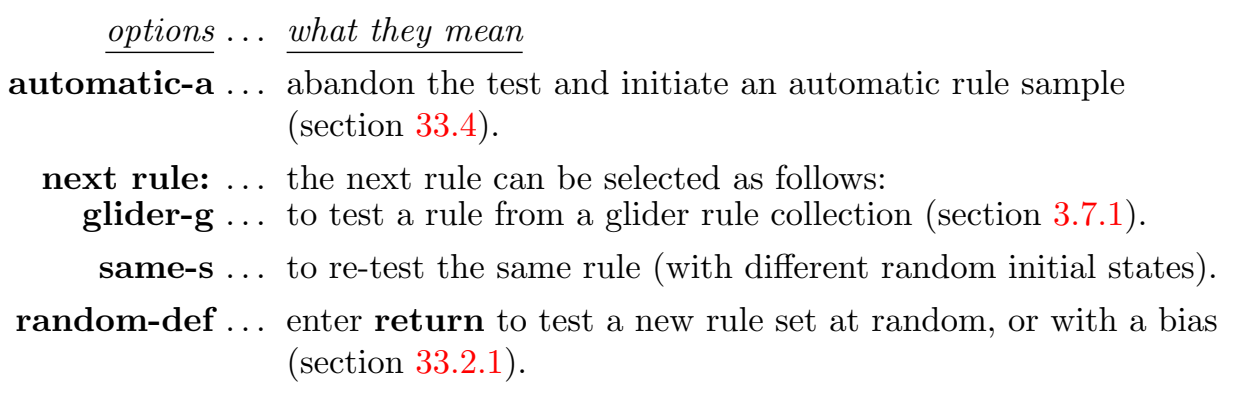

\subsection{Creating an automatic rule sample}

Enter a in sections 33.2 or 33.3 to create an automatic sample. A filename for the sample (.sta) will first be selected in a top-right window (default filename my_stat.sta, section 35.2 ), followed by a top-right prompt to append the data to an existing file, or start a new file,

my_sta.sta: append data-a, new file-(def), add + to pause: (or the selected filename) 


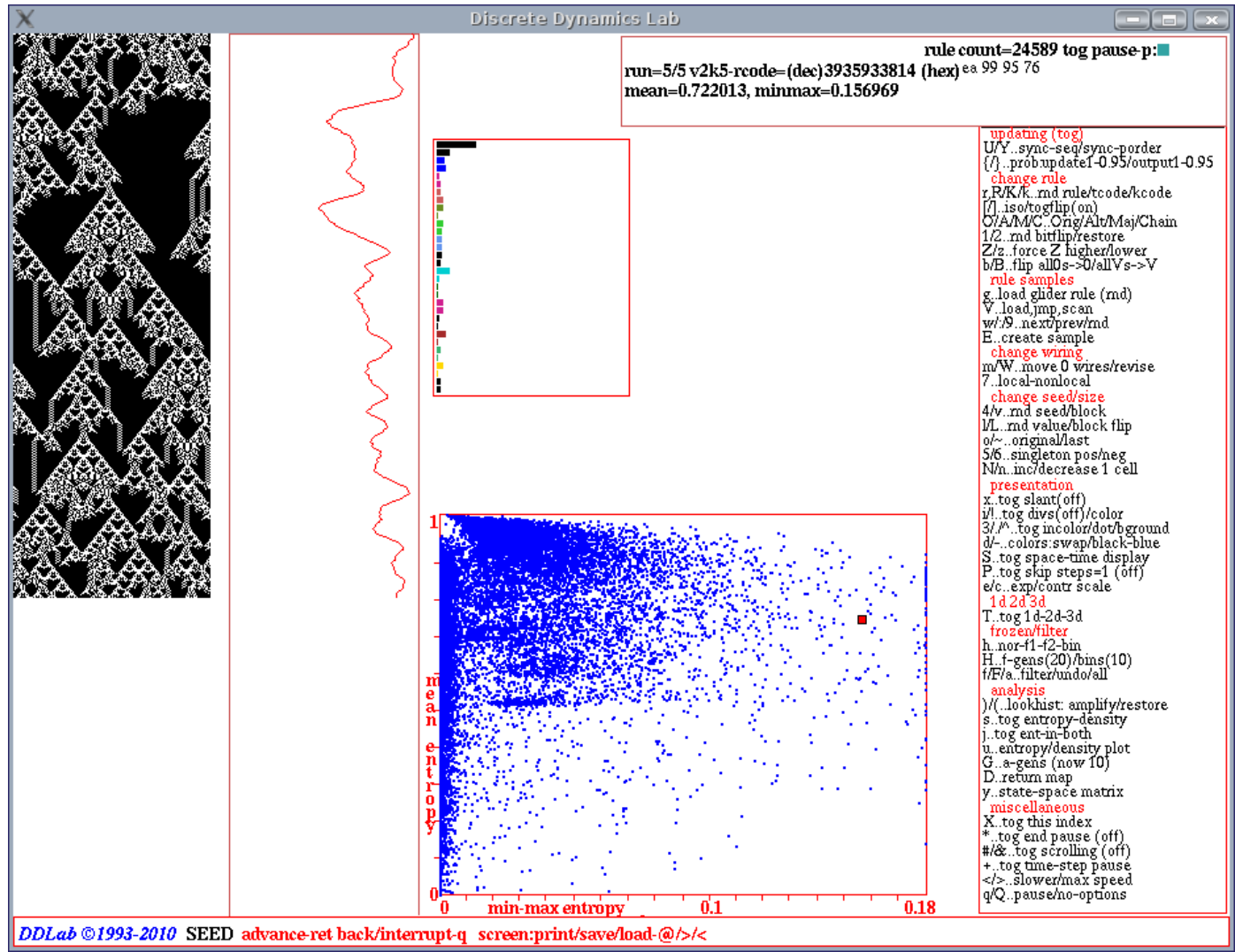

Figure 33.7: A snapshot of the DDLab screen while creating an automatic sample - this example for 1d $n=150 v 2 k 5$ CA. The default parameters in section 33.2 were applied, except that the sample is biased for random isotropic rules. The sample was paused at rule-count 24589, at rcode(hex)ea999576. The scatter-plot, mean entropy against entropy variability, is continuously updated in a lower-center window. After the runs for each rule are complete, a small red square is plotted. This is replace by a blue dot when the next rule is complete, and so on. Note that any variability above 0.18 is rounded down to 0.18

To pause after each new rule, enter or add + , i.e. a + to append data and pause. The automatic sample will then start, plotting the mean entropy against the entropy variability, one point for each new random rule in the scatter plot in a lower-center window (figure 33.7). After set of runs for each rule is complete, a small red square is plotted. This is replaced by a blue dot when the next rule is complete, and so on.

For each run, the following top-right window keeps track of the count of rules so far, with a reminder that a pause can be set on-the-fly with key $\mathbf{p}$.

\section{rule count $=1457$ tog pause-p}

Initially there is no pause between each new rule, however if a pause is activated with on-the-fly key $\mathbf{p}$, at the pause the top-right window will include the data as in section 33.3, for example, 


\section{rule count $=24569$ tog pause-p: \\ run $=5 / 5$ v2k5-rcode $=($ dec $) 3935933814$ (hex)ea 999576 \\ mean $=\mathbf{0 . 6 7 3 5 0 5}, \operatorname{minmax}=\mathbf{0 . 1 6 3 5 9 4}$ (or sdev - as in figure 33.7)}

If the pause is set, enter return (or $\mathbf{p}$ to toggle the pause off) to continue with the next rule. The sample will continue indefinitely until paused with $\mathbf{p}$, or interrupted with $\mathbf{q}$ (section 32.14 enter $\mathbf{q}$ again to backtrack). If interrupted, the sample can always be continued later by appending a new sample run to an existing filename.

To considerably speed up computation, turn off the space-time graphics with on-the-fly toggle S (section 32.9.8). Note that most on-the-fly options such as "presentation" (section 32.9) and "frozen/filter" (section 32.11) will work while the sample is in progress, but other on-the-fly options should be used with caution as the consequences are unpredictable.

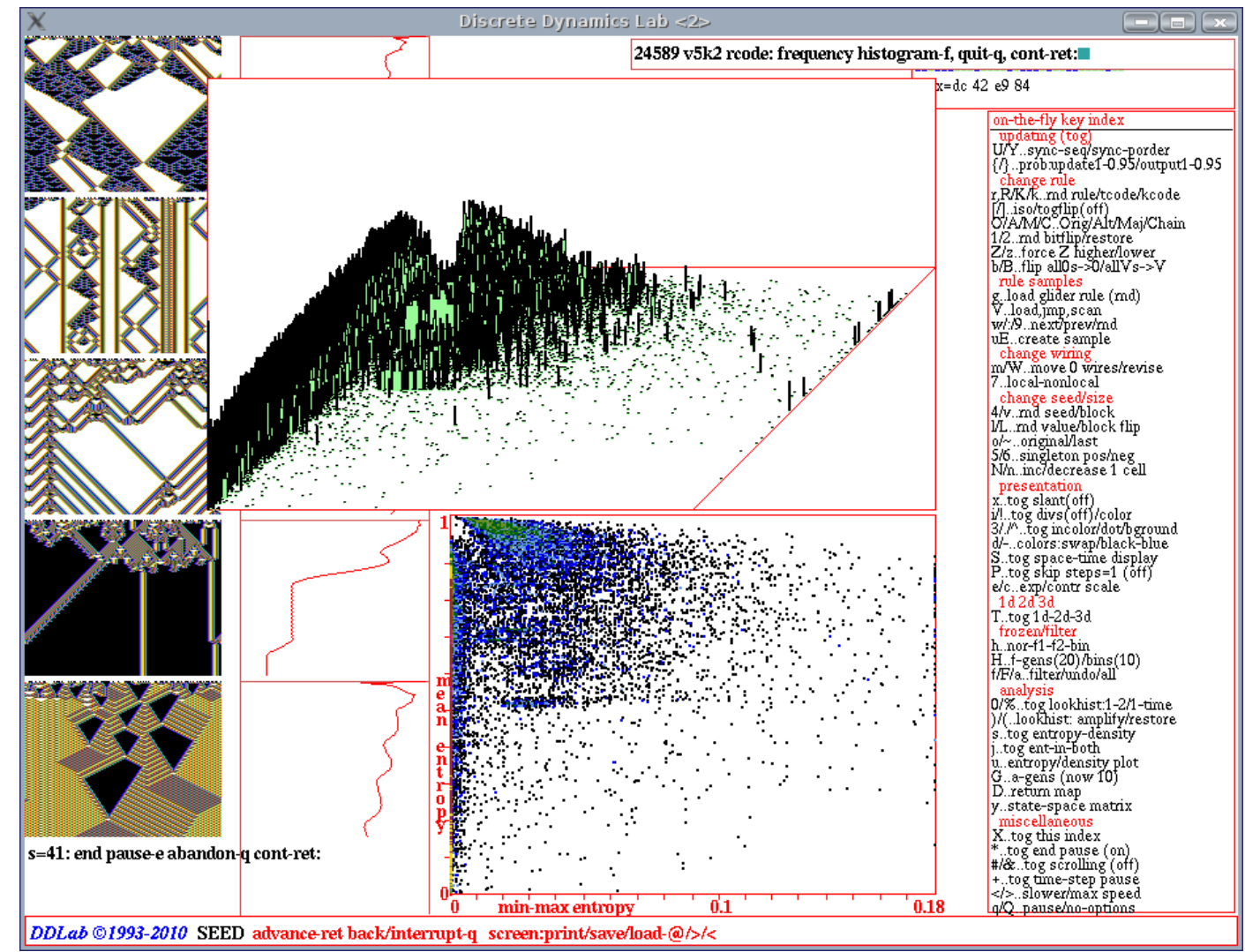

Figure 33.8: A snapshot of the DDLab screen after loading an automatic sample of 24589 rules, for $1 \mathrm{~d} n=150 v 2 k 5$ isotropic CA, created in figure 33.7 (file v2k5iso.sta). The sample was sorted by min-max entropy. Left: space-time pattern in blocks of 120 time-steps showing sequential examples,

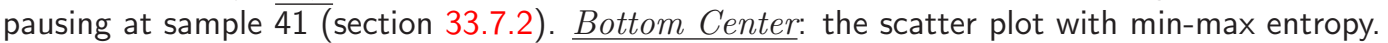
Top Center: the $2 \mathrm{~d}$ histogram with the $z$-axis in log form. 


\subsection{Loading, sorting and displaying a sample}

To load an automatic sample, enter on-the-fly key V..load,jmp,scan while space-time patterns are running, or when interrupting space-time patterns (section 32.14) enter $\mathbf{E}$ to load, or $\mathbf{K}$ to "keep" a sample that is currently active (section 32.16.2).

If a sample is not currently active, the following top-right prompt precedes loading the file to set the type of entropy variability (min-max or sdev). This is just to correctly label the graph,

entropy variability, sdev-s, min-max -(def):

If a sample is currently active, entering on-the-fly key V..load,jmp,scan results in the following top-right prompt,

rule sample: load new-n, rule index/scan 1-24589: (for the sample in figure 33.7)

Enter $\mathbf{n}$ for a new sample, otherwise the space-time patterns of successive rules can be scanned, from rule index 1 (the default, enter return) or from any rule index (enter a number) - this is described in section 33.7.2.

Selecting the loading of a new sample above, is followed by the prompt for selecting the filename (section 35.2). Note that any filename will load, because the file does not record $[v, k]$ (section 33.11), so loading a file that was created with conflicting parameters will result in a meaningless scatter plot.

Once the filename is selected, the following top-right list/sort/display prompt is presented,

list: all-l coords-c, sort-s backtrack-q

min-max plot: Z-Z lda-L ent-(def) smalldots+d: ( $\mathbf{Z}$ and $\mathbf{L}$ for rcode only)

options... what they mean

list: ... to list the sample (sections 33.8), which allows selecting a rule from the list (section 33.8.1)).

all-1 ... to list the sample starting from index 1.

coords-c ... to list at, or near to, given scatter plot coordinates (section 33.9), also done by probing with the mouse (section 33.6.1).

sort-s ... to sort the sample - necessary for an unsorted sample for effective listing or scanning space-time patterns. A prompt for a new filename (.sta) for the sorted file will be presented (section 35.2), which can be the same as before. The rules are sorted firstly by decreasing variability (min-max or sdev), then by decreasing mean entropy for each measure of variability for a large sample this might take a little time.

backtrack-q ... to backtrack to showing space-time pattern, keeping the active sample.

min-max plot: ... (or sdev plot:) show the scatter plot (variability, min-max or sdev on the $x$ axis) in a lower center window (section 33.6), which can the be "probed" with the mouse (sections 33.6.1) to list and select rules.

$\mathbf{Z}-\mathbf{Z}$... (rcode only) to plot variability against the $Z$ parameter (figure 33.14 Top). 
lda-L ... (rcode only) to plot variability against $\lambda$-ratio (figure 33.14 Bottom).

ent-(def) ... (the default) enter return to plot variability against mean entropy.

smalldots $+\mathbf{d} \ldots$ enter $\mathbf{d}$ (or $\mathbf{Z d}$ or $\mathbf{L d}$ ) for the above plots, but with smaller dots. For a low resolution DDLab screen (less than 640 pixels wide) small dots is the default, so the prompt reads bigdots $+\mathbf{d}$.

Once the scatter plot is displayed, further options include probing the plot with the mouse and listing/selecting rules (section 33.6.1), and displaying a $2 \mathrm{~d}$ frequency histogram, where the vertical axis represents rule frequency.

\subsection{The rule sample scatter plot}

Enter return in section 33.5 to display the scatter plot of mean entropy against entropy variability (min-max or sdev) in a lower center window. For rcode only, $\mathbf{Z}$ or $\mathbf{L}$ can be entered for alternative plots, where mean entropy is replaced by either the $Z$-parameter (figure 33.14 top), or by $\lambda$-ratio (figure 33.14 below).

Rules are represented by colored square points on a $256 \times 256$ grid, and the scatter plot color scheme indicates the frequency of rules falling on each square (figure 33.9).

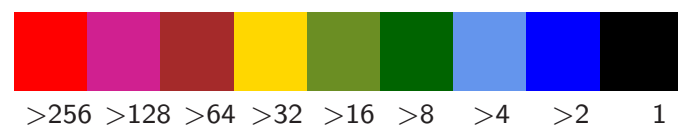

Figure 33.9: The scatter plot color scheme indicates the frequency of rules falling on each square of the $256 \times 256$ grid. Unoccupied squares remain white.

Scatter plot reminders/options appear in the following top-right prompt,

probe/select rules: point-click left mouse button frequency histogram or backtrack -q

Click on or near a point to probe the scatter plot (section 33.6.1), or enter $\mathbf{q}$ to show the scatter plot as a frequency histogram (section 33.6.2), or to backtrack to the list/sort/display prompt (section 33.5) where further backtracking restores space-time patterns.

\subsubsection{Probing the scatter plot with the mouse, and selecting rules}

The mouse pointer changes direction (to North-West) within the scatter plot, and a small topcenter window shows the pointer coordinates, for example $\mathrm{x}=\mathbf{8 4} \mathbf{y = 2 1 3}$. Clicking the left mouse button anywhere in the plot produces a top-right window (section 33.9), listing rules at that point, if any. The list can be expanded by setting a radius around the point to expand the coordinate area to include more rules - also useful to capture an isolated rule from a shaky mouse click. A rule can be selected from the list, and its space-time patterns run, as described in section 33.8.1. Enter $\mathbf{q}$ (or click again) to delete the list, and click the plot again at a new position to show a new list. The same list can be produced by setting the coordinates (section 33.9). 


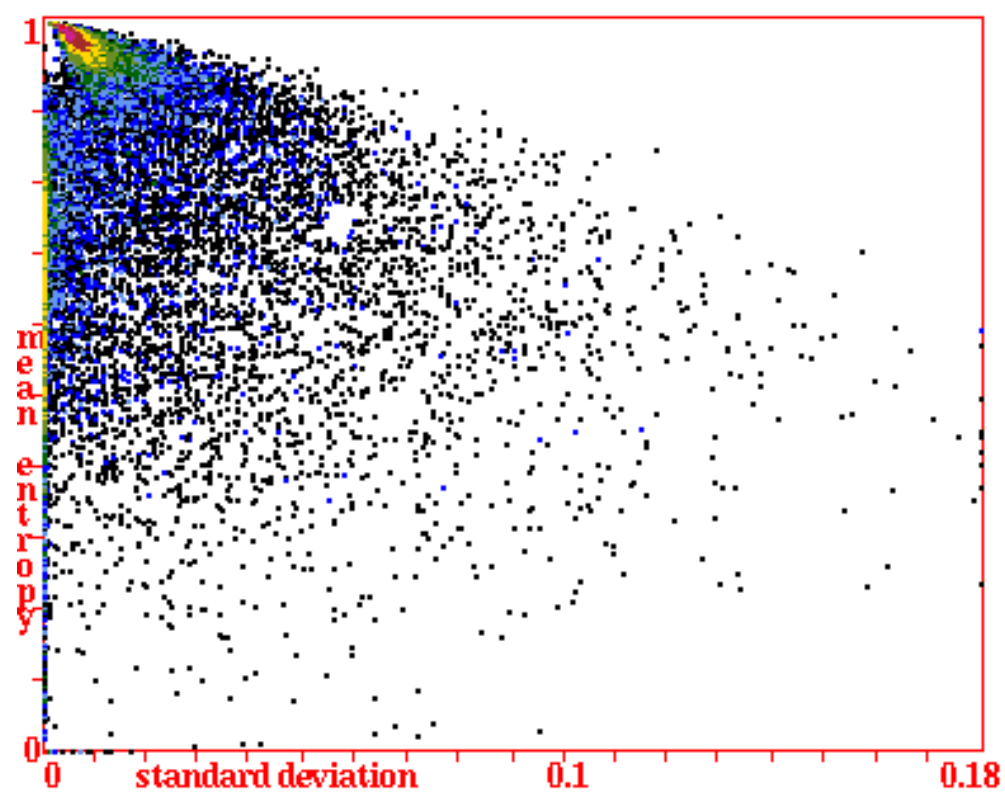

Figure 33.10: The rule sample scatter plot, showing a 1d rcode sample with 17680 unbiased random rules (file v2k5ss.sta), sorted by standard deviation entropy. A color scheme indicates frequency. To select a rule anywhere on the plot, click on a point with the left mouse button for a list, then select the rule (sections 33.9, 33.5).

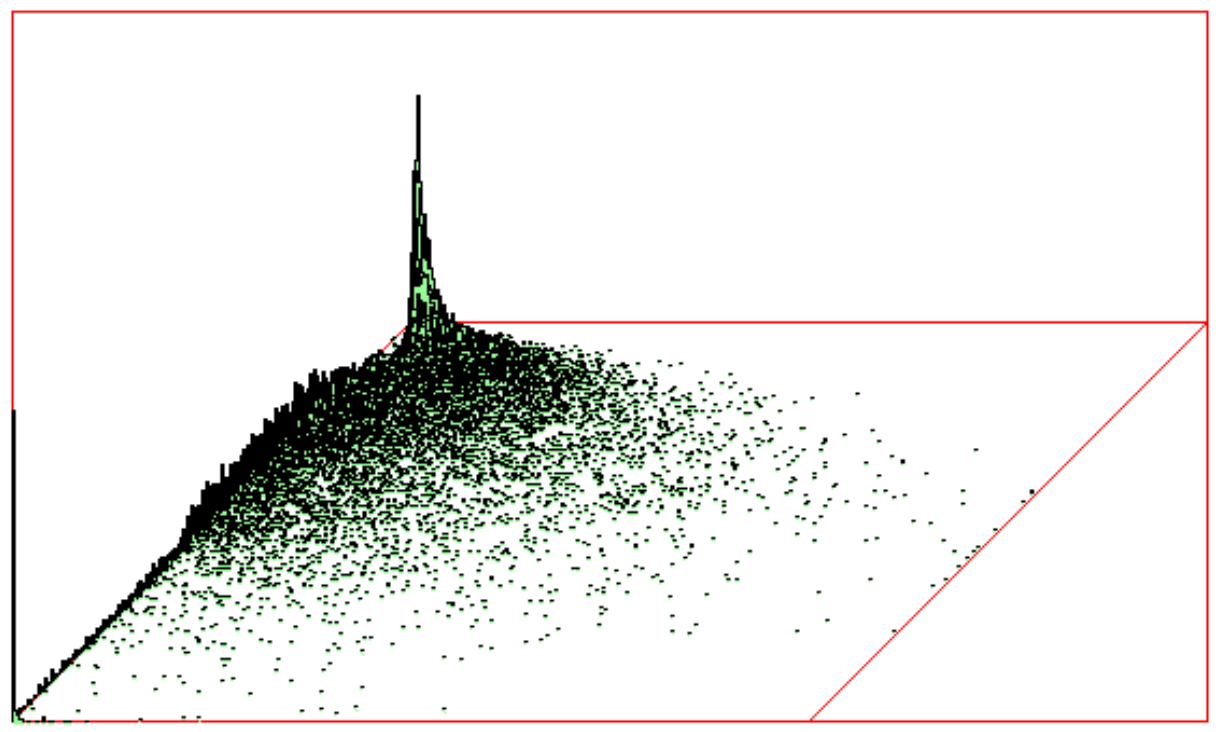

Figure 33.11: The $2 \mathrm{~d}$ frequency histogram of the rule sample scatter plot in figure 33.10. The vertical axis is the frequency of rules falling on each square of the grid - here $256 \times 256$. 


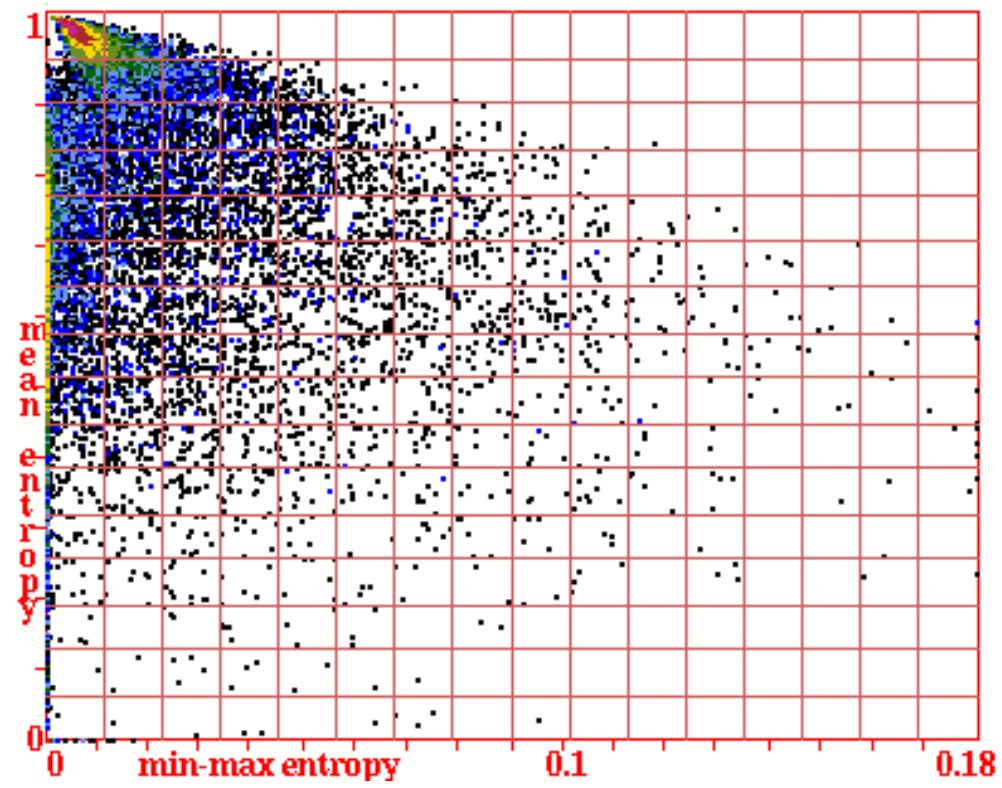

Figure 33.12: The rule sample scatter plot - the same as in figure 33.12 , but with a $16 \times 16$ superimposed grid - by entering grid-g in section 33.6.2.

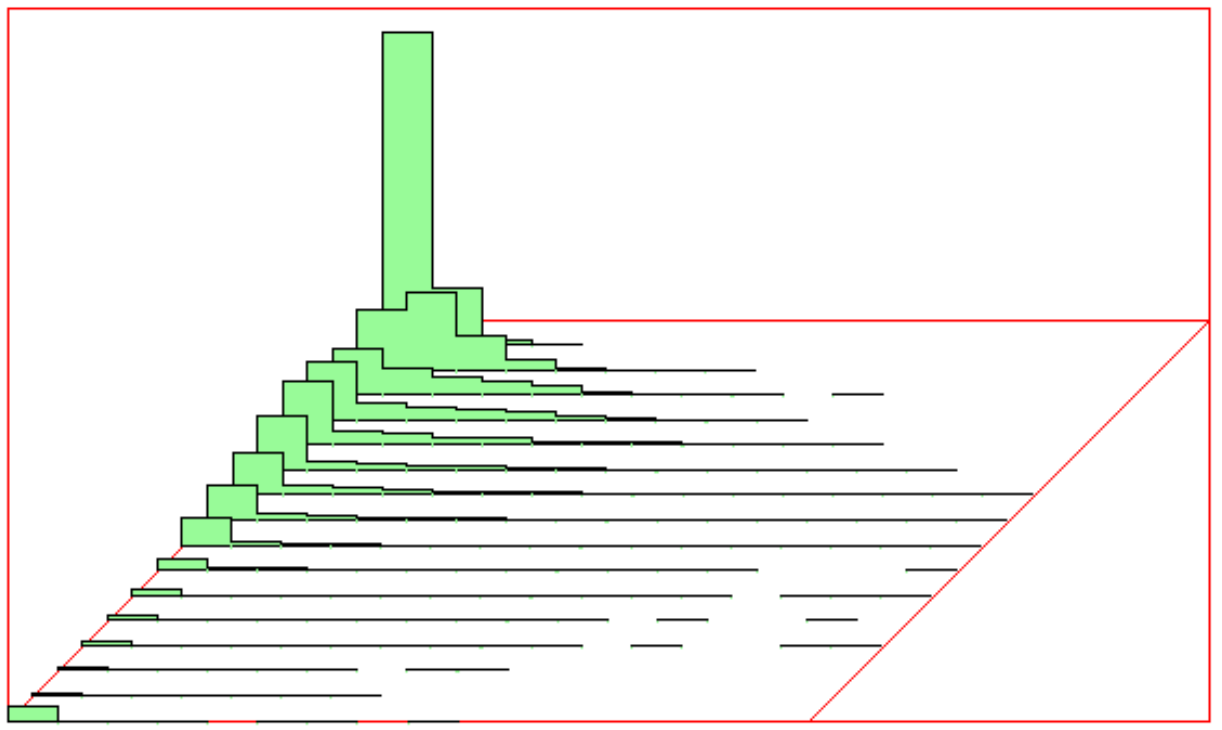

Figure 33.13: The $2 \mathrm{~d}$ frequency histogram of the rule sample scatter plot in figure 33.10 redrawn at a lower resolution, $16 \times 16$, selected in subdiv in section 33.6.2. The vertical axis is the frequency of rules falling on each square of the grid. This figure also illustrates pausing after each row with step-s or show\%-S in section 33.6.2. 


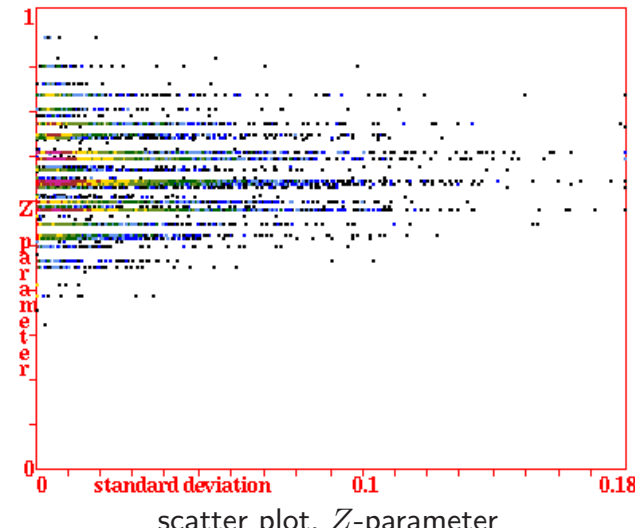

scatter plot, $Z$-parameter

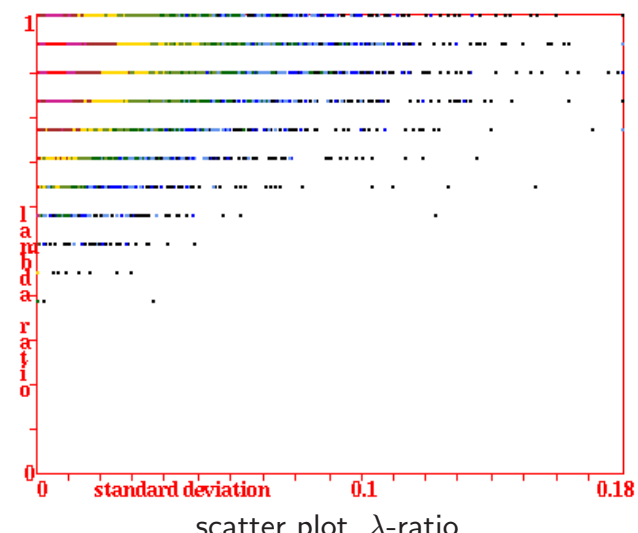

Figure 33.14: Alternative scatter plots and $2 \mathrm{~d}$ frequency histograms (rcode only) where mean entropy is replaced by: Top: the $Z$-parameter, and Bottom: $\lambda$-ratio, for the same rule sample as in figure 33.12. Listing by plot coordinates or probing with the mouse apply as usual (section 33.9).

\subsubsection{The scatter plot as a $2 \mathrm{~d}$ frequency histogram}

The $2 \mathrm{~d}$ frequency histogram of the scatter plot includes a vertical axis showing the frequency of rules falling on each square of a notional grid laid over the scatter plot - the maximum resolution of this grid, $256 \times 256$, can be reduced.

If $\mathbf{q}$ is entered in section 33.6 the following top-right prompt is presented,

17680 v2k5 rcode: frequency histogram-f, cont-ret: (for the sample in figure 33.10)

Enter return to skip the histogram and backtrack to the prompt in section 33.5.

Enter $\mathbf{f}$ to select the $2 \mathrm{~d}$ frequency histogram of the scatter plot (figure 33.11), which will appear in a top-center window - the following top-right prompts are presented in sequence to specify the presentation and other options,

freq hist, subdiv $(16,32,64,128,256-($ def $)$ :

save data-d, step-s, and show\%-S: freq log-l: grid-g: 

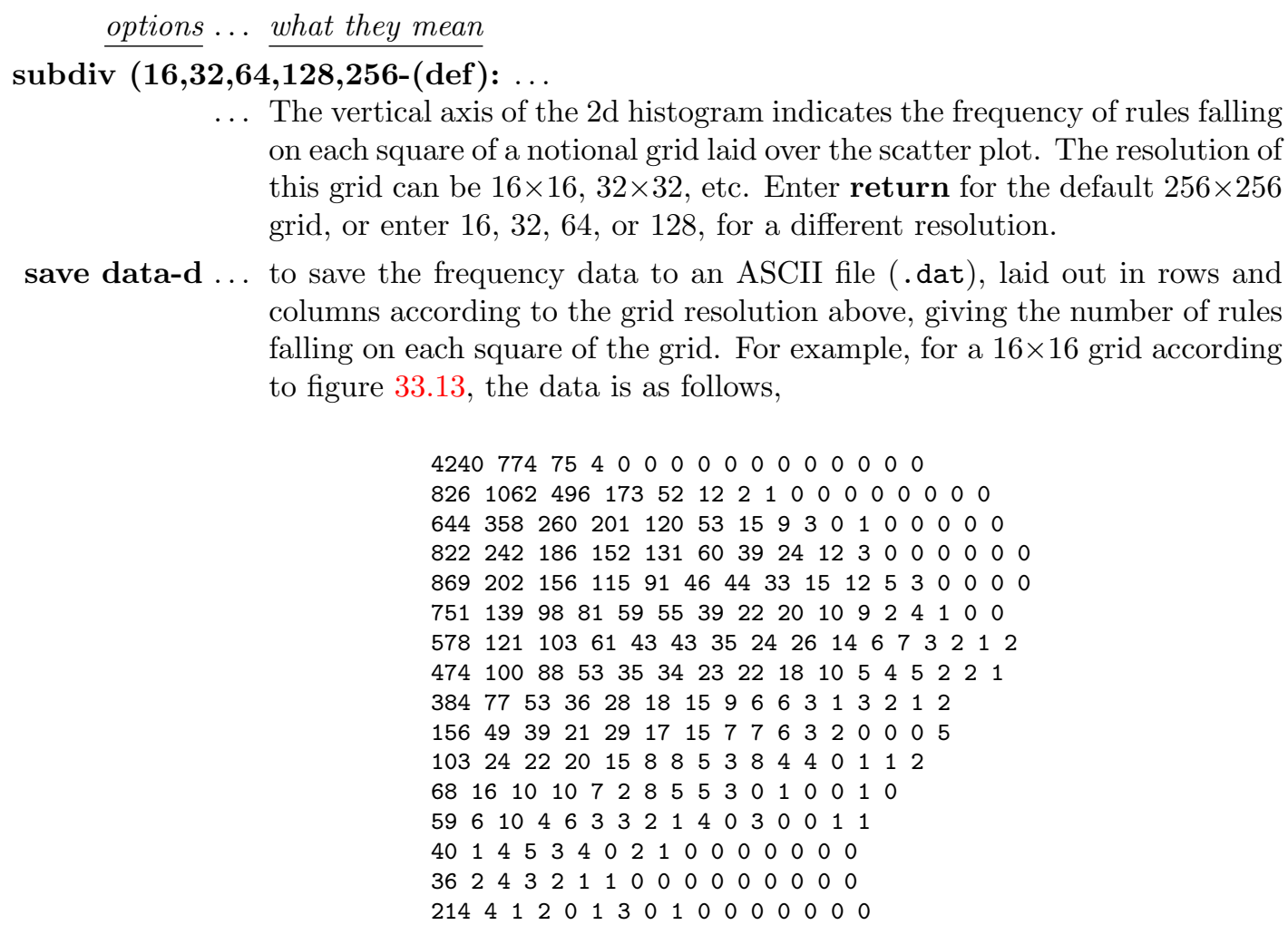

step-s, and show\%-S: ...

... enter $\mathbf{s}$ or $\mathbf{S}$ to pause after each row in the $2 \mathrm{~d}$ histogram, to see its structure in greater detail, then enter return for each next row. If $\mathbf{S}$ is entered, the height data for successive rows is displayed as a fraction of the maximum height, in a top-right window.

freq log-l: ... for a log scale of the vertical axis.

grid-g: ... to overlay a grid, selected in subdiv above, on the scatter plot, as in figure 33.12 .

Once these options are complete, the $2 \mathrm{~d}$ histogram of the scatter plot is drawn in a top-center window, and the histogram prompt in section 33.6.2 reappears. Enter $\mathbf{f}$ to reselect the histogram, or return (or q) to backtrack to the prompt in section 33.5 - further backtracking will restore space-time patterns.

\subsection{Scanning sample space-time patterns}

If a sample is currently active, space-time patterns, in any presentation ( $1 \mathrm{~d}, 2 \mathrm{~d}$ or $3 \mathrm{~d}$ ), can be scanned from the sample. For more meaningful results the sample should have been sorted in section 33.5. 


\subsubsection{Scanning on-the fly}

The simplest method of scanning is on-the-fly with the following keys,

$$
\text { w/:/9..next/prev/rnd }
$$

Enter $\mathbf{w}$ for the next sample index, enter : (colon) for the previous index, or $\mathbf{9}$ for a random index. Each rule change also sets a random initial state, and space-time patterns continue until a new on-the-fly key hit, or if interrupted. Information on the new rule, including the sample index, appears in a top-right window when the key is hit.

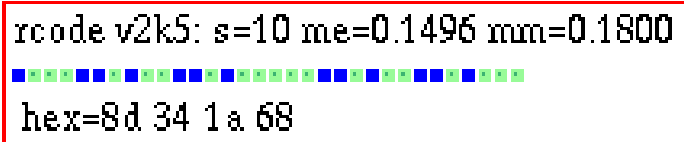

Figure 33.15: Information that appears in a top-right window when scanning sample space-time patterns with on-the-fly keys $\mathbf{w} /: / \mathbf{9}$, showing $[v, k]$, the sample index $(\mathrm{s}=10)$, the mean entropy (me), $\min -\max (\mathrm{mm})$ or the standard deviation (sdev), and the rule as a bit/value-pattern and in hex (from the sample in figure 33.7).

To start scanning from a selected rule index, enter on-the-fly key V..load,jmp,scan, then enter the rule index at a top-right prompt, for example,

rule sample: load new-n, rule index/scan 1-24589 (222-def): (sample in figure 33.8)

\subsubsection{Scanning automatically in blocks of time-steps}

To scan automatically in equal blocks of time-steps, or to just start from a new rule index, enter on-the-fly key V..load,jmp,scan, which gives the following top-right prompt, for example,

rule sample: load new-n, rule index/scan 1-176398 (17-def): (sample in figure 33.5)

Enter a number for a new rule index, or return for the current index which is shown (or $\mathbf{n}$ for a new sample). If, for example, 33 is entered for the rule index - the following prompts allow scanning automatically in blocks of time-steps,

\section{scan in blocks from sample index 33 -i:i set time-steps(def 150):}

Enter return to reject blocks and continue from the selected index. Enter $\mathbf{i}$ to scan in blocks - then the prompt to set the number of block time-steps is also presented (default 150), and space-time patterns will run, starting with the selected sample index, which changes automatically to the next index after each block of time-steps. For 1d space-time patterns, if scrolling is set to $o f f^{3}$ there will be a gap between each block, but no gap otherwise. To pause after each screen-full of blocks, set the end pause $o n^{4}$. The pause occurs when no more blocks will fit, with the following bottom-left prompt, which is presented below the last block, as in figure 33.16 and other DDLab screen snapshots in this chapter,

\footnotetext{
${ }^{3}$ Scrolling can be toggled on/off in section 31.2.6, or on-the-fly (\#/\&..tog scrolling, section 32.13.3).

${ }^{4}$ Pausing 1d space-time patterns when scrolling is off can be set on-the-fly (*..tog end pause (on), section 32.13.2), and also in section 31.2.7, or from the interrupt prompt (section 32.13.4).
} 


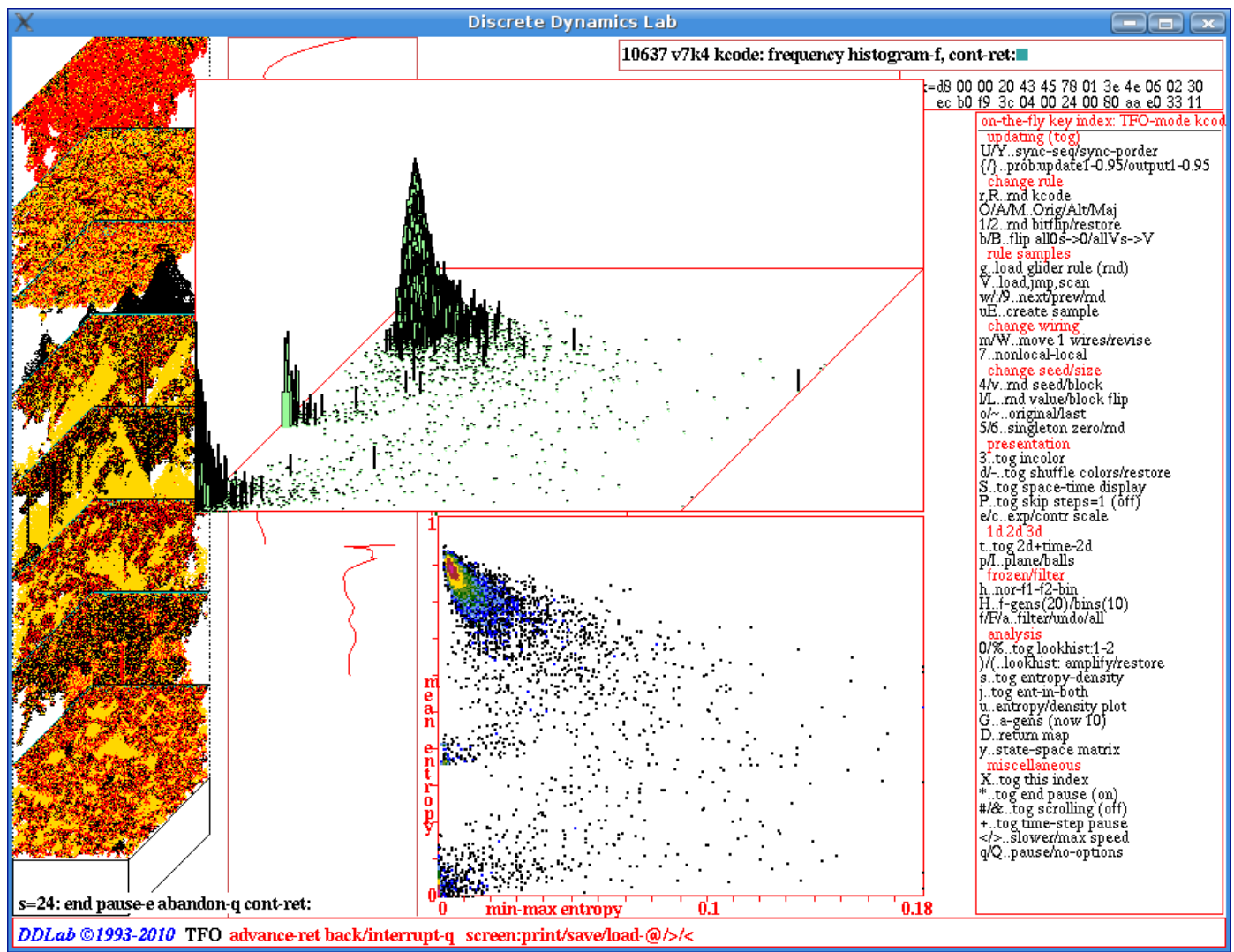

Figure 33.16: A snapshot of the DDLab screen, showing a 2d kcode sample (TFO-mode) with 10637 rules (file $v 4 \mathrm{k} 72 \mathrm{~d} 52$. sta), sorted by min-max entropy. The random rules have a percentage value-bias 3-0: 521616 16, and outputs of all uniform neighborhoods to the neighborhood value (section 33.2.1), resulting in a greater frequency of complex rules than in an unbiased sample. Left: $2 \mathrm{~d}$ space-time patterns $88 \times 88$ in blocks of 100 time-steps, paused at rule index 24 . Bottom Center: the scatter plot with min-max entropy. Top Center: the $2 \mathrm{~d}$ histogram with the $z$-axis in log form.

\section{$\mathbf{s}=\mathbf{2 4}$ : end pause-e abandon-q cont-ret: (as in figure 33.16)}

The rule index of the last block is given by $\mathbf{s}=\mathbf{2 4}$. Enter return twice for the next set of blocks - the interrupt prompt (section 32.13.4) is presented first (showing the next rule) - here other options can be activated including on-the-fly options (on-the-fly-y, section 32.16.9) where the scatter plot and its 2d histogram can be added to the presentation (as in figures 33.3, 33.5, $33.8,33.16$ ). Enter e to undo the block end pause, $\mathbf{q}$ to abandon blocks and backtrack.

\subsubsection{Scanning $2 \mathrm{~d}$ or $3 \mathrm{~d}$ samples}

A sample created for a $2 \mathrm{~d}$ or $3 \mathrm{~d}$ CA can also be scanned in ways analogous to those described in sections 33.7.1, 33.7.2, especially if the space-time presentation is transformed to $1 \mathrm{~d}$ with on-the-fly key $\mathbf{T}$ (section 32.10 .1 ), otherwise the $2 \mathrm{~d}$ or $3 \mathrm{~d}$ pattern is presented as a "movie" of successive time-steps. 


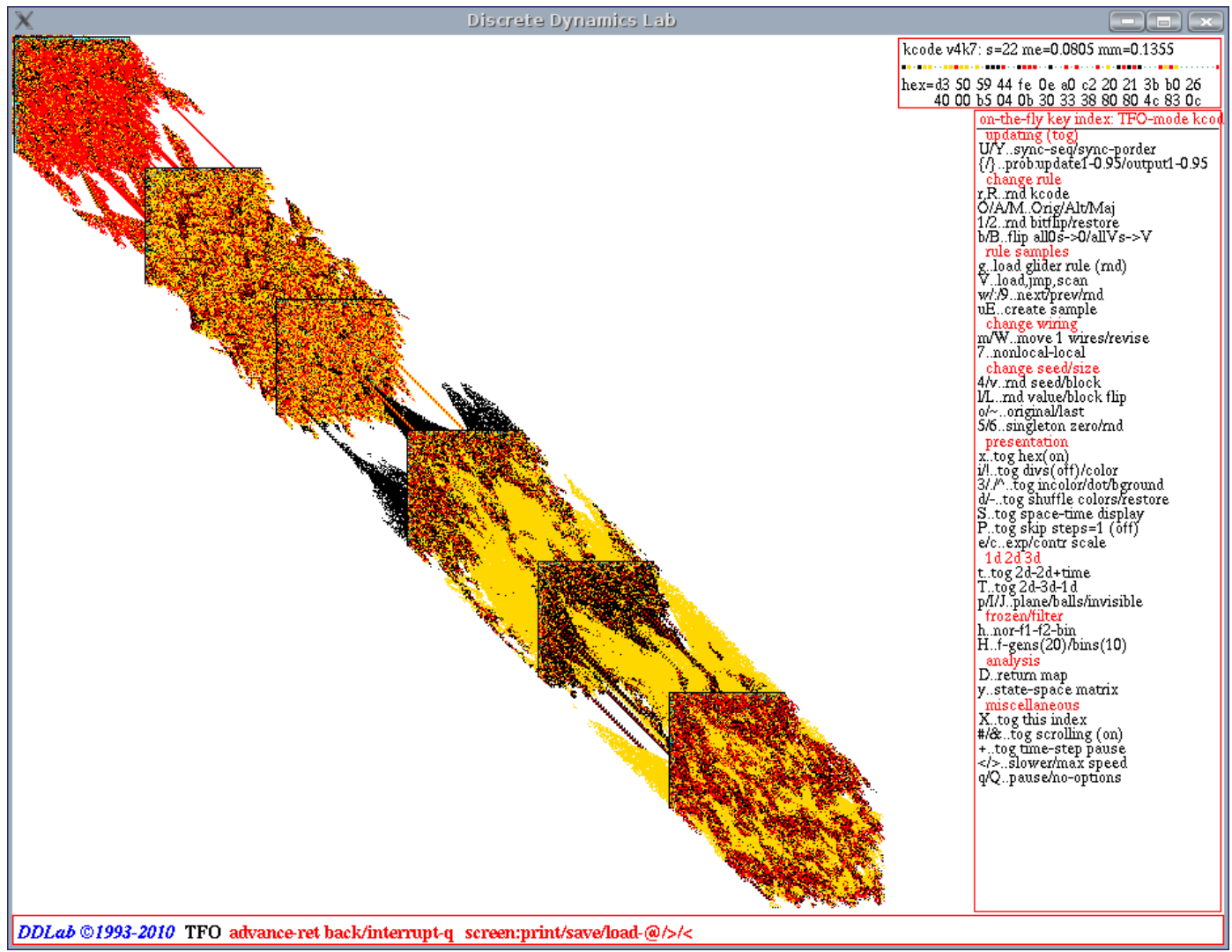

Figure 33.17: A snapshot of the DDLab screen while scanning a $2 \mathrm{~d}$ CA $88 \times 88$ in blocks of 100 time-steps scrolling diagonally, for biased $v 4 k 7$ kcode in figure 33.16.

A $2 \mathrm{~d}$ presentation can also be shown as either vertical or diagonal time-steps. For vertical timesteps (on-the-fly key t..tog 2d-2d+time, section 32.10.2), scrolling can be either on or off and the same methods apply for automatically scanning in blocks as in section 33.7.2, including setting a pause (figure 33.16). For diagonal time-steps (on-the-fly keys \#/\&..tog scrolling) which is confined to scrolling only, scanning in blocks also applies (figure 33.17).

\subsection{Listing a sample}

Enter $\mathbf{l}$ is entered in section 33.5 to list the rules in a sample, presented in a top-right window (figure 33.18). In a sorted list, rules are ordered by decreasing variability (min-max or sdev), then by decreasing mean entropy within each measure of variability, otherwise the list is in the order saved.

The title in the list window (e.g. $10637 \mathrm{v} 4 \mathrm{k} 7$ rules mean stan-dev) shows the number of $[v, k]$ rules in the sample. The rules are then listed, as many as will fit in the window, with a rule index list on the left, followed by the mean entropy and variability (min-max or sdec), and the rule in hex - as much as will fit. Sets of rules separated by horizontal lines have the same variability. The following prompt is show at the foot of the list, 


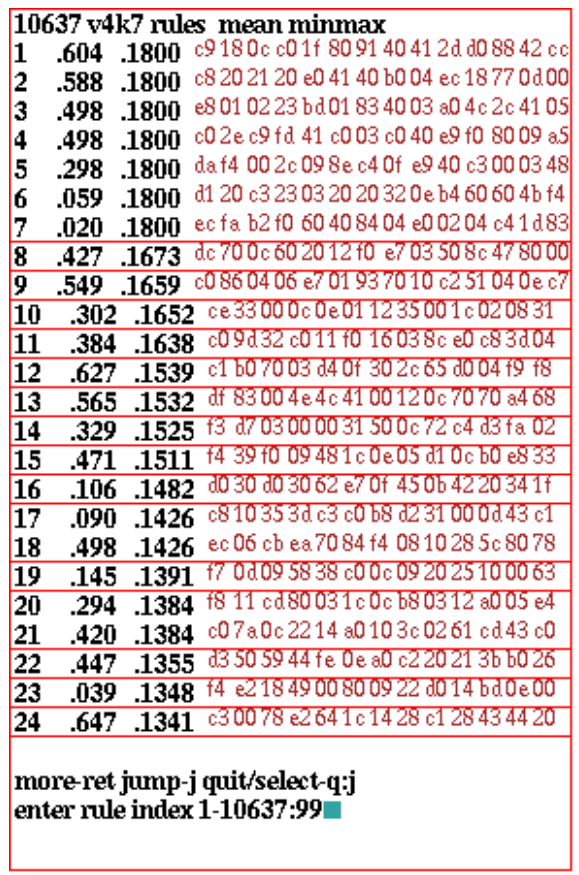

default listing from index 1 , and requesting a jumping to 99

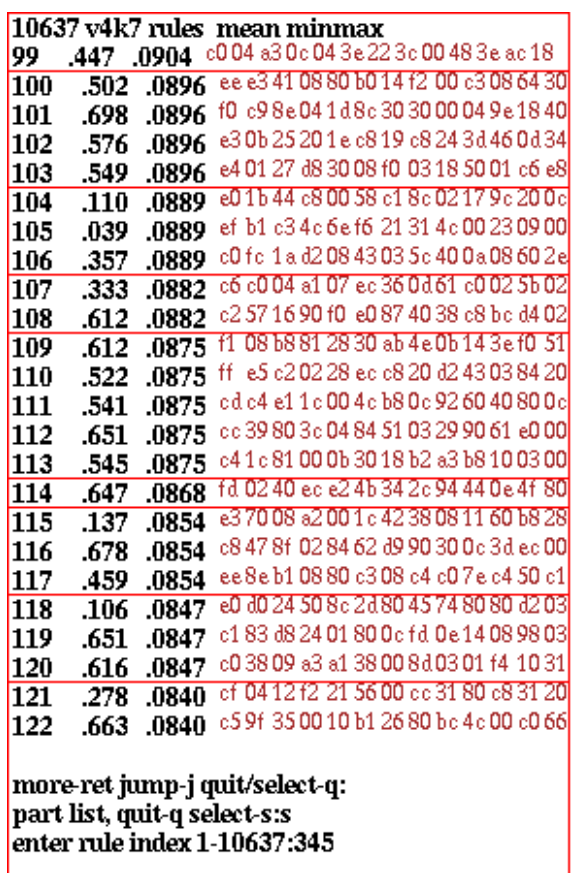

listing from index 99, and selecting rule 345 (not necessarily in the list)

Figure 33.18: Listing the sorted rule sample in figure 33.16 with $10637 v 4 k 7$ kcodes. Columns from left to right: rule index number, mean entropy, variability (min-max or sdev), and the rule in hex - as much as will fit. The list order is firstly by decreasing variability $v$, then by decreasing mean entropy within each measure of $v$. Sets of rules separated by horizontal lines have the same $v$ (max 0.18 because any $v>0.18$ is reset to 0.18 ). Various option are presented below the (partial) list: $\mathbf{m}$ to see more, $\mathbf{j}$ to jump to an index, $\mathbf{s}$ to select a rule.

\section{more-ret jump-j quit/select-q:}

options ... what they mean

more-ret ... if there are more rules in the sample, enter $\mathbf{m}$ to see the next batch.

jump-j ... to jump to a new rule index. The following extra prompt is presented,

enter rule index 1-10637: (for example)

Enter the rule index number which becomes the first in the new list.

quit/select-q ... if $\mathbf{q}$ is entered, the following extra prompt is presented,

part list, quit-q select-s:

Enter $\mathbf{q}$ to backtrack, or $\mathbf{s}$ to select a rule and see its space-time patterns (section 33.8.1 below). 

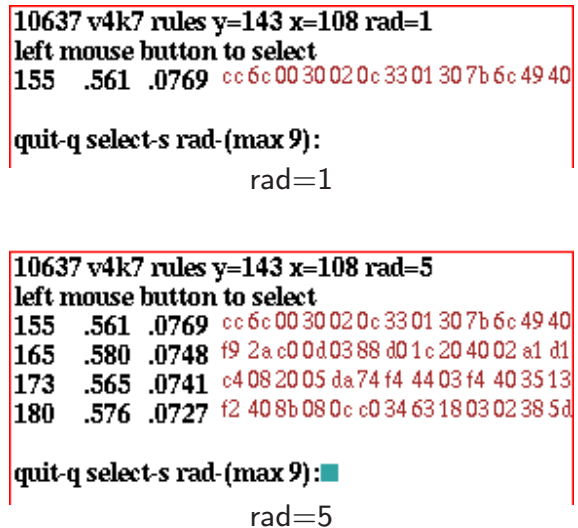

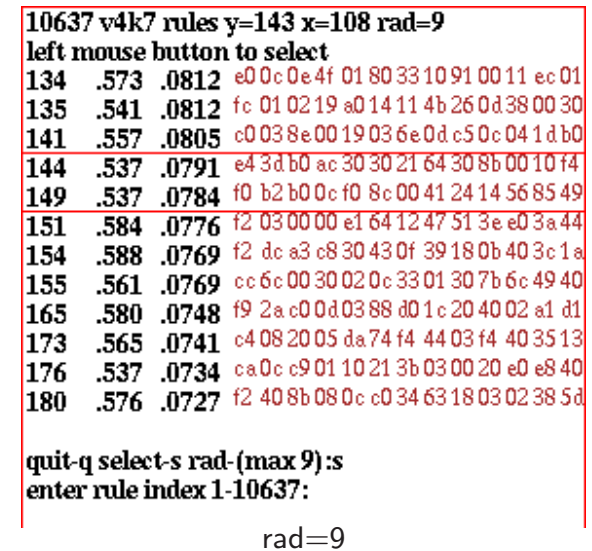

$\mathrm{rad}=9$

Figure 33.19: Listing the sorted rule sample in figure 33.16 by plot coordinates or probing with the mouse. The layout is the same as in figure 33.18, The coordinates $(y=143 x=108)$ were selected by clicking with the mouse (or by entering the values) with the default radius $=1$. The radius was expanded to 5, then to 9 to capture more rules on the scatter plot. Columns from left to right: rule index number, mean entropy, variability (min-max or sdev), and the rule in hex - as much as will fit. Various option are presented below the list: $\mathbf{q}$ to backtrack, $\mathbf{s}$ to select a rule (section 33.8.1), or rad-( $\max 9)$ to change the radius. A new mouse-click or return restores the scatter plot prompt (section 33.6) ready for a new mouse-click.

\subsubsection{Selecting a rule from the list}

Enter s in section 33.8 or 33.9 to select a rule from the list. The following extra prompt is presented at the foot of the list window,

enter rule index 1-10637: (for example)

Enter any rule index number within the range. The program reverts to the prompt in section 33.5. Enter q (backtrack to the interrupt prompt - section 32.14), then enter return to show the space-time patterns of the selected rule.

\subsection{Listing by plot coordinates or probing with the mouse}

Entering $\mathbf{c}$ in section 33.5, or clicking the mouse within the scatter plot in section 33.6.1, allows the rule or rules at, or adjacent to, chosen coordinates to be listed.

If $\mathbf{c}$ is entered, the following top-right prompts are presented in sequence,

\section{mean entropy pos 0-255: $\quad$ min-max pos 0-255: (or sdev)}

Enter the coordinates to list the rule/s at the grid reference, a number (0 to 255$)^{5}$ in each case. The same can be achieved by clicking on the plot with the left mouse button (section 33.6.1). The list will appear as in figure 33.19.

\footnotetext{
${ }^{5}$ The $y, x$ coordinates are plotted according to unsigned char values, so the mean entropy $(0$ to 1$)$ and the variability (0 to 0.18 ) are coarse-grained to the range 0 to 255 . Any variability above 0.18 is reset to 0.18 .
} 
$\mathbf{r a d}=\mathbf{1}$ indicates that only rules at the specific coordinate selected are shown, there may be none. rad-(max 9) allows expanding the coordinate area to include more rules (figure 33.19) enter a radius from 1 to 9 .

\subsection{Rule samples files included with DDLab}

The (sorted) rule sample files used as examples in this chapter are included in the file dd_extra.tar.gz (section 3.7) and listed in section 3.7.4.

\subsection{Rule sample encoding}

A rule sample file (.sta) is a binary file of an unsigned char array, recording (A) each rule, (B) its mean entropy, and $(\mathrm{C})$ the entropy variability (min-max or sdev). The file records A,B,C for each rule in the sample. In a sorted list, rules are ordered by decreasing variability, then by decreasing mean entropy within each measure of variability, otherwise the list is in the order saved.

The encoding of A,B,C is as follows: A (the rule-table) consists of bits as described in section 16.16, but omitting the two leading bytes for $v$ and $k$. B and $\mathrm{C}$ are both whole numbers $(0$ to 255$)$ and are stored as one byte each. The file does not record $[v, k]$ so loading a file that was created with conflicting $[v, k]$ will result in a meaningless scatter plot. 


\title{
Chapter 34
}

\section{Learning, forgetting, and highlighting}

\author{
not in TFO-mode.
}

Attractors classify state-space into broad categories, the network's "content addressable" memory in the sense of Hopfield[12]. Furthermore, state-space is categorized along transients, by the root of each subtree forming a hierarchy of sub-categories. This notion of memory far from the equilibrium condition of attractors greatly extends the classical concept of memory by attractors alone[26, 27].

DDLab provides tools for "sculpting" the basin of attraction field towards desired categories and sub-categories by methods that automatically attach/detach one or more "aspiring pre-images" to/from a "target-state". The results and side affects of learning can be seen most clearly for a basin of attraction field, but the methods (allowing larger networks) also apply to a single basin, a subtree, or simply to a space-time pattern running forward.

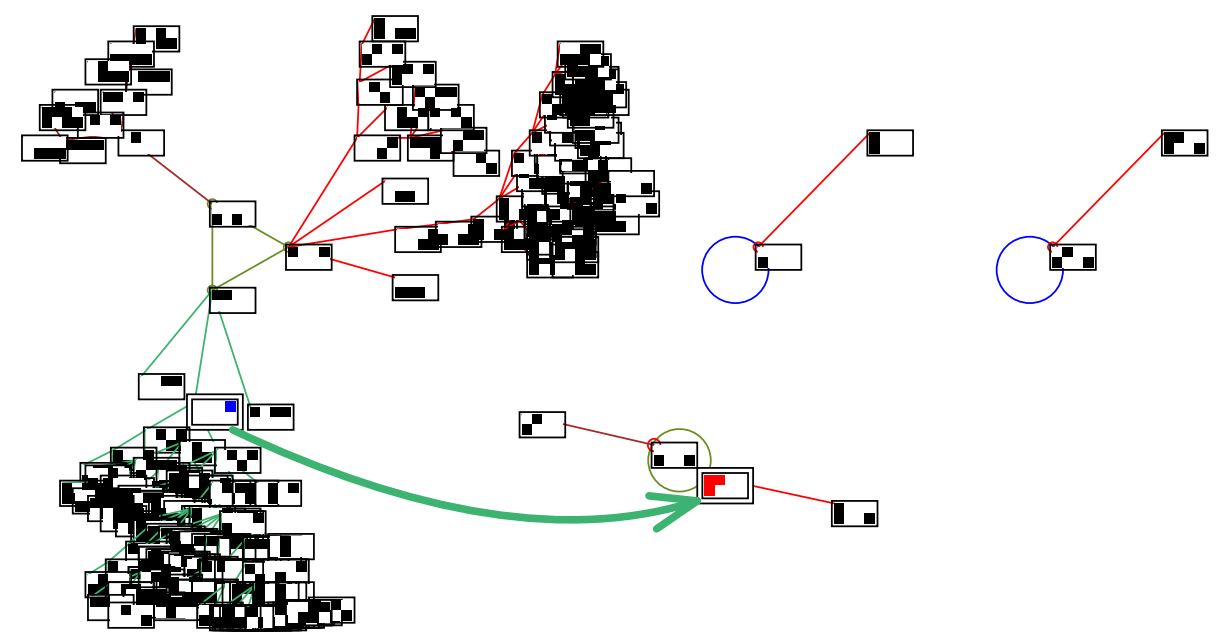

Figure 34.1: The basin of attraction field of a RBN, $v 2 k 4, n=8$, before learning. The red (arrowed) state has been selected as the target, and the blue state $\square$ (at the tail of the arrow) as the aspiring pre-image with "highlight only". Both have a double outline. The difference between the actual successor state $\square$ and the target state $\square$ is one bit (element 3), which should minimize side effects. Note that the basin layout was rearranged within the jump-graph, section 20.7. 


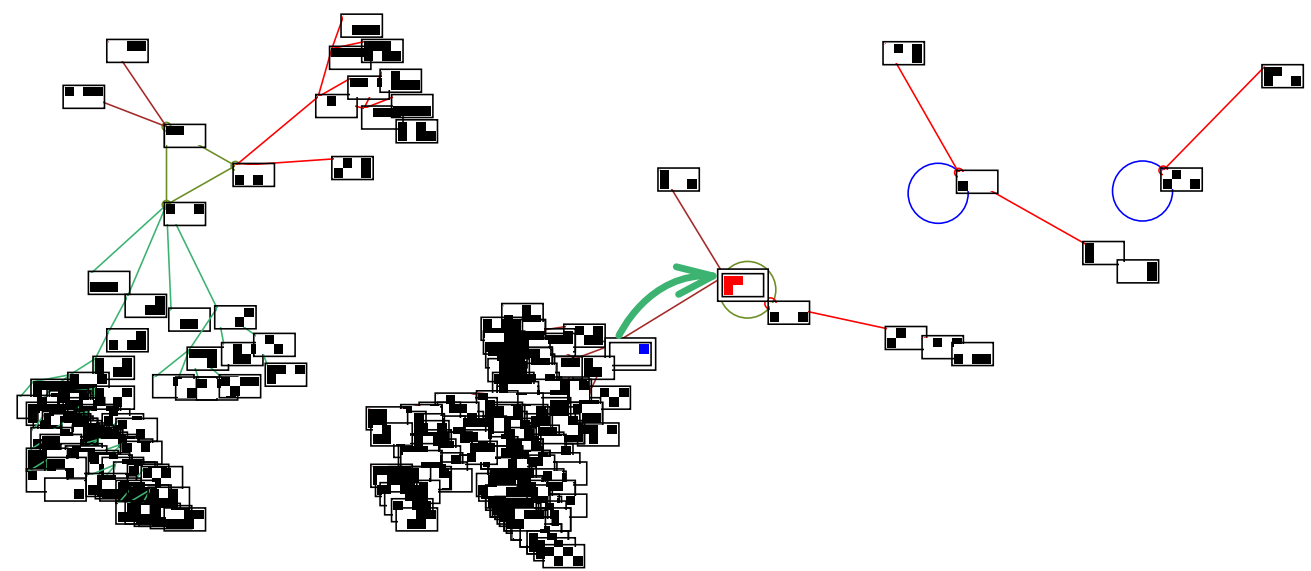

(a) The result of learning by rule bitflips, and the side effects. There is just one possible solution, which must succeed. The moved state's former subtree has been mostly transplanted.

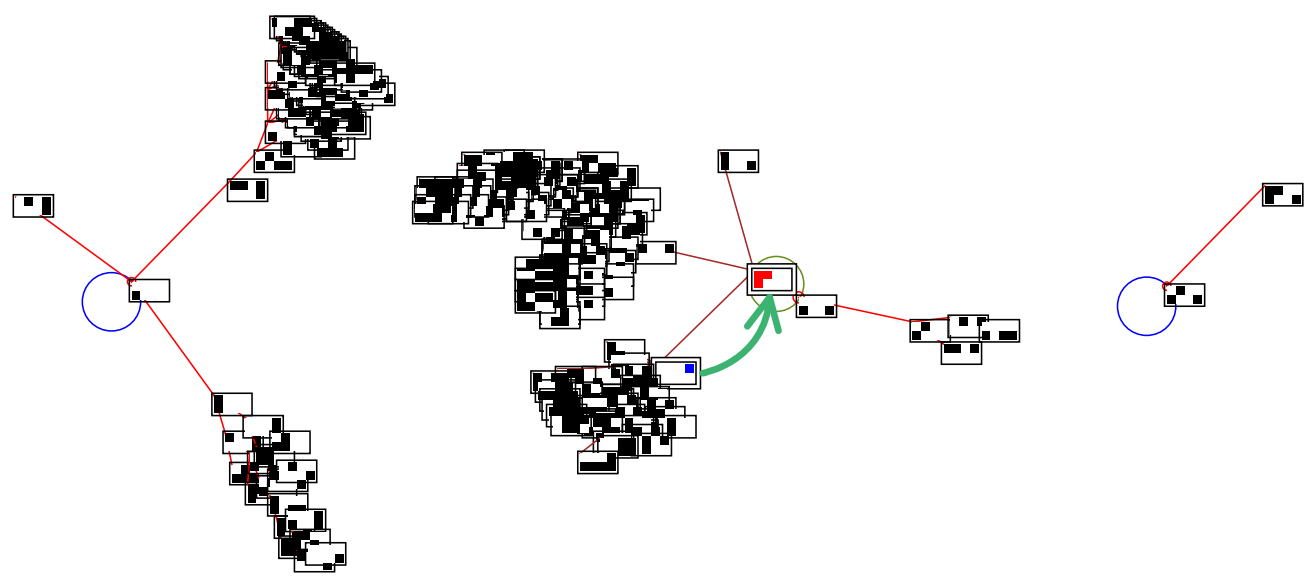

(b) The result of one example of learning by wire moves, with greater side effects. There are multiple possible solutions, but there may also be no solution by wire moves.

Figure 34.2: The basin of attraction field of a RBN, $v 2 k 4, n=8$, after learning (figure 34.1).

(a) By bit-flips. (b)By wire moves. The target state and its pre-image are highlighted with a double outline. Note that the order and orientation of the basins has changed.

\subsection{Learning/forgetting methods}

The learning methods apply to rcode only (i.e. not in TFO-mode), and work by adapting network architecture - mutating rules and/or moving wires. One or more states can be added or deleted as pre-images of a given state. Adding or deleting pre-images is analogous to learning or forgetting.

It turns out that the rule mutation algorithm is bound to learn a list of aspiring pre-images without forgetting the state's pre-existing pre-images - those not on the list may also be learnt 
3. 2. 1. 0. rcode(hex)

\begin{tabular}{|c|c|}
\hline 7: 3 & $\begin{array}{llll}3 & 1 & 6 & 0\end{array}$ \\
\hline 6: 1 & 1044 \\
\hline 5: 5 & $\begin{array}{llll}5 & 0 & 0 & 2\end{array}$ \\
\hline 4: 1 & 1125 \\
\hline 3: 5 & $\begin{array}{llll}5 & 1 & 7 & 4\end{array}$ \\
\hline 3 & $\begin{array}{llll}3 & 0 & 0 & 5\end{array}$ \\
\hline 4 & 4233 \\
\hline & $\begin{array}{llll}3 & 6 & 5 & 4\end{array}$ \\
\hline
\end{tabular}

Figure 34.3: The RBN network for figure 34.1 before learning. Because the difference between the actual successor state and the target state is confined to element 3 , learning changes effect this cell only. The new rule at element 3 (figure 34.2(a) is rcode(hex) b0c7 - one bitflip. The new wiring at element 3 (figure 34.2(b) is 5172 , one wire move.

as a side effect. There is just one specific rule scheme mutation, which is bound to succeed. By contrast, there are many alternative wire moves that may achieve the desired result, though success is not guaranteed, and pre-existing pre-images may be forgotten[26].

Any change, especially in a small network, usually has significant side effects ${ }^{1}$, but this can be beneficial because of generalization, were a subtree is transplanted together with the attached pre-image as in figure 34.2. The revised attractor basin can be immediately re-drawn to show the results and side affects of learning. The algorithms for forgetting pre-images cause less severe side effects because forgetting requires only minimal changes to network architecture.

The wiring algorithm requires nonlocal wiring. $2 \mathrm{~d}$ or $3 \mathrm{~d} \mathrm{CA}$ are always treated as nonlocal, but $1 \mathrm{~d}$ local wiring needs to be redefined as nonlocal in the learning prompts (section 34.5) or preset as nonlocal (section 12.4.1). The rule algorithm requires a rulemix set in chapter 14 note that a single rule can be set up as a rulemix (section 14.4.3).

\subsubsection{Highlighting states in attractor basins}

The nodes corresponding to a list of states can be highlighted in attractor basins - highlighting has no effect for space-time patterns. Highlighting shows up the results of learning and forgetting, but is also useful (without learning) to see how a particular set of states is distributed in the basin of attraction field, single basin or subtree, and how the distribution changes when network parameters are altered. The highlighted presentation is illustrated in figure 34.4 for different types of node display ${ }^{2}$.

For binary $(v=2)$ networks, the target node is shown in red and aspiring pre-images in blue - for highlighted nodes shown as bit patterns, these are the colors of 1s, otherwise 1s are shown black. For $v \geq 3$ networks, highlighted nodes shown as bit patterns correspond to values. Nodes not highlighted may be suppressed entirely. The highlighting feature may be used for any network architecture, including local 1d CA with compression on (section 26.1).

\subsubsection{Basin layout for learning}

The default layout of the basin of attraction field may need some adjustment for a good presentation for learning. Setting the layout is described in section 25.2, but can be readjusted with much greater flexibility in the jump-graph (section 20.7) - this was done for figure 34.1.

\footnotetext{
${ }^{1}$ These learning methods are not good at creating categories from scratch, i.e. solving the inverse problem or reverse engineering. A rudimentary solution to that is provided in section 18.7.4. However, the learning/forgetting methods are useful for fine tuning a network which is already close to where its supposed to be.

${ }^{2}$ The node display is selected in section 26.2 and is not affected by the presentation for selecting the target and pre-image states in sections 34.3 and 34.4.
} 

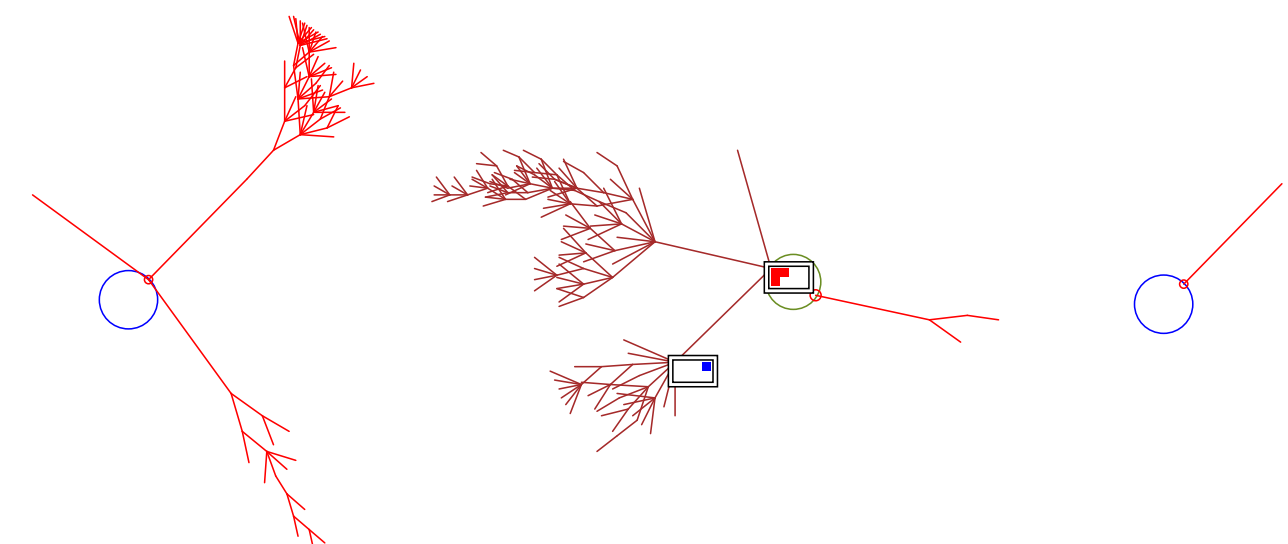

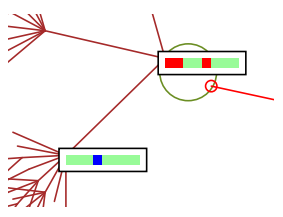

1d bit pattern

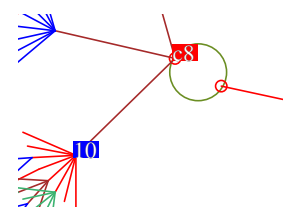

hexadecimal

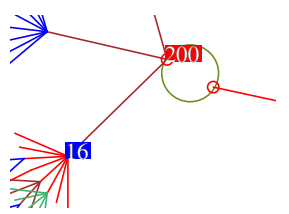

decimal

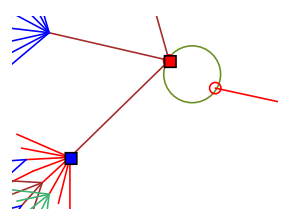

spots

Figure 34.4: Top: the basin of attraction field of the RBN in figure 34.2(b), $v 2 k 4, n=8$, learning by wire moves. The target and pre-image nodes (states) are shown as $2 \times 4$ bit patterns and highlighted with a double outline - other nodes are suppressed. Below: a fragment of the second basin showing alternative presentations of highlighted nodes (states). The presentation will follow the node display selected in section 26.2 irrespective of the presentation for selecting the target and pre-image states in sections 34.3 and 34.4 , but other nodes can be suppressed if required.

\subsection{Selecting the learn/forget/highlight window}

Once rule/s have been set, whenever the network is reviewed in the lower-left wiring graphic (section 17.3), the learn/forget/highlight window and functions (section 34.3) can be activated. The upper-right wiring graphic reminder (section 17.4) includes the following option (highlighting does not apply for space-time patterns),

\section{... highlight/Learn-l/L or for space-time patterns ... Learn-L}

Enter $\mathbf{L}$ for the learn/forget/highlight functions. For attractor basins, enter $\mathbf{l}$ to highlight selected states. Sections 34.2.1 and 34.2.2 describe how to get to this point in DDLab - the sequence of prompt windows is summarized as below,

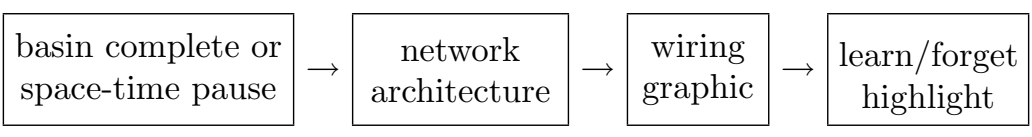

Note that changes to wiring and rules can be made directly from the wiring graphic, which can be done in conjunction with automatic learning/forgetting. 


\subsubsection{Selecting the wiring graphic}

The wiring graphic (section 17.3) can be reached from the top-right network architecture prompt (section 17.1), for example,

1d network $(n=150)$, review/revise/learn, wiring and rcode graph-g, matrix: revise-m view-M prtx-Mp graphic: 1d:timesteps-1 circle-c 2d-2:

Enter 1, c, 2 (or $\mathbf{3}$ for 3d) for different presentations of the wiring graphic (e.g. figures 17.2, $17.3,17.10,17.13)$.

\subsubsection{Selecting the network architecture prompt}

The network architecture prompt (section 17.1) can be reached as follows,

initial prompts ... during the main prompt sequence after rules are set - the network architecture prompt appears automatically (section 17.1).

attractor basins ... when a basin of attraction field, single basin or subtree is completed or abandoned, the following top-left "Attractor basin complete prompt" appears (section 30.4),

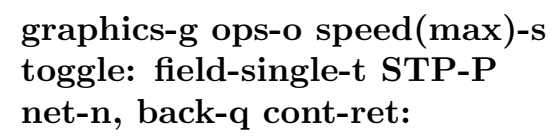

enter net-n,

alternatively ops-o followed by net-n (section 30.5),

space-time patterns ... when interrupting space-time patterns, the top-right "Space-time pattern interrupt/pause prompt" (section 32.16) appears, for example,

rule:save/load/revise/rnd/trans-s/l/v/r/t net-n sample:load/keep-E/K

PScript-P Z:high/low-Z/z canal-C g-rules(rnd/seq)-G

state:rev-e rnd/Ham/blk/orig/last-R/H/k/o/ sng:pos/neg-5/6 save/load-S/L

graph-g/p, border-B filter-f skip-X pause-N step-x/+ top-T

on-the-fly-y hide-W no-ops- $Q$ back-q cont-ret:

enter net-n for the network architecture prompt.

\subsection{Select the target state}

Enter $\mathbf{L}$, or $\mathbf{l}$ (for highlighting only) in section 34.2 for the "target state" prompts in a lower left window, similar to "The seed prompt" (section 21.1) - the various methods of setting states are described in chapter 21.

The initial prompts sets the "target state" — to which "aspiring pre-images" will attempt to directly attach or detach themselves by means of learning/forgetting algorithms. Prompts are presented in two separate windows, one above the other. The upper window indicates that the target state is to be set, 
for attractor basins, $\mathbf{L}$ or $\mathbf{l}$ selected in section 34.2 learn/forget/highlight: set target state or highlight only: set target state for space-time patterns, $\mathbf{L}$ selected in section 34.2, where highlighting does not apply learn/forget: set target state

The lower window presents the first of a series of prompts to select the target state, for example,

Select target stare (v2 $1 \mathbf{d} \mathbf{n}=14)$, win-w empty-e fill-f prtx-x (for 1d, $v=2$ ) rnd-r bits1d-b bits2d-B hex-h dec-c repeat-p load-l (def-B):

Select the target state as if selecting a seed in section 21.1 - the prompts are the same, but the default selection method is initially bits2d-B (sections 21.4, 21.4.6).

\subsection{Select aspiring pre-image/s}

Once the target state has been selected, one or more states are specified as its "aspiring pre-images" (i.e. direct predecessors). The learning/forgetting algorithms will attempt to directly attach (learn) these states, or detach (forget) existing pre-images of the target state. Alternatively the "aspiring pre-images" and the "target state" can be just highlighted. The set of aspiring pre-images can be an arbitrary list of states, a range of decimal equivalents (if applicable section 21.6), or can be based on Hamming distance, parity, or the previous list may be repeated. Prompts are presented in two separate windows, one above the other - the upper prompt appears first as follows,

pre-images: as before-b parity-p range-r Hamm-h1/h2 list-(def 1): (parity-p and Hamm-h2 for $v=2$ only)

These options are summarized below, with further details in the sections indicated,

options ... what they means

before-b ... to repeat a previous set of aspiring pre-images (parity, range or Hamm). If a list was previously selected, the new list defaults in section 34.4.4 will correspond to the previous list.

parity-p ... ( $(v=2)$ to select states based on odd or even parity (section 34.4.1).

range-r ... to specify a range of states according to their decimal equivalents, but within the limits of max- $n$ in section 21.6.

Hamm-h1/h2 ... enter h1 for states with Hamming distance 1 from the target state. For $v=2$, h2 gives a Hamming distance of both 1 and 2 (section 34.4.3).

list-(def 1) ... enter return to specify just one aspiring pre-images, or a number to specify the size of an arbitrary list of pre-images (section 34.4.4).

Once set, the target state and the set of aspiring pre-images (however selected) are reviewed as decimal equivalents (section 34.4.5). 


\subsubsection{Odd or even parity}

\section{binary $v=2$ systems only}

In a binary $v=2$ network, odd and even parity signifies that the total of $1 \mathrm{~s}$ in a state's bitstring is odd or even ${ }^{3}$ (a zero total of 1 s has even parity). By default, parity applies to the whole bitstring (size $n$ ) if $n \leq 20$, otherwise only the 20 leading bits are considered. A smaller number of leading bits can also be specified.

Enter $\mathbf{p}$ in section 34.4 to automatically select aspiring pre-images with the given parity.

The following upper prompt is presented,

parity: odd-1, or even-(def):

Enter return for even parity, 1 for odd parity. A further upper prompt allows the parity to relate to a leading part only of the network,

$$
\text { enter part system size (def 10): (for } n=10)
$$

If a size $x \leq n(\max x=20)$ is entered, states with the given parity relating only to cell indexes 0 to $x$ - 1 will be selected. Figure 34.5 shows the results for entering 7 in a $n=10$ network.

$$
\begin{aligned}
& \text { enter part system size (def } 10): 7 \\
& \text { target }=0 \text {, pre } \text { images }=64 \text {, parity }=e \text { ven, } \text { max state }=126 \text {, cont-ret: } \\
& 126,125,123,120,119,116,114,113,111,108,106,105,102,101,99,96,95,92,90,89,86,85,83,80,78,77,75,72,71,68,66,65,63,60,58,57,54, \\
& 53,51,48,46,45,43,40,39,36,34,33,30,29,27,24,23,20,18,17,15,12,10,9,6,5,3,0,
\end{aligned}
$$

Figure 34.5: Selecting even parity for the leading 7 bits in a 10 bit network. A list of the $2^{7} / 2=64$ states with even parity are displayed in decimal. This is one kind of review list covered in section 34.4.5.

\subsubsection{Range of decimal equivalents}

Enter $\mathbf{r}$ to automatically set a range of aspiring pre-images, set between two selected decimal values within the limits $\max -n$ in section 21.6. For a basin of attraction field, $n$ will be within these limits, but if $n \geq \max -n$ (likely for space-time patterns), so outside the range of allowed decimal equivalents, only max- $n$ leading bits/values are considered. The following (upper) sequence of prompts is presented to set the start and end decimal equivalents, and the "step" or increment size (default 1),

range of pre-images (def 1-8, max 255), start: end: $\quad$ step: (for $n=8)$

\subsubsection{Pre-images according to Hamming distance}

Enter $\mathbf{h} \mathbf{1}$ in section 34.4 to automatically set the aspiring pre-images based on a Hamming distance of 1 , where states differ by just 1 bit/value from the target state. For $v=2$ only, enter $\mathbf{h} \mathbf{2}$ for a Hamming distance of both 1 and 2 .

\footnotetext{
${ }^{3}$ The parity does not relate to the state expressed as a decimal value, but simply to the total of $1 \mathrm{~s}$ irrespective of their position in the bitstring.
} 


\subsubsection{List of aspiring pre-images}

Enter return to specify just one aspiring pre-images, or a number to specify the size of an arbitrary list of pre-images, at the (upper) prompt (section 34.4),

... list-(def 1):

A prompt, or series of prompts to select the pre-image/s will be presented in the lower window. These prompts are the same as for selecting a seed (section 21.1), except for the heading,

\section{Select aspiring pre-image $1 \ldots$}

for a list, this is followed by ...

Select aspiring pre-image $2 \ldots$

Select aspiring pre-image $3 \ldots$ etc.

The default selection method is setting $2 \mathrm{~d}$ bits (sections 21.4 and 21.4.6).

\subsubsection{Review target state and aspiring pre-images}

Once the target state (section 34.3) and aspiring pre-images (section 34.4) have been set, information about the selection will be displayed in the lower window. If the system size is within the limits for decimal, $n \leq \max -n$ (section 21.6), the states in decimal will also be included. Figure 34.5 gives an example for parity. Examples for a range of decimal equivalents, Hamming distance, and an arbitrary list, are given below for $v=2, n=8$,

a range of decimal equivalents, section 34.4 .2

target $=240$, pre-images $=16$, range $2-255$, step $=16$, cont-ret:

$2,18,34,50,66,82,98,114,130,146,162,178,194,210,226,242$,

the set of 1-bit mutants, Hamming distance=1, section 34.4 .3

target $=240$, pre-images $=8,1$-bit mutants, step $=16$, cont-ret:

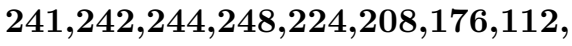

an arbitrary list of 5 states, section 34.4 .4

target $=240$, pre-images $=5$, cont-ret:

$50,167,152,88,94$

If there are more states than will fit in the window, the following prompt allows more to be displayed, or to abandon the display and continue,

\section{more-m cont-ret:}

Enter $\mathbf{q}$ to backtrack to section 34.4 and reset the aspiring pre-images.

\subsection{Learn, forget, or highlight only}

Once the target state (section 34.3) and aspiring pre-image/s (section 34.4.4) have been set, reviewed and accepted (section 34.4.5), prompts are presented to learn, forget, or just highlight the aspiring pre-images (as well as the target state). Note that highlighting does not apply for space-time patterns. 


\subsubsection{1d CA - local wiring and highlighting}

For a 1d CA (with local wiring and a single rule), or a network with 1d local wiring and mixed rules, a prompt (in the upper window) first allows the wiring to be redefined as nonlocal,

1d CA, if $\mathbf{L}$ was selected in section 34.2 (if $\mathbf{1}$ was selected, highlight only applies) local 1d: nonlocal wiring-w:

$1 d$ local wiring and mixed rules local 1d, rulemix: nonlocal wiring-w:

Enter $\mathbf{w}$ to redefine the wiring as nonlocal, allowing learning by wire moves, and suppressing compression in attractor basins (section 26.1). If return is entered to conserve local wiring, subsequent options differ for space-time patterns or attractor basins. For space-time patterns, the program skips directly to the last prompt before exiting the learning routine (section 34.9).

For attractor basins, there is a reminder that although learning is deactivated, the highlighting options remain,

\section{for attractor basins}

... highlight only-(def):

Enter return to skip directly to the highlighting options (section 34.8). Compression in attractor basins is suppressed (section 26.1).

Highlighting is the only possibility for a network were the wiring is defined as local 1d, using the local $1 \mathrm{~d}$ reverse algorithm (section 2.18). Note that $1 \mathrm{~d}$ local wiring can also be redefined so that it is treated as nonlocal in section 12.4.1. Highlighting on its own can be useful to show how a selected set of states is distributed in attractor basins.

\subsubsection{Nonlocal wiring - learn, forget, or highlight only}

For networks that do not have 1d local wiring, or if $\mathbf{w}$ was entered in section 34.5.1 above, or for any other network with nonlocal wiring, including $2 \mathrm{~d}$ and $3 \mathrm{~d} \mathrm{CA}^{4}$, the following prompt is presented,

\section{for attractor basins \\ highlight only-h, forget-f, learn-(def): \\ for space-time patterns, where highlighting does not apply forget-f, learn-(def):}

Enter return or $\mathbf{f}$ to learn or forget i.e. add/remove the aspiring pre-image/s to/from the existing pre-image fan of the target state. When the attractor basin is redrawn according to the altered network, the target and aspiring pre-image states will be highlighted (section 34.1.1).

Enter $\mathbf{h}$ to just highlight the selected states (without learning or forgetting) - the program skips directly to the last prompt before exiting the learning routine (section 34.9).

\footnotetext{
${ }^{4} 2 \mathrm{~d}$ and $3 \mathrm{~d}$ CA "local" wiring is treated as nonlocal in DDLab's algorithms.
} 


\subsubsection{Learning/forgetting by wire moves or bit/value-flips}

If learning or forgetting was selected in section 34.5.2, a prompt (in the upper window) is presented to proceed either by moving wires or flipping bits/values in rule-tables.

For a single rule network, only learning/forgetting by wire moves is possible ${ }^{5}$, and the prompt is as follows,

learn by wire-moves only-(def): (or forget ...)

Enter return to proceed by wire-moves (section 34.7).

Otherwise, for networks with nonlocal wiring and a rule-mix, and also networks with mixed- $k$ which by definition has both, the prompt is as follows,

for binary, $v=2$, networks

learn by wire-moves -w, bit-flips-(def): (or forget ...)

for multi-value, $v \geq 3$, networks

learn by wire-moves $-\mathrm{w}$, value-flips-(def): (or forget ...)

Enter return for bit/value-flips (section 34.6), or w for wire-moves (section 34.7).

Enter q to backtrack.

\subsection{Learning/forgetting by bit/value-flips}

The bit/value-flip learning algorithms[26] change a specific bit/value in the rule-table at each cell position where there is a mismatch between the actual successor cell of the aspiring pre-image and the same cell in the target state. This takes care of that specific mismatch for all future aspiring pre-images, so pre-existing pre-images cannot be "forgotten", i.e. detached as pre-images from the target state, by learning more states as pre-images.

However other states not on the list of aspiring pre-images may also be learnt, so side effects will occur elsewhere in the attractor basin. To "forget" a pre-image, just one of a set of possible bit/value-flips is sufficient, chosen from the set at random. As fewer network changes are needed there will be fewer side effects.

If bit-flips or value-flips was selected in section 34.5.3, the required changes to the network will be made, and the following (upper) prompt is presented,

for attractor basins
learn/forget/highlight more-m:
for space-time patterns, where highlighting does not apply
learn/forget more-m:

Enter $\mathbf{m}$ to repeat the procedure from section 34.5, otherwise the network as it stands is accepted. For attractor basins the node highlighting options follow (section 34.8). For spacetime patterns the program skips directly to the last prompt before exiting the learning routine (section 34.9).

\footnotetext{
${ }^{5}$ section 14.4.3 describes how to set up a single rule network that is treated as a rulemix.
} 


\subsection{Learning/forgetting by wire-moves}

The wire-move learning algorithms[26] move a single input wire at each cell position where there is a mismatch between the actual successor cell and the same cell in the target state. There is a choice of wires to select from the pseudo-neighborhood, and a choice of new coupling positions, some of which will correct the mismatch. The aspiring pre-image order, the pseudo-neighborhood order, and the coupling order, are all shuffled at random, and the first successful move is selected (if it exists). This may cause pre-existing (or just learnt) pre-images to be forgotten, i.e. detached as pre-images from the target state.

If "just learnt" states are forgotten when learning the next pre-image, the next wire-coupling alternative in the shuffled order will be tried. If none succeed the algorithm continues with the next aspiring pre-image. On completing the learning pass, the state/s learnt, and not learnt, are listed by their decimal equivalents, if within the limits for decimal, $n \leq \max -n$ (section 21.6), otherwise the states are symbolically represented by " $\mathrm{x}$ ".

The following examples are for binary $(v=2)$ networks,

failing to learn one pre-image by re-wiring

target state $=73$,

$193,<-$ no

re-learn by wire-moves -w, bit-flips -b: (or re-forget ...)

cont-ret:

tried rewiring again, successfully learnt one pre-image

target state $=73$,

$193,<$-ok

success - aspiring pre-image learnt (or ... forgotten)

cont-ret:

learning multiple pre-images, for Hamming distance 1 from state 8

target state $=8$,

$136,0<-$ no

$\mathbf{2 4 , 7 2 , 1 2 , 9 , 1 0 , 4 0 , < - o k ~ r e - l e a r n ~ b y ~ w i r e - m o v e s ~ - w , ~ b i t - f l i p s ~ - b : ~ ( o r ~ r e - f o r g e t ~ . . . ) ~}$

learning multiple pre-images, space-time pattern $n=55$, Hamming distance 1

target state $(\mathbf{n}=\mathbf{5 5})$ (outside decimal limits - states represented symbolically)

$\mathrm{x} \times \mathrm{x} \times \mathrm{x} \times \mathrm{x} \times \mathrm{x} \times \mathrm{x} \times \mathrm{x} \times \mathrm{x} \times \mathrm{x} \times \mathrm{x} \times \mathrm{x}<-$ no

$\mathrm{x} \times \mathrm{x} \times \mathrm{x} \times \mathrm{x} \times \mathrm{x} \times \mathrm{x} \times \mathrm{x} \times \mathrm{x} \times \mathrm{x} \times \mathrm{x} \times \mathrm{x} \times \mathrm{x} \times \mathrm{x} \times \mathrm{x} \times \mathrm{x} \times \mathrm{x} \times \mathrm{x} \times<-$-ok

re-learn by wire-moves -w, bit-flips -b: (or re-forget ...)

if all multiple pre-images are learnt (or forgotten)

success - all aspiring pre-images learnt (or ... forgotten)

When learning by wire-moves,

$<$-ok indicates learnt states, <-no unlearnt states

If there are unlearnt states remaining, options are offered to try to re-learn again by wire-moves, or by bit/value-flips (section 34.6) which is bound to succeed[26]. 

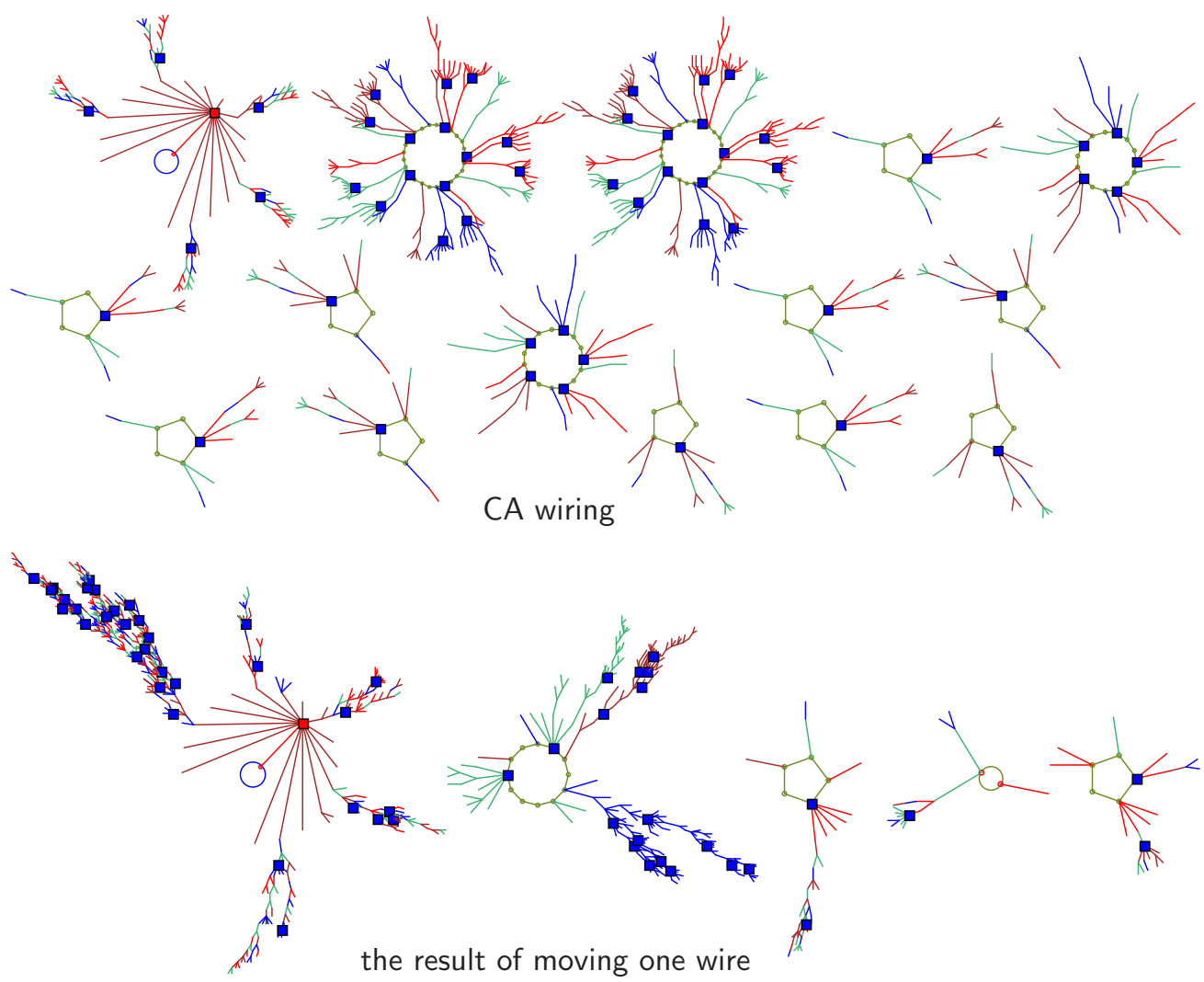

Figure 34.6: Top: the basin of attraction field of the CA $v 2 k 3$ rcode 110, $n=10$ (compression suppressed). Bottom: the result of moving one wire, cell 4 wiring 543 changed to 593 , done by "hand rewiring" in section 17.8.4. The node type selected was spot in section 26.2. In both fields, the target state (all-1s) is highlighted in red, together with states Hamming distance 1 and 2 from the target state (h2 section 34.4) highlighted in blue. All other states are suppressed. Note that the basin layout was rearranged within the jump-graph, section 20.7 .

\section{re-learn by wire-moves -w, bit-flips -b:}

If $\mathbf{w}$ is selected, the last $<-\mathbf{n o}$ state will be learnt first, followed by the set of shuffled $<-\mathbf{o k}$ states, then the remaining shuffled <-no states. On completing the pass the resultant listing and prompts are repeated. Note that for a large system with a large list of aspiring pre-images, a wire-move learning pass may take an unpredictably long time.

To forget a pre-image just one appropriate wire-move is required. Forgetting is generally much easier and quicker than learning. As fewer network changes are needed there will be fewer side effects. Note that in the forgetting procedure <-ok indicates states still attached to the target state, i.e. not forgotten, and <-no indicates states successfully forgotten, for example if all are $<-$ no the following prompt is presented below the listed states,

\section{success - aspiring pre-image forgotten} cont-ret: 

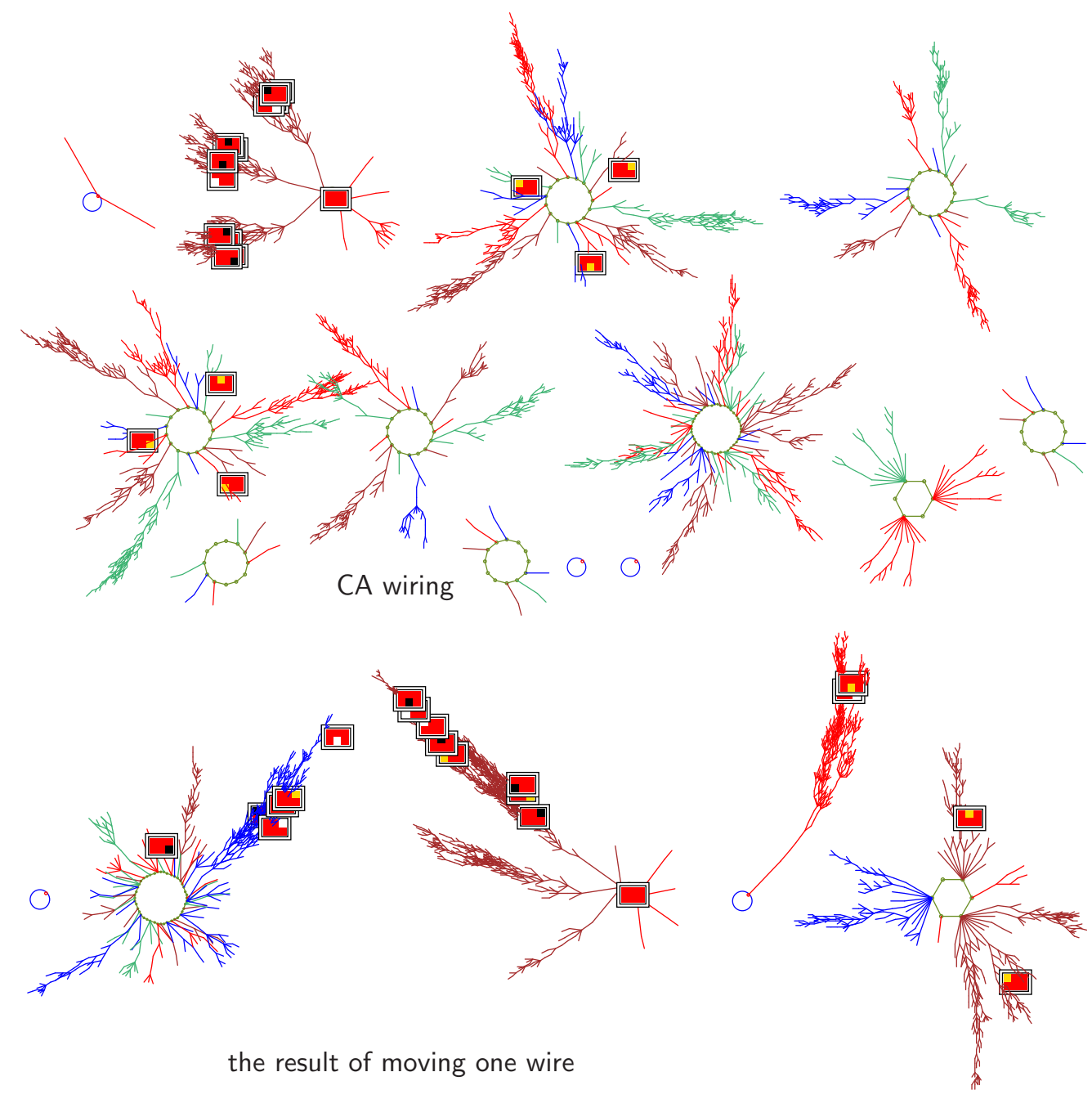

Figure 34.7: Top: The basin of attraction field of a CA $v 4 k 3, n=6$ (compression suppressed). Bottom: the result of moving one wire, cell 2 wiring 321 changed to 351 , done by "hand rewiring" in section 17.8.4. The node type selected was $\mathbf{2 d - B}$ in section 26.2. The highlighted states are the allred target (all-2s), together with states Hamming distance 1 from the target state (h1 section 34.4). All other states are suppressed. Note that the basin layout was rearranged within the jump-graph, section 20.7. (rcode(hex)96570e1b37b0b8d6b3189e967bac51ac).

If return is entered at the various prompts above, the following (upper) prompt is presented,

learn/forget/highlight more-m: or for space-time patterns learn/forget more-m:

Enter $\mathbf{m}$ to repeat the procedure from section 34.5, otherwise the network as it stands is accepted. For attractor basins the node highlighting options follow (section 34.8). For spacetime patterns the program skips directly to the last prompt before exiting the learning routine (section 34.9). 


\subsection{Highlighting options}

attractor basins only

Once learning is complete, or if highlight only was selected in section 34.5, the nodes representing the target state and the set of aspiring pre-images can be highlighted in the attractor basin - the particular form of highlighting depends on the node type selected in section 26.2.

The following prompt is presented,

highlight: none-n, suppress all other nodes-s:

Enter $\mathbf{s}$ to suppress the display of nodes other than highlighted nodes. Enter return to highlight the selected nodes and show other nodes normally according to the selected node type. Enter $\mathbf{n}$ not to highlight, but show all nodes as normal. Examples are shown in figures 34.4, 34.6 and 34.7.

\subsection{Learning/forgetting/highlighting complete}

When learning/forgetting or highlighting is complete, the lower-left wiring graphic (section 17.3) and the "wiring graphic reminder" (section 17.4) reappear. Within the wiring graphic, the results of learning/forgetting can be re-examined and changes made, for example with "hand rewiring" (section 17.8.4) or "revising the rule" section 17.8.10.

Enter return to exit the wiring graphic, and revert to the top-left "attractor basin complete" prompt (section 30.4), or the pause options for space-time patterns (section 32.16).

Enter return to redraw the attractor basin (or restart space-time patterns) with the new (learnt) parameters. Attractor basins will be drawn with the specified highlighting. Note that after the attractor basins have been drawn, if return is entered again, the network will be changed by the mutations set (by default) in chapter 28. This shows how a set of highlighted states is redistributed for a succession of mutated attractor basins (figures 34.6, 34.7). However, when "sculpting" the basin of attraction field, it is safer to first deactivate mutation in section 28.1. 


\section{Chapter 35}

\section{Filing}

DDLab has functions for saving and loading a variety of DDLab specific file types, which have default three letter extensions, for example myrul_v3.rul. PostScript files generated in DDLab have a two letter extension .ps.

DDLab was first developed in DOS, and DOS filename conventions still apply for all versions, including Linux-like operating systems. A DOS filename has up to eight characters (starting with a letter) and up to three characters in the extension following the dot.

In DDLab the lowercase letter $\mathbf{q}$ cannot be included because it is used for backtracking or deleting input. The filename extension is not entered as part of the filename - it will be automatically added according to the type of file.

This chapter lists the file types, and describes the methods for saving and loading files. The following file types are described in more detail in other sections of the manual, including their encoding, and loading constraints for compatibility with the current (base) network.

Network architecture files ... (.mix, .w_s, .r.s, .wrs) Described in detail in chapter 19. Encoding: sections 19.3 and 19.3.1. Constraints that apply to loading different types of file into a base network: section 19.4.1.

Single rcode, kcode or tcode files ... (.rul .vco .tco) Encoding: section 16.16. $[v, k]$ loading constraints: the value-range $v$ and neighborhood size $k$ in the file must be the same as in the base.

The seed or state (and rule-table) ... (.eed), for the seed or initial state or the ruletable saved as a seed, or a defined patch. Encoding: section 21.9. Size/dimension/value-range loading constraints: section 21.7.1. 


\subsection{File types, default filenames, and extensions}

The DDLab specific file types, default extensions, and default filenames are listed below.

type

K-MIX

WIRING ONLY

SINGLE RCODE SINGLE KCODE SINGLE TCODE

RULE TABLE

RCODE SCHEME KCODE SCHEME TCODE SCHEME

WIRING+RULE SCHEME default filename

description

$\operatorname{my} \operatorname{mix} . \operatorname{mix}$

The neighborhood-mix, (sections 9.4, 9.11.3, 19.5). Encoding: see section 19.3.1.

mywso.w_s The wiring scheme (chapter 19). Encoding: sections 19.3.

myrul_v2.rul ... for rcode (for example)

myvco_v3.vco ... for kcode (for example)

mytco_v4.tco ... tcode (for example)

etc. A single rule, rcode, kcode or tcode, for a given $v$ and $k$ (chapter 16, and sections 30.5.1, 32.16.1). Encoding: section 16.16. The default filename includes $v$.

When setting a rule, kcode, or tcode, in chapter 16, the most recent will be automatically saved, with the filename 1_v2k3.rul, 1_v2k3.vco,1_v2k3.tco - including both $v$ and $k$ - these rules can then be automatically reloaded.

mytbl_v2.tbl The rcode-table, or in TFO-mode the kcode-table or tcodemytbl_v3.tbl table, for a given value-range $v$, can be saved as an ASCII etc.

file (section 16.2). The default filename depending on $v$.

myrso_v2.r_s ... for rcode (for example)

myrso_v3.r_v ... for kcode (for example)

myrso_v4.r_t ... for tcode (for example)

etc.

The rcode, kcode, or tcode, scheme (with corresponding extensions .r_s, .r_v, .r_t (chapter 19). For homogeneous $k$ only, otherwise use the WIRING+RULE SCHEME below. The default filename includes the value-range $v$. Encoding: sections 19.3 .

mywrs_v2.wrs ... for rcode (for example)

mywrs_v3.wrv ... for kcode (for example)

mywrs_v4.wrt ... for tcode (for example)

etc. Both wiring and rules (chapter 19).

Encoding: sections 19.3.

The default filename includes the value-range $v$.

NETWORK DATA my_net.dat ... for the network data as an ASCII file (section 19.6). 
SEED

BASIN DATA

PRE-HIST-DATA

HIST-DATA

EXHAUSTIVE

ORDER-DATA

STAT-SAMPLE

GRAPH LAYOUT my_grh.grh

SCREEN etc.

my_prh.prh mysee_v2.eed A state bit/value-string, including the value-range $v$, size $n$, mysee_v3.eed dimension ( $1 \mathrm{~d}, 2 \mathrm{~d}$ or $3 \mathrm{~d}$ ), and the $i, j, h$ coordinates (sections mysee_v4.eed 30.5.2, 32.16.4). Encoding: section 21.9. The default filename includes the value-range $v$. As above, but the default filename changes to mypch_vx when saving a patch - part of a state.

my_data.dat An ASCII file for the field/basin/subtree and list of states data (sections 27.3-27.5.2, 19.6). Encoding: ASCII.

Data for the pre-image histogram. (section 24.6.5). Encoding: a binary file of an unsigned long array, recording each in-degree frequency, from zero onwards.

my_his.his

Data for various other histograms (sections 9.11.1, 17.8.13, 31.5.2, 31.6.5, 31.7). Encoding: a binary file of an unsigned short array, recording the height of each histogram bar, from zero onwards.

myexh_v2.exh An exhaustive mapping, listing each state and it successor myexh_v3.exh (section 29.8.1, 29.7.2, 18.1.2). Encoding: a binary file of a myexh_v4.exh char array recording a list of $v^{n}$ successor bit/value strings, etc. $\quad$ each in $v^{n} / 8$ bytes (minimum 1 byte), where the pre-image is the array index, from zero onwards. The default filename includes the value-range $v$.

my_order.ord For the sequential updating order. (section 29.9, 29.9.2). Encoding: a binary file of an unsigned short array, recording the sequential order according to the array index, from zero onwards.

mystat.sta The statistical sample of automatically classified rule-space, (chapter 33, sections 33.4, 33.5, 32.16.2). Encoding: section 33.11 .

The layout of the network-graph (section 20.2), or the attractor jump-graph (section 20.3). Encoding: a binary file of a signed short array, recording the $x, y$ coordinates of each graph node according to the array index, from one onwards.

myimage.nat The screen image, (section 5.6.1).

Encoding: (the same for all platforms) has a DDLab specific format too involved to explain here. A . nat file, saved on a given platform can be successfully loaded back into DDLab on the same platform, even if the screen size has been changed. If saved on one platform and loaded back to another, the results are less reliable. 


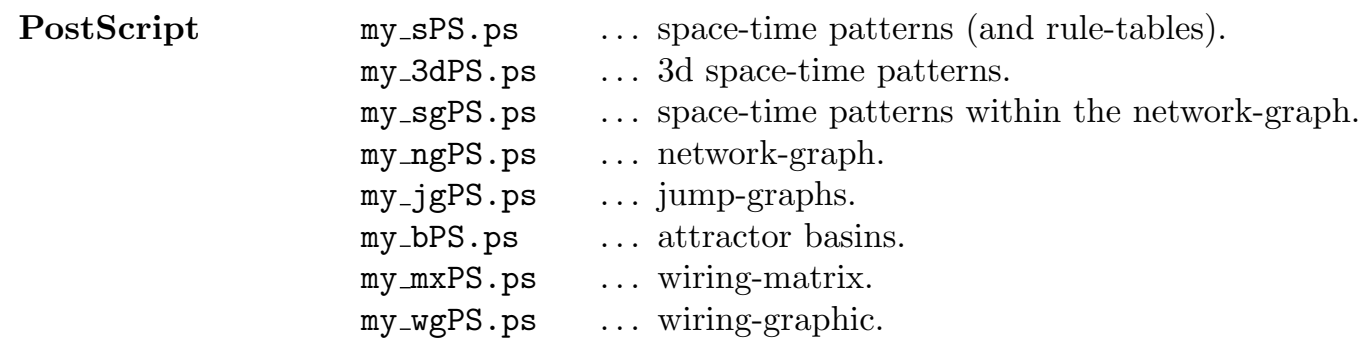

These are vector PostScript files described in chapter 36 . The files can be loaded in GhostView (Linux command gv), and included in LaTex documents. Encoding: ASCII - in the PostScript language.

\subsection{The filing prompt}

In general when saving or loading a file, the following filing prompt appears in a top-right window,

SAVE [or LOAD] [type] - filename (no ext) .[extension] will be added change dir-d, now: [current path] list-?, quit-q, default [filename]:

for example,

loading a $v=3$ seed in Linux-like systems

LOAD SINGLE RULE-filename (no ext) .rul will be added change dir-d, now:/home/sussex/ddlabm07/ddfiles: list-?, quit-q, default myrul_v3:

saving a $v=3$ seed in $D O S$

SAVE SEED-filename (no ext) .eed will be added change dir-d, now: C: $\backslash$ WATCOM $\backslash$ ddlabm07

list-?, quit-q, default mysee_v3:

To load or save, enter a legal filename, up to 8 characters starting with a letter but excluding $\mathbf{q}$, and without the filename extension, which is automatically added, or enter return to accept the default filename. When the filename is entered, say rule193 to load a single rule, the lowest line of the prompt will respond with,

\section{filename=rule193.rul REVISE-q cont-ret: (for example)}

Enter return to load the filename, or $\mathbf{q}$ to revise. If the file is not found, the following message appears in a top top-right window,

file error rule193.rul, cont-ret: (for example)

Enter return to continue. 


\subsection{Loading constraints on the value-range $[v, k]$ — rules}

When loading a single rule file (.rul, .vco, .tco, .tbl) into a single rule base (section 16.14), the $[v, k]$ parameters (value-range and neighborhood size) of the file and the current base must be equal, otherwise the following error messages are displayed in a top top-right window,

current-v not equal to file-v

file-v(3) != base-v(2), can't load, not loaded! cont-ret: (for example)

current- $k$ not equal file- $k$

not loaded! file-k(5) not equal to base-k(7), cont-ret: (for example)

Enter return to continue.

Note that a single rule file can be loaded at a give position in mixed rule network provided file- $k \leq$ base- $k$ - enter $\mathbf{v}$ from the wiring graphic, section 17.4.

For networks with mixed rules, non-local wiring, or mixed-k (.r_s, .r_v, .r.t, .wrs, .wrv, .wrt) the loading constraints, including network size and dimensions, are described in section 19.4.1.

\subsection{Loading constraints on the value-range $[v, n]$ — seeds}

When loading a seed the value-range $v$ of the file would usually be equal to the current (base) $v$, but a file with a different $v$ can still be loaded, in which case the following prompt appears,

file-v(2) != base-v(8), abandon-q, load anyway-ret: (for example)

The size and dimensions of the file and base networks can also differ — section 21.7.1 provides further details.

\subsection{Changing the directory}

The current directory is shown in the filing prompt (section 35.2), and can be changed by entering $\mathbf{d}$ - the following top-right prompt appears, for example,

example for Linux-like systems

current path /home/sussex/ddlabm07/ddfiles, CHANGE dir new path or subdir:

example for DOS

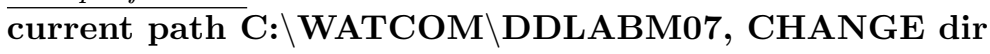

new path, (ie c: $\backslash$ dir $\backslash$ subdir): 
In Linux-like systems enter the sub-directory name, or ".." (dot dot) for the parent directory. In DOS, enter the full new path, (i.e. c: \newdir $\backslash$ newsub $\backslash$ mydir). Directory names are limited in the same way as filenames -8 characters starting with a letter but excluding $\mathbf{q}$.

The filing prompt (section 35.2) will reappear with the new path, which will stay current until changed again. If the new subdirectory or path is missing, or has an illegal name, the following error message appears,

can't change to [illegal name]

The program reverts to the filing prompt (section 35.2).

\subsection{List files}

To list the files with the current filename extension (in the current directory) enter ? (question mark) in the filing prompt (section 35.2). A list will be displayed in a narrow window on the right of the DDLab screen (figure 35.1). The following prompt appears at the bottom of the list,

list again-a (if the list fits on the screen) more-m (if there are too many files to fit) or filename

: (the filename (without extension) can be entered here

If there are too many files to fit, enter $\mathbf{m}$ to see more. A filename (without extension) may be selected in this window, or enter return and select the filename in the filing prompt (section 35.2).

\section{LOAD SINGLE K-CODE-filename (no ext) .veo will be added change dir-d, now: /home/andy/sussex/ddlabm06/ddfiles: list-?, quit-q, default myvco_v3:?}

v3k6x1.veo
v3k6x2.veo
v3k7w1.veo
v3k7w2.veo
1_v3k7.veo

Figure 35.1: Listing files with by entering? (question mark) in section 35.2. Only the files with the current filename extension and in the current directory are listed. 


\section{Chapter 36}

\section{Vector PostScript capture of DDLab output}

Graphic output in DDLab can be captured in vector PostScript graphics files, which can be scaled without degrading the resolution - this is preferable for publication quality images over bitmap graphics. Most figures in this manual are vector PostScript graphics, others are bitmap graphics ${ }^{1}$.

Vector PostScript files are available for the following DDLab graphic output, listed below with the default filenames. Instructions for creating the files are given within the manual in the relevant sections indicated.

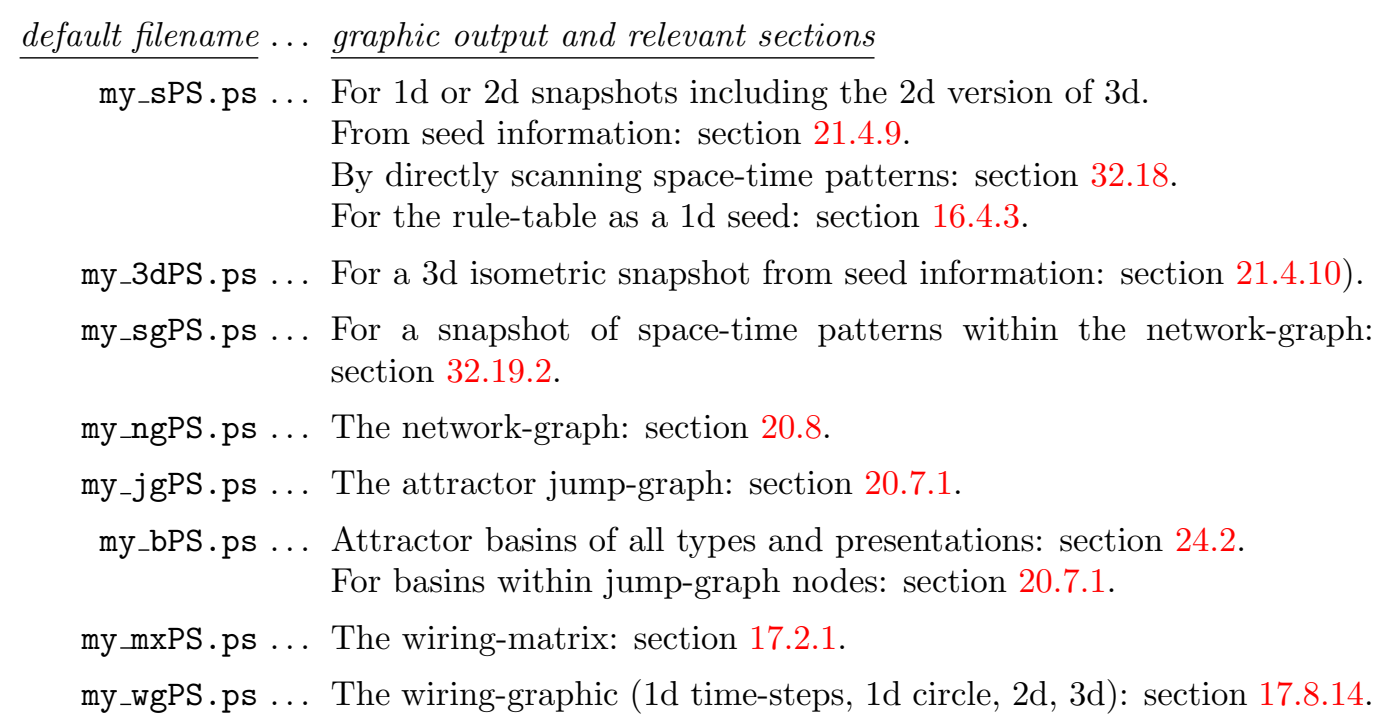

The vector PostScript methods work in both Linux-like systems and DOS. A top right prompt for saving to PostScript allows presentation alternatives. Then the top-right filing prompt (section 35.2) appears for naming the file with the extension .ps added automatically, with default filenames as listed above.

\footnotetext{
${ }^{1}$ Bitmap graphics are captured with the XV program (http://www.trilon.com/xv/).
} 
Functions in DDLab generate these files automatically according to the current presentation of the DDLab graphic, but also with various options for the PostScript image itself such as color or greyscale, and more options for the presentation of space-time patterns or snapshots, such as divisions between cells.

There is no limit on the size of a PostScript file, so for images with many components, lines, nodes etc. proceed with caution, especially for attractor basins, to avoid excessively large files in such cases a bitmap image might be preferable (section 2.14). Note that if an attractor basin is interrupted, the PostScript file of part of the graphic generated up to that point will still be valid.

The PostScript file cannot be viewed within DDLab - use GhostView.

\subsection{Cropping and editing vector PostScript}

Vector PostScript files are ASCII files, plain text files in the PostScript language, so can be easily edited, allowing the image to be cropped, images merged and annotations added. Instructions for the PostScript language can be found online ${ }^{2}$, so there is no need to delve further except to note how the image can be cropped, and the width of lines amended.

\subsubsection{Cropping the PostScript image}

The PostScript image can be cropped by editing the second line of the ASCII PostScript file to define a new BoundingBox with the bottom-left and top-right coordinates, for example,

\%\%BoundingBox: 2040924670

The required coordinates can be found by a readout of the cursor position when the file is opening in GhostView.

Another way of cropping is within the LaTex graphics command, for example,

\epsfig\{file=filename.ps, bb=81 85437 255, clip=, width=1 \linewidth $\}$

Where "bb=81 85437255 " is an example of the clip coordinates ( 0,0 is bottom-left in PostScript) and "clip=" forces LaTex to ignore anything outside the clip limits.

\subsubsection{Amending the width of lines}

The width of lines in various types of image generated by DDLab as vector PostScript (or converted to PDF) may look reasonable on the computer screen, but not in a high resolution printout, or vice versa. The defaults are a best compromise, but can be amended in the ASCII PostScript file by editing the value of "setlinewidth", usually in the fourth line of the file - presently either .7 or .3 , for the following file types according to the default filenames at the start of this chapter,

$$
\begin{aligned}
.7 \text { setlinewidth } \ldots & \text { my_ngPS.ps, my_jgPS.ps, my_bPS.ps, } \\
& \text { my_mxPS.ps, my_wgPS.ps (for links and outlines) } \\
.3 \text { setlinewidth } \ldots & \text { my_sPS.ps, my_3dPS.ps, my_sgPS.ps (for division lines and grid lines) }
\end{aligned}
$$

\footnotetext{
${ }^{2}$ For example, the PostScript Language Tutorial and Cook Book (http://www-cdf.fnal.gov/offline/PostScript/BLUEBOOK.PDF).
} 


\section{Chapter 37}

\section{Glossary}

Some of the labels, acronyms, and technical terms used in the manual, in alphabetical order.

\section{$\underline{\text { term }} \ldots \underline{\text { what it means }}$}

active cell ... the cell position that is highlighted in the wiring graphic (section 17.3).

attractor ... or attractor cycle, in a basin of attraction - made up of cycle of repeating states (chapter 23).

attractor basin ... collective term used for any of the following: basin of attraction field, single basin, tree or subtree (see also "state transition graph").

asynchronous updating ...

... where cells update in a predetermined or random sequence, or partial order, by the notion of time-step still applies.

basin of attraction ... the set of states that flow to an attractor, including the attractor itself (chapter 23).

basin of attraction field ...

... the basins of attraction comprising state-space (chapter 23).

block ... a block of cells in $1 \mathrm{~d}, 2 \mathrm{~d}$, or $3 \mathrm{~d}$, highlighted in the wiring graphic (section 17.3).

CA ... Cellular Automata: a local neighborhood of $k$ inputs (1d, 2d, 3d) and one rule (but possibly a mix of rules to extend the definition).

CA-wiring ... same as local wiring below.

cell index... (or network index) the way the network cells are numbered (sections 10.2.1 - 10.2.3), but cell index usually refers to a $1 \mathrm{~d}$ network, or a $1 \mathrm{~d}$ version of a $2 \mathrm{~d}$ or $3 \mathrm{~d}$ network.

DDN ... Discrete Dynamical Networks: as RBN, but allowing a value-range $v \geq 2$. Binary CA are a special case of RBN, and RBN and multi-value CA are special cases of DDN.

effective $k \ldots$ can be smalled than actual $k$ because of redundant wiring (section 18.7.3).

elementary rules ... 1d $v 2 k 3$ rcode make up the 256 elementary rules[22]. 
equivalence classes ... rules can be equivalent to each other by symmetry transformations, for example the 256 elementary rules collapse to 88 equivalence classes[25] (section 18.5). There will be more transformations for $v \geq 3$ or greater dimensions.

exhaustive algorithm ...

... finding pre-images by first creating a list of exhaustive pairs, each state in state-space and its successor. (section 29.7).

exhaustive pairs ... each state in state-space and its successor, for the exhaustive algorithm (section 29.7).

field ... as in basin of attraction field (chapter 23).

FIELD-mode ... to show the basin of attraction field, which does not require an initial state (the seed).

full rule-table ... rcode, where the rule-table (look-up table) lists the output for every possible neighborhood string.

garden-of-Eden state ...

... a state having no pre-images, also called a leaf state.

$G$-density ... the fraction of leaf states in a subtree, basin or field - a measure of convergence in network dynamics (section 24.9).

hex ... an abbreviation with two meanings according to the context: "hexagonal" referring to a 2d lattice or neiborhood, or "hexadecimal" referring to a numbering system for rules, as opposed to decimal.

homogeneous- $k$... same as $k$-hom.

history limit ... the number of time-steps recorded when checking for the attractor (section 29.3.1).

$i, j \ldots$ the size of a $2 \mathrm{~d}$ network, width $\times$ depth, or columns $\times$ rows.

$I, J \ldots$ the cell coordinates in a $2 \mathrm{~d} \mathrm{i}, \mathrm{j}$ network.

$i, j, h \ldots$ the size of a 3 d network, width $\times$ depth $\times$ height, or columns $\times$ rows $\times$ levels.

$I, J, H \ldots$ the cell coordinates in a $3 \mathrm{~d} \mathrm{i}, \mathrm{j}, \mathrm{h}$ network.

input-entropy ... the Shannon entropy of the input-frequency distribution in space-time patterns (section 31.4).

input-frequency ... the frequency of rule-table lookups, or neighborhood size blocks, in space-time patterns (section 31.4) usually presented as a histogram (e.g. figure 33.1).

jump graph ... a graphic representation, which can be manipulated, of the probability of jumping between basins of attraction (chapter 20).

$k \ldots$ the number of inputs to a cell, or size of the neighborhood.

$k$-hom ... homogeneous- $k$, where each cell in the network has the same number of inputs, or neighborhood size, $k$. Note that a homogeneous- $k$ network can be created as a $k$-mix network if required. 
$k_{\text {Lim }} \ldots$ the maximum size of $k$ (input wires to a cell) currently supported in DDLab, which depends on $v$ and is more generous if TFO-mode is active (section 7.2).

$k$-mix ... or mixed- $k$, where each cell in the network can have a different number of inputs, or neighborhood size, $k$.

kcode ... a k-totalistic rule, where the lookup-table lists the output for each combination of frequencies of values (colors) in the neighborhood, with $S=(v+k-1) ! /(k ! \times(v-1) !)$ entries.

$k_{\max } \ldots$ a deliberate selection of the maximum $k$ in a $k$-mix. Note that $k_{\max } \leq k_{\text {Lim }}$.

$\lambda$-parameter ... for $v=2$, the fraction of $1 \mathrm{~s}$ in the rcode-table. In general, the fraction of non-quiescent values[17] (section 24.9).

$\lambda_{\text {ratio }} \ldots$ a normalized version[25] of the $\lambda$-parameter to compare directly with the $Z$-parameter. For $v=2, \lambda_{\text {ratio }}=$ the total of the lesser value $\left(T_{L}\right)$ (1s or 0 s) divided by $S / 2$, in general, $\lambda_{\text {ratio }}=T_{L} /(S / v)$.

leaf state ... a state having no pre-images, also called a garden-of-Eden state.

local wiring ... or CA-wiring, where inputs may arrive from the local neighborhood as defined in chapter 10. Local wiring may be set up as nonlocal if required.

lookup-table ... a list of the outputs of possible neighborhoods, also known as the rule or rule-table (rcode, kcode or tcode) as defined in chapter 13.

$\max -k \ldots$ same as $k_{\max }$ above.

$\max -n$. . maximum network size that can be expressed in decimal (section 21.6).

max-S . . maximum rule-table size that can be expressed in decimal (section 16.6).

min-max ... a measure of variability, the maximum up-slope - the rise in entropy following a minimum value (section 33.1 and figure 33.2) - the alternative is sdev - standard deviation.

mixed- $k$... same as $k$-mix above.

$n \ldots$ the size of the network, or system size.

$n_{\text {Lim }} \ldots$ the maximum size of the network. In FIELD-mode $n_{\text {Lim }}$ varies according to the value-range $v$ (section 7.3). In SEED-mode or TFO-mode $n_{\text {Lim }}=65025$ (section 8.3).

$n_{\text {exhL }} \ldots$ the maximum size of the network for the exhaustive testing algorithm (section 29.7).

neighborhood ... the cells that are included in a given cell's update logic or transition rule. The "neighborhood" usually signifies a local CA type neighborhood of nearest (+ next nearest) neighbors in $1 \mathrm{~d}, 2 \mathrm{~d}$ or $3 \mathrm{~d}$ (pre-defined in chapter 10).

network dimensions ... 1d, 2d, or $3 \mathrm{~d}$, depending on the neighborhood (chapter 10).

network-graph ... a graphic representation of the network, which can be manipulated, showing all nodes and connections (chapter 20). 
network index ... (or cell index) the way the network cells are numbered (sections 10.2.1 10.2.3).

network size ... the mumber of cells or elements in the network, also refered to as "system size".

nonlocal wiring ... where inputs may arrive from from anywhere, but may also be biased. Local wiring may be set up as nonlocal.

outer-totalistic rules ... where a different totalistic rule (tcode or kcode) applies according to the value of the center cell, so $v$ rules. Applies to TFO-mode only, and not for mixed- $k$. (section 13.7).

partial order updating ...

... where a subset of cells updates synchronously, in parallel, followed by the next subset - then the "state" is the configuration after each updated subset (section 31.3.3).

pattern density ... the fractions of different values in space-time patterns (section 31.4). pseudo-neighborhood ...

... required for nonlocal wiring where inputs to a cell can arrive from anywhere. The nonlocal inputs are wired to a notional CA (local) neighborhood (in $1 \mathrm{~d}, 2 \mathrm{~d}$ or $3 \mathrm{~d}$ ), allowing the rule to be applied to this indexed pseudo-neighborhood (section 10.1.1).

pre-images ... a state's immediate predecessors.

$R$... the number of bytes required to encode a rule-table (section 16.16).

RBN ... Random Boolean Networks: random wiring of $k$ inputs (but possibly with mixed- $k$ ) and a mix of rules (but possibly just one rule).

rcode ... the full rule-table (lookup-table) listing the outputs for every neighborhood string, with $S=v^{k}$ entries.

\section{reaction-diffusion rules ...}

... or excitable-media[9], where cells may be either resting, excited, or refractory, and change according to thresholds and values, resulting in waves, spirals and related patterns.(sections 13.8, 13.8.2, and 14.2.1).

rule ... any kind or rule or rule-table, including rcode, kcode or tcode, as defined in chapter 13.

rulemix ... where each cell in the network can have a different rule (chapter 14).

rule-table ... a list of the outputs of possible neighborhoods, also known as the lookuptable (rcode, kcode or tcode) as defined in chapter 13.

rule scheme ... the actual rulemix (chapter 14).

$S \ldots$ the size of a rule-table (section 16.16).

sdev ... the standard deviation of the entropy - a measure of variability (section 33.1 and figure 33.2) - the alternative is min-max.

seed ... the initial state set in SEED-mode or TFO-mode (chapter 21.

SEED-mode ... when not in TFO-mode, to run forward for space-time patterns, or 
generate a single basin of attraction, or a subtree, which requires an initial state or seed.

sequential updating... where each cell is updated in turn in some arbitrary order - then the "state" is the configuration when all $n$ updates are complete (sections 29.9, 31.3.2).

space-time patterns ... the forward dynamics shown as a pattern for each successive timestep, which can be presented in many alternative formats described in chapters 31,32 .

state ... usually refers to the global state, the bit/value string, as opposed to the cell state or cell value/color.

state transition graph ...

... the graph representing an attractor basin, but the following terms are also used for the various types of state transition graph: basin of attraction field, single basin, tree or subtree (see also "attractor basin").

STP-dimensions ... the dimensions of the space-time pattern presentation, which are not necessarily the underlying network dimensions.

subtree ... part of a transient tree in a basin of attraction defined by its root (chapter 23).

synchronous updating ...

... where all cells in the network update simultaneously, in parallel.

system size ... the mumber of cells or elements in the network, also refered to as "network size".

tcode ... a t-totalistic rule, where the lookup-table lists of the outputs of all the possible totals when the values in the neighborhood are simply added, with $S=k(v-1)+1$ entries. (section 13.6.3).

TFO-mode ... stands for "totalistic forwards only" and constrains DDLab to run just forwards to generate space-time patterns (attractor basin functions are suppressed). TFO-mode allows greater value-range $v$ and neighborhood size $k$ (section 7.2), but the rules are limited to various types of totalistic rule (tcode and rcode), outer-totalistic rules, and reaction diffusion rules (not full rule-tables - rcode). TFO-mode requires an initial state or seed.

totalistic rules ... rules that depend on the totals of each value, in the neighborhood, not the pattern of values. There are two main types, k-totalistic defined by kcode and t-totalistic defined by tcode.

tree ... or transient tree in a basin of attraction, consists of all the states and merging links (trajectories) defined by the tree's root on the attractor (chapter 23).

trajectory ... the sequence of state in forward dynamics (chapter 23).

transient ... a trajectory leading to an attractor (chapter 23).

uniform state ... a global state (bit/value string) were all cells have the same value (color) (section 26.1.2). 
update-tag ... (from 0 to $n$-1) in sequential updating (section 29.9 way that cell indexes are listed from left to right and updated.

$v_{\text {bits }} \ldots$ the number of bits required to encode a rule-table output or cell value; 1 bit for $v=2,2$ bits for $v=3$ or 4, 3 bits for $v-5$ to 8 (section 16.16).

$v 2 k 3, v 3 k 7$ etc $\ldots$ the notation for showing the value-range and neighborhood size.

value-range, $v \ldots$ the number of possible cell value, or colors, or internal states, or the size of the "alphabet" (chapter 7, sections 21.9,16.16).

variability ... of the entropy, from "Classifying rule space" (chapter 33), either min-max or sdev.

wiring ... the connections between network elements or cells, either local or non-local. For CA the local wiring creates the network geometry, 1d, 2d or $3 \mathrm{~d}$.

wiring scheme ... the actual wiring in the network (chapter 12).

$Z$-parameter ... A static parameters from the rcode-table giving the probability that the next unknown cell in the CA reverse algorithm is determined[25, 32, 42]. High $Z$ predicts chaos, low $Z$ predicts order (section 24.9, figure 24.10). To tune $Z$ see sections 16.3 and 32.5.5. 


\section{References}

[1] Adamatzky,A, (Ed) (2002) "Collision-Based Computing", Springer, London. x

[2] (2000) Albert,R., H.Jeong, and A-L.Barabasi, (2000) "Error and attack tolerance in complex networks", Nature, vol 406. 78, 182

[3] Bilotta, E., A.Lafusa, and P.Pantano, (2003). "Is self-replication an embedded characteristic of the artificial/living matter?", in Artificial Life VIII, eds. Standish and Bedau, MIT Press, 38-48. 100

[4] Burraston,D., and A.Martin, (2006) "Digital Behaviors and Generative Music", Special Issue, Leonardo Electronic Almanac Vol 14, No. 7-8. http://leoalmanac.org/journal/Vol_14/lea_v14_n07-08/dburastonamartin.asp 2

[5] Byl,J., (1989) "Self-Reproduction in small cellular automata." Physica D, Vol. 34, pages 295299. 29

[6] Conway,J.H., (1982) "What is Life?" in "Winning ways for your mathematical plays", Berlekamp,E, J.H.Conway and R.Guy, Vol.2, chap.25, Academic Press, New York. 112, 145

[7] Das,R., M.Mitchell, and J.P.Crutchfield, (1994) "A Genetic Algorithm Discovers Particlebased Computation in Cellular Automata", In Y. Davidor, H.-P. Schwefel, and R. Mnner, eds., Parallel Problem Solving from Nature III, Springer-Verlag, 344-353. 261, 269, 371

[8] Derrida,B., G Weisbuch, (1986) "Evolution of overlaps between configurations in random Boolean networks", J.Pysique, 47, 1297. 246

[9] Greenberg,J.M., and S.P.Hastings, (1978) "Spatial patterns for discrete models of diffusion in excitable media", SIAM J. Appl. Math. 34. 11, 102, 108, 484

[10] Gutowitz,H.A., ed., (1991) "Cellular Automata, Theory and Experiment", MIT press.

[11] Harris,S.E., B.K.Sawhill, A.Wuensche, and S.Kauffman, (2002) "A Model of Transcriptional Regulatory Networks Based on Biases in the Observated Regulation Rules", COMPLEXITY, Vol.7/no.4, 23-40. x, 2, 14, 124, 136, 246, 271, 364, 371, 380

[12] Hopfield,J.J. (1982) "Neural networks and physical systems with emergent collective computational abilities", Proceedings of the National Academy of Sciences 79:2554-2558. 459

[13] Hordijk,W., J.P.Crutchfield, M.Mitchell, (1998) "Mechanisms of Emergent Computation in Cellular Automata", In A.E. Eiben, Th. Back, M. Schienauer, and H-P. Schwefel (eds.), Parallel Problem Solving from Nature, Springer-Verlag, 613-622. 188, 261, 269, 371 
[14] Kauffman,S.A.,(1969) "Metabolic Stability and Epigenesis in Randomly Constructed Genetic Nets", Journal of Theoretical Biology, 22, 437-467.

[15] Kauffman,S.A.,(1993) "The Origins of Order, Self-Organization and Selection in Evolution", Oxford University Press. 5

[16] Langton,C.G., (1986) "Studying Artificial Life with Cellular Automata", Physica D, 22, 120149. x, 2, 14, 246, 380

[17] Langton,C.G., (1990) "Computation at the Edge of Chaos: Phase Transitions and Emergent Computation", Physica D, 42, 12-37.

[18] Li,W., (1989) "Complex Patterns Generated by Next Nearest Neighbors Cellular Automata", Comput. \& Graphics Vol.13, No.4, 531-537. x, 136, 154, 270, 271, 423, 483

[19] Li,W., and N.H.Packard, (1990) "The Structure of the Elementary cellular automata rule space", Complex Systems, 4(3), 281-297. 418

[20] Somogyi,R., and C.Sniegoski, (1996) "Modeling the Complexity of Genetic Networks: Understanding Multigenetic and Pleiotropic Regulation", COMPLEXITY, Vol.1/No.6, 45-63. 389, 397

[21] Shmulevich,I., H.Lhdesmki, E.R.Dougherty, J.Astola, W.Zhang, (2003) "The role of certain Post classes in Boolean network models of genetic networks", Proceedings of the National Academy of Sciences of the USA, Vol. 100, No. 19, 10734-10739. x, 2, 380

[22] Wolfram,S., (1983) "Statistical Mechanics of Cellular Automata", Reviews of Modern Phisics, vol $55,601-644.122$

[23] Wolfram,S., ed. (1986) "Theory and Application of Cellular Automata", World Scientific. 1, $102,141,188,189,481$

[24] Wolfram,S., (2002) "A New Kind of Science", Wolfram Media, Champaign, IL. x

[25] Wuensche,A., and M.J.Lesser. (1992) "The Global Dynamics of Cellular Automata; An Atlas of Basin of Attraction Fields of One-Dimensional Cellular Automata", Santa Fe Institute Studies in the Sciences of Complexity, Addison-Wesley, Reading, MA. x

[26] Wuensche,A., (1994) "The Ghost in the Machine; Basin of Attraction Fields of Random Boolean Networks", in Artificial Life III, ed C.G.Langton, Santa Fe Institute Studies in the Sciences of Complexity, Addison-Wesley, Reading, MA. x, xi, 2, 6, 8, 18, 21, 23, 34, 68, 136, 141, 154, 186, 189, 190, 256, 270, 275, 276, 288, 290, 327, 397, 423, 482, 483, 486

[27] Wuensche,A., (1994) "Complexity in One-D Cellular Automata; Gliders, Basins of Attraction and the Z Parameter", Santa Fe Institute Working Paper 94-04-025. 7, 21, 23, 24, 327, 329, $459,461,468,469$

[28] Wuensche,A., (1996) "The Emergence of Memory: Categorisation Far From Equilibrium", in Towards a Science of Consciousness: The First Tuscon Discussions and Debates, eds. Hameroff SR, Kaszniak AW and Scott AC, MIT Press, Cambridge, MA, 383-392. x, 2, 7, 271, 361, 398, 459

[29] Wuensche,A., (1997) "Attractor Basins of Discrete Networks; Implications on self-organisation and memory", Cognitive Science Research Paper 461, Univ. of Sussex, D.Phil thesis. x, 2 
[30] Wuensche,A., (1998) "Genomic Regulation Modeled as a Network with Basins of Attraction", Proceedings of the 1998 Pacific Symposium on Biocomputing, World Scientific, Singapore. 329, 333, 334, 335, 371, 380, 437

[31] Wuensche,A., (1998) "Discrete Dynamical Networks and their Attractor Basins", Proceedings of Complex Systems '98, University of New South Wales, Sydney, Australia. x, 2, 124, 364, 380

[32] Wuensche,A., (1999) "Classifying Cellular Automata Automatically; Finding gliders, filtering, and relating space-time patterns, attractor basins, and the $Z$ parameter", COMPLEXITY, Vol.4/no.3, 47-66. 327

[33] Wuensche,A., (2000) "Basins of Attraction in Cellular Automata; Order-Complexity-Chaos in Small Universes", COMPLEXITY, Vol.5/no.6, 19-25. (based on an art exhibition in collaboration with Chris Langton) x, 2, 7, 8, 18, 23, 28, 70, 71, 136, 270, 272, 361, 398, 399, 419, $423,437,486$

[34] Wuensche,A., (2001) "The DDLab Manual" (for ddlabx24), http://www.ddlab.org. 2

[35] Wuensche,A., (2002) "Basins of Attraction in Network Dynamics: A Conceptual Framework for Biomolecular Networks", in "Modularity in Development and Evolution", eds G.Schlosser and G.P.Wagner. Chicago University Press 2004, chapter 13, 288-311. x, xi

[36] Wuensche.A.,(2002), "Finding Gliders in Cellular Automata", in "Collision-Based Computing", ed. A.Adamatzky. Springer, London. 380

[37] Wuensche,A., (2003) "Discrete Dynamics Lab: Tools for investigating cellular automata and discrete dynamical networks". Kybernetes 32, 77-104.

[38] Wuensche,A., (2004), "Self-reproduction by glider collisions: the beehive rule", Alife9 Proceedings, eds J.Pollack et al., pages 286-291. MIT Press. xi

[39] Wuensche,A., (2005) "Glider Dynamics in 3-Value Hexagonal Cellular Automata: The Beehive Rule", Int. Journ. of Unconventional Computing, Vol.1, No.4, 2005, 375-398. 437

[40] Wuensche,A., A.Adamatzky, (2006), "On spiral glider-guns in hexagonal cellular automata: activator-inhibitor paradigm", International Journal of Modern Physics C,Vol. 17, No. 7,10091026. xi, 28, 84, 106, 107, 361, 437

[41] Wuensche,A., (2009), "Cellular Automata Encryption: The Reverse Algorithm, Z-Parameter and Chain-Rules", Parallel Processing Letters (PPL), Vol 19, No 2, 283-297. xi, 2, 28, 84, 107, 116, 201, 414

[42] Wuensche,A., (2009), "Discrete Dynamics Lab: Tools for investigating cellular automata and discrete dynamical networks", "Artificial Life Models in Software, 2nd Ed.", eds. M.Komosinski and A.Adamatzky, chapter 8, 215-258, Springer. 147, 271, 314

[43] Wuensche,A., (2010), "Complex and Chaotic Dynamics, Basins of Attraction, and Memory in Discrete Networks", ACTA PYSICA POLONICA B, Vol 3, No 2, 463-478.

$270,397,486$ 


\section{Index}

1d neighborhood, 83

1d space-time patterns

ring of cells, scrolling, 39

1d-2d-3d space-time patterns, 408-409

2 d neighborhood, 83

2 d neighborhood(, 83

2d+time space-time patterns, 409

3d neighborhood, 85

acronym glossary, 5

active cell, 160

adjacency-matrix, 224-226

Altenberg, Lee, 145

asynchronous updating, 358-359

attractor basins, 5, 14

basin of attraction field, 32, 34

changes on-the-fly, 343-350

changing parameters, 32

computation, 70

data, 299-313

display of, 288-298

drawing, 343-350

equivalent CA dynamics, 288-291

errors, 302, 345, 346

fan angle, 296, 297

final options, 322-342

graphic conventions, 252-257

highlighting, 461

idea of, 252, 253

in-degree frequency, 264-268

layout, 281-287

layout in jump-graph, 219

mutation, 314-321

node display, 292-296

on-the-fly options, 280, 346

orientation, 296, 297

output parameters, 258-280

pausing, 299-313 range of sizes, 34

revising the seed, 349

single, 35, 322-324

speed, 261, 280, 345, 348

subtree, 322

attractor cycles, 255

attractor histogram, 371-379

data, 376

density classification problem, 377

fuzzy attractors, 380

jump-graph, 379

pausing, 375

print/save, 377

rescaling, 375

sorting, 379

attractor jump-graph, 204-226, 379

defining a block, 217

jump-table, 224-226

probabilistic "ant", 217-219

attractor states

print/save, 377

backtracking in DDLab, 59

backward space-time patterns, 33, 278-279

scrolling, 278

basin of attraction field, 32, 34

in jump-graph PostScript, 221

layout in jump-graph, 219

network too big for, 90

or initial state, 9

or seed, 61,62

biased random wiring, 94-97

confine to zone, 94

distinct, 96

links to $3 d$ layers, 96-97

local treated as random, 95

release wires from zone, 95

same random everywhere, 97 
self-wiring, 96

suppress periodic boundary conditions, 96

bitmap graphics, 17, 479

block, 160

density, 231-232

Boolean networks, 1

Byl, J., 29

CA, see cellular automata

canalyzing, 117, 124-130, 349, 425

$\lambda$ and $P$ data, $277-278$

Derrida plot, 125, 250

frequency, 127-130

homogeneous $k, 126$

mixed $k, 127-129$

single rcode, 191

single rule, 191

cell color

by value or neighborhood, 353

cell scale, 356

cellular automata, 1, 5

chain-rules, 117, 147

classifying rule space, 399-401, 426, 437-458

sample file encoding, 458

sample plot data, 452

color

basin edges, 298

nodes, 294

command line arguments, 54

complement rcode, 189

complementary values, 154

complex rules, 357

compression of equivalent CA dynamics, 288291

suppress, 289, 291

computational limitations

attractor basins, 70

convert coordinates

$1 \mathrm{~d}-3 \mathrm{~d}, 86,87$

Conway, John, 112, 145

copying a rule to part of the network, 183

copyright, 29

damage between two networks, 364

histogram, 366-370

Das, Raja, 269, 372

DDLab accessing, 25-29

at SourceForge, 25

backtracking, 59

copyright, 29

latest versions, 25

license, 29

manual, 25

previous versions, 29

prompts, 59

quitting, 31

registration, 29

skipping forward, 31

source code, 25

UNREGISTERED banner, 29, 55

web sites, 25

DDN, see discrete dynamical networks

deleting a cell, 171

density classification problem, 269, 371 attractor histogram, 377

density of seed or block, 231-232

density-bias, 111, 134

Derrida plot, 163, 246-251

canalyzing, 125, 250

coefficient, 250

options, 247

parameters, 248

directory, changing, 477

discrete dynamical networks, 2, 3, 5, 49

measures, 8

parameters, 4,7

DOS, 2, 27, 53, 54

memory window, 58

resolution, 63

virtual memory, 59

DOSBox, 27, 53

dragging nodes or fragments

network-graph or jump-graph, 212-217

duplicate the network and seed, 365

edge color, 298

effective $k, 192$

effective $k=0,76,114,192$

emergent computation, 269

encryption, 147

entropy

of space-time patterns, 361, 419

single cell, 363 
entropy/density plot, 420

equivalence classes, 189

equivalent CA dynamics, 288-291

equivalent rules with greater $k, 191$

errors in attractor basins, 302, 345, 346

excitable media, 108

exhaustive map, 192

exhaustive reverse algorithm, 23, 329-335

exit DDLab, 60

fan angle in attractor basins, 297

FIELD-mode, 9, 61, 62

network size limits, 67

file encoding

$k$-mix, 196

rule sample, 458

seed, 245

single rule, 149

wiring/rulemix, 195

filing, 16, 184, 194-202, 473-478

$k$-mix, 76,81

attractor basin data, 306

data, 17

directory, 477

graph layout, 222

list files, 478

loading $k$-mix networks, 201

network architecture, 194-202

network parameters, 17

networks and sub-networks, 196

prompt for saving and loading, 476

random map, 334

screen image, 17,55

seed, 237-241

value-range constraints, 477

wiring/rulemix, 195

filtering space-time patterns, 192, 410, 414-416, 428

first prompt, $61-65$

flashing cursor speed, 65

font size/weight, 64-65

forgetting, 459

forwards only

totalistic, 7

forwards-only

constraining to, 61,62

TFO-mode, 9
Fredkin, E., 146

frozen generation size, 355

frozen regions in space-time patterns, 410-414

frequency bins, 413-414

generations, 413

full rule-table

binary, 102

multi-value, 104

functions summary, 9-24

fuzzy attractors, 21, 380

G-density

against $Z$ or $\lambda_{\text {ratio }}, 273$

against network size, 272

$G$-density, 270

game-of-Life, 42, 145

outer-kcode, 108, 112-114

seeds, 28

generation size

frozen, 355

input-entropy, 363

pattern density, 363

geometry of network, 85-87, 91

glider rules, 357, 398

glossary of acronyms, 5

glossary of technical terms, 481-486

graph layout PostScript, 222

graphics, 16

bitmap, 17

vector PostScript, 17, 479-480

graphics setup, 63, 430

DOS, 31

Linux-like systems, 31

Windows command line, 31

Hamming distance, 246, 248, 427

hexagonal neighborhood 2d, 82, 83

highlighting, 459

states in attractor basins, 461

histograms

$2 \mathrm{~d}$ rule sample, 451

attractor, 371-379

canalyzing frequency, $127-130$

cell wiring out-degree, 158, 164

damage, 366-370

in-degree, 264-268

partial pre-image stack, 326-329 
skeleton, 380-385

wiring input, 184-185

wiring output, $184-185$

history limit, 323

Hopfield, J., 459

hypercube wiring, 92

in-degree frequency histogram, 264-268

attractor basins rescaling, 268

cut-off, 264

data and prompts, 267

log plot, 268

indexing

1d networks, 86

2d networks, 86

3d networks, 87

initial choices, 9

initial state, see seed

input-entropy, 361-419

generation size, 363

on-the-fly options, 363

single cell, 363

input-frequency, 361

inverse problem, 192

invert the kcode, 357

invert the rcode, 188

isotropic rules, 117, 133, 389, 395, 396, 442

jump-graph, 204-226, 379

activate, 207, 260

basins at nodes, 219

dragging nodes or fragments, 212-217

jump-table, 225

PostScript, 222

reminder, 208

jump-table, 224-226

$k$, see neighborhood

$k$-matrix, 121

$k$-mix, 10, 75-81, 90

at random, 79

changing the size, 182

copy rules automatically, 119

effective $k, 192$

effective $k=0,76,114,192$

file encoding, 196

filing, 76, 81, 201-202

greater $k, 191$ increasing $k_{\max }, 80$

$k_{\text {Lim }}, 75$

$\max -k, 192$

normal distribution, 77

percentage of different $k \mathrm{~s}, 78$

power-law distribution, 77-79

reviewing, 80-81

within network architecture, 81

saving, 202

setting by hand, 77

showing the distribution, 78

specifying, 76, 77

transform, 187

where all $k$ 's are the same, 119

with uniform $k, 79$

$k=0,76,114,192$

$k_{\text {Lim }}, 7,9-11,67,106,107,163$

$k_{\max }, 80$

$\mathrm{k}$-totalistic rules, 106

Kauffman, Stuart, 5, 14

kcode, 104, 106

matrix, 107

mult-value, 105

outer-totalistic, 108

swapping values, 152

with increased $k, 149$

kill a cell, 183

Lafusa, Antonio, 100

$\lambda$-parameter, 111, 134, 270-277

weighted average, 180

Langton, Chris, 29, 271

layout of attractor basins, 281-287

pause, 286-287

preview, 281

learning and forgetting, 181, 459-472

aspiring pre-images, 464-466

parity, 465

highlighting only, 461

target state, 463

learning, forgetting, and highlighting, 24

Lesser, Mike, xi

Li, Wentian, 418

Liapunov exponent, 21, 250

license, 2, 29

Life-like rules, 112-114

limit backward steps, 325 
line spacing, 64-65

DOS, 65

Linux-like operating systems, 2, 52, 63

Linux-like versions, 26

list files, 478

loading, see filing

screen image

DDLab format, 56

loading sub-networks, 199

local CA neighborhood, 82-87

$1 \mathrm{~d}, 83$

$2 \mathrm{~d}, 83$

$2 \mathrm{~d}$ hexagonal and square, 82

$3 \mathrm{~d}, 85$

lookup-table, 102-107

majority rules, 116, 120, 133, 142-144 shifted, 144

matrix

kcode, 107

wiring, 158-160

$\max -k, 80,201$

reducing to max network- $k, 192$

measures

on global dynamics, 21

on local dynamics, 19

memory window DOS, 58

$\min -\max , 440$

minimum $k, 75$

Moore neighborhood, 11, 84, 145

multi-value

1d space-time patterns, 41

neighborhood matrix, 121

mutation, 18, 314-321

of attractor basins, 314-321

on one screen, 283,318

space-time patterns

current state, 403-404

rules, 394-397

wiring, 402

$n_{\text {Lim }}, 67$

negative equivalent rcode, 189

neigborhood

file loading constraints, 477

neighborhood, 10, 75-87, 90

$k$-mix, 76, 90 $k_{\text {Lim }}, 10$

$1 \mathrm{~d}, 75,83$

$2 \mathrm{~d}, 83$

cubic, 11

$2 \mathrm{~d}$ hexagonal or square, 82

$3 \mathrm{~d}, 85$

changing $k, 182$

effective $k=0,76,114,192$

greater $k, 191$

$k$-mix, 10, 75-81

$k_{\text {Lim }}, 75$

$k_{\max }, 67$

periodic boundary, 4

pseudo, 4, 82

neighborhood matrix, 121

binary, 103

multi-value, 105

network

$1 \mathrm{~d}$ indexing, 86

$2 \mathrm{~d}$ indexing, 86

3d indexing, 87

network architecture, 156-185, 349, 425, 430

filing, 194-202

network-graph, 206

sub-networks, 196

network geometry, 85-87, 91

network size, 10

1d, 68-74, 93

2d and $3 \mathrm{~d}, 89,93$

limits, 7, 70, 323

too big for a basin field, 90

network-graph, 204-226

1d, 2d, 3d, 85-87

adjacency-matrix, 224-226

defining a block, 217

dragging nodes or fragments, 212-217

PostScript, 222

probabilistic "ant", 217-219

reminder, 206

space-time patterns, 433-436

neutral order

components, 24, 339

field, 341

subtree, 340

node

colors, 294

display in attractor basins, 253, 292-296 
noisy updating, 42, 358-359

nonlocal wiring, 82, 91-99

reverse algorithm, 23

on-the-fly changes

attractor basins, 280, 343-350

options summary, 346-347, 386

bottom title bar, 388

classifying rule space, 437-458

space-time patterns, 386-436

current state, 403-404

options summary, 393

rules, 394-397

wiring, 402

orientation of attractor basins, 297

outer-kcode

game-of-Life, 108, 112-114

reaction-diffusion, 112

outer-totalistic kcode or tcode, 112-114

output parameters

attractor basins, 258-280

space-time patterns, 351-385

partial order updating, 23, 361

partial pre-image histogram, 347

partial pre-image stack, 326

local wiring, 327

nonlocal wiring, 327-329

pattern density, 361, 419

generation size, 363

on-the-fly options, 363

single cell, 363

pause options

layout of attractor basins, 286-287

space-time patterns, 424-436

periodic boundary conditions, 82,85

suppress, 96

platforms for DDLab, 2

Post functions, 117, 122-123, 314

PostScript

amending linewidth, 480

cropping, 480

editing, 480

graph layout, 222

graph snapshot, 428

jump-graph, 222

jump-graph basins, 221 network-graph, 222

network-graph space-time pattern, 436

rule-table, 139-140

seed, 234, 237-241

space-time patterns, 426, 431

wiring graphic, 185

wiring matrix, 159

PostScript files, 17, 479-480

power-law distribution

of $k, 78-79,181$

of outputs, 181

pre-images

backwards space-time patterns, 278-279

early exit in pre-image fan, 344

exhaustive pairs, 330

in attractor basins, 252-253

in-degree frequency, 264-268

learning and forgetting, 181, 459-472

list of states, 311

partial pre-image stack, 327-328

reverse algorithms, 21-23

uniform states, 290

printing

data, see terminal

screen image, 17, 55-57

in DOS, 57

Linux-like systems, $56-57$

$\mathrm{XV}, 57$

probabilistic

"ant" in network or jump graph, 217-219

space-time patterns, 42, 358

updating, 358-359

prompts in DDLab, 59

pseudo-neighborhood, 4, 82, 173, 177

quick start examples, 30-51

random Boolean networks, 1, 5, 49

random map, 2, 23, 332-335

data, 335

saving/loading, 334

random number seed, 65

random wiring, 82, 91-99

range- $n, 1 \mathrm{~d}, 68$

$\mathrm{RBN}$, see random Boolean networks rcode

binary, 102 
multi-value, 104

reaction-diffusion, 148

reaction-diffusion, 102, 108-109

outer-kcode, 109, 112

rcode, 109, 148

threshold interval, 109

recursive

inputs, 168

outputs, 168

reflection equivalent of rcode, 189

registration, 29

return map, 354, 420

reverse algorithms, 21-23

1d local wiring, 23

exhaustive, 329-335

nonlocal wiring, 23

reorder to optimize, 329

partial pre-image stack

local wiring, 327

nonlocal wiring, 327-329

reverse engineering, 192

ring of $1 \mathrm{~d}$ cells, 39, 406, 434

rule scheme, $4,110-123$

filing, 194-202

rule-table, 102-107

rulemix, 101, 110-123

by hand, 117

canalyzing, 124

complete rules automatically, 119

copy rules automatically, 119

for large networks, or large k, 120

just one rule, 116

methods for setting, 114-115

random, 116

rules, 11, 131-155

Altenberg, 41, 42, 117, 133, 144, 145, 371

bias when classifying, 442

canalyzing, 117

chain, 117, 147

clusters, 189

combinations, 101

density-bias, 111, 134

encoding, 149

filing a single rule, 148

filing transformed rule, 188

game-of-Life, 112-114, 141, 145

in hex, 141 isotropic, 117

$\lambda$-parameter, 111, 134

majority, 116, 120, 142-144

outer-totalistic, 101, 108

PostScript, 139-140

reaction-diffusion, 102, 108-109

repeating last, 148

revising and copying, 183

set as bits or values, 136

show in terminal, 150

single, 110, 131-155

totalistic, 104

transform, 155, 183, 186-193

types, 100-109

value-bias, 135

sample file encoding

rule sample, 458

saving, see filing

attractor basin data, 306

attractor states, 377

rule sample plot data, 452

screen image, 55-57

DDLab format, 56

Linux-like systems, 56-57

$\mathrm{XV}, 57$

scale-free networks, 78, 182

scatter plot, 448

color scheme, 448

screen image

file, $55-57$

screen-saver demo, 48, 270

scrolling

network-graph, 408

ring of $1 \mathrm{~d}$ cells, 39, 406

space-time patterns, 421-422

sculpting attractor basins, 24, 459-472

sdev, 440

seed, 12, 227-245

as bits or values, $233-241$

complement, 154

density, 231-232

file encoding, 245

filing, 237-241, 243, 245

in decimal, 243

in hex, 242

loading constraints, 244 
or basin of attraction field, 9, 61, 62

PostScript, 234, 237-241

random, 230-232

value-bias, 230, 231

SEED-mode, 9, 61, 62

sequential updating, 23, 335-342

attractor basin, 338

list all orders, 337-338

neutral order

components, 339

field, 341

subtree, 340

space-time patterns, 359, 394

single basin

history limit, 323

of attraction, 323-324

single rule, 131-155

file,revise,transform, 349, 425

size

limits on $v$ and $k, 67$

of font and line spacing, 64

of frozen generations, 355

of the DDLab screen, 16, 63

of the network, 10

$2 \mathrm{~d}$ and $3 \mathrm{~d}, 89$

limits, 7, 67, 70

limits for exhaustive algorithm, 331

limits for FIELD-mode, 67

limits for seed in decimal, 243

of the rule-table, 102-107

of trailing time-steps, 363

range of $1 d, 68$

skeleton histogram, 380-385

data, 383

parameters, 380

pausing, 382

sorting, 385

skip time-steps, 429

solidify the rule, 189

source code for DDLab, 2, 25

space-time patterns, $5,13,37-48$

1d PostScript, 431

1d multi-value, 41

1d scrolling, 356

1d-2d-3d, 408-409

2d+time, 409

analysis, 416 asynchronous updating, 358-359

attractor histogram, 371-379

backwards, 278-279

changes on-the-fly, 386-436

options summary, 386-393

classifying rule space, 399-401, 437-458

damage, 364

damage histogram, 366-370

density/entropy plot, 420

dimensions, 356

drawing, 386-436

duplicate the network and seed, 365

filtering, 192, 410, 414-416, 428

fix border, 428

frozen regions, 355, 410-414

frequency bins, 413-414

generations, 413

Hamming distance, 427

input-entropy, 361-419

generation size, 363

single cell, 363

network-graph layout, 427, 433-436

noisy updating, 42, 358-359

output parameters, 351-385

partial order updating, 361

pattern density, 361, 419

pause options, 424-436

PostScript, 426

probabilistic updating, 358

return map, 354, 420

scrolling, 421-422

sequential updating, 359, 394

skeleton histogram, 380-385

skip time-steps, 407, 429

sound, 408

speed, 422

state-space matrix, 354, 420

time-step pause, 356, 422

speed

attractor basins, 261, 280, 345, 348

limitations, 70

space-time patterns, 422

square neighborhood 2d, 82, 83

starting DDLab, 52-60

state, see seed

state-space matrix, 33, 261-264, 354, 420

sub-networks, 12 
loading in set position, 199

subtree, 36, 257, 322

forward before backwards, 323

subtree=basin, 304, 305

suppress equivalents, 289

trees and subtrees, 291

swapping kcode values, 152

t-totalistic rules, 107

tcode, 104, 107

mult-value, 105

outer-totalistic, 108

terminal, 52

exhaustive pairs, 331

attractor basin data, 306

attractor states, 377

jump-table or adjacency-matrix, 225

network data, 202-203

rule data, 150-152

TFO-mode, 9, 10, 41, 61, 62

time-steps

skip, 429

totalistic rules, 104, 105

constraining to run forwards-only, 61, 62

TFO-mode, 9

transform rcode, 155, 183, 186-193

canalyzing, 191

complement, 154, 189

effective $k, 192$

exhaustive map, 192

greater $k, 191$

invert, 188

$\max -k, 192$

negative equivalent, 189

reflection equivalent, 189

solidify, 189

transient trees, 255

uniform states, 256

type of rule, 100

uniform states, 288, 289

pre-images, 290

transient trees, 256

UNREGISTERED banner, 29

untangle wiring, 171

updating

noisy, 42 partial order, 23, 361

probabilistic, 358-359

sequential, 23, 359

value-bias, 135, 230, 231

value-range, $4,10,66-67$

file loading constraints, 477

limits on $k, 67$

limits on $n, 67$

prompt, 66

vector graphics, 17, 479-480

von Neuman neighborhood, 11, 84

wiring, 11, 91-99

1d, 2d and 3d, 93

by hand, $97-98$

graphic, 160

1d, 164-171

2d, $172-176$

3d, 175-179

reminder, 161-163

hypercube, 92

input frequency histogram, 184-185

local, 93

local treated as random, 93

nonlocal, 82, 91-99

output frequency histogram, 184-185

periodic boundary conditions, 82,85

quick settings, $88-90$

random, 82, 94-97

biased, see biased random wiring

reviewing, 98-99

untangle, 171

wiring matrix, 158-160

wiring scheme, 4, 91-99

filing, 194-202

loading, 88

wiring/rulemix: file encoding, 195-196

Wojtowicz,Mirek, 146

Wolfram, S., 102, 188

xterm, see terminal

$\mathrm{XV}, 57$

$Z$-parameter, 134, 270-277, 397, 425

weighted average, 180 